\title{
Next generation turbine system study
}

\section{Phase I program}

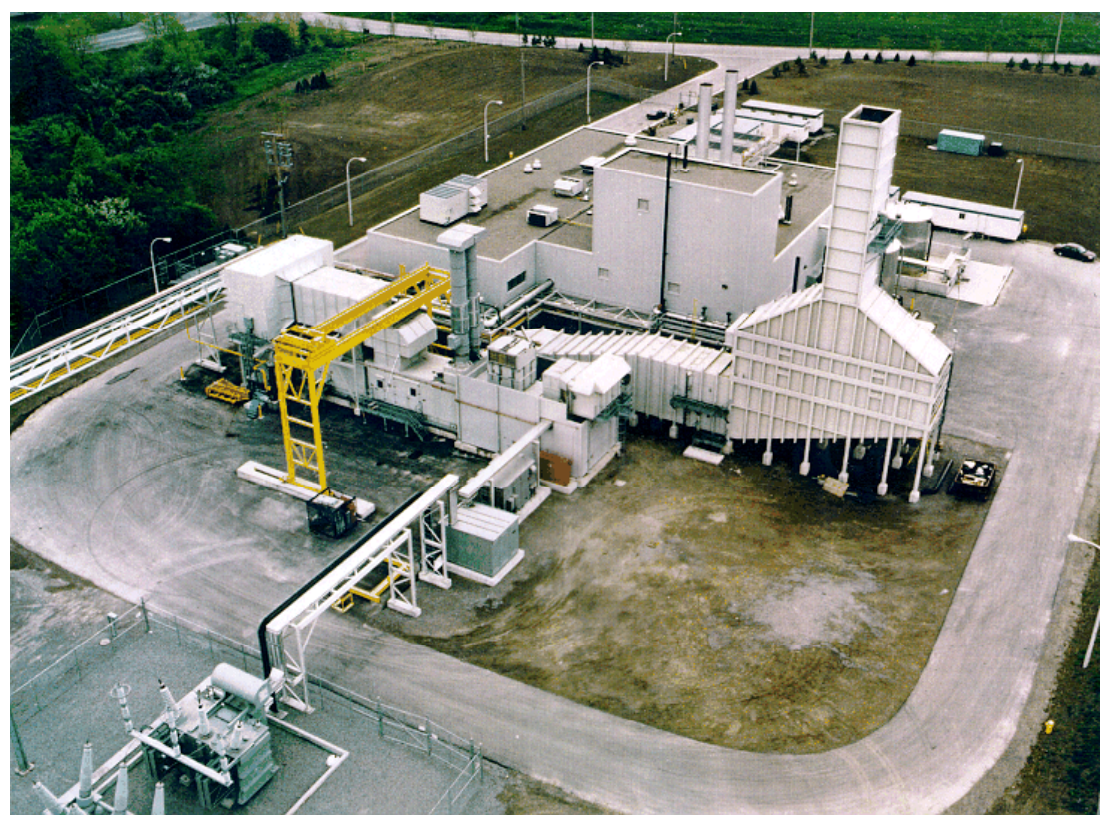

\section{Final technical report}

\section{For the period January 2001} to December 2001

\section{Issued:}

February 2002

Work performed under contract:

DE-AC26-00NT40852

Submitted by:

Rolls-Royce Corporation

P.O. Box 420

Indianapolis, IN 46206-0420

Program manager:

Frank Macri

Phone: 317-230-3281

Fax: 317-230-3542

Submitted to:

U.S. Department of Energy

National Energy Technology Laboratory Morgantown, WV 26507-0880

EDR 19776 


\title{
Next generation turbine system study
}

\section{Phase I program}

\author{
Final technical report
}

\section{For the period January 2001 to December 2001}

Issued:

February 2002

Work performed under contract:

DE-AC26-00NT40852

Submitted by:

Rolls-Royce Corporation

P.O. Box 420

Indianapolis, IN 46206-0420

Program manager:

Frank Macri

Phone: 317-230-3281

Fax: $317-230-3542$

Submitted to:

U.S. Department of Energy

National Energy Technology Laboratory

Morgantown, WV 26507-0880

\section{EDR 19776}

\section{DISCLAIMER}

This report was prepared as an account of work sponsored by an agency of the United States Government. Neither the United States Government nor any agency thereof, nor any of their employees, makes any warranty, express or implied, or assumes any legal liability or responsibility for the accuracy, completeness, or usefulness of any information, apparatus, product, or process disclosed, or represents that its use would not infringe privately owned rights. Reference herein to any specific commercial product, process, or service by trade name, trademark, manufacturer, or otherwise does not necessarily constitute or imply its endorsement, recommendation, or favoring by the United States Government or any agency thereof. The views and opinions of authors expressed herein do not necessarily state or reflect those of the United States Government or any agency thereof. 


\section{Abstract}

Rolls-Royce has completed a preliminary design and marketing study under a Department of Energy (DOE) cost shared contract (DE-AC26-00NT40852) to analyze the feasibility of developing a clean, high efficiency, and flexible Next Generation Turbine (NGT) system to meet the power generation market needs of the year 2007 and beyond.

Rolls-Royce evaluated the full range of its most advanced commercial aerospace and aeroderivative engines alongside the special technologies necessary to achieve the aggressive efficiency, performance, emissions, economic, and flexibility targets desired by the DOE. Heavy emphasis was placed on evaluating the technical risks and the economic viability of various concept and technology options available. This was necessary to ensure the resulting advanced NGT system would provide extensive public benefits and significant customer benefits without introducing unacceptable levels of technical and operational risk that would impair the market acceptance of the resulting product. Two advanced cycle configurations were identified as offering significant advantages over current combined cycle products available in the market. In addition, balance of plant (BOP) technologies, as well as capabilities to improve the reliability, availability, and maintainability (RAM) of industrial gas turbine engines, have been identified. A customer focused survey and economic analysis of a proposed Rolls-Royce NGT product configuration was also accomplished as a part of this research study.

The proposed Rolls-Royce NGT solution could offer customers clean, flexible power generation systems with very high efficiencies, similar to combined cycle plants, but at a much lower specific cost, similar to those of simple cycle plants. 


\section{Table of Contents}

Abstract

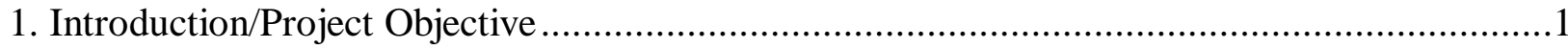

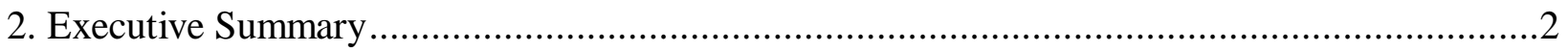

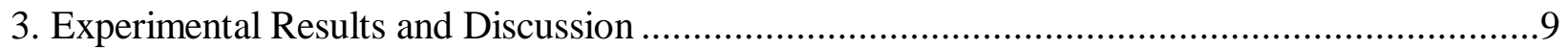

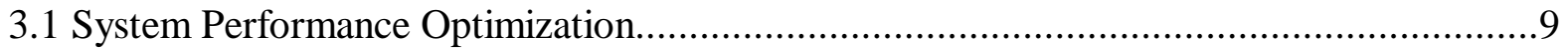

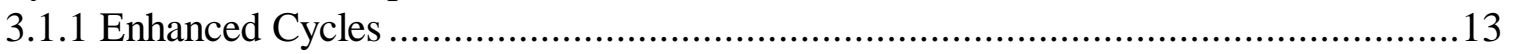

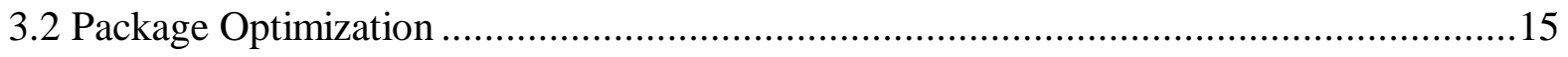

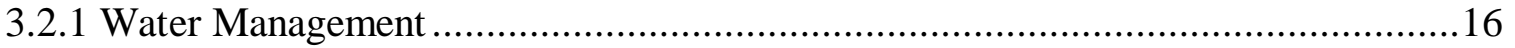

3.2.2 Once Through Steam Generator ................................................................... 17

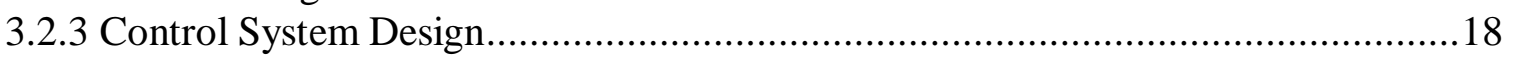

3.3 NGT Marketing/Economic Analysis .............................................................. 19

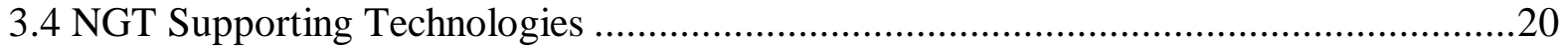

3.4.1 Combustion Emissions Considerations.......................................................20

3.4.2 Materials Requirements for High Steam Content Application.............................21

3.4.3 Reliability and Maintainability (RAM) Optimization Studies and More Electric

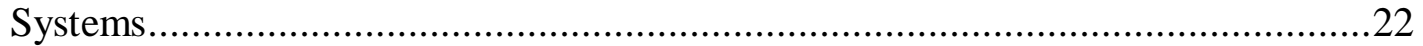

3.4.4 Advanced Rolls-Royce Recuperator Technology ........................................23

3.4.5 Advanced Controls Sensors and Diagnostics .............................................28

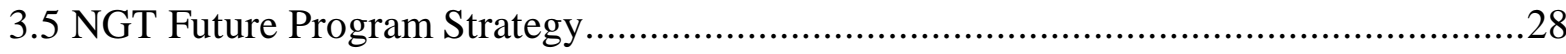

3.6 Phase I Program Final Technical Briefing ........................................................29

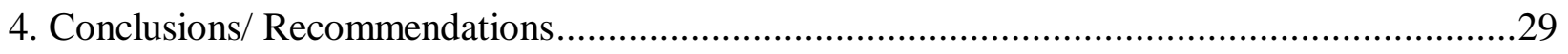

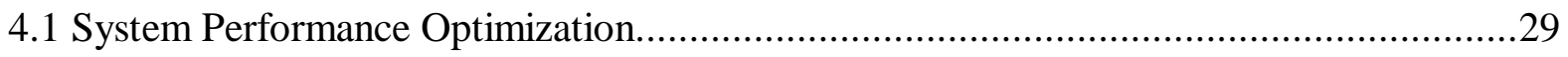

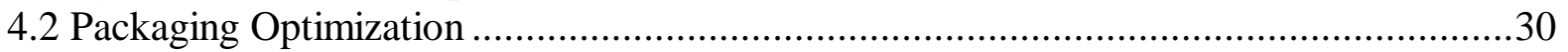

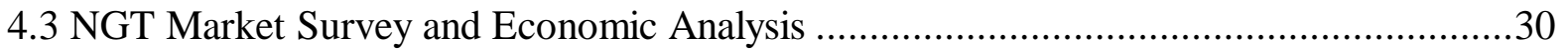

4.4 NGT Supporting Technologies ..................................................................... 31

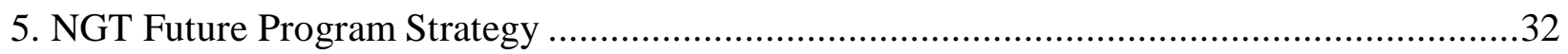

Appendix A. System Performance Optimization: Cycle Analysis/Turbomachinery Selection Appendix B. Packaging Optimization: Next Generation Turbine System Controls Appendix C. Packaging Optimization

Appendix D. NGT Market/Economic Analysis: PB Power Report Appendix E. NGT Market/Economic Analysis: DFM Consulting Report Appendix F. NGT Market/Economic Analysis: SRC Report Appendix G. NGT Supporting Technologies: RAM Technologies/More Electric Studies Appendix H. NGT Supporting Technologies: More Electric Systems Studies Appendix I. NGT Supporting Technologies: Spiral Wrap Recuperator Study Appendix J. NGT Supporting Technologies: Sensor/Controls Diagnostics Appendix K. NGT System Study: Phase I Program DOE Review 


\section{List of Illustrations}

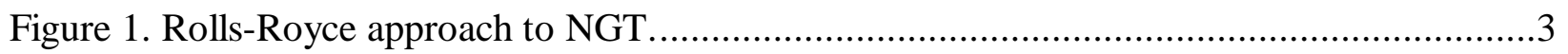

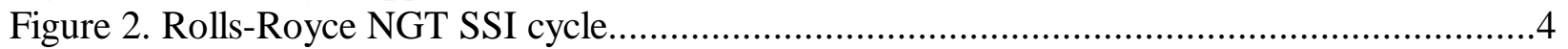

Figure 3. Rolls-Royce BOP layout for SSI cycle. ……......................................................

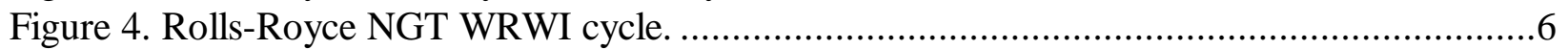

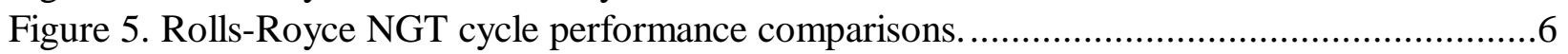

Figure 6. Rolls-Royce customer survey feedback. .............................................................

Figure 7. Rolls-Royce NGT market economic summary.......................................................

Figure 8. Potential NGT core engine configuration. ……......................................................... 10

Figure 9. Simple cycle, IC and ICR gas turbine efficiency as a function of pressure ratio............11

Figure 10. Simple cycle, IC, and ICR gas turbine efficiency as a function of power/unit turbine

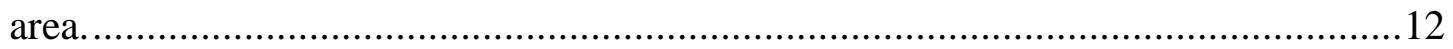

Figure 11. Exhaust steam condensation system using ambient air cooling..................................17

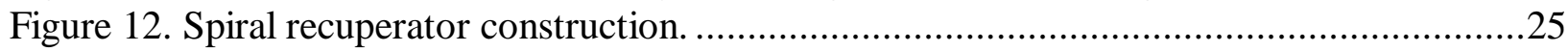

Figure 13. Automated assembly and reduced parts count. ..................................................26

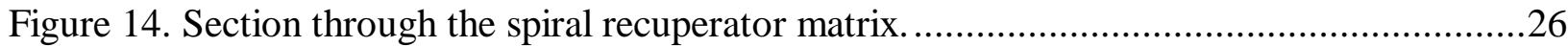

Figure 15. Microturbine design incorporating spiral recuperator.................................................27

Figure 16. 20 MW concept featuring multiple installation of the spiral recuperator.....................27

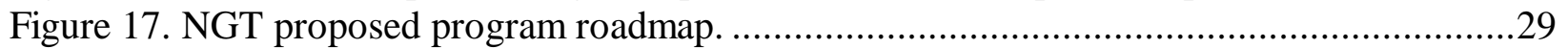

\section{List of Tables}

Table I. Major items required to enhance a simple cycle plant. 


\section{Acronym List}

\begin{tabular}{|c|c|}
\hline ALARP & as low as reasonably practicable \\
\hline BOP & balance of plant \\
\hline${ }^{\circ} \mathrm{C}$ & degrees Celsius \\
\hline $\mathrm{CO}_{2}$ & carbon dioxide \\
\hline DLE & dry, low emission \\
\hline DOE & Department of Energy \\
\hline $\begin{array}{l}\text { EPRI } \\
{ }^{\circ} \mathrm{F}\end{array}$ & $\begin{array}{l}\text { Electric Power Research Institute } \\
\text { degrees Fahrenheit }\end{array}$ \\
\hline HP & high pressure \\
\hline HRSG & heat recovery steam generator \\
\hline IC & intercooled \\
\hline ICR & intercooling and recuperation \\
\hline IP & intermediate pressure \\
\hline IPP & intermediate power producer \\
\hline $\mathrm{kW}$ & kilowatt \\
\hline $\mathrm{lb} / \mathrm{s}$ & pounds per second \\
\hline LPM & lean, premix \\
\hline MW & megawatt \\
\hline NGT & Next Generation Turbine \\
\hline NOx & nitrous oxide \\
\hline OTSG & once through steam generator \\
\hline ppm & parts per million \\
\hline ppmv & parts per million volume \\
\hline RAM & reliability, availability, and maintainability \\
\hline RRESI & Rolls-Royce Energy Systems Incorporated \\
\hline SCR & selective catalytic reactors \\
\hline SRC & Strategic Research Center \\
\hline SSI & super steam injection \\
\hline WIWR & wet intercooling and wet recuperation \\
\hline
\end{tabular}




\section{Introduction/Project Objective}

This final technical report describes the programmatic and technical results for all efforts related to DOE contract DE-AC2600NT40852, Next Generation Turbine (NGT) Systems Study, conducted by Rolls-Royce in Indianapolis, IN. This Phase I feasibility study has focused on identifying candidate flexible gas turbine cycles and BOP systems based on existing Rolls-Royce turbomachinery modules and validated technologies to achieve the specified NGT/Vision 21 goals. In addition to using existing Rolls-Royce aeroderivative core engine technology, innovative technologies pertaining to the gas turbine configuration and the plant have been reviewed for potential development and integration into a pilot demonstrator system in subsequent DOE program phases. This NGT Phase I feasibility study program also includes results from various marketing and economic evaluation studies accomplished to ensure that effective market penetration for a 35 to $150 \mathrm{MW}$ power generation product can be achieved.

A descriptive summary of the primary task elements completed under the Phase I study effort is detailed in the following subsections.

Program Management-This task element comprises efforts associated with general program management for all aspects of the study effort. It includes programmatic and financial reporting, as well as ensuring deliverables are completed within the period of performance.

\section{NGT Market Evaluation and Engine} Cycle Development - This part of the program involves conducting studies of various cycle configurations to down select an optimized product system. Economic and market study evaluations are also performed concurrently with the plant system cycle development.
NGT Component Technology

Evaluation-This task element involves analyzing various candidate gas turbine engine technologies to be further developed under subsequent phases of the program.

System Skid (BOP) Evaluation-This task element evaluates the BOP technologies that should be integrated into the proposed product configuration.

\section{NGT Recuperator Technology}

Evaluation - Since wet recuperation is an important aspect of the final NGT product configuration, this effort accomplishes utilization of the Rolls-Royce patented spiral recuperator technology within the NGT program. This includes system performance requirements and the identification of potential U.S. based manufacturers.

The objectives of the Phase I study include the following elements:

- Completion of combustion studies to confirm flexible low emissions operation.

- Identification of critical technologies in materials, combustion, controls, and aerodynamic cycle analysis.

- Completion of a follow-on strategy along with a cost estimate for a development program beyond Phase I.

- Evaluation of more electric systems for turbomachinery concepts.

- Evaluation of controls and diagnostics technologies.

- Summarization of the public benefits of a proposed NGT system to the U.S.

- Completion of a customer focused survey on an optimum NGT plant concept.

- Completion of an economic model to assess the merits of proposed NGT configurations. 


\section{Executive Summary}

Rolls-Royce is pleased to report the results achieved from the Rolls-Royce Next Generation Gas Turbine (NGT) Systems Feasibility Study performed under DOE contract DE-AC26-00NT40852. For this study, the Rolls-Royce task was to determine the feasibility of developing flexible gas turbine systems with a greater than $30 \mathrm{MW}$ power rating that can achieve the following stated DOE goals:

- Compared to 1999 state-of-the-art systems, the proposed system shall include:

- A $15 \%$ or higher improvement in net system efficiency

- Improvement in turndown ratios

- A $15 \%$ or higher reduction in $\mathrm{COE}$

- Improved service life

- Reduction of emissions (carbon and $\mathrm{NOx}$ )

- A $15 \%$ or higher reduction in operations and maintenance costs

- A $15 \%$ or higher reduction in capital costs

- Increased flexibility (minimum 400 starts/year).

- Improvement in RAM

- Capability to use multiple fuels

For this feasibility study, the full range of the most advanced Rolls-Royce commercial aerospace and aeroderivative engines was evaluated alongside the special technologies necessary to achieve the aggressive efficiency, performance, emissions, economic, and flexibility targets desired by the DOE. Throughout the study, Rolls-Royce placed heavy emphasis on evaluating the technical risks and the economic viability of the many concept and technology options available. This was necessary to ensure the resulting advanced NGT system would significantly benefit the end customers without introducing unacceptable levels of technical and operational risk that would impair the market acceptance of the resulting product. The proposed Rolls-Royce NGT solution would offer customers a very high efficiency power generation system, similar to combined cycle plants, but at a much lower specific cost, similar to those of simple cycle plants.

Rolls-Royce evaluated several approaches for achieving the performance targets desired by the DOE. These included:

1. Increasing pressure ratios and firing temperatures

2. Intercooling and recuperation (ICR)

3. Wet intercooling and wet recuperation (WIWR)

4. Super steam injection (SSI).

Rolls-Royce has considerable experience with each of the concepts evaluated, with the exception of wet intercooling and recuperation. The $50 \mathrm{MW}$ Trent is the highest pressure ratio simple cycle gas turbine engine in the market today. The Rolls-Royce WR-21 is the only intercooled and recuperated engine in the marketplace and is currently on order for the UK Royal Navy's Type 45 next generation frigates. Rolls-Royce has also been producing steam injected $501-\mathrm{K}$ engines since 1984 and has delivered approximately 100 engines that have accumulated over 2 million hours of operation. All of these products are efficiency leaders in their power class. For the NGT study, Rolls-Royce evaluated significantly advanced versions of these concepts.

Figure 1 illustrates the various simple cycle and advanced cycle concepts reviewed in this study. Although increasing the pressure ratio 
and temperatures of the simple cycle machine like the Rolls-Royce Trent would improve the overall system efficiency to the mid to high $40 \%$, the high pressure/high temperature materials and cooling technologies required are not mature and are considered high risk. Fuel flexible dry low emissions combustion, already quite difficult to achieve in today's engines, becomes even more difficult as the pressures are further increased. Because of the high technical risks and the relatively lower payoffs associated with this approach, the advanced cycles were considered more promising in achieving the DOE NGT goals.

A higher technology version of the RollsRoyce WR-21 ICR engine was also evaluated. Due to the nature of the ICR cycle, improvements would only allow the overall system to achieve the mid $40 \%$ efficiency. This approach was also not deemed feasible due to the relatively small payoffs.
By introducing water into the gas turbine cycle, large improvements in the overall system efficiency, in the order of 51 to $53 \%$, could be achieved. System power also goes up substantially, thereby allowing the specific capital cost $(\$ / \mathrm{kW})$ to be reduced. The WIWR concept utilizes an intercooler humidifier and a recuperator humidifier to allow greater heat recovery. The SSI concept uses a steam generator (instead of a recuperator) to recover energy from the engine exhaust. The increased system power is achieved from the increase in mass flow due to the water injected. Both these concepts provide adequate performance benefits to justify the large development investments required.

A parallel shaft WIWR was also reviewed. But this approach would have reduced the development investment, the added complexity of the system, the compromised

\section{High P.R. Simple Cycle}

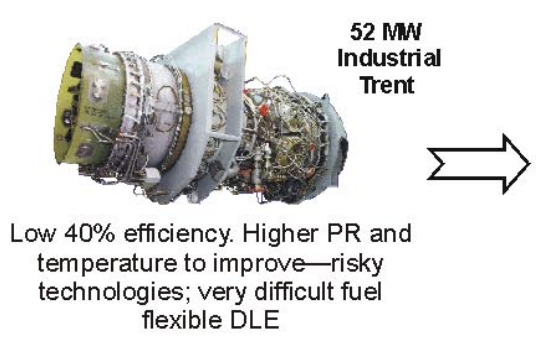

ParalleI Shaft WIWR
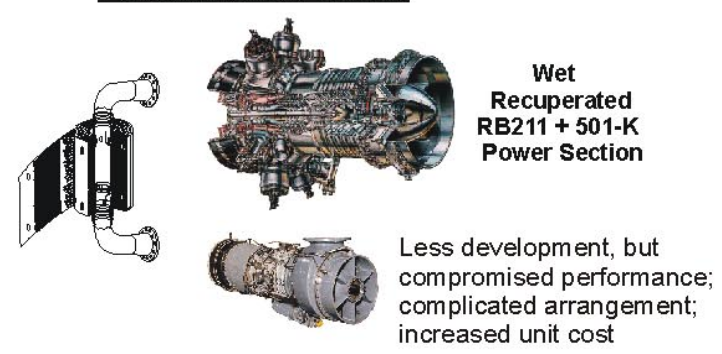

Wet I.C. Wet Recuperated (WIWR)

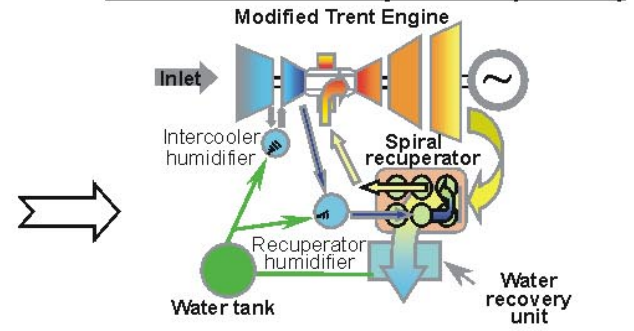

Low $50 \%$ efficiency. Large development; high recuperator durability risk; difficult to control
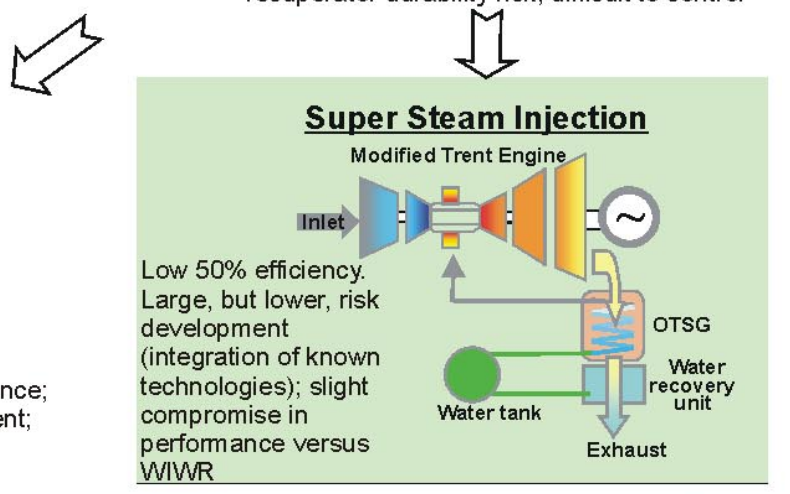

TE02-0091

Figure 1. Rolls-Royce approach to NGT. 
performance, and the increased unit costs made this approach unattractive.

Selection by Rolls-Royce of the preferred go-forward concept for the NGT product was very much a balanced tradeoff between performance gains, economic benefits to the end customer, technical risks, and market potential. Figure 2 illustrates the SSI concept and the anticipated performance and cost of the overall system. The plant utilizes a modified Rolls-Royce Trent engine with advanced technologies and accommodations for SSI, a fast response heat recovery steam generator to extract energy from the gas turbine exhaust, and a water recovery unit in the exhaust. The water recovery unit is included in the overall system to nullify water consumption requirements and ground water environmental implications. A water-polishing unit is also included to ensure that only clean water enters the steam generator and the engine.
The SSI plant will generate $73 \mathrm{MW}$ of electrical power, achieve a $>50 \%$ thermal efficiency, emit less than 10 ppm NOx, be able to achieve very quick starts ( $<15$ minutes), and be priced at $\$ 400$ to 450 per $\mathrm{kW}$ on a turnkey basis. As the NGT plant will be powered by an aeroderivative engine, the SSI plant will be capable of multiple daily start/stop cycles without the significant increase in maintenance requirements and costs encountered by heavyweight combined cycle plants. Figure 3 illustrates the overall plant layout of the SSI plant.

The WIWR concept was also considered a viable NGT option, as this approach provided the highest overall system performance. In this system, the Rolls-Royce patented spiral recuperator is used to extract useful energy from the engine exhaust. A humidifier is utilized to intercool the compressor, and a second humidifier is used

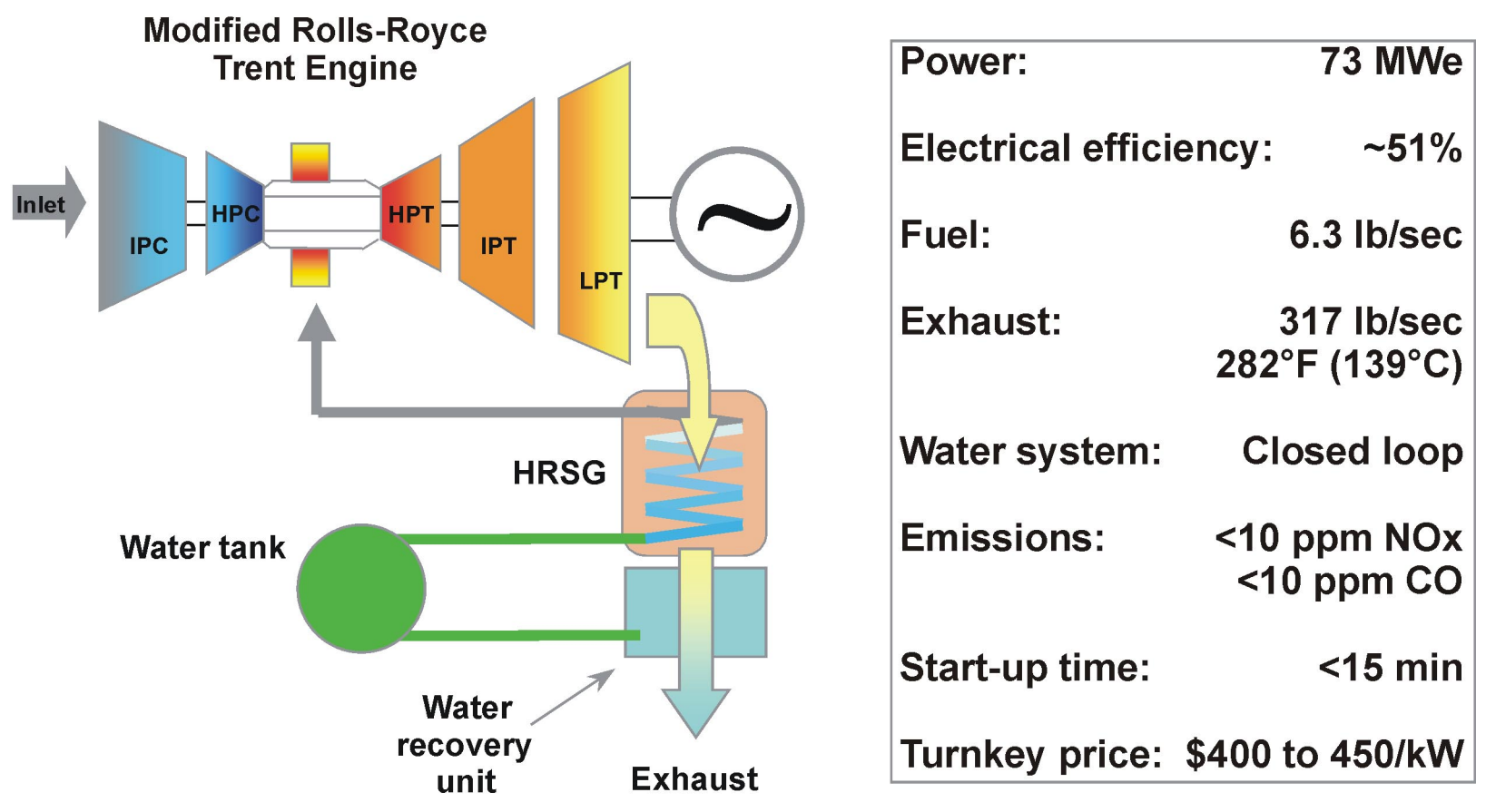

Figure 2. Rolls-Royce NGT SSI cycle. 


\section{Plant Layout—Super Steam Injected}

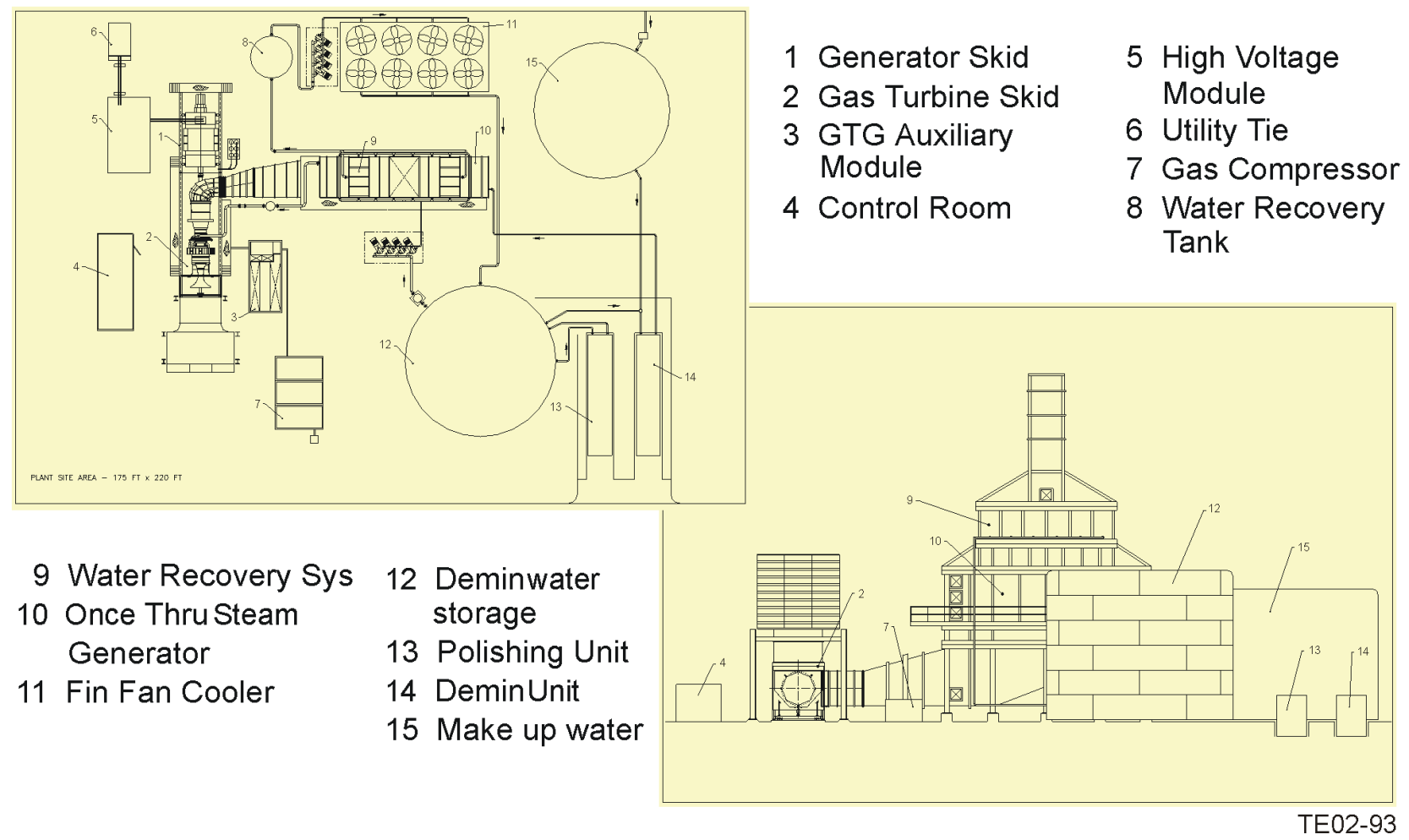

Figure 3. Rolls-Royce BOP layout for SSI cycle.

to cool the air at the compressor outlet/ recuperator air inlet. A water recovery unit is also employed in the overall system. Figure 4 illustrates the WIWR plant and its performance characteristics.

Compared to the SSI concept, the WIWR plant produces about $10 \%$ more power and is a little more efficient. However, the overall system is more complicated and involves higher technical risks and more development, especially in the areas of recuperation, humidification, and overall system control dynamics.

Figure 5 compares the Rolls-Royce NGT systems to current products in the marketplace. The Rolls-Royce NGT SSI and WIWR are both superior to the simple cycle machines with approximately $25 \%$ efficiency improvements while maintaining the same levels of $\$ / \mathrm{kW}$ turnkey plant price and similar levels of operational flexibility required for peaking and intermediate duty operation. Compared to combined cycle plants, the NGT systems maintain the efficiency levels of combined cycle plants while providing customers with a $30 \%$ reduction in $\$ / \mathrm{kW}$ turnkey plant price. The NGT systems also provide customers with additional operating flexibility not viable with combined cycle plants (e.g., rapid starts, ability to tolerate high number of start/stop cycles).

As a part of the study, Rolls-Royce contracted with PB Power to perform a survey with the following objectives:

1. Determine the market acceptability for the proposed Rolls-Royce NGT concepts.

2. Understand the customer purchasing decision factors and economic evaluation techniques. 


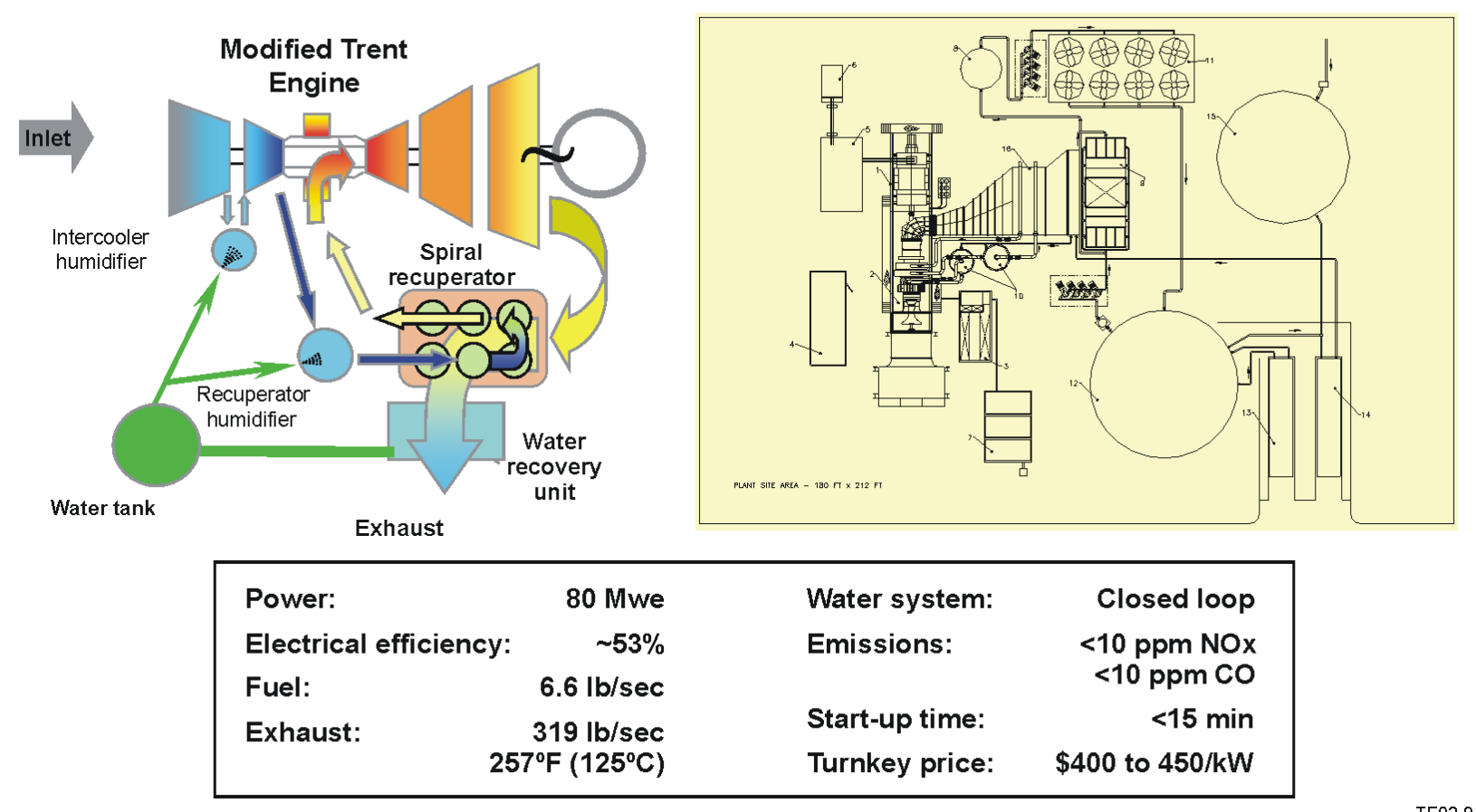

Figure 4. Rolls-Royce NGT WRWI cycle.

\begin{tabular}{|c|c|c|c|c|c|c|c|c|c|c|}
\hline & \multicolumn{3}{|c|}{ Simple Cycle } & \multicolumn{3}{|c|}{ Wet Cycle } & \multicolumn{4}{|c|}{ Combined Cycle } \\
\hline & $\begin{array}{c}\text { GE } \\
\text { LM6000 } \\
\text { PD (DLE) }\end{array}$ & $\begin{array}{l}\text { R-R } \\
\text { Trent } \\
\text { DLE }\end{array}$ & $\begin{array}{c}\text { GE } \\
\text { Fr6FA }\end{array}$ & $\begin{array}{c}\text { GE } \\
\text { LM6000 } \\
\text { Sprint }\end{array}$ & $\begin{array}{c}\text { R-R } \\
\text { NGT80-SI } \\
\text { (Super Stm } \\
\text { Inj) }\end{array}$ & $\begin{array}{c}\text { R-R } \\
\text { NGT80- } \\
\text { WIWR } \\
\text { (Wet IntrCool } \\
\text { / Wet Recup) } \\
\end{array}$ & $\begin{array}{l}\text { GE } \\
\text { LM6000 } \\
\text { C.C. }\end{array}$ & $\begin{array}{c}\text { R-R } \\
\text { Trent } \\
\text { C.C. }\end{array}$ & $\begin{array}{c}\text { GE } \\
\text { Fr6FA } \\
\text { C.C. }\end{array}$ & $\begin{array}{c}\text { GE } \\
\text { Fr7EA } \\
\text { C.C. }\end{array}$ \\
\hline Power (MW) & 43.1 & 51.2 & 70.1 & 47.3 & 73 & 80 & 56.4 & 66 & 107.4 & 130.2 \\
\hline Efficiency (\%) & $41.4 \%$ & $41.6 \%$ & $34.2 \%$ & $41.4 \%$ & $51.0 \%$ & $53.0 \%$ & $52.5 \%$ & $54.3 \%$ & $53.2 \%$ & $50.2 \%$ \\
\hline $\begin{array}{l}\text { Turbogenerator } \\
\text { Price }(\$ / k W)\end{array}$ & $\$ 366$ & $\$ 303$ & $\$ 285$ & $\$ 298$ & & & & & & \\
\hline \begin{tabular}{|l} 
Turnkey \\
Price $(\$ / \mathrm{kW})$
\end{tabular} & 6 & $\$ 485$ & $\$ 456$ & $\begin{array}{c}\$ 430 \\
\text { (no water } \\
\text { recov) }\end{array}$ & $\begin{array}{c}\$ 400-450 \\
\text { (incl water } \\
\text { recov) }\end{array}$ & $\begin{array}{c}\$ 400-450 \\
\text { (incl water } \\
\text { recov) }\end{array}$ & & $\$ 650$ & $\$ 730$ & $\$ 514$ \\
\hline Flexibility & High & High & High & High & High - & High - & Med/Lo & Med/Lo & Low & Low \\
\hline
\end{tabular}

The Rolls-Royce NGT solution:

Source: Gas Turbine World Handbook

" 25\% improvement in efficiency of simple cycle machines

" $\sim 30 \%$ improvement in capital cost $(\$ / \mathrm{kW})$ of combined cycle plants

" Maintains operational flexibility of simple cycle machines

Figure 5. Rolls-Royce NGT cycle performance comparisons. 
3. Understand the customer perspective regarding specific technologies to be employed for the NGT system.

4. Estimate the market potential for the proposed Rolls-Royce NGT product(s).

PB Power instituted a number of discussions with 17 independent power producers to elicit their opinions of the NGT concepts. The respondents were chosen to cover a wide range of the North American markets and included companies such as El Paso, BP, Calpine, Cogentrix, Conoco, and Trigen. The responses received suggest that a majority of these developers believe a market would exist for an NGT-type product and the Rolls-Royce NGT concept appears to be appropriate for this future marketplace. Figure 6 summarizes some of the survey results.

Rolls-Royce also launched a detailed customer based economics analysis to

\section{Distributed generation growth?}

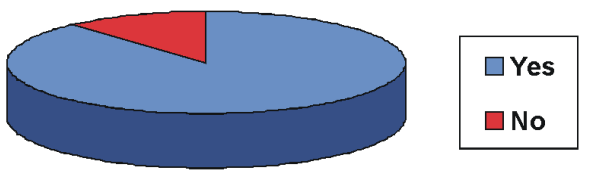

Future mid-merit market?
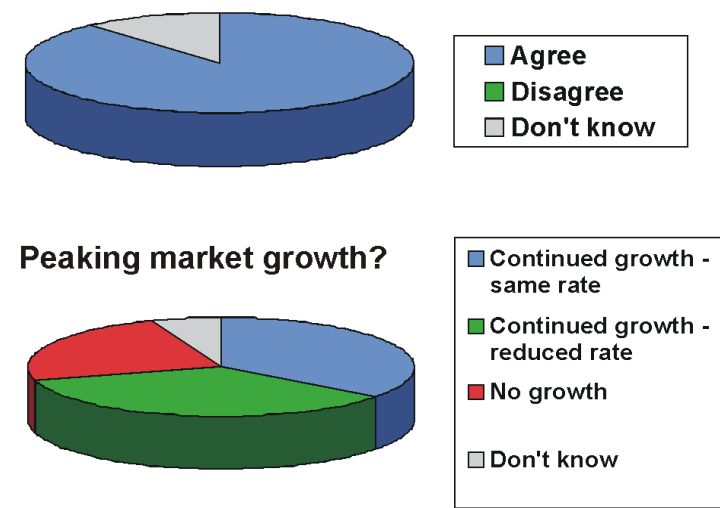

determine the economic performance of the two Rolls-Royce NGT options and determine whether these customer economics are likely to offer competitive advantage when compared with alternative simple cycle and combined cycle power plants. The analysis considered the full life cycle costs that the customer would incur including initial capital costs, fuel costs, operation and maintenance costs, annual charges (e.g., connection fees, etc), network infrastructure costs, and environmental levies. The analysis also considered the customer's revenue stream from the sale of the electricity generated. Other factors included were the discount rate, inflation rate, and machine availability rates. The output of the economics analysis included the cost of electricity generated, the customer's internal rate of return, and the customer's investment payback period.

\section{Market perceived for the Rolls-Royce NGT?}

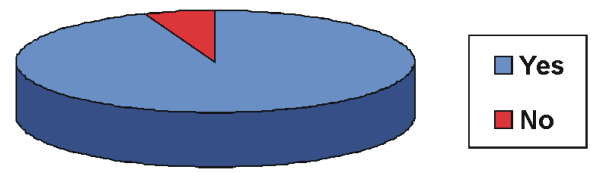

70 to $80 \mathrm{MW}$ size?

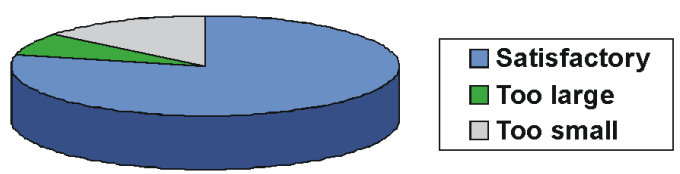

How fast should start time be?

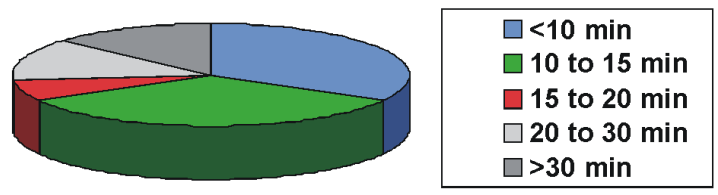

Figure 6. Rolls-Royce customer survey feedback. 
The economics were analyzed for three customer operating modes: baseload (8300 hours per year), intermediate duty (4500 hours per year), and spot market trading. In the spot market trading mode, an hourly basis the spot market price is compared with the marginal cost of generation. For those cases where the spot price exceeds the marginal generation cost, the engine is assumed to run. The spot market price at that time is captured as income to the plant. The spot market trading analysis utilized historical hourly pricing from New England, PJM, California, and the UK power exchanges to determine the economic impact to the customer.

The analysis also included five market scenarios, all of which were derived from the DOE's draft scenarios for natural gas planning. The base case scenario uses the reference case data taken from the IEA's Annual Energy Outlook 2001 and assumes an electricity selling price of 6 cents $/ \mathrm{kWh}$ and a wholesale gas price of $16.5 \%$ above the predicted wellhead price. The base case is considered to be the scenario that is most likely to occur in

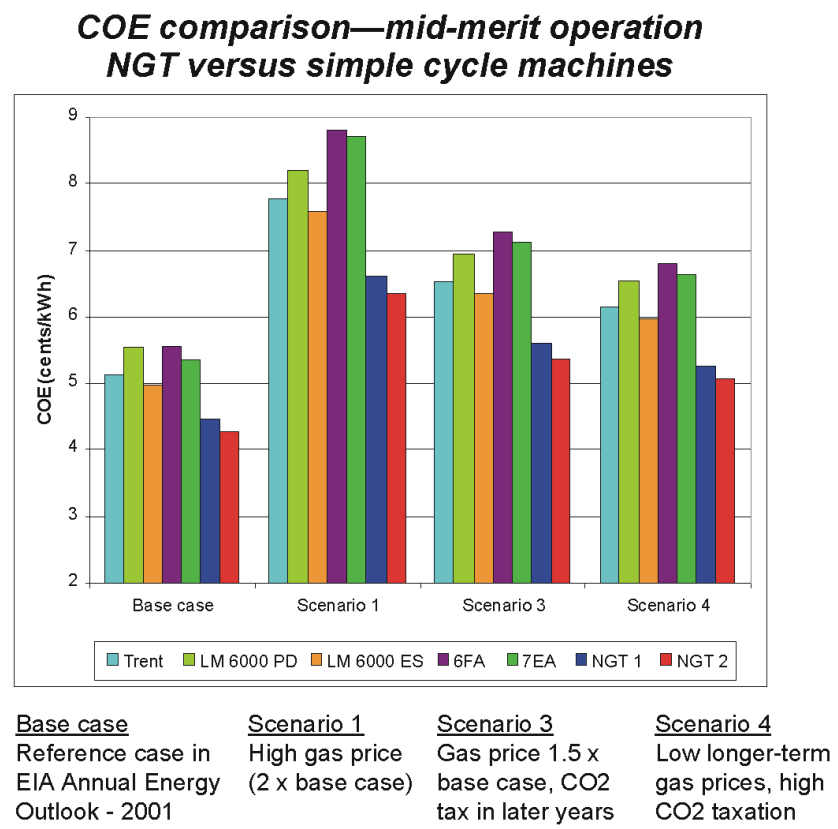

practice. Other scenarios included a high gas price scenario, a scenario with some carbon $\left(\mathrm{CO}_{2}\right)$ taxation in the later years, and the Kyoto scenario, which includes a significant $\mathrm{CO}_{2}$ levy.

The study concluded that both NGT arrangements are likely to offer significant commercial advantage in comparison with other currently available machines of similar power rating, including combined cycle variants. These commercial advantages are a direct consequence of the high efficiency of the NGT solutions, which is comparable to a typical combined cycle machine, and their low capital cost, which is comparable to a typical simple cycle machine. Figure 7 summarizes some of the customer economics analysis results.

The Rolls-Royce NGT concepts and technologies will provide substantial public benefits if developed and deployed. Because of the NGT system's higher efficiencies, considerable savings in fuel and $\mathrm{CO}_{2}$ emissions can be realized. Assuming 34

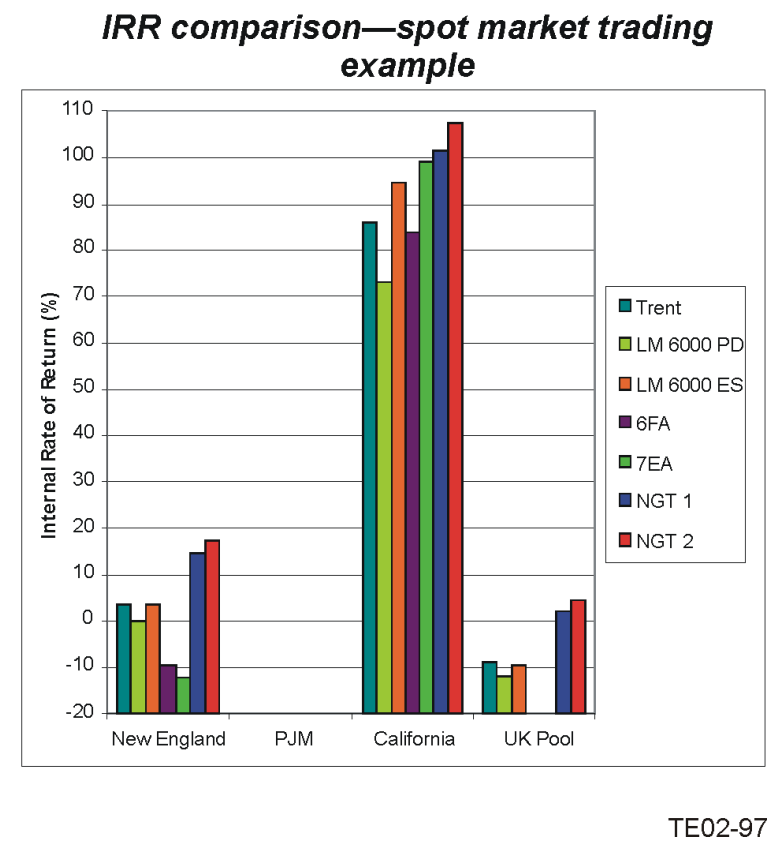

Figure 7. Rolls-Royce NGT market economic summary. 
Rolls-Royce NGT units per year are installed in the U.S., a 15-year cumulative savings in $\mathrm{CO}_{2}$ emissions would equal 630 million metric tons when compared to coal plants or 150 million metric tons when compared to simple cycle gas turbine plants. This would equate to $\$ 12.6$ billion and $\$ 3$ billion savings in $\mathrm{CO}_{2}$ trading credits, respectively, assuming a trading credit of $\$ 20 /$ metric ton. Fuel savings of $\$ 8.3$ billion or 2.6 trillion cubic feet of natural gas could also be achieved in this 15year period. Since the Rolls-Royce NGT system will be manufactured in the U.S., over $\$ 2$ billion per year in exports could be generated.

In summary, Rolls-Royce is encouraged by the results of this study. The Rolls-Royce NGT concepts have been approached from an economic viability perspective by leveraging available hardware and technologies to lower risk, investment, and time-to-market and applying these in innovative ways to develop a solution providing customers with improved return on investment while providing extensive public benefits. Rolls-Royce's NGT concepts meet the DOE NGT goals, seem to be appropriate for the future marketplace, provide substantial economic benefits to operators, and provide substantial public benefits.

\section{Experimental Results and Discussion}

\subsection{System Performance Optimization}

The best aeroderivative engines now available for electrical power generation exploit high pressure ratio, in the region of 30 , and high turbine temperature to attain high levels of power/weight and thermal efficiency. The electrical efficiency at the terminals reaches $41 \%$. Using this figure as datum, the DOE call for $15 \%$ improvement in efficiency requires the attainment of $47 \%$ at the terminals or 48 to $49 \%$ shaft efficiency.
Another very important parameter targeted by DOE is capital cost, typically expressed as $\$ / \mathrm{kW}$, targeted to reduce $15 \%$. In the Rolls-Royce proposal, this was translated to offering combined cycle efficiency at simple cycle cost.

Rolls-Royce has considered using existing turbomachinery in their core NGT cycle concepts. One such configuration studied is an advanced aeroderivative Trent engine known as the MT30. The MT30 is detailed in Figure 8.

Two heat exchange devices, commonly cited as potential sources of efficiency improvement, are intercoolers and recuperators. These have been successfully applied to the WR21 naval gas turbine, in a joint Northrop/Rolls-Royce program, to retain greater than $40 \%$ efficiency over a very wide power range. Though valuable in solving the Navy's special needs, the resulting plant would not be cost effective in commercial power generation.

Intercoolers are placed at intercompressor positions. They benefit thermal efficiency by reducing the drive work required by downstream compressors to deliver their design pressure ratio but detract from efficiency by wasting heat developed in the upstream compressor and through their own pressure losses. There is an optimum split in the pressure rise of a cycle at which to place the intercooler; too early and it has little cooling to do, too late and it does not decrease temperature in much of the compression process. The net effect of a well placed intercooler on cycle efficiency is usually a small gain. Sometimes they are attractive because they allow a given high-pressure spool to be used with generous supercharging without raising compressor late-stage component temperatures much higher. In this manner, a small core can support a high power level, a potentially cost-effective 


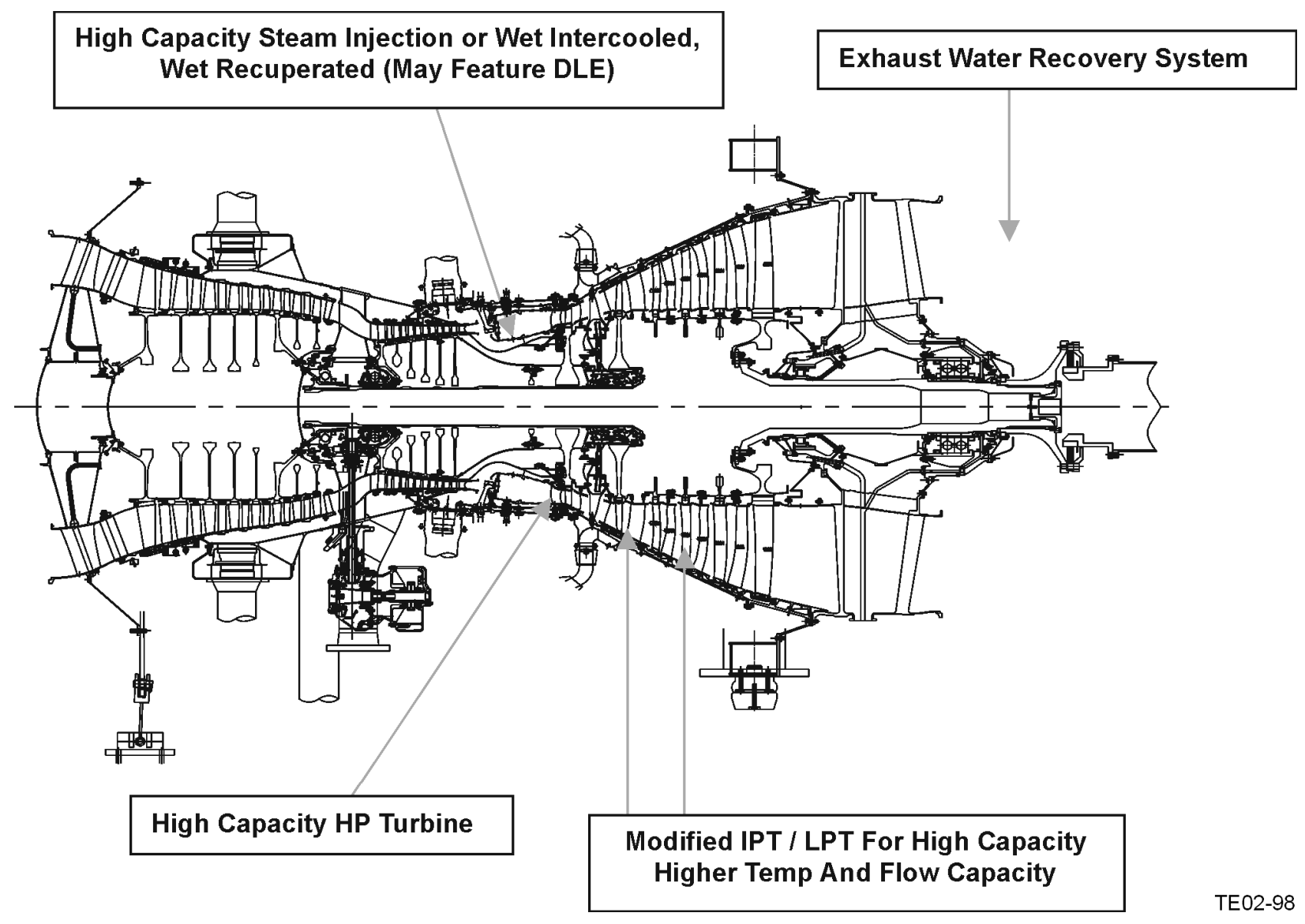

Figure 8. Potential NGT core engine configuration.

solution in some cases. Most modern aero engines are quite high in pressure ratio, so further increase leads to problems such as increased combustion emissions and severe volumetric lag effects during transients, particularly load sheds. Intercoolers are seldom attractive in gas turbines, taken alone.

Recuperators, in theory, are much more effective in enhancing efficiency. Their function is to transfer some exhaust heat into the airflow leaving the compressor, raising its temperature, and substituting for an increment of fuel flow. Used as the only device added to a simple cycle machine, there are limits to their effective range. They are at their best in low pressure ratio, normal turbine temperature engines, because the exhaust temperature of such engines is much higher than compressor discharge temperature, making generous heat transfer possible. Higher pressure ratio cycles result in increased compressor exit temperature and decreased turbine exhaust temperature, so the temperature difference becomes small or even reverses. Depending on turbine temperature level, the best efficiencies generated by a simply recuperated engine occur around a pressure ratio of 5. This is not a cost-effective design region because, at lowpressure level, all the expensive hot parts are large in relation to the power developed.

Using an intercooler and recuperator together is effective in gaining efficiency in a higher pressure ratio engine. By resetting the temperature near to ambient partway through the compressor system, the intercooler effect results in reduced compressor discharge 
temperature, allowing heat to be regained from the exhaust once more. Using this combination, the optimum cycle pressure ratio proves to be about 25 at current turbine temperatures.

Simple cycle, intercooled (IC) and ICR cycle efficiencies evaluated using simplifying assumptions are shown in Figure 9.

The simple cycle curves show that pressure ratios in the region of 30 give nearmaximum efficiency at high turbine temperature, and improvements of efficiency with turbine temperature increase are small, partly due to the concurrent increase in turbine cooling air penalty. Low levels of cooling flow have been assumed, so this penalty is less than $2 \%$ efficiency at $2600^{\circ} \mathrm{F}$. The curves have been cut off at 36 pressure ratio because this is both where late stage compressor components become too hot to be cost effective and where combustion emissions become worse, particularly with liquid fuels that tend to preignite. The highest pressure ratio fielded in liquid fueled gas turbine with dry, low emission (DLE) capability (LM2500) is 29.

IC cycles ease these problems, permitting the use of higher pressure ratio cycles but worsening the rate at which the engine becomes mismatched below full power. The consequence of this is that bigger bleeds or extra variables have to be deployed, thus the engine becomes more complicated and less flexible. Figure 10 shows a scatter of IC results, reflecting variations brought about by shifting the bleed plane through the compression process. Intercooling alone is unable to provide a good enough efficiency to meet DOE targets.

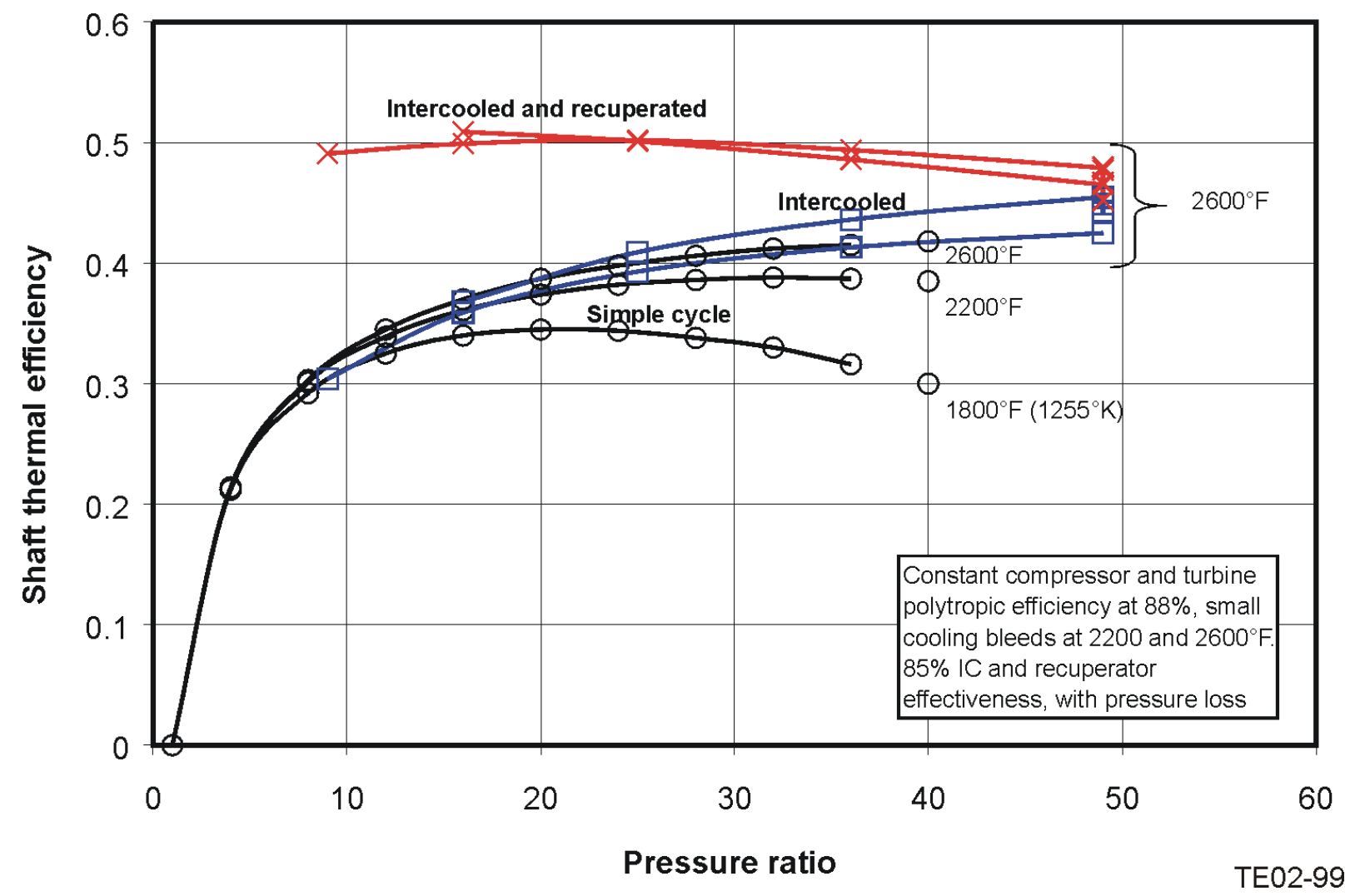

Figure 9. Simple cycle, IC and ICR gas turbine efficiency as a function of pressure ratio. 


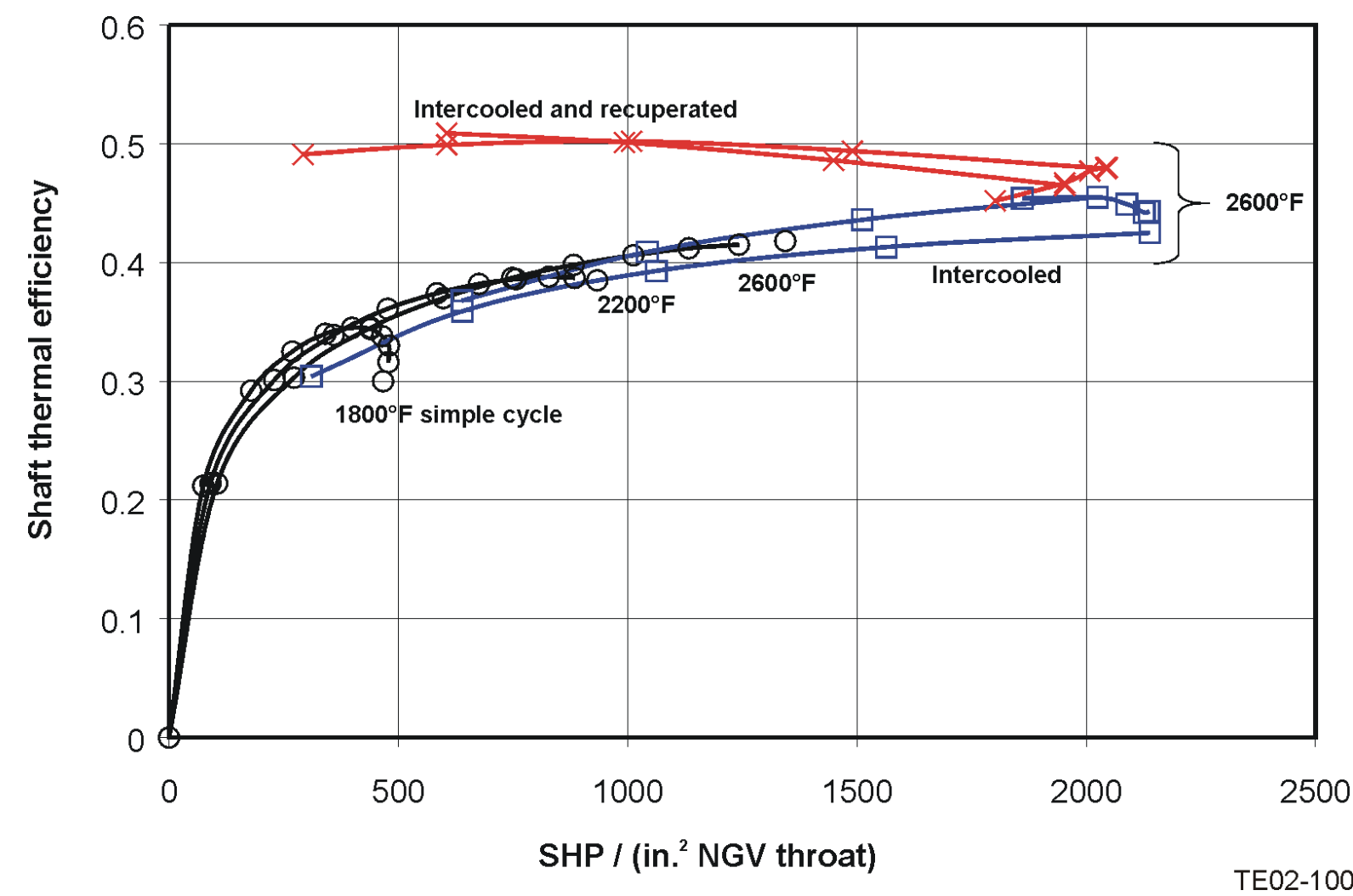

Figure 10. Simple cycle, IC, and ICR gas turbine efficiency as a function of power/unit turbine area.

The ICR results, also with various bleed planes, show their best efficiency over the range 10 to 30 pressure ratio for $2600^{\circ} \mathrm{F}$ turbine temperature. The peak level is about $50 \%$ shaft efficiency and is the only cycle shown that just qualifies for the DOE $+15 \%$ efficiency target level (the WR21 has bigger cooling flows and does not quite reach this level). A cycle in the region of 25 pressure ratio is typical of large aeroderivative engine cores. The Rolls-Royce family of large aero engines is unique in utilizing a two-spool core, with a fairly even split of pressure ratio between the two spools. This is well suited to IC operation as it provides a natural split in the compression system, facilitating the introduction of IC components at a position providing good thermodynamic benefits, as in the WR21.
The efficiency data of Figure 9 have been replotted versus power per unit area of first turbine nozzle throat in Figure 10. The point of this plot is that increased power per unit area means increased power for an engine derivative retaining a particular HP turbine. In Figure 9, it can be seen that a 25 pressure ratio ICR engine achieves $50 \%$ efficiency, whereas its simple cycle 25 pressure ratio counterpart achieves only $40 \%$. Tracking data points into Figure 10 shows that the ICR engine develops perhaps $10 \%$ more power than the simple cycle engine.

The cost of the ICR unit is, however, raised significantly by the intercooling and recuperating components. Even using the low figures assumed for the cost reduced spiral wrap recuperator system, the added turnkey cost is in the region of $30 \%$, whereas the fuel 
efficiency is only raised $25 \%$ and the power raised $10 \%$, so the $\$ / \mathrm{kW}$ is raised about $20 \%$. As a general guide, it seems the value of a generating plant increases proportional to its fuel efficiency, suggesting a small net advantage for the ICR unit. Rather than being a decisive step forward in value for money, the ICR engine is a small advance in exchange for a big increase in unit cost and complexity.

Air bottoming cycles do not gain enough to pay for the large heat exchangers required to extract exhaust heat or the large turbomachinery required to convert this relatively low grade heat into power. It is concluded that a cycle involving a second working fluid, or a supplementary cycle, is required.

\subsubsection{Enhanced Cycles}

While retaining a well proven open cycle turbomachine as the primary cycle element, the recognized possibilities are:

- Water enhanced cycles:

- Combined cycle-reference case, good but too expensive.

- Steam injection in which steam is raised from exhaust heat and reinjected into the combustor zone.

- Humidified - in which water is evaporated in the gaspath prior to turbine entry to add mass flow.

- Spray cooling — often used at inlet to counter the effect of high ambient temperature

- Alternate fluid primary and bottoming cycles:

- e.g., lower boiling point than water to minimize cycle heat rejection

- Chemical recuperation:
- Upgrading the fuel calorific value using exhaust energy and steam (after Jack Janes)

Cycles using alternate working fluids, often poisonous or hazardous, can be around $10 \%$ more efficient than air/steam based systems but have to meet much higher standards of integrity for safety reasons. The result has sometimes been described as being as expensive as a nuclear plant.

Chemical recuperation was previously studied by Rolls-Royce under contract to the Electric Power Research Institute (EPRI) and found not to be cost effective. Therefore, this study concentrated on the various ways of using water for cycle enhancement.

Combined cycles using a steam bottoming cycle are attractive as they do not closely interweave the air and steam cycles, thus the steam cycle can, in principle, always be seen as a bonus. In particular, failure of some element in the steam circuit need only reduce plant power by a fraction because the gas turbine can usually still be operated. In addition, steam cycle equipment is familiar and proven, so a combined cycle does not necessarily involve system development with the attendant risks. However, aeroderivatives tend to be the least rewarding for the addition of the steam cycle because they have the highest pressure ratios. This provides very efficient gas turbines but leaves little heat to be recovered from the exhaust. A large industrial frame engine can be expected to support at least a $50 \%$ increase in total power (and fuel efficiency) from a steam bottoming cycle, whereas a high grade aeroderivative offers only 20 to $30 \%$. Based on Gas Turbine World figures, combined cycle plant $\$ / \mathrm{kW}$ is most often about $50 \%$ higher $\$ / \mathrm{kW}$ than the simple cycle baseline, indicating the added features are not very cost effective for aeroderivatives. 
Humidification or steam injection cycles share the important characteristic that water vapor is added to the gaspath ahead of the turbine and is then raised to full normal turbine entry temperature. This temperature is about 1500 to $1600 \mathrm{~K}$ in modern aeroderivatives, rather than the 700 to $800 \mathrm{~K}$ exhaust temperature. The great benefit of this feature is that in a fully implemented system engine power output typically rises by $100 \%$. This suggests that both of these approaches may be more cost effective than a traditional combined cycle, particularly for aeroderivatives. Though both these families of solution eliminate the need for the expensive steam turbine plant of a combined cycle, they still have a significant inventory of added equipment, even for the simplest embodiments (Table I).

Exhaust water flow, in the region of 50 $\mathrm{lb} / \mathrm{s}$, must be recovered for several reasons. Potential customers cited this requirement:

- To minimize water treatment cost (recycled water only needs polishing)

- To ease or enable local water use permitting

- To avoid large steam plume or fog formation
Exhaust water recovery requires very large, expensive, condensing heat exchangers because it has to be recovered from about 250 $\mathrm{lb} / \mathrm{s}$ of gas flow at small temperature difference, yielding a high percentage recovery (about 80\%).

The rest of the performance discussion, given in Appendix A, details the properties and value of a variety of wet cycles based on those discussed above and considers the various ways of generating steam or water vapor. Attention has been paid to performance, cost qualities, development timescale, and customer acceptance in reaching a recommendation for the best configuration that is most compliant with the DOE solicitation goals.

In the choice of preferred configuration approach, risk perceptions played a major role, as follows:

- Previous, and active, experience with recuperators shows they add seriously to the potential for overspeed at load shed due to their large energy storage both in terms of pressurized gas mass and the heat energy in their metal. The system requires large, fast-acting dump valves for adequate control, which are expensive

Table I. Major items required to enhance a simple cycle plant.

\begin{tabular}{|l|l|}
\hline \multicolumn{1}{|c|}{ Steam injection cycles } & \multicolumn{1}{c|}{ Humidified cycles } \\
\hline $\begin{array}{l}\text { Exhaust boiler (heat exchanger) } \\
\text { ORSG requires boiler quality water }\end{array}$ & Exhaust recuperator (heat exchanger) \\
\hline & $\begin{array}{l}\text { Humidifier pressure vessels or foggers } \\
\text { Foggers require premium water } \\
\text { Humidifiers accept boiler quality water or lower }\end{array}$ \\
\hline & Complex connection to recuperator \\
\hline Larger capacity high pressure turbine & Larger capacity high pressure turbine \\
\hline Larger expansion ratio power turbine & Larger expansion ratio power turbine \\
\hline Small fast valve for load shed & Large fast valves for load shed \\
\hline Water treatment to required purity & Water treatment to required purity \\
\hline Exhaust water recovery & Exhaust water recovery \\
\hline
\end{tabular}


and tend to complicate the control system because their openings often have to be timed and conditioned relative to other bleed valves to avoid precipitating surges. Recuperator use has been deferred for this reason. Ultimately, recuperators may become attractive because they offer the best efficiency by a modest margin.

- Humidifier vessels contribute to energy storage through pressurized gas mass. Though they potentially confer the advantage of requiring only relatively low purity water, they have also been deferred due to the overspeed control risk.

- Water injection spray systems have been made to work, but have bad failure cases. Failure to sustain very fine droplet sizes results in rapid blade erosion if any spray is placed ahead of a compressor. Imperfect water purification results in rapid fouling, particularly of any downstream hot surfaces, which results in performance loss. Cleaning is then often an expensive operation.

- Simple intercooling by heat exchanger becomes very expensive because it requires extensive structure change away from production standard parts to accommodate the heat exchangers. It gives rather little efficiency gain and significant power loss and is hard to justify on a cost-benefit basis. There is an energy storage hazard with off-engine (large) heat exchangers and a possible fouling problem with onboard (highly compact) exchangers.

Steam raising in the exhaust system, by contrast, is a well established technology, affords relatively easy overspeed control, because the flow can be chopped rapidly without hazard, and provides a much enhanced system efficiency, only a few points below the highest efficiency candidate.
As well as the intended application for stand-alone distributed generation, an exhaust steam enhanced generating system can be very attractive for operations requiring a variable process steam supply. The steam output is used in the turbine when not required in the process. The use of a once through steam generator (OTSG) can be turned to extra advantage if an exhaust duct burner is fitted, permitting a high enough steam generation rate to satisfy maximum turbine capacity and some process steam. Use of this burner would be influenced by the current relative values of gas, electricity, and steam, providing excellent operations flexibility.

Appendix A examines system hot day performance and shows, as is normal with water-enhanced cycles, better results than simple cycle engines. One of the simpler conclusions coming from the study was that the most suitable aeroderivative at a pressure ratio level is the biggest flow aeroderivative, because the system complication cost changes little with power level and the capacity oriented components (e.g., boiler) costs scale up slower than power level. Raising flow by supercharging an existing system much beyond a pressure ratio of 30 is not found to be helpful. This is partly due to the need to move to a more complex engine (one more spool in our case) and partly due to problems such as emissions control at very high pressure.

\subsection{Package Optimization}

Though the gas turbine engine and associated power turbine are the key empowering components dominating the performance of the system in generating electricity, they only account for about onethird of the plant cost. It follows that a good quality, affordable plant depends on an excellent BOP design. Many of the major components of the proposed plant are common in simple cycle generating sets: 
- Air inlet ducting with snow and dust ingestion protection

- Fuel compression system

- Fuel metering components

- Fuel and plant management control system

- Skid and enclosure structures with ventilation and fire control features

- Engine auxiliaries (e.g., start system)

- Lube system skid

- Exhaust diffuser, ducting, and silencers

- Safety and diagnostic sensor system

- Alternator

- Utility interconnect and high voltage breakers

Optimization of the above system set is an established procedure and has resulted in an array of commercial plant sizes for which cost and price vary rather consistently with plant power size. Continuous cost reduction can still be achieved, based on improvements of each technology involved and its method of application. Appendix B points out how some $30 \%$ of controls related cost can be eliminated from the control system in an evolutionary manner.

However, in addition to the familiar inventory above, the high efficiency plant configurations proposed to meet the goals DOE set for the NGT have large additional equipment items to achieve extra cycle functions, namely:

- Water purification

- Heat recovery from the turbine exhaust stream, either by boiler or recuperator

- Water vaporization, either by boiler or humidifier column

- Features to inject water or steam back into the gas turbine gas path
- Water recovery from the exhaust stream, efficient enough to avoid the need for substantial makeup

- Water storage to support running at times when full recovery is impractical

Though none of these items are truly novel, this application requires careful selection of options to keep the system reliable and affordable.

\subsubsection{Water Management}

The cycles proposed use nearly $50 \mathrm{lb} / \mathrm{s}$ treated water and the sheer cost of treating this much "raw" water makes it essential to recover the majority so that only a small makeup is required. Potential customers also report the limitations large-scale water consumption would place upon deployment and permitting, indicating recovery is essential. It is found that the treatment of makeup water is most economically handled by using treatment trailers that return to a remote facility to be recharged, thus avoiding the capital requirement to set up a complete local water treatment plant.

The engine combustion process continuously creates water and this sets the maximum level that may be released from the exhaust stack without the requirement for any make up. Unfortunately, the saturated humidity level equals the amount created by combustion when the exhaust temperature is $104^{\circ} \mathrm{F}$; so final stack exhaust gas stream temperature would sometimes have to be near or below ambient to achieve full recovery. Although there are several ways a chiller could be incorporated into the system to avoid this problem, it is considered more cost effective to provide substantial tank storage to supply the sporadically required makeup water. An exhaust gas cooling system, using atmospheric air as the heat sink (using an intermediate water loop) has been designed having about 
$40^{\circ} \mathrm{F}$ differential between the final exhaust temperature and ambient (Figure 11). The atmospheric air stream is raised nearly $80^{\circ} \mathrm{F}$ and is a little more than 10 times larger. Assuming these numbers on a $105^{\circ} \mathrm{F}$ day would give a stack exhaust temperature of $145^{\circ} \mathrm{F}$ and a cooling tower air exit temperature of $185^{\circ} \mathrm{F}$.

The stack exhaust will carry away about $30 \mathrm{lb} / \mathrm{s}$ more water than is being made by combustion (less any humidity being taken into the engine intake), so a reserve tank of demineralized water is required. The plant layout includes a 50,000- $\mathrm{ft}^{3}$ tank, which would support roughly 30 hours continuous running at this extreme condition. Some makeup water is required when day temperature exceeds $70^{\circ} \mathrm{F}$, and has only $1 \%$ humidity. The final engine stack exhaust is always saturated and would generate a prodigious plume if no corrective action were taken. The obvious solution is to mix the stack and cooling tower exhausts, yielding a low humidity, hot exhaust. This feature is not included in the plant layouts.

\subsubsection{Once Through Steam Generator}

The conventional exhaust heat recovery steam generator (HRSG), having hot water and steam drums connected by many heating tubes, is a well established design for recovering exhaust heat. For the proposed system, however, it has a number of disadvantages, which are discussed in Appendix C, the most pertinent of which is that it takes upwards of one hour to reach full steam conditions.

The OTSG dispenses with water and steam drums, reducing the startup time by a factor of at least two. The fluid phase inside the tubes changes from being primarily liquid with steam bubbles to varying between liquid, mixed, and vapor, leading to a higher tube wall temperature. This situation requires the uses of more costly tubes and much higher purity makeup water, but it does allow the steam rate to be varied, within limits, independent of exhaust gas conditions. This is not acceptable for an HRSG, where reduced steaming requires some exhaust gas be diverted around the HRSG by extra flap valves
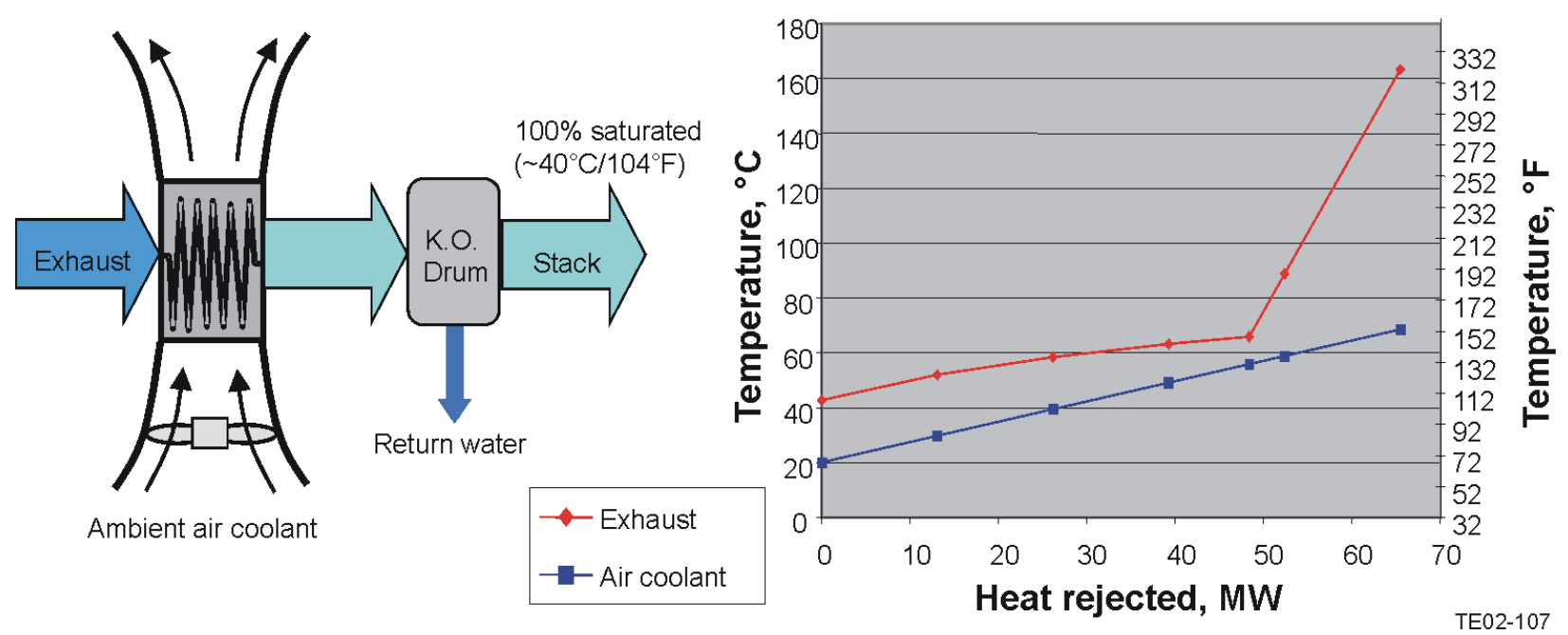

Figure 11. Exhaust steam condensation system using ambient air cooling. 
and ducts. The OTSG is simpler than an HRSG but requires additional control; for instance, to avoid an excessive inrush of cool water into heating tubes that have previously settled to a higher temperature at a lower steam setting. The cost of the two systems is very similar.

A range of choices to be examined more closely is in the management of steam generation with system power output variation. Appendix C indicates OTSG steam output should not be cut down too low and a circuit could be provided to dump steam that might be in excess to the requirements, such as may occur transiently when the power level is controlled downward. If minimum OTSG flow requirements are removed, the water pump for the OTSG becomes somewhat akin to a fuel injector for the engine, as any water injected is rapidly converted to steam and then transferred to the engine unless it is spilled.

The proposed engine configuration features a free power turbine, so the gas generator speed is a variable that can be changed by steam or fuel flow changes, and the combined effect of the two must be controlled accurately including during transients. Whether it is more appropriate to exercise control by controlling water input rate and avoiding steam spillage or to have a water flow rate fixed by boiler concerns and controlling a steam "waste gate," is yet to be determined. It is significant that these concerns do not really arise in our previous experience with the 501-KH engine because the latter is of single-shaft, synchronous alternator configuration.

\subsubsection{Control System Design}

Appendix B provides the control system approach. Appendix B discusses several improvements in approach contributing to first-cost reduction, lower maintenance costs, and improved reliability.
Whereas it has been typical practice to centralize control functions, which keeps the simplest block diagram for the system, this has a disadvantage in a mid-sized (50 to $150 \mathrm{MW}$ ) plant. Such a plant is large enough to require transporting in several separate truck sized modules. Combined with the need to minimize the number of connections made in traditional analog instrument and actuator lines, this arrangement requires a major on-site effort to install data lines and calibrate systems, particularly sensors. On-site installation is much more expensive than shop installation.

Several technology-based hardware improvements are proposed:

- Change to distributed control, using several modern, low cost CPU containing units. Each is largely shop connected to its immediate sensors and actuators.

- Use of the incoming generation of "intelligent sensors." These are defined by the properties of being self-calibrating, self-diagnosing, and providing digital data output. These properties greatly decrease plant commissioning labor and increase reliability, by elimination of analog signals, which are open to contamination.

- More electric actuator technology, where applied, has similar calibration and reliability merits.

Using the previous approach, interconnection of the major modules is simplified to relatively non-emanding digital data lines and simple power cables.

Software improvements are to be introduced at the same time. The two major changes introduced will be:

- To speed development, obtain independence from proprietary electronics, and give maximum flexibility with universal code recognition, all of 
which come from the use of a graphical input code (Simulink is presently preferred) used to generate " $C$ " operating code. The input code serves both to build the control system characteristics and to represent the plant behavior so the control code can be tested in depth with a minimum of effort. This is critically important to the relatively complex proposed plant.

- Given the complex interactions between fuel input and steam generation, presenting more opportunities for inaccurate control than a standard gas turbine plant, it is intended to advance the control feedback approach from reactive to predictive. Appendix B gives the following illustration of the virtue of predictive control feedback:

- "Notice that the (predictive) strategy is very similar to the control strategy used in driving a car. The driver knows the desired reference trajectory for a finite control horizon, and by taking into account the car characteristics (mental model of the car) decides which control actions (accelerator, brakes, and steering) to take in order to follow the desired trajectory. If the car driving analogy is extended, as has been done by one of the commercial (predictive control) vendors, in their publicity, the (reactive) way of driving a car would be equivalent to driving the car just using the mirror."

Appendix B also addresses safety evaluation using the ALARP (as low as reasonably practicable) risk mitigation approach and a preview of the system reliability.

Interestingly, the reliability analysis presented points to sensors, especially thermocouples, as being a leading source of unreliability. This is can be alleviated by using multiple sensors for the same nominal parameter and an intelligent fault identification circuit - a multiple thermocouple with attached logic constitutes an intelligent sensor with improved reliability.

\subsection{NGT Marketing/Economic Analysis}

Under the Phase I program study, three independent economic and customer market survey studies of proposed Rolls-Royce NGT product configurations were completed. The first study involved a detailed customer market survey performed by PB Power Inc. under contract with Rolls-Royce. This effort involved direct power-generation end user contact and interviews to obtain feedback on Rolls-Royce NGT industrial power plant concepts, as well as North American market attitudes on distributed power generation. Information from this study was used to develop an understanding of the market potential for proposed Rolls-Royce NGT product configurations.

The customer market survey conducted under this contract concluded that most intermediate power producer (IPP) companies consider there will be a market for a 70 to 80 MW relatively high efficiency gas turbine. The main market for NGT is considered the midmerit and peaking market. Principle attributes identified for an NGT product to be successful in the identified market sectors are low capital cost, high efficiency, high availability, and reliability. Water availability was also a crucial factor in the study, since both NGT product configurations feature wet cycle concepts. Having a closed-loop cycle system for water injection was identified as a favorable feature. The customer market survey also noted that the Rolls-Royce brand name carries considerable influence, but the name is more associated with aerospace products. The final 
report completed by PB Power is included in Appendix D for review.

The second project accomplished under this portion of the NGT Phase I study includes an engineering Value Model marketing assessment conducted by DFM Consulting.

The Value Model concept permits a scoring in profit per unit dollars for technology components and power generation configurations (used for this study). A higher score indicates a better configuration. The difference in the scores of the two configurations is a rating of the technology. Any technology with a positive rating is of interest, but higher ratings indicate more promising technologies. Value models were developed as a part of this study for two primary NGT cycle configurations and a proposed spiral wrap recuperator technology. A summary of the research effort is included in Appendix E.

As a part of this contract, the Rolls-Royce Strategic Research Center (SRC) was also retained to conduct an economic and market assessment of proposed NGT product configurations. The goal of this study was to determine economic performance of NGT configurations from the perspective of the end user and assess the competitive advantage of an NGT product relative to existing products.

The customer based economic analysis concluded that both NGT arrangements are likely to offer significant commercial advantage in comparison with other currently available machines of similar power rating, including combined cycle variants.

A detailed summary of this economic analysis is included in Appendix F.

\subsection{NGT Supporting Technologies}

In addition to developing an optimum gas turbine cycle and plant configuration to address the Vision 21 goals as a part of this study, Rolls-Royce Corporation also investigated some key technology areas requiring integration into the advanced cycle design. Specifically, these areas included:

- Low emissions combustion technology

- Advanced materials requirements

- RAM and more-electric systems concepts

- Advanced recuperator technology studies

- Advanced controls sensors and diagnostics

\subsubsection{Combustion Emissions Considerations}

There have been several observations of gas turbine emissions changes resulting from the introduction of water in various forms prior to the combustion process. Steam injected (STIG and Cheng) cycles were introduced some years ago, before the present focus on ultra-low emissions at any price. The typical experience was to find that the gas turbine NOx emissions, already low (100 to 200 ppmv) compared to most power generation plant was further reduced by a factor in the region of 5 to an excellent level of 10 to 25 ppmv.

Assuming the steam is uniformly mixed with combustor entry airflow, the impact of steam on NOx emissions has several components:

- The steam is relatively inert and it dilutes the incoming oxidant stream much as lean, premix (LPM) dilutes the fuel gas stream, lowering the maximum gas temperatures realized locally.

- Steam has roughly twice the specific heat of air, which again constrains peak temperatures such as might occur in a locally stoichiometric region. 
- The presence of steam has a favorable effect on chemical kinetics influencing the formation of NOx.

- The much increased power level per unit dry airflow decreases the accounted emissions corrected to $15 \% \mathrm{O}_{2}$ exhaust.

Although there has been some concern that $\mathrm{CO}$ emissions might rise with the introduction of steam, this has not yet proved to be a problem.

There are several existing combustor standards that could be used for the proposed engine, ranging from relatively low cost aero equipment to substantially more expensive industrial staged DLE units. Aero standard units, even with the more recent reduced emission liners and a high steam injection rate, are considered unlikely to improve all the way to "single digit NOx" though they might make the 25 ppmv level.

The axially staged LPM low emissions combustion system supplied with the current industrial RB211 generator set supports good turndown, is warranted to keep NOx emissions below 25 ppmv over a range of power output, and normally produces significantly lower values. It is expected that high percentage steam injection will be beneficial and that, with retuning of the fuel staging and dilution air distribution, should be consistently in the single-digit category. Detailed evaluation was not considered to be sufficiently decisive or indecisive to justify a detailed chemical kinetics and design assessment for this study. Eventually, emissions performance should be checked throughout the sustained operating envelope, both by computational modeling and by combustor rig calibration to account for such influences as cold days and turndown. The combustion liner sections may not be interchangeable with the simple cycle engine components because they must accommodate about 20 to $30 \%$ more flow without incurring high losses.

If local permitting required inappropriately low levels of emission, selective catalytic reactors (SCR) for $\mathrm{CO}$ and NOx could be fitted to the exhaust system. The presence of large quantities of steam will slightly increase the capacity of SCRs required but will not prevent their functioning.

\subsubsection{Materials Requirements for High Steam Content Application}

Rolls-Royce Corporation familiarity with high steam content combustion engines arises mainly from the program to create and support the 501-KH5 industrial engine product line. This is a nominal $4 \mathrm{MW}$ engine when operating in simple cycle mode, which reaches over $7 \mathrm{MW}$ when operating with steam reinjection amounting to nearly $20 \%$ added mass flow.

In simple cycle operation, the added fuel mass flow is about $2 \%$ of the airflow, which combusts to about $4 \%$ water mass flow. The impact of steam addition of about $20 \%$ causes only a factor of about 6 on the concentration of steam taking part in reactions in the hot section of the engine. Our product experience does not point toward any serious chemical corrosion consequence of running with the high steam content. This remark is qualified by the fact that the turbine entry temperature in the $501-\mathrm{K}$ is about $200^{\circ} \mathrm{C}$ less than that of the proposed NGT product.

Steam chemical content is definitely important because much of the dehydrated residue of mains water is comprised of minerals that will melt and stick to the turbine blades and bring operating efficiency down rapidly. On-line cleaning is only partly effective for turbine blades, due to the airfoil section shape. Two steps have been found necessary to prevent this class of deposits: 
Using makeup water treated to regular boiler clean standard, it is essential to ensure a knockout drum is placed between the boiling surfaces and the combustor steam delivery points. This ensures no droplets deliver traces of impurity present in the boiler water standard to the internal surfaces of the gas turbine hot end. Elimination of droplets also prevents accumulative mechanical damage to hot rotating parts, particularly where these use thermal barrier coatings to achieve good service life.

Structural problems have been experienced, resulting from the attachment of high-pressure steam pipes, which tend to be immensely rigid, to aeroderivative style casings, which are lightweight and fragile by comparison. These can be solved by good practice rather than material changes.

The presence of $20 \%$ more steam in the combustion air raises the working fluid specific heat by about $10 \%$. In the chosen configuration, cooling air is drawn from a point before the steam is added, which therefore retains its original, lower specific heat. This leads to the possibility of slightly inadequate turbine cooling and life degradation due to local overtemperature. The NGT combustor delivery temperature profile will change from the aero patterns, even if the steam is uniformly distributed.

With steam injection systems there is an option to distribute the injected steam selectively in and around the combustion zone and to even displace the dry cooling air coming from the compressor, because the steam delivery pressure can be higher than compressor discharge pressure. This option can be used to preserve or even improve the acceptable engine firing temperature, whereas the wet recuperated system can only deliver uniformly mixed mainstream flow and has no overpressure to displace the cooling air.
Other turbine material issues arise indirectly from the use of steam in the cycle, no matter how it is produced, due to smaller temperature drops in the early turbine stages. The compressors work close to their normal operating points and require their normal input power in the absence of intercooling, but the turbines have about $20 \%$ increased mass flow and $10 \%$ increased specific heat. This results in 30\% less temperature drop through the HP and IP turbines to provide the required power, with the consequences that the IP turbine blade runs about $100^{\circ} \mathrm{C}$ hotter and the first power turbine blade runs about $180^{\circ} \mathrm{C}$ hotter. This takes the uncooled high duty alloy IP turbine blade into a range where it needs minimal cooling. It is anticipated a simple, single-pass, radial passage cast into the exiting airfoil form will provide adequate cooling. The first power turbine blade can remain uncooled but will have to be manufactured in the higher duty alloy already deployed for the IP turbine blade.

\subsubsection{Reliability and Maintainability (RAM) Optimization Studies and More Electric Systems}

To address the issue of identifying critical technologies that could improve the RAM of industrial gas turbine engines, Rolls-Royce Corporation utilized the services of FosterMiller Corporation to conduct a thorough investigation on this topic. Foster-Miller was also called upon to research the issue of more electric machinery components and the associated benefits to the end user if these types of systems (e.g., magnetic bearings) were incorporated in an advanced industrial gas turbine engine combined cycle plant.

Foster-Miller's approach to research the area of RAM and more electric machinery was focused on a general research of the subject matter to identify current trends in the field, and also (more importantly) direct interviews 
with end users to get their perspectives and feedback on what are currently related reliability problems with existing technology industrial gas turbine engines. In addition to addressing specific areas within the gas turbine for improved reliability techniques, a matrix of specific problems and recommended technologies was developed to address improved RAM. A summary of these findings is included in Appendix G.

Since the primary Rolls-Royce NGT cycle configurations feature a wet cycle concept utilizing a large proportion of direct steam injection or humid air cycles for improved efficiency and power augmentation, FosterMiller also conducted research on RAM issues as they pertain to steam injection, which is commonly used in industrial gas turbine applications. Foster-Miller again conducted research with end users and other engineering firms that deal with steam injection technology and concluded that water quality is critical in maintaining effective performance and durability in a steam cycle gas turbine engine; therefore, necessitating careful design of the steam delivery and heating system. This is most important in the hot section gas path in which water droplet size has is proportional to combustion and turbine damage. Steam injection also results in increased turbine blade loading, which must facilitate higher creep strength materials.

RAM issues with a steam cycle are also addressed in Appendix G.

Foster-Miller also conducted research in the potential for using more electric systems in advanced gas turbine cycles. As a part of this research, a trade-off analysis of using electric machinery systems that focused on the two areas of:
- Ancillary equipment_-gas turbine mechanical systems (bearings, pumps, actuators)

- Electric power equipment-electric generators, power transmission devices

A detailed summary including the benefits and hurdles with various more electric system technologies is also detailed in Appendix $\mathrm{H}$.

In addition to the work performed by Foster-Miller, Rolls-Royce Energy Systems Incorporated (RRESI), which has extensive background and experience in the design and manufacture of industrial gas turbine packaging systems, also conducted extensive research in the field of more electric technologies targeted for 50 to $100 \mathrm{MW}$ industrial engine applications. The research conducted by RRESI centered on the following technologies:

- Shaft power transfer

- Electric starter generators

- Active magnetic bearings

- Power electronics-grid interface / direct drive generators

The detailed study summarized in Appendix $\mathrm{H}$ includes a matrix conclusion identifying the applicability to Rolls-Royce products and the economics of developing the proposed more electric technologies.

\subsubsection{Advanced Rolls-Royce Recuperator Technology}

A critical technology considered for application in this Phase I NGT preliminary design study was an advanced recuperator concept termed spiral wrap and developed by Rolls-Royce plc. The advantages of this recuperator technology include lower cost of manufacture, improved durability, and simplicity in the overall design. The spiral wrap concept permits the ability to scale rolled 
recuperator modules for small-to-large size industrial gas turbine engines. This technology also offers a critical advantage for the microturbine industry.

Rolls-Royce has developed a new recuperator specifically designed to address the problems of strength when subjected to thermal cycling and the high cost associated with production complexity. Unlike existing recuperators, the spiral wrap recuperator is made from three continuous sheets of metal wound into a spiral form. The spiral wrap recuperator is believed to be the first recuperator in the world to be made in a continuous production process.

The recuperator achieves a significant increase in thermal fatigue resistance relative to state-of-the-art recuperators through to its compliant structure and careful design of detail features. A previous technology program has already proven the design, manufacture and testing of some recuperator cores. The success of this earlier work has led to the continuation of the program to address the application of this technology to engines from microturbines of $30 \mathrm{~kW}$ up to large industrial and marine gas turbines of $20 \mathrm{MW}$.

Rolls-Royce believes there is a significant market for these recuperators and in particular in the microturbine market where the ability to wrap a circular recuperator around the engine has special advantages. On the back of the business plan, Rolls-Royce is planning the launch and manufacture of these components in a new facility. To this end a study was completed by IBIS Associates under the NGT program, which has specifically looked at planning the recuperator manufacture. The work by IBIS has included a review of potential suppliers in the U.S., a cost study to identify which manufacturing activity controls cost, and a fresh review to understand if any new manufacturing methods should be adopted. The final report from IBIS is included Appendix I.

IBIS collected data from potential suppliers and tooling manufacturers and used this to help compile a cost simulation of the manufacturing process. The simulation was run to identify where the manufacturing costs lie. This simulation was in turn used to test different scenarios and to identify cost improvements. The work helped to identify some cost reduction strategies, potential suppliers, and partners who could help build the recuperator business.

The cost model has shown that material represents the dominant cost. This verifies previous work and enables cost reduction activity to concentrate on material reductions.

The review of the manufacturing process outlined in the IBIS report includes a preliminary review of different welding methods. This highlighted the need to revisit the options of seam and plasma welding.

In establishing the recuperator business, a range of potential suppliers will be needed to provide either individual tools or complete automated solutions. Selection of these suppliers is a time consuming and critical task to the success of the recuperator business. The inquiries IBIS has made are a valued start to the planning of recuperator manufacture in the U.S. Further work lies ahead on this business development, including substantial investment in capital plant, machinery development, product development, and product testing and validation.

Unlike previous gas turbine recuperators, the Rolls-Royce spiral recuperator has a very low parts count and is welded together rather than brazed. This avoids the costs of brazing furnace time and braze alloy. The spiral recuperator is made as a continuous process involving very little labor. Figure 12 shows the 
general construction of the spiral recuperator and demonstrates how the matrix is created in the spiral form. The figure also shows the flow arrangement within the recuperator, with compressor air entering the top and flowing down, while the exhaust gas from the engine enters the spiral at the bottom and flows upwards. Such a counterflow arrangement is essential for high heat transfer.

Traditional recuperators are manufactured from a coil of sheet delivered to the workshop area, which is then cut into smaller rectangular pieces. These are rejoined to form a stack either using a brazing cycle or a welding process. This technique inherently causes a high parts count and high labor content. In comparison, the spiral recuperator can be made from only two sheets with a single fin sheet dispersed between them.

Figure 13 shows how the spiral recuperator utilizes the raw material as a coil and simply unwinds and then rewinds the sheet with another to form a recuperator. This allows the continuous, highly automated process, with minimal parts count and low labor input. The recently completed technology acquisition program has demonstrated this manufacturing process can produce spiral recuperators to a high quality and with minimal labor intervention.

Figure 14 shows a more detailed section through the matrix. As can been seen, there are corrugations on the hot gas side and a

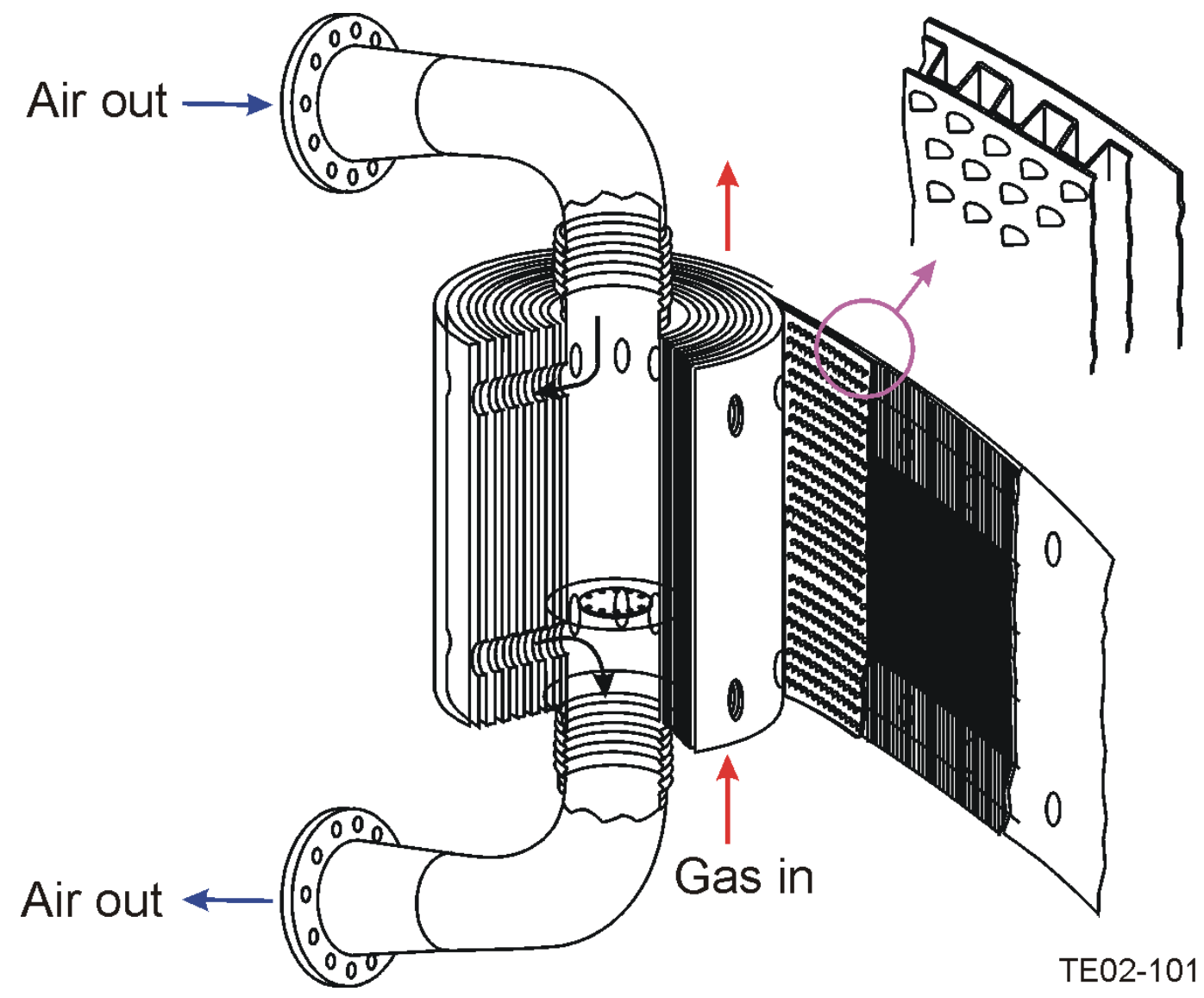

Figure 12. Spiral recuperator construction. 


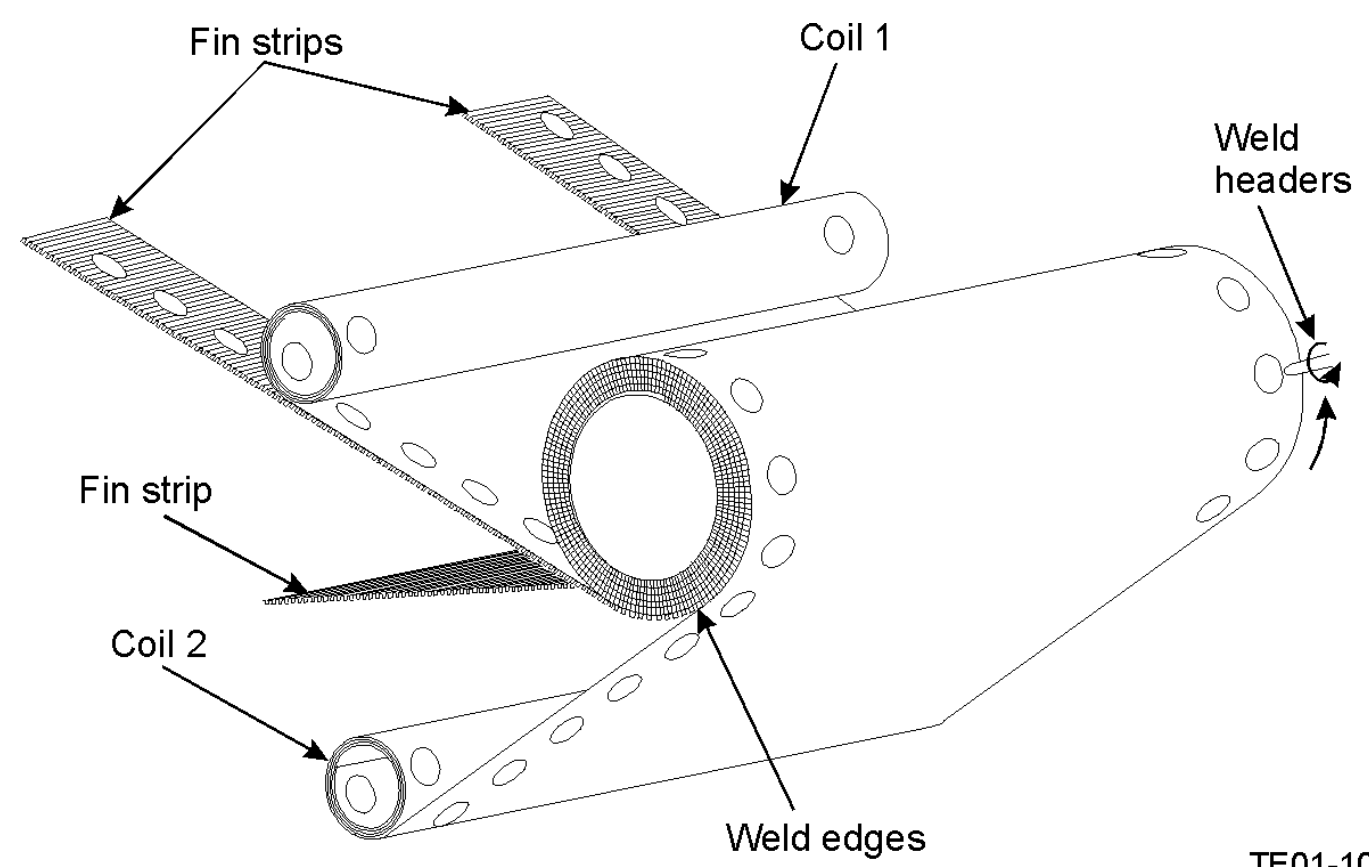

TE01-102

Figure 13. Automated assembly and reduced parts count.

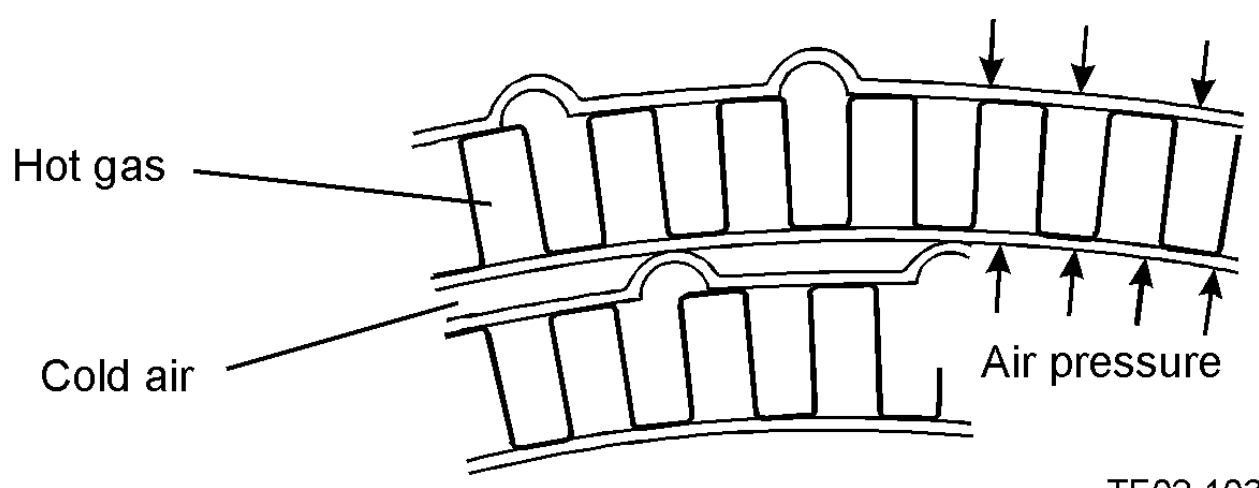

TE02-103

Figure 14. Section through the spiral recuperator matrix.

narrow primary surface on the higher-pressure cold side. These heat transfer surfaces are optimized to give the best overall engine efficiency for cost and pressure drop and allow very high surface area densities to be achieved. One unusual feature of this arrangement is that the fins on the gas side of the matrix are not physically attached to the pressure retaining sheets. Figure 14 shows that instead of using a physical connection such as braze or weld, the high-pressure air is used to maintain contact between the gas side fins and the adjacent sheet. This helps with thermal compliance in coping with rapid thermal transients.

An initial technology demonstrator was manufactured in 1996, and more recently a second and a third unit have been made to demonstrate production techniques and quality. The second and third units have allowed the development of the key manufacturing processes, such as welding, to production standard. 
Recuperator testing has successfully validated the recuperator design methods and provided sound evidence that this recuperator can withstand both the thermal shocks and naval marine mechanical shocks of $20 \mathrm{~g}$ force.

The recuperator was originally designed in 1990 to fit a concept microturbine (Figure 15). For microturbines to become successful, it is essential low cost recuperation is achieved and heat is retained within the engine architecture. A recuperator wrapping around the engine, such as the Rolls-Royce spiral design, contains and saves heat otherwise lost in radiation. A further benefit of a wraparound recuperator is that it serves as a containment ring in the event of a turbine disk burst. Microturbine size recuperators are currently under development.

Machines of up to $20 \mathrm{MW}$ have previously been built and studied. A recuperator arrangement as shown in Figure 16 is considered for machines of large size (20 to $150 \mathrm{MW}$ ) like an advanced NGT concept requiring several recuperator cores with an array of piping. The modularity of the design facilitates easy removal and replacement of recuperator elements when in service.

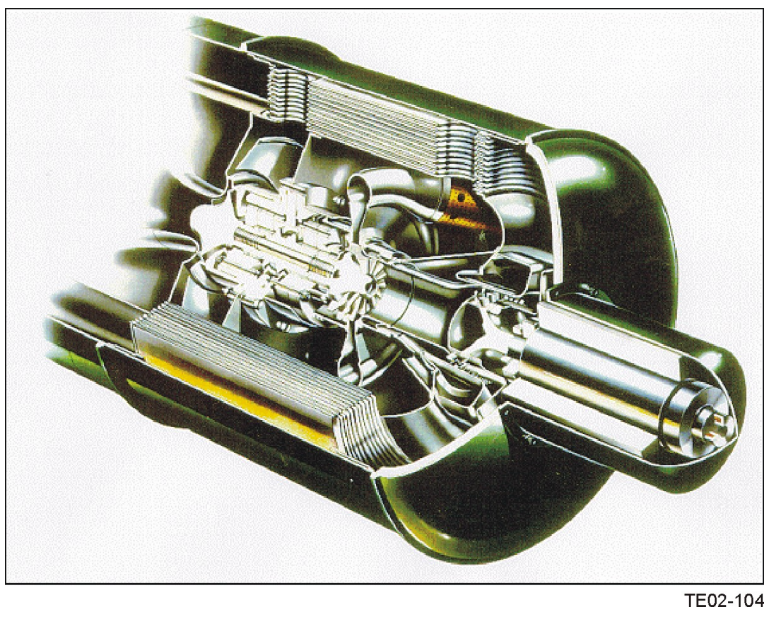

Figure 15. Microturbine design incorporating spiral recuperator.

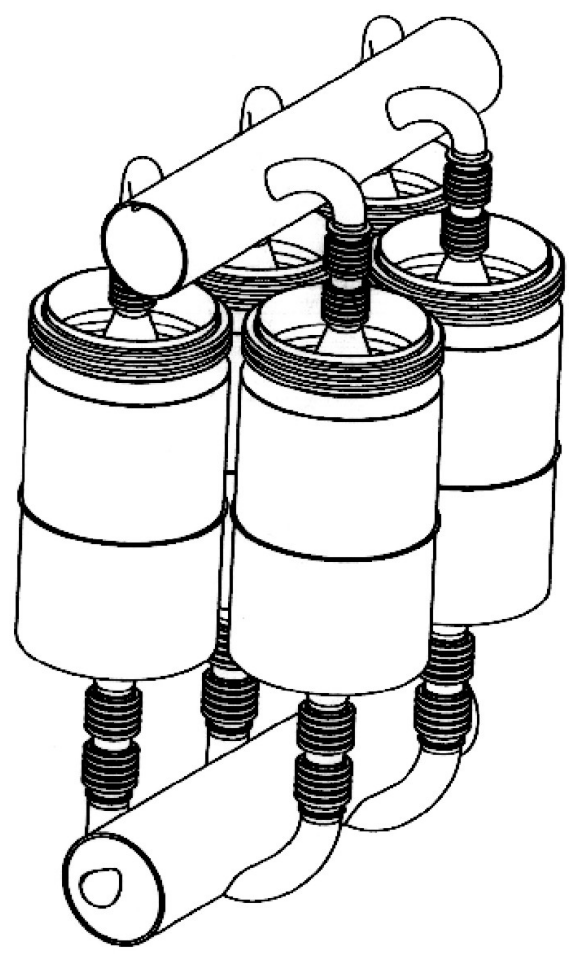

Figure 16. $20 \mathrm{MW}$ concept featuring multiple installation of the spiral recuperator. 


\subsubsection{Advanced Controls Sensors and Diagnostics}

A major focus in improving the reliability of industrial gas turbines is associated with the ability to diagnose engine degradation by use of advanced sensors and electronic control logic and algorithms. Many times, significant downtime and repair costs can be avoided if gas turbine component distress is identified in the early stages before a major component failure occurs. As a part of this preliminary design study, Distrigen Advisory Group was contracted to conduct research into control and monitoring technologies to improve RAM. Much of the research conducted by Distrigen also focused on the users perspective, so realistic assessments could be made along with determining what owners really want and what areas need attention.

This study concluded that operators do not want a complex array of sophisticated sensors. If possible, keeping the system simple is best (in essence, less is more). The study also identified several potential research areas for improving sensor technologies. These included:

- Fuel/air ratio sensors

- Gas leakage monitors

- Gas quality sensor

Other critical areas for more expanded development included plant control algorithms for improved decision support information. These comprise issues such as an ability to override less critical control system functions under certain conditions (something that has much debate on warranties) and risk analysis.

Lastly, the field of diagnostic and prognostic control technologies was also researched. This research concluded that the current technology in industrial gas turbines is limited. Efforts should be concentrated on using historical trend information to suggest probable causes for various situations. Advanced software could suggest areas where parts have failed or components are malfunctioning. This study effort is detailed in Appendix J.

\subsection{NGT Future Program Strategy}

As part of this study, Rolls-Royce Corporation also reviewed the potential for a follow on to the Phase I preliminary design effort under the NGT program. Table II details a potential roadmap for an NGT product utilizing existing Rolls-Royce Trent aeroderivative gas turbine technology and the associated new technology development areas for integration of a wet cycle concept.

While Rolls-Royce is encouraged by the results obtained from this study, there are some concerns about making a significant investment towards a next generation industrial turbine and BOP product configuration. These specific concerns are:

- Uncertainty with the industrial gas turbine mid-merit market capability in 5 to 7 years.

- Rapidly changing market economics in the energy field.

- Magnitude of the investment required (despite a DoE cost share initiative).

- High risk technology programs required (e.g., low emissions coal gas derivative).

- Uncertainty with the funding environment in the DOE for the NGT program as part of a fossil fuels development initiative.

Rolls-Royce utilizes a product design and development methodology known as The Derwent Process to move a technology concept towards a product development effort as well as the entire product the life cycle. Accordingly, further investigation must be accomplished within the DOE stage 2 phase (also know as a Derwent stage 0) before the 
initial tollgate into a Derwent Stage 1 development effort (noted by the yellow triangles in Figure 17). Completion of a Derwent Stage 3 effort would essentially permit full-scale production of the advanced industrial gas turbine product.

Some of these issues must be addressed before Rolls-Royce Corporation can commit to a large-scale industrial gas turbine development effort.

\subsection{Phase I Program Final Technical Briefing}

As required by this contract, a final NGT Phase I program briefing with DOE was accomplished in October 2001. The presentation material is included in Appendix K.

\section{Conclusions/ Recommendations}

The following conclusions and recommendation were derived from this study:

\subsection{System Performance Optimization}

The DOE NGT efficiency solicitation goal is close to $50 \%$, which will not be achieved by any planned simple cycle gas turbine.

An intercooled and recuperated engine with economized cooling flows can achieve $50 \%$ efficiency but at high system cost per kilowatt. Further recuperator technology advance would be required to make this solution affordable.

Evaporation of water vapor, using waste heat and its injection into the combustor zone, doubles engine power output, raises efficiency, and can decrease system cost per kilowatt.

The original proposal to use two turbines in parallel to accept the increased engine mass flow proved less cost effective than increasing main engine turbine capacity.

The lowest technical risk embodiment uses a OTSG in the exhaust to raise steam and

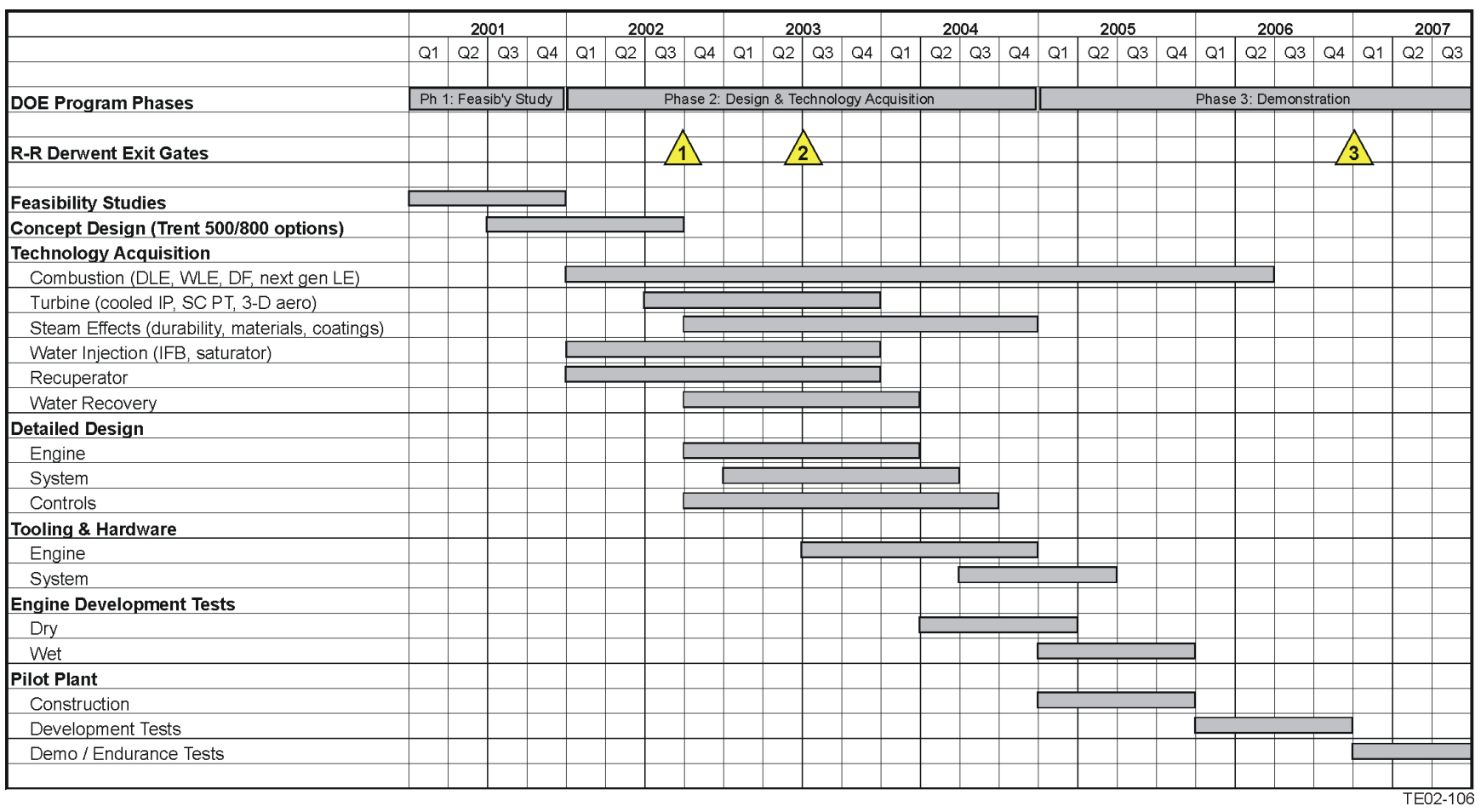

Figure 17. NGT proposed program roadmap. 
reinject it into the combustor zone of a twospool Trent aero core. This achieves about $51 \%$ efficiency. This system achieves combined cycle efficiency at simple cycle cost.

The most efficient version uses the same core with a recuperator and evaporative water vapor intercooling and aftercooling to achieve $54 \%$ efficiency, but at higher risk and cost.

Both enhanced cycles give superior hot day performance

\subsection{Packaging Optimization}

The recirculated cycle water flow requires a large condensing system to recover a sufficient water fraction from the humid engine exhaust. For extremes of weather, some on-site water storage is required.

For rapid system power up, a OTSG, having no boiler drum storage, will be used in place of the more common HRSG.

The OTSG requires a higher standard of water purity than an HRSG. This is most economically provided by purification trailers, regenerated off site.

A distributed control system approach will eliminate much onsite electronic installation, reducing assembly cost and facilitating trouble-free plant commissioning.

Increased system complexity tends to reduce control accuracy. This effect will be averted by upgrading to predictive control feedback and using smart sensors to improve control data quality with minimum complication.

\subsection{NGT Market Survey and Economic Analysis}

Based on the market survey conducted with IPPs, a market for the 70 to $80 \mathrm{MW}$ class of industrial gas turbine with high efficiency and rapid start-up capabilities. Most respondents in the survey believe future power plants will be smaller in capacity due to transmission grid constraints and future plants must be more flexible in operation to meet baseload, mid-merit, and peaking operation.

The main market for an NGT industrial product configuration appears to be in the mid-merit and peaking operation.

Major drivers for a successful NGT product include low capital cost, high efficiency, and improved RAM. A rapid startup capability was observed to be important as well as dual fuel operation. In addition, delivery for NGT product plants should be 12 months.

Water availability was observed to be a major issue with all respondents from the survey. A wet cycle configuration operating on a closed-loop system as proposed by RollsRoyce was viewed as attractive.

Initial product plant configurations were seen as high risk ventures, which underscores the necessity of a DOE sponsored initiative supporting a pilot plant demonstration phase.

The Rolls-Royce brand name was observed favorably in the market, however, the greatest recognition was associated with aerospace gas turbines - all industrial Rolls-Royce engines are aeroderivitive based turbines. The Rolls-Royce technical standing was highly regarded.

Key NGT technologies identified as having high technical risk during the survey were the water recovery system and a recuperator.

Economic analysis performed with the assumption of an NGT product configuration in simulated market scenarios seem to suggest that both of the NGT cycle arrangements considered offer significant commercial advantages and margins. 
A Value Model for Rolls-Royce NGT product configurations, including a component level assessment of the spiral wrap recuperator technology, has been established as a part of this study contract. The Value Model has identified critical design factors that are important to develop the greatest profit drivers.

\subsection{NGT Supporting Technologies}

A matrix of problem areas and recommend corrective action to improve RAM in gas turbines has been developed as a part of this study and is included in Appendix G. Specific RAM technologies include optical spectrometry, pyrometers to evaluate blade/vane metal temperatures, ultrasonic cleaning for improved debris removal, and eddy current sensors.

RAM improvements in reference to steam injection were also reviewed. In general, water quality with steam injection is critical in improving the reliability of the engine. Careful attention must also be paid to the steam and water delivery system. Many early problems with steam injection occur as a result of water droplets impinging on hot section components, which results in erosion damage and thermal gradients. In some cases, higher creep strength turbine materials may be required to accommodate higher blade loading.

More electric machinery options with an NGT product configuration include magnetic bearings, adjustable frequency drives, electric actuators, and high voltage generators. While each technology does offer advantages, each presents unique constraints. An NGT product configuration featuring magnetic bearings in the inline shaft system was not considered for further research and development. A cost study for proposed more electric turbomachinery concepts was also performed and is included in Appendix $\mathrm{H}$.
A manufacturing study conducted on the spiral wrap recuperator system considered for one NGT cycle configuration was accomplished and provided insight into a cost effective methodology for high volume manufacture. Based on the analysis performed and the results from the cost study, material cost is the controlling factor in the spiral wrap system manufacture. Special attention must be paid to material usage and scrap minimization. Utilization of plasma cutting welding techniques appears to yield greater economic benefits than conventional cutting and joining methods.

Analysis of the process flow for manufacture of the spiral system was also optimized as a part of this study. Finally, a list of recommended U.S. based suppliers was developed to provide manufacturing services or special machines for high volume manufacture of the spiral wrap recuperator. Results of this study also pertain to a potential microturbine sized recuperator system.

A review of sensors and diagnostic monitoring techniques for turbomachinery applications performed as a part of this study concluded that the needs of the operator are critical when implementing improved controls and sensor systems. Simplicity in sensing and control technologies for the operator is beneficial. Several sensing technologies that can improve RAM on gas turbines have been identified and are presented in Appendix $\mathrm{J}$.

The presence of a large steam fraction in the combustion zone is very helpful in minimizing emissions. However, the favored specification of ultra-low emissions, effectively regardless of cost, will probably require significant combustor development.

No materials problems are expected to result directly from the introduction of large quantities of steam. Minor problems arise from temperature distribution changes arising from 
cycle changes and steam distribution profile effects.

\section{NGT Future Program Strategy}

A proposed followon NGT strategy incorporating the Rolls-Royce Derwent

Process for transition of research and development technology programs towards full-scale product development was developed as a part of this research study. However, there are several variables concerning the future of an NGT program in Rolls-Royce that must be resolved before a commitment on the Phase II program can be achieved. 


\section{Appendix A System Performance Optimization: Cycle Analysis/Turbomachinery Selection}




\section{Wet Cycle Refinement - Summary}

Wet cycles are attractive because they offer substantial power and efficiency gains over conventional cycles. Power rises because water increases both the mass flow and the average specific heat of the turbine working fluid without requiring compression work. The efficiency improves because the water returns heat from the turbine exhaust to the cycle.

In a fully implemented system, the extra mass flow entering the turbines usually requires a turbine capacity increase, at least for the first stage, to prevent the compressor surge margin from being decreased unacceptably. Part of the approach toward achieving DOE cost objectives, adopted in the Rolls-Royce proposal, is to minimize the requirement for high cost non-standard turbine parts. The cycle suggested in the proposal avoided this need by providing additional turbine capacity in a second parallel-connected machine. If the primary machine is the RB211 industrial engine and the parallel unit is the combustor-expander section of the 501-KB7S, the proposed NGT plant could be based on two standard industrial production skids. Detailed assessment showed that, though considerable improvement can be attained this way, the resulting plant does not meet the DOE cost goal and is marginal in terms of efficiency.

Wet recuperated cycles on a single flowpath were found to offer attractive levels of efficiency. If the air is additionally saturated part way through the compression the efficiency is further enhanced as is the power output. Alternatively, water can be introduced to the cycle as steam raised in a Once Through Steam Generator (OTSG). Power and efficiency were once again found to be attractive, though intercooling did not offer further SFC gains. The steam injected engine was chosen as the preferred NGT candidate. Power and efficiency are high and development risk is considered low - the concept is an amalgamation of existing technologies. The wet intercooled, wet recuperated concept is promoted as a downstream development for NGT. It offers enhanced efficiency, power and reduced $\$ / \mathrm{kW}$, though this is offset by increased technological risk.

The steam injected engine was investigated at off design conditions. It was found to offer superior power lapse rate on hot days when compared to traditional machines. The ability to vary steam flow independently allows unique potential for actually boosting power on hot days. Issues surrounding the changes to engine components in a steam injected engine were explored. No unmanageable technological risks were identified.

\section{Parallel Turbine Layout (See Appendix K, Slides 37-38)}

Introducing large amounts of water downstream of the HP compressor results in the turbine flows being substantially larger than those in the compressors. The extra turbine mass flows require that the turbines be resized to suit. Not only is the mass flow higher, the specific heat of the water is much larger than that of air and its presence increases the average specific heat of the fluid. For each shaft, to drive the compressor at the same working point requires less temperature drop across the turbine. A lower temperature drop in each of the turbine stages means that the entry temperature to the downstream turbines increases. The flow capacity of the HP turbine must be increased, the flow capacity of the IP is little changed and the capacity of the power turbine must be reduced. The IP and Power turbines may also require extra cooling or material change to retain acceptable life at their increased operating temperatures. 
It is possible to use existing, unaltered turbo-machinery and avoid these changes if the extra mass flow is diverted to a parallel combustor/turbine unit. This arrangement was described in the proposal. It was modeled with an RB211 primary machine operating at a stator outlet temperature (SOT) of $1525 \mathrm{~K}$ with an overall pressure ratio of $21: 1$. Of several existing candidates within Rolls-Royce, the Allison 501 was identified as the most suitable parallel turbine.

The cycle efficiency, at around 48\%, was, in NGT terms, disappointing. The 501 is limited to an SOT of $1350 \mathrm{~K}$ and an expansion ratio of 20:1 is far larger than that for which it was designed. The result is that the flow exits the turbine at very high velocity and thus high kinetic energy. The subsequent loss of this energy results in poor efficiency for the second shaft. An alternative strategy of throttling the flow upstream of the turbine results in a similar loss of energy and once again the poor second shaft efficiency drags down the efficiency of the overall cycle.

The parallel turbine concept has further drawbacks. The addition of a second combustor and turbine increases complexity and cost. The $\$ / \mathrm{kW}$ initial cost will become unattractive and the system required to control the plant would be complex.

As a result of these drawbacks the concept of using a parallel turbine to swallow the extra wet cycle mass flow was abandoned.

\section{Two Spool Wet Recuperated Cycle(See Appendix K, Slides 39-41)}

Recuperated cycles achieve high efficiency by returning much of the exhaust heat to the cycle. The saturator downstream of the compressors adds water to the high pressure flow and cools it, enabling the recuperator to extract more heat from the exhaust gas. Additional exhaust heat is recovered by the economizer downstream of the recuperator, which is used to warm the feedwater and thereby increases the amount of water that can be vaporized in the cycle.

The saturator, recuperator and economizer constitute a complex interlinked system and the overall cycle pressure ratio influences how they interact. As pressure ratio rises so does the compressor exit temperature and hence more water can be absorbed in the saturator. The extra water rapidly increases the cycle power (Appendix K, Slide 41) and also decreases the stack temperature. The extra heat recycled from the exhaust improves the efficiency. However, rising pressure ratio is accompanied by a fall in turbine exit temperature and a consequent fall in the temperature of recuperator air-side delivery. This increases the amount of fuel that must be burnt to achieve the SOT and the result is poorer efficiency.

Three turbo-machinery options were modeled to cover a range of cycle pressure ratios. Firstly, the Industrial RB211, with 7 stage IP compressor at a PR of around 21:1. If an extra stage is added to the IP compressor the pressure ratio rises to around 27:1. The addition of the Industrial Trent LP Compressor upstream of the IP Compressor further raises the PR above 33:1. The results of the study are shown in Appendix K, Slide 41. The efficiency falls steadily with pressure ratio, more rapidly above a pressure ratio of 30:1.

\section{Intercooled, Wet Recuperated Cycle (See Appendix K, Slides 42-43)}

Intercooling is traditionally employed in a simple cycle gas turbine to raise the power with little effect on SFC. The cooled inlet to the HP compressor reduces the work done by that component but the cooler delivery air requires more fuel to heat it to the SOT. In a dry 
recuperated cycle intercooling boosts efficiency because, despite the HP compressor delivery air being cooler, the recuperator air -side exit temperature will remain the same. The power boost from intercooling is not offset by extra fuel burn and hence SFC improves.

Appendix K, Slide 42 shows both wet and dry intercooling as applied to the wet recuperated cycle. The wet intercooled layout has additional water feed from the economizer that saturates the HP compressor inlet flow. The flow is again saturated downstream of the HP compressor. The dry intercooler features a conventional heat exchanger between the IP and HP compressors to cool the air with no addition of water.

Appendix K, Slide 43 shows that both dry and wet intercooling improve cycle efficiency. The HP compressor work falls but the recuperator effect avoids extra fuel being burnt to achieve the SOT. The dry intercooled, wet recuperated cycle suffers a severe drop in power compared to the non-recuperated case. The HP compressor delivery air is cooler than in the non-recuperated case and is thus less capable of evaporating water in the saturator. The cycle water / gas ratio is lower and hence the cycle power falls.

The wet intercooler has a different effect on the cycle. As the liquid water evaporates into the flow the phase change cools the mixture, giving the desired intercooling effect. However, the liquid water from the economizer is warm and has a high specific heat capacity (Cp). When mixed with the air-flow in the wet intercooler the effect is to raise the amount of heat energy in the flow (enthalpy) even though the temperature falls. At HP compressor exit the total flow enthalpy is higher for the wet intercooled case than it is for the case with no intercooling and the wet intercooled case is therefore capable of absorbing extra water at this point. Despite the intercooling effect the wet intercooled, wet recuperated cycle can still achieve a high water / gas ratio and therefore cycle power.

Note also that the dry intercooler removes heat from the cycle and loses it to the environment. In contrast, the wet intercooler returns heat to the cycle via the water warmed in the economizer. The recovery of cycle heat in the wet intercooled case versus the removal of cycle heat in the dry intercooled case is the explanation for the higher cycle efficiencies obtained for the wet intercooled case on Appendix K, Slide 43.

Dry intercooling was rejected because, despite offering a small efficiency gain, the cost to cycle power is unacceptably large. Wet intercooling however, offers not only a useful power gain, but also an impressive efficiency boost over the already high efficiency of the wet recuperated cycle.

\section{Steam Injected Cycles (See Appendix K, Slides 44-46)}

In this arrangement (Appendix K, Slide 44) a Once Through Steam Generator (OTSG) is located in the exhaust downstream of the power turbine. It is used to raise steam that is mixed with the compressor delivery air upstream of the combustor. Unlike a Heat Recovery Steam Generator (HRSG), the phase boundaries within the OTSG can move. This improves the flexibility of the plant, particularly during transients and permits the amount of steam generated to be varied at will by controlling the water input rate, down to zero (running dry) if necessary. 
Appendix K, Slide 45 shows the effect on power and efficiency of pressure ratio in a steam injected cycle. For all pressure ratios, as the steam flow rises so does the power. There is, however, a steam flow above which the efficiency starts to fall. When steam flow is below this value there is enough heat in the turbine exhaust to superheat the steam in the OTSG to within a few degrees of the turbine exhaust temperature (the OTSG pinches at steam exit). As steam flow is increased there comes a point at which the available heat in the exhaust is no longer sufficient to heat the steam to the temperature where it pinches at exit. The OTSG pinches internally and the steam delivery temperature starts to fall. Falling steam temperature reduces the combustor inlet temperature and hence the efficiency of the cycle starts to drop.

Note that the fall in efficiency with steam flow is reasonably slow. If it were desirable to increase power with some cost to efficiency, this would be entirely possible. The data indicates that power boosts of 5-10\% are achievable with less than $0.5 \%$ deterioration in SFC. An example of what can be achieved by re-optimizing the cycle for power rather than efficiency is shown on Appendix K, Slide 45a.

Appendix K, Slide 45 also indicates that, in contrast to the wet recuperated cycle, the efficiency of the steam injected option increases with increasing pressure ratio and the cycle water consumption at peak efficiency falls. (Note that, for this study, the inlet air mass flow was held constant). These trends result from interplay between several factors. These include the effect of pressure ratio on compressor delivery and turbine exhaust temperatures, and also the effect of water pressure on phase boundary locations within the OTSG. Nevertheless, the slide shows that at a Pressure Ratio of 27 the steam injected cycle is operating at a favorable point. Appendix K, Slide 46 shows the efficiency and power curves for an 8 stage IP compressor based steam injected machine operating at approximately this pressure ratio. At this time the shaft efficiency and power were predicted to be $52 \%$ and $75 \mathrm{MW}$ respectively.

\section{Steam Injected Cycles with Intercooling (See Appendix K, Slides 48-49)}

Appendix K, Slide 48 shows the effect of dry intercooling on the steam injected cycle at constant inlet air mass flow as steam flow is varied. It will be noticed that, despite offering a substantial power boost for a given steam flow, the dry intercooled machine has rather poor efficiency. The power increase is due to the reduction of HP compressor work but the result is a far cooler compressor delivery temperature. With no recuperator this temperature reduction needs to be made up by burning extra fuel. Note also that the efficiency is further reduced by the pressure drop across the intercooler air side.

Appendix K, Slide 49 shows the effect of wet intercooling. The power boost is even larger than for the dry intercooled case for two reasons. Firstly, the wet intercooler itself adds water to the flow. Secondly, once this extra water has passed through the cycle it carries heat that allows more steam to be raised in the OTSG. This further increases the amount of water in the cycle for a given inlet air mass flow. Despite the increase in power, the efficiency falls with wet intercooling. Once again this is as a result of the cooler HP compressor exit temperature.

The other issue in mitigating against intercooling with the steam injected concept is the effect on turbine inlet temperatures. The very large power boosts resulting from intercooling, and the 
reduced work in the HP compressor, together result in uneconomically high temperatures at inlet to the IP and Power turbines.

\section{Bottoming Compressor (See Appendix K, Slides 50-51)}

In this configuration a compressor operating in the exhaust system, downstream of the recuperator and economizer and discharging to ambient, reduces the back pressure on the turbines. The cycle power output is increased without a rise in cycle temperature because the compressor inlet temperature is less than that entering the late sections of the power turbine. The power gain from using this compressor can be increased further by using an intercooler between the economizer and the compressor intake.

Appendix K, Slide 51 shows the effect on power and efficiency of a wet recuperated cycle as the inlet temperature to the bottoming compressor (i.e. the intercooler effectiveness) is varied. It will be noticed that the cycle with bottoming compressor does indeed deliver a substantial power boost regardless of the intercooler effectiveness.

The efficiency of the cycle, however, is reduced over most of the intercooler effectiveness range. The turbine exhaust temperature is reduced as the expansion ratio rises and therefore the recuperator air-side exit temperature falls, requiring greater heat input to the combustor to achieve the SOT. The extra power generated is not great enough to improve overall efficiency unless the bottoming compressor inlet temperature is very low.

The kinks in the efficiency and power curves are explained by water condensing out in the intercooler as the flow is cooled below the saturation temperature. The condensed water is removed from the cycle, reducing both the mass flow and the specific heat capacity of the flow the bottoming compressor must pass. It is only when the temperature becomes low enough to condense out most of the water that the efficiency rises above that of the wet recuperated cycle without bottoming compressor.

There are significant practical drawbacks to the addition of a bottoming compressor. The power turbine will require an additional or enlarged last stage and diffuser system due to the increased volume of the exit flow. More importantly, the very large (reaching 75' height), normally flat sided exhaust structures will now have to be reconfigured and reinforced to withstand large compressive loads.

The bottoming compressor was rejected as it was felt that the power and efficiency gains were not sufficient to justify the re-engineering of major plant features and added expense.

\section{Cycle Selection (See Appendix K, Slides 53-57)}

The studies into the various wet cycles suggested 3 cycle configurations worthy of further review. These were:

- Wet recuperated engine

- Wet intercooled, wet recuperated engine

- Steam injected engine. 
These cycles were based upon turbo-machinery derived from the RB211/Trent family. Each could be specified with either a 7 stage or 8 stage IP compressor, giving a total of 6 variants in all. The 6 variants are summarized, and their constituent components tabulated, on Appendix K, Slide 53. Note that the ' 80 ' variants are based upon the 8 stage compressor, the ' 60 ' variants on the 7 stage compressor, the numbers referring to the power output in MW rounded up to the nearest 10 .

Appendix K, Slide 54 shows the 6 variants compared on power, efficiency, power turbine entry temperature, water flow rate, HP turbine capacity change and perceived risk of implementation. It is clear that the ' 80 ' variants with the 8-stage compressor have substantially higher powers. One of the prime objectives of the NGT project is to deliver a low $\$ / \mathrm{kW}$ initial cost. It is far easier to achieve low $\$ / \mathrm{kW}$ on a cycle with high power than on a low power cycle. The higher pressure ratio of the 8 stage IP compressor has other benefits. The IP and Power turbine inlet temperatures are reduced and the steam-injected engine has higher efficiency at high pressure ratios. For all of these reasons an 8 stage IP compressor is preferred.

Though the wet recuperated cycles show a decline in efficiency with increasing pressure ratio, the rate is slow in this region. It was considered unwise to add an Industrial Trent LP spool and push the pressure ratio even higher, The risks of developing a combustor operating at high watergas ratio and an elevated pressure were considered too great.

The wet recuperated cycles, with and without wet intercooling are considered to involve similar degrees of risk. Both the intercooler and saturator are similar devices whether they administer the water through spray nozzles or via a saturator tower. The wet intercooler variant enjoys an efficiency advantage of around $2 \%$ points and is thus preferred to the non-intercooled version.

The comparison of the steam injected and wet intercooled, wet recuperated cycle is tabulated on Appendix K, Slides 56 and 57. It is felt that, on balance, the lower risks inherent in the development of the steam injected option outweigh the modest advantages in power and efficiency of the wet intercooled, wet recuperated cycle. The wet intercooled, wet recuperated option has not been discarded but, rather, is seen as a potential downstream development, using the same engine components as the steam injected engine.

\section{Off Design Performance}

The design point study has shown that both the steam injected and wet intercooled, wet recuperated versions of the NGT machine show promising power and efficiency characteristics. The next phase of the work takes the study a step further to understand in more depth the behavior of the cycle at different ambient conditions and at part load.

The first step was to undertake a major review of the modeling assumptions and to set up a new, more accurate model based upon real component characteristics. The NGT80SI cycle is shown, in diagrammatic form on Appendix K, Slide 60, which includes representations of the water recovery and treatment plant. The predicted cycle performance resulting from this study was a net cycle power of 73.3MW at an electrical efficiency of $51.1 \%$. 


\section{Effect of Ambient Temperature on Steam Injected Cycle (See Appendix K, Slides 62-64)}

Appendix K, Slide 62 shows the effect of ambient temperature on the electrical net power of the steam injected cycle with 8 stage IP compressor as compared to a simple cycle machine represented by the Industrial Trent. The right hand chart shows the powers normalized to power developed on an ISO day. The chart shows that on a hot day the power of the NGT80 SI cycle falls at a rate about $30 \%$ lower than the simple cycle machine. Both the NGT80SI and simple cycle curves are the result of calculations at constant stator outlet temperature (SOT). The reason for this improved power lapse is that on hot days the water-gas ratio of the cycle rises, the OTSG raises more steam relative to the air mass flow. Two factors contribute to this. Firstly, the temperature of the feed-water for the OTSG is assumed to rise with ambient temperature and secondly, the power turbine exit temperature rises. On an ISO day the water / gas ratio at the combustor is around 0.165 . At elevated temperatures, a limit on water-gas ratio of 0.2 has been applied. This is believed to be a reasonable limit below which combustion should not become difficult. The limitation is achieved by reducing the water flow at ambient temperatures above $35^{\circ} \mathrm{C}$.

At ambient temperatures below $15^{\circ} \mathrm{C}$ the power output of the NGT80SI steadily falls but the output of the simple cycle continues to rise down to temperatures of $0^{\circ} \mathrm{C}$. In cold ambient conditions the non-dimensional speed of the IP compressor rises. For any given compressor there is a limit on non-dimensional speed which is applied to ensure mechanical integrity. At $15^{\circ} \mathrm{C}$ the simple cycle machine has some margin on non-dimensional speed and thus ambient temperature can fall to $0^{\circ} \mathrm{C}$ before it must be limited by a reduction in SOT. It is this SOT reduction which causes the developed power to level off.

To maximize the ISO power of the NGT80SI and thus achieve minimum $\$ / \mathrm{kW}$, the nondimensional speed of the IP compressor is very close to the mechanical limit at $15^{\circ} \mathrm{C}$. As temperature falls, therefore, there is no margin and the non-dimensional speed must be limited, which is done by reducing SOT. The alternative strategy, of reducing steam flow, was found to lead to reduced part load efficiency, the reasons are discussed later.

Appendix K, Slide 63 shows electrical efficiency of the same machines over the same range of ambient temperatures. It will be noticed that the response of electrical efficiency of the steaminjected cycle to ambient temperature is impressively flat. It remains above $50 \%$ over the entire ambient range between $-25^{\circ} \mathrm{C}$ and $+45^{\circ} \mathrm{C}$. Further, on hot days, the NGT80SI efficiency lapse is far slower than for a conventional cycle. The reason for this is the rising water / gas ratio in the steam-injected machine. Likewise, on colder days it is the falling water / gas ratio, in conjunction with the requirement to limit IP compressor speed by reducing SOT, which explains the steady fall in electrical efficiency.

On a moderately hot day $\left(20-30^{\circ} \mathrm{C}\right)$, the IP compressor slows down and the water-gas ratio is still comfortably below the limit of 0.2 . If required, these margins can be exploited by increasing steam flow up to the limiting water-gas ratio to increase power at some cost to efficiency (Appendix K, Slide 64). Appendix K, Slide 45 shows there is a steam flow at which peak cycle efficiency occurs. At this steam flow there is just enough heat in the exhaust to superheat the 
steam to within a few degrees of the turbine exhaust temperature. Increasing steam flow beyond this point results in increased power but the delivered steam is now somewhat cooler than the turbine exhaust and hence efficiency falls.

At $25^{\circ} \mathrm{C}$ it was found that a power boost of $8 \%$ could be achieved, at a cost of only $0.25 \%$ efficiency, by raising the steam flow by $14 \%$. This plant flexibility could be especially useful in hot regions where power demand rises on hot days due to air-conditioning loads.

\section{Part Load/ Cold Day Strategies (See Appendix K, Slides 65-67)}

A reduction in power of a conventional cycle, using a free power turbine, requires lower fuel flow, with a corresponding reduction in turbine temperature and cycle efficiency. The steam injected engine offers the flexibility to reduce power by an alternative strategy; the steam flow rate can be reduced whilst maintaining constant SOT. Appendix K, Slide 65 shows normalized efficiency versus normalized power for a simple cycle machine and the NGT80SI. Curves are shown for the NGT80SI with the power controlled by SOT reduction and reduction of steam flow. It is clear from this diagram that the ability to control power by reducing steam flow does not offer an advantage. The efficiency fall off with steam flow reduction is faster than that for either the simple cycle machine or the NGT80SI controlled by SOT reduction but starts at a higher level such that NGT80SI engine has better part load performance than the simple cycle.

As has previously been discussed, a similar requirement to limit engine power, or more specifically, IP compressor speed, occurs at low ambient temperatures. Once again, the option to control the engine in this condition by reducing the steam flow is available. Appendix K, Slide 66 shows the results of a study into this possibility. It is found that varying the steam flow is not advantageous. At low ambient temperatures, following this strategy results in a faster lapse in both power and efficiency. In simple terms as the steam flow is reduced, the cycle becomes more like a dry simple cycle machine. It will be recalled that the efficiency of dry simple cycle machines is up to $20 \%$ worse than wet machines and reducing steam flow bridges this gap. A wet engine at low SOT performs considerably better (in terms of both power and efficiency) than a dry simple cycle engine at high SOT.

Appendix K, Slide 67 shows the SOT reduction required to achieve power turndowns for the simple cycle machine and the NGT80SI. It will be noticed that for a given reduction in power, it is necessary to reduce the SOT by less for the NGT80SI. The advantage of this is that SOT reduction results in higher production of $\mathrm{CO}$. By maintaining a higher SOT at a given percentage load, the NGT80SI can achieve greater turndown without breaching pollutant limits. The reason for this is that when NGT80SI SOT is reduced, the water-gas ratio falls with it as a consequence of falling turbine exhaust temperatures. This reduces power output in its own right and thus for a given power turndown less SOT reduction is needed.

Studies into the behavior of the steam injected cycle in different ambient conditions and at part load have shown that maintaining adequate surge margin is unlikely to be an issue. 


\section{The Wet Intercooled, Wet Recuperated Cycle (NGT80WIWR) (See Appendix K, Slides 68)}

The wet intercooled, wet recuperated cycle is considered to be a downstream development of the steam injected engine with lower $\$ / \mathrm{kW}$ and higher power and efficiency. To ensure that this was indeed the case a new model of the wet intercooled, wet recuperated model was set up in the same rigorous manner adopted for the model of the steam injected machine. A representation of the plant and the predicted net power and electrical efficiency are shown on Appendix K, Slide 68.

\section{The NGT80WIWR Cycle, Response to Ambient Temperature (See Appendix K, Slides 69-70)}

In modeling the WIWR cycle, the saturation of the air both upstream and downstream of the HP compressor is to be achieved by saturator towers. These units are inflexible in as much as the quantity of water vapor they deliver to the flow is always that flow which causes saturation. Appendix K, Slide 69 shows the effect this inflexibility has on a hot day. The increase in temperature of the feed-water and the intake air increase the amount of water absorbed into the cycle. In common with the steam injected cycle, the water / gas ratio begins to approach the limit of 0.2 . In contrast to the steam injected cycle the water-gas ratio cannot be controlled by varying the water flow (saturator wicks must not be allowed to dry out). It should also be noted that the sensitivity of water / gas ratio to ambient temperature is higher for this cycle. With no reduction in SOT the water-gas ratio reaches 0.2 at $32^{\circ} \mathrm{C}$ compared with $35^{\circ} \mathrm{C}$ for the steam injected engine. One means for holding the water-gas ratio down is to reduce the SOT. This reduction in SOT causes the rapid reduction in power and efficiency for the WIWR cycle above $30^{\circ} \mathrm{C}$.

Though it has not been investigated to date, it may be possible to mitigate this effect. Partially bypassing the economizer will reduce the saturator feed temperatures and hence the water-gas ratio.

\section{The NGT80WIWR Cycle, Part Load Efficiency (Appendix K, Slide 71)}

Appendix K, Slide 71 shows that the part load efficiency lapse of the wet intercooled, wet recuperated cycle is far superior to that of either the NGT80SI or simple cycle machines as represented by the Industrial Trent. The reason for this is the greater sensitivity of water / gas ratio to ambient temperature for this cycle. As ambient temperature falls, the water / gas ratio falls and thus at any given percentage power, the SOT will be higher for the WIWR engine than for the steam injected engine. The cycle thus benefits from higher simple cycle efficiency and increased heat recovery at part load. This appears to be at odds with the conclusion drawn previously, that for the steam injected engine it is better to reduce SOT than water / gas ratio. The difference is that, in the steam-injected engine, only the water is heated by the turbine exhaust gas stream. Reducing this water flow means that the heat extracted from the exhaust falls and thus the stack temperature rises. In the recuperated engine both the water introduced in the intercooler and saturator and the combustor delivery air are heated by the exhaust stream. Reducing the watergas ratio does not reduce the temperature drop in the turbine exhaust and therefore the stack temperature does not rise. It is because of this that the wet recuperated, wet intercooled cycle has a significant efficiency advantage at part load. 


\section{Engine Component Adaptation Issues (See Appendix K, Slides 73- 76)}

The proposed steam injection solution requires a number of changes to the design of existing Rolls Royce turbines. A preliminary study has been undertaken of the implications of these changes. The intention has been to ensure that the proposals are achievable without undue risk and do not require technology that is not presently available within Rolls Royce. The greater turbine mass flow of the steam-injected cycle requires that the swallowing capacity be increased, by around $20 \%$.

As described, the greater mass flow and specific heat capacity of the flow through the turbines results in a reduced temperature drop across each turbine stage. The result is higher temperatures at inlet to both the IP and Power turbines. To accommodate these temperature increases, the IP rotor blade will require some cooling. The preferred solution is casting a simple core into the existing form. It is likely that the Power turbine will remain uncooled which means that single crystal material will probably be required. This may impose some restriction on airfoil geometry.

Were the wet intercooled, wet recuperated machine to be developed it would be necessary to remove flow downstream of the HP compressor for saturation and subsequent heating in the recuperator. A preliminary study was undertaken to determine how practical this would be. Forming manifolds on the engine as per the WR21 (Appendix K, Slide 74) would require increased engine length. This is not judged to be a high risk but would reduce parts count commonality with aero machines.

An alternative is to extract air from the HP compressor diagonally outwards through the rear of the combustor casing via ducts passing between adjacent combustor discharge nozzles (Appendix K, Slide 75). These would combine in a ring manifold outside the engine casing. Return would be via a similar manifold feeding pipes entering the front of the combustor casing. CFD analysis suggested that this configuration would have acceptable pressure drop.

Appendix K, Slide 76 summarizes the relative merits of using spray nozzles or saturator towers to saturate the flow both upstream and downstream of the HP compressor. The saturator tower is preferable as it performs its own water treatment and the number of spray nozzles required to pass the water flows would be excessive. 


\section{Appendix B \\ Packaging Optimization: \\ Next Generation Turbine System Controls}




\begin{tabular}{|c|c|c|c|}
\hline \multirow{3}{*}{\begin{tabular}{|l|} 
ROLLS \\
DD \\
TII \\
ROYCg \\
\end{tabular}} & \multicolumn{2}{|l|}{$\begin{array}{r}\text { Security Classification: }{ }^{\# 01} \\
\text { Unclassified }\end{array}$} & \\
\hline & \multicolumn{2}{|l|}{$\begin{array}{l}\text { Security Classification:\#01 } \\
\qquad \text { Unclassified }\end{array}$} & \\
\hline & \multicolumn{2}{|c|}{$\begin{array}{l}\text { ROLLS-ROYCE plc } \\
\text { TECHNICAL REPORT }\end{array}$} & \\
\hline Discipline\#10 & Document Number\#52 & Report Number\#12 & Issue No.\#13 \\
\hline \multicolumn{4}{|l|}{ Title\#15 } \\
\hline Authors\#20 & \multicolumn{2}{|l|}{ Telephone: } & \\
\hline
\end{tabular}

Summary $\# 60$

The proposed Next Generation Gas Turbine (NGGT) Control System for Rolls-Royce Corporation is based on open architecture and communications using commercial off-theshelf technology where possible. This document outlines the:

-Hardware and software components required

-Preliminary reliability of system

-Cost savings of system

-fuel and steam control systems

-Advanced control techniques

-Load management study

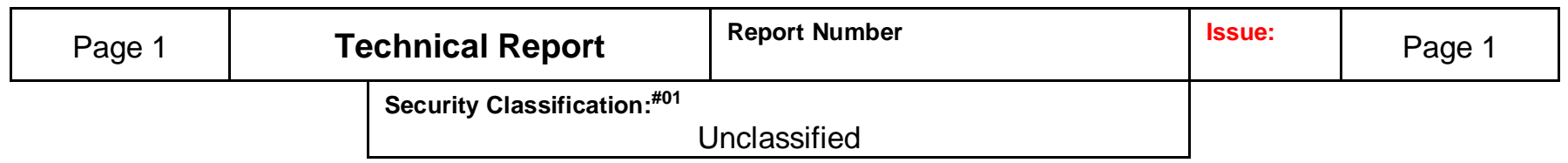




\section{INTRODUCTON}

This document describes the overall hardware and software architecture for the control system, reliability, cost analysis, fuel and steam control philosophy, and load management study.

\section{SCOPE OF DOCUMENT}

The aim of this document is to provide a top-level system description, based on the controls work of the NGGT. The document will provide a basis for producing specifications for the individual system components.

This document assumes that the plant being controlled is a typical gas turbine package, comprising a gas turbine, Generator, and associated package equipment. This does not however preclude the use of components of the system for the control of other equipment, as considered appropriate.

\begin{tabular}{|c|c|c|c|c|}
\hline Page 2 & Technical Report & Report Number & Issue: & Page 2 \\
\hline & \multicolumn{2}{|c|}{ Security Classification:\#01 } & & \\
\hline
\end{tabular}




\section{OVERVIEW OF CONTROL SYSTEM CONFIGURATION}

\subsection{Background to architecture design}

The NGGT Controls study has sought to identify technologies and software implementation tools and techniques, which can be used to produce a control system, which is modular. In particular, this has resulted in a focus on commercially available PC hardware, transportable/open software programming languages, and Fieldbus communications for input/output plant interfaces.

The safety system is separated from the main control system and is based on qualified components designed specifically for safety applications. It is therefore not based on open PC technology (at this time). It will, however provide suitable interfaces for communications with the ECS/PCS controllers.

\subsection{Main Components}

The NGGT Control System Controller will comprise a number of distinct interconnected components or subsystems. The principal components are shown in the tables below, and are more fully described later in this document.

\begin{tabular}{|l|l|l|}
\hline \multicolumn{2}{|c|}{ NGGT Control System } \\
\hline \multicolumn{2}{|c|}{ Real Time Controllers } & Data Server/Local HMI (DSH) \\
\hline \multicolumn{1}{|c|}{ ECS } & \multicolumn{1}{|c|}{ PCS } & Ethernet interface 1 (ECS/PCS) \\
Fieldbus interface & Fieldbus interface & Ethernet interface 2 (remote systems) \\
ECS distributed I/O System & PCS distributed I/O System & Windows OS \\
RTOS & RTOS & Standard HMI tools \\
OS+ & OS+ & \\
Application (Simulink) & Application (IEC) & \\
IEC61131-3 soft PLC & IEC61131-3 soft PLC & \\
Ethernet Interface (PCS/DSH) & Ethernet Interface (ECS/DSH) & \\
& & \\
\hline
\end{tabular}

\begin{tabular}{|l|l|}
\hline \multicolumn{2}{|c|}{ Safety Related System (SRS) } \\
\hline \multicolumn{1}{|c|}{ Programmable Shutdown System } & Overspeed Detection Unit \\
\hline Safety PLC & Overspeed unit \\
Local I/O & Signal repeaters \\
Remote I/O (via Safety bus-P) \\
Trip relays
\end{tabular}

\begin{tabular}{|c|c|c|c|c|}
\hline Page 3 & Technical Report & Report Number & Issue: & Page 3 \\
\hline & \multicolumn{2}{|c|}{$\begin{aligned} \text { Security Classification: }{ }^{\# 1} & \\
& \text { Unclassified }\end{aligned}$} & & \\
\hline
\end{tabular}




\subsection{ECS/PCS/SRS/DSH Functions}

- ECS is the Engine Control System, providing control of the Gas Generator, DLF Fuel Control System, and Steam Injection System, with its own specific application code

- PCS is the Package Control System, providing control of engine ancillaries (e.g. oil systems), waste-heat boilers, Generator, evaporator, water recovery systems.

- SRS is the Safety-Related System which independently monitors and protects the unit to a specified safety integrity level

- DSH is the Data Server/local HMI which provides data services to external systems, and an operator interface

\subsection{Selected Technology and Supplier Base}

\subsubsection{ECS/PCS Hardware Platforms}

\subsubsection{PC Hardware}

The NGGT Control System will developed with the ability to be implemented on a number of selected PC platforms and fieldbuses. This allows different market requirements to be satisfied, ensures that hardware can be multi-sourced.

PC-based controllers will conform to the basic $\times 86$ generic standard. This includes a wide range of industrial $\mathrm{PC}, \mathrm{PC} / 104$, CompactPCl and single board computer products. There is therefore no single selected manufacturer for the ECS/PCS hardware.

Examples of PC systems which may be considered are:

- "Industrial" PC

- $\mathrm{PC} / 104$

- CompactPCI

- Single Board Computer

\begin{tabular}{|c|c|c|c|c|}
\hline Page 4 & Technical Report & Report Number & Issue: & Page 4 \\
\hline & \multicolumn{2}{|c|}{ Security Classification:\#01 } & & \\
\hline
\end{tabular}




\subsubsection{PC Bus Technology and PC Specification}

The various PC hardware platforms identified above will include a variety of different buses within their internal architecture, each of which will offer varying speed, and performance, for example ISA, PCI, IDE, EIDE, SCSI, AGP, PCMCIA, to name some examples.

\subsubsection{ECS/PCS Fieldbuses}

It will be possible to interface a number of fieldbus systems to the controller, and employ a number of different $\mathrm{I} / \mathrm{O}$ systems for interfacing the fieldbus to the engine and package instrumentation. The choice of these will be determined by the application and subject to agreed validation processes.

The NGGT control could have the following fieldbuses:

- Profibus DP

- CANopen

- ControlNet

In addition, there is a desire to incorporate intelligent instruments within the control scheme. Examples of these are Foundation Fieldbus and Profibus PA instruments transmitters. $\Rightarrow$ Why we gonna do this? Because of the Improved Reliability, Accuracy, and Diagnostics

\subsubsection{Fieldbus Network Configuration Tools}

A software tool is required for configuring the fieldbus network. This tool will be used to configure network communications, nodes, etc.

It is likely more than one network configuration tool will be required in order to cover the full range of fieldbuses and I/O systems.

\begin{tabular}{|c|c|c|c|c|}
\hline Page 5 & Technical Report & Report Number & Issue: & Page 5 \\
\hline & \multicolumn{2}{|c|}{ Security Classification:\#01 } & & \\
\hline
\end{tabular}




\subsubsection{Data Server/Local HMI Hardware Platform}

The DSH will take the form of a PC. As the DSH is not critical to control of the package or engine.

\subsubsection{Safety-Related System Hardware Platform}

The SRS will be based on an IEC 61508 Industry Approved System

\subsubsection{Software Development Tools}

The software shell will be developed using standard commercially available tools, and programmed in $\mathrm{C} / \mathrm{C}++$. These languages ensure maximum scope for portability of the software to other platforms in the future if necessary.

Engine control embedded applications will be developed using the Simulink and Stateflow products from The Mathworks Ltd. The resultant models will be converted into C source code using the Real Time Workshop product from the same company.

\subsection{Overall System Architecture for a Typical GT Package}

Figure 1 (below) shows a simplified block diagram of the system; showing a typical installation comprising an engine package, control system and safety system. Transmitters and actuators are hardwired to fieldbus input/output modules, which are connected to the ECS and PCS controllers. The SRS inputs and outputs are wired separately, and there are both hardwired and fieldbus interfaces between the SRS and the NGGT Control System.

\subsection{ECS/PCS/DSH Architecture}

Figure 2 (below) shows the overall hardware architecture of the NGGT Control System (in block diagram form only), assuming all level 1 and level 2 functions are included. This provides a context for the software component descriptions included later in the document.

(Note: This diagram does not necessarily reflect the final design of any particular application, but sets out the principles on which the system is based).

\begin{tabular}{|l|l|l|l|l|}
\hline Page 6 & Technical Report & Report Number & Issue: & Page 6 \\
\hline
\end{tabular}



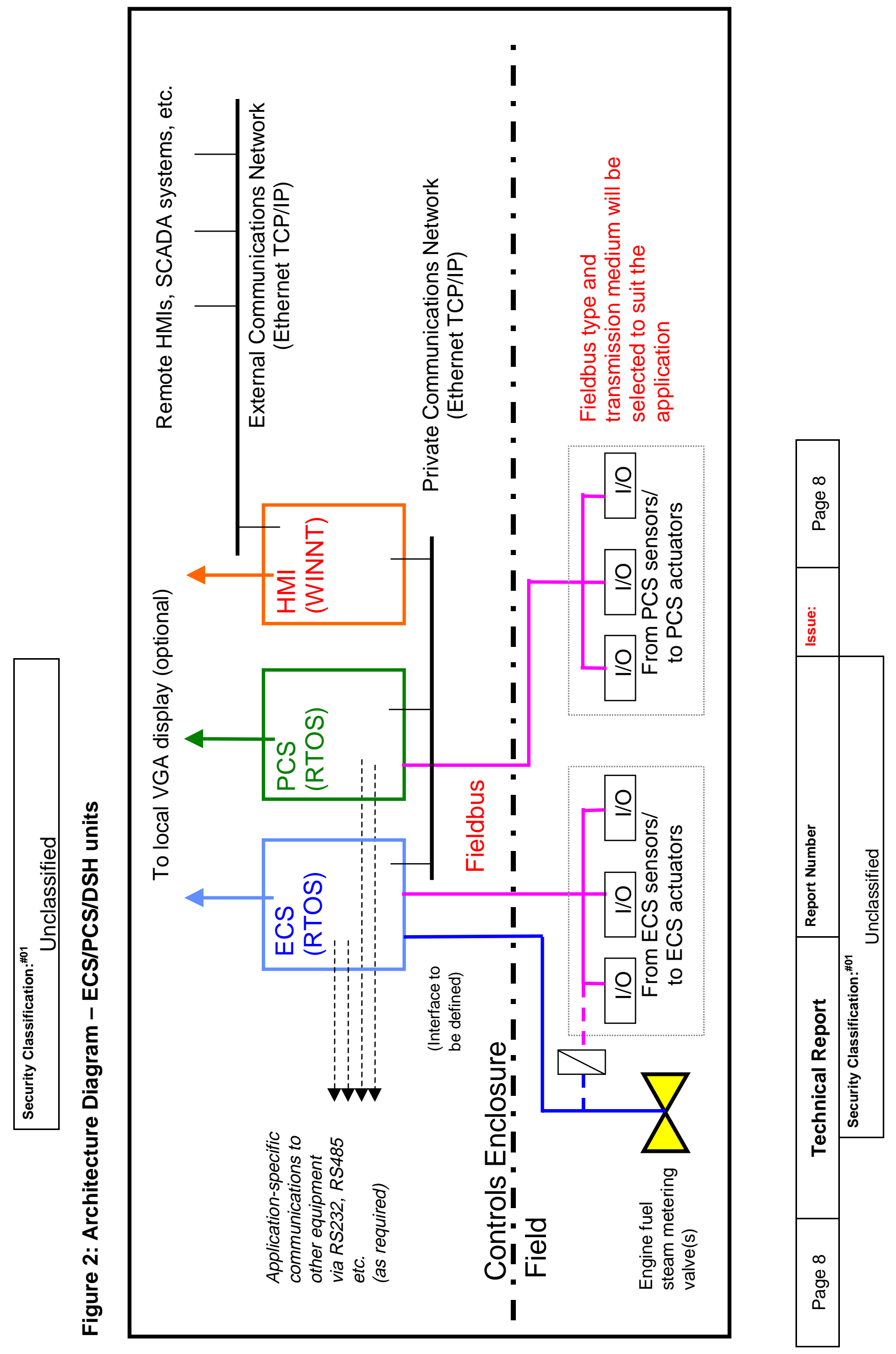


\subsubsection{Description of Architecture Diagram}

3.6.1.1 The system is simplex in nature, and comprises a number of sub-systems as described earlier in this document. (see section ... for Reliability)

3.6.1.2 CPUs can exchange data via the private communications network shown. This will be an Ethernet physical layer running TCP/IP protocols. The high-level protocol will be fully defined in a separate document.

3.6.1.3 Separate ECS and PCS fieldbuses segregate the (fixed) ECS configuration from the (potentially variable) PCS configuration. Different fieldbuses operate on different principles (e.g. master/slave or producer/consumer messages). Some fieldbuses allow additional CPUs to "listen" on a fieldbus without adding to the network traffic; therefore, this aspect is subject to detailed design considerations.

3.6.1.4 The power supply arrangement for the system may vary according to the application, and will be selected according to input voltage, reliability and current capacity requirements. Dual-redundant $100 \%$ rated PSU's may be considered to improve system availability on certain controller configurations.

3.6.1.5 The CPUs will be selected according to required performance and features, as determined by the application. Passive cooling techniques will be used rather than fan cooling.

3.6.1.6 The DSH CPU may optionally be an "external" PC connected to the PCS/ECS via Ethernet, rather than a control cubicle-mounted system. The DSH will run the Windows NT operating system (or similar). The HMI application itself may be based on a full SCADA package or could optionally run only an OPC server or other data server utility with one or more remote clients.

3.6.1.7 Each CPU will include both dynamic RAM and non-volatile memory. For ECS/PCS controllers, the NVM will be in form of flash or similar solid-state memory. For the DSH $\mathrm{CPU}$, a high-capacity rotating drive may be considered to be more appropriate.

3.6.1.8 The exact nature of the interface to the SRS may vary between applications, and will be defined when more detailed design work is completed. One factor in determining this is the available range of interface modules provided by the SRS manufacturer.

3.6.1.9 The local display, if required, will be a monitor connected to the video adapter (VGA port) on the CPU. PC peripherals can be shared between the CPUs by means of a COTS KVM switch, if desired.

3.6.1.10 Some I/O may be required locally in the controller enclosure (e.g. supply distribution monitoring, cubicle temperature monitoring, time sync. pulse etc). It is assumed this will be a local fieldbus I/O drop.

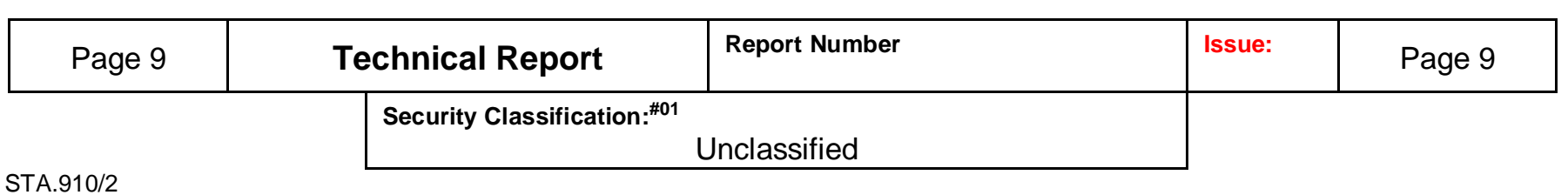


If time synchronisation between CPUs is required, the signal may be derived either from a real-time clock system (providing a serial time code or similar), or in the form of a periodic pulse connected to an input module.

\begin{tabular}{|l|l|l|l|l|}
\hline Page 10 & Technical Report & Report Number & Issue: & Page 10 \\
\hline & Security Classification:\#01 & \\
\cline { 2 - 3 } & Unclassified &
\end{tabular}




\subsection{Main Software Components ECS/PCS}

\section{Figure 3: Software Component Layering}

Application

Developmet

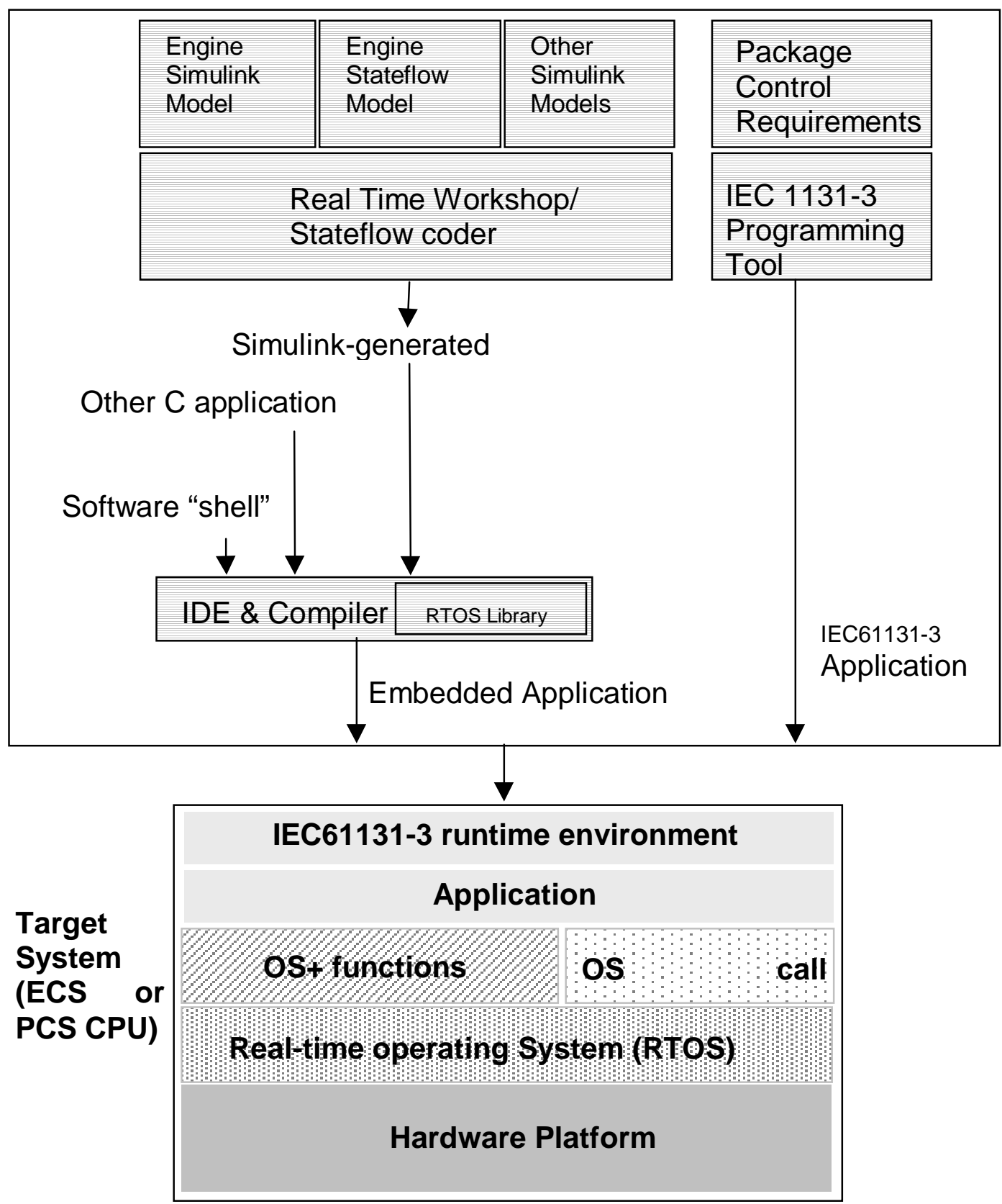




\subsubsection{Description of Software Component Diagram}

3.7.1.1 The ECS control algorithm will be modelled using Mathworks Simulink/Stateflow tools. The control model will be verified against the engine model in the Simulink environment.

3.7.1.2 The Mathworks Real-Time Workshop and Stateflow coder tools will be used to auto code the control algorithm into ANSI C source code. This code will not be targeted for a particular operating system, but generated in "embedded" format, in order to ensure that the source files are open/transportable. The ECS C code algorithm will include a defined I/O interface which will be independent of both the hardware and the target operating system.

3.7.1.3 The Simulink pictures-to-code environment can optionally be used for other (nonengine control) applications if required (shown as "Other Simulink Models" on the diagram).

3.7.1.4 For the PCS/non-engine control requirements, the application code may be written in IEC61131-3 languages, giving high visibility and ease of modification in the field. This code will be executed on the PCS CPU by a compatible IEC61131-3 runtime system. The IEC61131-3 environment will also have the capability of executing " $\mathrm{C}$ " routines which can be called as function blocks from within IEC61131-3 programs.

3.7.1.5 The IEC 61131-3 Program is a international standard program. This program language is used on different brand of control systems so that every one is familiar with the IEC 611313-3 Program. This program language is easy to learn and handle.

3.7.1.6 A suitable compiler/linker will be used to generate the executable code for the target CPU, in conjunction with the appropriate RTOS support library and IEC61131-3 runtime library (if used).

3.7.1.7 A defined set of functions (shown, as "software shell" and "OS+" will be required to operate above the RTOS. These are defined later in the document. The OS+ layer will be capable of running on different PC platforms and acts as an interface between the application and the physical controller.

3.7.1.8 In order to make the system design as open and modular as possible, operating system calls will be declared indirectly, and a separate table of call definitions will be maintained, referencing the RTOS-specific call definitions.

3.7.1.9 The RTOS will include debugger support for use during the development phase.

\begin{tabular}{|r|c|l|l|l|}
\hline Page 12 & Technical Report & Report Number & Issue: & Page 12 \\
\hline \multicolumn{2}{|c|}{ Unclassified } & & \\
\cline { 2 - 3 } STA.910/2 & Security Classification:\#01 &
\end{tabular}




\subsubsection{Watchdog Task}

The watchdog task monitors each OS+ function for successful completion within its allocated time. The watchdog task has the capability to suspend the application task on detection of a task-overrun event or failure to complete.

\begin{tabular}{|c|c|c|c|c|}
\hline Page 13 & Technical Report & Report Number & Issue: & Page 13 \\
\hline & \multicolumn{2}{|c|}{$\begin{array}{l}\text { Security Classification: } \# 01 \\
\text { Unclassified }\end{array}$} & & \\
\hline
\end{tabular}




\subsection{Interface Summary Table}

The following list summarises the interfaces which will be used for exchange of data both internal and external to the NGGT Control System, along with the proposed protocol. In some cases, more than one option is presented. This allows, for example the PCS to take the form of an external PLC

\begin{tabular}{|c|c|l|l|l|}
\hline Page 14 & Technical Report & Report Number & Issue: & Page 14 \\
\hline
\end{tabular}




\begin{tabular}{|c|c|c|c|}
\hline A-end & Z-end & Interface Type & Protocols \\
\hline ECS & PCS & Ethernet & $\begin{array}{l}\text { Modbus serial } \\
\text { Modbus TCP/IP }\end{array}$ \\
\hline ECS or PCS & External eqpt. & $\begin{array}{l}\text { Ethernet } \\
\text { Serial }\end{array}$ & $\begin{array}{l}\text { Modbus serial } \\
\text { Modbus TCP/IP }\end{array}$ \\
\hline ECS & $\mathrm{DSH}$ & Ethernet & Modbus TCP/IP * \\
\hline PCS & DSH & Ethernet & Modbus TCP/IP \\
\hline ECS/PCS/DSH & Local Display & PC video port & $\begin{array}{c}\text { Standard VGA, mouse and } \\
\text { keyboard }\end{array}$ \\
\hline ECS & ECS I/O & Fieldbus & As required for selected fieldbus \\
\hline ECS & FMV & tba & Depends on what is provided \\
\hline PCS & PCS I/O & Fieldbus & As required for selected fieldbus \\
\hline ECS/PCS/DSH & $\begin{array}{l}\text { Time } \\
\text { reference }\end{array}$ & $\begin{array}{l}\text { Serial Time Code, } \\
\text { Digital sync pulse }\end{array}$ & $t b c$ \\
\hline PCS & $\begin{array}{l}\text { Maintenance } \\
\text { tool }\end{array}$ & $\begin{array}{l}\text { Ethernet, } \\
\text { Serial }\end{array}$ & See maintenance tool specification \\
\hline ECS & $\begin{array}{l}\text { Maintenance } \\
\text { tool }\end{array}$ & $\begin{array}{l}\text { Ethernet, } \\
\text { Serial }\end{array}$ & See maintenance tool specification \\
\hline DSH & $\begin{array}{l}\text { SCADA } \\
\text { clients, } \\
\text { Level 3+ }\end{array}$ & Ethernet & $\begin{array}{c}\text { SCADA-specific protocol, } \\
\text { optional SCADA web server, } \\
\text { OPC (DCOM) }\end{array}$ \\
\hline ECS & SRS & Fieldbus & Fieldbus node on I/O system \\
\hline
\end{tabular}

Notes:

1. The Modbus protocol may be expanded to include additional non-standard functions, if deemed necessary

2. * This interface may not be required, if all DSH data is transferred via the PCS 


\subsection{Data Server/Local HMI (DSH) Functions}

The DSH will perform the following functions:

- Acquisition of data from controller(s)

- Upload facility for controller log files (e.g. pre/post trip logs)

- Logging of controller data (diagnostic and process)

- System status and diagnostics screens

- Upload/download of application programmes

- Parameter tuning for selected (non-engine control) functions

- Mimic screens for engine monitoring

- Data screens for engine control algorithm monitoring

- Mimic screens for package monitoring/control as required

- Data server for remote client connection

\subsection{ECS/PCS Controller Software Structure}

The following structure diagrams should be read in conjunction with the preceding system description. The purpose of the diagrams is to represent the relationships between the various modules of the control system, and thus assist with module specification.

\begin{tabular}{|c|c|l|l|l|}
\hline Page 16 & Technical Report & Report Number & Issue: & Page 16 \\
\hline \multicolumn{2}{|c|}{ Unclassified } & & \\
\cline { 2 - 3 } & \multicolumn{2}{|c|}{ Security Classification:\#01 } &
\end{tabular}




\section{Figure 4: Context Diagram}

Note: This diagram indicates data flow between a single NGGT Control System and peripheral/external equipment.

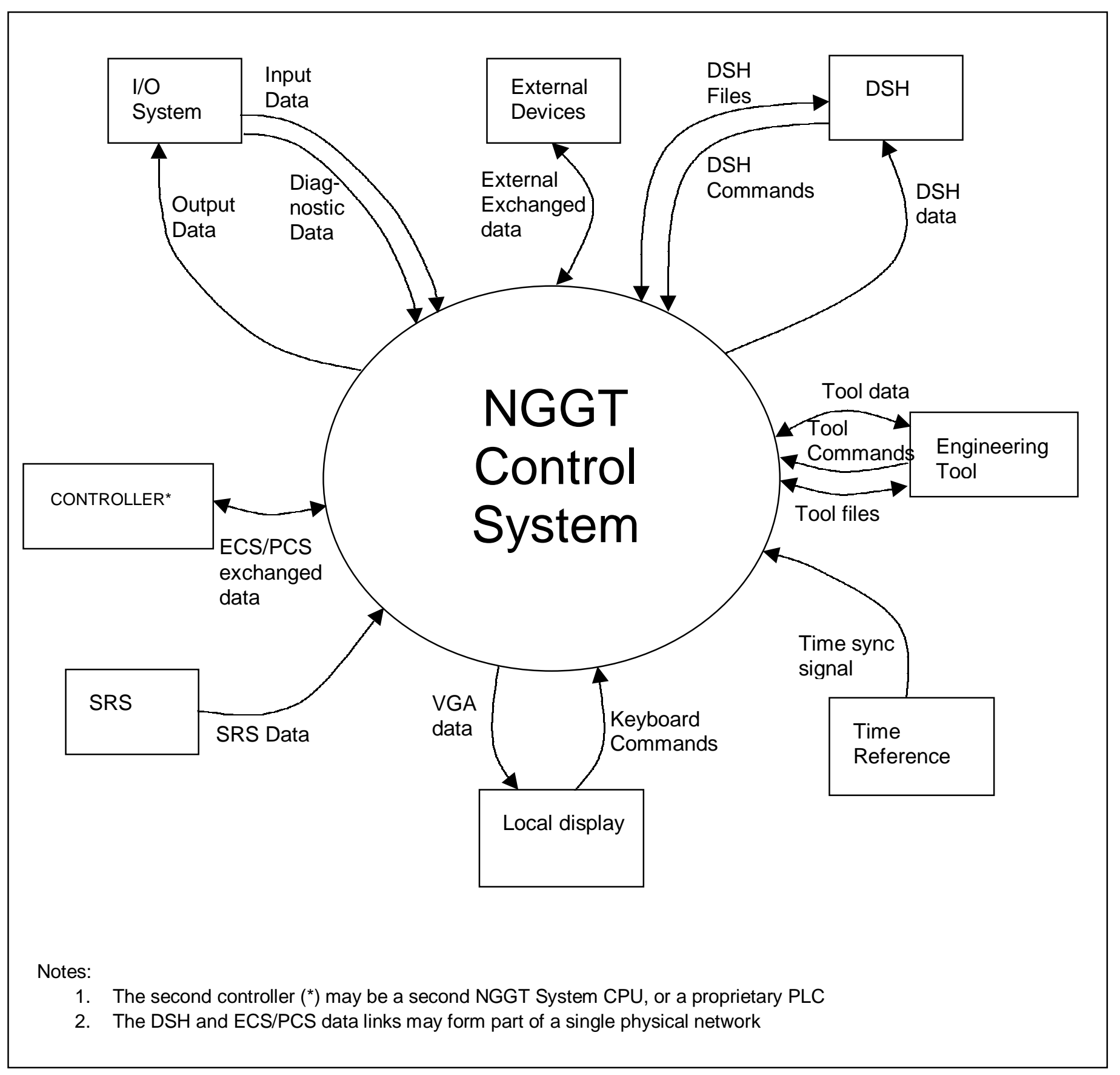

\begin{tabular}{|c|c|c|c|c|}
\hline Page 17 & Technical Report & Report Number & Issue: & Page 17 \\
\hline & \multicolumn{2}{|c|}{ 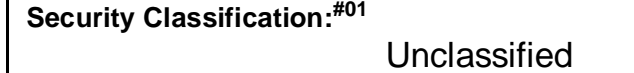 } & & \\
\hline
\end{tabular}




\section{Figure 5: Level 0 Data Flow Diagram - NGGT System Controller}

Note: This diagram indicates the major data flows within a NGGT System controller. Straight arrows indicate links to external devices (see context diagram). The diagram demonstrates how the database is used as the central holding point where all controller data is maintained.

Processes 17, 18 and 20 (applications) do not form part of the software shell (see IEC61131-3 and Embedded Applications section 3.11)

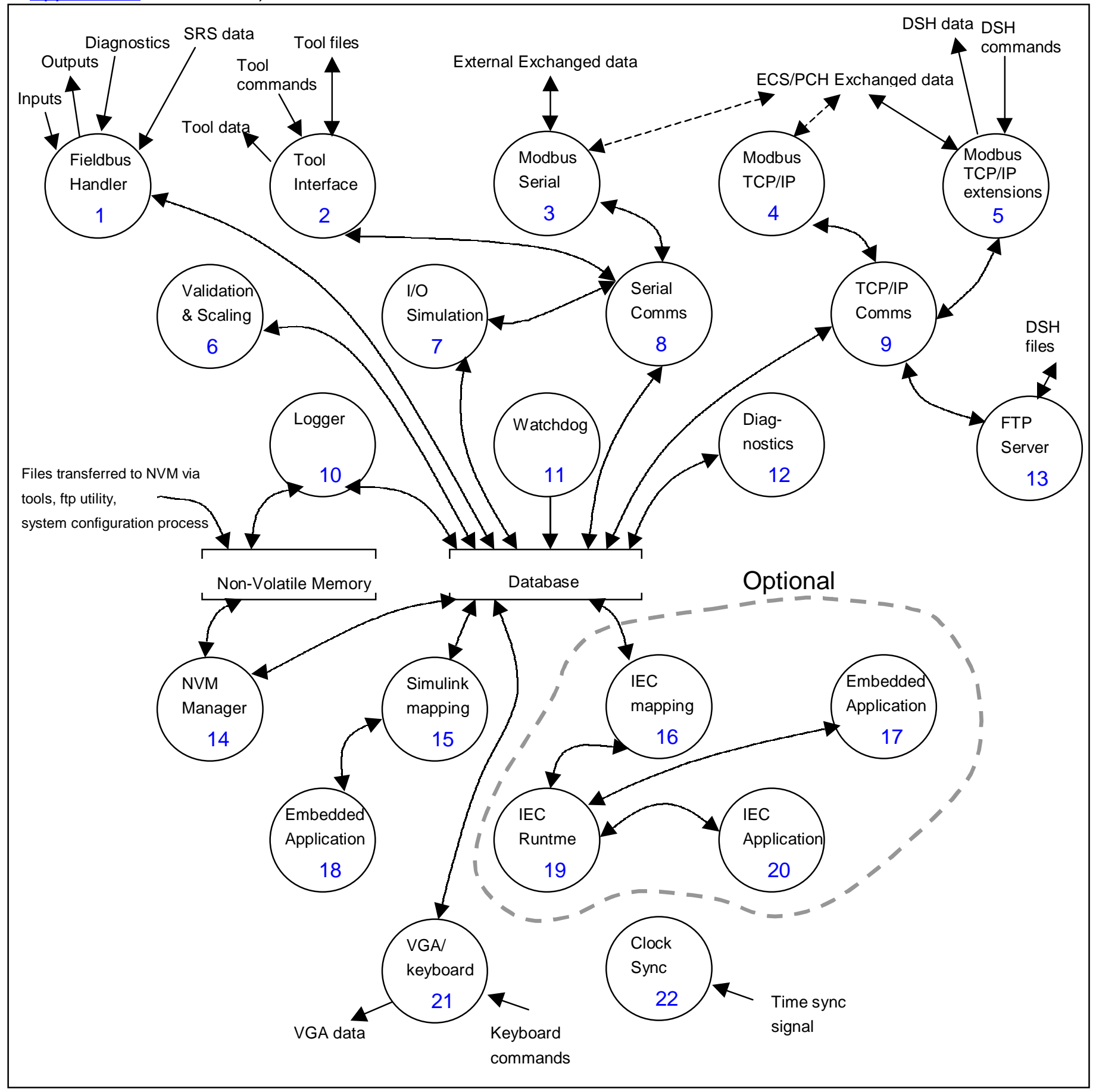

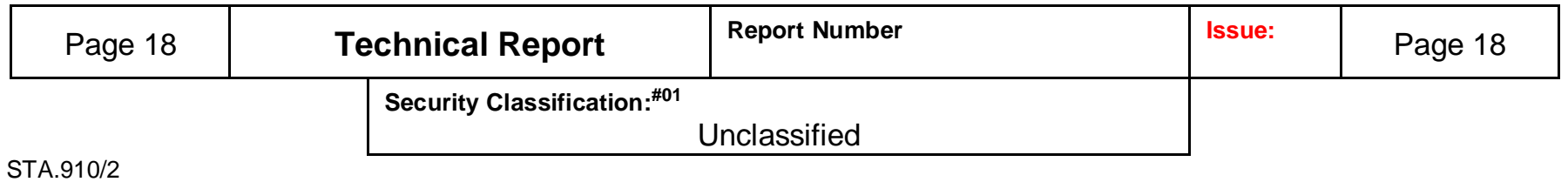




\subsection{Task Scheduling}

The controller tasks will be scheduled by the task scheduler. This is not shown on the data flow diagram, which does not show time dependencies.

\subsection{PCS Software Structure}

The PCS, for R-R Energy business applications, will be programmed using the proprietary IEC61131-3 programming languages provided by the vendor. The software will be structured by means of programme organisation units (POUs) as directed by the IEC standard.

The IEC61131-3 runtime system software is COTS and its structure will be documented in the vendor's literature.

Where gaps appear in document table references, this has been done to maintain consistent numbering with earlier versions of this document.

The above list does not include algorithm/logic definition for the PCS and ECS applications. These are to be addressed separately in other documents.

\subsection{IO Count}

\begin{tabular}{|l|r|r|r|r|r|r|r|r|r|}
\hline & Panel & Driver & MCC & $\begin{array}{c}\text { GG Lube } \\
\text { Oil }\end{array}$ & $\begin{array}{r}\text { PT/GEN } \\
\text { Lube Oil }\end{array}$ & Generator & $\begin{array}{c}\text { Water } \\
\text { Recovery }\end{array}$ & $\begin{array}{c}\text { Steam } \\
\text { System }\end{array}$ & Boiler \\
\hline Al & 2 & 34 & & 2 & 2 & 12 & 10 & 10 & 2 \\
\hline AO & 2 & 4 & & 2 & 2 & 2 & 2 & 2 & 2 \\
\hline DI & 16 & 16 & 16 & 8 & 8 & 16 & 16 & 8 & 8 \\
\hline DO & 16 & & 24 & 8 & 8 & 8 & 8 & 8 & 8 \\
\hline Total & $\mathbf{3 6}$ & $\mathbf{5 4}$ & $\mathbf{4 0}$ & $\mathbf{2 0}$ & $\mathbf{2 0}$ & $\mathbf{3 8}$ & $\mathbf{3 8}$ & $\mathbf{2 8}$ & $\mathbf{2 0}$ \\
\hline
\end{tabular}

In this System are used:

74 Analog Inputs

18 Analog Outputs

112 Digital Inputs

88 Digital Outputs

\begin{tabular}{|c|c|l|l|c|}
\hline Page 19 & Technical Report & Report Number & Issue: & Page 19 \\
\hline
\end{tabular}




\section{SAFETY SYSTEM}

\subsection{Introduction}

A safety system can not be designed until the complete system is designed. Therefor it is necessary to design the complete system before the safety system to know which method for the safety system will be used.

Assuming a hazard occurs, this failure will be categorised as a catastrophic event. If the predicted likelihood of this event is such that the calculated risk is the highest risk class, the risk needs to be reduced. If the risk/consequence cannot be lessened. To an acceptable level with a simplex system, redundant systems will need to be implemented.

The following is the method that will be used.

\subsection{Discussion}

The aim of IEC 61508 is to provide a route for implementing electrical, electronic or programmable electronic technology in such a way that an acceptable level of safety is achieved.

The first premise of the standard is that there will be equipment intended to provide functionality e.g., electricity generation or chemical processing and there is a system that controls it. These are known as the "equipment under control" (EUC) and EUC control system respectively.

The second premise is that safety functions throughout are to be provided to reduce the risks posed by the EUC and the EUC control system. IEC 61508 refers to the SRS as being a separate entity to the EUC and EUC control system. However, in reality, the SRS may be contained within the EUC control system. This is acceptable as long as the SRS function is sufficiently partitioned i.e. independent, and so cannot be affected by the EUC control system functions.

A fundamental tenet of the standard is that it is not valid to assume that if the EUC and EUC control system are built well and are reliable, they will be safe. They must be built to be safe and operated safely. The safety functions to be included in the systems, should be based upon an assessment and understanding of the safety risks posed by inadvertent behaviour of the EUC and EUC control system.

The standard gives guidance on good practice; it gives recommendations; it recognises that safety cannot be based upon retrospective proof, but must be demonstrated in advance. It recognises that there can never be perfect safety (zero risk). The recommendations are not restricted to technical affairs but include the assessment of all activities throughout the entire life of a system from conception to decommissioning. IEC 61508 is a standard for the management of safety and defines the safety lifecycle.

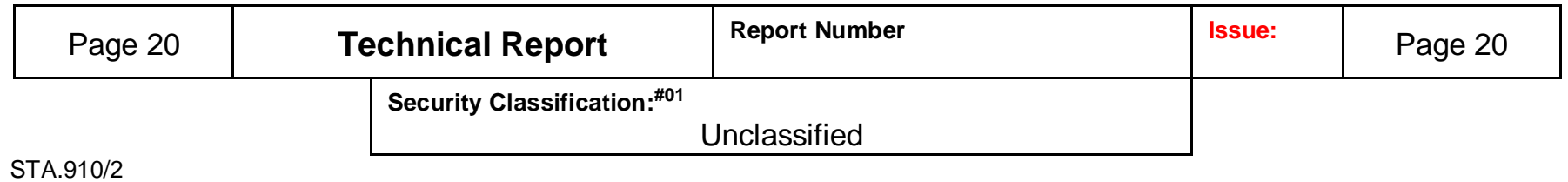


It should be stressed that IEC 61508 is a generic standard. It is intended to be used as the basis for writing more specific i.e. sector-specific or application-specific, standards. However, in the absence of any sector-specific or application-specific standard it may be used as the applicable standard.

In order to understand the risks posed by the EUC and EUC control system, a requirement of the standard is to perform hazard and risk assessment. It is left to the user to decide how to do this but the standard does offer guidance. It gives definitions of consequence, likelihood, risk, risk classification etc.

\subsection{Consequence Categories}

An example of consequence categories are shown in the following table:

Consequence Categories

\begin{tabular}{|l|l|}
\hline Category & Definition \\
\hline Catastrophic & multiple deaths \\
\hline Critical & $\begin{array}{l}\text { a single death, and/or multiple severe injuries or severe occupational } \\
\text { illnesses }\end{array}$ \\
\hline Marginal & $\begin{array}{l}\text { a single severe injury or occupational illness and/or multiple minor } \\
\text { injuries or minor occupational illnesses }\end{array}$ \\
\hline Negligible & at most a single minor injury or minor occupational illness at worst \\
\hline
\end{tabular}

\subsection{Likelihood Categories}

An example of likelihood categories of accidents are shown in the following table:

\section{Accident Likelihood (frequency)}

\begin{tabular}{|l|l|l|}
\hline $\begin{array}{l}\text { Likelihood } \\
\text { Category }\end{array}$ & $\begin{array}{l}\text { Definition } \\
\text { (during operational life considering all instances } \\
\text { of the system) }\end{array}$ & $\begin{array}{l}\text { Numerical range } \\
\text { failures/operating hour }\end{array}$ \\
\hline Frequent & many times in system lifetime & $>10^{-3}$ \\
\hline Probable & several times in system lifetime & $10^{-3}$ to $10^{-4}$ \\
\hline Occasional & once in system lifetime & $10^{-4}$ to $10^{-5}$ \\
\hline Remote & unlikely to occur in system lifetime & $10^{-5}$ to $10^{-6}$ \\
\hline Improbable & very unlikely to occur & $10^{-6}$ to $10^{-7}$ \\
\hline Incredible & cannot believe that it could occur & $<10^{-7}$ \\
\hline
\end{tabular}

Note: the likelihood categories can be defined qualitatively or quantitatively.

\begin{tabular}{|c|c|c|c|c|}
\hline Page 21 & Technical Report & Report Number & Issue: & Page 21 \\
\hline & \multicolumn{2}{|c|}{$\begin{aligned} \text { Security Classification: }{ }^{\sharp 01} & \\
& \text { Unclassified }\end{aligned}$} & & \\
\hline
\end{tabular}


The consequence and likelihood categories can be combined to form the following risk matrix:

\begin{tabular}{|l|l|l|l|l|}
\hline $\begin{array}{c}\text { Consequence } \\
\text { Likelihood }\end{array}$ & Catastrophic & Critical & Marginal & Negligible \\
\hline Frequent & & & & \\
\hline Probable & & & & \\
\hline Occasional & & & & \\
\hline Remote & & & & \\
\hline Improbable & & & & \\
\hline Incredible & & & & \\
\hline
\end{tabular}

\subsection{Risk, Tolerable Risk and the Alarp Principle}

IEC 61508 defines risk for a particular event as:

$$
\text { risk = likelihood of event } \mathrm{X} \text { consequence of event }
$$

e.g. if a system failure is likely to occur once in 10 years (or 0.1 times per year) and could cause 2 deaths, the risk associated with this failure is $0.1 \times 2=0.2$ deaths/year.

The risk posed by a hazard may thus be reduced if either the consequence or likelihood (or both) are reduced. A catastrophic hazard occurring frequently is the worst case. A negligible hazard with an incredibly low likelihood is the least serious case. These are diagonal opposites of the above matrix.

Nothing has zero risk, nothing can be called " $100 \%$ safe", although in an ideal world, it is nice to get as close to this figure as possible. Thus, it becomes necessary to think about what levels of risk are tolerable or even acceptable. Tolerable risk indicates a willingness to live with a risk so as to secure certain benefits, at the same time expecting it to be kept under review and reduced as and when this can be done. Cost would probably come into the picture in that any potential reduction in risk would be compared with the cost needed to achieve it.

Having listed each identified hazard in the risk matrix above, IEC 61508 then recommends that they are assigned to one of four risk classes (see table below). Each risk class broadly equates to the ALARP levels shown above.

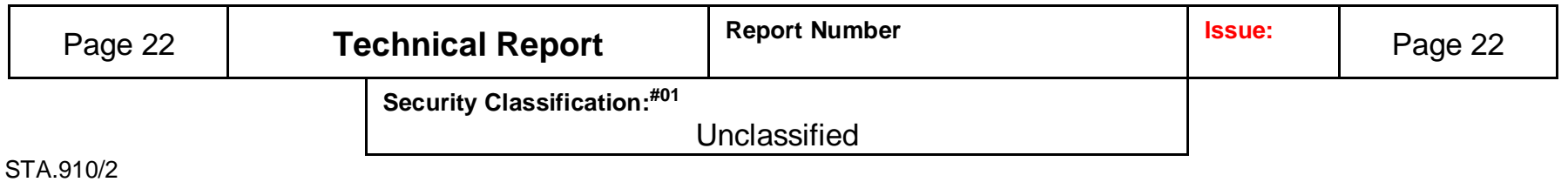




\begin{tabular}{|l|l|}
\hline Risk class & Interpretation \\
\hline Class I & Intolerable risk: unacceptable in any circumstance \\
\hline Class II & $\begin{array}{l}\text { Undesirable risk: tolerable only if risk reduction is impracticable, or } \\
\text { if the costs are grossly disproportionate to the improvement gained }\end{array}$ \\
\hline Class III & $\begin{array}{l}\text { Tolerable risk if the cost of risk reduction would exceed the } \\
\text { improvement gained }\end{array}$ \\
\hline Class IV & $\begin{array}{l}\text { Negligible risk: acceptable as it stands but may need to be } \\
\text { monitored }\end{array}$ \\
\hline
\end{tabular}

- Risk class I lies in the intolerable region

- Risk class II lies in the upper part of the ALARP region near to the intolerable region

- Risk class III lies in the lower part of the ALARP region, below class II

- Risk class IV lies in the broadly acceptable region

Note: system-specific risk classes can be established which further refine these classes i.e. so there are more than four risk classes.

Each cell of the risk matrix, and hence its corresponding hazards should be given a risk class based upon an agreed risk classification table; an example of which is shown below:

\begin{tabular}{|l|l|l|l|l|}
\hline $\begin{array}{c}\text { Consequence } \\
\text { Likelihood }\end{array}$ & Catastrophic & Critical & Marginal & Negligible \\
\hline Frequent & I & I & I & II \\
\hline Probable & I & I & II & III \\
\hline Occasional & I & II & III & III \\
\hline Remote & II & III & III & IV \\
\hline Improbable & III & III & IV & IV \\
\hline Incredible & IV & IV & IV & IV \\
\hline
\end{tabular}

This table shows that there are several class I risks. These risks are not acceptable and so thus have to be reduced. The class II \& III risks may be acceptable and the class IV risks broadly acceptable.

In IEC 61508, the link between risk and the appropriate risk-reducing action is provided by EUC safety-related systems (SRSs) and their safety integrity levels (SILs).

\subsection{Safety Integrity Levels}

Safety integrity is defined in the standard as:

the likelihood of a safety-related system satisfactorily performing the required safety functions under all the stated conditions, within a stated period of time.

\begin{tabular}{|c|c|c|c|c|}
\hline Page 23 & Technical Report & Report Number & Issue: & Page 23 \\
\hline & \multicolumn{2}{|c|}{$\begin{aligned} \text { Security Classification: }{ }^{\# 01} & \\
& \text { Unclassified }\end{aligned}$} & & \\
\hline
\end{tabular}


A safety integrity level (SIL) is defined as:

a discrete level ( 1 = lowest, $4=$ highest $)$ for specifying the safety requirements of safety functions i.e. the safety-related systems.

Notes:

1. although a SIL is derived from an assessment of risk, it is not a measure of risk,

2. it is not correct to refer to a system or an individual item of equipment as having a SIL without also referring to its context of operation

3. SILs define the amount of rigour that should be used to reduce risks,

4. meeting the requirements of a SIL gives confidence not proof.

Therefore, in the above matrix, to reduce a class I risk to (say) class II or class III, the SRS needs to be developed using the appropriate SIL so that the whole inclusive EUC system risk is reduced to the appropriate level.

For example, if the overall total EUC system failure rate is not to exceed $10^{-9} /$ hour, and the (EUC and EUC control system) has a failure rate of $10^{6} /$ hour, the independent SRS needs to have a probability of failing to operate not exceeding $10^{-3}$.

It is important to stress that the numerical values in the accident likelihood table and the values in the SIL tables for low demand and high demand/continuous operation need to be stated before the analysis.

The IEC 61508 is intended to be a generic standard and various industrial-sector specific standards, compliant with IEC 61508, exist for the development of safety-critical and safetyrelated systems. Each standard specifies the measures and techniques required to produce a system for that particular sector. In general terms, the more effort put into making a product safe, the greater the confidence we can have in the safety of that product. In terms of SILs, this means that the more measures and techniques used, as recommended in IEC 61508, the higher the SIL that can be claimed for the overall system. In reality, however, since the risk and particularly, perception of risk, is different between the various industrial sectors, different amounts of safety effort are required to claim the same SIL more effort is required to claim SIL 4 in the nuclear industry than SIL 4 in the automotive industry.

\begin{tabular}{|c|c|c|c|c|}
\hline Page 24 & Technical Report & Report Number & Issue: & Page 24 \\
\hline & \multicolumn{2}{|c|}{ Security Classification: ${ }^{\# 01}$ Unclassified } & & \\
\hline
\end{tabular}




\subsection{Perception of Risk}

The principle of ALARP describes the way in which risk it treated legally by the Helth and Safety Executive (HSE). The concept is that all reasonable measures will be taken in respect of risks that lie in the "tolerable region" to reduce them further until the cost of risk reduction is grossly disproportionate to the additional benefit gained.

It is at this point that the concept of "cost per life saved" arises. Industries and organisations are reluctant to state specific levels of "cost per life saved" which they would regard as becoming grossly disproportionate to a reduction in risk

Perception of risk is certainly influenced by the circumstances. A far higher risk is tolerated from voluntary activities than involuntary activities (people feel that they are more in control of the situation on roads than on a railway). Furthermore, the risk associated with multiple, rather than single, fatalities is expected to be much lower in the former case.

Another factor is the difference between individual and societal (population) risk. An incident with multiple fatalities is perceived less acceptable than the same number occurring individually. Thus, whereas say, a $10^{4}$ level of risk is tolerated for vehicles (single death voluntary), even say, a risk level of $10^{-8}$ might not satisfy everyone in the case of a nuclear power station incident (say 1000 deaths - involuntary) even though the risk/individual is now reduced to $10^{-5}$.

\subsubsection{Example of ALARP}

The figure below shows how, for a particular industry or application, the intolerable, tolerable and acceptable regions might be defined and how the tolerable region can be seen to reduce as the number of fatalities increases. Thus for a single fatality (left-hand vertical axis), frequencies of $10^{-5}$ to $10^{-3}$ are regarded as ALARP. Above $10^{-3}$ is regarded as an unacceptable (intolerable) risk and below $10^{-5}$ is an acceptable risk.

However, and this is only an example, for more fatalities, both the lower and upper ALARP level become more stringent, the tolerable risk is continually reduced from $10^{-5}$ per individual (lower level) and $10^{-3}$ per individual (upper level) as the consequence severity (no. of fatalities) increases. The vertical distance between the upper and lower levels for a particular fatality figure illustrates the reduction in tolerability. This reflects the effect of perception of risk regarding involuntary exposure and societal risk.

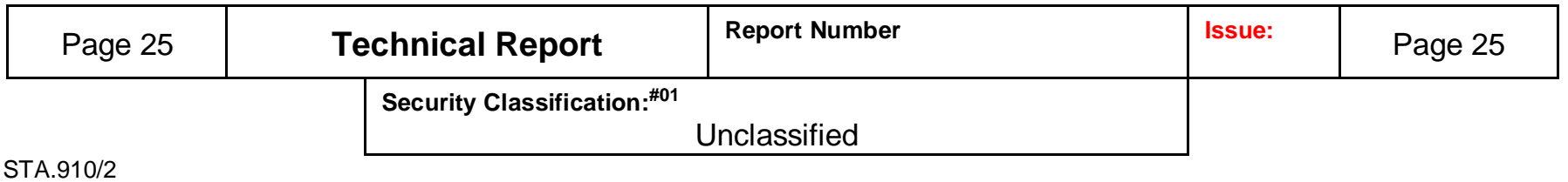




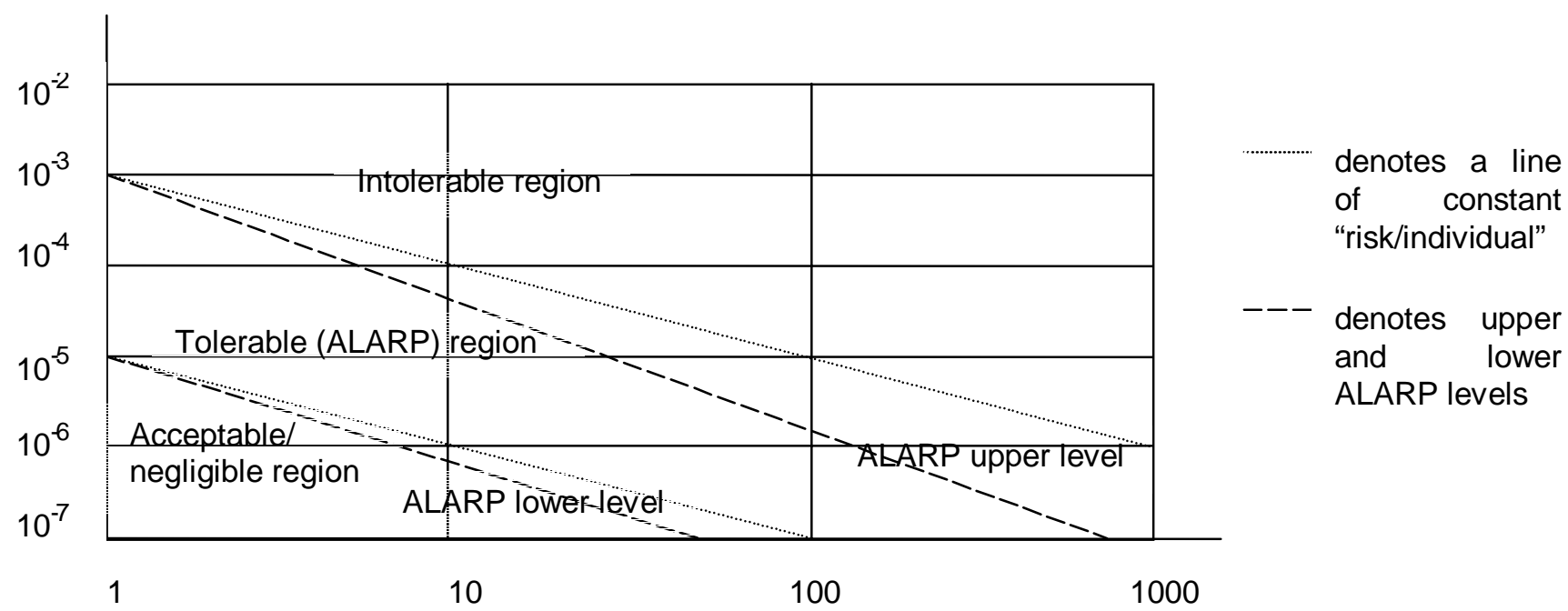

If is for the reason of perception of risk, different risk exposure types etc. in the various industrial sectors that there are different consequence category definitions for each industrial sector. Hence SILs (1 to 4 ) differ in each industrial sector i.e. considerably more safety- effort is required to claim SIL 4 in the nuclear sector, than is required to claim SIL 4 in the automotive sector. Again, to re-iterate, for IEC 61508 compliance, justification must be shown for any safety claim figure.

\subsection{NGGT Application}

Regarding NGGT for the Systems, Safety \& Reliability Group think that IEC 61508 should be applied in a methodical manner within the framework of a compliant safety management process. The safety management process for the full product lifecycle, as defined in IEC 61508 , should show how identified hazards will be addressed using safety cases and not just be reliant upon numerical arguments. The following method should be adopted.

1. with the EUC and EUC control system at a suitably "frozen" design state, the combined (EUC and EUC control system) hazards should be identified and agreed upon.

2. define the consequence of each hazard using established consequence categories.

3. define likelihood of each hazard using established likelihood categories.

4. define the risk class of each hazard using the established risk class table.

All class I risks are not tolerable and therefore need to be reduced. Class II and Class III risks will need to be further assessed for possible risk reduction compliant with ALARP. Risk reduction may be achieved by the introduction of an EUC safety-related system (SRS).

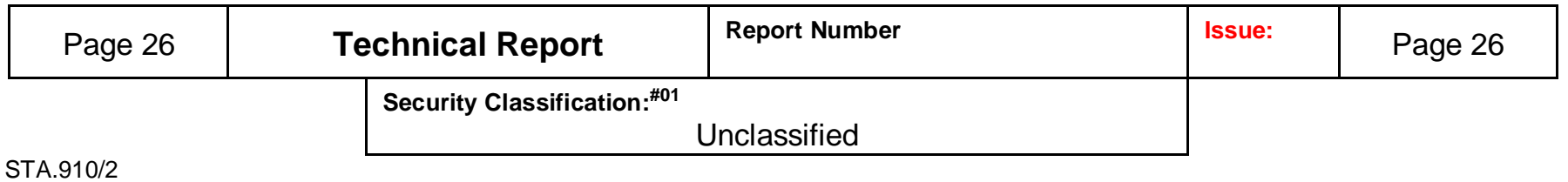


If the SRS is independent and developed to a certain SIL then the whole system may be claimed to be developed to a SIL one greater than the sub-system with the highest SIL, with the proviso that the highest SIL that can be claimed is SIL 4. For example:

SIL 1 (sub-system 1) and SIL 2 (sub-system 2) $\Rightarrow$ SIL 3 (combination of sub-systems 1 \& 2) SIL 2 (sub-system 1) and SIL 2 (sub-system 2) $\Rightarrow$ SIL 3 (combination of sub-systems 1 \& 2) SIL 1 (sub-system 1) and SIL 3 (sub-system 2) $\Rightarrow$ SIL 4 (combination of sub-systems 1 \& 2) SIL 2 (sub-system 1) and SIL 3 (sub-system 2) $\Rightarrow$ SIL 4 (combination of sub-systems 1 \& 2) SIL 2 (sub-system 1) and SIL 4 (sub-system 2) $\Rightarrow$ SIL 4 (combination of sub-systems 1 \& 2)

Note: we have made the following assumptions:

1. the (EUC and EUC control system) i.e. combined, equates to sub-system 1,

2. the EUC safety-related system equates to sub-system 2 ,

3. the combination of the (EUC and EUC control system) and EUC safety-related system is analogous to tow independent channels,

4. some form of common cause analysis (CCA), which will prove there are no random or systematic failures that can effect both sub-systems, will demonstrate independence between channels. It is interpreted from IEC 61508 that the amount of rigour put into CCA is dependent upon the sector-specific risk i.e. more rigorous CCA is required for nuclear applications compared with automotive applications. And importantly, the SIL claimed for the overall system (combination of sub-systems), should be the level used for determining the level of CCA required for each sub-system. For example, if SIL 3 is claimed for the overall system, which consists of an independent combination of two sub-systems (SIL 1 and SIL 2 respectively), then the overall system should have a "SIL 3 level" depth of CCA performed on it.

\subsubsection{Example}

A hazard is unaccommodated engine overspeed caused by the (EUC and EUC control system) leading to high-energy debris. Assuming this is categorised as a catastrophic event. If the predicted likelihood of this event is such that the calculated risk is class I then the risk it needs to be reduced. It is important to note that the lowest failure rate that can be claimed for the (EUC and EUC control system) without SIL level is $10^{-5}$ per hour. This value if claimed needs to be justified. Also, assume that the consequence cannot be lessened i.e. it stays at high-energy debris. Thus, the only way to reduce the risk is to reduce the likelihood of the event.

If this risk needs to be reduced to $<10^{-5}$ / hour then there is required risk reduction of $\mathrm{c} .10^{4}$. According to the SRS low demand mode of operation table in IEC61508, the risk reduction can be achieved by the introduction of an independent SRS developed to SIL 4.

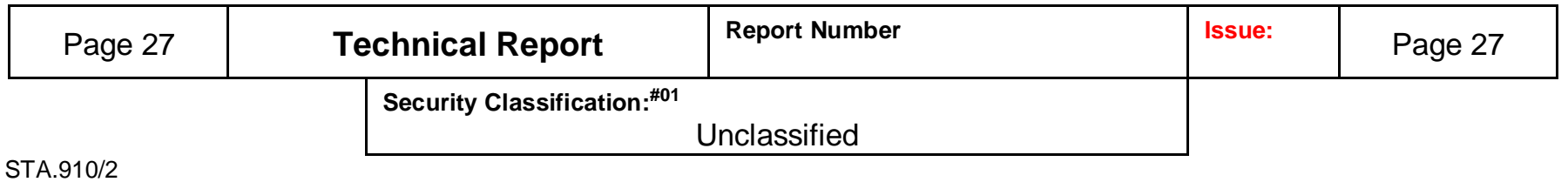




\section{COST PLAN}

\section{$5.1 \quad$ Introduction}

Moving from a centralized system to a distributed control system and the proprietary design to commercial of the shelf (COTS) was choosen to reduce product cost which would offset the initial higher Development cost.

The target is to reduce the product costs $25 \%$ to meet the Goals NGGT.

This will be possible if a distributed system is used instead of a centralized system. Thereby the interconnecting wiring costs and the commissioning and startup time will be reduced dramatically.

Due to commercial of the shelf (COTS) the product can adapt quicker to the consumer requirement and to the market changes, also a lower material cost can be obtained.

If corresponding software is used which pictures to code the development time will be lessened furthermore the simulation on the PC can rapidly convert to real time application, a reduction of the total cost is the consequence.

A other option to reduce the product cost is to use the IEC 1131 Compliant, it allows the transportation of the system code from one control environment to a other control environment without any other devices.

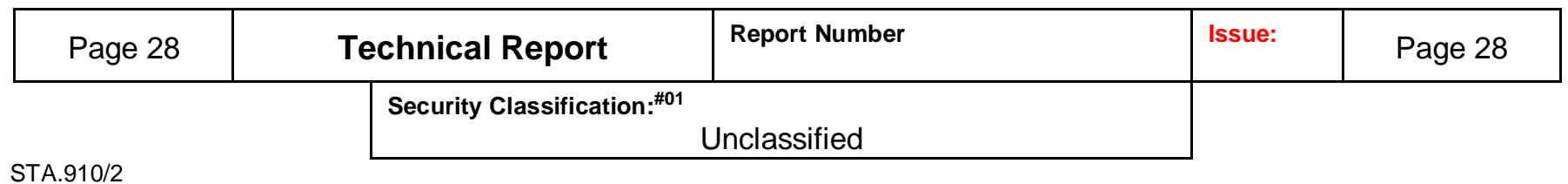




\subsection{Discussion}

The segmentation of the costs of a "Today's" control system is shown in the Figure below:

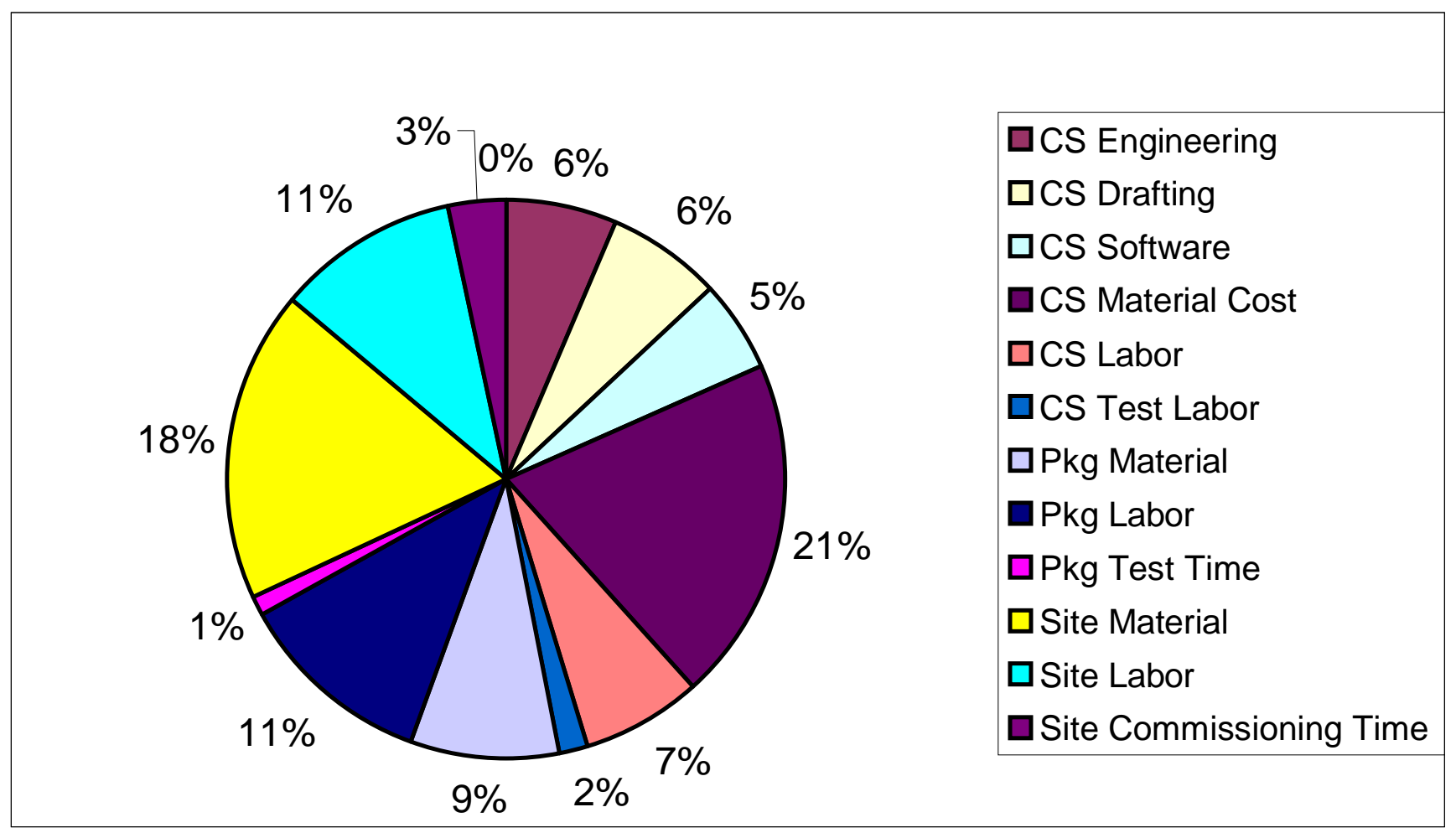

The manufacturing cost of the NGGT control system compared to the "today's" control system can be abated by reducing the material cost's with COTS and reducing the site material cost's. As a result of using a Remote IO the wiring on the NGGT control system is much less compare to the "Today's" control system, in fact this will reduce the site material costs.

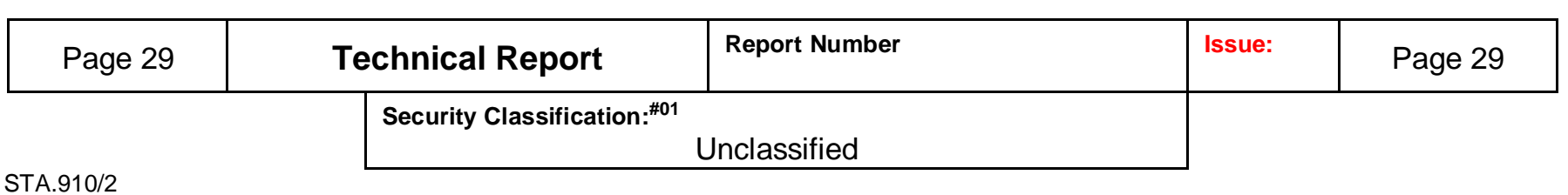


The cost of a Next Generation Gas Turbine (NGGT) Control System Product Cost compare to a Traditional Control System is shown in the Figure below:

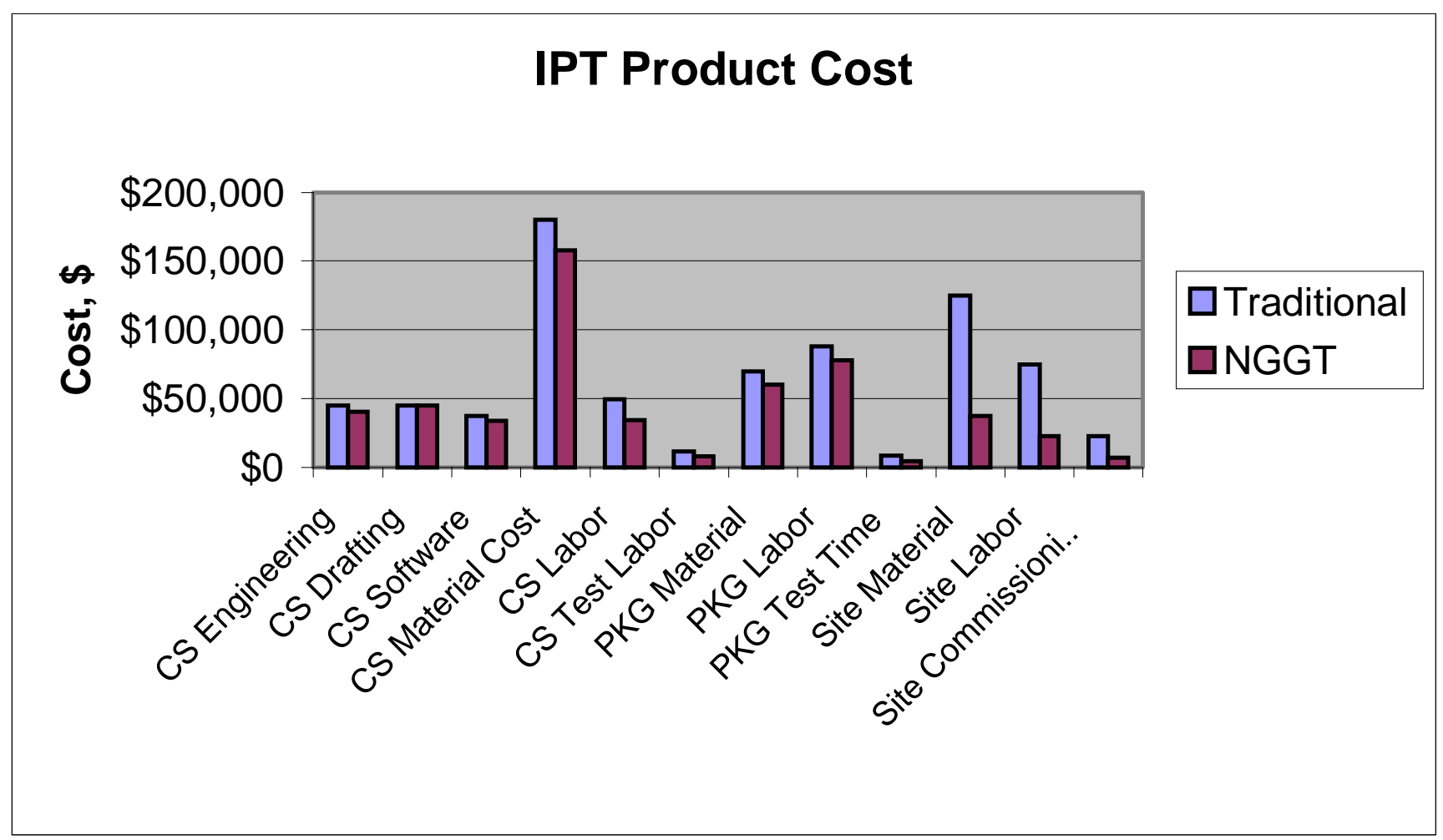

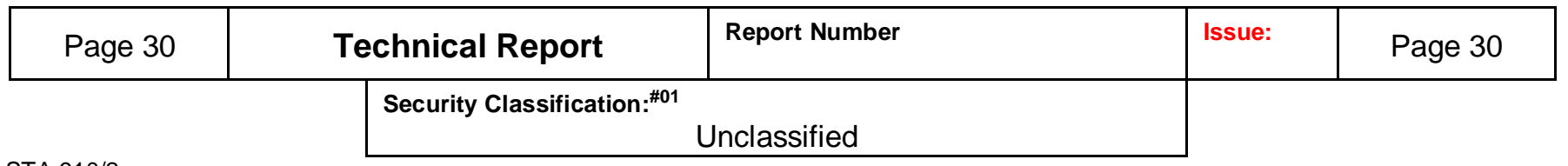


The following Figure shows the Cost and the Saveings from a Traditional Control System to a Next Generation Gas Turbine Control System.

$\begin{array}{rrr}\text { Control System Engineering } & \text { Traditional } & \text { NGGT } \\ \text { Engineering } & 45,000 & 40,500 \\ \text { Drafting } & 45,000 & 45,000 \\ \text { Software } & 37,500 & 33,750 \\ & & \\ \text { Control System Manufacturing } & & \\ \text { Material Cost } & 180,000 & 157,800 \\ \text { Manufacturing Labor } & 49,300 & 34,510 \\ \text { Test Labor } & 11,560 & 8,092\end{array}$

Package Installation

$\begin{array}{rrr}\text { Material } & 70,000 & 60,000 \\ \text { Labor } & 88,000 & 78,000 \\ \text { Test Time } & 8,400 & 4,200\end{array}$

Site Installation

$\begin{array}{rrr}\text { Material } & 125,000 & 37,500 \\ \text { Labor } & 75,000 & 22,500 \\ \text { Commissioning Time } & 22,400 & 6,720 \\ & & \\ \text { Total, \$ } & \mathbf{7 5 7 , 1 6 0} & \mathbf{5 2 8 , 5 7 2}\end{array}$

Savings, \%

30.1901844

Savings, $\$$ 228,588

Reduced Install Time

Savings, \$

42,000

Total Savings, \$

270,588

\begin{tabular}{|r|c|l|l|c|}
\hline Page 31 & Technical Report & Report Number & Issue: & Page 31 \\
\hline
\end{tabular}




\subsubsection{Cost Benefit Analysis}

Estimate a $50 \%$ time reduction in commissioning from 2 weeks to 1 week, at a conservative $\$ 6 \mathrm{~K}$ a day, this is $\$ 42 \mathrm{~K}$ savings.

\subsubsection{Summary}

Assume \$3 million extra to develop and validate NGGT Control vs a Traditional Control System.

\begin{tabular}{|c|c|c|}
\hline Year & Units & Savings \\
\hline $0-3$ & 0 & $-3,000,000$ \\
\hline 4 & 2 & $-2,458,000$ \\
\hline 5 & 5 & $-1,103,000$ \\
\hline 6 & 10 & $1,607,000$ \\
\hline $7-10$ & 20 & $23,287,00$ \\
\hline
\end{tabular}

Over a 10 year period 23 million dollars would be saved.

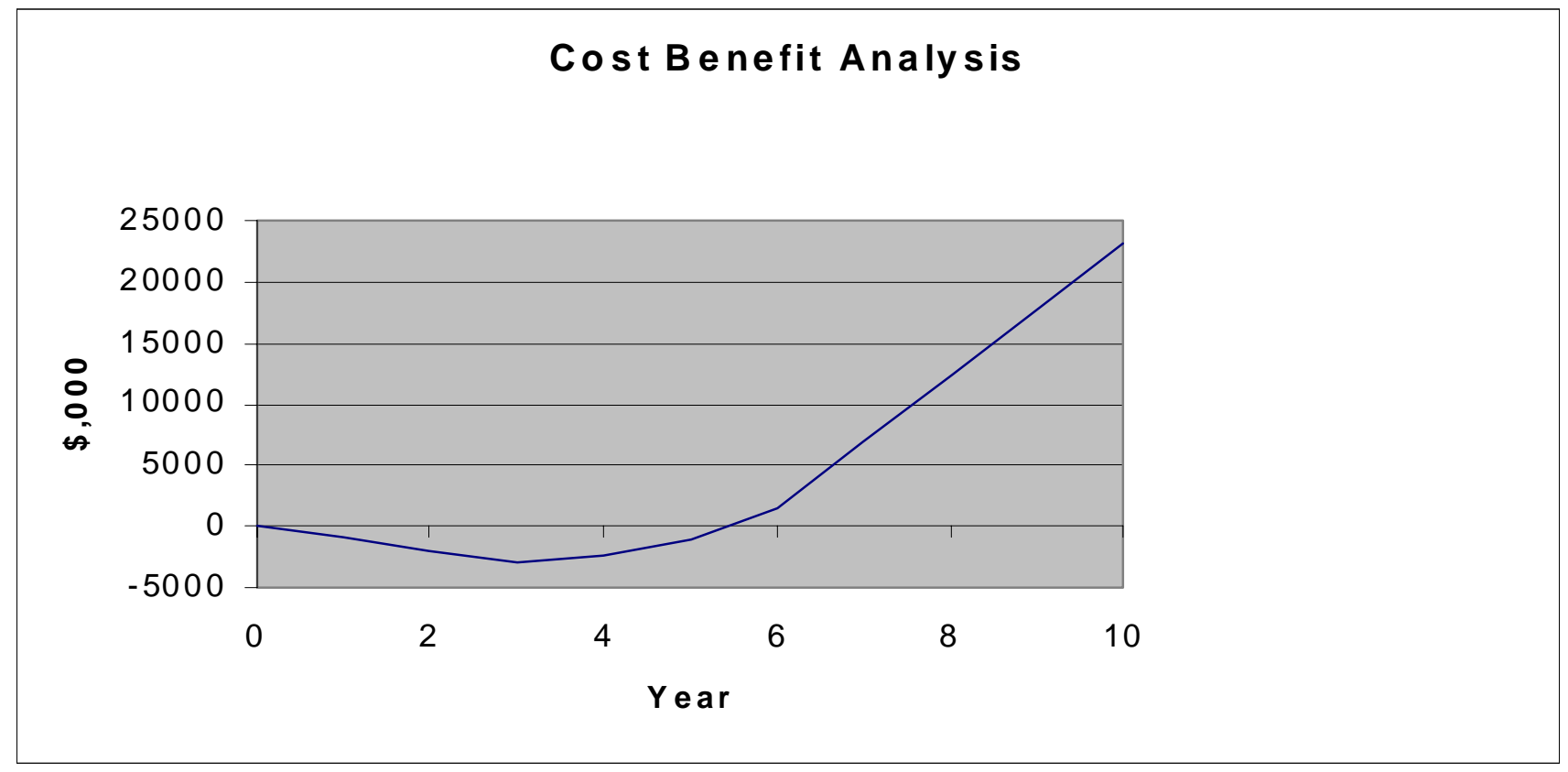




\section{PRELIMINARY RELIABILITY STUDY}

\subsection{Introduction}

The NGGT preliminary design differs significantly from the traditional design in that it will be using COTS (commercial off the shelf) equipment and a majority of the equipment will be in the form of remote $\mathrm{IO}$. Since the preliminary design is a departure from the traditional system it is necessary to perform a preliminary reliability study to ensure the reliability goals can be attained.

A typical goal is a MTTF of 30,000 per gas turbine subsystem. This study will consider the following sub-systems to demonstrate that the preliminary design will be able to meet the reliability targets:

Engine Control System (ECS)

Package Control System (PCS)

Boiler/Water Recovery System (BCS)

The goal is to obtain a MTTF of 10,000 for this three combined Systems.

\subsubsection{Discussion}

The preliminary design is based around Allen Bradley Flex IO, ControlNet, and an Open Pentium based controllers. Allen Bradley has supplied MTTF numbers for the Flex 10 line and ControlLogix platform. The Allen Bradley numbers are derived from their installed field base (i.e. number of boards shipped and when verse the number of returns).

\begin{tabular}{|c|c|c|}
\hline Flex IO Module & Description & MTTF \\
\hline 1794-PS1 & AC\DC Power Supply & $2,465,733$ \\
\hline 1794-ACN15 & ControlNet Module & 704,730 \\
\hline 1794-IB16 & 24 VDC 16 Input Module & $25,010,475$ \\
\hline 1794-OB16 & 24 VDC 16 Output Module & $4,428,410$ \\
\hline 1794-IF2XOF2I & Isolated $2 \mathrm{Al} \backslash 2 \mathrm{AO}$ Module & 646,880 \\
\hline 1794-IB8 & 24 VDC 8 Isolated Input Module & $1,285,440$ \\
\hline 1794-OB8EP & 24 VDC 8 Fused Digital Outputs & $2,489,149$ \\
\hline 1794-IJ2 & 2 Frequency Input Module & 543,574 \\
\hline 1794-IT8 & 8 Thermocouple Input Module & 272,113 \\
\hline$?$ & ControlNet to FF Linker & 475,709 (Estimated) \\
\hline 1794-OM8 & 220 VAC Digital Output Module & $2,466,880$ \\
\hline \multicolumn{3}{|l|}{ ControlLogix } \\
\hline 1756-OCX & Open Pentium Controller & 104,140 (Estimated) \\
\hline 1756-PAS2 & 220\120 VAC Power Supply & 931,000 \\
\hline 1756-A4 & 4 Slot Rack & $6,400,000$ \\
\hline 1756-CNB & Control Net Bridge & 992,000 \\
\hline
\end{tabular}

\subsubsection{Engine \& Package Control System}

\begin{tabular}{|c|c|c|c|c|}
\hline Page 33 & Technical Report & Report Number & Issue: & Page 33 \\
\hline \multicolumn{3}{|c|}{ Security Classification: ${ }^{\# 01}$ Unclassified } & & \\
\hline
\end{tabular}


The preliminary design has the ECS, PCS and BCS sharing remote 10 nodes using ControlNet. This was done to provide the lowest cost and most reliable system. Therefore the target MTTF for the 3 combined systems will be 10,000 hours. The remote IO was divided into 9 Systems:

\begin{tabular}{|l|l|r|r|r|r|r|r|r|}
\hline & \multicolumn{3}{|c|}{ Panel IO } & \multicolumn{2}{c|}{ Driver IO } & \multicolumn{2}{c|}{ MCC IO } & \multicolumn{2}{c|}{ GG Lube Oil IO } \\
\hline & Nr. & \multicolumn{1}{|c|}{ MTTF } & Nr. & MTTF & Nr. & MTTF & Nr. & MTTF \\
\hline \multicolumn{1}{|c|}{$2,465,733$} & 1 & $2,465,733$ & 1 & $2,465,733$ & 1 & $2,465,733$ & 1 & $2,465,733$ \\
\hline 1 CN & 1 & 704,730 & 2 & 352,365 & 1 & 704,730 & 1 & 704,730 \\
\hline 1CN to FF & & & & & 1 & 475,709 & 1 & 475,709 \\
\hline $16 \mathrm{DI}$ & 1 & $25,010,475$ & & & 1 & $25,010,475$ & & \\
\hline $16 \mathrm{DO}$ & 1 & $4,428,418$ & & & & & & \\
\hline 8 DO & & & & & 3 & 829,716 & 1 & $2,489,149$ \\
\hline 2 Al/AO & 1 & 646,880 & 2 & 323,440 & & & 1 & 646,880 \\
\hline 8 DI & & & 2 & 642,720 & & & 1 & $1,285,440$ \\
\hline 2 FRQ IN & & & 3 & 181,190 & & & & \\
\hline 8 TC/RTD IN & & & 3 & 90,704 & & & & \\
\hline & & & & & & & & \\
\hline
\end{tabular}

\begin{tabular}{|c|c|c|c|c|c|c|c|c|}
\hline & \multicolumn{2}{|c|}{ PT/GEN Lube Oil IO } & \multicolumn{2}{|c|}{ Generator } & \multicolumn{2}{|c|}{ Water Recovery } & \multicolumn{2}{|c|}{ Steam System } \\
\hline & $\mathrm{Nr}$. & MTTF & $\mathrm{Nr}$. & MTTF & $\mathrm{Nr}$. & MTTF & $\mathrm{Nr}$. & MTTF \\
\hline $1 \mathrm{PS}$ & 1 & $2,465,733$ & 1 & $2,465,733$ & 1 & $2,465,733$ & 1 & $2,465,733$ \\
\hline $1 \mathrm{CN}$ & 1 & 704,730 & 1 & 704,730 & 1 & 704,730 & 1 & 704,730 \\
\hline $1 \mathrm{CN}$ to $\mathrm{FF}$ & 1 & 475,709 & 1 & 475,709 & 1 & 475,709 & 1 & 475,709 \\
\hline $16 \mathrm{DI}$ & & & 1 & $25,010,475$ & 1 & $25,010,475$ & & \\
\hline $16 \mathrm{DO}$ & & & & & & & & \\
\hline $8 \mathrm{DO}$ & 1 & $2,489,149$ & 1 & $2,489,149$ & 1 & $2,489,149$ & 1 & $2,489,149$ \\
\hline $2 \mathrm{Al} / \mathrm{AO}$ & 1 & 646,880 & 1 & 646,880 & 1 & 646,880 & 1 & 646,880 \\
\hline $8 \mathrm{DI}$ & 1 & $1,285,440$ & & & & & 1 & $1,285,440$ \\
\hline $2 \mathrm{FRQ}$ IN & & & 1 & 543,574 & & & & \\
\hline 8 TC/RTD IN & & & 1 & 272,113 & 1 & 272,113 & 1 & 272,113 \\
\hline MTTF Total & & 150,325 & & 87,498 & & 104,284 & & 96,832 \\
\hline
\end{tabular}

\begin{tabular}{|r|c|l|l|c|}
\hline Page 34 & Technical Report & Report Number & Issue: & Page 34 \\
\hline
\end{tabular}




\begin{tabular}{|l|l|r|}
\hline & \multicolumn{2}{|c|}{ Boiler } \\
\hline & Nr. & MTTF \\
\hline $1 \mathrm{PS}$ & 1 & $2,465,733$ \\
\hline $1 \mathrm{CN}$ & 1 & 704,730 \\
\hline $1 \mathrm{CN}$ to FF & 1 & 475,709 \\
\hline $16 \mathrm{DI}$ & & \\
\hline $16 \mathrm{DO}$ & & \\
\hline 8 DO & 1 & $2,489,149$ \\
\hline $2 \mathrm{Al} / \mathrm{AO}$ & 1 & 646,880 \\
\hline 8 DI & 1 & $1,285,440$ \\
\hline 2 FRQ IN & & \\
\hline 8 TC/RTD IN & & \\
\hline & \multicolumn{3}{|c|}{} \\
\hline MTTF Total & \multicolumn{3}{|c|}{} \\
\hline
\end{tabular}

The total MTTF for the IO's is shown in the figure below

\begin{tabular}{|l|r|}
\hline IO Box & \multicolumn{1}{|l|}{ MTTF } \\
\hline Panel IO & 275,011 \\
\hline Driver IO & 40,925 \\
\hline MCC IO & 193,353 \\
\hline GG Lube Oil IO & 150,325 \\
\hline PT Lube Oil IO & 150,325 \\
\hline Generator & 87,498 \\
\hline Boiler & 150,325 \\
\hline Water Recovery & 104,284 \\
\hline Steam System & 96,832 \\
\hline Total & $\mathbf{1 1 , 8 2 8}$ \\
\hline
\end{tabular}

\begin{tabular}{|c|c|l|l|c|}
\hline Page 35 & Technical Report & Report Number & Issue: & Page 35 \\
\hline
\end{tabular}


The open controller for ECS will have his own processor, power supply, rack and ControlNet card. PCS and BCS will both have a processor, power supply, rack, and ControlNet card.

\begin{tabular}{|l|l|}
\hline Device & MTTF \\
\hline Power Supply & 931,000 \\
\hline Processor & 104,140 \\
\hline 4 slot rack & $6,400,000$ \\
\hline ControlNet Card & 992,000 \\
\hline Total & $\mathbf{8 4 , 4 5 3}$ \\
\hline
\end{tabular}

The total MTTF for ECS, PCS, BCS and IO is 9,240 hours.

\subsection{Summary}

Without going in to a detailed reliability study it appears that the NGGT preliminary design will the reliability target. It is recognized that this preliminary study does not include all of the components, but includes the major items that will affect reliability. All of the numbers used assume any fault will force a shutdown. If market requires greater reliability a duplex system could be provided.

\begin{tabular}{|r|c|l|l|c|}
\hline Page 36 & Technical Report & Report Number & Issue: & Page 36 \\
\hline \multicolumn{2}{|c|}{ Unclassified } & & \\
\cline { 2 - 3 } STA.910/2 & \multicolumn{2}{c}{ Security Classification:\#01 }
\end{tabular}




\section{DEVELOPMENT OF CONTROL PHILOSOPHY}

\subsection{Dry Low Emission Control}

\subsubsection{Introduction}

The legislative requirements to limit or regulate the pollutants emitted from land based fossil fuelled engines is becoming more stringent. This has resulted in the radical redesign of the combustion system employed on high pressure ratio aero derived gas turbines. The RollsRoyce Industrial NGGT, has been developed to minimise emission using a pre-mixed lean burn staged combustion technique. This has been designated NGGT Dry Low Emission (DLE)

\subsubsection{DLE Combustion System}

The pollutants emitted from gas turbine engines are directly related to the temperature at which the air and fuel reactants are burnt in their combustion systems. The principle pollutants are oxides of nitrogen (NOx), which begin formation at high temperatures and with rates which grow exponentially with increasing temperatures; carbon monoxide ( $\mathrm{CO}$ and unburned hydrocarbons (UHC) which are produced due to incomplete or weak combustion, typically at low combustor temperatures.

\begin{tabular}{|c|c|c|c|c|}
\hline Page 37 & Technical Report & Report Number & Issue: & Page 37 \\
\hline & \multicolumn{2}{|c|}{$\begin{aligned} \text { Security Classification: }{ }^{\# 1} & \\
& \text { Unclassified }\end{aligned}$} & & \\
\hline
\end{tabular}


The only viable method of limiting the production of $\mathrm{NOx}$ and $\mathrm{CO}$ emissions is by controlling the combustion temperature. There are two techniques which are available to control temperature, namely "rich-burn rapid quench" and "pre-mix lean burn". a both avoid the high temperature generated at near stoichiometric conditions. The terms "rich" and "lean" convey the magnitude of the fuel to air mixture strength with respect to stoichiometric conditions, the NGGT DLE employs the pre-mix lean burn option.

However, the employment of "lean" fuel to air mixtures requires operating the combustion system in closer proximity to the reactants weak extinction limit. Therefore, to provide low emissions over a wide operating range demands that the air to fuel ration (AFR) is modulated in some way to sustain combustion particularly at part load conditions where the mixture would be too weak to burn.

The idealised low emission combustor design would have the ability to modulate the air and fuel flow being used in the combustion process. Such designs were discarded on economic and reliability criteria.

A compromise is to divide the combustor into a number of discrete combustion stages, where at any power condition the proportion of the total air flow to each stage is fixed. Fuel can now be injected into each of these stages as a function of the power output demand and combustor inlet conditions. Thus, the ratios of the fuel and air reactants in each stage can be individually apportioned to control their respective temperatures of combustion within the close temperature bands established to meet the emission target.

The greater the number of stages the closer is the approximation to the idealised system, and increases the turndown of the pre-mix lean burn operating range. However as the air supply to the combustor is finite, an optimum number of stages can be determined, the NGGT DLE has three stages.

Whenever operational conditions determine that pre-mix lean burn is not practicable, (starting and low power operation), the Primary stage incorporates a diffusion flame type injector.

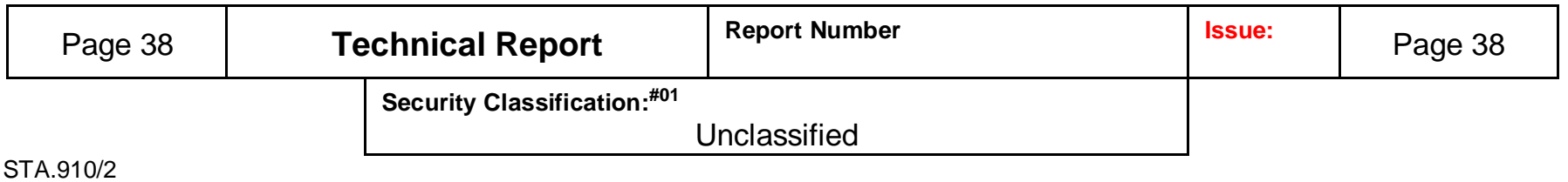




\subsubsection{NGGT DLE Staged Combustion description}

The DLE combustion system designed for the Industrial NGGT is a Staged Combustor in Series, which consists essentially of a number of separate combustion zones arranged in cascade, Primary, Secondary and Tertiary respectively, each independently fuelled, where the fuelling and the required number of active stages depends upon the ambient temperature and load range which must be covered.

With this design only the fist stage operates closer to the weak extinction conditions, as the burnt products are cascaded through the combustor and any subsequent stage receives preheated gases. Though the temperature is much reduced, the preheat significantly improves their respective weak extinction margins. Consequently this extends the range of pre-mix lean burn operation and turndown.

The NGGT DLE design has 9-off combustors which are projected radially outwards of the engine casing, replacing the inherited aero annular combustor. Each combustor is separate and independent, i.e. there are no flame tube interconnectors, and each combustor requires its own individual ignition source.

Located adjacent to each of the main combustors are separate gas fuelled Torch combustion chambers each of which are ignited form an electrical ignition source. The resulting combustion in the Torch chamber propagates a flame into the main combustor Primary Stage where it ignites the fuel emitted from a diffusion flame type injector.

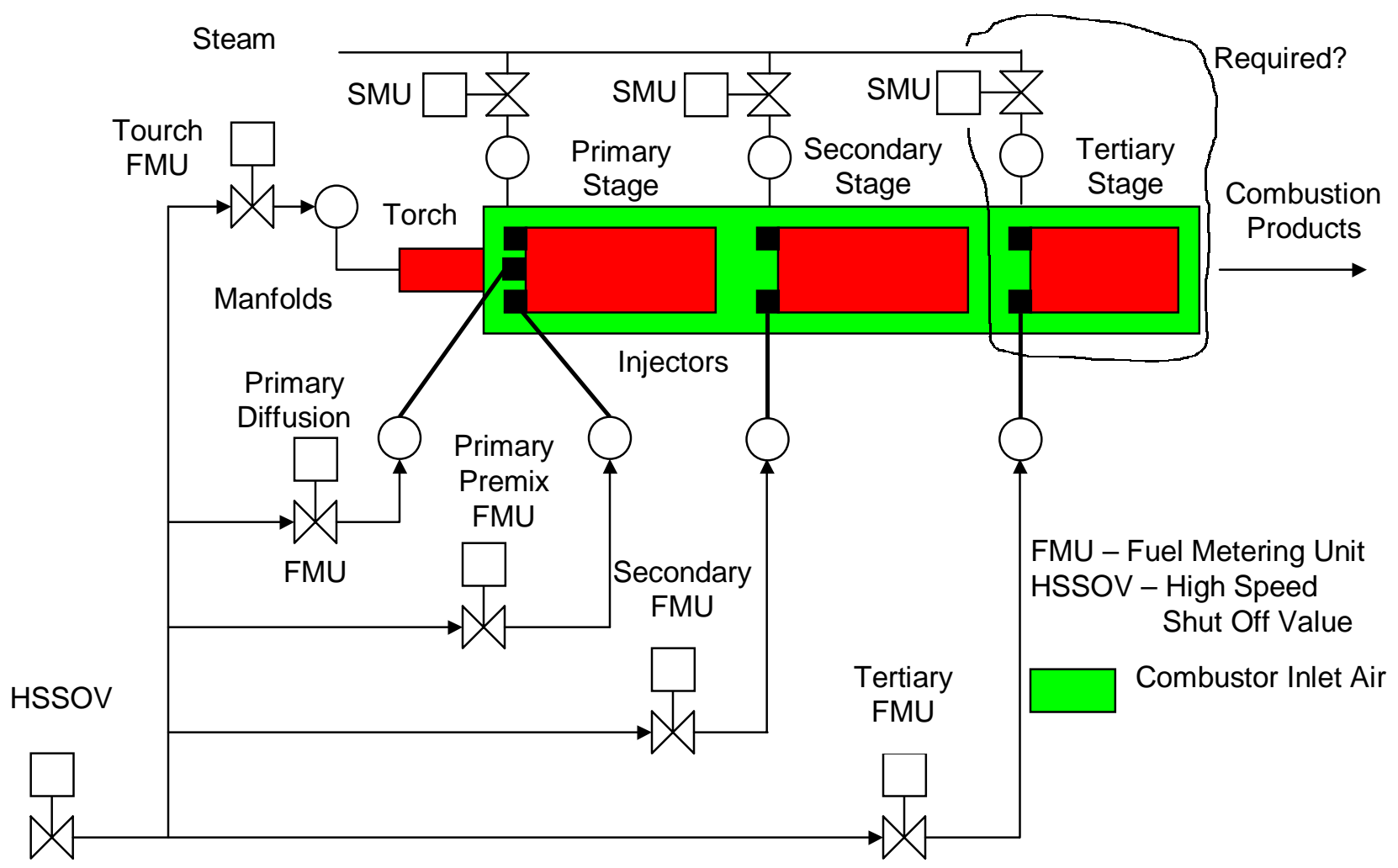

\begin{tabular}{|c|c|c|c|c|}
\hline Page 39 & Technical Report & Report Number & Issue: & Page 39 \\
\hline \multicolumn{3}{|c|}{$\begin{array}{l}\text { Security Classification: }{ }^{01} \text { Unclassified }\end{array}$} & & \\
\hline
\end{tabular}


The primary zone is fuelled form a diffusion flame injector, for starting and low power operation, until lean burn is selected. Fuelling of this zone is then transferred to a pre-mix type injector. During the normal operational cycle of the engine the control scheme is required to transfer between the two Primary Stage combustion modes of diffusion and pre-mix lean burn without loss of availability.

The pre-mix injector carefully pre-mixes the air and fuel reactants in ratios weaker than stoichiometric before they enter the combustrion zone. Similar pre-mix injectors fuel the Secondary and Tertiary zones.

As the Primary Stage does not receive any preheat, as the air reactant is at a relatively low temperature (650 to $750 \mathrm{~K}$ ), its turndown range is too small, less than $10 \%$ variation in AFR. Consequently the Primary Pre-mix Stage has been designed to operate at a constant zone temperature, approximately $1800 \mathrm{~K}$ to minimise emissions, thereby its stability margin does not become dependant on the operating conditions, and the narrower limit is therefore acceptable.

Whenever pre-mix lean burn operation is selected the Primary and Secondary pre-mix stages are always concurrently active to achieve the required power turndown.

When the Tertiary Stage is active the temperature of the Secondary combustion zone is modulated between its lower and upper limits of $1600 \mathrm{~K}-1800 \mathrm{~K}$ respectively to produce Tertiary combustion temperatures within the same band, thus minimising the levels of $\mathrm{CO}$ and UHC emissions whilst preventing any further NOx being produced.

Though each of the combustors are independent the fuel manifolding system located circumferentially around the engine connects each fuel injection lane of the nine combustors together. Thus the control scheme is only required to individually modulate the fuel to each combustion stage, as each of the five manifolds performs the distribution to each combustor.

\begin{tabular}{|c|c|l|l|l|}
\hline Page 40 & Technical Report & Report Number & Issue: & Page 40 \\
\hline
\end{tabular}




\subsubsection{Control Scheme Strategy}

The primary task of the combustor irrespective of whether it is a conventional or staged combustor is to provide the energy to drive both compressor and power turbines. The overall temperatures rise across the combustor will be identical between both types of combustor, hence the turbine inlet conditions will be no different with a staged combustor.

Therefore, the basic control loop, see the figure below, for the staged combustor is essentially the same "governors in parallel" scheme currently used on conventional engines. In such schemes the total engine fuel flow demand, Wfe, is scheduled by Lowest and Highest Wins Gate logic selecting the appropriate output demand form the various engine limiters, governors and transient schedules.

For the staged combustor Wfe is apportioned by the Staging Scheme to each of the respective combustion stage's fuel metering units $\mathrm{Wf}_{1}, \mathrm{Wf}_{2}, \mathrm{Wf}_{3}$ etc.

The thermodynamic heat balance of the control loop remains the same, and if the dynamics of the individual fuel metering units are identical, the response of the engine will also be similar.

The selection of individual fuel metering units for the staged scheme provides maximum flexibility to schedule the individual stages of the combustor consistent with variations in power output demands and ambient conditions and their effect upon the combustion operating point. The methodology employed to apportion the individual AFRs within each stage requires that the strategy of the "staging scheme" does not affect the forward path dynamics of the flow demand loop with delays or lags that may impair both engine and the system performance.

The control scheme is required to modulate the heat input to the engine to meet changing lead demands, no matter how sudden, but as the lean burn combustion process demands operation in closer proximity to weak extinction limits, the staging scheme must respond with adequate margins to ensure combustion is always sustained.

The staging scheme strategy is required to automatically enable the Primary Diffusion Injector whenever the conditions are outside the range of pre-mix lean burn operation but transfer back whenever the conditions are satisfactory.

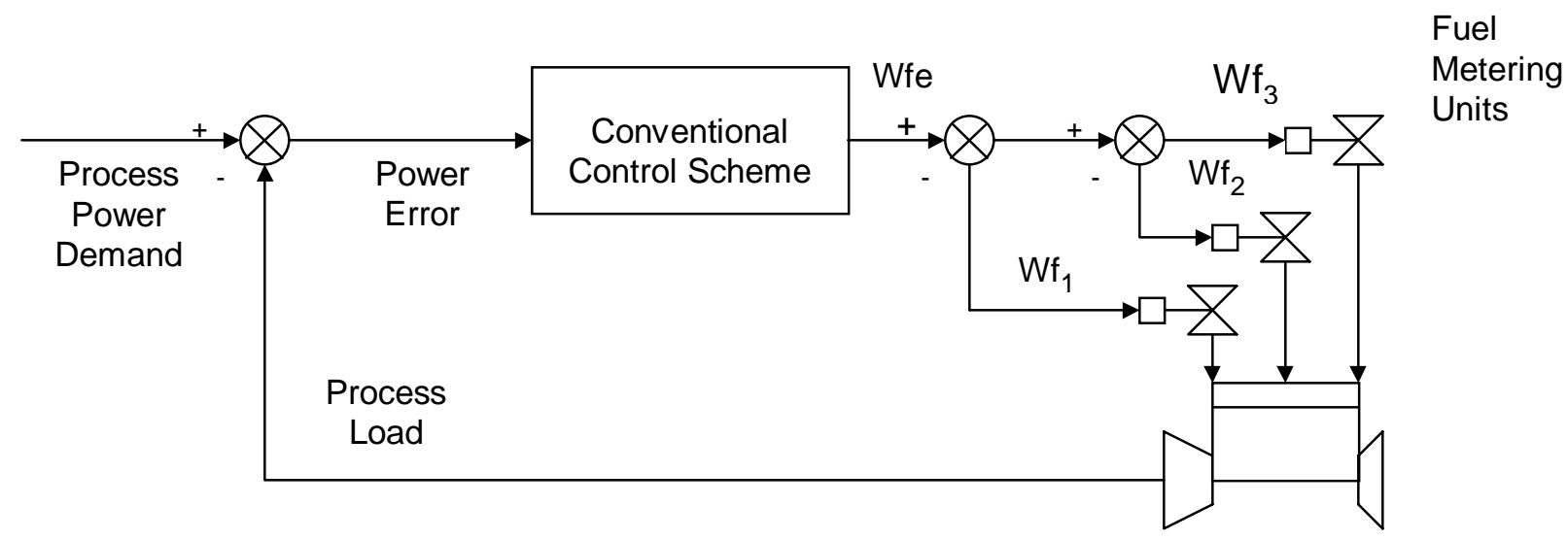

\begin{tabular}{|c|c|c|c|c|}
\hline Page 41 & Technical Report & Report Number & Issue: & Page 41 \\
\hline & \multicolumn{2}{|c|}{$\begin{aligned} \text { Security Classification: }{ }^{\# 01} & \\
& \text { Unclassified }\end{aligned}$} & & \\
\hline
\end{tabular}




\subsection{Steam Control}

The steam control controls the injection of the steam into the primary combustion zone and secondary combustion zone, as shown in the figure below. The Steam flow will also help reduce emmisions. The injected steam would act as added humidity. With the injected steam in the primary and secondary combustion zone a much better control of the combustion temperature during load transients is possible.

To control the steam in the combustion zone and control the combustion temperature consequently leads to a lower pollutant emission.

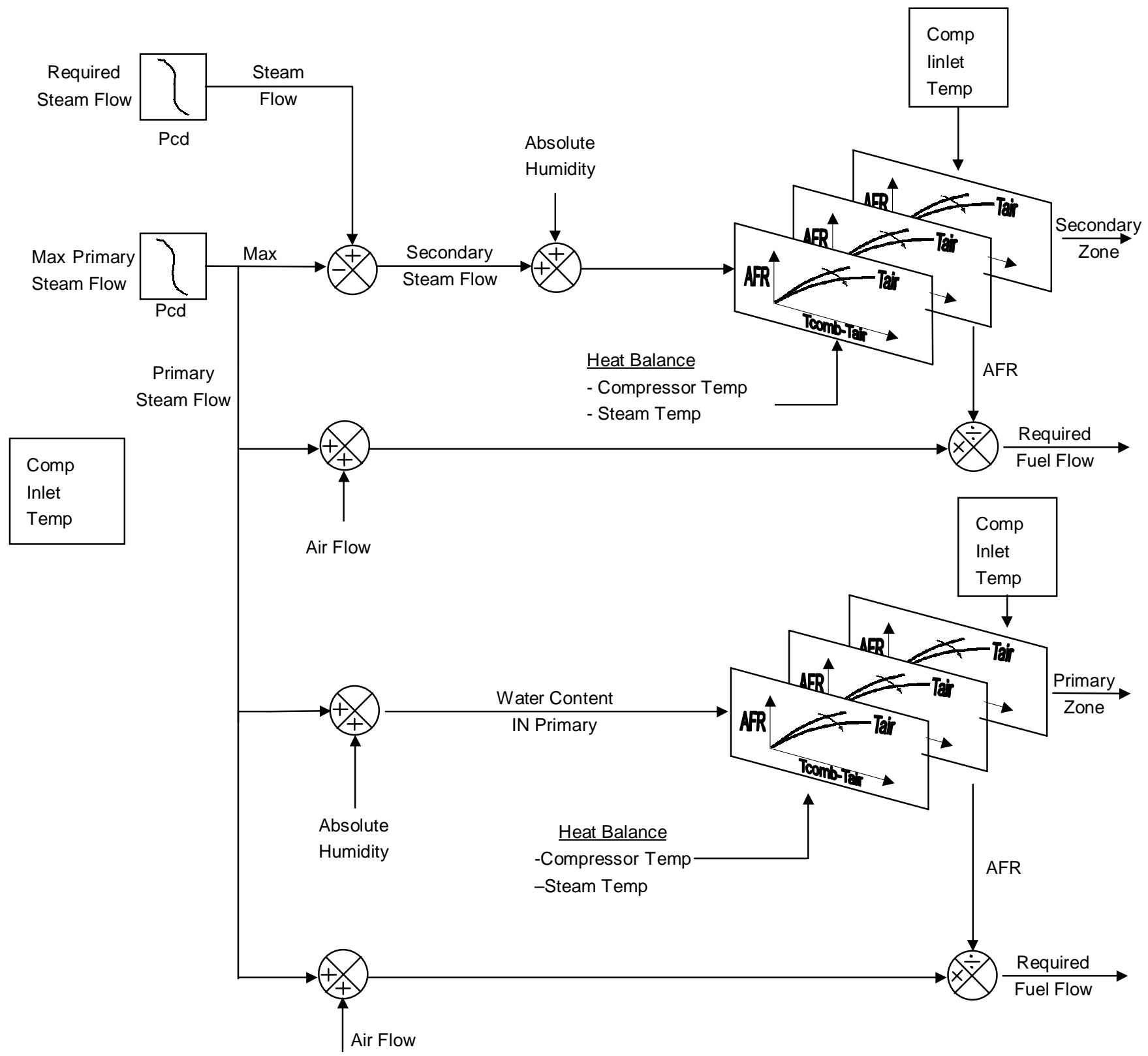

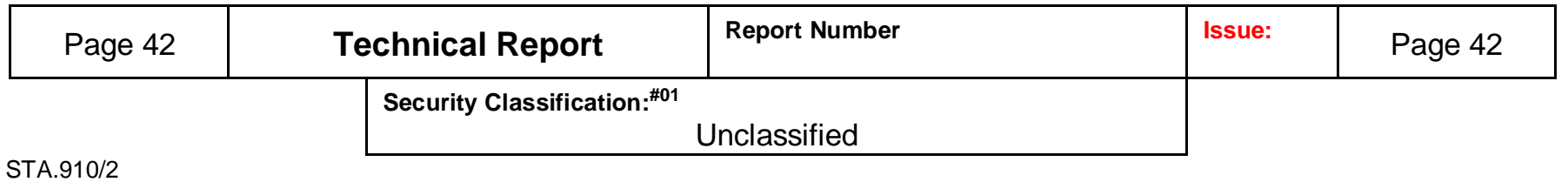




\subsection{Boiler Control}

\subsubsection{Once-through forced-flow boiler}

In once-through forced-flow boiler, the water generally flows from the economizer to the furnace-wall tubes, then to the gas-convection-pass enclosure tubes and the primary superheater. Usually, the transition to the vapor phase (if operation is below the critical pressure) begins in the furnace circuits and depending upon the operating conditions and the design, is completed either in the gas-convection-pass enclosure or in the primary superheater. The steam from the primary superheater passes to the secondary (and possibly to a tertiary) superheater. One or more reheaters are provided to reheat the low-pressure steam.

\subsubsection{Boiler Circulation}

Adequate circulation in the steam-generating section of a boiler is required to prevent overheating of the heat-absorbing surfaces, and it may be provided naturally by gravitational forces, mechanically by pumps, or by a combination of both methods.

Natural circulation is produced by the difference in the densities of the water in the unheated downcomers and the steam water mixture in the heated steam generating tubes. This density differential provides a large circulating force. The downcomers and the heated circuits are so designed that the friction, or resistance to flow, through the system balances the circulating force at the desired total circulating flow.

In forced-flow once-through boiler, the water from the feed supply is pumped to the inlet of the heat-absorbing circuits. Evaporation, or change of state, takes place along the length of the circuit, and when evaporation is completed the steam is superheated. These units do not require steam or water drums and, in most cases, use relatively small-diameter tubes. The boilers can be started rapidly owing to the elimination of the drums and the reduced amount of metal. The water flow to the unit is the same as the steam output, and fluid velocities greater than those needed for natural- or assisted-circulation units must be used at full load so as to maintain adequate velocities at the low loads and, thus, satisfactory tube-metal temperatures at all loads.

The transition from a liquid to a vapor at, or above, the critical steam pressure of 3,206 lb/in. (225 kgf/cm•) is dependent upon temperature and takes place without a change in density. Thus, separation of steam and water is impossible and forced-flow once-through boilers must be use.

\begin{tabular}{|r|c|l|l|l|}
\hline Page 43 & Technical Report & Report Number & Issue: & Page 43 \\
\hline \multicolumn{2}{|c|}{ Security Classification:\#01 Unclassified } & & \\
\cline { 2 - 3 } STA.910/2 & \multicolumn{2}{|c|}{}
\end{tabular}


Security Classification: $\# 01$

Unclassified

Forced-flow once-through boilers must be operated above a specified minimum flow - usually one-quarter to one-third of full-load flow - in order to maintain adequate water velocities in the furnace-wall tubes. However, the turbogenerator can be operated at any lead by the use of a bypass system that diverts the excess flow to a flash tank for heat recovery. the bypass system also can be used as a pressure relieving system, as the source of low-pressure steam to the turbine during start-up, and as a means of controlling steam temperature to the turbine during hot restarts.

Combined circulation units utilize forced once-through flow with flow recirculation in the furnace walls to provide satisfactory water velocities during start-up and low-load operations. In this design, some of the water at the exit of the furnace circuits is mixed with the incoming feedwater, flows to and through a circulating pump, and then passes to creases the water velocities in the furnace tubes at low loads, and since recirculation is not used at the higher loads, there is no increase in velocity or in the resistance to flow at the higher loads.

\subsubsection{Operating Controls}

The need for operating instruments and manual or automatic controls varies with the size and type of equipment, method of firing, and proficiency of operating personnel.

Safe operation and efficient performance require information relative to the water level in the boiler drum; burner performance; steam and feedwater pressures; superheated and reheated steam temperatures; pressure of the gas and air entering and leaving principal components; feedwater and boiler-water chemical conditions and particle carryover; operation of feed pumps, fans, and fuel burning and fuel-preparation equipment; relationship of the actual combustion air passing through the furnace to that theoretically required for the fuel fired; temperatures of the fuel, water, gas and air entering and leaving the principal components of the boiler unit; and fuel, feedwater, steam and air flows so as to monitor operating conditions continuously and to make such adjustments as might be necessary.

Control of the various functions to maintain the desired operating conditions may be accomplished on small-capacity boilers by the manual adjustment of valves, dampers, and motor speeds. Most oil- and gas fired package boilers are equipped with automatic controls to purge the furnace, to start and stop the burners, and to maintain the required steam pressure and water level. the operating requirements of utility and large industrial boilers dictate the sue of automatic controls for the major variables, such as feedwater flow, firing rate, and steam temperature. The type of boiler and its components generally establishes the basic mode of control. Analog controls of either the pneumatic or the electric type are available. Digital control is being used more extensively.

Sequence controls often are applied in the start-up of utility boilers to program the furnace purge, burner light-off, and burner control. Interlocks are essential to ensure the proper staring and firing sequence and to alarm or automatically shut down the unit in the event of the failure of essential auxiliaries.

\begin{tabular}{|c|c|c|c|c|}
\hline Page 44 & Technical Report & Report Number & Issue: & Page 44 \\
\hline \multicolumn{2}{|c|}{ Unclassified } & & \\
\cline { 2 - 3 } STA.910/2 & Security Classification: ${ }^{* 01}$ &
\end{tabular}




\section{CONTROL TECHNIQUES}

\subsection{Predictive Control}

Predictive control techniques employ advanced controllers of the digital type that utilize a (usually computerized) model of the system, including the process, to predict the reaction of the system to variations of the load variable of the set point. The result is used to calculate the optimum output of the compensator (controller) to the manipulated variable.

Consider a control strategy that uses only the current value of the system output as feedback and another control strategy that uses knowledge of the future behaviour of the output for calculating the feedback signal. Which control strategy is expected to perform better? Clearly, the answer is the second control strategy, as the necessary action can be taken beforehand if the future behaviour of the system can be predicted.

Predictive Control has three basic steps:

\section{Output prediction:}

a model of the system is used to predict the future behaviour of the output based on the available data at the current time.

\section{Control calculation:}

a future control signal is calculated so that the predicted output is as close as possible to the desired future output.

3. Closing the feedback loop:

the above two steps are carried out in open-loop fashion, and the loop is closed by applying the current value of the proposed future control signal, calculated in the previous step, to the system and discarding the rest.

Controller design in model predictive control is based on the predicted behaviour of the process over the predicted horizon. Values of the manipulated variables are computed to ensure that the predicted response has certain desirable characteristics. One sampling period after the application of the current control action, the predicted response is compared with the actual response. Using corrective feedback action for any errors between actual and predicted responses, the entire sequence of calculations is then repeated at each sampling instant. The control objective is to have the corrected predictions approach the set point as closely as possible.

Predictive control methods are useful, for example, in the design of controllers for multiple input, multiple output (MIMO) systems, where standard design methods for standard controllers cannot be used efficiently. An example would be when the process exhibits unusual dynamic characteristics or when it is crucial to meet constraints on the manipulated and/or controlled variables.

\begin{tabular}{|c|c|c|c|c|}
\hline Page 45 & Technical Report & Report Number & Issue: & Page 45 \\
\hline \multicolumn{2}{|c|}{ Unclassified } & & \\
\cline { 2 - 3 } STA.910/2 & Security Classification:\#01 & &
\end{tabular}




\subsection{Model Predictive Control MPC}

All the control action of the classical control strategy is based on the reaction of past errors. For the Model Predictive Control (MPC) a model is used to predict the future plant output, based on past and current values and on the proposed optional future control actions. These actions are calculated by the optimizer taking into account the cost function (where the future tracking error is considered) as well as the constraints.

The process model plays, in consequence, a decisive role in the controller. The chosen model must be capable of capturing the process dynamics so as to precisely predict the future outputs as well as being simple to implement and to understand.

The optimizer is another fundamental part of the strategy as it provides the control actions. If the cost function is quadratic, its minimum can be obtained as an explicit function of past inputs and outputs and the future reference trajectory. In the presence of inequality constraints the solution has to be obtained by more computationally taxing numerical algorithms. The size of the optimization problems depends on the number of variables and on the prediction horizons used and usually turn out to be relatively modest optimization problems which do not require sophisticated computer codes to be solved. However the amount of time needed for the constrained and robust cases can be various orders of magnitude higher than that needed for the unconstrained case and the band width of the process to which constrained MPC can be applied is considerably reduced.

The methodology of all the controllers belonging to the MPC family is characterized by the following strategy:

1. The future outputs for a determined horizon, called the prediction horizon, are predicted at each instant using the process model. These predicted outputs (for the NGGT the Power, Airflow and the Temperature) depend on the known values (Steam I \& II and Fuel I \& II) and on the future control signals, which are those to be sent to the system and to be calculated. As shown in figure below.

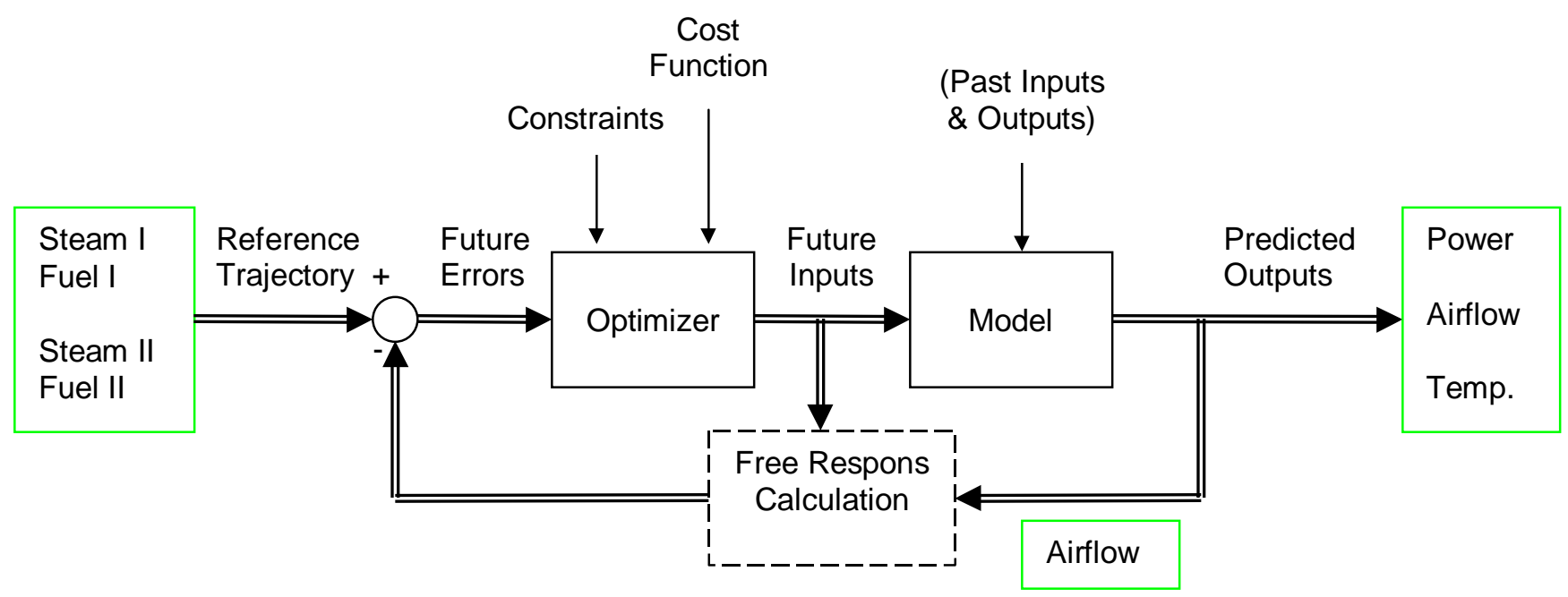

\begin{tabular}{|c|c|c|c|c|}
\hline Page 46 & Technical Report & Report Number & Issue: & Page 46 \\
\hline & \multicolumn{2}{|c|}{ Security Classification:\#01 Unclassified } & & \\
\hline
\end{tabular}


2. The set of future control signals is calculated by optimizing a determined criterion in order to keep the process as close as possible to the reference trajectory (which can be the setpoint itself or a close approximation of it). This criterion usually takes the form of a quadratic function of the errors between the predicted output signal and the predicted reference trajectory. The control effort is included in the objective function in most cases. An explicit solution can be obtained if the criterion is quatratic, the model is linear and there are no constraints, otherwise an iterative optimization method has to be used. Some assumptions about the structure of the future control low are also made in some cases, such as that it will be constant from a given instant.

3. The control signal is sent to the process whilst the next control signals calculated are rejected, because at the next sampling instant is already known and step 1 is repeated with new value and all the sequences are brought up to date.

Notice that the MPC strategy is very similar to the control strategy used in driving a car. The driver knows the desired reference trajectory for a finite control horizon, and by taking into account the car characteristics (mental model of the car) decides which control actions (accelerator, brakes and steering) to take in order to follow the desired trajectory. Only the first control actions are taken at each instant, and the procedure is again repeated for the next control decisions in a receding horizon fashion. Notice that when using classical control schemes, such as PIDs, the control actions are taken based on past errors. If the car driving analogy is extended, as has been done by one of the commercial MPC vendors, in their publicity, the PID way of driving a car would be equivalent to driving the car just using the mirror. (((This analogy is not totally fair with PIDs, because more information (the reference trajectory) is used by MPC. Notice that if a future point in the desired reference trajectory is used as the setpoint for the PID, the differences between both control strategies would not seem so abysmal. 


\subsection{Observer}

In the classical method of design feedback is most generally obtained from one variable, the output. Only when inner feedback loops are used for system compensation is more than one variable employed for feedback control.

The conventional controller incorporating $P+D, P+l$, phase lead or phase lag algorithms is generally unable to control independently all system poles. Since the number of free parameters available for adjustment is restricted to two or three in most cases. Therefore, if the process can be described in state vector form, it is logical to extend the power of the classical design approach by providing full state feedback. This leads to the concept of state variable feedback and is the basis of most design techniques in modern control theory.

Many of the analytical design procedures reduce to problems of:

- finding a suitable variable to manipulate to enable the system output to be changed from some initial state to any other desired state in a finite time and

- being able to estimate, with confidence, the process state from observations made over a finite time of the output response.

These two properties are classified as controllability and observability respectively.

The arrangement shown in the Figure below is a block diagram representation of a system in which each state variable is fed back through a fixed gain. The state vector for the $\mathrm{n}$ dimensional process is fed back through a fixed a constant matrix.

The system can be shown to be controllable, i.e. it is possible to change the system state from some initial state to a desired equilibrium state in a finite time interval by means of the process input. This gives rise to a design procedure known as pole-placement.

Although most physical systems are controllable, there are some exceptions and care must be taken in making all-inclusive general statements. In particular, lack of controllability may not be apparent when a transfer function description is used to model a process.

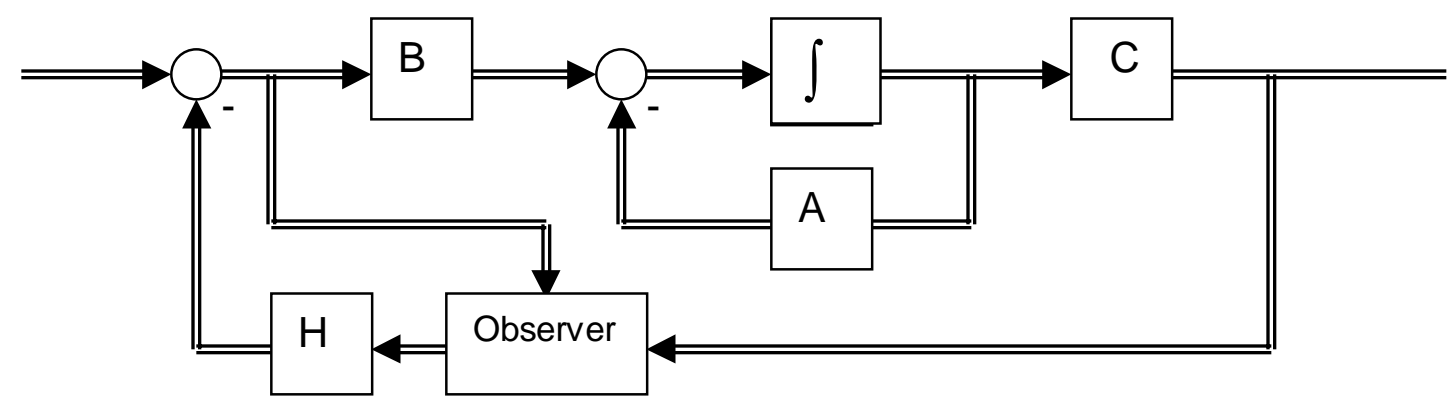




\section{LOAD MANAGEMENT STUDY \\ 9.1 Fuel/Valve system Configuration affects}

\subsubsection{Introduction}

The configuration of the fuel system mechanical components and response of each component affects the safety and performance of the gas turbine package. The objective of this document is to outline the varying effects of system configuration for the NGGT power generation package.

\subsubsection{Model}

The model being used as the basis for analysis was created in the MATLAB/SIMULINK environment. MATLAB and SIMULINK are software modeling programs created by The Mathworks Inc.. The core of the model contains the following:

1. NGGT Gas Generator

2. Power Turbine and Load Model

3. Fuel Control Logic

4. Fuel System Piping Model

5. Component Modeling

\subsubsection{Assumptions:}

- Direct Drive 2 pole Generator

- 3600 RPM Operations Speed

- Approximately 50 MW per Unit

\begin{tabular}{|r|c|l|l|c|}
\hline Page 49 & Technical Report & Report Number & Issue: & Page 49 \\
\hline
\end{tabular}


9.1.4 Pictures of the Model

NGGT-Power Generation

SIMULATION

Valve Setpoint

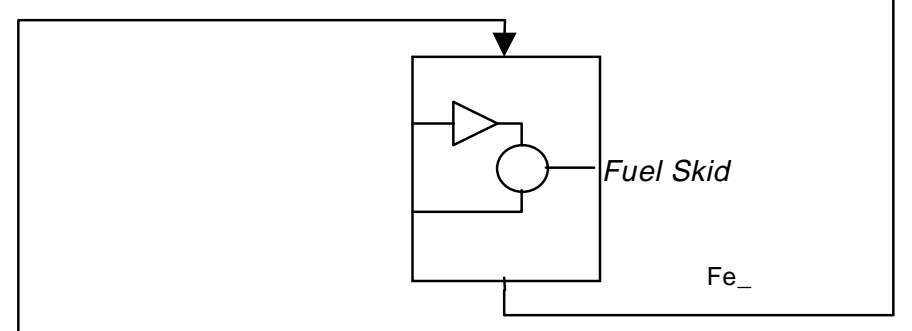

N1

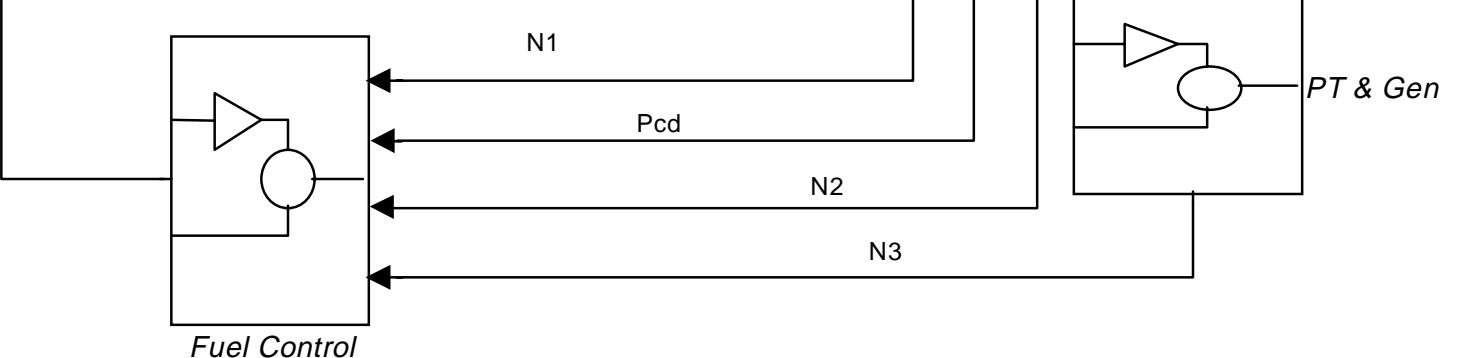

Page 50

Technical Report

Security Classification:\#01

Unclassified
Gas

Generator

$\mathrm{Fe}$

T6

P6

M6
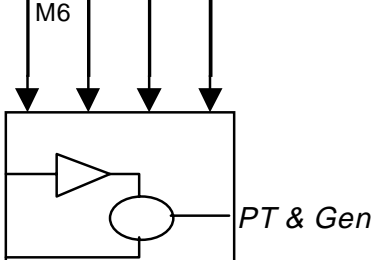

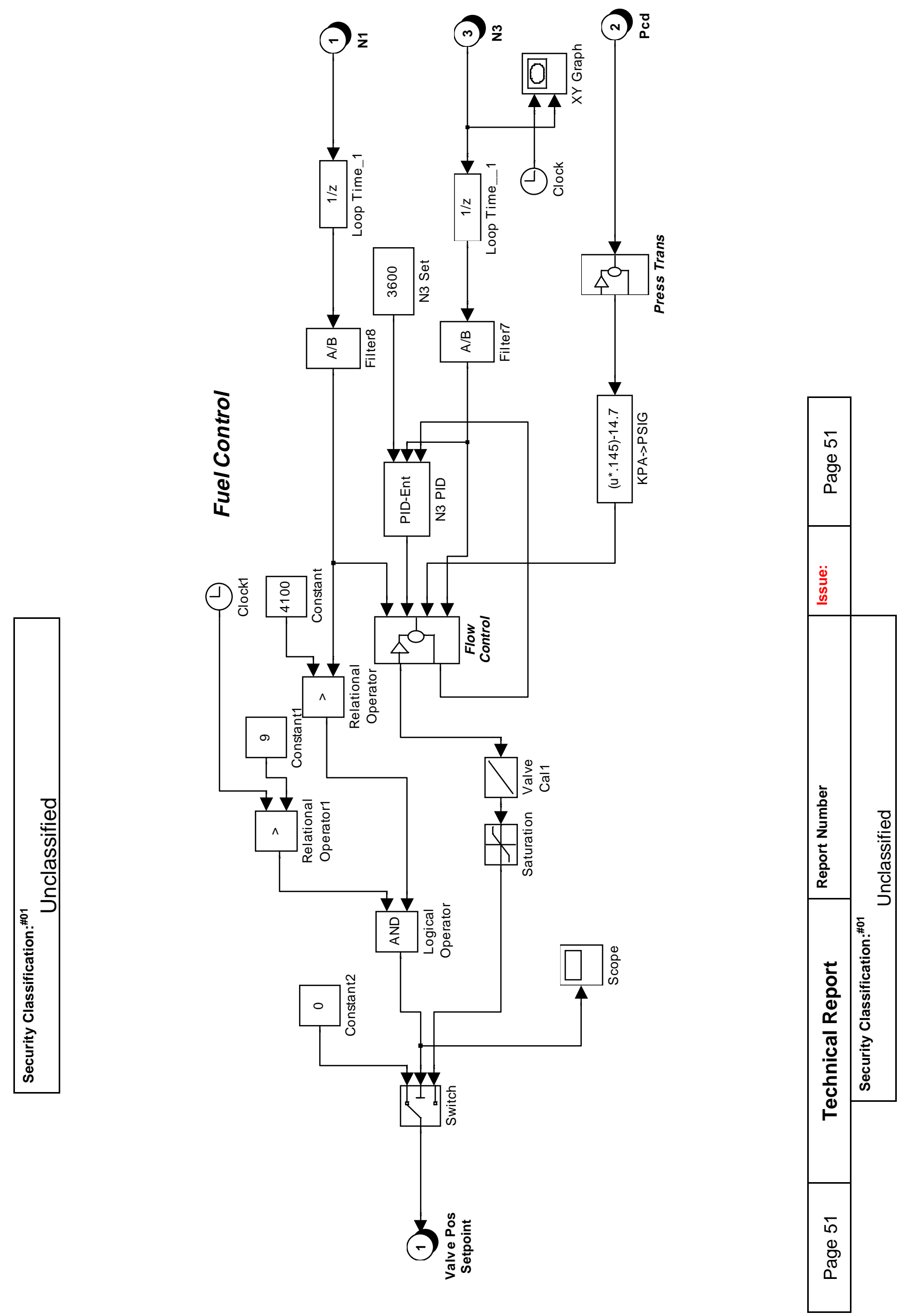


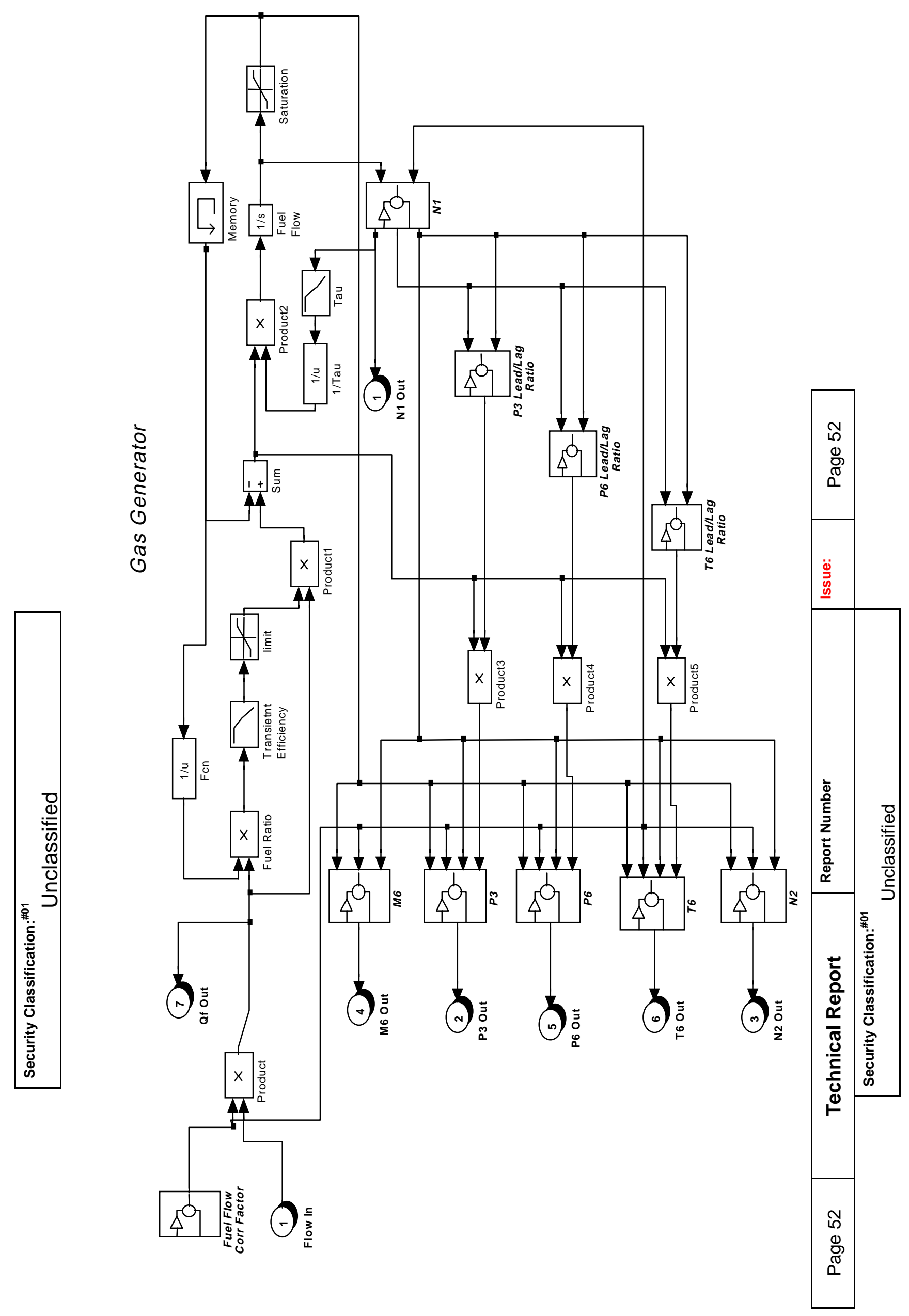




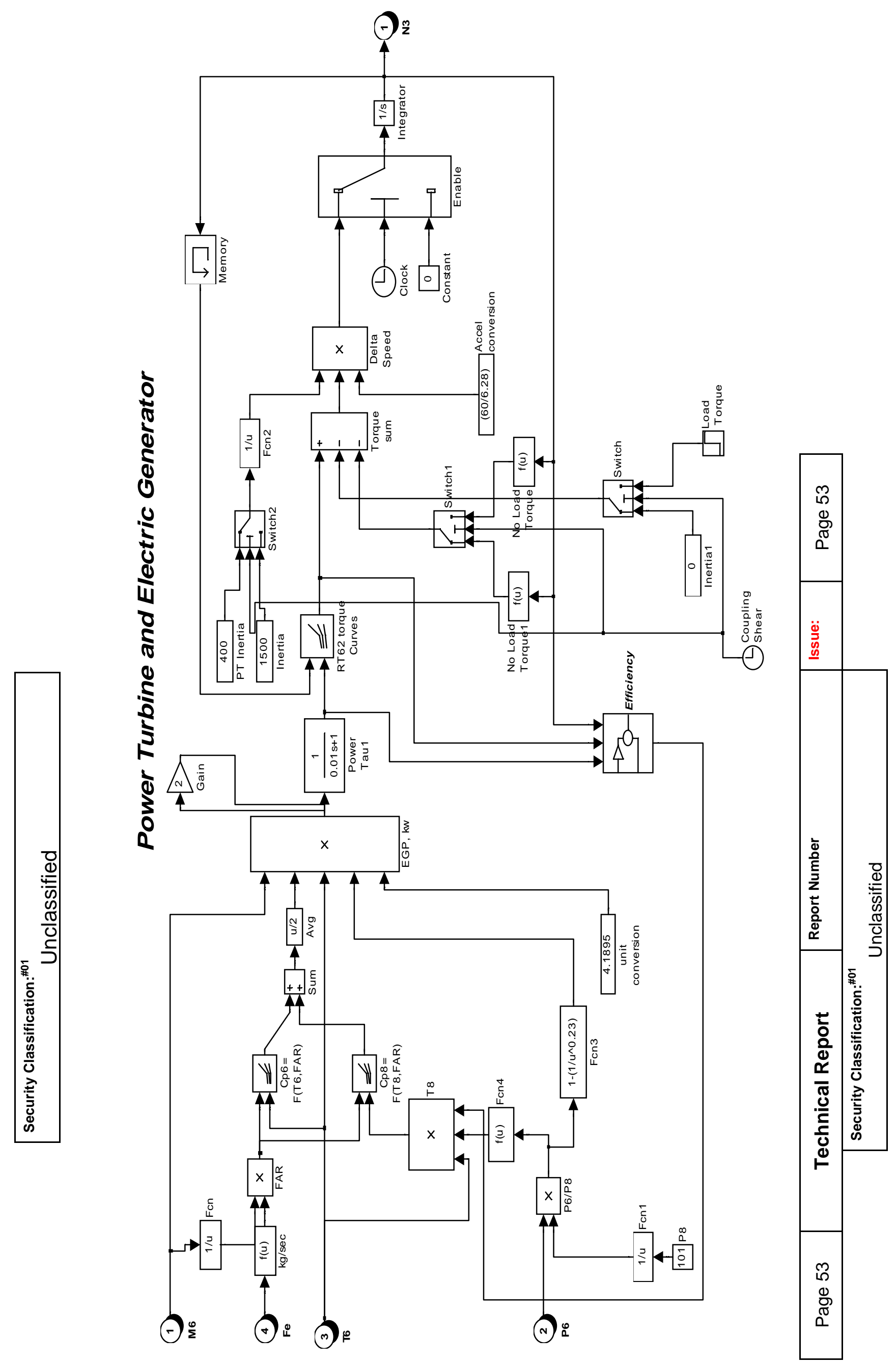




\subsubsection{Requirements}

The design speed of the unit is 3600 RPM. The trip speed is usually $10 \%-15 \%$ above the design speed. API 616 states the maximum speed attained after an overspeed trip is $110 \%$ of the trip speed. This requirements should provide guidance during mechanical component design.

\subsubsection{Dynamic Analysis}

The dynamic analysis will define what volumes and instrument effect. Furthermore shows the dynamic analysis the consequence of $100 \%$ loss of load.

\begin{tabular}{|c|c|c|c|}
\hline Inertia & $\begin{array}{l}\text { Manifold } \\
\text { Volume }\end{array}$ & Valve Slew Rate & Frequency Shift \\
\hline \multirow[t]{4}{*}{$500 \mathrm{~kg}-\mathrm{m} \bullet$} & 3000 in. & $\begin{array}{r}200 \mathrm{~ms} \\
100 \mathrm{~ms} \\
40 \mathrm{~ms} \\
20 \mathrm{~ms}\end{array}$ & $\begin{array}{l}40.28 \\
38.36 \\
36.45 \\
36.10\end{array}$ \\
\hline & $2000 \mathrm{in} \bullet$ & $\begin{array}{r}200 \mathrm{~ms} \\
100 \mathrm{~ms} \\
40 \mathrm{~ms} \\
20 \mathrm{~ms}\end{array}$ & $\begin{array}{l}37.49 \\
35.92 \\
34.13 \\
33.78\end{array}$ \\
\hline & $1000 \mathrm{in} \bullet$ & $\begin{array}{r}200 \mathrm{~ms} \\
100 \mathrm{~ms} \\
40 \mathrm{~ms} \\
20 \mathrm{~ms} \\
\end{array}$ & $\begin{array}{l}37.62 \\
33.69 \\
31.88 \\
31.53\end{array}$ \\
\hline & 500 in॰ & $\begin{array}{r}200 \mathrm{~ms} \\
100 \mathrm{~ms} \\
40 \mathrm{~ms} \\
20 \mathrm{~ms}\end{array}$ & $\begin{array}{l}36.57 \\
32.49 \\
30.69 \\
30.33\end{array}$ \\
\hline \multirow[t]{3}{*}{$\begin{array}{ll}1000 & \mathrm{~kg}- \\
\mathrm{m} & \end{array}$} & 3000 in॰ & $\begin{array}{r}200 \mathrm{~ms} \\
100 \mathrm{~ms} \\
40 \mathrm{~ms} \\
20 \mathrm{~ms}\end{array}$ & $\begin{array}{l}23.50 \\
21.02 \\
20.00 \\
19.79\end{array}$ \\
\hline & $2000 \mathrm{in} \bullet$ & $\begin{array}{r}200 \mathrm{~ms} \\
100 \mathrm{~ms} \\
40 \mathrm{~ms} \\
20 \mathrm{~ms}\end{array}$ & $\begin{array}{l}21.99 \\
19.68 \\
18.63 \\
18.42\end{array}$ \\
\hline & $1000 \mathrm{in} \bullet$ & $\begin{array}{r}200 \mathrm{~ms} \\
100 \mathrm{~ms} \\
40 \mathrm{~ms} \\
20 \mathrm{~ms}\end{array}$ & $\begin{array}{l}20.76 \\
18.38 \\
17.30 \\
17.10\end{array}$ \\
\hline
\end{tabular}


Security Classification:\#01

Unclassified

\begin{tabular}{|c|c|c|c|}
\hline $\begin{array}{ll}1000 & \mathrm{~kg}- \\
\mathrm{m} \bullet & \end{array}$ & $500 \mathrm{in} \bullet$ & $\begin{array}{r}200 \mathrm{~ms} \\
100 \mathrm{~ms} \\
40 \mathrm{~ms} \\
20 \mathrm{~ms} \\
\end{array}$ & $\begin{array}{l}20.08 \\
17.66 \\
16.62 \\
16.41 \\
\end{array}$ \\
\hline \multirow[t]{4}{*}{$\begin{array}{l}1500 \\
\mathrm{~m} \bullet\end{array}$} & 3000 in॰ & $\begin{array}{r}200 \mathrm{~ms} \\
100 \mathrm{~ms} \\
40 \mathrm{~ms} \\
20 \mathrm{~ms}\end{array}$ & $\begin{array}{l}15.97 \\
14.58 \\
13.75 \\
13.61\end{array}$ \\
\hline & 2000 in• & $\begin{array}{r}200 \mathrm{~ms} \\
100 \mathrm{~ms} \\
40 \mathrm{~ms} \\
20 \mathrm{~ms}\end{array}$ & $\begin{array}{l}15.28 \\
13.54 \\
12.78 \\
12.64\end{array}$ \\
\hline & $1000 \mathrm{in} \bullet$ & $\begin{array}{r}200 \mathrm{~ms} \\
100 \mathrm{~ms} \\
40 \mathrm{~ms} \\
20 \mathrm{~ms}\end{array}$ & $\begin{array}{l}14.44 \\
12.50 \\
11.81 \\
11.67\end{array}$ \\
\hline & $500 \mathrm{in \bullet}$ & $\begin{array}{r}200 \mathrm{~ms} \\
100 \mathrm{~ms} \\
40 \mathrm{~ms} \\
20 \mathrm{~ms}\end{array}$ & $\begin{array}{l}13.89 \\
12.15 \\
11.46 \\
11.25\end{array}$ \\
\hline \multirow[t]{4}{*}{$\begin{array}{ll}2000 & \mathrm{~kg}- \\
\mathrm{m} \cdot & \end{array}$} & $3000 \mathrm{in} \bullet$ & $\begin{array}{r}200 \mathrm{~ms} \\
100 \mathrm{~ms} \\
40 \mathrm{~ms} \\
20 \mathrm{~ms}\end{array}$ & $\begin{array}{l}12.30 \\
11.02 \\
10.46 \\
10.35 \\
\end{array}$ \\
\hline & 2000 in॰ & $\begin{array}{r}200 \mathrm{~ms} \\
100 \mathrm{~ms} \\
40 \mathrm{~ms} \\
20 \mathrm{~ms}\end{array}$ & $\begin{array}{r}11.57 \\
10.29 \\
9.73 \\
9.61\end{array}$ \\
\hline & $1000 \mathrm{in} \bullet$ & $\begin{array}{r}200 \mathrm{~ms} \\
100 \mathrm{~ms} \\
40 \mathrm{~ms} \\
20 \mathrm{~ms}\end{array}$ & $\begin{array}{r}10.88 \\
9.57 \\
9.00 \\
8.89\end{array}$ \\
\hline & $500 \mathrm{in} \bullet$ & $\begin{array}{r}200 \mathrm{~ms} \\
100 \mathrm{~ms} \\
40 \mathrm{~ms} \\
20 \mathrm{~ms} \\
\end{array}$ & $\begin{array}{r}10.51 \\
9.20 \\
8.63 \\
8.52 \\
\end{array}$ \\
\hline
\end{tabular}

\begin{tabular}{|c|c|c|c|c|}
\hline Page 55 & Technical Report & Report Number & Issue: & Page 55 \\
\hline \multicolumn{3}{|c|}{ Security Classification: ${ }^{\# 01}$ Unclassified } & & \\
\hline
\end{tabular}


The expected Inertia is $1500 \mathrm{~kg}-\mathrm{m} \bullet$ with a valve response time from $100 \mathrm{~ms}$ and a manifold volume of 3000 in. .

\begin{tabular}{|ll|l|l|l|}
\hline \multicolumn{1}{|c|}{ Inertia } & $\begin{array}{c}\text { Valve } \\
\text { Response }\end{array}$ & $\begin{array}{c}\text { Manifold } \\
\text { Volume }\end{array}$ & $\begin{array}{c}\text { Frequency } \\
\text { Shift }\end{array}$ \\
\hline $\mathbf{1 5 0 0}$ & $\mathbf{k g -}$ & $\mathbf{1 0 0} \mathbf{~ m s}$ & $1000 \mathrm{in \bullet}$ & $12.5 \%$ \\
$\mathbf{m \bullet}$ & & & $2000 \mathrm{in} \bullet$ & $13.61 \%$ \\
& & & $\mathbf{3 0 0 0} \mathrm{in \bullet}$ & $\mathbf{1 4 . 4 4} \%$ \\
& & & $4000 \mathrm{in} \bullet$ & $15.28 \%$ \\
\hline
\end{tabular}

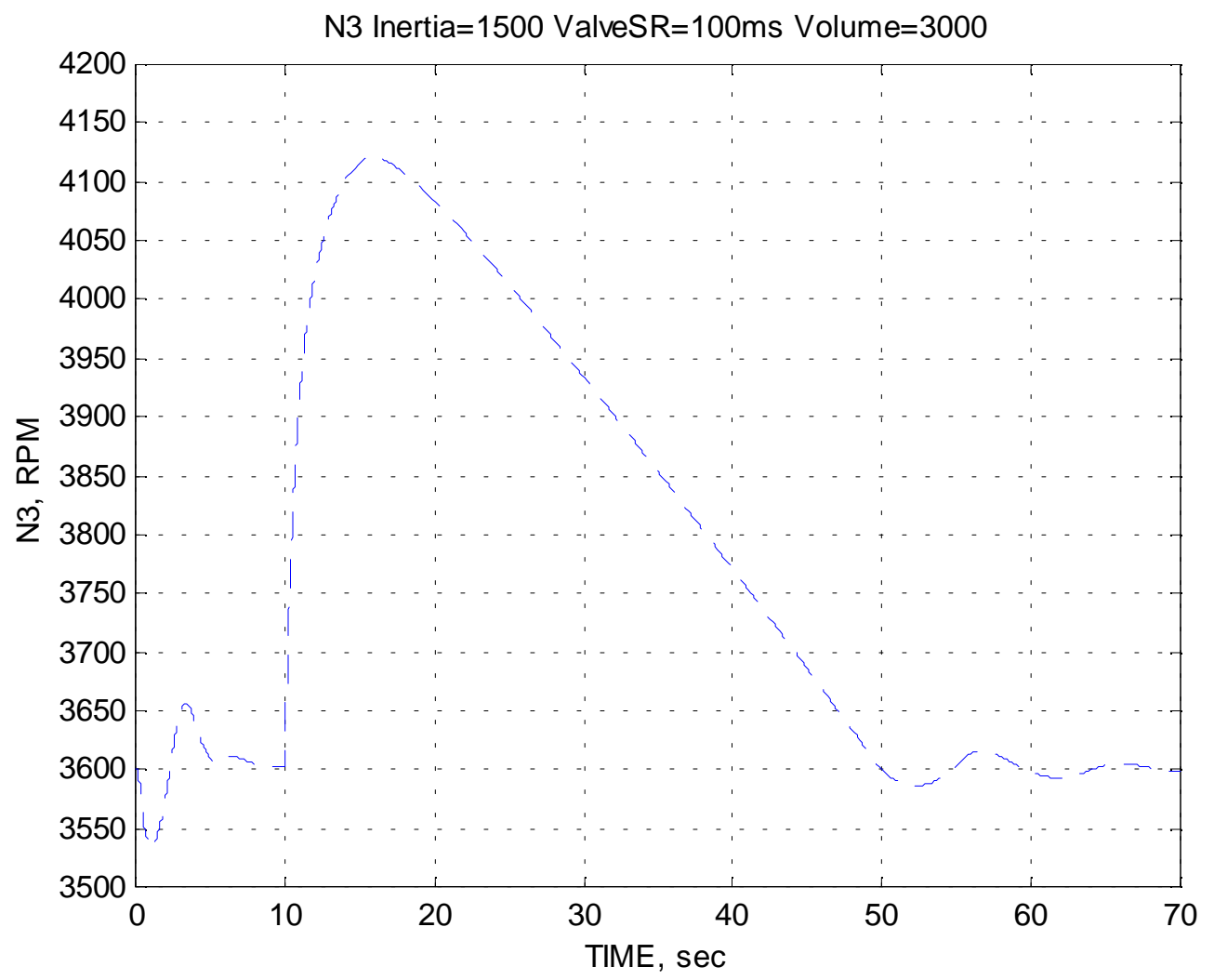

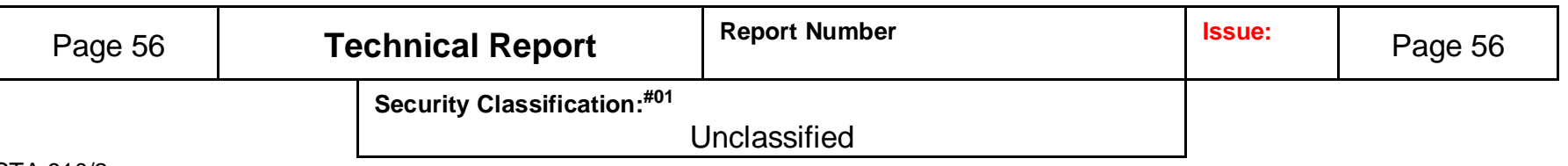




\subsubsection{Safety Analysis - Shaft Shear}

The Trip on overspeed is set at $15 \%$ above the rotate speed

Isolation value / Safety System Respone

\begin{tabular}{|c|c|c|c|c|c|}
\hline Inertia & $\begin{array}{c}\text { Manifold } \\
\text { Volume }\end{array}$ & $\begin{array}{c}\text { Isolation } \\
\text { Valve } \\
\text { Response }\end{array}$ & $\begin{array}{c}\text { Trip } \\
\text { Speed }\end{array}$ & $\begin{array}{c}\text { API 616 } \\
110 \%\end{array}$ & Max N3 \\
\hline $\begin{array}{ll}1500 & \mathrm{~kg}- \\
\mathrm{m} \bullet & \end{array}$ & 3000 in॰ & $\begin{array}{r}20 \mathrm{~ms} \\
50 \mathrm{~ms} \\
100 \mathrm{~ms} \\
200 \mathrm{~ms}\end{array}$ & $\begin{array}{l}4140 \mathrm{rpm} \\
4140 \mathrm{rpm} \\
4140 \mathrm{rpm} \\
4140 \mathrm{rpm}\end{array}$ & $\begin{array}{l}4554 \mathrm{rpm} \\
4554 \mathrm{rpm} \\
4554 \mathrm{rpm} \\
4554 \mathrm{rpm}\end{array}$ & $\begin{array}{l}5144.5 \mathrm{rpm} \\
5166.5 \mathrm{rpm} \\
5230.3 \mathrm{rpm} \\
5386.3 \mathrm{rpm}\end{array}$ \\
\hline
\end{tabular}

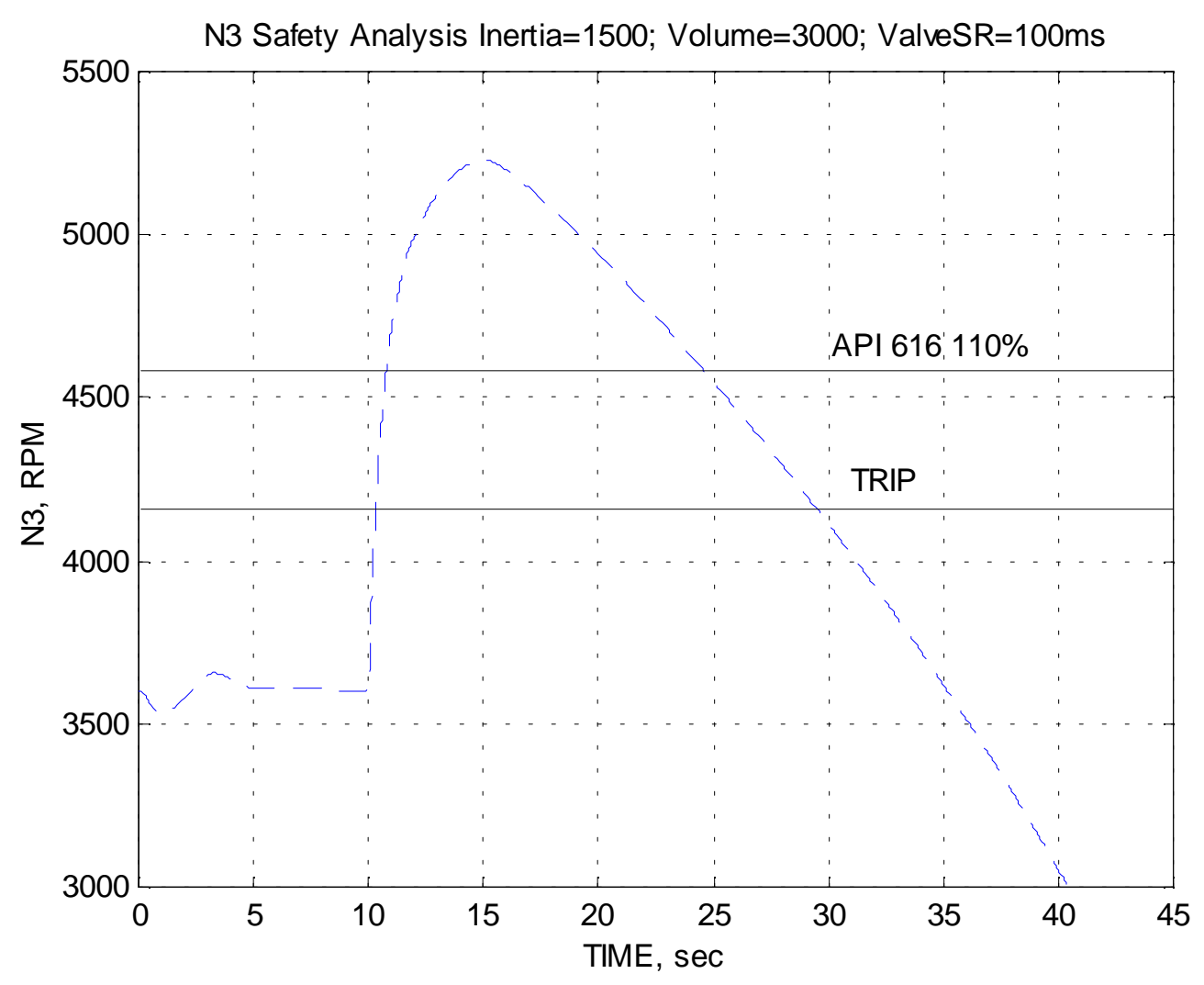

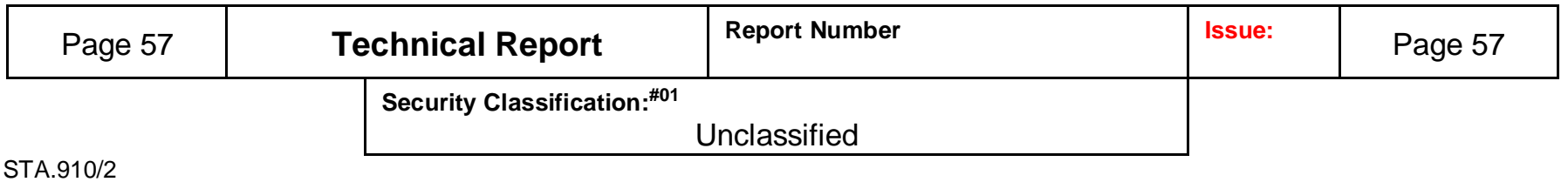


Manifold Dump Valve

\begin{tabular}{|l|l|c|c|c|c|}
\hline \multicolumn{1}{|c|}{ Inertia } & $\begin{array}{c}\text { Manifold } \\
\text { Volume }\end{array}$ & $\begin{array}{c}\text { Dump } \\
\text { Valve } \\
\text { Response }\end{array}$ & $\begin{array}{c}\text { Trip } \\
\text { Speed }\end{array}$ & $\begin{array}{c}\text { API 616 } \\
\mathbf{1 1 0} \%\end{array}$ & Max N3 \\
\hline $1500 \mathrm{~kg}-$ & $3000 \mathrm{in} \bullet$ & $20 \mathrm{~ms}$ & $4140 \mathrm{rpm}$ & $4554 \mathrm{rpm}$ & $4882.5 \mathrm{rpm}$ \\
$\mathrm{m}$ & & $50 \mathrm{~ms}$ & $4140 \mathrm{rpm}$ & $4554 \mathrm{rpm}$ & $4928.0 \mathrm{rpm}$ \\
& & $100 \mathrm{~ms}$ & $4140 \mathrm{rpm}$ & $4554 \mathrm{rpm}$ & $5044.3 \mathrm{rpm}$ \\
& & $200 \mathrm{~ms}$ & $4140 \mathrm{rpm}$ & $4554 \mathrm{rpm}$ & $5301.8 \mathrm{rpm}$ \\
\hline
\end{tabular}

N3 Safety Analysis Dump-Valve Inertia $=1500$; Volume $=3000$; ValveSR $=100 \mathrm{~ms}$

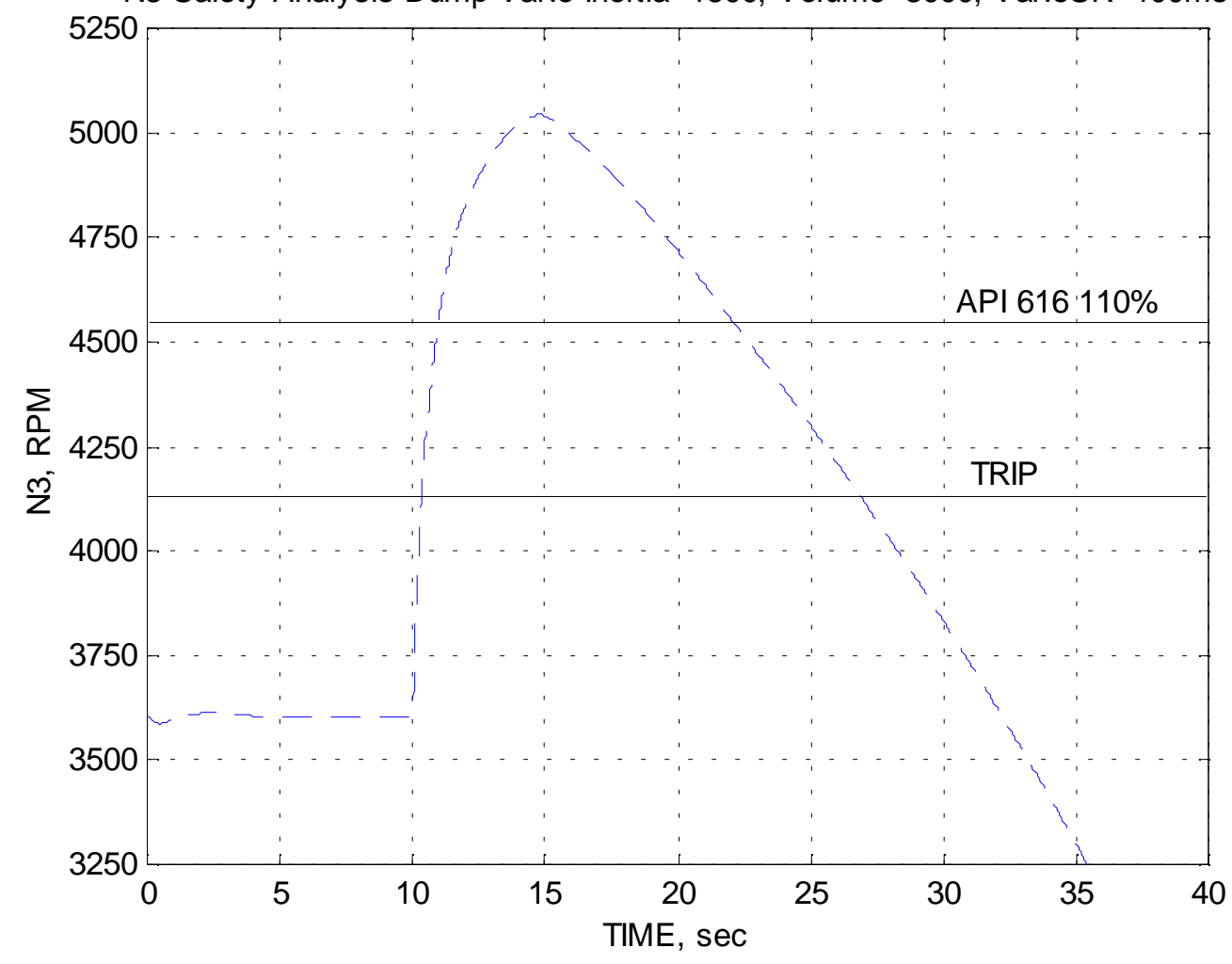

Page 58

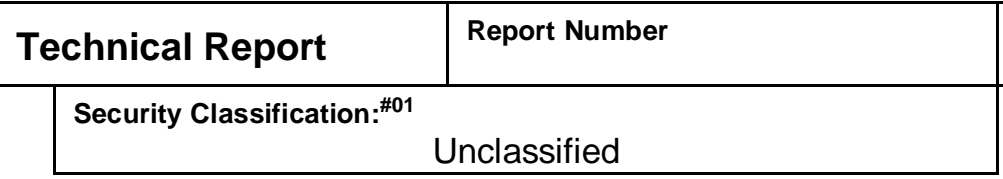

Issue:

Page 58 


\section{Appendix C \\ Packaging Optimization}




\section{Executive Summary}

Rolls Royce Energy Systems Inc. is in the business of packaging and marketing GTGs (gas turbine generators). RRESI was given the task of studying and evaluating current market demands and the latest technologies being introduced for GTGs. This report covers the main plant mechanical equipment selection and design theories for each of the two cycles selected by the NGT (Next Generation Turbine) team: full steam injection and wet intercooled/wet recuperated. To meet the highest next generation standards for these cycles, new technologies will be needed to meet plant efficiencies with environmentally suitable systems.

The main goal of the study was to deliver a preliminary design proposal for each system that optimizes machine performance, efficiency and first cost, and that best matches the results put forward in the separate marketing survey. The primary areas of concentration were:

- Low capital cost

- Highest thermal efficiency

- High reliability and availability

Special emphasis was applied to several other parameters determined by experience to provide overall package competitiveness on an installed-at-customer's-site basis. Each of these key design parameters is discussed in this report with a criteria sheet outlining primary design goals.

\section{Introduction}

As part of the DoE Next Generation Turbine program, Rolls-Royce Corp. asked RRESI to produce preliminary package concepts for proposed gas turbine cycles. Approaches followed were in line with the specific goals of the funded program and based on systems meeting both near-term market needs and advanced technology to ensure reliability and life cycle cost.

During the early stages of Phase I, several cycles were investigated; two were selected for more detailed study. Each of these concepts was developed to the point at which a fairly accurate benefit/cost analysis could be produced.

This report by RRESI reflects the results of several months of study and presents findings for follow-on evaluation.

\section{General Cycle Descriptions}

\subsection{Full Steam Injection (SI)}

Unlike previous steam injected gas turbine systems (including our Allison 501 KH machine), the rotating turbine components will be designed to optimize power and efficiency while being injected with the maximum recoverable heat produced by the turbine exhaust. 
Cycle conditions:

$$
\begin{aligned}
& \text { Pressure Ratio } \\
& \text { Power Output }
\end{aligned}
$$

Electrical Efficiency

Water Flow Rate
27

72.6 MWe

$51.9 \%$

$45 \mathrm{lb} / \mathrm{s} @ 764 \mathrm{~K}$

\subsection{Wet Intercooled/Wet Recuperated (WIWR)}

This cycle uses humidified air injection into the turbine between the LP and HP compressor sections and upstream of the combustor. The thermal energy used for this supply is produced in separate recuperator and economizer sections located in the turbine exhaust duct.

Cycle conditions:

\author{
Pressure Ratio \\ Power Output \\ Electrical Efficiency \\ Water Flow Rate
}

27

76.2 MWe

$53.8 \%$

$45 \mathrm{lb} / \mathrm{s} @ 460 \mathrm{~K}$

\section{General Design Guidelines}

In the design of the gas turbine generator and associated balance of plant we sought to utilize state of art features of current packaging techniques while incorporating the latest safety concepts under review by both the North American and European regulatory committees.

There were four major areas that needed to be addressed from RRESI's standpoint for the design and the development of this type of equipment.

(1) Package Cost - including materials cost, manufacturing cost and package design cost

(2) Development Issues - including major sub-component development testing ,turbomachinery and first article site development testing.

(3) Installation Costs - including installation hours, site commissioning and testing

(4) Operational Issues - including reliability and availability

Standard modular philosophy was applied to all of the major components and sub-systems. Standardization to the highest logical level allows for subsequent packages to be built with minimal engineering input.

When applying the module concept to the package design, one area of potential conflict must be addressed: accessibility versus footprint. While a major benefit of the module concept is to provide suitable maintenance access, the design must also take into consideration the footprint or area required for installation. The footprint will have an impact on the future suitability of the package's applications in or near existing facilities and will also impact installation costs. An optimum balance was attempted with typical layouts shown for each plant. (See Figures 1 through 4). 


\section{NGT80 WI \& WR - Plant Layout (Top View)}

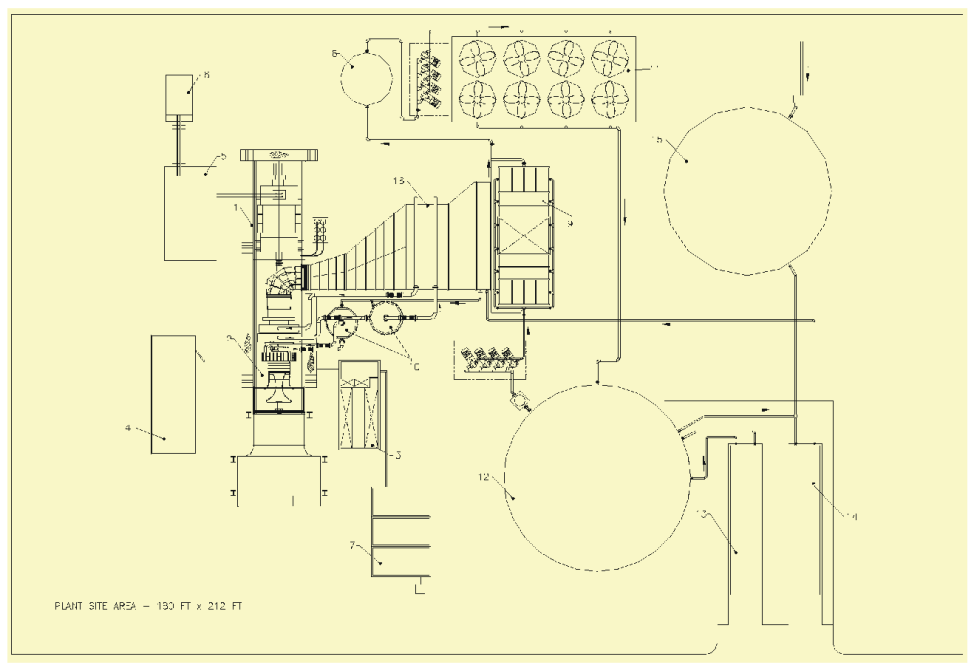

\begin{tabular}{|rl|}
\hline 1 & Generator Skid \\
2 & Gas Turbine Skid \\
3 & GTG Auxiliary Module \\
4 & Control Room \\
5 & High Voltage Module \\
6 & Utility Tie \\
7 & Gas Compressor \\
8 & Water Recovery Tank \\
9 & Water Recovery System \\
10 & Humidification Towers \\
11 & Fin Fan Cooler \\
12 & Demin Water Storage \\
13 & Polishing Unit \\
14 & Demin Unit \\
15 & Make up Water Tank \\
16 & Recuperator \\
\hline
\end{tabular}

Figure 1

\section{Next Generation Turbines}

\section{NGT80 WI \& WR - Typical Plant Layout (Side View)}

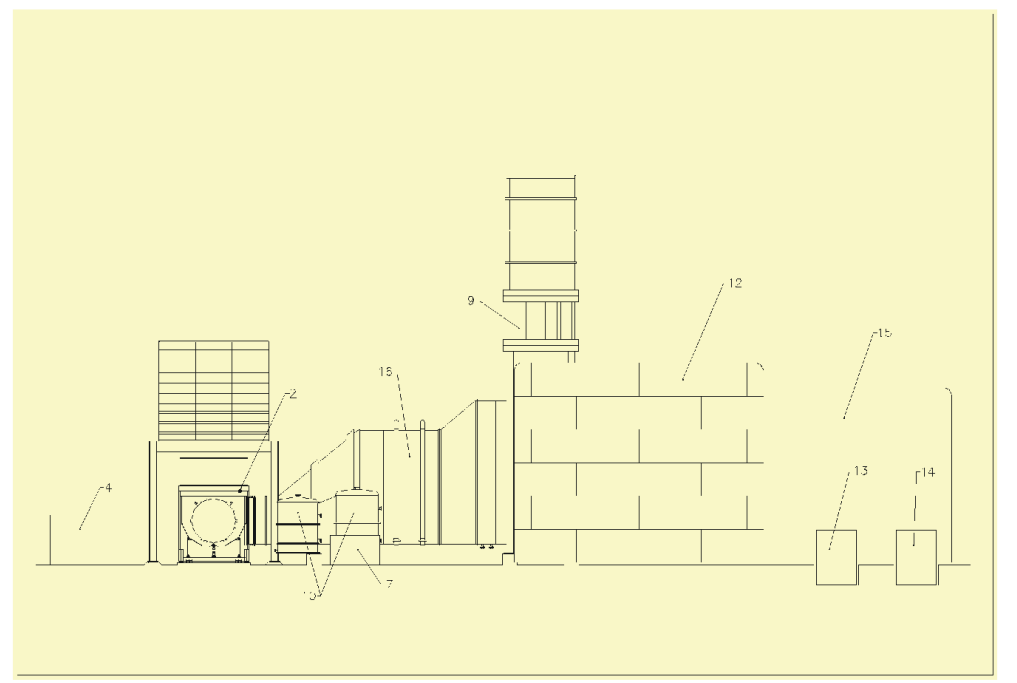

2 Gas Turbine Skid

4 Control Room

7 Gas Compressor

9 Water Recovery

10 Humidification Towers

12 Demin Water Tank

13 Polishing Unit

14 Demin Unit

15 Make up Water Tank

Figure 2 
Next Generation Turbines

\section{NGT80 S.I. - Plant Layout (Top View)}

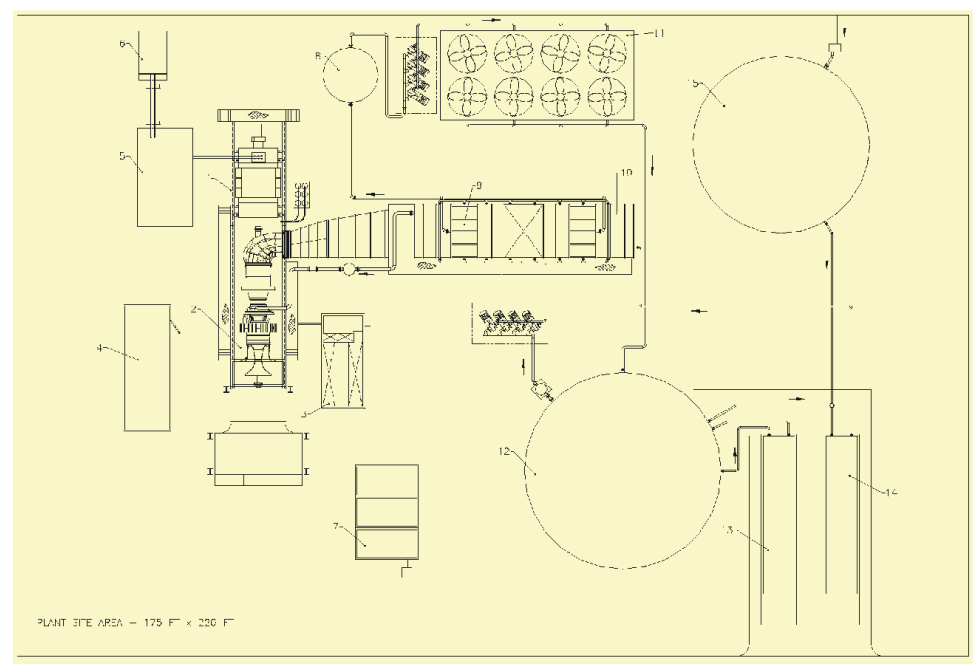

1 Generator Skid

2 Gas Turbine Skid

3 GTG Auxiliary Module

4 Control Room

5 High Voltage Module

6 Utility Tie

7 Gas Compressor

8 Water Recovery Tank

9 Water Recovery Sys

10 Once Thru Steam

Generator

11 Fin Fan Cooler

12 Demin water storage

13 Polishing Unit

14 Demin Unit

15 Make up water

Figure 3

Next Generation Turbines

\section{NGT80 S.I. - Typical Plant Layout (Side View)}

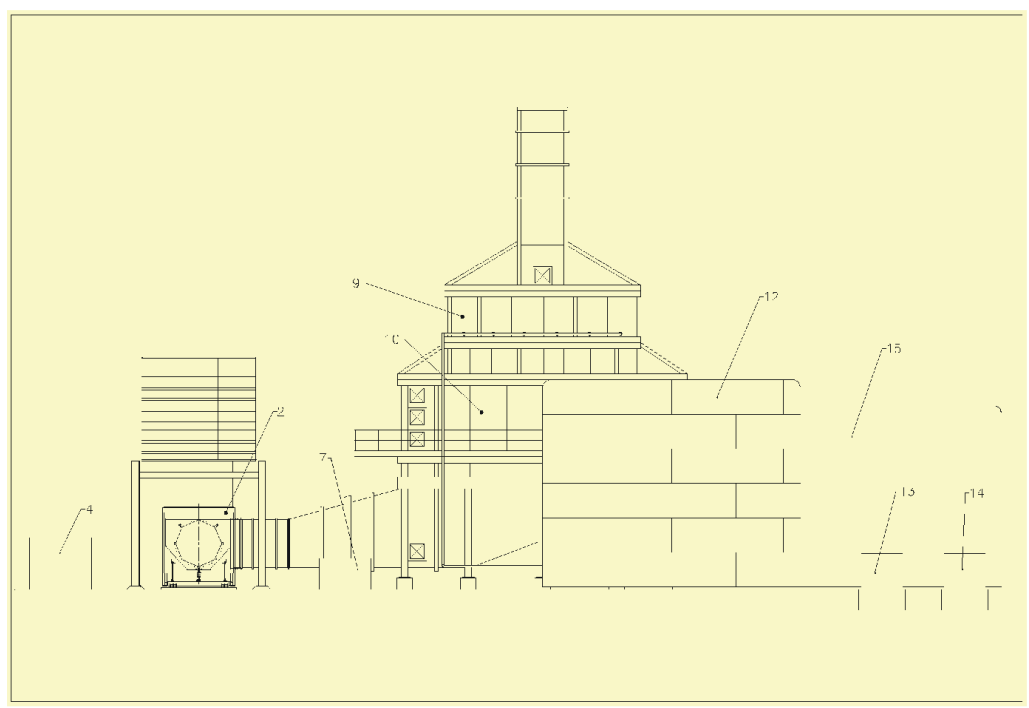

2 Gas Turbine Skid

4 Control Room

7 Gas Compressor

9 Water Recovery Sys

10 Once Thru Steam

Generator

12 Demin Water Tank

13 Polishing Unit

14 Demin Unit

15 Make up Water Tank

Figure 4 


\section{Codes and Standards}

All packages must conform to the Health and Safety Standards applicable for the territory in which the unit is to be installed.

Primary structure, enclosures, ventilation hoods and ducts shall be in accordance with Uniform Building Code (UBC) Seismic Zone 4. Internal components and systems shall be designed to (UBC) Seismic Zone 1. For the standard plant site, specific seismic requirements shall be a non-pre-engineered option.

The design will provide provisions that allow the package to be installed in environments where the ambient temperature could be as low as $-20^{\circ} \mathrm{F}$ or as high as $110^{\circ} \mathrm{F}$.

The package shall be designed for wind loading of $100 \mathrm{mph}$ gusts and snow loading of 30 $\mathrm{lbs} / \mathrm{sq}$. $\mathrm{ft}$. The standard altitude range for the package shall be -100 feet below sea level to +5000 feet above sea level.

Standards are required to be defined for tropicalization of the package and for any special requirements associated with coastal or high airborne contaminate content environments.

The basis for the plant layout and area classifications shall be per the recommended practices outlined in the National Fire Protection Association (NFPA) publication 497A. All equipment shall be suitable for use in the assigned area classification, including fault conditions, start, stop, and standby operating modes.

\section{Functional Descriptions}

\subsection{Gas Turbine Driver Skid}

The GTG skid will consist of a structural steel baseplate with an acoustical enclosure over the gas generator and power turbine. It will have minimal terminal points and contain engine handling beams for heavy maintenance tasks. The engine removal route will be forward through the intake plenum which will allow splitting of the turbine modules (axially) within the package. The design will offer the benefit of not requiring a separate (local) maintenance facility. The power turbine section will remain in place for most activities, thereby retaining major component alignment and reducing overall work content. The effect on civil foundation and overhead steel for supporting engine from above or below will be incorporated in the initial design.

\subsection{Driven Generator Skid}

The generator skid will be designed as a separate module with its own lubrication system to supply generator bearings. A separate jacking oil system will be provided to assure safe shut down and start-up of the rotating equipment. The module will provide its own generator cooling air circuit and supply air for the low speed coupling tunnel.

\subsection{Control Module}

The control module will house all the local control equipment in an acoustic, weather-tight, self-supporting enclosure. The control room will be environmentally controlled for dependable operation and human occupation when needed and will provide EMC protection as appropriate. 


\subsection{Emissions}

The gas turbine will be configured with a DLE combustion system that will achieve less than $10 \mathrm{vppm}$ Nox and less than $10 \mathrm{vppm}$ CO during normal operation on natural gas fuel. Oil system vents (GT \& Generator) shall also be considered and meet environmental legislation applicable to the location.

\subsection{Fuel Gas Conditioning}

Natural gas fuel supply pressure will be assumed as high pressure pipeline gas at $350 \mathrm{psig}$. Fuel gas compression will meet working level requirements of the gas generator and be provided by either two $100 \%$ or three $50 \%$ compressors to assure system availabilities.

\subsection{Noise}

Near field - noise emissions from equipment shall not exceed $85 \mathrm{~dB}(\mathrm{~A})$. Far field - the package noise abatement systems will be designed to achieve three standards of maximum sound pressure level on a per unit basis, measured at 400 feet from the geometric center of the GTG package. The basic design will not exceed $65 \mathrm{~dB}(\mathrm{~A})$, or $80 \mathrm{~dB}(\mathrm{~A})$ in the $31.5 \mathrm{~Hz}$ octave band.

\subsection{Availability}

The plant design and material selection will adequately support an overall availability guarantee of $98 \%$. A reliability case will be prepared to show the need for, and sensitivity of, redundant systems and equipment.

\subsection{Maintainability}

To ensure highest possible plant availability the design will address the issues relating to routine, preventive and corrective maintenance. The gas turbine replacement total turn time target will be 12 hours without the need of external independent carnage. Gas turbine combustion inspections or replacements will be achievable within the acoustic enclosure by the use of special product tooling. Maintenance access, lifting requirements and efficient use of lay down space will be considered and analyzed during design.

\subsection{Plant Operation}

The plant will intended to be operated as "mid-merit" or "base continuous" depending on the plant location and application.

\subsection{Auxiliary Power Black-out}

Black starting will not be required as a standard. (Note: Black starting, when required, will use a suitable standby generator for station power.) Black stopping will be required as standard. Black stop can occur under fault conditions. The plant must then come to rest safely and without damage. Essential protective and monitoring systems (e.g., fire protection, ventilation and lubrication, if necessary) must continue to operate long enough to avoid damage. 


\subsection{Steam Generator}

The SI cycle will require high pressure steam with particular attention paid to water impurities and water carry-over. Key to the successful operation of the NGT machine will be its ability to withstand multiple starts and the rapid start-up requirement (less than 30 minutes). The system will also be designed to operate in colder climates. Existing field units are preferred with similar duty cycle to assure system reliability.

\subsection{Recuperator Section}

The WIWR cycle will include a Rolls-Royce spiral recuperation section located down stream of the exhaust diffusion duct. The unit will be factory assembled with a single horizontal split line minimizing field welding. Particular attention will be applied to the thermal expansion of connection piping as well as purging requirements for non-operational conditions in cold climates.

\subsection{Water Recovery Section}

Due to the large amount of water use by each cycle under consideration, recovery of this natural resource will be necessary. Results of the customer survey also indicate this feature would greatly enhance the ability to permit equipment. The plant will recover approximately $75-80 \%$ of the exhaust steam water content to make it a self-sufficient system. The plant will have a 24 hour supply of demineralized water on hand to handle any upset condition.

\subsection{H.V. Utility Interconnect Module}

This module will be factory assembled and contain all the necessary electrical gear required for interconnection to the utility. The unit will be self supporting and field mounted to a separate concrete pad.

\subsection{Design Life}

The design life will be 25 years, allowing 400 start-stop cycles per annum. Paint and other protection systems should be adequate for 10 years between maintenance. Galvanized surfaces should be adequate for 25 years.

\subsection{Site Conditions}

The typical site is assumed to be "green field" with 350 psig gas available at the site boundary, no additional power export lines required, average soil conditions, satisfactory space available and reasonable environmental limits (Nox limit of $15 \mathrm{ppm}$ on gas was used).

\subsection{Transportation}

The GTG and auxiliaries will be capable of being transported by road within North America. The OTSG, recuperator and water recovery sections will be designed for transport by rail. All equipment will be provided with temporary restraint as appropriate for shipping purposes. 


\section{System-Key Features}

\subsection{Gas Turbine Driver Skid}

The approximate dimensions of the G/T driver skid will be $41 \mathrm{ft}-4$ in $\mathrm{L}$ x $14 \mathrm{ft} \mathrm{W}(12.6 \mathrm{M} \mathrm{x}$ $4.3 \mathrm{M})$.

The gas generator will be a derivative of the Trent 800 with twin spools air coupled to the power turbine. The GG will be basically cantilevered off of the P/T frame supported by a " $1 G$ " vertical mount located at the aft LPC flange.

The power turbine module will provide the main turbine mounts reacting thrust, vertical, side and torsional loads. All major drive train components will be aligned to the P/T module. The P/T rotor will be supported on anti-friction bearings similar to the GG and will use a common lube system.

The low speed drive coupling will be a dry type flexible diaphragm (non-lubricated). It will connect directly to the power turbine shaft and the generator rotor. The coupling will be shielded from the turbine exhaust heat by an insulated tube which also will provide the flow path for cooling air to the PT rear sump area.

The G/T skid will be supported on a structural steel base plate which will transfer the drive train loads to the mounting foundation. The skid will be rigid enough to handle transport and off loading loads.

The G/T enclosure will be a one piece welded acoustic enclosure designed to meet near and far field sound levels. Enclosure will be rigidly bolted to the skid base structure and will provide protection of equipment during transport.

The enclosure ventilation will be designed to operate with negative pressure provided by three $50 \%$ fans mounted on the roof section in a separate housing. Fan discharge air will be silenced to meet overall noise requirements.

\subsection{Combustion Air System}

Until site specific details are available, the unit standard design will be based upon a 3-stage system with high efficiency barrier filters, pre-filters and rain separators. The inlet silencer is integral to the inlet duct system and incorporates a "Low Loss" parallel/staggered turning vane assembly which will allow the air flow to be directed into the G/T with minimal distortion and constant area to avoid system losses. (See Figure 5)

\subsection{Gas Turbine Exhaust System}

The exhaust turning/diffuser chosen for this application is one with minimal pressure loss with performance recently field proven on the 50MW RR-Trent machine. The design consists of eight conical diffuser sections which turn the gas stream around the load coupling into rectangle side exit opening. This MAHD (Multi-Annular Hybrid Diffuser) delivers an even gas velocity to the recuperator or the OTSG system. The additional benefit of this low-loss/uniform flow design is the additional reduction in low frequency noise which is the most costly to attenuate. (See Figure 6) 
Next Generation Turbines

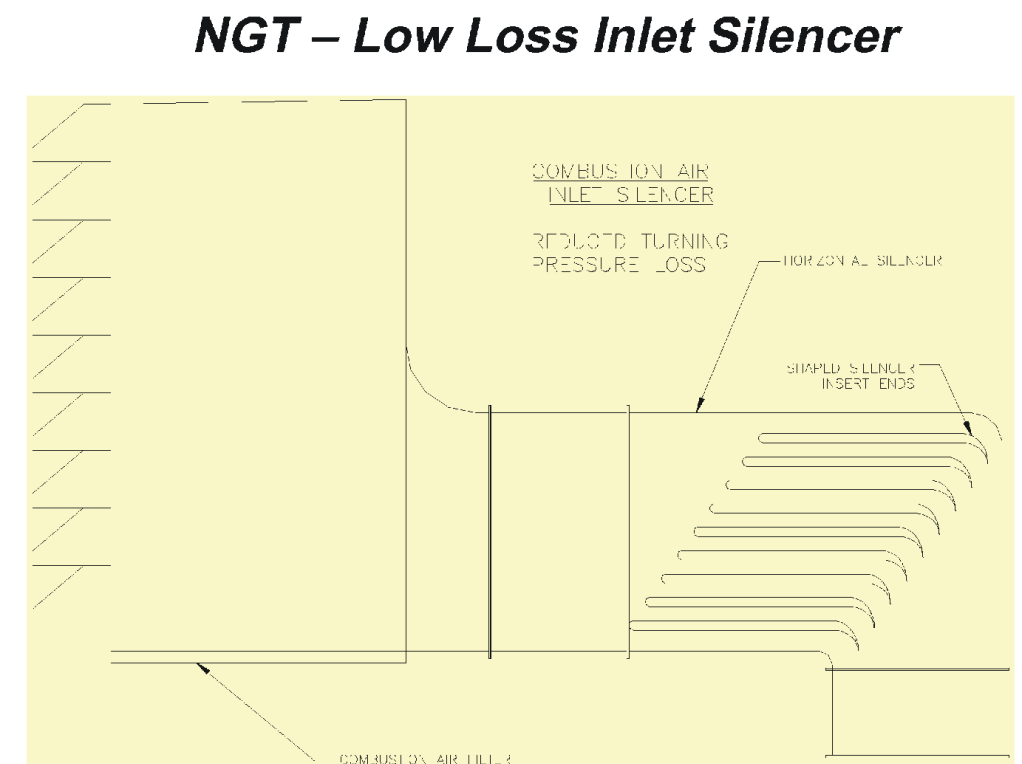

Figure 5

Next Generation Turbines

\section{NGT - MAHD (Multi-Annular Hybrid Diffuser)}

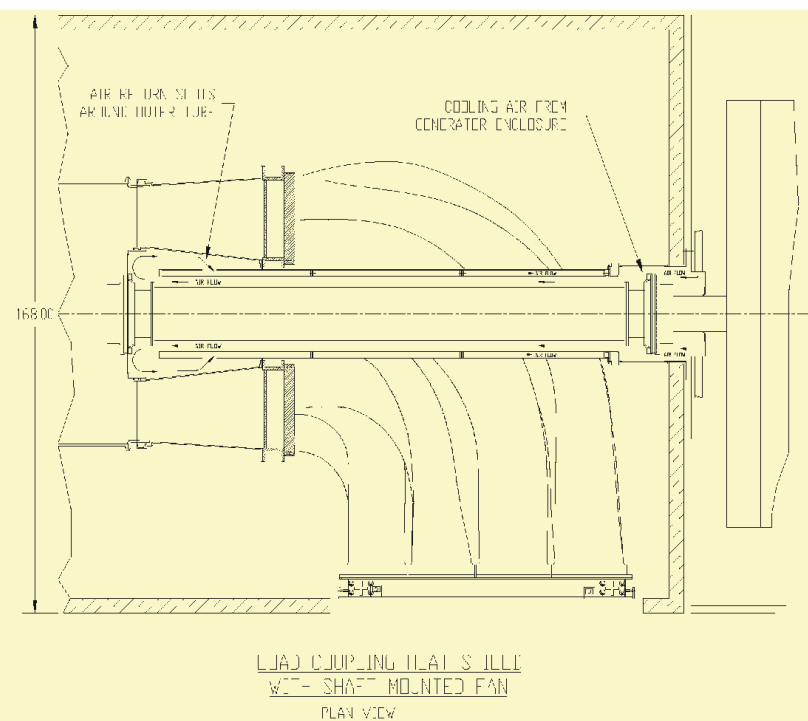

Figure 6 


\subsection{Generator Skid}

The generator skid will be field bolted to the G/T driver skid with common width and height dimensions. The length of this module will be approx. $26 \mathrm{ft}-6 \mathrm{in}$.

The generator standard is a 13,800 volt two-pole synchronous speed (3600 RPM) design. It will be air cooled via shaft mounted fans with a separate air filtration and discharge system. The generator bearings (sleeve type) will be fed by a mineral lubrication system supplied by the generator manufacturer. The jacking oil system will be used to assure safe start-up and shutdown on the machine. This system also allows ease of blade inspection during normal borescope operations.

\subsection{Gas Turbine Auxiliary Skid}

The auxiliary skid will house the support systems for the GTG unit. Approximate dimensions of this module will be $24 \mathrm{ft} \mathrm{L} \mathrm{x} 11 \mathrm{ft} \mathrm{W} \mathrm{x} 9 \mathrm{ft} \mathrm{H}$. Major systems contained in this module will be as follows:

G/G \& P/T Lubricating Oil System - the G/T lube oil system will be synthetic and will lubricate the three gas generator bearing chambers, the internal auxiliary gearbox and the two bearing chambers of the P/T. Supply flow will be directed through a duplex filter and into each manifold for distribution. Oil scavenged from the bearings via a strainer will be routed to a cooled heat exchanger before returning to the main oil reservoir.

Start System - the start system will be an electric/hydraulic pump driving a motor mounted on the G/T auxiliary gearbox.

Gas Fuel System - the gas fuel metering, control and isolation skid will be located adjacent to the main GTG and forward of the hot turbine section and G/T gas supply manifolds. High pressure gas will be supplied to the separate fuel forwarding skid via three $50 \%$ reciprocating compressors located in a convenient location between the customer's gas metering station and the turbine generating unit.

Fire Suppression System - the extinguishant gas will be $\mathrm{CO}_{2}$ with separate zones for the $\mathrm{G} / \mathrm{T}$ skid, generator skid, auxiliary skid and control room. Each protected area will be provided with detectors, discharge nozzles, off skid gas storage bottles with fire shutter louvers on all openings.

\subsection{Gas Turbine Control Module}

The separate power and control module will be located near the GTG to minimize interconnect wiring and will have approximate dimensions of $32 \mathrm{ft} \mathrm{L} \mathrm{x} 12 \mathrm{ft} \mathrm{W} \times 10.5 \mathrm{ft} \mathrm{H}$.

The module will contain all the necessary equipment for local control of the generating unit and support systems. The module will be factory assembled, minimizing field interconnection, and will ship as a single lift unit for placement onto a concrete pad with pre-located conduits. 


\subsection{High Voltage Module}

This module will contain all the necessary equipment for interconnection to the local utility. The unit will be factory assembled and shipped as a single lift module for placement onto a separate foundation pad. The module will contain the following major components:

- Main unit breaker

- Sub-voltage breaker

- Auxiliary transformers

- PTs, CTs and lighting arrestors

- Batteries and charger

- Utility connection and switch for step-up transformer

\subsection{Steam Generator}

The common systems in use today for STIG or CCCT plants are the OTSG (Once Through Steam Generator) and the typical HRSG (Heat Recovery Steam Generator). Key to the system selection for the NGT duty cycle was the need to achieve rapid starts which more or less ruled out the drum type boiler. It was also a system with the least amount of stored energy and believed to place less operational demands on facility staff. The results of this comparison are shown below:

- The OTSG can tolerate a more rapid start-up (only limited by thermal inertia of tubing and casing etc.)

- It can reach a balanced output in under 30 minutes

- As such, an OTSG start can be hastened by applying supplemental firing using a duct burner. The same technique cannot be applied to an HRSG start.

- The OTSG can also be operated in "dry mode' which allows the elimination of a by-pass stack

- Hot re-start is also possible, if the feed water is introduced in a careful controlled manner

- The absence of items like drums and deaerators etc., simplifies the monitoring of safety and safety control systems required. Emergency stop will be simpler due to the minimization of stored energy in the system.

\subsection{Water Treatment System}

The exact analysis cannot be determined until final site selection. However, whether it is City Mains, Surface, Borehole, etc., the quality and quantities needed are generally known and used for initial design purposes. Based upon these requirements and the planned plant operation, the best solution determined for this application would be the use of portable process trailers. This type of installation is becoming more popular with both peaking and permanent installations. Some of the general advantages of this system are listed below:

- Reduced plant cost making amount of debt financing less and projects more viable (estimated savings for this installation $\approx \$ 400,000.00$ ) 
- Benefit of having treatment plant only when needed to handle operational requirement

- Minimizes operational staff so existing manpower can focus on other activities

- Improve quality of treatment by added flexibility to treat any changes in facility operational requirements

- Improved reliability and predictability of the water treatment plant operation will extend boiler/turbine maintenance periods

- Minimal site chemical and waste product handling

- Plant flexibility to take advantage of future improvements in RO and other treatment technologies along with ease to meet new environmental requirements

- Minimize plant storage requirements with added safety of redundant trailers to assure availability of treatment system

The water quality requirements for the OTSG are shown in Figure 7 and are compared with the more standard steam drum type design. This is of less significant when considering that normal operation will only require treatment of recovered water. The expected water analysis of the recovered water is shown in Figure 8.

\subsection{Water Recovery System}

The main objective of the water recovery system was to recover enough water from the turbine exhaust stream to allow the NGT concept to operate without make-up water. After review of various systems, there were essentially two practical options evaluated in detail.

(1) Direct condensing with air (exhaust-air cooler in exhaust). Simplified schematics (Option 1) shown in Figures 9 and 10.

(2) Water quench condensation (water quench in exhaust), followed by quench water-air cooled. Simplified schematics (Option 2) shown in Figures 11 and 12.

Preliminary analysis of these systems indicated the "Quench" system to have several advantages over the "Direct" system as indicated below.

- Temperature rise in air coolant (of quench based system) is relatively low:

- $13^{\circ} \mathrm{C} / 23^{\circ} \mathrm{F}$ versus $43^{\circ} \mathrm{C} / 77^{\circ} \mathrm{F}$

- Air mass flows consequently large (in relative terms $>3 x$ )

- Auxiliary power consumption possibly greater (again $>3 x$ )

- Design of quench condensing system is more sensitive to increases in ambient temperature

- Aspect ratio of direct condensing heat exchanger is very sensitive to pressure loss requirements

In summary, the "Direct Condensing" system appears to be the most practical means of recovery and will be analyzed in more detail by follow-on study. The key points for this conclusion are as follows:

- Analysis indicated that both systems will be roughly the same cost 
Next Generation Turbines

\section{Key Features - Water Treatment System Water Quality Guidelines (max values)}

- Typical WHRG

Silicon
Particulate
Cation Conductivity
Total Dissolved Solids
Total Solids
$\mathrm{Na}, \mathrm{K}, \mathrm{Ca}$, and $\mathrm{Mg}$
ph Range

$20 \mathrm{ppm}$
$10 \mathrm{ppm}$
$0.1 \square \mathrm{s} / \mathrm{cm}$
$50 \mathrm{ppm}$
$.15 \mathrm{ppm}$
$2 \mathrm{ppm}$
$8.0-9.5$

Figure 7
- Typical OTSG/Steam Injection

$\begin{array}{lc}\text { Silicon } & 20 \mathrm{ppb} \\ \text { Particulate } & 3 \mathrm{ppm} \\ \text { Cation Conductivity } & 0.25 \square \mathrm{s} / \mathrm{cm} \\ \text { Total Dissolved Solids } & 50 \mathrm{ppb} \\ \text { Total Solids } & 0 \\ \mathrm{Na}, \mathrm{K}, \mathrm{Ca} \text { and } \mathrm{Mg} & 50 \mathrm{ppb} \\ \text { ph Range } & 9.3-9.6\end{array}$

Next Generation Turbines

\section{Key Features - Water Treatment System}

- Based upon steam injected operating data collected from current RR turbines. The condensate water can easily be put back into the cycle with little treatment.

TEST DATA:
Contaminants

$\begin{array}{lc}\mathrm{CaCo}_{3} & 25 \mathrm{ppm} \\ \text { ph } & 4 \\ \text { Conductivity } & 42 \square \mathrm{S} \\ \text { Chlorides } & 5.3 \mathrm{ppm} \\ \text { Sulfates } & 1.5 \mathrm{ppm}\end{array}$

Figure 8 


\section{Option 1 - Direct Condensing}

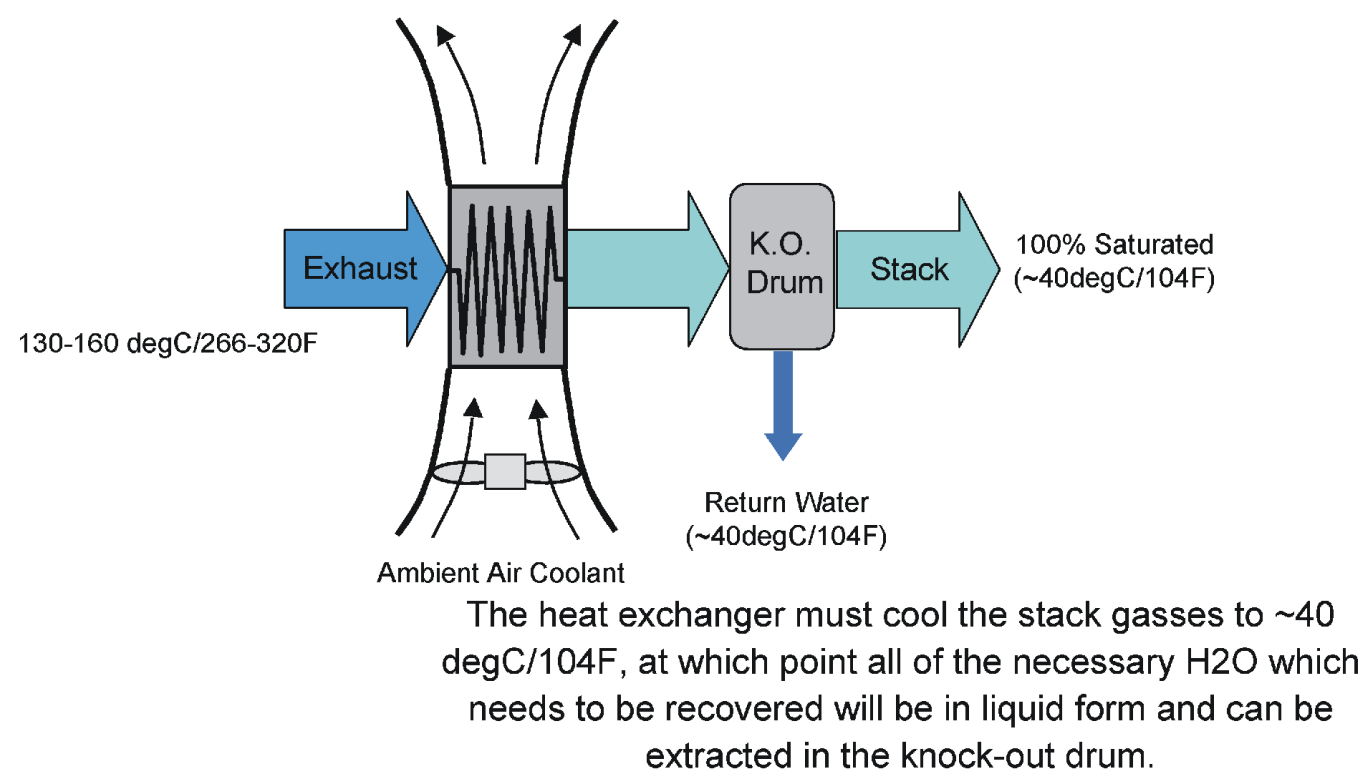

Figure 9

\section{Next Generation Turbines}

\section{Option 1 - Direct Condensing at Ambient of 20degC/68F}

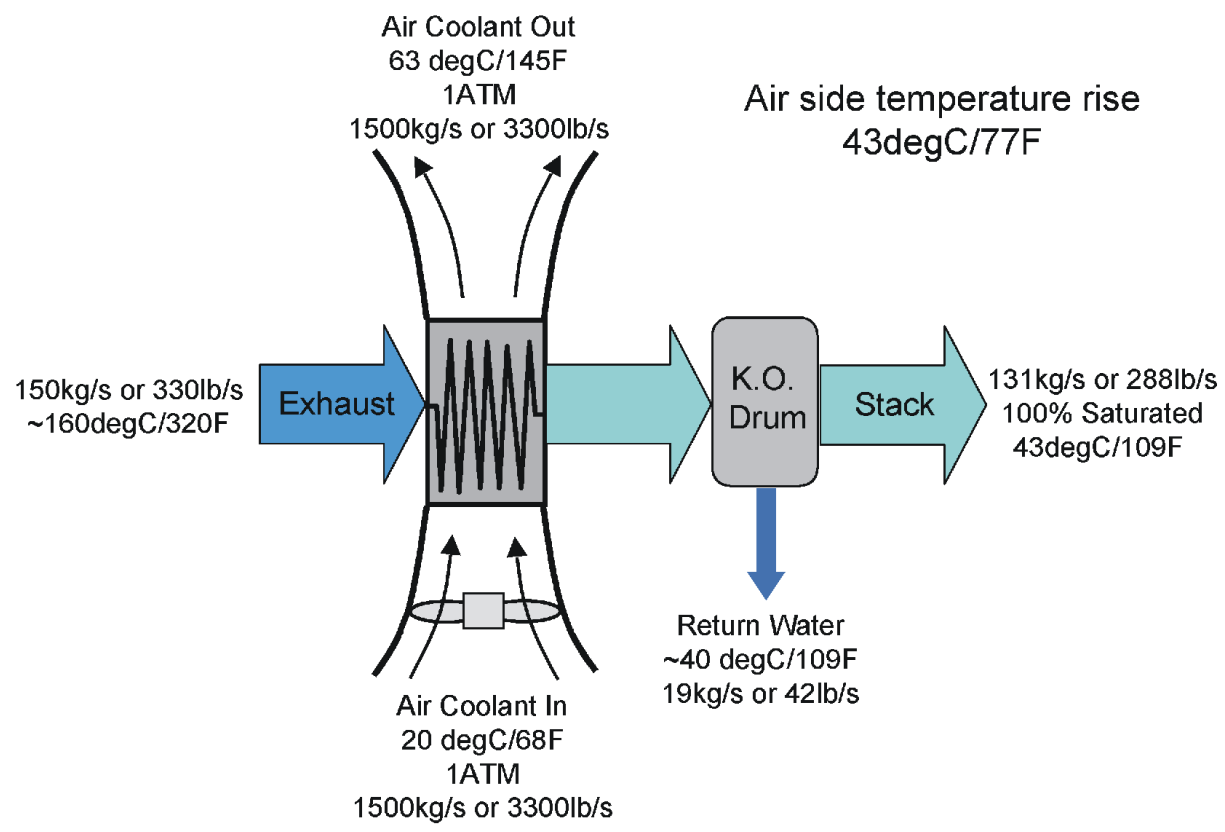

Figure 10 


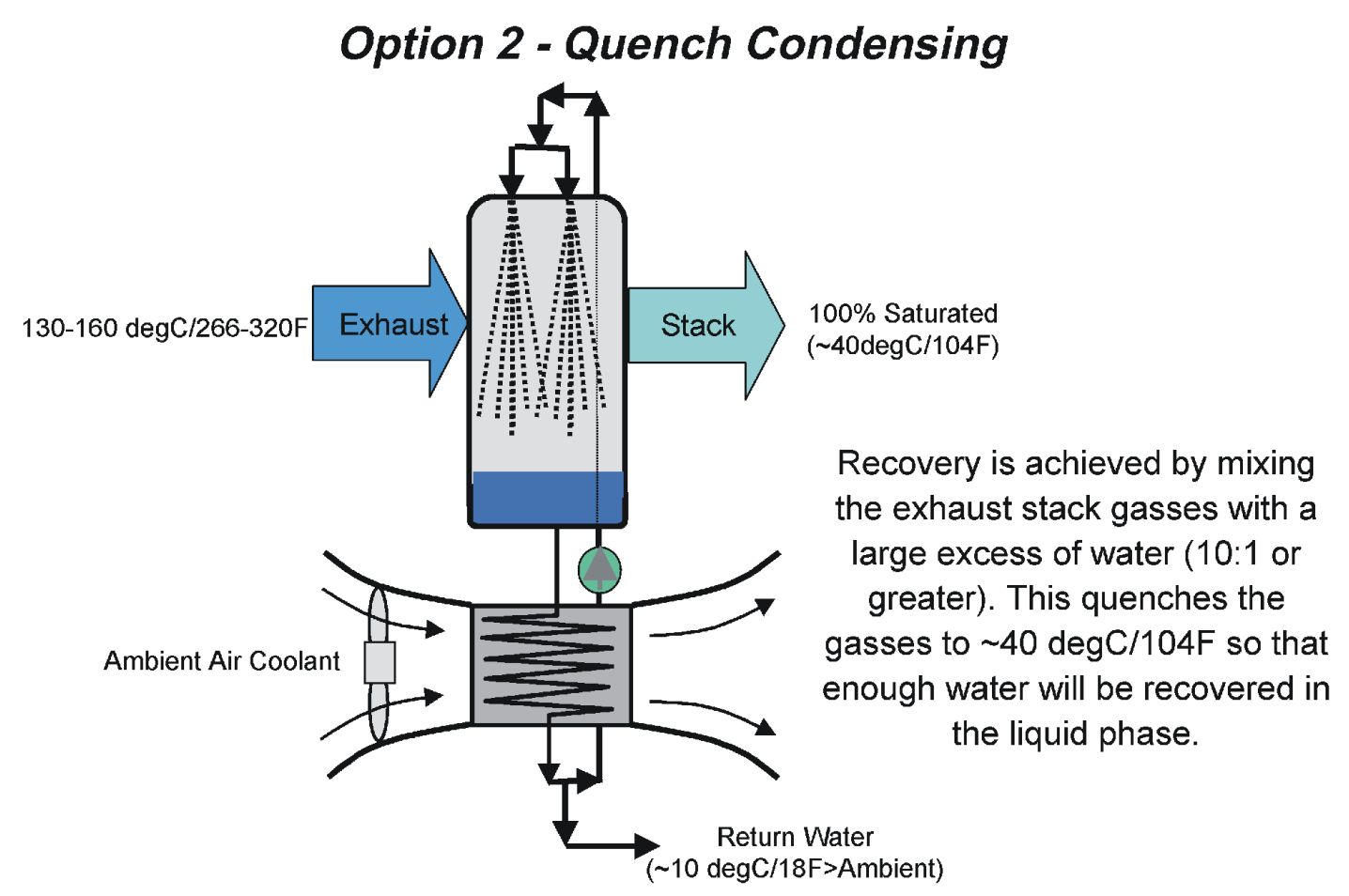

Figure 11

Next Generation Turbines

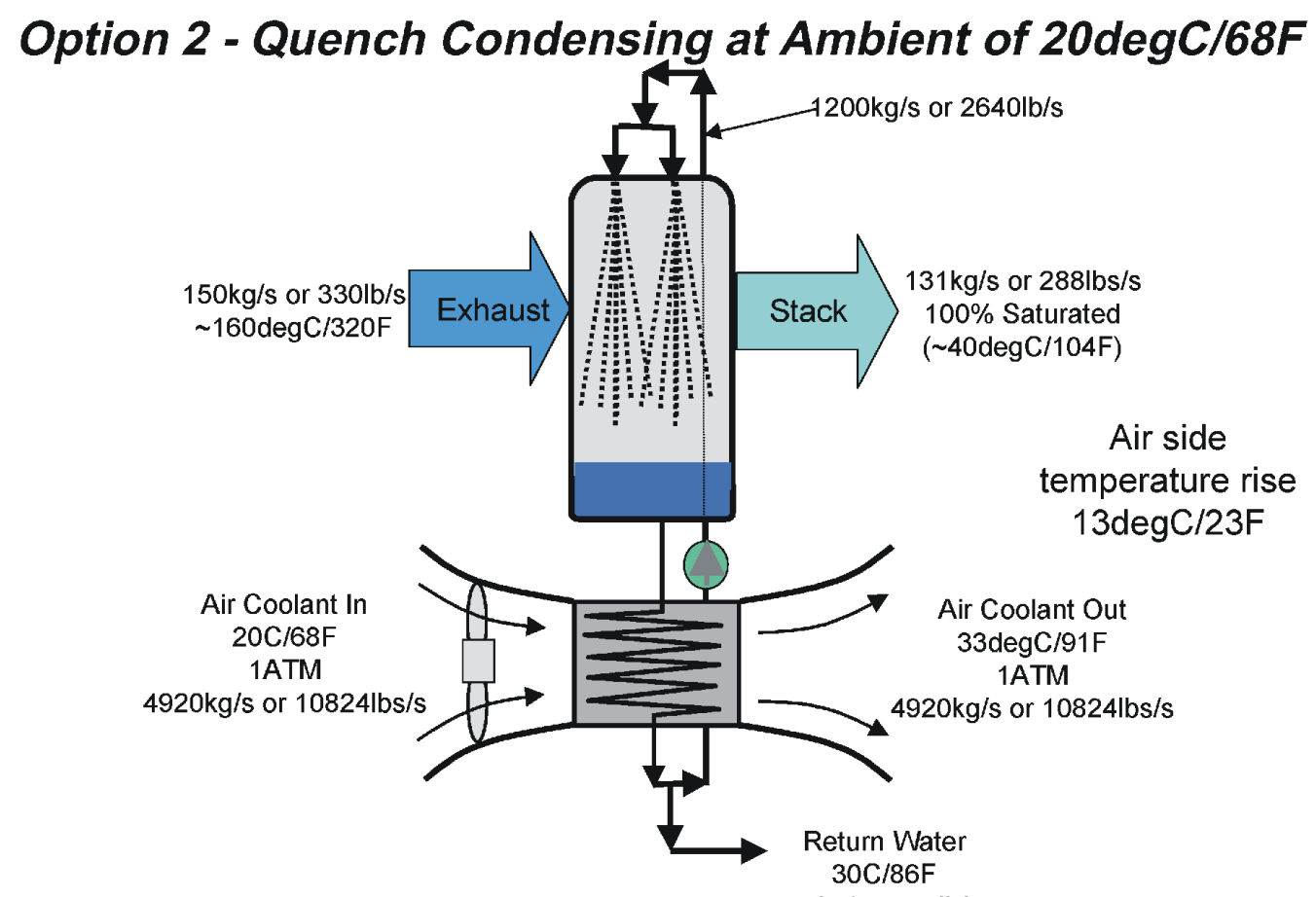

Figure 12 19kg/s or 421b/s 
Both system costs are dominated by the costs of the fixed surface heat exchanger

- The direct condensing system size and auxiliary power requirements is less sensitive to ambient temperature fluctuations

- The direct system has a lower auxiliary power requirement due to the significantly lower air coolant flow rates required for operation

- In both cases the stack gases will be $100 \%$ saturated. As such there is a risk of plume formation. This needs to be assessed in more detail.

\section{Equipment and Plant Cost Analysis}

Material cost analysis was based upon firm quotations as well as estimates utilizing past experience. Package engineering costs were estimated from previous projects which involved roughly the same amount of machinery, taking advantage where possible of existing hardware, which will be incorporated into this package. An example of this would be the MAHD exhaust system. Project contingencies and normal product margins were based upon current industry standards.

The BOP material was generally based upon vendor estimates with larger cost tolerance applied to newly designed hardware (e.g., water recovery system and recuperator section).

The development cost associated with the new turbo-machinery was a separate analysis and only applied to the plant cost as a single line item for investment recovery.

Results of this analysis are shown below based upon a $\$ / \mathrm{kW}$ installed cost basis.

- $\quad$ Trent 80 S.I.

$\$ 442.60$

- $\quad$ Trent 80 W.I.W.R.

$\$ 437.60$

\section{Demonstration Testing}

Rolls-Royce has conducted initial discussions with a major U.S. utility to provide a Beta site for the first operational test. This particular site already has many of the required infrastructure services which would minimize the cost of demonstration, and is located close to the engineering resources needed to support this work. Preliminary costing has been studied and incorporated into follow-on program plans. 


\section{Appendix D NGT Market/Economic Analysis: PB Power Report}




\section{Rolls-Royce NGT SURVEY}

IPP Market Survey

For:

Rolls-Royce Energy Systems Inc.

MAY 2001

PB Power, Inc.

11757 Katy Freeway, Suite 600

Houston, TX 77079

281-589-5801 Fax: 281-496-3873 


\begin{tabular}{|l|l|l|}
\hline REPORT PREPARED BY: & M N Arnold & 23 May 2001 \\
\hline REPORT APPROVED BY: & K P Taylor & 23 May 2001 \\
\hline \multicolumn{2}{|l|}{ Final Report Issued to Rolls-Royce } & 23 May 2001 \\
\hline
\end{tabular}




\section{LIST OF ABBREVIATIONS}

\begin{tabular}{ll} 
CCGT & Combined Cycle Gas Turbine \\
CO & Carbon monoxide \\
D & Day \\
DOE & Department of Energy \\
EPA & Environmental Protection Agency \\
Gal & Gallon \\
Gpm & Gallons per minute \\
GT & Gas turbine \\
IPP & Independent Power Project \\
KW & Kilowatts \\
LADWP & Los Angeles Department of Water and Power \\
IbS & Pounds \\
MS & Microsoft \\
MW & Megawatts \\
MWh & Megawatt-hour \\
NA & North America \\
NGT & Next Generation Turbine \\
NOx & Oxides of nitrogen \\
O \& M & Operations \& Maintenance \\
OEM & Original Equipment Manufacturer \\
PBP & PB Power Inc. \\
R-R & Rolls-Royce plc \\
SCR & Selective Catalytic Reduction \\
sec & Second \\
T\&D & Transmission \& distribution \\
US & United States of America \\
USGS & US Geological Survey \\
WRR & Water Resource Region \\
WT & Water Treatment \\
\hline
\end{tabular}




\section{CONTENTS}

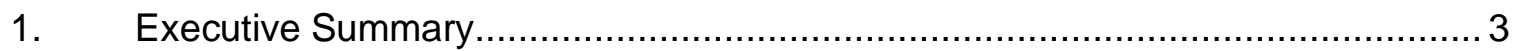

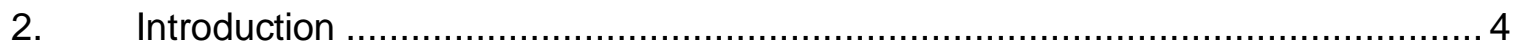

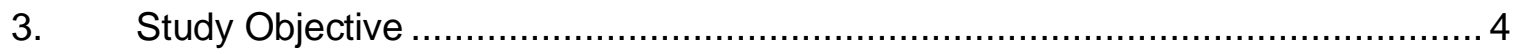

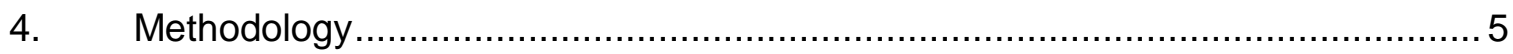

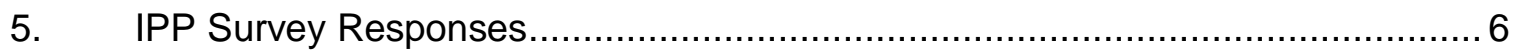

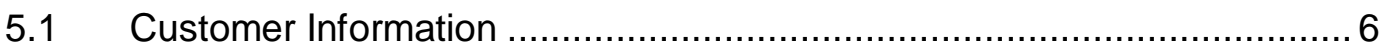

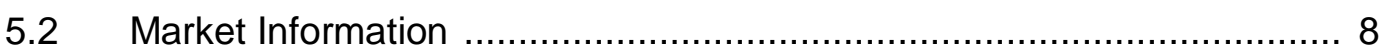

5.3 NGT Market Potential ................................................................. 10

5.4 Pro-forma Issues .................................................................... 14

5.5 Service Offerings/Incentives …................................................... 16

$5.6 \quad$ Water Usage \& Recovery ........................................................... 18

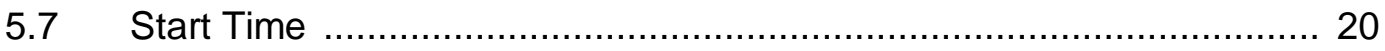

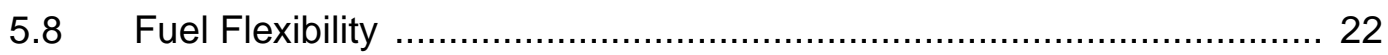

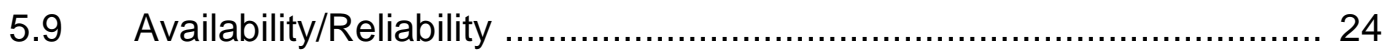

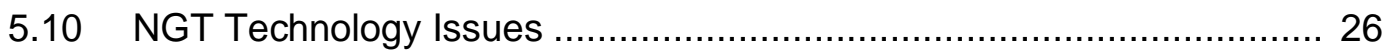

6. Availability and Permitting of Water in US ..................................................... 28

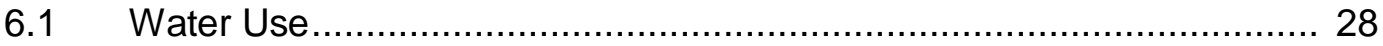

6.2 Permitting \& Conservation Programs/Requirements .......................... 30

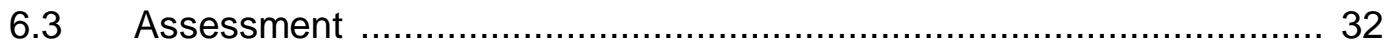

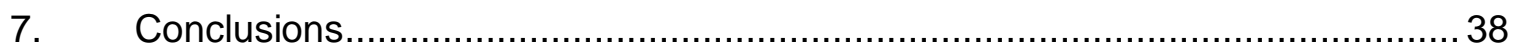

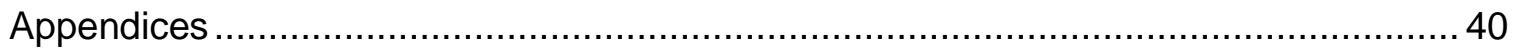




\subsection{Executive Summary}

Rolls-Royce has commissioned PB Power to carry out a limited market survey of the North American power generation market attitudes to the acceptability of Rolls-Royce's NGT concepts of super steam or water injection or water recuperation. PB Power instituted a number of discussions with IPP developers, to elicit their opinions on the NGT concepts. The respondents were chosen to cover a wide range of the NA market.

The limited survey concludes NGT will have a market in the mid-merit and peaking sectors. The attributes NGT must have are; competitive capital cost, high efficiency, and high availability. Start-up time should be within 15-30 minutes. Dual fuel capability will be required by some of the market. Delivery period should be about 12 months. Maintenance contracts and lease engine programs should be offered.

The availability and permitting of power plants to consume large volumes of water is difficult and will continue to be difficult in the future. The zero-water consumption concept of the NGT would be highly valuable in the market. A NGT plant consuming $45 \mathrm{lbs}$ per second of water is not likely to obtain any sales in the United States.

Selling new technology into the power generation market will be a challenge. Introduction of NGT will most likely require Rolls-Royce to assume the majority of technology risk by offering guaranteed performance and availabilities.

The water recovery system and recuperator are considered the more technically challenging developments for Rolls-Royce. However, the Rolls-Royce reputation is highly regarded such that Rolls-Royce are considered by the industry to be capable of successful development of the NGT concept. 


\section{Introduction}

The US DOE is working with industry to jointly fund a development program to produce advanced gas turbine systems, known as the "Next Generation Turbine" (NGT) program. This program is a follow-on to the DOE Advanced Turbine Systems nine-year program that aided development of the "H" class machines. A survey performed for the DOE in 1998/1999 concluded that about $124 \mathrm{GW}$ of new generating capacity will be needed in the US within the next two decades to meet an ever increasing demand and replace old inefficient power plants. Of this new capacity, approximately 90 per cent is envisaged to be combustion turbine based power plant. The DOE is promoting the development of gas turbine generators that can attain greater than 50 per cent thermal efficiency, low emissions, reduced O\&M costs, improved service life and the ability to start and stop frequently. To meet such challenging goals, RollsRoyce have been investigating developments to recuperate and water or steam inject the RB211 and/or Trent to achieve increased power outputs and significant improvements in thermal efficiencies. Rolls-Royce studies to date indicate that this concept can achieve power levels between 70-80 MW at 51-53 per cent thermal efficiency, dependent upon which core engine is used and the level of technology utilized.

To enable Rolls-Royce to determine the targets necessary to meet the US market's perceived requirements for output, efficiency, acceptable technology risk and capital cost a survey of the US market has been undertaken.

This report by PB Power Inc. reflects the professional opinion of PBP. It is understood that PBP make no warranties or guarantees, actual or implied, and bears no responsibilities for the ultimate commercial, technical, economic or financial performance of the project in any manner whatsoever.

\section{Study Objective}

The objective of the study is to:

- Determine market acceptability for proposed R-R NGT concepts;

- Understand customer purchasing decision factors and economic evaluation techniques;

- Understand customer perspective regarding specific technologies; and

- Estimate market potential for proposed R-R NGT product(s). 


\section{Methodology}

Based upon achieving the objectives, Rolls-Royce developed a preliminary NGT market survey document that outlined the questions the survey attempted to answer. PB Power and R-R jointly refined the questionnaire to those questions we believed the IPP companies would be willing and able to respond to. To be able to explain the R-R NGT concepts, Rolls-Royce produced a short MS PowerPoint presentation for PB Power to use to describe the NGT system to each participant IPP company.

Through our contact and involvement with a number of major IPP companies active in the US and overseas market we developed with Rolls-Royce a list of potential candidate survey participants of about 30 potential companies based and active in the US. Selection of 17 was based upon our impression of which companies were likeliest to be forthcoming with their opinions and provide as much coverage of the US as possible. Senior engineers based in our Houston, Boston, New York and San Francisco offices hold these contacts, and it was these engineers who formed the survey team. These team members maintain regular contact with many of the prominent and active developers as well as smaller 'briefcase' developers. In selecting the companies and their personnel to interview we took cognizance of these factors:

- Has the technical background and seniority to understand the delivered NGT concept and can judge his company's expected attitude to the questions posed.

- Will be willing to allocate adequate time for discussions otherwise responses are likely to be ill thought through. A typical interview took about two hours or more.

Each team member made contact with the people within the IPP company regarded as the more appropriate to provide feedback, and meetings were arranged. In order for the survey candidates to provide the feedback we desire, it was necessary to commence the meeting by delivering the Rolls-Royce prepared NGT presentation, the survey's goals and objectives, and describes the Rolls-Royce NGT concepts. Thereafter, we instigated a discussion and directed the debate so as to answer the pre-defined set of questions. These questions are included in Appendix A.

Following each visit, the survey team member generated a meeting report. Each meeting report was e-mailed to each team member and the Rolls-Royce project manager to gauge response, keep all team members advised and prompt any changes to our approach judged necessary.

The NGT concept may use up to $45 \mathrm{lbs}$. per second of demineralized water. One aspect of the survey is to assess attitudes to such water usage. As well as surveying power generation companies, for further background, we have investigated the likely availability and permitting issues associated with consumption of water for power generation within the US. Such issues are becoming more prominent in a number of states when obtaining permits. The data and 
opinions resulting from the research and interview phase have been analyzed and conclusions made.

\section{IPP Survey Responses}

We have tabulated the responses from our interviews with the companies for analysis. Each topic issue of our questionnaire is analyzed separately in this section.

\subsection{Customer Information}

We have conducted discussions with a number of prominent IPP companies. The interviews were carried out during the period mid-March through end April. The companies surveyed in order of survey were:

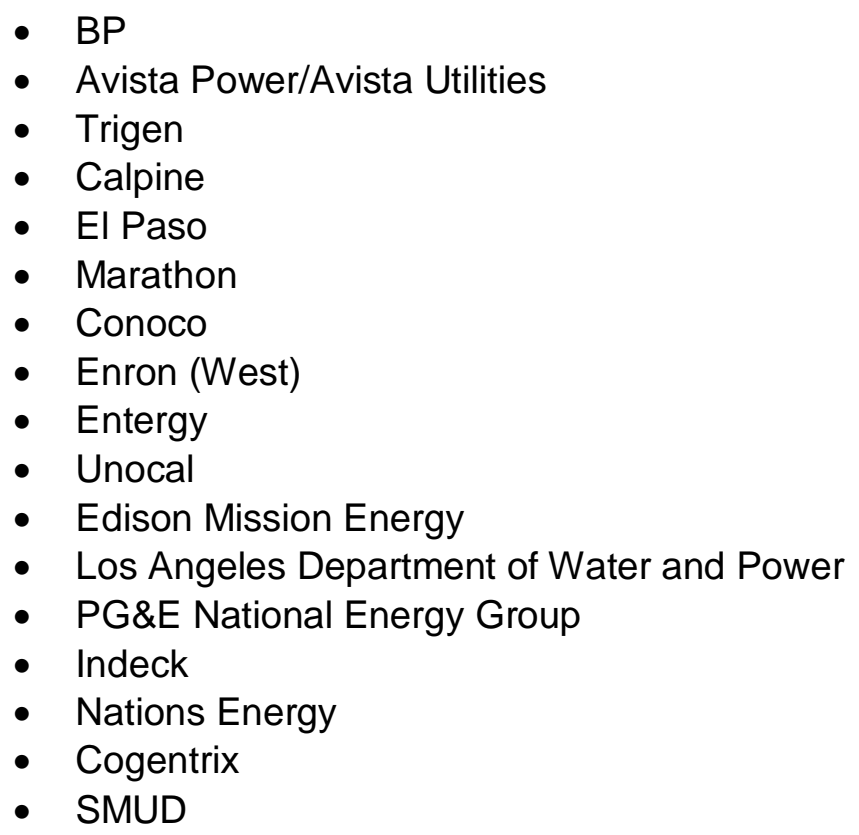

Their main markets have been in the US since many US based developers have reined in their international developments to concentrate on the active US market. However, a number have international developments in SE Asia, Middle East, Europe, Latin America and Africa.

Their current plant portfolios range from LM 6000 (<50 MW) through ' $F$ ' and 'G' frame units (> $250 \mathrm{MW}$ ). The power plant type sectors encompassed by our respondents are large CCGT, open-cycle peaking plants and cogeneration applications.

Very few of our respondents expected to use multiple small units, citing economics as the main reason. One company, Company E, expected to use multiple units for some of their cogeneration plants, justifying redundancy for security of steam production as their main reason. 


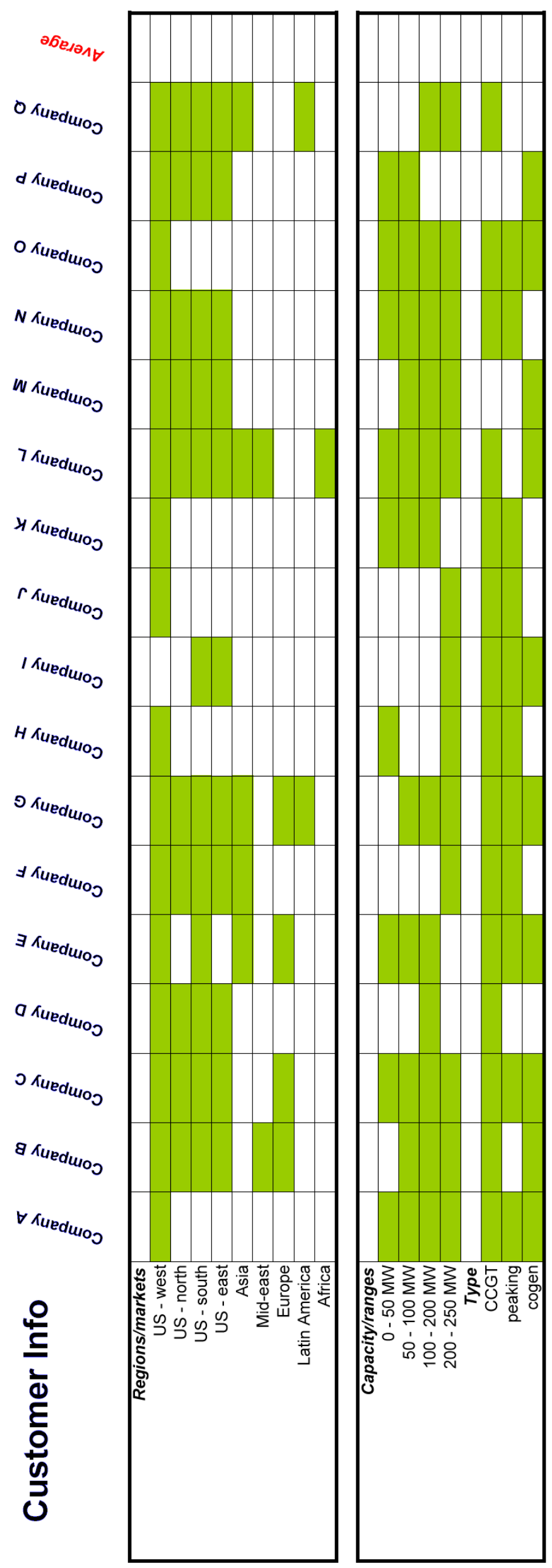

\begin{tabular}{|c|c|c|c|}
\hline $\mid$\begin{tabular}{ccc}
$\circ 0$ & $\circ$ \\
\hdashline & $\infty$ & $\infty$ \\
0 & $\infty$
\end{tabular} & 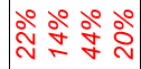 & 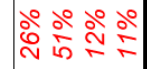 & $\frac{\dot{z}}{2}$ \\
\hline ঃิ응 ঃ̊ & ○̊ ঃㅇㅇㅇㅇ & ㅇํㅇㅇㅇㅇ ஃ & 今̊ \\
\hline 응 ঃㅇㅇ & 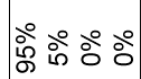 & ஃ̊ ஃㅇํㅇㅇํ & 今े \\
\hline 옹 응 움 & 응 응 ᄋ̊ ঃे & 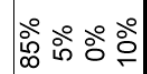 & î̀ \\
\hline 옹 응 음 & ㅇํㅇ ㅇํㅇ ㅇํㅇ ㅇํㅇ & 응 옹융 & 今̊ \\
\hline 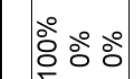 & 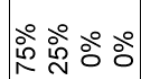 & ○ㅇㅇㅇํㅇ ঃ & 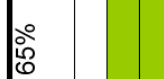 \\
\hline 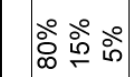 & 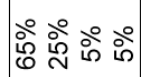 & ㅇํㅇ ㅇํㅇ ㅇํㅇ & 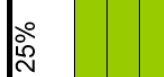 \\
\hline 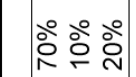 & 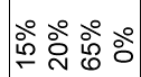 & 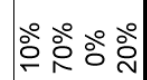 & 今े \\
\hline ㅇํㅅ 융 & 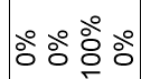 & 융 ㅇํㅇㅇํㅇํํ & ○े \\
\hline 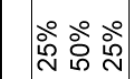 & 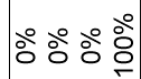 & 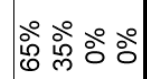 & 今े \\
\hline 응 & 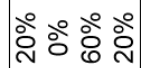 & 응 ㅇㅇㅇㅇㅇㅇ & in \\
\hline 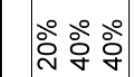 & 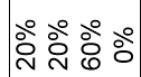 & ஓ̊ ঃ̊ ঃ̊ ০̊ & 今̀ \\
\hline ঃ̊ ঃ̊ & ㅇํㅇ융 ㅇํㅇ & 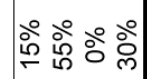 & ○े \\
\hline 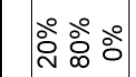 & 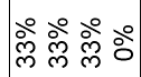 & ㅇํㅇ 융ㅇㅇ & ○े \\
\hline ○̊ ঃㅇㅇํ & ஃ̊ ঃㅇㅇㅇ ஃ̊ & 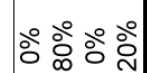 & 今े \\
\hline 옹 응 음 & 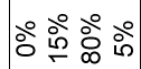 & 응 ㅇํㅇ ㅇํㅇㅇํㅇ & in \\
\hline 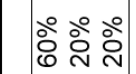 & ঃ̊ ঃ̊ ঃ̊ ০̊ & 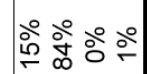 & i̊ \\
\hline 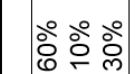 & 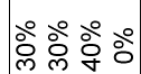 & 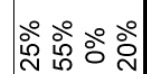 & 今̊ \\
\hline 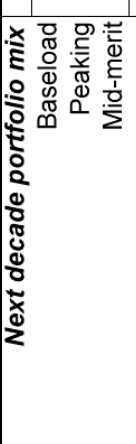 & 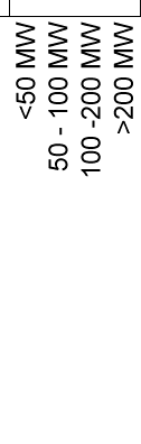 & 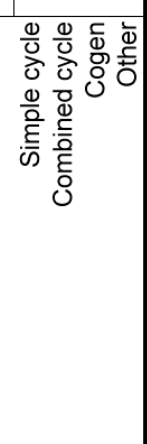 & 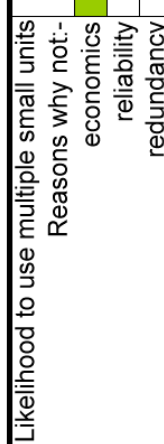 \\
\hline
\end{tabular}




\subsection{Market Information}

Most respondents considered that there will be growth in the baseload market and the peaking market. Many also considered there will be a market for mid-merit power plant; some respondents refer to such plant as intermediate power. This is a power plant that typically starts up twice a day, probably Monday - Friday, to capture the higher power prices associated with the shoulder peaks of the morning and evening. Such power plants typically operate about 4500 hours per year. Some respondents consider many of the current operational and most future baseload CCGT will have to service this mid-merit market, the current high gas prices mean many baseload CCGT plants are unable to compete against the low-cost nuclear and coal-fired baseload plants. However, a few of the respondents did not see such a mid-merit market and were not convinced such a market sector will evolve in the US.

Most of our respondents considered the peaking plant market would continue to grow in the short-term, say over the next three to five years. These respondents were evenly split over whether the growth will be at a lesser pace than has been experienced over the past three years, or at the current frenetic rates continuing over a longer time-frame. Four respondents considered there would be no growth in the peaking market due the high numbers recently installed causing market saturation and due to the baseload capacity currently in construction that will shortly become operational.

All respondents reported the US electricity transmission system was experiencing constraints and that it is becoming more difficult to find sites for large power plants near to pipelines having sufficient gas capacity. All agreed the transmission system was in dire need of upgrading and expansion, but all recognized there were inadequate financial incentives to encourage such works. To overcome such transmission constraints, there has been much talk within the industry that smaller power plants will be connected to the distribution system, rather than the transmission system, more local to the load centers. Virtually all respondents foresee growth in distributed power generation. However, some see such plants as being less than $10 \mathrm{MW}$, and others see larger power plants up to $75 \mathrm{MW}$. 


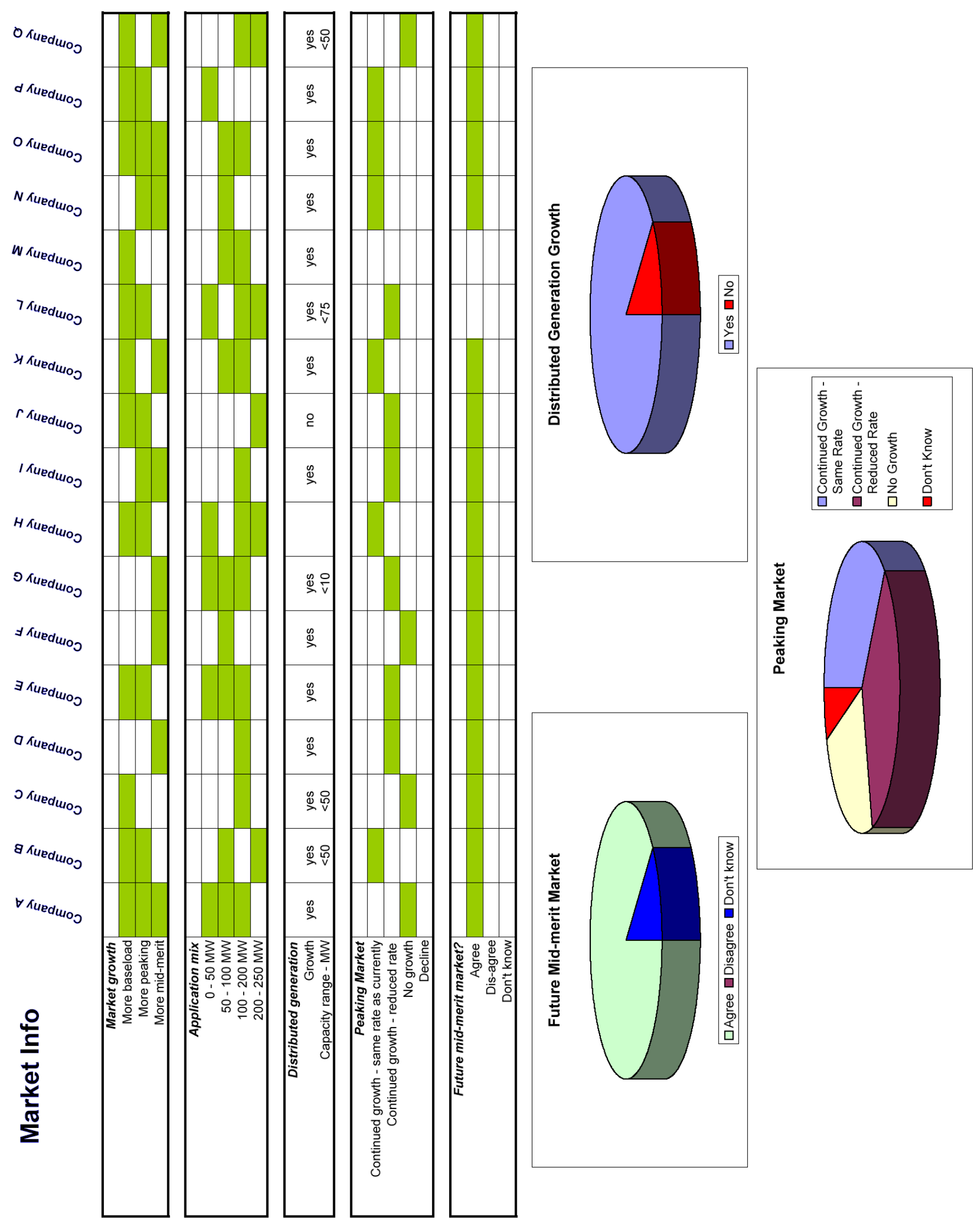




\subsection{NGT Market Potential}

Nearly all respondents see a future market for a relatively high efficiency, 70-80 MW gas turbine of about $\$ 500 / \mathrm{kW}$ capital cost, although our respondents were nearly equally split on whether this unit size is satisfactory or too small. Most could not see any network constraints to NGT, although Company L considered that this unit size was too large to connect to the distribution system. None could see any specific regulatory constraints to NGT. Some respondents indicated that this capacity would require applying for air permits via a more onerous route compared to a simpler procedure for power plants of less than about $50 \mathrm{MW}$ output, but none saw this as a significant issue.

Most respondents consider the NGT concept will be more suited to the mid-merit market sector due to its relatively competitive price, high efficiency and its' ability to frequently, rapidly start-up to full load without significant consequential maintenance burden. Some consider there could be opportunities for some baseload applications, perhaps within metropolitan areas where currently poor efficiency, must-run power plants are located for load and frequency support. Some respondents also felt NGT could find opportunities in the peaking sector, although a number considered NGT was too complex and costly within this sector. Others argued that such a plant can satisfy the goals of a peaking plant, but it also allows the developer some assurance the plant could compete in the mid-merit market later in the event the peaking market declines.

It is clear that as there is little usable heat remaining in the turbine exhaust that NGT will have no attraction to the cogeneration market.

The general consensus was that the key attributes required for a NGT plant for the identified markets are:

- Low capital cost

- High thermal efficiency

- High reliability and availability

Start-up time was considered important for peaking power plant, but less so for mid-merit market. Emissions are considered important, but only to the extent that they must meet air permit regulations; there is no economic advantage in bettering the regulation limits.

Attributes that did not score as particularly important were unmanned operation, minimum plant footprint, fuel flexibility and improved part load operation. Whilst some thought unmanned operation for a peaking plant was an advantage, few considered a NGT plant of the described complexity as being practical for unmanned operation. As long as the plant does not require any more staff than is currently typical for a power plant of this capacity, manning was not perceived as a significant issue. The plant footprint size was not considered a major issue except perhaps in urban locations, when noise and visual impact become concerns. Some respondents would 
require dual fuel capability but most considered the additional expense, complication and longer, more difficult permitting for a dual fuel power plant not worthwhile. Improved part load performance did not score highly. Few could see any advantage for a 70-80 MW power plant to operate at part load for any significant periods. However, a number expressed the opinion that if the super steam or water injection process also ameliorates the fall off in power output with higher ambient temperatures this would be a significant advantage.

Most respondents desired a delivery period of 12 months. Most agreed that delivery periods faster than this may not aid the overall installation time as other issues, many referred to permitting timescales, would become the critical path. 


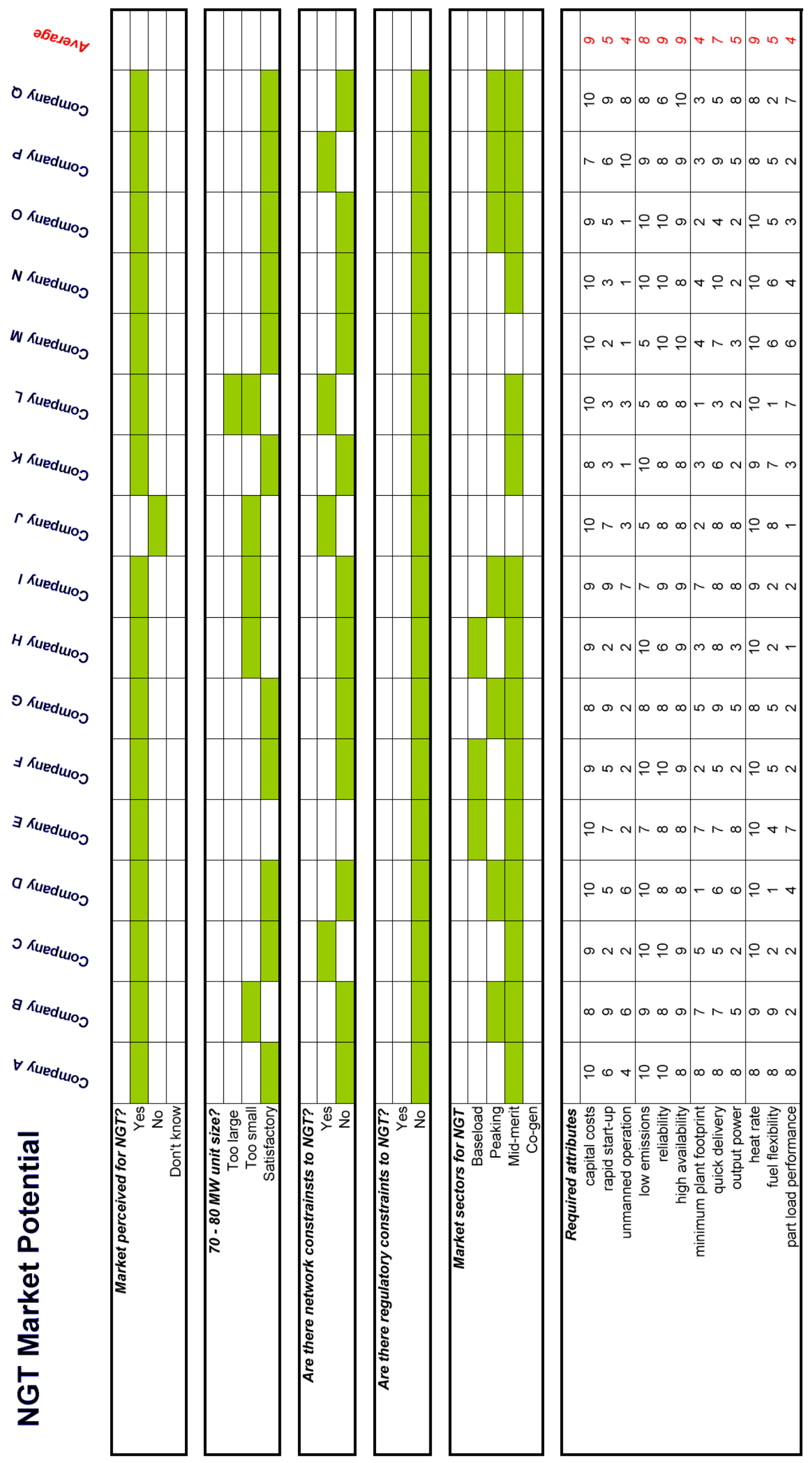




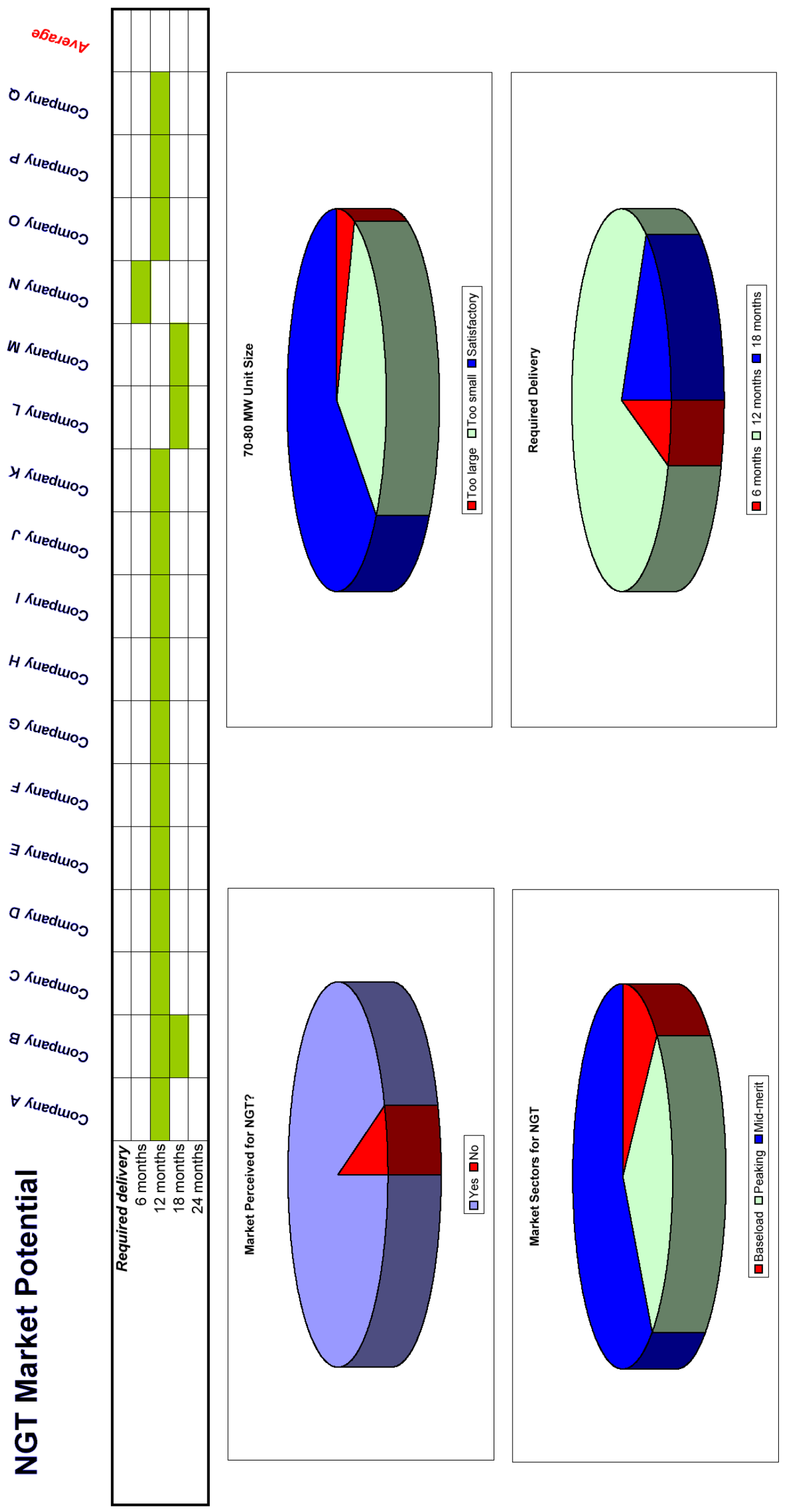




\subsection{Pro-forma Issues}

Our questions of their pro-forma considerations confirmed earlier conclusions that the main perceived markets are the peaking and mid-merit market sectors. Significant issues that impact their pro-forma returns for these markets are chiefly:

- Capital cost

- Heat rate

- High reliability and availability

- Water consumption

- O\&M costs were significant to some, and less so to others. Output power and low manning are not regarded as significant factors.

Virtually all respondents described themselves as risk adverse and would not purchase any power plant until regarded as proven, some citing the problems of obtaining financing for unproven plant, others simply wary of new technology following the various problems all the major gas turbine manufacturers have recently experienced in bringing new technology to the market. Some quoted a value of incremental rate of return that would be required for NGT, but most stated the caveat that this was after the first machines had been installed by some other entity. 


\begin{tabular}{|c|c|c|c|c|}
\hline${ }^{2} \sigma_{e_{\text {, }}}$ & $n \stackrel{\infty}{q} f$ & $\infty \infty$ & 央 & \\
\hline O kuedulo? & ০응 & 으은 & $\infty \sim \infty)$ & \\
\hline$d k_{\text {ueduo }}$ & 0 ○ & $\infty \begin{array}{lll}\infty & \infty & 0\end{array}$ & $\infty$ 요 $\infty$ 웅 & \\
\hline 0 Kueduo & $\stackrel{2}{\circ}$ & $a \wedge-$ & 0 웅ㅇㅇㅜ N & \\
\hline$N_{\text {Kuedulo }}$ & $\circ \infty$ ని & $\circ \infty-$ & 응으 으은 N & \\
\hline$w^{1}$ ured $_{u_{u_{0}}}$ & 용요 웅 & $\circ \infty-$ & $\wedge$ 응의 & \\
\hline 7 Kueduoj & 는 융요 잉 & ㅇN & ம $\infty \propto$ & \\
\hline$H_{1}$ Kued $_{w_{10}}$ & 으 & $\infty \infty-$ & $\wedge$ 응우 $\sigma$ N & \\
\hline$r$ Kuedulo & 000 & 응 & $\circ \infty \infty$ 웅ㅇ & \\
\hline 1 Nued $\mathrm{u}_{u_{\mathrm{O}} \mathrm{J}}$ & $\circ$ ○ & 으우 & $\infty \wedge ㅇ$ & \\
\hline H kueduos & 웅ㅇ & $\begin{array}{ll}0 & N\end{array}$ & 으응 0 응 & \\
\hline O אueduo & $\sim \stackrel{\infty}{\infty} 8$ & $\infty \begin{array}{lllllllll}\infty & \infty & 0\end{array}$ & $0 \circ \infty \infty$ & \\
\hline$\exists$ Kued $_{\mathrm{u}_{\mathrm{u}_{\mathrm{O}} \mathrm{O}}}$ & 우 웅ㅇ & $a \wedge N$ & $1 ㅇ ㅡ ㅇ ㅇ ㅇ ㅇ$ & \\
\hline$\exists$ Kued $_{u_{u_{0}}}$ & 잉요 & or 0 & $\infty \wedge ? 0$ & \\
\hline O Kueduoj & OR요 & $ㅇ ㅜ ㅇ$ & $ㅇ ㅡ ㅇ \div 0$ & \\
\hline 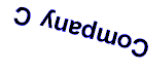 & 응 is & $\begin{array}{ll}\infty & \sim\end{array}$ & 응ㅇㅇㅇㅇㅡ N & \\
\hline 8 Kueduo & ○ 요요 in & $\infty \wedge i$ & $\mid \begin{array}{lllll}\infty & \infty & \infty & \infty & 0\end{array}$ & \\
\hline$\forall$ Kued uloj & ¿ & $\circ \quad \forall$ & $\sigma$ 응 잉 & \\
\hline $\begin{array}{l}0 \\
\frac{0}{2} \\
0 \\
\frac{\infty}{0} \\
\frac{\sigma}{E} \\
\frac{E}{0} \\
\frac{0}{0} \\
\frac{0}{2}\end{array}$ & 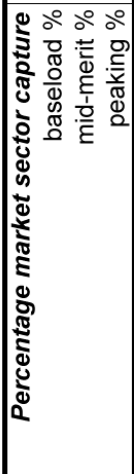 & 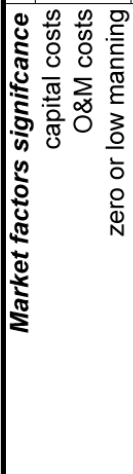 & 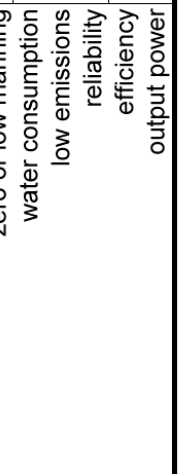 & 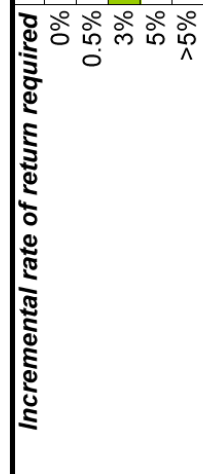 \\
\hline
\end{tabular}




\subsection{Service Offerings/Incentives}

All respondents considered service offerings important for the reduction of project risk. Most would require maintenance contracts and many were interested in a lease engine program. None of our contacts were interested in O\&M contracts on the basis they either operate their power plants themselves or competitively bid this function out to third party operations companies.

When asked the question of what incentives would be necessary to launch NGT, all were definite that availability and performance guarantees, supported by substantial liquidated damages, would be required. A number would also require Rolls-Royce to have equity participation to reinforce commitment to the project. Most made the comment that they would need to ensure all the technology risk was taken by the OEM, that they were not prepared to be a test bed for manufacturers. A significant number finance power plant projects off balance sheet and could not obtain bank finance, either competitively or at all, unless the banks regarded NGT as acceptable technology risk. 


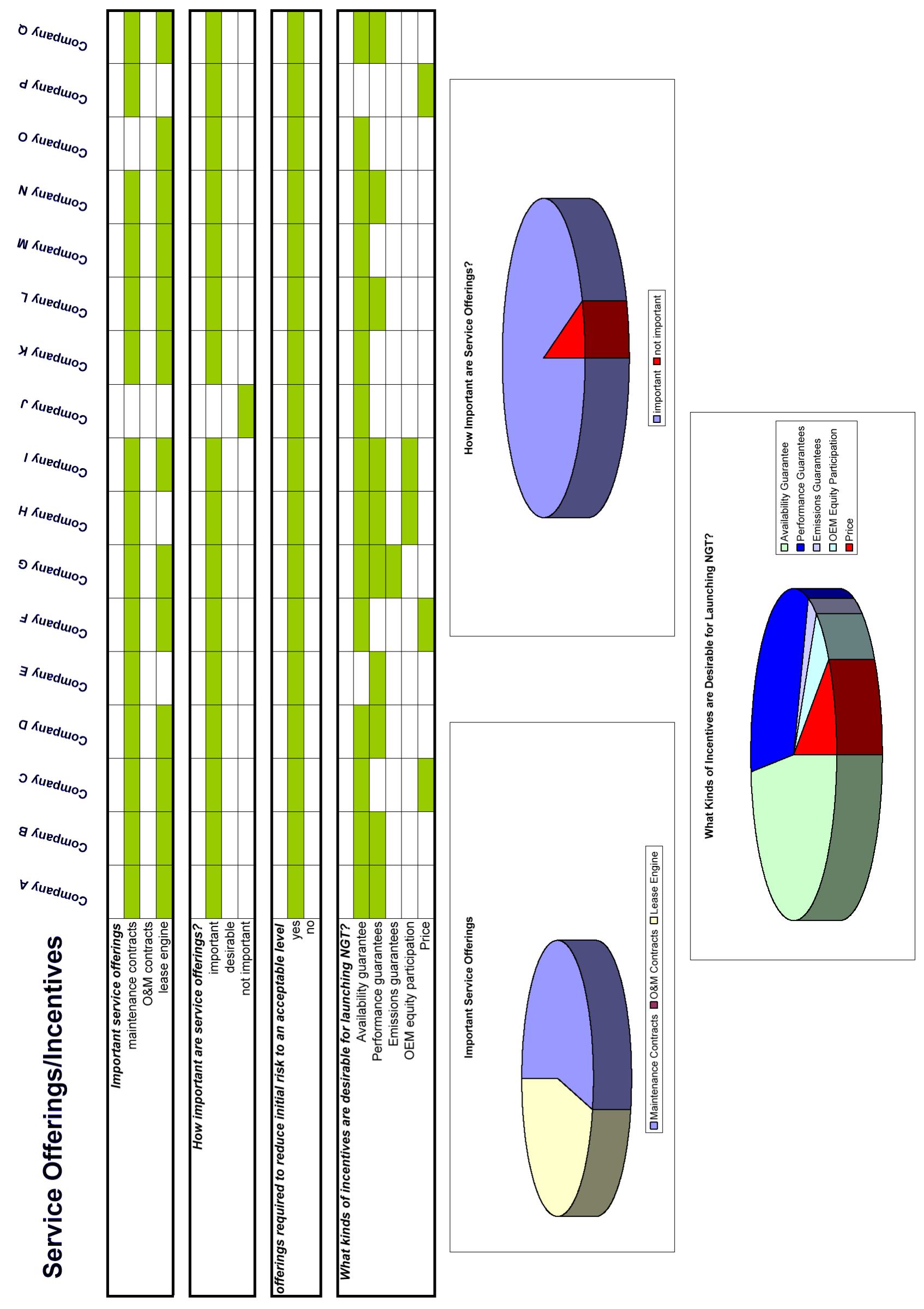




\subsection{Water Usage \& Recovery}

Our respondents were divided on whether the water recovery system will limit sales of NGT. Of course, their responses were based upon the vague description provided to them, and as such a system of water recovery from the exhaust of a gas turbine is unknown in power generation, their experience upon which to base their opinions was very limited. Half ventured that if such system is proven to work then there is no fundamental reason why sales will be discouraged. Power plant operators are used to managing systems and this will just be another one. Others considered the extra complication will limit sales, more so in the peaking plant sector, but all agreed any power plant system that resulted in zero water consumption was extremely valuable in the US market, and in many overseas locations. All were resolute that a 70-80 MW power plant consuming $45 \mathrm{lbs}$. per second of water was unacceptable for economic reasons (costs of raw water, water treatment plant for $400 \mathrm{gpm}$ and WT plant chemical consumption), the difficulty in locating sources for such a water demand and the difficulty in permitting it. Utilization of water resources in the US is becoming a very emotive issue with the local population, triggering protest movements, introducing permitting delays and bad publicity for the development company. Some respondents stated it was company policy to be seen as "good neighbours" and therefore it was corporately unacceptable to base developments on such water consumption rates. 


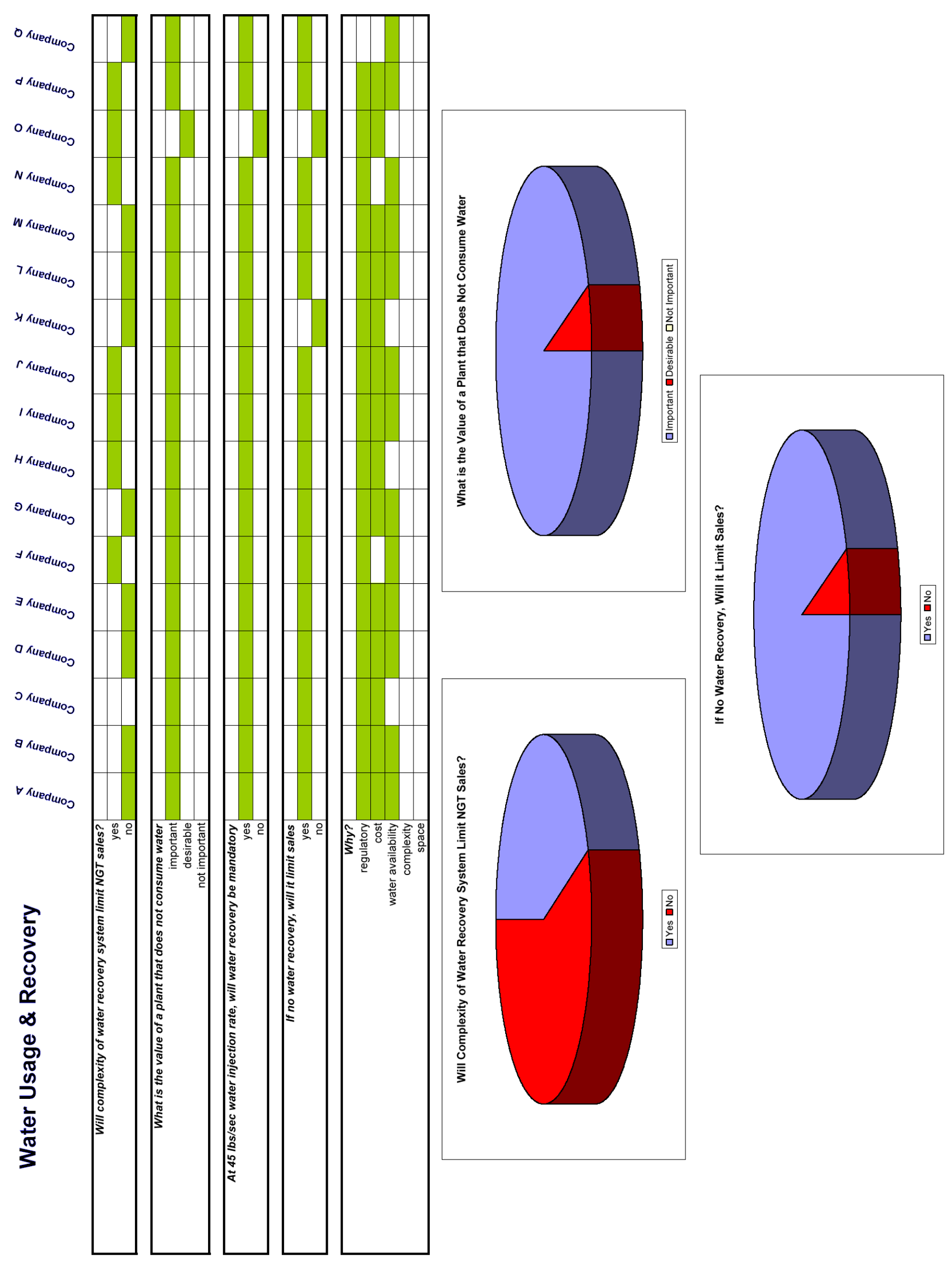




\subsection{Start Time}

Most of our contacts considered rapid start time was important for power plant operating as peaking plant, but less so for the mid-merit and baseload sectors. Most considered a start time of 10-15 minutes was desirable with others finding 15-30 minutes acceptable. Logic seems to suggest that if a plant is to start up perhaps twice a day to capture mid-merit dispatching then the start up time should be reasonably rapid otherwise overall heat rate will be affected. 


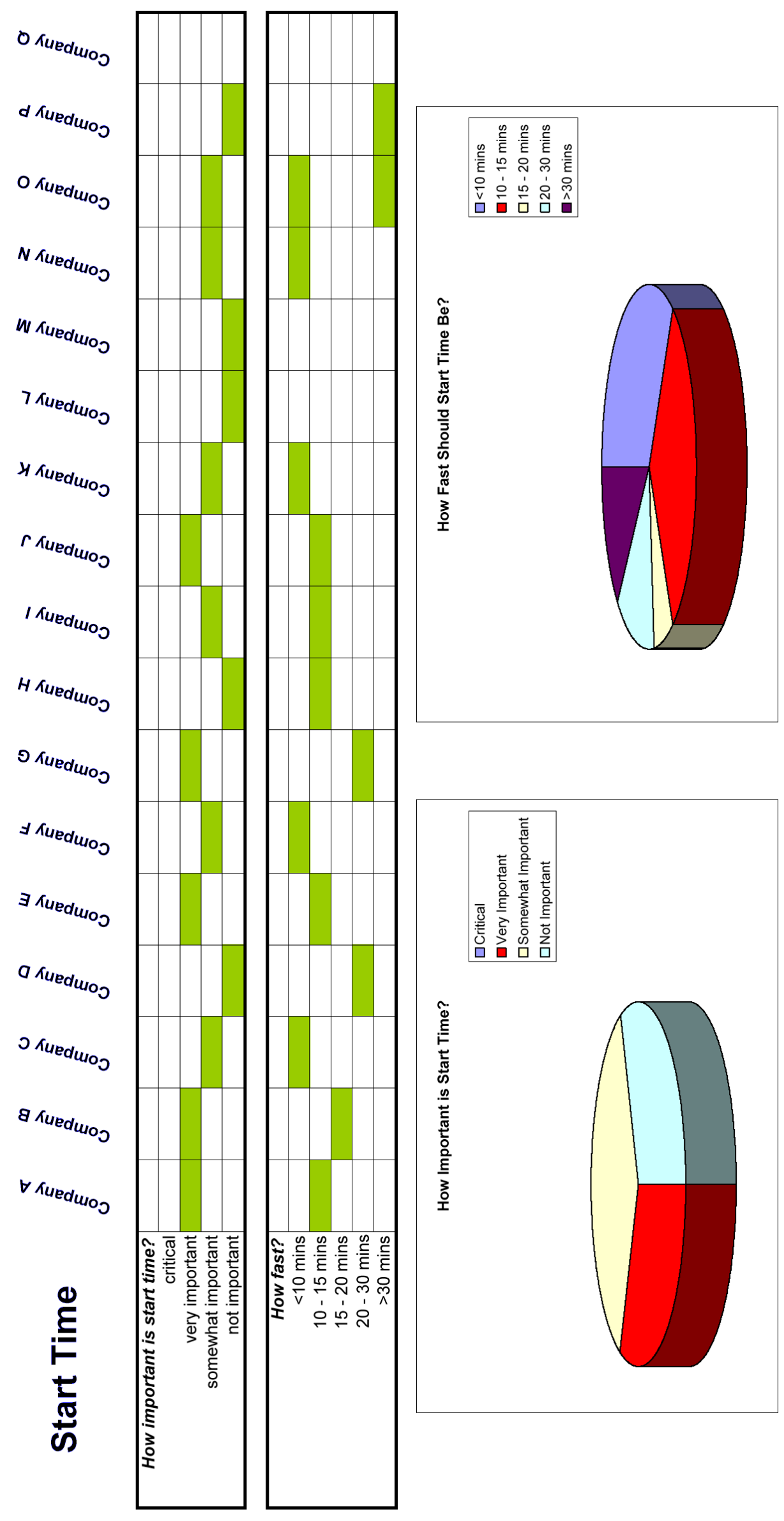




\subsection{Fuel Flexibility}

Our contacts were equally divided on whether dual fuel flexibility would be required. Since we were requiring them to project their opinions forward five years, many appeared concerned about what will be the reliability of the gas supply with the current high gas prices and lack of new pipeline constructions being undertaken. Some saw the ability of being able to burn distillate fuel as a means of hedging against high gas prices. We are not convinced such scenarios will prevail. It generally happens that distillate fuel prices rise if gas prices remain high, and permitting of power plant for significant operating hours on liquid fuel will be difficult unless there is a significant change in EPA regulations.

All respondents consider \# 2 distillate as the primary back up fuel with some noting the advantage of burning naphtha or refinery fuels in specific applications. Most prefer fuel changeover whilst the unit operates on load, but if this is not possible, it should be achievable within one hour of shutdown. 


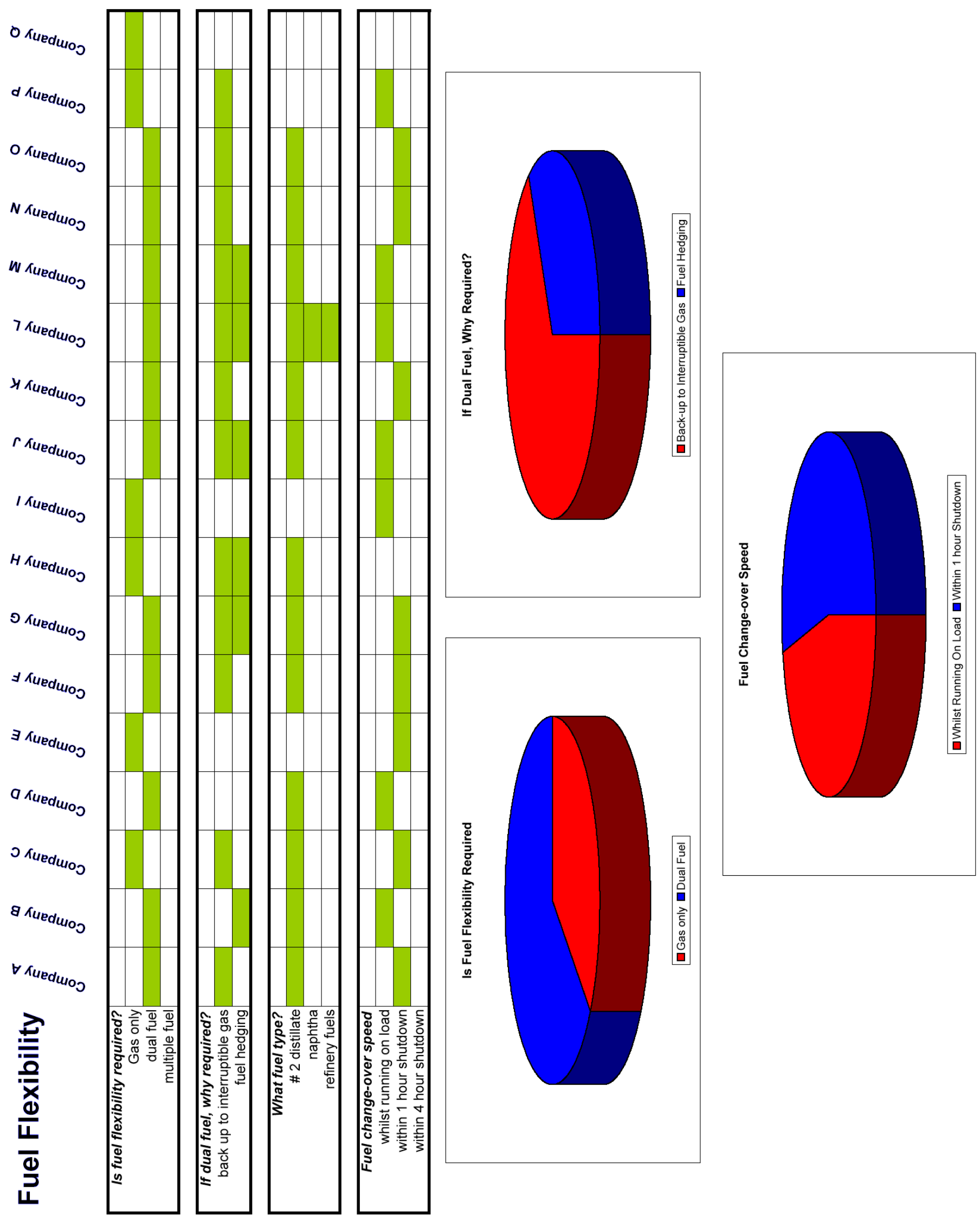




\subsection{Availability/Reliability}

Our respondents all have high expectations for availability and reliability of power plant. Their desire is for availabilities of better than 96 per cent and forced outage rates less than 2-3 per cent.

Generally, most are satisfied with maintenance intervals currently offered by the GE LM 6000 gas turbine of 25,000 hours between turbine change-out when burning gas fuel. Most also considered a lease engine program important to facilitate improved availabilities and as a riskmitigation measure against unexpected long downtime periods.

Few considered there would be many applications for a unit to be able to switch between grid and island mode. The main applications for such operation were considered to be for power plant supplying process plants, which would probably be cogeneration applications and therefore not likely to be NGT plants. 


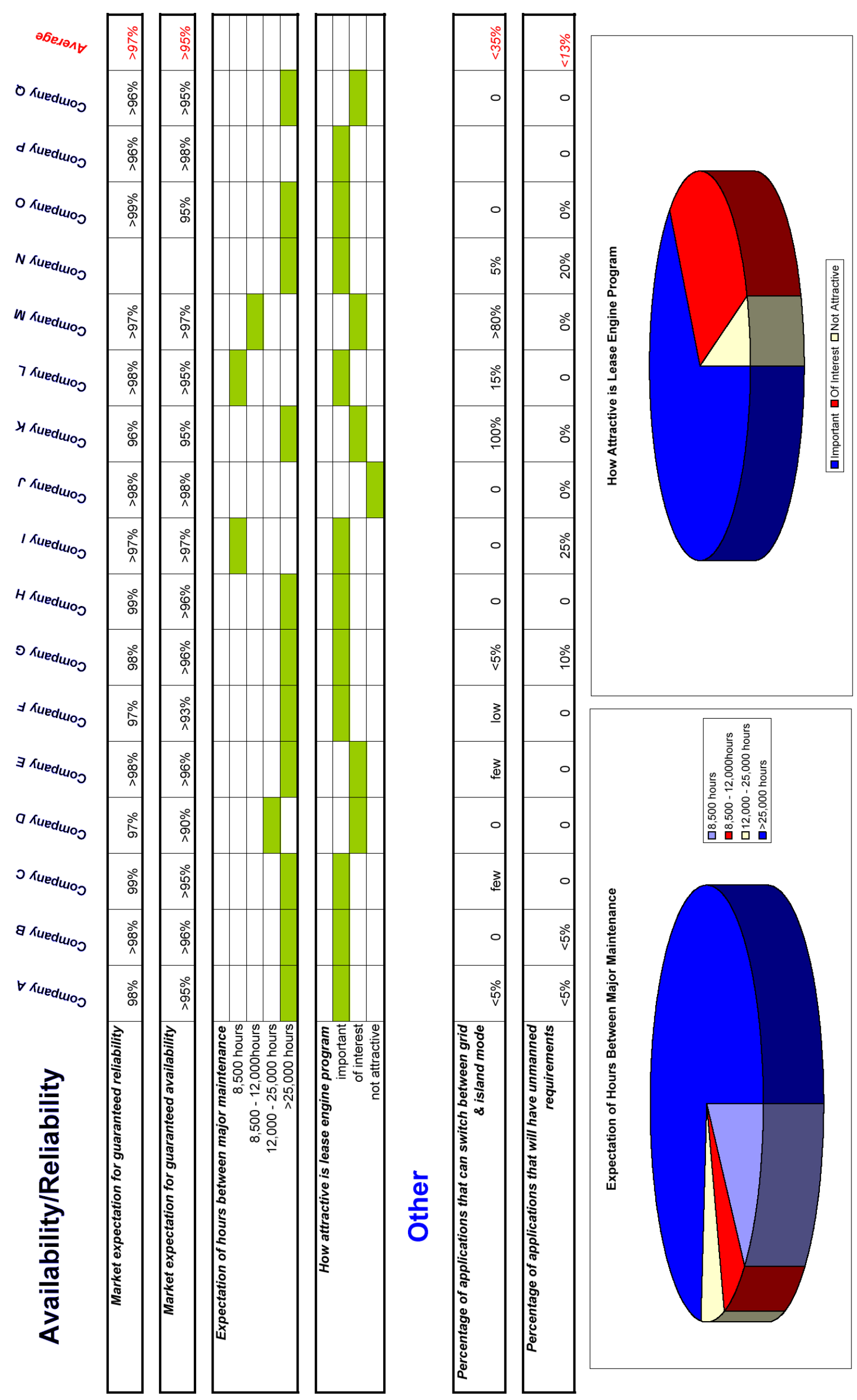




\subsection{NGT Technology Issues}

All respondents advised they were risk adverse and none considered themselves likely to be the first to install NGT. All required proven power plant, either for financing reasons or that they were generally wary of new technology following their experiences with problems initially encountered with the latest gas turbines offered by the major manufacturers.

The name of Rolls-Royce clearly carries substantial credence in the industry. All were aware of the company and had a high regard for the Rolls-Royce reputation, however this was mainly related to their aerospace products and none of our respondents considered Rolls-Royce a first tier manufacturer of gas turbines for power generation. Some were aware of the emission problems being experienced with the industrial Trent gas turbine and questioned R-R's commitment to the power generation side of their business since it appeared this emission issue was taking an inordinately long time to resolve. A number queried whether a British company has an adequate US based spares and service network to rapidly respond to their needs.

The key items considered likely to carry the major risk for NGT development were the water recovery system and recuperator. The SCR and once-through HRSG boiler are generally regarded as proven technology. Some respondents considered the super steam and water injection concept risky due to the massive volumes injected, whilst others saw no real issue.

Virtually all respondents felt Rolls-Royce were sufficiently capable of managing the engineering development of the NGT concepts. The Rolls-Royce reputation carries considerable influence. 

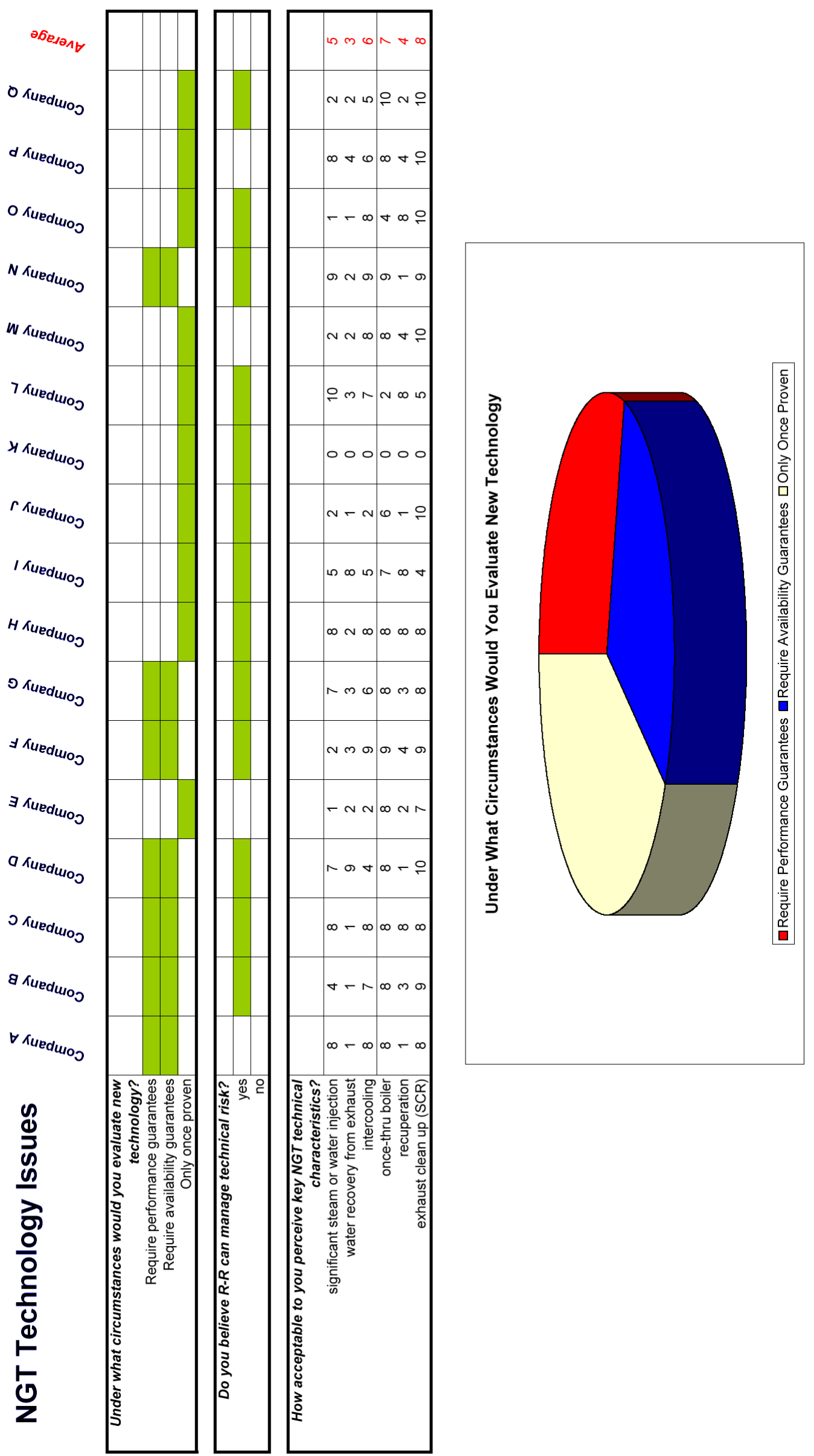


\section{Availability and Permitting of Water in US}

There is a critical need for power system technologies in the US that can utilize indigenous resources at ultra-high efficiencies without harmful emissions. This need for innovative system designs, which is fueled by the rapid growth in demand for clean, reliable and affordable power, faces increasing public interests towards sustainable development, and progressively more stringent regulatory requirements.

Environmental challenges faced by the NGT program include current and potential future regulatory requirements to reduce emissions of nitrogen oxides (NOx), sulfur dioxide (SO2), fine particulates, carbon dioxide (CO2), and other liquid, solid and gaseous pollutants, and the conservation of natural resources due to tightening land and water supplies. This section of the report reviews the environmental challenges associated with the management of water supplies and the potential impacts to the thermoelectric power industry.

\subsection{Water Use}

\section{National Water Use}

Newly released statistics on water use by the U.S. Geological Survey (USGS) show that the nation is using less water-- 402 billion gallons per day (bgd) for all offstream water-use categories (public supply, domestic, commercial, irrigation, livestock, industrial, mining, thermoelectric power), which is 2 percent less than in 1990 and nearly 10 percent less than in 1980 , despite a continuous increase in population over that same time period.

The USGS has compiled and reported national water-use statistics once every 5 years since 1950 from three principal sources: groundwater, freshwater and saline surface water [the most recent report being published in 1995]. The USGS data, reported by major watersheds called water resource regions, covers instream use at hydroelectric plants, withdrawals for delivery to offstream locations, and consumptive use, which is the portion of a withdrawal that evaporates, transpires or is incorporated into an end product, becoming unavailable for use by others. The portion of the withdrawal that is not consumptively used either returns to the stream (return flow) or contributes to groundwater storage.

After continual increases in the nation's total use of surface and ground water for the years reported from 1950 to 1980, water use declined and has remained fairly constant since the mid1980 s, according to the USGS report.

\section{Regional Water Use}

A comparison of total withdrawals by water resource region (WRR) indicates that the California, South Atlantic-Gulf, and Mid-Atlantic regions account for one-third of the total water withdrawn 
in the US. The largest amount of irrigation occurs in the California, Pacific Northwest, and Missouri regions; and the largest withdrawals (fresh and saline) for thermoelectric power occur in the Mid-Atlantic and South Atlantic-Gulf regions. A similar comparison by state indicates that California accounts for the largest withdrawals, 45,900 Mgal/d, followed by Texas, Illinois, and Florida.

\section{Thermoelectric Power Water Use.}

During 1995, the most water (190,000 Mgal/d, of which 57,900 Mgal/d was saline) was withdrawn for thermoelectric power cooling. Thermoelectric power is by far the largest water use in the Eastern US. The eight eastern water-resource regions, led by the Mid-Atlantic region, account for 75 percent of the total water withdrawn for thermoelectric power cooling. The highly populated states of Illinois, Texas, New York, Florida, and California use the most water for thermoelectric power. Illinois leads the Nation, nearly double Texas, in the use of freshwater for thermoelectric power.

The total quantity of water used for thermoelectric power generation during 1995 was approximately 3 percent less than during 1990. Records demonstrate that the withdrawal of waters for thermoelectric cooling decreased significantly between 1975 and 1990, and has declined steadily since as a result of improved efficiency. The improved efficiency has occurred partly by greater reuse of withdrawn water (power plants converted from once-through cooling to re-circulating cooling systems). In contrast to the recent decline of total withdrawals, production of electricity has continued to rise.

Withdrawals for thermoelectric power account for 39 percent of freshwater use for all offstream categories and represent 47 percent of combined fresh and saline withdrawals. Pubic suppliers only delivered about $100 \mathrm{Mgal} / \mathrm{d}$ of water to thermoelectric plants during 1995; this accounted for less than 1 percent of total public-supply withdrawals. Fossil fuel thermoelectric plants account for 71 percent of total thermoelectric withdrawals [nuclear power: 29 percent; geothermal: less than 1 percent].

Surface water was the source for more than 99 percent of total thermoelectric withdrawals, and about 31 percent of the surface-water withdrawal was saline. About 2 percent of the water withdrawn during 1995 was consumptively used as a result of once-through, cooling-tower, or pond-cooling. Less water is required when cooling is recycled through cooling towers or ponds, but a high percentage of the cooling water is evaporated (consumptive use), usually more than 60 percent. When the water is withdrawn for cooling is used only once before it is returned to the source, significantly more water is required, but evaporation is low (less than 3 percent).

Trends. Looking at the percentage of change in water use by category for 1990 and 1995 shows that public supply increased by 4 percent, rural domestic and livestock use increased 13 percent, thermoelectric power use was down 3 percent, other industrial use was down 3 percent, irrigation was down 2 percent, total withdrawals were down 2 percent, hydroelectric power use was down 4 percent, and the use of reclaimed wastewater was up by 36 percent. 
Future water use in the US is estimated by extending past trends in basic water-use determinants such as future population and rate of change in energy use per person and in water use per kilowatt-hour produced. Those trends are largely encouraging. Over the past 35 years, withdrawals in industry and at thermoelectric plants have steadily dropped per unit of output. If these trends continue, which includes decreased agricultural and domestic withdrawals, aggregate withdrawals in the US over the next 20 years may remain below 10 percent of the 1995 level, despite a 41 percent expected increase in population. However, not all areas of the US are projected to fare as well. Of the $20 \mathrm{WRRs}$, withdrawals in seven are projected to increase by from 15 to 30 percent above 1995 levels. Most of the substantial increases are attributable to domestic and public or thermoelectric use. The largest percentage increases occur in regions of greatest population increase [Pacific Northwest, California, Lower Colorado, Texas-Gulf, Mid-Atlantic, South Atlantic-Gulf and Ohio] and where production at freshwater plants is currently less important [Pacific Northwest].

\subsection{Permitting \& Conservation Programs/Requirements}

Often taken for granted when supplies are plentiful, water has become the focus of increasing controversy as growing populations and changing values continue to place increasing demands on supplies and river systems, resulting in water use and management conflicts throughout the country. Yet, actual physical scarcity of water is not the key issue in most regions. Rather, there is enough water to meet society's needs, but there are few regulatory requirements [e.g. facility siting, supply, efficiency requirements] and market incentives for conserving water or for encouraging efficient allocation among competing users.

Debate over water resources revolves around the issue of how best to plan for and manage the use of this renewable, yet sometimes scarce and increasingly sought after, resource.

Approaches to management of water resources in the US include structural and non-structural methods. Structural water resource management includes enhancing water supplies, by building, for example, new storage or diversion projects, expanding old ones, or funding water reclamation and reuse facilities. Non-structural water resource management emphasizes the need to manage existing supplies more efficiently -- through conservation, revision of policies that encourage inefficient use of water, and establishment of market mechanisms to allocate water according to users' willingness to pay. The non-structural water resource management approach, particularly programs that employ conservation and policies effecting facility design/operation, presents a challenge to the permitting of new power generation facilities.

The stringency of non-structural programs is fittingly influenced by population trends [increasing v. decreasing] and water availability [arid v humid regions; drought susceptibility; quality/quantity; competing demands; etc]. The stringency of these programs is also an indication of the attitudes [and regulatory environment and public acceptance] towards water usage for power generation. As implied by current and projected water usage, the most stringent programs and requirements [the thermoelectric industry may encounter whilst permitting new facilities] are prescribed by states located within the water resource regions with 
the greatest populations and largest thermoelectric water withdrawals: Pacific Northwest, California, Lower Colorado, Texas-Gulf, Mid-Atlantic, South Atlantic-Gulf and Ohio. Water resource programs prescribed within these regions includes but is not limited to power plant siting programs, conservation planning programs, water resource planning, water availability programs, and efficiency-based conservation programs. The programs considered presenting the most demanding permitting and design requirements are the power plant siting and efficiency-based conservation programs:

\section{Power Plant Siting Programs.}

Water use criteria of power plant siting programs includes requirements for evaluating preferred siting conditions. Reviewing an assessment that the applicant must complete regarding the plant's planned use of, and potential effect on, a water resource generally comprises the evaluation. An assessment typically includes: sources of water supply; total amount to be used by facility; ability of the water resource to meet the needs of the merchant power plant and other users; alternative sources of water supply [e.g. reclaimed, irrigation return flows, naturally brackish water, once-through sea-water]; and conservations measures proposed by the power plant for reducing the plant's effect on water resources. Applications may be denied if a proposed site does not have reasonable access to a proven water supply sufficient for plant operation and/or where the use of a water resource results in material adverse effects on a water resource. Known, active water resource regions with established power plant siting programs includes the Mid-Atlantic [State of Maryland]; Ohio [State of Indiana]; Upper Mississippi [State of Minnesota]; South Atlantic-Gulf [State of Florida]; Pacific Northwest [State of Washington]; and California.

\section{Efficiency-based Conservation Programs.}

All states carry out some form of water-related conservation planning that vary in a number of ways and is typically oriented towards water quality planning. Water quality planning is not linked or integrated with quantity planning, the latter being the subject of this review. Whereas the primary intent of the water conservation planning programs is to assist the State better manage its water resources, the intent of efficiency-based conservation programs is to move users to the greatest level of water use efficiency economically attainable given the use of the latest available water conservation technology. Efficiency programs range from the development and implementation of conservation plans that specify facility practices, methods or techniques to achieve stated goals of water conservation including monitoring and reporting requirements to programs that prescribe high-level efficiency requirements such as increasing number of cooling tower cycles or cycles of concentration. The conservation-efficiency programs are typical of arid and drought-susceptible water resource regions, such as States of Texas and Arizona.

Albeit water resource management is considered most stringent in areas of growing populations and total water withdrawals, water use by power generation facilities is invariably subjected to regulation in most, if not all, states. The regulation in the less regulated states however, most 
commonly involves the registration of a new project [generalized, not industry specific] that requires a water volume over a prescribed significant standard [e.g. States of Indiana and North Carolina requires the registration of facilities withdrawing 100,000 gpd].

\subsection{Assessment}

The projected water usage of the Rolls Royce NGT concept is estimated to be upwards to 45 pounds per second or 466,560 gallons per day, not including cooling tower make up water requirements. Comparatively, and whilst considering the requirement for makeup and cooling tower water, which may increase the facility needs upwards a range of $10-25 \%$ depending upon the quality of the water supply, the water requirement of the Rolls Royce NGT type is likely to be considered significant by permitting agencies and community observers. However, the prospective of public endorsement and ensuing regulatory permitting is invariably dependent upon the time of which the generation facility enters the market. Specifically, entry into the market of a Rolls Royce NGT type gas turbine at present time and possibly projecting into calendar year 2010, the regulatory and public opinion would be expected to be equivalent to the water use and availability setting presented herein; whereas the most demanding permitting and design requirements (e.g. siting and efficiency-based conservation) are located within regions of high populations, greatest water withdrawals and regions characteristic of aridity or drought susceptibility, and the least demanding requirements (e.g. facility registration) are located within regions of the opposite (e.g. low populations and ample water supplies). Projecting the regulatory trend post calendar year 2010 , it is reasonable to speculate that the most demanding programs of siting and efficiency-based programs will be a regional standard. Consequently, there is justifiable cause for concern over the adequacy of water supplies and development of new generation facilities employing the Rolls Royce NGT concept. Water resource management approaches throughout the U.S. are changing noticeably as more and more water suppliers and planning agencies are beginning to explore efficiency improvements through regulatory requirements [affecting design, permitting and operations], implementing options for managing demand, and reallocating water among users to reduce projected gaps and to meet future needs. 


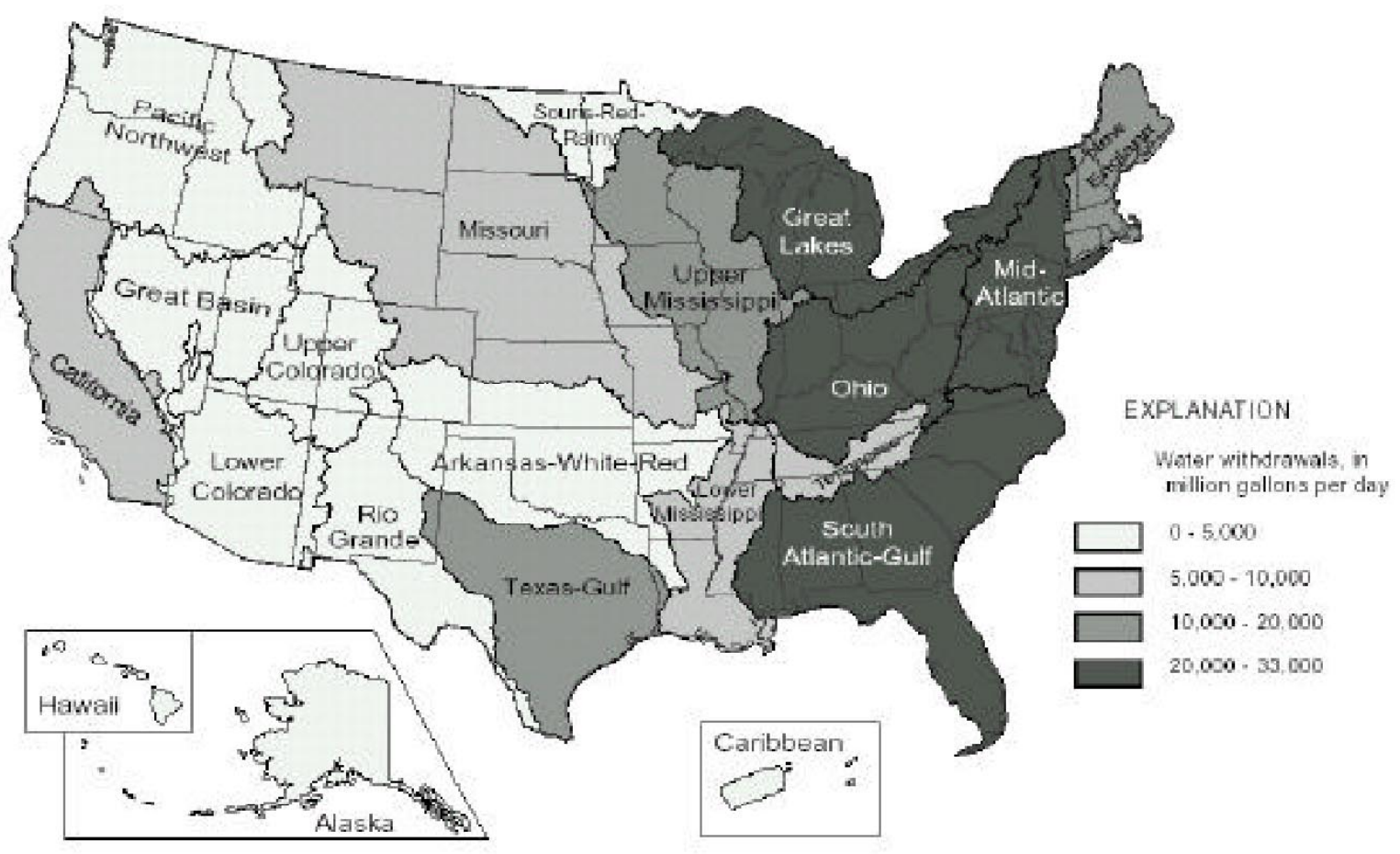

Thermoelectric Fresh and Saline Water Withdrawals by Water Resource Region, 1995

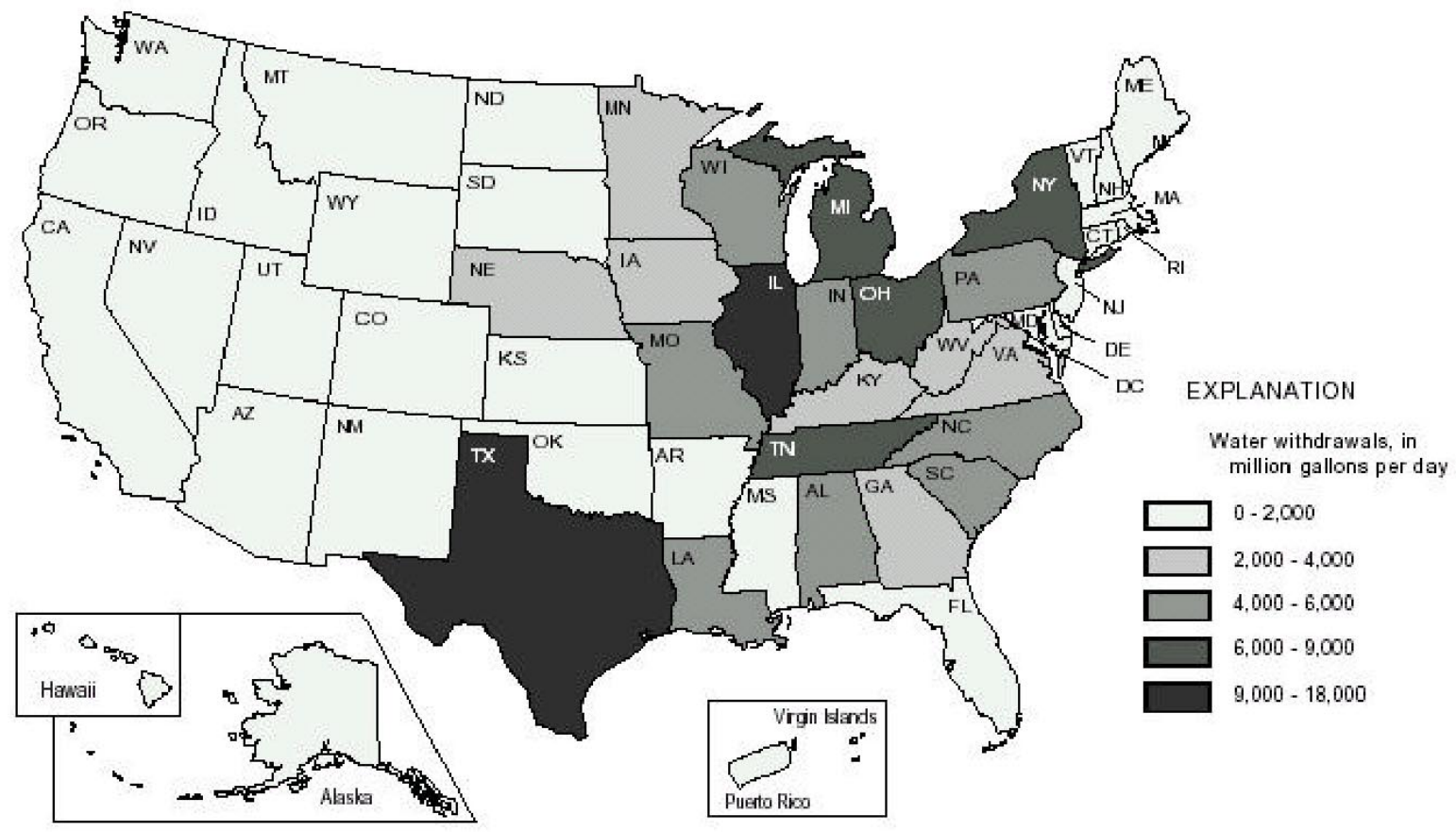

Thermoelectric Fresh Water Withdrawals by State, 1995 


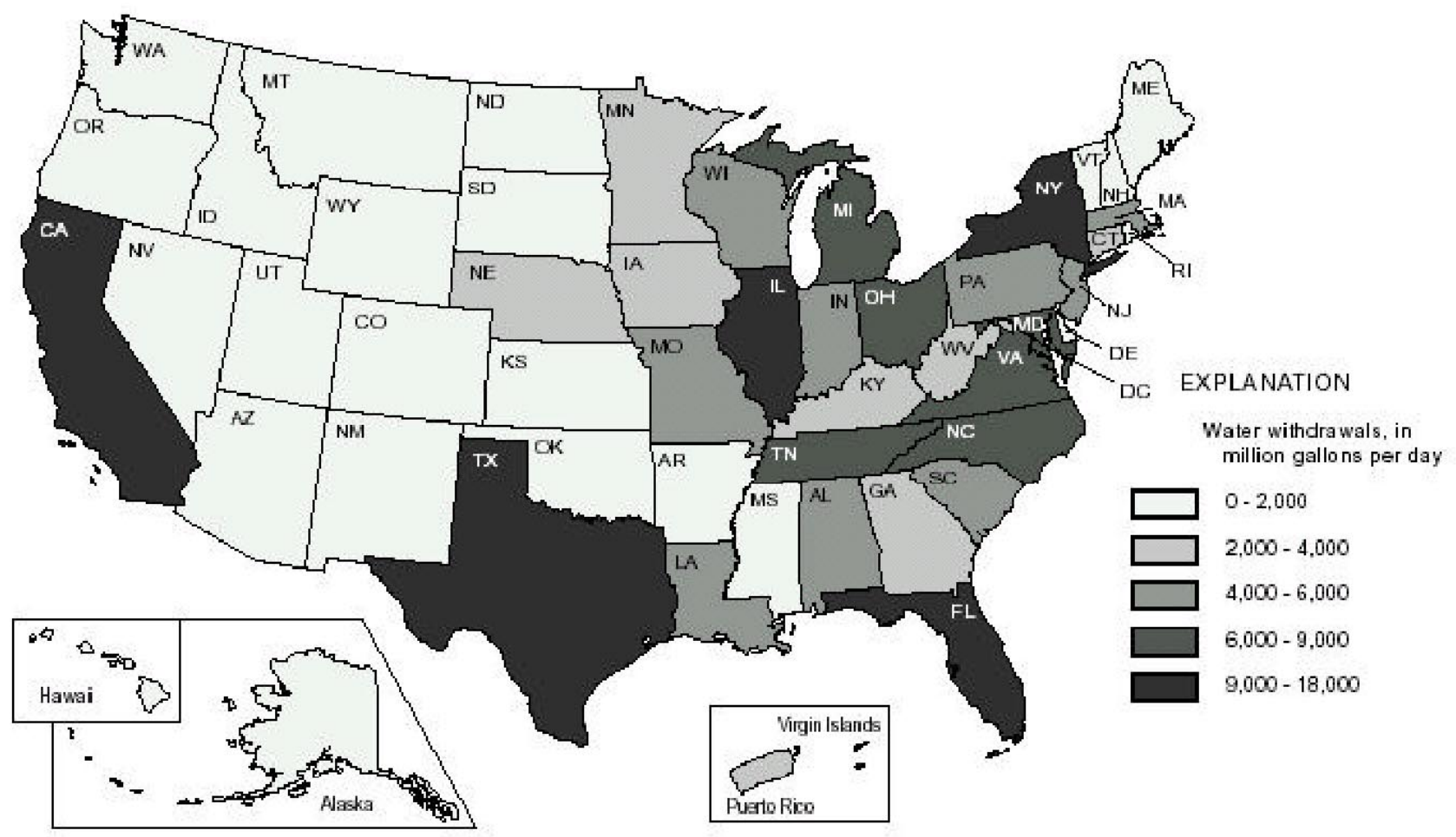

Thermoelectric Fresh and Saline Water Withdrawals by State, 1995 
Thermoelectric Fresh and Saline Water Use by State, 1995

[Figures may not add to totals because of independent rounding. Mgal/d = million gallons per day; $\mathrm{kWh}=\mathrm{kilowatthour]}$

\begin{tabular}{|c|c|c|c|c|c|c|c|c|c|c|}
\hline \multirow{4}{*}{ STATE } & \multicolumn{9}{|c|}{ ALL THERMOELECTRIC POWER WATER USE, in Mgal/d } & \multirow{4}{*}{$\begin{array}{l}\text { POWER } \\
\text { GENERATED, } \\
\text { in million } \mathrm{kWh}\end{array}$} \\
\hline & \multirow{2}{*}{\multicolumn{4}{|c|}{$\begin{array}{c}\text { Self-supplied withdrawals, by source and type } \\
\begin{array}{c}\text { Ground } \\
\text { water }\end{array}\end{array}$}} & \multirow{3}{*}{$\begin{array}{c}\text { Public- } \\
\text { supply } \\
\text { deliveries } \\
\text { Fresh }\end{array}$} & \multicolumn{4}{|c|}{ Total use } & \\
\hline & & & & & & \multirow{2}{*}{$\begin{array}{l}\text { Withdrawals } \\
\text { and } \\
\text { deliveries } \\
\text { Fresh }\end{array}$} & \multicolumn{3}{|c|}{ Consumptive use } & \\
\hline & Fresh & Fresh & Saline & Total & & & Fresh & Saline & Total & \\
\hline $\begin{array}{l}\text { Alabama . . . . . . A } \\
\text { Alaska . . . . . . . } \\
\text { Arkansa. . . . . } \\
\text { California }\end{array}$ & $\begin{array}{r}6.0 \\
4.2 \\
42 \\
5.2 \\
3.6\end{array}$ & $\begin{array}{r}5,190 \\
26 \\
20 \\
1,770 \\
202\end{array}$ & $\begin{array}{r}0 \\
0 \\
0 \\
0 \\
9,430\end{array}$ & $\begin{array}{r}5,190 \\
26 \\
20 \\
1,770 \\
9,630\end{array}$ & $\begin{array}{l}0 \\
0 \\
0 \\
5.3\end{array}$ & $\begin{array}{r}5,200 \\
31 \\
62 \\
1,770 \\
211\end{array}$ & $\begin{array}{l}32 \\
3.1 \\
54 \\
28 \\
9.7\end{array}$ & $\begin{array}{r}0 \\
0 \\
0 \\
0 \\
19\end{array}$ & $\begin{array}{l}32 \\
3.1 \\
54 \\
28 \\
29\end{array}$ & $\begin{array}{r}85,300 \\
3,770 \\
65,300 \\
37,400 \\
76,000\end{array}$ \\
\hline $\begin{array}{l}\text { Colorado....... } \\
\text { Connecticut .... } \\
\text { Delaware . . . . } \\
\text { D.C. ...... } \\
\text { Florida . . . . . . }\end{array}$ & $\begin{array}{r}22 \\
.2 \\
.2 \\
0 \\
21\end{array}$ & $\begin{array}{c}93 \\
760 \\
534 \\
9.7 \\
615\end{array}$ & $\begin{array}{r}0 \\
3,180 \\
740 \\
0 \\
11,000\end{array}$ & $\begin{array}{c}93 \\
3,940 \\
1,270 \\
9.7 \\
11,600\end{array}$ & $\begin{array}{l}14 \\
1.0 \\
.5 \\
0 \\
3.6\end{array}$ & $\begin{array}{c}128 \\
761 \\
535 \\
9.7 \\
640\end{array}$ & $\begin{array}{r}41 \\
5.9 \\
.2 \\
.8 \\
56\end{array}$ & $\begin{array}{l}0 \\
74 \\
2.9 \\
0 \\
0\end{array}$ & $\begin{array}{l}41 \\
80 \\
3.1 \\
8 \\
56\end{array}$ & $\begin{array}{r}30,600 \\
27,500 \\
6,060 \\
189 \\
149,000\end{array}$ \\
\hline $\begin{array}{l}\text { Georgia . . . . . . } \\
\text { Hawaii . . . . . . } \\
\text { Idaho . . . . . . } \\
\text { Illinois. . . . . . . } \\
\text { Indiana }\end{array}$ & $\begin{array}{l}4.8 \\
67 \\
0 \\
11 \\
11\end{array}$ & $\begin{array}{r}3,040 \\
0 \\
0 \\
17,100 \\
5,680\end{array}$ & $\begin{array}{r}33 \\
903 \\
0 \\
0 \\
0\end{array}$ & $\begin{array}{r}3,070 \\
903 \\
0 \\
17,100 \\
5,680\end{array}$ & $\begin{array}{l}0 \\
0 \\
0.3 \\
5.2 \\
0\end{array}$ & $\begin{array}{r}3,040 \\
67 \\
0 \\
17,100 \\
5,690\end{array}$ & $\begin{array}{c}145 \\
0 \\
407 \\
114\end{array}$ & $\begin{array}{l}0 \\
9.0 \\
0 \\
0 \\
0\end{array}$ & $\begin{array}{c}145 \\
9.7 \\
0 \\
407 \\
114\end{array}$ & $\begin{array}{r}92,700 \\
6,370 \\
0 \\
147,000 \\
105,000\end{array}$ \\
\hline $\begin{array}{l}\text { lowa .......... } \\
\text { Kansas . . . . . } \\
\text { Kentucky } \\
\text { Louisiana . . . . . } \\
\text { Maine ... . . . }\end{array}$ & $\begin{array}{r}15 \\
14 \\
38 \\
31 \\
.7\end{array}$ & $\begin{array}{r}2,110 \\
1,250 \\
3,410 \\
5,450 \\
30\end{array}$ & $\begin{array}{r}0 \\
0 \\
0 \\
0 \\
105\end{array}$ & $\begin{array}{r}2,110 \\
1,250 \\
3,410 \\
5,450 \\
135\end{array}$ & $\begin{array}{l}3.0 \\
.8 \\
0 \\
0 \\
.9\end{array}$ & $\begin{array}{r}2,130 \\
1,270 \\
3,440 \\
5,480 \\
31\end{array}$ & $\begin{array}{r}10 \\
58 \\
203 \\
222 \\
3.5\end{array}$ & $\begin{array}{l}0 \\
0 \\
0 \\
0 \\
1.7\end{array}$ & $\begin{array}{c}10 \\
58 \\
203 \\
222 \\
5.2\end{array}$ & $\begin{array}{r}32,600 \\
38,100 \\
70,600 \\
54,200 \\
4,600\end{array}$ \\
\hline $\begin{array}{l}\text { Maryland ....... } \\
\text { Massachusetts .. } \\
\text { Michigan ..... . } \\
\text { Minnesota . . . . } \\
\text { Mississippi . . . }\end{array}$ & $\begin{array}{l}1.8 \\
46 \\
3.0 \\
1.9 \\
42\end{array}$ & $\begin{array}{r}358 \\
150 \\
8,370 \\
2,090 \\
220\end{array}$ & $\begin{array}{r}6,000 \\
4,370 \\
0 \\
0 \\
112\end{array}$ & $\begin{array}{r}6,360 \\
4,520 \\
8,370 \\
2,090 \\
333\end{array}$ & $\begin{array}{l}0 \\
0 \\
0 \\
1 \\
2.2\end{array}$ & $\begin{array}{r}360 \\
196 \\
8,370 \\
2,090 \\
265\end{array}$ & $\begin{array}{r}3.7 \\
0 \\
126 \\
48 \\
27\end{array}$ & $\begin{array}{l}48 \\
6.0 \\
0 \\
0 \\
3.6\end{array}$ & $\begin{array}{c}52 \\
6.0 \\
126 \\
48 \\
31\end{array}$ & $\begin{array}{l}43,200 \\
34,000 \\
96,700 \\
41,300 \\
26,100\end{array}$ \\
\hline $\begin{array}{l}\text { Missouri ..... . . } \\
\text { Montana . . . . . . } \\
\text { Nebraska . . . } \\
\text { Nevada. . . . . } \\
\text { New Hampshire. }\end{array}$ & $\begin{array}{l}9.5 \\
0 \\
4.4 \\
6.3 \\
.8\end{array}$ & $\begin{array}{r}5,540 \\
22 \\
2,350 \\
21 \\
228\end{array}$ & $\begin{array}{r}0 \\
0 \\
0 \\
0 \\
877\end{array}$ & $\begin{array}{r}5,540 \\
22 \\
2,350 \\
21 \\
1,110\end{array}$ & $\begin{array}{l}0^{.2} \\
0 \\
1.5 \\
.3\end{array}$ & $\begin{array}{r}5,550 \\
22 \\
2,350 \\
28 \\
229\end{array}$ & $\begin{array}{r}51 \\
22 \\
12 \\
28 \\
4.3\end{array}$ & $\begin{array}{l}0 \\
0 \\
0 \\
8.3 \\
0\end{array}$ & $\begin{array}{l}51 \\
22 \\
12 \\
37 \\
4.3\end{array}$ & $\begin{array}{r}63,600 \\
8,770 \\
23,800 \\
18,900 \\
14,000\end{array}$ \\
\hline $\begin{array}{l}\text { New Jersey...... } \\
\text { New Mexico .... } \\
\text { New York .... } \\
\text { North Carolina ... } \\
\text { North Dakota ... }\end{array}$ & $\begin{array}{l}1.9 \\
9.3 \\
0 \\
.1 \\
.3\end{array}$ & $\begin{array}{r}578 \\
46 \\
6,570 \\
5,860 \\
879\end{array}$ & $\begin{array}{r}3,780 \\
0 \\
6,490 \\
1,550 \\
0\end{array}$ & $\begin{array}{r}4,360 \\
46 \\
13,100 \\
7,420 \\
879\end{array}$ & $0_{0}^{.1}$ & $\begin{array}{r}605 \\
56 \\
6,570 \\
5,860 \\
880\end{array}$ & $\begin{array}{c}4.4 \\
48 \\
170 \\
57 \\
25\end{array}$ & $\begin{array}{r}32 \\
0 \\
130 \\
17 \\
0\end{array}$ & $\begin{array}{r}36 \\
48 \\
300 \\
74 \\
25\end{array}$ & $\begin{array}{l}23,600 \\
29,100 \\
76,100 \\
93,400 \\
26,300\end{array}$ \\
\hline $\begin{array}{l}\text { Ohio ........... } \\
\text { Oklahoma ...... } \\
\text { Oregon ....... } \\
\text { Pennsylvania ... } \\
\text { Rhode Island ... }\end{array}$ & $\begin{array}{l}19 \\
3.5 \\
0 \\
6.2 \\
0\end{array}$ & $\begin{array}{c}8,170 \\
121 \\
9.0 \\
5,920 \\
0\end{array}$ & $\begin{array}{r}0 \\
0 \\
0 \\
0 \\
275\end{array}$ & $\begin{array}{c}8,170 \\
121 \\
9.0 \\
5,920 \\
275\end{array}$ & $\begin{array}{l}0 \\
1.2 \\
0 \\
1.6 \\
0\end{array}$ & $\begin{array}{c}8,190 \\
126 \\
9.0 \\
5,930 \\
0\end{array}$ & $\begin{array}{c}336 \\
60 \\
7.8 \\
239 \\
0\end{array}$ & $\begin{array}{l}0 \\
0 \\
0 \\
0 \\
5.5\end{array}$ & $\begin{array}{c}336 \\
60 \\
7.8 \\
239 \\
5.5\end{array}$ & $\begin{array}{r}135,000 \\
44,700 \\
3,620 \\
168,000 \\
278\end{array}$ \\
\hline $\begin{array}{l}\text { South Carolina ... } \\
\text { South Dakota ... } \\
\text { Tennessee .... } \\
\text { Texas . . . . . . . } \\
\text { Utah . . . . . . }\end{array}$ & $\begin{array}{l}39 \\
3.4 \\
0 \\
59 \\
0\end{array}$ & $\begin{array}{c}4,770 \\
1.9 \\
8,300 \\
9,530 \\
48\end{array}$ & $\begin{array}{r}0 \\
0 \\
0 \\
3,870 \\
0\end{array}$ & $\begin{array}{c}4,770 \\
1.9 \\
8,300 \\
13,400 \\
48\end{array}$ & $\begin{array}{c}0 \\
0 \\
29 \\
0 \\
0\end{array}$ & $\begin{array}{c}4,810 \\
5.4 \\
8,300 \\
9,620 \\
48\end{array}$ & $\begin{array}{r}51 \\
.1 \\
297 \\
47\end{array}$ & $\begin{array}{r}0 \\
0 \\
0 \\
12 \\
.3\end{array}$ & $\begin{array}{l}51 \\
.1 \\
309 \\
47\end{array}$ & $\begin{array}{r}74,200 \\
2,800 \\
73,800 \\
259,000 \\
31,600\end{array}$ \\
\hline $\begin{array}{l}\text { Vermont ........ } \\
\text { Virginia ....... } \\
\text { Washington .... } \\
\text { West virginia ... } \\
\text { Wisconsin . . . . }\end{array}$ & $\begin{array}{r}.4 \\
.4 \\
.5 \\
.5 \\
5.8\end{array}$ & $\begin{array}{r}452 \\
3,890 \\
375 \\
3,010 \\
5,820\end{array}$ & $\begin{array}{r}0 \\
2,730 \\
0 \\
0 \\
0\end{array}$ & $\begin{array}{r}452 \\
6,620 \\
375 \\
3,010 \\
5,820\end{array}$ & $\begin{array}{l}0 \\
0 \\
0 \\
.2 \\
.1\end{array}$ & $\begin{array}{r}453 \\
3,890 \\
376 \\
3,010 \\
5,830\end{array}$ & $\begin{array}{r}4.0 \\
8.8 \\
10 \\
122 \\
58\end{array}$ & $\begin{array}{l}0 \\
0 \\
0 \\
0 \\
0\end{array}$ & $\begin{array}{r}4.0 \\
8.8 \\
10 \\
122 \\
58\end{array}$ & $\begin{array}{r}4,400 \\
50,900 \\
13,300 \\
79,100 \\
44,700\end{array}$ \\
\hline $\begin{array}{l}\text { Wyoming ....... } \\
\text { Puerto Rico. . . } \\
\text { Virgin Islands ... }\end{array}$ & $\begin{array}{l}1.0 \\
2.2 \\
0\end{array}$ & $\begin{array}{r}219 \\
0 \\
0\end{array}$ & $\begin{array}{r}0 \\
2.260 \\
173\end{array}$ & $\begin{array}{r}219 \\
2,260 \\
173\end{array}$ & $\begin{array}{l}0 \\
2.2 \\
8\end{array}$ & $\begin{array}{r}220 \\
4.4 \\
8\end{array}$ & $\begin{array}{r}50 \\
.7 \\
2\end{array}$ & $\begin{array}{l}0 \\
0 \\
0\end{array}$ & $\begin{array}{r}50 \\
.7 \\
2\end{array}$ & $\begin{array}{r}38,600 \\
15,800 \\
771\end{array}$ \\
\hline Total ........... & 565 & 131,000 & 57,900 & 189,000 & 100 & 132,000 & 3,310 & 369 & 3,680 & $2,690,000$ \\
\hline
\end{tabular}


Thermoelectric Water Use by Energy Source and Water Resource Region, 1995

\begin{tabular}{|c|c|c|c|c|c|c|c|c|c|c|c|c|}
\hline \multirow{4}{*}{ REEGION } & \multicolumn{6}{|c|}{ FOSSIL FUEL } & \multicolumn{6}{|c|}{ NUCLEAR } \\
\hline & \multicolumn{4}{|c|}{$\begin{array}{l}\text { Whithdrenegls, } \\
\text { by soures and type }\end{array}$} & \multirow{2}{*}{\multicolumn{2}{|c|}{$\begin{array}{l}\text { Consumptive } \\
\text { use }\end{array}$}} & \multicolumn{4}{|c|}{$\begin{array}{l}\text { Withdrawals. } \\
\text { by souroe and five }\end{array}$} & \multirow{2}{*}{\multicolumn{2}{|c|}{$\begin{array}{l}\text { Consumptive } \\
\text { use }\end{array}$}} \\
\hline & \multirow{2}{*}{$\begin{array}{l}\text { Ground } \\
\text { waler } \\
\text { Fresh }\end{array}$} & \multicolumn{3}{|c|}{ surface water } & & & \multirow{2}{*}{$\begin{array}{l}\text { Ground } \\
\text { Weter } \\
\text { Fresh }\end{array}$} & \multicolumn{3}{|c|}{ Surface water } & & \\
\hline & & Fresh & Saline & Total & Fresin & Seline & & Fish & Sallne & Tolal & Fresh & Saline \\
\hline $\begin{array}{l}\text { New England } \\
\text { Mld-Atlanitic } \\
\text { South Atlantic-gialf } \\
\text { Great Lakes ......... } \\
\text { Ohio }\end{array}$ & $\begin{array}{l}46 \\
9.6 \\
39 \\
70 \\
70\end{array}$ & $\begin{array}{r}684 \\
8,490 \\
11,200 \\
15,300 \\
22,500\end{array}$ & $\begin{array}{r}5.450 \\
10.900 \\
9.290 \\
0 \\
0\end{array}$ & $\begin{array}{r}5,+500 \\
19,400 \\
20,500 \\
15,300 \\
22,500\end{array}$ & $\begin{array}{r}14 \\
915 \\
220 \\
180 \\
810\end{array}$ & $\begin{array}{c}\frac{82}{150} \\
3.6 \\
0 \\
0\end{array}$ & $\begin{array}{r}0.1 \\
1.2 \\
40 \\
0^{2}\end{array}$ & $\begin{array}{r}9.36 \\
4,140 \\
6,340 \\
7,520 \\
65\end{array}$ & $\begin{array}{r}3,340 \\
8,790 \\
3,360 \\
0 \\
0\end{array}$ & $\begin{array}{r}4.270 \\
12,900 \\
9.700 \\
7,520 \\
65\end{array}$ & $\begin{array}{l}3.2 \\
93 \\
124 \\
249 \\
29\end{array}$ & $\begin{array}{c}5.0 \\
68 \\
17 \\
0 \\
0\end{array}$ \\
\hline 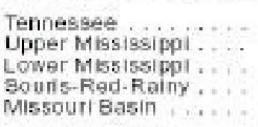 & $\begin{array}{r}0 \\
20 \\
3 \pi \\
9 \\
28\end{array}$ & $\begin{array}{r}4,750 \\
12,300 \\
5,600 \\
7,700\end{array}$ & $\begin{array}{l}0 \\
0 \\
9 \\
9 \\
0\end{array}$ & $\begin{array}{r}4,750 \\
72,300 \\
5,600 \\
30 \\
T, 700\end{array}$ & $\begin{array}{r}11 \\
163 \\
223 \\
0 \\
161\end{array}$ & $\begin{array}{l}0 \\
0 \\
0 \\
0 \\
0\end{array}$ & $\begin{array}{c}9 \\
3.4 \\
32 \\
0 \\
.4\end{array}$ & $\begin{array}{r}2,240 \\
B, 690 \\
1,020 \\
1,080\end{array}$ & $\begin{array}{l}0 \\
0 \\
0 \\
0 \\
0\end{array}$ & $\begin{array}{r}2,240 \\
6,690 \\
1,020 \\
1,000\end{array}$ & $\begin{array}{c}1.5 \\
225 \\
30 \\
0 \\
11\end{array}$ & $\begin{array}{l}0 \\
0 \\
0 \\
0 \\
0\end{array}$ \\
\hline $\begin{array}{l}\text { Arkansas-brhite-Fed } \\
\text { Texas-Guif } \\
\text { Fio Grande } \\
\text { Upper colorado . . . . . } \\
\text { Lower colorado }\end{array}$ & $\begin{array}{r}37 \\
49 \\
16 \\
0 \\
45\end{array}$ & $\begin{array}{c}3,150 \\
4820 \\
22 \\
146 \\
17\end{array}$ & $\begin{array}{r}3,870 \\
0 \\
0 \\
0\end{array}$ & $\begin{array}{c}3,150 \\
8,680 \\
2.2 \\
146 \\
17\end{array}$ & $\begin{array}{r}149 \\
228 \\
14 \\
130 \\
57\end{array}$ & $\begin{array}{r}0 \\
12 \\
0 \\
0 \\
0\end{array}$ & $\stackrel{0}{0}_{0}^{0}$ & $\begin{array}{r}969 \\
2,820 \\
0 \\
0 \\
0\end{array}$ & $\begin{array}{l}0 \\
0 \\
0 \\
0 \\
0\end{array}$ & $\begin{array}{r}969 \\
2,820 \\
0 \\
0 \\
0\end{array}$ & $\begin{array}{r}14 \\
2 \mathrm{E} \\
0 \\
0 \\
0\end{array}$ & $\begin{array}{l}0 \\
0 \\
0 \\
0 \\
0\end{array}$ \\
\hline 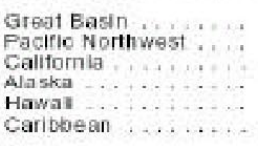 & $\begin{array}{r}2.5 \\
4 \\
3.5 \\
4.2 \\
67 \\
2.2\end{array}$ & $\begin{array}{r}21 \\
25 \\
190 \\
26 \\
0 \\
0\end{array}$ & $\begin{array}{r}9 \\
9 \\
4.730 \\
0 \\
903 \\
2,440\end{array}$ & $\begin{array}{r}21 \\
26 \\
4.920 \\
26 \\
903 \\
2.440\end{array}$ & $\begin{array}{r}23 \\
3.2 \\
9.4 \\
3.1 \\
.7 \\
.9\end{array}$ & $\begin{array}{l}0 \\
0 \\
2.8 \\
0 \\
9.0 \\
0\end{array}$ & $\begin{array}{l}0 \\
1 \\
1 \\
0 \\
0 \\
0 \\
0\end{array}$ & $\begin{array}{r}0 \\
368 \\
12 \\
0 \\
0 \\
0\end{array}$ & $\begin{array}{r}0 \\
4,650 \\
0 \\
0 \\
0\end{array}$ & $\begin{array}{r}30 \\
+710 \\
0 \\
0 \\
0\end{array}$ & $\begin{array}{l}0 \\
9.8 \\
3 \\
0 \\
0 \\
0\end{array}$ & $\begin{array}{l}0 \\
9 \\
1.3 \\
0 \\
0 \\
0\end{array}$ \\
\hline Total ............ & 496 & 97,000 & 37,600 & 135,000 & 2,500 & 263 & 78 & 34,300 & 20,200 & 54.500 & 815 & 62 \\
\hline
\end{tabular}

Thermoelectric Fresh and Saline Water Withdrawals by Water Resource Region, 1995

[Figures may not add to fotals because of independent rounding. Mgalid = million gallons per day: KWh = kilowatthour]

\begin{tabular}{|c|c|c|c|c|c|c|c|c|c|c|}
\hline \multirow{4}{*}{ REGION } & \multicolumn{9}{|c|}{ ALL THERMOELECTRIC POWER WATER USE, in Mgal d } & \multirow{4}{*}{$\begin{array}{l}\text { PQWER } \\
\text { GENERATED, } \\
\text { in million kWưh }\end{array}$} \\
\hline & \multicolumn{4}{|c|}{ Self-supplied withdrawals, by scurce and typer } & \multirow{3}{*}{$\begin{array}{l}\text { Pulvic- } \\
\text { supply } \\
\text { deliveries } \\
\text { Fresh }\end{array}$} & \multicolumn{4}{|c|}{ Tolal use } & \\
\hline & \multirow{2}{*}{$\begin{array}{l}\text { Ground } \\
\text { water } \\
\text { Fresh }\end{array}$} & \multicolumn{3}{|c|}{ Surface water } & & \multirow{2}{*}{$\begin{array}{c}\text { Withdrawals } \\
\text { and } \\
\text { dellueries } \\
\text { Fresh }\end{array}$} & \multicolumn{3}{|c|}{ Consumptyouse } & \\
\hline & & Fiesh & Saline & Tolal & & & Fresh & Saline & Total & \\
\hline $\begin{array}{l}\text { Now England ..... } \\
\text { Mid-Atlantic ...... } \\
\text { South Atlantic-Gulf } \\
\text { Great Lakes....... } \\
\text { Ohio............ }\end{array}$ & $\begin{array}{l}48 \\
11 \\
79 \\
7.6 \\
70\end{array}$ & $\begin{array}{r}1,620 \\
12,600 \\
17,500 \\
22,800 \\
22,600\end{array}$ & $\begin{array}{r}8,800 \\
19,700 \\
12,700 \\
0 \\
0\end{array}$ & $\begin{array}{l}10,400 \\
32,400 \\
30,200 \\
22,800 \\
22,600\end{array}$ & $\begin{array}{r}2.3 \\
27 \\
5.6 \\
.1 \\
.3\end{array}$ & $\begin{array}{r}1,670 \\
12,700 \\
17.600 \\
22,800 \\
22,600\end{array}$ & $\begin{array}{r}17 \\
189 \\
344 \\
429 \\
838\end{array}$ & $\begin{array}{r}88 \\
213 \\
20 \\
0 \\
0\end{array}$ & $\begin{array}{l}105 \\
401 \\
365 \\
429 \\
838\end{array}$ & $\begin{array}{r}84,600 \\
259,000 \\
478,000 \\
219,000 \\
451,000\end{array}$ \\
\hline $\begin{array}{l}\text { Tennessee ......... } \\
\text { Upper Mississippi } \\
\text { Lower hilssissippi ... } \\
\text { Sauris-Red-Rainy } \\
\text { Missout Basin }\end{array}$ & $\begin{array}{r}0 \\
24 \\
69 \\
0 \\
9\end{array}$ & $\begin{array}{r}6,990 \\
19,000 \\
6,670 \\
38 \\
8,770\end{array}$ & $\begin{array}{l}0 \\
0 \\
0 \\
0 \\
0\end{array}$ & $\begin{array}{r}6,900 \\
19,000 \\
6,670 \\
80 \\
8,770\end{array}$ & $\begin{array}{l}0 \\
7.4 \\
1.1 \\
0 \\
4.7\end{array}$ & $\begin{array}{r}6,990 \\
19.100 \\
6,740 \\
38 \\
6,810\end{array}$ & $\begin{array}{r}13 \\
388 \\
253 \\
0 \\
172\end{array}$ & $\begin{array}{l}0 \\
0 \\
0 \\
0 \\
0\end{array}$ & $\begin{array}{r}13 \\
386 \\
253 \\
0 \\
172\end{array}$ & $\begin{array}{r}76,600 \\
211.000 \\
78.100 \\
396 \\
167.000\end{array}$ \\
\hline $\begin{array}{l}\text { Arkansas-Whlte-Red. } \\
\text { Texas-Gulf ......... } \\
\text { Flo Grande } \\
\text { Upper Golorado. . . . . } \\
\text { Lower Colorad . ... }\end{array}$ & $\begin{array}{r}37 \\
50 \\
16 \\
0 \\
45\end{array}$ & $\begin{array}{c}4.140 \\
7.630 \\
2.2 \\
146 \\
17\end{array}$ & $\begin{array}{r}0 \\
3,870 \\
0 \\
0 \\
0\end{array}$ & $\begin{array}{c}4.140 \\
11.500 \\
2.2 \\
146 \\
17\end{array}$ & $\begin{array}{c}28 \\
13 \\
0 \\
0 \\
1.5\end{array}$ & $\begin{array}{r}4.200 \\
7.700 \\
18 \\
146 \\
64\end{array}$ & $\begin{array}{r}163 \\
252 \\
14 \\
130 \\
57\end{array}$ & $\begin{array}{r}0 \\
12 \\
0 \\
0 \\
0\end{array}$ & $\begin{array}{r}163 \\
264 \\
14 \\
130 \\
57\end{array}$ & $\begin{array}{r}143.000 \\
224.000 \\
7,780 \\
94,000 \\
62,400\end{array}$ \\
\hline 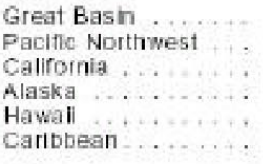 & $\begin{array}{r}2.6 \\
5 \\
3.6 \\
4.2 \\
67 \\
2.2 \\
\end{array}$ & $\begin{array}{r}21 \\
384 \\
202 \\
26 \\
0 \\
0\end{array}$ & $\begin{array}{r}0 \\
0 \\
9,430 \\
0 \\
903 \\
2440 \\
\end{array}$ & $\begin{array}{r}21 \\
384 \\
9,630 \\
25 \\
903 \\
2,440 \\
\end{array}$ & $\begin{array}{l}0 \\
0 \\
5.3 \\
.15 \\
.3 \\
2.2 \\
\end{array}$ & $\begin{array}{r}24 \\
385 \\
211 \\
31 \\
67 \\
4.3\end{array}$ & $\begin{array}{r}23 \\
18 \\
9.7 \\
3.1 \\
7 \\
9 \\
\end{array}$ & $\begin{array}{c}8.6 \\
0 \\
19 \\
0 \\
9.0 \\
0 \\
\end{array}$ & $\begin{array}{r}32 \\
18 \\
29 \\
3.1 \\
9.7 \\
9\end{array}$ & $\begin{array}{r}16,300 \\
17,000 \\
76,000 \\
3,770 \\
6,370 \\
16,500 \\
\end{array}$ \\
\hline Total , ........ . . , i. & 565 & 131,000 & 57,900 & 189000 & 100 & 132,000 & 3,310 & 369 & 3.690 & $2.690,000$ \\
\hline
\end{tabular}




\section{Thermoelectric Power Water Use by State, 1995}

[Figures may not add to totals because of independent rounding. All values in million gallons per day]

\begin{tabular}{|c|c|c|c|c|c|c|c|c|c|c|c|c|}
\hline \multirow{4}{*}{ STATE } & \multicolumn{6}{|c|}{ FOSSIL FUEL } & \multicolumn{6}{|c|}{ NUCLEAR: } \\
\hline & \multicolumn{4}{|c|}{$\begin{array}{l}\text { Withdrawals, } \\
\text { by source and type }\end{array}$} & \multirow{2}{*}{\multicolumn{2}{|c|}{$\begin{array}{l}\text { Consumptive } \\
\text { use }\end{array}$}} & \multirow{3}{*}{$\begin{array}{l}\text { Ground } \\
\text { water } \\
\text { Fresh }\end{array}$} & \multicolumn{3}{|c|}{$\begin{array}{l}\text { withdrawals. } \\
\text { by source and type }\end{array}$} & \multirow{2}{*}{\multicolumn{2}{|c|}{$\begin{array}{c}\text { Consumptive } \\
\text { use }\end{array}$}} \\
\hline & \multirow{2}{*}{$\begin{array}{l}\text { Ground } \\
\text { water } \\
\text { Frash }\end{array}$} & \multicolumn{3}{|c|}{ Suriace water } & & & & \multicolumn{3}{|c|}{ Surtace water } & & \\
\hline & & Frash & Sallne & Total & Fresh & Saline & & Fresh & Soline & Total & Fresh & Saling \\
\hline Alabama ........ & 6.0 & 4,330 & 0 & 4,330 & 30 & 0 & 0 & 862 & 0 & 862 & 1.7 & 0 \\
\hline Alaska ........... & 4.2 & 26 & 0 & & 3.1 & $\theta$ & 0 & 0 & 0 & 0 & 0 & 0 \\
\hline Arizona ......... & 42 & 20 & 0 & 20 & 54 & 0 & 0 & 0 & 0 & 0 & 0 & 0 \\
\hline Arkangas ....... & 5.2 & 798 & 0 & 798 & 27 & 0 & 0 & 967 & 9 & 967 & 1.2 & 0 \\
\hline Californta & 3.5 & 190 & 4,730 & 4,920 & 9.4 & 2.8 & 1 & 12 & 4,690 & 4,710 & 3 & 1,3 \\
\hline Colorado. & 22 & 93 & 0 & 93 & 41 & 0 & 0 & 0 & 0 & 0 & 0 & 0 \\
\hline Connecticut..... & .1 & 276 & 882 & 1.160 & 5.9 & 74 & 1 & 484 & 2,300 & 2,780 & 0 & 0 \\
\hline Dolawaro ........ & 2 & 534 & 740 & 1,270 & 2 & 2.9 & 0 & 0 & 0 & 0 & 0 & 0 \\
\hline D.c. $+\ldots \ldots \ldots$ & 0 & 9.7 & 0 & 9.7 & 8 & 0 & 0 & 0 & 0 & 0 & 0 & 0 \\
\hline Flonda .......... & 21 & 615 & 9,140 & 9. 760 & 54 & 0 & .3 & 0 & 1,810 & 1,810 & 1.2 & 0 \\
\hline Goorgla. ........ & 3.18 & 2,010 & 33 & 2,950 & 52 & 0 & 1.0 & 122 & 0 & 122 & 03 & 0 \\
\hline Hawail , . . . . . & 67 & 0 & 903 & 903 & .7 & 90 & 0 & 0 & 0 & 0 & 0 & 0 \\
\hline Idaho ............ & 0 & 0 & 0 & 0 & 0 & 0 & 0 & 0 & 0 & 0 & 0 & 0 \\
\hline |llinols . . . . . . . . & 9.5 & 9.570 & 0 & 9.570 & 144 & 0 & 1.3 & 7.520 & 0 & 7.520 & 263 & 0 \\
\hline Indiana ......... & 11 & 5.680 & 0 & 5,680 & 114 & 0 & 0 & 0 & 0 & 0 & 0 & 0 \\
\hline$\ldots \ldots \ldots$ & 13 & 2,100 & 0 & 2,100 & 7.8 & 0 & 2.0 & 8.1 & 0 & 8.1 & 26 & 0 \\
\hline Kansas . . . . . . & 14 & 1,230 & 0 & 1,230 & 45 & 0 & 0 & 22 & 0 & 22 & 13 & 0 \\
\hline Kentucky ........... & 38 & 3,410 & 0 & 3,410 & 203 & 0 & 0 & 0 & 0 & 0 & 0 & 0 \\
\hline Louislang . . . . . . & 31 & 4.430 & 0 & 4,430 & 212 & 0 & 1 & 1,020 & 0 & 1,020 & 10 & 0 \\
\hline Maine ......... & 7 & 30 & 105 & 135 & 3.5 & 1.7 & 0 & 0 & 0 & 0 & 0 & 0 \\
\hline Maryland ........ & 1.6 & 358 & 2,780 & 3,140 & 3.7 & 32 & 2 & 0 & 3,220 & 3,220 & $a$ & 16 \\
\hline Massachusetts ... & 46 & 150 & 3,910 & 4,060 & 0 & 0 & 0 & 0 & 454 & 454 & 0 & 6.0 \\
\hline Michlgan ........ & 3.0 & 6,030 & 0 & 6,030 & 50 & 0 & 1 & 2,340 & 0 & 2.340 & 76 & 0 \\
\hline Minnesote . . . . . . & 1.8 & 1.210 & 0 & 1.210 & 28 & 0 & .1 & 886 & 0 & 886 & 20 & 0 \\
\hline Mississippi ...... & 10 & 220 & 112 & 339 & 8.0 & 3.6 & 32 & 0 & 6 & 0 & 19 & 0 \\
\hline Missouri . . . . . . & 9.1 & 5.520 & 0 & 5.520 & 40 & 0 & 4 & 21 & 9 & 21 & 11 & 0 \\
\hline Montana ....... & 0 & 22 & 0 & 22 & 22 & 0 & 0 & 0 & 0 & & 0 & 0 \\
\hline Nebraska ...... & 4.4 & 1,290 & 0 & 1,290 & 12 & 0 & 0 & 1,080 & 0 & 1.060 & 0 & 0 \\
\hline Nevada .......... & 6.2 & 21 & 0 & 21 & 28 & 0 & 0 & 0 & 0 & 0 & 0 & 0 \\
\hline Naw Hampshire. . & 6 & 228 & 292 & 521 & 4,3 & $\theta$ & 0 & 0 & 585 & 505 & 0 & 0 \\
\hline Naw Jersey ...... & 1.2 & 578 & 980 & 1,560 & 3.7 & 99 & 7 & 0 & 2,800 & 2.600 & .7 & 22 \\
\hline New Mexico...... & 8.3 & 46 & 0 & 46 & 48 & 0 & 0 & 0 & 0 & 0 & 0 & 0 \\
\hline New rork ....... & 0 & 5,140 & 5.470 & 10.600 & 103 & 109 & 0 & 1,420 & 1,010 & 2.440 & 68 & 20 \\
\hline North Carolina & 1 & 3,210 & 0 & 3,210 & 56 & 0 & 0 & 2660 & 1.550 & 4,210 & 1.5 & 17 \\
\hline North Dakota..... & 0 & 879 & 0 & 879 & 20 & 0 & 0 & 0 & 0 & 0 & 0 & 0 \\
\hline ohlo & 19 & 8.040 & 0 & 8,040 & 309 & 0 & 0 & 137 & 0 & 137 & 27 & 0 \\
\hline oklanoma ...... & 3.5 & 121 & 0 & 121 & 60 & 0 & 0 & 0 & 0 & 0 & 0 & 0 \\
\hline olegon .......... & $\theta$ & 8.0 & 0 & 9.0 & 7.8 & 0 & 0 & 0 & 0 & 0 & 0 & 0 \\
\hline Ponnsylvania. ... & 5.2 & 3,870 & 0 & 3.870 & 120 & 0 & 0 & 2,050 & 0 & 2.050 & 179 & 0 \\
\hline Fhode Isiand. . . . & 0 & 0 & 275 & 275 & 0 & 5.5 & 0 & 0 & 0 & 0 & 0 & 0 \\
\hline South Carollna . . & 4 & 1.290 & 0 & 1,290 & 23 & 0 & 39 & 3,470 & 0 & 9.470 & 28 & 0 \\
\hline South Dakota ... & 2.6 & 1.9 & 0 & 1.9 & 1 & 0 & 0 & 0 & 0 & 0 & 0 & 0 \\
\hline Tennessog . . . . & 0 & 6.830 & & 6,830 & 5 & 0 & 0 & 1.470 & 0 & 1.470 & 0 & 0 \\
\hline Texas .......... & 58 & 6.710 & 3,870 & 10,600 & 271 & 12 & .8 & 2,820 & 0 & 2,820 & 26 & 0 \\
\hline Utahi _........ & 0 & 48 & 0 & 48 & 47 & 0 & 0 & 0 & 0 & 0 & 0 & 0 \\
\hline Vermont ....... & 4 & .5 & 0 & .5 & -7 & 0 & 0 & 452 & 0 & 452 & 3.2 & 0 \\
\hline Virginia .... . . . . . . & 1 & 1.820 & 973 & 2,790 & 8.8 & 0 & 3 & 2,080 & 1.760 & 3,830 & 0 & 0 \\
\hline Washington...... & 4 & 17 & 0 & 17 & 4 & 0 & -1 & 358 & 0 & 358 & 9.8 & 0 \\
\hline Wost virginia & 5 & 3,010 & 0 & 3,010 & 122 & 0 & 0 & 0 & 0 & 0 & 0 & 0 \\
\hline Wisconsin . . & 5.6 & 3,860 & 0 & 3,860 & 39 & 0 & 1 & 1.970 & 0 & 1.970 & 20 & 0 \\
\hline Wyoming . . . . . . & 1.0 & 210 & 0 & 219 & 50 & 0 & 0 & 0 & 0 & 0 & 0 & 0 \\
\hline Puerto Rico...... & 2.2 & 0 & 2,260 & 2,260 & .7 & 0 & 0 & 0 & 0 & 0 & 0 & 0 \\
\hline Virgin Islands , & 0 & 0 & 173 & 173 & 2 & 0 & 0 & 0 & 0 & 0 & 0 & 0 \\
\hline Total $\ldots \ldots \ldots$ & 486 & 97.0003 & 37,600 & 135,000 & 2,500 & 263 & 78 & 34,300 & 20,200 & 54,500 & 815 & a2 \\
\hline
\end{tabular}




\section{Conclusions}

Most IPP companies consider there will be a market for a 70-80 MW relatively high efficiency gas turbine able to rapidly start up daily. Whilst few companies utilize multiple small units, many of our respondents consider future power plants will have to become smaller in capacity (less than $500 \mathrm{MW}$ ), due to transmission grid constraints, and that future power plants will need to be more flexible in operation to meet baseload, mid-merit and peaking operation as market conditions dictate throughout the life of a plant.

The main market for NGT is considered to be the mid-merit and peaking market. There is probably only limited application in the baseload sector and no applications for cogeneration power plants.

Important attributes required for NGT to be successful in the identified market sectors are low capital cost, high efficiency, high availability and reliability. $O$ \& $M$ costs can be significant to many of our respondents. A rapid start-up time is expected to be advantageous for these sectors, the market requiring start times of 15-30 minutes. Necessity for dual fuel capability varies between our respondents, some considering the current high gas prices will require them to utilize oil fuels to hedge against fuel price risk. Small plant foot-print, part-load operation, and unmanned operation are not considered significant attributes.

Delivery period should be of the order of 12 months.

Maintenance contracts and lease engine arrangements are attractive to the industry as a means of reducing project risk.

Our US water availability survey and all our respondents confirm that one of the major obstacles to installing new power plant is availability of water. The IPP market is agreed that a plant that can operate without consuming water is very valuable, and extremely attractive. An NGT plant consuming $45 \mathrm{lbs}$ per second of water would not obtain many sales in the US market.

Selling new power plant technology in the US will be a challenge pending sufficient operation to demonstrate proven plant. The market and financial institutions are risk adverse. The first NGT plants will require Rolls-Royce to offer significant guarantees and warranties, as incentives.

The Rolls-Royce brand name carries considerable influence. However, the market's perception of Rolls-Royce is associated with aerospace gas turbines. Whilst Rolls-Royce's involvement in the power generation market is known by all, none considered Rolls-Royce as a major player. The Rolls-Royce technical standing is highly regarded such that most of our respondents considered Rolls-Royce equal to the task of developing the NGT concept.

The NGT characteristics considered carrying the most technical risk are the water recovery system and recuperator. 


\section{Appendices}

Appendix A Questionnaire 


\section{APPENDIX A \\ Rolls Royce Energy Systems Inc. \\ NGT Customer Survey \\ Discussion Topics}

\section{Customer Info}

- What market niches are you currently active in, or planning to be active in:

- Regions / markets

- Capacity range(s)

- Type (CCGT, peaking, cogen)

- What is your anticipated development portfolio mix over the next ten years?

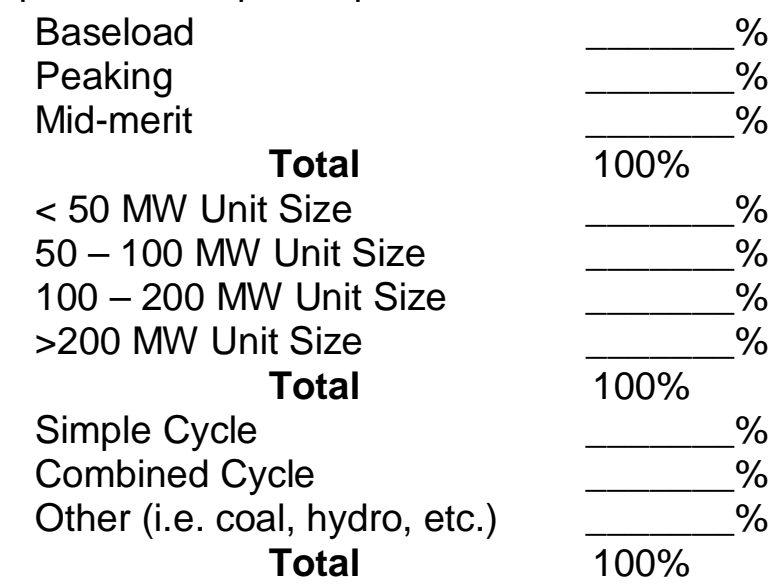

- For your larger development projects, how likely are you to use multiple smaller units versus single larger units? $\%$. Why (i.e. economics, reliability, redundancy, etc.)?

\section{Market Info}

- How do you expect the power generation markets that you are active in to grow over the next 5 to 10 years?

- What application mix (baseload, peaking, mid-merit) do you anticipate for this future growth?

- What power range mix (<50 MW, 50-100 MW, 100-200 MW, >200 MV) do you anticipate for this future growth?

- With the current transmission grid system constraints making it difficult to locate large base load power plants, and the low level of new power lines expected to be installed in the next few years, are applications for smaller, relatively high efficiency power plants connected at distribution voltages expected to be more prevalent in 5+ years time?

- Will the recent surge in the sales of peaking plants in the US market impact the future sales of peaking plants in this market or will this trend continue? 
- Do you anticipate that there will be a market for power plants that can start-up daily, or maybe twice daily, to capture peak and mid-merit tariffs?

\section{NGT Market Potential}

- Do you perceive a market for 70-80 MW high efficiency power generator units in 5+ year time frame?

- For the market sector(s) you are active in, is 70-80 MW unit size too small, too large, satisfactory?

- Are there unique network constraints / barriers for the 70-80 MW power range?

- Are there regulatory constraints / barriers for the 70-80 MW power range?

- What market segment (baseload, peaking, mid-merit) do you feel the NGT will best satisfy?

- What are the key attributes wanted from this type of gas turbine for this market sector? - Is it capital cost, rapid start-up, unmanned operation, low emissions, reliability, high availability, minimum plant footprint, quick delivery, output power, heat rate, fuel flexibility, part power performance (efficiency, emissions, etc.)? (Rank each attribute on scale $0-10$, with 0 lowest - 10 highest)

- What would be an acceptable time frame for delivery and installation (time from order to operation)?

- What products will compete with the NGT in your purchase decisions?

- How do you perceive Rolls-Royce's capability in the power generation market?

\section{Pro-forma Issues}

- At the targeted NGT plant capital cost and quoted efficiencies, what percentage of the market sector(s) identified could be captured? Base load peaking $\%$. $\%$, mid-merit $\%$,

- For the market sector(s) identified, what are the factors which significantly influence the project decisions/returns (i.e. capital, O\&M costs, zero or low manning levels, water consumption, emissions level, efficiency, power level, start-up time, etc.)? (Rank each attribute on scale $0-10$, with 0 lowest -10 highest)

- How would you evaluate trade-offs between acquisition cost, operational cost and projected benefits associated with the NGT product?

- What incremental increase in your rate of return $(0 \%,+0.5 \%, 1.0 \%, 3.0 \%, 5.0 \%$, etc.) would be necessary to support a decision to invest in a NGT type power plant?

\section{Service Offerings / Incentives}

- What service offerings are important or preferred (i.e. maintenance contracts, operation \& maintenance contracts, lease engines, etc.)?

- How important are service offerings likely to be in your NGT purchase decision?

- Are service offerings required to reduce the initial risk to an acceptable level?

- What kinds of incentives are desirable for launching new products like the NGT? How are these incentives valued? 


\section{Specification Issues}

- Water usage \& recovery:

- Will the additional complexity of a water system limit the potential sales of the NGT?

- What is the value of a plant that does not consume water?

- With the projected levels of water injection rate (45 lbs/sec), will water recovery be mandatory?

- If no water recovery is used, will the projected levels of water usage (45 lbs/sec) limit the potential sales of the NGT? Why (i.e. regulatory, cost, availability of water, complexity, space, etc.)?

- Is evaluation of water use and water recovery purely based on economic parameters (i.e. capital cost, cost of treating water, cost of recovery, etc.)?

- What is your cost of water (untreated potable)? What region?

\section{- Start Time}

- How important is start time to full power in your purchase decisions (critical, very important, somewhat important, not important)?

- If start time is important, how fast must the NGT plant be capable of achieving full power (<10 min, 10-15 min, 15-20 min, 20-30 min)?

\section{- Fuel Flexibility}

- Is fuel flexibility required? (Gas only, dual fuel, multi-fuel)

- If dual fuel is required, is the requirement for the second fuel for back-up use only or for continuous duty?

- If dual fuel is required, is the requirement due to the benefits of lower priced interruptible gas supply or is the requirement due to desire to hedge against uncertain gas fuel prices?

- If dual fuel is required, is there a secondary fuel preference? What fuel?

- If dual fuel is required, what is the required speed for the fuel change-over (while running, with shutdown in 1 hour, 4 hours)?

- Availability / Reliability

- What is the market expectation for guaranteed reliability and availability levels?

- What is the expectation for consecutive hours that a plant will run prior to planned maintenance in various applications?

- How attractive is a lease engine program?

- Other

- What percentage of your future applications is likely to have a requirement to be able to switch between operating in grid mode and operating in island mode?

- What percent of your projects will have unmanned requirements?

\section{NGT Technology Issues}

- How do you assess, calibrate and value incremental risks associated with a new technology, product, system, plant, etc.? 
- Under what circumstances would you evaluate new technology (i.e. a NGT product) for a project compared to 'proven' technology?

- For the key characteristics of the proposed R-R NGT concept (massive steam/water injection with re-designed turbine to accept such volumes, water recovery and exhaust recuperation) - do you consider R-R has adequate technical expertise and experience to develop the NGT power plant such that the technical risk, whilst real, is accepted as manageable by R-R?

- How acceptable do you perceive the key NGT technical characteristics? Rank acceptability associated with the following: (Rank each attribute on scale $0-10$, with 0 lowest acceptability - 10 highest acceptability)

- Significant steam or water injection

- Water recovery from exhaust

- Once-thru steam generator

- Recuperator

- Intercooling

- Exhaust clean-up (SCR) 


\section{Appendix E NGT Market/Economic Analysis: DFM Consulting Report}




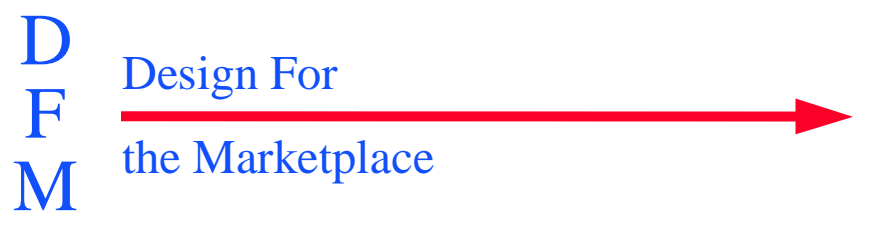

\title{
DFM Consulting
}

Post Office Box 247

Urbana IL 61803

Toll Free: (888) 643-4393

fax: (217) 367-5864

email:Paul@DFMConsulting.com

\section{Next Generation Turbine Value Model}

\author{
Paul D. Collopy
}

November 2001 


\section{Table of Contents}

$1 \quad$ NGT Value Model — Executive Summary 3

2 Value Model Overview 5

$\begin{array}{lll}3 & \text { NGT Value Model Operation } & 7\end{array}$

$4 \quad$ NGT Value Model Inputs $\quad 14$

$5 \quad$ NGT Value Model Assumptions $\quad 16$

6 Sensitivity Study on Value Model Assumptions 22

$7 \quad$ NGT Value Model Software Design 34

$8 \quad$ Recuperator Component Value Model 44

9 Development Cost Methodology 45

10 NGT Value Model Conclusions 45

11 References $\quad 48$ 


\section{$1 \quad$ NGT Value Model - Executive Summary}

In February, 2001, Rolls-Royce Corporation contracted DFM Consulting to provide a value model for the US Department of Energy Next Generation Turbine program. The goal of this model is to assist RollsRoyce in technology assessments, trade studies and parametric studies by evaluating power generation configurations based on their figures of merit. The value model focuses on the power generation application, capturing the business motivations of a power production company to pay for a power generation system primarily based on the profits it will generate in service.

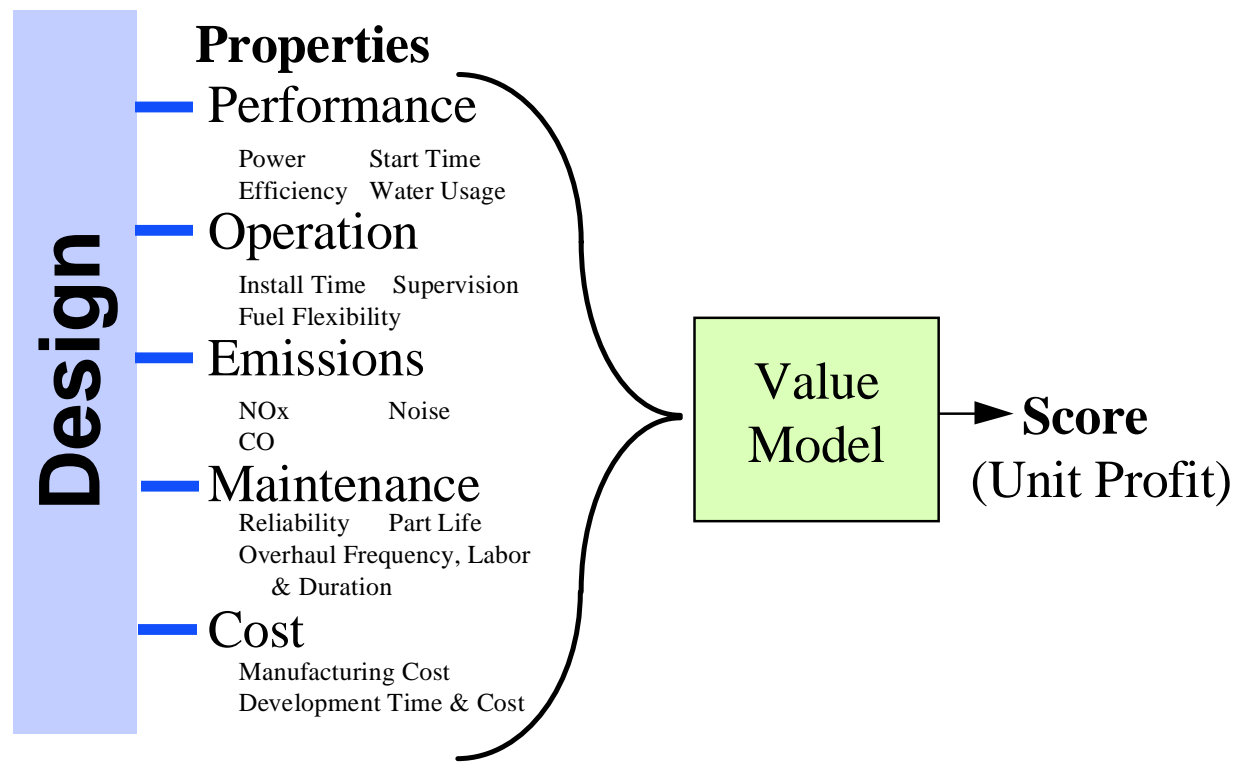

Scoring designs with a value model

The value model gives a score to every power generation configuration-a higher score indicates a better configuration. The value model can also rate technologies: a system is configured with the technology used in the most advantageous way, and is configured without the technology. The difference in the scores of the two configurations is a rating of the technology. Any technology with a positive rating is of interest, but higher ratings indicate more promising technologies.

Because the score is denominated in dollars of profit per system, the technology rating is the profit added per system. Based on the anticipated number of systems sold, this rating can be used to justify investment in developing the technology. In fact, the value model can estimate the net present value of the NGT program with and without the technology. The difference is the net present value of the technology to the program, which is a key consideration when choosing how much money to invest in developing the technology. 
The value model is an essential element of the advanced design process shown below:

1. starting from the top, an existing design is improved, either by adding new technology or by choosing a more promising point in a parametric design space (a point might be a combination of boiler pressure, rotor inlet temperature, compressor pressure ratio, or other parameters);

2. the parameters and technology are elaborated into a rough system design using a cycle model and a rough layout;

3. the design is analyzed with the performance model, development cost model, manufacturing cost analysis, and so on, to generate figures of merit;

4. finally, the value model is used to assign a single dollar value (score) that accounts for the impact of all the figures of merit.

By cycling through the advanced design process, the NGT design team can find the best engine configuration in the parametric design space. The advanced design process, incorporating the value model, is a precursor to an automated optimized design process by replacing the "Improve" step with an optimal search technique in the parametric design space.

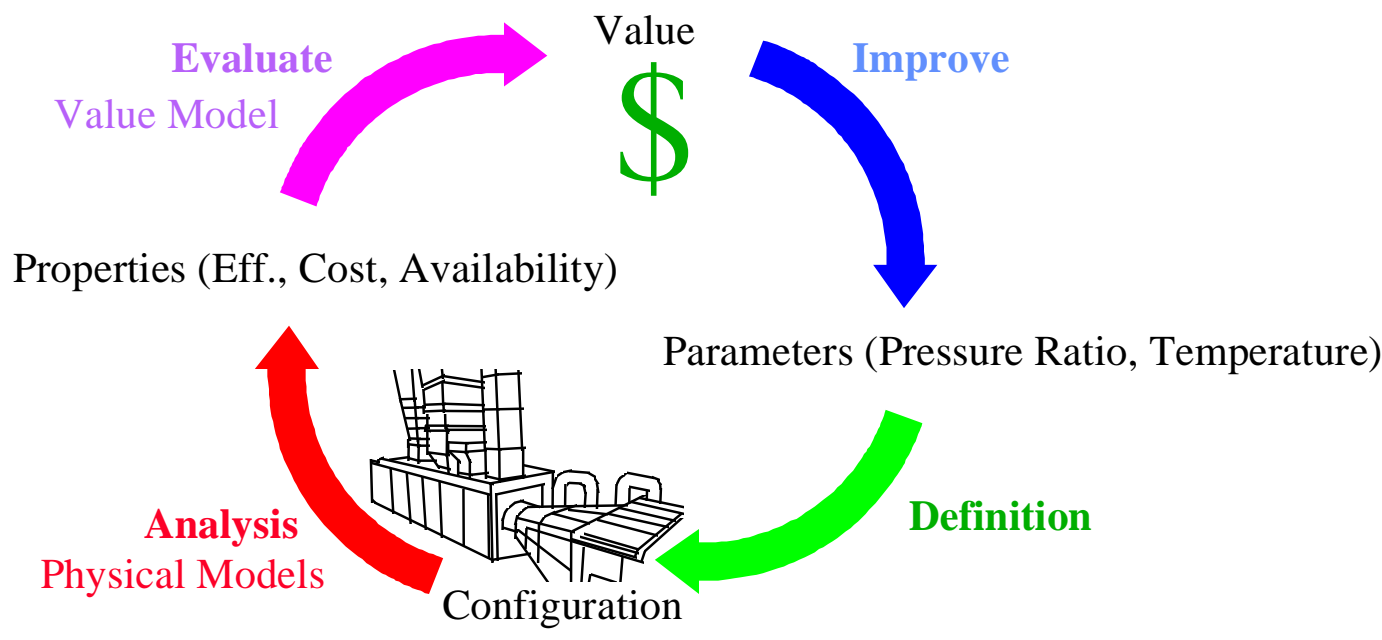

Advanced design process 


\section{Value Model Overview}

The purpose of a value model is to serve as a guidance technology ${ }^{1}$ for design and technology development. That is, the value model points the way for improving design. By evaluating possible designs in a parametric design space, the parametric description of the best design can be estimated. Also, by rating technologies with the value model, the best suite of technologies can be identified. The value model can be used to guide overall system architectural design or the detailed design of individual components.

This section will give an overview of how the value model works. A product creates value by functioning for the user. A power generation system operated by an independent power producer (IPP) creates value by generating electricity for profit. Electricity provides revenue for the IPP. Fuel and other operating costs are deducted from this revenue, leaving the IPP with an operating profit. This is indicated along the top of Figure 2-1.

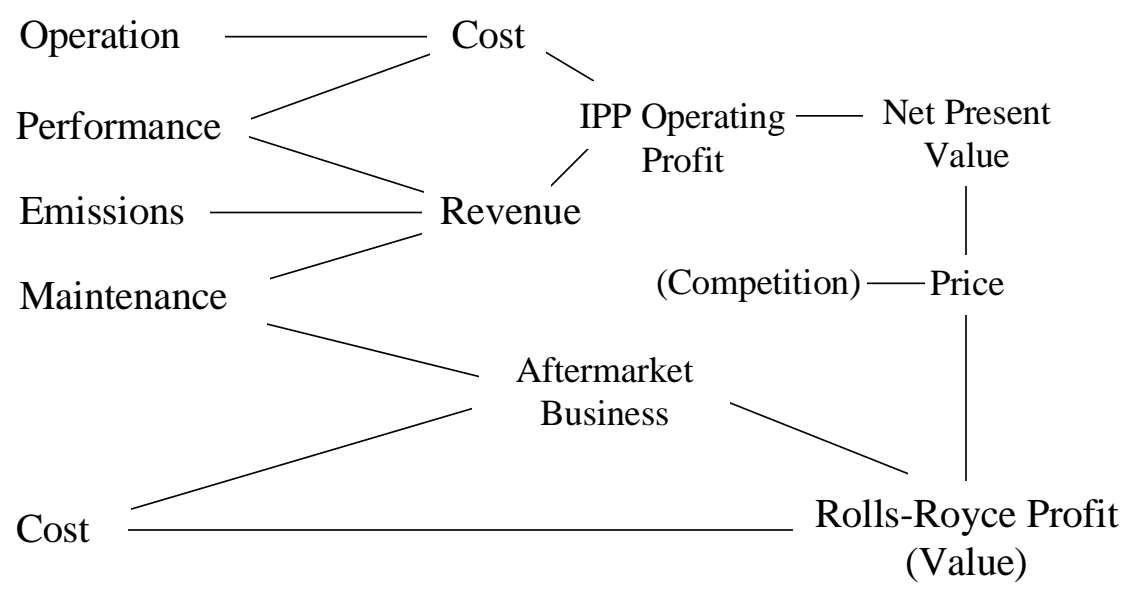

Figure 2-1: Value Model Overview

The price that an IPP is willing to pay for a generation system is the net present value of the profit the system will earn for the IPP minus an adjustment determined by the price of competing generation systems. The adjustment is the net present value which would be earned by operating the best competing system minus the price of that system. This is somewhat complex, but the important point is that, for every dollar change in the net present value of the IPP profit, the price also changes by one dollar in the same direction.

In this model, all maintenance for the life of the generation system is assumed to be performed by RollsRoyce (or its contractors) under a service warranty. The warranty is considered part of the price, and therefore the Aftermarket Business shown in Figure 1-1 is pure cost to Rolls-Royce, for parts and labor. By this means, the model provides the proper motivation to the design: to maximize part life and minimize diagnostic and overhaul labor. This approach is superior to business plans which assume spare part sales with markups of several hundred percent, and therefore show an illusory profit for designs in which expensive parts fail frequently.

The system price, minus this aftermarket maintenance cost, minus the manufacturing cost plus an allowance for amortized development cost is the unit profit to Rolls-Royce, the ultimate figure of merit. (Development cost amortization is tuned to make this unit profit trend with the net present value to RollsRoyce of the product program.)

Most attributes of the generation system design fit smoothly into this economic perspective. Following are a few that do not:

${ }^{1}$ Carliss Baldwin and Kim Clark, Design Rules, MIT Press, 2000. 
- Environmental impact (CO and NOx emissions and noise). The model addresses the impact of these factors by attenuating the value of the ultimate product, the electricity produced, as the environmental measures exceed critical value. This is consistent with assuming that environmental factors are appearance issues that do not directly impact economics but are strongly associated with perceived quality in the customer's mind. ${ }^{1}$

- Reliability. This is also treated as a perceived quality rather than a direct economic impact, on the assumption that the customer's distaste for an unreliable generation system exceeds the dollar and cents impact that could be easily assessed.

- Development time. Long product development cycles result in concrete economic impact, such as late returns on investment, reduced sales and profits due to competitive cost disadvantages, and reduced prices due to increased variance between customer needs anticipated during design and actual needs of customers during production. To simplify the model, all these effects are combined into a single reduction in profit that is linear with the length of the development program.

In form, the value model accepts a set of twenty inputs that describe a proposed design and calculates a single output. Operation of the model is described in Section 3 and the inputs are reviewed in Section 4.

Value model calculations are based on twenty-seven assumptions, such as the prevailing price of fuel. These assumptions are reviewed in Section 5. The model can be improved by gathering more data to increase the certainty of these assumptions. Section 6 documents a sensitivity study that indicates which assumptions are worth investigating more fully.

Section 7 describes in detail the software design of the model and the calculations used. Section 8 describes in detail the cogen value model. Section 9 discusses a risk based development cost methodology that can be used to relate risk to value in the model.

Section 10 enumerates the conclusions of the NGT value model study. Section 11 provides some references for further information on value modeling, value based acquisition, and related topics.

\footnotetext{
1 The method is adapted directly from "Methods for Analyzing the Value of Automobiles," by J. A. Donndelinger and Harry E. Cook of the University of Illinois. Copyright 1997 by The Society of Automotive Engineers, Inc. Warrendale, PA. In the article, the critical value method is used to evaluate automobile interior noise, among other factors.
} 


\section{$3 \quad$ NGT Value Model Operation}

The NGT Value Model is a Microsoft Excel spreadsheet workbook. Although the model is delivered in Excel 97, it is developed in Excel 5.0 and therefore can be provided in version 5.0 or 7.0.

\subsection{Summary Sheet}

The NGT Value Model can be operated completely from the Summary worksheet. Inputs are entered in the yellow cells. These inputs are described in detail in Section 4. Up to four cases can be entered simultaneously in the parallel columns for easy comparative analysis. All inputs in a column must be set to some reasonable value. If an input, such as OpLabor, is not relevant in a particular study, all cases (columns) should use the same baseline value, so that the input's impacts will cancel out.

The gray cells near the top of the sheet, labeled UnitProfit, are the scores for each case. A higher score is better. The difference between scores of different cases is relevant. Ratios of scores are meaningless because the zero value is arbitrary.

\subsection{Model Sheet}

When you run a study and observe the different outputs, you will immediately wonder what aspect of the design is responsible for the difference. This can be analyzed on the Model sheet where the actual calculations are done. Every intermediate calculation is reported on this sheet, and the areas in which two cases diverge can be quickly identified. Using the flow charts and algorithms in Section 7 of this report, the differences can be analyzed and the causes determined.

\subsection{Business Plan Sheets}

At the bottom of the Summary worksheet are three outputs from the business plan analysis: Net Present Value, Internal Rate of Return and Return on Capital. These are only calculated for the business case in the first column. They are provided to give some additional insight into the significance of the UnitProfit score at the top of the column. These values are calculated in the Business Plan (\$) worksheet. This worksheet provides a very simple business case analysis of the first case on the Summary sheet. System price is determined from the value model. The plan assumes that unit profit will be used to enhance market share, so that market share increases as unit profit rises, from a minimum share of one-third to a maximum share of two-thirds. A negative unit profit should cause the production program to be canceled (production volume equal to zero), but to avoid dividing by zero the minimum production volume is one unit. Production is scheduled based on the completion of development (DevTime input). Production rampup and many other details of the business plan are borrowed from Brad Pokosh's plan for the NGT.

The Business Plan $(\mathfrak{E})$ worksheet is the identical plan rendered in pounds sterling, using a currency exchange rate. The exchange rate and many other assumptions and calculations used to generate the business plan are located on the Busiplan Frame worksheet. Only a minimum of calculation is performed on the Business Plan worksheets themselves.

\subsection{Assumptions Sheet}

The Assumptions worksheet contains the twenty-seven key numerical assumptions on which the value model calculations are based, which are discussed in detail in Section 5. These assumptions are in blue fields. Also on this sheet are

- Six factors calculated in the Business Plan (\$) worksheet and used in the value model. If there were no business plan sheet, these six would be assumptions. Instead the business plan is used to calculate these based on the four business plan assumptions (the last four blue cells).

- Four constants. These are not assumptions since their values are fixed.

- Ten quality parameters. These are simply restatements of the first ten assumptions in forms more easily used by the model: the midpoints and slopes of five logistic curves. A pair of quality assumptions (90\% and $99 \%$ point) determines a pair of quality parameters (slope and midpoint) 
which determines a single logistic curve. The relation of the pair of assumptions to the pair of quality parameters is illustrated in Figure 3-1. The reason that the assumptions are converted to the parameters is that, although the parameters are appropriate for simple calculation of the logistic curves, the assumptions are much more tangible values to the user. The midpoint of the logistic curve is far down the curve from where any good design will be, and the slope of the curve at the midpoint is equally remote from the part of the curve that is used.

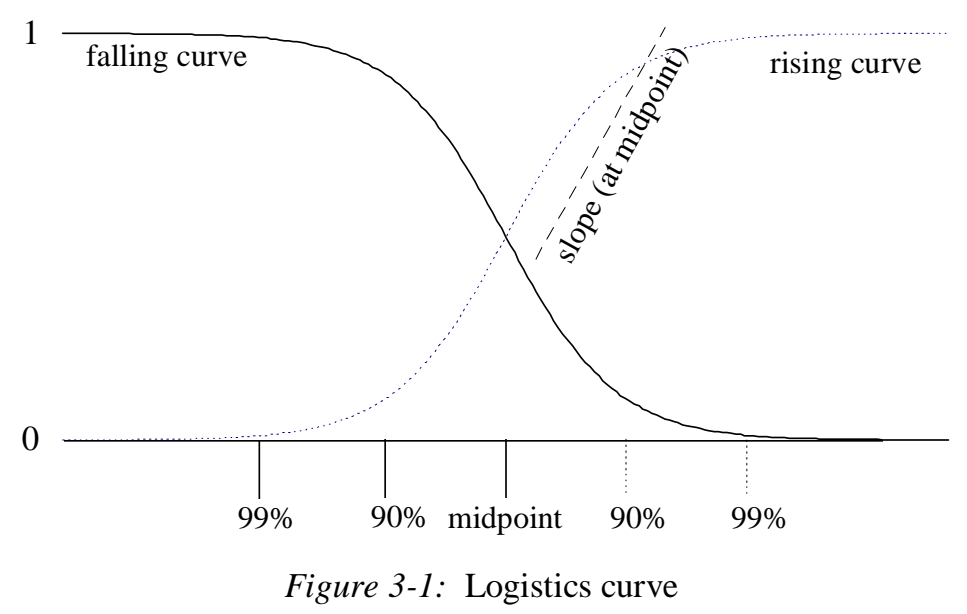

Notice in Figure 3-1 that, for the falling curve, the 99\% point is where the curve first noticeably departs from one. Thus, for any values left of the $99 \%$ point, the value of the function is essentially one. Since the curve is a multiplier, it has essentially no effect for values less than the $99 \%$ point. These values correspond to high quality designs. To the right of the $90 \%$ point, the falling curve begins to drop precipitously. These values represent low quality. The right markings of $90 \%$ and $99 \%$ correspond to the rising curve. In this case, values to the right of the $99 \%$ mark are high quality and values to the left of $90 \%$ are low quality. Thus, the rising logistics curve is used to describe qualities where more is better, such as reliability, and the falling curve describes qualities where more is worse, such as noise.

The actual logistics curves determined by the quality parameters are plotted on the Charts worksheet as documented below.

\section{Modifying Assumptions}

The spreadsheet model is designed to allow users to modify assumptions. However, this should be done with care. If you modify assumptions in the course of a trade study, parametric study, or technology evaluation, such that some results were determined with one setting of an assumption and some with another, this will usually obscure the meaning of the results of the study. Also note that if you change assumptions, you will usually want to change them back to the original values. Therefore you should make such changes on a temporary copy of the value model, or you should carefully document the changes so you can retrace your steps.

\subsection{Cogen Sheet}

Although the NGT product does not include steam cogeneration (sometimes known as "combined heat and power" or CHP), other products do. To generalize the power generation value model so that it can be used with any product, a cogeneration system value model is provided. This model treats cogeneration as an optional feature that a customer could select with a power generation system. Thus, the power generation system is assumed to exist, and the cogeneration system is evaluated in terms of what it adds and detracts from the basic power generation package.

Cogeneration adds a supply of heat energy in the form of steam. This steam is treated as if the system operator sells by the BTU. The steam price per BTU is the price of gas per BTU, since gas is an alternative way to generate the same heat energy. Gas price per BTU is an assumption of the basic model, and this assumption is imported into the cogen model. The supply of steam is based on 
- the amount of gas energy which is not converted to electricity by the power plant and

- the ability of the cogen unit to extract this waste heat.

There is no fuel cost for cogen steam. Therefore, the system provider can charge a price near the net present value of the heat energy produced, if the price includes a full warranty. The surplus value of cogen to the system provider is the net present value of the energy produced less the net present value of the system maintenance cost, less the manufacturing cost, less the amortized share of the development cost. This is the calculation performed on the cogen worksheet. It is documented in detail in Section 7.

\subsection{Charts Sheet}

Charts is a sheet that plots the five quality curves (for reliability, CO NOx, noise, and start time) and the electrical power demand curve.

\section{Electrical Power Demand}

Electric Power Demand

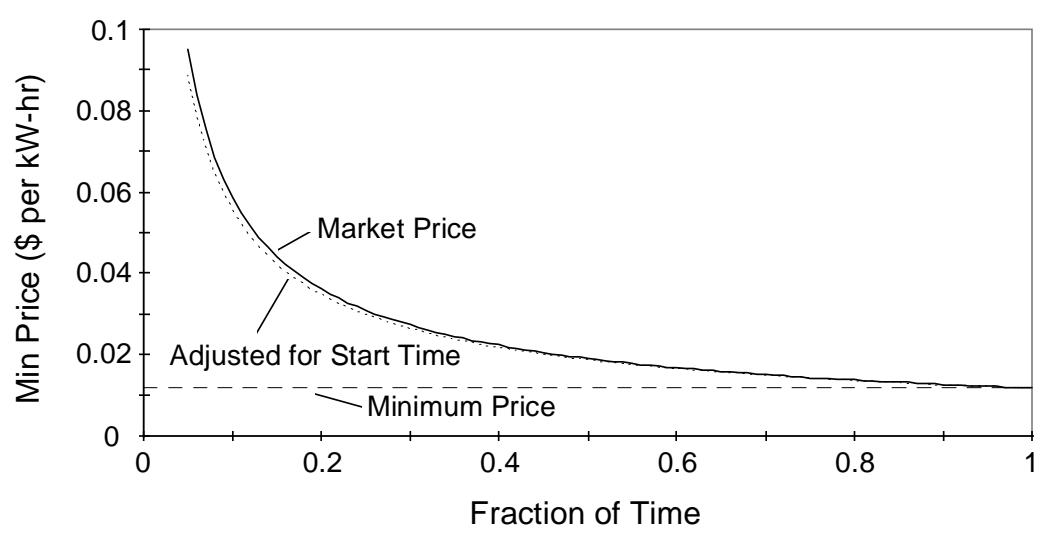

Figure 3-2: Electrical Power Demand Curve

Demand is the price set by the market. Electrical power demand fluctuates because of the needs of consumers and the capabilities of suppliers. Consumers collectively want significantly more power at some times than at others, and they highly value the availability of power at the time they want it. Therefore the market is willing to pay a high marginal price for electricity at periods of high demand. The industry cost structure has matched this demand by providing power plants with various duty cycles. Each plant can amortize its capital cost only over the period when it operates. Thus, the cost of generating the excess electricity during high demand periods is higher because the equipment which generates the excess electricity is amortized over fewer operating hours. This naturally leads to the hyperbolic-shaped demand curve shown in Figure 3-2.

Electricity providers who wish to service the brief periods of high demand (the left side of Figure 3-2) must respond rapidly to power requests from the grid. Generating plants with long start times will not be able to service the shortest period, highest priced demand. The dashed line indicates the electricity price reduction because of the start time requirements of the generating system (based on the start time in case 1 on the summary sheet).

As the NomElecPrice and DemandSlope assumptions are set, the Electrical Power Demand curve on the charts sheet should be observed to be sure that the resulting demand curve meets expectations. 


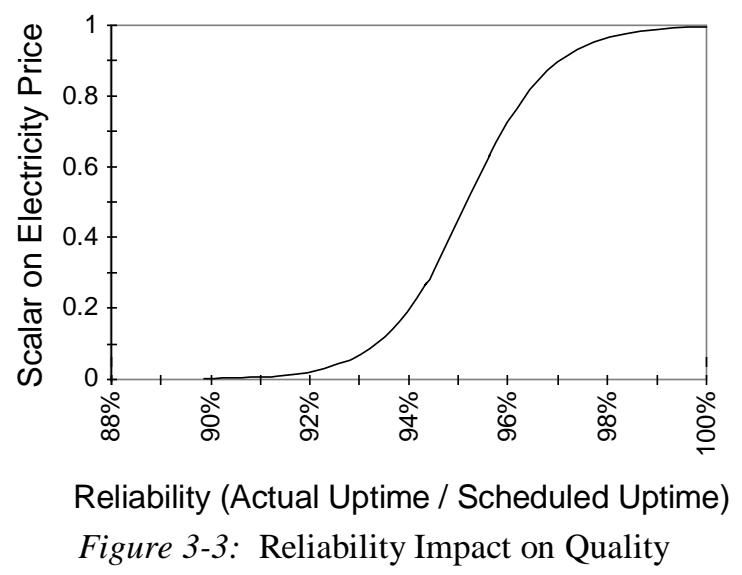

Reliability is the ratio of operating time to the sum of operating time and time unavailable due to unscheduled maintenance. Low reliability has a negative impact on customers that is far greater than the economic impact of missed revenue opportunity. It chaotically disrupts operations and can give the power supplier a bad reputation with its customers. This makes reliability a subjective property of the power generation system. The value model uses quality curves for subjective properties. The quality curve shown in Figure 3-3 is a hyperbolic tangent (also known as a logistic curve). The value model uses the value of the curve as a scalar applied to electricity price. That is, if the reliability were only $95.5 \%$, this would have a negative impact equivalent to a $50 \%$ reduction in the sale price of electricity. The curve is determined by two assumptions: Reliability 90 and Reliability 99 . Reliability 90 is the value on the horizontal axis at which the curve crosses 0.9 on the vertical axis. Reliability 99 is the value on the horizontal axis at which the curve crosses 0.99 on the vertical axis. After setting these two assumptions, it is wise to look at the Reliability Impact on Quality curve to see whether this curve, used by the model, appears reasonable.

\section{CO Quality Curve}

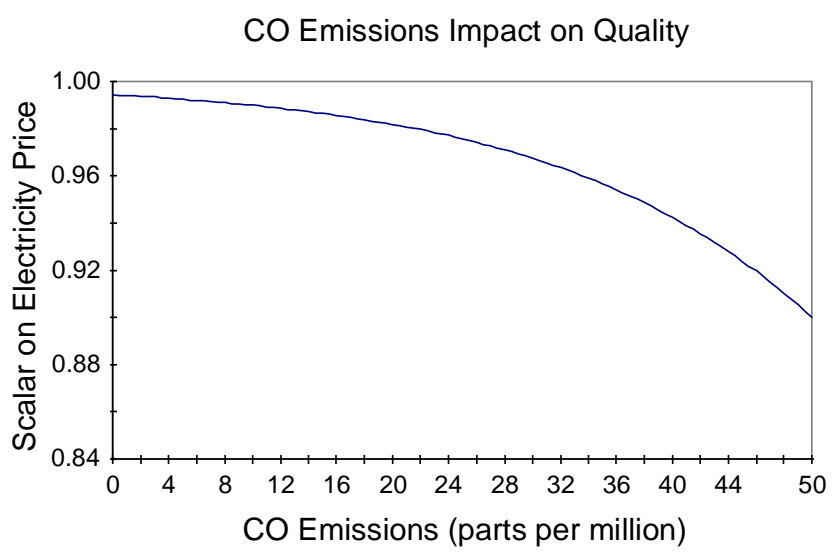

Figure 3-4: CO Quality Curve

The Carbon Monoxide emissions quality curve is like the Reliability Curve in Figure 3-3 except that, whereas more reliability is good, more $\mathrm{CO}$ emissions are bad. The impact of $\mathrm{CO}$ emissions is twofold: on the one hand, high emissions can reduce sales because high emissions plants may not be permitted to operate in many areas. On the other hand, the plant manufacturer and operator would like to be good citizens and protect the environment, beyond the levels that economic considerations would dictate. The first consideration, sales and permitting, seems to dominate.

This curve is also a hyperbolic tangent between 0 and 1 . The curve is determined by two assumptions: CO90 and CO99. CO90 is the value on the horizontal axis at which the curve crosses 0.9 on the vertical axis. CO99 is the value on the horizontal axis at which the curve crosses 0.99 on the vertical axis. After 
setting these two assumptions, it is wise to look at the CO Quality curve to see whether this curve, used by the model, appears reasonable.

\section{NOx Quality Curve}

NOx Quality Impact

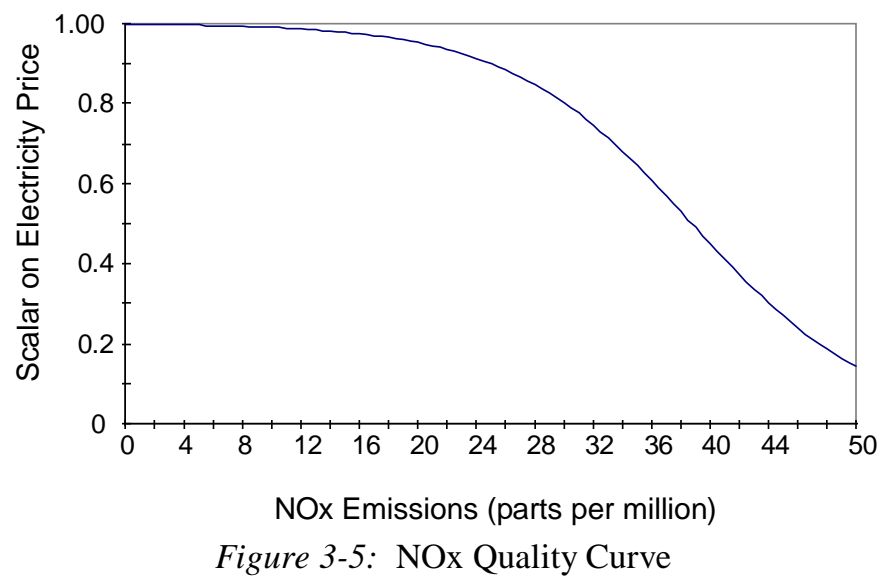

The oxides of nitrogen emissions quality curve is very much like the $\mathrm{CO}$ quality curve in Figure 3-4 above. The curve is determined by two assumptions: NOx90 and NOx99. NOx90 is the value on the horizontal axis at which the curve crosses 0.9 on the vertical axis. NOx99 is the value on the horizontal axis at which the curve crosses 0.99 on the vertical axis. After setting these two assumptions, it is wise to look at the NOx Quality curve to see whether this curve, used by the model, appears reasonable.

\section{Noise Impact on Quality}

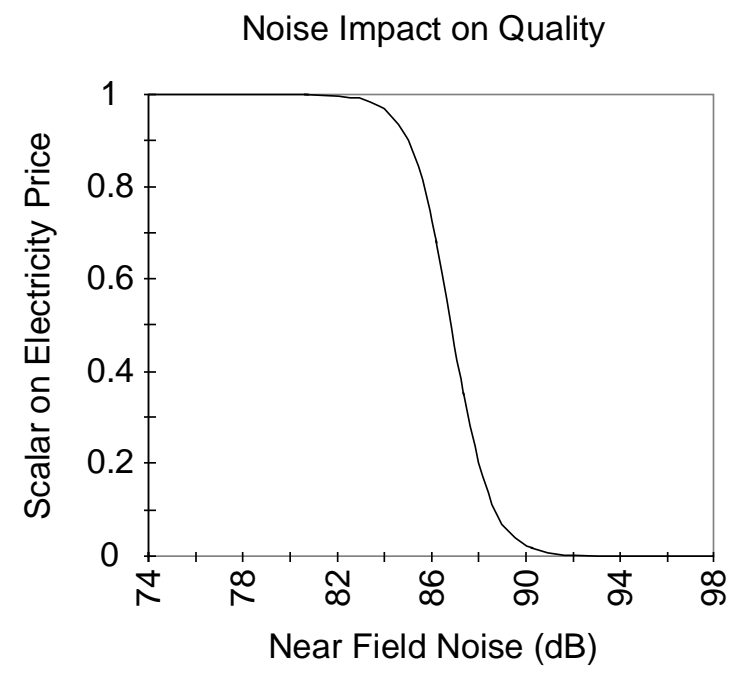

Figure 3-6: Noise Impact on Quality

Noise has a purely qualitative impact on power generation system price. High noise levels make the generation station a bad neighbor and an unpleasant and possibly unhealthy place to work. The value model uses quality curves for subjective properties. The quality curve shown in Figure 3-6, a hyperbolic tangent, is determined by two assumptions: Noise90 and Noise99. Noise90 is the value on the horizontal axis at which the curve crosses 0.9 on the vertical axis. Noise99 is the value on the horizontal axis at which the curve crosses 0.99 on the vertical axis. After setting these two assumptions, it is wise to look at the Noise Impact on Quality curve to see whether this curve, used by the model, appears reasonable. 


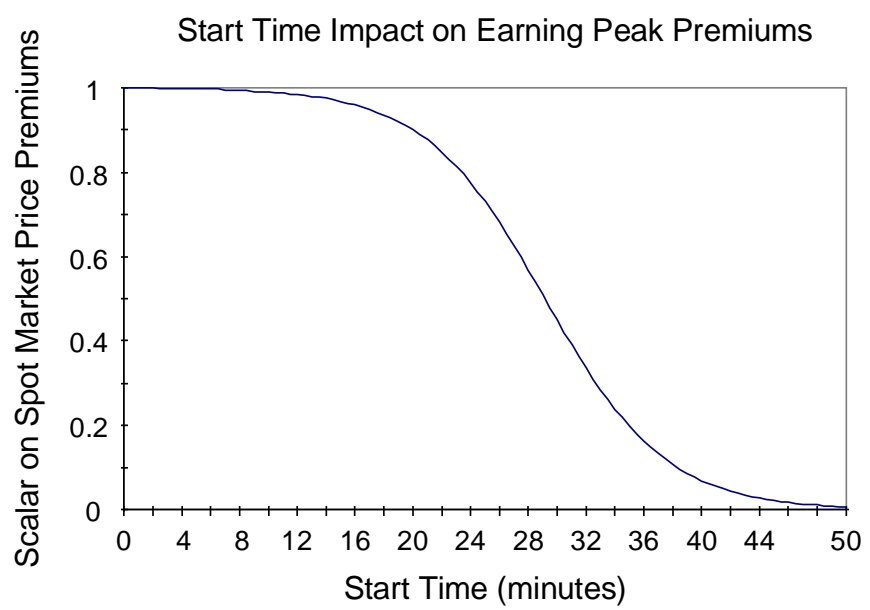

Figure 3-7: Start Time Quality Curve

\section{Start Time Impact on Quality}

It is understood that long start times reduce revenue opportunities for peaking power generators, but beyond the general effect, none of the parties interviewed had a notion as to any details of the relationship. Therefore this has been modeled with a quality curve, as shown in Figure 3-7. The curve yields a scalar which is applied to the upper part of the electricity demand curve as illustrated in Figure 3-2. The quality curve is a hyperbolic tangent determined by two assumptions: StartTime90 and StartTime99. StartTime90 is the value on the horizontal axis at which the curve crosses 0.9 on the vertical axis. StartTime99 is the value on the horizontal axis at which the curve crosses 0.99 on the vertical axis. After setting these two assumptions, it is wise to look at the Start Time Impact on Quality curve to see whether this curve, used by the model, appears reasonable.

\subsection{Ranges Sheet}

The Ranges sheet is used to capture the uncertainty bands for each of the assumptions on the Assumptions sheet. An uncertainty band is defined by a lower limit and an upper limit. The assumption value should lie on or between the limits.

The range sheet is used for a quasi-manual process to perform sensitivity studies. Column $\mathrm{h}$ on the sheet is marked "use." When this column is blank, the linear model on the next sheet is derived from the assumptions in column $\mathrm{c}$, which are the normal assumptions found on the assumption sheet. If an $\mathrm{H}$ is put in a cell in column $\mathrm{h}$, the upper bound of the assumption range is used for the assumption in that row to derive the linear model. An $\mathrm{L}$ in the cell causes the lower bound for the assumption in that row to be used. In this way the impact of uncertainty in the assumptions on the linear model can be evaluated.

\subsection{Linear Sheet}

The Linear sheet contains a linear value model that approximates the full value model for inputs near the inputs on the summary sheet in the leftmost case. The linear model is created by perturbing each of the inputs to the value model and capturing the change induced in the output (surplus value). The change in output divided by the perturbation in the input is an approximation of the partial derivative of the output to the input. Such a partial derivative is calculated for each input to the value model. Perturbations are performed in the vicinity of the inputs to the leftmost case on the summary sheet. The resulting partial derivatives are listed in the Gradient column in the linear model. The model works by multiplying each of the inputs by the corresponding element in the gradient. The products are shown in the third column, labeled Product. The sum of all these products, plus a constant shown at the bottom of the column, gives the linear approximation to Surplus Value.

The Linear Model is the heart or kernel of the value model. The gradient summarizes the essential characteristic of the model: the relationship of inputs to the output. Also, the gradient contains the only factors from the value model that are necessary to create component value models (see Collopy [2001] and Section 8 of this report). 


\subsection{Busiplan Frame Sheet}

The business plan frame contains the calculations behind the business plans presented on the third and fourth worksheets.

\subsection{Notes Sheet}

The notes sheet tracks changes, problems and discrepancies in the model. 


\section{$4 \quad$ NGT Value Model Inputs}

The inputs to the value model can be considered in five groups: Performance, Operation, Emissions, Maintenance and Cost. The inputs are described in table 4-1.

\begin{tabular}{|c|c|c|c|}
\hline Name & Description & Units & Notes \\
\hline \multicolumn{4}{|l|}{ Performance } \\
\hline Power & Output Power & MWatts & Rated \\
\hline HeatRate & Thermal Efficiency at full power & BTU/kW-hr & BTU's of fuel heating value req'd per kW-hr of elec \\
\hline StartTime & Time up to Power & minutes & from turnon to full rated power \\
\hline Water & Water Consumption & $\mathrm{gal} / \mathrm{sec}$ & at rated power \\
\hline \multicolumn{4}{|l|}{ Operation } \\
\hline OpLabor & Operator Labor & ratio & Hours of Operator Labor per Operating Hour \\
\hline AltFuel & Alternate Fuel Capability & True/False & Ability to run on heating oil, coal gas or other \\
\hline Footprint & Footprint Area & $\mathrm{sq} \mathrm{ft}$ & Perimeter of skid plus required clearances \\
\hline \multicolumn{4}{|l|}{ Emissions } \\
\hline NOx & NOx at Full Power & ppm vol & Parts per million in exhaust gas, volumetric \\
\hline $\mathrm{CO}$ & $\mathrm{CO}$ at Full Power & ppm vol & Parts per million in exhaust gas, volumetric \\
\hline Noise & Noise at Full Power & deciBels & near field \\
\hline \multicolumn{4}{|l|}{ Maintenance } \\
\hline Reliability & Mean Time between Failure & hours & oper hrs between unscheduled maint events \\
\hline Downtime & Downtime per Unsch Maint Event & hours & \\
\hline OverhFreq & Mean Time between Overhauls & hours & operating hours between overhauls \\
\hline OverhDur & Downtime per Overhaul & hours & \\
\hline OverhLabor & Labor per Overhaul & hours & \\
\hline PartLife & Mean Part Life & hours & wear-out life of parts weighted by cost \\
\hline \multicolumn{4}{|l|}{ Cost } \\
\hline MfgCost & Manufacturing Cost & $\$ 000$ & 250th unit cost in $2001 \$$ \\
\hline OTSParts & Off the Shelf Parts & none & $\%$ by cost of parts that are learned out \\
\hline DevCost & Development Cost & $\$ 000000$ & Nonrecurring cost from program launch \\
\hline DevTime & Development Time & years & Program launch to first sale \\
\hline
\end{tabular}

Table 4-1: Value Model Inputs

While many of these inputs are self-explanatory, some require more definition:

- HeatRate - Heat rate should be for natural gas operation where the natural gas heating value is consistent with the gas price assumption (see Section 5). Currently, the gas price is based on a heating value of 1,030 BTU per cubic foot.

- OpLabor - This is essentially the requirement for on-site supervision of the power plant. If every unit requires continuous supervision, the input would be 1.0 , or perhaps 1.1 to allow overlap at shift changes. If an off-site supervisor can monitor 10 plants, the value might be 0.1 . This should be an average over the types of installations anticipated.

- AltFuel - This value should be FALSE if the turbine can only operate on natural gas. It should be TRUE if the turbine can also run on standard \#2 distillate or some other alternative fuel.

- PartLife - Part Life is the cost-weighted average part life for the generation system. It is calculated by the following formula:

$$
\frac{\text { MfgCost }}{\text { PartLife }}=\sum_{\text {system }} \frac{\text { Individual Part Cost }}{\text { Individual Part Life }}
$$

Note that the individual terms in this sum are the hardware components of part maintenance cost, in dollars per operating hour, and the sum is total system spare part cost. The reason that Part 
Life is used instead of Maintenance Cost is that it simplifies use of the model. When the MfgCost input is changed, maintenance cost impact in the model is properly adjusted without requiring a separate action by the user.

- MfgCost - Manufacturing cost is learned out (250th system) cost, including packaging, water treatment, exhaust scrubbing, plant civil analysis, installation and startup.

- OTSParts - This input is the percent of MfgCost to which learning curves do not apply, because they are commercial off-the-shelf parts or because they are shared with other programs, such as an aero engine component that is used unchanged (the learning all occurs on the aero program).

- DevCost - This includes all non-recurring costs from the beginning of detailed design on, including all test articles that cannot be subsequently sold, or the discount taken on such articles in subsequent sale.

- DevTime - Time in years from the beginning of detailed design to the time when revenue is received for the first system sold. 


\section{$5 \quad$ NGT Value Model Assumptions}

The value model assumptions are summarized in Table 5-1.

\begin{tabular}{|c|c|c|c|}
\hline Name & Nominal Value & Description & Units \\
\hline \multicolumn{4}{|l|}{ Quality } \\
\hline Reliability 90 & $97.0 \%$ & Reliability serious impact & none \\
\hline Reliability99 & $99.0 \%$ & Reliability initial impact & none \\
\hline StartTime90 & 20 & Time required to start serious impact & minutes \\
\hline StartTime99 & 10 & Time required to start initial impact & minutes \\
\hline $\mathrm{CO} 90$ & 50 & $\mathrm{CO}$ emissions serious impact & ppm vol \\
\hline CO99 & 10 & CO emissions initial impact & ppm vol \\
\hline NOx90 & 25 & NOx emissions serious impact & ppm vol \\
\hline NOx99 & 10 & NOx emissions initial impact & ppm vol \\
\hline Noise90 & 85 & Noise serious impact & $\mathrm{dB}$ (near field) \\
\hline Noise99 & 83 & Noise initial impact & $\mathrm{dB}$ (near field) \\
\hline \multicolumn{4}{|l|}{ Performance } \\
\hline NomElecPrice & $\$ 0.045$ & Avg price to purchase from IPP & $\$$ per kWhr \\
\hline DemandSlope & $50 \%$ & Normalized slope of elec demand & none \\
\hline GasPrice & $\$ 3.25$ & Cost to operator & \$ per million BTU \\
\hline BestFuel & 0.95 & Scalar to Gas cost for Alt fuel & ratio \\
\hline WaterPrice & $\$ 3.80$ & Cost to operator (untreated water) & $\$$ per 000 gal \\
\hline \multicolumn{4}{|l|}{ Price } \\
\hline OpLabRate & $\$ 20.00$ & Boiler operator loaded labor cost & $\$$ per hr \\
\hline WaterPermit & $\$ \quad 20$ & Capital cost to use water & $\$ 000$ per gpm \\
\hline OpDiscRate & $16.0 \%$ & Operator Cashflow discount rate & none \\
\hline Competition & $\$ 53$ & Reservation Price less Actual Price & $\$ 000$ per MWatt \\
\hline \multicolumn{4}{|l|}{ Maintenance } \\
\hline OpporMaint & $60 \%$ & Avg part life consumed at replacement & none \\
\hline MaintLabRate & 50 & Loaded labor rate for overhaul tech & $\$$ per hour \\
\hline \multicolumn{4}{|l|}{ Business Plan } \\
\hline $\begin{array}{l}\text { LearningCurv } \\
\mathrm{e}\end{array}$ & $90 \%$ & Cost improv't rate during production & none \\
\hline Preload & 6 & Production preload to learning curve & units \\
\hline Market Size & 67 & Annual demand, incl competitors & units \\
\hline RRDiscRate & $11.6 \%$ & Rolls-Royce cashflow discount rate & none \\
\hline EarlyEntry & 360 & Impact of late product introduction & $\$ 000000$ per yr \\
\hline MaintShift & 2.0 & Sale date to start of maint costs & years \\
\hline
\end{tabular}

Table 5-1: Assumptions

The following subsections discuss these assumptions one group at a time. The data discussed was provided by the PB Power customer survey and by Richard Knight, Bob Moritz, Dennis Rayome, Larry Davis, Brad Pokosh, Paul Fletcher, Jim Oswald, Mike Raines and Martyn McArthur. 


\subsection{Quality}

These ten assumptions are parameters for the five logistics curves ${ }^{*}$ used in the value model. Reliability is a rising logistics curve. The other four are falling curves. The $99 \%$ value is the point at which the quality impact is just noticeable. The $95 \%$ value is the point at which the quality impact becomes very serious, making the design unacceptable.

Reliability90

Nominal: $\quad 97 \% \quad$ Range: $96 \%-97 \%$

Larry Davis believes that customers will demand a guarantee of $96 \%$ reliability.

Reliability99

Nominal: $\quad 99 \% \quad$ Range: $99 \%-99.5 \%$

Larry Davis believes that customers desire a reliability level of $99 \%$. They are not likely to pay a premium price for marginal improvements above 99\%. (The economic impact of earning more revenue for reliability levels above $99 \%$ is accounted for elsewhere in the model. The quality factor is for the additional subjective impact on price.) Unocal said system reliability should be $96 \%$. Cogentrix, Entergy and Nations Energy asked for 97\%. Avista, BP Global Power, El Paso, Indeck, Marathon and Trigen expressed a requirement for 98\% reliability. Calpine and Enron expect 99\% reliability. Several customers asked for reliability equal to or greater than the LM6000.

\section{StartTime 90}

Nominal:

20 minutes

Range: 20 - 40 minutes

StartTime 99

Nominal: $\quad 10$ minutes $\quad$ Range: 7 - 20 minutes

Calpine said 10 minute starts are required for ancillary services markets. Edison Mission Energy, Enron, Los Angeles Department of Power, PG\&E and SMUD also said 10 minutes starts would be required. Avista mentioned a 10 to 15 minute startup would be required, but this could vary by markets and specific requirements for ancillary service contracts. Conoco and Indeck also said that 10 to 15 minutes is an acceptable range. BP Global Power said 15 minutes would be needed for ancillary services and 20 minutes to support trading. Cogentrix and El Paso said the desirable range is 20 to 30 minutes. Unocal said 30 minutes would be adequate.

CO90

Nominal: $\quad 50 \mathrm{ppm} \quad$ Range: $15-50 \mathrm{ppm}$

Emissions requirements in most markets are currently at $50 \mathrm{ppm}$ or better. A system that put out more than $50 \mathrm{ppm}$ would not be permitted in most markets. However, the high end of what is tolerable must include $50 \mathrm{ppm}$ since systems at this level are being sold today. This data was supplied by Larry Davis and confirmed by Martyn McArthur.

CO99

Nominal: $\quad 10 \mathrm{ppm} \quad$ Range: $9-10 \mathrm{ppm}$

The most conservative vision of the near future, according to Larry Davis, is a requirement for $10 \mathrm{ppm}$ max emissions of $\mathrm{CO}$ and NOx. Power producers are not considered likely to pay higher prices for systems that significantly improve upon this limit, such as emitting $5 \mathrm{ppm}$.

* See Figure 3-1 and the following text in Section 3 for more information about logistics curves.

NGT Value Model 
NOx90

Nominal: $\quad 25 \mathrm{ppm} \quad$ Range: $15-25 \mathrm{ppm}$

Emissions requirements in most markets are currently at $25-29 \mathrm{ppm}$ or better. A system that put out more than $25 \mathrm{ppm}$ would not be permitted in most markets. However, the high end of what is tolerable must include $25 \mathrm{ppm}$ since systems at this level are being sold today. This data was supplied by Larry Davis and confirmed by Martyn McArthur.

NOx99

Nominal: $\quad 10 \mathrm{ppm} \quad$ Range: $9-10 \mathrm{ppm}$

The most conservative vision of the near future, according to Larry Davis, is a requirement for $10 \mathrm{ppm}$ max emissions of $\mathrm{CO}$ and NOx. Power producers are not considered likely to pay higher prices for systems that significantly improve upon this limit, such as emitting $5 \mathrm{ppm}$.

Noise 90

Nominal: $\quad 85 \mathrm{~dB} \quad$ Range: $84-90 \mathrm{~dB}$

Noise99

Nominal: $\quad 83 \mathrm{~dB} \quad$ Range: $82-84 \mathrm{~dB}$

Larry Davis stated that the noise level would need to be less than $85 \mathrm{~dB}$, near field or far field, to be competitive. He suggested the range of $83 \mathrm{~dB}$ to $85 \mathrm{~dB}$ is the range of interest, with $83 \mathrm{~dB}$ meeting the highest customer expectations.

\subsection{Performance}

The NomElecPrice is the average price across all IPPs that they can expect to be paid for baseload electricity during the time the generation systems are produced, ignoring general inflation.

NomPremium is the average increment added to the price of electricity during peak demand periods, ratioed to the NomElecPrice. "Peak demand periods" must correspond to the PartHrperYr assumption (see Price subsection below). In other words, if part time systems are assumed to run 4,000 hours per year (mid-merit operation) then NomPremium should reflect the average premium that would be earned over the highest demand 4,000 hours of the year. This would be a lower average premium than would be earned over a shorter duty cycle such as the highest demand 1,000 hours of the year.

GasPrice and WaterPrice are self-explanatory, except to note that the price of water is for untreated water, since the cost of a purification plant should be included in the generator system manufacturing cost. It is reasonable to include in these costs an allowance for amortized cost of permitting and piping fuel and water to the plant.

BestFuel is the average cost of fuel given the ability to switch between natural gas and \#2 distillate (heating oil) divided by the average cost of fuel using only natural gas. This ratio must be less than or equal to one, under the assumption that the operator will always choose the cheapest fuel.

NomElecPrice

Nominal: $\quad \$ 0.045$ per kW-hr $\quad$ Range: $\$ 0.040-0.060$

DemandSlope

Nominal: $\quad 50 \% \quad$ Range: $14 \%-90 \%$

Martyn McArthur said that electricity prices range from $2-3 £$ per MW-hr to $150 £$ per MW-hr. This translates to a range from $\$ 0.003$ to $\$ 0.225$ per $\mathrm{kW}-\mathrm{hr}$. Richard Knight suggested a flatter curve, with a minimum of $\$ 0.05$ and $\$ 0.055$ at the midpoint (50\%). Dennis Rayome said that peaking operators earn $\$ 0.30$ per $\mathrm{kW}-\mathrm{hr}$, slightly above the top of McArthur's range. The assumptions used give a lower 
minimum that Richard's (about \$0.022) but about the same slope (not as steep as Martyn's). The lower minimum yields reasonable profits with the projected gas price of $\$ 3.25$ per MBTU, which is somewhat lower than current prices.

GasPrice

Nominal: $\quad \$ 3.25$ per million BTU Range: \$ $3.00-5.00$

Richard Knight recommended the value between $\$ 3.00$ and $\$ 3.50$ based on a CERA study, which he considers the best analysis in the industry. This is a projection for four to five years out after a peak near $\$ 4.75$ in 2001. Larry Davis proposed a range of $\$ 4.00$ to $\$ 6.00$ per MBTU, which is reasonable for 2001 but is not expected to hold up in the future as supply responds to the high prices. Dennis Rayome cited a value of $\$ 9.00$ per MBTU. Cincinnati Gas \& Electric retails gas at \$1.60 per MBTU. These values seem extreme.

\section{BestFuel}

Nominal:

0.95

Range: $0.90-0.95$

Dual fuel is often used simply to allow the plant to purchase gas at interruptible rates, even though the supply may never be interrupted. The interpretation here is that dual fuel allows the lower, interruptible rates and the option of using fuel oil when prices make oil more economical. The result of both these effects is to reduce the mean cost of fuel.

None of the parties interviewed by PB Power or DFM Consulting had any input about the amount of the fuel cost reduction with dual fuel. Because many customers said they would not use dual fuel, the reduction is presumably small. The sensitivity study indicated that this assumption is among the least important to value model operation, so further research is not strongly warranted.

\section{WaterPrice}

Nominal: $\quad \$ 3.80$ per 1,000 gallons Range: $\$ 1.00-5.00$

Larry Davis stated that water in wet cycle plants runs about 5\% of the cost of fuel. $\$ 3.80$ makes the LM6000 water cost equal 5\% of the fuel cost. Richard Knight recommended $\$ 0.001$ per $\mathrm{kg}$ which translates to $\$ 3.80$ per 1,000 gallons. Entergy said water prices in the Eastern US were $\$ 3$ to $\$ 5$ per 1,000 gallons. Dennis Rayome quoted a price of $\$ 0.00264$ per lb., which equals $\$ 22$ per 1,000 gallons, but this does not line up with the other data, probably because he was considering pre-treated water. Conoco cited prices of $\$ 0.13$ (Lake Charles, LA) and $\$ 0.50$ to $\$ 1.00$ per 1,000 gallons (Corpus Christi, TX) for untreated water. SMUD said local water (Sacramento) was much less than $\$ 1$ per 1,000 gallons.

\subsection{Price}

These six assumptions are self explanatory except for Competition. Competition is the impact of competitors on price. Roughly, it is the highest price the generation system could earn in a monopoly market minus the price it will actually earn in the real market.

\section{OpLabRate}

Nominal: $\quad \$ 20.00$ per hour $\quad$ Range: $\$ 10.00-\$ 40.00$

None of the interviews provided any input on labor rates.

WaterPermit

Nominal: $\quad$ Range: $\$ 1,000-40,000$ per gpm

\footnotetext{
* For a more thorough explanation, including explanations of Reservation Price and Surplus Value, see the paper "Surplus Value in Propulsion System Design" at www.dfmconsulting.com/research.htm
} 
Richard Knight said water permitting would typically run $11 \%$ of total installation cost. Avista noted that permitting and water rights are usually major issues.

OpDiscRate

Nominal: $\quad 16.0 \% \quad$ Range: $12 \%$ to $20 \%$

Martyn McArthur said that, across different customers, this could range from 6\% to 25\% (note that the range of $12 \%$ to $20 \%$ is the uncertainty range of the average of all the customers). Utilities would fall in the $6 \%$ to $8 \%$ range, and oil and gas companies closer to $25 \%$. He suggested using a range of $12 \%$ to $15 \%$. Mike Raine estimated the discount rate to be $15 \%$ to $20 \%$. Dennis Rayome earlier said that his customers usually required a $25 \%$ to $30 \%$ rate of return to justify investments. McArthur believes this is typical of the oil and gas customers that buy the smaller systems which Rayome marketed, but is not applicable to NGT-class systems.

\section{Competition}

Nominal: $\quad \$ 53,000$ per MWatt

Competition is a tuning coefficient more than an assumption. It can be used to zero out the surplus value of the baseline case in a comparison so that the surplus value results for other cases are actually deltas from the baseline. The value of $\$ 53 \mathrm{~K}$ was chosen to fix the normalized price of the LM6000 Sprint at $\$ 500 / \mathrm{kW}$, which is a value that Rolls provided to PB Power.

\subsection{Maintenance}

During an overhaul, some parts will be replaced even though they are not worn out to avoid the risk that they might where out prior to the next scheduled overhaul. This practice is called opportunistic maintenance. Although it reduces the frequency of overhauls, it increases the cost of spare parts, since parts are replaced more frequently. OpporMaint is the ratio of the average life of parts at the time when they are replaced divided by the design life of the parts.

MaintLabRate is self explanatory.

OpporMaint

Nominal: $\quad 60 \% \quad$ Range: $50 \%-75 \%$

This is essentially the rate used at an earlier value study at Rolls-Royce, Indianapolis. The sensitivity analysis indicated this is one of the least important assumptions.

\section{MaintLabRate}

Nominal: $\quad \$ 50.00$ per hour $\quad$ Range: $\$ 40-\$ 80$ per hour

None of the interviews provided any input on labor rates.

\subsection{Business Plan}

LearningCurve is the ratio of the cost of part number $2 n$ to the cost of part $n$, for all parts which are not learned out at the beginning of the program. The ratio is fairly constant according to the economic theory manufacturing.

Preload is the number of systems manufactured on normal production lines prior to the manufacture of the first system sold for revenue.

LearningCurve

Nominal: $\quad 90 \% \quad$ Range: $86 \%-94 \%$

This is a typical aerospace industry value. 
Nominal: $\quad 6$ units $\quad$ Range: 1 to 10 units

The development estimate prepared in Mt. Vernon implies that 6 units or parts of units would be built during development.

\section{MarketSize}

Nominal:

67 units

Range: 30 to 200 units

Brad Pokosh's business plan shows 20 units per year sold in mid-production. Assuming a $30 \%$ market share, this implies a market of 67 units per year.

\section{RRDiscRate}

Nominal: $\quad 11.6 \% \quad$ Range: $11.0 \%$ to $11.6 \%$

Brad Pokosh's business plan uses $11 \%$, but Dean Roberts on an earlier study had cited a value of $11.6 \%$. Dean stated very clearly that the $11.6 \%$ was a real discount rate (suitable for use in constant dollar studies). Brad uses his $11 \%$ with a $2 \%$ inflation rate, which implies a real discount rate of $9 \%$. Dennis Rayome uses an $8 \%$ hurdle for investment decisions. By industry standards, this is a very low discount rate.

\section{EarlyEntry}

Nominal:

$$
\$ 360,000,000 \text { per year Range: } \$ 130 \text { to } \$ 500 \text { million per year }
$$

An early study for the AE30xx aircraft engine went into considerable depth to determine this factor, which turned out to be $\$ 360 \mathrm{M}$ per year, or about $\$ 1 \mathrm{M}$ per day. The prime driver was the gross income stream. Brad Pokosh's business plan shows a gross income stream very similar to the AE30xx, so the same factor probably fits. This assumption was determined to be non-critical in the sensitivity study, so no further investigation was pursued.

\section{MaintShift}

Nominal: $\quad 2.0$ years $\quad$ Range: 2 to 4 years

Two years is the shift between production ramp-up and aftermarket ramp-up shown in Brad Pokosh's business plan. 


\section{$6 \quad$ Sensitivity Study on Value Model Assumptions}

In this section are shown tornado diagrams that were generated in an analysis of the sensitivity of the value model to the assumptions on the Assumption Sheet. Each diagram plots a partial derivative from the Gradient in the Linear Model (see section 3.8 Linear Sheet). For example, the EngineLife diagram is a bar graph of variations in the partial derivative of Surplus Value to variations in assumptions. Every bar is formed by varying one assumption from its lower bound to its upper bound and plotting the resulting variation in the partial derivative. The vertical line on the graph is the value of the partial derivative when all assumptions are at their nominal values. Because the Linear Model captures the essence of the value model, the sensitivity of the coefficients of the linear model (the partial derivatives) to changes in the assumptions essentially measures the sensitivity of the model to the assumptions.

Table 6-1 shows the nominal values, lower bounds and upper bounds for each assumption. These are shown on the Ranges Sheet in the value model spreadsheet workbook.

\begin{tabular}{|c|c|c|c|c|c|}
\hline \multirow{2}{*}{$\begin{array}{r}\text { Name } \\
\text { Quality }\end{array}$} & Nominal Value & \multicolumn{2}{|c|}{ Upper Bound } & \multicolumn{2}{|c|}{ Lower Bound Units } \\
\hline & & & & & \\
\hline Reliability90 & $97.0 \%$ & & $97.0 \%$ & & $96.0 \%$ none \\
\hline Reliability99 & $99.0 \%$ & & $99.5 \%$ & & $99.0 \%$ none \\
\hline StartTime 90 & 20 & & 40 & & 20 minutes \\
\hline StartTime99 & 10 & & 10 & & 7 minutes \\
\hline CO90 & 50 & & 50 & & 15 ppm vol \\
\hline CO99 & 10 & & 10 & & 9 ppm vol \\
\hline NOx90 & 25 & & 25 & & 15 ppm vol \\
\hline NOx99 & 10 & & 10 & & 9 ppm vol \\
\hline Noise90 & 85 & & 90 & & $84 \mathrm{~dB}$ (near field) \\
\hline Noise99 & 83 & & 84 & & $82 \mathrm{~dB}$ (near field) \\
\hline \multicolumn{6}{|l|}{ Performance } \\
\hline NomElecPrice & \$ 0.045 & $\$$ & 0.060 & $\$$ & $0.040 \$$ per kWhr \\
\hline DemandSlope & $50 \%$ & & $90 \%$ & & $14 \%$ none \\
\hline GasPrice & $\$ 3.25$ & $\$$ & 5.00 & $\$$ & $3.00 \$$ per million BTU \\
\hline BestFuel & 0.95 & & 0.95 & & 0.90 ratio \\
\hline WaterPrice & $\$ 3.80$ & $\$$ & 5.00 & $\$$ & $1.00 \$$ per $000 \mathrm{gal}$ \\
\hline \multicolumn{6}{|l|}{ Price } \\
\hline OpLabRate & $\$ 20.00$ & $\$$ & 40.00 & $\$$ & $10.00 \$$ per $\mathrm{hr}$ \\
\hline WaterPermit & 20 & $\$$ & 40 & $\$$ & $1 \$ 000$ per gpm \\
\hline OpDiscRate & $16.0 \%$ & & $20.0 \%$ & & $12.0 \%$ none \\
\hline \multicolumn{6}{|l|}{ Maintenance } \\
\hline OpporMaint & $60 \%$ & & $75 \%$ & & $50 \%$ none \\
\hline MaintLabRate & 50 & & 80 & & $40 \$$ per hour \\
\hline \multicolumn{6}{|l|}{ Business Plan } \\
\hline LearningCurve & $90 \%$ & & $94 \%$ & & $86 \%$ none \\
\hline Preload & 6 & & 10 & & 1 units \\
\hline MarketSize & 67 & & 200 & & 30 units \\
\hline RRDiscRate & $11.6 \%$ & & $11.6 \%$ & & $11.0 \%$ none \\
\hline EarlyEntry & 360 & & 500 & & $130 \$ 000000$ per yr \\
\hline MaintShift & 2.0 & & 4 & & 2 years \\
\hline
\end{tabular}

Table 7-1: Ranges used for Assumptions in the Sensitivity Analysis 
NomElecPrice

\section{DemandSlope}

\section{OpDiscRate}

\section{GasPrice}

\section{MarketSize}

Figure 7-1: Assumptions which contribute the most to uncertainty in the value model

Figure 7-1 summarizes the results of the sensitivity analysis. The chart plots the frequency at which assumptions occur at or near the tops of the tornado diagrams which follow. The five assumptions shown in Figure 7-1 dominate the uncertainty in the model - the next highest assumption had only half the frequency of MarketSize. The top two, NomElectricPrice and DemandSlope, shape the electricity demand curve. The shape of the electricity demand curve impacts the relative advantages of expensive highefficiency plants versus inexpensive low-efficiency plants. The next factor, OpDiscRate, relates to the time period within which the operator must recover the capital cost of the plant. Therefore, it to directly impacts the relative worth of plant efficiency versus plant cost. Gas price also plays directly into yield and the efficiency versus cost trade. Market size is critical for the trade of development cost versus manufacturing cost, since it determines over how many production units the development can be amortized.

In a nutshell, the study indicates that an understanding of future gas and electricity prices, and particularly the variation between base and peak electricity prices (which is strongly influenced by the distribution of efficiency across the capital stock of generation plants) is essential to determining the design of future systems. Also, the financial stability and organization of the power producers (reflected

\section{Power}

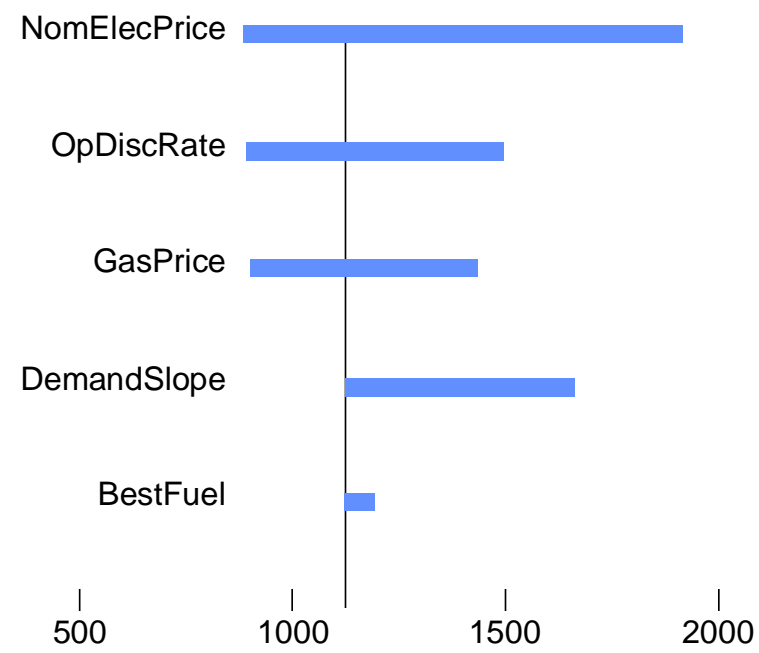

\$ 000 Unit Profit per MW

NGT Value Model

Figure 7-2: Uncertainty in the derivative of unit profit to power Consulting 


\section{HeatRate}

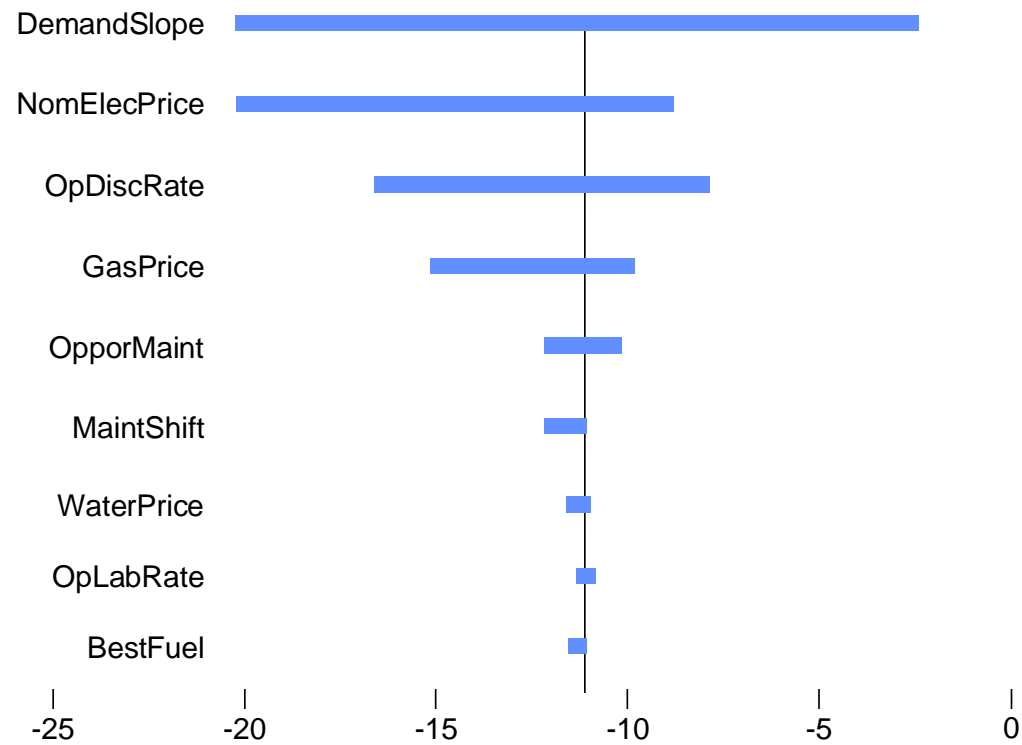

\$ 000 Unit Profit per (BTU per kW-hr)

Figure 7-3: Uncertainty in the derivative of Unit Profit versus HeatRate

in their cashflow discount rates) is essential to determining the types of systems they will buy. Government-supported utilities have a much longer time horizon and are much more willing to invest in expensive, high-efficiency plants than fly-by-night IPP's.

Each tornado diagram measures the partial derivative of the model output to one of the model inputs under various values of assumptions. Each assumption is set at its lowest and highest possible value (see section 3.7, Ranges Sheet) while all other assumptions are held at their nominal values. The blue bars in the diagram illustrate the change in the derivative as the assumptions vary from their low extreme to their high extreme.

\section{StartTime}

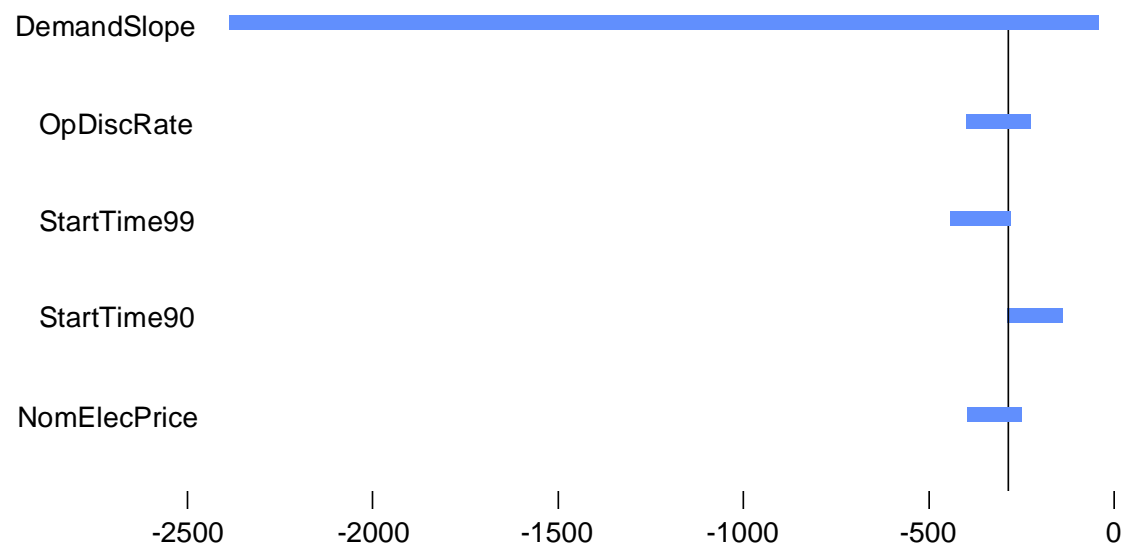

$\$ 000$ Unit Profit per Minute

Figure 7-4: Uncertainty in the derivative of Unit Profit to StartTime 
Water

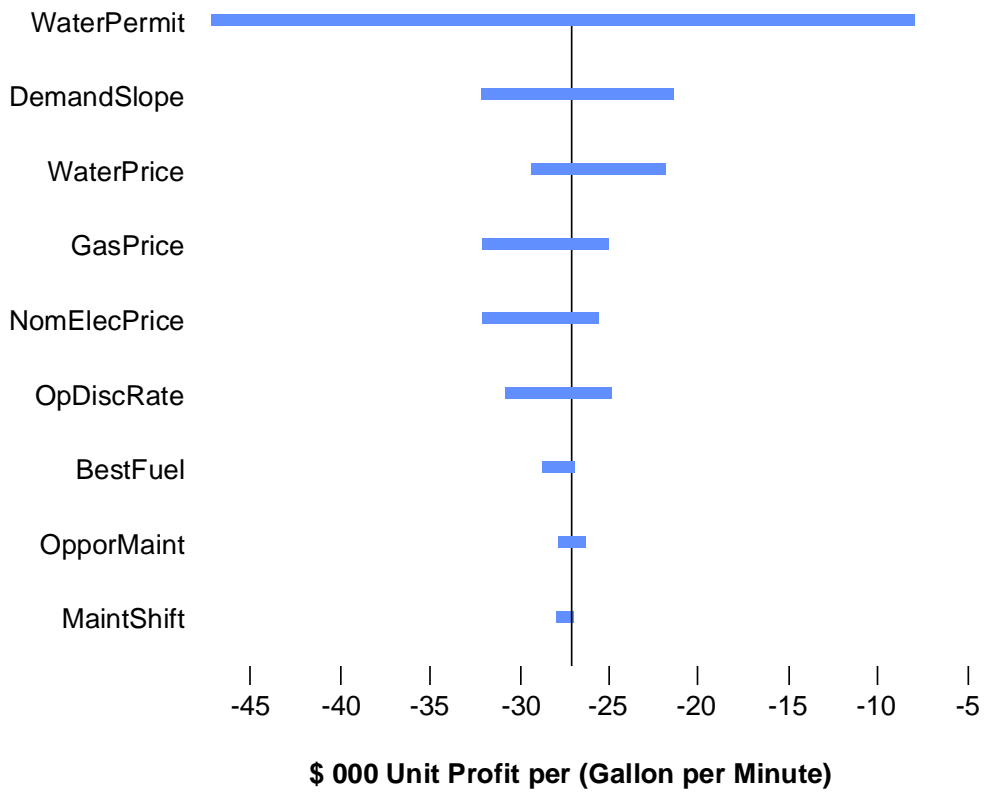

Figure 7-5: Uncertainty in the derivative of Unit Profit to Water

In Figure 7-2, the left end of the bar labeled "GasPrice" is the value of the derivative of surplus value to power when the Gas Price assumption is set to the top of its uncertainty range, $\$ 5.00$, while all other assumptions are at their nominal values. The vertical black line indicates the value of the derivative when GasPrice is at its nominal value. The right end of the bar marks the derivative when GasPrice is at its minimum value. The bars are ranked in descending order of length. Short bars are not shown, although the sensitivity calculations were done for all assumptions for all derivatives. Thus the upper bars indicate the assumptions whose uncertainty contributes the most to uncertainty in the model.

\section{OpLabor}

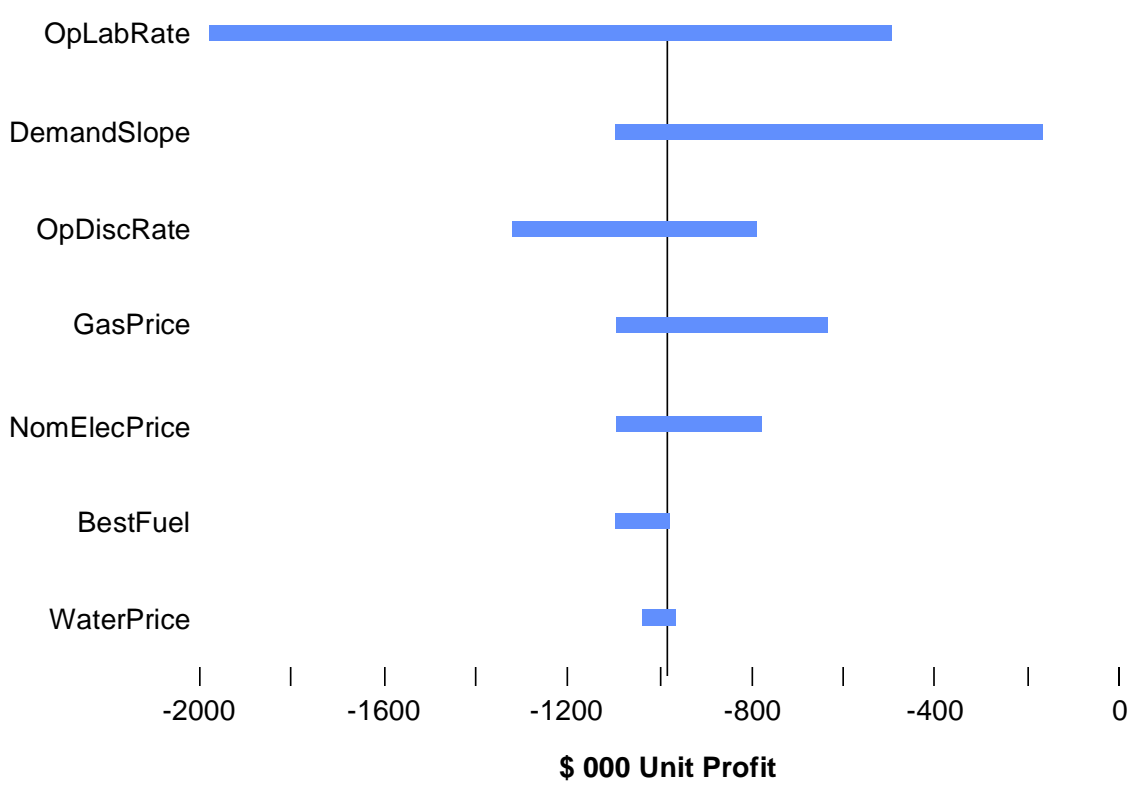

Figure 7-6: Uncertainty in the derivative of Unit Profit to OpLabor 


\section{AltFuel}

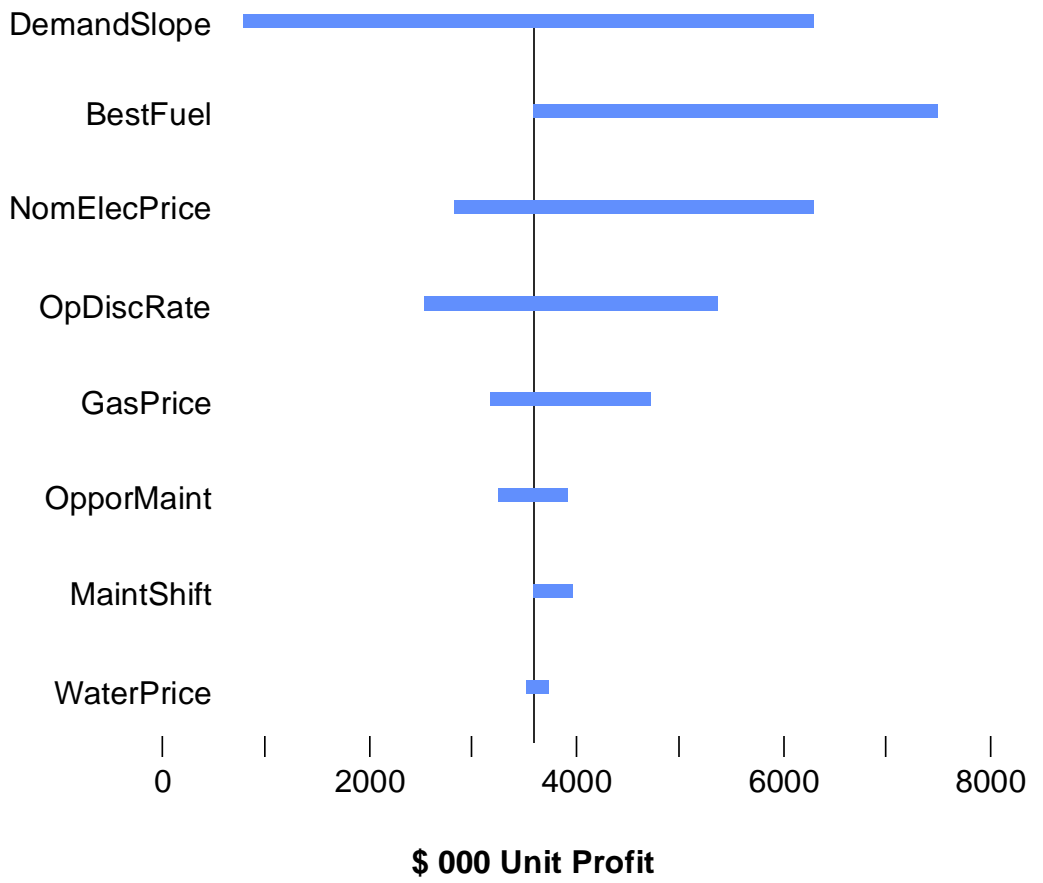

Figure 7-7: Uncertainty in the derivative of Unit Profit to AltFuel

\section{Install}

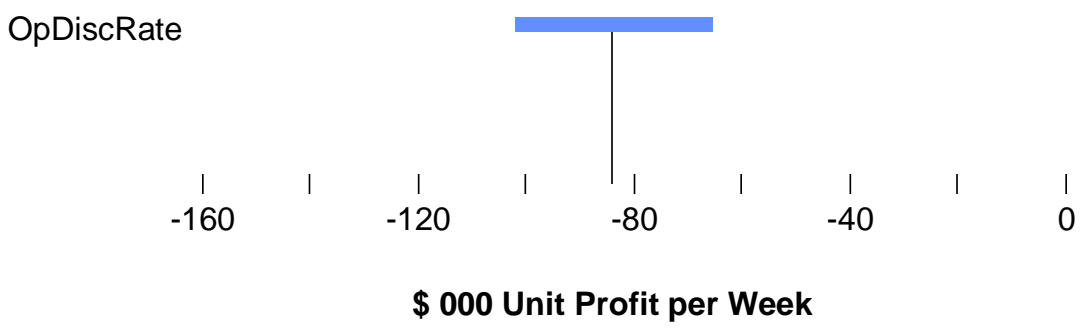

Figure 7-8: Uncertainty in the derivative of Unit Profit to Install 


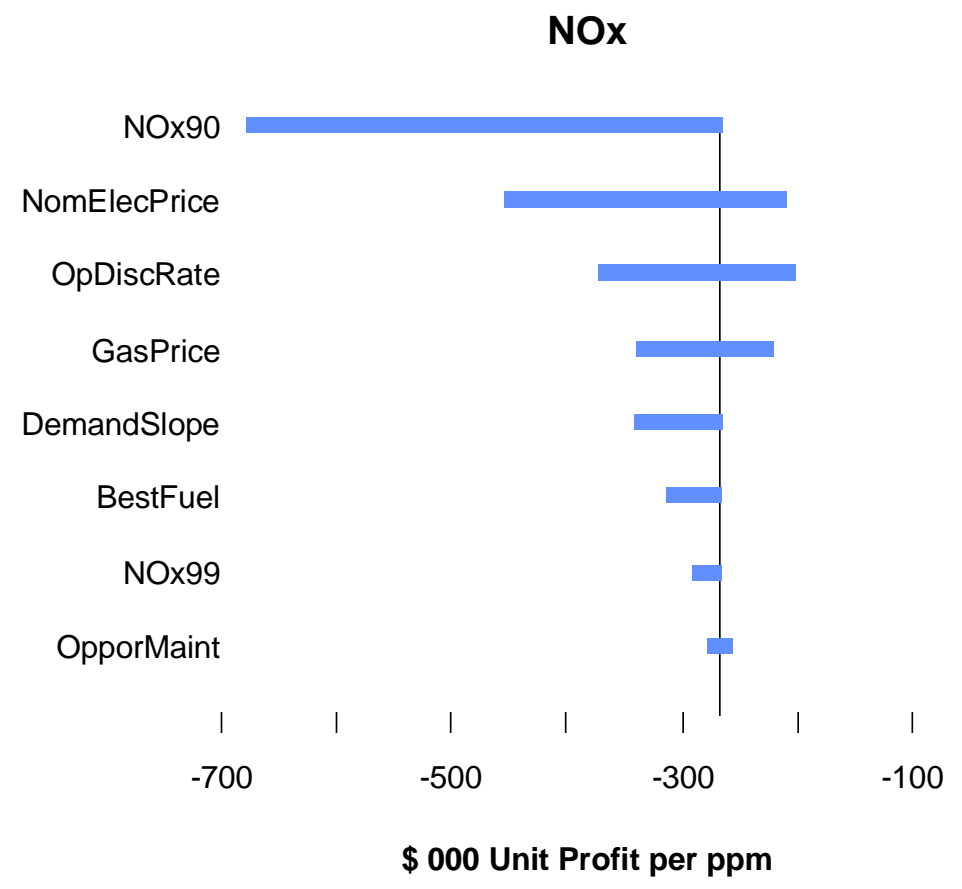

Figure 7-9: Uncertainty in the derivative of Unit Profit to NOx

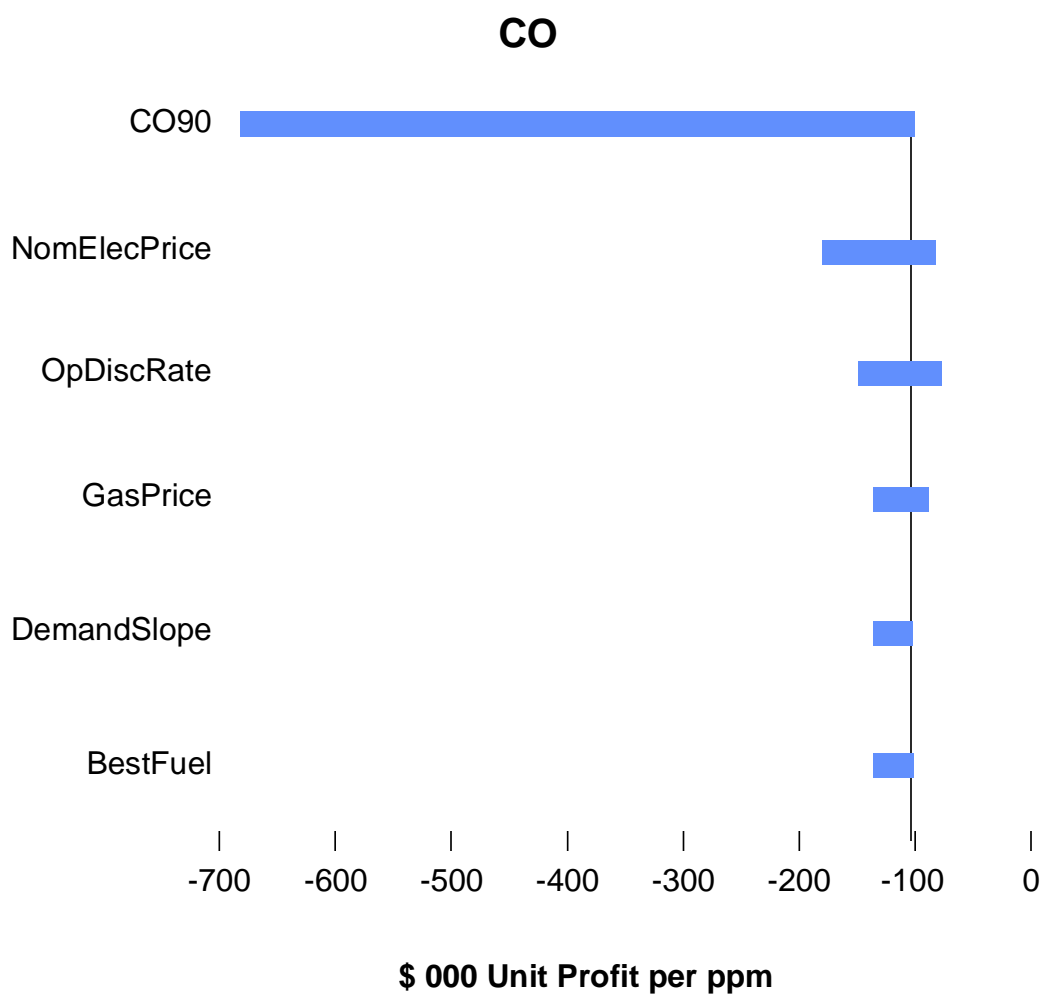

Figure 7-10 Uncertainty in the derivative of Unit Profit to CO 


\section{Noise}

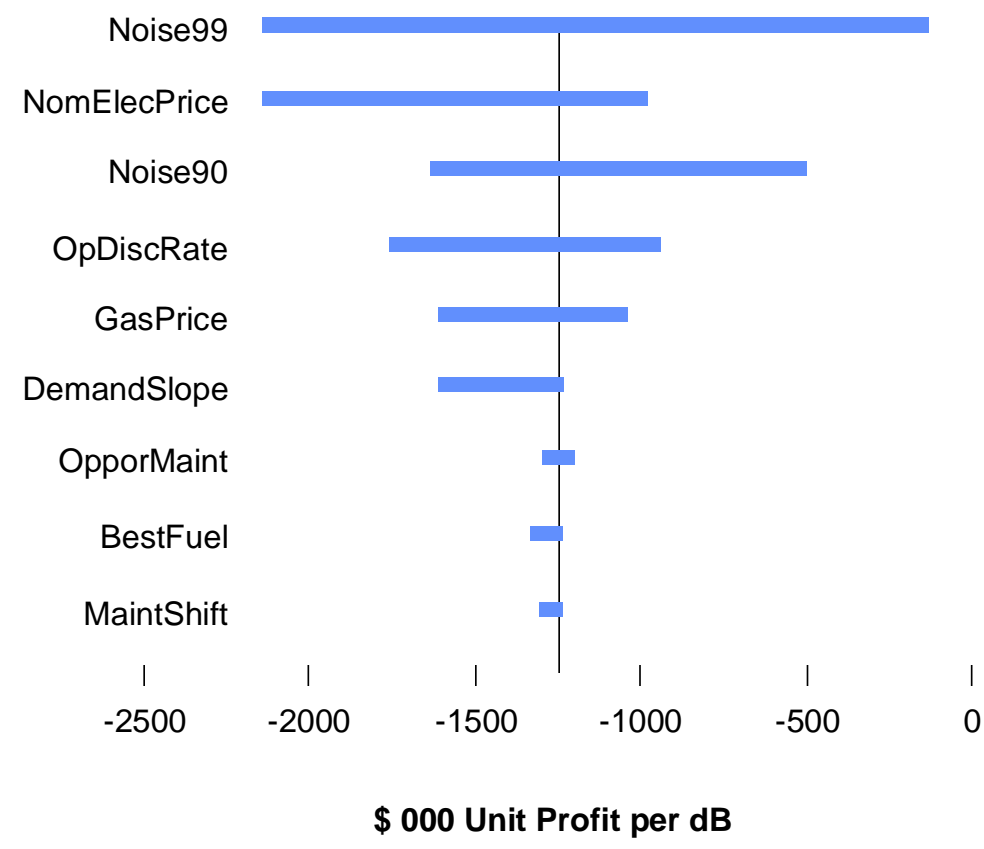

Figure 7-11: Uncertainty in the derivative of Unit Profit to Noise

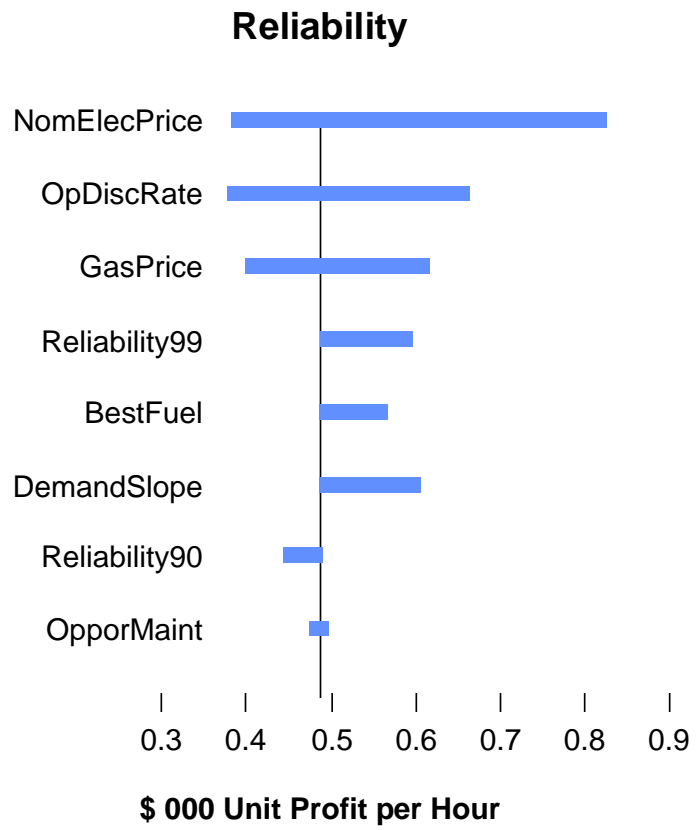

Figure 7-12: Uncertainty in the derivative of Unit Profit to Reliability 


\section{Downtime}

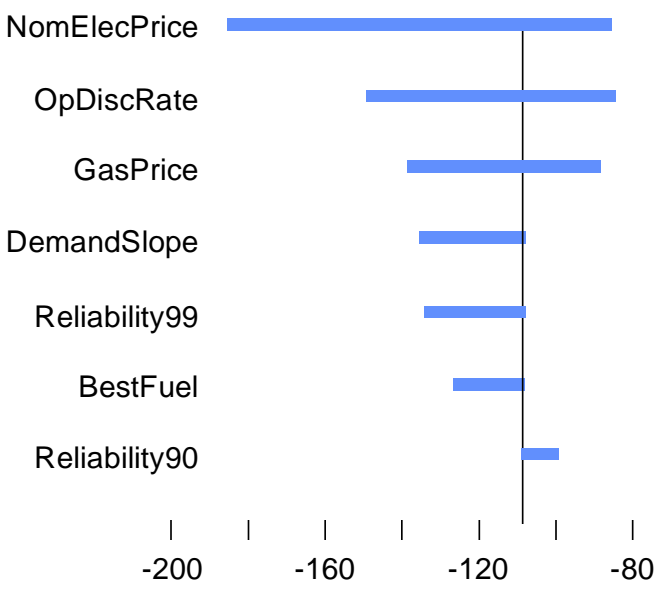

\$ 000 Unit Profit per Hour

Figure 7-13: Uncertainty in the derivative of Unit Profit to Downtime

\section{OverhFreq}

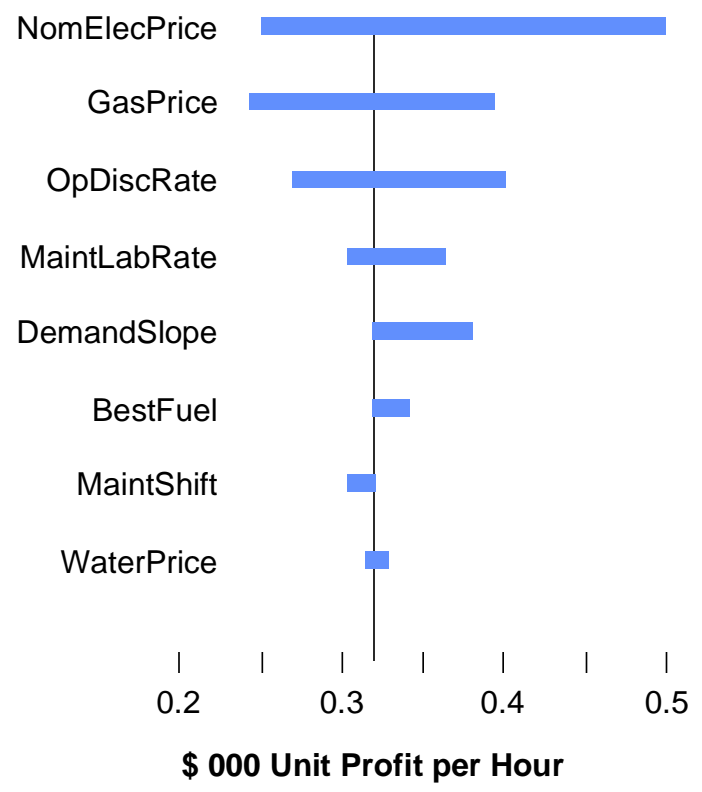

Figure 7-14: Uncertainty in the derivative of Surplus Value to OverFreq 


\section{OverhDur}

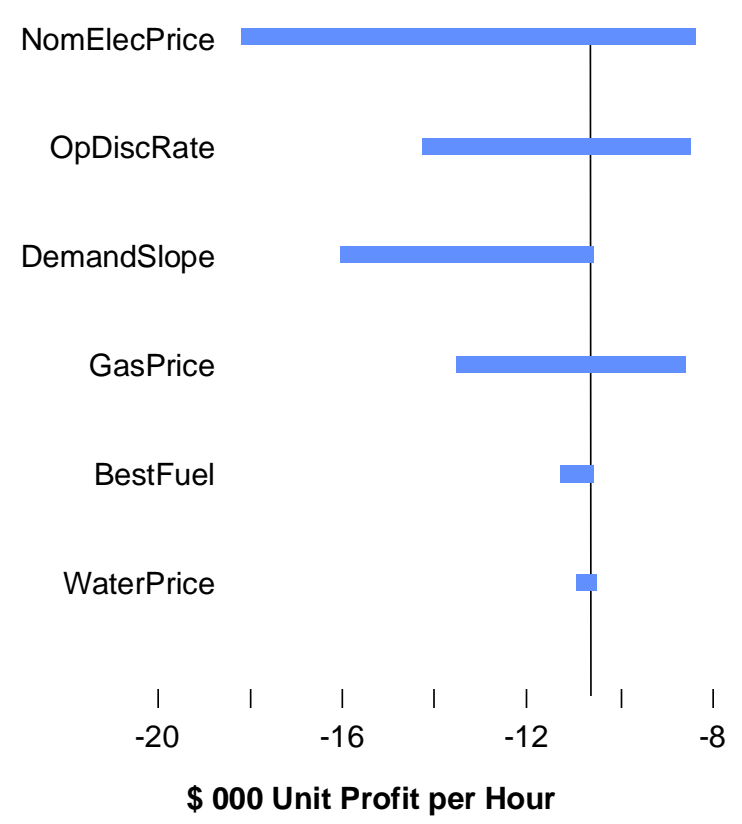

Figure 7-15: Uncertainty in the derivative of Unit Profit to OverhDur

\section{OverhLabor}

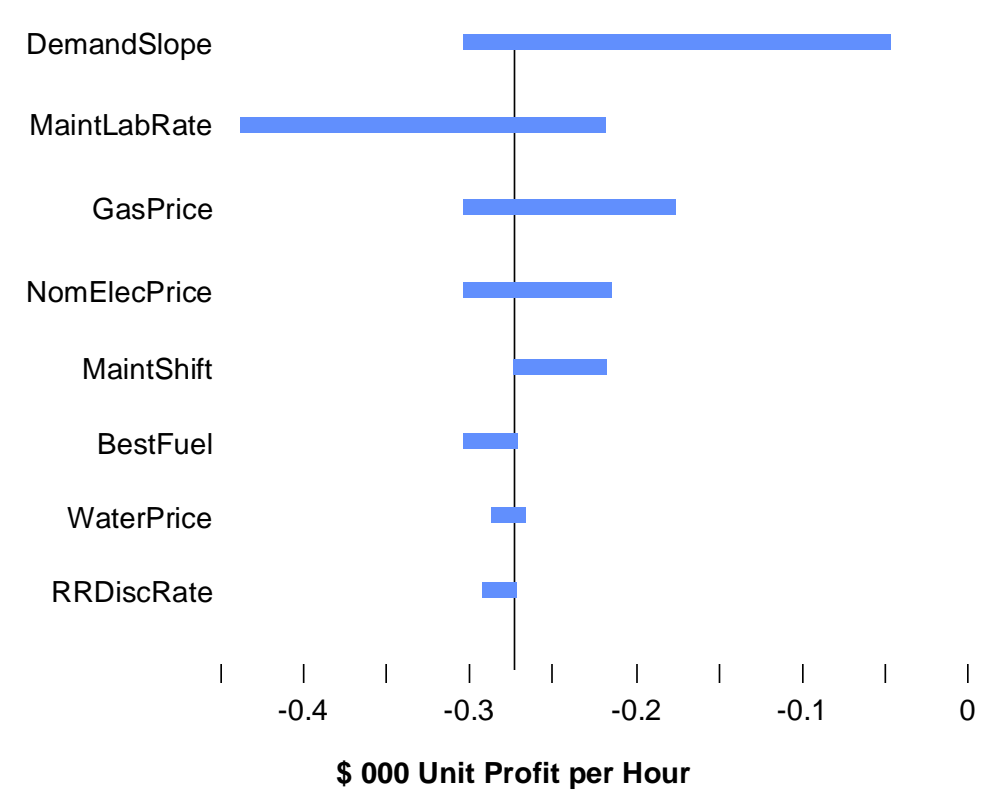

Figure 7-16: Uncertainty in the derivative of Unit Profit to OverhLabor 


\section{PartLife}

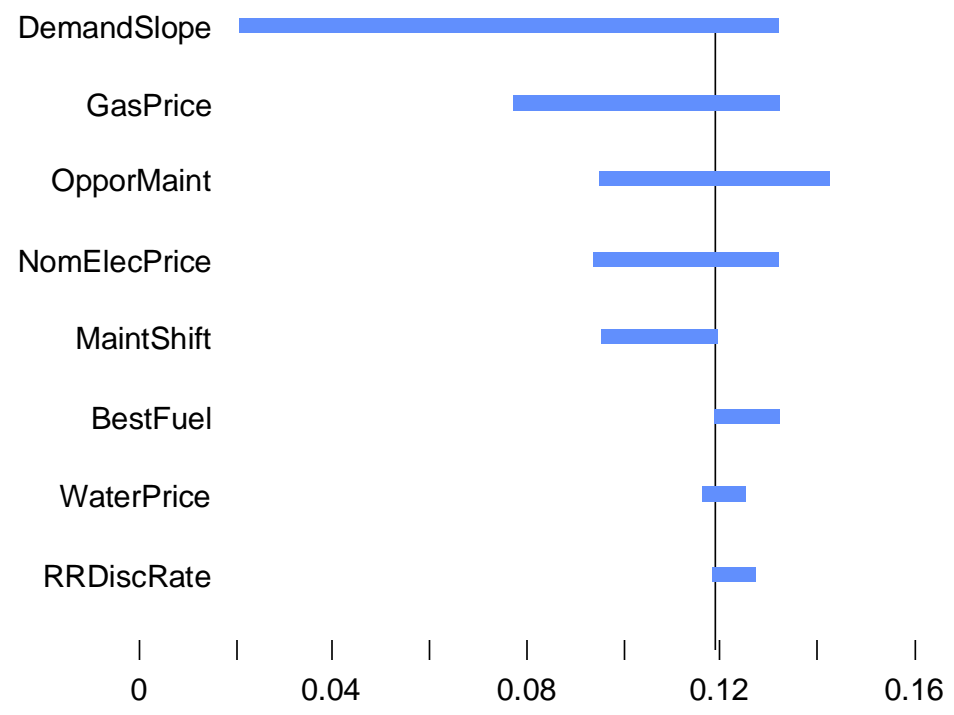

\section{\$ 000 Unit Profit per Hour}

Figure 7-17: Uncertainty in the derivative of Unit Profit to PartLife
MfgCost

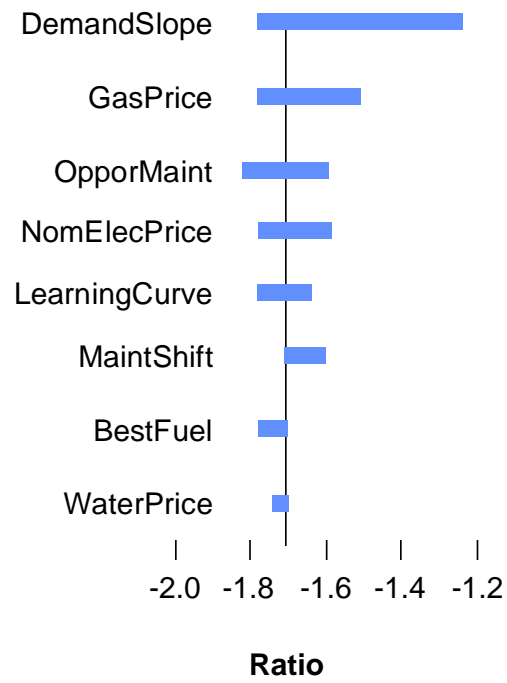

Figure 7-18: Uncertainty in the derivative of Unit Profit to MfgCost

\section{OTSParts}

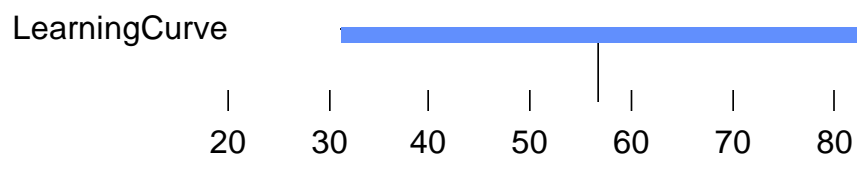

\section{\$ 000 Unit Profit per \% Off-the-Shelf Parts}

Figure 7-19: Uncertainty in the derivative of Unit Profit to OTSParts 


\section{DevCost}

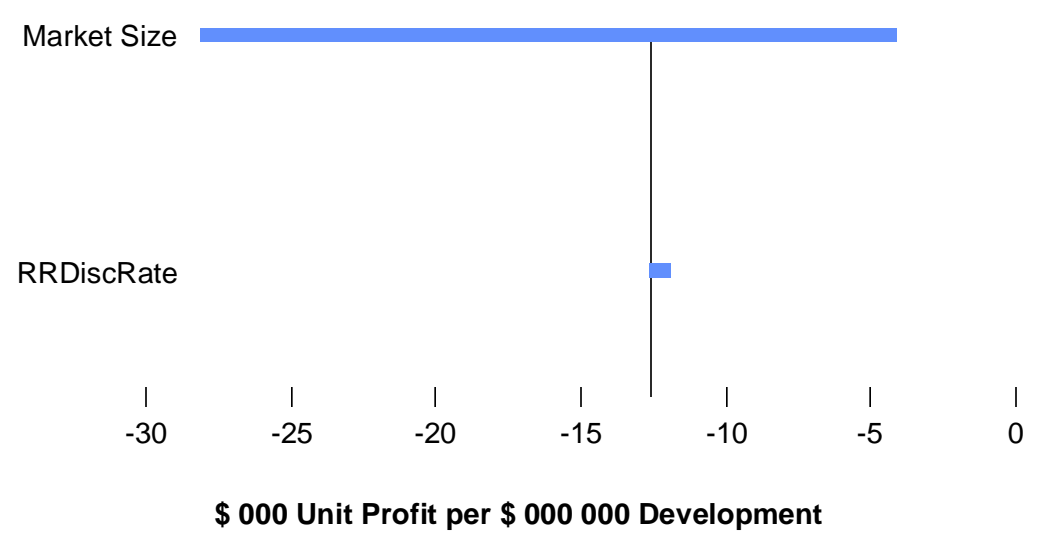

Figure 7-20: Uncertainty in the derivative of Unit Profit to DevCost

\section{DevTime}

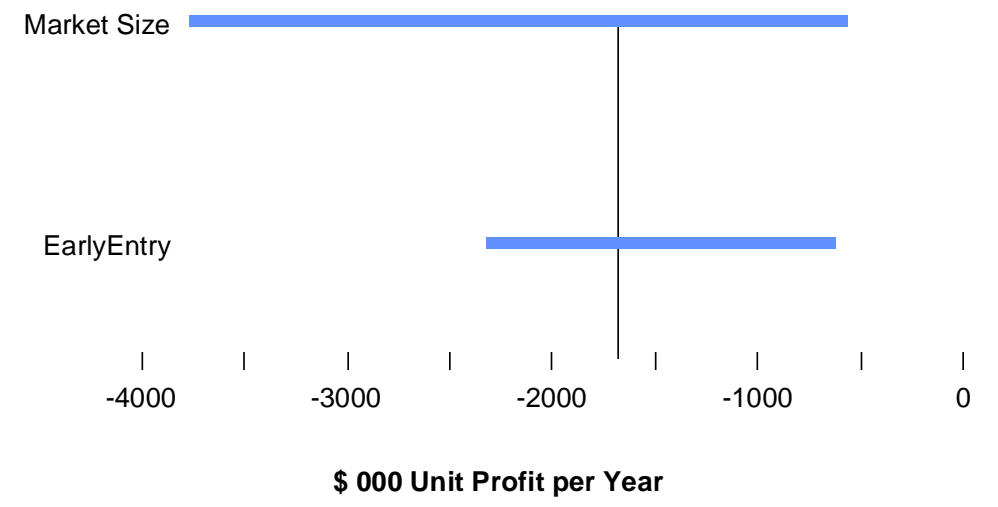

Figure 7-21: Uncertainty in the derivative of Unit Profit to DevTime 


\section{$7 \quad$ NGT Value Model Software Design}

This section completely documents the calculations in the NGT Value Model, such that the model worksheet can be reconstructed from the data herein. The design is modularized. Most bottom level modules (modules which do not incorporate other modules) are named by the output variable. Every output variable from every module and every intermediate variable within each module corresponds to a cell on the Excel worksheet. Bold face is used to identify a group of input variables (see Section 4), as a substitute for writing out the individual variable names. Names followed by a "\#" are assumptions (see Section 5), which are treated as constants in the model.

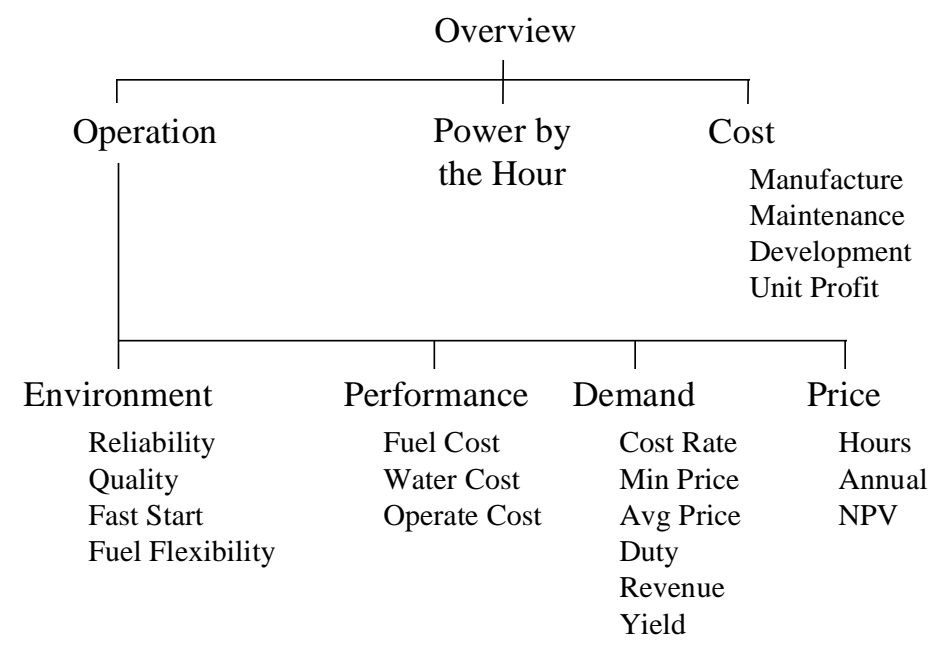

Figure 7-1: Module Structure

This is a list of all the modules in the model, showing which modules include which other modules. Only modules at the bottom of the tree (modules which do not include other modules) perform calculations.

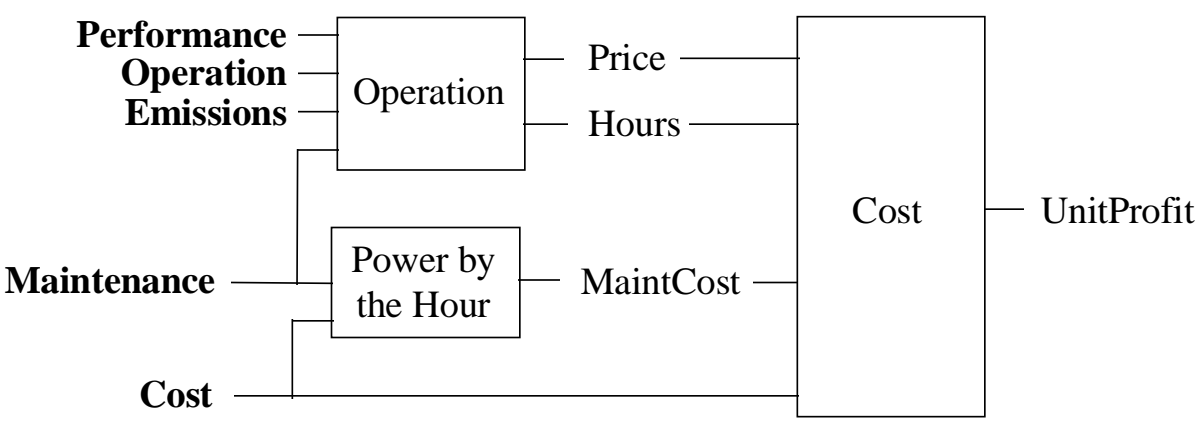

Figure 7-2: Data Flow Overview

This and all the figures to follow are Data Flow Diagrams. The flow is always left to right. Inputs enter on the left and outputs leave on the right. UnitProfit is the single output of the entire model. Note that the boldface names are groups of variables. 

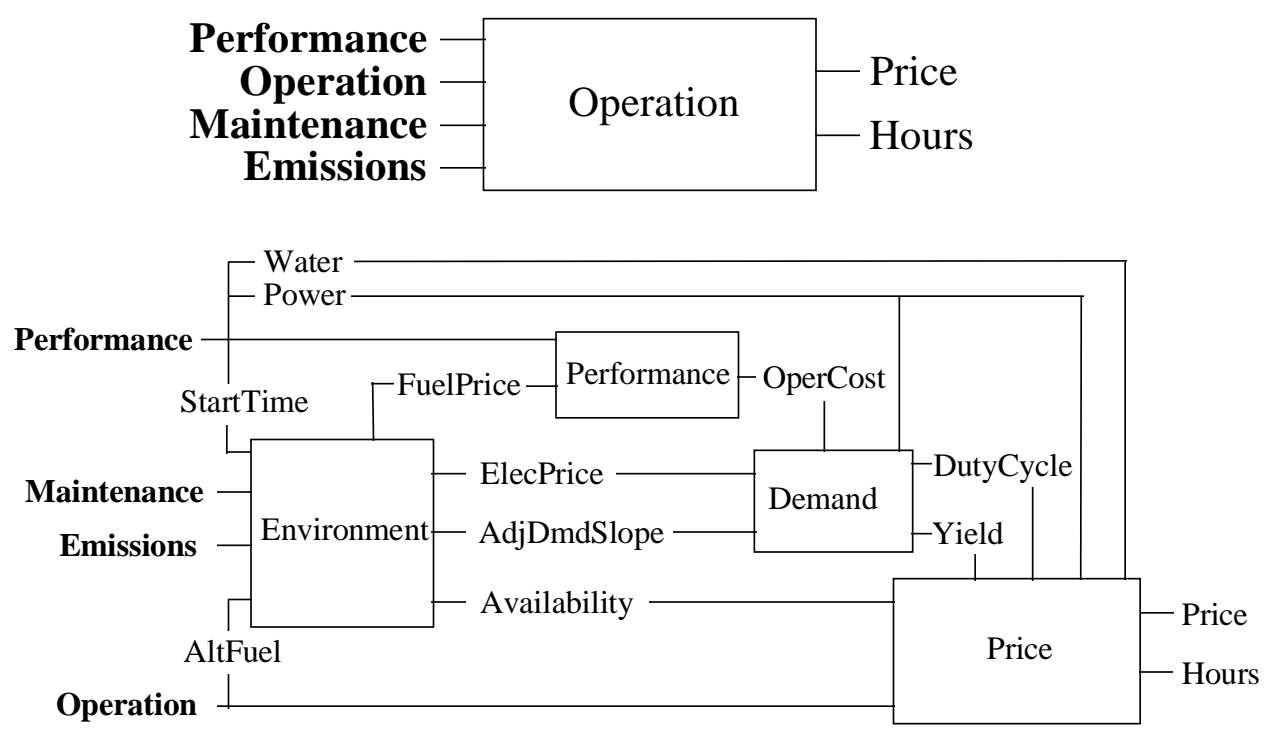

Figure 7-3: Operation

Operation describes the operation of the Independent Power Producer (IPP) who purchases the NGT system. This module calculates the market price of the NGT based on revenues and costs to the IPP. Like the charts that follow, the upper section of Figure 7-3 is a black-box diagram of the Operation module. The lower section shows the processes inside the module, which in this case are the data flows among subsidiary modules.
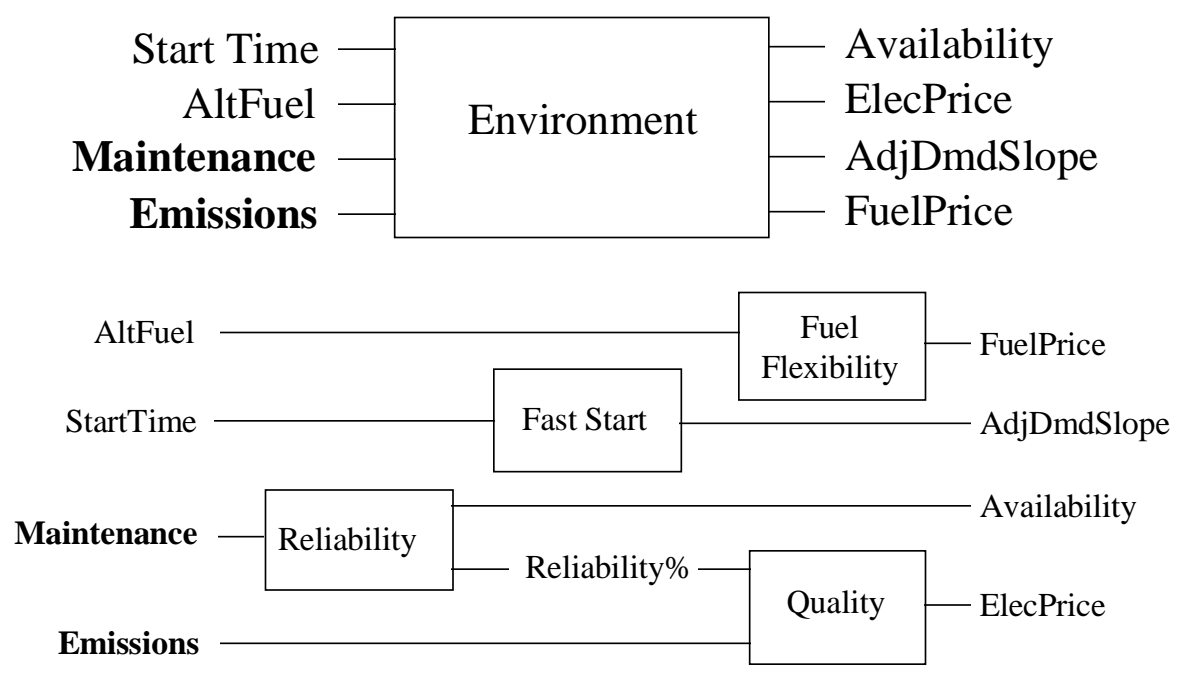

Figure 7-4: Environment

Environment addresses quality issues and fuel flexibility. Emissions quality and reliability directly impact the desirability of operating the generation system. This is modeled by reducing the effective price of the plant output, the electricity price, based on quality. Start time is also a quality measure, but it only affects peaking units, so it is applied to the electricity demand slope which modifies the electricity price during peak operation. AltFuel reduces the effective fuel price by permitting an alternative in times of natural gas scarcity. 


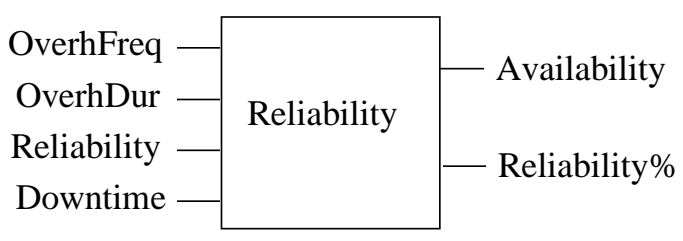

$$
\begin{aligned}
& \text { Reliability } \%=\frac{\text { Reliability }}{\text { Reliability + Downtime }} \\
& \text { Availability }=\frac{\text { OverhFreq }}{\text { OverhFreq + OverhDur }} \cdot \text { Reliability\% }
\end{aligned}
$$

Figure 7-5: Reliability

Reliability is the percent of time that the system actually runs out of the period of time with the IPP plans to run the system. The difference between Reliability $\%$ and $100 \%$ is the fraction of potential operating time that the system is shutdown for unscheduled maintenance. Availability is a similar measure, but also removes the time that the system is down for scheduled maintenance.

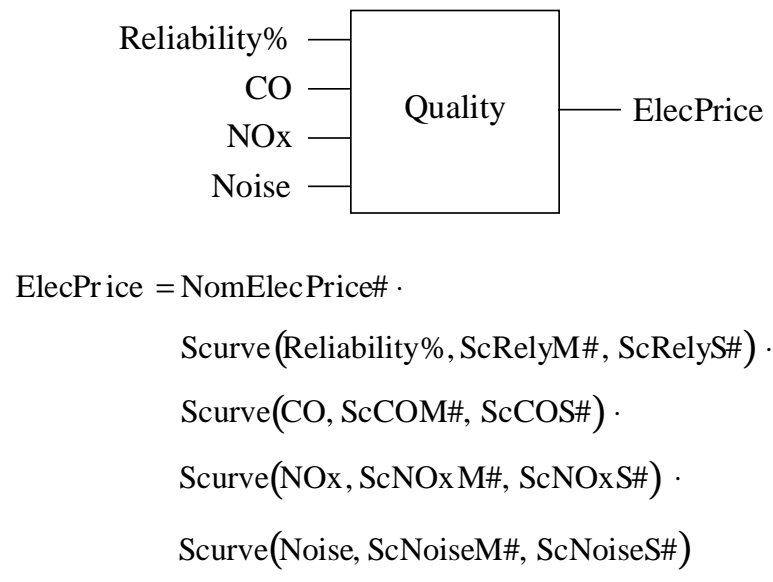

Figure 7-6: Quality

The Scurve routine implements the logistics curve shown in Figure 3-1 in Section 3. The constants ending in M\# are the midpoints of logistics curves; ending in S\# are the slopes of the curves.

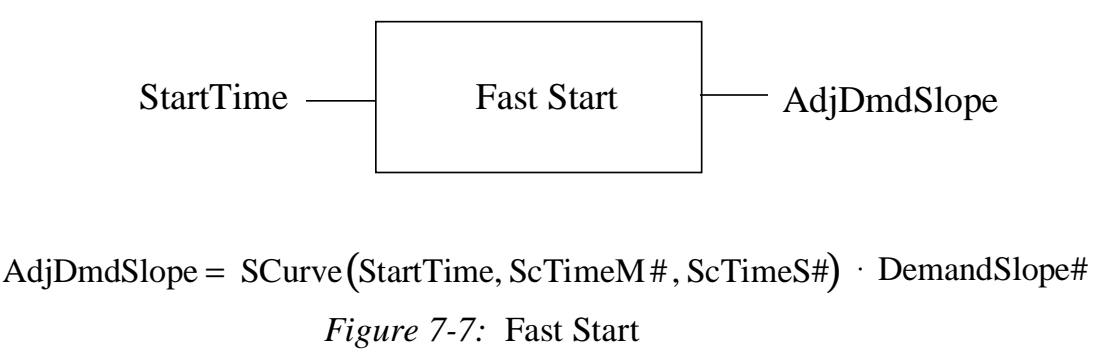

Fast start adjusts the slope of the electricity demand curve depending upon the start time. If the start time is very fast (less that \#StartTime99), the adjusted demand curve essentially follows the market demand curve, because the generating system can meet all market requests. If the start time is slow, the demand curve flattens, so that the generating system can still participate in the base load market but is less and less a participant in the peaking market. 


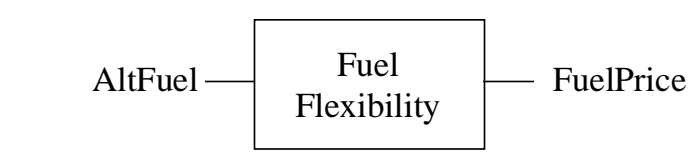

If AltFuel then

$$
\begin{aligned}
& \text { FuelPrice }=\text { BestFuel\# } \cdot \text { GasPrice \# } \\
& \text { else } \\
& \text { FuelPrice }=\text { GasPrice } \#
\end{aligned}
$$

Figure 7-8: Fuel Flexibility

The gas price constant is modified (reduced) if alternative fuels can be used.
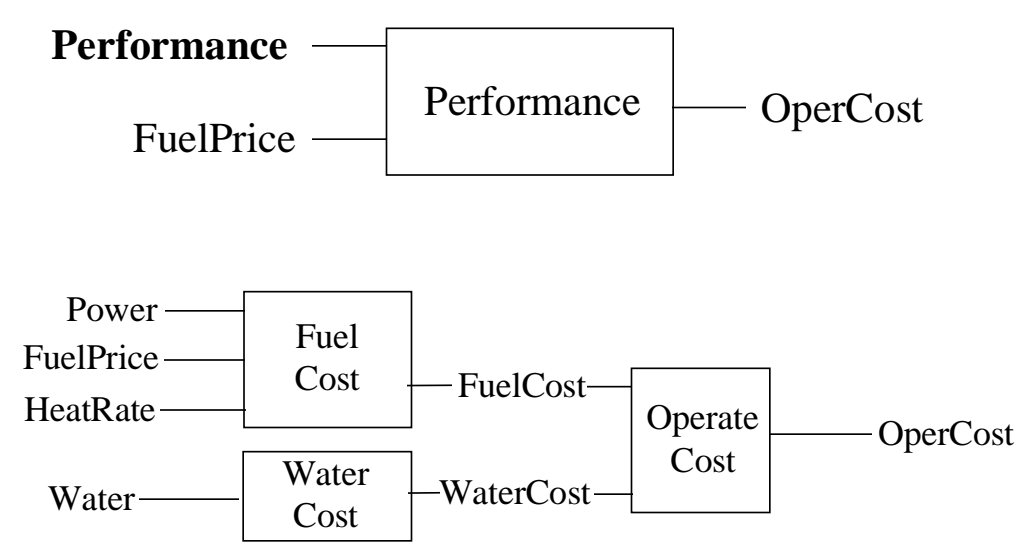

Figure 7-9: Performance

Performance determines the operating cost of the generating plant on a basis of thousands of dollars per hour.

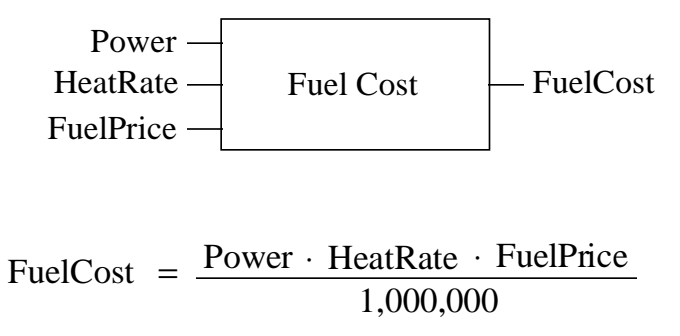

Figure 7-10: Fuel Cost

The factor of 1,000,000 is required to balance the units used: $\$ 000 / \mathrm{hr}=\mathrm{MW} \times \mathrm{BTU} / \mathrm{kw}-\mathrm{hr} \times \$ / \mathrm{MBTU}$

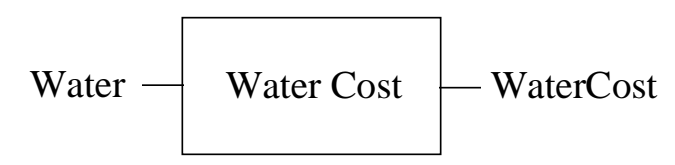

\footnotetext{
WaterCost $=$ Water $\cdot$ WaterPrice\# $\cdot$ MegaMinPerHr\#

Figure 7-11: Water Cost
} 


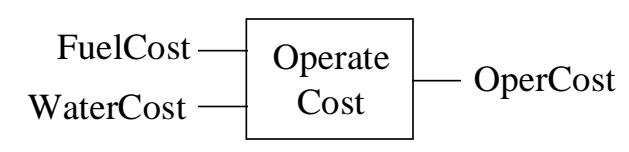

OperCost $=$ FuelCost + WaterCost

Figure 7-12: Operate Cost
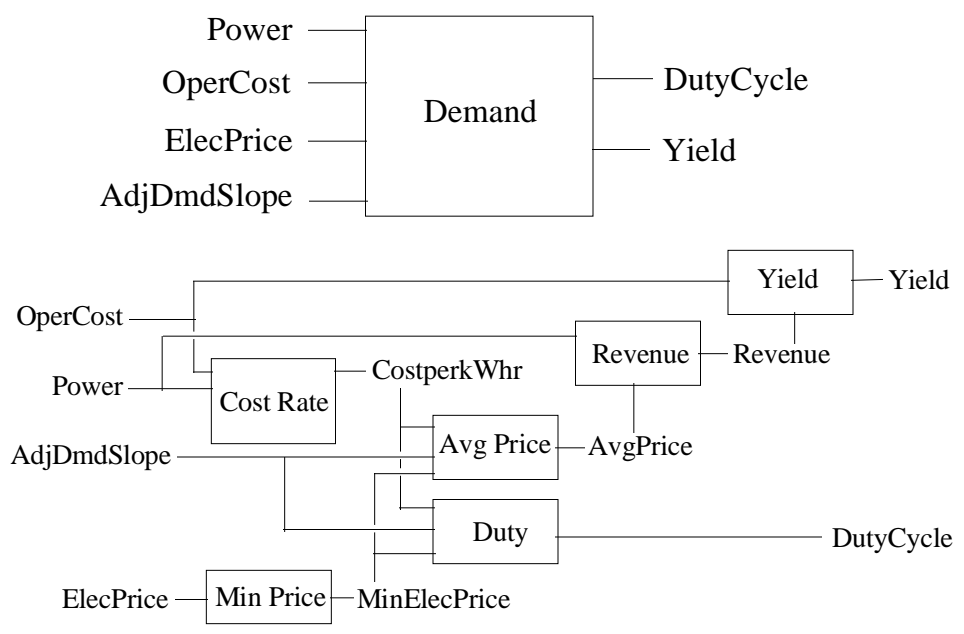

Figure 7-13: Demand

Based on operating cost, the demand module determines from the electricity demand curve what fraction of the time the plant can operate profitably. It then determines the average electricity price for that fraction of time. From the price and cost, it determines the hourly yield (profit).

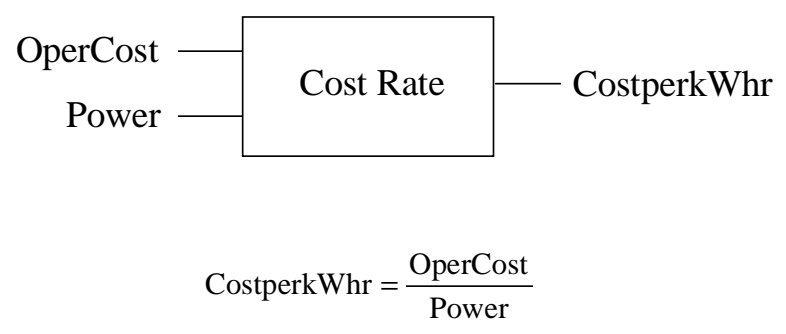

Figure 7-14: Cost Rate

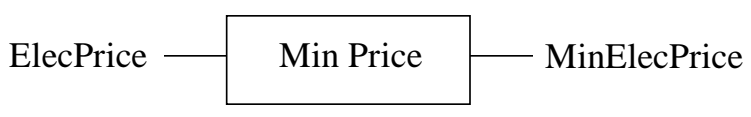

MinElecPrice $=(1-$ DemandSlope\# $) \cdot$ ElecPrice

Figure 7-15: Min Price

Min Price is the price of electricity at times of lowest demand. The slope of the hyperbolic demand curve determines the value of the curve at $100 \%$ versus the average value (ElecPrice). 


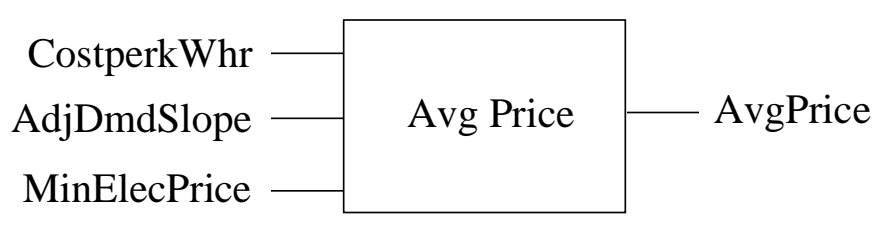

If MinElecPrice $\geq$ CostperkWhr then

$$
\begin{aligned}
& \text { AvgPrice }=\frac{\text { MinElecPrice }}{1-\text { AdjDmdSlope }} \\
& \text { else } \\
& \qquad \text { AvgPrice }=\frac{\text { CostperKwhr }}{1-\text { AdjDmdSlope }} \\
& \text { endif }
\end{aligned}
$$

Figure 7-16: Avg Price

Avg Price calculates the average of the demand over the fraction when it is profitable to operate the plant. The first case in the If test applies if it is profitable to run the plant full time (base load). The second case applies when the plant runs part time.

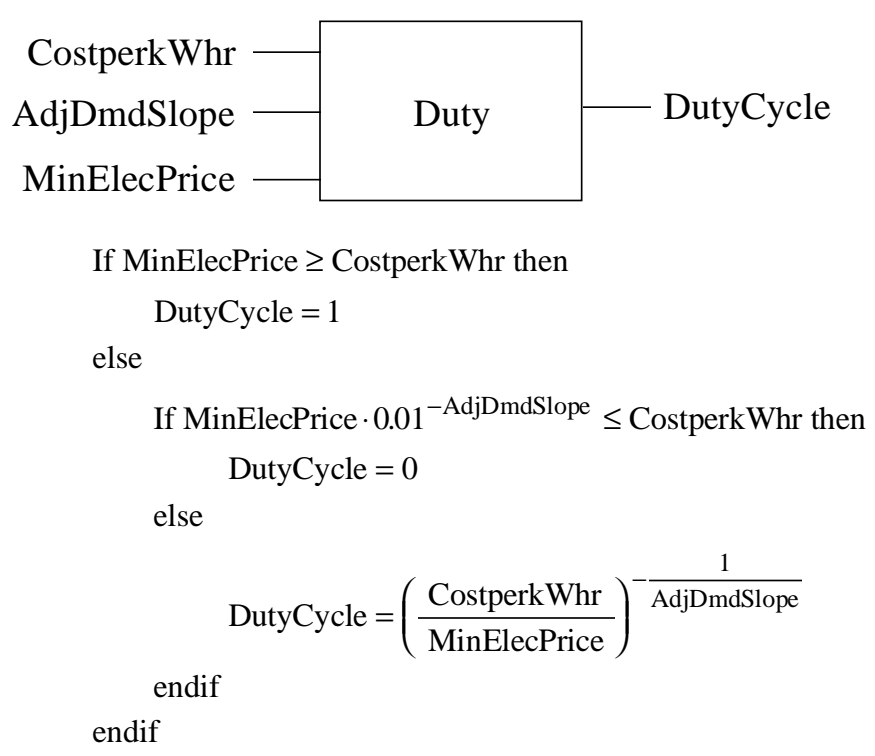

Figure 7-17: Duty Cycle

Duty Cycle calculates the fraction of time when it is profitable to run the plant. This is the fraction of time when the electricity price exceeds the cost to generate the electricity. 


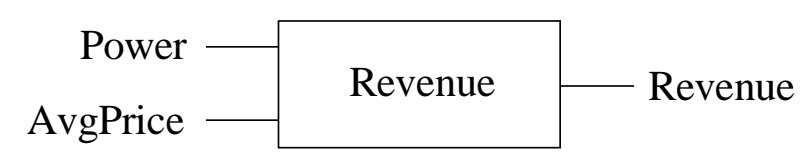

Re venue $=$ AvgPrice $\cdot$ Power

Figure 7-18: Revenue

Revenue converts price per kilowatt-hour to plant total revenue per hour of operation.

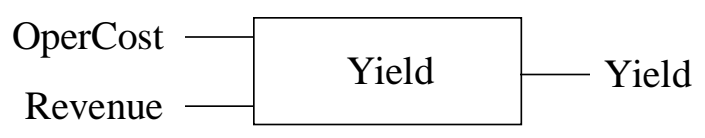

Yield $=$ Revenue - OperCost

Figure 7-19: Yield
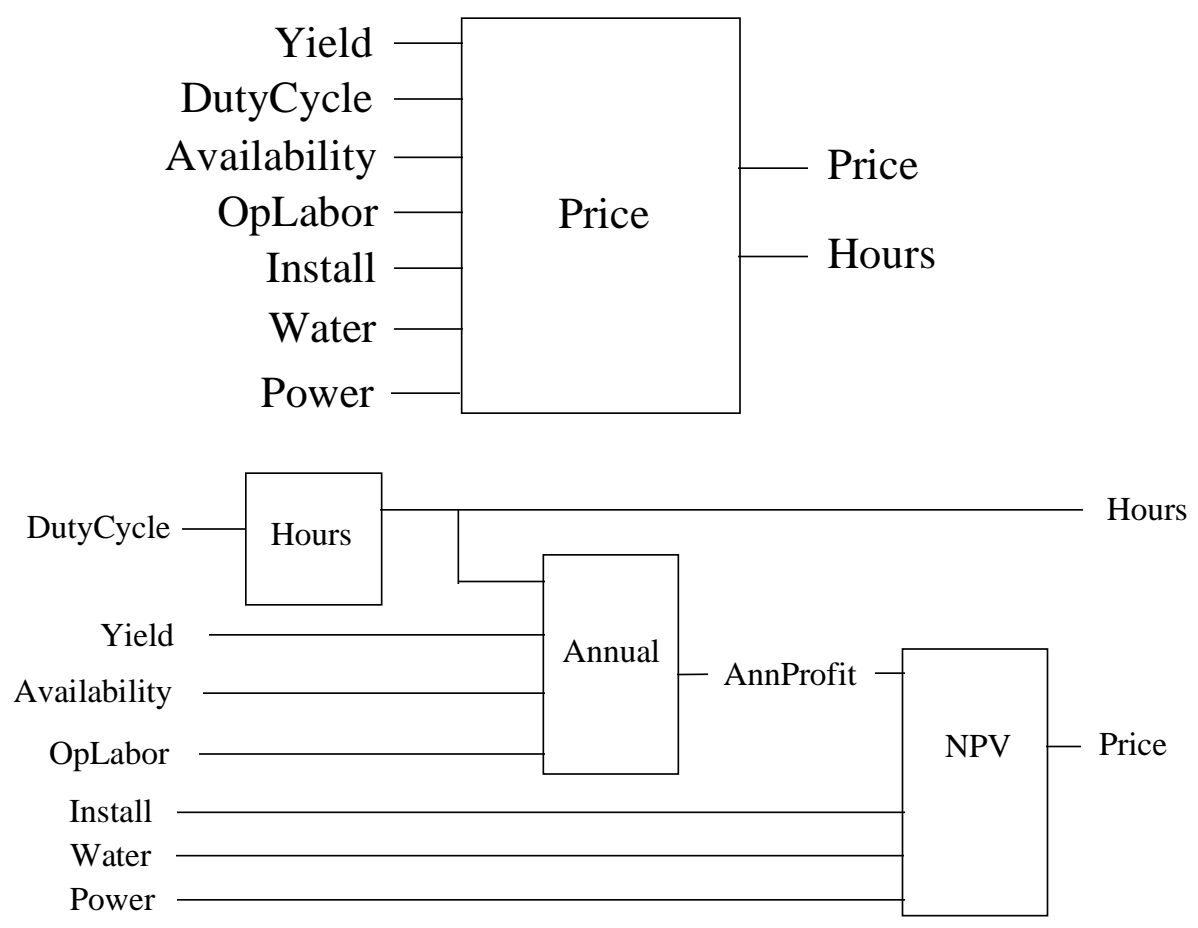

Figure 7-20: Price

Price is determined from the net present value of operator profit minus the capital costs required to site and install the plant. 


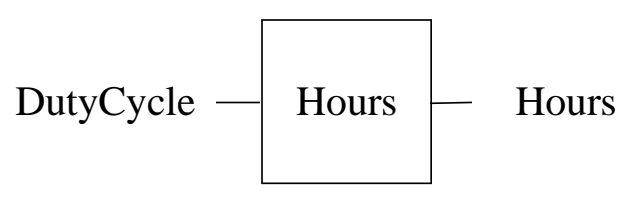

Hours $=$ HrperYr\#· DutyCycle

Figure 7-21: Hours

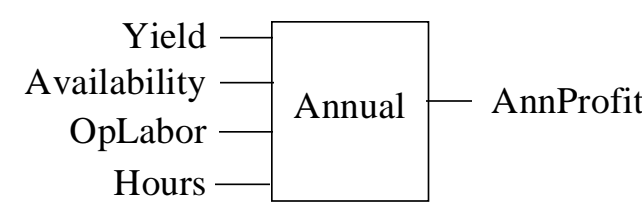

$$
\begin{aligned}
\text { AnnProfit }= & \text { Yield } \cdot \text { Hours } \cdot \text { Availability } \\
& \quad \text { OpLabor } \cdot \text { 0.001 } \cdot \text { OpLabRate\# } \cdot \text { Hours } \\
& \text { Figure 7-22: Annual }
\end{aligned}
$$

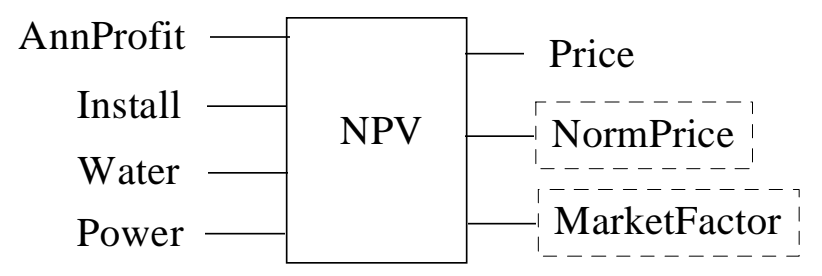

$$
\begin{aligned}
& \text { MarketFactor }=\text { Competition\#. Power } \\
& \text { Price }=\frac{\text { AnnProfit }}{\text { OpDiscRate\# }} \\
& \text { - MarketFactor · }\left(2-\left(\frac{1}{1+\text { OpDiscRate\# }}\right)^{\frac{\text { Install }}{52}}\right) \\
& \text { - Water } \cdot \text { WaterPermit\# } \\
& \text { NormPrice }=\frac{\text { Price }}{\text { Power }}
\end{aligned}
$$

Figure 7-23: NPV

Annual profit is calculated based on hourly profit adjusted for supervision labor. Reservation price is the net present value of profit. For simplicity, the system is assumed to run forever and there is consequently no salvage. Thus, net present value is annual profit over discount rate. The cost of constructing the facility is deducted to evaluate footprint area. However, this has only a trivial impact on the model (footprint is unimportant overall).

NormPrice (Normalized Price) and MarketFactor are output just to give visibility into the calculation. 


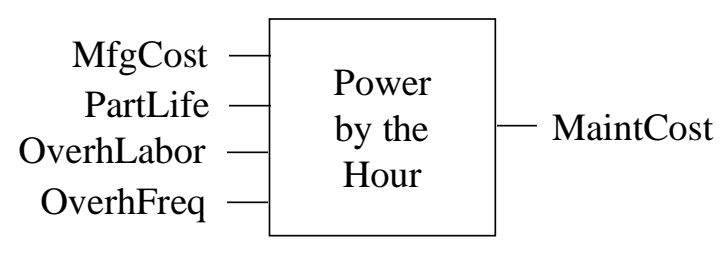

$$
\begin{aligned}
\text { MaintCost } & =\frac{\text { MfgCost }}{\text { PartLife } \cdot \text { OpporMaint\# }} \\
& +0.001 \cdot \frac{\text { OverhLabor }}{\text { OverhFreq }} \cdot \text { MaintLabRate\# }
\end{aligned}
$$

Figure 7-24: Power by the Hour

Maintenance cost per hour is part cost plus labor cost. Ideally, part cost is manufacturing cost over design life, but this is adjusted for opportunistic maintenance. Labor only addresses overhaul, because the labor associated with unscheduled maintenance is insignificant.
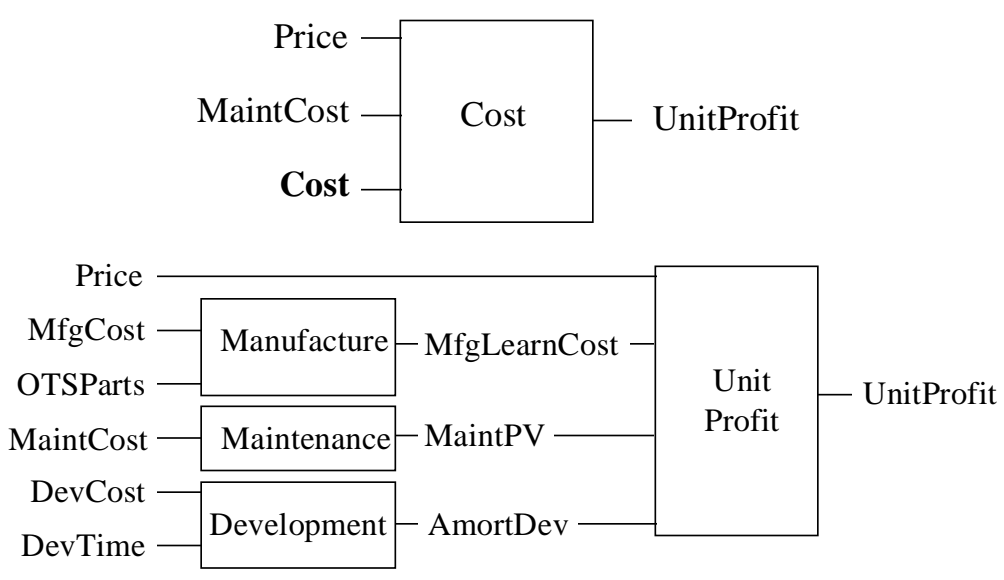

Figure 7-25: Cost

Profit to Rolls-Royce is price less manufacturing, maintenance and development cost.

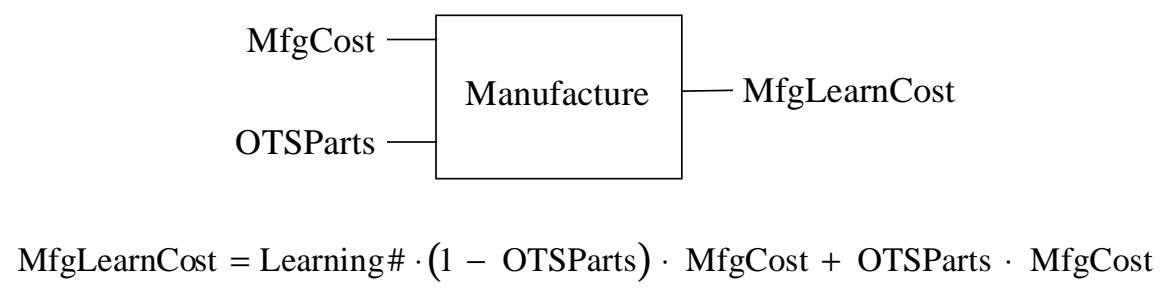

Figure 7-26: Manufacture

Learning is selectively applied to parts that are unique to the NGT program. 


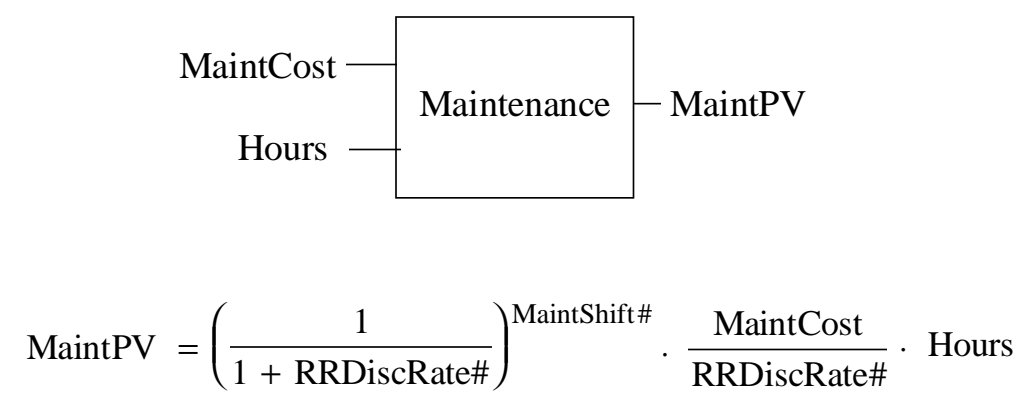

Figure 7-27: Maintenance

The system is assumed to operate forever, for simplicity, which simplifies the net present value calculation (Maint Cost per year / discount rate). Since maintenance does not really kick in until somewhat after the sale, this present value is also adjusted to the Maintenance Center, the time when maintenance really starts relative to the sale.

$$
\begin{aligned}
& \begin{array}{l|l|}
\text { DevCost }- \text { Development } & - \text { AmortDev } \\
\text { DevTime } &
\end{array} \\
& \text { AmortDev }=\frac{1,000}{\text { Volume\# }} . \\
& \text { (DevCost } \cdot\left(1+\text { RRDiscRate\#) }{ }^{\text {DevCenter \# · DevTime+ Pr odCenter \# }}\right. \\
& \text { + EarlyEntry\#·(DevTime-6)) }
\end{aligned}
$$

Figure 7-28: Development

Development cost is discounted to the average (center) time when it occurs. Early Entry rewards short development programs and punishes long ones.

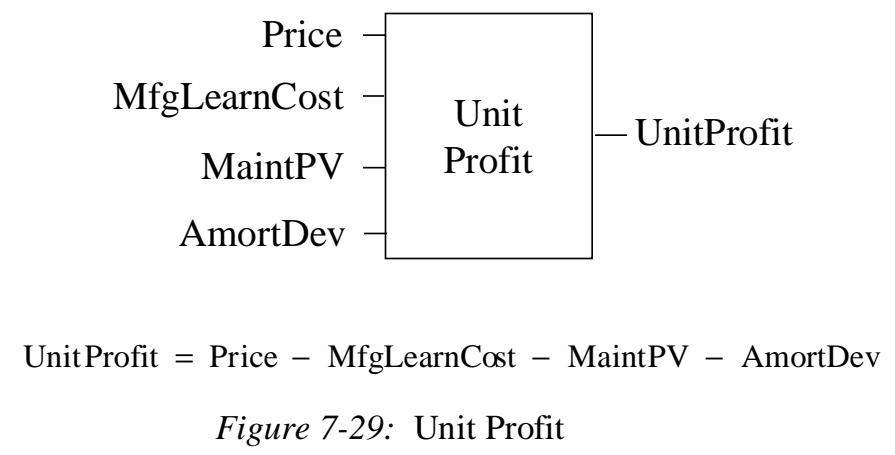

Unit Profit is the ultimate metric of the goodness of the design. 


\section{Recuperator Component Value Model}

Once a whole system value model is available, linear value models can be developed for each component. Table 8-1 shows a linear value model for the recuperator that was derived from the NGT value model. When every component is designed to maximize value using linear component value models derived from a single system value model, the final integrated system will be the best possible system (given the component partitioning), as measured by the system value model. This property is called distributed optimal design, and is the primary reason for developing value models. A distributed optimal design can reduce the cost of a complex product by half or increase the performance a corresponding amount.

\begin{tabular}{lrrrr} 
Name & Status & Gradient & \multicolumn{2}{c}{ Product } \\
Effectiveness & $93.5 \%$ & 54.1 & $\$ 5,056$ \\
PressureDrop & $2.4 \%$ & 279 & $\$$ & 670 \\
Reliability & $20,000 \mathrm{hrs}$. & 0.00249 & $\$$ & 50 \\
ComponentLife & $40,000 \mathrm{hrs}$. & 0.0410 & $\$ 1,639$ \\
MfgCost & $\$ 1,470$ & -1.40 & & $\$(2,052)$ \\
DevCost & $\$ 11,400$ & 5.259 & & $\$ 59,949$ \\
Recuperator Value & & & & $\$ 65,313$
\end{tabular}

Table 8-1: Recuperator Value Model

A component value model is derived as follows (see the paper "Economic-Based Distributed Optimal Design" referenced in Section 11 for details):

Given a system value model $\pi$ that inputs a vector of properties $\stackrel{\square}{\mathrm{x}}$, and given a composition function $\mathrm{h}$ that inputs component properties $\vec{z}$ and outputs the corresponding system properties $\mathrm{x}$, and given $\mathrm{a}$ particular component (the recuperator) whose properties are described by the vector $y$, a subset of $z$, then the value of the component can be calculated as

$$
v\left(\frac{\square}{y}\right)=\sum_{j}\left(\sum_{i} \frac{\partial \pi}{\partial x_{i}} \cdot \frac{\partial x_{i}}{\partial y_{j}}\right) \cdot y_{j}
$$

When the partials are taken for a fixed design (such as a preliminary design) the summation in the parentheses is a constant so the value function is linear. Table 8-1 was calculated according to formula (8 - 1). The elements of y are listed in the column Status. The sums of the products of the partial derivatives are listed in the column Gradient. The outer summation is accomplished in Table 8-1 by multiplying for each row the Status cell by the Gradient cell to get the Product cell. Summing the Product column (the outer sum in formula $(8-1)$ gives $v(\mathrm{y})$, the component value.

The elements of $\frac{\partial \pi}{\partial x_{i}}$ are listed in the Gradient column in the linear NGT value model on the Linear Sheet of the Excel spreadsheet val-ngt.xls (see section 3.8). Therefore, to create the recuperator value model it is only necessary to determine the partial derivatives of the composition function. That is, we need only answer the question, "How do the properties of the recuperator impact the properties of the NGT system?"

The manufacturing cost of the recuperator is assumed to have a one-to-one impact on the manufacturing cost of the NGT system. The same goes for the development cost. The partial derivative of system reliability to recuperator reliability is easily calculated to be the square of the ratio of system reliability to recuperator reliability. The partial derivative of system life to recuperator life is the square of the ratio of system life to recuperator life times the ratio of recuperator manufacturing cost to system manufacturing cost. More difficult are the partial derivatives of system power and efficiency to recuperator effectiveness and pressure drop. These derivatives were provided by Paul Fletcher. The result of these calculations is shown in Table 8-1. 


\section{$9 \quad$ Development Cost Methodology}

\section{Technical Risk}

The most important driver of development cost is technical risk. Technical Risk is the possibility that elements of the current design may not perform as intended in the actual product. Virtually all new products contain some degree of technical risk, insofar as they incorporate any novelty at all. The risk in a particular element of the design is expressed by the Probability of Failure, $\mathrm{P}_{\mathrm{f}}$. This is the numerical probability that the element will not perform as intended. If it does not perform properly, the design must be redone. Therefore, if the cost of the design is $\mathrm{C}$, the expected cost of reworking the design appears to be

$$
\mathrm{P}_{\mathrm{f}} \cdot \mathrm{C}
$$

so that the total cost of designing the component is

$$
\mathrm{C}+\mathrm{P}_{\mathrm{f}} \cdot \mathrm{C}=\mathrm{C} \cdot\left(1+\mathrm{P}_{\mathrm{f}}\right)
$$

The total cost is actually better estimated as

$$
\text { Total Cost }=\frac{\mathrm{C}}{1-\mathrm{P}_{\mathrm{f}}}
$$

which captures the possibility that the revised design may carry technical risk and may need to be redone once more. The difference between the two expressions is

$$
\frac{C \cdot P_{f}^{2}}{1-P_{f}}
$$

which, when $\mathrm{P}_{\mathrm{f}}$ is large (close to one), can be quite profound.

A good development program will uncover all design failures of this sort prior to product release. Therefore, all rework of this nature will appear in the actual cost of the program.

\section{Basic Cost}

The Basic Cost, $\mathrm{C}$, can be interpreted as the cost of creating the plans for operating, manufacturing and maintaining the product. For products like NGT, the manufacturing plans (drawings or product definition) dominate. Thus, the basic cost is strongly related to the information content of the product definition. However, NGT will not be designed on a clean sheet of paper (few products are). Much of the product definition will be borrowed from previous programs. Therefore the essence of the design cost (prior to considering risk) is the information content of those parts of the product definition that are new relative to previous programs.

Basic cost is best estimated by comparison with similar programs. Every program has risk, so the risk level of each component of the reference program must be assessed, and then the basic cost of each component can be derived from the historical cost by back-calculating through equation $(9-1)$. The reference basic cost must then be compensated based on the information content of the component to be estimated versus the reference component. If the information is represented in a logarithmic form, a straight proportion of cost to information should give a reasonable estimate.

\section{Testing}

Tests are performed to uncover design failures. If there were no technical risks, testing would be unnecessary. When a problem is uncovered by a test, the design must be corrected and the test repeated. The probability that a problem will be uncovered is the product of the probability that there is a latent problem (technical risk) and the probability that the test can find the problem (test effectiveness). The cost of redesign is related to the probability that a problem will be uncovered and to the original cost of 
designing the part. Once the component has passed a test, the technical risk is reduced according to the test effectiveness.

$$
\mathrm{P}_{\mathrm{f}}^{\prime}=\mathrm{P}_{\mathrm{f}} \cdot\left(1-\mathrm{P}_{\mathrm{TE}}\right)
$$

Comprehensive tests, such as engine tests and demo plants, can uncover a high percentage of problems. These tests are expensive and time-consuming, however, so that the necessary retests become a major burden in time and money. Often component and rig tests are performed prior to engine test to try to uncover and correct some failures in a less costly environment.

Thus, the decision to conduct a test is based on trading three properties of the test:

1. The cost and schedule impact of the test.

2. The fraction of the design failures that the test will uncover.

3. The cost of implementing design changes to correct the failures uncovered by the test.

Early testing is a "pay me now or pay me later" proposition. Good risk assessments are necessary to construct a cost-effective test program and to estimate the cost of the program. It is essential to remember that every test is expected to entail a redesign activity, else the test is useless. The risk reduction achieved by the test is directly proportional to the redesign effort that the test occasions.

These, then, are the essential elements of the development cost methodology illustrated in the spreadsheet developed for the NGT program:

- Basic cost, from the amount of new product definition information

- Technical Risk

- Testing Cost and impact on Risk 


\section{NGT Value Model Conclusions}

The NGT value model development provided concrete results in two areas:

1. Demonstration of the value modeling methodology

2. Determination of design trade factors

\section{Demonstration of value modeling methodology}

A major conclusion of the value modeling portion of the NGT program is the demonstration that value models can be developed and applied constructively to engineering design programs of this sort.

First, this program has demonstrated that formal value modeling methodology can be applied to power generation design systems to assist in conceptual design, design trade studies, and technology evaluation.

Second, a component value model for the recuperator was derived from the NGT Value Model, demonstrating the derivation of consistent component value models from system value models. This is the first such demonstration on any program to our knowledge, and is a necessary component of distributed optimal design, a methodology that has the potential of reducing the manufacturing cost of this class of systems by tens of percent.

Third, a risk-based development cost methodology was outlined, the first such extensive formal application of probability of success measures to estimating the cost of the development of complex systems. This methodology has promising extension to automotive, aerospace and defense industries.

\section{Determination of design trade factors}

More specific results of this exercise concern the determination of numerical trade factors which can guide the design of this NGT concept and are essential to optimizing the design. The Linear sheet of the value model Excel workbook contains a linearized version of the value model at the baseline design point. This linear model consists of the partial derivatives of the model output, Unit Profit, with respect to each of the model inputs. Near the baseline design point, the linear model duplicates the behavior of the full value model. Each input is multiplied by its corresponding partial derivative and the products are summed together. A constant bias is added and the result is an approximation of the Unit Profit output. Table 10 - 1 below lists all the partial derivatives from the linear model.

Each partial derivative can be regarded as the trade factor between the input and Unit Profit. For example, the partial derivative of Unit Profit with respect to Power is 965 thousand dollars per megawatt, from Table 10-1. This means that, all other measures being equal, a design that produces 81 megawatts should earn about one million dollars more profit per unit than a design that produce 80 megawatts.

Because of the chain rule of derivatives, trade factors between any two inputs can be determined by taking the ratio of their derivatives. For example, the derivative of Unit Profit with respect to Heat Rate is -10.3 thousand dollars per (BTU per kW-hr). The derivative of Unit Profit with respect to Manufacturing cost is 1.78 (dimensionless). Therefore, the derivative of Manufacturing Cost with respect to Heat Rate is 10.3 divided by -1.78 which equals 5.8. The units are thousands of dollars per (BTU per kW-hr).

Here is how to apply such a trade factor. Say that a set of changes are proposed which increase system cost but reduce heat rate. Divide in each case the change in cost by the change in heat rate. If the ratio is greater than 5.8 thousand dollars per (BTU per kW-hr), the change may be a good idea. If not, it is too expensive.

For more complicated decisions involving several properties changing at once, it is much simpler to use the value model in trade study mode. If the unit profit increases with the change, it is a good idea. If not, it should be dropped. 
Name Partial

Derivative

Performance

Power

964.937

HeatRate

$-10.3044$

StartTime

$-3902.93$

Water

$-32.3615$

\section{Operation}

OpLabor

AltFuel

Install

$-1095.75$

4538.793

$-11.6047$

Emissions

NOx

$\mathrm{CO}$

Noise

$-252.625$

$-100.002$

$-1204.03$

Maintenance

Reliability

2.206693

Downtime

$-247.416$

OverhFreq

0.022074

OverhDur

$-3.39072$

OverhLabor

$-0.12641$

PartLife

0.127545

\section{Cost}

$\begin{array}{lr}\text { MfgCost } & -1.77681 \\ \text { OTSParts } & 5258.72 \\ \text { DevCost } & -12.5882 \\ \text { DevTime } & -1732.24\end{array}$

Table 10-1: Value Function Gradient 


\section{References}

The following references provide additional information on developing and using value models.

\subsection{DFM Consulting references}

All these references, with their abstracts, are available at the website www.dfmconsulting.com/research.htm

Collopy, Paul D. "A System for Values, Leadership and Communications in Product Design," pages 95 98 in International Powered Lift Conference Proceedings, P-306, SAE Publications, Warrendale, PA, 1997.

A brief overview of the need for value modeling and distributed optimal design.

Collopy, Paul D. "Surplus Value in Propulsion System Design Optimization," AIAA 97-3159. American Institute of Aeronautics and Astronautics, Reston, VA, 1997.

Addresses value modeling methodology.

Collopy, Paul D. "Economic-Based Distributed Optimal Design," AIAA 2001-4675. American Institute of Aeronautics and Astronautics, Reston, VA, 2001.

Application of value modeling to distributed optimal design.

VanAtta, Richard; Lippitz, Michael; Collopy, Paul; Hartfield, Brad; and Richmond, Noah. Complex Product Realization 2020: Key Issue Areas. Institute for Defense Analyses, Alexandria, VA, 1999.

Addresses a wide span of state of the art design techniques, including value-based techniques.

Collopy, Paul D. "Joint Strike Fighter: Optimal Design through Contract Incentives." Pages 335-346 in 1999 Acquisition Reform Symposium Proceedings, Defense Systems Management College, 1999.

An introduction into value-based acquisition and the development of military value models.

\subsection{Other references}

Cook, Harry E. Product Management. Chapman \& Hall, New York, 1997.

Addresses more sophisticated pricing models, value modeling for consumer products, and quantitative market research methods for gathering data to support value models.

Lippitz, Michael J.; O'Keefe, Sean; and White, John P. "Advancing the Revolution in Business Affairs." Pages 165 - 202 in Keeping the Edge: Managing Defense for the Future. Edited by Ashton B. Carter and John P. White, Preventive Defense Project, Ashton B. Carter and William J. Perry, codirectors, Cambridge MA and Stanford CA, 2000.

Details the process and rationale for value-based acquisition (procurement based on value models).

Baldwin, Carliss and Clark, Kim. Design Rules. MIT Press, 2000.

Provides a coherent perspective on complex product design and the need for "guidance technologies" such as value models.

Collopy, Paul D. Replanning in Organizations. Dissertation for the Stanford University School of Engineering, 1998.

Provides the theoretical foundation for the mathematics of cost of risk in Section 9. 


\section{Appendix $F$ NGT Market/Economic Analysis: SRC Report}




\title{
TECHNICAL REPORT
}

\begin{tabular}{|l|l|l|}
\hline Discipline or report series & $\begin{array}{l}\text { Document number } \\
\text { DNS 82883 }\end{array}$ & $\begin{array}{l}\text { Issue } \\
\text { Strategic Research Centre Report }\end{array}$ \\
\hline Title \\
$\begin{array}{l}\text { Next Generation Turbines, contract DE-AC26-00NT40852 } \\
\text { Economic and Market Evaluation Final Report }\end{array}$ \\
\hline $\begin{array}{l}\mid l \\
\text { Authors } \\
\text { Richard Knight }\end{array}$ & $\begin{array}{l}\text { Telephone: } \\
69409\end{array}$ & $\begin{array}{l}\text { Date } \\
\text { Nov } 2001\end{array}$ \\
\hline
\end{tabular}

\begin{abstract}
Report Outline
This report is part of the deliverables of DOE contract DE-AC26-00NT40852, and summarises market appraisals performed by Rolls-Royce in order to determine the likely attractiveness of its NGT options to the energy market. The outputs of the following study is presented:

- Customer based economic appraisals

From the economic appraisals it is concluded that both R-R NGT arrangements are likely to offer significant commercial advantage in comparison with currently-available machines of similar power rating.
\end{abstract}




\section{CONTENTS}

SUMMARY

3

1. INTRODUCTION

4

2. CUSTOMER-BASED ECONOMIC APPRAISALS

5

$2.1 \quad$ Introduction

5

2.2 Engines considered and assumptions used

5

2.3 Engine operating modes

6

2.4 Market scenarios

6

$2.5 \quad$ Method of analysis

7

$2.6 \quad$ Results of analysis

8

2.6.1 Cost of delivered electricity analysis

8

2.6.2 Internal rate of return analysis

13

2.6.3 Payback period

15

2.7 Conclusions from economic studies

16

APPENDICES

17 


\section{SUMMARY}

This report is part of the deliverables of DOE contract DE-AC26-00NT40852, and summarises market appraisals performed by Rolls-Royce in order to determine the likely attractiveness of its NGT options to the energy market. The outputs of the following market based study is presented:

- Customer based economic appraisals

Using standard financial techniques, the customer based economic study has determined the potential economic performance of the two Rolls-Royce NGT options from the perspective of the customer / enduser. This establishes the likely competitive advantage offered by these solutions in comparison with existing alternatives. For the assumptions detailed in section 2, it is concluded that:

( B Both NGT arrangements are likely to offer significant commercial advantage in comparison with other currently-available machines of similar power rating, including combined cycle variants. The magnitude of the advantage varies with scenario, but is at least $5 \%$ when compared with alternative combined cycle machines, and at least $10 \%$ when compared with alternative simple cycle machines. In many cases the margins are significantly greater.

( These commercial advantages are a direct consequence of the high efficiency of the NGT solutions which is comparable to a typical combined cycle machine, and their low capital cost which is comparable to a typical simple cycle machine. 


\section{INTRODUCTION}

This report is part of the deliverables of DOE contract DE-AC26-00NT40852, and summarises market appraisals performed by Rolls-Royce in order to determine the likely attractiveness of its NGT options to the energy market. Although the studies concentrate on the specific case of the United States, many of the features of NGT will also be attractive to other markets elsewhere.

The work is focused on the following economic research for the NGT program:

(i) Customer-based economic appraisals, with the specific objectives:

( To determine the economic performance of the two Rolls-Royce NGT options from the perspective of the customer / end user.

u To determine whether these economics are likely to offer competitive advantage to RollsRoyce when compared with alternative machines.

The outcome of these two studies are discussed in detail in section 2 . 


\section{CUSTOMER-BASED ECONOMIC APPRAISALS}

\section{$2.1 \quad$ Introduction}

This section considers the economics of the proposed NGT options in comparison with alternative machines in the region 40-130 MW, all of which are existing products available on the market today. This performance has been determined in a variety of market scenarios as described in section 2.4. In effect, the studies attempt to establish whether the NGT solutions offer sufficiently attractive economic performance to make them the engine of choice for customers in preference to the options that are already available on the market place today. They also provide an indication of the likely margins that would exist between the NGT options and the alternatives if NGT becomes available.

\subsection{Engines Considered and Assumptions Used}

The economic performance of eleven different engine configurations has been studied. These, along with a summary of the performance and cost assumptions used in the studies are shown in Table 1 . The data for existing machines has been taken from Gas Turbine World [1]; data for the NGT options has been determined by Rolls-Royce's internal calculations.

\begin{tabular}{|l|l|c|c|c|}
\hline Engine & Configuration & $\begin{array}{c}\text { Power output } \\
(\mathrm{kW})\end{array}$ & Efficiency $(\%)$ & $\begin{array}{c}\text { Capital cost } \\
(\$ / \mathrm{kW})\end{array}$ \\
\hline Trent & Simple cycle & 51,190 & 41.6 & 485 \\
\hline Trent & Combined cycle & 66,000 & 54.3 & 650 \\
\hline LM 6000 PD & Simple cycle & 43,100 & 41.4 & 586 \\
\hline LM 6000 PD & Combined cycle & 56,400 & 52.5 & 658 \\
\hline LM 6000 ES & Spray water injected & 48,000 & 42.5 & 430 \\
\hline Frame 6FA & Simple cycle & 70,150 & 34.2 & 456 \\
\hline Frame 6FA & Combined cycle & 107,400 & 53.2 & 730 \\
\hline Frame 7EA & Simple cycle & 85,400 & 32.8 & 365 \\
\hline Frame 7EA & Combined cycle & 130,200 & 50.2 & 514 \\
\hline NGT 1 & Steam injected & 73,000 & 51.0 & 438 \\
\hline NGT 2 & Wet & 80,000 & 53.0 & 415 \\
\hline
\end{tabular}

Table 1 - Engine Performance and Cost Assumptions

On first inspection it can be seen that the predicted efficiency and capital cost of both NGT configurations compare favourably with the alternatives. They both offer efficiency of the same order as equivalent combined cycle machines, and capital cost of the same order as equivalent simple cycle machines.

In the absence of reliable data, the fixed and variable operations and maintenance costs for all machines have been assumed to be the same $(\$ 15 / \mathrm{kW} /$ year and 0.2 cents $/ \mathrm{kWh}$ respectively). To the first approximation this assumption is reasonable given that all machines are gas turbine based. However, in 
practice it is possible that more complex machines, such as NGT and the spray water injected LM 6000 ES, may cost more to operate and maintain. This is an area for further work. However, it is worth noting that as fuel and capital costs are by far the most dominant factors in gas turbine power generation plant economics, any variations in O\&M costs are not likely to be significant.

The availability of all machines has been assumed to be $95 \%$, including scheduled and unscheduled outages.

From the financial perspective, a discount rate (cost of borrowing) of $11 \%$ and a $3 \%$ inflation rate have been assumed throughout the studies.

The analysis includes the costs associated with network connection and network upgrade. Default network costs taken from published utility information (UK and US) have been included for this purpose. For these studies it has been assumed that $1 \mathrm{~km}$ of new network infrastructure is required to interconnect each machine to the electricity grid. Additionally, annual grid connection charges of $\$ 50 / \mathrm{kW} / \mathrm{year}$ have been assumed.

\subsection{Engine Operating Modes}

Three engine operating modes were considered for each engine option under consideration:

Baseload operation - This assumes that plant runs continuously within the constraints of equipment availability (95\%), hence the engines are assumed to run for $8760 \times 0.95=8322$ hours per year. In baseload all energy generated is assumed to be sold to the market at a fixed selling price under each market scenario. This might represent a long-term electricity supply contract. The cost of generation varies with time as a consequence of variable fuel prices and environmental levies (see section 2.4).

Mid-merit operation - In this operating mode the engines are all assumed to run for 4500 hours per year. This represents a typical operational regime for a merchant plant. Again all electricity generated is assumed to be sold into the market at a fixed price under each market scenario.

Spot market trading - This models a plant which is scheduled to respond to fluctuations in energy spot market price, and for this purpose hour-by-hour spot market price data is required. On an hourly basis the spot market price is compared with the marginal cost of generation; for those cases where the spot price exceeds the marginal generation cost the engine is assumed to run. The spot market price at that time is captured as income to the plant. For these studies four sets of 1999/2000 market data have been used as examples - New England, PJM, California and the UK. The respective pricing within these markets is shown in Appendix 1 for information purposes.

\subsection{Market Scenarios}

Five market scenarios have been studied, all of which are derived from the DOE's draft scenarios for natural gas planning. Appendices 2, 3 and 4 summarise the pricing and other data assumptions used for each scenario.

The base case scenario uses the reference case data taken from the IEA's Annual Energy Outlook 2001 and assumes an electricity selling price of $6 \mathrm{cents} / \mathrm{kWh}$. The price of gas payable by electricity generators applicable to the base case has been assumed to be $16.5 \%$ above the predicted wellhead 
prices (this is the average inflation above wellhead price paid by generators in the US over the previous 10 years according to IEA data). The base case scenario is considered to be the scenario that is most likely to occur in practice.

Apart from scenario 2, all remaining scenarios assume a fixed selling price for electricity derived from the base case selling price. Scenario 2 (generation market power case) assumes that the selling price for electricity varies as a function of the California spot market price during 2000.

Scenario 3 includes a small amount of carbon $\left(\mathrm{CO}_{2}\right)$ taxation which takes effect in later years. Scenario 4 (the Kyoto case) includes a significant $\mathrm{CO}_{2}$ levy which has the effect of improving the relative performance of the more efficient machines.

\subsection{Method of Analysis}

The analysis uses three standard financial techniques to assess the relative economic performance of the machines under consideration:

Cost of delivered electricity analysis - This rationalises the total costs of a power generation scheme over its operational lifetime into an equivalent time-discounted cost per delivered kWh of energy. Input factors include:

( Capital cost

( Fuel costs

( Operations and maintenance costs

u Annual charges (eg connection fees, etc)

( Network infrastructure costs

( Environmental levies

Internal rate-of-return (IRR) analysis - The IRR is a time-discounted measure of the value of an investment. It is defined as the rate of discount which equates the net present value of cash flows to zero. It can be considered to be a measure of the financial return on total project investment.

Payback period analysis (discounted) - The discounted payback period determines the time required to recover the initial investment outlay from the project's future cash flows, taking account of the time value of money. 


\subsection{Results of Analysis}

\subsubsection{Cost of Delivered Electricity Analysis}

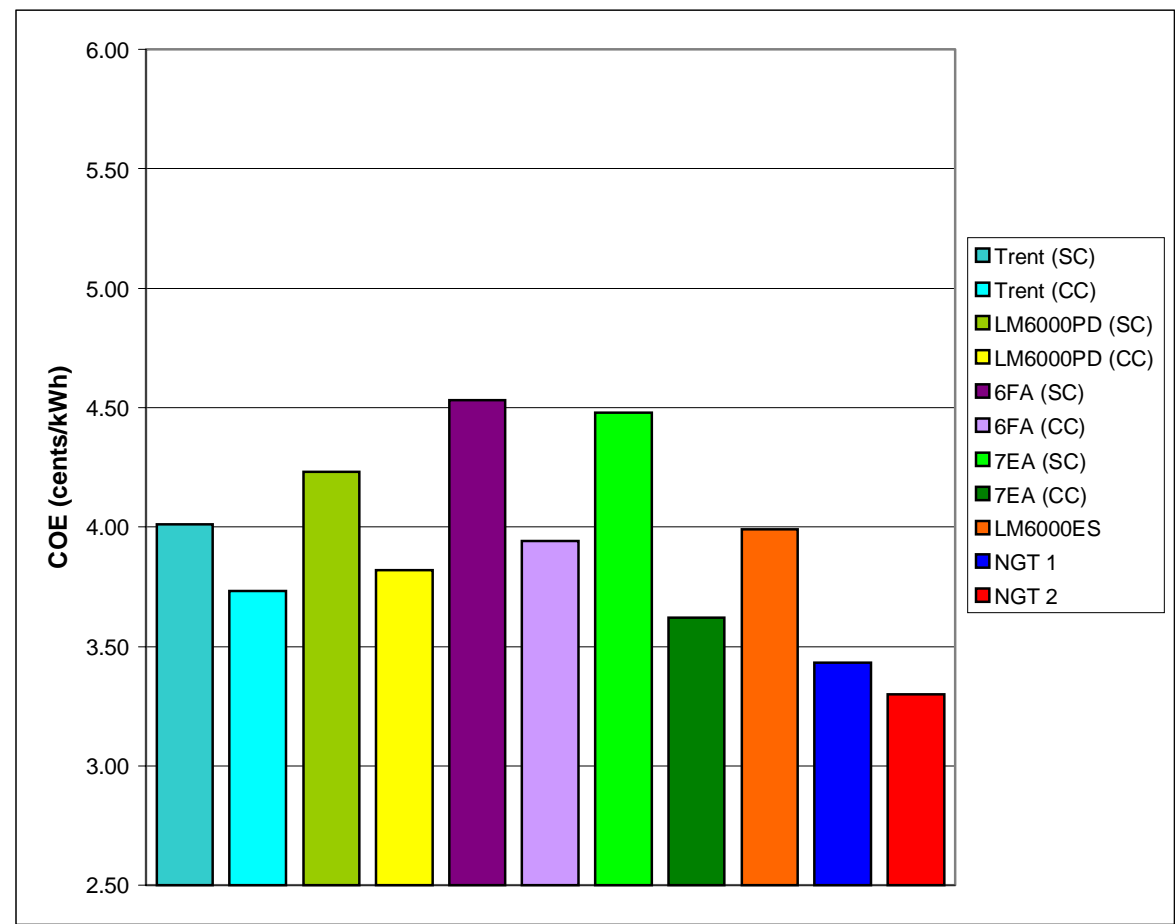

Figure 1 - Base Case, 10 Year Project, Baseload Operation

Figure 1 compares the cost of delivered electricity (in cents $/ \mathrm{kWh}$ ) of all the machines considered as part of this study. The figures relate to the base case with all machines operating in baseload (ie $95 \%$ availability) over a 10-year project lifetime.

The main point to note is that both NGT arrangements have a clear COE advantage over all the other machines, the closest competitor being the combined cycle Frame 7EA. NGT 1 (steam injected) outperforms the combined cycle 7EA by around 6\%, whereas NGT 2 (wet intercooled/recuperated) is superior by around $10 \%$. NGT 2 fares best as a direct consequence of its high efficiency and low $\$ / \mathrm{kW}$ installed cost.

As a general comment, in baseload operation all of the combined cycle machines fare well in comparison with their simple cycle equivalents. This is due to the high plant utilisation in baseload making fuel costs much more significant than $\$ / \mathrm{kW}$ capital costs in determining overall lifetime economic performance. As the combined cycle variants have significantly higher efficiencies than their simple cycle equivalents it makes them much more attractive. 


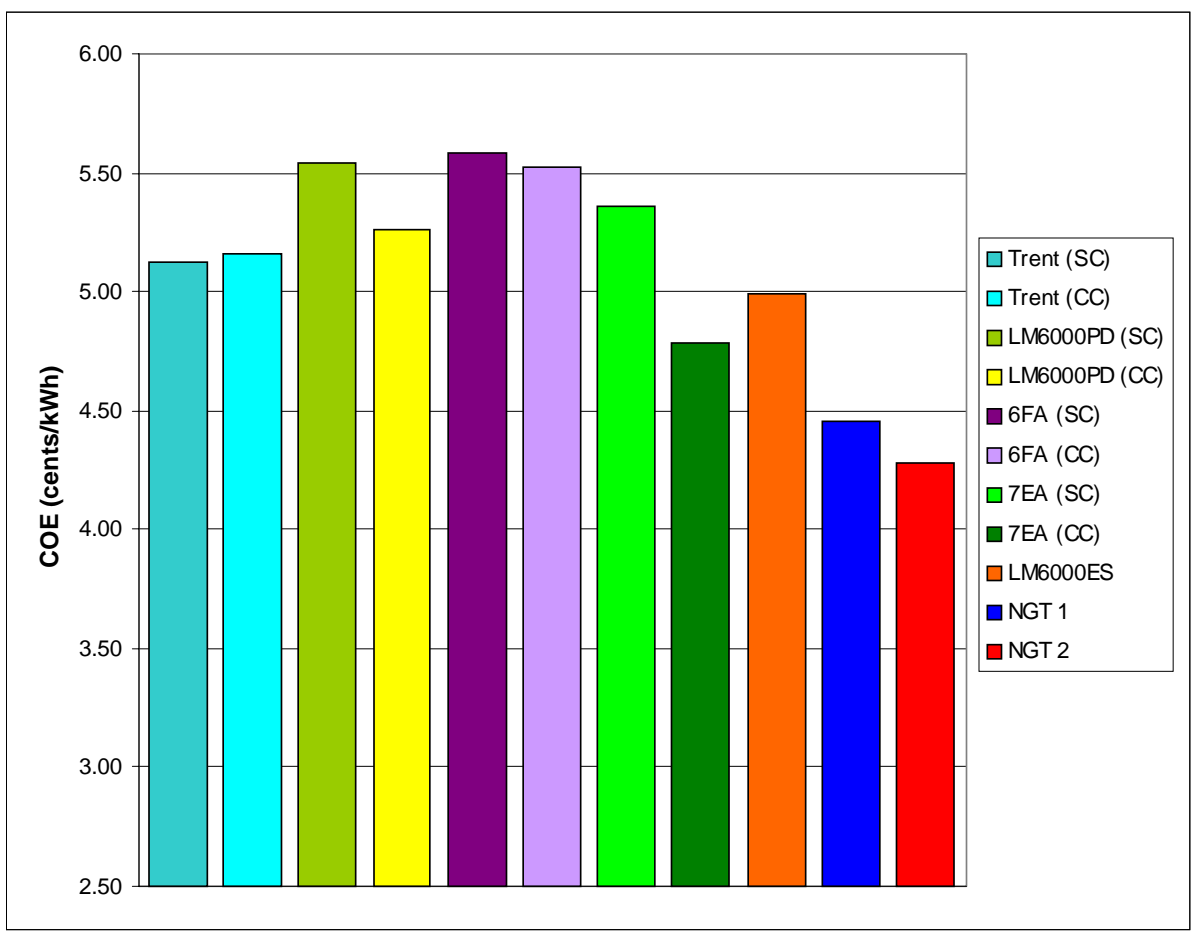

Figure 2 - Base Case, 10 Year Project, Mid Merit Operation

Figure 2 again provides a comparison of all machines considered in this study in the base case over a ten-year project duration. However, this time the assessment is made of their performance in mid-merit operation (4500 hours operation per year).

As a result of the reduced plant utilisation compared with the baseload case, the cost of electricity of all machines has increased. The amount of this increase is different for each machine, varying with the capital cost of the plant. The other contributory factors such as fuel and operations and maintenance are the same in baseload as they are in mid merit as they are levied on a cents/kWh basis.

Once more the economic advantage of the NGT options is immediately apparent, and again it is the combined cycle Frame 7EA that is the nearest competitor. In percentage terms, NGT's advantage over the combined cycle 7EA is slightly greater in the mid-merit case. The reason for this is the higher $\$ / \mathrm{kW}$ capital cost of the 7EA compared with the NGT solutions, and the consequent larger proportional increase in COE for the combined cycle 7EA in the mid-merit case.

The final point to note is that the relative performance of the combined cycle machines in comparison with their simple cycle equivalents is proportionally worse in the mid-merit case than it is in the baseload case. This again is a consequence of the increased contribution of capital costs to the COE figures in mid-merit, and the higher capital costs of the combined cycle machines in comparison with their simple cycle equivalents. 


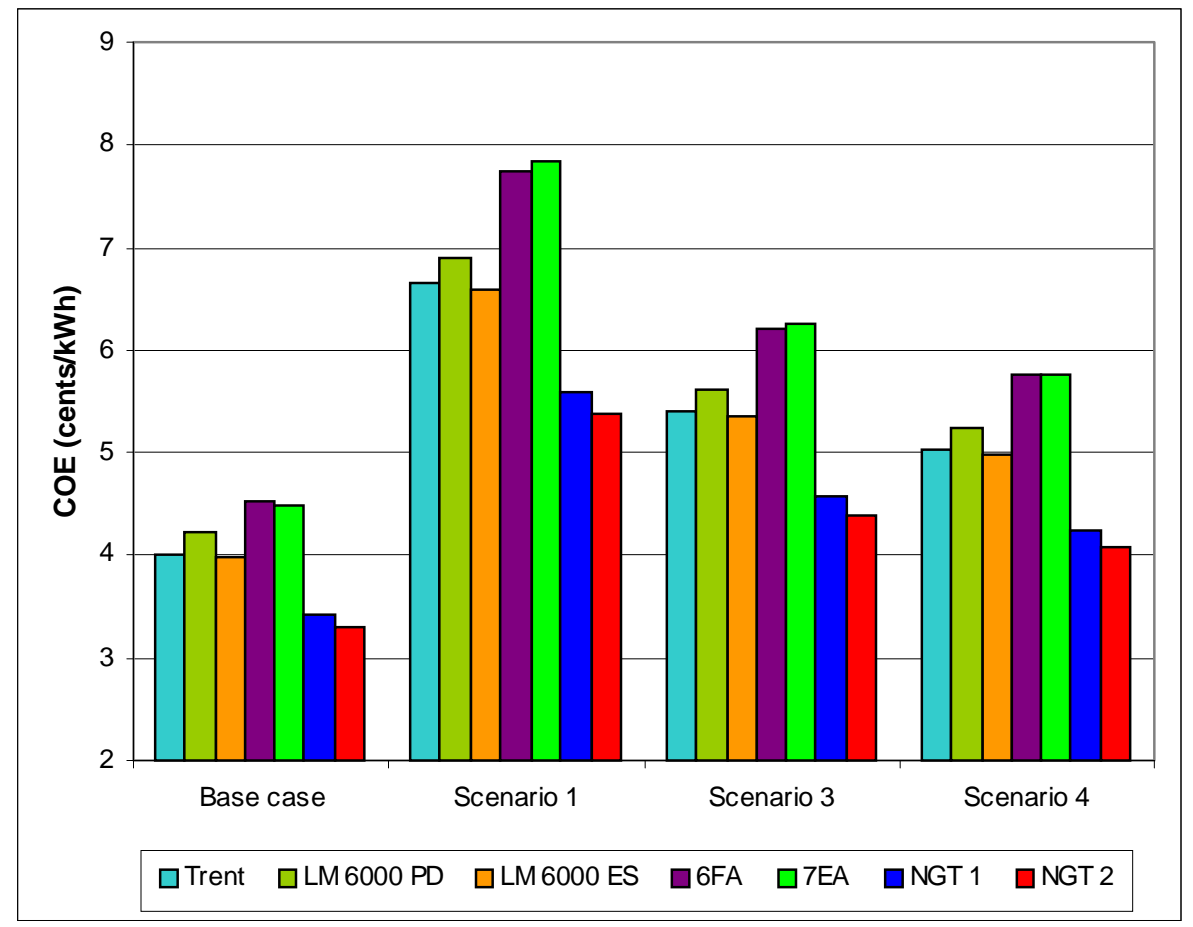

Figure 3 - NGT versus Simple Cycle Machines (Baseload, 10 year project)

Figure 3 provides a comparison of NGT's baseload COE performance compared with the simple cycle variants of the competing machines, for differing market scenarios over a 10-year project timeframe. The spray water injected LM 6000 ES has also been included within this category for the purposes of this report. Market scenario 2 (Generation market power - California case) has not been included as under that scenario plant utilisation and hence COE is variable and depends on the spot market prices of the specific market under consideration. The performance of the machines under scenario 2 are considered in section 2.6.2 later.

For the market scenarios above, NGT's superiority in comparison with the simple cycle variants of the competing plants is significant and clear. NGT 1 outperforms the nearest alternative (the LM 6000 ES) by at least $17 \%$ in all scenarios, with NGT 2 providing an advantage of at least $22 \%$.

The margins between the NGT options and the simple cycle alternatives are greater in those scenarios where gas prices and environmental levies exceed those of the base case. This is a consequence of the much superior overall efficiencies of the NGT options resulting in the fuel cost and environmental levy COE contributions of NGT being much smaller than those of the simple cycle machines. This is particularly apparent in Scenario 1 where the cost of fuel has been assumed to be double that of the base case. 


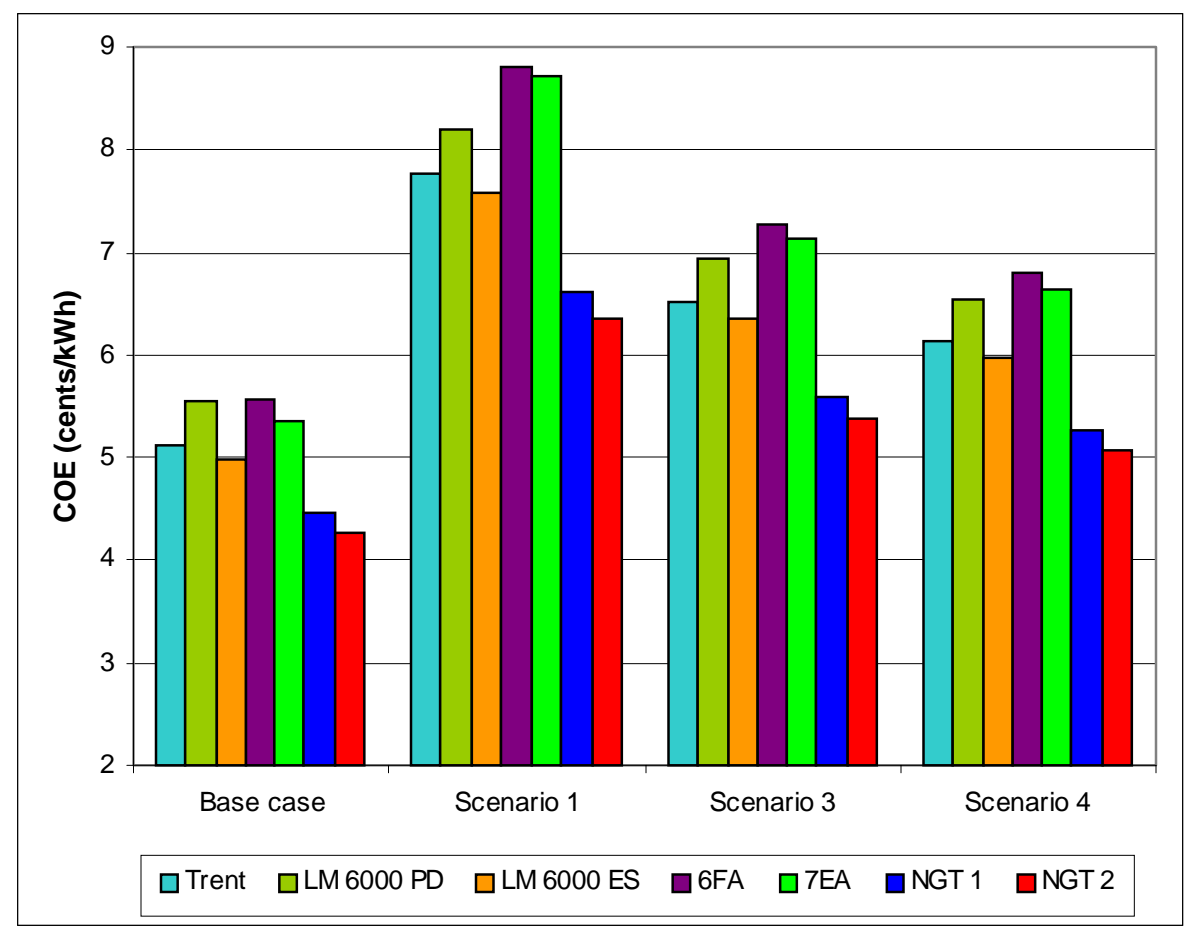

Figure 4 - NGT versus Simple Cycle Machines (Mid Merit, 10 year project)

Figure 4 provides a comparison of NGT's Mid-Merit COE performance compared with the simple cycle variants of the competing machines, for differing market scenarios over a 10-year project timeframe.

As with Figure 2, the reduced utilisation under mid-merit operation has the effect of increasing the COE of all machines in proportion with the $\$ / \mathrm{kW}$ installed capital cost. The margin between the NGT options and the Trent, LM 6000 and Frame 6FA machines has remained essentially the same as the baseload case in percentage terms as the capital costs of these machines are similar to those of NGT. Hence any changes brought about by the reduced utilisation are reflected to a similar degree in all these machines. However, for the simple cycle 7EA machine, which has a significantly lower capital cost than both NGT machines, the margin between it and the NGT options has reduced in mid-merit when compared with the baseload case. Notwithstanding this, the margins between NGT and the alternatives remain substantial.

Figures 5 and 6 compare the performance of NGT with the combined cycle variants of the competing machines, for the differing market scenarios over a 10-year timeframe. The margins between NGT and the combined cycle machines appear fairly small, however in all of the market cases summarised in Figures 5 and 6, both NGT machines out-perform the alternative combined cycle machines. This is a very important issue as it can be concluded that the performance of R-R's NGT concepts in COE terms are at the very least as good as, if not better than, alternative combined cycle machines in the same power range.

In relation to Figures 5 and 6, the 7EA once more provides the closest competition to the NGT options, although in baseload operation there is very little to choose between it and the Trent and LM 6000 PD combined cycle machines. This is primarily because there is little to choose in efficiency terms between these machines and it is fuel cost that dominates the COE. However, in mid-merit the relatively low capital cost of the combined cycle 7EA makes the margins between it and the combined cycle Trent and LM 6000 PD machines more significant as capital cost has a more significant contribution to COE than in baseload. 


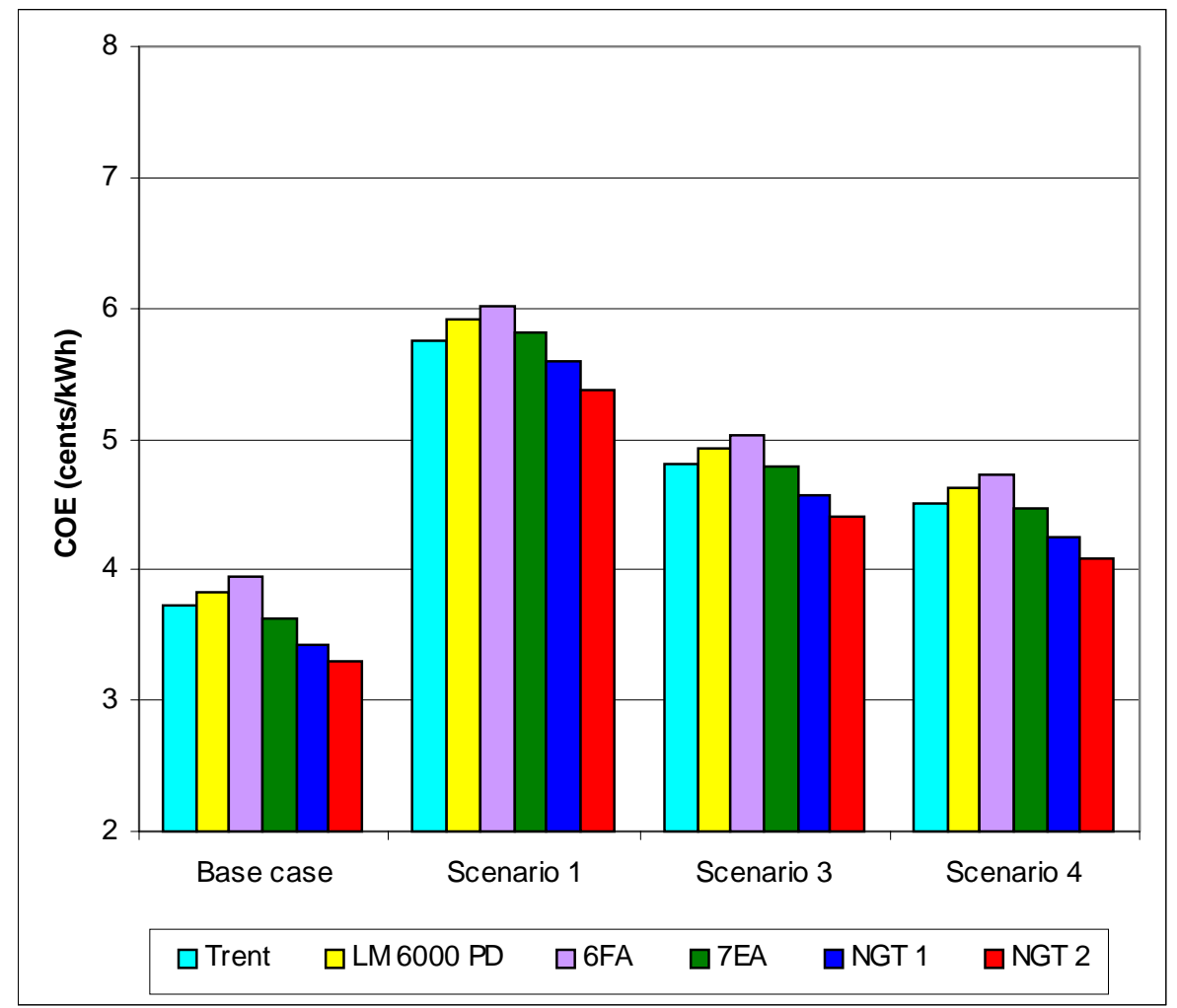

Figure 5 - NGT versus Combined Cycle Machines (Baseload, 10 year project)

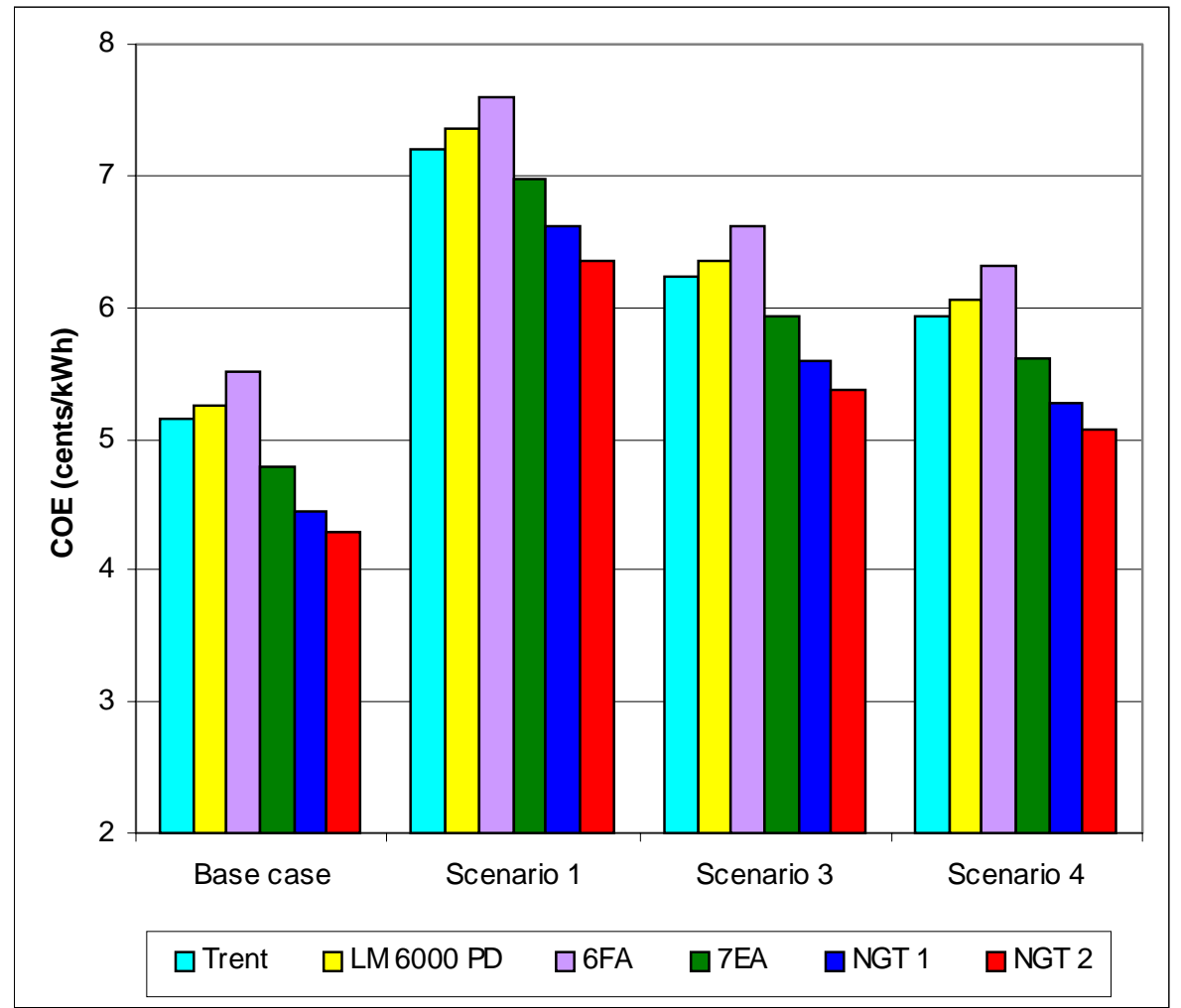

Figure 6 - NGT versus Combined Cycle Machines (Mid Merit, 10 year project) 


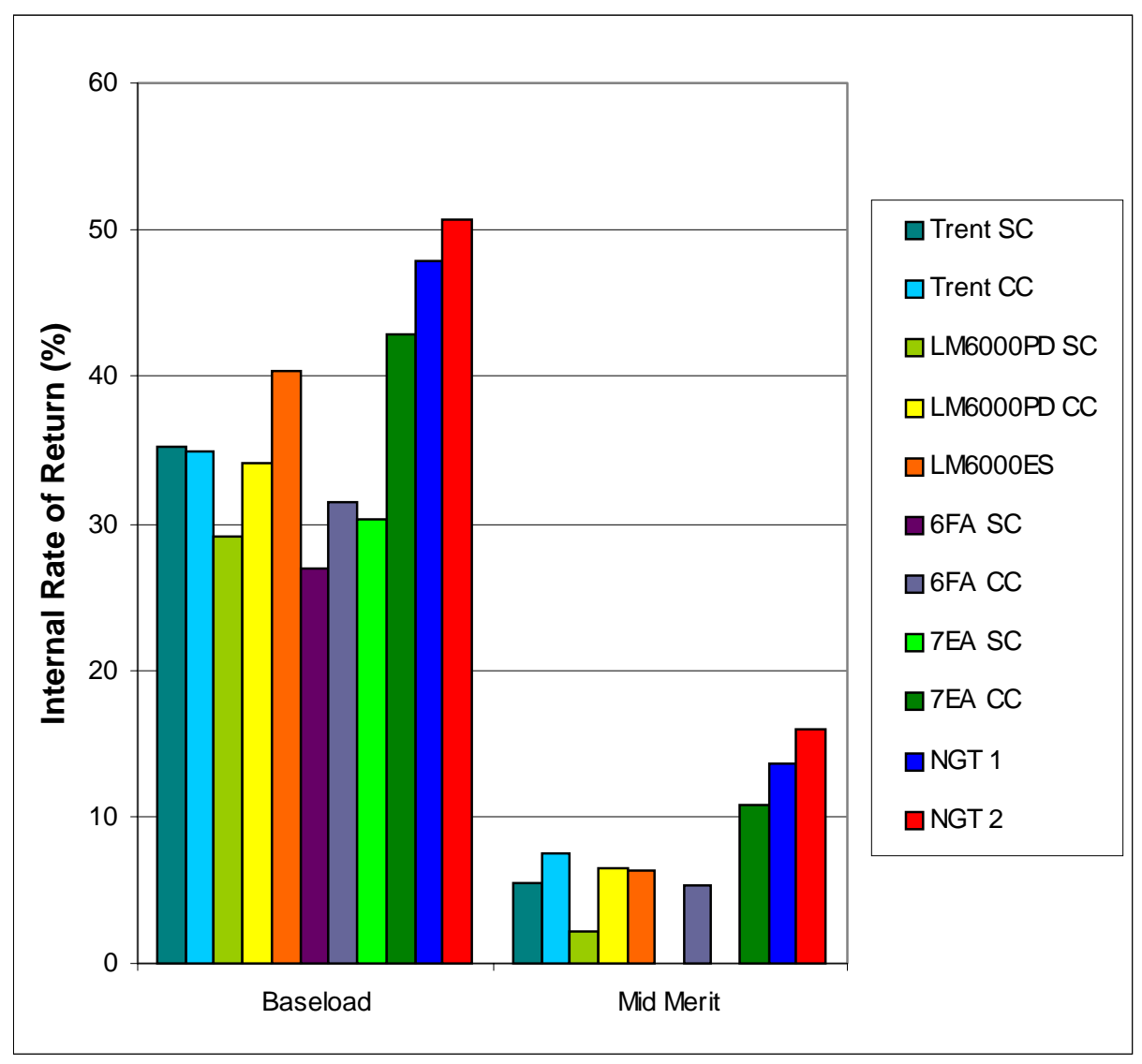

Figure 7 - IRR Comparison of all Machines, Base Case, 10 year Project

Figure 7 provides an indication of the potential return on investment that the installation of each machine will produce over a ten-year period using the base-case market scenario data. It includes baseload operation data (on the left) and mid-merit operation data (on the right). It also assumes an electricity selling price of 6 cents/kWh for all energy generated.

In baseload operation all machines return an IRR of greater than $25 \%$ which would generally be considered satisfactory in terms of a return on capital investment. Both NGT machines have a clear advantage over all other alternatives, with only the combined cycle 7EA and the LM 6000 ES (sprint version) producing IRR within 10 percentage points of the NGT solutions. The reason for NGTs' superior performance is once more their combination of high efficiency and relatively low capital cost in comparison with the alternatives. It is interesting that the LM 6000 ES is seen to provide good IRR performance when its COE performance under the analysis of the previous section indicated that its performance was average at best. The reason for this improvement is the low assumed cost of the LM 6000 ES when compared with the other non-NGT machines $(\$ 430 / \mathrm{kW})$, and as IRR is effectively a measure of return on capital, the low capital cost makes the IRR figures more attractive. However, even with this the margin between the LM 6000 ES and the NGT options still remains large with NGT's superiority being around 7 percentage points. 
Overall the picture in mid-merit operation (Figure 7) is somewhat less attractive as the reduction in plant utilisation produces a significant fall in IRR when compared with the baseload case, although it is clear that the NGT options still have a clear advantage over the alternatives. Only the NGT machines and the combined cycle 7EA are able to provide a return of greater than $10 \%$. This is an indication that a market selling price of 6 cents/kWh for 4500 hours operation per year is on the margin of economic acceptability for the majority of the machines under consideration.

In order to provide an indication of the likely performance of each of the machines in a more volatile market (which would equate to scenario 2 - generation market power), the financial returns of machines operating in the spot market trading situation (refer to section 2.3 for details) were assessed. It was assumed that only the simple cycle variants would be capable of responding quickly enough to respond to hour-by-hour changes in spot market pricing and therefore only the simple cycle and NGT machines were considered in this part of the study. The results of this are presented in Figure 8 . The four markets considered were:

\section{New England \\ PJM (Pennsylvania, New Jersey, Maryland) \\ California \\ UK Pool}

The hourly spot market prices for 1999/2000 were used as the market pricing reference data for each of the four markets in which the machines are assumed to operate. More detail regarding the price levels for these markets over the selected year can be obtained from Appendix 1.

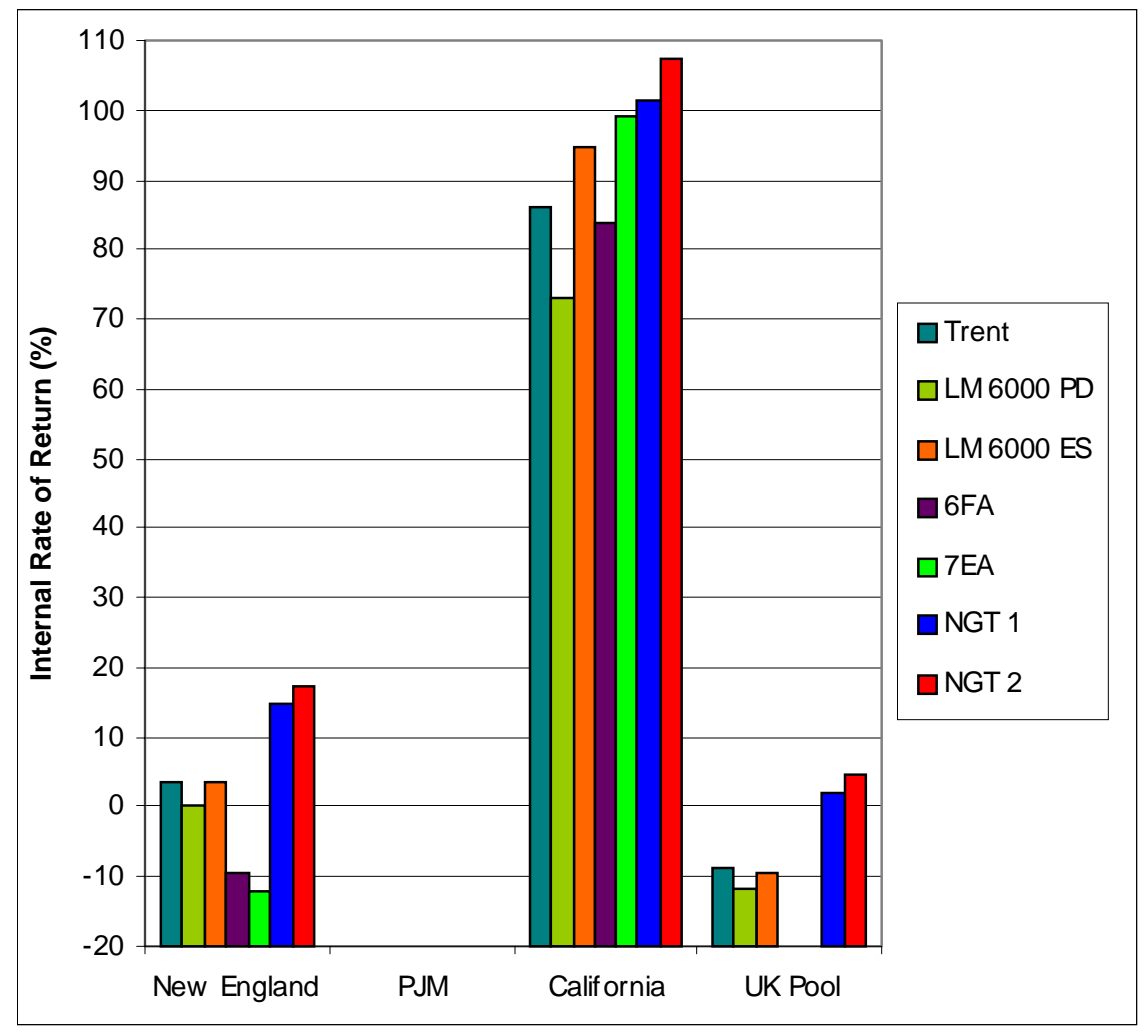

Figure 8 - Spot market trading comparison 
The first point to note is that the markets with higher average pricing and which are more volatile (eg California primarily) offer the most to spot market traders. This is of course as would be expected given that each hour of plant operation would generally attract a high market price for energy exported.

Once more it is the NGT machines that have the advantage in comparative terms, although in the California case the margins between NGT and the 7EA and the LM 6000 ES are relatively small. This is a consequence of the low capital costs of the 7EA and the LM 6000 ES offsetting their poorer efficiencies.

\subsubsection{Payback Period}

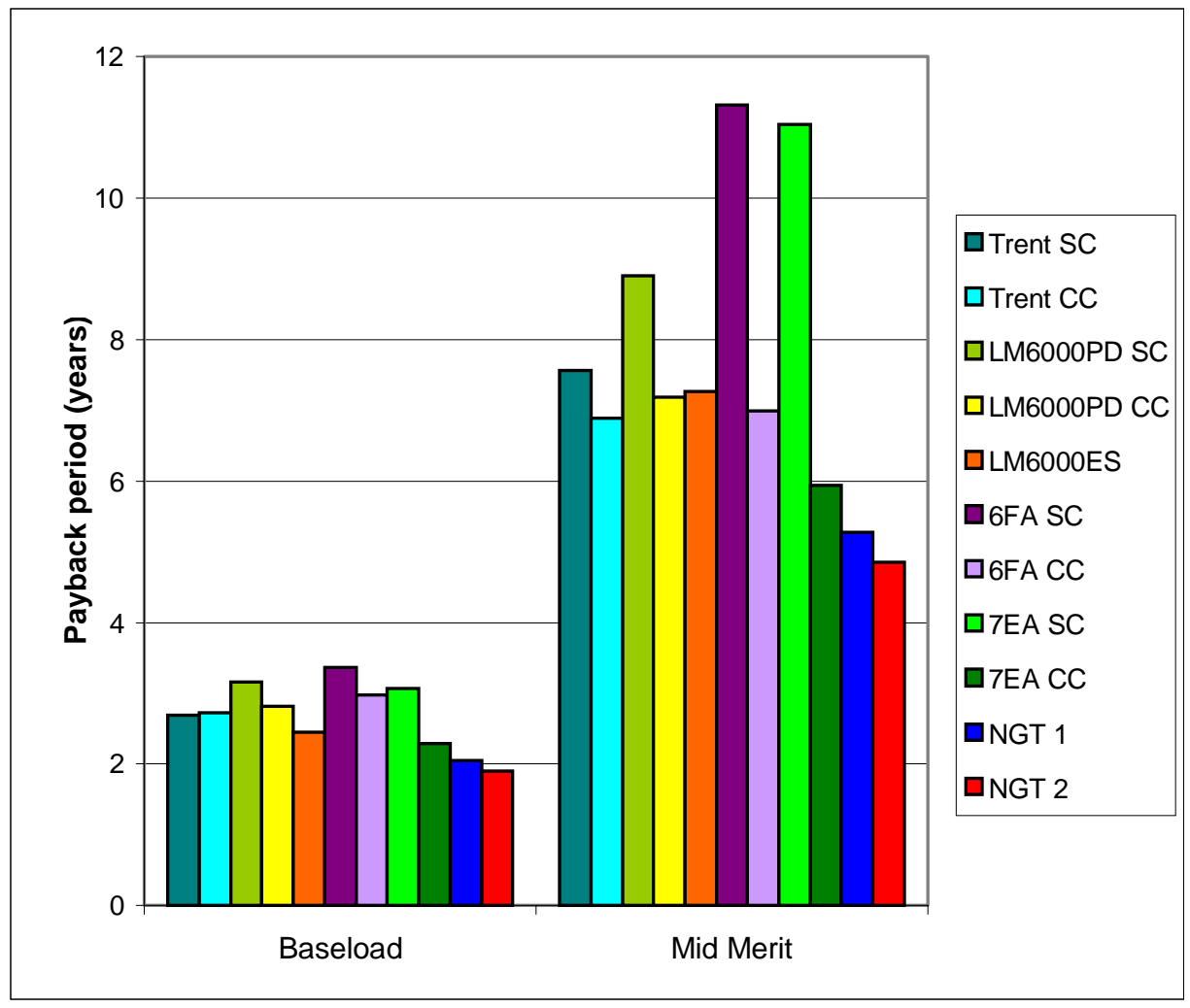

Figure 9 - Base Case Payback Period Comparison

Figure 9 shows the likely payback period for NGT and the other machines considered in the base case. In baseload, with the 6 cents/kWh electricity selling price, all machines pay back quickly. The NGT options pay back the most quickly, after a period of around 2 years.

In mid-merit the payback period increases for all machines as a result of the reduced utilisation and consequent reductions in income. The payback time advantage of the NGT machines in comparison with the alternatives is more clearly visible in the mid-merit case. This is a consequence of the lower mid-merit COE and relatively low \$/kW installed capital cost of NGT which makes the profit made on each kWh sold greater than the equivalent profit per kWh for the alternative machines. 


\subsection{Conclusions from Economic Studies}

For the assumed technical performance and cost parameters, and for the assumed operating and market conditions:

( Both NGT arrangements are likely to offer significant commercial advantage in comparison with other currently-available machines of similar power rating, including combined cycle variants. This advantage has been observed in all market scenarios considered and under varying modes of operation. The magnitude of the advantage varies with scenario, but is at least $5 \%$ when compared with combined cycle machines, and at least $10 \%$ when compared with simple cycle machines. In many cases the margins are significantly greater.

Q These commercial advantages are a direct consequence of the high efficiency of the NGT solutions (which is comparable to a typical combined cycle machine) and their low capital cost (which is comparable to a typical simple cycle machine). 
APPENDIX 1 - SPOT MARKET PRICE VARIATIONS

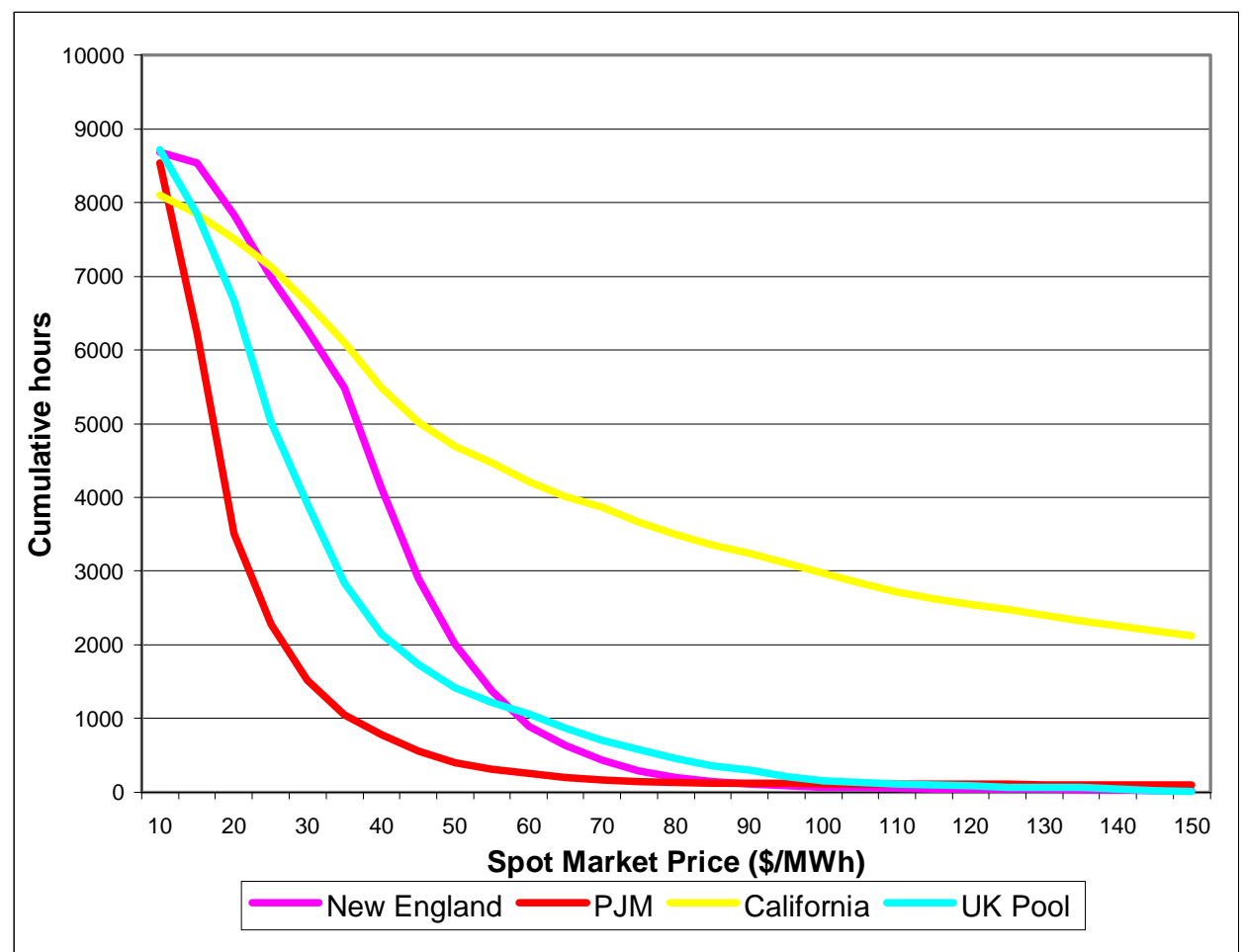

New England Market

Average price $=$

$\$ 41 / \mathrm{MWh}$

Standard deviation $=$

$\$ 21 / \mathrm{MWh}$

Volatility $=$

LOW

$\underline{\text { PJM Market }}$

Average price $=$

$\$ 28 / \mathrm{MWh}$

Standard deviation $=\$ 72 / \mathrm{MWh}$

Volatility $=$

MEDIUM

\section{Californian Market}

Average price $=$

\$99/MWh

Standard deviation =

$\$ 107 / \mathrm{MWh}$

Volatility $=$

$\mathrm{HIGH}$

$\underline{\text { UK Market }}$

Average price $=$

\$34/MWh

Standard deviation $=\$ 22 / \mathrm{MWh}$

Volatility $=$

LOW 


\section{APPENDIX 2 - MARKET SCENARIO INPUT DATA}

\begin{tabular}{|c|c|c|c|c|}
\hline Scenario & Overview & Electricity Price & Gas Price & Emissions $\left(\mathrm{CO}_{2}\right)$ Levy \\
\hline Base Case & $\begin{array}{l}\text { Reference case from } \\
\text { EIA Annual Energy } \\
\text { Outlook } 2001\end{array}$ & 6 cents/kWh & $\begin{array}{l}\text { Falls initially then } \\
\text { increases slowly }\end{array}$ & None \\
\hline Scenario 1 & $\begin{array}{l}\text { High gas demand and } \\
\text { high gas supply costs }\end{array}$ & $\begin{array}{c}\text { Higher than the base } \\
\text { case. } 125 \%, 150 \% \text { and } \\
175 \% \text { base case values } \\
\text { studied }\end{array}$ & Double the base case & None \\
\hline Scenario 2 & $\begin{array}{c}\text { Generation market } \\
\text { power (California case) }\end{array}$ & $\begin{array}{l}\text { High variability. } 50 \% \text {, } \\
75 \% \text { and } 100 \% \text { of } 2000 \\
\text { California prices studied }\end{array}$ & $\begin{array}{c}\text { Higher than the base } \\
\text { case. } 125 \%, 150 \% \text { and } \\
175 \% \text { base case values } \\
\text { studied }\end{array}$ & None \\
\hline Scenario 3 & $\begin{array}{l}\text { Globalisation and rapid } \\
\text { economic growth }\end{array}$ & $\begin{array}{c}\text { Higher than the base } \\
\text { case. } 125 \%, 150 \% \text { and } \\
175 \% \text { base case values } \\
\text { studied }\end{array}$ & $\begin{array}{l}50 \% \text { higher than the } \\
\text { base case }\end{array}$ & $\begin{array}{c}\text { Small levy staring in } \\
\text { 2006, increasing after } \\
2015\end{array}$ \\
\hline Scenario 4 & Kyoto agreement & $\begin{array}{c}\text { Higher than the base } \\
\text { case. } 125 \%, 150 \% \text { and } \\
175 \% \text { base case values } \\
\text { studied }\end{array}$ & $\begin{array}{c}\text { Near-term gas prices } \\
\text { increase but fall over } \\
\text { time with focus on } \\
\text { efficiency }\end{array}$ & Significant levy \\
\hline
\end{tabular}


APPENDIX 3 - MARKET SCENARIO GAS PRICE ASSUMPTIONS

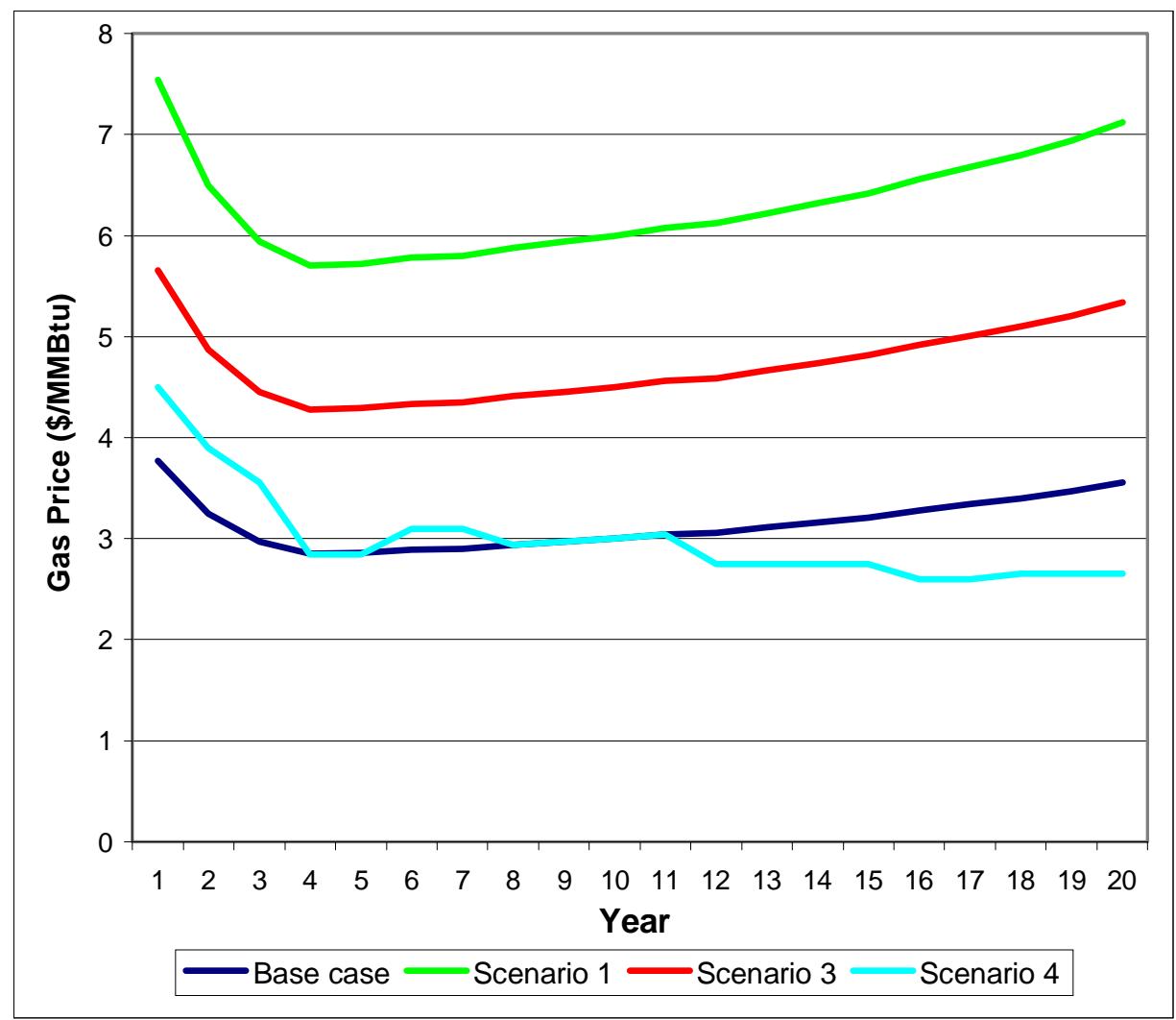

Note that scenario 2 figures are not included as this contains three gas price scenarios $(125 \%, 150 \%$ and $175 \%$ of the base case values). 
APPENDIX 4 - MARKET SCENARIO ENVIRONMENTAL LEVIES

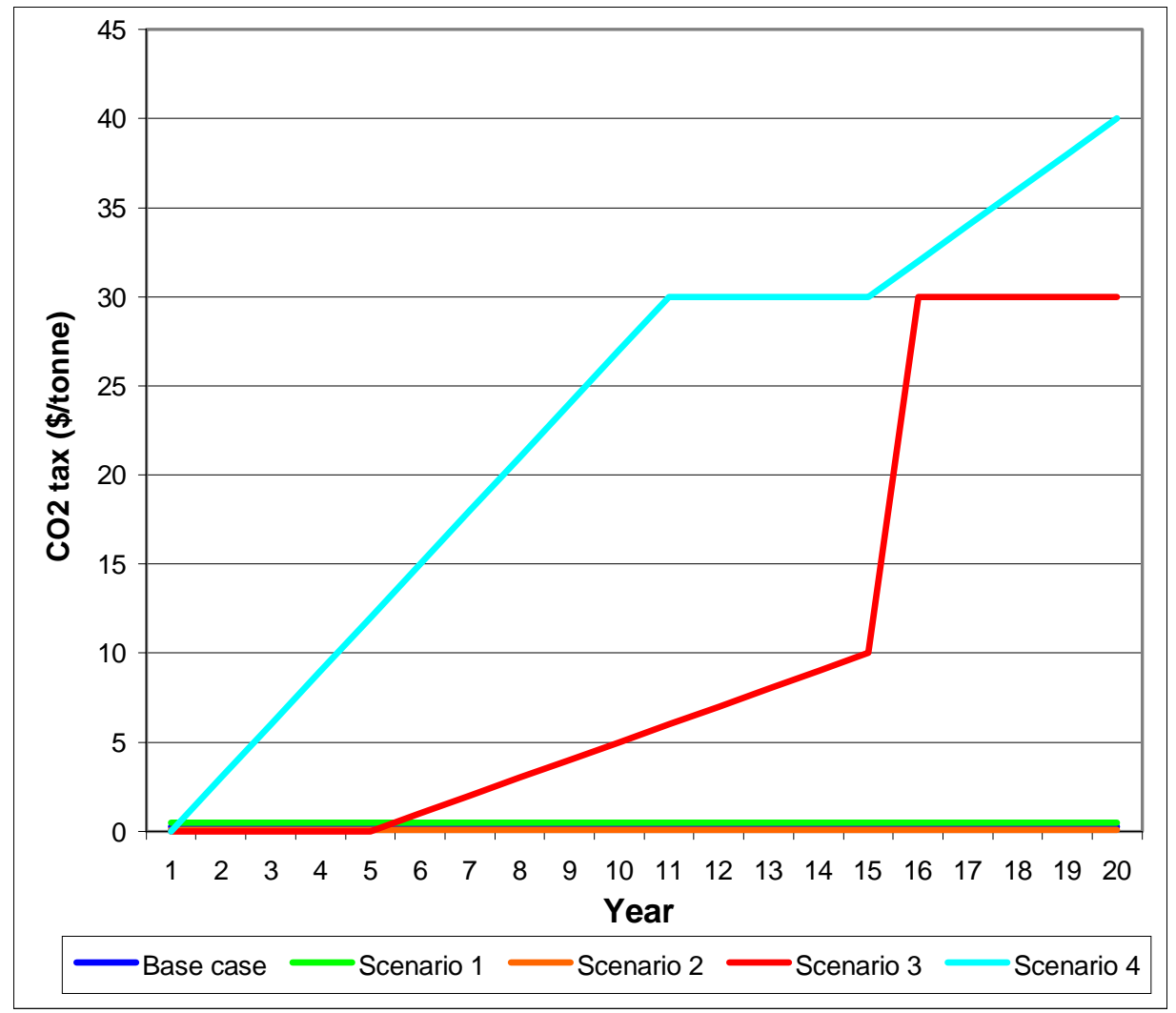

Note that in the base case, scenario 1 and scenario 2 there is no environmental levy. 


\section{Appendix G NGT Supporting Technologies: RAM Technologies/More Electric Studies}




\section{RAM PROBLEMS CURRENTLY ENCOUNTERED IN THE FIELD}

\subsection{Overview and Current Market Conditions}

There are dozens, if not hundreds, of ways a gas turbine can fail. In this report, our purpose is not to provide an exhaustive list of every conceivable failure mode, or even every failure mode that has ever been encountered in the field. Our survey of the technical literature very quickly showed the folly of that approach. What we have done for this report is integrate the results of the literature survey with conversations with current gas turbine operators to come up with the failure modes that seem the most common. More importantly, we have focused on failure modes that could conceivably be monitored or corrected for in the field, rather than those that could only be corrected for in the design phase of a new gas turbine.

Overall market conditions effect RAM in a variety of ways.

The recent "boom" in gas turbine sales has gas turbine OEMs operating at maximum capacity, which has had the effect of decreasing the availability and increasing the lead times of spare parts. This sales boom has had many causes, among them: higher cycle efficiencies, lower capital and start-up costs, faster installation times, the ability to use multiple fuels, including natural gas, and favorable emissions rates, which make obtaining siting permits easier and more cost-effective.

For all these cost and performance advantages, however, newer gas turbines are also seen as carrying higher operating risks as new designs are introduced into service without a long operating history behind them. This has led to the more common use of long-term service agreements between operators and the OEMs. Operators see these agreements as a way to get the OEM to share the risks inherent in an unproven design. New F-class, as well as the upgrades to the older E-class machines have failed more often than anticipated - more than 5 percent of them catastrophically.

High energy prices have encouraged power generators to increase the power available from their existing assets through the use of new technologies with short operating histories such as inlet fogging and steam injections. It has also increased the incentive for operators to find ways to push maintenance outages as far into the future as possible, as well as to concentrate their outages in low usage seasons.

Many plants designed as peaking units are seeing duty cycles closer to "cycling" than "peaking" duty. Since thermomechanical fatigue is the primary cause of hot section component failure, this has led to unforeseen maintenance difficulties. The differing duty cycles have also made it harder for users to predict failures.

In general, power producers are no longer satisfied with the classic method of doing major maintenance at fixed hourly intervals. Instead, they want to move toward condition-based maintenance. For example, many operators wish to push combustion inspections from the classic $8,000 \mathrm{hr}$ interval to $12,000 \mathrm{hr}$, or eliminate them entirely. Several operators have switched to condition-based maintenance on the combustion inspection by monitoring emissions levels. When NOx emissions increase substantially, they know it's time for a combustion inspection. 


\subsection{Fuel/Combustion Systems}

\subsubsection{Problems with Dry, Low NO x Combustors}

Increased restrictions on NO $\mathrm{x}$ emissions have led to the widespread adoption of dry, low-NOx combustor designs. The DLE combustor creates a lean diffusion flame which is so lean that it's on the edge of being quenched during normal operation. This makes these combustors very sensitive to fluctuations in compressor exit pressure and in fuel delivery pressure.

With a lean-fuel mix, the combustor flame burns on the thin edge of not having enough fuel to keep burning, and a phenomenon analogous to a flickering candle sets up pressure oscillations like a series of very rapid small explosions rather than a steadily burning flame. These oscillations can resonate with the vibration modes of the combustion unit and, literally, shake it to pieces.

Fluctuations in compressor exit pressure can occur very quickly as a result of rapid changes in barometric pressure, temperature, and humidity. Fluctuations in fuel delivery pressure can result from clogged fuel nozzles or randomly occurring non-homogeneities in fuel composition. Indeed, some operators report that just several tenths of a percent of an impurity in a fuel supply can cause performance problems.

A sudden loss of fuel pressure, or a sudden increase in compressor exit pressure, can cause the flame front to move back and forth in the combustor can. If the flame front travels too far upstream (flashback), towards the fuel nozzles, the nozzles can be damaged by excessive temperature. Many operators are now blaming a rash of flashback incidents on a lack of superheat, or looked at another way, an excess of humidity or lack of dryness in pipeline delivered gas. If the flame front travels too far aft, the transition pieces and the first stage nozzles can also be damaged by the excessive temperatures.

Even if the flame does not travel far enough to harm components, pressure fluctuations can lead to unwanted vibrations in the thin ceramic shells of the combustion liner and transition pieces. These are normally referred to as buzzing and humming, as it produces audible noise. These vibrations can, over a short time, cause cracks in the combustion liner and transition pieces, leading to early failure.

In order to avoid serious potential internal damage, gas turbines are set to trip (i.e., to shut down) in the event of some abnormality in either the fuel system or in a variety of other parameters such as emissions levels, exhaust gas temperatures, or flame detector indications. In the GE 7F fleet, for example, a variety of trips have been caused by a sudden increase in NO x emissions and a sudden spread in exhaust gas temperature. (The GE 7F is equipped with approximately 27 thermocouples positioned at the exhaust. A difference in temperature reading between any two greater than some preset amount will trigger a trip.)

In case of unusual emissions or temperatures, the combustor cans and fuel nozzles must be inspected for thermal damage. Even when no signs of damage are ultimately found, the inspection can take an average of around $30 \mathrm{hr}$.

Because of the sensitivity of the flame and the possibility of serious damage, many machines are designed to trip when even potential problems are found with the fuel delivery system. Some of the most common causes of forced outages in the GE 7F fleet, for example, are due to problems detected in the fuel delivery system. Examples of failures include: failure of a gas control valve to 
open or close when called upon, abnormal pressure drops detected in the fuel filtration system, and a power loss to the preheaters now commonly installed in natural gas delivery systems. Even though in none of these cases is there necessarily any indication of actual problems in the combustor, an imperfectly functioning fuel delivery system could lead to a condition in which serious damage could occur faster than the unit's ability to shut itself down.

The delicacy of the flame produced by dry, low NO x combustor designs, along with the stiff penalties paid from even accidental non-compliance with emissions limits, has necessitated a very close monitoring of the fuel system and combustor performance. This has resulted in an increased number of trips due to combustion or fuel system operational variations.

A machine trip has consequences that go beyond lost power revenue and the cost of man-power to perform necessary inspections. The biggest root cause of failure in the hot section of the modern gas turbine is thermomechanical fatigue due to thermal cycling. A machine trip which results in a sudden and rapid decrease in temperature severely shortens the life of the hot section components.

\subsubsection{Problems with Heavy Hydrocarbon Condensate}

In an ideal world, the natural gas delivered over the pipeline would be 100.00 percent $\mathrm{CH} 4$ and nothing else. In reality, however, there is always a finite proportion of heavier hydrocarbons present. These heavier hydrocarbons tend to settle in fuel storage tanks and in low sections of piping that can act as traps. During times of high fuel flow rates (i.e., during peak demand periods), these trapped heavy hydrocarbons can be sucked into the fuel line in large enough quantities to condense into their liquid state. Most gas turbine combustors are not designed to burn liquid/gas mixtures, especially not liquids that tend to polymerize at temperatures above about $700^{\circ} \mathrm{F}$.

The liquids entrained into the fuel system can cause blocked fuel nozzles and form residues on combustion liners, transition pieces, and turbine blades. These problems usually manifest themselves to the operator as increased emissions levels and/or exhaust has temperature spreads.

A common but incomplete solution to this problem is to preheat the incoming natural gas to vaporize any liquids that have condensed. Even with preheated systems, however, problems are still encountered at high flow rates. Another simple solution is to increase the size of the traps or the storage tanks on the site to be able to store more heavy hydrocarbons, and then to do regular inspections of the hydrocarbon levels in the tanks. This is adequate, but labor intensive, and an automated system would be preferred.

\subsubsection{Problems with Fuel Impurities}

As operating temperatures in gas turbines continue to increase, the units become more and more susceptible to hot corrosion attack from impurities in the fuel used. Major contributors to hot corrosion attack commonly found in fuels are the alkali and alkaline earth salts - sodium, calcium, and potassium. Gas turbine operators put high quality specifications on their fuel suppliers, but this is often not enough. For a variety of reasons, this problem has been increasing in severity in recent years. 


\subsection{The Compressor Section}

For the most part, operators see the hot section of the turbine as being the life-limiting, and certainly the budget-busting, section of the machine. Most turbine maintenance discussion in the industry revolves around problems with the turbine rotating components, especially those in the first stage. The compressor section, however, has maintenance problems of its own that are being exacerbated by recent trends.

Compressor problems fall into two broad categories: problems that result in gradual performance degradation, and problems that can result in sudden, catastrophic failure.

\subsubsection{Problems Resulting in Gradual Performance Degradation}

\section{Fouling}

As the compressor ingests large amounts of air, it also ingests fine solid and liquid particulates. These particulates build up to form deposits on both the stationary and rotating blades. This phenomenon is called compressor fouling.

The deposits decrease the mass flow rate through the compressor and reduce the aerodynamic efficiency of the blades, which leads to higher compressor exit temperatures and lower compressor efficiency. Even the best practical filtration systems cannot eliminate the problem.

Fouling occurs quickly. Depending on environmental conditions such as humidity and particulate count, gas turbines can lose 3 to 5 percent of their power output in a matter of weeks. Without taking any corrective measures, power output lost due to fouling can easily be 10 percent in less than 6 months.

The direct effects of compressor fouling include:

- Loss in revenue due to lower power output.

- Increase in fuel costs due to higher heat rate.

- Reduction in life of hot section components due to higher turbine inlet temperatures.

- Increased rotor imbalance leading to higher vibrational loads and shorter bearing life.

It is estimated that 80 to 90 percent of the power lost during the operation of the gas turbine is due to compressor fouling, and is therefore potentially recoverable by cleaning the deposits off of the compressor.

Fouling is removed using a combined regimen of on-line and off-line water washes which remove some of the deposits. Much of the fouling remains, however, using even the most stringent of current techniques. This is evidenced by the dirty appearance of most compressors when the casing is removed for overhaul.

\section{Erosion and Pitting}

Erosion of the compressor blades has always occurred to some extent in gas turbines, but in recent years pitting and erosion have worsened due to the increasingly common practice of injecting water into the inlet to increase power output. A properly designed and mounted inlet fogging system should be placed upstream of the inlet filters so that, while it will decrease the wet 
bulb temperature of the incoming airstream, it is not injecting liquid droplets directly into the compressor inlet.

Any liquid droplets larger than approximately $100 \bullet-$ will, over time, cause erosion and pitting in compressor blades. These droplets can come from either poorly designed on-line washing systems, or from improperly mounted inlet fogging systems. Inlet fogging systems generally operate at lower pressures, and thus higher droplet sizes, than well designed water washing systems.

\subsubsection{Problems Resulting in Sudden Catastrophic Failure}

To increase compressor efficiency, and therefore overall efficiency, airfoil cross sections are becoming thinner, blade tip clearances are becoming smaller, and new "3-D" airfoil designs are being introduced. At the same time, compressor pressure ratios are increasing. Historically, compressors have run with compression ratios of about 12:1. But new designs are pushing the compression ratios to $14: 1$ and even 18:1.

New airfoils for compressors are being designed with advanced CFD tools resulting in drastically different shapes than the previous " $2 \mathrm{D}$ " generation. At this early stage of development, this has resulted in increased problems with aerodynamically induced vibration modes (most commonly, "flutter"). This has led to an increase in cracking problems for these newer compressor designs. Closing radial gaps has increased the frequency of blade rubs in the compressor, and increased the need for dimensional precision and stability in casings.

All of this puts unprecedented loads on a given compressor blade. In addition, as with many aspects of recent turbines, new designs are being "tested in the field," or so say operators who encounter unexpected problems on new machines. As a result, compressor blade cracking and chipping are becoming more common, even as FOD is becoming less common in industrial settings.

These problems are often fleet specific or vintage specific. For example, mid-80's vintage GE 7EAs experienced problems in the field in the aft end of the compressor, while early 90's FAs experienced problems in the forward end. Toward the end of the 90's, problems with the aft end of EA retrofits were beginning to resurface (but due to a different cause).

The conventional way to deal with these problems is to perform more borescope inspections over a larger area of the compressor section than operators would like. One SWPC 501F plant in New England, for example, has to do a detailed inspection of its compressor section every few hundred hours to monitor cracking problems in their newly designed compressor section. Another GE 7FA plant in Florida must continually monitor cracking in their compressor section as well. Many turbines were not designed with a great deal of access to the compressor, since outages are conventionally thought of as being driven by the hot section.

Borescope inspection in the compressor is complicated by many factors. There are upwards of 17 stages in the compressor of a large industrial gas turbine. Having borescope ports at every single stage is impractical, leaving many areas of the compressor unavailable for visual inspection. The axial clearance between stages is much smaller than in the turbine section, making borescopes difficult to maneuver. And many problems common to compressor blades are difficult to detect and quantify visually. Fine cracks, even serious ones that could be signs of imminent failure, are 
difficult to see with certainty without using ultrasonic or florescent dye penetrant techniques. Operators have reported that they are often uncertain when, during a compressor borescope inspection, they see what they think to be a crack. They are unable, sometimes, to confirm that it is a crack and, if they believe it so, to determine its severity (i.e., its length and depth).

\subsection{The Turbine Section}

Since the hot section of the turbine is exposed to the harshest conditions, maintenance decisions are most often driven by the condition of parts in this section. Most often, the goal of improving RAM is achieved by operators' attempts to extend the repair/maintenance interval and by attempts to extend the number of service intervals before replacement of critical parts.

The most important trend in gas turbine design over the last two decades is the constant increase in firing temperature. As demand for more efficient and more powerful turbines has increased, OEMs have responded by continually pushing turbine inlet temperatures higher and higher. This has put the most strain on the materials, coatings, and blade cooling techniques used in the hot section. It has also driven up the prices of new turbine parts by more than a factor of 3 over the last decade. This increase in the price of new parts has spurred the non-OEM repair and refurbishment industry to invent and develop a number of new repair techniques and has encouraged operators to attempt to repair parts as much as possible.

\subsubsection{Oxidation-Resistant and Thermal Barrier Coatings}

Typical first stage nozzles and first stage buckets are first coated with an oxidation-resistant bond coat (so called because part of its function is to bond the upper TBC coating to the base metal) made of a PVD deposited or plasma sprayed MCrAlY or CoCrAlY material. Over this bond coat is plasma sprayed a ceramic thermal barrier coating, usually yttria-stabilized zirconia. The TBC's primary function is to maintain a 50 to $75^{\circ} \mathrm{F}$ temperature gradient across its thickness, thus lowering the temperature of the base metal. The TBC is porous, and the MCrAlY coating underneath functions as an oxidation resistor.

Due to the difference in thermal expansion coefficient between the TBC and the metal system underneath, thermal cycling of the gas turbine results in coating cracks over time. This exposes the bond coat to local higher temperatures and locally increases its rate of oxidation. Thermal cycling also leads to spallation of the bond coat (i.e., small, oxidized "chunks" of alumina become debonded from the coating and flake off, usually taking some of the TBC with them).

Even without thermal cycling, the coatings have only a finite life. Erosion and exposure to a corrosive atmosphere are constantly thinning the TBC. And as the MCrAlY loses its aluminum (the element responsible for its oxidation resistance), it ceases to protect the base metal.

Operators use borescope inspections to visually track the condition of these coatings over time. Their effort is primarily directed at crack detection and evaluation. Whenever a crack is seen in the coatings, as they often are, the critical issue is its depth. Cracks that are solely in the TBC are less critical to performance. Indeed, some OEMs say to expect a measure of "craze cracking," a web-like pattern of thin surface cracks like those seen in the mud of a dried riverbed, after only a few cycles. Deeper cracks, ones that extend into the base metal, are more of a problem. Not only will they result in a scrapped blade at the next overhaul, but there is a serious danger of blade separation causing damage to the rest of the blade row. 
At present, it is very difficult to evaluate the depth of a given crack visually. Operators, OEMs and third party inspectors often make educated guesses based on the shape and pattern of the cracks to decide if they are "just in the coating" or if they extend into the base metal. Some argue that the "dried mud" pattern indicative of craze cracking is almost always shallow and harmless. Others argue that any crack visible by borescope is too deep to be craze cracking.

A coating may have little remaining life even if a visual inspection reveals no cracking. The TBC overcoat is constantly thinning due to simple erosion, and the aluminum in the bond coat is constantly being depleted by continued oxidation. There is currently no way to measure the remaining $\mathrm{TBC}$ thickness or the remaining aluminum in the coating, and parts are often retired before it is necessary due to this lack of knowledge.

\subsubsection{Complex Internal Cooling Passages}

Most early stage nozzles and buckets in the newest turbine engines use internal passages to circulate cooling air throughout the interior of the blade, as well as holes at the leading edge, trailing edge, and the tip for "film cooling" purposes. If these passages or holes are blocked, or if a crack penetrates through the blade wall to compromise these passages, immediate and severe blade damage can occur.

There is little an operator can do in service to evaluate the condition of these passages. A visual borescope inspection can reveal evidence of overheating on various areas of blades that may indicate a blocked passage. Some fiberscopes are small enough to enter a cooling hole if it is unblocked, but articulation of these scopes is limited.

Cooling passages are especially susceptible to blockage during the recoating process undergone during refurbishment.

\subsubsection{Overall Remaining Life Assessment}

With new parts being an expensive proposition and a catastrophic failure being an even more expensive one, every effort is made to assess the remaining life of the turbine blades during overhaul. With the rotor removed from the machine, a detailed non-destructive evaluation is performed on every blade. Dye penetrant, ultrasonic and eddy current techniques are used to find and evaluate the length and depth of any cracks found in the blades. Blade wall thicknesses are measured to ensure enough wall thickness remains to withstand mechanical stresses. Usually, at least one blade in a row is "sacrificed" to destructive metallurgical evaluation. Only a microstructural evaluation using standard metallurgical techniques can reveal how much the microstructure has "aged," and thus how much fatigue and creep strength remains.

This technique can give the operator a sense of how many hours the blades may continue to be used without excessive risk of catastrophic failure. This, combined with the best estimate available of the operating temperature history, is currently the best way of estimating remaining part life. Unfortunately, it can only be done during overhaul, with the rotor removed.

For newer, higher firing temperature, machines, the operating temperature history is also conventionally difficult to measure accurately. Over the last decade, firing temperatures have increased beyond the capabilities of conventional thermocouples that were once used to measure turbine inlet temperature. Today, it is more common to back-calculate the turbine inlet temperature based on measured exhaust gas temperatures. Clearly, this calculation depends on a 
lot of assumptions about the behavior of each turbine stage, and is unlikely to accurately detect localized hot spots. Calculations of remaining life based on exhaust gas temperature measurements must be very conservative because of this inaccuracy.

\subsection{Control Systems}

The control system of a modern gas turbine is designed not only to run the system, but to shut down the machine in the event of a condition that might lead to expensive failure. To this end, it monitors a great number of parameters including: vibration levels, temperatures, pressures, emissions, flame detection, and valve positions. The controller is designed to trip the machine if any of these parameters falls outside of normal levels.

Unfortunately, there are a fair number (perhaps as high as 30 percent) of trips that could be categorized as "false alarms:" spurious or noisy sensor readings, a temporary loss of excitation to a sensor, or a software or computer card communication error. Each of these can cause a trip, and in each case there is nothing wrong with the gas turbine itself. Most of these "false alarms" are easily and quickly rectified, with the machine back running in less than an hour. But the thermal cycle, especially in the case of a very rapid cooldown, does unnecessary damage to the hot section components.

\subsection{A Note on Sources}

The material in this report comes from the variety of sources described below:

\subsubsection{Published Information}

- Journals such as Power, Turbomachinery International, Power Engineering, Gas Turbine World, Diesel and Gas Turbine Worldwide.

- Conference proceedings from the IGTI (ASME) Turbo Expo and the University of Texas Turbomachinery Symposium.

\subsubsection{Interviews with Industry Experts}

- O\&M vendors such as Advanced Combustion Technology, Sermatech Gas Path, TurboCare, Hickham Industries, and Liburdi Engineering.

- End Users such as PG\&E, Reliant Energy, Duke Energy, Dominion Energy, Sithe Energies, Florida Power \& Light, and Massachusetts Municipal Wholesale Electric.

- Other Vendors and Institutions in the field such as Southwest Research Institute, BWD Turbines, Gas Turbine Materials Associates, Land Infrared, Meggitt Avionics, and Jentek Sensors.

- $\quad$ OEMs such as Pratt \& Whitney Power Systems and Rolls Royce Power Systems. 


\section{NEW RAM IMPROVEMENT TECHNOLOGIES IN DEVELOPMENT}

Many of the new technologies for improving RAM in development are being designed to achieve one overall goal: to enable operators to make better and better estimates of the remaining life of their hot section components, both the combustor and the turbine. The hot section is the highestcost section of the turbine to maintain and repair, as well as the section that operates closest to its operating limits. There are, additionally, several technologies in development for dealing with various other problems that cannot be grouped into one category.

\subsection{Technologies for More Reliable Fuel/Combustor Systems}

\subsubsection{Optical Spectrometry Sensors for Detecting Compounds in the Flame}

Optical spectrometry is a technique that uses an optical sensor to measure the emission spectra of hot bodies. The flame of a gas turbine contains a variety of compounds including combustion products, impurities, fuel, and air. Measuring the frequency distribution spectrum of the flame can yield information about the quantities of the various compounds in it. Currently, this technique is being used to measure impurities such as sodium, calcium, and other alkali salts that have been a prevalent problem in fuel oils. These alkali salts attack and crack welds. They generally show up as green spots on the TBCs of combustion liners and transition pieces.

The same technique could be used, however, to deliver on-line measurements of pollutants such as $\mathrm{NO} x$ and CO. Currently, both of these emissions are measured in the stack, where they are detected anywhere from 3 to $10 \mathrm{~min}$ after they have been formed. Measuring their presence closer to the source can yield more information about what exactly causes, say, changes in emissions rates. It could also be used for more accurate control of ammonia injection in an SCR, in which the delay in NO x measurement can often lead to inappropriate ammonia injection. The system could also be used to detect the presence of heavy hydrocarbons in the flame.

To use optical spectrometry to detect a variety of compounds requires complex optical filtration technologies, as well as some basic research and calibration for the various compounds desired. In principle, however, the technology could be a powerful tool for gas turbine operators to know exactly which impurities, and in what quantities, have gone through their machines. Ultimately, the technique could be used to measure dynamic pressure in real time, which would enable operators to control acoustic vibrations.

\subsubsection{Optical Pyrometry for Measuring Flame Temperature and Position}

Conventional flame detectors are essentially UV detectors and operate in an "on/off" mode (i.e., they either detect a flame or they don't). Using newer infrared imaging techniques could improve greatly upon this simple function. Rather than simply detecting "flame or no flame" as in current flame detectors, an IR imaging chip could detect temperature distribution over the area it "sees" and thus detect the center of the flame front and track its movements.

As was stated in Section 1 of this report, modern dry low NO x combustors are susceptible to flame instabilities that can cause the flame to flash forward, toward the first stage turbine nozzles, or flash back, toward the fuel nozzles. Either way, damage is caused when the flame front moves. Additionally, combustor vibration is a problem that results in combustion liner and transition piece damage (usually cracking and coating degradation) over time. To control combustor vibration, it is helpful to be able to measure it. IR optical flame detection could help measure flame vibration 
in real time as well as be able to detect which way the flame is tending to move. This helps in assessing damage when the flame front moves too far upstream or downstream. Currently, operators know they've had some sort of flame movement "event," but don't know how far or which way. Their only option is to perform a difficult inspection with the machine off-line if they want to ensure that their machine is undamaged.

\subsubsection{Precision Electric Fuel Metering}

A sensor that could detect the position of the flame in real time could be used to feedback to a precision electric fuel metering valve with rapid response. This could be used to actively damp combustion vibrations due to flame front vibration. It could also be used to prevent flame propagation upstream towards the first stage nozzles by reducing fuel pressuring in response to fluctuations in compressor exit pressure. The frequency response of both the sensor and the actuator would have to be fairly high for this to be feasible.

\subsubsection{Fuel Supply Conditioning Technologies}

As mentioned in Section 1, heavy hydrocarbons and liquid impurities in the fuel supply can condense and cause serious problems in the combustion system. For example, a gas turbine had just come off a combustion inspection in which all combustion hardware had been replaced with new components. Within three weeks, the NOx emissions had become unacceptably high. The operator had no choice but to shut the unit down and do an extensive visual inspection. The inspection revealed extensive "coking" on the surface of the liners and transition pieces where heavy hydrocarbons had condensed, polymerized, and burned holes through the liners. Heavy hydrocarbon liquids had also clogged several fuel nozzles. The resulting combustion imbalance severely damaged the crossfire tubes as well.

The simplest way to avoid hydrocarbon condensation is to pre-heat the incoming fuel enough to prevent condensation when the fuel is pressurized. The problem with this method is to determine the amount of pre-heat required. It depends greatly on the type of heavy hydrocarbons present. Conventionally, the amount of heavy hydrocarbons are expressed in categories: the amount of $\mathrm{C}$ compounds, $\mathrm{C} 2$ compounds, $\mathrm{C} 4$ compounds, and $\mathrm{C} 6$ and above compounds. The problem is that grouping all the "C 6 and above" compounds together does not give fine enough resolution to solve the problem. Fuels with identical analyses by the above method have dewpoints as far apart as $100^{\circ} \mathrm{F}$. A better method is to be able to detect hydrocarbons on-line up to $\mathrm{C} 14$.

Another low-tech method is to place a "catch tank" in the fuel system somewhere in the line after the fuel is pressurized. The idea of a tank is that liquids will settle to the bottom, and then they can be drained. OEMs currently recommend both procedures. They do, however, have their limitations.

Real-time detection of impurities and heavy hydrocarbons in the fuel can reveal when the pre-heat should be increased to counter a temporary increase in these contaminants.

\subsection{Technologies for More Reliable Compressor Systems}

\subsubsection{Cleaning Technologies to Improve Fouling Removal and Minimize Erosion}

One concept that would improve cleaning performance would be to use an ultrasonic cleaning technique which has been proven to eliminate deposits of all kinds. Aircraft engine compressors have been cleaned using ultrasonic techniques with great success. Of course, to use this 
technology as a substitute for current water washing technologies would require the engineering of a system to "dam" the compressor at the inlet and at some latter stage. The fouled compressor sections could then be filled with a water/detergent solution and ultrasonic transducers deployed. Within 1 to $2 \mathrm{hr}$, the built-up deposits on the compressor blades could be loosened and removed. The compressor can then be rinsed, the enclosure removed, and the unit returned to service.

The primary technical barrier to the success of this concept is the mechanical design of the aft "damming" system for sealing off the compressor from the rest of the turbine without disassembling the machine. The axial clearance between blades is less than half an inch, and access to the interior of the compressor is limited. Probably the most accessible areas are the bleed annuli usually located about 1/3 of the distance from the inlet and exit (see Figure 1).

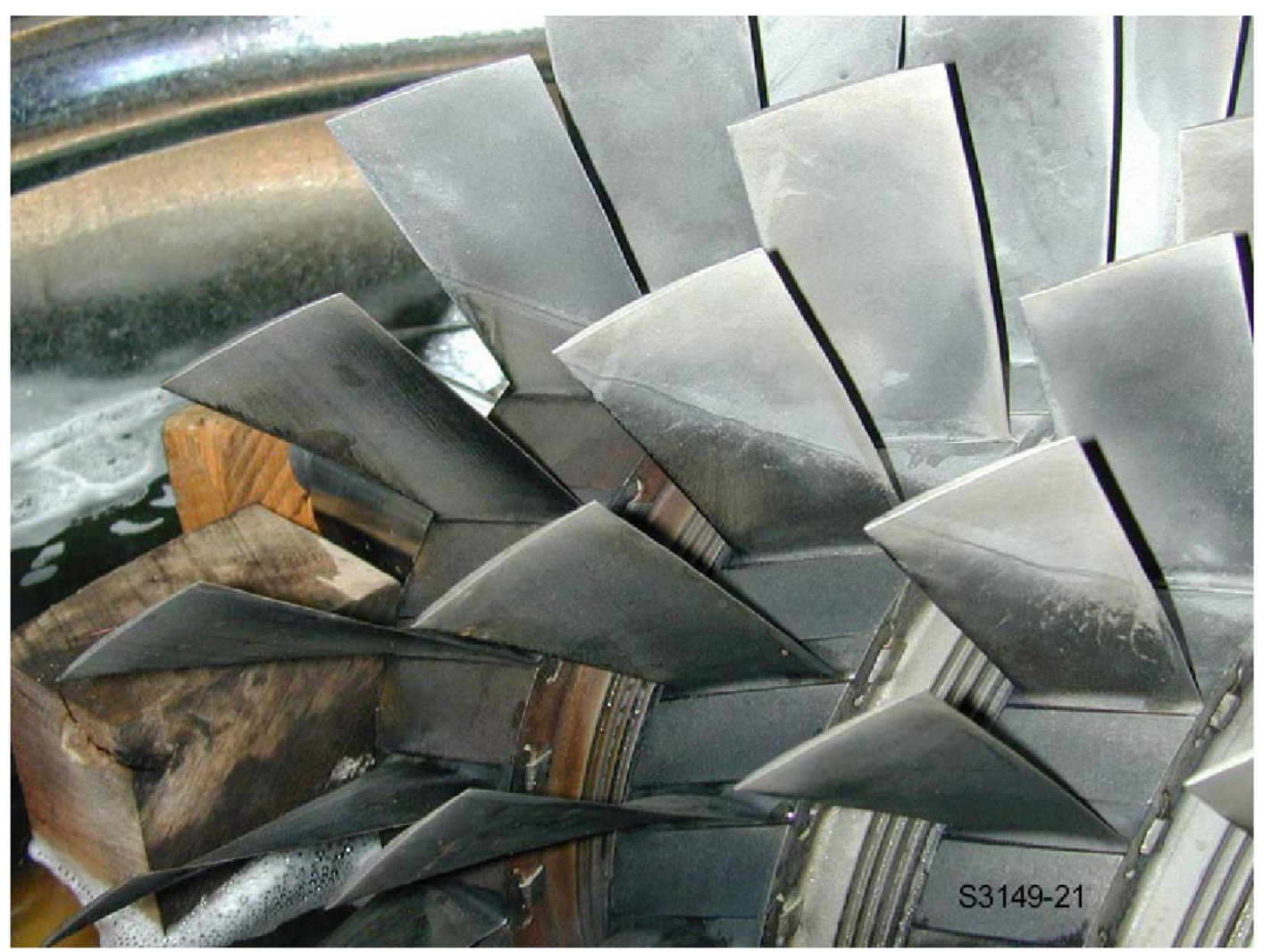

Figure 1. Illustration of the effectiveness of ultrasonic cleaning. The top has been cleaned, the bottom has not. 
The ultrasonic technique would, if practicable, result in complete removal of compressor fouling with no erosion or pitting. If it could be done in a similar time period to a conventional off-line water wash (also known as a crank wash), it could lead to much improved compressor performance, and longer hot section life.

\subsubsection{Improved Compressor Inspection Technologies}

\section{Improved Access Tool}

A tool that could be inserted at the inlet of the compressor, travel down the compressor to any stage, and then be articulated circumferentially to inspect all of the blades, would enable an operator to have full access to the compressor without necessitating the construction of over 40 borescope ports. Recall that an average sized industrial compressor can have over 17 stages. To see just the rotating components on such a machine would require at least 17 borescope ports. It would be impossible with conventional tools to see all the stationary components. Standard practice on the turbine end, which has many fewer stages, is to place borescope ports on at least four circumferential positions to afford some access to the stationary blades.

This tool could be developed through the modification of existing steerable lance technologies (see Figure 2). 


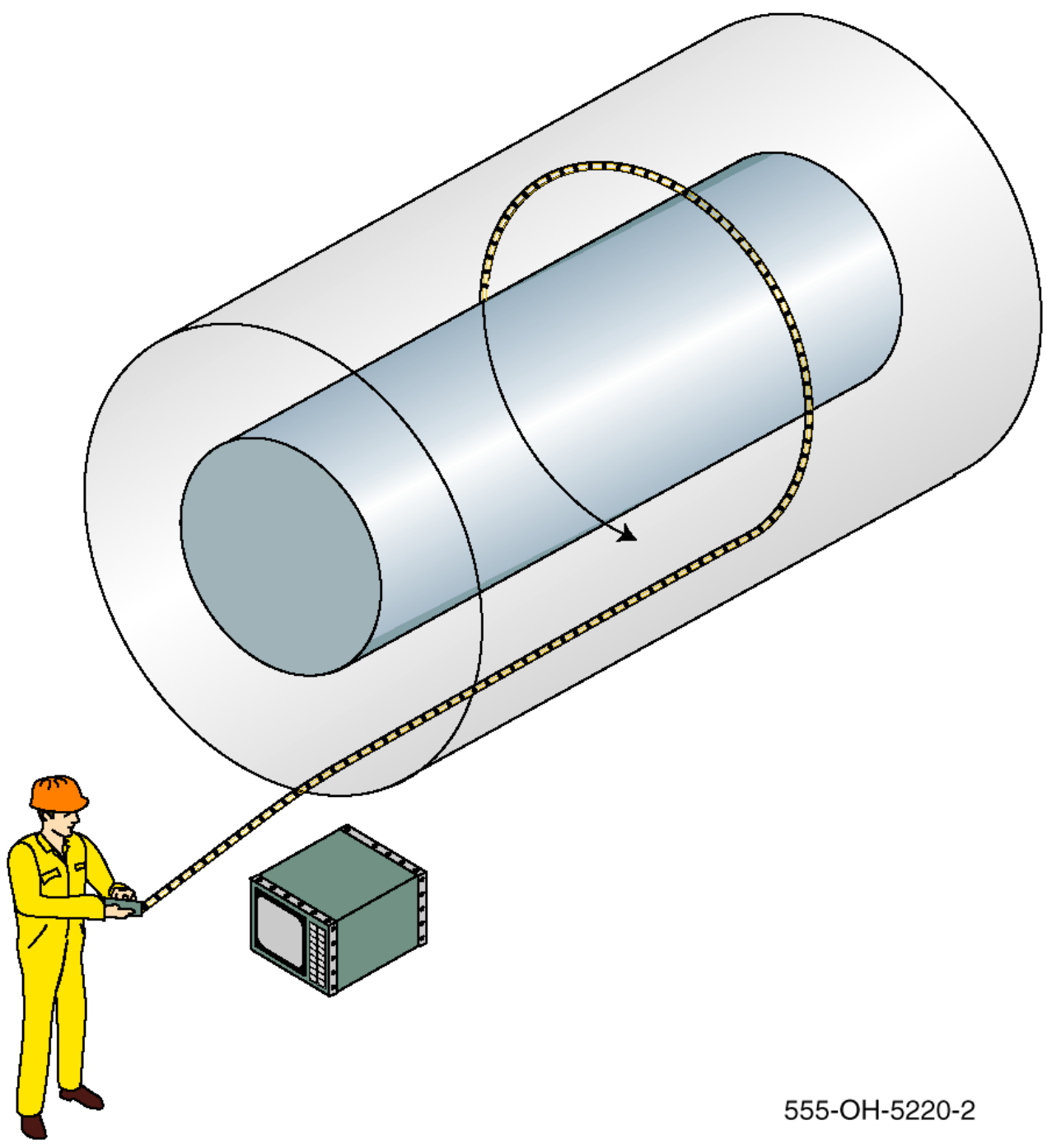

Figure 2. Concept for a compressor inspection tool that would access the entire compressor
independent of built-in borescope ports.

Improved Sensor Technologies

The steerable tool described in the previous section could deliver a variety of sensor technologies to detect and measure cracks. Conversations with experienced borescope inspectors suggest that it is often difficult to evaluate the severity and probable consequences of cracks with only a visual inspection. Shadows thrown by hundreds of blades in a poorly lit compressor can make it difficult to determine whether a particular feature on a particular blade is even a crack at all.

Adding an ultrasonic or eddy current sensor to an inspection tool would remove this subjective ambiguity from the inspection process. Such sensors are currently used on the benchtop when the rotor has been removed from the casing to evaluate necessary repairs. Tools could be developed that could deliver them to the rotor while it was still in place.

Inspectors would then be able to measure the length and depth of cracks to determine their severity objectively. 


\subsection{Hot Section Remaining Life Assessment Technologies}

There are two major related problems that improved life assessment technologies aim to solve: catastrophic failures, and high turbine blade scrap rates.

In the worst case scenario, a blade can become so damaged during operation that a large chunk of it separates from the rotor and destroys many other blades before the machine can be shut down. Typical gas turbine control systems are equipped with vibration sensors to detect the imbalance that can be caused by a blade separation (among other things) and trip the machine to contain the damage. Most of the time, however, a blade separation causes millions of dollars in damage and lost revenues.

Most of the time, operators are conservative enough in their maintenance schedules to avoid a catastrophic failure. The more common, but related, problem is that of a high turbine blade scrap rate when the machine is overhauled. During a conventional overhaul, the blades are evaluated individually to determine whether they can be repaired or replaced. A repaired blade can cost 1/10 th to $1 / 3$ rd the amount of a new blade, so operators have a strong motivation to perform an overhaul late enough to maximize their revenue, but early enough to avoid a high scrap rate.

The key question facing operators is: how does one know before an overhaul how much longer a blade can be operated before it either fails catastrophically or becomes scrap?

\subsubsection{Predictive Maintenance Software}

One approach under development is using software to continually monitor thermodynamic conditions in the hot section in real time. Using this data, along with a mathematical model of material behavior based on empirical studies, the algorithm can make a prediction about remaining life.

Math models relate time and temperature to the metallurgical changes in the material that lead to properties degradation. So you need to know the initial conditions (i.e., the presence of various phases in the material), and the operating speeds and temperatures continuously, in order to predict part life. Even a short period of undocumented operation at unusually high turbine inlet temperatures, for example, can drastically shorten expected blade life. Therefore, the continuous predictive approach is superior to an "average" calculation based on typical operating speeds and temperatures.

There are two kinds of difficulties encountered in optimal implementation of a software-based predictive maintenance routine: the lack of fully developed mathematical models for every failure mode, and the lack of exact knowledge of necessary thermal parameters.

For example, the Larson-Miller creep life model is commonly used to model the remaining creep life of the combustion liners and turbine blades, and this life is taken to be solely a function of temperature and operating speed (i.e., stress). However, most modern turbine components are life limited by thermomechanical fatigue or coating degradation, and often fail long before they reach the end of their creep life. Good models for these other two common failure modes are not as well developed. Both are more than solely functions of temperature.

As for the measurement of blade temperatures, a convective heat transfer model, coupled with estimates of turbine stage performance, is used to calculate the metal temperatures on the turbine blades, rather than measuring their temperature directly (as seen in Section 1). Because firing 
temperatures have become too high for conventional thermocouples, most currently operating machines depend on exhaust gas temperature measurements to derive an estimate of the temperature of the first stage nozzles and blades. The errors and inaccuracies inherent in these estimates limit the utility of prognostic modeling techniques.

\subsubsection{Improved Hot Section Temperature Sensors}

In order to optimize the effectiveness of any real-time prognostic tool, the temperatures of the hot section components, most specifically the 1 st stage turbine buckets, should be known as accurately as possible. Measuring the temperature of the turbine blades in real time would have the following benefits:

- Increasing the accuracy of the predictions made by prognostic algorithms.

- Immediate detection of blocked or non-functioning cooling channels which could lead to blade overheating and possible separation.

Optical pyrometry is the main technique used. For more than a decade, optical pyrometry has been used to test gas turbine prototypes in OEM test beds. More recently, the technique has been used in the field to take high speed measurements of rotating blade temperatures.

There are two distinct techniques, both broadly referred to as "pyrometry," that are currently under development: one measures the temperature of the hot gas, and the other purports to directly measure blade surface temperatures.

The first technique uses high temperature fiber optics to look at the spectrum of a piece of emissive material suspended in the gas flow field. By examining two particular spectral frequencies, the temperature of the body, and hence the temperature of the hot gas, can be measured. Conax Buffalo is one company working on this kind of technology, and has demonstrated that its system can give accurate temperature measurements for at least $8000 \mathrm{hr}$. The emissive material is placed in a sapphire rod to prevent the formation of deposits that may alter its surface emissivity.

The second technique looks directly at the emission spectra of the turbine blades themselves. A system sold by Land Infrared consists of 32 high-speed, high-precision optical pyrometers positioned circumferencially around the first rotating turbine stage. The system is claimed by the company to have a resolution of $1^{\circ} \mathrm{F}$ over its operating range of 1200 to $2000^{\circ} \mathrm{F}$. The problem with looking directly at the turbine blade is that the surface emissivity of the blade is effected in an unknown way by the surface characteristics of the blade. Impurities, deposits, cracks, and other features that modify the surface can effect the emission spectrum. Nevertheless, this technique can be very useful in detecting unusually high temperatures in a given blade, and in detecting sudden changes in the temperature of a given blade that might indicate a clogged cooling passage. Thus, even if the system is not as accurate as claimed, it can still detect certain faults before conventional techniques would.

Neither of these technologies directly measures the exact temperature of greatest interest to turbine operators: the temperature of the blade parent material. The first technology measures the gas temperature, and the second measures the temperature at the surface of the TBC. From either of these temperatures, the parent material temperature can be estimated, but what is ultimately desired is an actual direct measurement. 
One technique that has been suggested to measure the temperature of the parent material directly is a variant of the second technology described above. The ceramic TBCs that are used become translucent to particular wavelengths of light above a certain temperature. A pyrometer system that could detect these wavelengths, and could calibrate and convert them to a temperature, would be measuring the temperature of the parent material beneath the TBC.

\subsubsection{Increased Inspection}

Visual inspection of hot section components has been used as a maintenance tool for decades, but its utility is limited. Parts are visually inspected for obvious damage. If the damage is below some minimally acceptable level, the part remains in service for another inspection interval. For this process to work, it is important that damage does not progress to failure rapidly from a minimally detectable level that might be missed in an inspection. It must be determined during the inspection that a given part can last longer than an inspection interval with some degree of certainty.

If the inspection interval were shorter, this would be an easier decision, and ultimately parts could be operated for more of their useful life. This would simply require more inspections. However, the cost of each inspection, and the analysis that accompanies it, must be taken into account. If inspections were faster and less expensive, then shorter inspection intervals would be practical.

Most turbine components are easily accessible with conventional borescope tools. However, the leading edges of the first stage turbine nozzles are difficult to access in can-type combustor designs. Only the trailing edge can be easily accessed, but most damage occurs on the hot leading edge. To solve this problem, and others like it, requires inspection tools with more flexibility and controllability. An example is shown in Figure 3. 


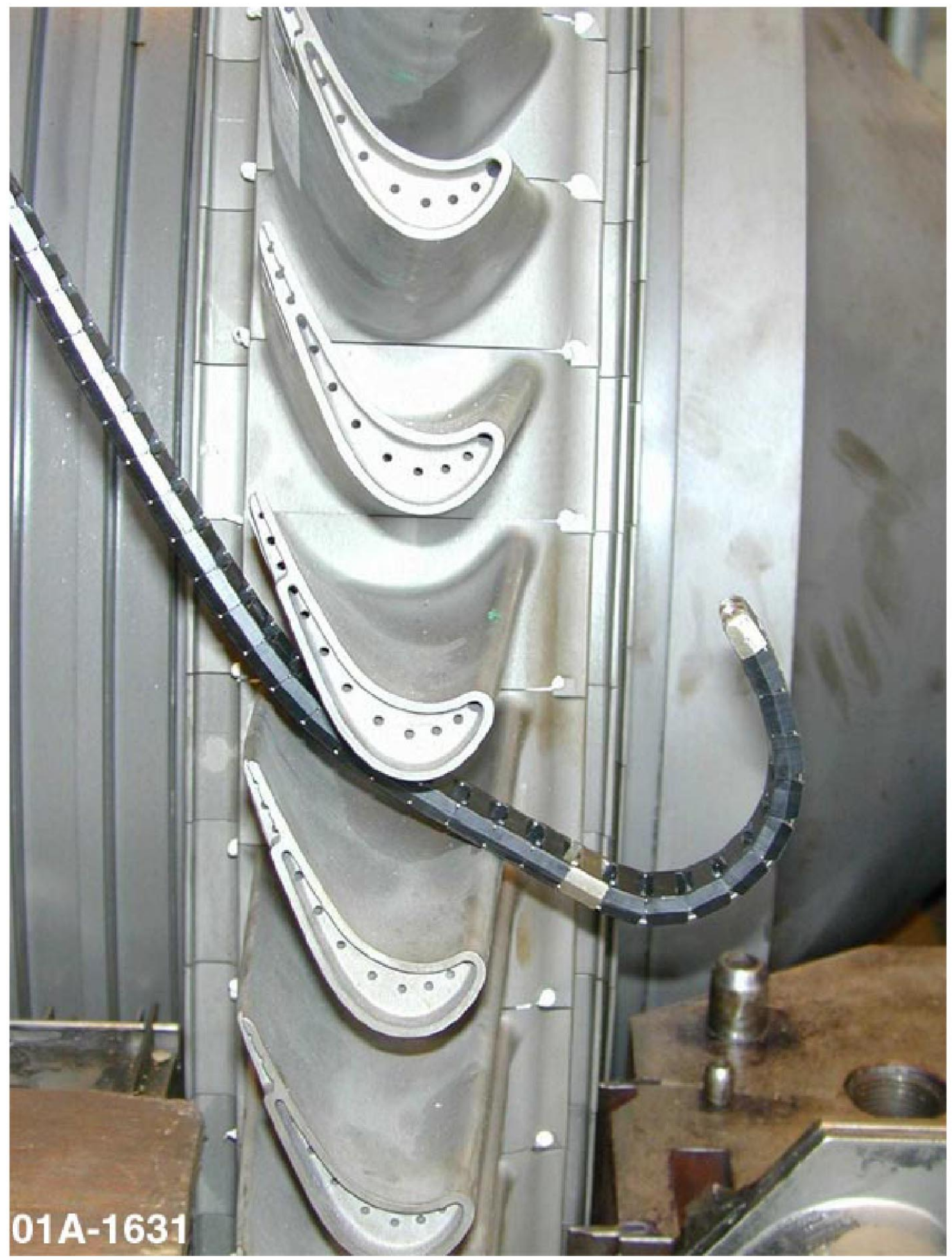

Figure 3. Turbine inspection tool. Note how the tool could approach from the trailing edge but turn around to inspect the leading edge. The tool could be fit with visual and NDE sensors. 


\subsubsection{Non-Visual Inspection Techniques}

There are a variety of failure modes in the hot section components that are either impossible or very difficult to visually detect. New inspection techniques that use non-destructive testing technologies to detect non-visible damage to components could increase part service life.

\section{Creep Damage}

Creep damage is dependent on time, temperature, and stress, and creep life is usually available as a general guideline from the manufacturer. This standardized life estimate is usually a conservative and safe limit under all operating conditions for the fleet. Any given real part will probably last longer than this estimated general life, unless it is subjected to operating conditions of more than average severity.

The question then becomes how to measure the extent of the creep damage already suffered by a component. The conventional approach relies on removing a turbine blade from a given row and subjecting it to a destructive metallurgical evaluation. Metallurgists look for such things as aging of precipitation hardened alloys, formation of intermetallic phases, carbide aging, and quantitative analysis of gamma prime size.

Inspection of the blades will not identify creep or fatigue damage unless it is in the very late stages where dimensional changes can be measured and tracked. The primary way of "measuring" creep damage in real time would be to measure the temperature of the blade as accurately as possible and integrate this data into a remaining life model using well-understood relations between time, temperature and remaining life.

\section{Hot Corrosion Damage}

Oxidation is the primary corrosion mechanism attacking metals at high temperatures. Special coatings are used, the primary purpose of which are to protect hot components from oxidation. The life and failure modes of these coatings will be discussed in the next section.

Other elements, mostly present as impurities in the fuel, are also responsible for hot corrosion. Among these are some mentioned earlier, calcium, potassium, and sodium, as well as sulfur compounds, lead and vanadium. The best way of dealing with this kind of corrosion is not to let it occur in the first place. Fuel filtration systems and on-line monitoring of fuel content will help alleviate gas turbine hot section damage from this particular mechanism.

\section{Coating Degradation due to Thermal Cycling}

As was seen in Section 1, oxidation and temperature resistant coatings are applied at least to first stage turbine nozzles and buckets, if not the aft stages as well. If these coatings are damaged or destroyed, the parent metal underneath will be subjected to temperatures and oxidation damage beyond their design limits and quickly fail.

Visual inspection of these coatings can reveal some cracks and spalling in the coatings. However, a common failure mechanism for these coatings is that they simply erode away, a process almost impossible to detect visually. Some non-destructive techniques are being developed which use eddy current or Meandering Winding Magnetometer (MWM) sensors to evaluate both the thickness of the remaining ceramic TBC and the remaining aluminum content in the bond coat. Integrated with a mathematical model to predict remaining life from knowledge of current 
thickness, these techniques could be used to give the most accurate estimate of remaining part life.

Visual inspection alone also cannot give an accurate estimation of crack severity. Even after $50 \mathrm{hr}$ of operation, a new coating can show craze cracking in the ceramic TBC. Most operators are alarmed the first time they see this, but OEMs reassure them that this cracking is "just in the coating" and is to be expected. But with visual inspection alone, an inspector cannot tell whether a crack is "just in the coating" or whether it extends deeper into the base material. If the crack extends into the base metal it is a much more serious problem. Such a crack could lead to blade disengagement from the rotor during operation and cause major damage to the entire rotor, or it could compromise the internal cooling passages which again could result in blade separation or melting. An eddy current or ultrasonic sensor deployed into the turbine would be the only way to make such a determination. Such sensors are already used on the bench when the components have been removed from the unit to evaluate repair options.

As in the case of compressors, non-visual crack detection methods could also help detect cracks that are difficult to see visually. From conversations with experienced inspectors, it seems that generally cracks on the leading and trailing edges are relatively easy to see because they show up with high contrast on the highly curved convex surface. On the concave surface of the body of the blade, however, it is very difficult to see cracks. It would be in these areas where non-destructive testing methods would be useful for crack detection and evaluation.

\subsubsection{Improved Inspection Summary}

Below is a summary of the applications for new inspection tools that would improve RAM in gas turbines.

\section{Combustion Hardware Visual Inspection}

What: Access combustion hardware (liners and transition pieces) with minimal disassembly (through a single flame detector, spark plug or pressure port), preferably without removing a combustion dome. Visually inspect liners, transition pieces, fuel nozzles and other hardware for burns or cracks. Inspect multiple cans from one access point by extending through the crossfire tubes. Inspect as many cans as possible from one insertion point.

Why: Dry low NOx combustors experience flame stability problems after which the damage to the combustor is unknown. Removing the combustion dome from a can is a laborious process. An inspection tool that could be easily inserted would enable operators to assess the condition of their combustors more cost effectively.

Where is Greatest Need: Gas turbines with dry low NOx combustion systems, primarily those designed with combustion cans (as opposed to annular or stack combustors, which are easier to access).

\section{First Stage Turbine Bucket NDE Inspection}

What: Access first stage turbine buckets through standard borescope holes. Deploy an ultrasonic or eddy current sensor along the main body of the blade (as opposed to the leading or trailing edge) to detect and measure cracks. The same tool could be used to deploy a sensor capable of measuring remaining coating thickness on the blade. 
Why: Cracks on the main body of the blade are difficult to see/evaluate with visual techniques because many of them just don't stand out in sharp contrast. Even when seen visually, their severity is difficult to evaluate. An NDE tool that could tell operators about the existence, length and depth of cracks would be a useful one for making maintenance decisions and assessing risks.

Where is Greatest Need: Virtually any gas turbine would be a candidate, but focus should be on newer machines with higher firing temperatures and fewer operating hours.

\section{First Stage Turbine Nozzle Leading Edge Inspection}

What: Access first stage turbine nozzle leading edge either through a combustor can port or through the standard trailing edge borescope holes. Visually inspect the entire circumference of the turbine nozzles from one access point (or as few as possible).

Why: In the can combustor design, the leading edge of the first stage turbine nozzles is difficult to see. Removing a combustor dome and going down the liner and transition piece is currently the only way, and this laborious process only affords access to a small fraction of the nozzles. Again, due to flame instability problems in DLN combustors, these nozzles can be damaged in operation.

Where is Greatest Need: Gas turbines with DLN can combustors would be the best market.

Any Stage Compressor Visual and/or NDE Inspection

What: Access any stage of the compressor from the inlet. Have the ability to go in axially and, upon reaching the desired stage, go around circumferentially to examine every blade with a videoprobe or an NDE crack detection technique.

Why: While the compressor is less of a problem than the hot section, it also generally has less access. Even a 17 stage compressor, for example, typically has borescope holes at only 3 to 5 axial locations. While most of the time this is adequate, in cases where there is an unusual and unknown problem this inability to see most of the compressor is a severe constraint.

Where is Greatest Need: Large, newer model, frame engines with long compressors. Newer models may be experiencing problems common to their class of machine. Both the newest GE and the newest SWPC machines have had compressor blade cracking problems reported.

\subsection{Other Technologies for Enhanced RAM}

\subsubsection{Improved Vibration Instrumentation}

Gas turbine operators have always relied on vibration instrumentation to monitor overall system stability. A sudden increase in vibration levels can indicate a loss of material somewhere on the rotor, a bearing problem, a sealing problem, or other kind of system imbalance. One of the complexities of using this technique has always stemmed from the subjective nature of interpreting vibration spectra, which makes setting simple alarm limits problematic.

Software that can better interpret the results of vibration analysis is one solution approach taken by Bently-Nevada. For example, vibration problems on machinery may be associated with particular harmonic orders or sub-harmonics. Various shaft modes and their harmonics occur at relatively low frequencies, much higher frequency vibrations are associated with the combustion system, harmonics associated with blade passing frequencies are usually somewhere in between these two extremes. Each machine ends up having its own harmonic signature. Monitoring this 
specific signature over time, and isolating the causes of the various components of the spectrum, enables a user to "tease" out a great deal of information about performance.

High temperature accelerometers capable of withstanding temperatures over $1000^{\circ} \mathrm{F}$ are being developed. In addition, improved data analysis tools will provide owner/operators with early fault detection that can prevent catastrophic failures resulting from continued operation after a malfunction occurs.

While usually smaller and lighter, aeroderivative gas turbines have the same basic design as industrial units, except for the common use of anti-friction bearings for radial and thrust loads. These roller bearings require less space and can handle higher speeds than journal bearings. Some aeroderivative designs have shaft speeds that exceed 10,000 rpm. Some "dual spool" units have a second lower speed shaft rotating inside the high-speed shaft. This system provides more efficient compression and combustion capabilities while maintaining a small footprint. Limited shaft access and extremely high temperatures on these machines make vibration monitoring systems difficult. Larger aeroderivative units may include accelerometers or velocity probes mounted inside the turbine casing on the actual bearing supports. The shorter transmission path of the vibration from the shaft surface to the bearing position provides a better indication of shaft vibration than externally mounted sensors. These vibration signals are split and filtered just like casing-mounted vibration transducers. Vibrations in specific frequency ranges are monitored to determine excitation associated with specific shaft speeds. Typical alarm setpoints of less than one inch per second for velocity signals and 2.0 mil pk-pk for displacement signals can be applied to both externally and internally mounted sensors.

\subsection{RAM Technology Assessment Conclusions}

Tables 1 and 2 summarize the findings of the RAM technology assessment. 
Table 1. Matrix of problems and solutions

\begin{tabular}{|c|c|c|c|}
\hline \multirow[b]{2}{*}{ Area } & \multirow[b]{2}{*}{ Problem } & \multicolumn{2}{|c|}{ Possible Solutions } \\
\hline & & Detection & Prevention \\
\hline \multirow{4}{*}{$\begin{array}{l}\text { Fuel/ } \\
\text { Combustion } \\
\text { System }\end{array}$} & Acoustic Vibrations (hum) & $\begin{array}{l}\text { Vibration analysis, focused } \\
\text { sensors }\end{array}$ & $\begin{array}{l}\text { Feedback control w/metering } \\
\text { valve }\end{array}$ \\
\hline & Flashback/Flashforward & $\begin{array}{l}\text { Optical flame position } \\
\text { detection }\end{array}$ & $\begin{array}{l}\text { Feedback control w/metering } \\
\text { valve }\end{array}$ \\
\hline & $\begin{array}{l}\text { Heavy Hydrocarbon } \\
\text { Condensate }\end{array}$ & Optical flame spectrometry & Pre-heating, filtration \\
\hline & Fuel Impurities & Optical flame spectrometry & Filtration \\
\hline \multirow[t]{3}{*}{ Compressor } & Fouling & $\begin{array}{l}\text { Cycle analysis, improved } \\
\text { sensors }\end{array}$ & Ultrasonic cleaning \\
\hline & Erosion and pitting & Visual inspection & Coating systems \\
\hline & $\begin{array}{l}\text { Blade cracking and } \\
\text { separation }\end{array}$ & Visual and NDE inspection & Design issue \\
\hline \multirow[t]{3}{*}{ Turbine } & $\begin{array}{l}\text { Coating failure - cracking } \\
\text { and spallation }\end{array}$ & $\begin{array}{l}\text { Visual and NDE inspection } \\
\text { Exhaust metal detection }\end{array}$ & $\begin{array}{l}\text { Better on-line temperature } \\
\text { control } \\
\text { Design issue }\end{array}$ \\
\hline & Blocked cooling passage & $\begin{array}{l}\text { Direct blade temperature } \\
\text { measurement }\end{array}$ & Design issue \\
\hline & Parent material failure & - & $\begin{array}{l}\text { Prognostic software and } \\
\text { temperature sensors }\end{array}$ \\
\hline Controls & Unnecessary trips & - & A.l. software \\
\hline
\end{tabular}




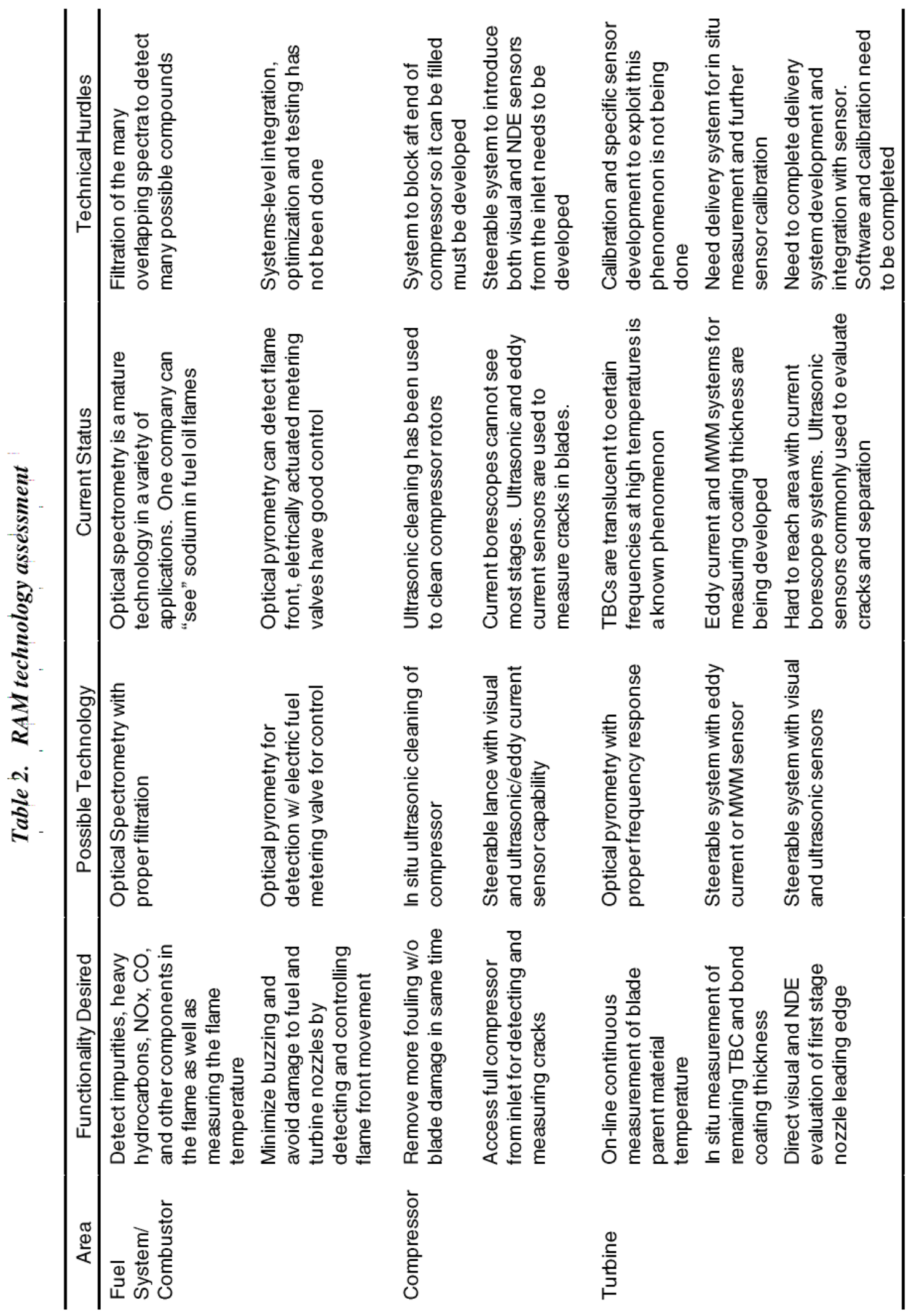




\section{MORE ELECTRIC MACHINERY}

\subsection{Introduction}

This section discusses the tradeoffs and emerging technologies related to the electric machinery used in the gas turbine power plant. It will focus on two areas:

- Ancillary Equipment: Electric power is used to run the auxiliary equipment required to operate the gas turbine. This auxiliary equipment performs a variety of functions. Pumps and filters provide fuel conditioning and delivery. Separate pumping and filtration systems provide lubricating and cooling oil conditioning and delivery for gearboxes and bearings. Hydraulic pumping systems provide pressurized fluid to actuators with various purposes from inlet guide vane actuation, to compressor bleed adjustment, to steam control valves for wet cycle gas turbines. Put together, the power lost in running the auxiliary equipment in a typical gas turbine power plant can approach 10 percent of the total power losses in the plant. The presence of many auxiliary pumps and systems also increases maintenance costs, plant initial costs, and plant footprint. It is therefore worthwhile to take a fresh look at this equipment to see if savings can be realized.

Electric actuators have long been looked to as an alternative to hydraulic and pneumatic systems. In general, insurance data shows that solid-state electronics, which are required to run electric actuators, are more reliable than reciprocating equipment and piping. This, combined with their advantages in cost, controllability and maintainability, make them an attractive alternative (see Figure 4).

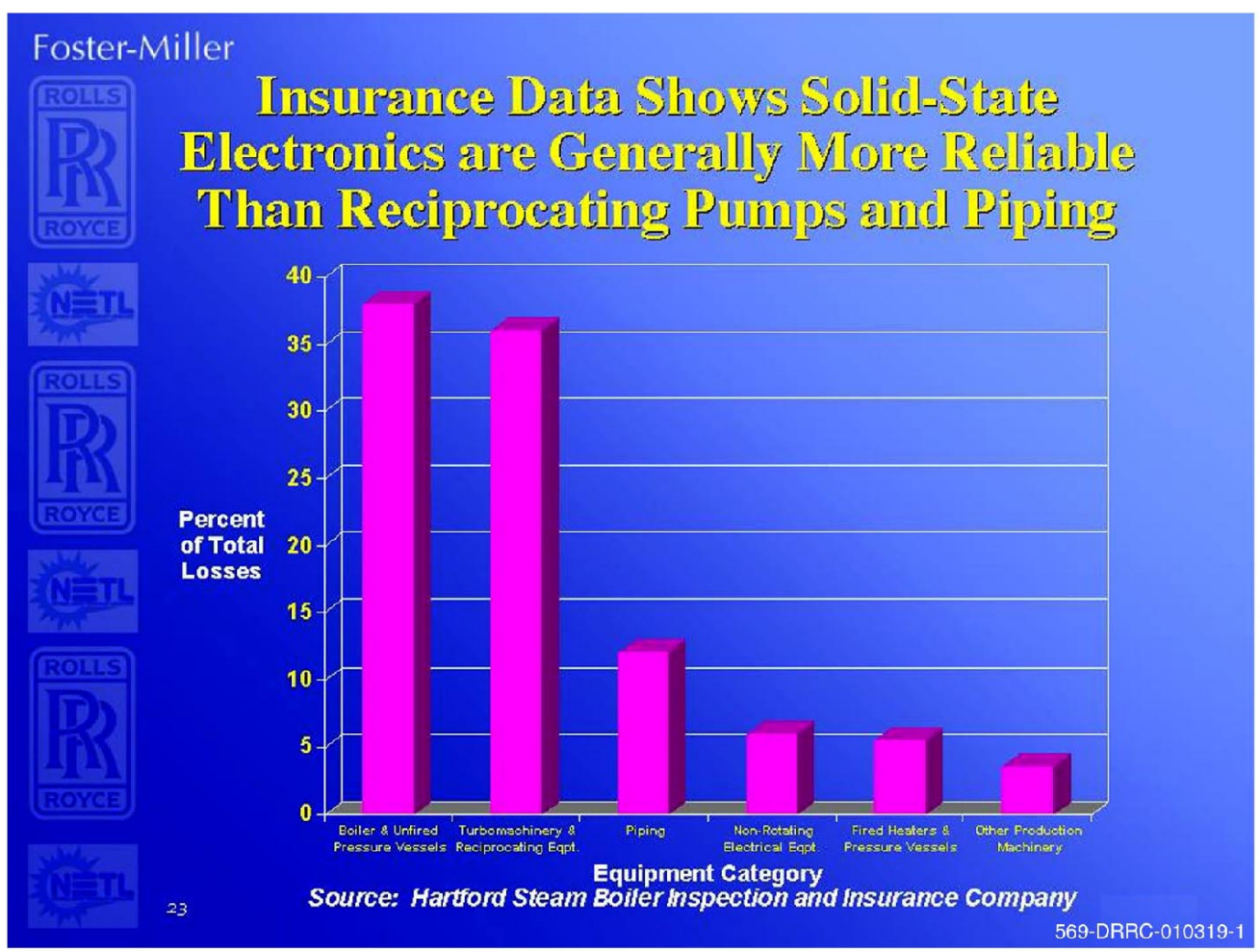

Figure 4. Insurance data shows solid-state electronics are generally more reliable than reciprocating pumps and piping. 
Magnetic bearings could be used to not only drastically reduce bearing losses, but also hold out the possibility of eliminating reciprocating journal bearing lubricating oil systems with solid-state electronic control systems. This document will report on the state of the art in both electric actuators and magnetic bearings for use in stationary gas turbine systems.

- Electric Power Equipment: Primary motive power from the plant comes from the spinning gas turbine shaft. This shaft turns an electric generator at $3600 \mathrm{rpm}$ to produce $60 \mathrm{~Hz}$ power (or $3000 \mathrm{rpm}$ to produce $50 \mathrm{~Hz}$ power). The generator's output voltage is generally around 13.8 to $22 \mathrm{kV}$, far below the desired transmission voltage. Thus, electric power passes through a step-up transformer to be brought to transmission voltages on the order of $138 \mathrm{kV}$ or higher. Between the output shaft of the gas turbine and the output of the step-up transformer, approximately 2 to 3 percent of the plant's output power is lost.

- Modern advances in motor/generator drives and inverters have made it possible to run the generator at any speed and electronically produce AC power at any desired frequency. This report will examine the possible advantages this technology may have at eliminating mechanical speed reduction systems or in gaining efficiency in the electrical system.

\subsubsection{Electric Actuators}

Historically, pneumatic or hydraulic systems have been used to actuate the various metering, bleeding, and shut-off valves that control the gas turbine. As illustrated in Figure 5, some of the primary uses for valves on the gas turbine are:

- Gas metering, conditioning and shut-off.

- Air metering and shut-off.

- Compressor bleed air adjustment.

- Water/steam injection system metering and shut-off.

- Bearing lubrication system regulation.

- Adjustable guide vane actuation. 


\section{Valves \& Controls in Gas Turbines}

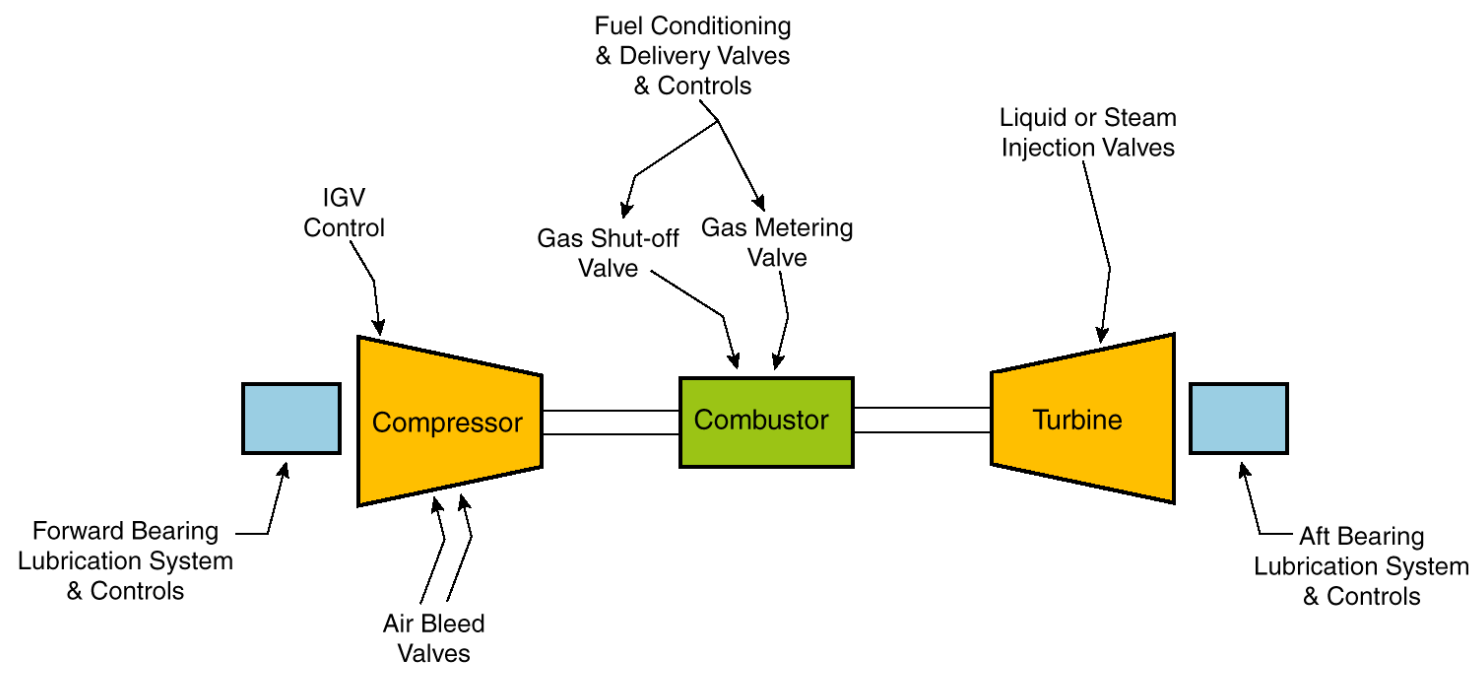

Figure 5. Some applications for actuators on a gas turbine.

To actuate this variety of valves and actuators using compressed air or hydraulic fluid requires an extensive ancillary pressure and pumping system, as well as complicated piping, filtration, and air/fluid conditioning equipment. This ancillary equipment adds to the total capital cost of the gas turbine generator system, it adds to the complication of the system and thus to the installation time, and it creates maintenance problems in the form of leaks and potential ancillary equipment failure. Even when this ancillary equipment does not directly fail, it tends to get in the way of easy access to the gas turbine. This increases turbine downtime because of the labor required to disconnect, blank-off, and physically move hydraulic and pneumatic piping and equipment.

Switching to electrically actuated valves could eliminate many of these problems, as well as provide for more accurate control of the overall system.

In practice, the advantages of electric actuation have been:

- Elimination of ancillary pumps and piping, and their replacement with more reliable solid-state electronics.

- Electric actuators are generally cheaper from a systems perspective. Though the actuators themselves tend to be more expensive, there is substantially reduced ancillary piping and system installation cost.

- Simplified maintenance and therefore reduced downtime. The failure mode is generally in an easily replaced sensor or "black box" electronic package. The lack of hard piping makes general plant maintenance easier.

- Elimination of hydraulic position errors for metering applications. Electric actuators are better at precision control.

The disadvantages or obstacles to use of electric actuators have been: 
- Limited availability of off-the-shelf actuators big enough for fuel metering applications on larger (>70 MW) frame engines.

- Metering and shut-off applications must be performed by separate valves. All-electric metering valves are generally actuated by a stepper-type motor that can be positioned precisely to a particular angular position. Shut-off applications are generally actuated by a solenoid actuator. The stepper motors are not fast enough to perform shut-off service, and the solenoids don't have the precision to be used in a metering application. Thus the two functions are best served by separate actuators.

- Limited availability of electric actuators with the speed and force required to actuate the Inlet Guide Vanes of even relatively small ( $25 \mathrm{MW}$ ) gas turbines. For example, Pratt \& Whitney has implemented almost all-electric actuation on their FT8, the prime mover of their $50 \mathrm{MW}$ TwinPac product. However, they have yet to find an off-the-shelf actuator for the IGVs on this machine.

\section{Conclusions}

- There are no real technical hurdles to implementing all-electric actuation. The primary hurdle has been supply and demand. Developing a custom motor is expensive for a low volume application.

- If the force requirement for the IGVs is too high, electric machinery becomes very large and thus cost-prohibitive and unwieldy. It is cheaper to develop very large forces in a smaller package in a hydraulic actuator.

- Some representative manufacturers of electric actuators for gas turbine applications include: Young \& Franklin, Woodward Controls, Precision Control Engineering, and Continental Controls Corporation. However, these are "packagers" in a manner of speaking. The real technical challenge is in the electric machine design, so the kind of firm that would really be adept at solving the problem would be a firm with a strong background in magnetics and electric machine design.

\subsubsection{Magnetic Bearings}

Magnetic bearings, with their elimination of mechanical systems and their vast reduction in bearing friction losses, have perennial appeal in the gas turbine industry. One can always find somebody that will advocate the use of magnetic bearings in gas turbines. In this section, we examine the pros and cons of this technology (see Figure 6). 

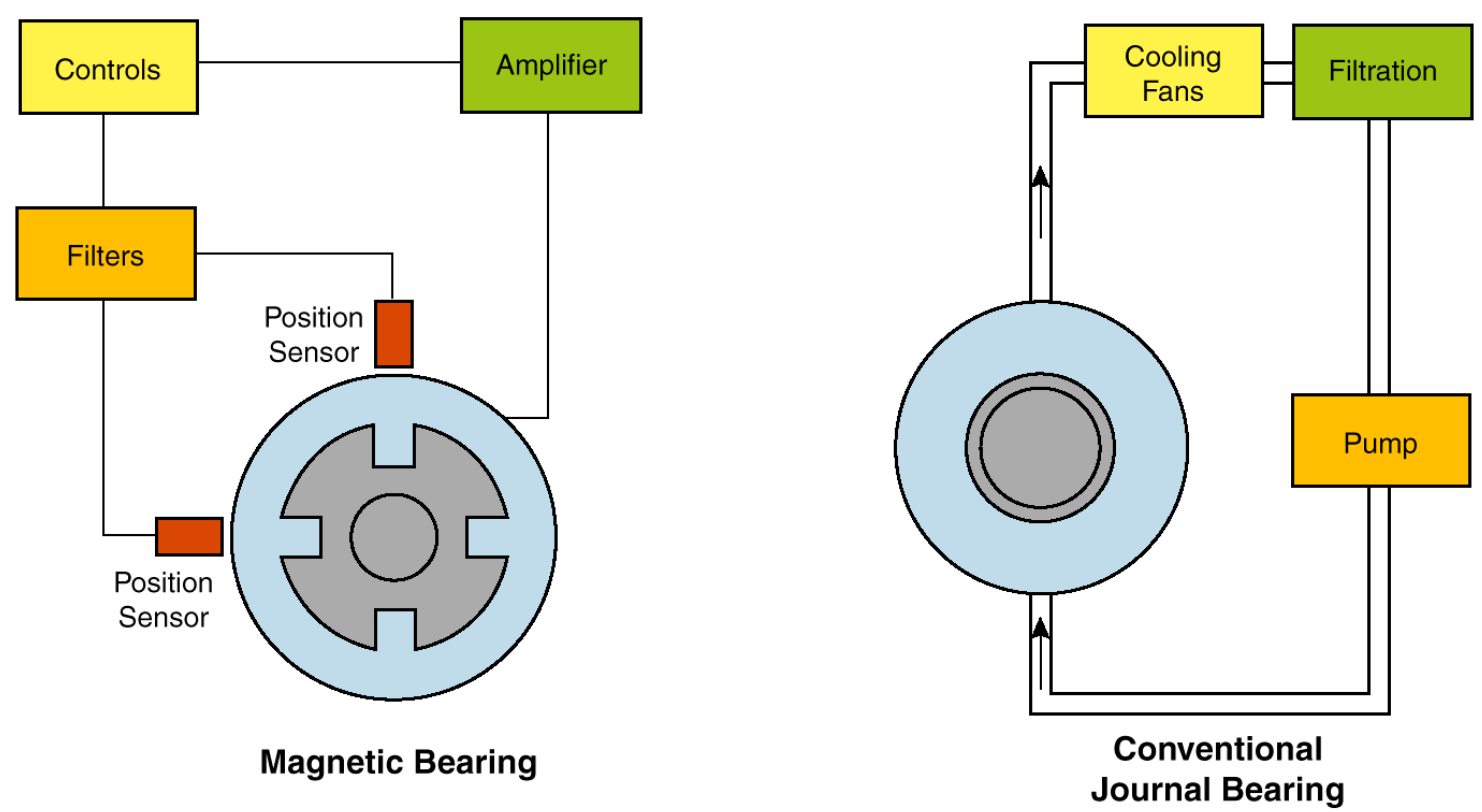

Figure 6. Comparison between magnetic and journal bearing systems.

\section{Current Bearing Configurations on Stationary Gas Turbines}

Non-aeroderivative stationary gas turbines use oil-lubricated journal bearings, typically one forward of the compressor and one aft of the turbine. In some very large gas turbines, notably the GE Frame 7 and Frame 9 designs, a "mid-frame" journal bearing is fitted in addition to those at either end of the turbine shaft. This mid-frame bearing is located at the discharge end of the compressor section, where temperatures are very high, exposing the oil to temperatures approaching $400^{\circ} \mathrm{C}$ in the bearing cavity areas. These temperatures will cause the lubricant in the bearings to:

- Decrease in viscosity, causing more heat as the oil loses its ability to support the load.

- Form a "varnish" residue and then "coke" at the elevated temperature. This "coking" will destroy the ability of the grease or oil to lubricate the bearing. It will also introduce solid particles into the lubricant.

Thus, oil cooling is crucial to the maintenance of this mid-frame bearing. This necessitates the use of an extra oil cooling system for this bearing, and results in this bearing causing more than its share of maintenance problems relative to the two outboard bearings which are generally quite reliable. The pumps and cooling fan required by the oil cooling system also represent power losses to the overall system.

Aeroderivative gas turbines used for stationary power generation are generally supported by rolling element bearings. Often, these bearings were not designed to be operated at full load, $24 \mathrm{hr}$ a day as required by, say, a base load power generating facility. They were designed for an aircraft duty cycle: full power for a brief time during takeoff, followed by partial power for a few hours, followed by shutdown and constant inspection. Thus the bearings in an aeroderivative engine require some special consideration, else they can become the biggest maintenance problem on the machine. 
When flight engines are modified to serve as stationary power sources, the bearing life problem is solved by pulling more compressor bleed air to cool the bearings to acceptable steady-state temperatures. This solves the issue of the bearings on the gas generator. This gas generator supplies hot, high pressure gases to a power turbine to make electric power. This power turbine is usually supported by journal bearings, the same as the entirety of a typical frame engine. These journal bearings can experience the same temperatures, and thus the same oil coking and cooling problems, as the mid-span bearing of a frame engine.

\section{Magnetic Bearings}

All sizes of magnetic bearings have been produced to date, though many larger sizes are not commercially available "off the shelf." One of the most important parameters determining the size of a bearing for a given machine is the pressure capability of the bearing. The maximum static force generated by the bearing is given by the projected area of the bearing, times the maximum pressure provided. Magnetic bearings are generally rated at $30 \mathrm{psi}$ compared with oil bearings rated at $75 \mathrm{psi}$, which tend to make magnetic bearings larger, taking up more room than equivalent oil bearings.

The basic magnetic bearing system is made up of the magnetic bearing actuators (permanent magnets or electromagnets or both), the position sensors, the filters, the control unit, and the amplifiers. Most permanent magnets have an upper operating temperature limit of approximately $200^{\circ} \mathrm{C}$, making their use in larger gas turbines, especially at mid-span, potentially problematic without extensive cooling. For a permanent magnet, this temperature limit is entirely materialdependent and cannot be altered without advances in material technology. Electromagnets are constructed of a copper wire coil wound around an iron core. The maximum operating temperature of an electromagnet is generally limited by the insulation that prevents shorting between the turns of the copper wire. Advances in insulation technology have increased this limit to at least $400^{\circ} \mathrm{C}$, and in some cases higher.

Auxiliary ("catcher") bearings are an essential part of most machine configurations using magnetic bearings. These are mechanical bearings that are used to hold the rotor when delevitated or for use in the event of magnetic bearing failure. The clearance between the rotor and auxiliary bearings is usually half the gap between the rotor and stator in the magnetic bearing. The air gap on a typical magnetic bearing can be anywhere between 5 to 25 thousandths of an inch. The smallest gap in the gas turbine should, of course, be smaller than the gap between the rotor and the catcher bearing to prevent damaging rubs.

In the event of a trip, a large frame-size gas turbine can take 20 to 30 min to coast down from its operating speed. This puts a performance demand on the auxiliary bearing to survive, uncooled, at high loads for this amount of time. To date, many have considered this requirement to be the most difficult to meet in designing magnetic bearing systems for large gas turbines that do not contain unacceptable large auxiliary bearings.

The bearing has electrical losses: eddy current, resistance, and hysteresis losses, and some windage losses on the rotor itself. But the overall energy loss in a magnetic bearing is an order of magnitude lower than that of a mechanical bearing doing the same job. Even for turbomachinery, involving radial bearings supporting rotating assemblies of up to $3,400 \mathrm{lb}$, power consumption is less than $4 \mathrm{~kW}$. This is because the power is transferred back and forth between the bearing coils, 
and capacitors which straddle the power amplifiers. The only real power consumed is due to eddy current losses in the bearing laminations, and resistance losses of the bearing coils and control system conductors. This can be compared to over $250 \mathrm{~kW}$ of power consumed by a conventional system, which represents the sum of the lube oil shear losses, pump and cooling fan power.

Due to the fact that magnetic bearings are a non-contact system, there is no mechanical wear on the bearing parts and they generally last a very long time as long as they're kept properly cooled. Reliability issues surrounding magnetic bearings generally revolve around the sensors and electronics required, as well as how many magnetic failures the auxiliary bearings can withstand before requiring replacement. Other than the auxiliary bearings, these components can generally be replaced without requiring machine disassembly. If auxiliary bearings could be developed that could last the span of time between overhauls, then magnetic bearings would require no new maintenance activity for their implementation.

Companies on the leading edge of magnetic bearing research and development include Revolve Corporation, Calnetix, Mohawk Innovative Technologies, Foster-Miller Technologies, and SatCon Technology Corporation.

\section{Benefits of Magnetic Bearings for Stationary Gas Turbines}

- Mechanical losses are typically $1 / 10$ th those of journal or rolling element bearings. However, journal bearing losses are fairly small compared to other losses in the power system, making this a marginal benefit.

- The oil lubrication and cooling system can be eliminated if magnetic bearings with high temperature capability are used. This has the double benefit of eliminating the power consumed by these systems, and improving the reliability of the overall system by replacing mechanical pumps and filters with more reliable electronic components.

- Magnetic bearings may have a higher temperature capability before they require ancillary cooling systems. For a magnetic bearing design using only electromagnets, the limiting technology is the insulation used on the copper windings. Conventional polymer-based high temperature insulations have a limit of about $200^{\circ} \mathrm{C}$. However, newer ceramic and mica insulations have been field tested with higher capabilities. Permanent magnets have temperature capabilities not much higher than 150 to $200^{\circ} \mathrm{C}$ before they lose magnetization, so their use in high temperature magnetic bearing designs is limited.

- Reliability and ease of maintainability are improved because external electronics are the primary point of failure. So long as the insulation on the coils of the bearing has the capability to withstand its temperature environment, the absence of mechanical contact makes the actual bearing assembly almost foolproof. The external sensors and electronics are usually the primary failure points of magnetic bearings. These components can be easily replaced without a great deal of disassembly. Compare to journal bearings where oil coking or mechanical failure requires turbine disassembly for repair or replacement.

\section{Drawbacks of Magnetic Bearings for Stationary Gas Turbines}

- Magnetic bearings are much larger than journal bearings because of their lower stiffness. For aeroderivative engines, replacing the rolling element bearings with magnetic bearings would require a great deal of re-design of the engine. This would make the engine too different from 
flight hardware: one might as well design a new engine! For the power turbine of an aeroderivative or for a frame engine designed exclusively for ground-based use, this is less of an issue.

- For magnetic bearings to be most useful, they would be required to eliminate the ancillary cooling pumps needed by conventional journal bearings. Further development is required to prove out insulation systems for magnetic bearings that can survive operating temperatures of at least $700^{\circ} \mathrm{F}$.

- The need for auxiliary mechanical "catcher" bearings to support the rotor in the event of magnetic bearing failure has always been a show-stopper for many in implementing magnetic bearings. They add to system complexity, detract from maintainability, and add to bearing losses. The necessity for catcher bearings in many applications mitigates or eliminates the benefits of having magnetic bearings in the first place. Classically, catcher bearings have been rolling element bearings that backed up the function of the magnetic bearing. However, some hybrid systems have been proposed, among them static journal or air bearings that could be activated in the event of magnetic failure. Unfortunately, one of the benefits of using magnetic bearings, that one would eliminate ancillary pumps and filters, would be mitigated with this approach. The catcher bearing issue is usually a sticking point to the acceptance of magnetic bearings.

\section{Conclusions on Magnetic Bearings for Stationary Gas Turbine Applications}

Application where magnetic bearings would have benefit:

- Journal bearings that are currently encountering reliability problems and requiring extensive ancillary cooling and lubrication because they are in a high temperature environment. For example: mid-span bearings on frame engines, and power turbine bearings on aeroderivative engines. There would be minimal benefit to replacing outboard bearings or bearings that currently operate in a lower temperature environment. Their reliability is generally good.

Benefits of magnetic bearings in these applications:

- High temperature magnetic bearings would eliminate the need for ancillary cooling and pumping systems. Their primary point of failure would be electronics, which are both generally more reliable than pumps and piping and easier to maintain/replace when they fail. Overall system efficiency would be improved through the reduction in bearing friction losses and the reduction in power required by ancillary equipment.

Technical hurdles in the way of achieving these benefits:

- New insulation systems would be required that would enable the bearing to operate un-cooled continuously at $700^{\circ} \mathrm{F}$. Early work in this area shows that this is feasible.

- An auxiliary bearing design which does not eliminate the benefits of using magnetic bearings would need to be developed. Mechanical bearings, fluid film bearings and passive magnetic bearings have all been suggested. More development is needed. A mid-span bearing might not require a catcher bearing at all.

\subsection{Adjustable Frequency Drives}

Please see Figure 7. 


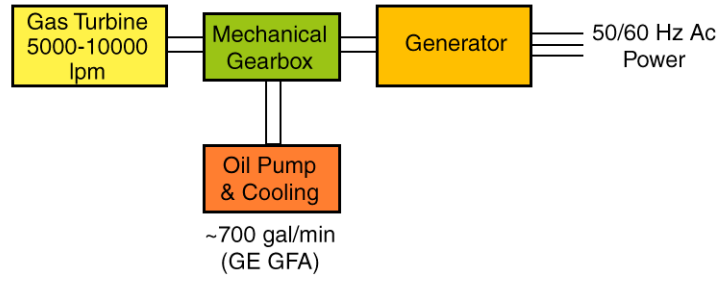

Conventional System

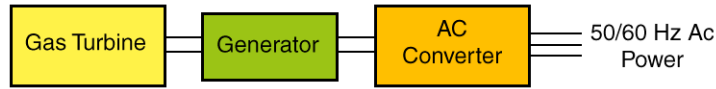

Adjusted Frequency Drive System

Figure 7. Conventional system versus one with an adjustable frequency drive.

\subsubsection{Introduction}

Some stationary gas turbines use a mechanical gearbox to reduce the shaft speed of the gas turbine (between 5000 to $10000 \mathrm{rpm}$ depending on design and size) to $3600 \mathrm{rpm}$ for producing 60 $\mathrm{Hz}$ power (or $3000 \mathrm{rpm}$ for producing $50 \mathrm{~Hz}$ power). The mechanical gearbox requires maintenance, and a lubricating oil system that has to eject the heat generated by the losses in the gearbox. The oil demand created by the cooling and lubricating needs of the mechanical gearbox can be quite substantial. The GE 6FA, for example, requires a total lubricating oil flow for both the gearbox and the various bearings of about $900 \mathrm{gal} / \mathrm{min}$. Of this, approximately $700 \mathrm{gal} / \mathrm{min}$ is required solely to service the gearbox, with the remainder needed for other purposes. In a typical aeroderivative engine which requires a gearbox, almost 75 percent of the lubricating oil flow is required to service the needs of the gearbox.

Classically, gearboxes have been responsible for a 2 to 3 percent decrease in overall system efficiency. However, newer gearboxes have pushed these losses down to about 1.5 percent. This does not include the lubrication and cooling system required, which would bring the number back up to 2 to 3 percent for the total gearbox system. Gearbox manufacturers often obscure just what they include in measures of gearbox "efficiency."

This reduction gear could be eliminated, replaced by a direct drive coupling so that the generator would run at the same speed as the gas turbine. The higher frequency output of the generator could then be electronically changed to the necessary 50 or $60 \mathrm{~Hz}$.

Adjustable speed controls date back more than 70 years, but only in the last 20 years have they become reliable and cost-effective. The development of adjustable speed controls has evolved with the advent of new, improved, and lower cost switching devices, from transistors to thyristors to GTOs and IGBTs. The evolution of switching devices has allowed higher power density applications with low harmonics and high efficiency. Continued development and increasing competition have reduced costs and increased reliability. Frequency converters have actually become simpler, because the new devices allow new topologies with fewer components.

\subsubsection{Mechanical Gearbox versus "Electronic Gearbox" Tradeoffs}

\section{Efficiency Tradeoff}

Efficiency is the first parameter to take into consideration when comparing these two systems. A mechanical gearbox can account for 1.5 to 3 percent of total plant losses if power required for ancillary cooling systems is taken into account. Electronic frequency inverters are not much more efficient, though recent and continuing advances in switching technology may make them so. 
However, one must look at the tradeoff from the systems perspective. Using an adjustable frequency inverter enables gas turbine and electric generator designers to choose the design speed of their machinery irrespective of the frequency required at the output. The question then becomes: does freedom to design for any rotational speed enable turbomachinery or electric machinery designers to make more efficient machines? Even if electronic inverters are less efficient than gearboxes as stand-alone devices, the power system as a whole could be more efficient if there are gains to be made by changing design speed.

When this question was posed to a variety of gas turbine designers from Rolls Royce, Pratt \& Whitney, and GE, it was decided that there was no real efficiency advantage to be gained by altering rotational speeds. The same question was posed to electric machinery designers at Pratt \& Whitney, Brush Electric Machinery, and SatCon Technology Corporation, a Cambridge-based advanced electric machinery company. Electric generators/motors do get more efficient with increasing rotational speed, but the difference is only appreciable when rotational speeds get very high. For example, conventional $3600 \mathrm{rpm}$ generators used in power generation applications are approximately 97 to 98 percent electrically efficient. Increasing this speed to 50,000 rpm would, theoretically, make this machine upwards of 99.5 percent efficient. This is a factor of 2 reduction in electrical losses in the generator, but it would be completely eliminated by the losses in the inverter from converting an $833 \mathrm{~Hz}$ signal to $60 \mathrm{~Hz}$ AC power.

\section{Efficiency Tradeoff Conclusion}

The technologies are approximately equivalent in terms of efficiency. Perhaps a 0.5 percent advantage could be given to one or the other in specific applications, but this would depend a great deal on the details of the specific system.

\section{Reliability Tradeoff}

As has been pointed out before, electronics are both generally more reliable than rotating machinery and easier to maintain when they do fail. In this particular case, however, the difference is marginal. Current gearboxes are a minimal maintenance issue in facilities in which they are used.

\section{Other Potential Benefits of Adjustable Frequency Drives}

- The use of an electronic drive would enable the generator to be used as a starter as well. This would have the benefit of eliminating the need for the extra starter motor and its attendant hardware.

- Electronic drives would enable the OEM to more easily make the switch between $50 \mathrm{~Hz}$ and $60 \mathrm{~Hz}$ power. Currently, OEMs must design and manufacture different sets of turbomachinery hardware to supply customers with systems capable of producing either standard frequency. The use of electronic gearboxes would make it very easy to change frequencies with just a simple change of off-the-shelf electronics.

- While higher speed turbomachinery is not necessarily more efficient than lower speed turbomachinery (assuming both are competently designed), it is generally smaller. Smaller turbomachinery is generally cheaper turbomachinery given the expensive alloys and materials used in modern gas turbines. The use of electronic gearboxes would enable manufacturers to use much smaller turbomachines for a given power output. 


\subsubsection{Adjustable Frequency Drives Conclusions}

Gearboxes are not often used in power generation applications. Turbomachinery is generally designed specifically for the application to run at the correct speed to give the desired frequency output. In fact, Pratt \& Whitney Power Systems stated that they would find it cheaper to just design a new power turbine than implement either a gearbox, mechanical or electrical. The question then becomes: is there any benefit to being able to design the power system to run at higher (or lower) speeds? There is minimal or no benefit in terms of efficiency. There may be some benefit in terms of cost, since faster rotating machinery is generally smaller and cheaper. And the OEM would only have to carry one set of turbomachinery designs for any desired electrical output frequency. It is the opinion of the author that this is not a particularly fruitful path to pursue.

\subsection{High Voltage Generators}

The output voltage of a conventional generator in a power plant is between 13.8 to $22 \mathrm{kV}$. This voltage is too low for electric transmission, which usually takes place at $69 \mathrm{kV}, 138 \mathrm{kV}$ or even higher. Therefore, between the generator and the transmission grid is a step-up transformer which brings the electric output up to the required transmission voltage. This transformer is roughly 98 to 99 percent efficient, but it is quite expensive. For a typical $50 \mathrm{MW}$ plant, the step-up transformer can run as much as $\$ 750,000$. The elimination of the step-up transformer would result in a cost and efficiency benefit. If the output voltage of the generator were already at the transmission voltage, there would be no need for the step-up transformer (see Figure 8).

\section{High Voltage Generator Eliminates Step-up Transformer}

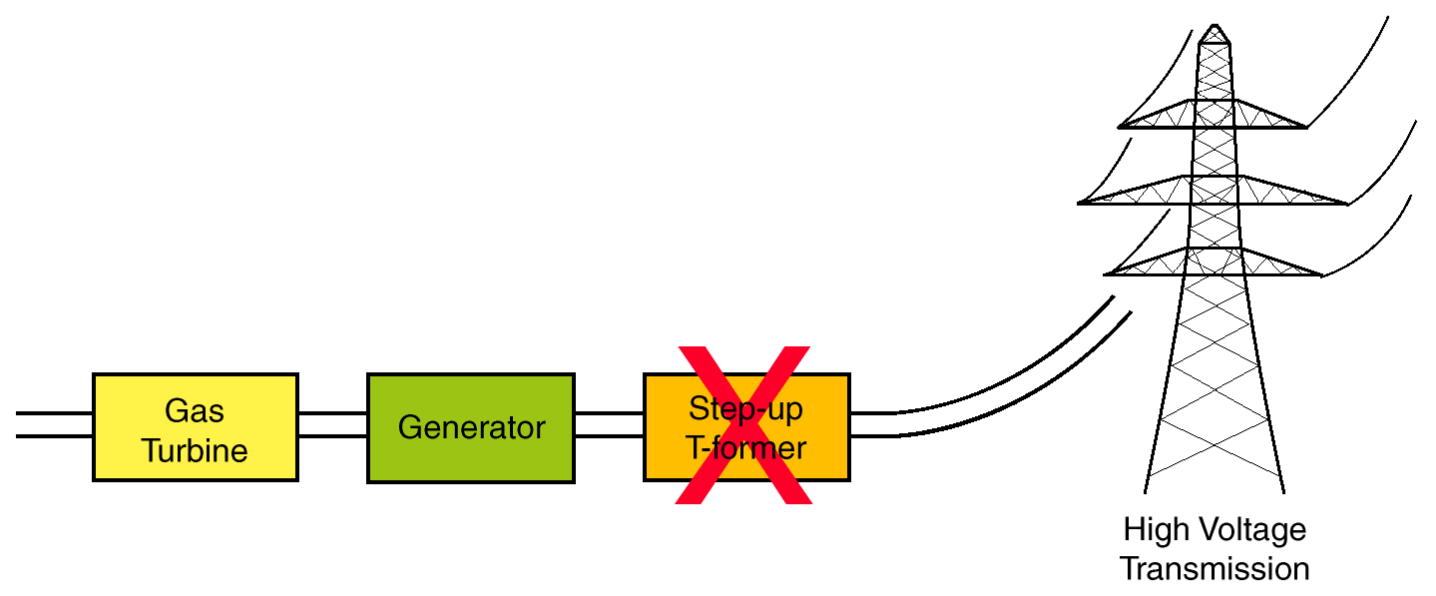

561-DRRC-010319-3

Figure 8. Power plant diagram showing step-up transformer. 


\subsubsection{Technical Hurdles to Implementing High Voltage Generators}

There are two primary technical issues involved in implementing high voltage generators. High Electric Fields

Most conventional generators are fabricated with long copper bars in the stator slots joined at the ends by copper arcs. This method creates sharp corners in the end turns, and inside the slots themselves, that results in very high electric fields. If a generator of this design were forced to operate at a higher voltage, the electric fields generated by these sharp corners would break down the insulation on the turns and at the corners inside the slots, cause arcing, and damage the machine.

Two changes in design are required. First, sharp corners in the copper windings need to be eliminated or mitigated to lower the maximum electric fields generated. The drawback of this is that it generally reduces the copper packing factor in the slots, which would necessitate some changes in electrical design. The second design change required is a new insulation system that could withstand the increased electric fields resulting from the higher voltages. Both design modifications in tandem could allow for higher voltage operation without increased risk of arcing.

ABB claims to have made such changes successfully in a recently tested generator. Their solution included using round conductors, rather than the usual rectangular conductors, and introducing a new insulation system made from cross-linked polyethylene. Thus, the motor is wound more like a conventional small motor. ABB claims a net gain of about 0.5 percent in overall plant efficiency. There is some question, however, of whether this number includes the effects of...

\section{Higher Harmonic Content}

Ideally, the AC power generated by a power plant should be a perfect $60 \mathrm{~Hz}$ sine wave. In the real world, for various reasons mostly related to the limitations of generator design that are beyond the scope of this report, the waveform emerging from the power plant is a combination of the fundamental frequency, $60 \mathrm{~Hz}$, and multiples of it, called harmonic frequencies. Harmonic distortion essentially converts a portion of the useful energy into high frequency energy that is no longer useful to most devices and is ultimately lost as heat.

The output of most conventional motors contain 4 to 5 percent harmonic content. Higher voltage designs run closer to 7 to 8 percent harmonic content. Again, care must be taken when talking about the "efficiency" of a power plant. Harmonic losses are experienced at the user end, when the user tries to run, say, a refrigerator motor where non $60 \mathrm{~Hz}$ portions of the signal are useless for producing mechanical work. We were unable to determine whether ABB's claim of a 0.5 percent efficiency improvement from the use of their generator included harmonic losses, or just measured total electrical output.

\subsubsection{Conclusions on High Voltage Generators}

\section{Efficiency Gains}

The end result of the efficiency tradeoff depends on the details of the system, but there is a potential for improvement. The elimination of the step-up transformer increases efficiency by 0.5 to 1 percent, but increased harmonic content may mitigate this gain. 


\section{Cost Advantage}

Elimination of the step-up transformer definitely eliminates a capital expense on the order of $\$ 500,000$ to $\$ 2$ million depending on plant size, which represents approximately 5 percent of the total cost of a new power plant. Assuming that the increase in cost of the high voltage generator is less than this, there should be a net cost savings as a result of their implementation.

U.S. companies that could design high voltage generators include Brush Electric Machinery, Electric Machinery, or Hitachi USA.

\subsection{More Electric Machinery Conclusion Summary}

\subsubsection{Electric Actuators}

\section{Benefits}

Clear cost savings, plant simplification, and reliability improvement

\section{Hurdles}

Need to develop high force actuators for IGV and large frame engine fuel metering applications

\subsubsection{Magnetic Bearings}

\section{Benefits}

For journal bearings operating in high temperature environments (i.e., mid-span or power turbine bearings), there is a reliability improvement and an efficiency gain from replacing them with magnetic bearings.

\section{Hurdles}

For the benefit to be realized, magnetic bearings must be able to operate continuously and uncooled at $700^{\circ} \mathrm{F}$. This probably requires the development of coil insulation capable of operating continuously at that temperature. The auxiliary bearing issue must also be resolved.

\subsubsection{Adjustable Frequency Drives}

\section{Benefits}

Potential for lower cost turbomachinery because of possible higher operating speeds, and the elimination of separate starter motors.

\section{Hurdles}

These drives are currently very expensive and are probably not efficient enough to be worthwhile, especially given the relatively minimal benefits.

\subsubsection{High Voltage Generators}

\section{Benefits}

Potential for overall plant efficiency improvement of 0.5 to 1 percent. Potential for cost savings of about 5 percent of overall plant capital cost.

\section{Hurdles}

Needs technical work in improved high voltage insulation systems and electric re-design to mitigate high electric fields. Possible issue with increased harmonic content that could mitigate efficiency gain. 


\section{STEAM INJECTION REPORT}

\subsection{Introduction}

There are two primary purposes for injecting steam into a gas turbine:

- First, to reduce thermal $\mathrm{NO} x$ by reducing flame temperature. Normally this requires only that about 3 percent of the working fluid be steam.

- Second, to increase power generation and thermal efficiency by extracting waste heat from the simple cycle and injecting larger amounts of steam into the mass flow somewhere upstream of the turbine. Generally, in such installations steam flow could be 15 to 20 percent of total turbine mass flow.

All of the design and maintenance issues relating to steam injection are present in both cases, but are clearly more serious in the case of steam injection for power increase. The use of steam injection for power increase will be our primary focus.

\subsection{Design Issues}

Most often in implementing steam injection in a gas turbine, an existing design is modified in order to accommodate the steam. In order to modify an existing gas turbine to use steam injection, several design changes must be made.

These are described below:

- The torque carrying capacity of the rotor must be increased to accommodate increased loads.

- The thrust balance will be changed, generally shifted aft, because the introduced steam will push on the turbine rotor. The mass flow will actually be greater through the turbine than through the compressor, so mass balances change.

- The surge margin of the compressor will decrease because the steam injection will force the engine pressure ratio to increase, which moves the compressor operating point closer to the surge line.

- Turbine blade heat transfer will increase because of the increased thermal conductivity of the steam/air mixture on the outside of the blade relative to the dry cooling air on the inside. This will cause the blade temperature to increase unless increased internal blade cooling mass flow is added and/or thermal barrier coatings are added.

- A feed water system for delivering demineralized water will need to be integrated into the plant. Approximately $1.0 \mathrm{gpm}$ to $1.5 \mathrm{gpm}$ of water is required for each $\mathrm{lb} / \mathrm{sec}$ of mass flow of the gas turbine engine.

- The generator power capacity will need to be increased by about 1.5 times, depending on the extent of the steam injection.

\subsection{Maintenance/Reliability Problems Encountered in the Field}

Each of the problems described below has been experienced in the field at some point since the introduction of steam-injected gas turbines. In some cases, a design or material change was made to correct the problem. In some cases, an operational change was made. Regardless of how these 
problems were solved after they were found, the lessons learned can be used to eliminate the root causes of most of the problems earlier in the design phase of a new steam-cycle gas turbine.

1. Shorter component life: For various reasons, the use of steam injection has resulted in shorter component life from the combustion hardware downstream to the last turbine stage. Some of the common problems encountered in the field, their root causes, and their solutions are listed in this section.

- Problem: Shorter first stage turbine bucket life: Higher blade temperatures will imply shorter blade life. For example, a $20^{\circ} \mathrm{F}$ increase in blade temperature will decrease the blade life by 50 percent. Higher blade temperatures result from the change in heat balance over the blade. The outside of the blade is in thermal contact with a steam/air mixture with a higher thermal conductivity than the pure cooling air on the inside surface of the blade.

- Typical Solution: Parts life impact from steam or water injection is related to the way the turbine is controlled. The control system on most base load applications reduces firing temperature as water or steam is injected. This counters the effect of the higher heat transfer on the gas side and results in no impact on bucket life. On some installations, however, the control system is designed to maintain firing temperature constant with water injection level. This results in additional unit output but it decreases parts life as previously described. Units controlled in this way are generally in peaking applications where annual operating hours are low or where operators have determined that reduced parts lives are justified by the power advantage. GE describes these two modes of operations as dry control curve operation and wet control curve operation, respectively.

- Problem: Shorter second and third stage turbine nozzle life: With the advent of steam injection, the repair intervals on both the rotating and the stationary turbine components were reduced, but for somewhat different reasons. For the rotating components, the reduction was primarily due to an increase in operating temperature resulting from the difference in gas thermal conductivity on the inside and outside of the blade. For the stationary components, higher aerodynamic loading on the turbine nozzles from the increased cycle pressure ratio resulted in higher than expected scrap rates. This was found to especially effect the second and third stage nozzles, which saw much higher scrap rates due to increased creep.

- Typical Solution: The introduction of GTD-222, a higher creep strength material, has effectively solved this problem.

- Problem: Shorter combustion hardware life: Shorter combustion hardware life is due to the fact that the combustion system will have more vibration problems due to the flame instabilities that typically result when steam or water are introduced into the combustion process. Combustion system vibration will affect the reliability of the TBC coatings on the combustion liners, transition pieces, first stage blades and vanes. It will create more wear at the interface joints of the liner and transition pieces and result in earlier crack formation. Both mechanisms of failure, coating flaking and joint/weld line cracking, have been seen in the field and have been attributed to increased vibration. 
- Typical Solution: Shorter combustion hardware change out intervals is the general short-term solution. Other solutions include the use of hardened material inserts to handle the wear problems at the joints and to design vibration dampening features and robustness into the vibrating hardware to prevent cracking.

2. Problems related to steam delivery: Steam must be delivered to the turbine with a uniform distribution and it must be dry (i.e., no droplets must be formed). The design of the steam delivery system is the key to delivering high quality steam. Some of the early problems with various steam delivery system designs are discussed in this section.

- Problem: Erosion due to low quality steam: Must ensure that steam is superheated enough so that no droplets form (i.e., must ensure that the steam is "dry"). Droplets will pit, erode, and create hot spots wherever they are formed. Increased erosion due to steam injection for power augmentation has been encountered in the field and has manifested itself as a decrease in efficiency that is detected when the steam is shut off. This is primarily due to erosion that has the effect of increasing clearances in the hot section.

- Typical Solution: The steam piping must be insulated and have automatic blow down and drain valves to allow for proper steam superheating and to prevent the formation of condensate. Water droplets in the steam injection system can cause major combustion system problems. Some intelligent thought needs to be put into the steam pipe layout, design and operation.

As an example, GE employs an integrally cast manifold in the combustor casing for the power augmentation steam injection, whereas Westinghouse utilizes an injection system that is located upstream of the combustion cans, and downstream of the compressor discharge, in an area referred to as the combustion wrapper.

Consequently, the Westinghouse design saturates the hot gases prior to entering the combustors, while the GE design mixes the gas/air mixture and the steam inside each individual combustion can.

It has been experienced in the field that the Westinghouse design offers an advantage over the GE design in a couple of ways. First, the GE design, with an integrally cast manifold on each of the combustion cans, has in the past allowed for liquid collection in the manifold, which, when it reaches critical mass, can then be ingested into the combustion system, and create problems with flame stability and or flashback. The Westinghouse system will not experience this problem.

In addition, the Westinghouse system requires only one hook up, compared to the GE design which requires a new ring header to be installed (a pipe the rings the compressor case) and a flex hose connection to each of the combustion cans. This may seem like a small matter, but to an operator the greater simplicity of fewer hook-ups to make and break during overhaul allows for greater maintainability (see Figure 9). 


\section{MS7001FA GAS TURBINE DLN2 COMBUSTION SYSTEM COMPONENTS}

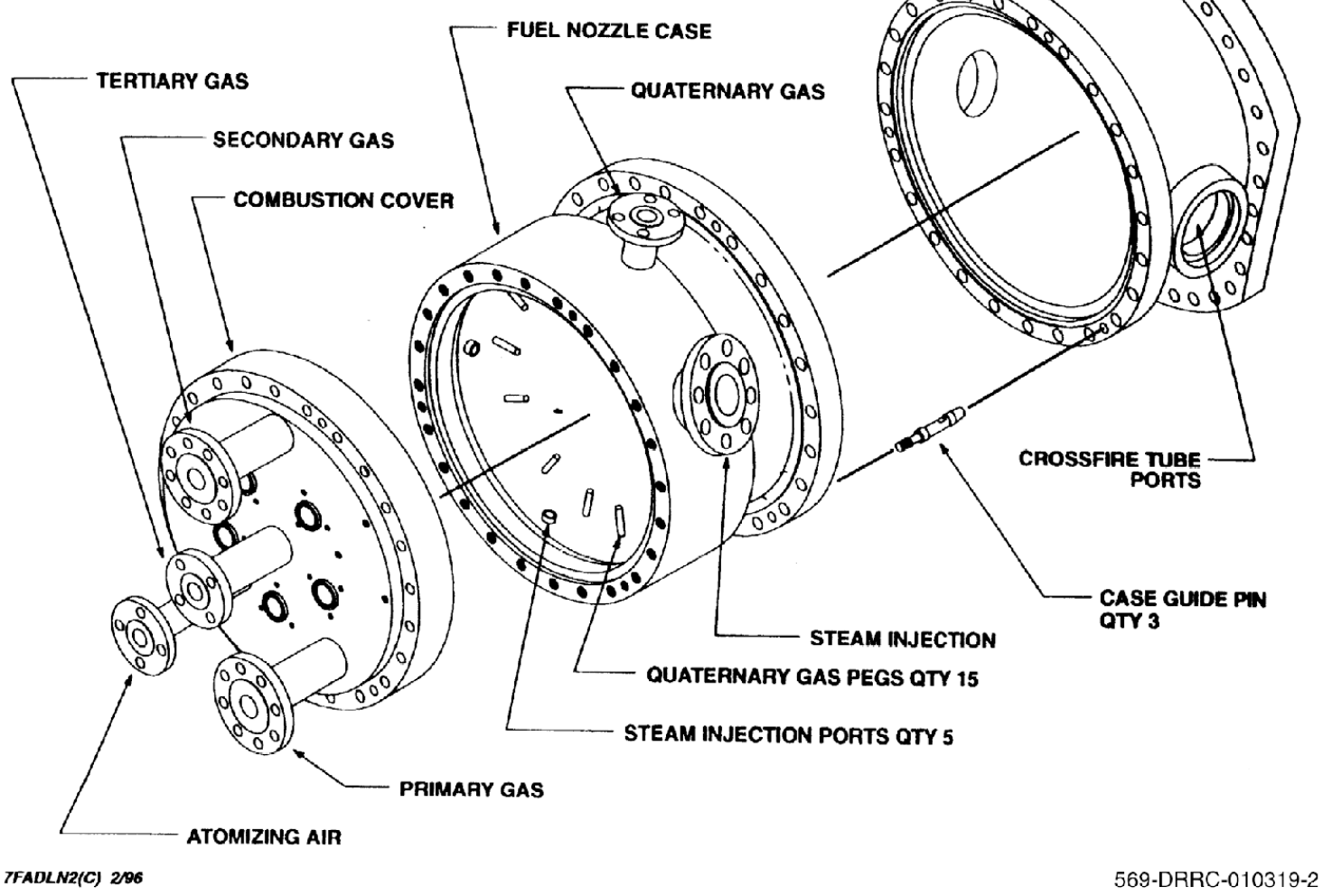

Figure 9. Schematic of the GE steam injection combustion dome.

- Problem: Nonuniform steam injection: Steam must be uniformly injected into each combustion chamber to prevent a damaging flow stimulus on the turbine blades. Steam injection non-uniformity can be caused by control valve leaks or differential injection nozzle erosion, the latter being the more common cause in the field.

- Typical Solution: Differential injection nozzle erosion in different cans is caused mostly by low quality steam. The best way to mitigate this is a change in steam delivery system design that results in more superheated steam into the system. For example, most machines using steam injection for $\mathrm{NO} \times$ abatement have designed the steam injection nozzles to be integral with the fuel nozzles to provide direct flame cooling. Nozzle erosion is the single biggest problem with this configuration.

- Problem: Steam control valve leakage: Steam control valve leakage has been a problem in several machines. OEMs have tried to get by with less expensive control valves and have not specified proper valve seat materials. In this situation, the high velocity steam during opening and closing can damage the valve seat. Once the seat is damaged, the valve leaks when closed.

- Typical Solution: Trying to cut system cost by specifying under-performing components is not always a good tradeoff in the long run. But this problem was a good illustration of the attention that needs to be paid to the steam delivery system. 
Overall the most common problems relating to steam injection in the field, as they are manifested to the operator (as opposed to being categorized by their root causes) are:

- Injection nozzle erosion.

- Leaky steam control valves.

- Combustion liner and transition piece cracking distress.

- Turbine blade and vane erosion and heat distress.

- Loss of efficiency (noted when the steam is shut off) more quickly than a comparable machine without steam injection.

A final common complaint from users about steam injection for power augmentation is the startup time factor. Steam injection designed for power increase does not allow for an immediate response to an increase in power demand. Even if the HRSG or other heat recovery device is "warmed up," it takes time to heat up the steam lines and manifolds so that the steam stays "dry" as it's injected into the cycle. It usually takes about an hour to implement steam injection, once an increase in power production is demanded. Obviously, this would not be as much of an issue with cycles in which the steam is meant to be on continuously for efficiency reasons, rather than as a response to changes in demand. Nevertheless, it was thought worth mentioning, since the comment was made by several operators (by some as an explanation as to why they did not use steam injection for power augmentation opting instead for, say, inlet fogging).

\subsection{Economics}

While gas turbines using steam injection have different particular maintenance problems than nonsteam injected gas turbines, none of the operators or experts interviewed by Foster-Miller felt that the overall maintenance costs of steam injected plants were any higher than those of a typical gas turbine plant. This is mainly due to the difficulties encountered in the field with dry, low-NOx combustors, which end up being just as problematic from a maintenance perspective as steam injection. Again, the problems are different, but the overall feeling is that they're roughly equivalent in terms of cost.

Thus the decision to implement steam injection is driven by initial capital costs with the differences in fuel efficiency factored in, along with the demands of the mission for which the machine is to be used (i.e., peaking, cycling, or base load). A general comparison of the typical costs is given below.

- Initial Capital Costs: A simple cycle gas turbine power plant in the $70 \mathrm{MW}$ to $80 \mathrm{MW}$ size will cost $\$ 300 / \mathrm{KW}$. A full combined cycle plant in the same size will cost $\$ 700 / \mathrm{KW}$. A steam injected gas turbine plant will cost about $\$ 500 / \mathrm{KW}$.

- Variable Costs: The fuel efficiency for these plants is typically 33 percent for simple cycle, 42 percent for steam injection and 50 percent + for combined cycle. The steam-injected gas turbine will have a much higher demineralized water cost than the full combined cycle.

\subsection{Conclusion}

- Steam injection is a relatively low cost method of achieving moderately high plant efficiencies. It requires less plant equipment and space; however, a high volume flow of good quality water 
is required. Also, relative to the combined cycle system, more maintenance is required for the gas turbine, however, this is balanced against not having a steam turbine/generator, condenser, circulating water system and cooling tower.

- Steam injection for high power augmentation can make economic and environmental sense for many power plant locations.

- Most potential maintenance problems associated with steam injection can be prevented through careful design of the steam heating and delivery system. Great care must be taken to ensure that only high quality dry steam is delivered to the gas turbine combustor. Many early problems with steam injected gas turbines resulted from inferior steam path design that ended up delivering water droplets that impinged on the combustion hardware.

- Most of the maintenance problems not related directly to low quality steam are familiar ones to gas turbine designers. The stationary turbine components will see higher blade loadings and thus require higher creep strength materials. Bucket and combustion hardware coatings must be designed for higher vibrational loads, or vibrations must be damped. This could require a substantial change in combustor geometry. 


\section{Appendix $\mathbf{H}$}

NGT Supporting Technologies:

More Electric Systems Studies 


\title{
TECHNICAL REPORT
}

\begin{tabular}{|l|l|l|l|}
\hline $\begin{array}{l}\text { Discipline or report series } \\
\text { CONTROL SYSTEMS ENGINEERING }\end{array}$ & Document number & Report number & Issue \\
\hline Title & \multicolumn{3}{|l|}{} \\
NGGT More Electric Systems Study & & \\
\hline & & \\
\hline
\end{tabular}

\begin{abstract}
Summary
This report details an investigation carried out by the authors as part of the NGGT, Ansty into the applicability of "More Electric" technologies to both the existing $R R$ Engines and a new, replacement engine configuration. The report considers both the economic and technical benefits of application of the following technologies to a 50-100 MW industrial gas turbine:
\end{abstract}

- Shaft Power Transfer

- Electric Starter Generator

- Active Magnetic Bearings

- Power Electronics Grid Interface/ Direct Drive Generator

Additional keywords

More Electric; Shaft Power Transfer ;Electric Starter Generator; AMBs; Power Electronics Grid Interface/ Direct Drive Generator; CVT

\section{Circulation:}

Approved

Retention category

by:

\begin{tabular}{|c|c|c|c|c|c|c|}
\hline \multicolumn{2}{|l|}{ Project } & \multicolumn{2}{|c|}{ Sub-system } & \multicolumn{2}{|c|}{ Process } & \multirow[t]{2}{*}{ Part number } \\
\hline Engine types $\# 24$ & Engine & s/vehicle name & & Vehicle & r/build $\$ 28$ & \\
\hline Material & Design definition & Design def. issue & CTP\#32 & ATP $^{\# 36}$ & Dept & File/folder $\# 48$ \\
\hline & & \multicolumn{4}{|c|}{ Security classification $\# 01$} & Page 1 of $58^{\# 56}$ \\
\hline
\end{tabular}




\section{TABLE OF CONTENTS}

$1 \quad$ INTRODUCTION .................................................................................................................

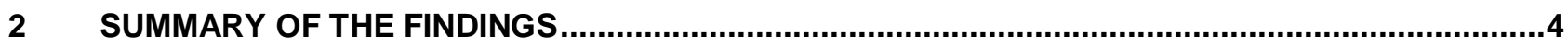

3 MOTIVATING FACTORS FOR CONSIDERING MORE ELECTRIC SYSTEMS ..............................6

LESSONS FROM RR-AEROSPACE INTEREST IN MORE ELECTRIC TECHNOLOGIES .............................................6

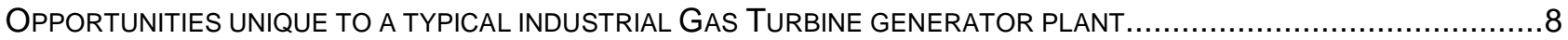

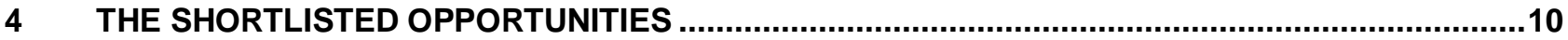

$5 \quad 50-100$ MW ENGINE ARCHITECTURE ISSUES.................................................................11

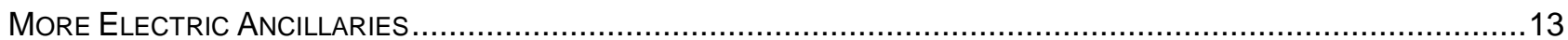

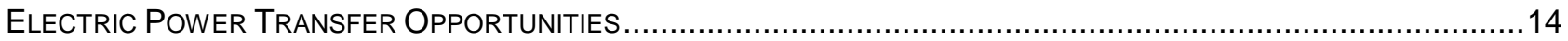

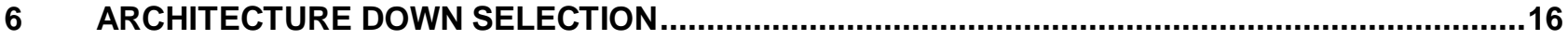

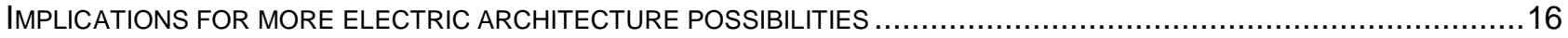

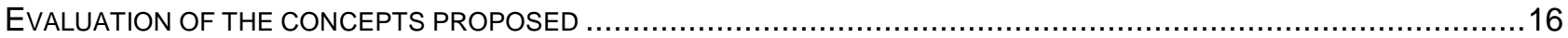

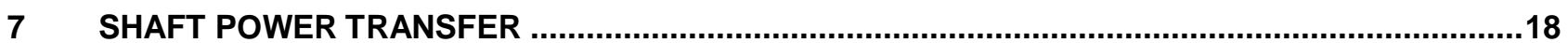

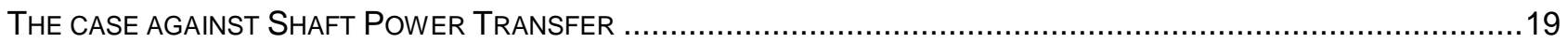

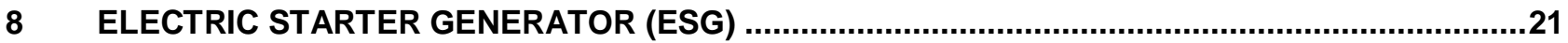

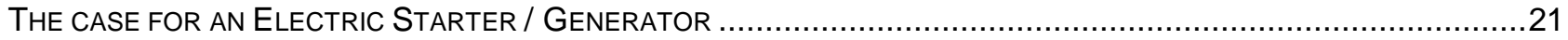

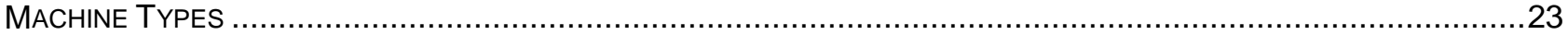

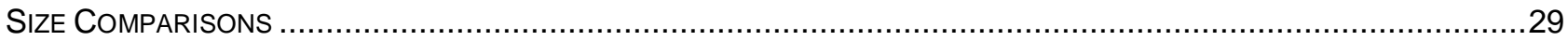

COST 33

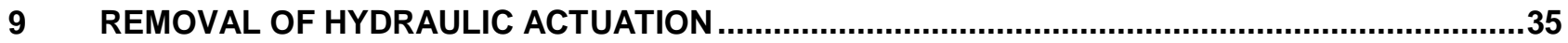

10 ACTIVE MAGNETIC BEARINGS

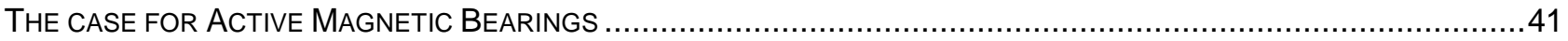

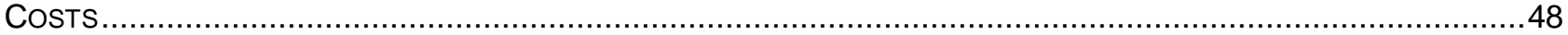

11 POWER ELECTRONICS INTERFACE AND / OR DIRECT DRIVE ALTERNATOR ..........................55

The CASE AgAinSt USING A POWER EleCtRonics INTERFACE/HIGH SPEEd GENERATOR ...............................56

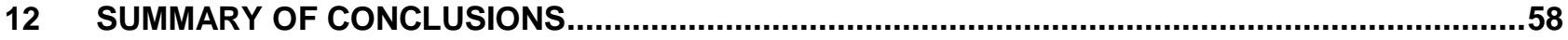

\begin{tabular}{|l|l|l|l|}
\hline Document number & Issue & Page 2 of 58 & \\
\hline
\end{tabular}




\section{INTRODUCTION}

Through consideration of the known design, performance and operational constraints of the 50-100 MW gas turbine based industrial plant, the authors have considered the potential of More Electric based technical solutions to provide product enhancement and differentiation opportunities.

This work was performed as part of a broader study into future product opportunities for Rolls-Royce in this sector of the market. Whilst the focus has predominantly been on a low cost engine for co-gen power generation applications, the requirements of the remainder of the market for an engine of this size, namely mechanical drive for the oil and gas industry and marine transportation, have also been considered.

This report outlines and evaluates the potential More Electric based ideas and assesses the possibility of applying them either as part of an upgrade to the existing RR Engines or as part of a new engine architecture. 


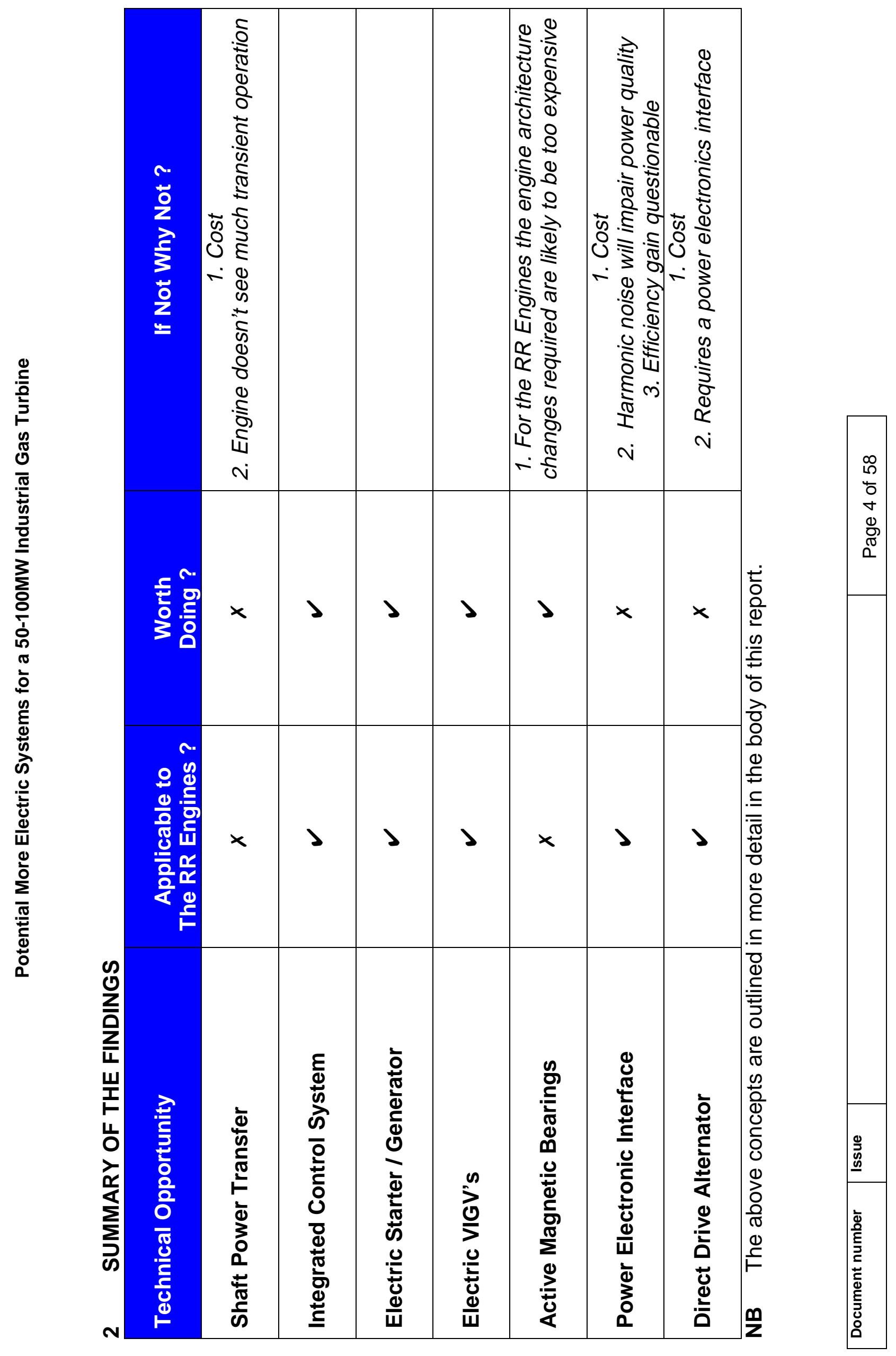




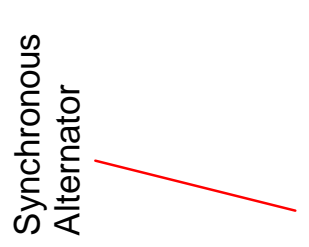

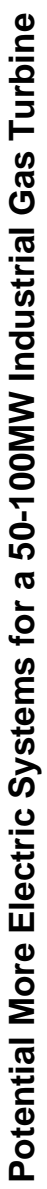
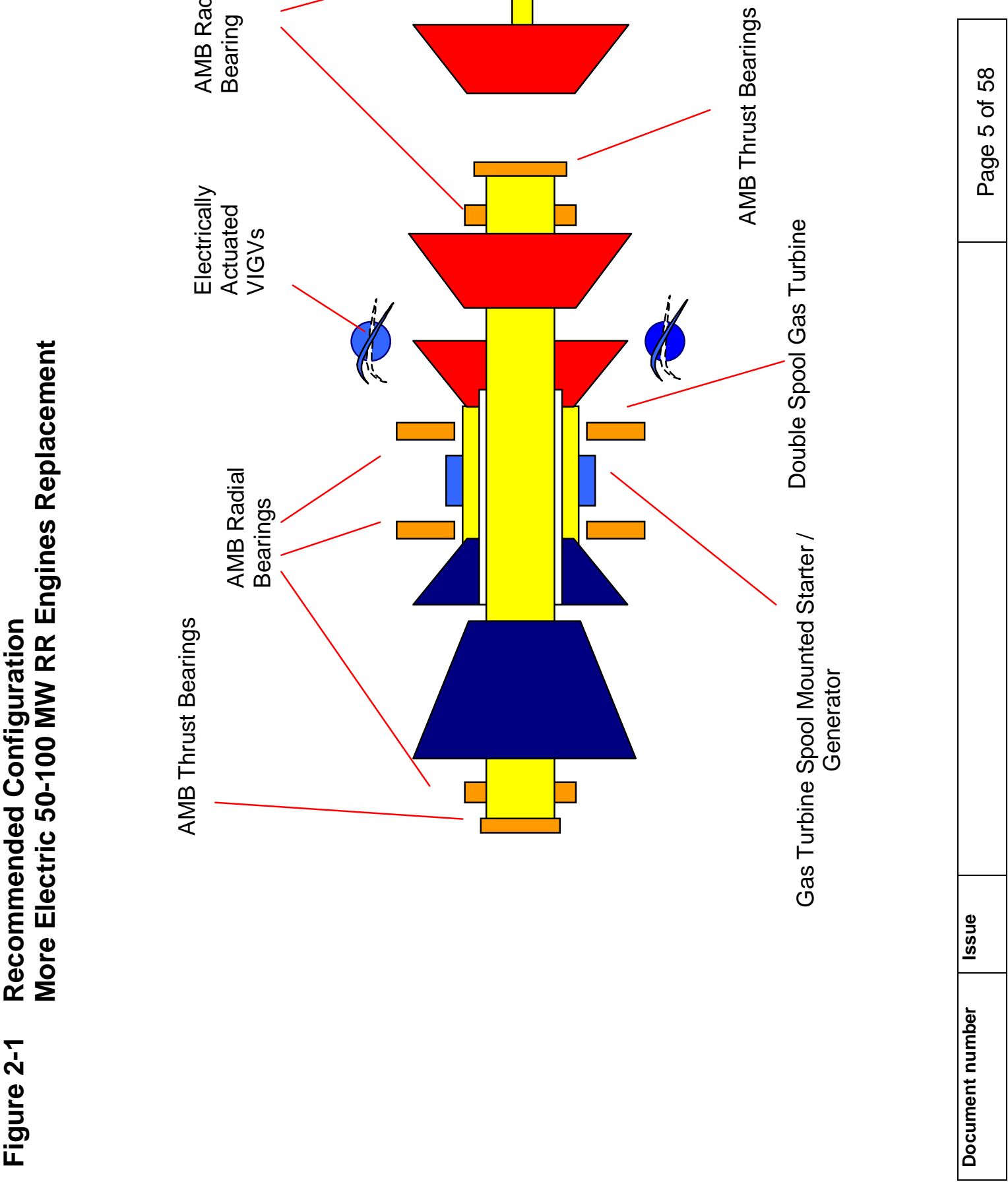


\section{MOTIVATING FACTORS FOR CONSIDERING MORE ELECTRIC SYSTEMS}

Recent developments in power electronics and electrical machines offer the potential to improve the performance of many existing electro-mechanical systems. Such improvements are possible through two principal means:

- The replacement of traditionally mechanical sub-systems with electrically based ones offering more control flexibility.

- The integration of traditionally separate mechanical and electrical functionality of a complex system to deliver a more optimal solution.

In Rolls-Royce, this premise is the basis of active projects in both the Aerospace and Marine sectors. In Aerospace the use of electrical ancillary systems and the potential for using embedded machines for starting and shaft power transfer is being considered. In Marine the integration of the propulsion and ship service systems through a common electrical distribution system has already delivered fuel economy benefits in the Type 23 class.

To date however, relatively little innovation of this sort has been proposed for industrial gas turbine generator sets, despite the relative safety and apparent suitability of this application environment for developing new technology. This could be seen as particularly surprising since a typical gas turbine generator set plant has an inherent "more electric" bias than equivalent aerospace or marine applications.

\section{Lessons from RR-Aerospace interest in More Electric Technologies}

A review of the principle theories and technologies being developed as part of the More Electric Aircraft / Engine initiatives raises opportunities that may have a relevance to an Industrial engine application. These can be summarised as follows:-

\section{- The integration of electrical machines into the engine}

The ability to motor or retard the engine using an electric motor / generator enables the following:

- Rapid start capability through direct drive of the HP shaft and potentially starting assistance of the IP and LP spools.

- Power transfer between spools, delivering enhanced compressor control and the possibility of removing the need for variable geometry vanes and handling bleed valves. 
Such motors can be mounted co-axially with the shafts and connected directly to them in the more demanding thermal environment inside the engine ('internal').

\section{- The application of Active Magnetic Bearings (AMBs)}

The complete, or partial, replacement the engine's oil bearings with AMBs may have the following benefits:

- Improvements in reliability, availability and scheduled maintenance over current bearing systems;

- The potential for removing the oil system(s) from not only the engine but potentially the plant.

- Reduction of fire risk and noxious emissions which could result from unintended oil consumption

- Better control of engine vibration.

- Inherent engine diagnostics, since changes in the feedback signals in the bearings used to control the levitation would provide indications about the changes in shaft dynamic performance.

Ultimately 'internal' motor/generators may be combined with AMBs into single units, offering useful reductions in weight, space requirements and parts count.

\section{- Replacement of Mechanically driven ancillaries with Electrically driven} alternatives

The replacement of mechanical ancillaries, such as fuel pumps and fuel compressors, with electrically powered ones is seen as a way of:

- Improving ancillary systems control

- Reducing costs through the deletion of mechanical transmission interfaces

- Simplifying the engine package.

- Minimising the engine to site interface requirements to ease installation and deliver reliability improvements.

- Increasing the potential to modularise the engine with associated benefits in assembly \& test, plug and play product variation opportunities, shipping, installation and maintenance. 
- Improve the control of ancillaries such as fuel and oil pumps through the use of electrical rather than mechanical drive.

\section{- The shift towards Distributed Control systems}

Local or Distributed control of the engine, plant and ancillaries, is seen as potentially delivering the following benefits:

- Enhanced safety through speed of response improvements and removal of plant wiring errors and harness connections failures

- Improved availability and reliability through the ability to intelligently and automatically reconfigure the plants operation

- Reduced installation costs

- Improved ease of use

- Greater information availability throughout the plant through the use of serial bus communications

\section{Opportunities unique to a typical industrial Gas Turbine generator plant}

As well as potentially enhancing the engine itself the technology has the potential to be applied to the rest of the plant:

- Application of a power electronic interface to the output of a gas turbine driven generator could ......

- Facilitate rapid engine start, potentially by using the alternator as a starter motor.

- Remove the need for a free power turbine by allowing the alternator to be directly coupled to the gas turbine.

- Allow a 50 or $60 \mathrm{~Hz}$ supply to be generated by a single shaft GT directly driving an alternator at speeds higher (or lower) than synchronous (1500 - $3600 \mathrm{rpm})$ without requiring a gearbox.

- Reduce the weight, the size and, potentially, the cost of the alternator by driving it at a higher speed.

- Allow the gas turbine speed to vary whilst still delivering a constant frequency output. 
- Allow the gas turbine to operate near the compressor surge line by using the ability to vary the rotational speed to actively control mass flow without the need for variable geometry stator vanes or air bleeds.

- Allow the gas turbine COT to remain high, even when the Gas Turbine is running at part load, by reducing the speed and hence mass flow.

- Allow a variable speed gas turbine, such as a marine drive application, to simultaneously drive an alternator for other applications, i.e. ship service loads, and hence deliver improvements in utilisation 


\section{THE SHORTLISTED OPPORTUNITIES}

All of the above can be categorised into six clear themes

Controls

Shaft Power Transfer

AMBs

Electric Ancillaries

Power Electronic Interface

High Speed Alternator
Control system improvements \& cost reduction

Engine handling flexibility through the use of electrical power transfer between the engines spools

Application of Active Magnetic Bearings to eliminate oil within the engine and, possibly, plant

The application of electrically driven ancillaries in place of hydraulic or mechanical systems.

Providing output frequency control to allow the gas turbine speed to change whilst maintaining a constant output speed

As above but with the additional benefit of being able to delete the gearbox.

Each of these possibilities can then be evaluated. 


\section{$5 \quad$ 50-100 MW ENGINE ARCHITECTURE ISSUES}

In order to assess the potential for these technologies one needs to appreciate the potential engine and plant configurations appropriate for the 50-100 MW power range.

Figure 5-1 Potential Configurations for a 50-100MW Industrial Gas Turbine 5-1(a) 2 Spool - Free Power Turbine Geared Hot End Drive

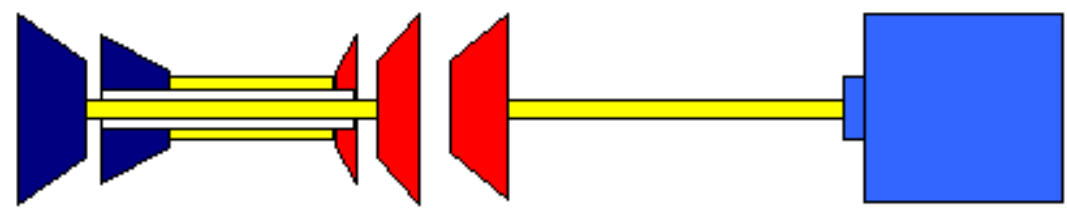

5-1(b) $\quad 2$ Spool - Free Power Turbine Geared Cold End Drive

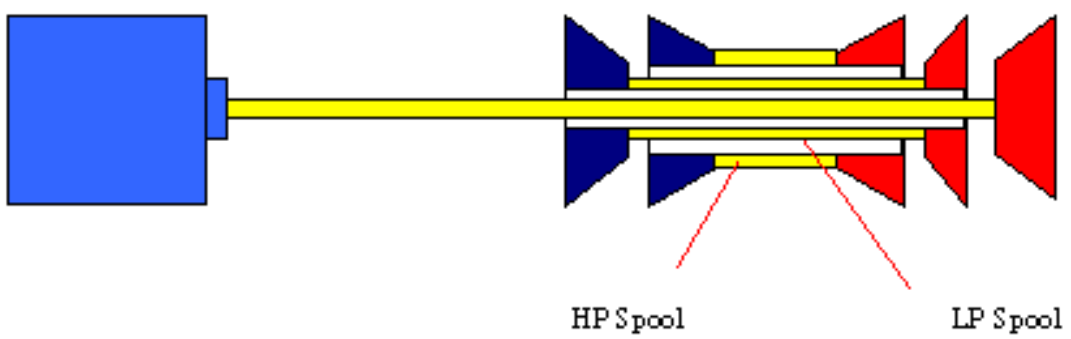


Table 5-1 Characteristics of alternative Gas Turbine configurations

2 Spool - Direct Drive

\begin{tabular}{|c|c|}
\hline Pros (+) & Cons (-) \\
\hline $\begin{array}{l}\text { - LP spool inertia maintains output } \\
\text { stability on application of load }\end{array}$ & - No potential for alternator self starting \\
\hline $\begin{array}{ll}\text { - } & \text { HP spool speed can vary with load } \\
& \text { Reasonable transient response }\end{array}$ & \\
\hline
\end{tabular}

\section{Spool - Free Power Turbine}

\begin{tabular}{|c|c|}
\hline Pros (+) & Cons (-) \\
\hline - Gas generator speed can vary with load & - No potential for alternator self starting \\
\hline $\begin{array}{l}\text { - Spool speed freedom allows better } \\
\text { aerodynamic design - potential to } \\
\text { remove handling devices }\end{array}$ & $\begin{array}{l}\text { - Transient lag as the gas generator } \\
\text { spool speeds change }\end{array}$ \\
\hline - Reduction in required gearbox ratio & - Free power turbine cost \& complexity \\
\hline & $\begin{array}{l}\text { - Potential for gas generator overspeed } \\
\text { on loss of load }\end{array}$ \\
\hline
\end{tabular}

Figure 5-2 Potential Power Electronic Interface Configurations

Shown for hot end drive configurations only for clarity but equally applicable to cold end drive.

\section{5-2(a) Direct Drive}

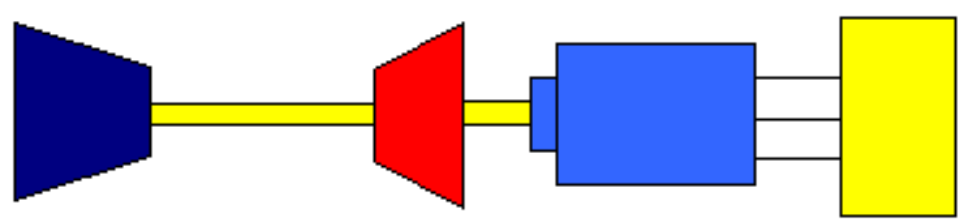

\section{5-2(b) Free Power Turbine}




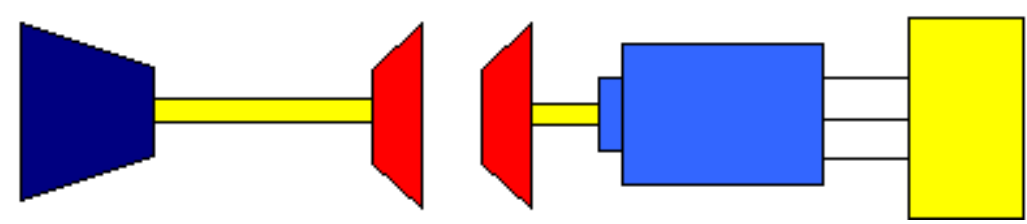

\section{More Electric Ancillaries}

The main motivation for switching to electric ancillaries is control flexibility. However in the case of an industrial engine, which tends to be operated in a much less transient regime than an aero engine, much of the demand for improved control in order to deliver engine handling benefits is superfluous.

A secondary motivation for an industrial engine may well be the possibility of removing much of the interface between the engine and the rest of the plant. The costs of installing off engine hydraulics for example can be significant.

The main ancillaries which may benefit from an electric approach are as follows:

\section{- Starter}

The starter motor is predominantly hydraulic and relatively expensive. Replacing this unit with an electric motor/generator delivers both a cost saving and potentially enhanced functionality (see Ch. 8)

\section{- Variable Inlet Guide Vanes \& Bleed Valves}

Assuming the starter is replaced the only remaining hydraulic systems on the engine are those required for engine handling. Both the on/off control of bleed valves and the variable position control required for VIGVs can be achieved electrically.

\section{- Bearings}

The advantages of using electrically powered Active Magnetic Bearings are discussed in Ch. 10.

\section{- Fuel Pump}

As the systems requiring an ancillary gearbox are gradually replaced with electrically driven ones then the justification for retaining the remainder becomes 
more difficult. In addition, the ability to control fuel pressure and flow independently of engine speed may be of value.

Electric Power Transfer Opportunities

As in the consideration of the benefits for enhanced control of the ancillaries, it is difficult to make a case for something specifically intended to improve transient engine handling if the engine spends the majority of its time at steady state base load.

However it may be that as elements of a power transfer system may already be in place, i.e. two electrical machines, the alternator and an electric starter, that a degree of power transfer capability is worthwhile. 
Figure 5-3 Electric Starter Configuration

Shown for hot end drive configuration only for clarity but equally applicable to cold end drive.

\section{Multi-Spool Machine}

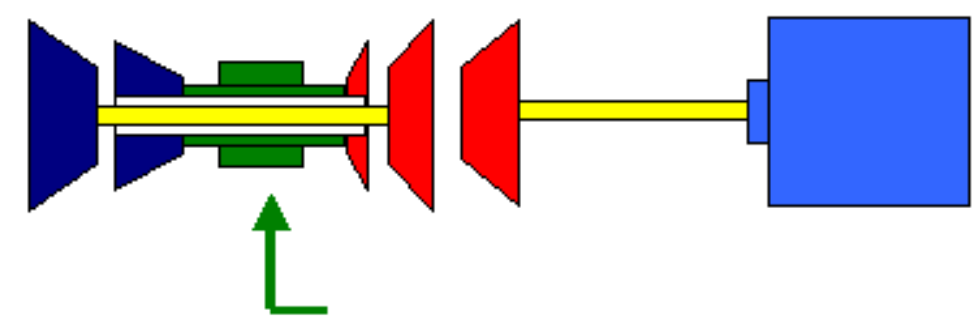

Figure 5-4 Power Transfer Architecture

Shown for hot end drive configuration only for clarity but equally applicable to cold end drive.

\section{Spool - Hot End Drive}

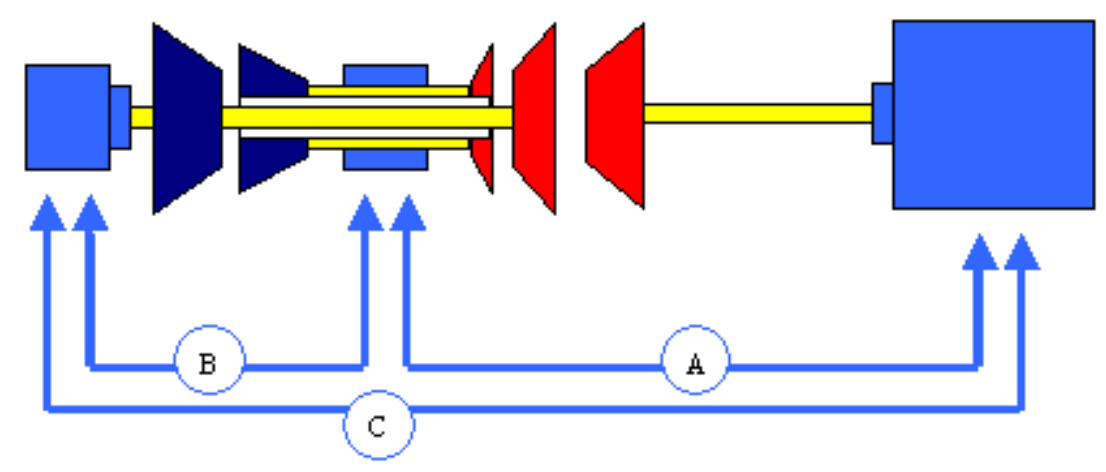

Power transfer possible in both directions between the :

- Starter/generator on the HP spool and the alternator (A)

- Starter/generator on LP shaft and an electrical machine on the LP shaft (B)

- Electrical machine on the LP shaft and the alternator (C) 


\section{ARCHITECTURE DOWN SELECTION}

The team performed an initial down selection of the alternative engine architectures and chose the double-shaft gas generator configuration shown in Figure 5-1(a).

In summary, the reasons for this selection were as follows

- A gas generator \& free power turbine based approach ensures maximum commonality between the power generation and mechanical drive configurations.

- The requirement to pass the drive shaft of the free power turbine back through the gas generator spool was a factor in the hot end drive decision. This results in the gas generator shaft being too large a diameter to make the cold end drive arrangement feasible.

\section{Implications for more electric architecture possibilities}

\section{- Control System}

Not affected

\section{- Shaft Power Transfer}

Not affected

- Active Magnetic Bearings

\section{- Electric Ancillaries}

- Power Electronic Interface / High Speed Alternator

Each of these solutions is applicable to this engine configuration. Whilst the use of a free power turbine configuration frees up the speed of the gas generator from having to run synchronously.

\section{Evaluation of the concepts proposed}

An explanation of the possible outline technical solution follows together with an assessment of the costs and benefits.

\begin{tabular}{|l|l|l|l|}
\hline Document number & Issue & Page 16 of 58 \\
\hline
\end{tabular}


A recommendation of each solutions potential is made and supported with additional evidence where available. 


\section{SHAFT POWER TRANSFER}

\section{OPPORTUNITY: SHAFT POWER TRANSFER}

Why?

\begin{tabular}{|l|l|l|}
\hline Performance & Improved Handling & $\begin{array}{l}\text { : Match air flows throughout engine } \\
\text { - Avoid surge in twin shaft engine } \\
\text { - Replace VIGVs and bleeds }\end{array}$ \\
\hline
\end{tabular}

\begin{tabular}{|l|l|l|}
\hline Functionality & Power Sharing & Swap power with the FPT \\
\hline
\end{tabular}

\section{Findings}

\begin{tabular}{|l|l|}
\hline $\begin{array}{l}\text { Applicable to existing RR } \\
\text { Engines ? }\end{array}$ & $\begin{array}{l}\text { No } \\
\text { Extensive engine architecture and casing changes } \\
\text { required }\end{array}$ \\
\hline Recommended ? & $\begin{array}{l}\text { Yes - but........ } \\
\text { Development of high temperature electrical machines } \\
\text { is an acknowledged technical risk }\end{array}$ \\
\hline
\end{tabular}

Figure 7-1 Power Transfer Arrangement

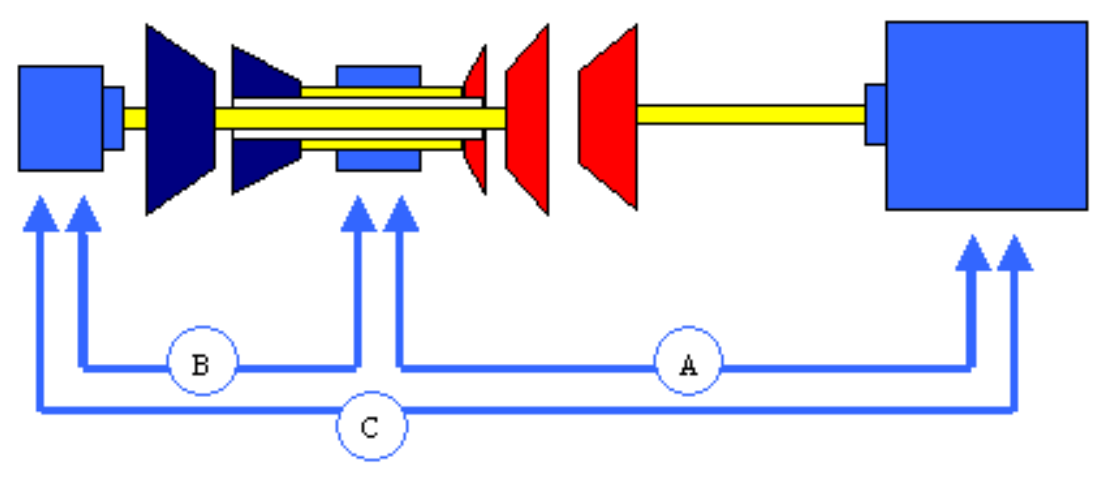


The case against Shaft Power Transfer

Shaft Power Transfer has been promoted for aero engines as a method of improving engine handling during transients and at part loads. In a power generation application the engine may experience a sudden loss of load due to an electrical failure of some part of the grid downstream of the generator set. A similar load loss is also possible in marine applications if the vessel's propeller comes out of the water in heavy seas.

In both of these cases, due to the sudden loss of load, fuel must be pulled off rapidly to avoid over speeding the free power turbine. Once the speed is under control the engine needs to be decelerated to idle to be available immediately for re-loading. This drop load condition is a challenge for both combustor stability and, for multiple spool engines, LP compressor operability.

The need to cope with and recover from this "external" event in both an industrial and a marine application is very different to the requirements for an aero engine. In the aero case the only way the engine can experience a sudden loss of load is through an "internal" catastrophic failure such as a fan loss or shaft breakage. As such in the aero engine case there is no prospect of recovering normal operation and so there is no requirement to maintain combustion. Furthermore the onset of surge is positively encouraged, as energy dissipated forward, away from the turbine, will not contribute to over speed.

Therefore, in general an industrial engine will see a much more constant load profile than an aero machine when it does see a transient the magnitude of load change can be much more severe. If an approach based on electrical power transfer is proposed to replace the existing aerodynamic controls using VIGVs \& BOVs the motor power requirements of an industrial engine could be much greater than an aero machine. Considering the likely cost constraints of an industrial engine and the relatively small value of any efficiency improvement in part load or transient operation this approach is unlikely to be viable.

The value of using the existing hardware, i.e. the starter and the alternator, to achieve some degree of power transfer is likely to be minimal. A detailed analysis of the benefit has not been made. However considering that:

- The starter motor power requirement is less than $1 \%$ of the full-load compressor power requirement.

$\&$

- The application of full starting power only reduces the time taken to accelerate between $75 \%$ and $95 \%$ load by less than $\sim 2 \%$,

The effect of power transfer at this magnitude is expected to be negligible. The fundamental idea behind enhancing the performance of the machine in particular regions of its operating regime using supplemental power from an electric drive is thought to be worthy of further study. However the concept is more likely to be suitable 
for propulsion applications which experience more frequent load transients and where cost is not such an overwhelming concern. 


\section{ELECTRIC STARTER GENERATOR (ESG)}

$\begin{array}{ll}\text { OPPORTUNITY: } & \text { REPLACE HYDRAULIC STARTER WITH ELECTRIC STARTER / } \\ & \text { GENERATOR }\end{array}$
Why ? GENERATOR

\begin{tabular}{|l|l|l|}
\hline Cost & $1^{\text {st }}$ Cost & $\begin{array}{l}\bullet \text { Delete accessory gearbox } \\
\text { Delete hydraulic starter }\end{array}$ \\
\cline { 2 - 3 } & Installation Costs & $\bullet$ Reduced engine/package interface \\
\cline { 2 - 3 }
\end{tabular}

\begin{tabular}{|l|l|l|}
\hline Reliability / availability & AMB supply & $\bullet$ Inherent supply if engine is rotating \\
\hline & $\begin{array}{l}\text { Brown out } \\
\text { protection }\end{array}$ & $\begin{array}{l}\text { Inherent supply for GT package } \\
\text { control }\end{array}$ \\
\cline { 2 - 3 }
\end{tabular}

\begin{tabular}{|l|l|l|}
\hline Functionality & Power Sharing & $\begin{array}{l}\text { Possible to swap power with the } \\
\text { FPT }\end{array}$ \\
\hline
\end{tabular}

\section{Findings}

\begin{tabular}{|l|l|}
\hline $\begin{array}{l}\text { Applicable to existing RR } \\
\text { Engines ? }\end{array}$ & $\begin{array}{l}\text { Yes - } \\
\text { Either on shaft end or through existing starter gear }\end{array}$ \\
\hline Recommended? & $\begin{array}{l}\text { Yes }- \text { but........ } \\
\text { Power transfer unlikely to be worthwhile due to } \\
\text { relatively small size of the machines }\end{array}$ \\
\hline
\end{tabular}

Figure 8-1 Embedded Starter Arrangement

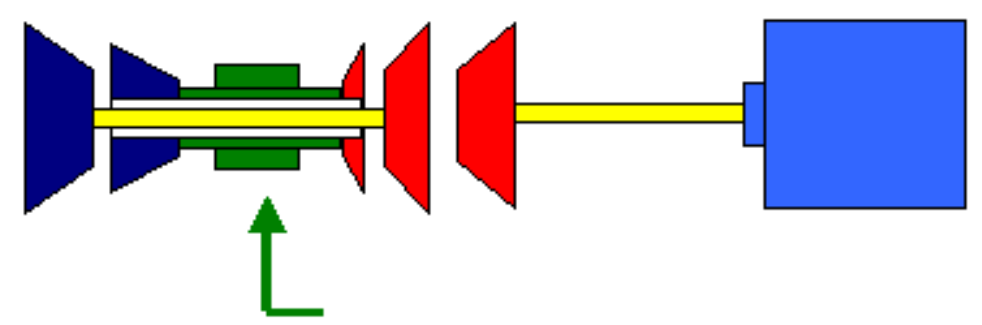

\section{The case for an Electric Starter / Generator}

Currently the engine is started by a hydraulic starter motor that provides drive to the gas generator shaft through some form of geared transmission. 
Replacing the relatively expensive hydraulic starter motor with an electrical machine should deliver a direct cost saving. High-speed machine technology would allow the starter to drive the gas generator directly, eliminating the need for a mechanical transmission. Further to these 'hardware' costs benefits there would be significant savings in both reduced installation and maintenance costs from the removal of the hydraulic system.

In terms of enhanced functionality, integration of a machine co-axially with the shaft offers the potential capability of continuous power generation, as long as the engine is rotating. This would allow the operation of the engine to continue in the event of loss of any primary power source. Such a feature would be an advantage in support of the argument for AMBs. The presence of an assured supply for the bearings will go some way to easing reliability concerns.

Discussions with Engine Packagers, such as RRESI, have indicated that there would be a real benefit in having a separate, engine driven source of electrical power to provide a high security supply to the gas turbine and the plant control system to maintain stability during brown out events.

\section{Embedded Starter / Generator Technology Issues}

There are a number of electrical machine types that can be considered for the embedded starter/generator. There are wide price differences among the various standard machine types, generally reflective of the degree of industrial usage. However, the novelty of this application coupled with annual anticipated requirements of relatively low volume might be expected to command a uniformity of pricing across the motor technologies.

On this basis, an exhaustive analysis of the various machine types has not been completed at this preliminary stage; this being an exercise for a subsequent, more detailed, engine design study. However, the salient points of the various machine types are outlined below. 


\section{Machine Types}

\section{Machine.}

The DC machine operates on the principle of the interaction of stationary magnetic stator and rotor fields. The stator of the machine consists of a non-rotating yoke, within which the field winding assembly is fitted. The field assembly consists of an even number of iron pole pieces around which coils are wound. The windings are organised in pairs to produce diametrically opposed north and south poles. Figure 82 shows a section through a four-pole machine. As the pole pieces sit proud of the stator frame the design is referred to as salient pole machine.

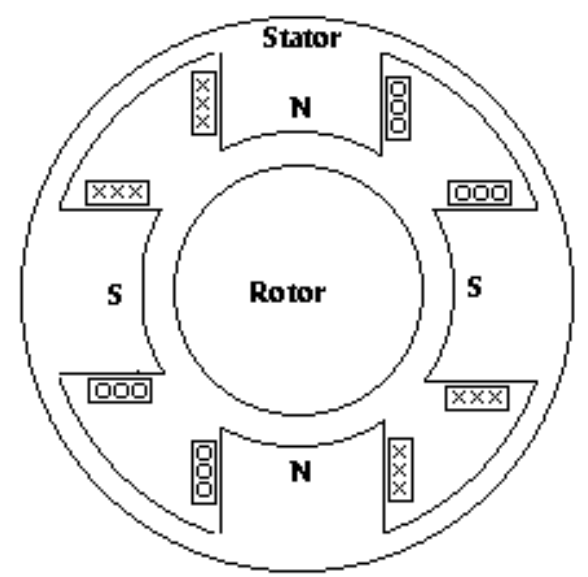

Figure 8-2 Section through a four-pole machine.

The stator yoke is usually the load-bearing member and is made of solid material. This is generally acceptable because the field flux is only varied at a slow rate and thus eddy current and hysteresis losses are negligible. However, separately excited machines, operated in field controlled mode, require a laminated rotor to avoid unacceptably high iron losses.

The main poles establish a very high radial magnetic flux density over a large proportion of the surface of the armature. This flux establishes a tangential force on the current carrying armature conductors resulting in rotor torque. To achieve this high flux density, the air gap is very small and the pole itself is often operated in the saturated region. Situated between the main poles smaller "interpoles", or "commutating poles", may be fitted. These are generally used in larger machines where they assist in current reversal in the armature windings by nulling the magnetic field at the point where the rotating commutator is swapping the polarity of the armature current. The flux density due to the interpoles should be linearly proportional to the armature current itself and thus the 
interpole-armature air gap is large to minimise the effects of variations in permeability within the magnetic circuit.

Current in the armature winding develops a magnetic field. This armature field will interact with the stator field, producing a significant distortion, or "armature reaction". To eliminate this effect, extra "compensating" windings are fitted to the stator main poles. These windings carry the armature current and produce a flux in opposition to the armature reaction flux, thus minimising the effect.

The rotor or armature of a large direct current machine carries a series of copper coils fitted into slots and retained by wedges and banding. Such a construction ensures that the rotor has no saliency. Each winding is not concentrated in one slot, but distributed about the rotor such that the flux density is uniform and broadly approximates a square wave about the circumference of the rotor. This prevents localised saturation of the rotor iron and keeps the forces exerted on the periphery of the rotor broadly constant. The rotor is constructed from soft magnetic laminations, to minimise eddy current losses.

The rotor also carries the commutator. A commutator is an assembly of isolated copper bars, individually connected to the armature winding via a "riser". A set of stationary, spring loaded carbon brushes contact the commutator, energising each consecutive armature winding as the rotor spins. In this way a stationary, torque producing magnetic field is established in the rotor. As with the main rotor bars, the commutator bars are retained by wedges and banding.

This construction limits the maximum centrifugal force that may be exerted on the bars before they move thus limiting the maximum effective rotational speed. A large industrial DC machine will typically have a maximum speed in the region of 4000rpm. The speed limitations of the commutator construction coupled with the necessary regular maintenance requirements associated with the brush gear, make this machine unsuitable for the embedded starter application.

\section{Induction Machine}

The induction machine operates on the principle of interaction of rotating stator and rotor fields. The non-salient stator of the induction machine is wound with either a three- phase or single phase winding such that a rotating magnetic field is generated. The rotor is made up as a cylinder of laminated core material with slots along its length. Conducting bars, of usually copper or aluminium, are fitted into the slots and at each end of the assembly, conductive shorting rings are braised to the conductor bars. The complete rotor assembly is termed a squirrel cage. The rotating stator magnetic field cuts the rotor conductors inducing an EMF, causing a current to flow in the short circuit rotor cage. This induced rotor current produces a rotor flux that is synchronous with the stator field generating it. Interaction of the two fields produces a torque, causing the rotor to rotate. As the rotor accelerates, the frequency of the induced current reduces, however the rotor and stator fields remain in synchronism. The difference in speed between the rotor speed and stator frequency is known as

\begin{tabular}{|l|l|l|l|}
\hline Document number & Issue & Page 24 of 58 \\
\hline
\end{tabular}


the 'slip' and is proportional to the frequency of the induced rotor current. The motor can never operate at synchronous speed because it relies on induction to generate the torque producing rotor field.

With the advent of power electronic inverter drives, the ability to vary the stator frequency and hence the speed of the induction machine, has been developed. Such progress has driven the development of high-speed induction machinery, with specialist machines in the MW range now being produced with speeds up to 14,000 rpm.

\section{Permanent Magnet Machine}

Brushless DC permanent magnet machines are capable of variable speed operation. In the brushless DC machine, the field is produced by a number of magnets located on the rotor. The armature windings are located on the stator. There is no mechanical commutator, hence these machines require a power electronic converter to sequentially switch the armature windings. The brushless DC machine generally has three or six windings, with concentrated turns. As such, the machine has locations of high magnetic flux at definite points on its circumference. This leads to inherent torque ripple. Low-speed ripple can be mitigated, to a limited extent, by axial skewing of the permanent magnets.

The motor may be of the inner or outer rotor type depending on the application. The inner rotor configuration is more suitable to high-speed operation and has the smaller rotor inertia. The outer rotor configuration can be useful in constant speed applications where the larger rotor inertia reduces speed fluctuations arising from torque ripple. Such a motor also has advantages from the cooling point of view, since the major heat producing element is the armature winding and its heat is mostly dissipated through the rotor.

An alternative configuration is the so-called disc, or axial flux, machine in which the active portion of the flux is axial and the winding length radial. Traditionally these machines are not favoured because the active length of the armature conductors is short and the velocity varies sharply along their length. The disc machine has to be large in diameter and short in axial length and benefits from being multiple pole. The three types of machine are shown in Figure 8-3.

There are three possible rotor structures for the conventional inner-rotor radial field machine, as shown in Figure 8-4. The surface-mounted magnet arrangement, as shown in Figure 8-5, is easy to construct, particularly with pre-formed sectional magnets. However, there are mechanical difficulties since the magnets must be glued to the rotor, limiting the maximum rotor speed. For high speed application an additional retaining ring, or rotor sleeve, is used. The ring increases the mechanical strength of the rotor but increases the effective air-gap. This causes a reduction in the magnet field strength or a requirement for deeper magnets. The sleeve can be manufactured from carbon-fibre in critical applications but stainless steel is commonly chosen. When using stainless steel additional losses in the rotor sleeve, due to slot 
ripple field components, must be considered. A drawback of using a sleeve is that stronger magnets are generally required, since the air gap flux density is equal to the magnet working flux density. This increases the cost of the machine.

The inset magnet configuration offers greater mechanical security to the magnets although a rotor sleeve may still be required in high-speed applications.

The interior magnet has a more complex mechanical and magnetic design. The magnets are located with their axes in the circumferencial direction so that the flux over a rotor pole arc is contributed to by two separate magnets. Higher flux densities are possible with such a configuration since it is possible for the flux density in the air gap to exceed the working flux density of the individual magnets. Under such circumstances the magnetic loading can be increased or, alternatively, a lower cost magnet such as a ferrite may be used.

The permanent magnet machine is capable of working at a better power factor and efficiency than the equivalent induction machine since the magnetising component of the armature current is not required. However, the cost of the rotor structure is typically high due to the permanent-magnet material and the rotor is generally less robust than that of the induction motor.
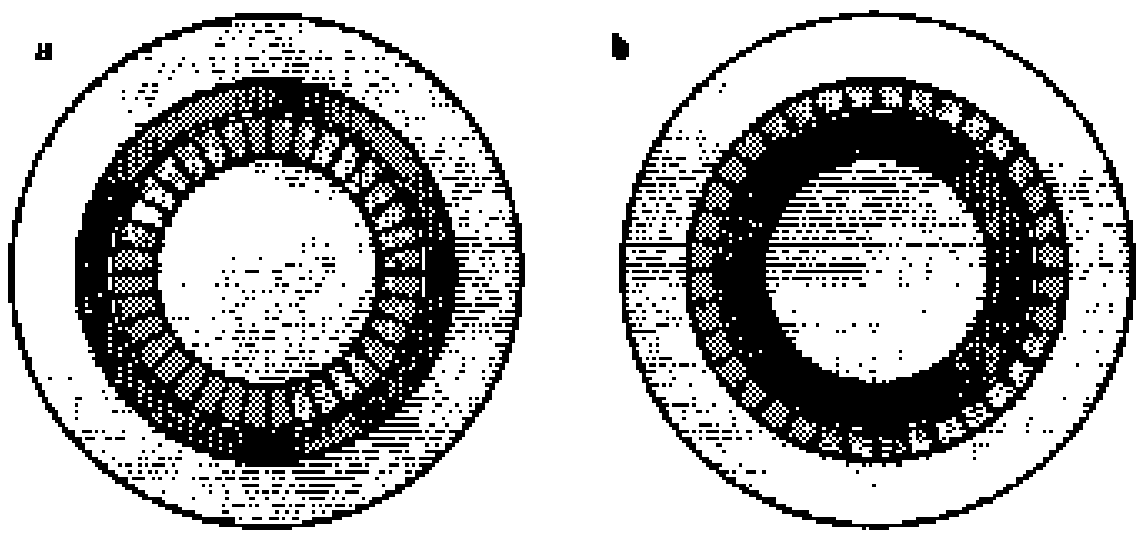

5
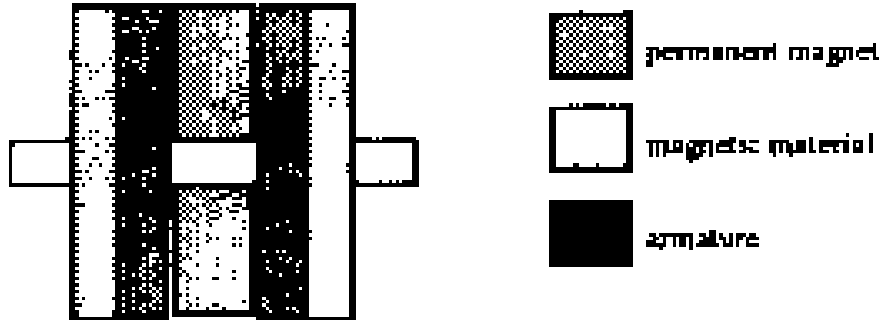
a) radial-field, inner rotor
b) radial-field, outer-rotor
c) axial-field

Figure 8-3 Permanent Magnet Machine Types 

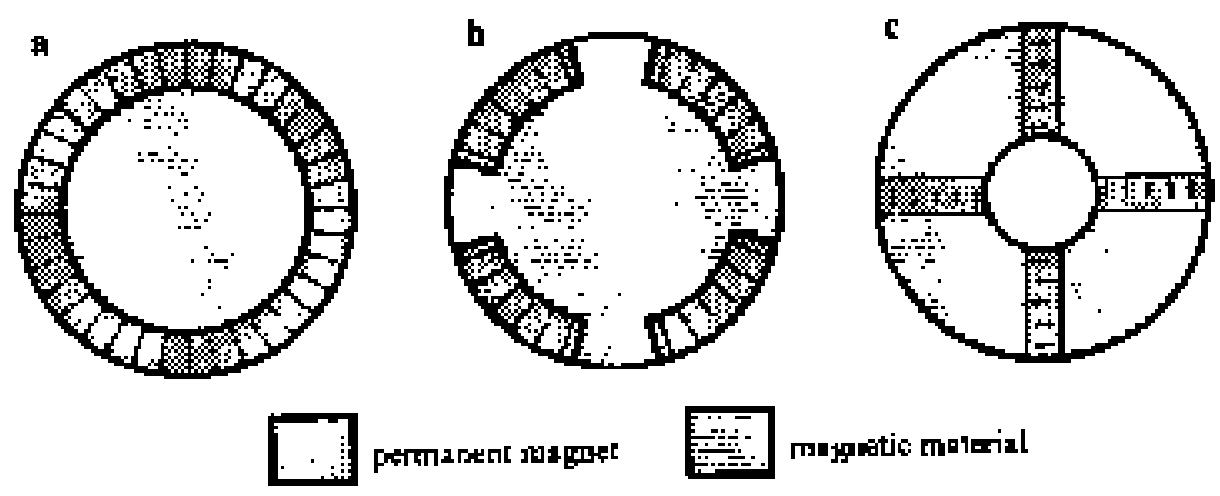

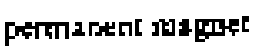

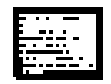

manpatic malcial
a) Surface
b) Inset
c) Interior

Figure 8-4 Radial Permanent Magnet Configurations

\section{Switched Reluctance Machine}

Switched Reluctance Motors (SRMs) operate on the principle of the natural tendency for a distorted magnetic field to align itself. The stator and rotor are formed with unequal numbers of axial poles or "teeth". The stator poles are wound in adjacent pairs to produce a N-S pole. When this winding is energised the magnetic field enters the nearest pair of rotor teeth. If the pair is not aligned with the stator pair then torque is produced.

Continuous motion is achieved by sequential switching using power electronics. Position knowledge is required to achieve continuous rotation.

The switched reluctance machine is a relatively recent development compared with the induction and permanent magnet machines. The machine has gained favour in certain niche applications including an engine embedded starter-generator developed as part of the US More Electric Aircraft initiative. The machine offers high fault tolerance and a rugged construction that is tolerant of harsh environments. The machine has no magnets or windings on the rotor; the excitation coming from the stator windings. Hence, a SRM has the potential to withstand a short circuit fault with no rotor-induced excitation to feed the fault. Also, the machine could continue to operate and produce useful power from the remaining windings. 


\section{Advantages of SR Machines:}

- Ability to run at very high speeds (tens of thousands of rpm)

- Speed or torque control modes

- High starting torque

- Almost constant torque up to base speed, constant power thereafter.

- High efficiency throughout speed range

- Mechanical simplicity / robustness. Low maintenance.

- Inherent reliability due to isolated coil structure.

\section{Disadvantages of SR Machines:}

- A lower power density than the permanent magnet machine

- SRM requires a relatively sophisticated power converter. 


\section{Size Comparisons}

Given the time constraints and scope of the study it was not considered necessary to complete detailed machine design at this stage; the intention being to outline size and cost to give a view on feasibility. As such, the machine size estimates given below are the result of simple scaling exercises on appropriate existing machine designs. The increased mechanical stress and electrical losses of higher speed operation have not been considered.

\section{Induction}

Standard industrial induction machines are designed for operation up to a nominal $3,000 \mathrm{rpm}$ from a $50 / 60 \mathrm{~Hz}$ mains supply. Inverter driven machines can be operated up to around 6,000 rpm using field-weakening techniques. Beyond this the losses of standard machines become excessive. Design of the starter for nominal operation at $8,500 \mathrm{rpm}$ would allow field weakened operation up to around 17,000 rpm, allowing for generation of $45 \mathrm{~kW}$.

To arrive at a size estimate for such a machine, a standard 2-pole industrial aircooled design, of Brook-Hansen manufacture, was used as a base. Brook-Hansen offer a V-DF255M-D 2-pole, 3,000 rpm, $45 \mathrm{~kW}, 400 \mathrm{~V}$, 3-phase machine in their standard range, suitable for operation in a $40{ }^{\circ} \mathrm{C}$ ambient. Such a machine has dimensions:

Typical Induction Machine

$\begin{array}{ll}\text { Stator Outside Diameter } & 448 \mathrm{~mm} \\ \text { Shaft Outside Diameter } & 55 \mathrm{~mm} \\ \text { Length } & 400 \mathrm{~mm} \\ \text { Mass } & 250 \mathrm{~kg}\end{array}$

Scaling was executed on the basis of conservation of the volume $x$ speed product. That is:

$$
\begin{gathered}
\text { Vlume }_{1} \times \text { Speed }_{1}=\text { Volume }_{2} \times \text { Speed }_{2} \\
D_{1}^{2} L_{1} N_{1}=D_{2}^{2} L_{2} N_{2} \\
\therefore D_{2}^{2} L_{2}=D_{1}^{2} L_{1} \frac{N_{1}}{N_{2}}
\end{gathered}
$$

From this, it is apparent that both the diameter and length can be reduced by a factor of: 


$$
\sqrt[3]{D_{1}^{2} L_{1} \frac{N_{1}}{N_{2}}}=0.71
$$

This yields a machine of:

\section{Induction Machine ESG Spec}

Stator Outside Diameter

Rotor Inside Diameter

Length

Mass
$286 \mathrm{~mm}$

$55 \mathrm{~mm}$

$255 \mathrm{~mm}$

$65 \mathrm{~kg}$

Note that no attempt has been made to account for the increased shaft diameter of $100 \mathrm{~mm}$ at this stage.

\section{Permanent Magnet}

At this early stage no decision has been made as to the most appropriate radial permanent magnet configuration. In order to size the machine for this exercise, an original design by I.Care et al was used as a base [DNS65535]. This machine was designed for $45 \mathrm{~kW}$ at 20,000 rpm, using rare earth magnets (Sm2Co17) and was oilcooled.

Typical PM Machine

$\begin{array}{ll}\text { Stator Outside Diameter } & 145 \mathrm{~mm} \\ \text { Shaft Outside Diameter } & 100 \mathrm{~mm} \\ \text { Length } & 50 \mathrm{~mm} \\ \text { Mass } & 6.5 \mathrm{~kg}\end{array}$


Starting of the compressor application of the new engine is estimated to need $45 \mathrm{~kW}$ up to approximately $8,500 \mathrm{rpm}$. Scaling was, again, executed on the basis of conservation of the volume $x$ speed product. That is:

$$
\begin{gathered}
\text { Volume }_{1} \times \text { Speed }_{1}=\text { Volume }_{2} \times \text { Speed }_{2} \\
D_{1}^{2} L_{1} N_{1}=D_{2}^{2} L_{2} N_{2} \\
D_{1}^{2} L_{1} N_{1}=D_{2}^{2} L_{2} N_{2}
\end{gathered}
$$

Assuming that $D_{1}=D_{2}$, then:

$$
L_{2}=L_{1} \frac{N_{1}}{N_{2}}
$$

This yields a machine of:

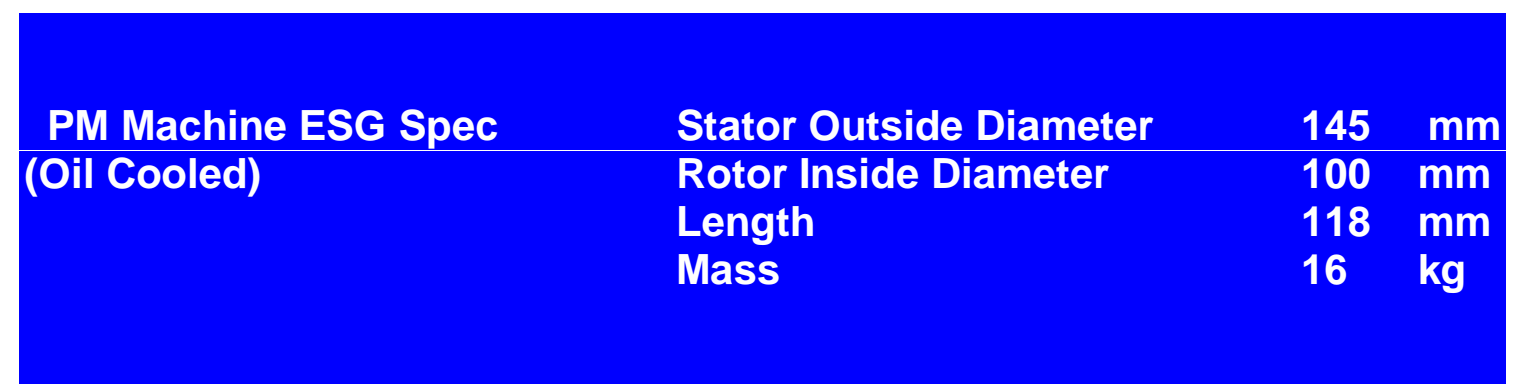

The mass estimate was arrived at by simply scaling the mass of the base machine with respect to volume.

\begin{tabular}{|l|l|l|c|}
\hline Document number & Issue & Page 31 of 58 \\
\hline
\end{tabular}




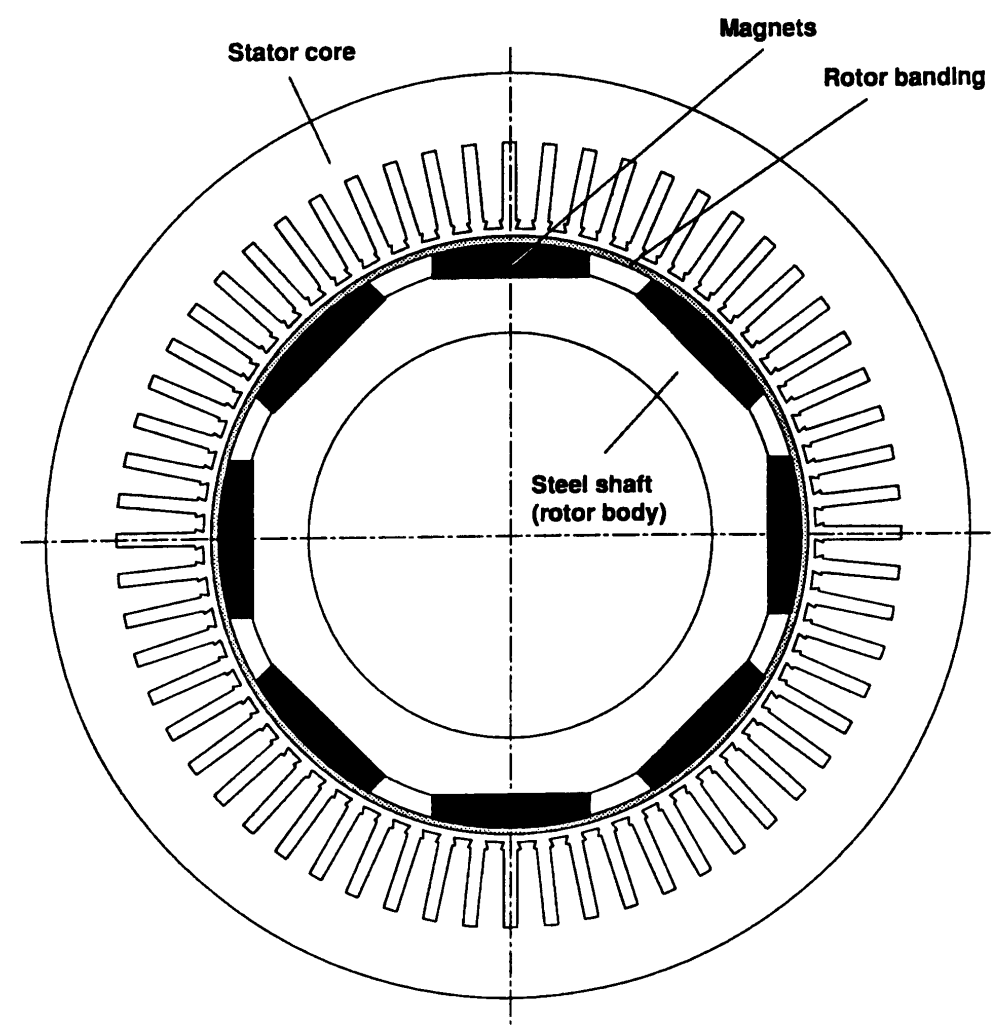

Figure 8-5 Typical Radial PM Machine Construction

With the overall aspiration of an oil-free engine, the incorporation of an oil-cooled machine is far from ideal. It was therefore decided to estimate the resulting size of an air cooled machine. The specific heat capacity of oil is approximately 1.8 times that of air. Therefore, assuming $100 \%$ volume increase for an air cooled machine then:

$$
2 D_{1}^{2} L_{1} N_{1}=D_{2}^{2} L_{2} N_{2}
$$

Assuming additional volume is achieved by increasing the outside diameter:

$$
2 D_{1}^{2}=D_{2}^{2}
$$




$$
\therefore D_{2}=\sqrt{2} D_{1}
$$

Such assumptions yield an air cooled machine of dimension:

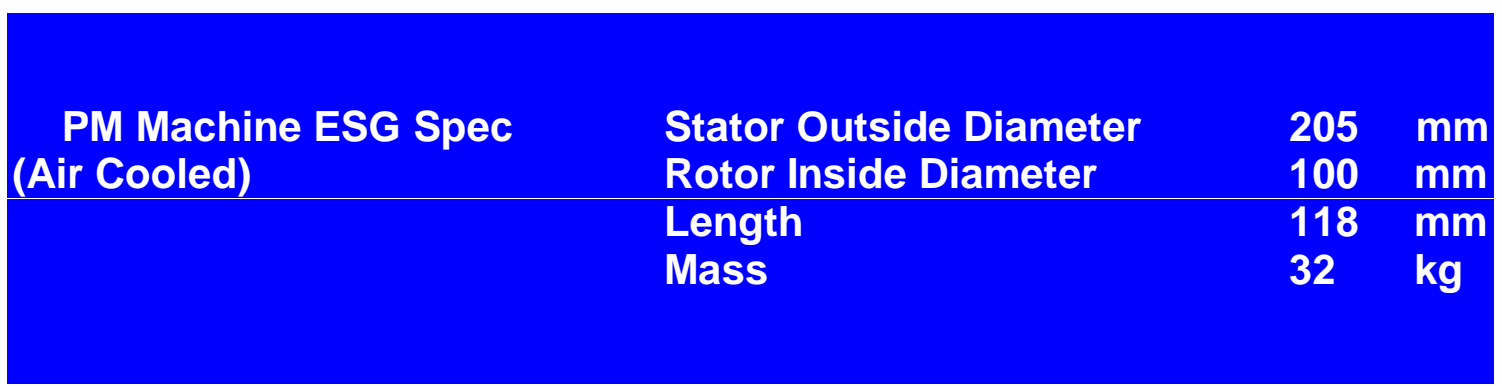

\section{Cost}

\section{Machine Cost}

An estimate of the cost of the embedded starter/generator was derived by considering the cost of a standard, caged induction machine in similar production volumes, the cost of permanent magnets and a factor to allow for the novelty of the machine and application. A standard D255 frame $45 \mathrm{~kW}, 3000 \mathrm{rpm}$ induction motor costs around US\$2,500. Therefore, the cost of a specially designed high-speed machine would be anticipated at around US $\$ 4,000$ in production volumes.

\section{Power Electronics Cost}

Standard commercial, air cooled power electronics will be suitable for this application as there are no substantial restrictions on weight or size. Power electronics for all machine types, except the SRM, are commercial off-the-shelf technology available from a number of manufacturers. A typical three-phase $400 \mathrm{~V}, 45 \mathrm{~kW}$, IGBT inverter drive costs around US $\$ 11,500$ in one-off quantities. A minimum discount of $10 \%$ would be expected for quantities in excess of 100, giving a price estimate in the region of US\$10,000. A typical $45 \mathrm{~kW}$ inverter drive system would be supplied in one $2100 \mathrm{~mm} \mathrm{H} \times 800 \mathrm{~mm} \mathrm{~W} \times 500$ mm D, floor mounted cubicle.

The requirement for an integrated electrical machine is in line with current aerospace development of embedded machinery for aero-engines. Whilst the environment is somewhat more benign, development issues might be expected to be broadly similar.

However machine mass, whilst impacting on rotor dynamics is not as undesirable as in aircraft applications. Furthermore, with the "cold end drive" engine general arrangements the machine will be located in a nominal ambient of $15{ }^{\circ} \mathrm{C}$ with an extreme of $50{ }^{\circ} \mathrm{C}$. Location in front of the engine compressor also provides a

\begin{tabular}{|l|l|l|l|}
\hline Document number & Issue & Page 33 of 58 \\
\hline
\end{tabular}


convenient forced vent cooling air flow of around $20.6 \mathrm{kgs}^{-1}$. However, the engine must be designed to cope with exposure to moisture and salt laden atmospheres together with the occasional cleaning fluid deluge. As such the machine will require coating and possible enclosing as generally associated with IP55 and higher industrial machines. 


\section{REMOVAL OF HYDRAULIC ACTUATION}

Presently, all actuation on the RR Engines is hydraulic with a hydraulic pump being connected to the accessory gearbox, which is in turn driven via a radial drive. On the existing RR Engines engines, there is no requirement for variables, so that hydraulics are only included for starting and actuation of bleed valves etc. Use of an integrated electric starter would allow removal of the radial drive. An oil pump is driven from the main, reduction gearbox. In removing the oil system, the oil pump is no longer needed.

All of the new engine configurations require variables as well as bleed valves. One possibility here is a return to pneumatic actuation. However, the sluggish responses of such systems, coupled with the necessary engine bleed requirements were contributing factors in the development of hydraulic actuation. The cost benefit of using an electric starter/generator together with pneumatic actuation is outlined in Table 9-1.

Competitors such as Nouvo Pignoni and ABB use electrical actuation engines such as the PGT5 and GT35 where electrification of all axes of actuation has proved economically viable as well as offering a greater precision of control.

The PGT5 is a $7 \mathrm{MW}$ engine. The IGV actuator fitted to such an engine is shown in Figures 9-1 and 9-2 below. This is a $4.77 \mathrm{Nm}, 1537 \mathrm{~W}, 325 \mathrm{~V}, 8520 \mathrm{rpm}$ permanent magnet driven actuator. Similar style rotary actuators are also available for bleed valve operation. A sprung extend electric VIGV actuator and controller from Moog Controls costs around US $\$ 13,000$, with a bleed valve actuator costing in the region of US $\$ 4,500$. The cost benefit of using an electric starter/generator together with electric actuation is outlined in Table 9-2. It can be seen that there is potentially an additional US\$ 5,500 saving through use of electric, as opposed to pneumatic, actuation. 


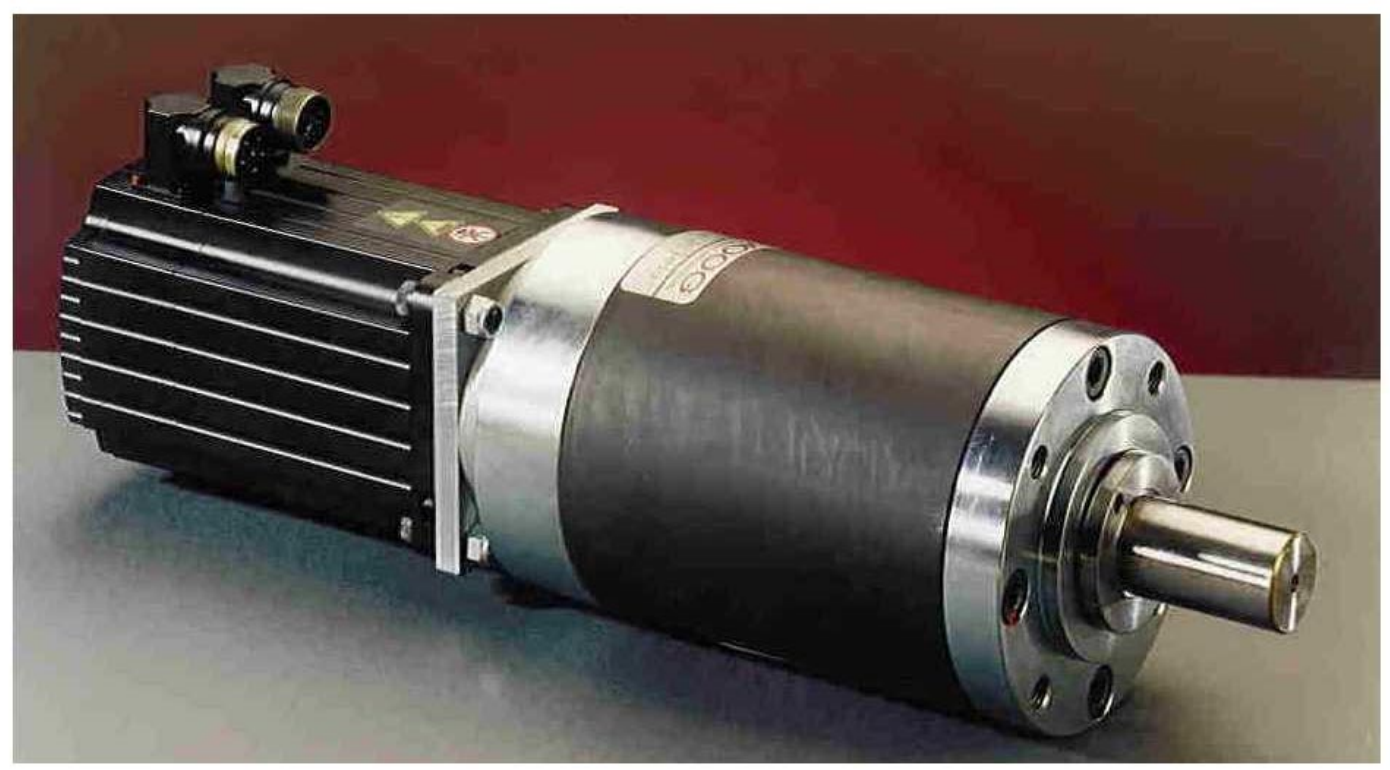

Figure 9-1 Brushless Permanent Magnet VIGV actuator assembly 
Potential More Electric Systems for a 50-100MW Industrial Gas Turbine

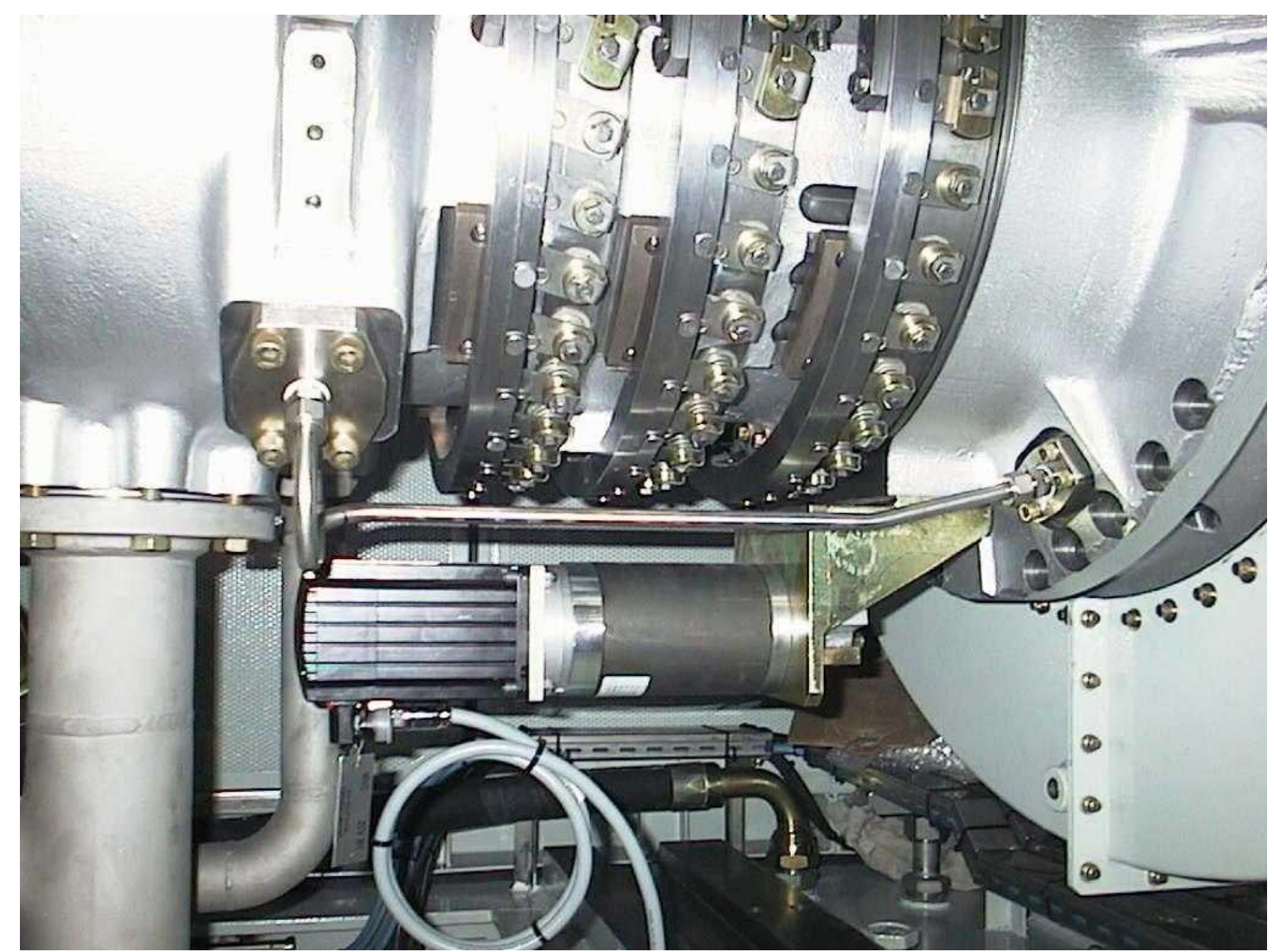

Figure 9-2 Electric VIGV actuator fitted to 7 MW Gas Turbine 
Potential More Electric Systems for a 50-100MW Industrial Gas Turbine

\begin{tabular}{|c|c|c|}
\hline Component (conventional engine) & Equivalent component (electric engine) & Saving (\$) \\
\hline Hydraulic pump (driven by oil pump motor) & $\begin{array}{l}\text { Replaced by bleed ducting (from HP compressor } \\
\text { via bleed valve) }\end{array}$ & 1000 \\
\hline Hydraulic system instrumentation & Pneumatic system instrumentation & 0 \\
\hline Hydraulic system controls & Pneumatic system controls & 0 \\
\hline Off engine hydraulic system pipework & Not required & 1500 \\
\hline $\begin{array}{l}\text { Off engine hydraulic system pipework } \\
\text { mounting }\end{array}$ & Not required & 1500 \\
\hline Hydraulic system non-return valves & Pneumatic system non-return valves & 0 \\
\hline $\begin{array}{l}\text { Hydraulic system flexible pipes (from pump } \\
\text { to engine) }\end{array}$ & Not required & 500 \\
\hline Hydraulic system engine mounted pipework & Pneumatic system engine mounted pipework & 0 \\
\hline $\begin{array}{l}\text { Hydraulic system engine mounted pipework } \\
\text { clipping }\end{array}$ & $\begin{array}{l}\text { Pneumatic system engine mounted pipework } \\
\text { clipping }\end{array}$ & 0 \\
\hline Hydraulic variable vane actuator & Pneumatic variable vane actuator & 0 \\
\hline Hydraulic starter & Electric motor/generator & 16000 \\
\hline Hydraulic motor control & Motor /Generator control system & -9000 \\
\hline Radial drive shaft & Not required & 1500 \\
\hline Internal starter gear & Not required & 600 \\
\hline Radial drive thrust bearing & Not required & 300 \\
\hline Radial drive inner location bearing & Not required & 500 \\
\hline Radial drive outer location bearing & Not required & 500 \\
\hline External starter gear & Not required & 600 \\
\hline Radial drive inner oil seals (2 off) & Not required & 150 \\
\hline Radial drive outer oil seals (2 off) & Not required & 150 \\
\hline Package totals & & 15800 \\
\hline
\end{tabular}

\section{Table 9-1 Estimated Cost Saving From Embedded Starter Generator \& Pneumatic Actuation}


Potential More Electric Systems for a 50-100MW Industrial Gas Turbine

\begin{tabular}{|c|c|c|}
\hline Component (conventional engine) & Equivalent component (electric engine) & Saving (\$) \\
\hline Hydraulic pump (driven by oil pump motor) & Not required & 3000 \\
\hline Hydraulic system instrumentation & Not required & 5000 \\
\hline Hydraulic system controls & Rotary Electric Bleed Valve Actuator & 5500 \\
\hline Off engine hydraulic system pipework & Not required & 1500 \\
\hline $\begin{array}{l}\text { Off engine hydraulic system pipework } \\
\text { mounting }\end{array}$ & Not required & 1500 \\
\hline Hydraulic system non-return valves & Not required & 1000 \\
\hline $\begin{array}{l}\text { Hydraulic system flexible pipes (from pump } \\
\text { to engine) }\end{array}$ & Not required & 500 \\
\hline Hydraulic system engine mounted pipework & Harnesses & 0 \\
\hline $\begin{array}{l}\text { Hydraulic system engine mounted pipework } \\
\text { clipping }\end{array}$ & Harness clipping & 0 \\
\hline Hydraulic variable vane actuator & Pneumatic variable vane actuator & -8000 \\
\hline Hydraulic starter & Electric motor/generator & 16000 \\
\hline Hydraulic motor control & Motor /Generator control system & -9000 \\
\hline Radial drive shaft & Not required & 1500 \\
\hline Internal starter gear & Not required & 600 \\
\hline Radial drive thrust bearing & Not required & 300 \\
\hline Radial drive inner location bearing & Not required & 500 \\
\hline Radial drive outer location bearing & Not required & 500 \\
\hline External starter gear & Not required & 600 \\
\hline Radial drive inner oil seals (2 off) & Not required & 150 \\
\hline Radial drive outer oil seals (2 off) & Not required & 150 \\
\hline Package totals & & 21300 \\
\hline
\end{tabular}

\section{Table 9-2 Estimated Cost Saving From Embedded Starter Generator \& Electric Actuation}


10 ACTIVE MAGNETIC BEARINGS

OPPORTUNITY: USE ACTIVE MAGNETIC BEARINGS (AMBS)

Why?

\begin{tabular}{|l|l|l|}
\hline Cost & $1^{\text {st }}$ Cost & $\begin{array}{l}\text { Reduced engineering cost } \\
\bullet \quad \text { No oil system required }\end{array}$ \\
\cline { 2 - 3 } & Installation Costs & $\bullet \quad$ No oil system fitting required \\
\cline { 2 - 3 }
\end{tabular}

\begin{tabular}{|c|c|c|}
\hline Performance & Oil free engine & $\begin{array}{l}\text { - } \text { Emissions reduction } \\
\text { - Reduction in parasitic bearing } \\
\text { losses } \\
\text { - Active tip control }\end{array}$ \\
\hline
\end{tabular}

\begin{tabular}{|l|l|l|}
\hline Reliability / availability & $\begin{array}{l}\text { Reduced } \\
\text { maintenance }\end{array}$ & No oil changes required \\
\hline \multirow{2}{*}{ Installation Costs } & $\begin{array}{l}\text { Inherent supply for GT package } \\
\text { control }\end{array}$ \\
\cline { 2 - 3 }
\end{tabular}

Findings

\begin{tabular}{|l|l|}
\hline $\begin{array}{l}\text { Applicable to existing RR } \\
\text { Engines? }\end{array}$ & $\begin{array}{l}\text { No } \\
\text { Extensive engine architecture and casing changes } \\
\text { required }\end{array}$ \\
\hline Recommended ? & $\begin{array}{l}\text { Yes - but........ } \\
\text { Development of high temperature AMBs is an } \\
\text { acknowledged technical risk }\end{array}$ \\
\hline
\end{tabular}


Figure 10-1 AMB Arrangement
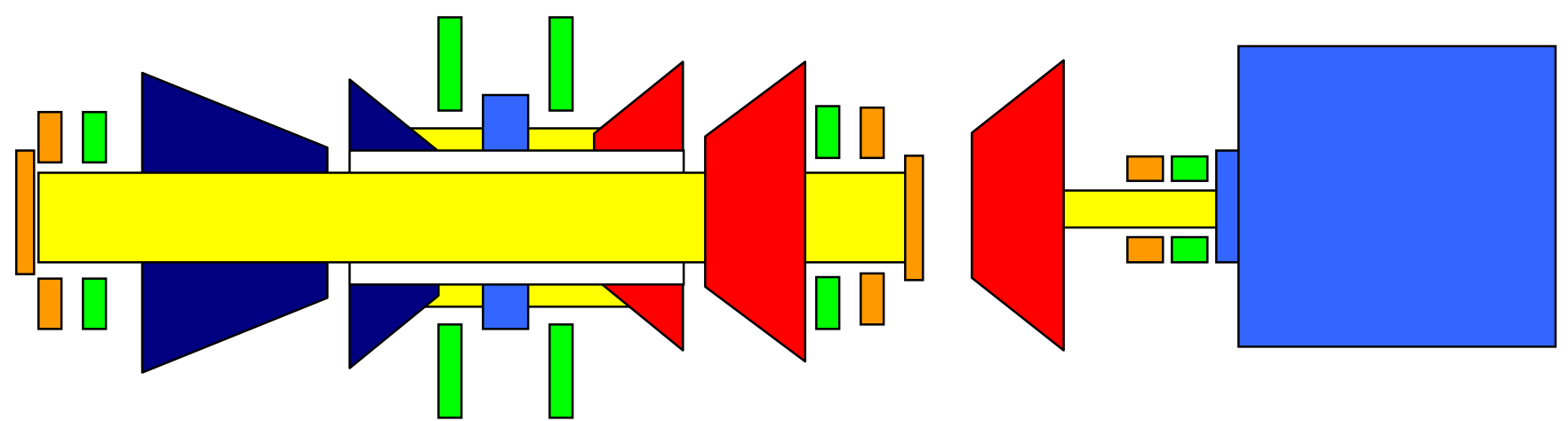

Radial AMB

Thrust AMB
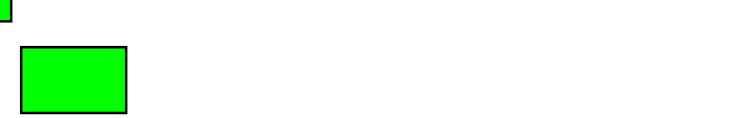

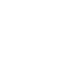


Potential More Electric Systems for a 50-100MW Industrial Gas Turbine

Typical radial AMB losses would be around $100 \mathrm{~W}$, with the losses of the large thrust bearing being around $500 \mathrm{~W}$.

\begin{tabular}{|l|c|c|}
\hline & Ball Bearing & $\begin{array}{c}\text { Roller } \\
\text { Bearing }\end{array}$ \\
\hline Oil Flow & 15 igph & 15 igph \\
\hline Pitch Circle Diameter & $72 \mathrm{~mm}$ & 72 \\
\hline Ball Diameter & $16 \mathrm{~mm}$ & - \\
\hline No of Balls & 14 & - \\
\hline Ball Material Code & ATY & - \\
\hline Inner Track Conformity & 1.037 & - \\
\hline Outer Track Conformity & 1.037 & - \\
\hline End Load & $4.45 \mathrm{kN}$ & - \\
\hline Catch Factor & 0.9 & 0.9 \\
\hline Coefficient of Spin Friction & 0.028 & - \\
\hline Nominal Contact Angle & 31 degrees & - \\
\hline Scavenge Efficiency & 2 & - \\
Factor & & 1 \\
\hline Heat Generation Factor & 1 & Side \\
\hline Feed Type & - & 2 \\
\hline Calculation Method & MTRR90141 & MTRR9014 \\
\hline & & \\
\hline & & \\
\hline
\end{tabular}

Table 10-1 Existing RR Engines Oiled Bearing Assumptions

6. Less critical balancing of production shafts and discs.

AMBs can allow the shaft to rotate about its natural centre of mass, rather than its geometric centre. Theoretically, less accurate balancing is required. In practice, the balancing accuracy will depend upon the clearance between the shaft and the back-up bearing. A typical tolerance for out of balance is $5 \%$ of the tip clearance required for thermal and centrifugal reasons. Hence, if the BUB is designed to operate once the 
shaft has moved $50 \%$ of its radial clearance, the shaft could be balanced $1 / 10^{\text {th }}$ as accurately ( 10 times as large an out of balance)

7. Rotative matching

Following on from the above, it may be possible that the increased tolerances preclude individual matching of components during refit.

8. Elimination of radial drive shaft and accessory gearbox.

9. No engine vibration monitoring system.

The AMB control system could be used to monitor engine vibration.

10. Vibration resonance control

The resonant frequency of a shaft depends on the location and stiffness of its supports as well as the shaft dimensions. The resonant frequency can be adjusted by changing the bearing stiffness. Such active tuning is not possible with conventional $\mathrm{RC}$ bearings. Such tuning may allow thinner shafts.

11. Active tip clearance control

It has been suggested that tip clearance could be controlled using Axial AMBs. Due to the angle of the blade tips, axial shaft position could be varied open or close the tip clearances. However, the effect would be small, and any effect on the compressor clearances would be offset by the resultant variation in turbine clearances.

12. Future potential

AMB technology for turbines is relatively immature. However, AMBs have been fitted in high volumes to turbomolecular pumping applications. Whilst the development costs for turbine ready AMBs may be quite high, the indication from the turbomolecular pumping market is that considerable volume discounts could be made. Also, at present the high temperature turbine environment is prohibitive. As the technology develops, the need for area cooling would reduce or eventually disappear.

What are the disadvantages?

1. Increased weight. 
2. Increased EMI and reduced EMC.

3. Technology development required - represents technical risk.

\section{Estimation of Bearing Sizes}

A spreadsheet tool has been developed by the authors to give approximate sizes of active magnetic bearings (AMBs). This tool has been used to size a number of bearings for both existing and new engines. The space envelope required by an AMB is a function of the load capacity and the material of manufacture.

Outline AMB sizes are shown in the following table for a number of potentially suitable materials. 


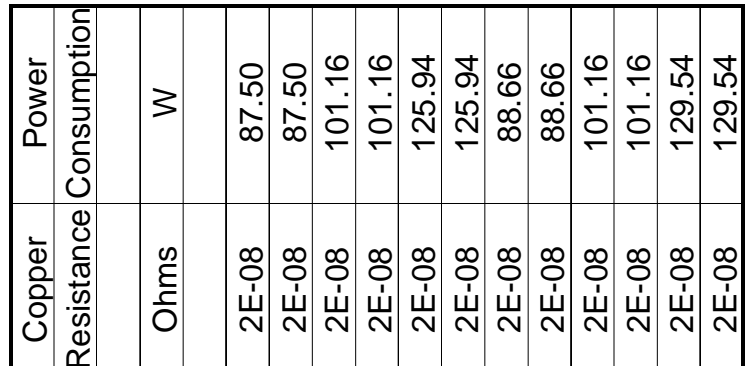

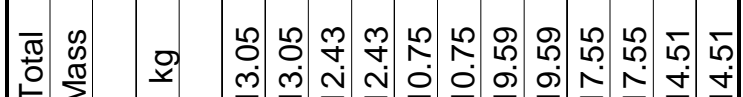

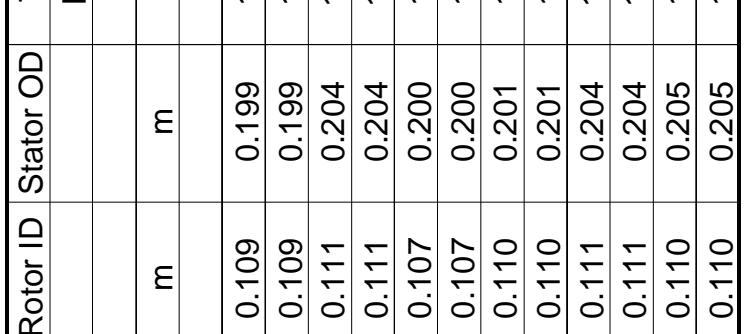

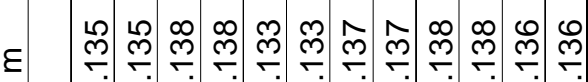

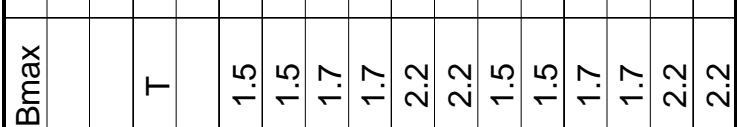




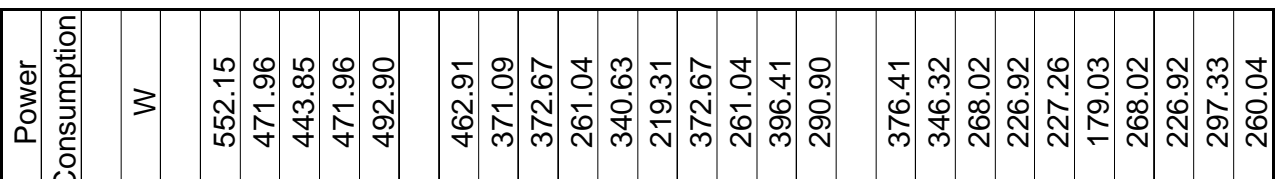

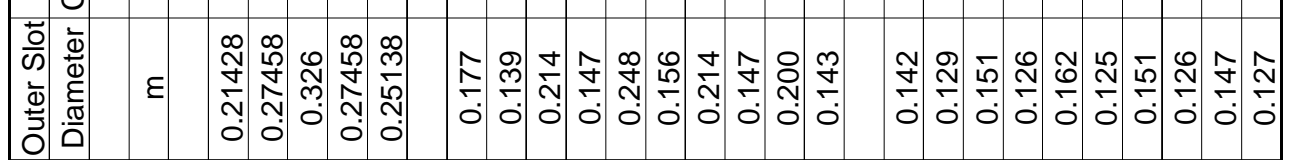

ఫ这

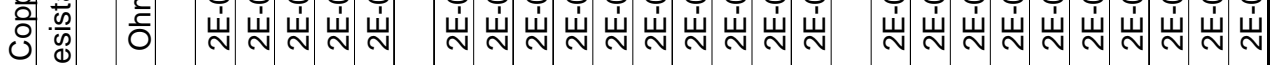

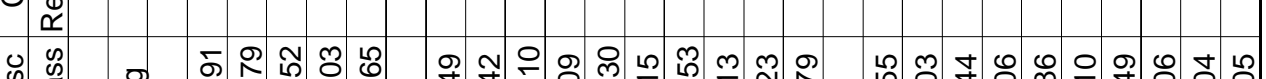

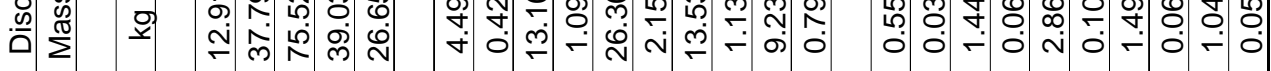

휴에

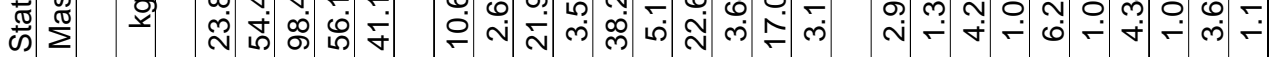

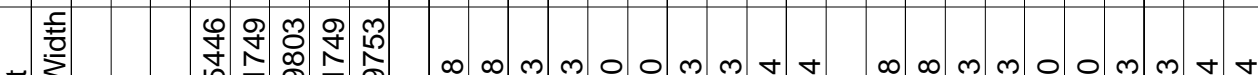

क

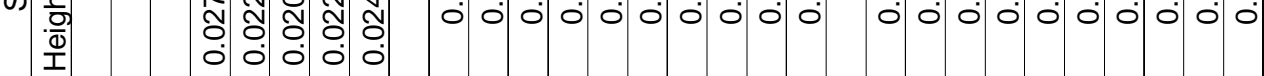

పั

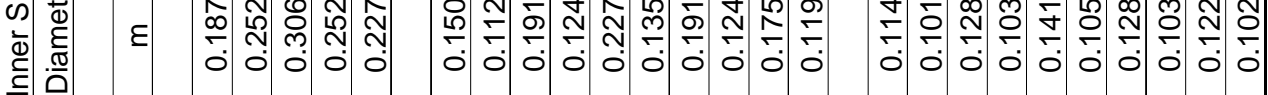

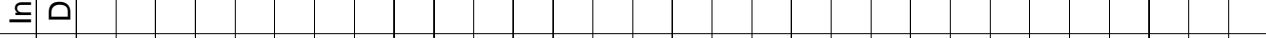

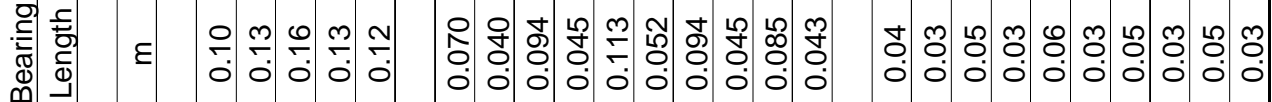

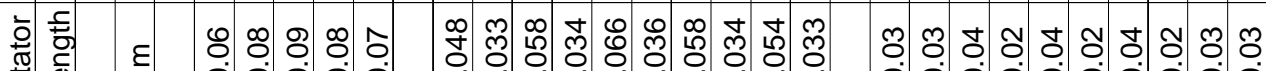

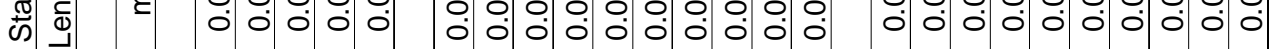

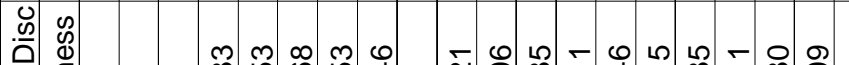

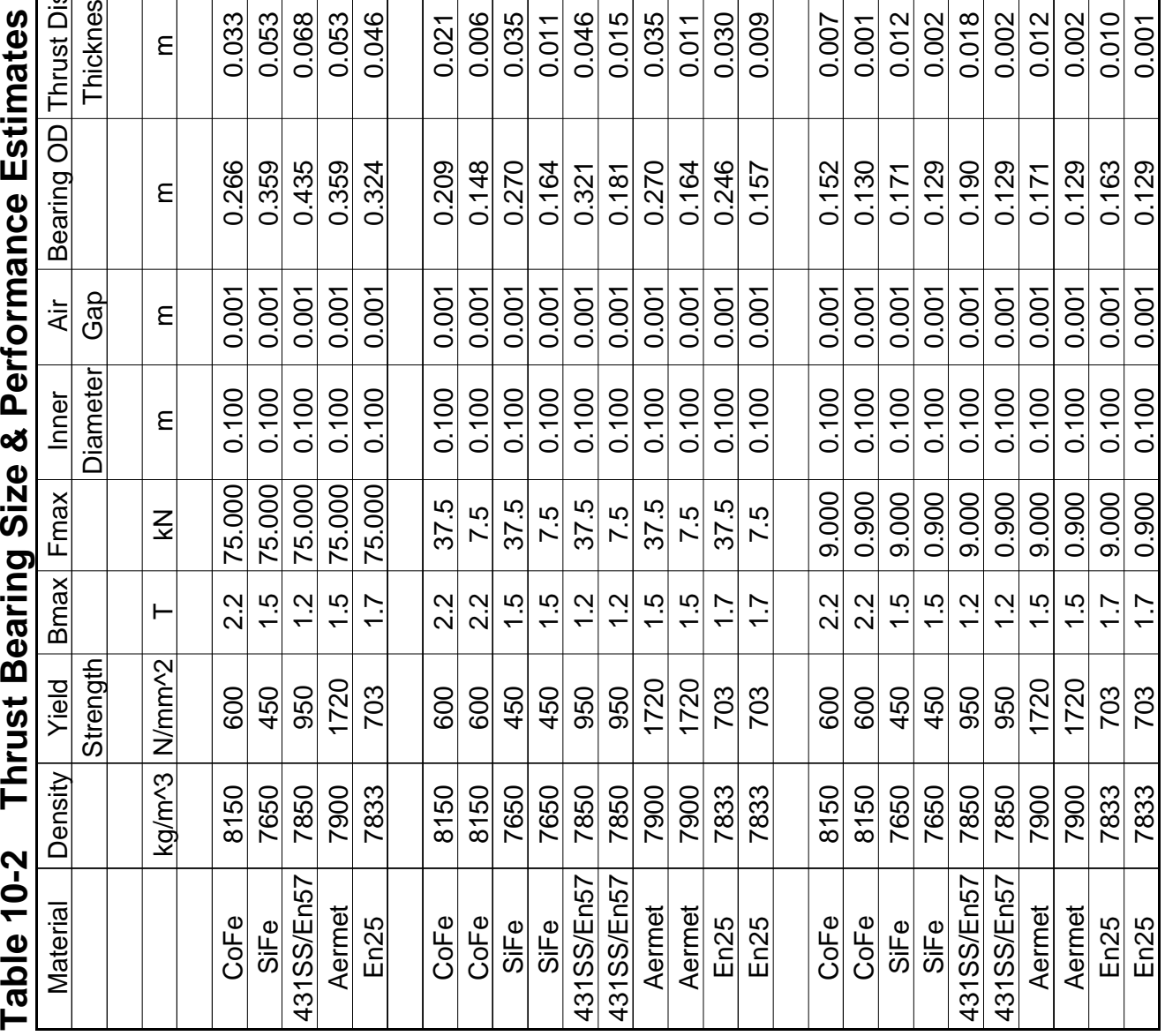




\section{Materials Issues}

Electrical steels have traditionally only been required in thin strip form. Generally, in reducing the losses of a classic rotating machine, the thinner the strip, or lamination, the better. However, in producing a thrust AMB larger thicknesses of material are required, that could only really be met by a bar or forged form of material. Data on such forms of electrical steel is not readily available but would be expected to show reduced strength properties over the already relatively poor strip forms. As an example, a typical martenesitic steel has a $0.2 \%$ Yield Strength of the order of 900 $\mathrm{N} / \mathrm{mm}^{2}$, whereas a typical Silicon iron lamination has a $0.2 \%$ yield Strength of around $350 \mathrm{~N} / \mathrm{mm}^{2}$.

There are stainless steels available in bar forms that offer good strength and reasonable magnetic performance. $431 \mathrm{SS} / \mathrm{En} 57$ has a $0.2 \%$ yield of $920 \mathrm{~N} / \mathrm{mm}^{2}$ and can offer a workable saturation flux density of up to around 1.2 T.

The high temperature magnetic performance of materials is supported by little empirical data. Whilst it is known that the performance generally reduces by a small amount as temperature increases, precise data is rare.

Air inside the gas turbine can be expected to be both water and salt laden depending on the environmental conditions of the operating site. Unless sealed to a high level, electrical machinery embedded within the turbine will have to cope with such corrosive atmospheres.

The vast majority of traditional electrical steels have poor resistance to corrosion. Traditionally, iron based electrical steels will corrode readily within such environments, unless they are surface treated in some way. Most electrical steels are suitable for coating in some way. However, such a coating would be expected to have a low permeability and, therefore, only a thin coating would be acceptable. Adhesion of such coatings will be of importance, considering the speed and diameters of the machines.

The environmental resilience of typical organic winding wire insulation varies depending on the particular insulating material. The vast majority of polyurethane and polyimide insulation commonly used demonstrate very good resistance to humidity, mineral oils and Freon22. Aromatic polyimide currently represents the organic insulation with the highest temperature withstand; with a Heat Index of 220 ${ }^{\circ} \mathrm{C}$ and a heat shock capability up to $250{ }^{\circ} \mathrm{C}$. The ceramic insulation currently suggested for high temperature windings, around $400{ }^{\circ} \mathrm{C}$, will degrade if exposed to moisture. 


\section{Back-Up Bearings}

Revolve Magnetic Bearings Inc., a Canadian AMB subsidiary of SKF, and S2M, a Seiko/SKF owned French AMB company, currently only supply rolling element backup bearings (BUBs). They offer either grease packed or dry-lubricated technologies. However, these companies only really have experience of such bearings in temperatures below $300{ }^{\circ} \mathrm{C}$; they feel they are unsuitable for the notional $400{ }^{\circ} \mathrm{C}$ environment that may be experienced in some installations.

Federal Mogul Magnetic Bearings, a UK AMB company, supply a sintered bush material known as "Deva". However, they felt that the high temperatures and relatively high thrust loads experienced in this application were beyond their AMB system capabilities. As such, they were not forthcoming with any detailed information, leaving the applicability of this BUB technology in question.

Present day back-up bearings are low-duty components, only intended for use if the AMB system fails. Back-up bearings are typically designed to survive a small number of short-duration "run-downs". As such, back-up bearings must be incorporated with replacement in mind. Maintenance and visible inspection is not such an issue as the AMB system can be used to systematically check for wear, especially with bush-type bearings.

\section{Costs}

\section{Initial Costs}

Budgetary costs of AMB systems were sought from existing $A M B$ manufacturers based on an assumed engine production of 200 per year. These requirements were based on an early, assumed typical load and speed assessment for a new engine concept. Whilst these were illustrative of the requirements of the final new engine, some adjustment was made to account for load variations. Only Revolve Magnetic Bearings Inc. responded in the time available for the study and their budgetary quotation was used as the basis for the final costing. As a qualifying check, an outline cost was obtained from S2M that was of a broadly similar order of magnitude. The final costs are assessed against the estimated cost of a traditional oil lubricated bearing system in Table 10-3.

Table 10-3 illustrates the cost savings of AMBs in a single shaft, axial, hot-end drive engine could be around US\$206K. $70 \%$ of this perceived saving is gained through assumed reduction labour requirements due to package simplification. The saving on parts alone is anticipated at around US\$ 62K.

Similar cost savings are anticipated for the single shaft centri-axial, hot-end drive design that has similar bearing requirements. 
Potential More Electric Systems for a 50-100MW Industrial Gas Turbine

Table 10-3

Estimated cost saving from applying Active Magnetic
Bearing technology to an Industrial 25-50 MW Gas Turbine -
Single Shaft Hot End Drive

\begin{tabular}{|c|c|c|c|}
\hline Component (conventional engine) & Equivalent component (electric engine) & Cost (\$) & Saving (\$) \\
\hline Baseplate & $\begin{array}{l}\text { Baseplate (Reduced size following engine } \\
\text { il_system deletion) }\end{array}$ & & 6875 \\
\hline Lube Oil System & $\begin{array}{l}\text { Lube Oil System (Reduction gearbox } \\
\text { retained) }\end{array}$ & & 42300 \\
\hline Miscellaneous & Miscellaneous (Reduced parts count) & & 13000 \\
\hline Labour & $\begin{array}{l}\text { Labour (Reduced parts count reduces } \\
\text { hours) }\end{array}$ & & 64932 \\
\hline Overhead & $\begin{array}{l}\text { Overhead (Reduced parts count reduces } \\
\text { residence time on site) }\end{array}$ & & 79548 \\
\hline Contingency & Contingency (Reduced parts reduces risk) & & 13600 \\
\hline Oil system engine mounted pipework clipping & Harness clipping & & 0 \\
\hline $\begin{array}{l}\text { External casing machining features to suit oil } \\
\text { system (Includes radial drillings) }\end{array}$ & & & 17500 \\
\hline \multirow[t]{2}{*}{$\begin{array}{l}\text { Internal casing machining features to suit oil system } \\
\text { (Includes bearing mount features) }\end{array}$} & $\begin{array}{l}\text { Holes through all bearing support struts to } \\
\text { allow access for harnesses and additionally } \\
\text { through the PT inlet strut to provide coolin } \\
\text { air for the Turbine radial AMB }\end{array}$ & & 0 \\
\hline & Back up bearing (6-off) & & -3000 \\
\hline Compressor radial roller bearing & Radial AMB (1.5 kN) & & -3400 \\
\hline Compressor location ball bearing & Thrust AMB (2 way) (1-off 9kN) & & 1000 \\
\hline Turbine radial bearing spring cage & Not required & & 1000 \\
\hline PT radial bearing squeeze film damper assy & Not required & & 1200 \\
\hline PT radial bearing spring cage & Not required & & 1000 \\
\hline PT rear radial roller bearing & Radial AMB (3 kN) & & -3500 \\
\hline Bearing chamber oil jets ( 5 off) & Not required & & 2500 \\
\hline Compressor location bearing chamber labyrinth (4 & Not required & & 2000 \\
\hline $\begin{array}{l}\text { Turbine radial and PT radial bearing chamber } \\
\text { labyrinth seals ( } 6 \text { off) }\end{array}$ & As for conventional engine & & 0 \\
\hline PT location bearing chamber labyrinth seals (4 off) & As for conventional engine & & 0 \\
\hline Package total & & & 206155 \\
\hline
\end{tabular}

The cold-end drive versions of these engines are essentially twin-shaft engines. As such an additional bearing is required to support the long, free power turbine shaft. This bearing has been sized to cater for a coaxial integrated starter/generator. The savings in utilising AMBs in such an engine arrangement are shown in Table 10-4. 
Potential More Electric Systems for a 50-100MW Industrial Gas Turbine

\section{Table 10-4 Estimated cost saving from applying Active Magnetic Bearing technology to an Industrial 25-50MW Gas Turbine - Two Shaft Cold End Drive}

\begin{tabular}{|c|c|c|}
\hline Component (conventional engine) & Equivalent component (electric engine) & Saving (\$) \\
\hline \multicolumn{3}{|l|}{ Electric main-shaft bearings } \\
\hline Baseplate & $\begin{array}{l}\text { Baseplate (Reduced size following engine oil system } \\
\text { deletion) }\end{array}$ & 6875 \\
\hline Lube Oil System & Lube Oil System (Reduction gearbox retained) & 42300 \\
\hline Miscellaneous & Miscellaneous (Reduced parts count) & 13000 \\
\hline Labour & Labour (Reduced parts count reduces hours) & 64932 \\
\hline Overhead & $\begin{array}{l}\text { Overhead (Reduced parts count reduces residence time } \\
\text { on site) }\end{array}$ & 79548 \\
\hline Contingency & Contingency (Reduced parts reduces risk) & 13600 \\
\hline Off engine oil system pipework & Bearing control system & -8500 \\
\hline Oil system engine mounted pipework & Electric harnesses & -3000 \\
\hline Oil system engine mounted pipework clipping & Harness clipping & 0 \\
\hline $\begin{array}{l}\text { External casing machining features to suit oil } \\
\text { system (lncludes radial drillings) }\end{array}$ & & 17500 \\
\hline \multirow[t]{2}{*}{\begin{tabular}{|l|} 
Internal casing machining features to suit oil system \\
(Includes bearing mount features)
\end{tabular}} & $\begin{array}{l}\text { Holes through all bearing support struts to allow access } \\
\text { for harnesses and additionally through the PT inlet strut } \\
\text { to provide cooling air for the Turbine radial AMB }\end{array}$ & 0 \\
\hline & Back up bearing (6-off) & -3000 \\
\hline Compressor radial roller bearing & Radial AMB $(1.5 \mathrm{kN})$ & -3400 \\
\hline Compressor location ball bearing & Thrust AMB (2 way) (1-off 9kN) & 1000 \\
\hline PT forward radial roller bearing & Radial AMB (3 kN) & -3500 \\
\hline \multirow[t]{2}{*}{ PT location ball bearing (2 off) } & Thrust AMB (2 way) (2-off $35 \mathrm{kN}$ ) & -10800 \\
\hline & $\begin{array}{l}\text { Turbine Radial AMB (Not required on conventional } \\
\text { engine) }\end{array}$ & -5800 \\
\hline Turbine radial bearing squeeze film damper assy & Not required & 1200 \\
\hline Turbine radial bearing spring cage & Not required & 1000 \\
\hline PT radial bearing squeeze film damper assy & Not required & 1200 \\
\hline PT radial bearing spring cage & Not required & 1000 \\
\hline PT rear radial roller bearing & Radial AMB (3 kN) & -3500 \\
\hline Bearing chamber oil jets (5 off) & Not required & 2500 \\
\hline $\begin{array}{l}\text { Compressor location bearing chamber labyrinth (4 } \\
\text { off) }\end{array}$ & Not required & 2000 \\
\hline $\begin{array}{l}\text { Turbine radial and PT radial bearing chamber } \\
\text { labyrinth seals ( } 6 \text { off) }\end{array}$ & As for conventional engine & 0 \\
\hline PT location bearing chamber labyrinth seals ( 4 off) & As for conventional engine & 0 \\
\hline Turbine radial bearing squeeze film damper assy & Not required & 1200 \\
\hline Turbine radial bearing spring cage & Not required & 1000 \\
\hline $\begin{array}{l}\text { PT cold-end drive radial bearing squeeze film } \\
\text { damper assy }\end{array}$ & Not required & 1200 \\
\hline PT cold-end drive radial bearing spring cage & Not required & 1000 \\
\hline PT cold-end drive rear radial roller bearing & Radial AMB (3 kN) & -3500 \\
\hline Bearing chamber oil jets ( 5 off) & Not required & 2500 \\
\hline $\begin{array}{l}\text { Compressor location bearing chamber labyrinth (4 } \\
\text { off) }\end{array}$ & Not required & 2000 \\
\hline $\begin{array}{l}\text { Turbine radial and PT radial bearing chamber } \\
\text { tabyrinth seals }(6 \text { off) }\end{array}$ & As for conventional engine & 0 \\
\hline PT location bearing chamber labyrinth seals ( 4 off) & As for conventional engine & 0 \\
\hline Package total & & 211555 \\
\hline
\end{tabular}


With this configuration the overall saving is anticipated at around US\$ $211 \mathrm{~K}$, with 68 $\%$ of this being due to anticipated labour saving. A saving of US\$68 K is anticipated on parts alone.

\section{Generator Bearings}

Historically, the Brush Electrical Machines BJ45.89 alternator bearings for the RR Engines package have been one of two types:

- Pressure fed sleeve - oil ring backup

- Rolling element - grease lubricated

The driven-end radial load is $17.28 \mathrm{kN}$, with a non-drive-end radial load of $22.67 \mathrm{kN}$. Brush Electrical Machines were unable to give thrust load figures but suggested that they would be very small.

These bearings could be replaced by AMBs and suitable BUBs. With the cold-end drive arrangement the bearings would run in a nominal ambient of $15^{\circ} \mathrm{C}$ with a hotday extreme of $50{ }^{\circ} \mathrm{C}$. The bearings could be fitted in external bearing housing assemblies, facilitating ease of access and maintenance. As such, alternator AMBs are well within the capabilities of existing AMB technology. Size estimates for such bearings are given in Table 10-5. In arriving at these figures it has been assumed that the alternator shaft at the bearing locations has an outside diameter of $160 \mathrm{~mm}$. No attempt has been made to optimize the space envelopes of the bearings as there are no obvious physical restrictions. 


\begin{tabular}{|c|c|c|c|c|c|c|c|c|}
\hline $\begin{array}{l}\bar{\Phi} \\
\vdots \\
0 \\
0\end{array}$ & 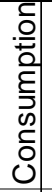 & 3 & $\begin{array}{l}\infty \\
\stackrel{0}{0} \\
\stackrel{n}{-} \\
\stackrel{n}{-}\end{array}$ & 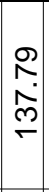 & $\begin{array}{l}\text { Oे } \\
0 \\
0 \\
0\end{array}$ & 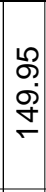 & $\begin{array}{l}\bar{n} \\
\dot{\sigma} \\
\stackrel{5}{-}\end{array}$ & 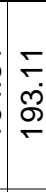 \\
\hline $\begin{array}{l}\frac{1}{\Phi} \\
\text { 잉 } \\
\text { 인 }\end{array}$ & 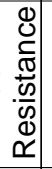 & $\left|\begin{array}{c}0 \\
\mathfrak{2} \\
\multicolumn{1}{c}{} \\
0\end{array}\right|$ & $\begin{array}{l}\infty \\
0 \\
\text { 山े } \\
\stackrel{1}{*}\end{array}$ & $\begin{array}{c}0 \\
0 \\
⿱ 1 \\
\stackrel{1}{\sim} \\
\text { | }\end{array}$ & $\begin{array}{c}\infty \\
0 \\
\stackrel{1}{\sim} \\
\stackrel{\sim}{n}\end{array}$ & 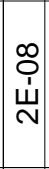 & $\begin{array}{c}\infty \\
0 \\
\text { ய } \\
\sim\end{array}$ & 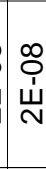 \\
\hline $\begin{array}{l}\frac{\pi}{0} \\
\frac{0}{0}\end{array}$ & $\mid \begin{array}{c}0 \\
\mathfrak{\infty} \\
\stackrel{\infty}{\Sigma} \\
\end{array}$ & 2) & $\begin{array}{l}m \\
\stackrel{m}{\infty} \\
m \\
\stackrel{\infty}{-}\end{array}$ & \begin{tabular}{c}
9 \\
+ \\
$\infty$ \\
0 \\
\hdashline \\
\end{tabular} & $\begin{array}{l}\hat{N} \\
\text { D. } \\
\text { T. }\end{array}$ & $\begin{array}{l}\hat{L} \\
\mathfrak{N} \\
\mathfrak{+} \\
\end{array}$ & $\begin{array}{l}\text { ñ } \\
\text { o̊ } \\
\text { o. }\end{array}$ & $\begin{array}{l}\frac{\sigma}{\tau} \\
\stackrel{+}{\circ}\end{array}$ \\
\hline $\begin{array}{l}0 \\
0 \\
\frac{1}{0} \\
\frac{\pi}{0} \\
0\end{array}$ & & $E$ & $\begin{array}{l}\text { m} \\
\text { p. } \\
0\end{array}$ & 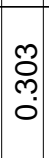 & $\begin{array}{c}\tau \\
\tilde{n} \\
0\end{array}$ & $\begin{array}{l}\text { న్ } \\
\text { N̦ } \\
0\end{array}$ & $\begin{array}{l}\mathscr{2} \\
\text { N̦} \\
0\end{array}$ & $\begin{array}{l}\text { న్ } \\
0\end{array}$ \\
\hline 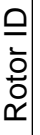 & & $\varepsilon$ & $\frac{\tau}{\Lambda}$ & $\frac{\pi}{\tilde{N}}$ & $\begin{array}{l}\frac{1}{N} \\
\frac{0}{0}\end{array}$ & 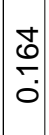 & $\begin{array}{l}\tilde{E} \\
0 \\
0\end{array}$ & $\frac{\mathscr{m}}{0}$ \\
\hline $\begin{array}{l}0 \\
0 \\
\grave{0} \\
\stackrel{0}{0} \\
\check{\Upsilon}\end{array}$ & & $\varepsilon$ & Nָ & $\begin{array}{c}m \\
\\
0\end{array}$ & $\begin{array}{c}\infty \\
\\
0 \\
0\end{array}$ & $\begin{array}{l}\text { ț } \\
\text { ஸ̦ } \\
0\end{array}$ & $\begin{array}{l}\text { ț } \\
\text { N̦ } \\
0\end{array}$ & 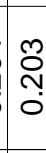 \\
\hline 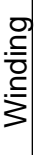 & 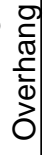 & $\varepsilon$ & $\begin{array}{l}0 \\
\text { D్ } \\
0 \\
0\end{array}$ & $\begin{array}{c}0 \\
\text { p. } \\
0 \\
0\end{array}$ & $\begin{array}{l}0 \\
\text { Oे } \\
0 \\
0\end{array}$ & $\mid \begin{array}{l}0 \\
\text { + } \\
0 \\
0\end{array}$ & $\begin{array}{l}0 \\
\text { பे } \\
0\end{array}$ & 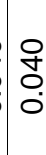 \\
\hline $\begin{array}{l}\text { 돔 } \\
\text { 임 } \\
\text { d. } \\
\end{array}$ & & $\varepsilon$ & $\begin{array}{l}0 \\
1 \\
\text { m. } \\
0\end{array}$ & 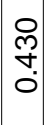 & $\mid \begin{array}{l}0 \\
\text { లె } \\
0\end{array}$ & $\begin{array}{l}0 \\
\infty \\
0 \\
0 \\
0\end{array}$ & $\begin{array}{l}0 \\
\text { N̦} \\
0\end{array}$ & 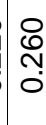 \\
\hline 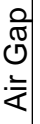 & & $\varepsilon$ & \begin{tabular}{|l|}
1 \\
0 \\
0 \\
0 \\
0
\end{tabular} & $\mid \begin{array}{l}\mid \\
\text { டி } \\
8 \\
0 \\
0\end{array}$ & $\mid \begin{array}{l}\mid \\
\\
8 \\
0 \\
0\end{array}$ & $\begin{array}{l}1 \\
\\
\\
\\
0 \\
0\end{array}$ & $\begin{array}{l}0 \\
0 \\
0 \\
0 \\
0\end{array}$ & $\begin{array}{l}0 \\
0 \\
0 \\
0 \\
0\end{array}$ \\
\hline 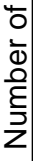 & $\begin{array}{l}\mathscr{\infty} \\
\frac{\mathscr{d}}{0} \\
0 \\
\end{array}$ & & $\infty$ & $\infty$ & $\infty$ & $\infty$ & $\infty$ & $\infty$ \\
\hline $\begin{array}{l}\stackrel{\times}{\widetilde{\Xi}} \\
\stackrel{E}{ \pm}\end{array}$ & & $\underline{z}$ & $\stackrel{\infty}{\sim}$ & 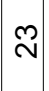 & $\stackrel{\infty}{-\infty}$ & N & $\stackrel{\infty}{-1}$ & $\stackrel{\sim}{N}$ \\
\hline 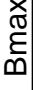 & & $\vdash$ & $\stackrel{n}{-}$ & ?ִ & $\stackrel{\text {. }}{-}$ & $\stackrel{\text { }}{\sim}$ & $\begin{array}{l}\stackrel{N}{N} \\
\sim\end{array}$ & $\underset{\sim}{\sim}$ \\
\hline$\stackrel{\frac{0}{0}}{\frac{1}{2}}$ & 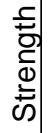 & $\left|\begin{array}{l}\mathcal{N} \\
\xi \\
\xi \\
\Sigma \\
Z\end{array}\right|$ & ○ी & 号 & $\begin{array}{l}0 \\
\text { N̦ }\end{array}$ & $\begin{array}{l}0 \\
\text { ஸे }\end{array}$ & 8 & 8 \\
\hline 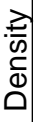 & & $\mid \begin{array}{l}m \\
2 \\
\xi \\
\delta \\
\underline{x}\end{array}$ & \begin{tabular}{|l}
0 \\
0 \\
0 \\
1
\end{tabular} & \begin{tabular}{|c}
0 \\
10 \\
0 \\
1
\end{tabular} & $\begin{array}{l}m \\
\mathscr{N} \\
\infty\end{array}$ & $\begin{array}{c}n \\
\tilde{N} \\
\infty \\
N\end{array}$ & $\frac{0}{\infty}$ & $\frac{\circ}{\infty}$ \\
\hline$\stackrel{\overrightarrow{\widetilde{\sigma}}}{\Sigma}$ & & & $\stackrel{ \pm}{\stackrel{亡}{\omega}}$ & $\frac{0}{\omega}$ & 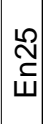 & $\begin{array}{c}\stackrel{\sim}{N} \\
\stackrel{w}{w}\end{array}$ & $\begin{array}{l}0 \\
\stackrel{1}{0} \\
0\end{array}$ & ப. \\
\hline
\end{tabular}




\section{Through Life Costs Benefit - No Oil Consumption}

Currently the RR Engines consumes approximately 0.33 gallons/ fired day, at a cost of around US\$ 64 ( $£ 40) /$ gallon. This is a running cost of approx.:

\section{US $\$ 21.12 /$ day $=$ US $\$ 0.88 / \mathrm{hr}=$ US $\$ 7708.8 /$ year}

It is assumed that the 8000 hour (Class B) maintenance can be reduced by around $16 \%$. This currently costs around US\$13760 per visit. Therefore, there is a potential saving of:

\section{US $\$ 13760 \times 0.16=$ US $\$ 2201.6 / 8000 \mathrm{hrs}=$ US $\$ 0.28 / \mathrm{hr}$.}

Hence around US $\$ 2410.7$ / year can be saved on maintenance.

Therefore, a total saving of US $\$ 10,119.5$ / year can be made by fitting AMBs, which is equivalent to US\$1.16 / fired hour.

\section{Comment On State Of Art \& Development Requirements}

The front end bearing chambers are assumed to be at a temperature of around 50 ${ }^{\circ} \mathrm{C}$. Stage 4 air is the simplest choice for cooling of the high temperature bearing areas of the engine if traditional labyrinth seals are to be used. Use of earlier stage air requires increasing numbers of labyrinth seals and increased air system complexity. An ISA standard ambient temperature is $15^{\circ} \mathrm{C}$. Each compressor stage approximately incurs an additional $50{ }^{\circ} \mathrm{C}$. Therefore, using Stage 4 air requires an ISA standard nominal requirement of around $215{ }^{\circ} \mathrm{C}$. However, during extremes of environmental conditions, this air may be expected to rise to a temperature close to $250{ }^{\circ} \mathrm{C}$. With an estimated increase of $30{ }^{\circ} \mathrm{C}$ due to ducting effects, the AMBs would be required to withstand area temperatures of around $280-300{ }^{\circ} \mathrm{C}$. The use of Stage 3 compressor air would reduce this upper limit to $250{ }^{\circ} \mathrm{C}$, but would require additional sealing, with an increased seal package axial length. Knowledge on the performance of magnetic circuit materials at such temperatures is limited as the majority of electrical machinery operates at temperatures below $180{ }^{\circ} \mathrm{C}$. Therefore, some reliability and lifetime testing of AMBs at representative temperatures would be necessary.

Carbon ring seals, as produced by manufacturers such as "Sealol", offer sealing to a pressure differential of at least 10 bar in a space not dissimilar to a traditional labyrinth seal. Use of such seals enables the use of Stage 1 air at a nominal temperature of around $65^{\circ} \mathrm{C}$ and a maximum operating temperature of $115^{\circ} \mathrm{C}$. This is within the capability of traditional Class $\mathrm{H}$ and the more recent " $\mathrm{H}+$ " insulation.

Heat-soak may become an issue after emergency shut-down where it may be inappropriate to continue to rotate the engine. In this case, there is no longer any cooling air available from the compressor and the temperature will become

\begin{tabular}{|l|l|l|c|}
\hline Document number & Issue & Page 53 of 58 \\
\hline
\end{tabular}


increasingly uniform across the engine. This results in an initial fall in temperature of the turbine areas and a general rise in the temperature of the cooler areas. Without redress the bearing area temperatures will begin to rise to a level potentially around $450{ }^{\circ} \mathrm{C}$. The main mechanism of "heat-soak" into the bearing areas is conduction along the shaft and into the bearing rotors, followed by radiation into the bearing chamber. Conduction into the bearing stators could be slowed by gradually playing cooling water or air onto the shaft before the bearing locations. This could be actuated by an emergency trip. Failure modes, which preclude engine rotation, must be considered in assessing the need for such measures. This work was not carried out as part of this study. 


\section{POWER ELECTRONICS INTERFACE AND / OR DIRECT DRIVE ALTERNATOR}

\section{OPPORTUNITY: UTILISE A POWER ELECTRONIC INTERFACE BETWEEN THE GT DRIVEN ALTERNATOR AND THE GRID}

Why?

\begin{tabular}{|l|l|l|}
\hline Cost ? & $1^{\text {st }}$ Cost & $\begin{array}{l}\text { Potential for using a smaller } \\
\text { alternator } \\
\text { Potential for FPT deletion }\end{array}$ \\
\hline
\end{tabular}

\begin{tabular}{|c|c|c|}
\hline Performance & Part load efficiency & - Mass flow control possible \\
\hline Functionality & Package stability & - Isolation from Grid transients \\
\hline
\end{tabular}

\section{Findings}

\begin{tabular}{|c|c|}
\hline $\begin{array}{l}\text { Applicable to existing RR } \\
\text { Engines? }\end{array}$ & Yes \\
\hline Recommended? & $\begin{array}{l}\text { No } \\
\text { - Power Electronics costs are prohibitively high } \\
\text { - Power Electronics efficiency eliminates GT } \\
\text { efficiency gain } \\
\text { - Concern over waveform quality in Grid } \\
\text { applications }\end{array}$ \\
\hline
\end{tabular}

Figure 11-1 Power Electronic Interface Arrangement

High Speed Alternator

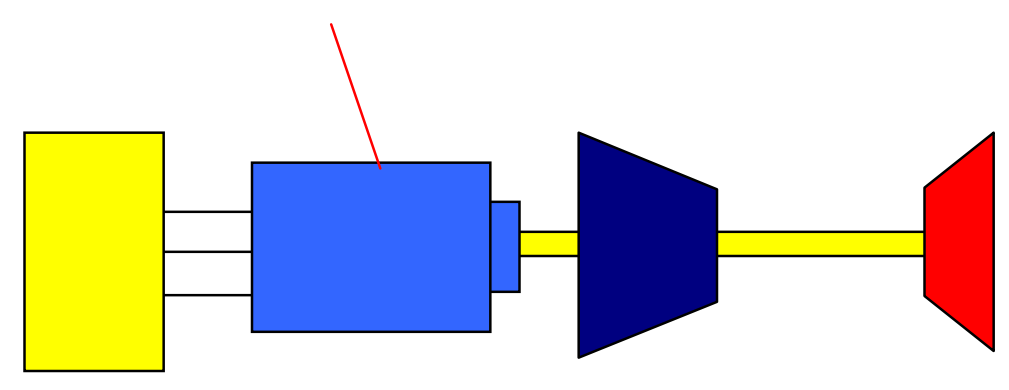




\section{The case against using a Power Electronics Interface/High Speed Generator}

The main motivation for considering a power electronics interface at the output of a Gas Turbine driven alternator is:

- The possibility of mass flow control through the gas turbine with subsequent simplification of the combustion system and the potential to improve part load efficiency

In the light of the apparent benefits the potential for extensive application of the technology would appear very promising. However in the above case the technology is attempting to displace or improve on a mature technical solution.

This study suggests that though technically feasible the technology cannot compete with the existing arrangement for the following reasons:

\section{Cost}

Despite being a relatively new technology the elements of a power electronics inverter (IGBTs, IC Control Boards, Contactors) are already built in a reasonable volume. An analysis of the economics suggests no clear indication of any reduction in the specific cost of a power electronics converter with size. This is due to a number of factors including:

- The large number of components

- The relatively small size of the market at this size offsetting other economies of scale,

\section{Power Output Quality}

In the situation at which the free power turbine is not rotating at synch speed, power conditioning is needed to align such generation with typical utility frequencies of 50 or $60 \mathrm{~Hz}$. Frequency conversion power electronics will inevitably create noise due to the necessary switching action. The use of such equipment raises concerns over compliance with G.5/3, the Electricity Council recommendation on "Limits For Harmonics In The United Kingdom Electricity Supply System", and other similar worldwide harmonic generation regulations.

G.5/3 only specifies harmonic limits for installations up to $1.5 \mathrm{MVA}$ for 12-pulse controlled converters at $11 \mathrm{kV}$ or ; the acceptable rating being lower for 6-pulse equipment. As such, the use of a high-speed alternator would require a detailed examination of existing harmonic current and voltage conditions and the conditions resulting from the new load, in line with Stage 3 of G.5/3. Such an investigation would need to consider: 
- The type and rating of the proposed plant,

- The rating and point of connection of any power factor correction or filtering equipment,

- The pulse number of the converter equipment, and

- Details of any phase shifting transformer usage as well as harmonic current production for the whole installation.

- The duty cycle of the plant, including particular times of day and duration of maximum harmonic production along with details of any short duration harmonics-

Assessment of the actual generated harmonics is impossible without knowledge of the supply conditions at the point of common coupling.

\section{Reliability / Availability}

The large numbers of components will do nothing to aid the machines relative reliability. In addition the failure mechanism of a complex and physically large power electronic device is not always obvious and so repair times could suffer. 

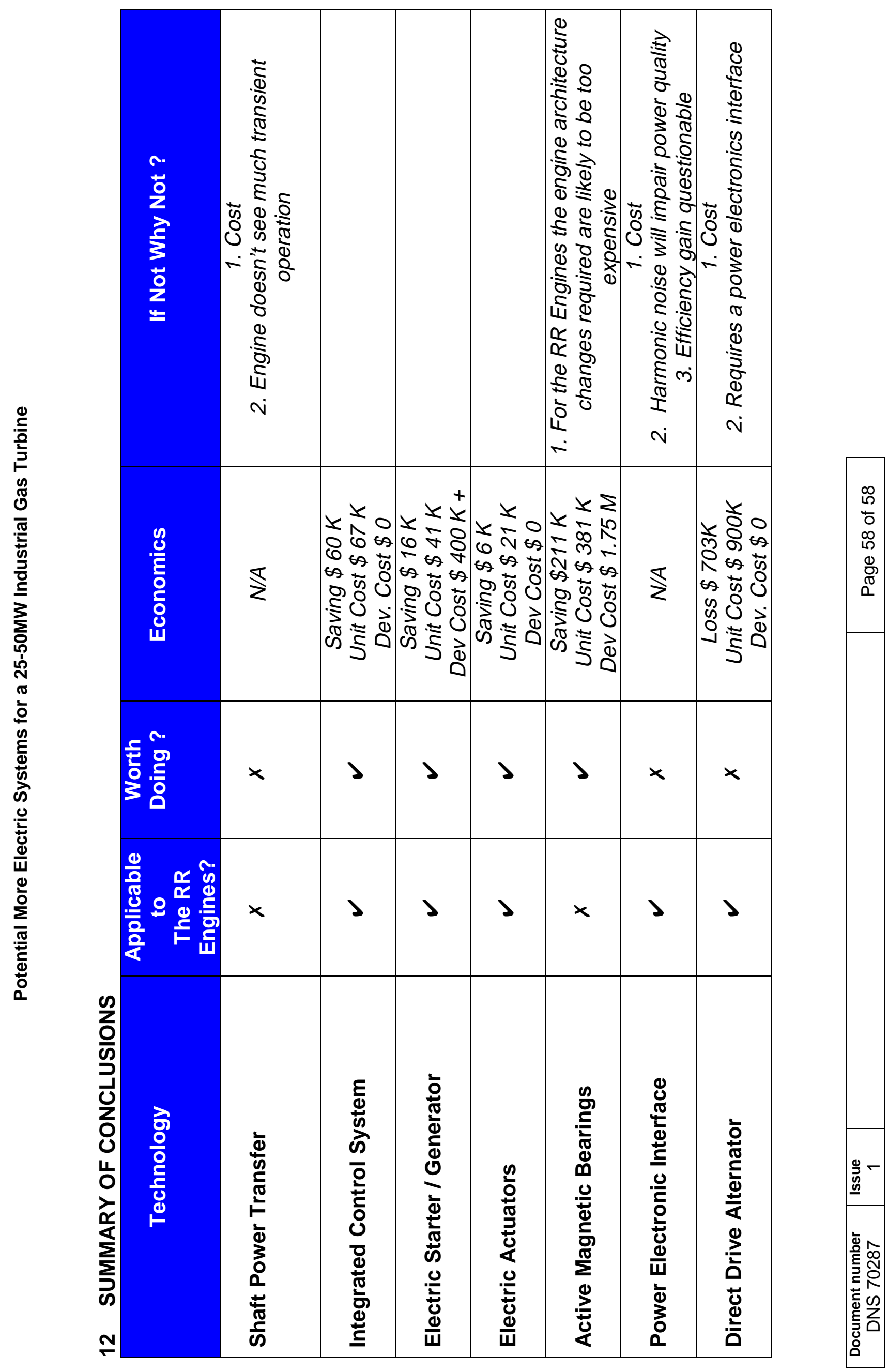


\section{Appendix I NGT Supporting Technologies: Spiral Wrap Recuperator Study}




\section{Final Report}

\section{Spiral Recuperator Manufacturing Study}

November 2001

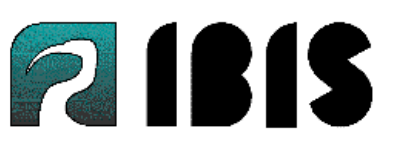

IBIS Associates, Inc.

1601 Trapelo Road, Suite 164

Wellesley MA 02451 USA

voice: $781-290-0400$

fax: 781-290-0454

email: IBIS@IBISassociates.com web: http://IBISassociates.com 


\section{Overview}

Rolls Royce contracted IBIS Associates, Inc. for assistance to develop and assess alternative manufacturing strategies for spiral recuperators. This assessment involved data collection, process cost simulation, and scenario modeling and analysis.

The first step in the program was to review and evaluate the spiral wrap recuperator core design. The starting point was the basic design and configuration information available in the Powergen 2000 paper authored by Oswald, Dawson, and Clawly of Rolls Royce plc, Ansty, UK. Further discussions and reviews of the functionality, design, and manufacturing concepts were carried out with Mr. Jim Oswald and with Mr. Dan Edwards of Rolls Royce, Indianapolis. With this concept as a starting place, IBIS outlined process flow diagrams for baseline and alternative manufacturing strategies. A list of production technologies and equipment requirements was then defined. Existing parts manufacturers, equipment suppliers, and other sources of knowledge relevant to the involved processes were identified and interviewed to collect process information needed for the analysis. A cost model structure capable of addressing all process alternatives was constructed and populated with the collected data. The recuperator core designs, as modified through review with manufacturing experts and the Rolls Royce design team were then run through the manufacturing cost model to assess production economics for baseline and alternative design and market scenarios.

The desired outcomes of this effort have been:

- An understanding of basic direct manufacturing cost structure of the core

- An evaluation of design and process cost reduction strategies

- An identification of potential manufacturing partners and technology sources 


\section{Product Description}

\section{Basic Concept}

The spiral wrap recuperator is a heat exchanger produced from rolling a three layer sandwich into a cylinder (see Figure 1). The sandwich consists of a flat bottom plate layer, a corrugated fin strip middle, and a dimpled top plate layer. As the cylinder is wound, the top plate of one wrap is seam welded to the bottom plate of the next outermost wrap, maintaining a separate hot zone (gas side) and cold zone (air side). The dimples in the top layer of the sandwich maintain a separation between the wraps, creating this gas side zone.

Before wrapping, holes are cut through all three layers of the sandwich and the top plate layer is welded to the bottom plate. The spacing of these holes is such that when the sandwich is wrapped, the holes line up in a radial pattern, connecting the air side cold zone layers to the central core. The welded top plate and bottom plate layers at each hole maintain separation between the zones.

In operation then, hot gas flows axially through the wrapped recuperator core, while cooler air flows in the core center, then radially out through the air passage holes, then along the air side zone, exiting through the air passage on the other side back to the core center (see Figure 2).

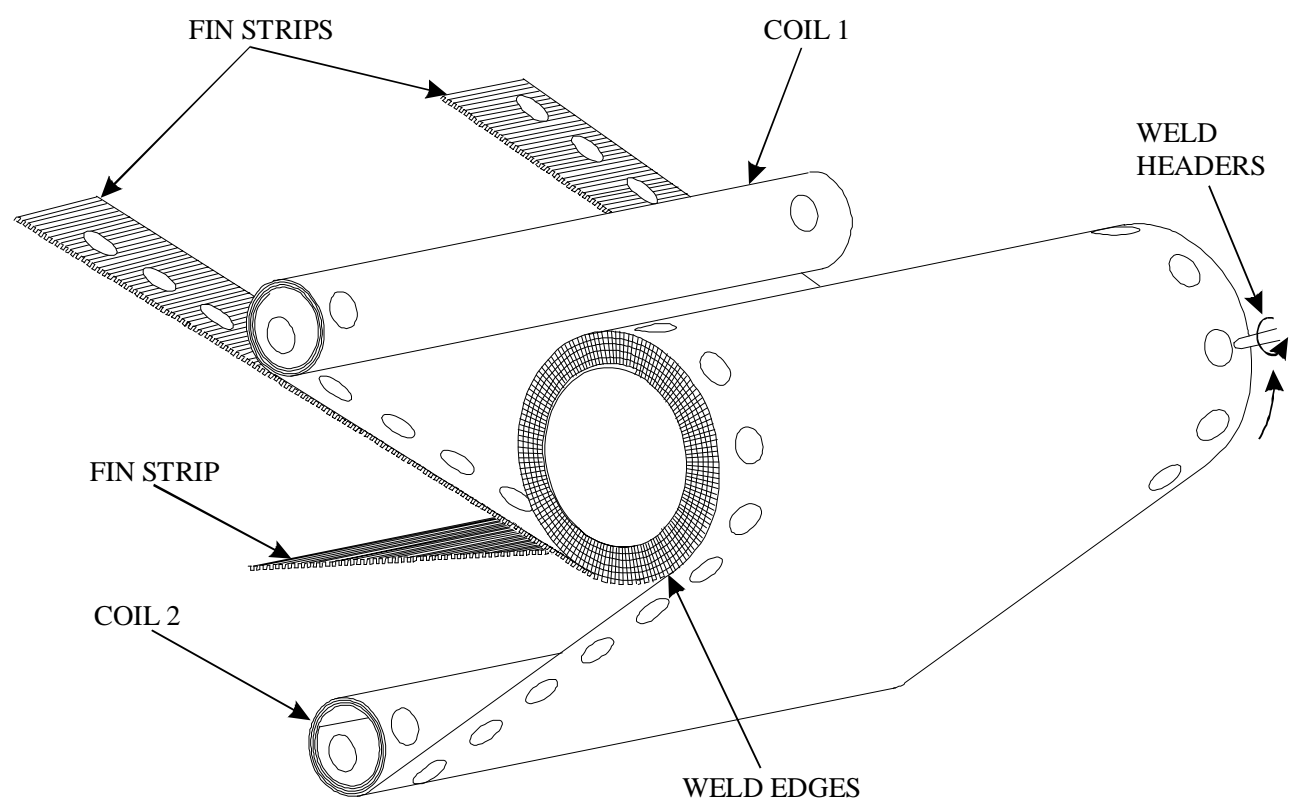

Figure 1 


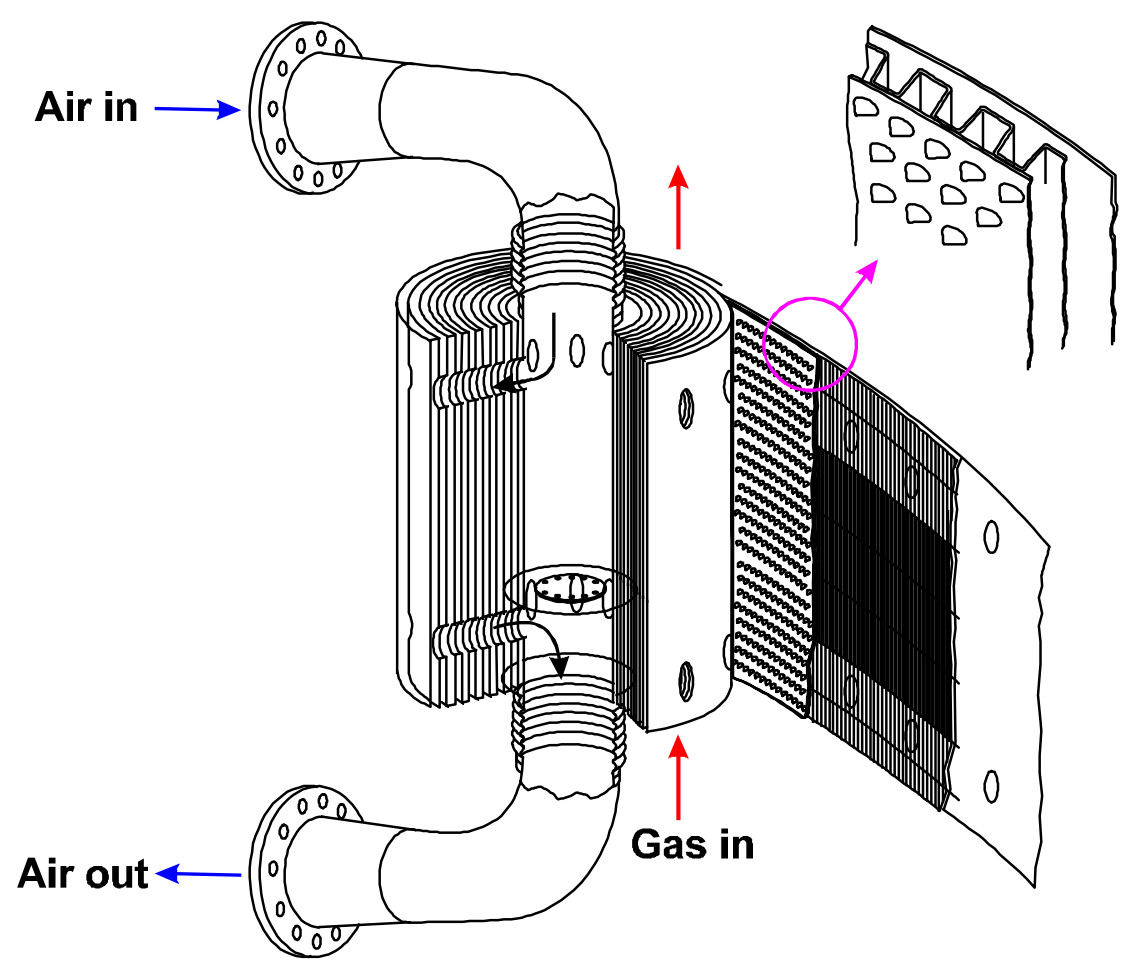

Figure 2

The two areas upon which the manufacturing analyses are most focused are the "header hole" (air passages between layers) design and fin design alternatives (ways of managing gas flow around header holes and through the recuperator).

\section{Header Holes}

The header holes provide a means of allowing air to flow from one layer to another through the gas side containing fins (see Figure 3). A "well" is stamped in the upper plate, which also contains dimples to maintain the spacing of the air side layer. "Gas turning" fins are employed on the gas side to flow exhaust around the well with minimal pressure drop, but also to keep the gas side layer from collapsing under the air side pressure (Dan Edward's analyses suggest a minimum fin spacing of approximately 0.25 "). The top plate well must be aligned correctly with the gas turning fin and brought together with the flat bottom plate. Holes can be cut through each of these three layers before they are brought together, but they must align perfectly as a welded air-tight seal is necessary (see Figure 4). It is thought that tolerances can be better maintained by stamping, laser cutting, or plasma arc cutting the holes through all three layers at once. If the subsequent welding through three layers presents any problems, the hole in the gas turning fin layer could be cut earlier, at a slightly larger diameter, since it is the weld between top layer and bottom that is critical. 


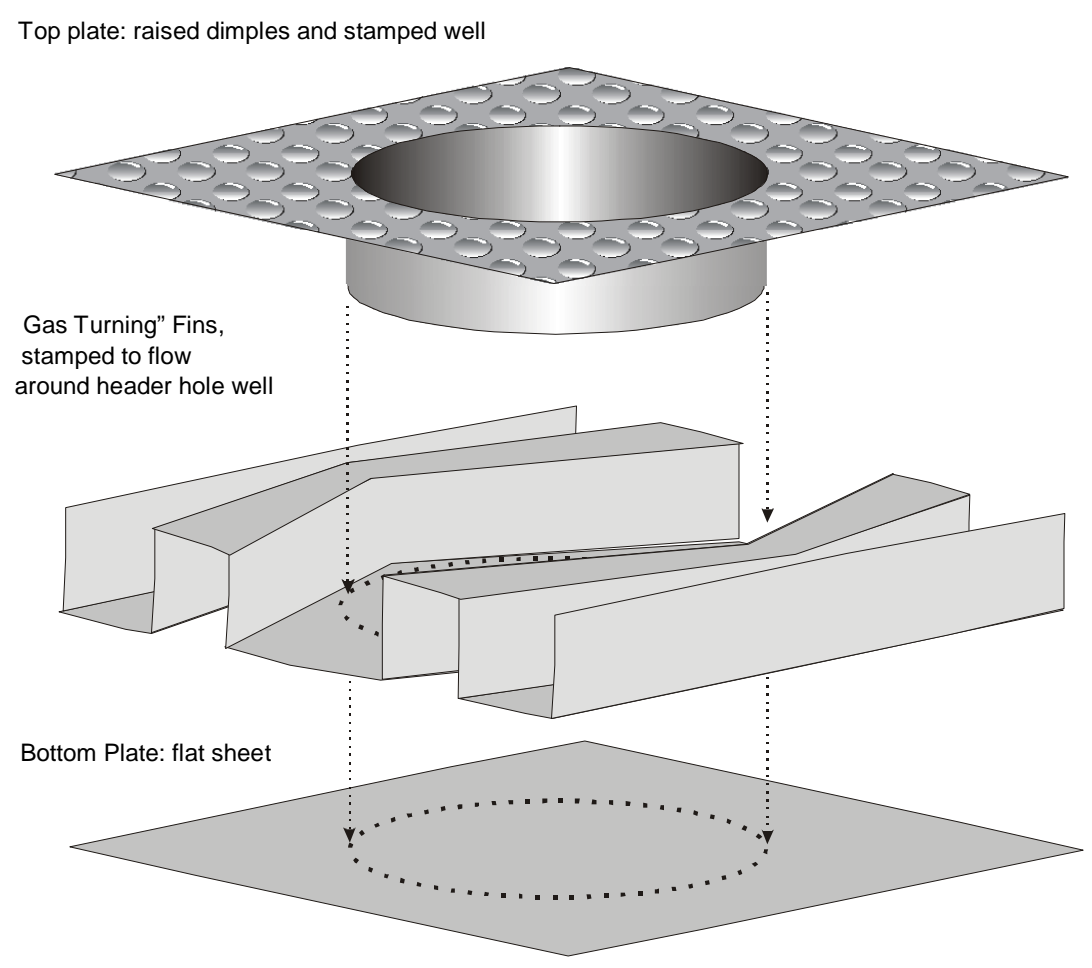

Figure 3

Top Plate, Fins, Bottom Plate joined, showing hole punched through sandwich and welded landing

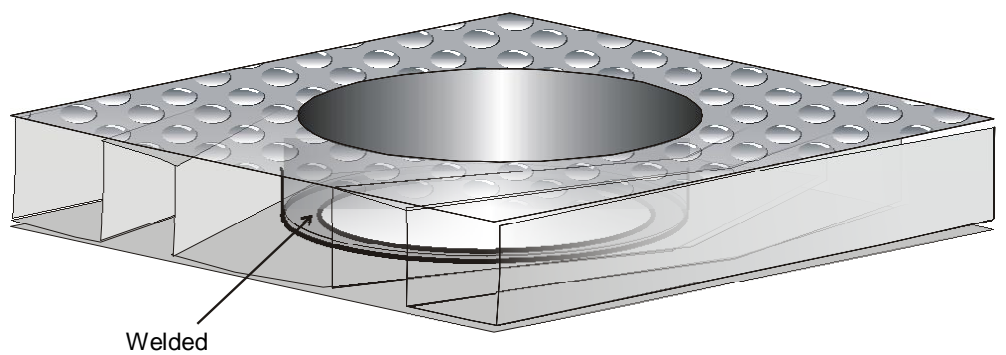

Figure 4

\section{Fin Strategies}

The ASME paper indicated a structure with a three separate fin strips; one central "main" fin strip, and two "gas turning" fin strips on the sides. Few details of these "gas turning" fins have been seen by IBIS, but it is understood that these contain non-linear channels to allow the exhaust to flow around the header holes without being blocked. This type of fin structure presents a problem of non-constant strip width as well as the fact that the spacing of the holes and gas turning fins is variable.

Three fin strategies have been developed to address alternative ways of producing the fin layer. The first involves three fin strips; a center main body strip of straight regular fins, 
and two side fin strips assembled from stamped "gas turning" sections alternating with regular fin material of graded lengths according to the as-rolled header spacing. The second involves these stamped gas turning sections bonded into a full width main body fin strip. The third would involve elimination of gas turning fins through tapered header holes and specially cut fin stock.

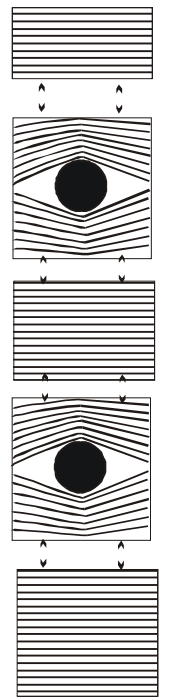

1.)

Assembled Fin Strip

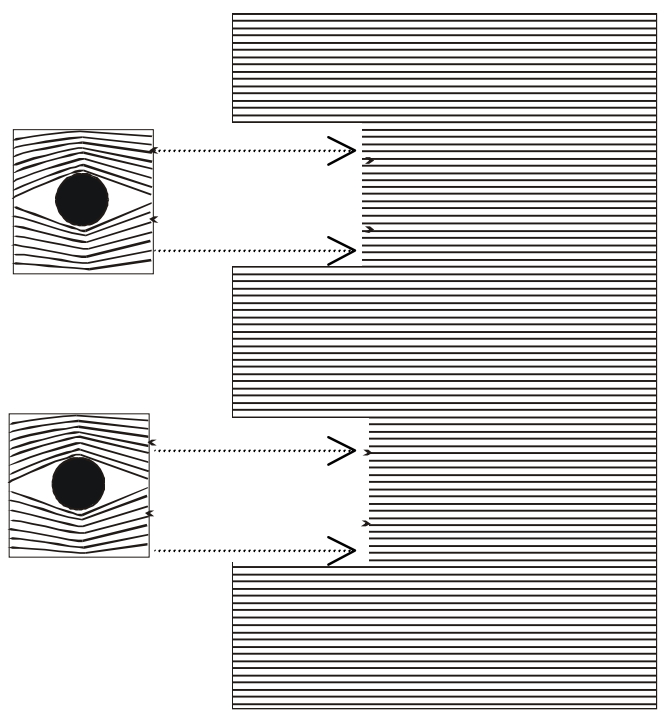

2.)

Full Width

Strip

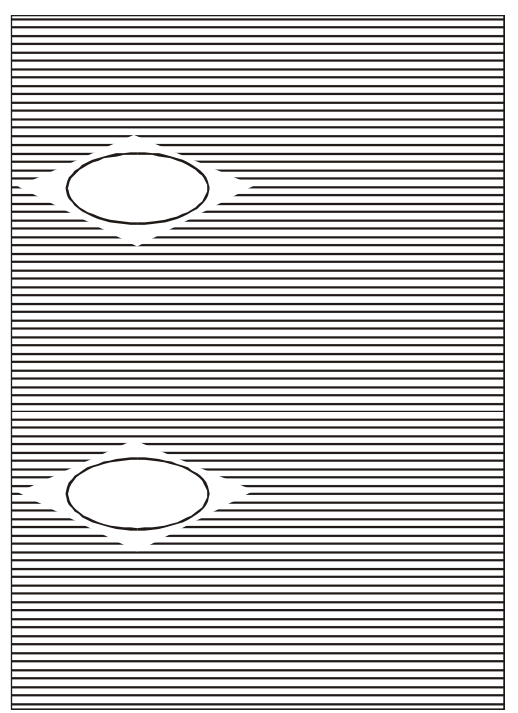

3.)

Tapered

Header holes

Figure 5

The following scenarios have been examined with the cost model to explore trade-offs of capital investment, labor intensity, and scrap losses.

\section{"Gas Turning” Fins}

To produce this "gas turning" fin strip, a complex two station, variably-indexing stamping line could be built, that would alternately stamp regular "straight" fins and then the curving "gas turning" fins at the appropriate spacing. As mentioned though, the cross sectional width of the "straight fins" will be less than that of "gas turning" fins, necessitating a wider coil, cutting of the fins, and scrap losses. 


\section{$\underline{\text { Assembled Fin Strip }}$}

An alternative that will be less capital intensive, but require more labor, would be to stamp the entire strip through conventional fin stamping, stamp the "gas turning" sections from discrete blanks, trimming them square and to width, and weld-splicing them into the fin strip at the appropriate positions.

\section{One Full Width Fin Strip}

If the trouble is being taken to weld these stamped "gas turning" fin sections into the strip, perhaps a single full-width fin strip could be used, stamping out rectangular sections on each side, where the stamped "gas turning" sections will be welded in. This would then result in a single fin strip to be employed in the rolling operation.

\section{Tapered Header Holes w/o "gas turning" fins}

Another option would require a more radical change in design, and in the course of this study has been evaluated by Rolls Royce in terms of gas flow, fin stress, and pressure drop, would allow for the elimination of "gas turning" fins and the complexity they bring to the process. It is currently assumed that the top plate of the sandwich contains both dimples and "wells" that bring the top plate in contact with the flat bottom plate and form the channels for the header holes. The "gas turning" fins are then needed to flow the gas around these circular wells. If the wells were formed with an aerodynamic profile shape, and a gap is left between the fins and the header well, the need for the complex gas turning fin structure could be avoided. This design is feasible, but incurs a greater pressure drop and loss of efficiency. In order to meet the same performance, a larger core is required. The economic trade-off between increased size and simplified manufacturing is discussed in the analysis section. 


\section{Manufacturing Processes}

The general layout of the manufacturing process conceived for the recuperator core is shown in Figure 6. The top plate is received in coil form with sheet thickness from 0.175 to $0.22 \mathrm{~mm}$ depending on the recuperator size. A full description of recuperator size dependent design parameters is compiled in Appendix B. Fin stock material is also received in coil form at $0.0762 \mathrm{~mm}$ thickness. The gas turning fin stampings are made from thicker material, the same as used for the top plate. After dimples, wells, and fins are stamped, the side fin strips are assembled and welded. All three material streams, along with a flat bottom plate sheet are degreased and fed into a continuous, automated, in-line system that cuts and welds the header holes, tests for weld integrity, and rolls and seam welds the core. The relative spacing between the header holes is critical for alignment in the as-wrapped recuperator. Therefore, the entire line between hole cutting and rolling/seam welding is to be controlled in a lockstep fashion. A low volume setup might involve a controlled indexing of the feed according to the hole spacings while a high volume process may justify a continuos line speed with computer controlled movement of stamping press and/or cutting/welding heads along a constantly moving strip material.
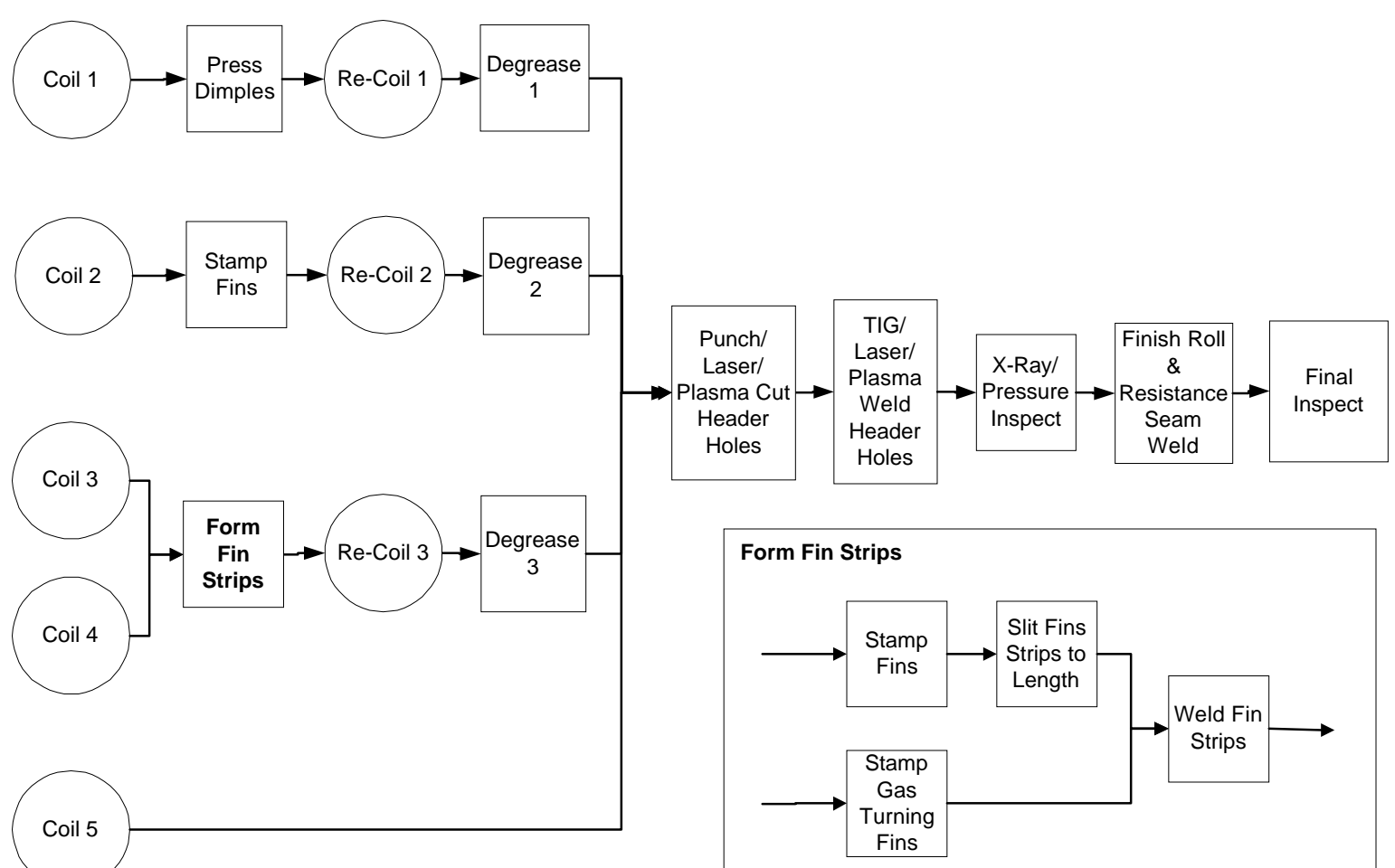

Figure 6 


\section{"Dimple" Features}

The creation of the air side dimple features on the ten mil thick passageway separating plates is a fairly straightforward metal forming application that may be done in one of two ways; either with a standard hydraulic press or with a system utilizing magnetism as the forming force.

\section{Hydraulic metal forming of 'dimples'}

The standard approach for the production of this type of feature is to use a hydraulic press to force the metal to take the form of a die. A single straight side stamping press will cost approximately $\$ 200 \mathrm{~K}$ per machine, not including auxiliary material handling equipment and tooling expenses. These presses can operate at a variety of speeds (defined by strokes per minute) and often are equipped to change speeds (variable speed stamping presses) depending on the product being run at that particular time. For simple dimensions such as the dimples the stroke speed could be very high (150 to 200 strokes per minute) but it should be a function of the tool size (amount of material formed per stroke) and the speed of the downstream collar forming press. It is estimated that 200 inches of material per minute would be close to the desired speed for this line and that it could be achieved with a 2" tool at 100 strokes per minute or a 4" tool at 50 strokes per minute.

The tooling for these features should be quite standard and cost an estimated $\$ 50 \mathrm{~K}$ per die. The tooling life for these dies will be enormous, primarily because of the simplicity of the features but also because of the thin nature of the material (0.010"). It is hard to say exactly how long one these tools will last before they need to be repaired or replaced, but a similar die that supplied for use in the production of automotive heat exchangers has lasted more than a year while being run two shifts per day and 200 strokes per minute.

\section{Magnetic metal forming of 'dimples'}

The use of magnetism to force a piece of metal into the form of a die is relatively novel approach developed by a company, Lourdes Systems, Inc., to improve on incumbent metal forming techniques. Lourdes' press technology uses an electromagnetic device, which when a pulse of energy is applied to its solenoid forces an armature out and drives the press mechanism. This technology has many advantages along with some disadvantages, that all seem to point to this application being a good fit.

The main disadvantage of this technology is its line speed. The maximum production rate for one of these systems is between 60 and 100 strokes per minute, while a mechanical press may operate as fast as 1200 strokes per minute. This has limited its use in high production volume industries such as automotive. Although the machines throughput is relatively low as compared to other press machine types, its stroke velocity is very high. This high stroke speed minimizes material deformation, lengthens tool life and produces features with much higher tolerances (reduces the radius produced in corner features and reduces material springback). 
Typical presses produced by Lourdes are manufactured for materials with a maximum coil width of 15.5 inches. A custom machine that could handle coils up to 36 inches in width would cost approximately $\$ 35-40 \mathrm{~K}$ and would include the press, table and controls. The speed of this machine for the application that I described would probably operate at a speed of about 30 strokes per minute.

The tooling component for this system is the same as the dies used in any other metal forming press and typically costs approximately $\$ 50,000$. The life cycle for a die that would be used in this operation is quite high, at around $1 \mathrm{M}$ strokes before requiring repair.

\section{Fin Production}

The creation of the gas side finning is a similar to the production of finstock for use in automotive heat exchanger applications. There are very few companies in North America that can claim to have more expertise in this manufacturing process than Robinson Fin, but other equipment manufacturers and similar metal forming production techniques may also be used to reveal specific manufacturing considerations. The sum of this information is presented below as a recommendation for production methods to manufacture this component of the Rolls Royce Spiral Wrap Recuperator.

\section{Finstock formation and material handling techniques}

The material thickness and fin feature sizes are consistent with plate recuperator finstock designs. As such, the expertise necessary to overcome issues pertaining to material deformation characteristics (i.e., material springback) and how they relate to overall dimensional tolerances is well developed. Equipment and tooling that needed to form the finstock material would therefore be 'off the shelf' if the fins were to be singulated into plates rather than wound in coil form.

Typical metal forming presses that are used to manufacture heat exchanger and recuperator finstock cost approximately a quarter of a million dollars apiece, while tooling may approach as much as $\$ 75,000$ per die. Due to the relatively thin nature of the material and based on data from finstock manufacturers using similar materials, the tooling should last a long time (approximately one million strokes).

In addition to these standard pieces of equipment, material handling machines (feeders and unwinders) will also be required. The feeding of the material appears to be a simple operation similar to conventional finning techniques. The winding of the finstock, however; will most likely take bit more process development. Although the material's gauge appears to be sufficient to prevent catastrophic feature deformation from the pressures that it will experience in coil form, the tension that is used to coil the material could be damaging to the fins. In order to avoid flattening the finstock the coil winder will require a servomotor that operates in tandem to the machine itself. Each material motor driven handling device may cost as much as $\$ 50,000$. 


\section{Header Holes}

After the three sheets: upper, lower, and fin stock are sandwiched together, the inlet and outlet header holes are perforated and welded. IBIS evaluated several options for this manufacturing sequence. Multitudes of companies were contacted to explore the feasibility of in-line variant hole punching. None of the contacts that IBIS spoke with was able to reference existing applications of this technology or suggest capable vendors. The alternative is CNC controlled operation that would be reconfigured with differently sized parts. Three scenarios were modeled for this sequence: standard punching of the holes and TIG welding, plasma cutting and welding, and laser cutting and welding.

Feedback on the preliminary analysis included questions about the ability of the various welding processes to weld three layers of steel at once. Further interviews with the equipment suppliers indicate that material thickness will not be a limiting factor for laser or plasma welding.

\section{Hole punching and TIG welding}

Hole formation was modeled as a standard pressing operation. As the inlet and outlet holes are horizontally offset, two stations will be needed. The stations designed for this operation will most likely operate at a rate of 1800 strokes / hour (30 strokes per minute), with a capital investment of $\$ 40,000$ each. Tooling is relatively simple, estimated at $\$ 30,000$, and expected to last over a year, or about 1,190 parts. The thickness of the stack and nature of the operation are not expected to place much strain on the die.

Once the holes are punched, they will be welded to ensure the integrity of the stack. Two welding options were proposed: TIG and plasma welding. TIG welding has several advantages over plasma welding: concentration of energy is greater, arc stability is improved particularly at low current levels, heat content is higher, the plasma has higher velocity, there is less sensitivity to variations in arc length, tungsten contamination is eliminated. A standard TIG welder would cost $\$ 70,000$, and operate at a rate of 7 inches per minute. A robotic welder could be used instead of the manual set-up, enabling increased design flexibility. This increases investment to $\$ 115,000$ and rates stay within the range of 7-15 inches per minute. There is no tooling associated with this operation and the only consumables are the tungsten tips and electrodes.

\section{Plasma cutting and welding}

Plasma cutting is not limited by thickness, although as with plasma welding, edge acuity is a concern. The expected kerf loss on a plasma cutter is $<1 / 2 \mathrm{~mm}$ for a $90-130 \mathrm{~mm}$ hole. These stations can be programmed in-line with the plasma welders (rates between 60-90 inches per minute depending on the system chosen). Equipment investment on the system 
is the same as a plasma welder, $\$ 30,000$ - $\$ 40,000$ for a standard system or 2-3 times that for the Arckinetics design.

Plasma welding holds several advantages over TIG welding. A standard welder cost between $\$ 30,000$ and $\$ 40,000$, a substantial reduction to the TIG systems. They have a longer orifice body life, and reduced rate of inert gas consumption. In addition, plasma systems do not require the same degree of welding knowledge, potentially reducing labor costs. Rates for a plasma welder are in the range of 60 inches per minute. A more advanced plasma welding technology is available, arckinetics. Arckinetics equipment costs 2-3 times more that a standard plasma welder, but rates improve to 70-90 inches per minute. Edge acuity may be an issue for plasma welders as the weld bead is typically 3-4 times the metal thickness.

\section{Laser cutting and welding}

Laser cutting offer a higher direct wattage than plasma cutting, but at a significant premium. The cost of a laser system can be broken down into component price estimates, such as $\$ 75-150 \mathrm{~K}$ for the bare laser. Periphery systems, such as inspection, cover gas and controls are approximately double this or approximately $\$ 300 \mathrm{~K}$. For a welding and cutting system, two heads are recommended for an additional $\$ 75-150 \mathrm{~K}$ per additional heads beyond one. Using these estimates, the total price for a dual head laser system is about $\$ 500-650 \mathrm{~K}$. The production rate for both processes is about 100 inches per minute, on a $500 \mathrm{~W}$ system.

There is some concern with laser cutting that the thickness of the material would increase equipment cost. As the thickness of the material that is to be cut increases, the distance over which the focal length of the laser changes also must change. This distance, along with laser power are directly related to equipment cost. In addition, while dimensional tolerance is much finer than a plasma cutter, with a witness of $0.004 "-0.005$ ", edge acuity may be a concern. As the laser penetrates the layers of materials while making the cut, the angle at which it is cutting changes. As it penetrates the bottom layer, the top layer may be damaged by the high power of the laser beam far away from the focal point.

\section{Seam Weld and Coil}

The final operation in recuperator manufacturing is to wind the coil and seam weld the layers. These operations would be performed in-line, and the end-winder would be rate dependent on the seam welder.

The layers are joined with a resistance weld. The estimated requirement for 0.010 " stainless steel is 5000 amps and $400 \mathrm{lbs}$. of electric force. Recommended coverage is 18 spots per inch, thereby producing a continuous seam. There are several forms of resistance seam welders. Newcor Bay City has a patented low inertia weld system that can weld at speeds of 60-70 feet per minute. These systems cost about $\$ 100 \mathrm{~K}$. Conventional AC resistance welders are in the range of $\$ 30-35 \mathrm{~K}$. These systems weld up to 50 inches per minute. A direct current system is recommended over an AC system. 
They are slightly more expensive, $\$ 40-45 \mathrm{~K}$, but can weld up to 100 inches per minute. All resistance welders have steep learning curves and rates are operator dependent. One source claimed that rates could double throughout the lifetime of the equipment.

Two seam welders would be used to join the opposite sides of the coil. A third seam welder may be used for the final horizontal seam. The overhead for the resistance seam welding operation is about $\$ 400 \mathrm{~K}$, with a final budget of $\$ 500 \mathrm{~K}$. This investment includes the end winder, a planishing station to even out the seams, and all necessary automation. The type of end winder required for this operation is a DC - powered roller with an expanding mandrel or clamshell design. These adjustments are necessary to alleviate tension from the fin stock, and maintain the spiral recuperator geometry. 


\section{Analyses}

A Technical Cost Model was developed that examines each operation in the proposed manufacturing flow. Data collected defining each operation, as well as alternative process technologies, was input into this model and economic simulations of production scenarios were analyzed.

\section{Baseline Analysis}

The baseline scenario examined in the following cost model results are for the largest size recuperator, an $1800 \mathrm{~kW}$ power rating, produced at high annual volumes of 1000 units per year. Although this volume is highly optimistic for a single recuperator size in the near term, it represents a production volume at which capital equipment and tooling are fully amortized and capacity is fully utilized and therefor a cost level that would be achieved under optimum conditions. For the baseline, the "assembled fin strip" design is assumed where header holes are mechanically punched and the top plate and bottom plate are TIG welded together. The impact of utilizing laser and plasma welding is discussed later.

The cost model results presented in Figures 7 and 8 show break down the manufacturing costs by element. This breakdown is overwhelming dominated by material cost, representing $77 \%$ of the total direct costs. A single $1800 \mathrm{~kW}$ core consists of several thousand lbs of stainless steel at $\$ 2.27$ to $\$ 3.86$ per $\mathrm{lb}$.

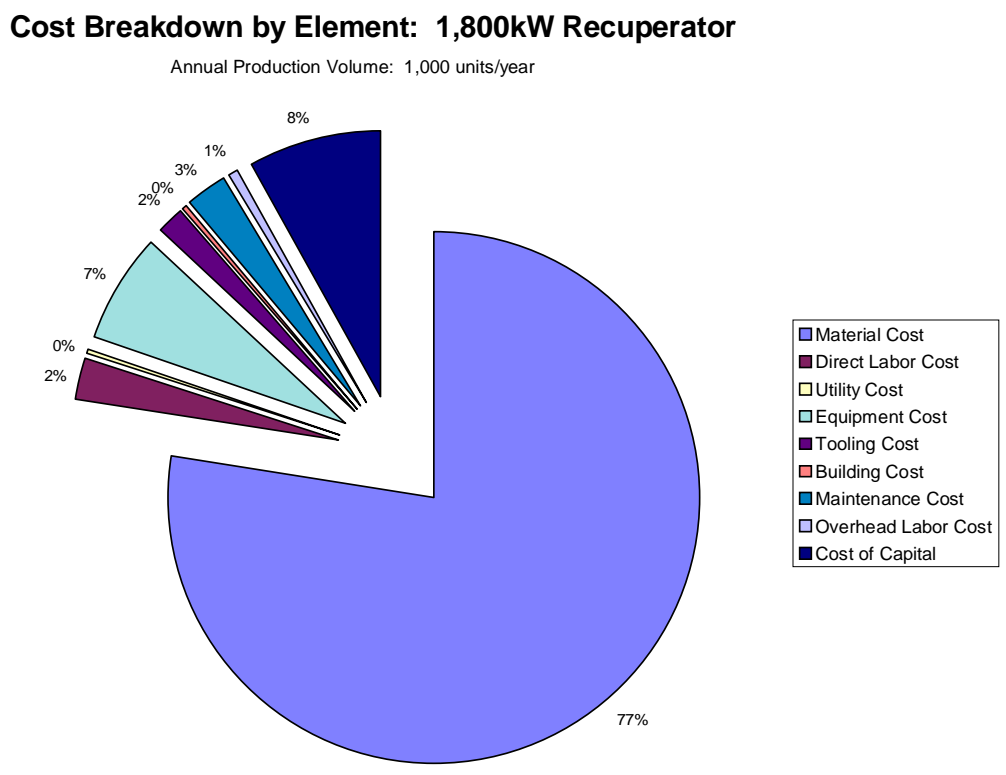

Figure 7 


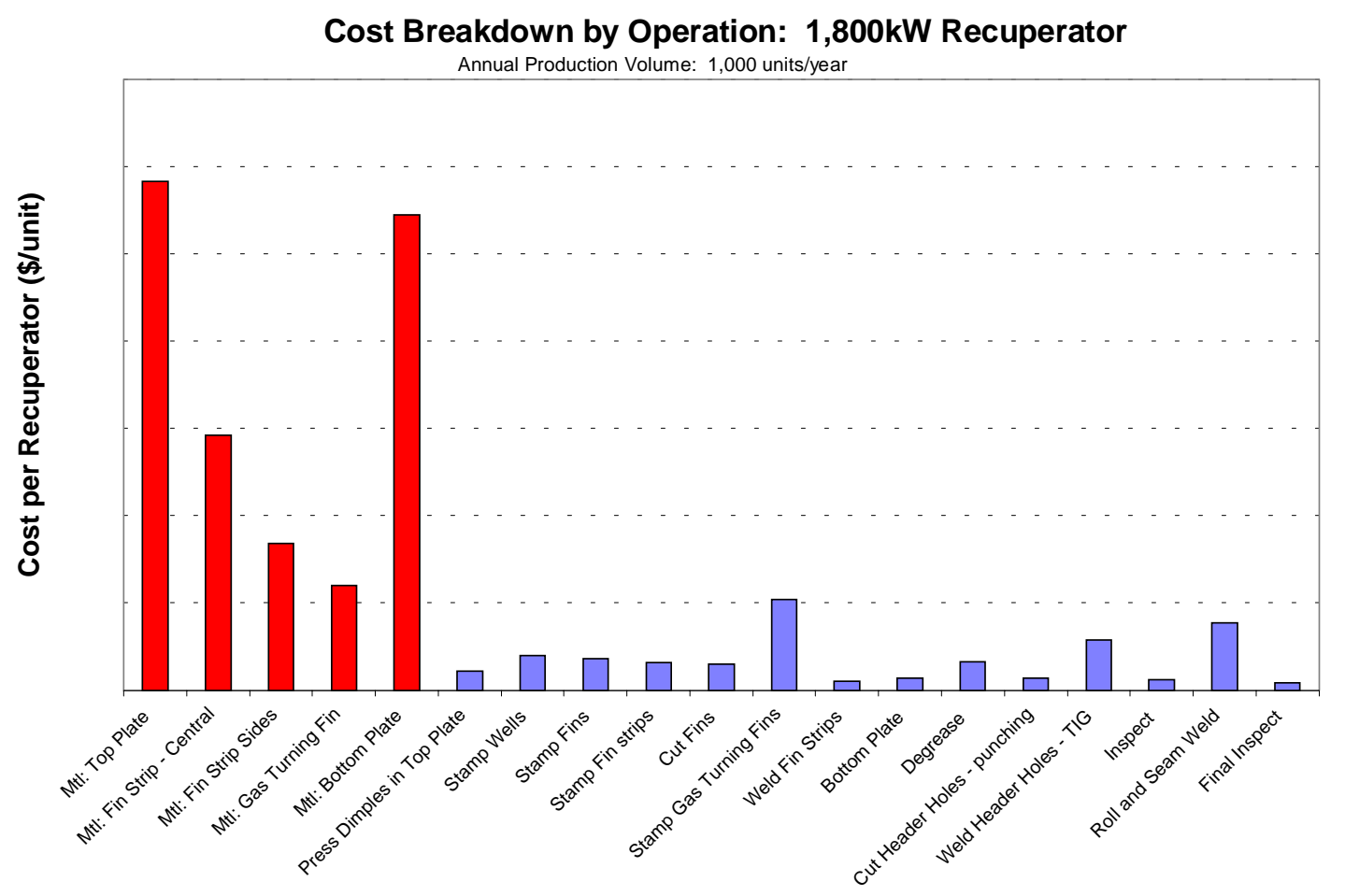

Figure 8

\section{Scenario Analysis}

The cost model was then used to understand the impact of annual production volume on part cost. The result of varying the production volume from 100 to 5000 units per year is shown in sensitivity curve shown in Figure 9. Also shown is the facility investment required to meet increasing production volume. The curves show that economies of scale are achieved at slightly over 1000 units per year. 


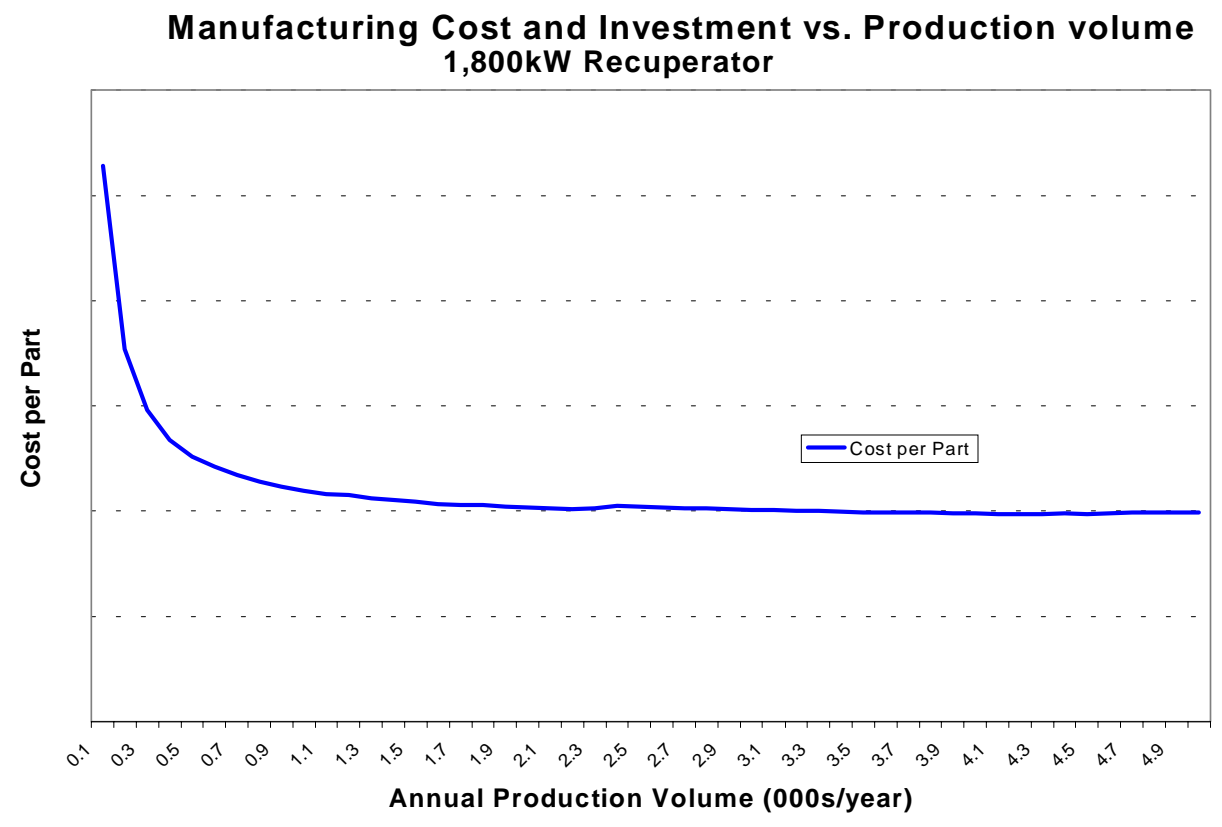

Figure 9

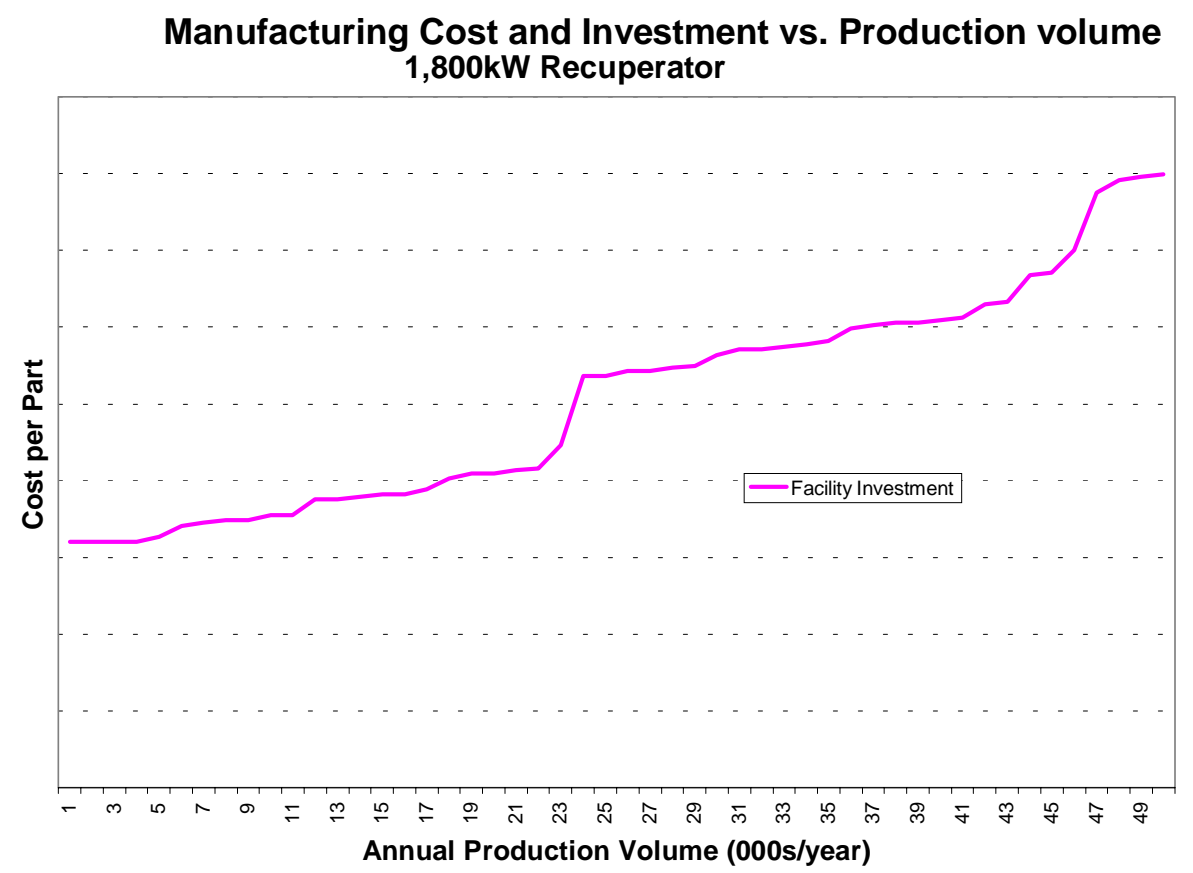

Figure 10 
A matrix of design parameters and calculations for all the potential recuperator sizes is shown in Appendix B. These parameters were used to generate the cost versus size comparison shown in Figure 11. Dividing these cost results by power rating yields the cost per kilowatt for each size shown in Figure 12. It should be noted that a production volume of 1000 units per year is constant for all sizes in this analysis.

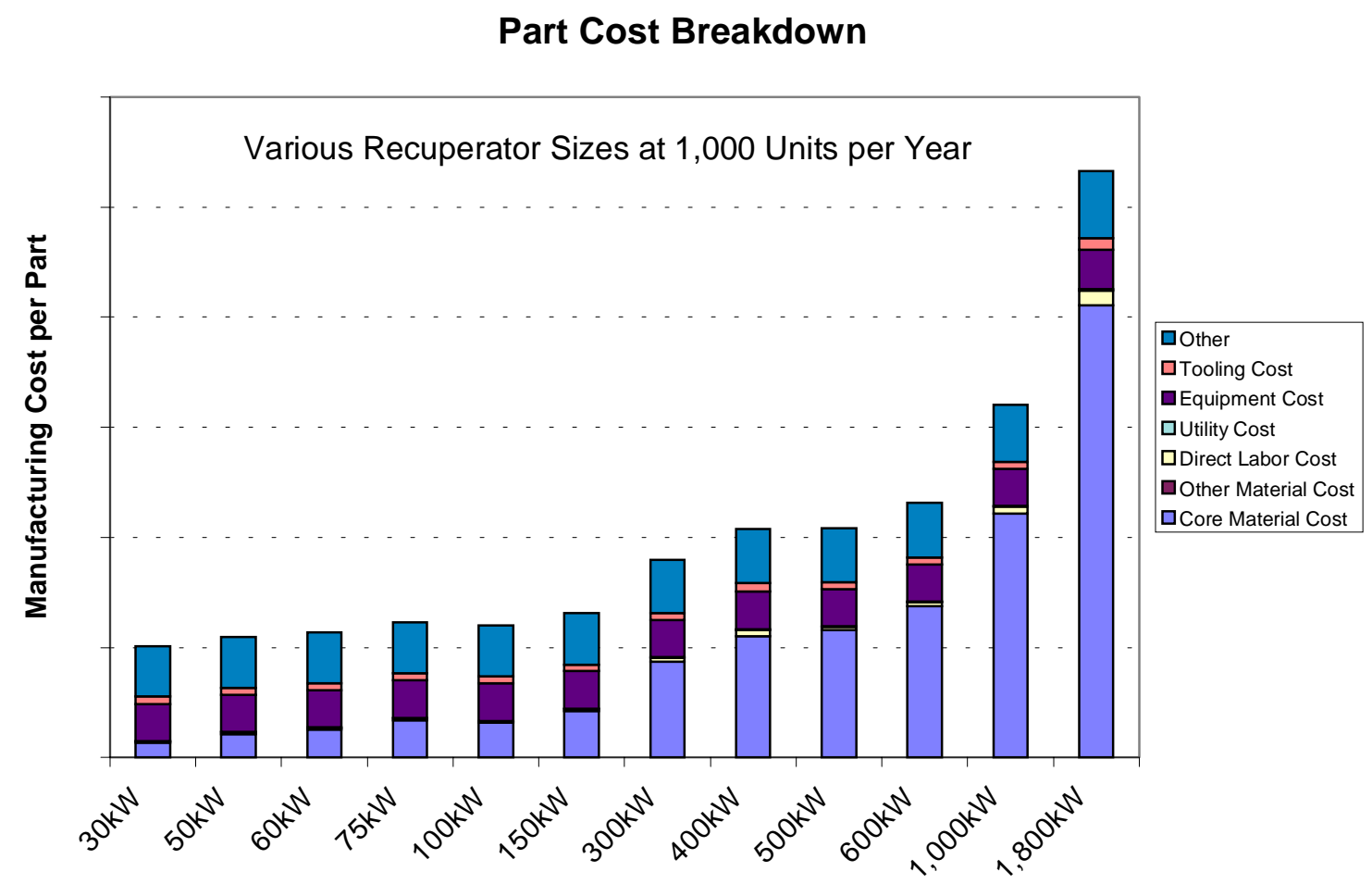

Figure 11 


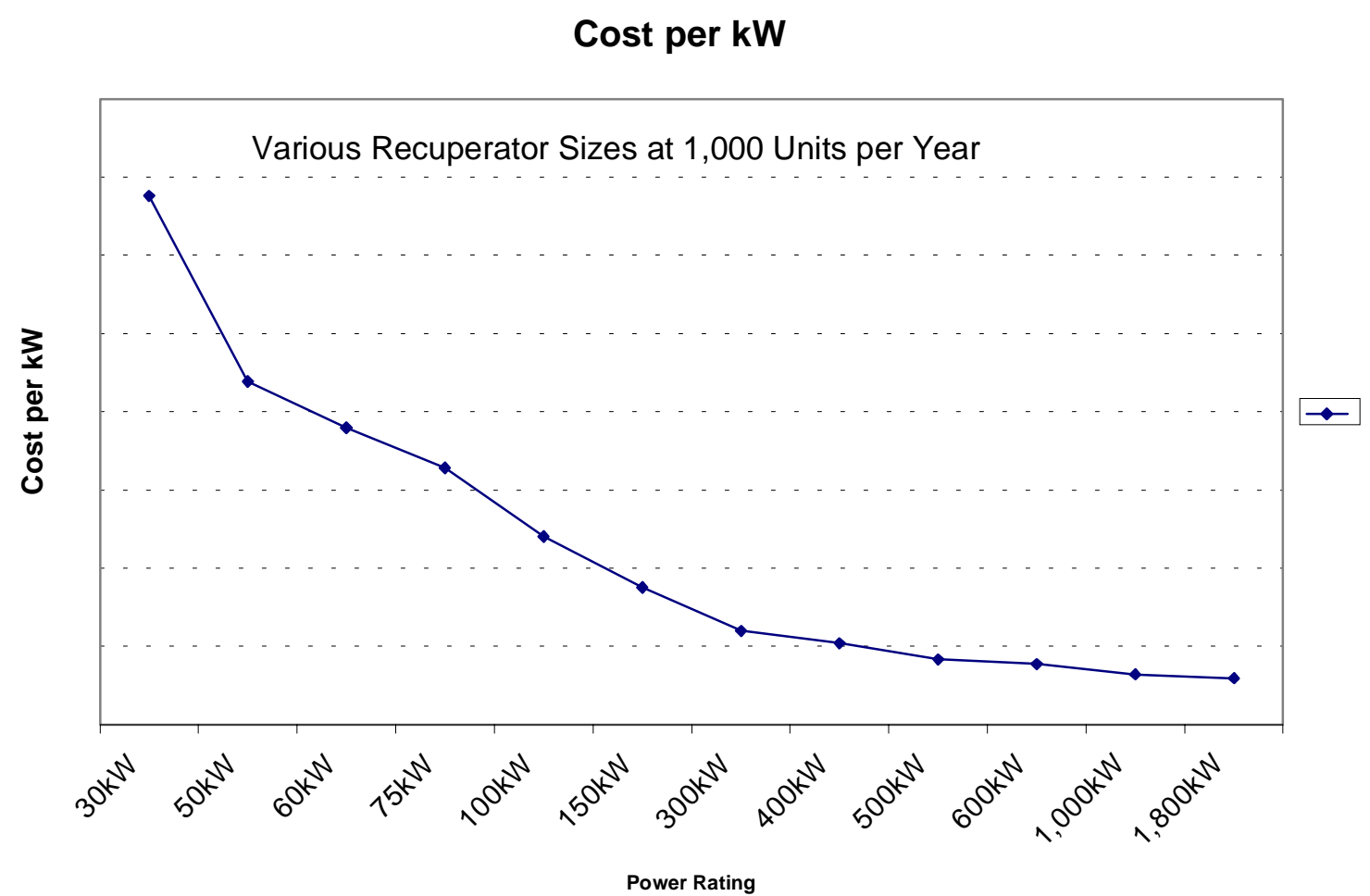

Figure 12

\section{Process Technology Alternatives}

For cutting the header holes, there were three technology options: stamping, laser cutting, and plasma cutting. For welding the top and bottom plates at these holes, there were also three options: TIG, laser, and plasma. Each technology has different rates, capital costs, and per piece costs. The baseline analysis presented above assumed stamping and TIG welding, so the model was used to understand the impact of the alternatives.

\section{Header Hole Cutting}

\begin{tabular}{|r|r|r|}
\hline Cutting Process & $\$ /$ part & investment \\
\hline Stamping & $\$ 70.38$ & $\$ 261,470$ \\
Laser & $\$ 244.69$ & $\$ 988,305$ \\
Plasma & $\$ 24.37$ & $\$ 111,470$ \\
\hline
\end{tabular}

Stamping has the lowest up front equipment cost, but when the cost of tooling over the entire product life is taken into account the total investment is much greater. Laser cutting is fast and accurate, but at the price of very expensive equipment that would take high 
volumes to be fully utilized. Plasma welding is slower, but much less expensive as well and seems the most likely candidate for production volumes in this range.

\section{Header Hole Welding}

\begin{tabular}{|r|r|r|}
\hline Welding Process & $\$ /$ part & investment \\
\hline TIG & $\$ 285.75$ & $\$ 720,320$ \\
Laser & $\$ 261.22$ & $\$ 1,237,240$ \\
Plasma & $\$ 40.90$ & $\$ 111,470$ \\
\hline
\end{tabular}

TIG welding has both costly equipment and is slow. Once again, laser welding is fast but at a very high price and plasma welding is the low cost route.

Implementing plasma cutting and welding would save approximately $\$ 290$ over the baseline case.

\section{Alternative Fin Strategies}

The two fin strategy alternatives to the assembled fin strip concept, the "full width strip" and the "tapered header hole", were pursued because they offered the potential of simplifying the complexity of the manufacturing process. In volume production, the costs of material are so dominant that the increased scrap and material costs of the alternatives overwhelms any benefits from reduced labor and equipment. Specifically, the scrap losses from the fin strip cutouts for the "full width strip" design cost an additional \$110.57 per core for the $1800 \mathrm{~kW}$ core. The "tapered header hole" concept, employing slotted fins to distribute gas flow, will require a larger core to maintain efficiency. Scaling Ansty's calculations on the $75 \mathrm{~kW}$ core to the $1800 \mathrm{~kW}$ core of the baseline analysis, this would result in approximately $\$ 1800$ increased material cost while saving less than $\$ 900$ in processing costs. 


\section{Conclusions}

\section{Manufacturing Costs}

The analysis showed that material cost is the dominant factor of direct manufactured cost. Therefore, particular attention must be paid to material usage issues, such as scrap and reject losses. While yield/reject losses are difficult to predict for a conceptual manufacturing process, scrap loss can be more closely estimated based on product design and understood processing routes. The two primary sources of engineered scrap are the side trim scrap of the three layers and cutouts the make the header holes. Scrap losses were calculated for all the different recuperator sizes. Because of different lengths, number and size of holes, and the number of wraps, scraps rates varied from $6 \%$ to $10 \%$ for side trim and $3.6 \%$ to $1.09 \%$ for hole scrap. Overall, this analysis showed that scrap losses for this design are relatively low, especially relative to stamped and brazed heat exchanger design that can generate significant and expensive material losses.

Because of the material cost dominance relative to processing costs, the baseline core design, with the most efficient material usage, though more complex process, proves to be the lease expensive in volume production. Employment of plasma cutting and welding appears to offer economic benefits over the cutting and joining alternatives.

\section{Manufacturing Resources}

The following equipment and service vendors are recommended for further consideration when moving forward with production plans for the spiral wrap recuperator. The following paragraphs outline the companies and their capabilities. More detailed interview notes and contact information are contained in Appendix A.

\section{Automation International}

Automation International is a world-class manufacturer of automated welding and assembly machines. They have the knowledge and capacity to handle all phases of equipment manufacturing. Automation International owns the trade names, "FEDERAL" Resistance Welding Equipment, "Berkeley-Davis" Arc Welding Equipment, and "SwiftOhio" welding products. Automation Internationals' relevant experience in seam welding and assembly is based on the following applications: catalytic converters, mufflers, fuel tanks, and duct work. For additional information reference the company website: www.automation-intl.com. 


\section{American and European Machinery}

American and European Machinery is full line welding supplier based in Ohio. Services of interest to the spiral recuperator design include resistance seam welding, plasma cutting, plasma welding, TIG welding, and necessary automation. Our contact had previous experience seam welding the M1A1 tank recuperator and had extensive background in related operations. American and European Machinery represents several foreign equipment vendors and our contact cited two for this particular applications: Serra, a full service welding equipment manufacturer headquartered in Barcelona; and Cemsa, a welding and assembly equipment vendor headquartered in Via Milano, Italy. A rough website is provided at www.aemachinery.com.

\section{Newcor Bay City}

Newcor Bay City is the specialty machines division of Newcor. Newcor is a $\$ 250 \mathrm{M}+$ company consisting of three divisions: precision machines products, rubber and plastic products, and special machines. Newcor Bay City specializes in welding and metal fabrication equipment. They were a main source of information for the resistance seam welding and coiling portion of recuperator manufacturing, focusing on their patented HyPak process. Additional production equipment is developed at Newcor for bending, blanking, crimping, notching, piercing, planishing, roll forming, shearing, spot welding, and other specialized operations. Newcor has nearly 70 years experience designing fabrication solutions in the following industries: automotive, appliance, aerospace, and steel. Newcors' Bay City, MI campus also houses a state-of-the-art research and development facility to focus on continual process improvements. For additional information their website is located at www.baycity.newcor.com

\section{Automated Tooling Systems}

Automated Tooling Systems provides factory automation solutions, process development and systems integration services to many industries including telecommunications, automotive, semiconductor, solar energy, fiber optics and consumer products. Their core competencies include optimizing a part for factory automation, engineering and product prototyping, and designing and implementing turnkey factory designs. ATS has 26 facilities worldwide, including factories in Europe, Asia, and across North America. Conversations with individuals at their Canadian based North American headquarters has yielded interest in pursuing product development with Rolls Royce on their Spiral Wrap Recuperator, based on existing knowledge at that factory pertaining to finstock manufacturing, laser cutting / welding and other metal forming processes. Automated Tooling Systems offers the most efficient means that we have found to test a prototype for full scale manufacturing practices, as well as providing a service that integrates individual vendors and service providers. The best way to approach ATS is to contact Dave Capindale, Applications Engineer at (519) 653 - 4483 extension 2336, dcapindale@atsautomation.com. 


\section{Alabama Laser}

Alabama Laser is a laser cutting and welding job shop and equipment manufacturer. Their systems provide high throughput solutions for cutting and welding sheet metal materials including carbon steels, stainless steels, nickel based alloys and titanium based alloys. Their in house job shop allows them the ability to research and develop specialty systems specific to a customer's part requirements. Alabama Laser has experience in providing laser systems for the automotive industry, as well as producing the machinery used to manufacture military tank turbine engine components. Alabama Laser is an experienced equipment manufacturer that also has the capability to research and develop new part designs, and manufacture prototypes at their own facility. Their website may be found at http://www.alspi.com/laser.htm.

\section{Phinney Tool and Die}

Phinney Tool and Die is a metal forming stamping press equipment and tooling manufacturer, primarily serving the automotive and residential heat exchanger markets. Their extensive background in these markets, as well as their in house prototype manufacturing capabilities make them the stand out choice for the development of the hole punching, and dimple forming processes. Phinney Tool and Die provided much of the information that we collected in regards to these processes including estimates for the feasibility of the alternative gas turning fin designs, material handling requirements, tooling and stamping presses. For further information on this company their website may be contacted at http://www.phinneytool.com/. 


\section{Appendix A: Interview Notes}

Fin manufacturing and design issues

\section{Engineered Materials Solutions}

Awadh Pandey, 508.342.2100

Engineered Materials Solutions (EMS), formerly a division of Texas Instruments, is most well known for their work in the catalytic converter metallic substrate industry. They specialize in roll-to-roll cladding of thin gauge ( $\sim 10$ mils thick) ferritic stainless steels. Dr. Pandey is a metallurgist in EMS' catalytic converter substrate (Durafoil ${ }^{\mathrm{TM}}$ ) product division.

We discussed the forming technologies that Rolls Royce may need to employ in order to manufacture their spiral wrap recuperator and concluded that some of the operations are analogous to catalytic converter substrate production. EMS, however, does not deal with any of these parallel operations. Most of their customers, the catalytic converter OEMs, are more adapted to form the coiled converter product and would be more useful to answer questions about welding, corrugating and otherwise forming large coils of thin gauge stainless steels.

\section{Alfa Laval Thermal Inc.} Jim Ritz, 717.453.8713

Alfa Laval is the world's largest heat transfer manufacturer, including plate, spiral and brazed-plate heat exchanger product forms. Their experience in manufacturing spiral products led me to contact their Lyken, PA. This individual is a process engineer and suggested that I speak with one of the company's marketing specialists.

\section{Alfa Laval Thermal Inc.}

Chris Wajciechoski, 804.236.1329

Alfa Laval manufactures large spiral wrap heat exchangers for the industrial and chemical processing industries. The spiral heat exchanger is produced by covering a plate or sheet's outer surface with rods, and then winding the plate around itself to form the coil. These rods act as separators between the coils subsequent layers. Chris believes that the Rolls Royce product will be manufactured very differently and that it will be hard for Alfa Laval to apply any of their manufacturing experience in a useful way.

The thinnest material that Alfa Laval uses in any of these spiral heat exchangers is a 12gauge ( 0.105 inch thick) material. The overall product weight, including headers and 
outer case, etc., is approximately $40 \mathrm{~K}$ pounds. The machinery that is used to coil this material would be much different than that which would be used to coil thin (5-10 mils thick) material, like Rolls Royce's.

The welding of most of their products is very labor intensive. In fact, a rule of thumb to describe the cost distribution for many of these products is $60 \%$ labor and $40 \%$ materials. The welding that would be used for materials approximately 5-10 mils thick would most likely be a less intense heat, such as TIG (tungsten inert gas) welding. This would be more easily automated than the welding needed for their larger, less intricate heat transfer products.

This person was able to suggest a couple of industry contacts that may be of more help to me. They include:

- The Heat Transfer Research Institute (HTRI) - a private company that researches ways to improve current manufacturer's heat exchanger designs.

- Plate coil manufacturers - this person feels that plate coils most closely resemble the product that I described, and that speaking with one of these manufacturers may be more useful. Two of the leading plate coil manufacturers are Mueller (Missouri), and Tranter (Houston, TX)

\section{Robinson Fin Machines, Inc.}

David Haushalter, 800.311.8409

Robinson Fin manufactures finstock materials of varying dimensions and with a maximum product width of 24 inches. The majority of their experience lies in supplying plate fin products to the recuperator industry. Some of their clients include Solar Industries, Capstone, and Ingersoll Rand. David is the VP of sales and marketing at Robinson.

Upon reviewing several Rolls Royce Spiral Wrap Recuperator drawings and schematics, David feels that Robinson is able to supply finstock, but limited in their ability to meet all of the current design requirements. The fin dimensions are fairly common to the recuperator industry and can be produced in supplied material for a cost of about $\$ 0.02$ per fin. This is not an exact estimate, but rather a rule of thumb works for finstock with a fairly low fin packing rate (under 25 fins per inch). A higher frequency of fins per unit length can be less expensive on a per fin basis. For example, a recently shipped fin product for an automotive application had 43 fins per inch and was about a.5 feet long. This product was sold for about $\$ 4.00$ per piece, or $\$ 0.005$ per fin.

The thin fin strips, which Rolls has designed for the sections surrounding the inlet and outlet holes, will be much different from anything that Robinson has done in the past. Dave explained that his current equipment is not able to produce the flow paths around the holes and that the small size of his company does not allow him to dedicate the time required to develop the needed machinery. We did discuss some alternate designs for 
these sections of the recuperator coil, such as welded separators to replace the fins around the holes, dimples or even welded bars. These options would seemingly accomplish all of the same tasks that the current finstock is intended to perform including; allowing for uninterrupted gas flow around the holes and through the fins and as a structural component that would keep the plates separated during high pressure gradients between the inner and outer chambers.

Dave also suggested some automotive automation equipment suppliers that may be able to design machines to perform the plate perforation process.

Continued contact with Robinson will hopefully fully explore the credibility of these alternative fin strip designs, as well as evaluate the requirements of the finstock, itself (i.e. fin height tolerance its affect on coil diameter and ultimately on predicting the distance between perforations).

\section{Automated Tooling Systems}

Rob Braccio, 905.469.6890

ATS manufactures custom automation equipment for a variety of industries including automotive. Their metal forming division is familiar with coil metal forming practices and is currently reviewing spiral wrap recuperator product drawings in an effort to supply specific equipment quotations.

\section{Dave Capindale, 519.650.6677}

Dave is an Applications Engineer for Automates Tooling Systems' metal forming equipment division. He was forwarded my request for information on the company's capabilities and relevant experience in regards to our client's spiral wrap recuperator design.

Currently, this division of ATS is involved in the production of plate recuperators for use in the microturbine industry. Although it is obvious that these plate products are very different from the spiral design that our client has developed, he feels that there are overlapping manufacturing processes and that their relevant process development experience developed could be useful.

In terms of specific manufacturing operations and equipment that this group is familiar with, Dave told me that they are currently customizing a fin making machine for the semiconductor industry and that they have worked with stamping presses, laser welding and resistive welding machines in the past. Dave is reluctant to give even budgetary quotes for the pieces of equipment that we feel will be needed for the spiral wrap recuperator production because he feels that they will be heavily modified versions of off the shelf machines or completely customized for this process.

He feels that along with ATS' many other concentrated divisions' bodies of expertise, they would be a good choice for a partner during the process development phase. To expedite 
this and to give us a better understanding of how well they match with this project's equipment requirements, Dave is sending a mutual confidentiality agreement for our client's review, as well as a package outlining the above mentioned projects and special equipment development capabilities.

\section{Laser Machining, Inc.}

Dave Plourde, Steve Haugen, Joel Decoch 1.800.77.LASER

Laser Machining is a laser cutting and welding equipment supplier with in house engineering services. LMI has experience in laser cutting and joining metal, wood and plastic materials for the automotive, aerospace and electronics industries. Much of the equipment that they supply these industries is custom designed to meet client's manufacturing tolerances and align with other in-line production equipment. I faxed LMI drawings of our client's spiral wrap recuperator product to get an idea of the operations that they envisioned to make up the manufacturing scenario and how they might be able to help.

IBIS Associates held a conference call with Dave, Steve, and Joel on August 20, 2001 to discuss the drawings and their experience with similar product forms. As a supplier of laser cutting and joining equipment to the electronics industry, LMI has a great deal of experience in designing machines to work with materials in coil forms. Their equipment has been built as stand alone stations, as well as integrated into a coil processing line with other converting machines. This knowledge of laser processing flexible materials and their experience in metal industries (i.e. automotive, and aerospace) with their ability to design, in-house, proprietary machines that meet client's product requirements appears to make LMI a resource for this development effort. That is assuming, however; that the manufacturing process requires laser cutting for the holes and laser welding of the header seams (inner hole diameter seam).

In their opinion, the manufacture of the holes will most efficiently be accomplished with a laser, the hole can then be laser welded and they can provide visual inspection systems, such as an x-ray inspection system. Some sort of inspection system will most likely be required prior to the final coiling step so that re-working of the welds is possible.

Budgetary numbers for a proposed laser welding and cutting system include:

- Rate:100 inches per min

- Witness (landing): 4-5 thousandths of an inch

- Power (Laser Size): 500 Watts

- Cover gas: typically high pressure Nitrogen

- Inspection System: X-ray

The cost of a laser system can be broken down into component price estimates, such as $\$ 75-150 \mathrm{~K}$ for the bare laser. Periphery systems, such as inspection, cover gas and controls are approximately double this or approximately $\$ 300 \mathrm{~K}$. For a welding and 
cutting system, two heads are recommended for an additional $\$ 75-150 \mathrm{~K}$ per additional heads beyond one. Using these estimates, the total price for a dual head laser system is about \$500-650K.

Laser Machining, Inc. has experience in processing similar multiple thin metallic materials in the production of batteries at Duracell. That particular line required 9 laser beams (heads) and cost in the low millions. A typical development program for LMI and one that they would prefer to follow includes three phases. First, preliminary product designs are evaluated by LMI and their in house engineering group develops a process design accordingly. Next, LMI would use their "pilot run" production facility to produce a couple hundred of the part. Finally, assuming that the first two phases are completed successfully, capital equipment is built and supplied to the client along with appropriate training and support phases.

\section{Lourdes Systems, Inc.}

\section{Rick Winter 1.631.234.7077}

Lourdes Systems manufactures metal forming presses for thin metal strip materials ( up to 0.055 inches). They have experience working with automotive and home use heat exchanger manufacturers including Visteon and Delphi. Most metal forming presses, such as those manufactured by Breuter of Austria, make use of hydraulics or electric servomotors to supply the metal forming force.

Lourdes' press technology uses an electromagnetic device, which when a pulse of energy is applied to its solenoid forces an armature out and drives the press mechanism. This technology has many advantages and even some disadvantages, that all seem to point to this application being a good fit. The main disadvantage of this technology is its line speed. The maximum production rate for one of these systems is between 60 and 100 strokes per minute, while a mechanical press may operate as fast as 1200 strokes per minute. This has limited its use in high production volume industries such as the automotive. Although the machines throughput is relatively low as compared to other press machine types, its stroke velocity is very high. This high stroke speed minimizes material deformation, lengthens tool life and produces features with much higher tolerances (reduces the radius produced in corner features).

Typical presses produced by Lourdes are manufactured for materials with a maximum coil width of 15.5 inches. A custom machine that could handle coils up to 36 inches in width would cost approximately $\$ 35-40 \mathrm{~K}$ and would include the press, table and controls. The speed of this machine for the application that I described would probably operate at a speed of about 30 strokes per minute.

The tools that are used to produce fins and dimple features that are this intricate and intended for materials that are this thin are available through a Rochester, NY company called Phinney Tool and Die. The tools are the most critical cost component for metal forming presses because they are so high in cost, their size along with the machines stroke 
speed determine line speed and their tool life (because of the high cost) becomes a crucial factor.

\section{Simons Rollforming Company}

\section{Chuck Simons 1.219.267.2175}

Simons Rollforming Company manufactures rollforming equipment and produces rollformed parts for high volume applications, such as those found in the automotive industry. I contacted them to discuss the feasibility of rollforming one or more of the components that make up the spiral wrap recuperator.

Chuck examined the preliminary sketches that I provided him with of the recuperator and its parts and determined that none of the parts are practical for the rollforming process. In order for a part to be considered for rollforming, one of the most important criteria is that its cross section be uniform. If the fins ran perpendicularly to the direction that they run now, this would be true. A second typical rollformed part characteristic is that after the coil of material is shaped, it is sheared into a smaller part length. He is not familiar with recoiling material once it has been shaped.

Mr. Simons is a good resource for other metal fabrication techniques, however and he offered his opinion of our initial manufacturing plan. The fin manufacturing will most likely be accomplished with an indexing hydraulic or electric servomotor driven stamping press. He cautioned that in his experience forming intricate shapes, such as the gas turning fin plates, often requires two stamping strokes. An initial action to form the material to the general shape of the part and another that will further form the part's corners (radius of curvature) to the product's specifications. The problem that often occurs when "double hitting" a material like stainless steel is that in the first stroke the heat from the forming anneals the material and decreases its ductility, often dramatically. If this occurs, then the second forming motion can damage or destroy the part rather than shape it.

In the case of joining the metal materials, especially around the holes, Mr. Simons feels that there are issues we also must consider. For example, laser cutting the header holes through the thickness of the plate (0.010"), finstock (0.003") and the dimpled plate (0.010") may be done, but the laser may be very expensive. As the thickness of the material that is to be cut increases, the distance over which the focal length of the laser changes also must change. This distance, along with laser power are directly related to equipment cost. Although he knows that lasers exist that can handle this thickness very easily our client must also consider edge acuity requirements. As the laser penetrates the layers of materials, making the cut, the angle at which it is cutting changes. As it penetrates the bottom layer, the top layer may be damaged by the high power of the laser beam far away from the focal point.

He recommended that I look at the July 2001 issue (volume 31, \# 7) of FMA (Fabricators and Manufacturers International) Magazine, which lists all of the top laser cutting and 
welding equipment and component suppliers in the metal fabrication industry. The magazine is also a comprehensive source for many other metal fabrication processes.

\section{Phinney Tool and Die Co., Inc.}

Carl Phinney 1.716.798.3000

\section{Conversation 1}

Phinney Tool and Die manufactures high precision metal stamping tools for the automotive and residential heat exchanger industries. I have faxed and mailed them descriptions of the recuperator parts for which we are seeking quotation on, and will hopefully hear back from them soon.

\section{Conversation 2, September 13, 2001}

After reviewing the product's components and estimated manufacturing processes, Carl feels that Phinney Tool and Die does have the experience to be of assistance. Phinney Tool and Die is a stamping die and tool manufacturer, but they also put together turnkey systems including stamping and forming equipment and material handling machines, such as coilers and un-coilers.

His first concern with the project is in regards to the production of the "gas turning fin plates". Carl feels that this component will be very difficult to manufacture and may be impossible, depending on the tolerances of some of the dimensions. As the piece of material is bent into the individually angled finstock, the amount of material that is gathered laterally will vary. If the part is produced from a uniformly dimensioned piece of material, the edges will wrinkle or rip. Also, the thickness of the material is of concern to Carl because it leaves very little room for any stretching of the material. Carl would like to propose to our client that alternative designs for this feature be considered and that prototype dies be produced in order to address these types of issues.

Mr. Phinney feel that the production of the dimples and the collars (header hole features) in the 0.010 " thick piece of material is a straightforward pressing operation. He envisions that two presses will be necessary because of the pitch differences in the placement of the collars. Carl estimated the process to look like the following diagram:

\section{Dimple and Header holer collar forming operations}

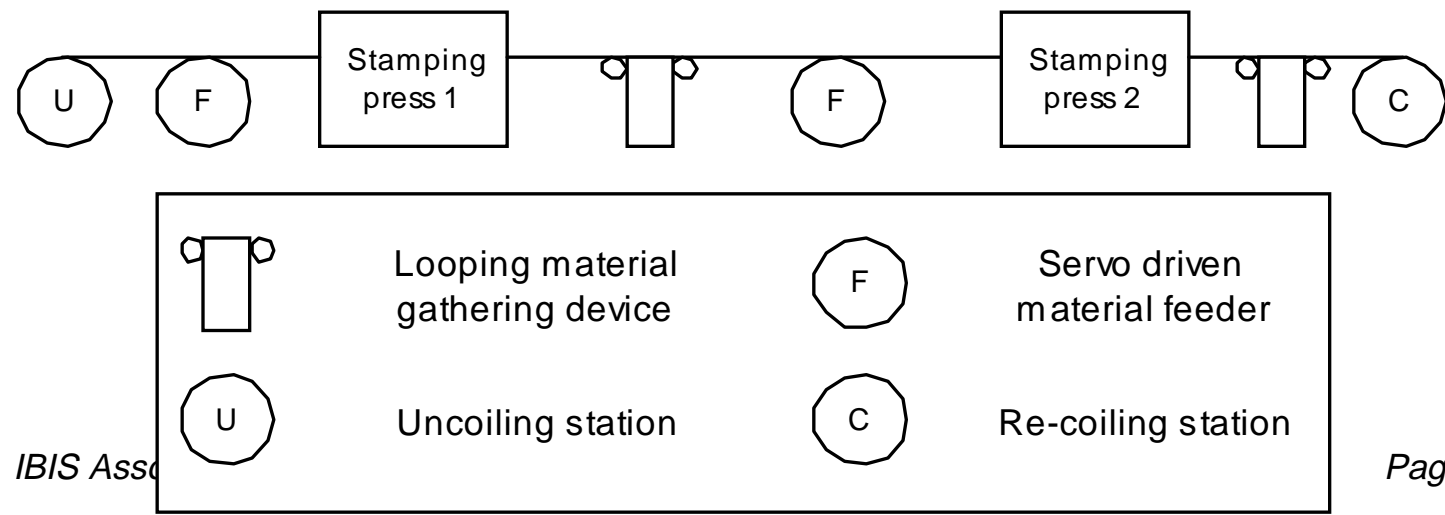


He estimates that each straight side stamping press, the first being for the formation of the dimples and the second for the header hole collars, will cost approximately $\$ 200 \mathrm{~K}$ apiece. These presses can operate at a variety of speeds (defined by strokes per minute) and often are equipped to change speeds (variable speed stamping presses) depending on the product being run at that particular time. For simple dimensions such as the dimples the stroke speed could be very high (150 to 200 strokes per minute) but it should be a function of the tool size (amount of material formed per stroke) and the speed of the downstream collar forming press. He estimates that 200 inches of material per minute would be close to the desired speed for this line and that it could be achieved with a 2 " tool at 100 strokes per minute or a 4" tool at 50 strokes per minute.

The tooling for these features should be quite standard and cost an estimated $\$ 50 \mathrm{~K}$ per tool. The tooling life for these dies will be enormous, primarily because of the simplicity of the features but also because of the thin nature of the material (0.010"). It is hard for him to say how long these tools will last before they need to be replaced, but he explained that a similar die that he supplied for use in the production of automotive heat exchangers has lasted more than a year while being run two shifts per day and 200 strokes per minute.

The material handling equipment shown in the above diagram (looping material gathering device, uncoil, re-coil and material feeding stations) is also necessary for this production line. He estimates that typical uncoiling and coiling machines cost approximately $\$ 20 \mathrm{~K}$ apiece, while the servo material feeders cost as much as $\$ 50 \mathrm{~K}$. As mentioned above the speed of the dimpling machine is a function of the speed of the downstream operation, or collar-forming machine. Since the pitch of the collars will be changing over the length of the material, the speed of the process will also change. To accommodate for this a material gathering or looping station will be used. This device gathers excess material from the first, faster process (dimple forming) and feeds it as it is needed to the slower indexing collar forming station.

An uncalculated cost in all of this will be the integration costs. When a line such as this one is built it must be put together, tested and calibrated before it is used for production. This is usually done at Phinney Tool and Die on their production floor and may cost as much as $\$ 150 \mathrm{~K}$.

\section{Hole punching and spacing}

Many perforated metal manufacturers have been contacted in the search for an analogous product or process involving variably spaced hole punching on continuous strip and/or in line coiling and winding of perforated product. No strong comparisons have yet been found, nor have the fabricators been aware of other processors or equipment with this capability. The following contacts have been the most helpful and have agreed to review schematics internally and to possibly identify additional leads. 


\section{Hendrick Manufacturing Company}

Jake, 800 225-7373

\section{Direct Metals Co.}

Steve Zaval

\section{Argus Steel Products}

877 333-5300

Diamond Manufacturing Co.

Marty Binetsky 800 233-9601

\section{Skidmore Engineering}

800 642-1773

\section{Hamlin Sheet Metal}

800 656-2004

\section{James River Steel}

800 825-0717

$\underline{\text { Hole welding and sandwich formation }}$

\section{Cloos-Robotic Welding}

Jim Berge, 847-923-9988

Jim Berge has been Vice President and General Manager of Cloos Robotic Welding since Oct. 2, 2000. Berge has been active in the robotic welding industry for 15 years, including five years with his own company, in which he provided consulting, training, and programming expertise to companies using robotic welding equipment.

About two-thirds of Berge's career has been on the manufacturing side of the business, working for end-users of robotic and automated welding equipment, making robots work on the factory floor. The remainder of his time has been in the marketing end, working for companies such as ABB Flexible Automation, and other suppliers of automated welding equipment. 
Berge has a bachelor of science degree in welding engineering from LeTourneau University and is the author of the book, "Automating the Welding Process," published in 1994 by Industrial Press. He is a member of AWS and has presented various papers and seminars regarding automated and robotic welding.

Cloos has been in the welding business since 1919. In the beginning, Carl Cloos designed and built welding power sources, laying the foundation for the modern MIG/MAG welding process. Mechanized welding machines filled the automation needs of Cloos customers worldwide. Then, in 1981, Cloos designed and began building its own arc welding robots.

Today, Cloos operates from subsidiaries worldwide, supplying true turn-key robotic welding systems, pre-integrated robotic welding cells, special purpose machines, and welding power sources to a multitude of industries. The United States subsidiary was established in 1985, and supplies complete turnkey systems, spare parts inventories, training and service from the Schaumburg, Illinois headquarters. A subsidiary of the U.S. operations -- located in Monterrey, Mexico -- provides service and support to the Mexican market, including spare parts and training services.

Jim will be providing IBIS with information regarding the automatic hole welding operation.

\section{Edison Welding Institute}

Dick Holdren, 614-688-5000

Dick Holdren has logged many years of welding experience since obtaining his bachelor of science degree in welding engineering from The Ohio State University in 1973. A member of the American Welding Society (AWS), he is a senior certified welding inspector and an ASNT Level III in MT, PT, and UT. His awards include CWI of the Year - - Columbus Section and District 7; ASNT Fellow; AWS Distinguished Member; and AWS District Meritorious Certificate. EWI provides practical welding and joining solutions through technical assistance, contract research, consultancy and training to 400 member companies worldwide.

Dick was faxed the schematics of the recuperator and we are scheduled to talk next week about possible manufacturing scenarios.

\section{Red Bud Industries}

Dean Linders, 618-282-3801

Red Bud is a supplier of coil processing equipment. They specialize in blanking equipment and multi-blanking equipment, but also supply other types of coil handling machines.

They should be able to provide information on how the separate coils should be handled and combined. 


\section{Alabama Laser Technologies \\ Mike Sharp 1.256.358.9055}

Alabama Laser Technologies manufactures laser cut and laser welded parts, as well as the respective equipment systems for a variety of industries and applications. Their in house job shop provides them with the ability to test systems as they develop them for a customer. Specifically, Alabama Technologies has experience cutting and welding steels, stainless steels, nickel alloys, and titanium alloys for the electronics, automotive and food processing industries.

Mike Sharp specializes in the design of laser welding systems for Alabama Technologies. Mike explained that he has experience developing automated laser welding systems for the manufacture of Avco Lycoming's M1 Tank engine recuperator.

Laser cutting stainless steel sheet, as required by our client's spiral wrap recuperator design, and subsequently welding three layers together along the inner diameter of the hole appears to be feasible, although testing is required to ensure that weld's strength is sufficient for the pressure of the system. The laser cutting operation is the most straight forward of the two proposed processes. An automated laser-cutting machine may be programmed to cut holes of varying size and pitch. The speed of the system is required to accurately estimate the laser's power requirement and cost.

The laser welding operation may be done in one of two ways; using a lap or annular weld, or through the use of a direct edge weld. A lap weld begins at one surface or material face and continues through the material until a full weld is completed. Typically, its seam or weld footprint is approximately $1.5 \mathrm{~mm}$ in width at the first surface and reduces gradually through the thickness of the material. In this case, an annular weld through 0.030 " of stainless steel would result in a weld footprint of about $0.5 \mathrm{~mm}$ on the second or bottom material face. These welds usually require approximately 0.005 " of material between the weld itself and the edge of the material. Although this weld penetrates through the materials it may leave small portions along the seam un-welded. This is dependent on the speed and power of the laser.

The second weld type, the direct edge weld, is slower and more difficult to achieve. It takes place, as it suggests, along the edge of the joining materials. In this case, the weld takes place along the inner diameter of the hole. The speed of the weld and size of its footprint are both a function of the laser's power, although it is usually accurate to describe its seam as smaller and more leak resistant than the lap weld.

In both cases, Mike suggests that a test be performed on a prototype part in order to accurately define the power requirement for the laser. 


\section{$\underline{\text { Rolling and seam welding }}$}

\section{New Southern Resistance Welding}

Tom Christianson: 888-950-6779

NSRW manufactures resistance weld equipment for automotive components. Our contact estimated the equipment necessary for a resistance seam weld of $2 \mathrm{SS}$ sheets, $0.2 \mathrm{~mm}$ thick. This thickness would require a 3-phase resistance welder, the primary demand is too large to be done in a single phase unit. The controller alone is $\$ 40,000$, and total equipment cost is about $\$ 170,000-\$ 190,000$. This would require up to 4000 pounds, or 2 tons of pressure. Required current would be about 50,000 AMPs. The electrode wheels are manufactured with Class $3 \mathrm{Cu}$ and are included in the budgetary estimate given. Additional tooling would be in the form of certain jigs to stabilize the part.

Note: IBIS has reason to believe out contact had made a conversion error. A phone call has been placed to reevaluate this data.

Sciacky, Inc.

James Miller: 708-594-3800

Sciacky, Inc. manufactures resistance seam welders for a range of automotive, military, and telecommunications applications. This application, 210 mil SS sheets seam welded, would require 7000 AMPs or $600 \mathrm{lbs}$. Force. This is a three phase unit. Single phase seam welders are really only used for mild steels in job shop applications. A bare bones estimate for this unit is $\$ 170 \mathrm{~K}$, additions could easily increase costs to $\$ 200 \mathrm{~K}$.

Recommended coverage for seam welds is 18 spots per inch, which overlap thereby forming a continuous seam. There units consist of two hot and cold cycles, and cooling is accomplished by a water well.

\section{NewCor Bay City}

Doug Cotes: 800-959-0627

NewCor Bay City manufactures resistance seam welders for catalytic converters. This uses a single sheet, which is wound to a coil and then seam welded. We discussed the potentiality of performing the same operation with multiple, non-uniform sheets. IBIS sent over some additional information and Mr. Cotes was going to evaluate NewCor's capabilities.

\section{Second Conversation}

Mr. Cotes believes New Cor can service the seam welding operation. Individual welders for this application would utilize Newcor Bay City's patented low inertia weld systems which could possibly weld at speeds of 60 - 70 feet per minute versus the conventional 30 - 40 feet per minute. Each of these welders would be at approximately $100 \mathrm{~K}$. 
NewCor BayCity also has metal fabrication potential. Additonal diagrams were provided to Mr. Cotes and IBIS is awaiting additional information on the system. At this point, a ballpark figure was 2-3million (which should not be granted much consideration at this point).

\section{Weldmation}

Jim Lippick: 248-585-0010

Weldmation manufactures metal fabrication for heavy and light-duty trucking industries. They have experience with catalytic converter winding and welding. Mr. Lippick was provided with schematics of the spiral recuperator manufacturing process, and he will contact IBIS with his opinions of the process.

\section{Second conversation}

IBIS held a conference call with Jim Lippick to explain the manufacturing problems with the recuperator. JMr. Lippick was relaying this information to his proposal team and is expected to provide budgetary numbers for the entire system and the seam welding operation by August $20^{\text {th }}$.

\section{UPDATE}

Weldmation has decided that the manufacturing demands of this project exceed their capabilities. While they might provide insight into a resistance seam welder, they are not able to provide an educated quote on this and other fabrication processes.

\footnotetext{
Automation Int'1

Kyle Dines: 217-446-9500

Automation Int'l manufactures resistance seam welders for catalytic converters. It is a similar operation, except recuperators have multiple sheets to be wound. Mr. Dines is evaluating a schematic that was sent over and he will be providing input on the equipment they could provide. The clamshell design would allow for non-uniform sheets to be wound without losing their shape. A Z-bar is inserted to maintain the weld location.
}

\section{Second Conversation}

Automation International provided an initial budgetary quote for the resistance seam welding equipment. The current requirement for 0.010 " thick stainless is $5000 \mathrm{amps}$ and $400 \mathrm{lbs}$. of electric force. Barring any design changes that would be required, the rough estimate for this seam welder would be $\$ 45,000$. The rate on the seam weldre is about 60 " per minute. The only consumables on this machine are the seam welders. 
For the $90 \mathrm{~mm}$. diameter header hole, Automation International recommends a TIG welder. This unit would cost in the range of $\$ 70,000$. These machines are entirely dedicated to the hole diameter, so an additional purchase would be needed for the $130 \mathrm{~mm}$ header hole. The rate on this equipment is $30 \mathrm{~s}$. for a $90 \mathrm{~mm}$. hole, or 7 inches $/ \mathrm{min}$. The only consumables would be the TIG torch tips and electrodes.

A robotic TIG welder could be used instead of the manual slide set-up. The investment for this machinery is about $\$ 115,000$. The rates are essentially the same, 7-15 inches /

min. This would not be a dedicated machine, with respect to hole dimensions, thereby proving more cost effective when compared to the manual welder.

\section{UPDATE}

Automation International also provided a list of advantages and disadvantages of TIG vs. plasma welding.

ADVANTAGES

1. Concentration of energy is greater

2. Arc stability is improved, particularly at low current levels

3. Heat content is higher

4. The plasma has higher velocity

5. There is less sensitivity to variations in arc length

6. Tungsten contamination is eliminated

DISADVANTAGES

7. Higher cost of equipment (generally two to five times higher)

8. Short life of orifice body

9. Need for greater welder knowledge

10. High rate of inert gas consumption

(Note: this is contradictory to existing data we have gathered on plasma arc machines which appear to be half the cost of TIG welders.)

\section{Third conversation}

Automation Int'l was contacted again to discuss automation for the entire recuperator line. The expected overhead for the resistance seam welding operation is $400 \mathrm{~K}$. This 
would integrate an end winder into th etwo resistance seam welding stations. The final cost for the process would be about 500K. Mr. Dines estimated that the type of endwinder that is required for this process is an powered roller with a DC motor. This would aleviate tension off the fin stock and also maintain it's geometry. This investment also includes a planishing station that would even out the weld seam. IBIS is not positive that this operation is necessary for the spiral wrapped recuperator.

\section{American and European Machinery}

Marty Keasal: 419-877-1001

Marty Keasal assisted in development of the gas turbine recuperator for the army M-1 series engine. He was responsible for the resistance seam weld, and although the design is different, Mr. Keasel believes he can add insight to the spiral recuperator we are evaluating. Within the M-1 series tank recuperator there are three types of welds used: Ebeam weld for the holes, MIG weld, and resistance seam weld. Mr. Keasel was provided with schematic outlining the manufacturing process and we are awaiting his input.

\section{Second conversation}

Mr. Keasal provided some estimates on throughput and investment for the resistance seam welding operation. From the diagrams provided, Mr. Keasal believes that the operation would require 4 seam welders, each $30 \mathrm{~K}-35 \mathrm{~K}$. A DC welder (which is recommended) is more expensive, $40 \mathrm{~K}-45 \mathrm{~K}$, with an amperage loading of 50-60kVa. Throughput on the machinery ranges from 50 inches per minute for an alternating current system (amperage requirement of $80-90 \mathrm{kVa}$ ) to 100 inches per minute for a direct current system. These units have a steep learning curve, and Mr. Keasel has seen operations where throughput doubles over the duration of it's lifetime.

For the header weld, Mr. Keasal recommends a plasma system over a TIG weld. The plasma system operates at 60 inches / minute. An arckinetics (advanced plasma weld technology) system operates at 70-90 inches / minute.

The end winder would require a special tension control and expanding mandrel. American and European Machinery is capable of fully integrating this equipment and partner manufacturers include Cemsa in Venice, and Serra in Barcelona, both of whom are actively engaged in continuous seam welding.

A conference call is scheduled for Monday to discuss American and European machinery's integrating potential.

\section{Third conversation}

A conference call was held August 20,2001 to discuss header welds, suggestions for punching techniques and potential bottlenecks in the operation. The weld bead dimensions for a plasma weld are approximately 3-4 times the metal thickness. Plasma 
weld systems are in the same price rance as resistance welders $(30 \mathrm{~K}-40 \mathrm{~K})$. The arckinetics system is marked up 2-3 times initial cost.

The hole may be cut with a laser or plasma beam. The edge acuity is not a major concern for the recuperator as the holes are only used for air flow. Expected kerf loss on a plasma cutter $\mathrm{s}<1 / 2 \mathrm{~mm}$ for a $90-130 \mathrm{~mm}$ hole. The plasma cutters could be assembled in line with the weld system, and are in the same cost range. The laser system offers higher direct wattage, $10^{6} \mathrm{~W} / \mathrm{cm}^{2}$, but at a significant premium (systems are in the range of $300 \mathrm{~K})$. An excellent supplier of laser cutting systems in the Boston area is Coherent General.

Marty has been in contact with Bill DeGroot, an equipment supplier in the greater Boston area. Bill has expereince with stainless steel fabrication in the aerospace industry. He will be contacting IBIS to discuss metal fabrication capabilities.

\section{UPDATE}

\section{Fourth conversation}

Marty Keasal was contacted again to discuss the limitation of plasma cutting. Plasma cutting was used for the M1A1 tank engine armor plates. These plates were 2" thick and previously cut with an GMAW torch. Plasma cutting is not limited by thickness, and a $3.2-3.5 \mathrm{~mm}$ stack would be feasible. Laser cutting is limited by stack thickness because of the voltage requirements of the laser. The only concern with plasma cutting is the kerf loss on the weld. A plasma cutter has a kerf of 0.010 " to 0.012 ". If the edge acuity of the header hole is not a major concern, then Mr. Keasal recommends exploring plasma cutting.

\section{Other companies contacted}


Coil Joining Technologies: 440-582-5006

Precision Arc Co, LLC: 203-332-7388

GWI Engineering: 616-459-8274

RWC, Inc: 989-684-4030

Stapla Ultrasonics Corporation: 800-647-5996

JRJohanson, Diamondback Technology: 805-544-3775

Sciacky, Inc: 780-594-3800

NSRW: 888-950-6779 


\section{Appendix $\mathbf{J}$}

\section{NGT Supporting Technologies: Sensor/Controls Diagnostics}


Final Report

Next Generation Turbines Contract DE-AC26-00NT40852

Control and Monitoring Capabilities that Benefit Reliability, Availability, and Maintainability

Prepared For:

National Energy Technology Laboratory, and Rolls-Royce

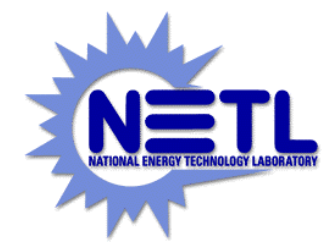

Rolls-Royce

November 2001

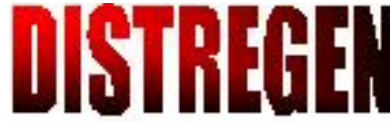

Advisory Group 


\title{
Table of Contents
}

\author{
Executive Summary
}

1 The Project and Project Process

2 Operators Needs and Wants

Site Visits

Operator Need and Wish List

3 Control Technologies

4 Potential Benefits of Control \& Monitoring Technologies for Meeting Operators Needs

$5 \quad$ Conclusions and Recommendations

\section{List of Tables}

Table 2-1

Sites Visited and Characteristics

Table 2-2

Site 1 Characteristics \& Issues

Table 2-3

Site 2 Characteristics \& Issues ..2-3

Table 2-4

Basic Operator Wants \& Needs

Table 3-1

Sensor \& Control Technology Strategy

Table 3-2

Condition Monitoring Technologies for Engine Monitoring.

Table 3-3

Condition Monitoring Technologies for Other Areas ..3-4

Table 3-4

High Temperature Sensing Needs $3-6$

Table 3-5

Control Capabilities of Equipment

Table 3-6

Diagnostic \& Prognostic Capabilities

Table 3-7

Other Plant Monitoring \& Control Systems

Table 4-1

Basic Operator Needs

Table 4-2

Operator Needs by Function - Turbine \& Combustion Systems

Table 4-3

Operator Needs by Function - Turbine \& Auxiliary Systems.

Table 4-4

Operator Needs by Function - Other Systems.

Table 5-5

Development/Improvement Projects

This report has been prepared for the use of the client for the specific purposes identified in the report. The conclusions, observations, and recommendations contained in the report constitute the opinions of Distregen Advisory Group. To the extent that preparation of this report, Distregen Advisory Group has relied upon the same to be accurate, and for which no assurances are intended and no representations or warranties are made. Distregen Advisory Group makes no certification and gives no assurances except as explicitly set forth in this report. 


\section{Executive Summary}

$\mathrm{T}$

This work effort is focused on the question: "What does the operator of turbine generation really want and need in control and monitoring capabilities to improve reliability, availability and maintainability (RAM)?"

The approach taken was to investigate potential control and monitoring technologies that may have an impact on RAM. Technologies that were investigated included those in common use, those in the early stages of commercialization, and those that are in the experimental stages. After a list of possible technologies was determined, two generation sites and a packager were visited to understand their viewpoint on control and capabilities needed to improve the RAM of the gas turbine system. This input was used along with capabilities of each of the technologies to explore the value of each of the technologies to operators of gas turbine power plants. The value of each of the technologies is analyzed from the viewpoint of how it provides value to the operator and maintenance staff with a bias towards improving RAM. Based on this analysis, recommendations were made for technologies that should be included in gas turbine systems as well as recommendations for development projects.

The site visits showed that many of the control and monitoring devices and software did not provide the value that was expected of them. This was also true of software put in place to improve the control and monitoring functions. Sensor inaccuracies and failures at the sites approached $25 \%$ in actual operation while software problems created situations where incorrect information confused the operation and maintenance staff. Some of the software problems directly related to extended shutdowns of the units involved.

The basic answer to the question of what operations wants in control and monitoring capabilities is the ability to get accurate and reliable information, have the ability to get the generation system up to full power quickly, and keep the system running. This points to a solution of keeping the control and monitoring system as simple for the operators to run as possible. Many sensors and overly sophisticated software confuses the operators and maintenance staff as well as creating a higher risk for problems that may have been missed in testing. Operators also want the ability intelligently defeat some of the controls in order to get up and running under certain circumstances.

In keeping with the operators desire to keep things simple the recommendations in this report are focused on simplifying the monitoring and control functions. The recommendations are focused in three categories: development/improvement of sensing technologies, development of tools to provide decision support information to the operator, as well as the development of expanded diagnostic and prognostic capabilities.

Development/Improvement of sensing technologies includes recommendations for an improved fuel to air ratio sensor, gas leakage monitor, and gas quality monitor. These sensing devices were chosen because of the impact they will have on the reliability, availability, and maintainability of the gas turbine system.

The development of tools to provide decision support information to the operator includes the capability to provide an assessment of risk that an operator takes on by any particular action as wells the capability to override less critical control system functions. 
The development of expanded diagnostic and prognostic capabilities is focused on providing information that is useful for gaining understanding of potential causes of problems. In addition, the goal is to suggest probable causes and identify parts that have failed or contributed to the problem.

Improved prognostic capabilities include the capabilities to identify potential operating problems before they occur as well as to identify potential equipment failure.

By keeping the control and monitoring systems simple, RAM will be improved from that of the average gas turbine system. By developing the sensing devices and software tools, RAM will improve to a much greater degree.

In a time of deregulated markets and volatile market prices, large improvements in RAM will have a large effect on the economics of a gas turbine system. The acceptance and implementation of these recommendations coordinated with a well designed gas turbine system will produce a package that will have high efficiency, high reliability, high availability, and low maintenance requirements. 


\section{Section 1}

\section{The Project and Project Process}

$\mathrm{T}$ The purpose of the work effort is to determine the best approaches towards improving gas-turbine engine reliability and maintainability (RAM). The effort will focus on integrated control, and monitoring systems capabilities. This section describes the process Distregen used in evaluating and assessing RAM technologies.

In order to provide this work effort in the most efficient manner, several tasks were performed as outlined below. These tasks, and methodology for performing these tasks, have been chosen to provide the best information for the least cost.

The major question addressed in this report is: "What does the operator of gas turbines really want and need in monitoring and control capabilities to improve reliability, availability and maintainability (RAM)?"

This question is answered with a focus towards the end user. What do they really want and need for better RAM operation of their equipment. Interviews were held with several entities to answer this question. The interviews focused not only on the turbine but also on the entire balance of plant. This focus helped the interviewees to express concerns that were widely varied as well as to express wants and needs that covered the entire facility. These wants and needs had a common theme at each facility and across all facilities investigated.

Part of this effort is to investigate potential control and monitoring technologies. The process used to discover potential RAM technologies started by identifying potential purveyors of RAM technologies. Potential RAM technologies were identified based on capabilities of these purveyors and practices identified in the literature. A detailed review of the literature concerning these technologies and purveyors was performed.

Next in the project process was to qualify and interview packagers so as to determine end users wants and needs for improved reliability, availability and maintainability of gas-turbine engine systems. A visit was paid to one of the top packagers for an in-person interview and review of their standard packages in order to address these concerns. In addition, interviews were held with personnel responsible for operation and maintenance of power generation stations in order to understand their concerns under real life conditions.

Identified during these interviews were major concerns for reliability and maintainability expressed by end users of generation and those held by practices of the packager based on many years of experience by the interviewed packager.

Based on information obtained during the investigation, a list of potential monitoring and control system sensors and functions was developed. These technologies are prioritized for their potential for meeting customers RAM needs. Each of the technologies has been investigated for their potential benefits as well as potential downside to implementing such technologies. Special focus has been given to what benefits they bring to customers and the relative importance of such benefits.

Each of the highest rated RAM technologies were evaluated for potential benefits to the end customer. Each of these technologies was compared based on the value equation in order to determine top recommended technologies as well as top combinations of technologies. These technologies were recommended based on the value provided to the operations and maintenance as well as the relative cost and ease in implementing these projects. 


\section{Operators Needs and Wants}

$\mathrm{S}$ everal sites were visited in order to gain a better understanding of the operators needs and wants. A packager was chosen because of their understanding of what their customers really want and need. Operation sites were chosen in order to solicit opinions about the operation of the gas turbine systems. Sites that were visited include a gas turbine packager and two different operating gas turbine facilities. These sites were chosen for their similarity to the sizes and types of gas turbine design that are being considered as part of this project.

\begin{tabular}{l|c|c|c|}
\multicolumn{1}{c}{} & \multicolumn{2}{c}{ Packager } & \multicolumn{2}{c}{$\begin{array}{c}\text { Site 1 } \\
\text { Northern U.S. }\end{array}$} & \multicolumn{1}{c}{$\begin{array}{c}\text { Site } 2 \\
\text { Southern U.S. }\end{array}$} \\
\cline { 2 - 4 } Characteristic \\
\cline { 2 - 4 } Turbine Type & Gas Turbine Packager & Peaking Power Plant & Peaking Power Plant \\
\cline { 2 - 4 } Rating & Various & GE Fr 7EA & GE LM6000 SPRINT \\
\cline { 2 - 4 } & $15-50 \mathrm{MW}$ & 4 at 75 MW Each & 4 at 45 MW Each \\
\cline { 2 - 4 } Comments & Ready to Run Packages & $\begin{array}{c}\text { Ongoing Problems } \\
\text { Provide Good Example } \\
\text { of Operator Needs }\end{array}$ & $\begin{array}{c}\text { Newly Started Unit } \\
\text { Shows Shakeout } \\
\text { Problems }\end{array}$ \\
\cline { 2 - 4 } & &
\end{tabular}

Table 2-1 - Sites Visited and Characteristics

\section{Packager}

The packager provides complete gas turbine packages in the 15 to 50 megawatt range. They provide these packages with complete control and monitoring packages. These packages tend to be standard for all size and types of gas turbines supplied. There is very little modification from the standard package. This keeps the cost down and provides a better level of reliability than packages that are highly customized. They provide complete operational testing before sending the gas turbine out to the field. This usually provides for easy startup of the gas turbine system.

This packager was chosen because of their long-term success in providing gas turbines to utilities and industry. They have been known to be successful in understanding their customers wants and needs. Their control and monitoring systems have been based on years of experience, understanding the operation of turbines, and operation of the balance of plant. A packager is ideal for providing this kind of understanding because they are often the first one called when problems occur at a facility and have to make good in order to fulfill the warranty requirements as well as to maintain their reputation.

This packager uses standard sensors with limited use of the more innovative sensors available. They also use PLC's (Programmable Logic Controllers), specialized electronics, and PC's (Personal Computers) to provide a more sophisticated operating controls and monitoring system based on a simple set of sensors. They use this equipment along with detailed models of the gas turbine system in order to provide a finer level of control and information to the operator. This method of monitoring and control allows them to display what is happening throughout the gas turbine despite a limited amount of sensors 


\section{Site 1 - Peaking Power Plant in Northern United States}

This site was chosen because it has gas turbines in the size range that are of interest for this project. The site has four GE Fr 7EA gas turbines rated at $75 \mathrm{MW}$ each. This large of a

Characteristics

Peaking Power Plant

GE Fr 7EA

4 at $75 \mathrm{MW}$ Each

\begin{tabular}{|l|}
\multicolumn{1}{c|}{ Issues } \\
\hline Installed Summer 1999 \\
\hline Computer, Control \& Monitoring Systems \\
\hline Flame Scanner Problems \\
\hline $\begin{array}{l}\text { Sensoring \& Diagnostics Software Too } \\
\text { Complex to Setup }\end{array}$ \\
\hline Only 75\% of Sensors Accurate at any Time \\
\hline Too Many Local Alarms \\
\hline $\begin{array}{l}\text { Requires Dedicated Person to Keep Facility } \\
\text { Running }\end{array}$ \\
\hline
\end{tabular}

\section{Table 2-2 - Site 1 Characteristics \& Issues} machine provides a benchmark of types of sensors and controls that are available. In these particular units, there was a standard (basic) set of sensors. The application used sophisticated computer diagnostic tools in order to determine what was really happening in the turbine and throughout the facility. These tools help to determine the source of problems within the turbine and balance of plant. They also point out inefficiencies in operation of the system.

It is useful to understand the control and monitoring issues that this facility has experienced. These issues have had a major impact on the reliability, availability, maintainability, and ongoing operation of the facility. Although the vendor of the equipment has been responsive, the problems still have a major affect on RAM.

The units were originally put in operation in the summer of 1999. The troubles that are discussed have been with the units from day one and are still ongoing issues. Software problems are still an issue for the control and monitoring systems. The sophisticated computer diagnostic tools and human machine interface have particular issues that need to be addressed. In particular, the operation software is complex to setup. This tool requires a sophisticated programming skill to make sure the software is setup correctly and completely. In addition, this software needs to be setup often. The setup time can be a day to two weeks. The issue here is that the setup is too complex to be handled by facility personnel easily. The vendor is usually called in to handle the setup. Because of the complexity of the software, interplay between field engineers and home office engineers must often take place. The second part that makes this more complex is that of the proprietary nature of the software. Software setup may be required for a number of reasons, most of which are not under the control of operations. This setup time may therefore occur at the time when the generator is desperately needed to be in operation. The major problem is with the human machine interface (HMI). Without a correctly working HMI package, control of the unit is lost.

More controls have created more problems in many different aspects. One aspect is that of the complexity of the software and electronic systems. A more mundane aspect is that of handling the wiring that needs to provide the information from these controls. For instance, getting wires back from a sensor is not a trivial matter. In this instance, several wiring runbacks were placed too close to high temperature sections and led to failure of these wires. (The same sort of thing has been known from an electromagnetic interference from being inappropriately located on the generator side of a turbine generator package) The complexity of more conduits leading wiring back to the control area has resulted in a higher probability of problems. 


\section{Section 2}

Another issue is that access to many sensors was difficult. This results in extended outages when replacing sensors. In some cases sensor replacement requires removal of major equipment.

At this particular site, they also had considerable trouble with an SRV valve providing incorrect feedback to the control system. Another ongoing problem is that of flame scanners. The type of flame scanner they use are Silicon Carbide flame scanners that are considered more accurate in assessing flame temperature and flame out conditions. However, these sensors have had a number of failures due to overheating situations. This provides for serious problems since these sensors are an important part of the input to the software tool to understand the issues behind the operation of the turbine.

Other issues include that sensors of all types fail or fall out of calibration on a regular basis. This leads to problems in feedback that undermines the goal of reliable and efficient operation. Once sensors do fail, the access for replacement of these sensors is often difficult. This problem is so bad that this site identified that $75 \%$ of the conditions shown by sensors are real and the rest are incorrect due to failed sensors or sensor connections.

Due to sensor failure and other items, this site still has 3 years of punch list items to go. That is, it is expected that most of the startup bugs will be out of the system 5 years after the units were initially started. Not all of these are control and monitoring system issues, but many of them are.

Another major concern is that of just too many alarms. The station is meant to be operated remotely from a large power plant located a short distance away. The operators at that station complain that there are far too many alarms occurring. They ignore these alarms because they do not have the resources to cover such situations. Instead, full time maintenance people are kept at the remote site to handle alarms as they occur. Unfortunately, the plan on the generation site was to have it as an unmanned site. These problems create a situation where the business case for putting in this type of peaking station is considerably worse than was originally considered.

Other characteristics of the situation at this site provide some insight to the operation. One of the things that is liked by the operations and maintenance staff is the ability of the operating software to allow forcing some functions to a logic " 1 ". This is the equivalent of putting a jumper across a contact. This allows the operator to keep in control of startup and to eliminate interlocks that they believe are erroneously causing startup problems.

Emissions are critical for the operation of this facility. They have some real-time information in order to keep emissions at the expected levels. This is backed up by an independent emission monitoring station that is used to provide emission levels.

Communication with the operators is via a SCADA (Supervisory Control and Data Acquisition) system. This system provides a large amount of data back to the operators.

The information gained at this site shows that despite the sophistication in the controls and monitoring available, a number of control and monitoring issues create a bad RAM problem. This is due to the problem of often having difficulty of getting information for efficient, economic operation of the gas turbine system under what should be normal operating conditions. 


\section{Site 2 - Peaking Power Plant in Southern United States}

The site has generation units in the size range and technology that is comparable to the size that is being considered for this project. The site has four GE LM6000 SPRINT units. These

Characteristics

Peaking Power Plant

GE LM6000 SPRINT

4 at $45 \mathrm{MW}$ Each

\begin{tabular}{|l|}
\multicolumn{1}{c|}{ Issues } \\
\hline Started Summer 2001 \\
\hline Trips are Still a Regular Problem \\
\hline Auxiliaries are Major Cause of Trips \\
\hline Gas Leaks Detected \\
\hline Troubleshooting Difficult \\
\hline
\end{tabular}

\section{Table 2-3 - Site 2 Characteristics \& Issues} units are rated at $45 \mathrm{MW}$ each. This type of unit provides lower emissions by a water spray method. Sensors used are just a basic sensor set providing a fundamental measurement system. These units do have sophisticated computer diagnostic tools similar to the other site but with a simpler version of software.

The units were in service for just a few months when the site visit was performed. This enabled us to get a better handle on the startup issues and problems that are of concern.

There are two units located at this location. The operator is located on site to control both of the units. The operator and maintenance staff interviewed identified that it was a good thing they were on site because a gas leak occurred early in the process. Because they were on site, they were able to identify the problem and solve it before causing potential catastrophic problems by starting up one of the turbines.

False trips are still an ongoing problem. False trips occur for many reasons. Auxiliary systems are the major causes of trips. The chiller system was one of the earliest sources of problems. Considerable rework of the sensors used and logic was needed to stop the trips from occurring. The SCR system is still causing a considerable number of unit trips.

They have found the sophisticated software tools to be helpful in understanding what was going on in the operation of the facility. Of particular use are the trending and historic recording tools. These tools provide assistance in troubleshooting where historic information is of use. However, they had a number of bad sensors and the software tool would give them some misleading information. In addition, they have not been trained on many of the tools. The software interface is not intuitively obvious. They are spending considerable time to update their knowledge on the capability of the software tools they have at their disposal. For instance, they noticed a transient vibration problem. Using the software tools, they were able to understand the extent of the problem and the historic trend of the problem.

As a side-point, the sophisticated computer tools provided a lot of information. Unfortunately, it was not easy to understand the logic behind the information since logic diagrams and other drawings were not easily available.

This site also has emissions as a critical function. This location has a stand-alone emission monitoring system to record emission levels and alert them to abnormal emission levels.

This new facility has had its fair share of operating problems due to startup issues. It is apparent that the operators like the sophisticated tools included with their operation computer system. They are insistent on the needs to have a local presence as opposed to a remote operation. They are continuing to do the shakedown on these units as they get through their first summer of operation. They have also had considerable problems with sensors and control systems that have made trips of the system to be of major concern. Their concerns 
about emissions make them be very careful on their normal operation. The lack of appropriate logic diagrams cause a major issue when they have to troubleshoot with troubleshooting time sometimes lasting hours longer than they would have expected with appropriate software tools and drawings.

\section{Operator Wants, Needs, and Don't Want Items}

Operators and maintenance people have expressed major concerns for reliability and maintainability of control and monitoring systems. Based on the investigations summarized above, other discussions, and experience a list of needs and wants that operators would like to see was developed. Operators need both information and controls to keep a facility up and running.

\begin{tabular}{|l|l|l|}
\hline \multicolumn{1}{|c|}{ Overall Needs } & \multicolumn{1}{c|}{ Monitoring } & \multicolumn{1}{c|}{ Controls } \\
\hline Overview of Information & Current & Get to Full Power Quickly \\
\hline Easily Access Detailed Information & Historic & Keep the System Running \\
\hline Alarm Prioritization & Trends & Fast Acting Protective Systems \\
\hline Easy to Quickly Troubleshoot & Documentation & Overrides with Risk Assessment \\
\hline
\end{tabular}

Table 2-4 - Basic Operator Wants \& Needs

Under the category of overall needs is that of information. There is a variety of information that is needed for reliable and efficient operation. First, they need an overview of data that is available. Correctly displayed data will let them better understand what's going on in the operation of the gas turbine system and where they need to be focused for assessing potential operational troubles. From this overview of information they need to easily drill down to more detailed data. This data would include sensor data, summarized and aggregated sensor data, sensor data corrected to remove inaccuracies as well as logic diagrams, schematics, etc.

Historic data shows the operating characteristic of the facility. This type of information allows comparison between different periods of time. Only by seeing changes in data can the operator understand whether the information is telling of a present or imminent problem.

Current data needs to be as accurate as possible. Much too often data is inaccurate due to sensors that are not working properly. For instance, in one of the sites visited only $75 \%$ of the data was considered accurate. Data needs to be screened for its accuracy and legitimacy and displayed in such a way as to not mislead the operator.

Taking the historic and current data together and displaying it as a trend is a powerful tool for the operator. By easily being able to identify trends, the operator can be on the lookout for various signs of problems. Trends often will display inefficient operation, deteriorating operating efficiency, and potential for part failure.

Incorporated with any data presentation is that of documentation. Documentation should be readily available in both an electronic and paper format to make it easier for troubleshooting problems. We have already discussed that incomplete documentation has led to long delays in restarting gas turbines that have tripped off line.

Another area of information that needs to be considered is that of alarms. Alarms need to be prioritized based on the importance of the alarm to the operation of the gas turbine. Not all alarms need to be displayed to the operator in a critical type fashion. They can be displayed as an issue that needs to be addressed. Alarm prioritization helps the operator to determine 


\section{Section 2}

the true sense of what the situation is with the gas turbine and auxiliary systems. Proper prioritization will help the operator in making a correct decision on when the gas turbine needs to be brought down or can continue running. History has shown that if all alarms have equal priority or almost equal priority the number of alarms quickly exceeds the number that an operator can find useful for understanding the problem. This usually results in all alarms being ignored by the operator. They then depend on the protective systems to help avoid failures.

Once alarms occur or other problems are recognized from the information displayed, operators need to find the source or sources of the alarm or information of concern. Appropriate prioritization of alarms result in faster troubleshooting of problems than displays showing all alarms. Items like logic diagrams, wiring diagrams, schematics available both in electronic and paper formats are of help with the trouble shooting. The more real-time information that can be displayed on this documentation, the easier and quicker will be the troubleshooting task.

From a controls standpoint there are just two simple things the operator expects the control system to accomplish. They are to get up to full power quickly and once that is accomplished to keep on generating electricity without interruption to the needs of dispatch. These two simple things can be accomplished in many different ways. The way most operators express it is to just keep the controls simple. Some operators know that controls get in the way of reliable and efficient operation. They cause more problems by being there than if they were not there in the first place.

Operators understand the need for controls and interlocks that keep the operator from accidentally causing a failure in parts of the gas turbine system or the gas turbine itself. What they don't like, is these controls can cause trips or long outages due to events that may be relatively minor or due to inaccurate sensors or other equipment. In order to help alleviate such problems, operators are looking for a way of taking an intelligent risk in order to get the gas turbine up and running when it is needed.

Not having the gas turbine up and running, can often cost the operator's organization thousands of dollars for every hour that the gas turbine is needed but is not running. They want tools that will help them make an intelligent decision to make the tradeoff between the economics of generating power under less than ideal circumstances, economics of turbine operation, efficiency of the system, and potential for damage to the gas turbine system.

In order to make such a decision the operator needs to understand whether the various systems that are part of the gas turbine meet minimal requirements for safe operation. They need to understand the tradeoffs for sub-optimal operation.

For instance, will operation shorten the life of the turbine or generator and how much will the life be shortened by operation? The answer to such a question can determine whether the operator should consider operation under the specific contract circumstances they are working under.

Once a tradeoff is determined, they need to have the ability to act on the tradeoff. In the old days, controls and logic systems were temporarily defeated by putting jumpers across certain contacts. Today, they need to have the ability to temporarily defeat certain software controls in order to get the turbine up and running. 


\section{Section 2}

When looking at a needs and wants list we would also like to look at what the operator does not want. These items are what operators have seen cause them much trouble in performing their primary role.

They do not want to see more complexity in the control systems. The more complex the system the more difficult it is to understand and the more difficult it is to troubleshoot problems. The more complex systems also have more components to fail and software bugs that need fixing.

Operators have been dealing with inaccurate information for years. It is most often better to have no information rather than inaccurate information. It would be very helpful if the monitoring system can help the operator to determine whether a piece of information is accurate or is suspect.

Operators and maintenance requires tools that are user friendly and require little training. The issue is that it may take several months or years before the operators are able to get the training that is needed to best use the tools. For instance, many gas turbine packages include tools that are quite sophisticated both for operations and for troubleshooting. However, because they require extensive training they are either not used or are used with the risk of the information portraying a result that can be easily misinterpreted.

Operators are not interested in more data. They get much more data than they can assimilate. In most operating environments, many screens of data are available. However, under most circumstances the operators use only two or three screens for most operations and troubleshooting situations. What they want is information that is of particular use under the present circumstances of operation. Useful information, as opposed to raw data, is focused on clarifying problems and identifying issues. When they are starting up the gas turbine system, they need different information than when they are running normally. When they are getting alarms, they need to have easy access to data applicable to the alarm and less information that is used during other operational circumstances.

Operators also do not need protective systems that produce trips or lockouts that are hard to trace. Such systems should easily identify the cause of the trip or lockout for simple devices and propose possible causes for trips or lockouts that are more complex. Often, the troubleshooting process for such circumstances takes hours. The result of the troubleshooting often being a relatively minor issue.

Operators really want simple controls, reliable sensors, and software that provides important information. The more bells and whistles, the more complicated the operational environment, the lower the RAM. Simple and intelligent control environments lead to higher reliability and availability as well as lower costs and time to maintain the gas turbine and auxiliary systems. 


\section{Control and Monitoring Technologies}

$\mathrm{I}$ n order to gain an understanding of how the needs and wants of the operators can be implemented we need to understand existing and potential control and monitoring technologies. The technologies considered will pertain to the entire gas turbine environment. That is, the gas turbine and auxiliary systems. These technologies include existing and potential new technologies that have a high potential to meet customers RAM concerns as expressed elsewhere in this document.

\section{Strategy for Implementing Sensor and Control Technologies}

Before looking at actual technologies that could be considered, lets first look at the strategy of implementing such sensors and controls.

\begin{tabular}{|c|c|}
\hline \multicolumn{2}{|r|}{ Sensor \& Control Technology Strategy } \\
\hline & $\begin{array}{l}\text { itor Critical Parameters - Pressure, } \\
\text { perature, Flow, etc. }\end{array}$ \\
\hline & $\begin{array}{l}\text { Automate Controls - Quick Reactions, } \\
\text { Lockout Operation }\end{array}$ \\
\hline Q & $\begin{array}{l}\text { Advanced Diagnostics \& Prognostics } \\
\text { Capabilities }\end{array}$ \\
\hline
\end{tabular}

Table 3-1 - Sensor \& Control Technology Strategy
The strategy is based on keeping things simple while providing useful information and control. The first item is to monitor critical parameters. Such parameters include such items as pressure, temperature, flow rates, etc. Parameters monitored should provide useful information about the gas turbine or critical auxiliary systems.

Controls must be automated to as full an extent as possible. This allows for quick reaction to changes in conditions. A fully automated set of controls will help prevent start up or continued operation under critical situations.

Advance diagnostic and prognostic capabilities are an important part of the strategy of implementing sensor and control technologies. These capabilities will allow for greatly decreased time in troubleshooting problems as well as understanding where potential problems may be forthcoming. Sequence of events recording is the first part of the diagnostic picture. Complete data on what happened during events or points in time are the first critical part of diagnosis. Sophisticated capabilities would provide not only rough data but also potential interpretations of the data. These interpretation could be based on experience with the local machine, experience with the type of machine, and results of problems that were historically experienced and are presently being experienced with similar machines.

Trending capabilities is a very useful tool for a variety of troubleshooting and predictive analyses that an operator may want and need to entertain. Trending capabilities provide historic data that show individual sensors as well as groups of sensors and other calculated data to provide a historic trend-line. Analysis of this trend-line will help the operator to understand what happened or assist him in predicting what the result of continued operation in the trend may be. An even better approach is to use the trend line to do some predictive modeling what the future trend may look like. This approach may be of great help in assessing what operational changes might be needed and what maintenance tasks need to be performed in a time frame based on what is seen in the trends. Such an approach may greatly increase reliability and availability. 


\section{Section 3}

The next stage in advanced diagnostic and prognostics capabilities is that of predictive capabilities. These can be thought of as using techniques such as artificial intelligence. Predictive capabilities can match up experiences through similar machines and auxiliary systems with data and trends that have been experienced by these machines. It would be able to point out to the operator potential problems with the gas turbine system, possible solutions to the problem, and what the results may be under different operating scenarios. This kind of tool has the potential for providing an "alarm" to tell the operator of an upcoming condition instead of alarming when the condition happens. This type of method gives the operator more flexibility to make intelligent decisions based not only on the gas turbine system parameters but also on the needs of the electric power system and economics of providing electric power under the present circumstances.

The strategy of sensor and control technology implementation is to make startup, normal operation, and abnormal operation easier for the operator to deal with.

\section{Monitoring and Control Technologies}

There are a variety of monitoring and control technologies that can be used for gas turbine systems. The tables on the following pages display some of the technologies that are presently implemented or can be considered for implementation in a gas turbine package.

The following table (Table 3-2) displays the condition monitoring technologies used for engine monitoring. The table displays a variety of technologies used for monitoring the condition of the engine. Each technology provides a certain type or types of measurement and/or provides a function that is important for the operation of a gas turbine.

Lubricating oil is an important material in the operation of a gas turbine. The oil must be

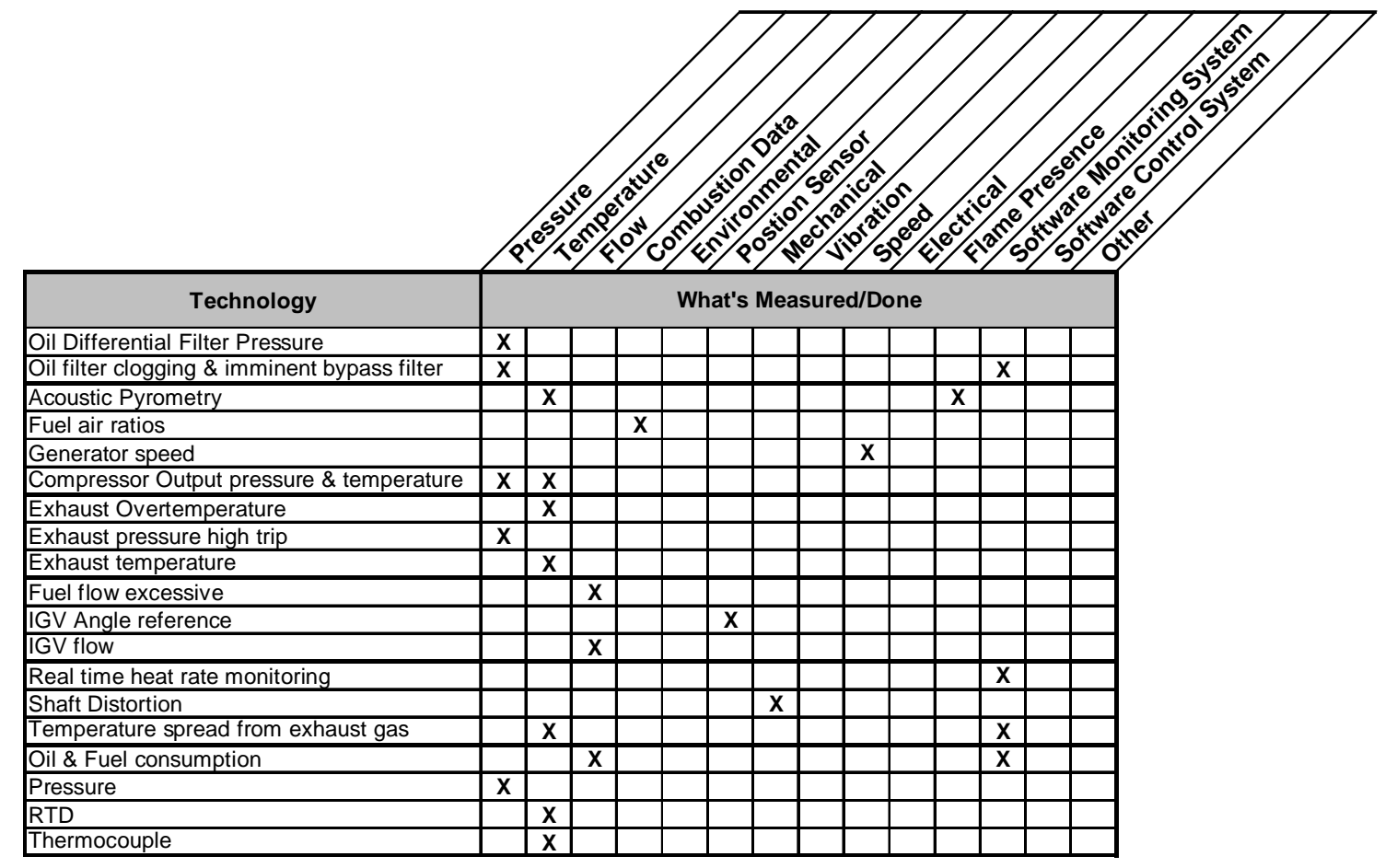

Table 3-2 - Condition Monitoring Technologies for Engine Monitoring 


\section{Section 3}

clean and provided to each of the lubricating points to the gas turbine at the appropriate pressure. Many modern gas turbines measure the difference in pressure across the filter in order to determine when an oil filter is clogged. This differential pressure system is alarmed to identify to the operator the need for maintenance of the filter. Bypassing of the filter so as to continue oil flow is a common method used in gas turbines and in some auxiliary equipment. This function has a high impact on RAM and therefore should be considered in any gas turbine package.

Acoustic pyrometry is a method in its early stage of development to provide a better function of flame detection and flame intensity. As this technology and other flame detection/intensity functions mature, these technologies will provide for better operating characteristics. Their biggest impact on RAM will be to quickly detect flameout conditions.

The fuel/air ratio is critical for the more efficient operation of a gas turbine. Unfortunately, at this time accurate and cost effective fuel/air ratio sensing is not readily available. The development of such a sensing system would be a strong positive to the RAM and efficient operation of a gas turbine.

Generator or turbine speed is a critical function to measure. Overspeed protection is critical to avoid excessive damage to the equipment and facility. Fortunately, this is a mature technology that should be in place on every turbine in existence.

Compressor pressure and temperature as well as the temperature and pressure of the gas turbine exhaust provide important information for the efficient operation of the gas turbine. Common technologies are employed to provide these measurements. Each of these technologies is relatively mature but have their tradeoffs in accuracy and cost. These are discussed for each type of sensor later in the discussion of this table.

Excessive fuel flow is just a common alarm used in many gas turbine systems using readily available flow information. This type of alarm especially when coupled with protective action provides greatly increased RAM since it alerts the operator to imminent problems with the machine.

Inlet guide vane control provides important information to the operator on the operation of the gas turbine. Common position sensors and flow sensors are used in such circumstances. These are mature technologies that are reasonably accurate.

In today's electric market environment real time heat rate monitoring is an important function for determining the economics of dispatching any particular unit. Presently, several inputs are used to calculate the heat rate. However, the calculation is only as good as the measurement devices and the massaging of these inputs to create a heat rate number. Further development of methods of more accurately measuring actual heat rate and predicting potential heat rate for the upcoming generating timeframe would be of great use to more economically dispatch the units. The impact on RAM is small, but the impact on the economics of operations of the gas turbine is large.

Technologies to measure shaft distortion are in their early stages of development. This technology would be of great advantage to increase the RAM of a gas turbine package. As the development of these technologies mature, the inclusion of these technologies would be important to implement for the increase of RAM.

One method used to determine what is happening in a gas turbine is that of measuring temperature spread at the exhaust of the turbine. This measurement, tied together with 


\section{Section 3}

appropriate software, provides a means to understand whether the gas turbine is operating appropriately. This method provides a great increase in RAM by using software to look inside an almost impossible to measure environment. Further development of this type of software algorithms would provide more detailed information with its increased RAM results.

Oil and fuel consumed are normally measured as part of most gas turbine packages. It is important for RAM that abnormal consumption of oil or fuel for operating conditions in place is treated as a critical situation.

The most common measurement on a gas turbine is that of pressure and temperature. RTD's typically handle temperature measurements in electrical equipment and other environments where the temperatures are tens of degrees greater than ambient temperature. Thermocouples are typically used in the gas path and other high temperature environment. All of these measurement devices are quite mature. However, they all need ongoing calibration. This means that the data from these devices normally has limited accuracy. Development of more accurate sensors is ongoing with some digital type sensors now being available off the shelf. The more accurate sensors in certain applications will provide a greatly improved level of RAM.

The following table (Table 3-3) displays condition monitoring of areas in the gas turbine generating facility other than the engine. This monitoring is typically of auxiliary equipment located in the station. Failure of almost any individual piece of auxiliary equipment may cause a shutdown of the gas turbine having a large negative impact on RAM.

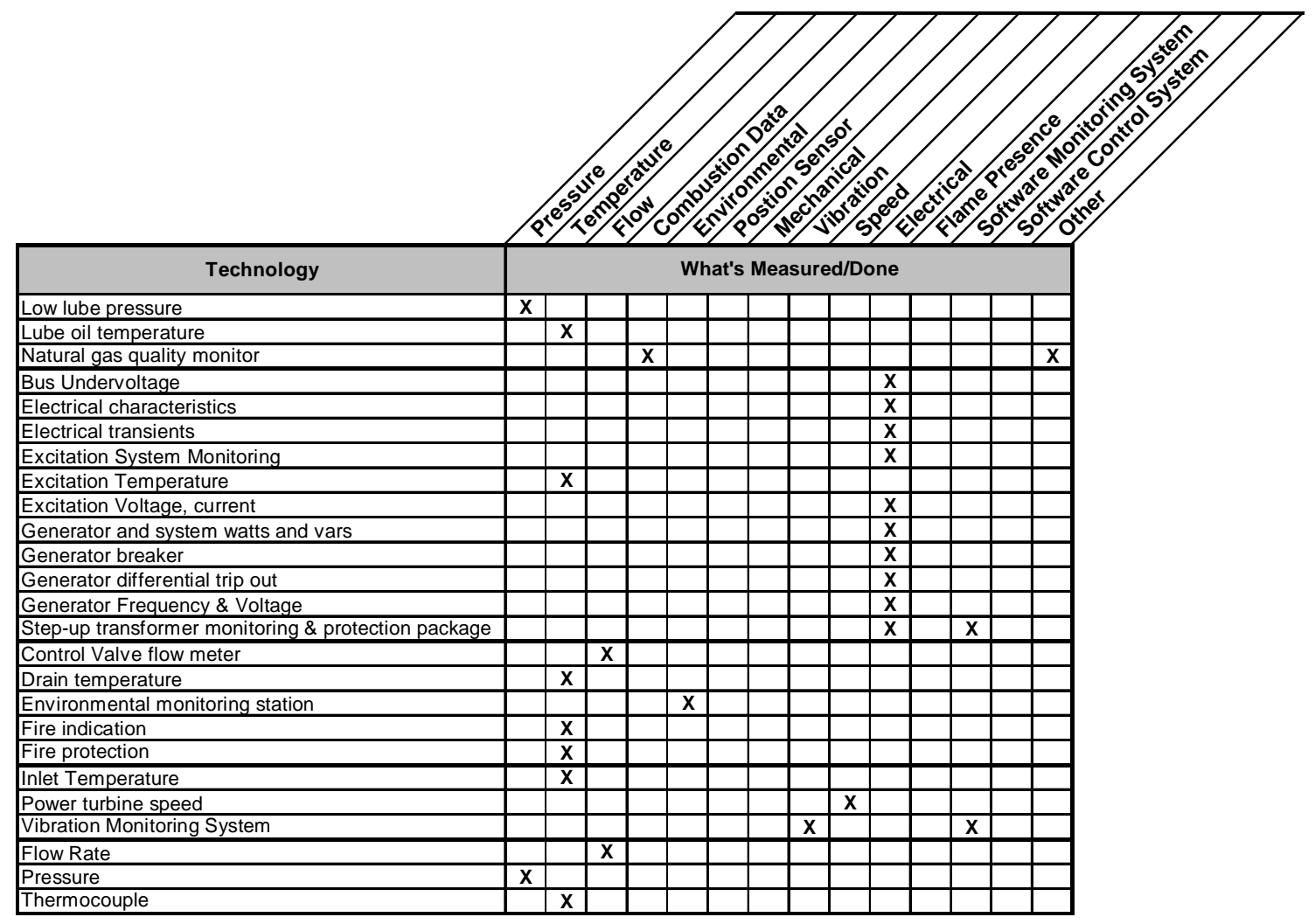

Table 3-3 - Condition Monitoring Technologies for Other Areas 


\section{Section 3}

Lubrication oil is used in many different types of equipment throughout a facility. Lubrication oil pressure and temperature are critical measurements for the RAM of auxiliary equipment. These are mature technologies that need to be implemented with the appropriate alarm and control systems.

The next few items in the table, from bus under-voltage to step-up transformer monitoring and protection are all part of the electrical system. These technologies are mature technologies that are standard in most installations. The amount of data available from these systems is quite large and at a high level of accuracy. To keep RAM at high levels it is important that protection packages are set properly, appropriate alarms are hooked up, and other information is fed back to the control center.

Control valves, flow meters, and drain temperatures are standard measurements that are incorporated in the control systems and provide data to the operator for more efficient operation of the gas turbine. These use common technologies commented on in the discussion of the previous table.

Emissions monitoring is one of the most critical issues for most of the generating facilities. State of the art monitoring systems are readily available off the shelf to provide accurate emissions data in both real-time and historic venues. Many facilities are limited by the total emissions they can produce. Therefore, real-time data incorporated in the control center with other operating parameters is an important piece of data to keep the gas turbine system available.

Fire indication and protection systems are critical in this highly flammable environment. Several different mature off-the-shelf technologies exist for high-speed recognition and control of a fire.

Power turbine speed is an important measurement, using common technologies. The generator is typically protected against overspeed situations through mature technologies. Power turbine speed is also an important quantity to be monitored before the generator is synchronized with the power system.

Vibration monitoring systems are mature. These systems use sophisticated equipment and software to determine whether the gas turbine system is operating properly, the level of alarm to be issued, or whether the turbine should be tripped off line due to excessive vibration.

Other measurements that are critical as feedback for control functions and ongoing monitoring include flow rate, pressure, and temperature. Comments on these areas can be found in the discussion of the previous table.

The following table (Table 3-4 on the next page) displays high temperature sensing needs. The most important of the high temperature sensing is that of flame detection and flame intensity. Flame detectors take on a variety of methods. Even the most mature of these technologies do not provide high accuracy and quick feedback. Research is continuing on many fronts to improve the accuracy and speed of flame detection. However, the best equipment available will increase the RAM of the system since the avoiding of a flameout condition is critical to the efficient operation of a gas turbine. 


\section{Section 3}

Flame intensity is even a more difficult measurement. Highly accurate methods of measurement are being implemented in some off the shelf products, but much research continues. At least one of the products used in the sites visited had ongoing problems with damage due to the high heat environment. The better measurements that can be obtained of flame intensity, the better the RAM and the efficiency of the gas turbine.

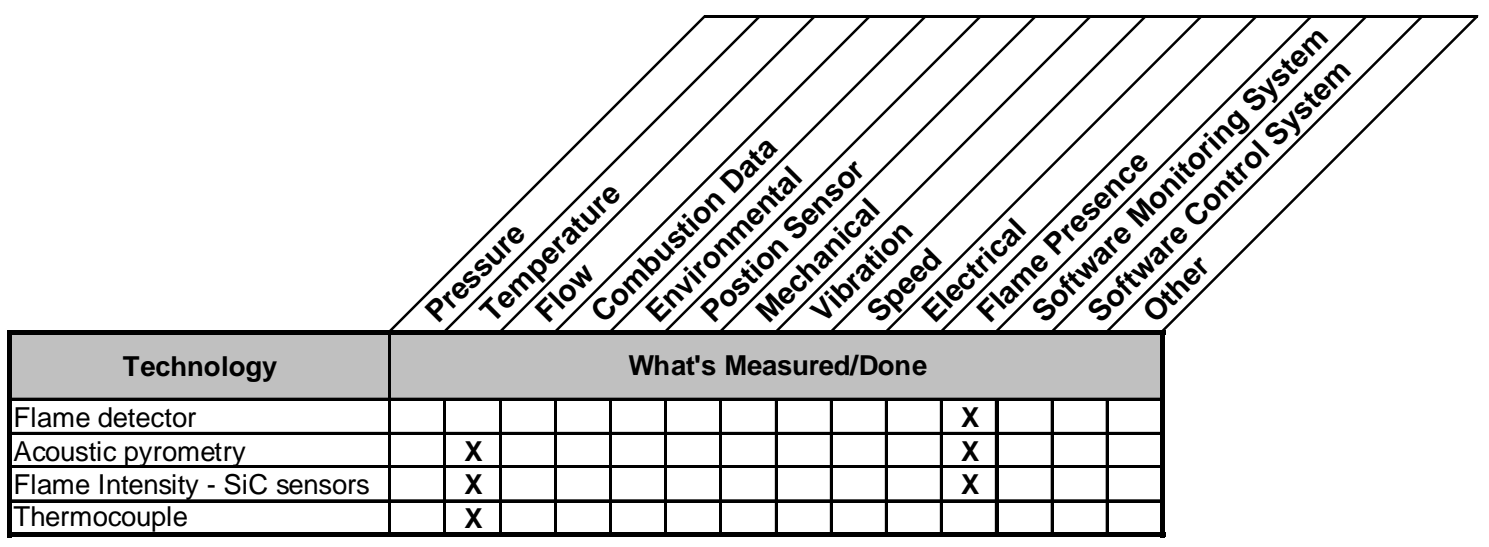

Table 3-4 - High Temperature Sensing Needs

The following table (Table 3-5) displays controls capabilities of gas turbine and auxiliary equipment. The control capabilities displayed range from common control capabilities implemented on many gas turbine systems to control concepts under research.

Several technologies are highlighted in the table that pertain to the combustion system. These controls are designed to sense whether a flame exists and its intensity. This data is used to control all the dynamics of operation of the combustors. Several efforts are being done to improve the control of the system. Active noise control is being actively pursued as an active combustion control technology. Other control technologies displayed are efforts that are continuing to perfect the operation of the combustion system. Fuel/air ratio control is

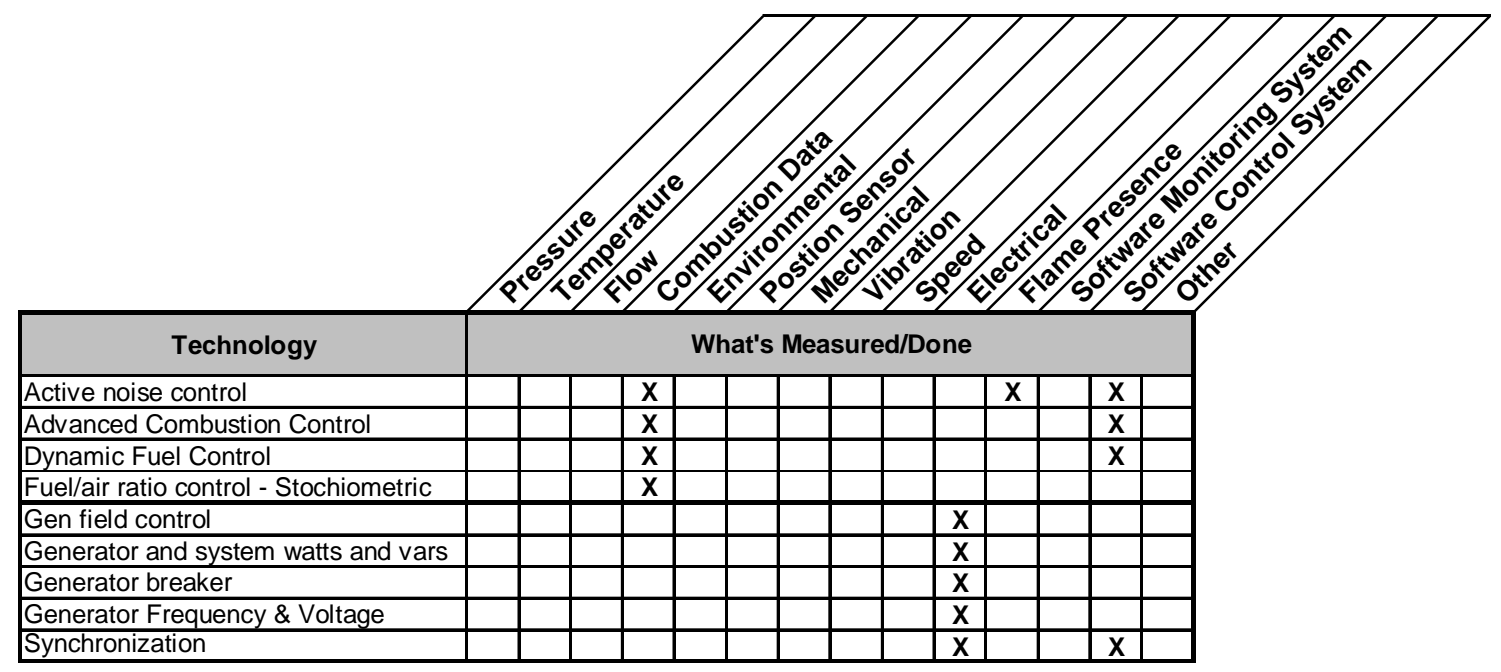

Table 3-5 - Control Capabilities of Equipment 


\section{Section 3}

an area that is ripe for further development.

The electrical system has a variety of controls so that the generator produces electrical energy of the correct voltage, frequency and in the correct amount of Megawatts as well as the correct amount of reactive power. These are mature technologies that produce highly accurate results.

The following table (Table 3-6) displays diagnostic and prognostic capabilities of monitoring and control systems.

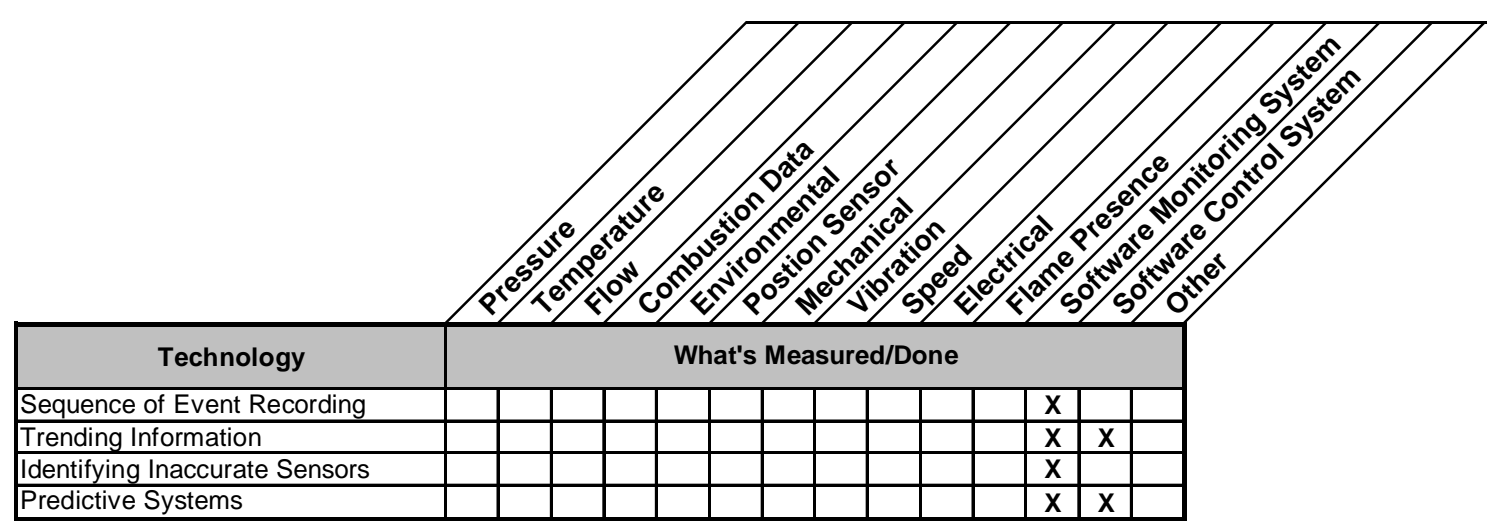

Table 3-6- Diagnostic \& Prognostic Capabilities

The most commonly used diagnostic package is that of sequence of event recorders. These standalone devices have been available for years. Many of the gas turbine software control systems provide essentially the same function incorporated in the software package itself. This is a very useful tool for understanding the history of an event and troubleshooting problems.

Some software packages included as part of the gas turbine monitoring software includes trending capabilities. The ability to see the trend of important parameters of the gas turbine operation can help an operator and maintenance to anticipate problems before they occur. This type of package along with active analysis can greatly improve RAM. Unfortunately, most operators and associated maintenance do not perform such an active analysis even if the software is readily accessible.

Some, but very limited work has been done on identifying inaccurate sensors and predictive systems. Most common sensors require considerable effort in calibrating. It is not uncommon to see $25 \%$ or more of the sensors to be inaccurate or not working at all. This provides data to the operator that is inaccurate and often misleading. More intelligent software packages would look for inconsistencies and identify potentially inaccurate sensors. This effort would greatly improve RAM. This improvement in RAM is due to the operator being able to more easily troubleshoot problems as well as more easily identify problems before they cause an outage. Such software would be a good development project.

Very few systems have even rudimentary predictive capability. This kind of capability is the follow-on to active analysis of information by the operator and maintenance personnel. Predictive software takes the current and historic information and develops a prediction of the operating behavior of the gas turbine system. The ultimate in this type of software would provide an alert of potential abnormal operation as well as alerts for changes in maintenance 


\section{Section 3}

schedule required to keep the gas turbine system running. Such a system would have a major impact on RAM as well as operating cost of the gas turbine system. RAM would be greatly improved since operating procedures could be changed to avoid exacerbating a problem and repairs could be done during a scheduled outage. RAM would also improve since outages would be scheduled with a particular problem in mind where appropriate equipment and labor would be ready to perform the assigned task. Costs would improve due to the higher probability of solving problems before they turn into expensive forced outages or damage to equipment. This effort would be a great development project to pursue both for its anticipated large improvement in RAM and operating cost improvement.

The major problem for operators with data and data analysis is that of getting the right amount of accurate information. Many gas turbine products provide vast amount of data with limited capabilities for checking the reasonableness of the data being forwarded by such a system. Individual sensors are still independent of any other data. When we start looking at software packages the solution is even more of a problem. At present most software systems have limited capability and are a long way from providing advanced solutions to meet operations needs. Software that is in place typically has many errors that cause many problems. The result of all this is that in today's environment operators are often given nonsensical results that take a lot of time for interpretation. This results in poor reliability and availability as well as excessive maintenance costs. Software discussed in this section would go a long way toward correcting this issue.

The following table (Table 3-7) displays other plant monitoring and control systems that may pertain to gas turbine operations.

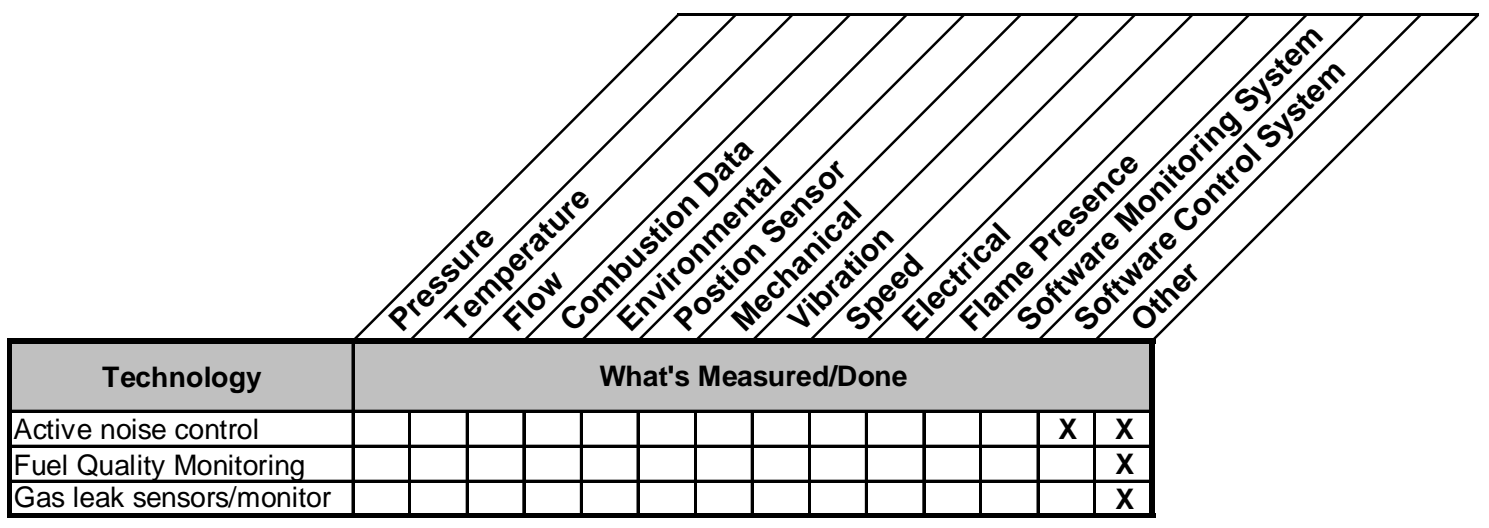

Table 3-7 - Other Plant Monitoring \& Control Systems

Active noise control in this context is a methodology for lowering the level of noise produced by gas turbines and auxiliary equipment by emitting a sound at each frequency out of phase with the noise. This tends to cancel out the noise in those particular frequencies. Although this is not a RAM issue, it has the possibility of decreasing the cost of sound barrier walls.

Fuel quality is a large issue in the efficient operation of a gas turbine. Natural gas has a large variability in heat content and impurities not only between different locations but also at the same location at different points in time. Fuel quality monitors would provide feedback that would confirm the fuel quality but would also provide the change of operations needed to better use the existing fuel. This has an impact on RAM since fuel quality can have an 


\section{Section 3}

extensive effect on turbine life. Some operating procedures can help offset different fuel quality in positive ways.

Gas leak monitoring is an important issue. It is not uncommon for gas leaks to occur due to the large amount of natural gas available to operate the turbines. RAM improvements can be had since an appropriate set of gas leak monitors would help to pinpoint possible sources of the gas leak more quickly than by other methods.

We have reviewed a variety of technologies that are either in common use or are in development. In the next section we will look at how each of these technologies provide benefits to RAM and meet operators needs. 


\section{Potential Benefits of Control \& Monitoring Technologies for Meeting Operators Needs}

I

$\mathrm{n}$ this section we will explore potential RAM benefits of several control and monitoring functions from the viewpoint of meeting operators needs. We reviewed in the previous section various technologies that are incorporated or are in the development stage for incorporating into gas turbine systems. These technologies provide a great many of function for the monitoring and control of gas turbine systems. However, the technologies may or may not meet the needs of the operators as we explored earlier in this document.

We have identified several operator needs, in Section 2 of this document, which should be addressed by the technology. Table 4-1 displays these operator needs. The needs have been categorized by overall needs, monitoring, and controls.

\begin{tabular}{|l|l|l|}
\hline \multicolumn{1}{|c|}{ Overall Needs } & \multicolumn{1}{c|}{ Monitoring } & \multicolumn{1}{c|}{ Controls } \\
\hline Overview of Information & Current & Get to Full Power Quickly \\
\hline Easily Access Detailed Information & Historic & Keep the System Running \\
\hline Alarm Prioritization & Trends & Fast Acting Protective Systems \\
\hline Easy to Quickly Troubleshoot & Documentation & Overrides with Risk Assessment \\
\hline
\end{tabular}

Table 4-1 - Basic Operator Needs

On the next few pages we will review the operator needs and map them to technologies that appear to meet or attempt to meet these needs. The discussion includes the potential benefit of implementing these technologies and relative value of these solutions to meeting operators needs.

In each of the tables that follow we look at a particular operator needs and map them against potential functions or technological solutions. Each of these solutions is also analyzed for how well they meet basic operator needs.

Table 4-2 (on the following page) takes a look at operator needs and potential function/technology solutions in the turbine and combustion systems. The first of the operator needs displayed in the table is that of being able to monitor and control the combustion operating characteristics. Most of the functions/technologies listed provide a fundamental information point that either is or can be used directly in the control system. Operator monitoring of this data can provide useful information on any manual setting the operator may want to use to better fine tune the combustion operating characteristics.

Monitoring of gas quality provides information so the operator or the system directly can operate properly for different heat content and impurity levels found in the fuel. Dynamic fuel control and advanced combustion control makes the operator's job easier by automating adjustments needed to improve the efficiency, the emissions, and the RAM of the machine.

Fuel/air ratio sensors and controls would greatly improve the combustion characteristics. Development of an advanced sensor would provide the opportunity for improved efficiency and improved RAM capabilities. 


\begin{tabular}{|c|c|c|c|c|c|c|c|c|c|c|c|c|}
\hline & & 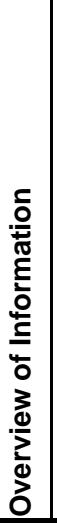 & 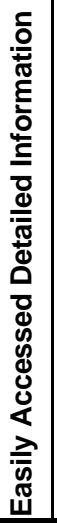 & 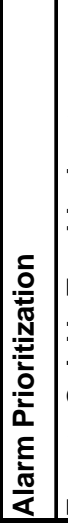 & 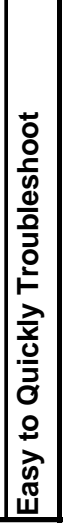 & 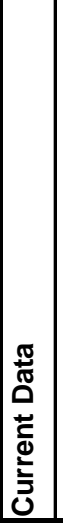 & 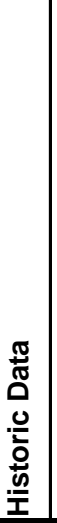 & 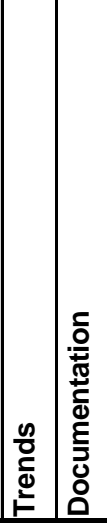 & 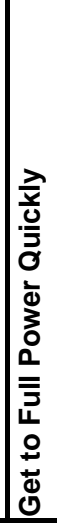 & 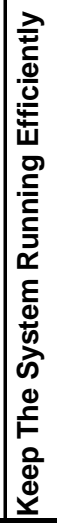 & 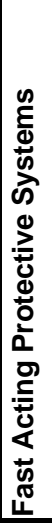 & 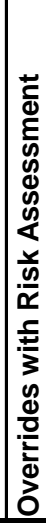 \\
\hline Operator Need & Function/Technology & \multicolumn{4}{|c|}{ Overall Needs } & \multicolumn{3}{|c|}{ Monitoring } & \multicolumn{4}{|c|}{ Controls } \\
\hline \multirow{9}{*}{ 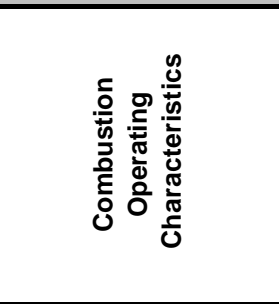 } & Gas Quality Monitor & & $\mathbf{X}$ & & & $\mathbf{X}$ & $\mathbf{X}$ & & & $\mathbf{X}$ & & \\
\hline & Dynamic Fuel Control & & & & & & & & $\mathbf{X}$ & $\mathbf{X}$ & & \\
\hline & Advanced Combustion Control & & & & & & & & $\mathbf{X}$ & $\mathbf{X}$ & & \\
\hline & Combustion Data & $\mathbf{X}$ & $\mathbf{X}$ & & & $\mathbf{X}$ & $\mathbf{X}$ & & & $\mathbf{X}$ & & \\
\hline & Fuel/Air Ratio Sensor \& Control & & $\mathbf{X}$ & & & $\mathbf{X}$ & & & & $\mathbf{X}$ & & \\
\hline & Acoustic Pyrometry & & $\mathbf{X}$ & & & $\mathbf{X}$ & & & & $\mathbf{X}$ & & \\
\hline & Flame detector & & & & & & & & & $\mathbf{X}$ & & \\
\hline & Flame intensity & & & & & $\mathbf{X}$ & & & & $\mathbf{X}$ & & \\
\hline & Flame Intensity - SiC sensors & & $\mathbf{X}$ & & & $\mathbf{X}$ & & & & $\mathbf{X}$ & & \\
\hline Turbine System & Compressor bleed valve postion trouble trip & & & & & & & & & & $\mathbf{X}$ & \\
\hline Control \& Protection & Discrete sequencing \& protection & & & & & & & & $\mathbf{X}$ & $\mathbf{X}$ & $\mathbf{X}$ & \\
\hline \multirow{16}{*}{ 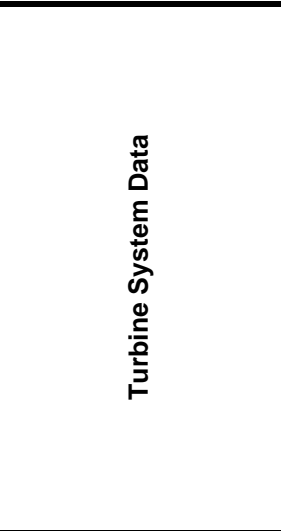 } & Flow Rate & & & & & $\mathbf{X}$ & & & & & & \\
\hline & Fuel flow excessive & & & & & & & & & & $\mathbf{X}$ & \\
\hline & Vane Flow & & & & & $\mathbf{X}$ & & & & $\mathbf{X}$ & & \\
\hline & Exhaust pressure high trip & & & & & & & & & & $\mathbf{X}$ & \\
\hline & Temperature spread from exhaust gas & $\mathbf{X}$ & $\mathbf{X}$ & & & & & & & $\mathbf{X}$ & & \\
\hline & Wheelspace temperature Measurement & & & & & $\mathbf{X}$ & & & & & & \\
\hline & Compressor Output pressure \& temperature & & $\mathbf{X}$ & & & $\mathbf{X}$ & & & $\mathbf{X}$ & $\mathbf{X}$ & & \\
\hline & Drain temperature & & $\mathbf{X}$ & & & $\mathbf{X}$ & & & & $\mathbf{X}$ & & \\
\hline & Exhaust Overtemperature & & $\mathbf{X}$ & & & $\mathbf{X}$ & & & & $\mathbf{X}$ & $\mathbf{X}$ & \\
\hline & Exhaust temperature & & $\mathbf{X}$ & & & $\mathbf{X}$ & & & & $\mathbf{X}$ & & \\
\hline & Inlet Temperature & & $\mathbf{X}$ & & & $\mathbf{X}$ & & & & $\mathbf{X}$ & & \\
\hline & Inlet Heating CV position & & & & & & & & & $\mathbf{X}$ & & \\
\hline & Engine Speed & & $\mathbf{X}$ & & & $\mathbf{X}$ & & & & $\mathbf{X}$ & $\mathbf{X}$ & \\
\hline & Power turbine speed & & & & & & & & & & $\mathbf{X}$ & \\
\hline & Shaft Distortion & & & & & $\mathbf{X}$ & & & & & & \\
\hline & Real time heat rate monitoring & $\mathbf{X}$ & $\mathbf{X}$ & & & $\mathbf{X}$ & $\mathbf{X}$ & & & $\mathbf{X}$ & & \\
\hline
\end{tabular}

Discussed earlier in this document is the importance of flame detection and flame intensity in the operation of a gas turbine system. The operator need for improved combustion operating characteristics would be successfully met by the use of the different flame detection and intensity sensors displayed here. The incorporation of these sensors to provide information to the operator and directly control aspects of the combustion system based on this feedback goes a long way toward meeting the operators need. Flame detection systems are important, but sensors that provide more detailed flame intensity provide a much better solution. History has shown, that further development needs to take place to have a higher accuracy flame intensity sensors. As we mentioned earlier, some commonly available products have been having troubles with overheating. This is of course creates a worse RAM situation than not having the device in the first place. 


\section{Section 4}

Operators need highly reliable turbine system control and turbine protection systems. There are a number of commonly available packages that are commonly used. Simple control and protection is easily accomplished to meet operator needs.

There are a variety of turbine system data sensors available. Some of the data sensors portrayed in the table are used as part of the feedback in the control system of most operating turbine systems. Other data sensors are available to portray the data to operations. In all cases, the concern is that of presenting the data in a format to keep the operator from being overwhelmed with data yet having precisely the data that is needed during troubleshooting. Accuracy of the data is also critically important to keep from misleading the operator with bad information. A higher level of RAM will result from better controls off of more accurate data sensors while appropriate display of accurate data will result in less misoperation of equipment due to operator concerns. This also would result in an improvement in RAM.

The last line item in turbine system data is that of Real Time Heat Rate Monitoring. This data will help the operator in making the decision on whether the generator should be operating under any given set of electric market conditions. This critical economic function will help the operator in making the tradeoffs between operating or not operating at any point in time.

Table 4-3 displays some of the functions/technologies used for monitoring and control of turbine and auxiliary system data. The functions/technologies displayed in this table provide

\begin{tabular}{|c|c|c|c|c|c|c|c|c|c|c|c|c|c|}
\hline & & 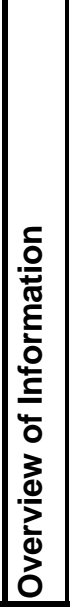 & 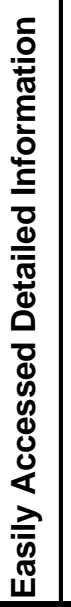 & 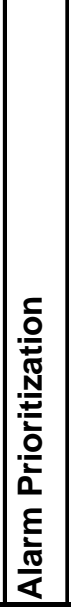 & 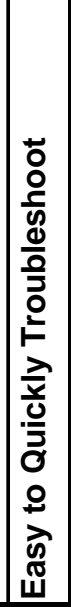 & 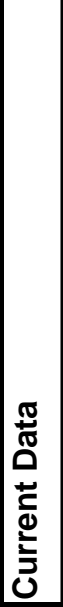 & 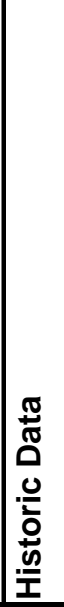 & 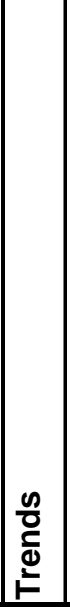 & 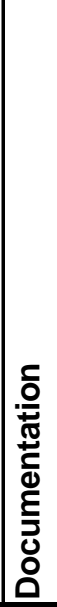 & 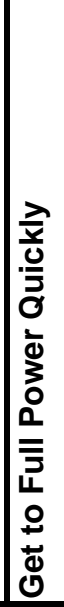 & 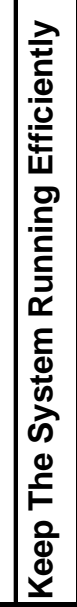 & 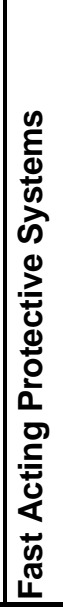 & 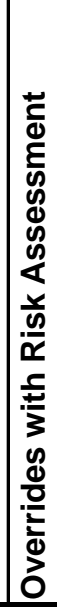 \\
\hline \begin{tabular}{|c|} 
Operator \\
Need
\end{tabular} & Function/Technology & \multicolumn{4}{|c|}{ Overall Needs } & \multicolumn{4}{|c|}{ Monitoring } & \multicolumn{4}{|c|}{ Controls } \\
\hline \multirow{8}{*}{ 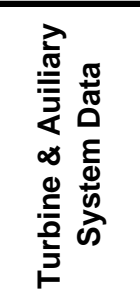 } & Low lube pressure & & & & & $\mathbf{X}$ & & & & & & $\overline{\mathbf{X}}$ & \\
\hline & Lube oil termperature & & & & & $\mathbf{X}$ & & & & & & $\mathbf{X}$ & \\
\hline & Oil filter clogging \& imminent bypass filter & & & & & & & & & & $\mathbf{X}$ & & \\
\hline & Control Valve flow meter & & $\mathbf{X}$ & & & $\mathbf{X}$ & & & & $\mathbf{X}$ & $\mathbf{X}$ & & \\
\hline & Pressure & & $\mathbf{X}$ & & & $\mathbf{X}$ & & & & $\mathbf{X}$ & $\mathbf{X}$ & & \\
\hline & Thermocouple & & $\mathbf{X}$ & & & $\mathbf{X}$ & & & & $\mathbf{X}$ & $\mathbf{X}$ & & \\
\hline & Oil \& Fuel consumption & & & & & $\mathbf{X}$ & $\mathbf{X}$ & & & & $\mathbf{X}$ & & \\
\hline & RTD & & & & & $\mathbf{X}$ & & & & & & & \\
\hline
\end{tabular}

Table 4-3 - Operator Needs by Function Turbine \& Auxiliary Systems 


\section{Section 4}

a means of monitoring, controlling and protecting various auxiliary equipment that provides critical service to the turbine. These devices are commonly used in such functions and have a reliable life. We have previously discussed the importance of oil pressure and temperature and the ability to assess whether an oil filter is clogged. These functions should be easily handled in today's environment. However, alarms are prevalent which require an operator to get the maintenance staff out to solve the oil problem or cause an outage. Automatic oil filter bypassing allows the machine to run while the filter is being replaced.

The set of sensors listed at the end of the table provides the operator with data concerning the auxiliary system critical for the turbine. The discussion for the previous table concerning accuracy of sensors applies to this application also.

Table 4-4 looks at operator needs that are not discussed in the previous two tables. In general, these cover the gas turbine as well as auxiliary equipment.

\begin{tabular}{|c|c|c|c|c|c|c|c|c|c|c|c|c|}
\hline & & 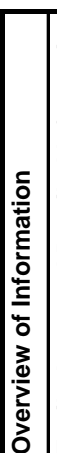 & 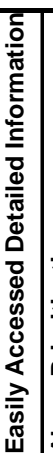 & & & & هُّ & 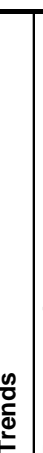 & & & & 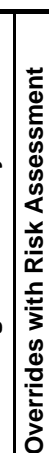 \\
\hline \begin{tabular}{|c|} 
Operator Need \\
\end{tabular} & Function/Technology & \multicolumn{3}{|c|}{ Overall Needs } & & \multicolumn{4}{|c|}{ Monitoring } & \multirow{2}{*}{\multicolumn{3}{|c|}{ Controls }} \\
\hline \begin{tabular}{|l} 
Assure Gas Leaks Not Present \\
Fire Protection Systems
\end{tabular} & Gas leak sensors/monitor & & & & & \begin{tabular}{|l|l} 
\\
\end{tabular} & & & & & & \\
\hline \begin{tabular}{|l} 
Fire Protection Systems \\
Emissions Monitoring
\end{tabular} & Fire protection & 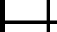 & & & & & & & & & $\underline{x}$ & \\
\hline $\begin{array}{l}\text { Emissions Monitoring } \\
\text { Vibration Information \& Protection }\end{array}$ & $\begin{array}{l}\text { Emissions monitoring station } \\
\text { Vibration Monitoring System }\end{array}$ & $|x|$ & $x$ & $\mathbf{x}$ & & $\frac{x}{x}$ & $\frac{x}{x}$ & $\frac{x}{x}$ & & $z$ & $\bar{x}$ & \\
\hline Lower Levels of Noise Pollution & Active noise control & & & & & $\mathrm{x}$ & & & & $\bar{x}$ & & \\
\hline \multirow{2}{*}{$\begin{array}{c}\text { Intelligent System Information \& } \\
\text { Alarms }\end{array}$} & \begin{tabular}{|l} 
Condition monitoring systems \\
$T$ Tonding inforytion
\end{tabular} & 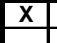 & $\mathbf{x}$ & $x$ & & & \begin{tabular}{l|l}
$\frac{x}{x}$ \\
\end{tabular} & $\frac{\mathrm{x}}{\mathrm{x}}$ & & $\underline{x}$ & & \\
\hline & $\begin{array}{l}\text { Trending information } \\
\text { Diagnostic and Prognostic software } \\
\end{array}$ & $\mathrm{x}$ & $x$ & $\bar{x}$ & $\bar{x}$ & & $\frac{\hat{x}}{\mathrm{x}}$ & $\frac{\hat{x}}{x}$ & & \begin{tabular}{l|l}
$x$ & 2 \\
\end{tabular} & & $\mathbf{x}$ \\
\hline \multirow{12}{*}{ 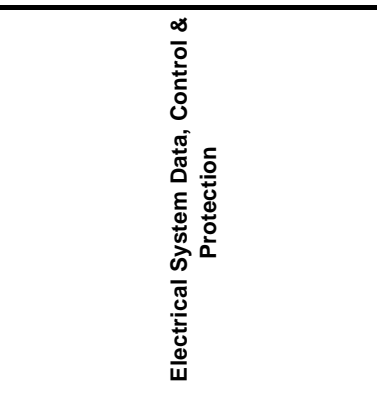 } & Bus Undervoltage & \begin{tabular}{|c|}
$x$ \\
\end{tabular} & - & & & X & & & & $\overline{\mathbf{y}}$ & $\bar{x}$ & 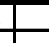 \\
\hline & Synchronization & $y_{y}$ & 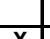 & & & & & & & \begin{tabular}{l|l}
$x$ & \\
&
\end{tabular} & & - \\
\hline & \begin{tabular}{|l} 
Electrical characteristics \\
Electrical transients
\end{tabular} & \begin{tabular}{|l|l|}
$x$ \\
$x$
\end{tabular} & $\begin{array}{ll}x \\
x\end{array}$ & - & & & $\mathbf{X}$ & & & $\lambda$ & - & — \\
\hline & Excitation System Monitoring & 4 & $\mathrm{x}$ & & & $\bar{x}$ & & & & $\bar{x}$ & $\bar{x}$ & \\
\hline & Excitation Temperature & 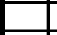 & $\mathbf{x}$ & & & $\mathrm{X}$ & & & & $\overline{2}$ & $\bar{x}$ & \\
\hline & Excitation Voltage, current & & $\mathbf{x}$ & & & $\mathrm{X}$ & & & & & $\bar{x}$ & \\
\hline & Generator and system watts and vars & $\mathrm{x}$ & $\mathbf{x}$ & & & & $\bar{x}$ & & & & $\bar{x}$ & \\
\hline & Generator Breaker & & & & & & & & & & $\bar{x}$ & \\
\hline & Generator Frequency \& Voltage & & $\mathbf{x}$ & & & & & & & & $\bar{x}$ & \\
\hline & Generator speed & & $\mathbf{x}$ & & & $\bar{x}$ & & & & & $\bar{x}$ & \\
\hline & Generator differential trip out & & & & & & & & & & $\bar{x}$ & \\
\hline & \begin{tabular}{|l} 
Step-up transformer monitoring \& protection package \\
Gen field control
\end{tabular} & & \begin{tabular}{l|l}
$x$ \\
$x$
\end{tabular} & & & \begin{tabular}{c|c}
$\mathbf{x}$ \\
$\mathbf{x}$
\end{tabular} & $\underline{\mathbf{X}}$ & & & & $\begin{array}{l}x \\
x\end{array}$ & - \\
\hline
\end{tabular}

Operators are quite concerned about gas leaks before starting up a turbine. Gas leaks have been found in locations visited. The operators now personally inspect the gas turbine area to ascertain that no gas leaks exist before startup. Such sensors would satisfy the concern of operators as well as decrease the amount of time it takes to start a turbine since this inspection requirement would disappear. 


\section{Section 4}

Operators have to closely monitor emission levels. There are strict requirements on the amount of emissions that are allowed monthly, seasonally, and yearly. The more real-time and accumulated emissions data available to the operator, the better the operator will be able to control the emissions level and be able to make intelligent decisions whether to operate the turbine based on emission limits and electric market economics. Therefore, increased emissions information can improve the availability of the generator.

Operators need access to a complete vibration information and protection system. Since vibration is such a critical part of assessing the state of rotating machinery, this provides the operator with automatic protection for critical function plus the ability to obtain data that will help in assessing problems and determining potential problems. Assessing vibration problems early will help to avoid catastrophic failure and to keep the time for repairs to a minimal level.

Operators would like to work in a less noisy environment. Active noise control besides helping with noise abatement issues would help the operators work in a less noisy environment. It is possible, that the masking out of certain sound frequencies will allow some sound frequencies to be more prevalent. This may allow the operator to hear sounds associated with potential failures that may have been otherwise drowned out by other noises coming from the machine.

Operators need system information to be presented in an easy to understand way. We have discussed in the previous chapter some of the details of how different types of intelligent system information and alarms would operate. Present tools are sophisticated, but do not provide the level of accuracy and user friendliness that meets operator needs. This area is ripe for considerable development. The focus of this effort is to provide information to the operator in order to make intelligent decisions.

In general, operator needs for accurate electrical system data as well as easy control and protection of the electrical system is easily taken care of with mature equipment in place at most stations. However, care must be taken to make sure that not too much data is provided. Confusion often takes place in this part of the station because too much data provides a confusion factor. The result is extended outages due to too much data.

In this section, we discussed the value of each of the functions and technologies identified in the earlier sections to meet the operators needs. The basic question is how does this improve RAM. First, the right information portrayed in an easy to understand way improves RAM. Unfortunately, most devices are put in place to provide data while information is what the operator needs. The net result is too much data that is worthless and not enough useful information.

Every additional sensor or monitoring device put in place increases the probability of a problem. These devices may cause confusion because of inaccurate sensors or because of interpretation by the operator of what the sensor really does measure. However, the judicious use of sensors with the appropriate sophisticated software tools will help meet the operators needs and improve the RAM of the gas turbine system.

Operator needs can be met by the judicious use of software tools along with appropriate monitoring and control devices. Sometimes advanced technology gets in the way of actual operation. The best method to meet operators' needs is to focus the use of technology on meeting a specific set of goals and to keep any interface with the operator simple. 


\section{Section 4}

In the following section, information portrayed in this document is drawn together to provide conclusions and recommendations. The conclusions and recommendations are focused on providing solutions to operators' needs. These solutions have been chosen for the high value they provide and the cost effectiveness of such solutions. 


\section{Conclusions and Recommendations}

$\mathrm{W}$ e have seen in this report that there are both a wide number of needs by operators and maintenance people as well as many potential sensors and controls that can be used in a gas turbine. The conclusions and recommendations offered in this section are an attempt to balance the many variables required to make an intelligent choice of controls and sensors.

Besides the needs of the operator, we also have considered the needs of the manufacturer and the packagers of gas turbines. There are many technical and legal considerations that must be taken. Operators of course want the unit to operate perfectly and when it does not have the ability to operate the unit at their will. Manufacturers and packagers need to balance the issues of RAM, price, technical capabilities of particular design and warranty issues. An operator that overrides the protection of a turbine or balance of plant system may get the immediate operation that is needed but may considerably shorten the life of the turbine or balance of plant system. The extreme case would be where overriding of an interlock may create a high risk of catastrophic failure.

We take a basic approach on how to evaluate what is best. First, we learned from operators that simple is best. Most gas turbine systems have too many sensors that provide too little value. They provide limited amounts of information, much of which is never used. However, due to the large number of sensor problems experienced, they provide issues in maintenance and timely startup of machines.

Operators also want such items as smarter alarming, easier interface with the system, and better predictive monitoring. It was also obvious from discussions that most operators felt there are far too many sensors. These sensors do not provide real information and have very limited value. Less is more, should be the operative word in the use of sensors.

\section{Conclusions}

With the above considerations in mind, we have reached the following conclusions. First, the needs of the operator are critical when implementing any technology. When a new technology or function is introduced, the data provided by the technology or function must be focused on the task at hand and presented in a meaningful way.

When different pieces of data are displayed, they need to be checked for consistency. Inconsistent data provides for increased time in trouble shooting effort as well as the potential for coming to an incorrect decision which may have a large affect on the availability of a machine.

To improve availability of gas turbines systems software tools needed to be provided, to operator and the maintenance staff, that are focused on assisting in understanding the history and trends of critical data concerning the operation of the turbine and auxiliary systems. Predictive function would go the next step towards increasing reliability and availability and decreasing maintenance costs. Predictive software would take the history of the machine and other similar machines in order to provide a series of recommendations of problems that should be mitigated. 


\section{Section 5}

Most importantly, any tool that is provided should be simple for the operator to use. Complex tools are of very little use since operators usually do not have the time or budget to learn how to use these tools. User friendly tools will allow the operator to more easily use advance software tools

Many existing sensing and control technologies are still troublesome to operators and maintenance. This is due to a variety of reasons including bad implementation and error prone software.

Any new sensing and control technology that is implemented should be put in place to focus on meeting a specific set of operational objectives.

\section{Recommendations}

These recommendations are based on the analysis of operational needs, monitoring and control equipment, as well as conclusions of this analysis. Table 5-1 portrays recommended development/improvement projects that should be considered. The recommendations include projects that are based on improvements in sensing technologies, improvements in decision

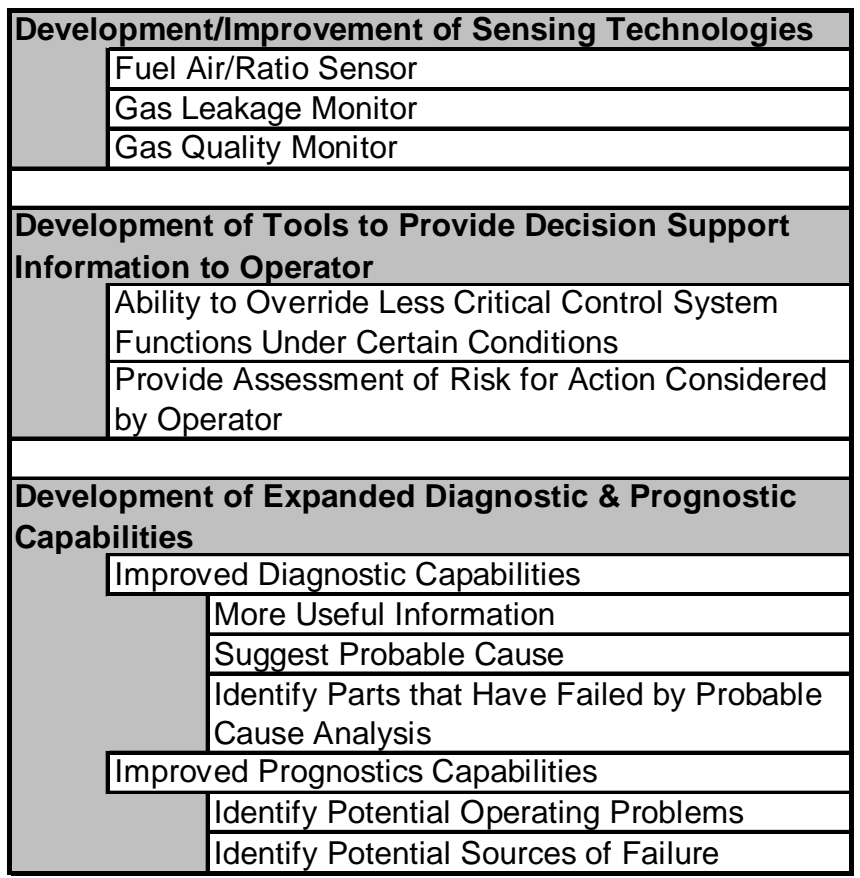

Table 5-1 - Development/Improvement Projects support information for the operator and maintenance staff, as well expanded diagnostics and prognostic capabilities.

\section{Sensing Technologies}

The first of these areas is that of development and/or improvement in sensing technologies.

An accurate fuel/air ratio sensor can provide better combustion control. This will result in better operating parameters and longer life for the combustors. This project would be a development project focused on developing an improved fuel/air ratio sensor that would directly connected into the control system. Such a sensor can more directly control the fuel and air mix to provide a better operating combustor. Data from this sensor could also be provided to historic data for troubleshooting

purposes.

The development of an improved gas leakage monitors would help to improve the availability of gas turbines and to more easily allow remote operation of the facility. The gas leakage monitor would help to alleviate some inspection time that some operators go through before they fire up the turbine. This would make the turbine available quicker than without such a monitor.

Gas quality is a major factor in the efficient and reliable operation of a gas turbine. The development of a gas quality monitor would provide information to the operator and possibly 


\section{Section 5}

to the control system concerning the heat content and the impurities in the gas provided to the turbine. Manual or automatic adjustments made to the operation of the turbine to take into account the actual fuel provided at that moment in time will increase efficiency, lower emissions and result in a more reliable machine that requires less maintenance.

\section{Decision Support Information}

Another area is the recommendation for the development of decision support information for the operation of a gas turbine. The functionality of such a development would be focused on allowing the operator to make intelligent decisions on overriding less critical control system functions under certain conditions. Some operating software has implemented this as a forced logic state that is the equivalent of jumpering a contact.

The problem with this type of functionality is that the operator may accidentally cause damage that possibly could lead to catastrophic failure or reduced equipment life. In order to avoid these kinds of problems this decision support information should include an assessment of risk for the actions considered by the operator. This assessment of risk would allow the operator to make a decision based on circumstances that he is experiencing and the tradeoffs of different actions. In some cases the operation considered would be so risky that the particular function could not be overridden. This type of decision support system would also be able to track what decisions are made for purposes of providing data for further history.

\section{Expanded Diagnostic \& Prognostic Capabilities}

Development of expanded diagnostic and prognostic capabilities could prove to be one of the most fruitful areas for improving RAM in a gas turbine system. Today's diagnostic capabilities are limited at best. Although there are some interesting tools available, they still provide only limited functionality.

The improved diagnostic capabilities should incorporate the full range of historic data and trending capabilities that is in place today in several software packages. However, the screens should be focused on providing useful information for the task at hand. Based on the inputs this software would be able to suggest probable causes for the type of situations at hand. Based on the probable cause analysis, the software would also suggest areas where parts have failed or where equipment is operating incorrectly. Improved diagnostic capabilities will greatly decrease the down time after events have occurred. Such software will lead to earlier diagnosis of some issues. This will result in less maintenance time and cost as well as higher reliability and availability for the machine or auxiliary device.

Development of prognostic capabilities is a development that can have a large impact on RAM. By using prognostic capabilities, forced outages can be greatly reduced. Since potential damage is identified before the damage becomes excessive, reliability and availability will be greatly improved while maintenance costs will be greatly reduced.

The focus of the prognostic capabilities is to identify potential operating problems and potential sources of failures. This can be accomplished by having a software tool that does an active analysis of historic information as well as current information. Historic information would include trends experienced on the gas turbine system as well as information concerning other similar gas turbine systems. Based on this information, the software would be able to provide a risk assessment of potential failures as well as providing suggestions for better operations from a RAM and efficiency standpoint. Prognostic capabilities would be used to assist the operator in better operation of the gas turbine. Prognostic capabilities would also be used to assist the maintenance staff in replacing parts before they fail and to perform maintenance function on an as needed standpoint. 


\section{Section 5}

The final recommendation is that any monitoring and control systems implemented should be as simple for operations and maintenance to understand as possible. A limited set of sensors should be available to provide specific information about the operation of the turbine and auxiliary equipment. Operational and other software should be designed to be user friendly for operations and maintenance personnel. The software should be able to provide screens with accurate data. This implies that erroneous data inputs will be handled by the software.

These recommendations are based on improving the Reliability, Availability and Maintainability as well as the efficiency of the monitoring and control systems of a turbine facility and its auxiliary systems. It is anticipated that the development projects and other efforts outlined in these conclusions and recommendation section will lead to great improvements in the next generation turbine. 


\section{Appendix K Next Generation Turbine System Study Phase I Program Department of Energy Review October 2001}




\section{Next Generation Turbine System Study}

\section{Phase I Program DoE Review}

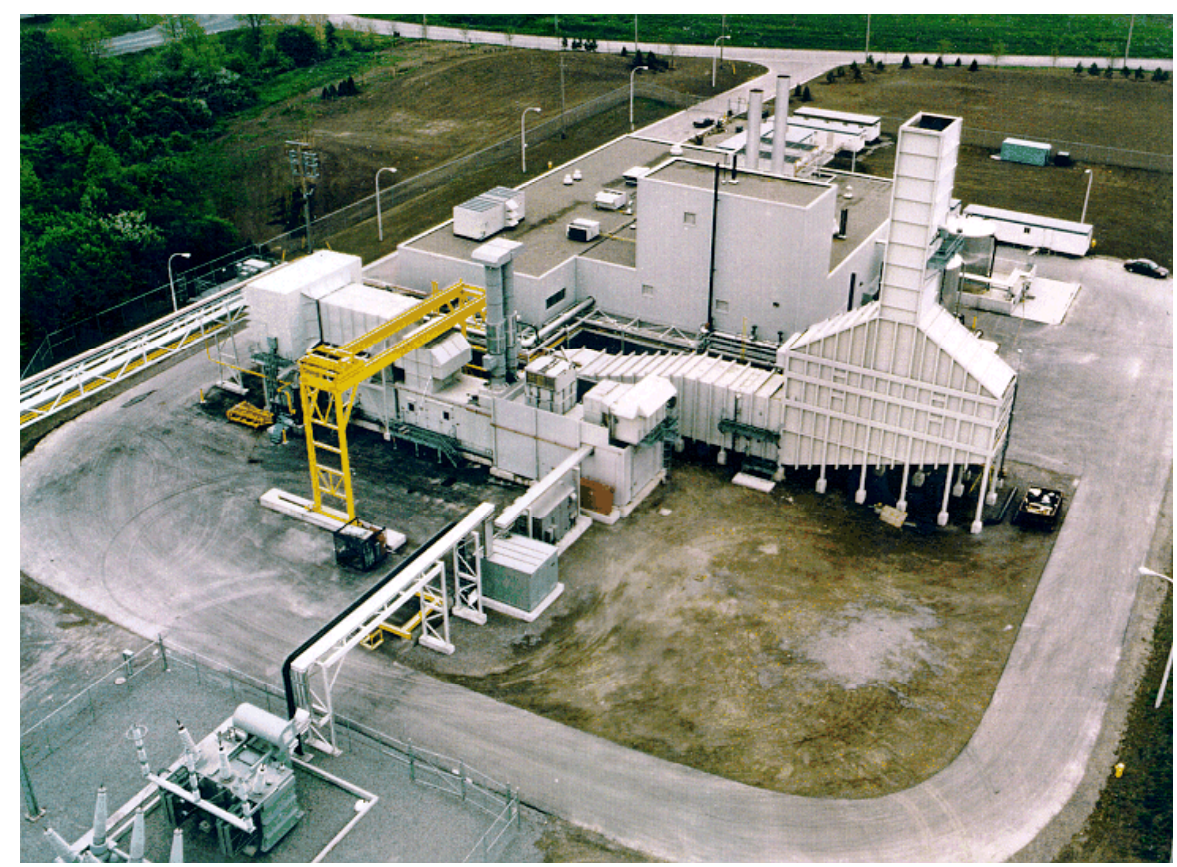

Issued: October 2001

Work Performed Under Contract:

DE-AC26-00NT40852

Submitted By:

Rolls-Royce Corporation

P.O. Box 420

Indianapolis, IN 46206-0420

Program Manager: Frank Macri

Phone: 317-230-3281

Fax: 317-230-3542

Submitted To:

U.S. Department Of Energy

National Energy Technology Center

Morgantown, WV 


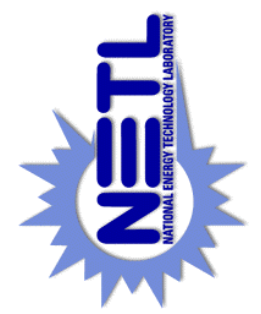

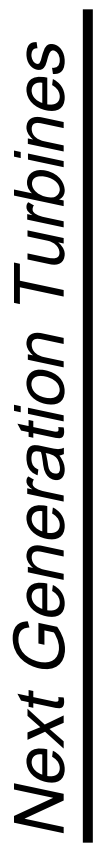
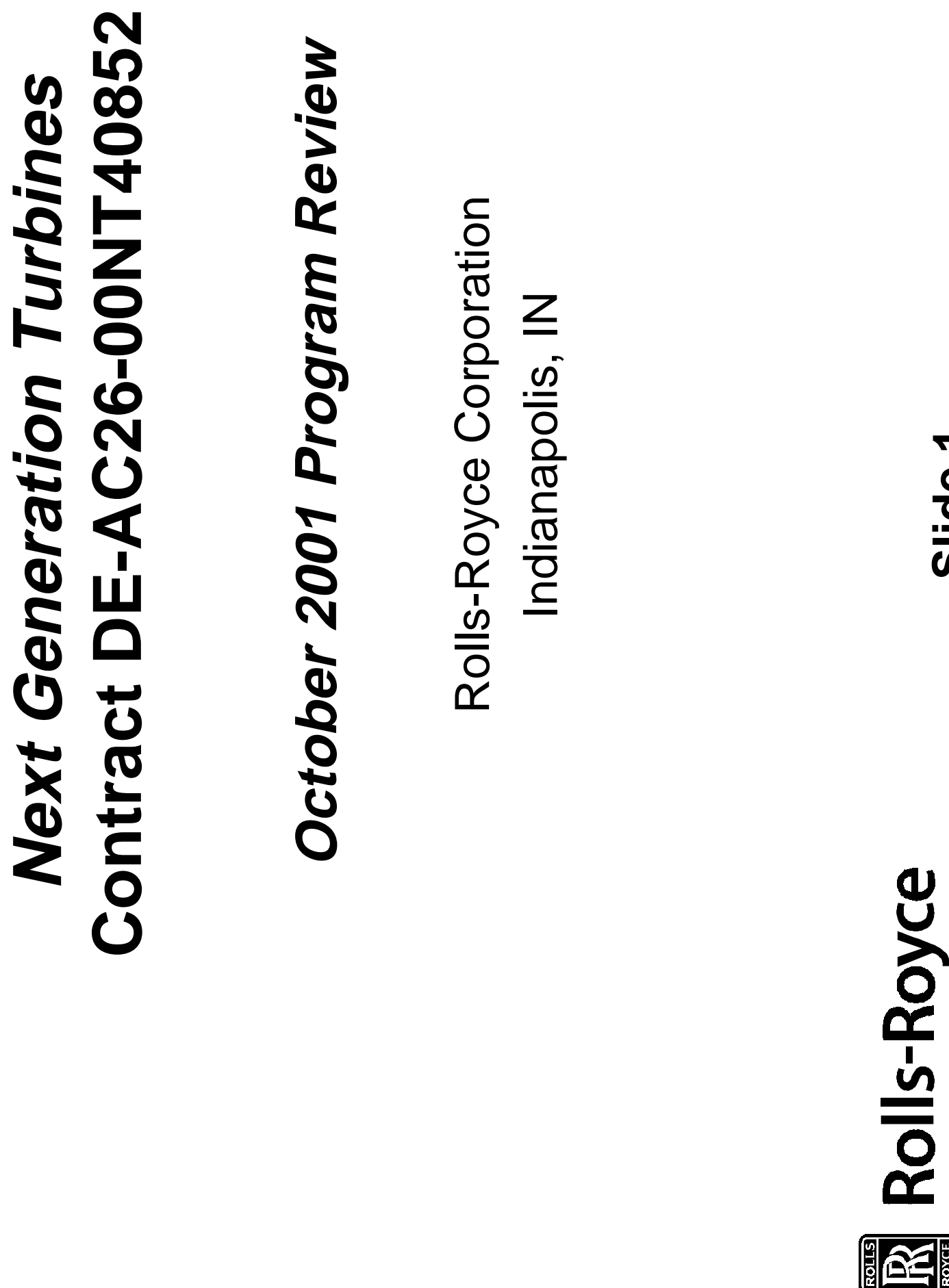

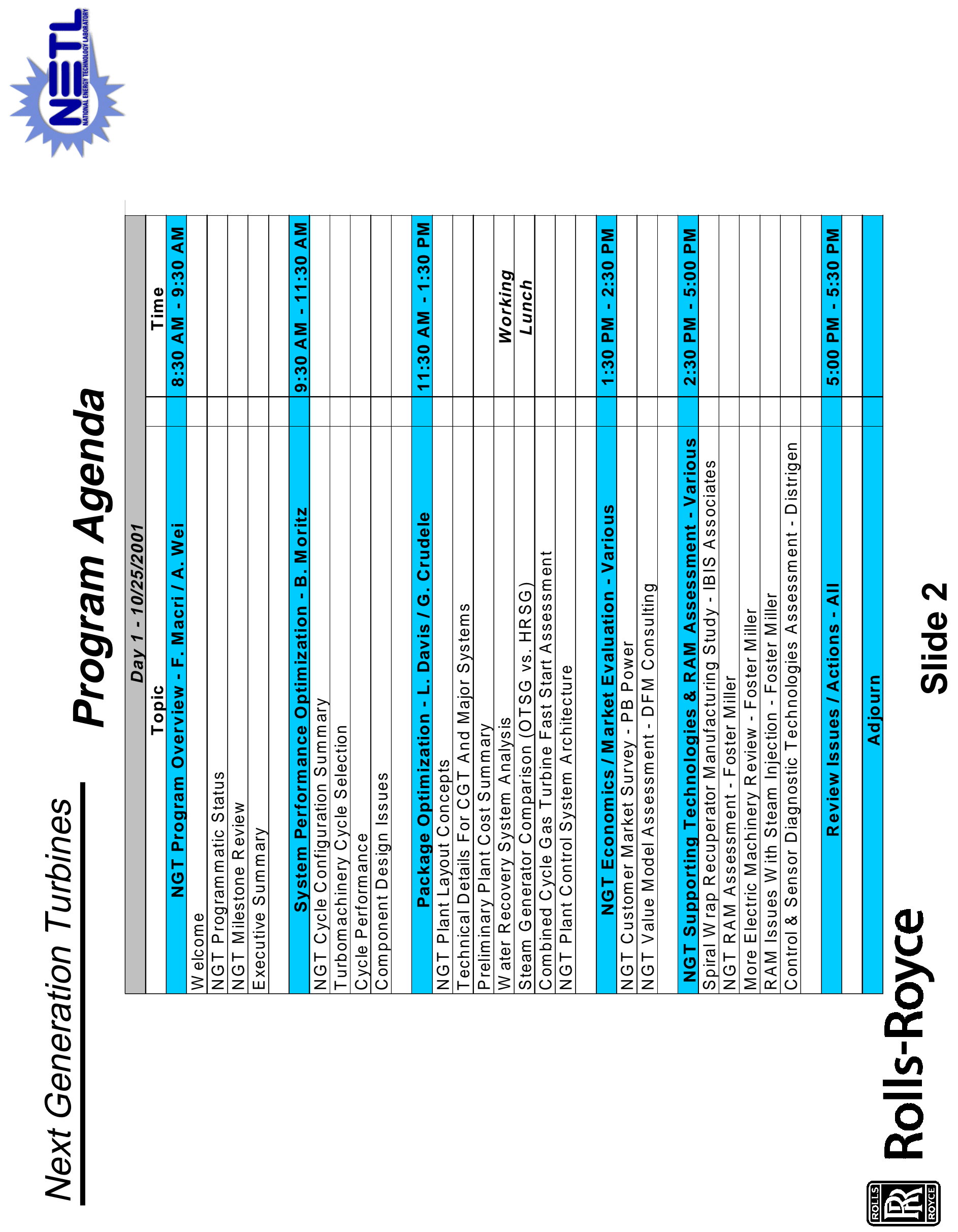


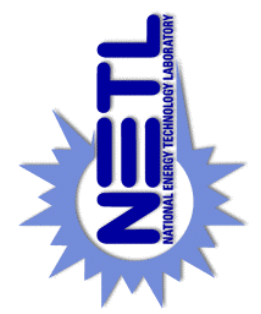

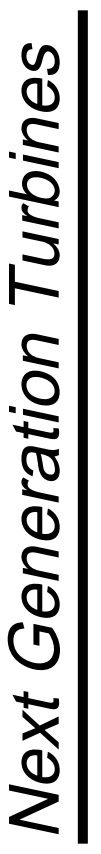

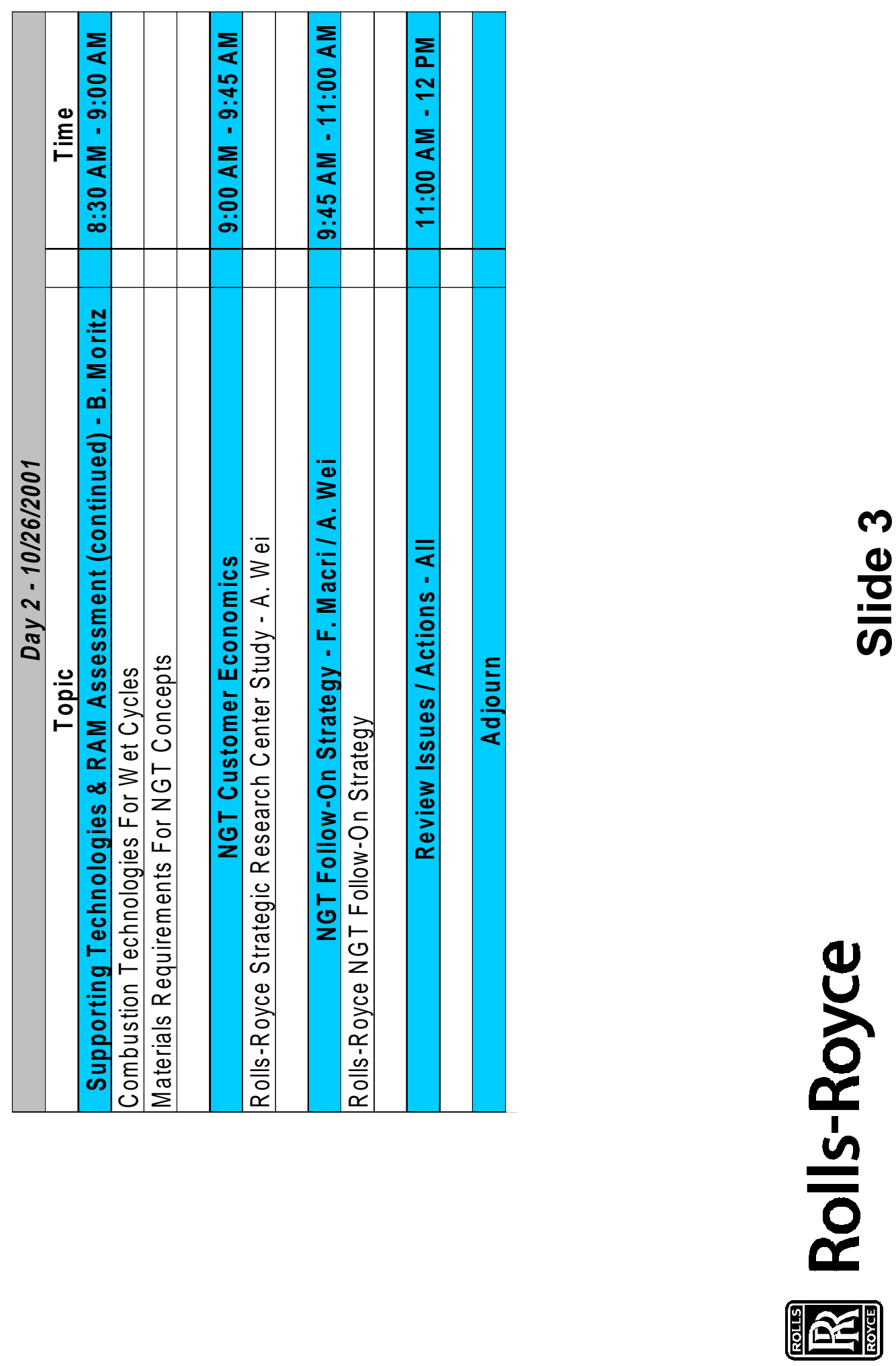




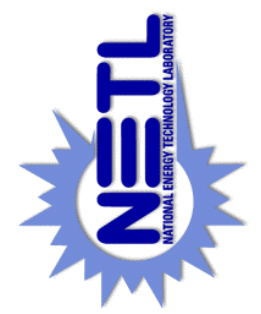

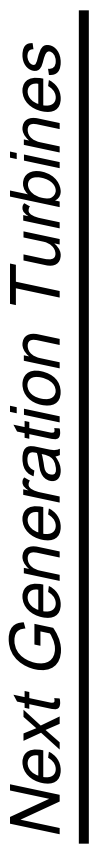
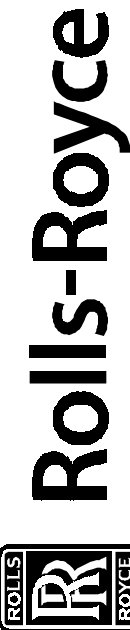


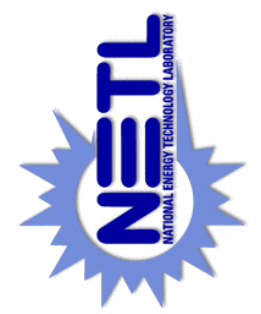

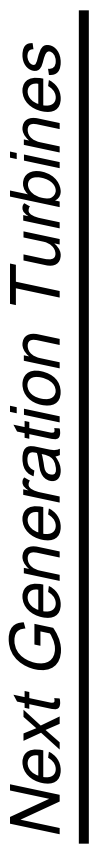
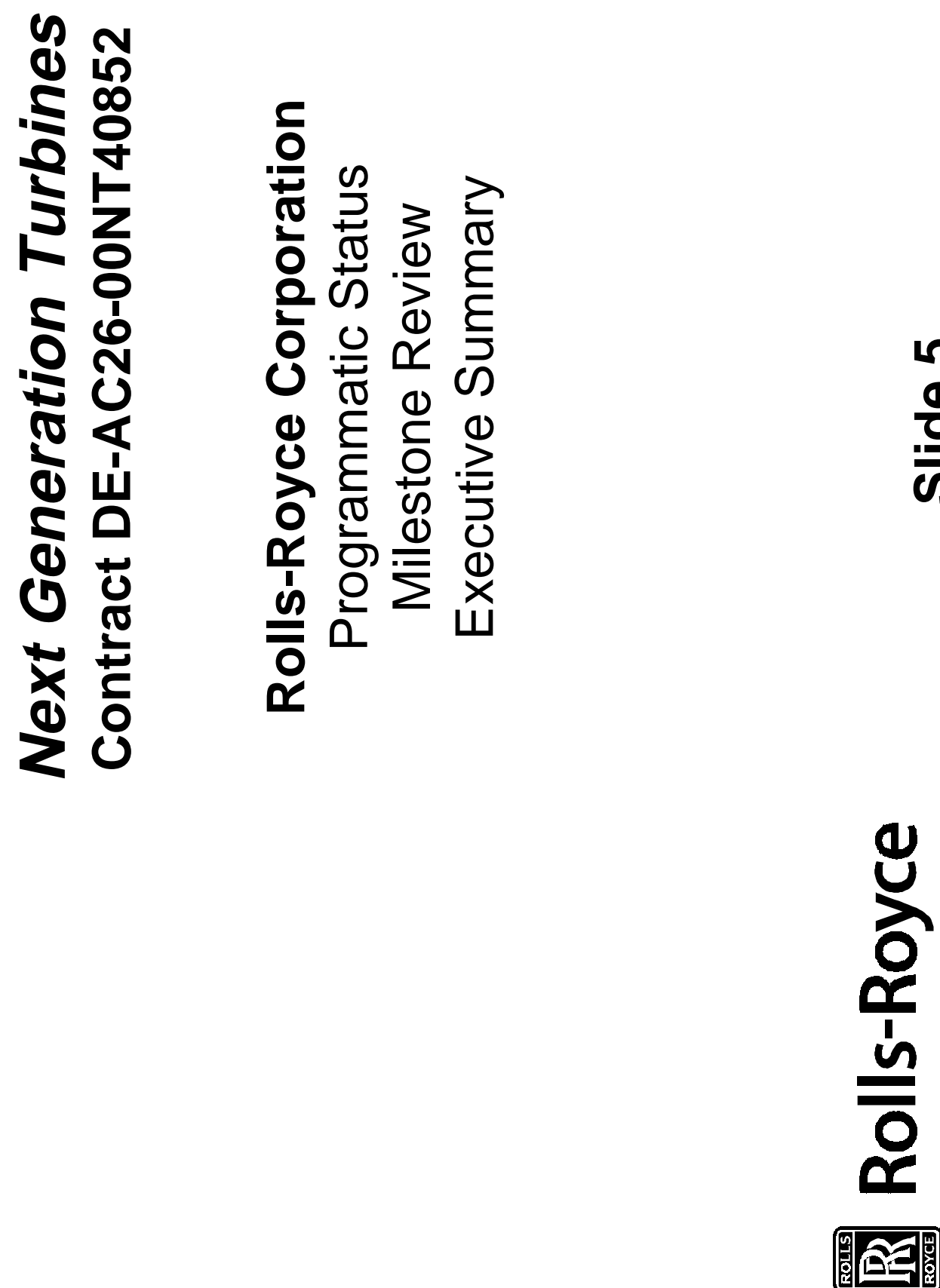


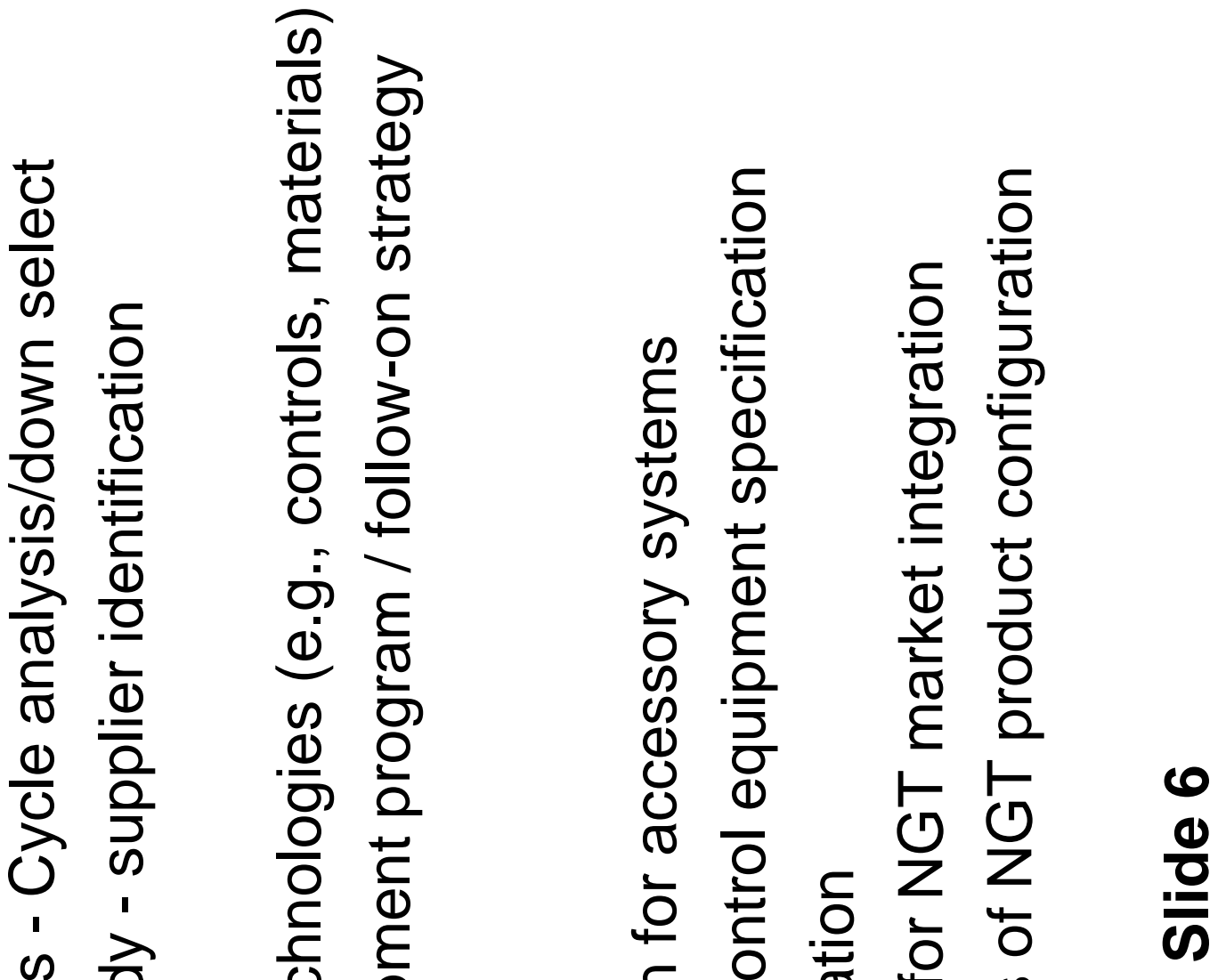

m 1 它

드

$(0$

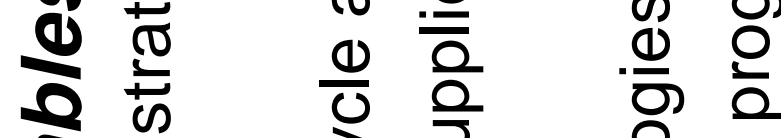

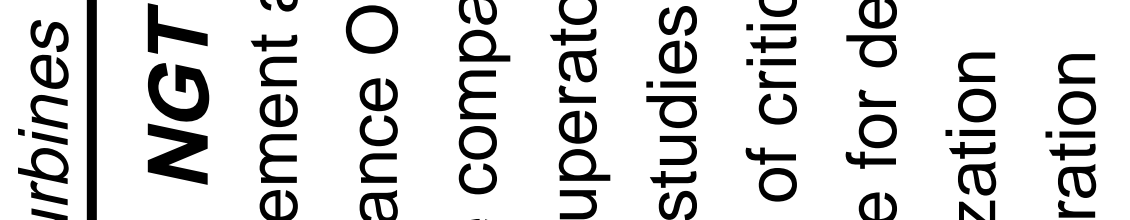

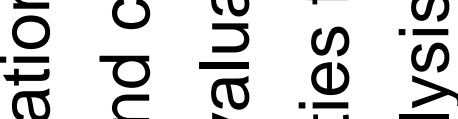

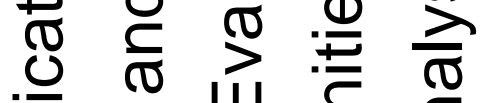

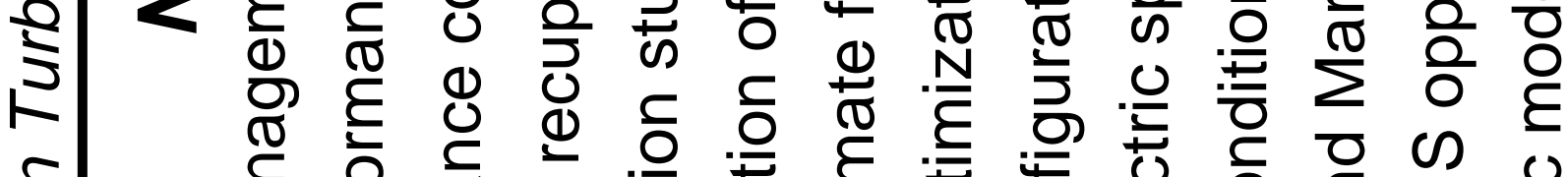

$F$

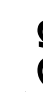

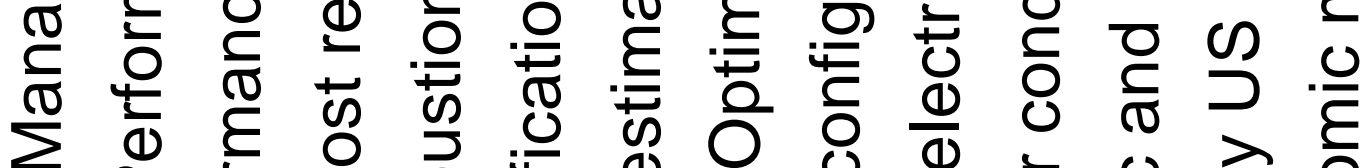

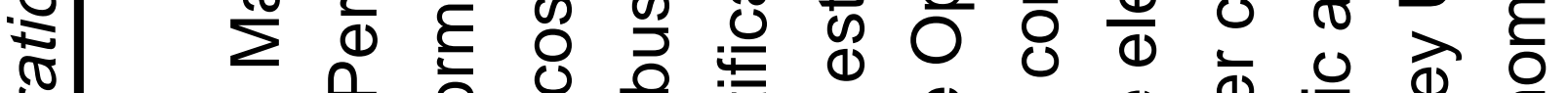

(1) $\varepsilon$ 일

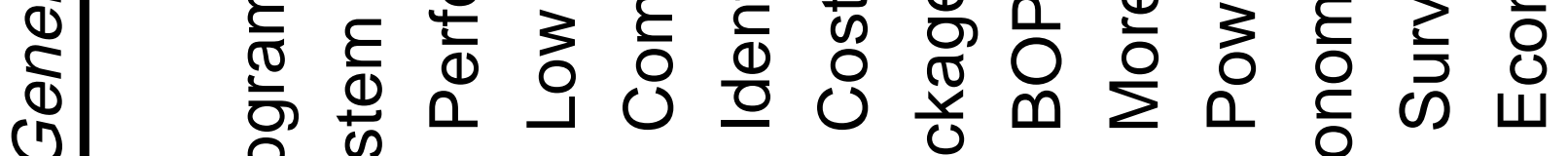

$\circlearrowleft$

은 $\stackrel{3}{\sim}$

O

ப

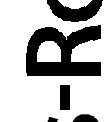

U

0

i

ㄷ 

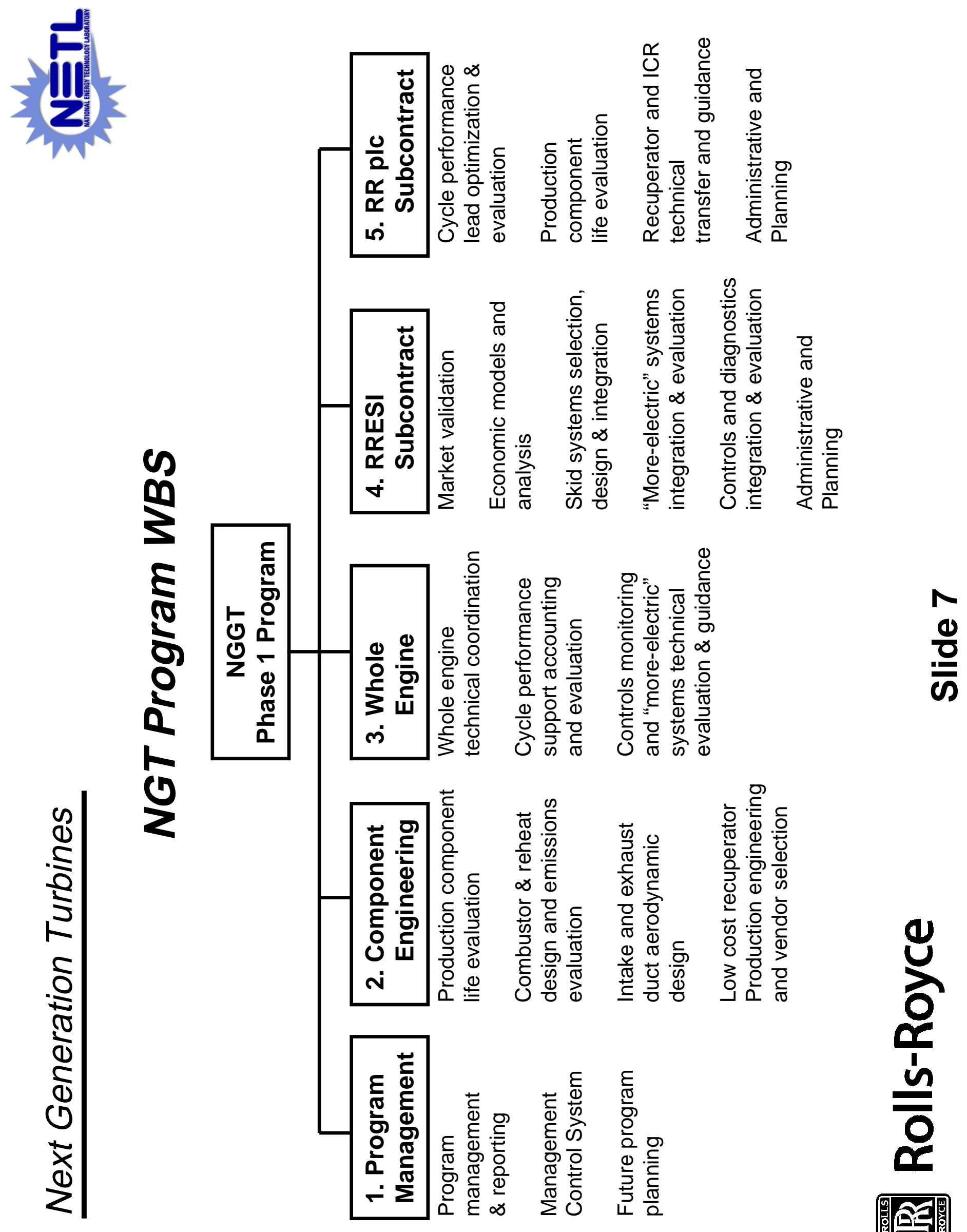

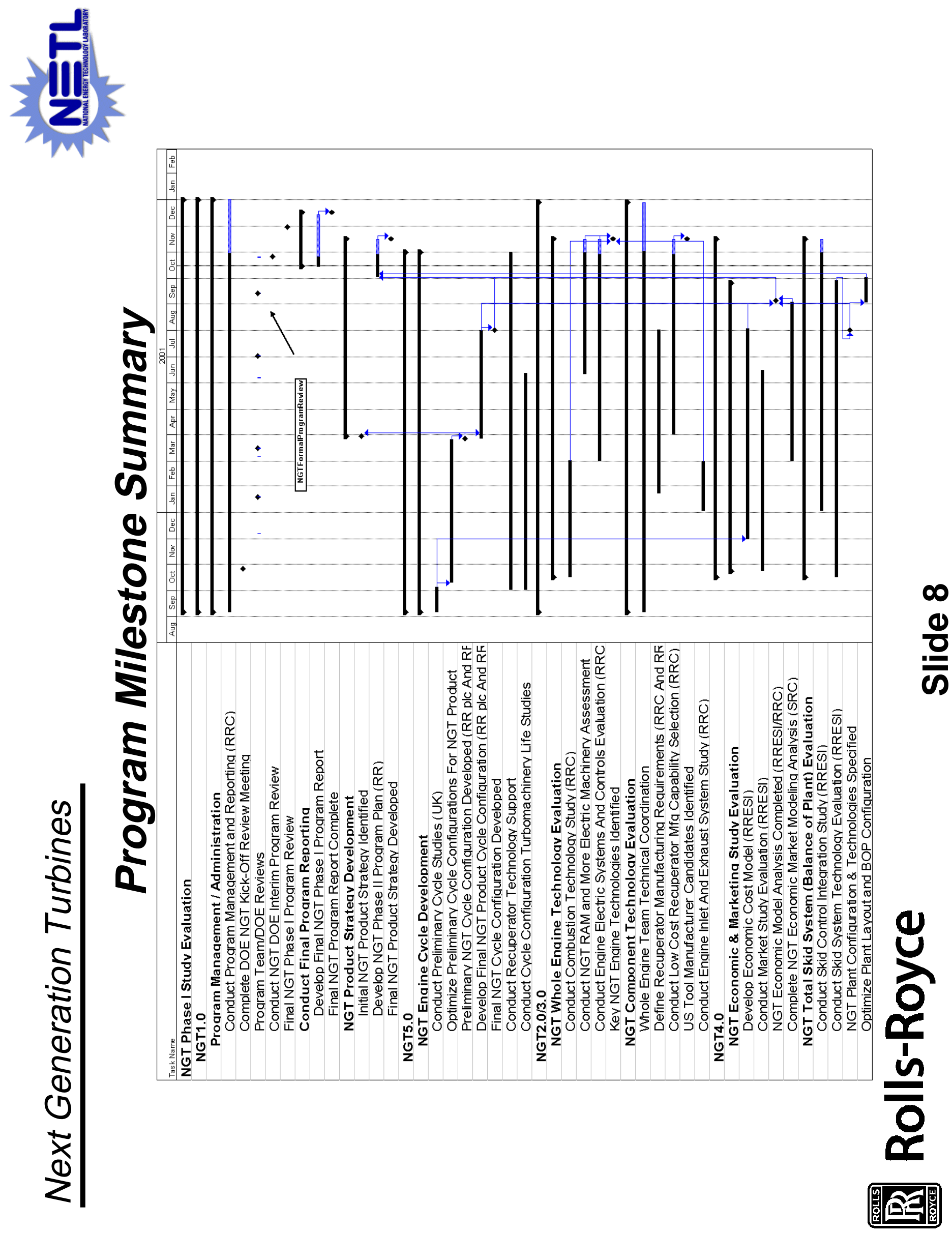

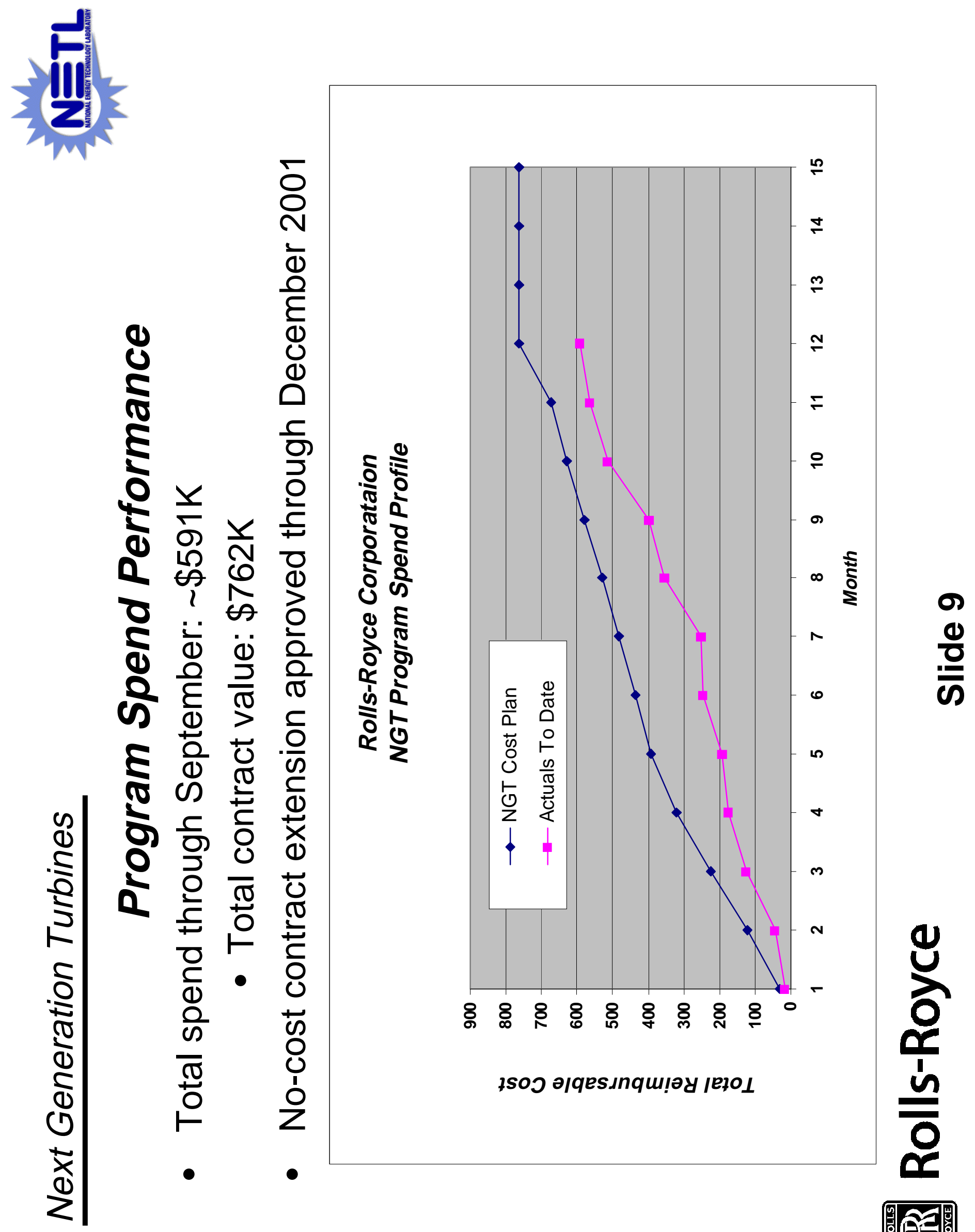

150J ә/qes.ınqu!əy |e10।

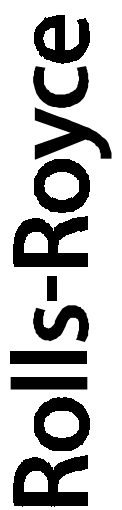




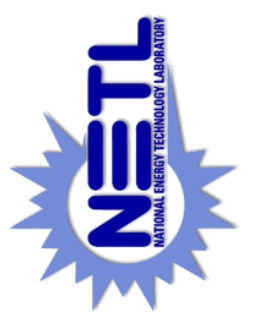

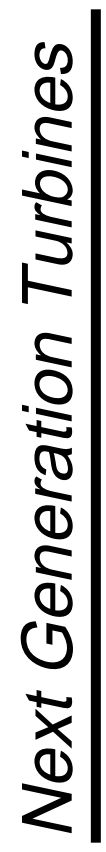

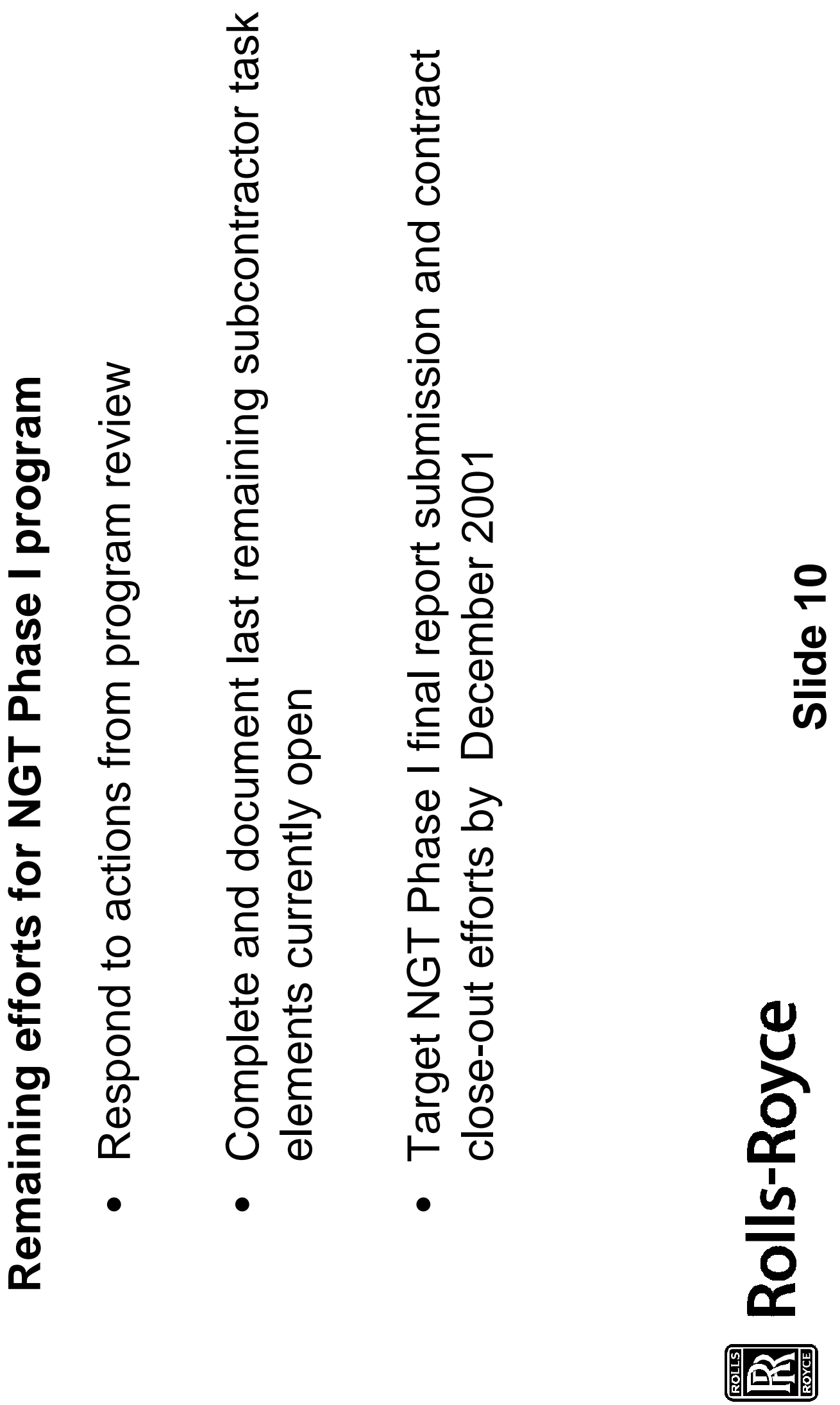




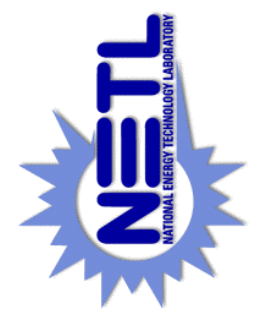

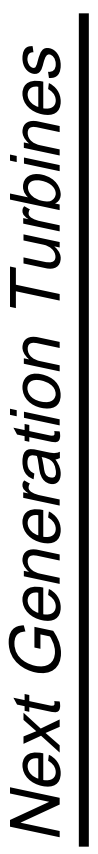

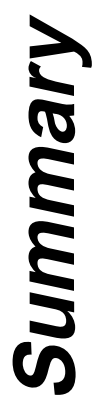

$\underset{1}{0}$

(

II
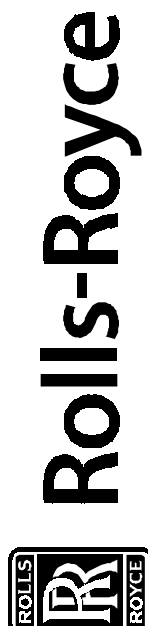

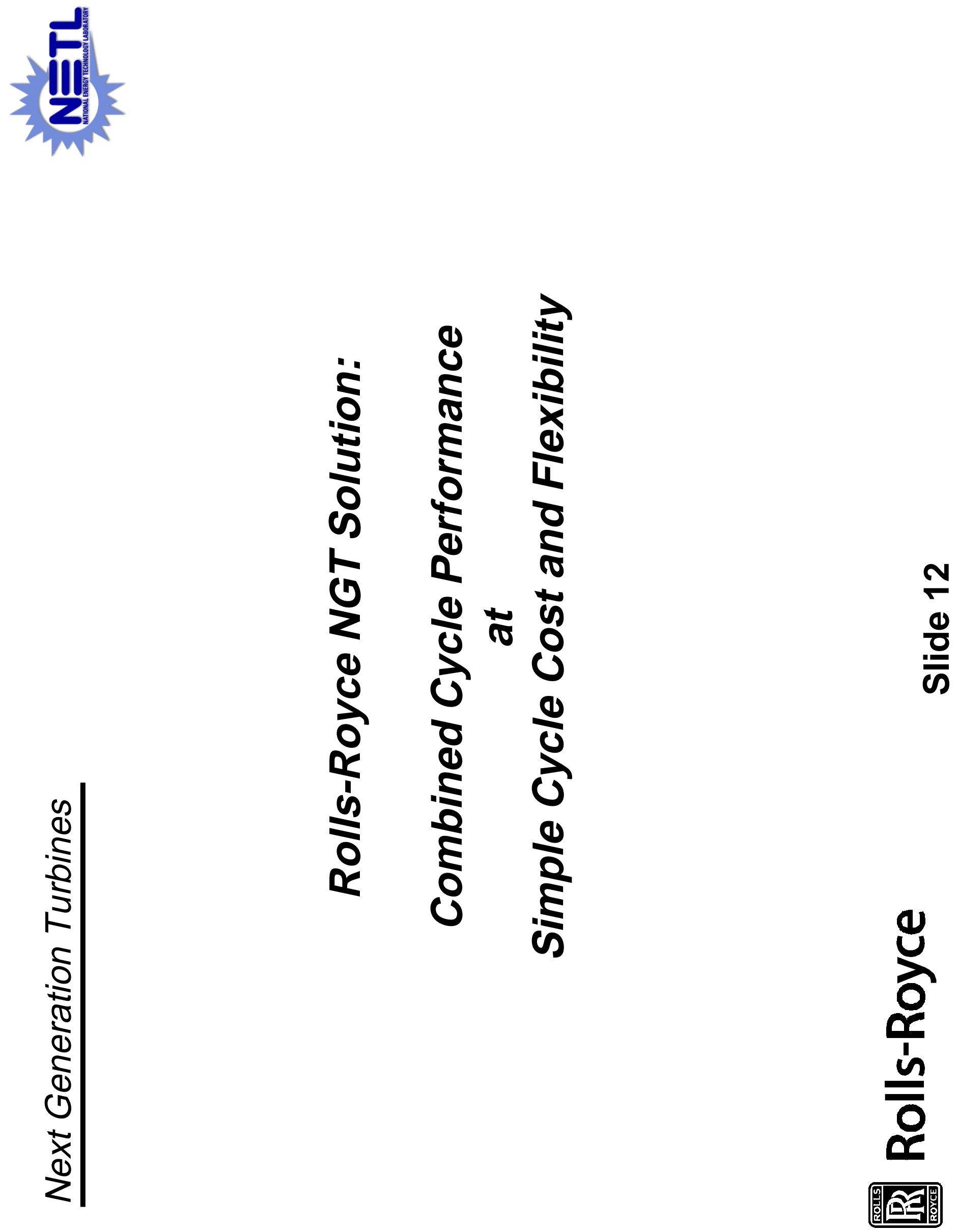

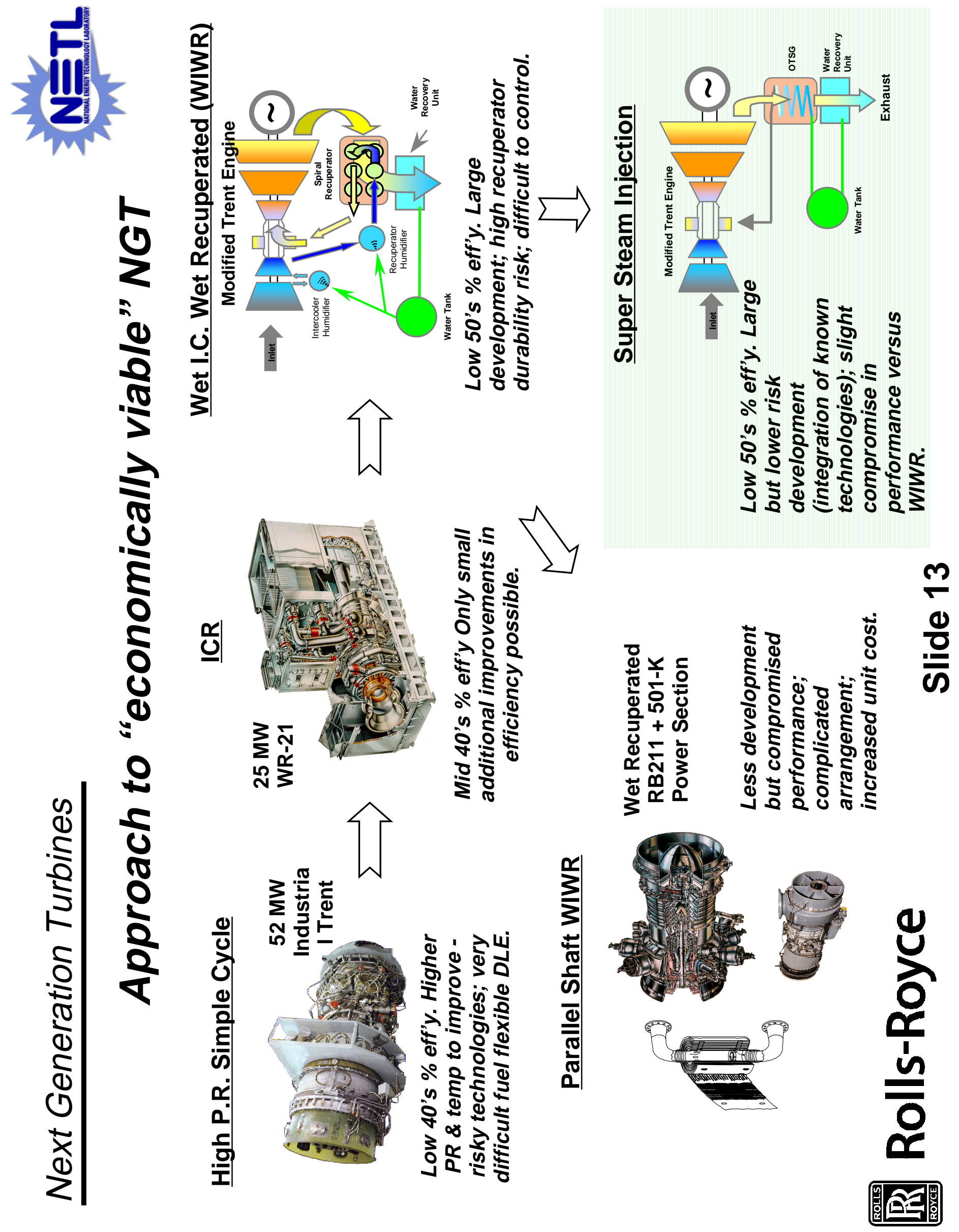

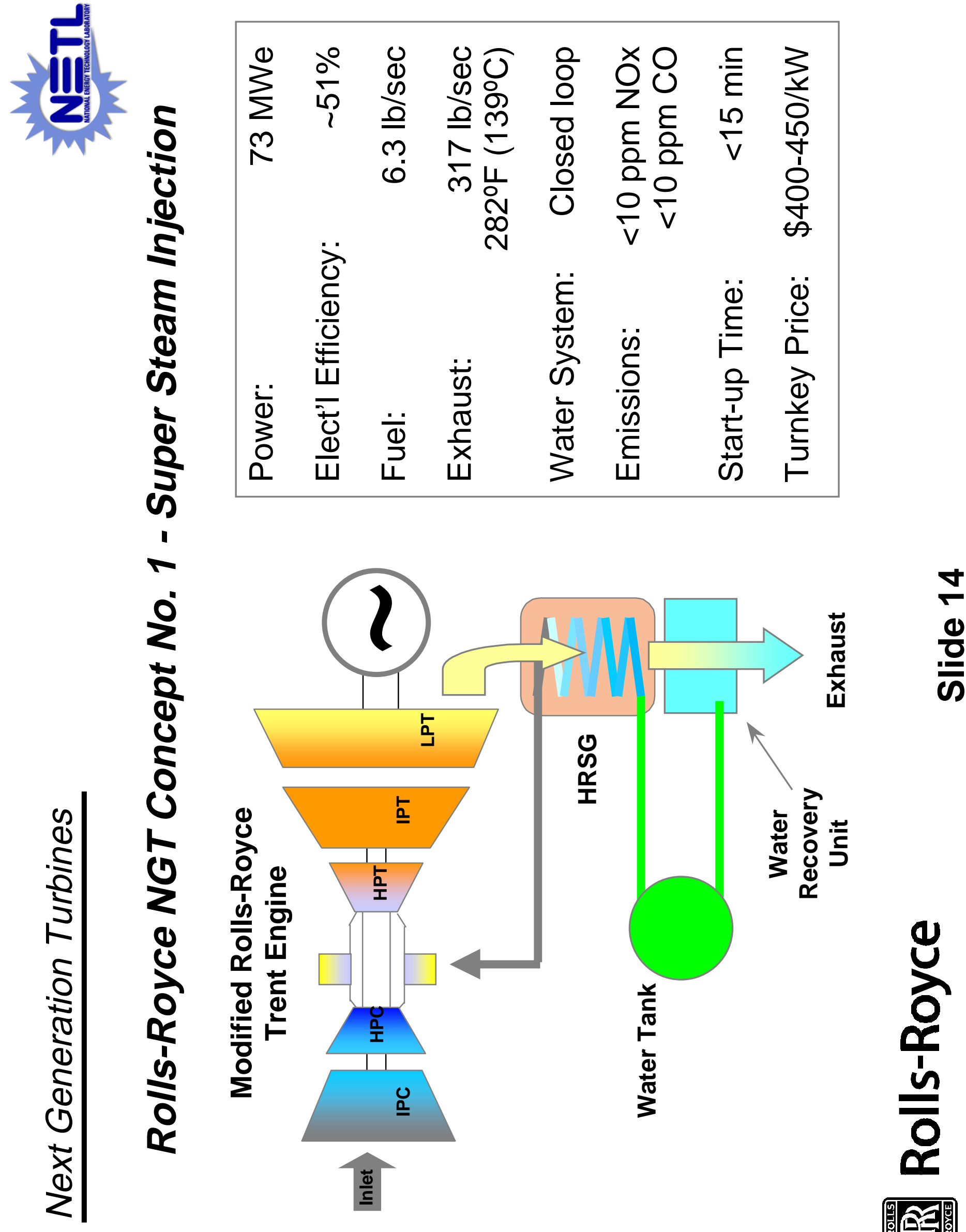


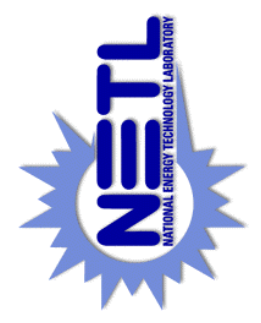

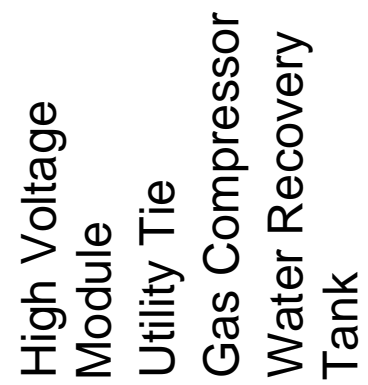

(1) $0 \wedge \infty$

d

(3)

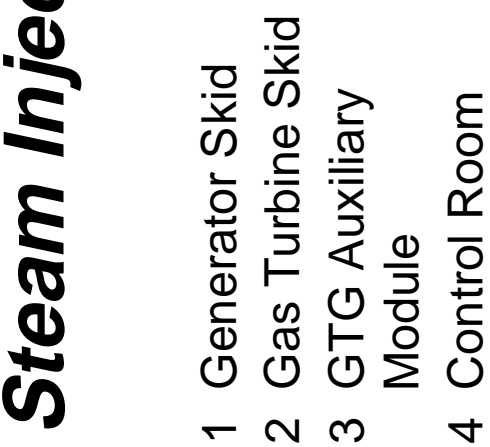

ฮ

2

ज

产

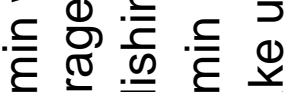

๑ั

$\frac{1}{5}$
$\frac{1}{5}$
$\frac{1}{2}$

$\square$

$\simeq \quad \stackrel{\square}{\square}$

के

ले हิ

गे $\frac{\Phi}{0} \frac{\bar{\Phi}}{0}$

ర્d

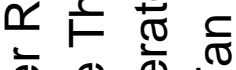

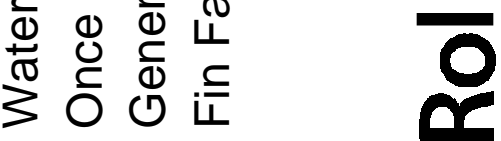

으 두

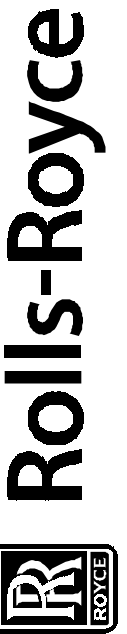



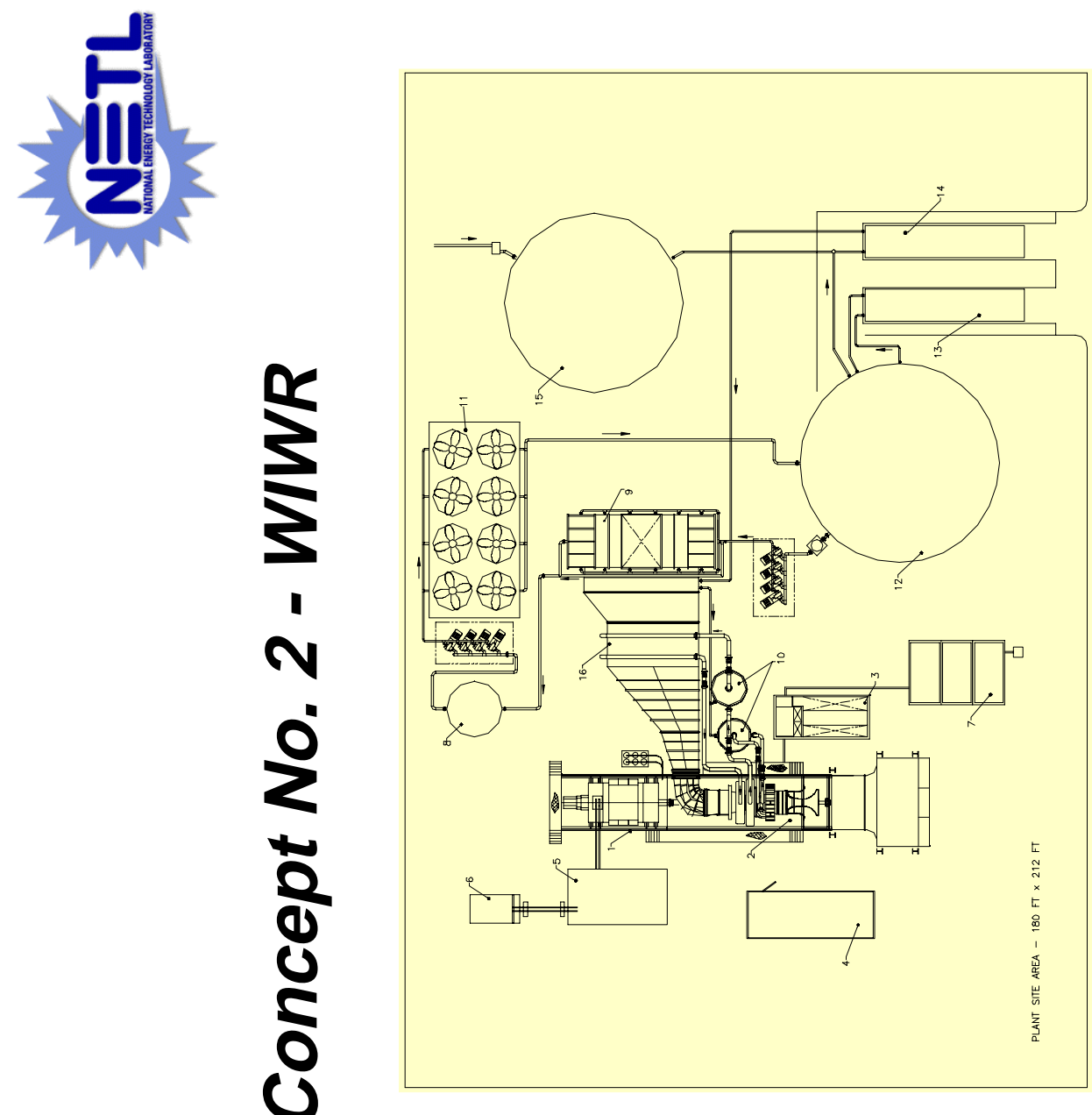

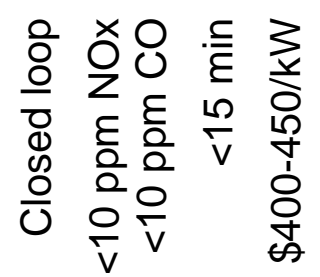

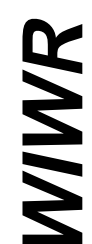

N

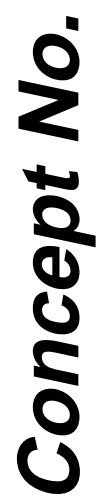

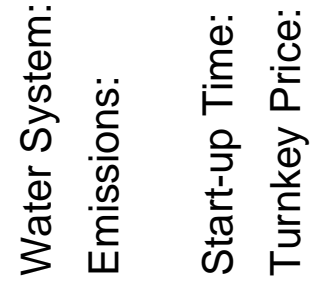
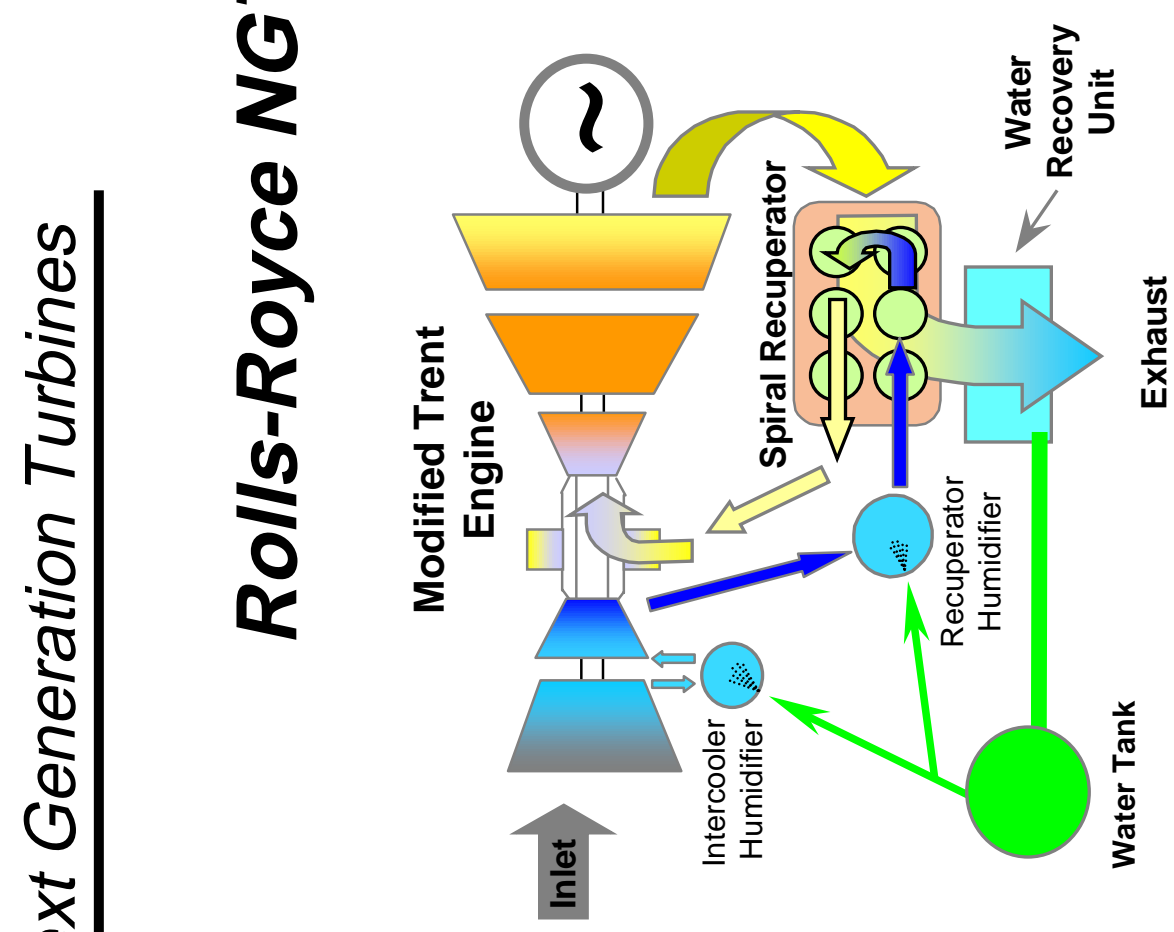

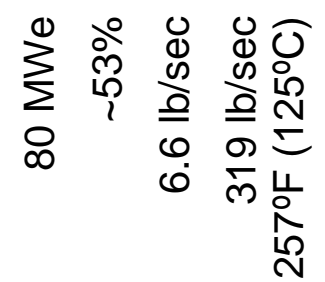
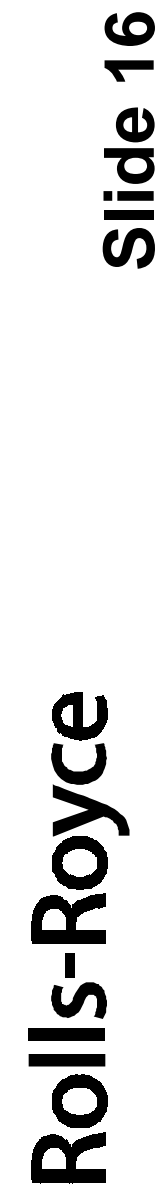

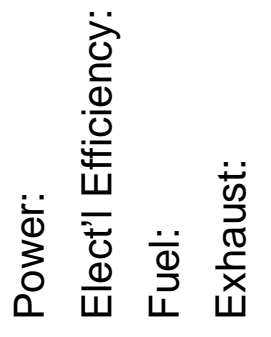

(1)

x

j 


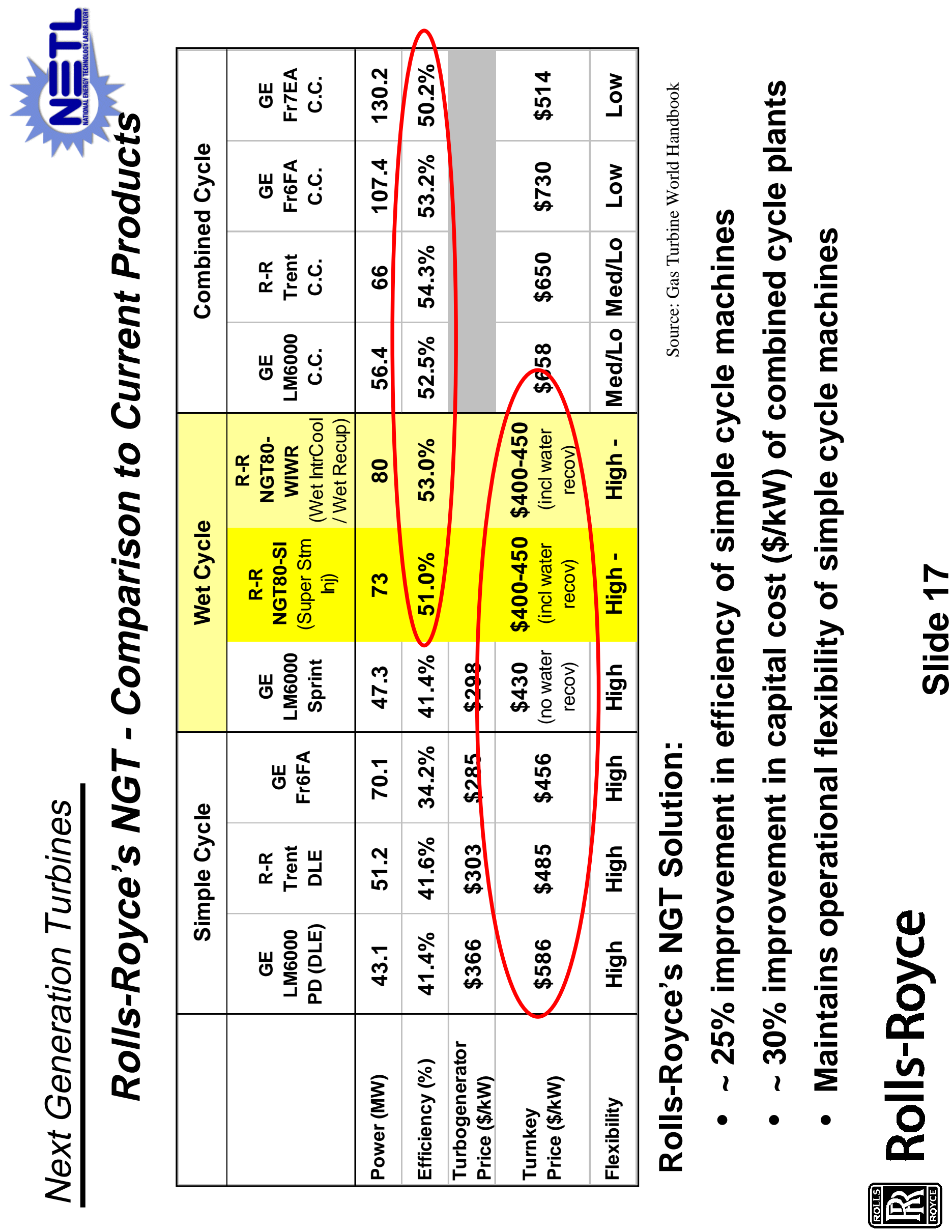




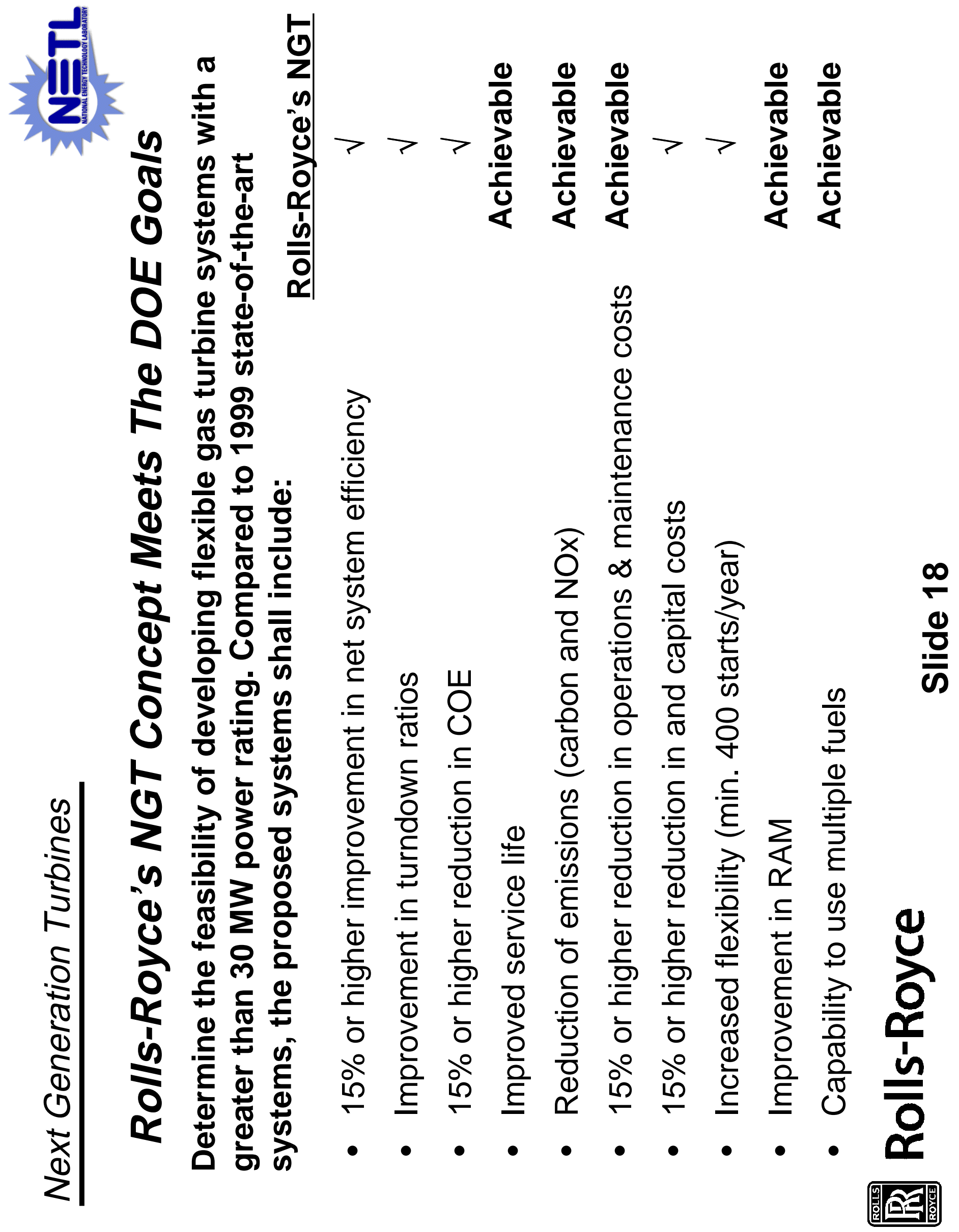




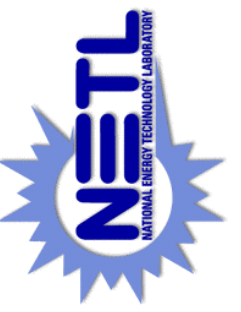

Z

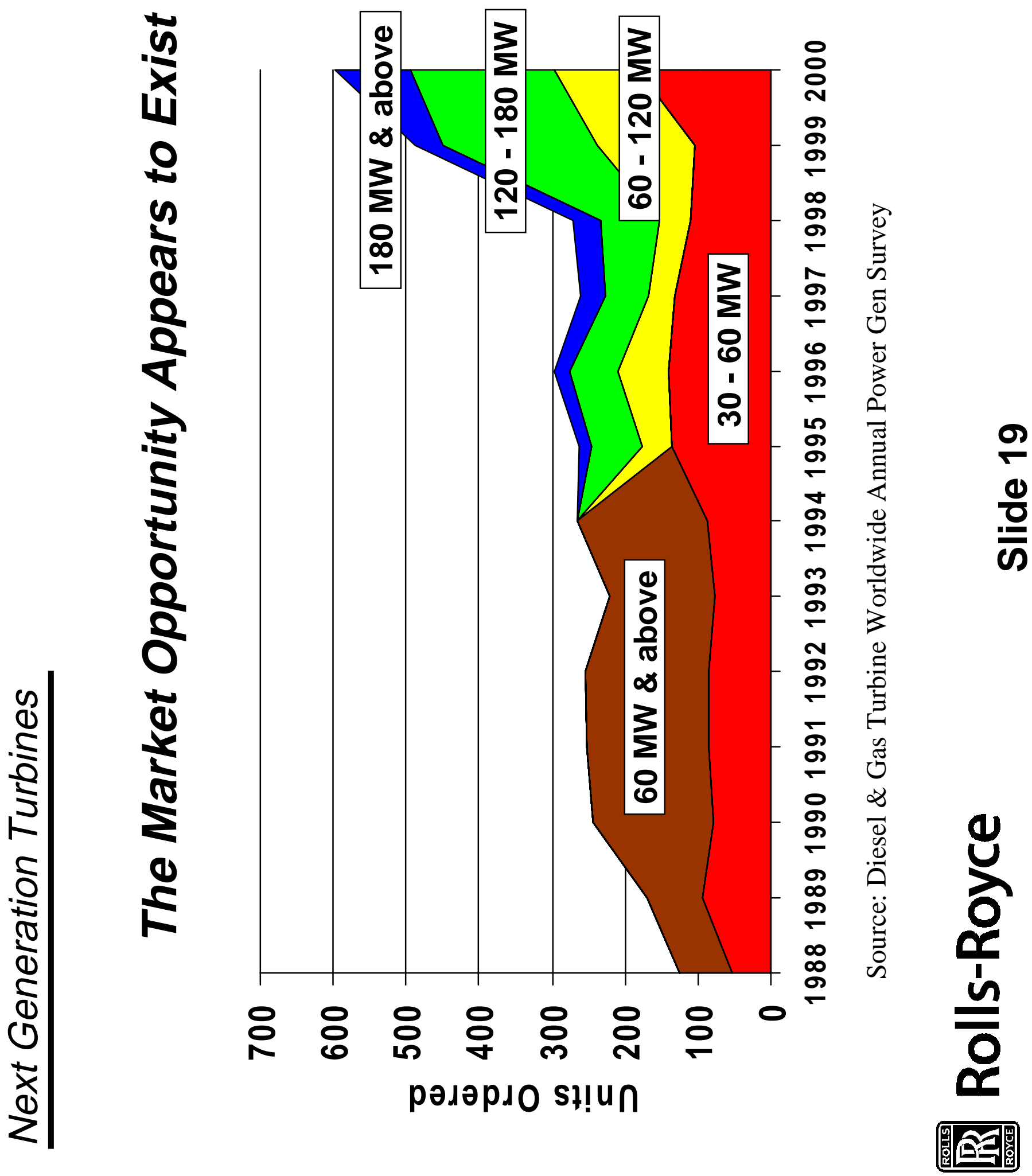



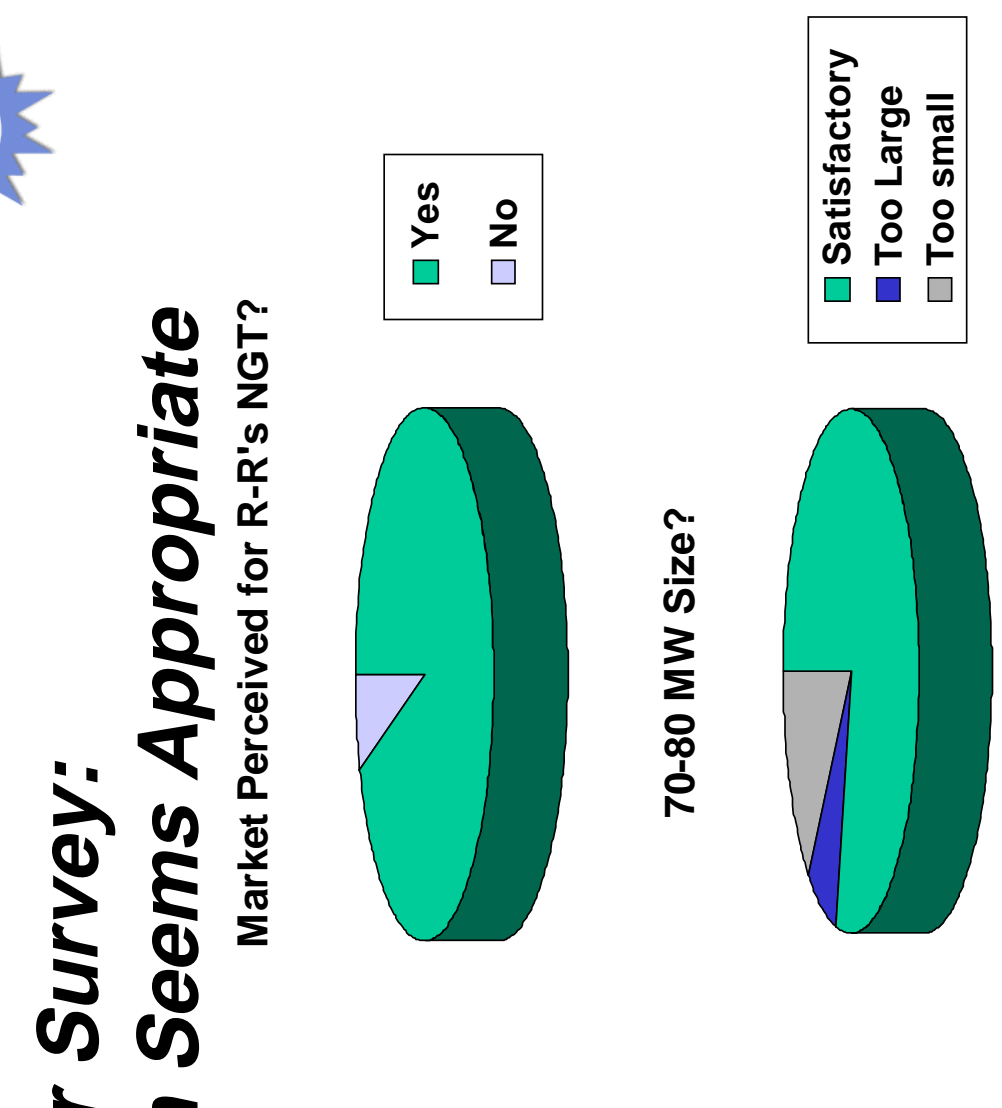

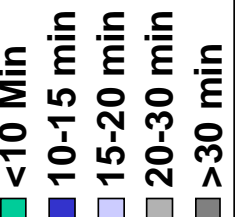
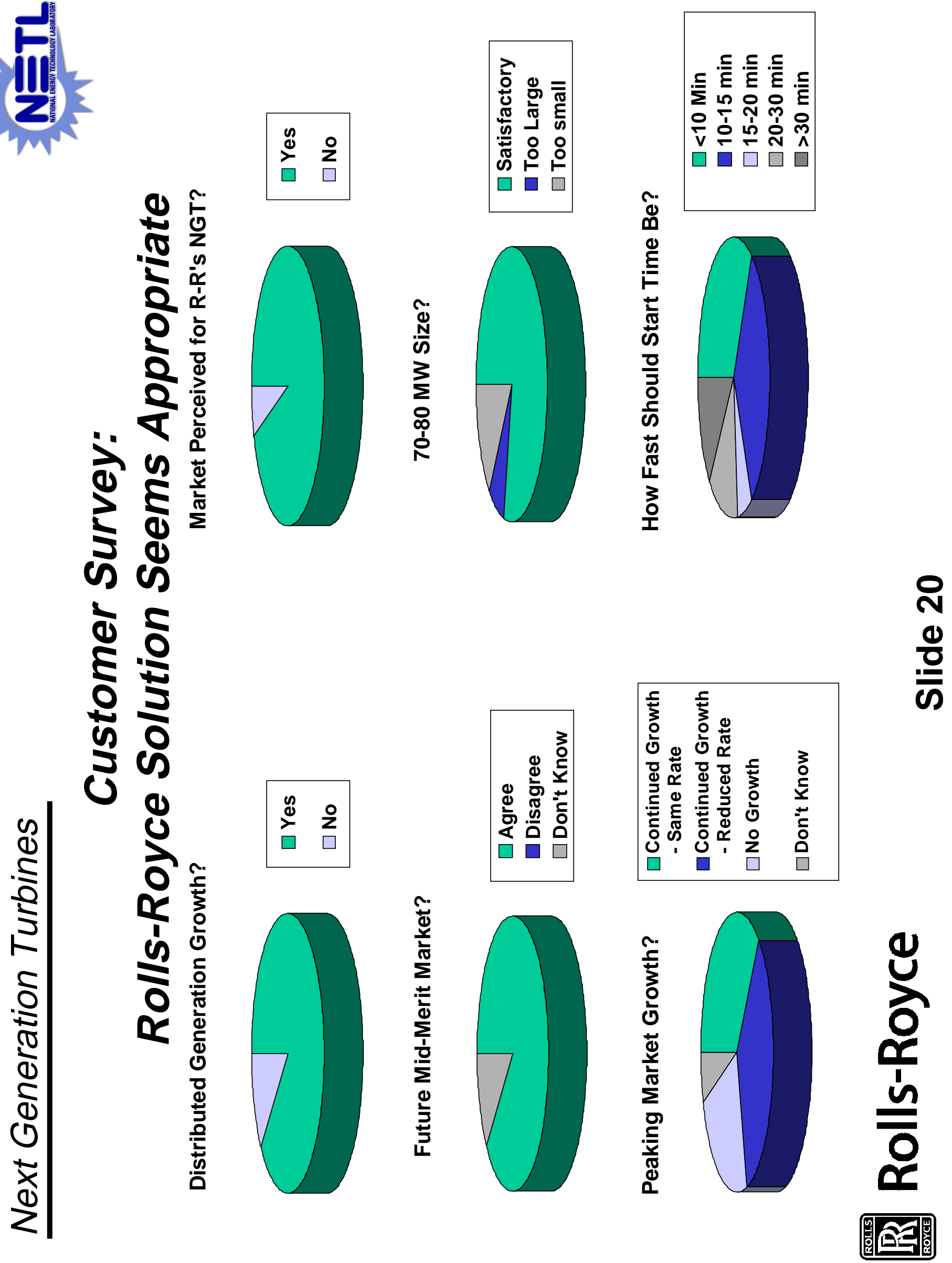


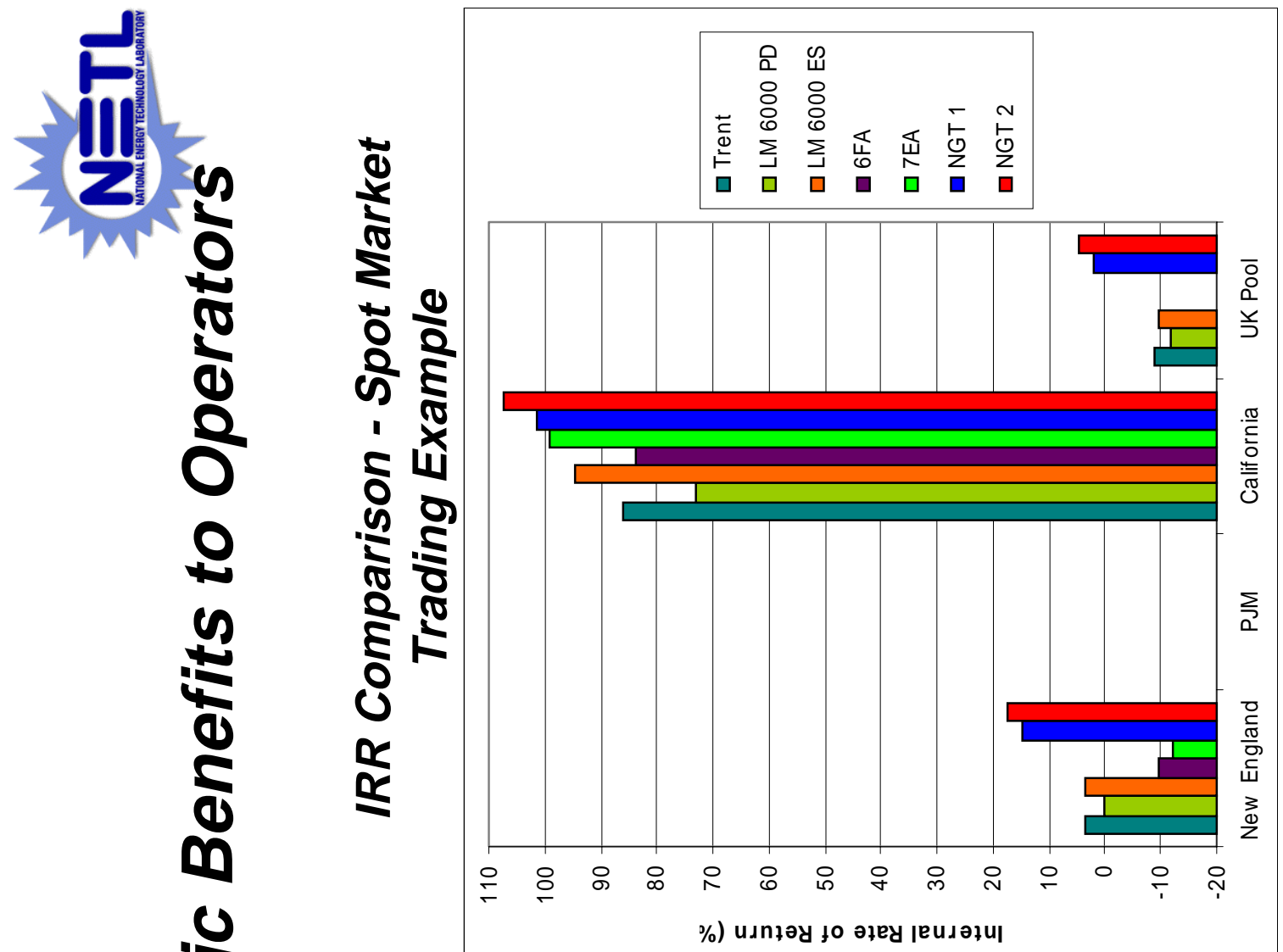

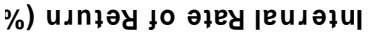
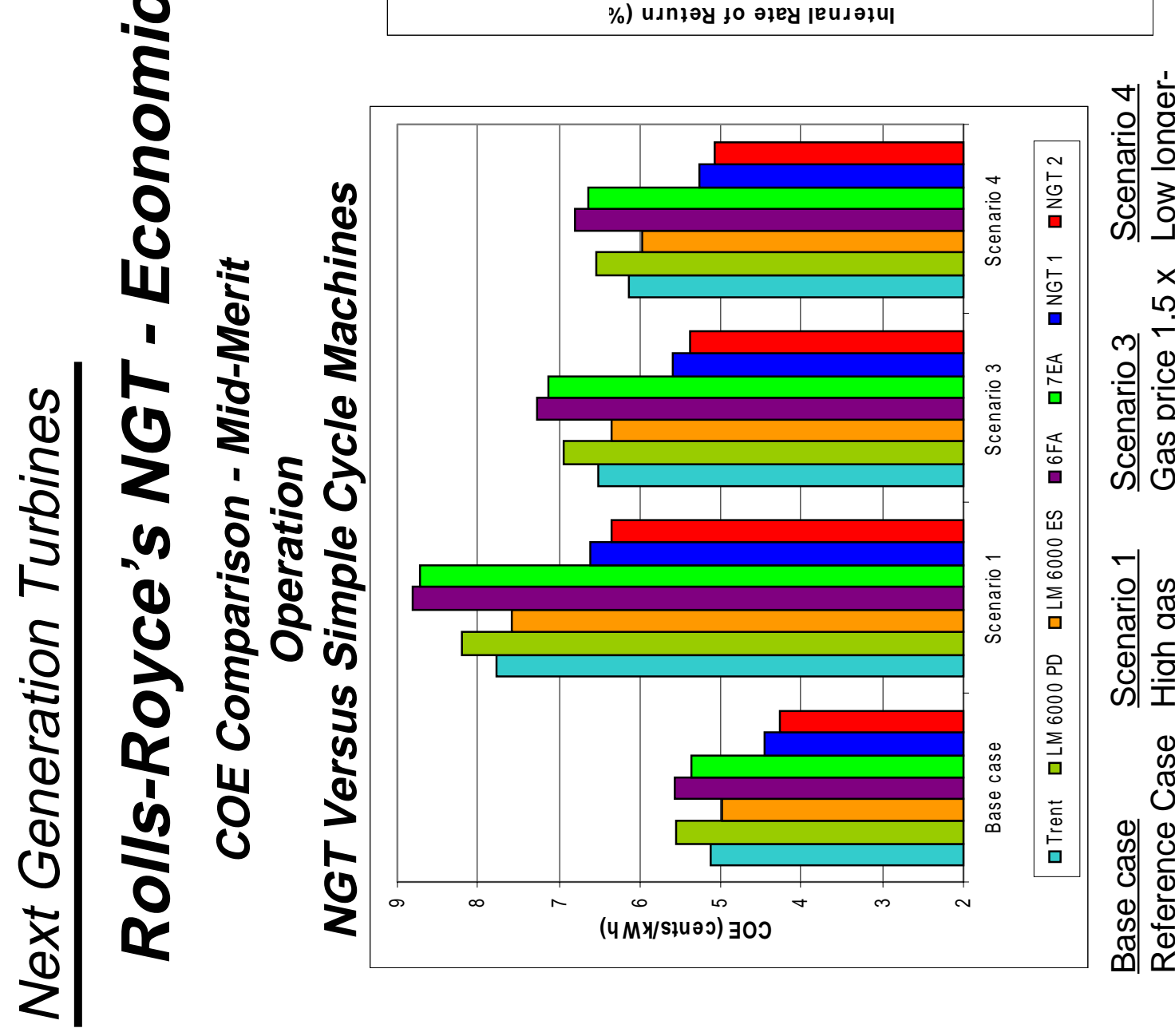

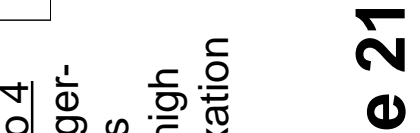

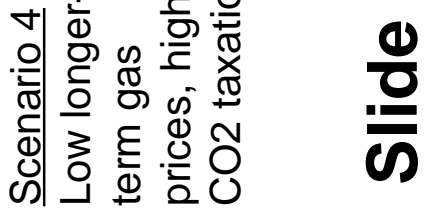

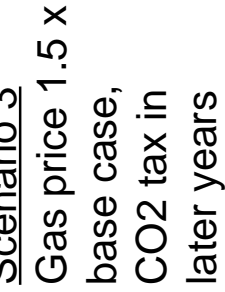

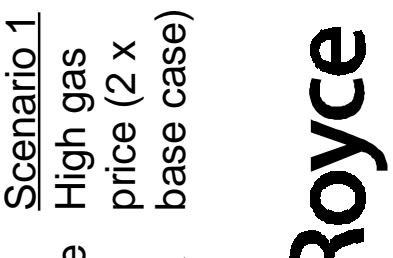

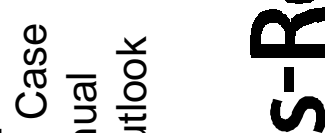
幽 过 0)

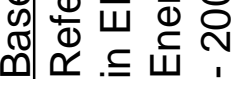




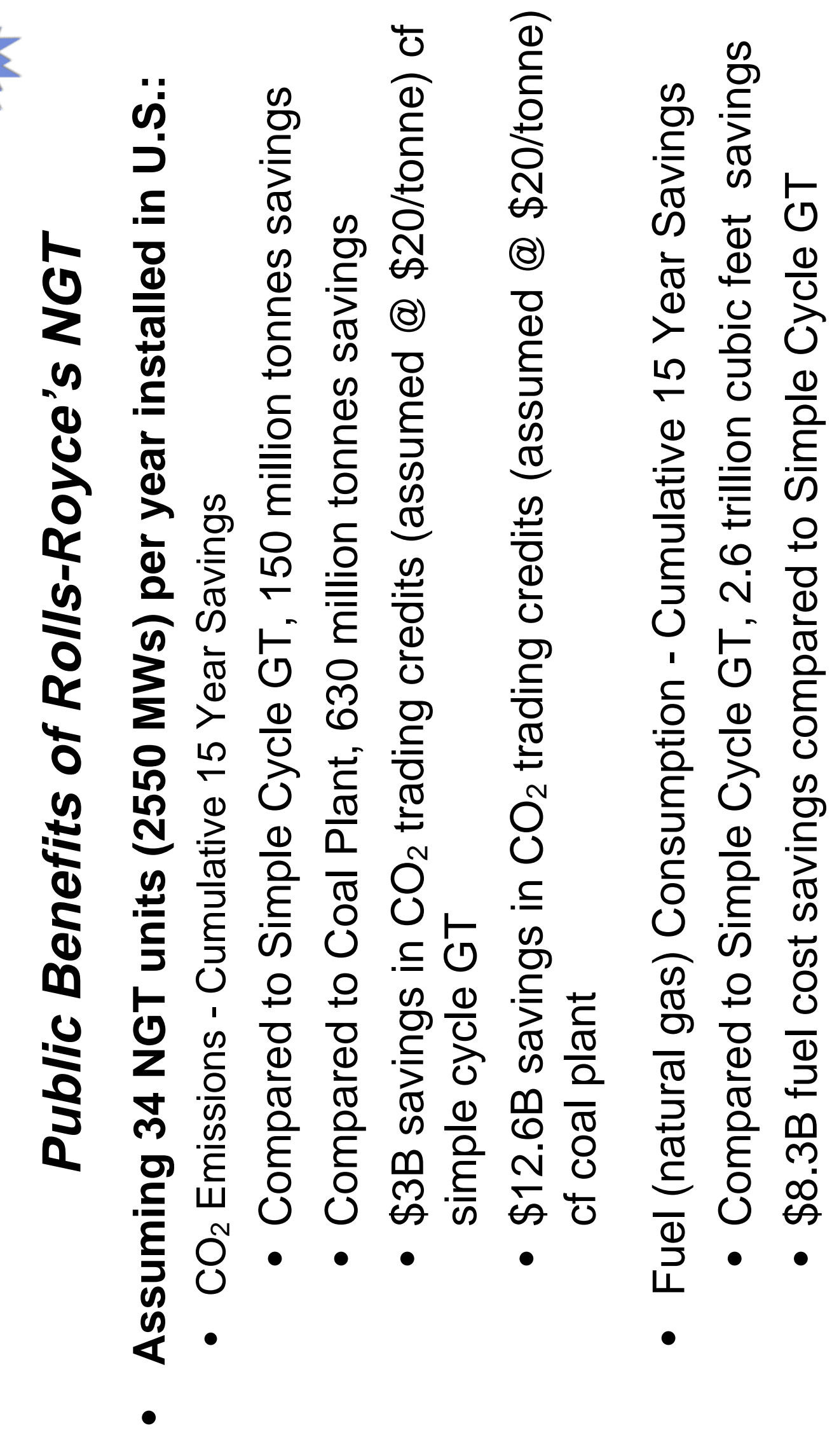

สิ
$\frac{0}{0}$
$\frac{0}{\infty}$

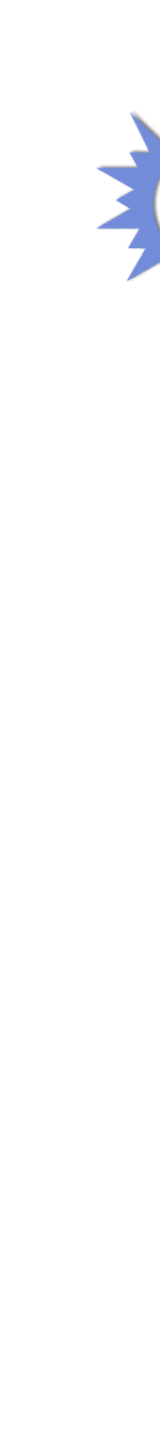

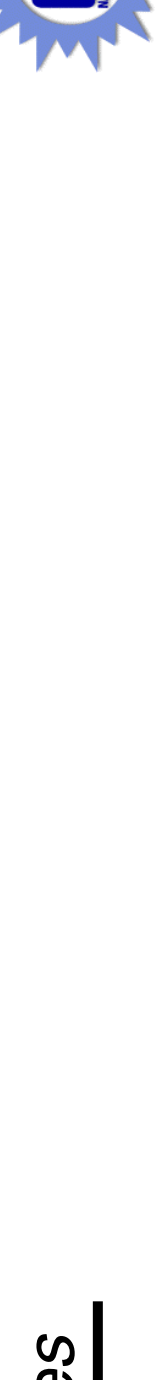

\% 2 g

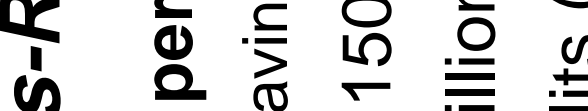

तथ $\omega$ है

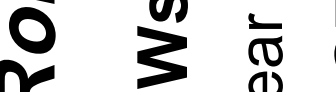

co

- 윤

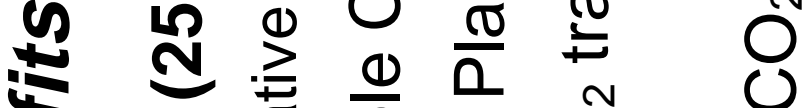

文

2 


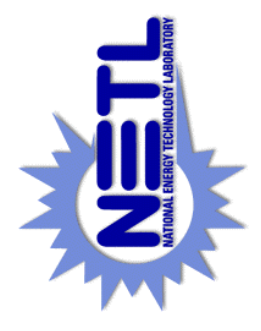

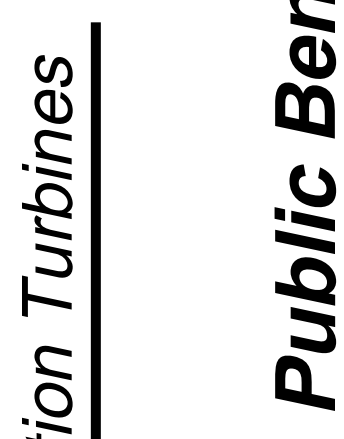
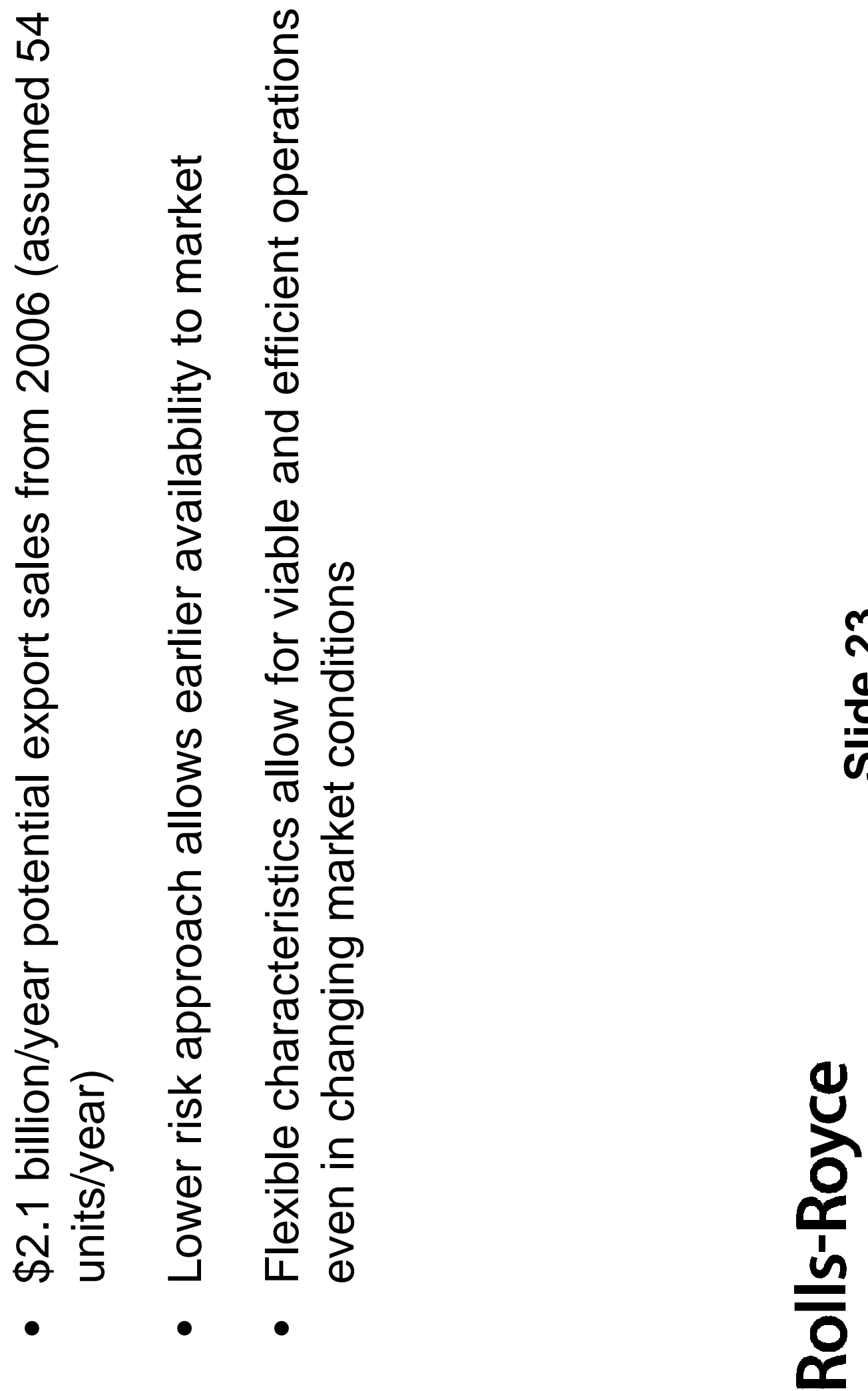


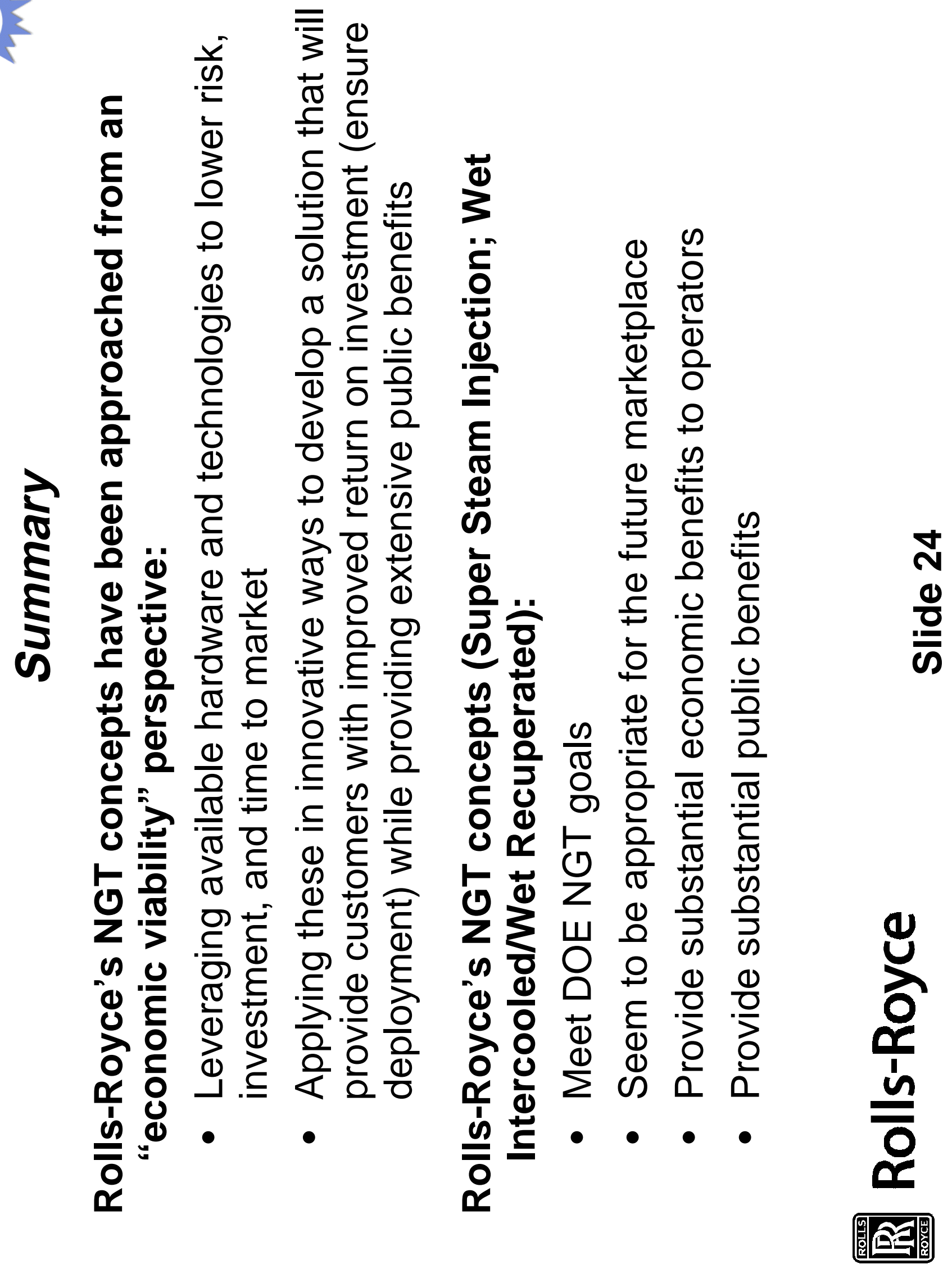




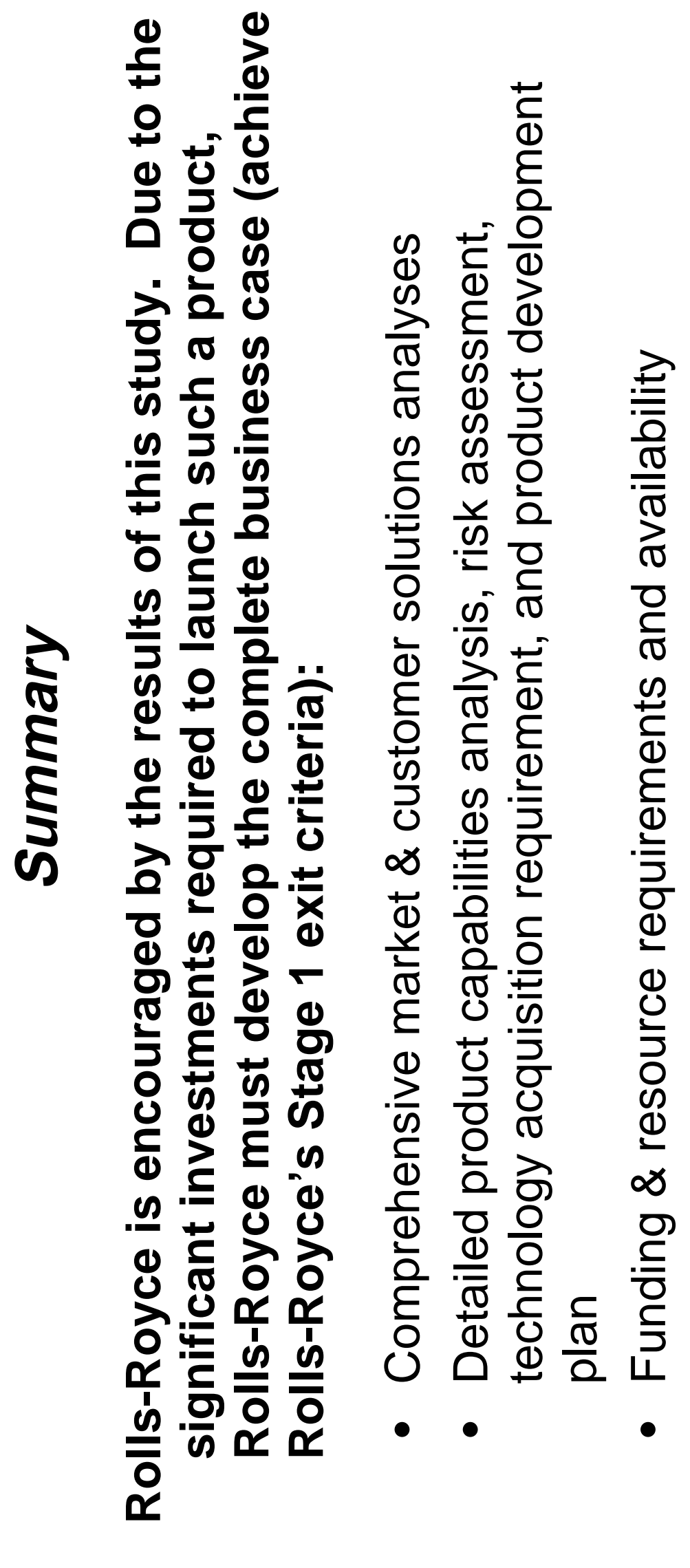




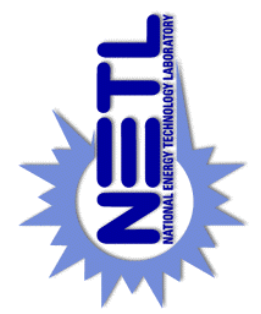

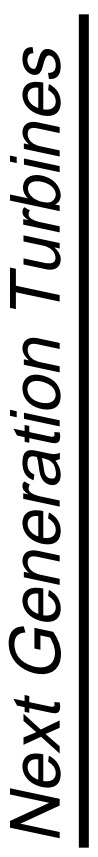
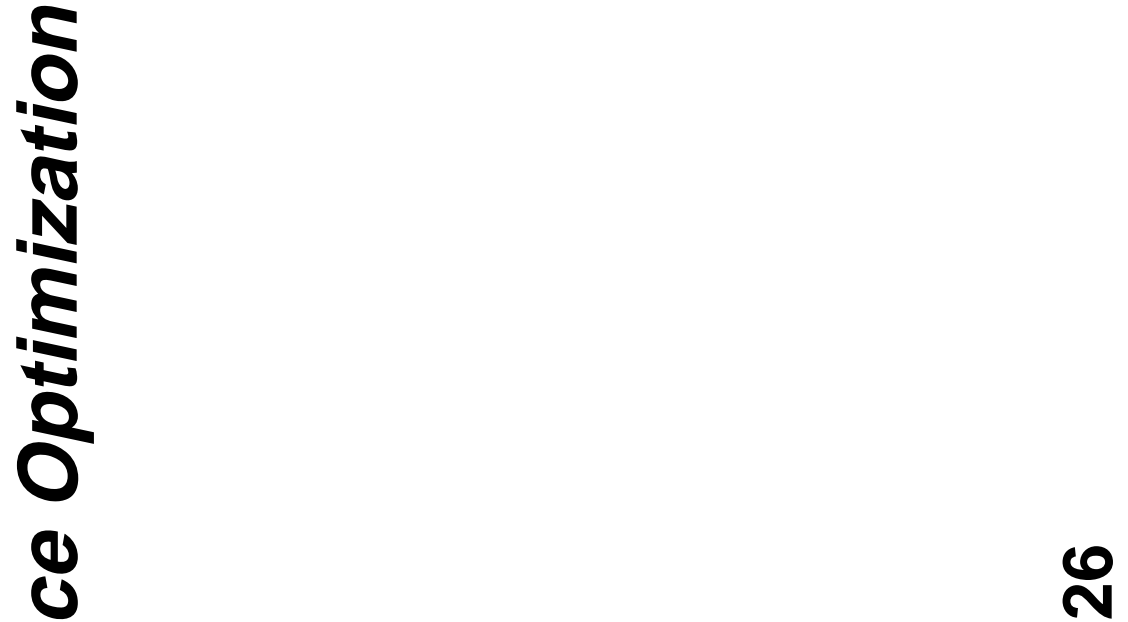

$\frac{0}{\circ}$

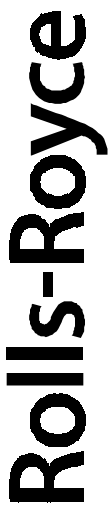

(1)

$\frac{5}{5}$

(D)

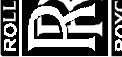



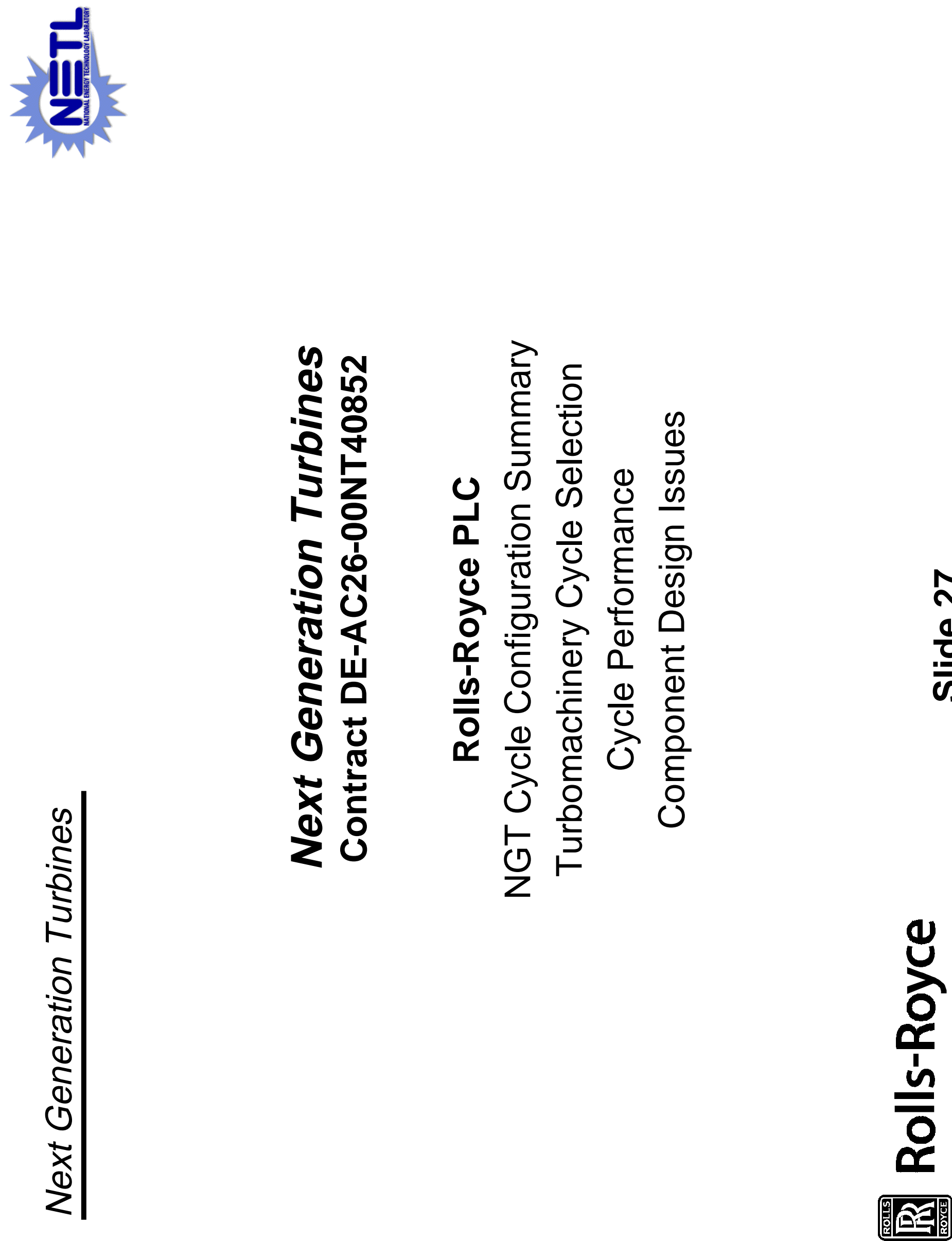


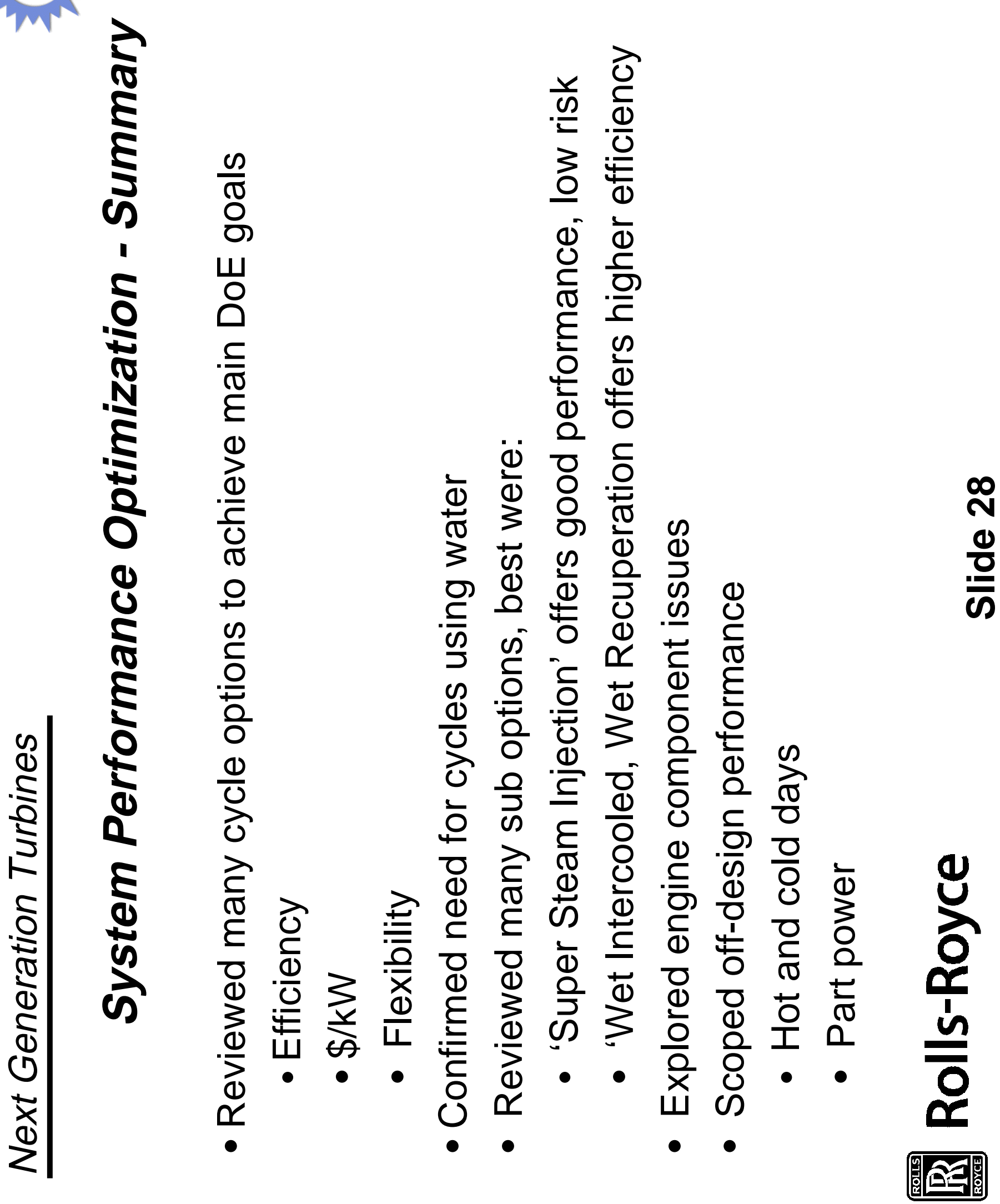




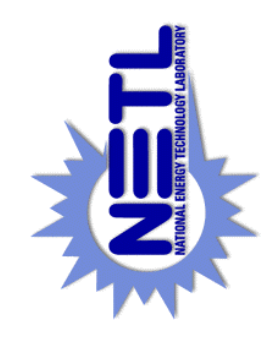

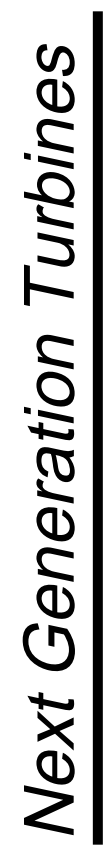
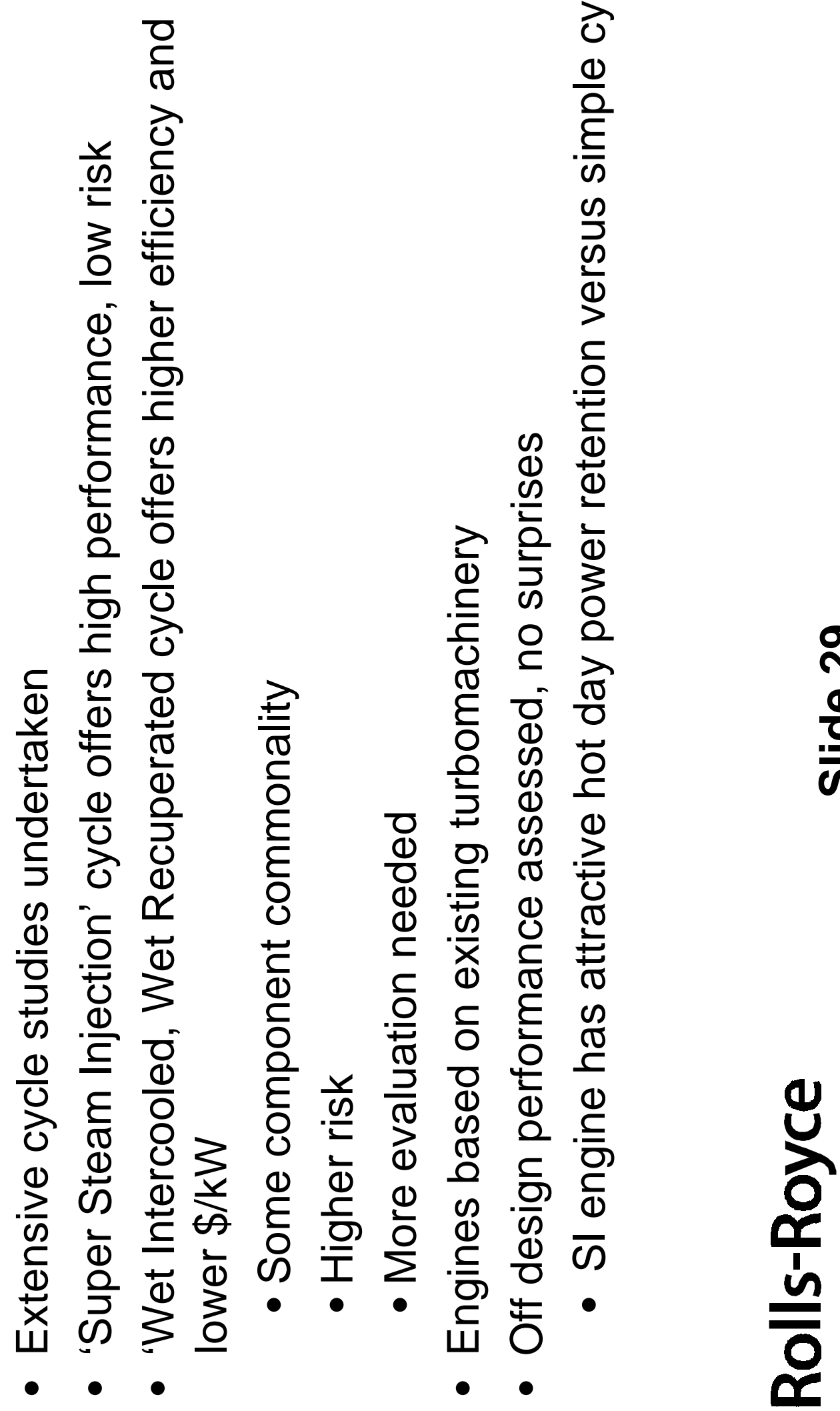


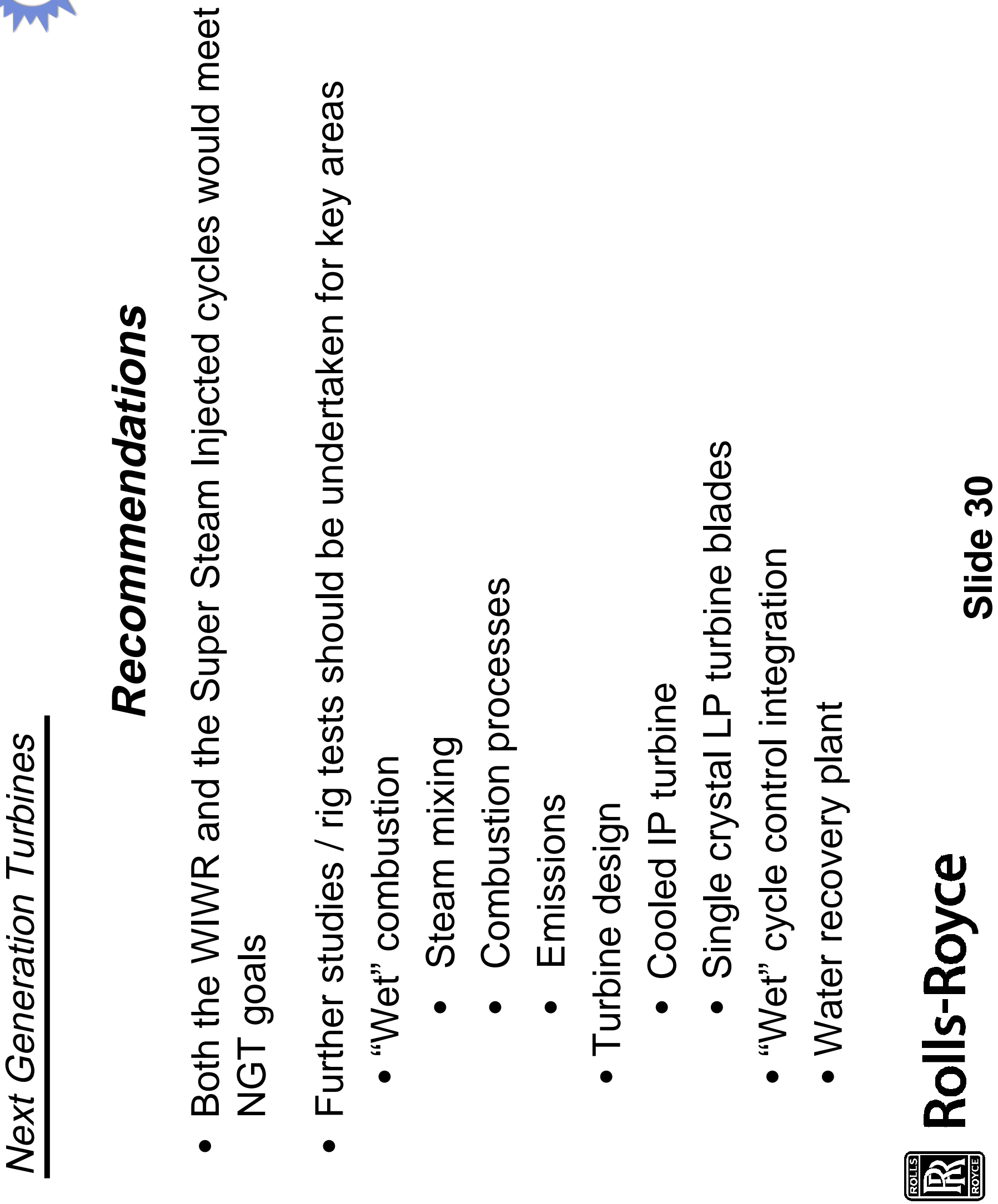




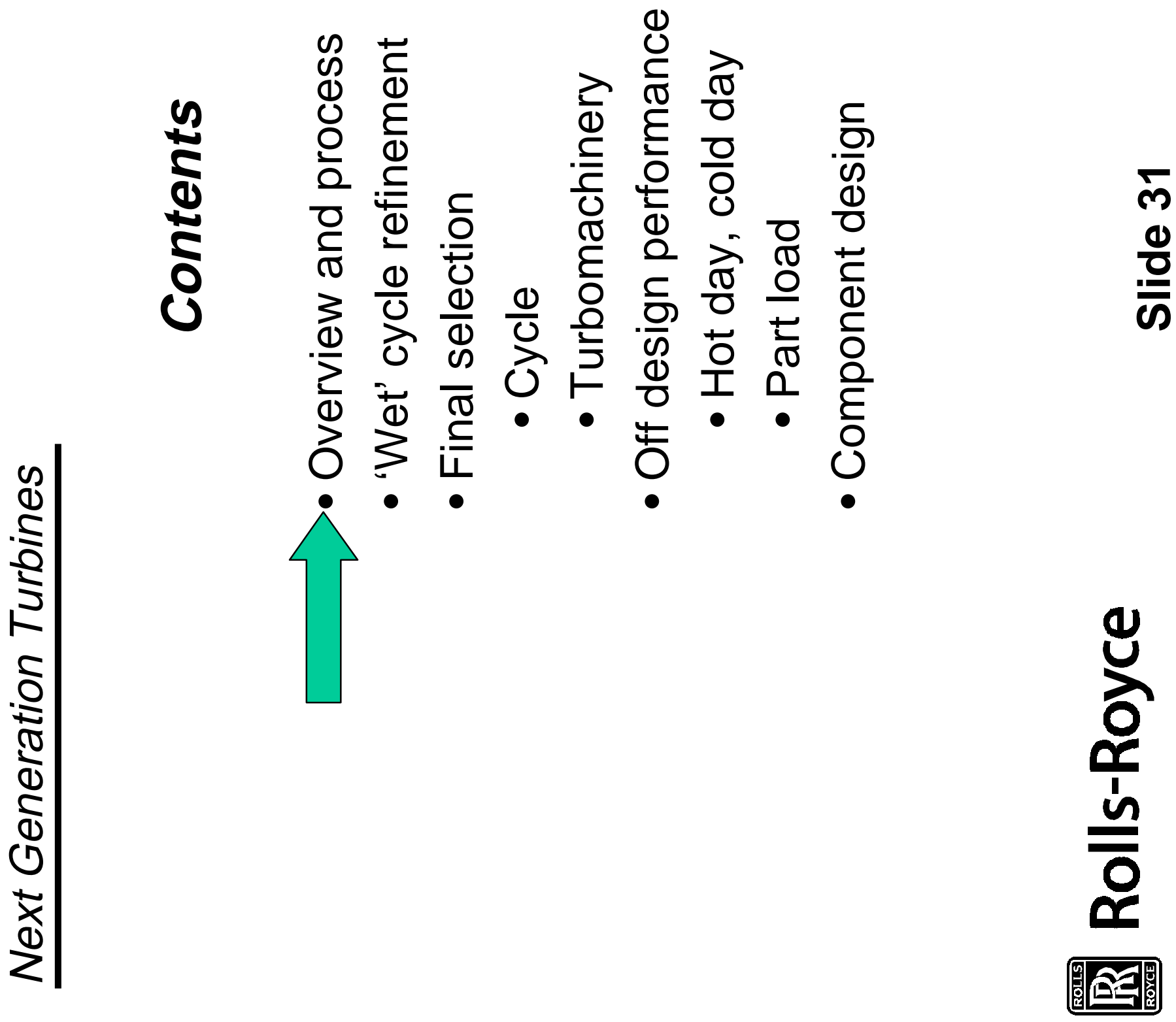




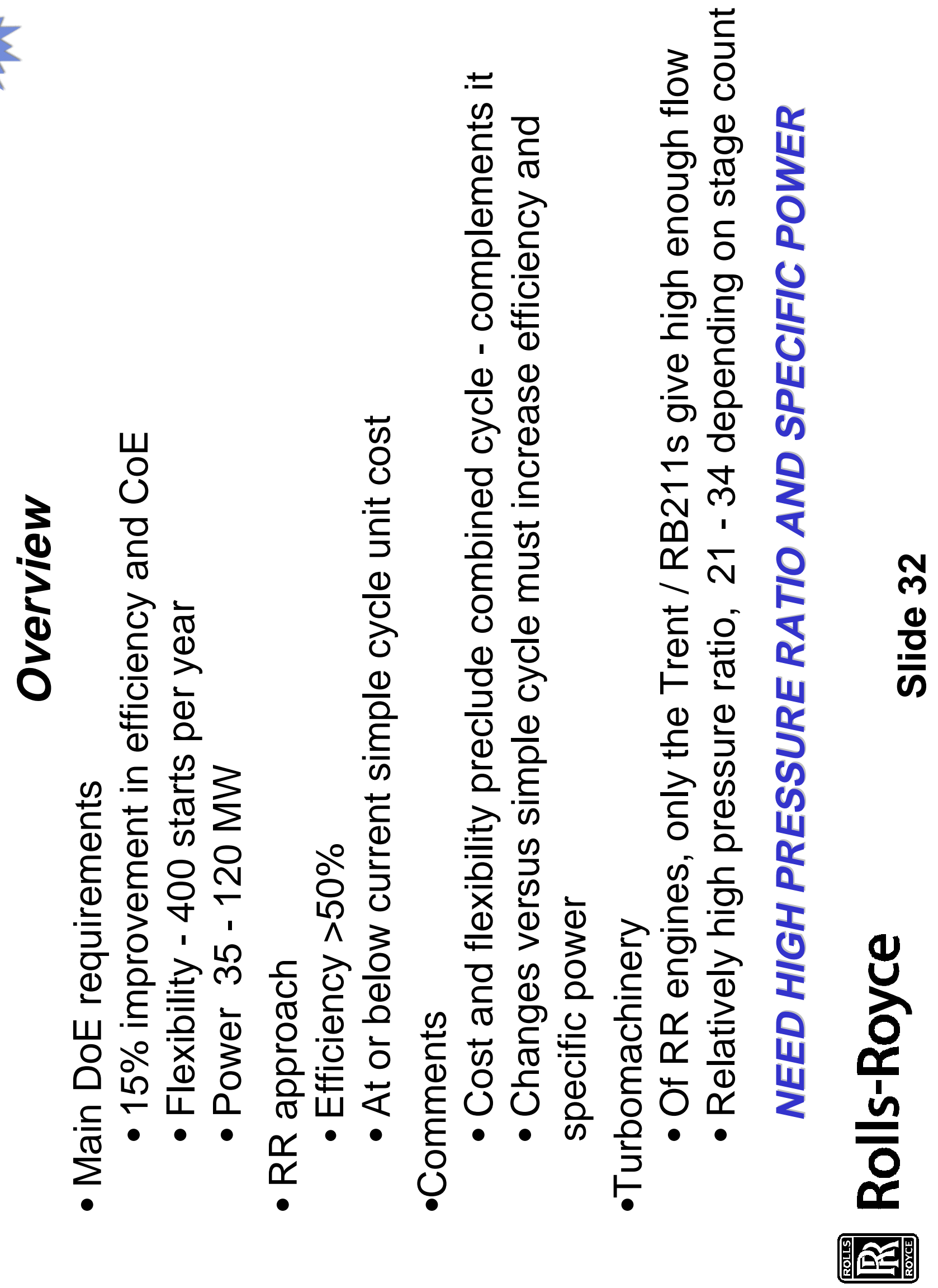




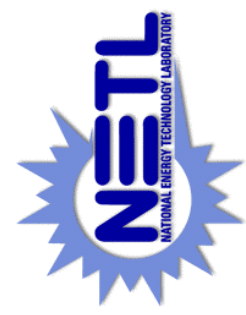

$\frac{3}{0}$

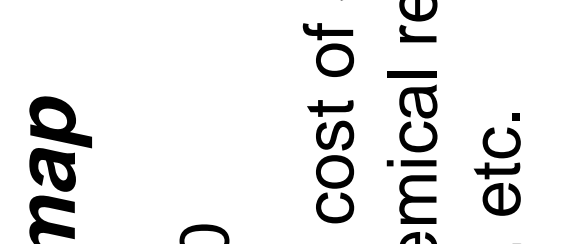
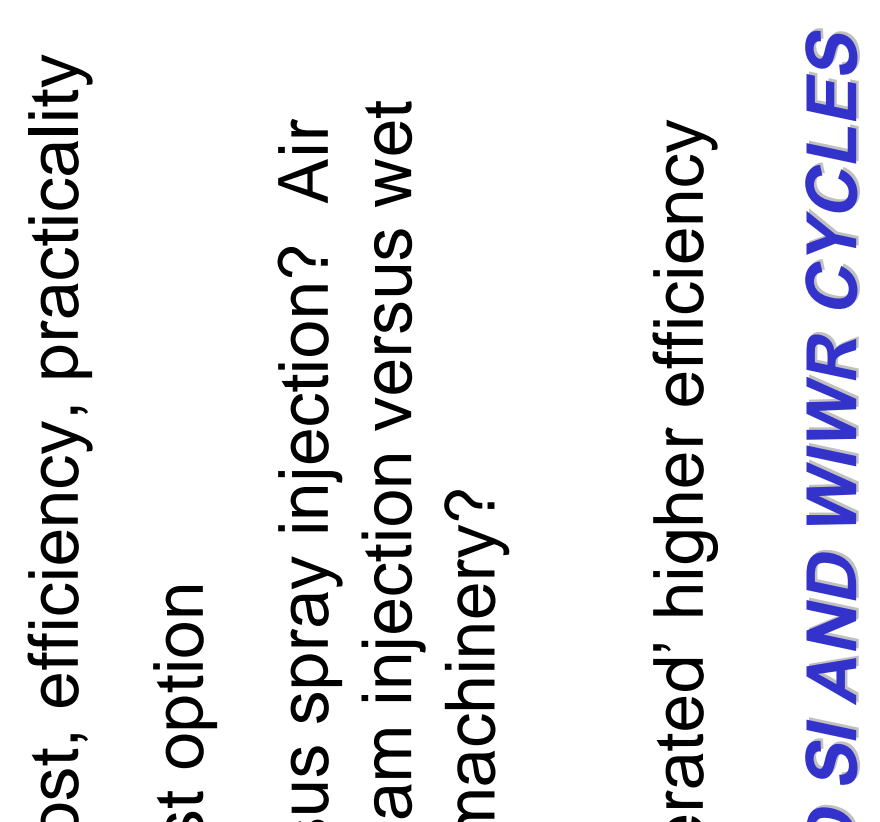

ป N 0

Q

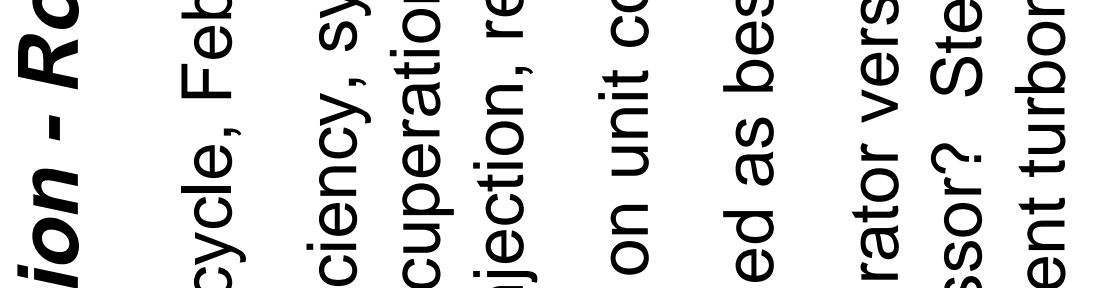

5 o

ष $\overline{8}$ क

ब $\frac{\pi}{0}$

市 둥

을

零

눙

(1)

要离

क 흠뭉.

ㄴ

m

c)

诺

$\frac{0}{0}$

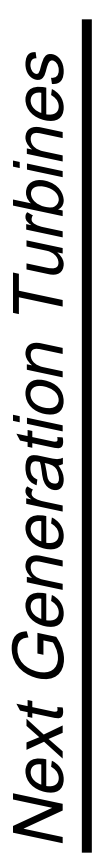

$3 \frac{\Phi}{\omega}$

తి

․ 휭 ․ㅡ응

U O

0

这

$\varepsilon$

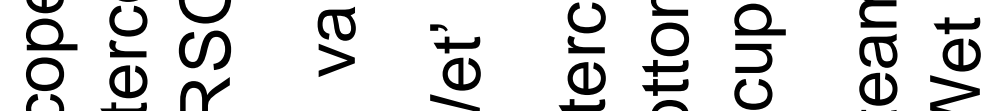

\

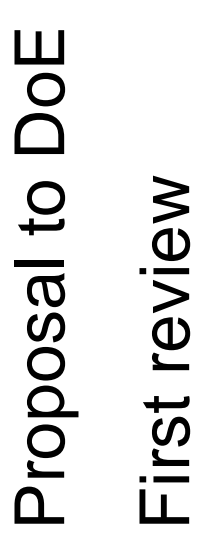

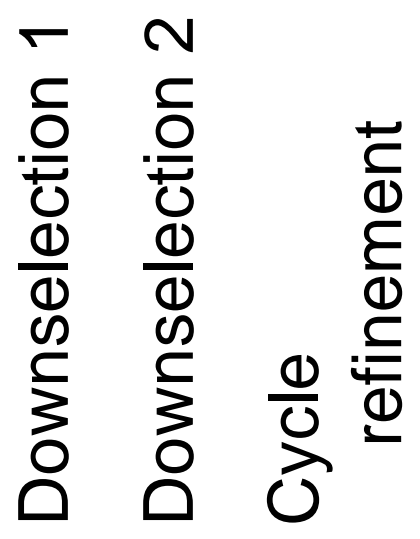

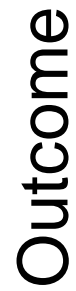

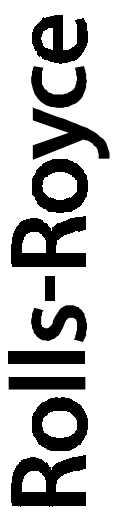




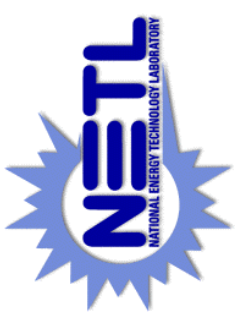

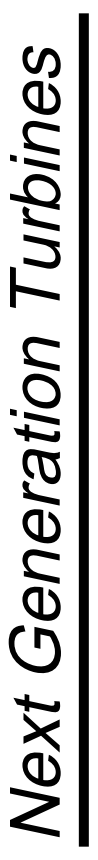
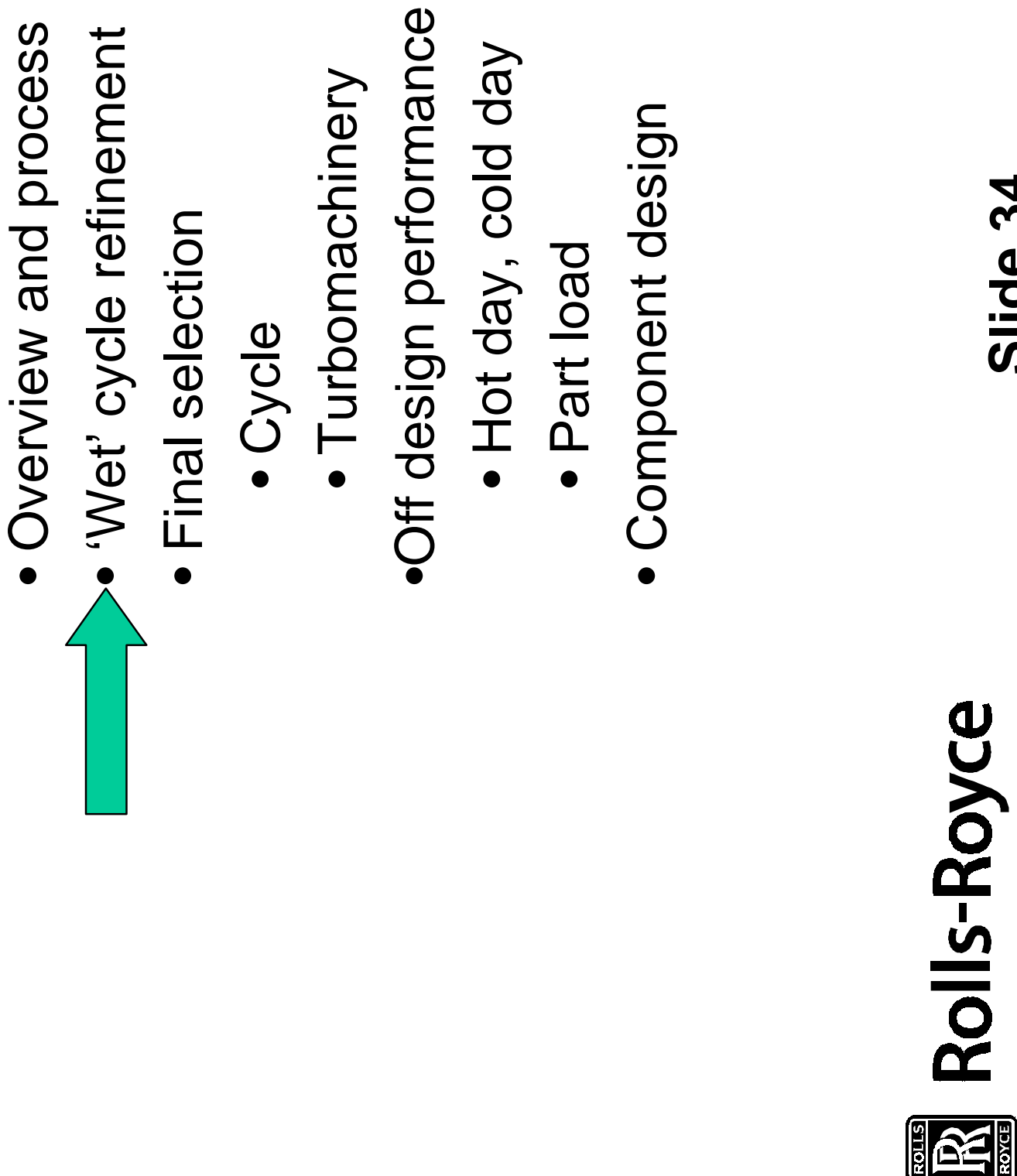

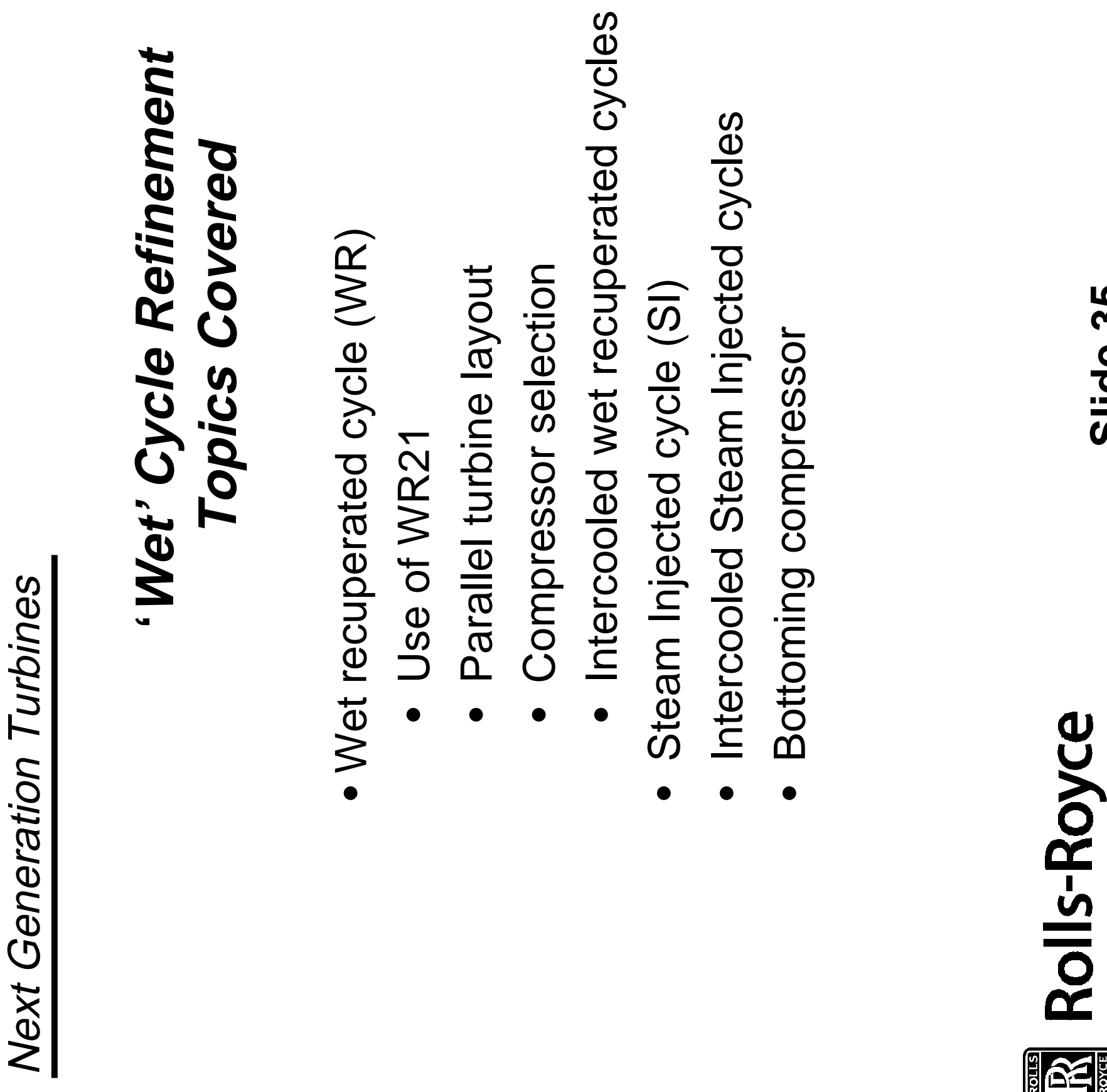

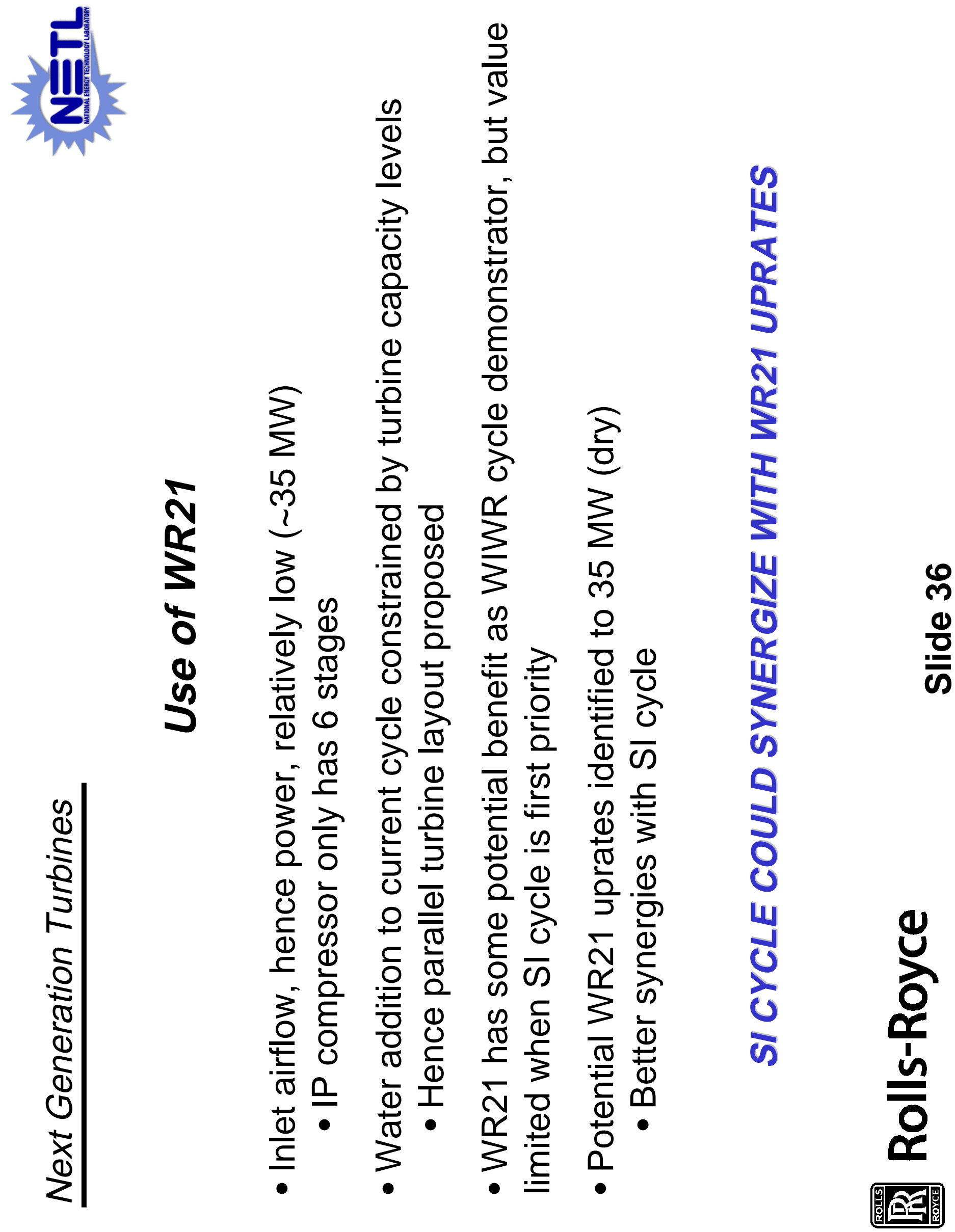


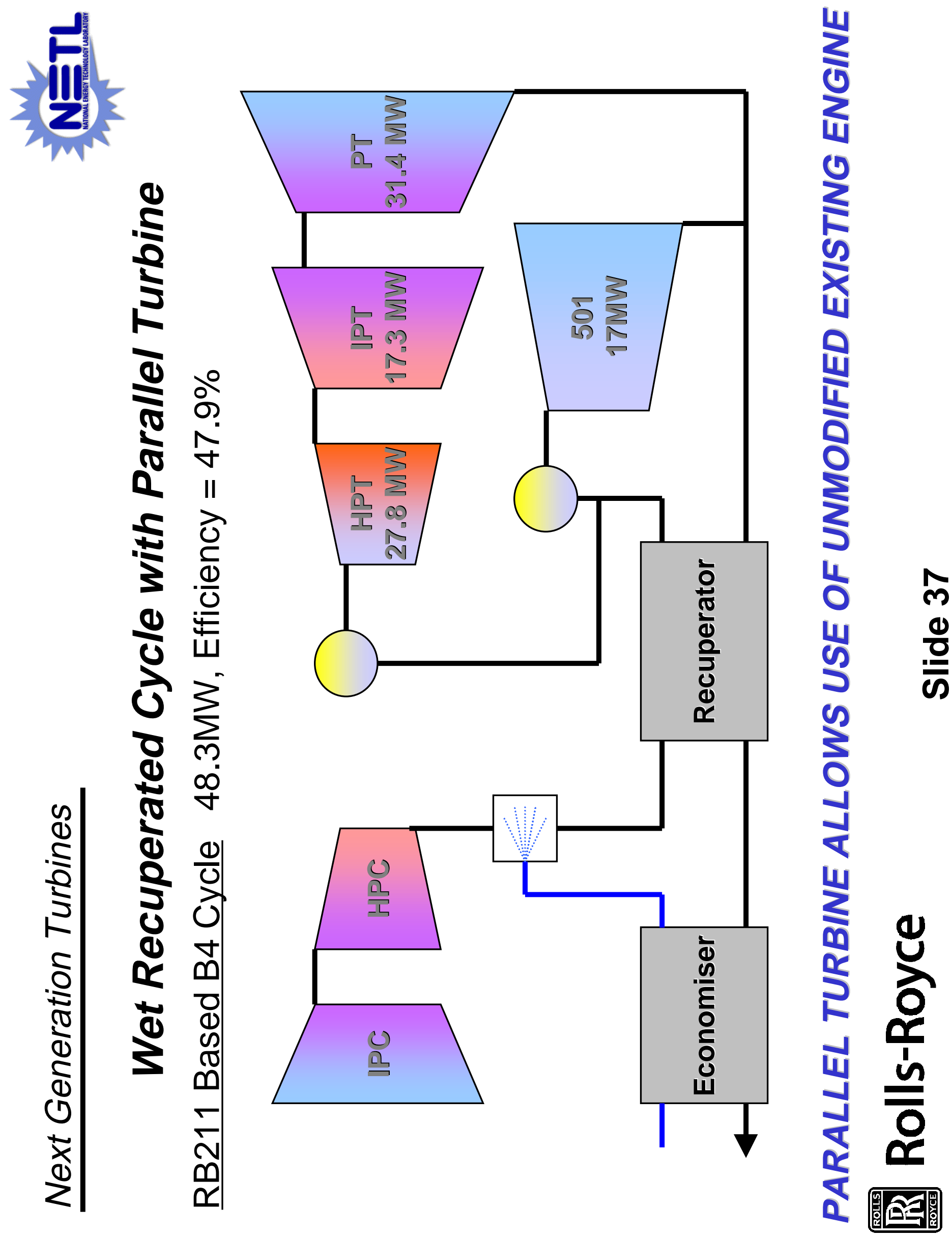




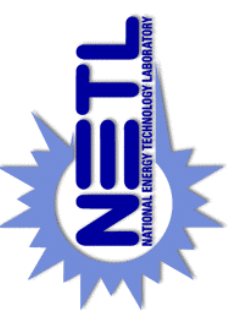

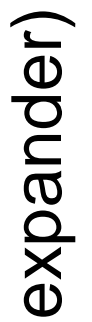
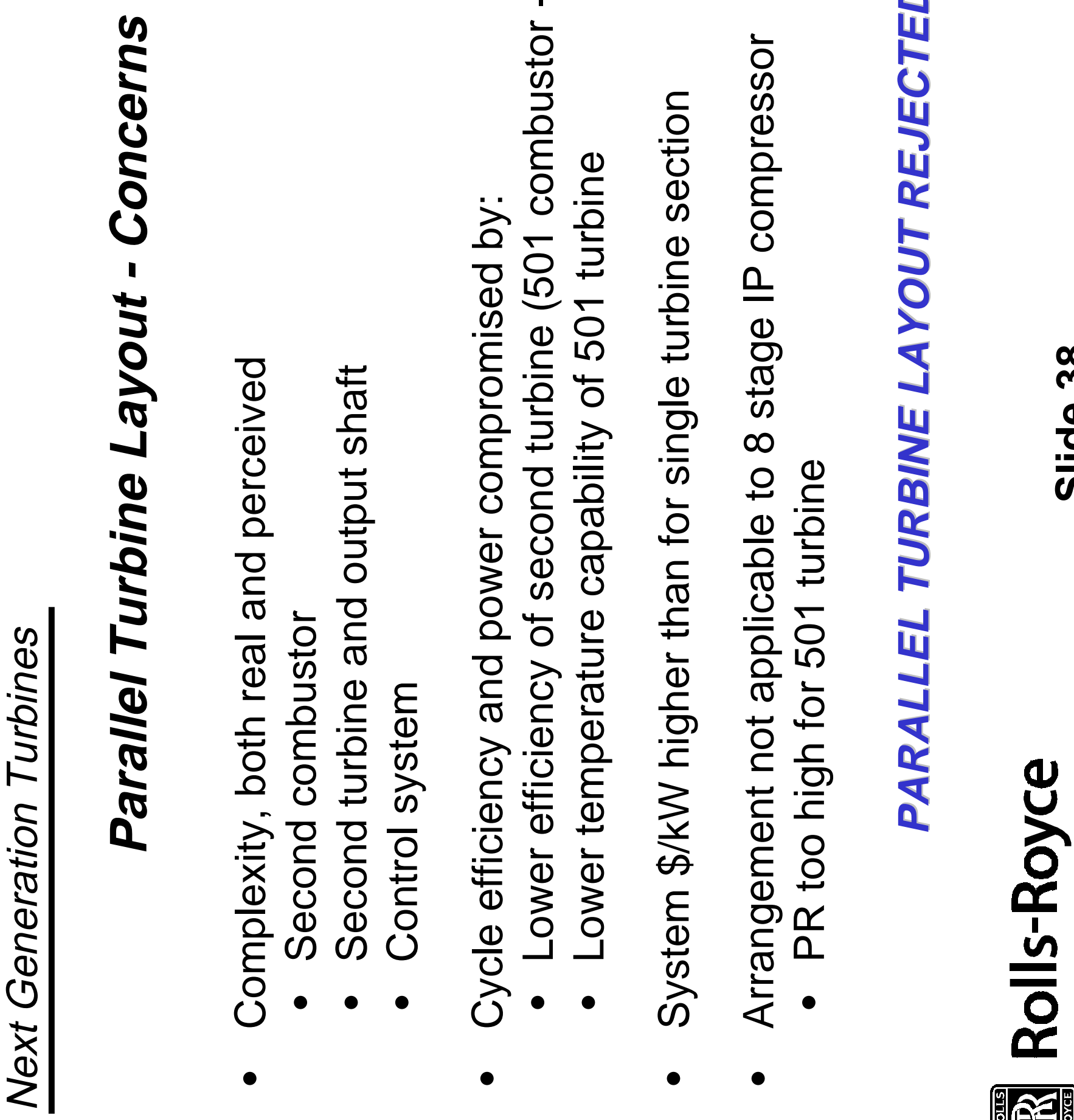

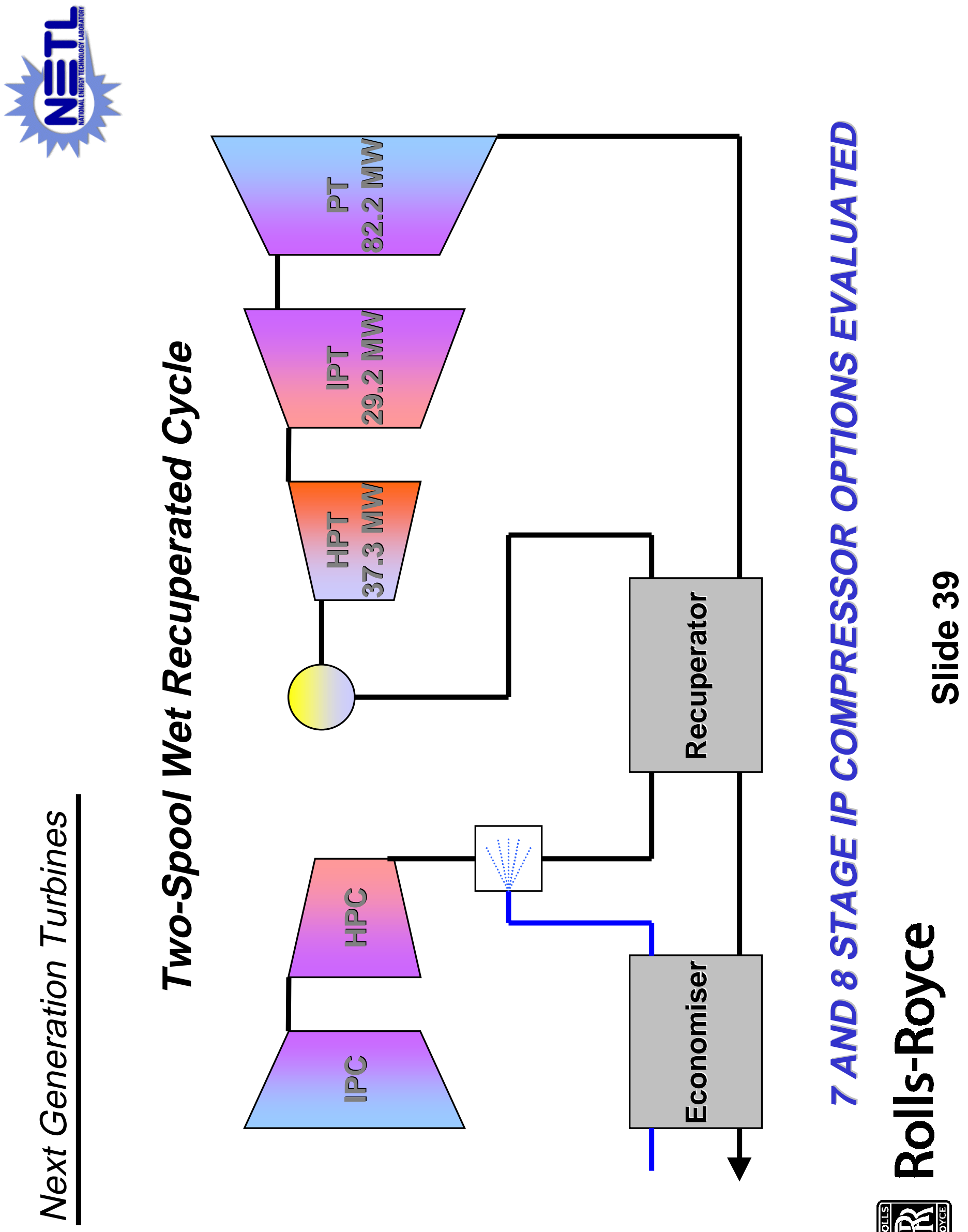

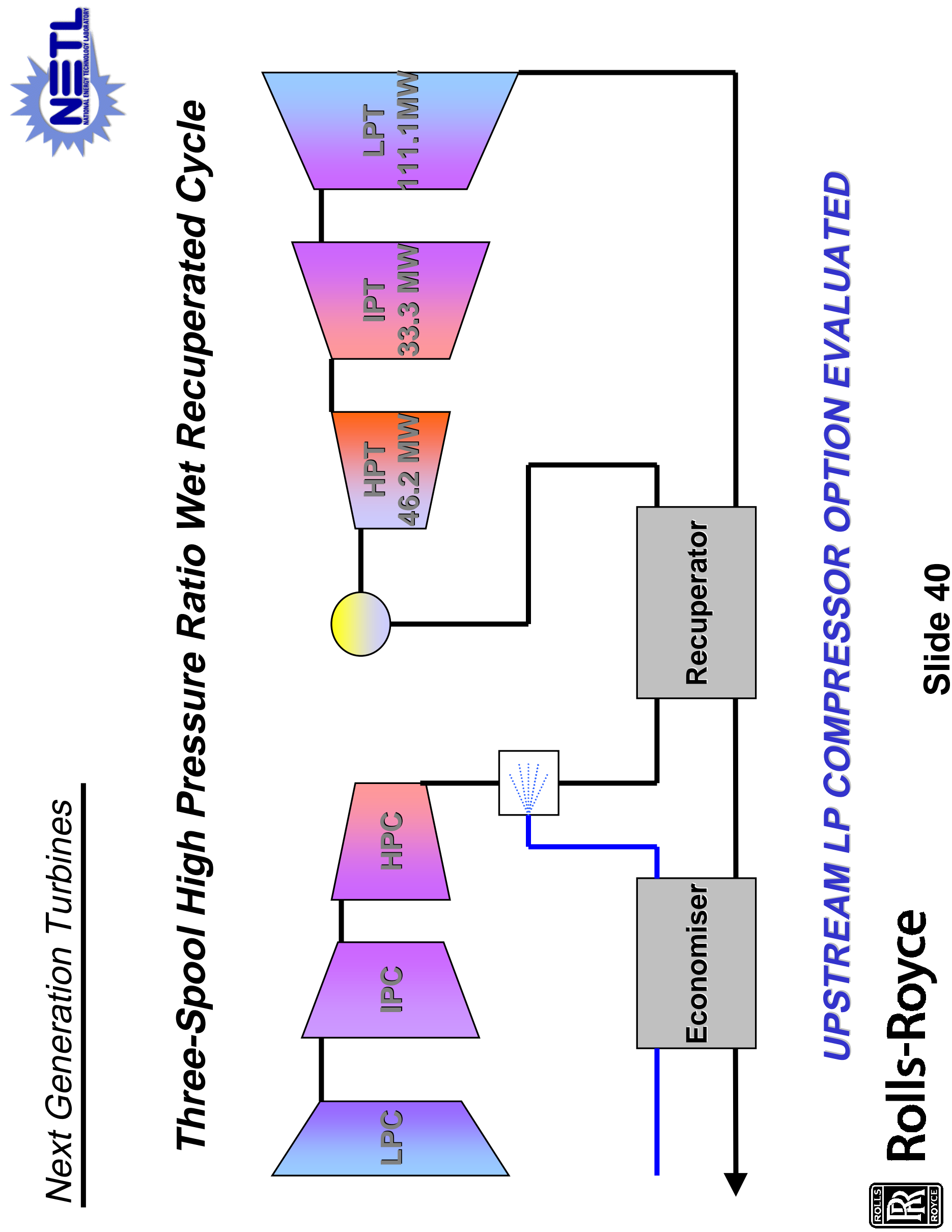


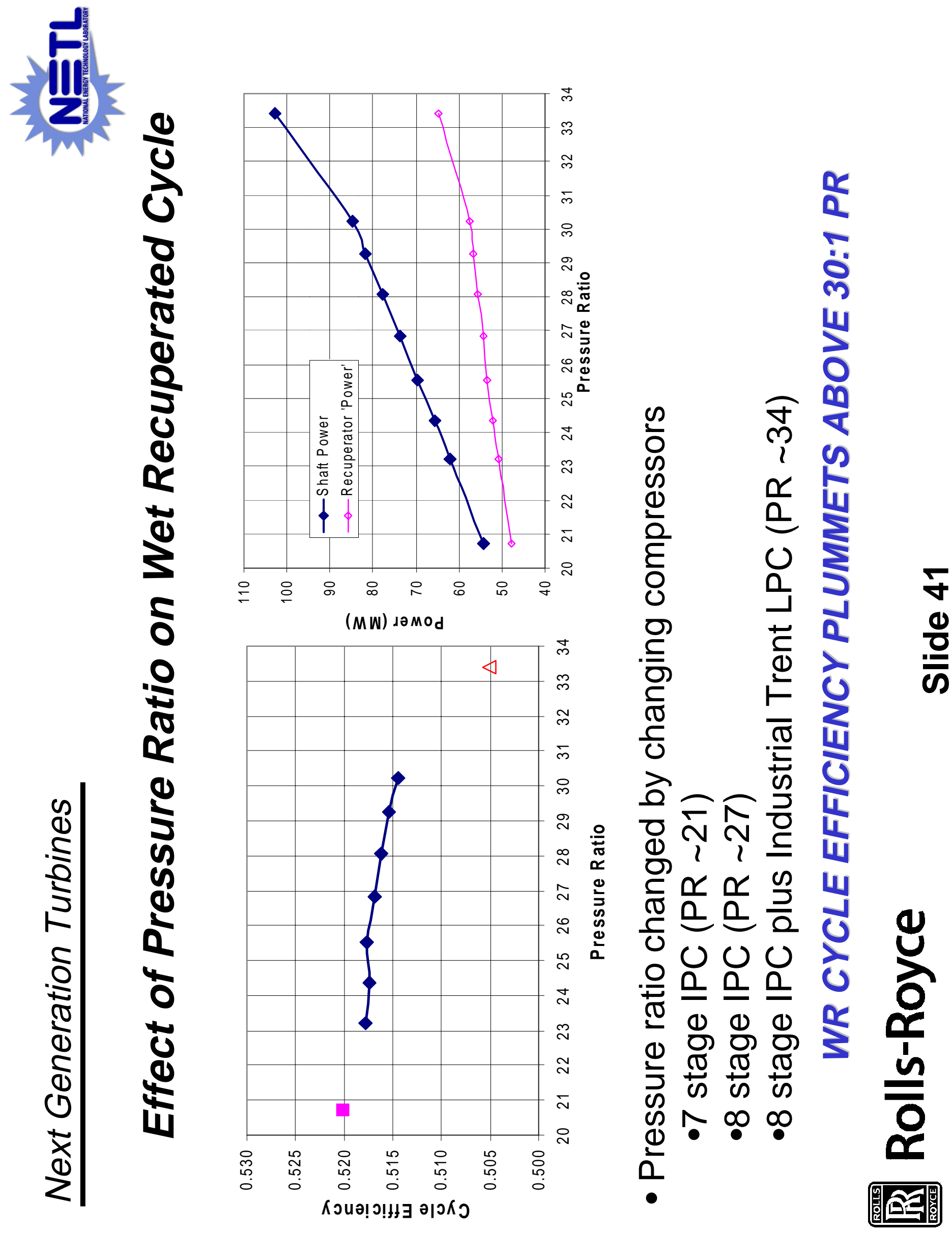




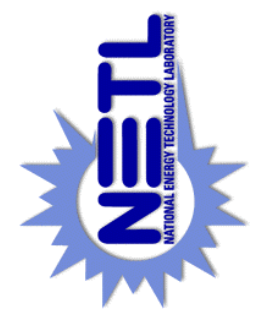

.

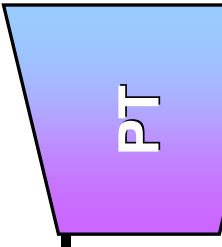

.
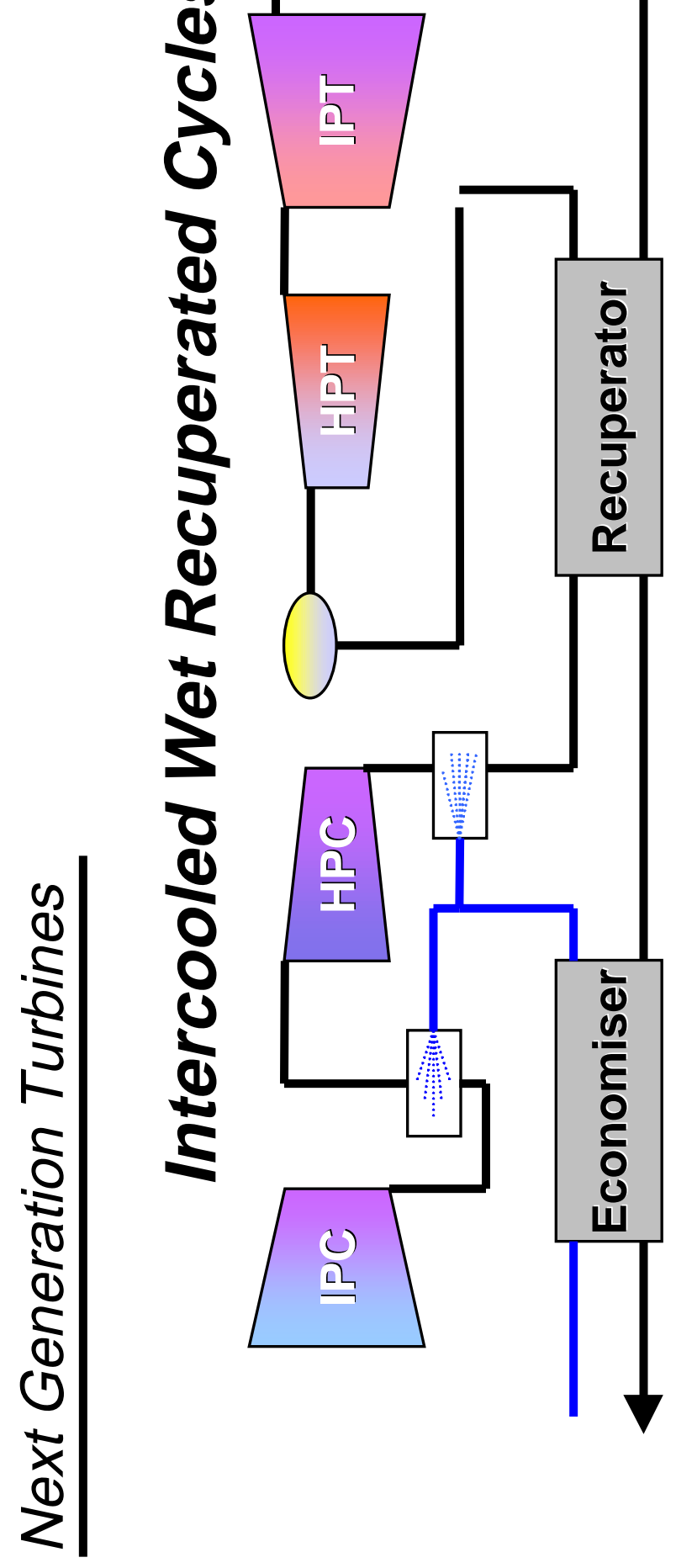
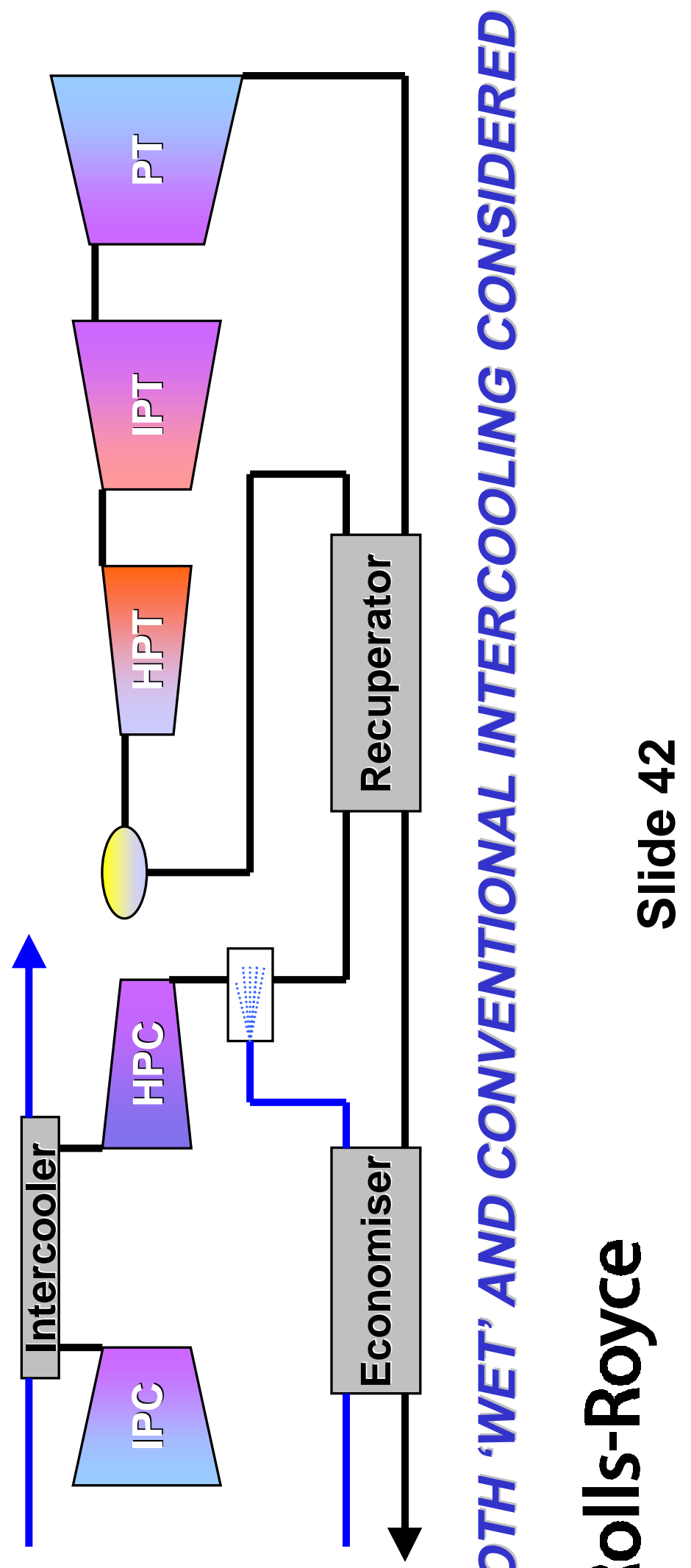

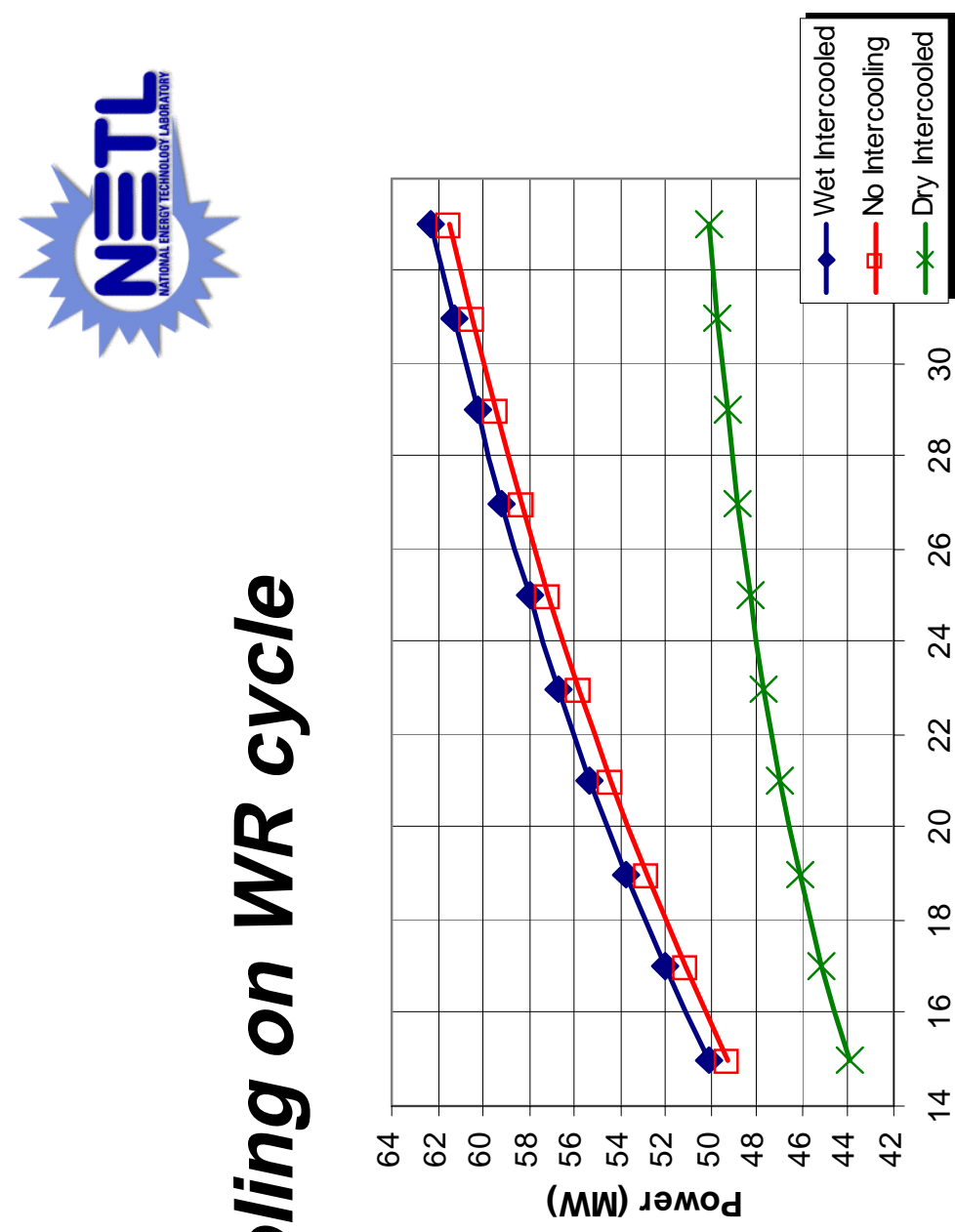

(MW) ләмоd

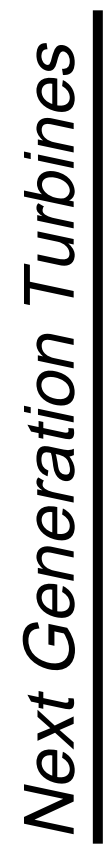

.

T

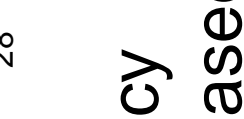

드

I $\frac{1}{0} \frac{1}{0}$

을 트

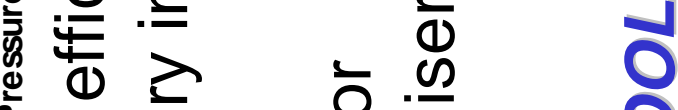

(1)

$\geqslant \geqslant$ (1)

눌

$\frac{1}{2}$

의

워

至

3 를

$\varepsilon$

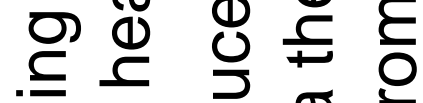

트들 문 드

응 훙

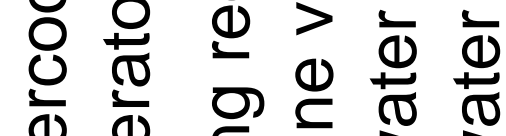

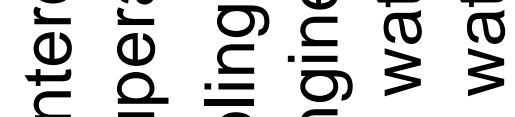

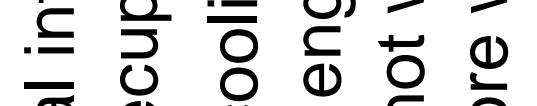

ธָธ ญ

으 $\stackrel{5}{ \pm}$ क

응 해

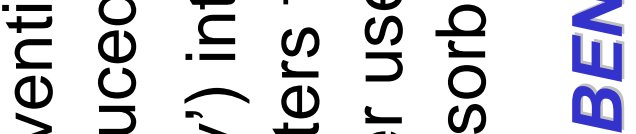

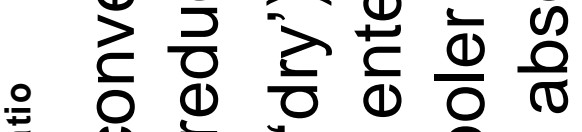

9

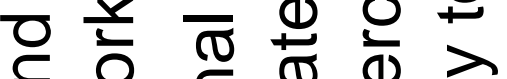

ชิ

ब记

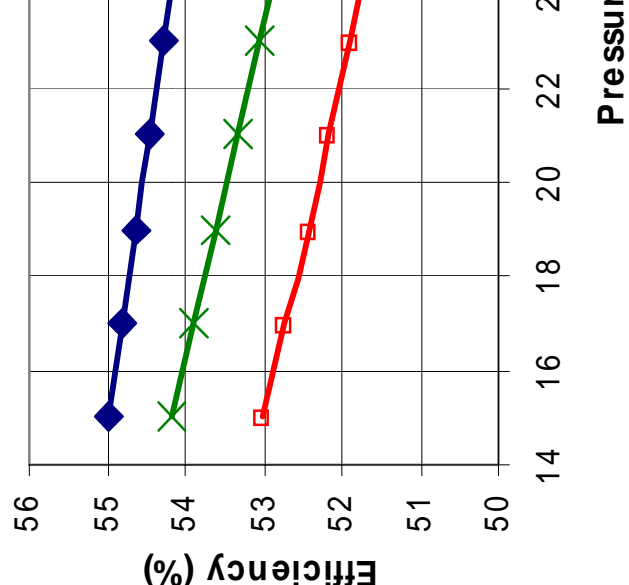

ส

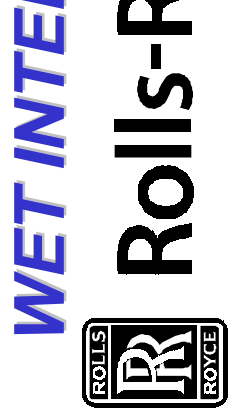




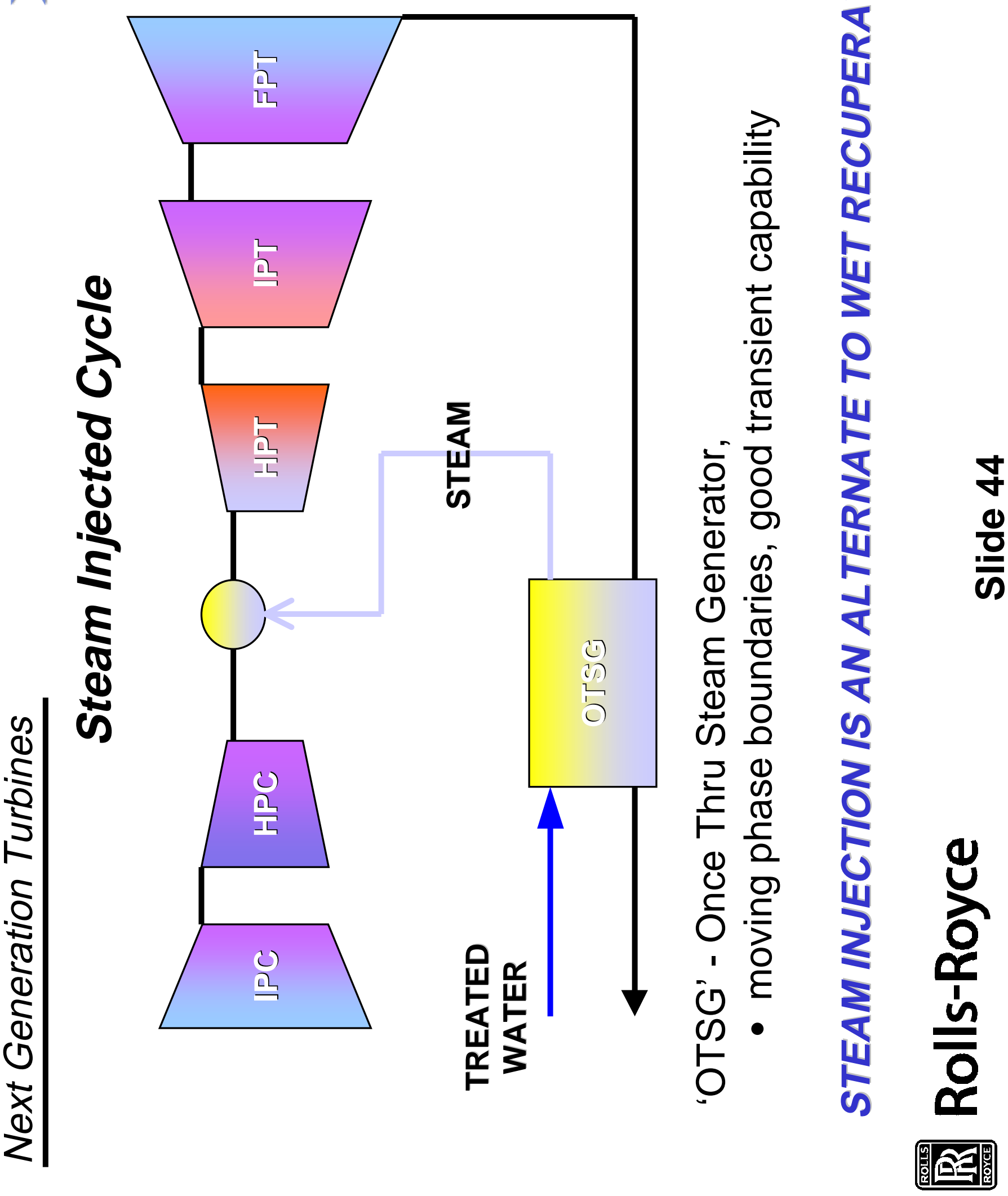



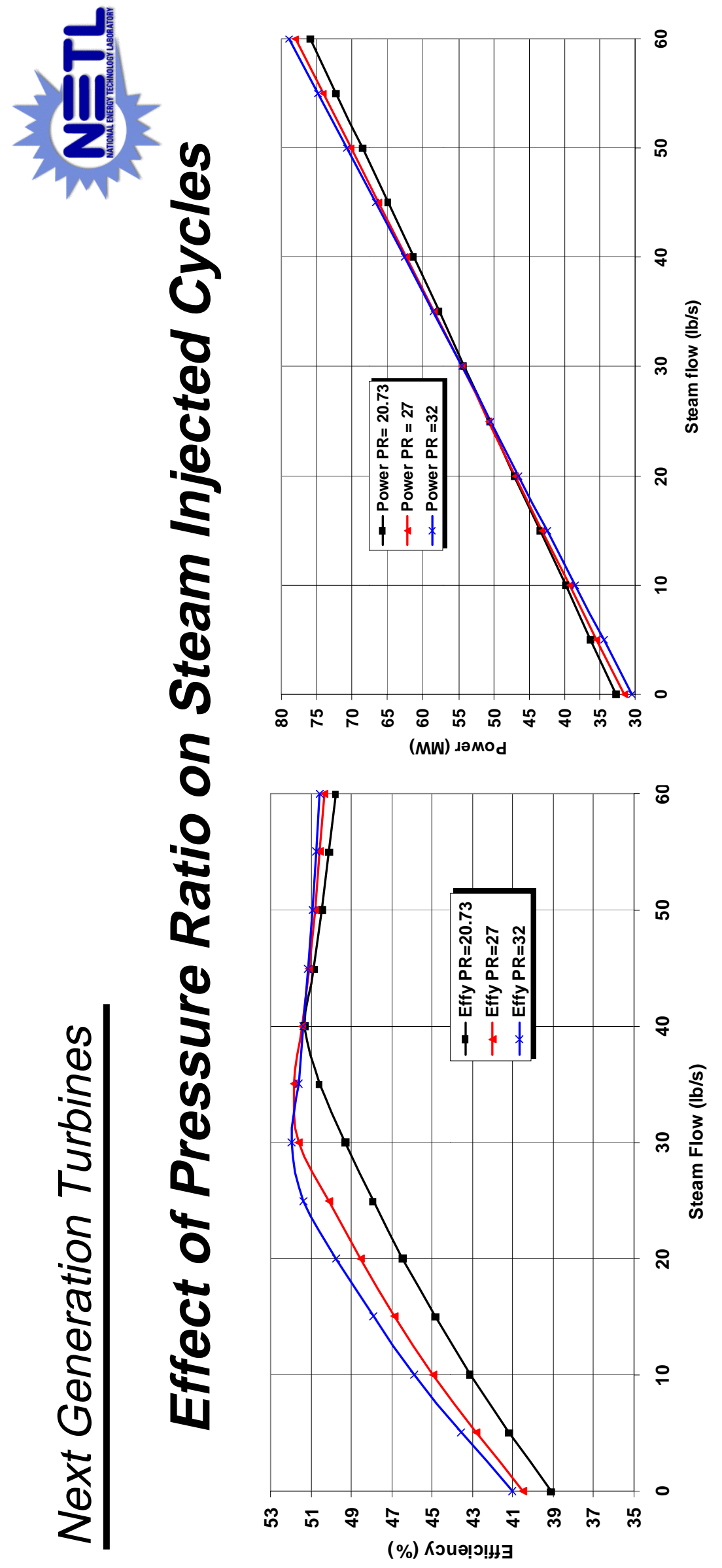

$\frac{1}{5}$

กิ ัั

त

들즐

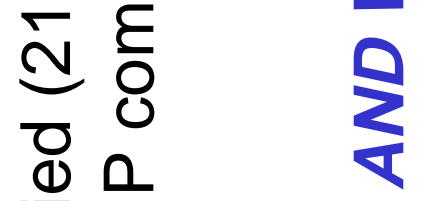

는

ชั

으 홍

즌

$\begin{array}{ll}\frac{1}{4} & \frac{1}{4} \\ \frac{1}{4} & \frac{0}{0}\end{array}$

(1) ำ

ต 으

凹

으 홍

14

$\frac{1}{8}$

0
$\frac{1}{0}$
$\frac{1}{2}$
$\frac{1}{2}$
$\frac{1}{2}$
$\frac{1}{2}$

สั

บ

ठํ

$\frac{0}{0}$

के

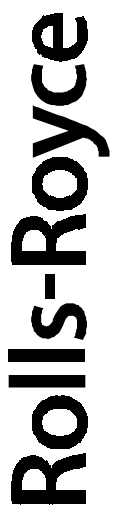



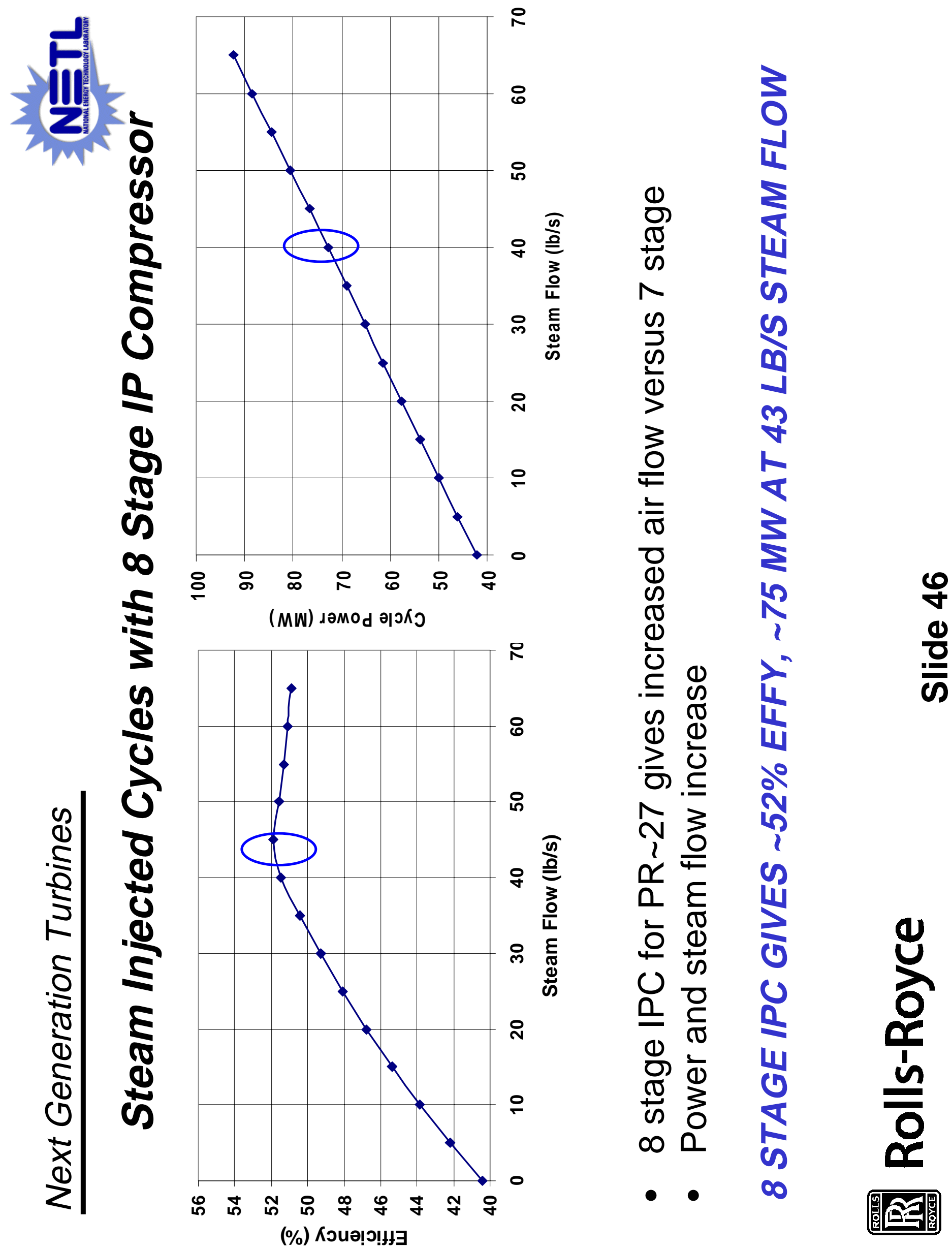


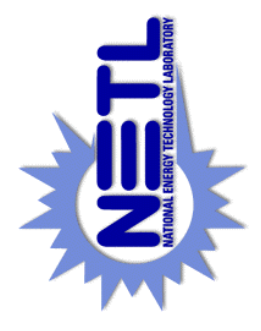

.
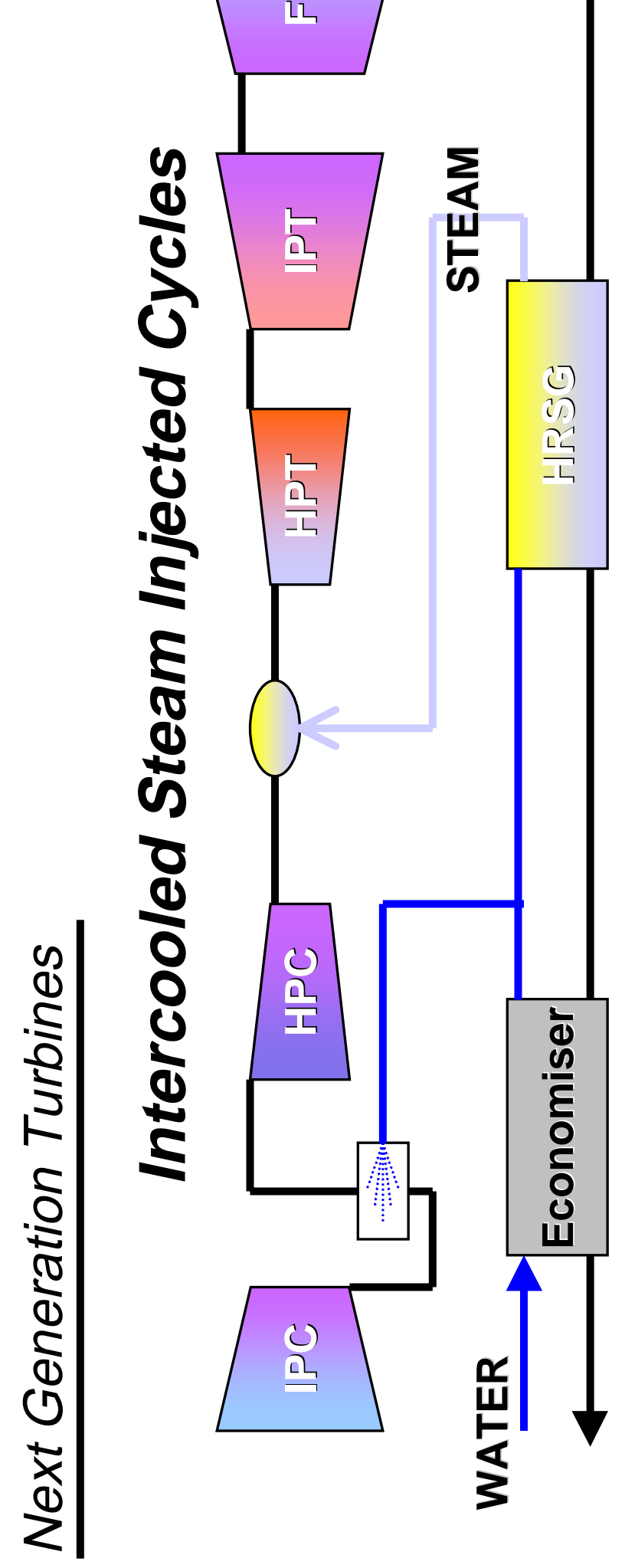

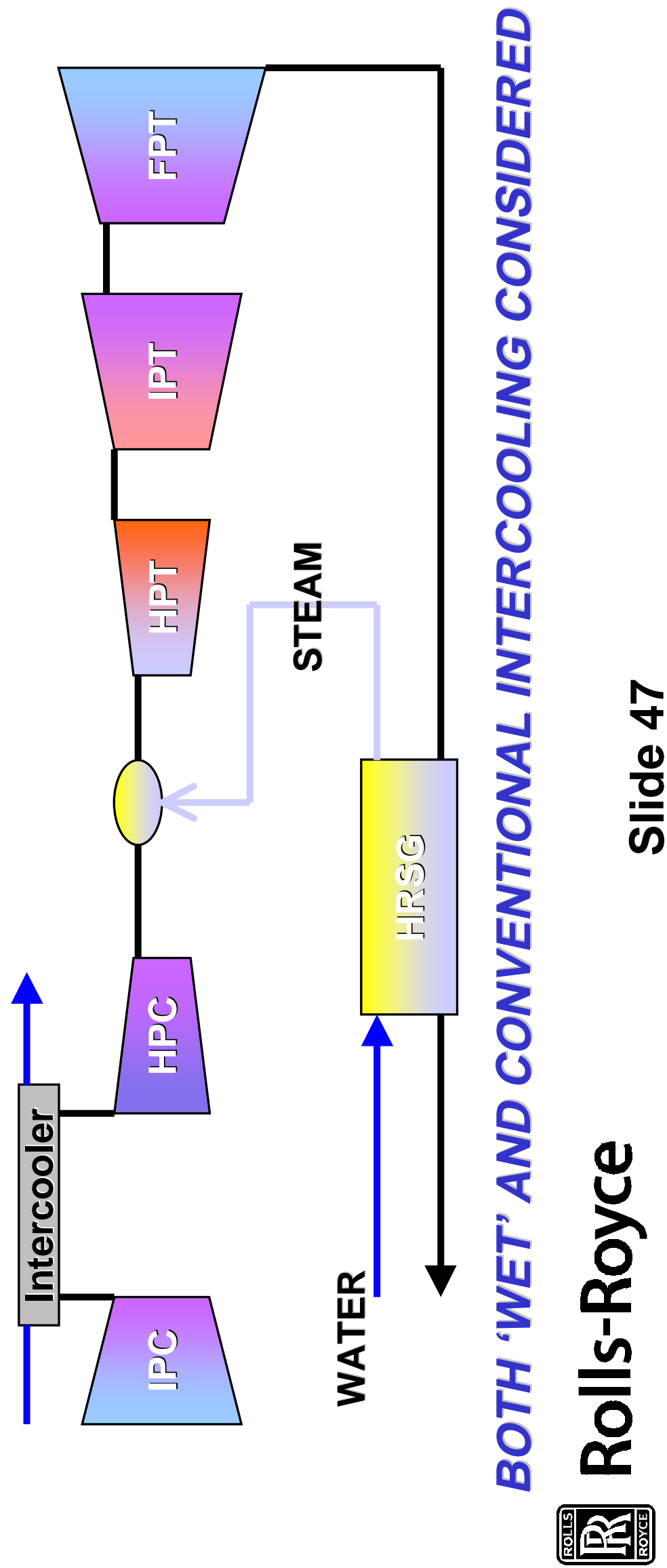



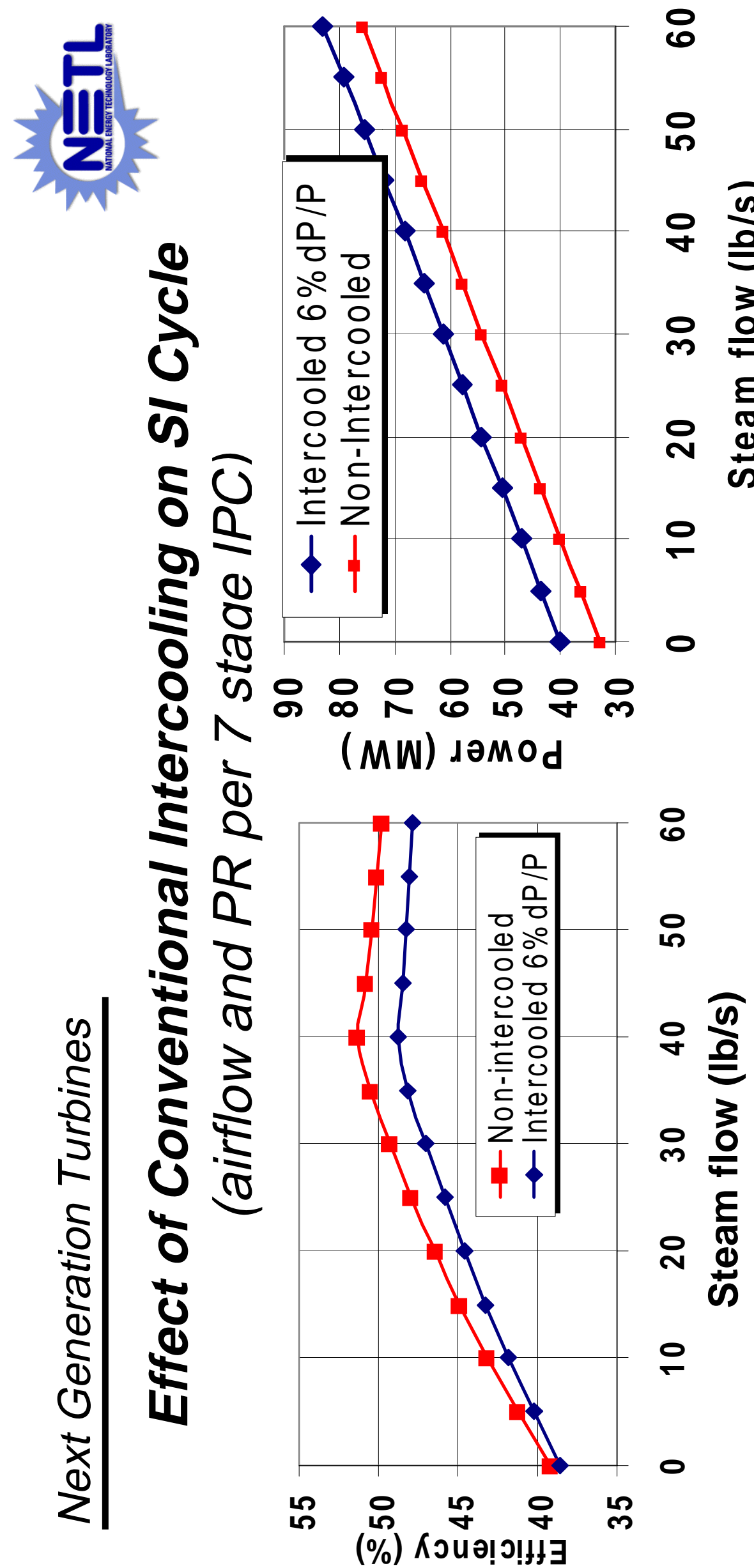

8

$\begin{array}{ll}0 & \frac{\omega}{2} \\ 0 & 0\end{array}$

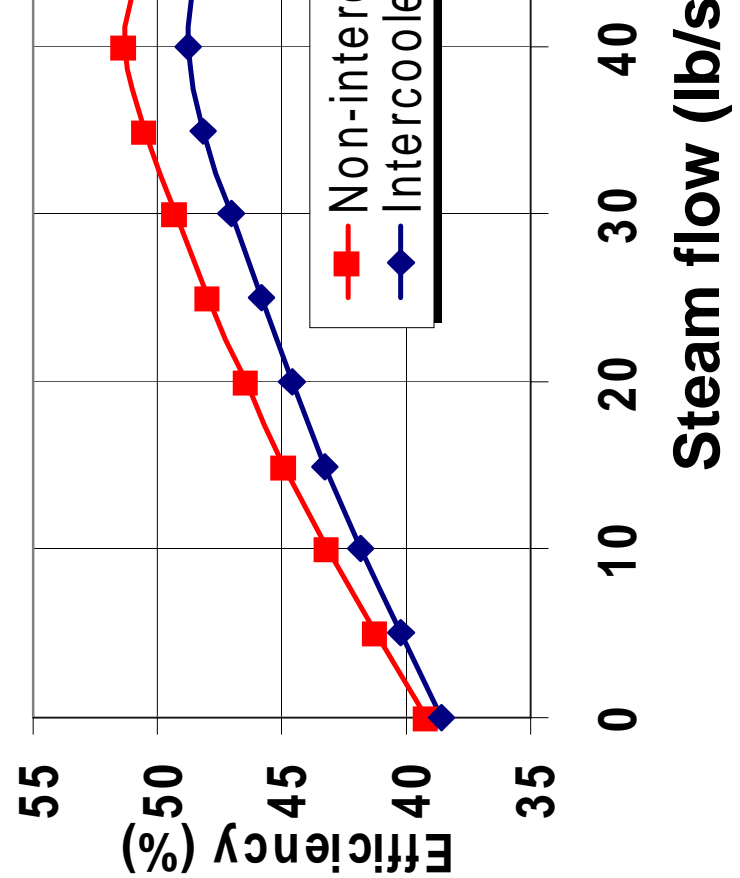

ד。

니 으 으

을 음

이

$\pm$

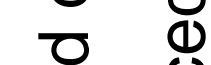

$\leqslant$

ชে ণ্

$\sum_{2}^{\frac{1}{2}} \underset{0}{0}$

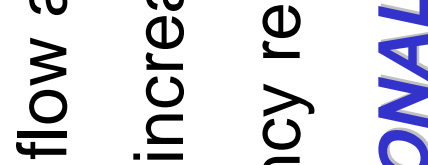

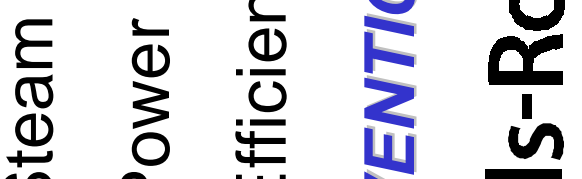

$\frac{1}{\infty}$

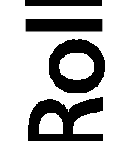



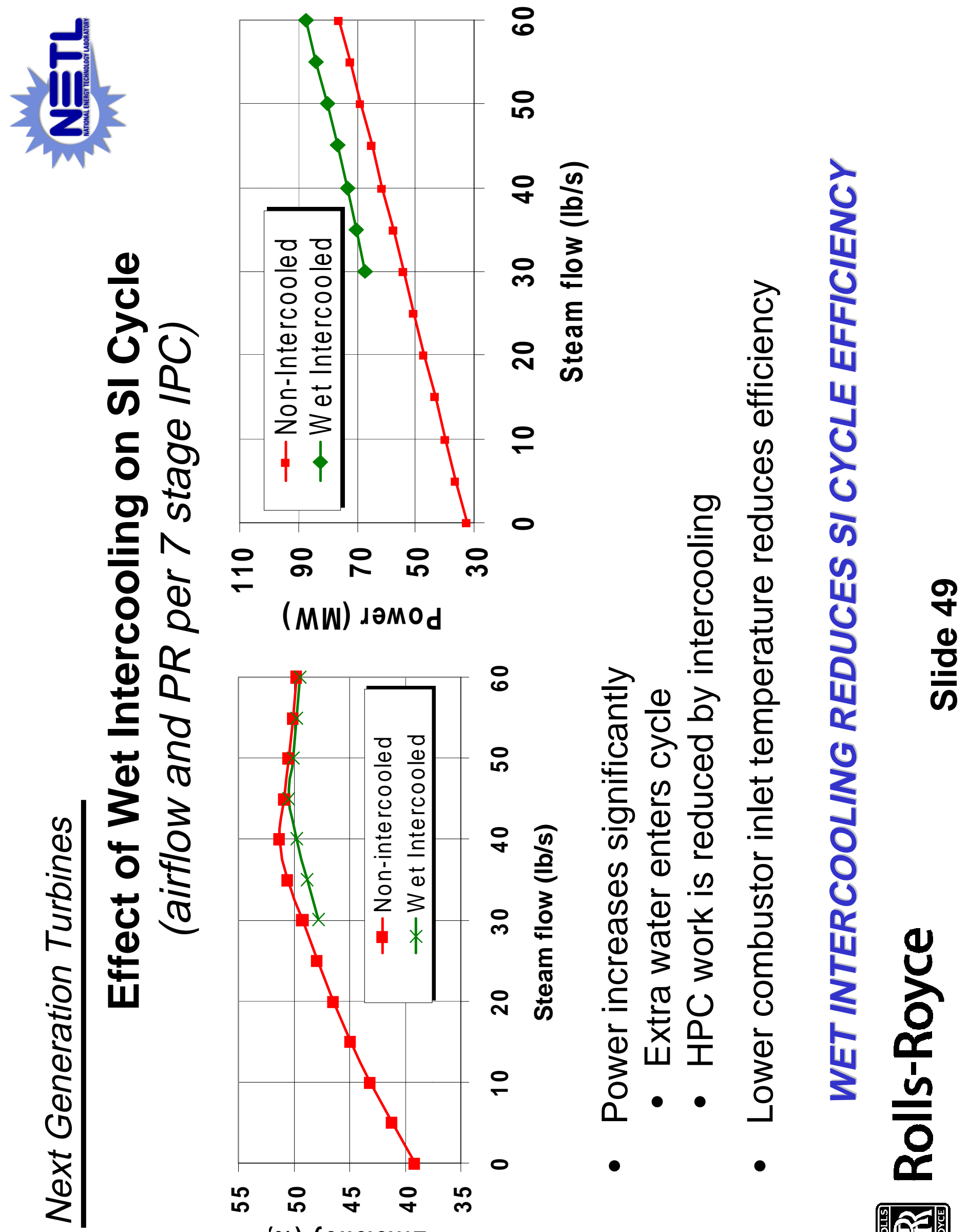

$\begin{array}{llllll}0 & 0 & 0 & 0\end{array}$

(MW) גәMOd

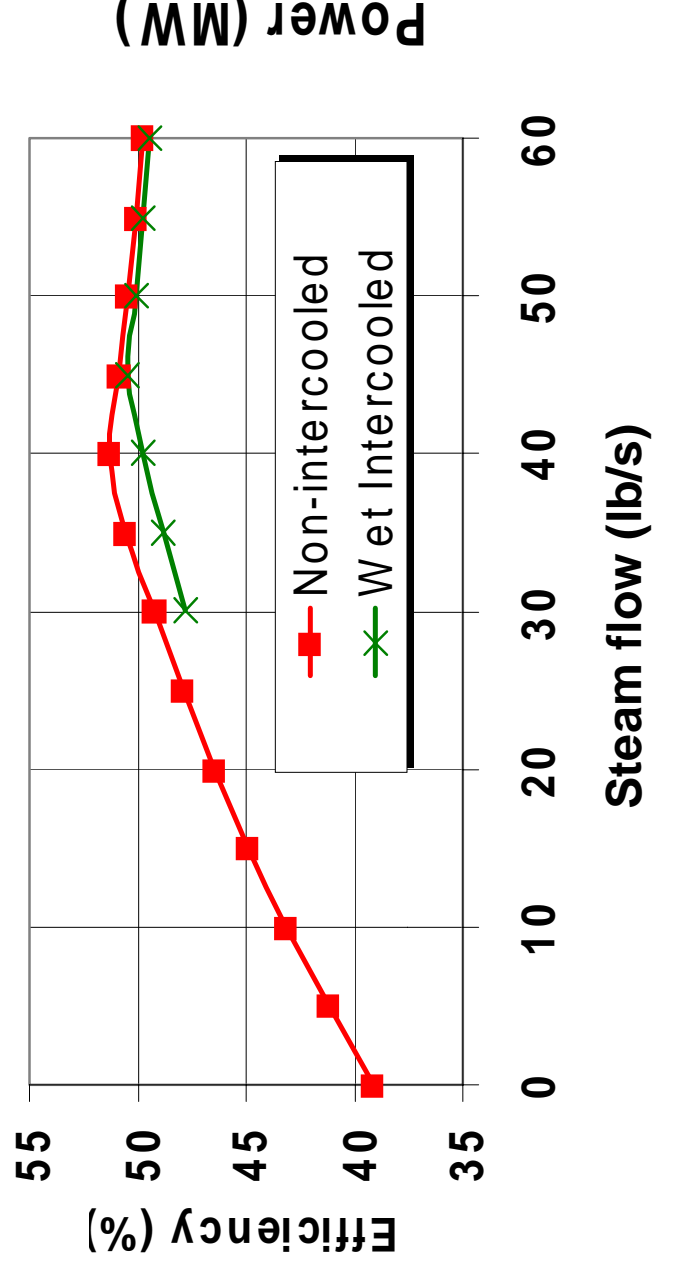

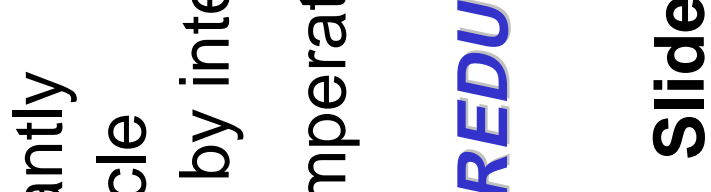

สู $\frac{0}{0}$ ह

응

등 ठํ क

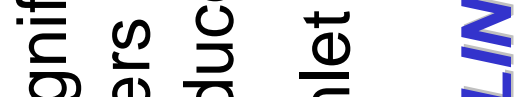

9

이

ต

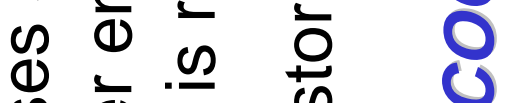

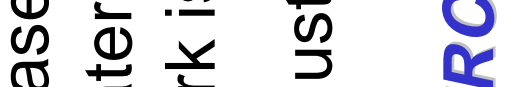

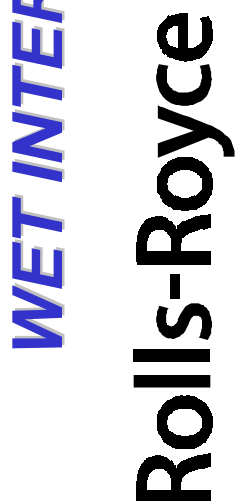

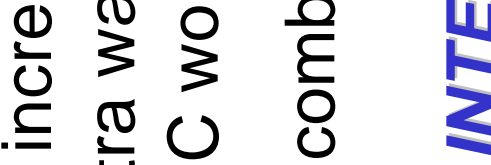

니ㄴㅗㅗ

$\sum_{0}^{\frac{1}{1}} \leq$

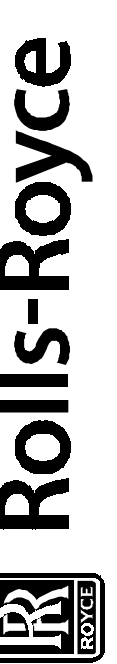



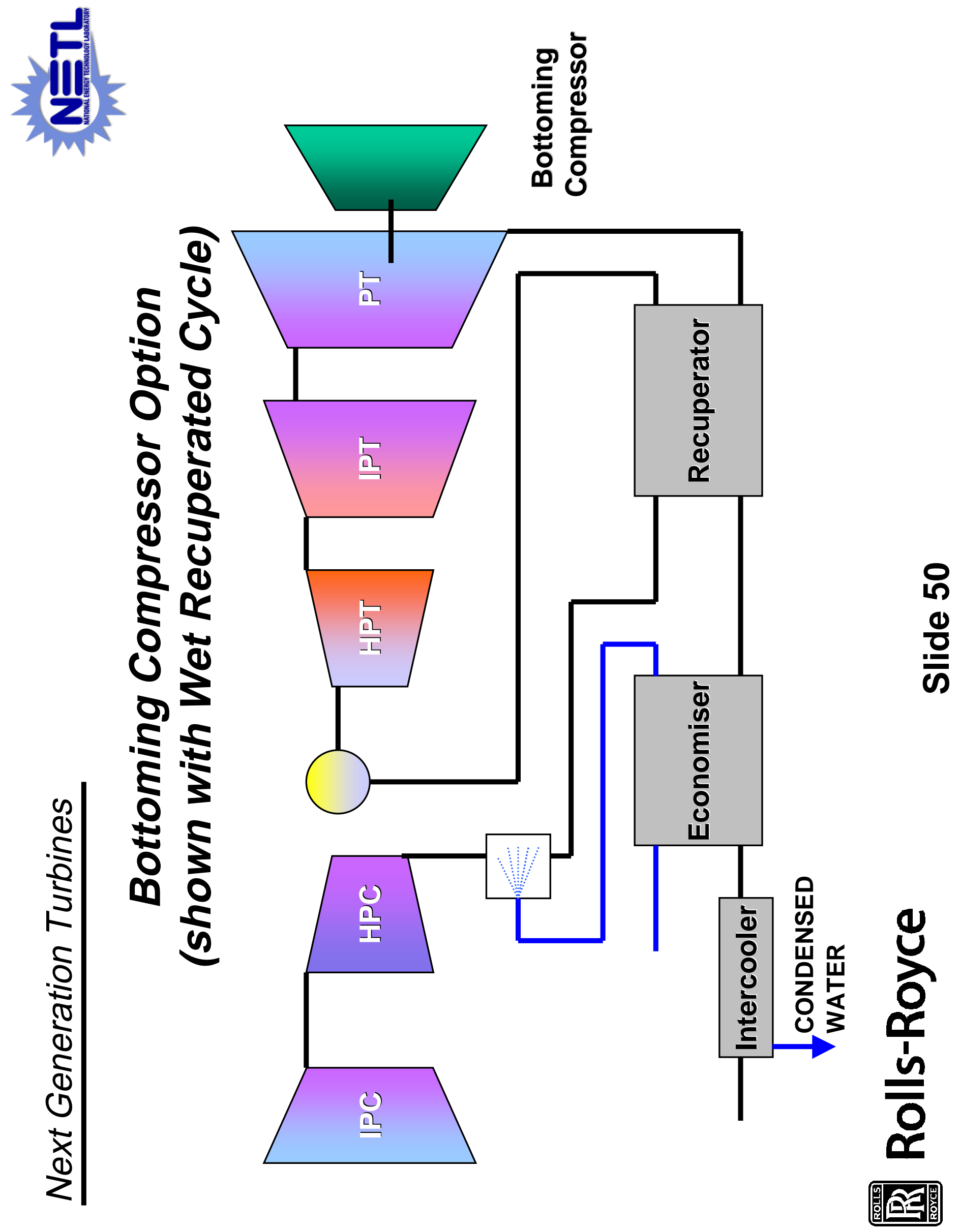

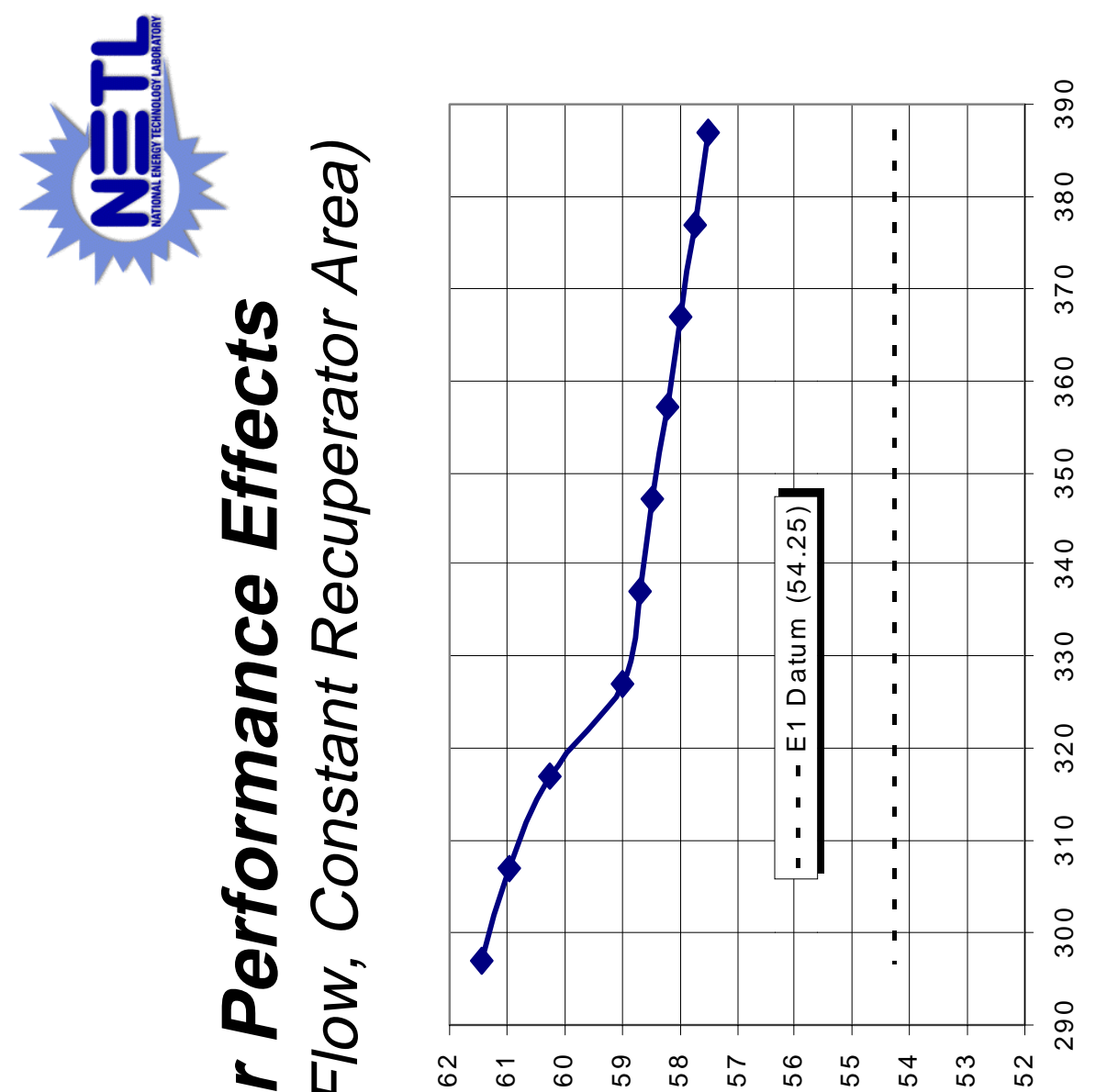

웅

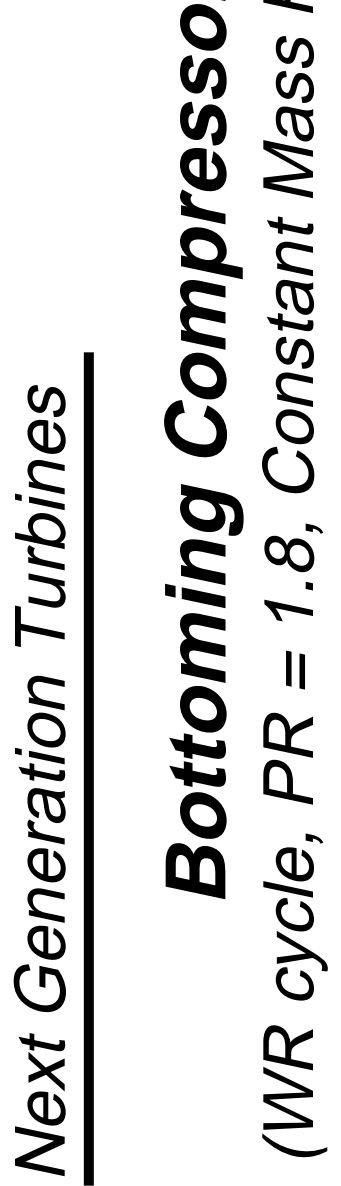

W) $\lambda ә \operatorname{Mod} ә$

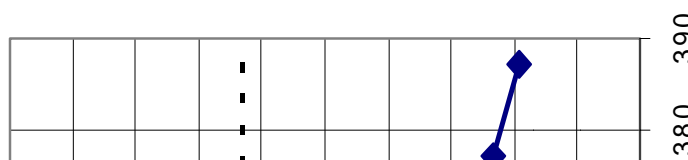

옹

$\stackrel{\Xi}{\subseteq} \quad \stackrel{\unlhd}{\subseteq}$

(1)

0

(1)

0

○ $\quad$ -

is च

ำ

ฮ

ஸ

光

$\frac{\bar{\tau}}{\times} \frac{\varepsilon}{\sigma}$

(1) (1)

$0 \pm$

(4)

(1)

10
$\frac{0}{6}$

(1)

II

$+\stackrel{0}{0}$

ac

U1

iv

(1) ㄴ

긍

02

0

0

(1) 1

ป $\frac{\text { ป }}{0}$

$\quad$

$\frac{\infty}{5} \div \frac{1}{1}$

$\frac{1}{0}$

(1)

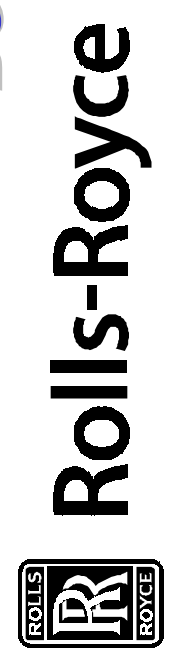

¿

ชิ

(\%) Кวนว!ว!н ヨ әюКว 


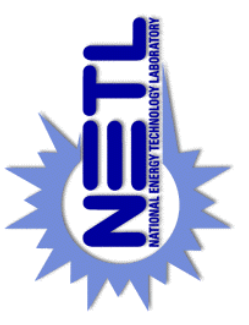

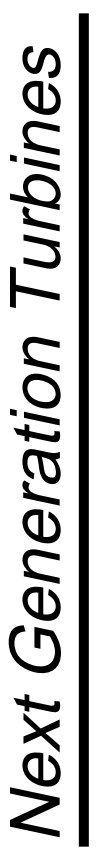
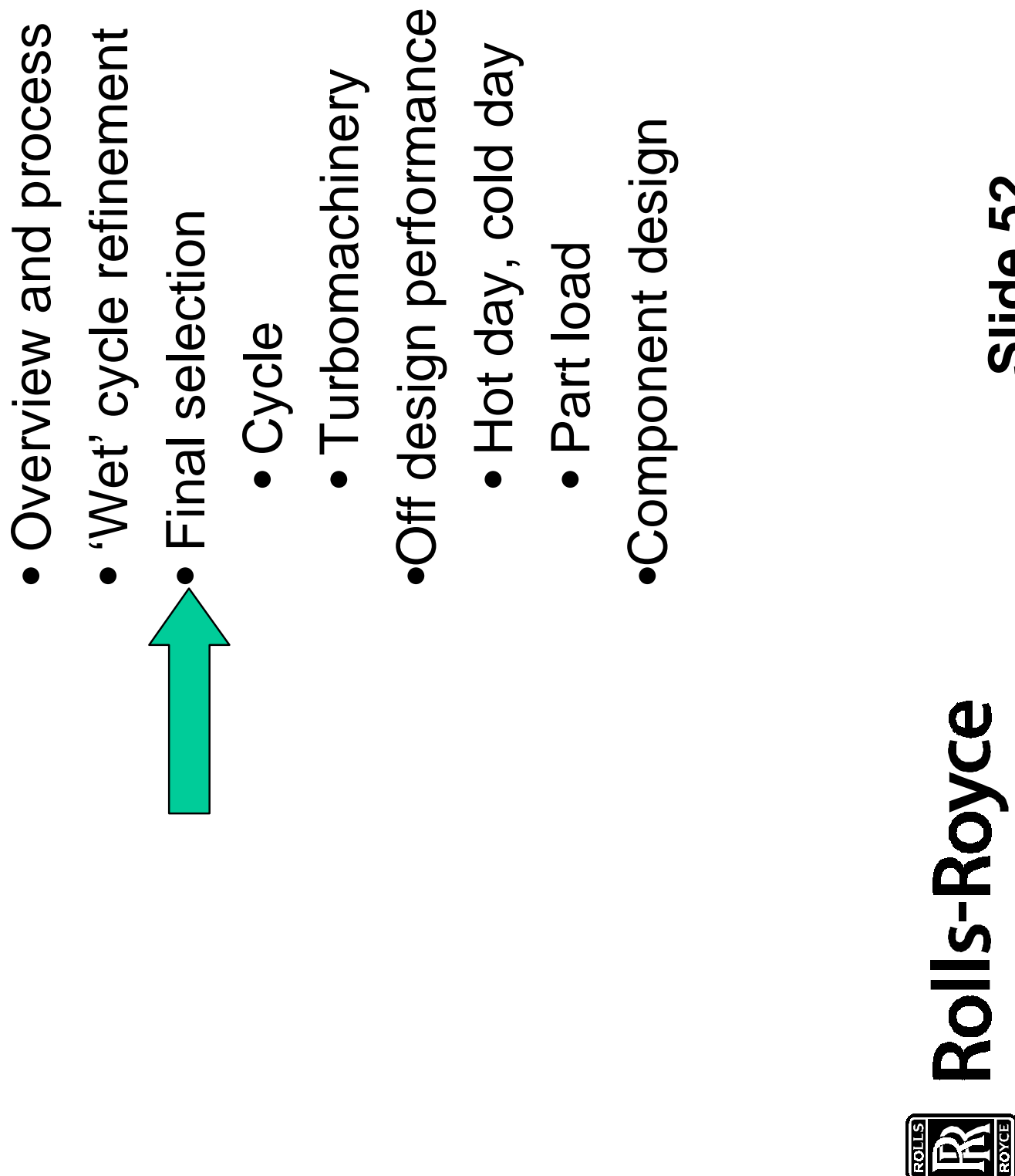

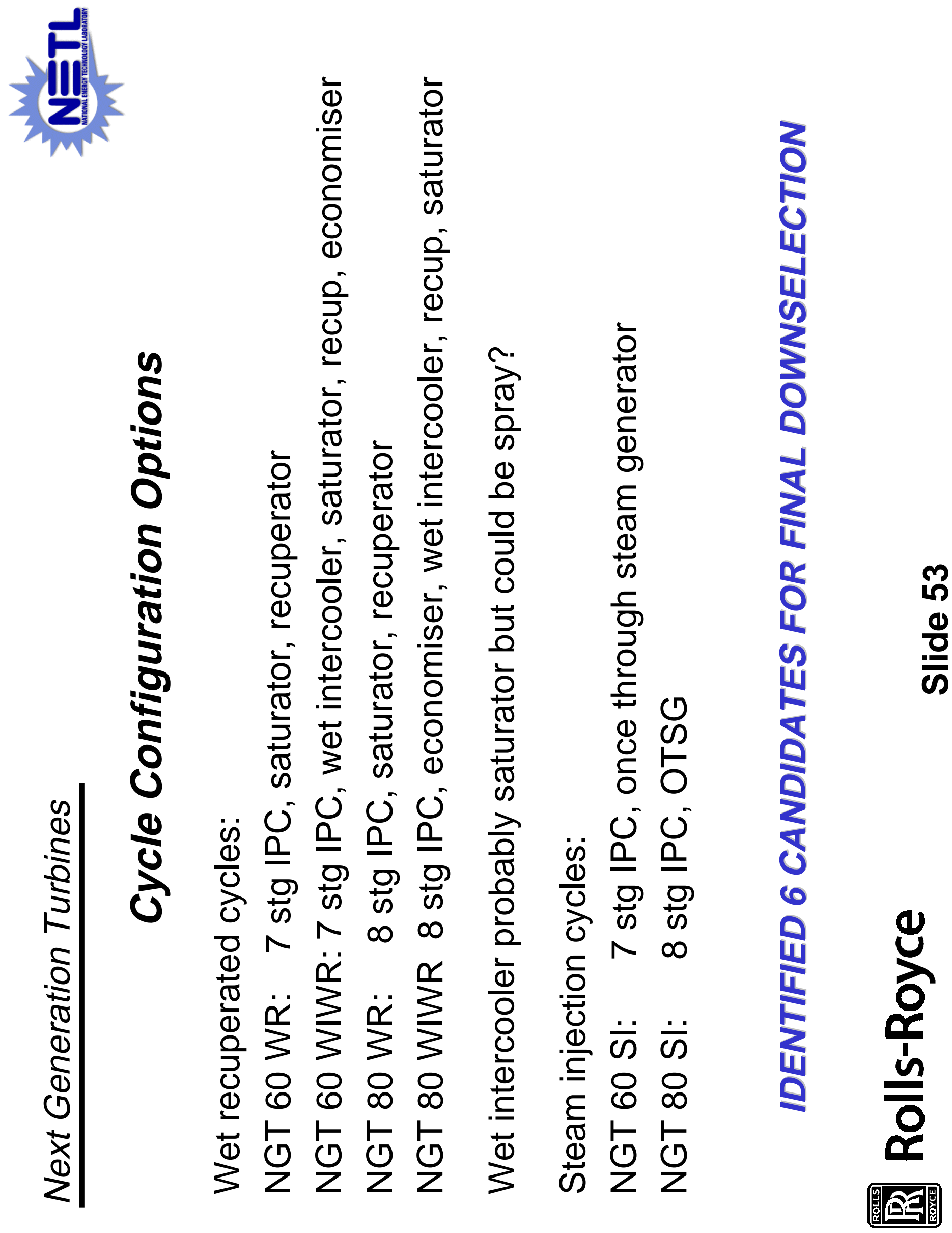

ถึ
$\frac{1}{0}$
$\frac{0}{0}$

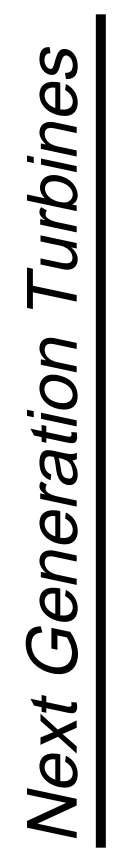




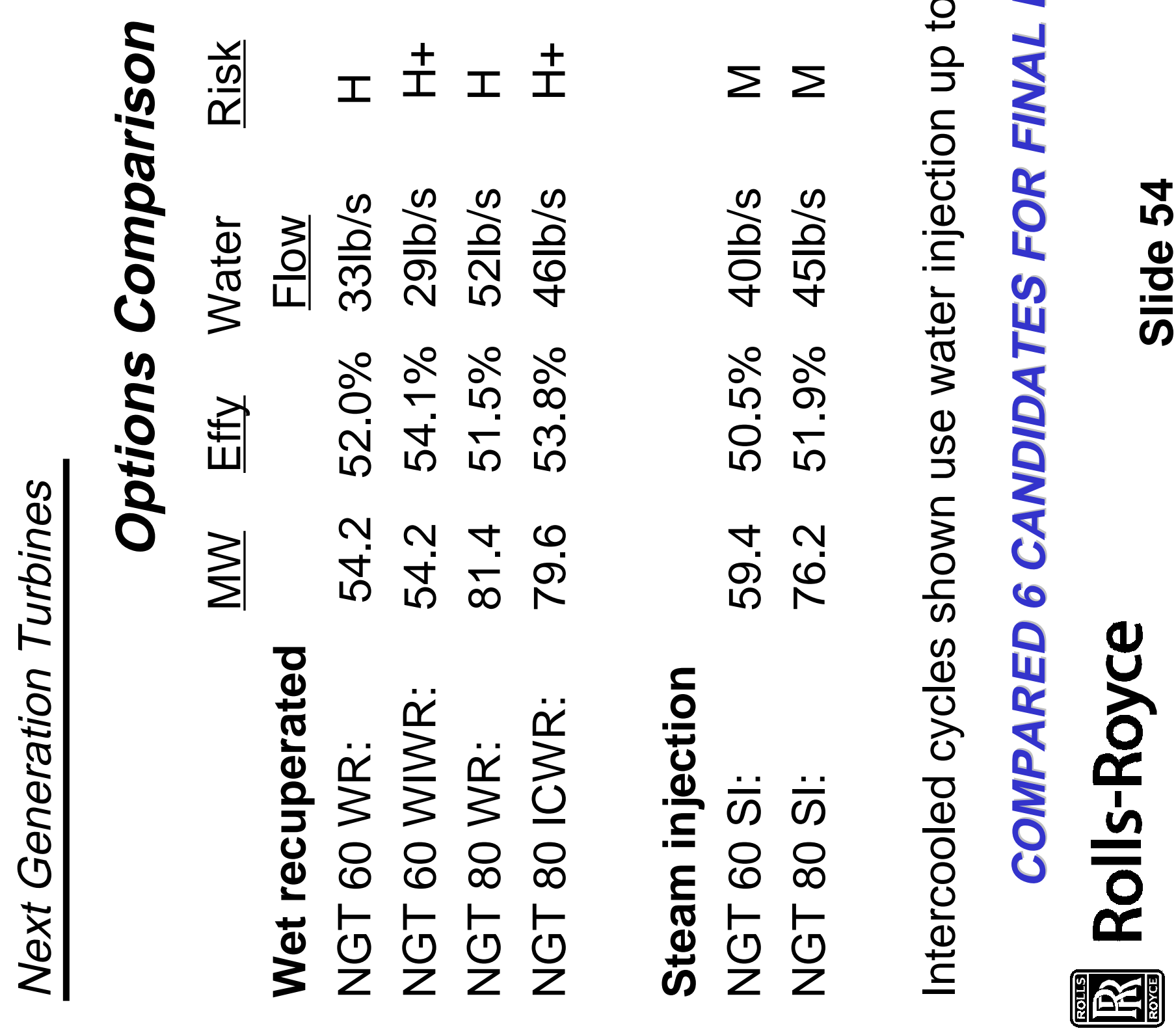



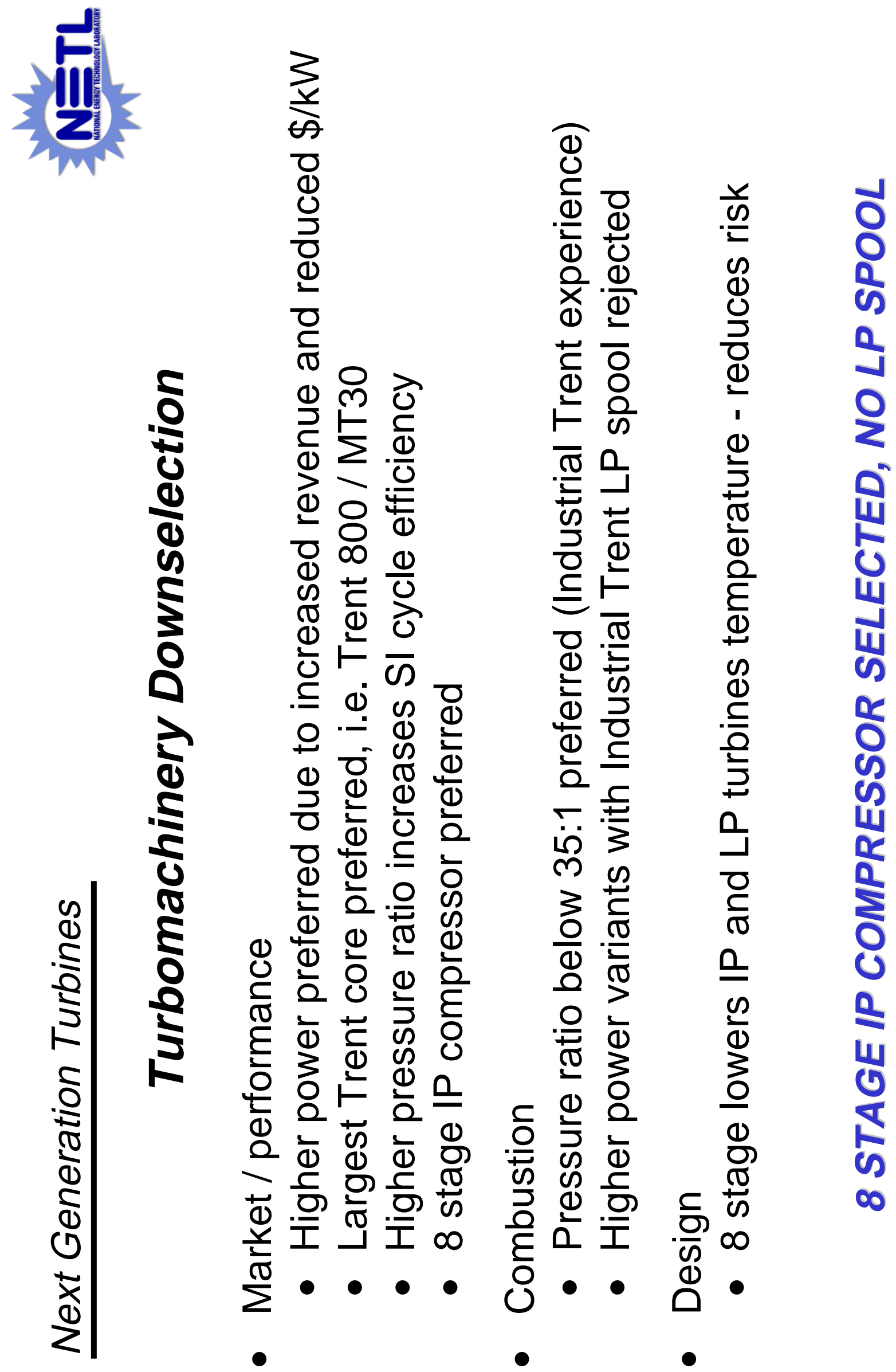

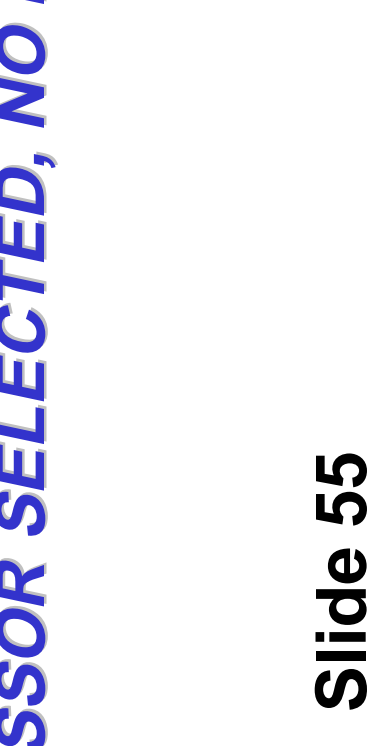

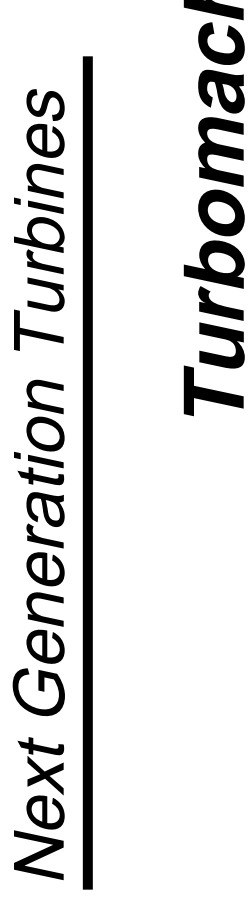

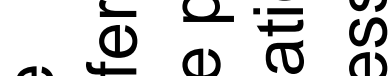

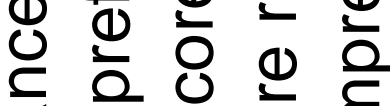

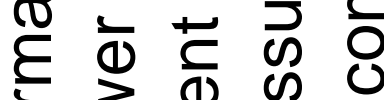

흔 은흐은

ㅎㅎㅎㅎㅇ

등 윤등 엔

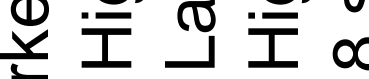

ह

प)

(1)

ら

둥

트응

范

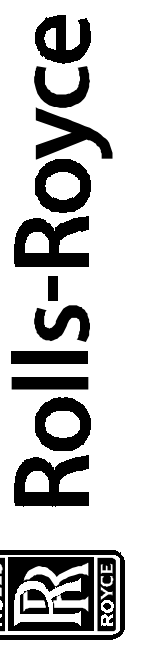




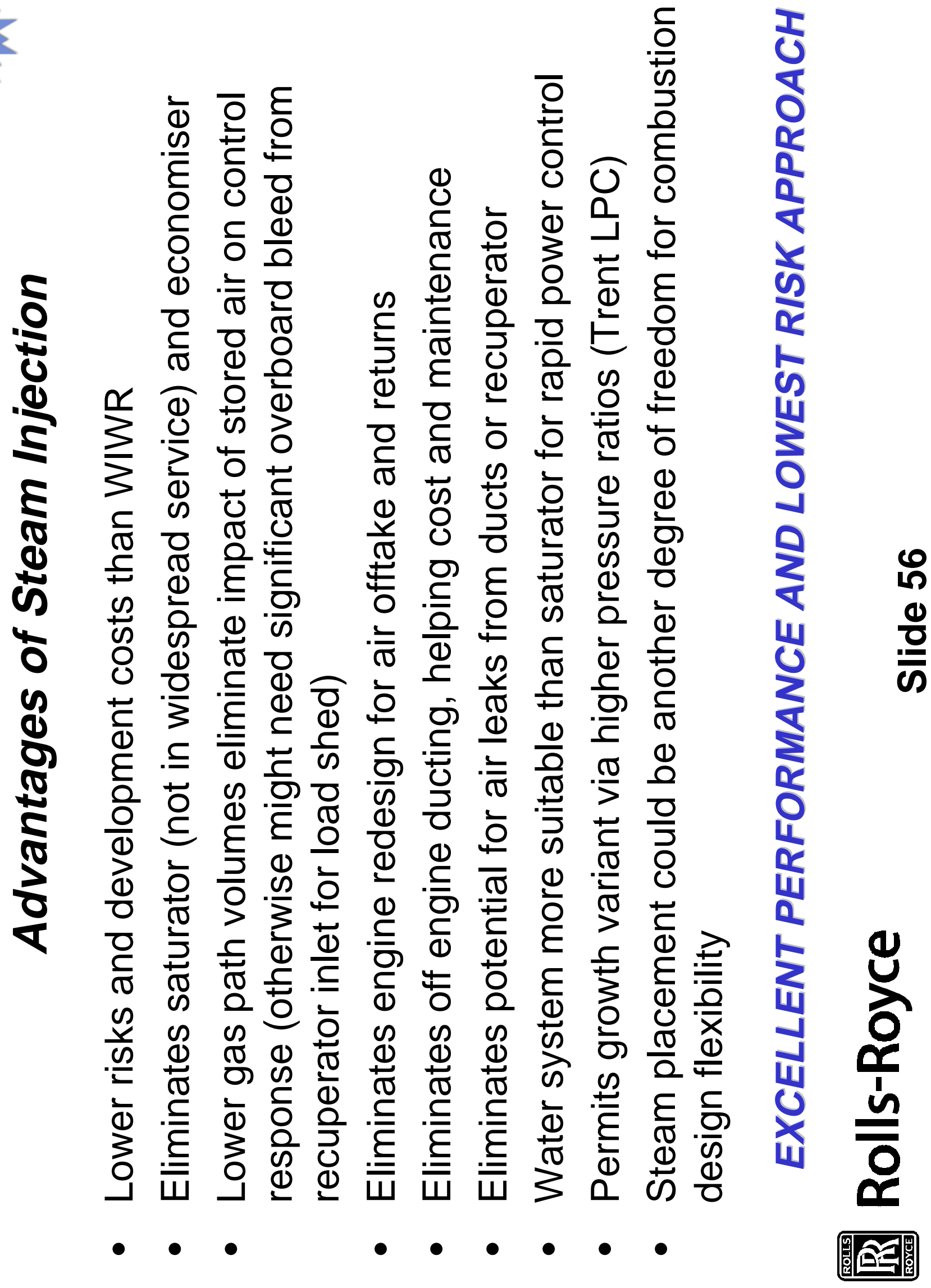




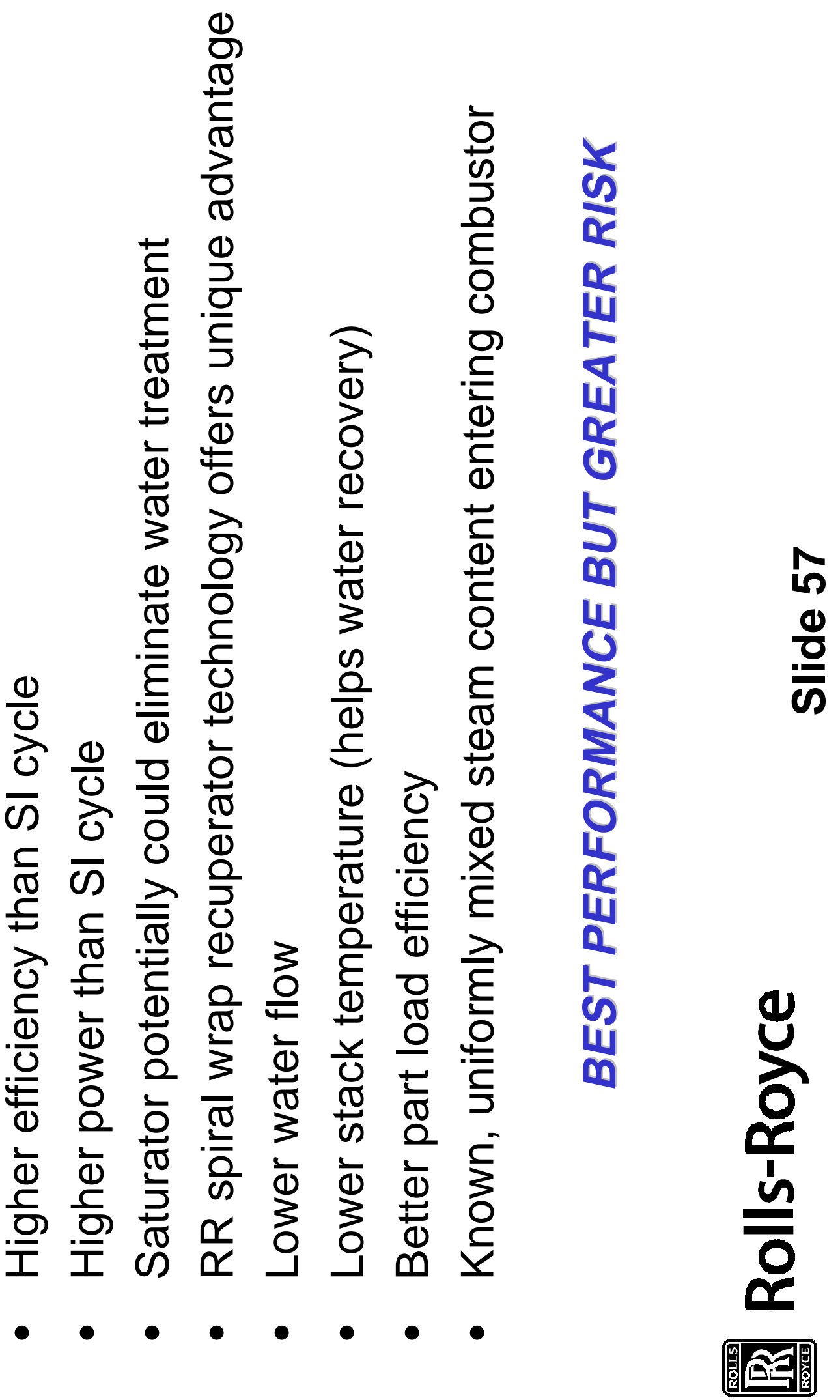




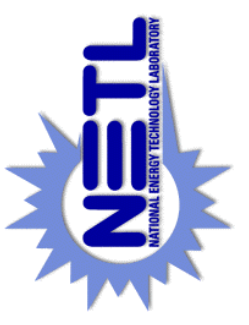

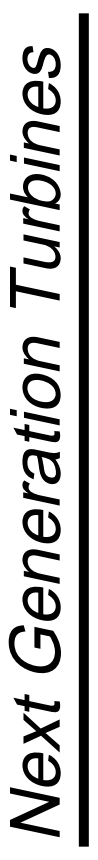
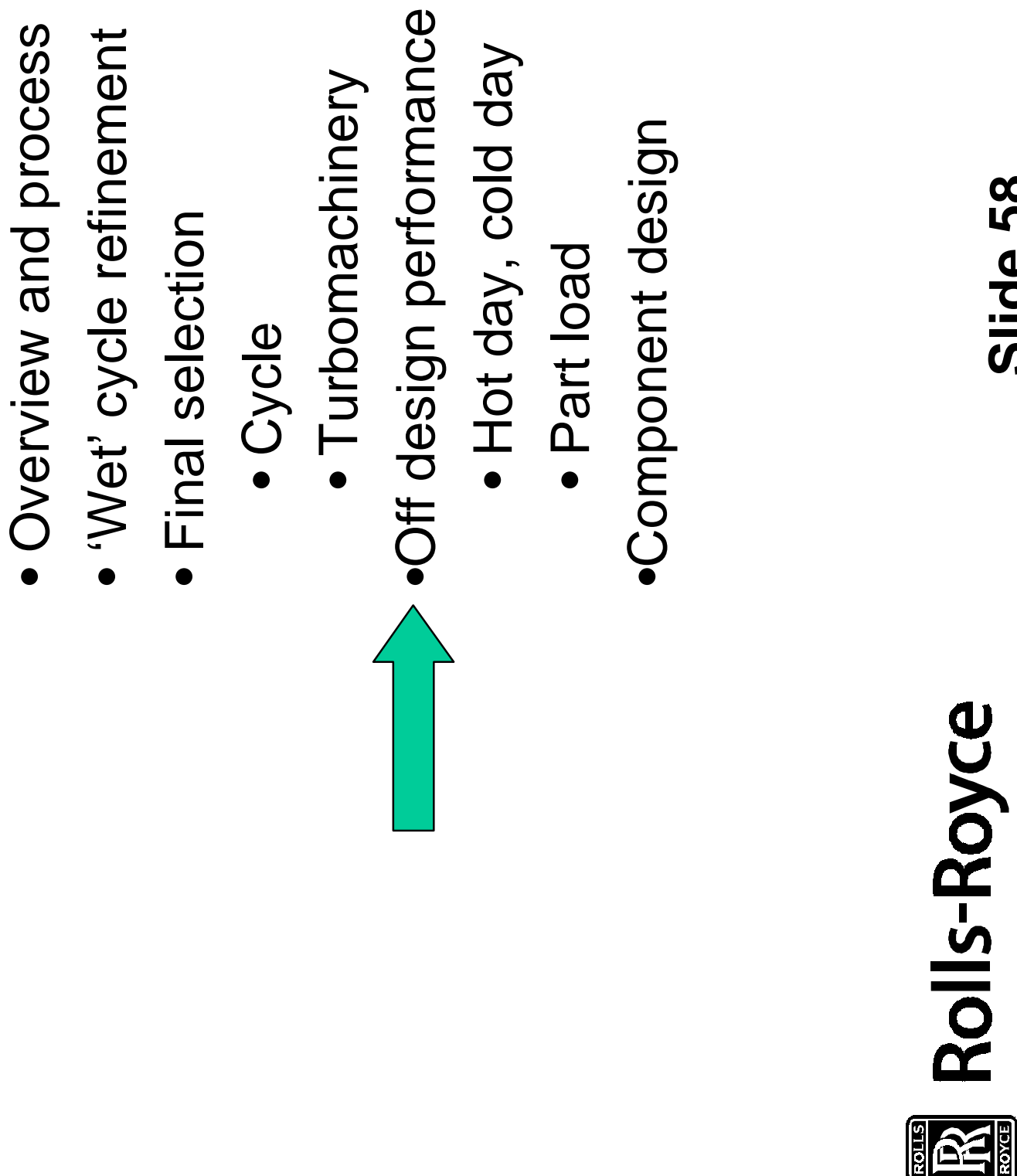


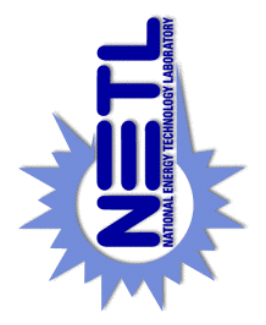

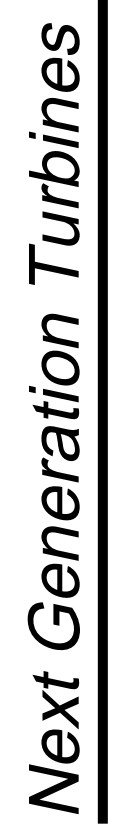
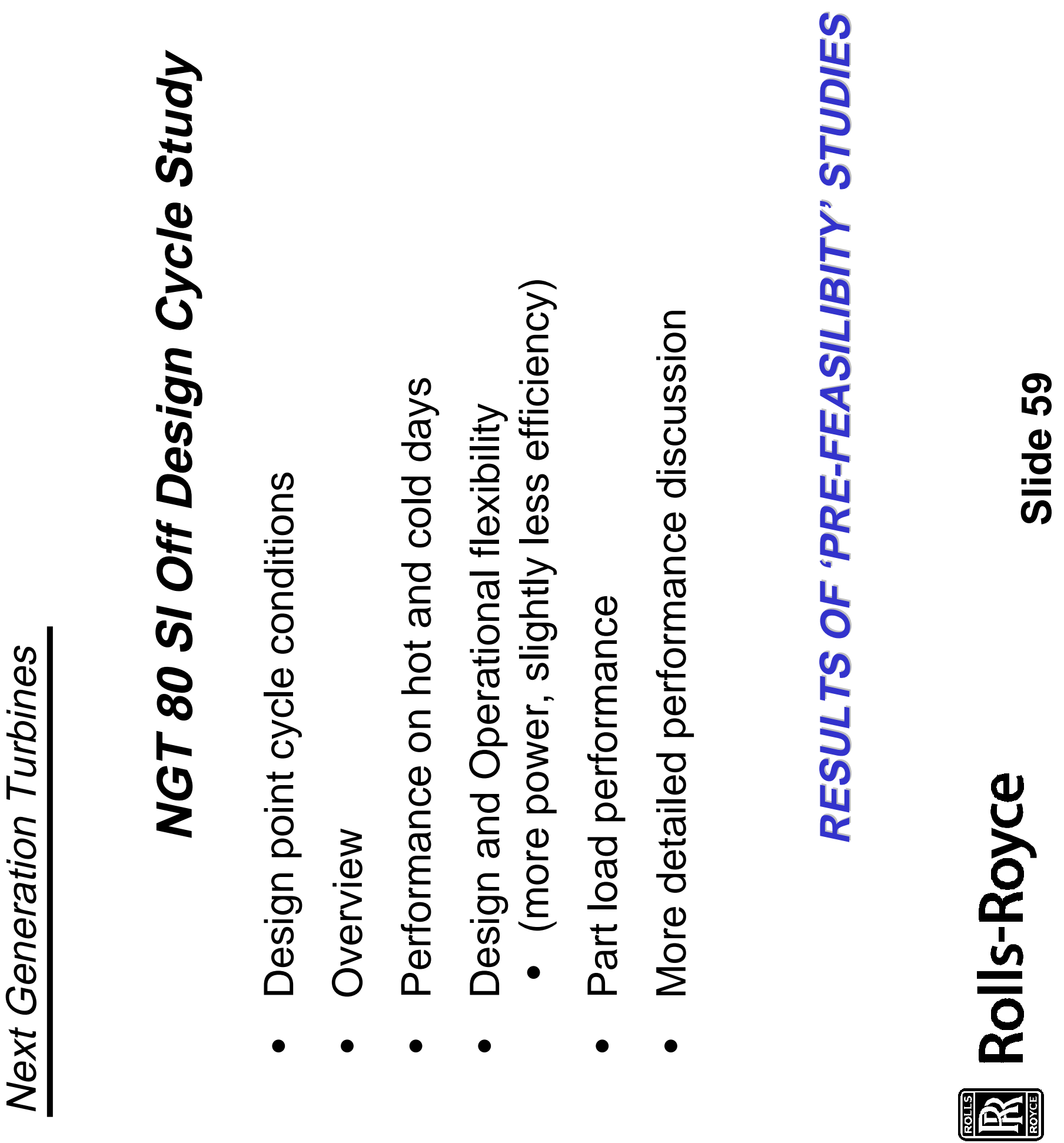


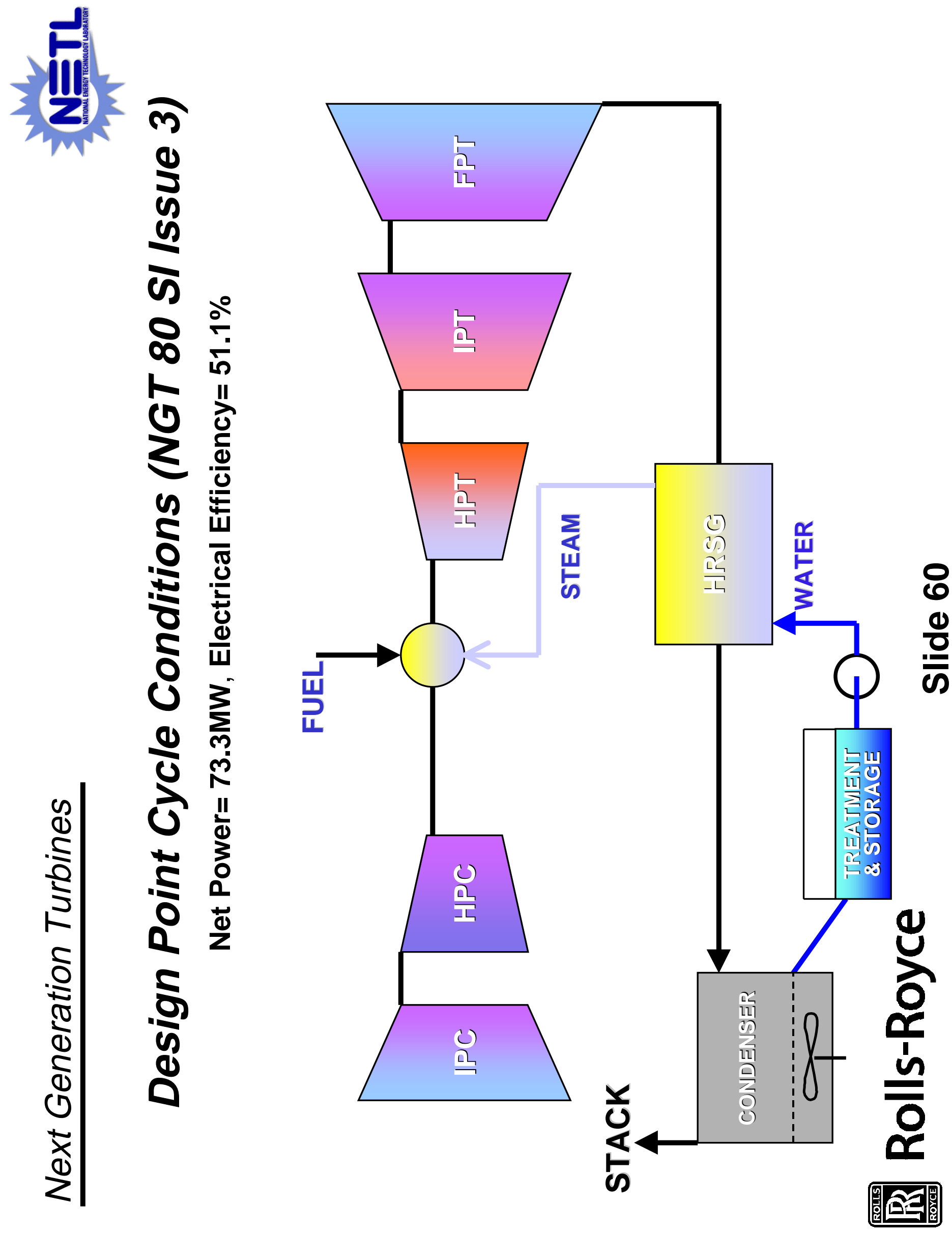




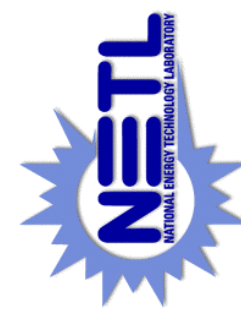

$\bar{\omega}$

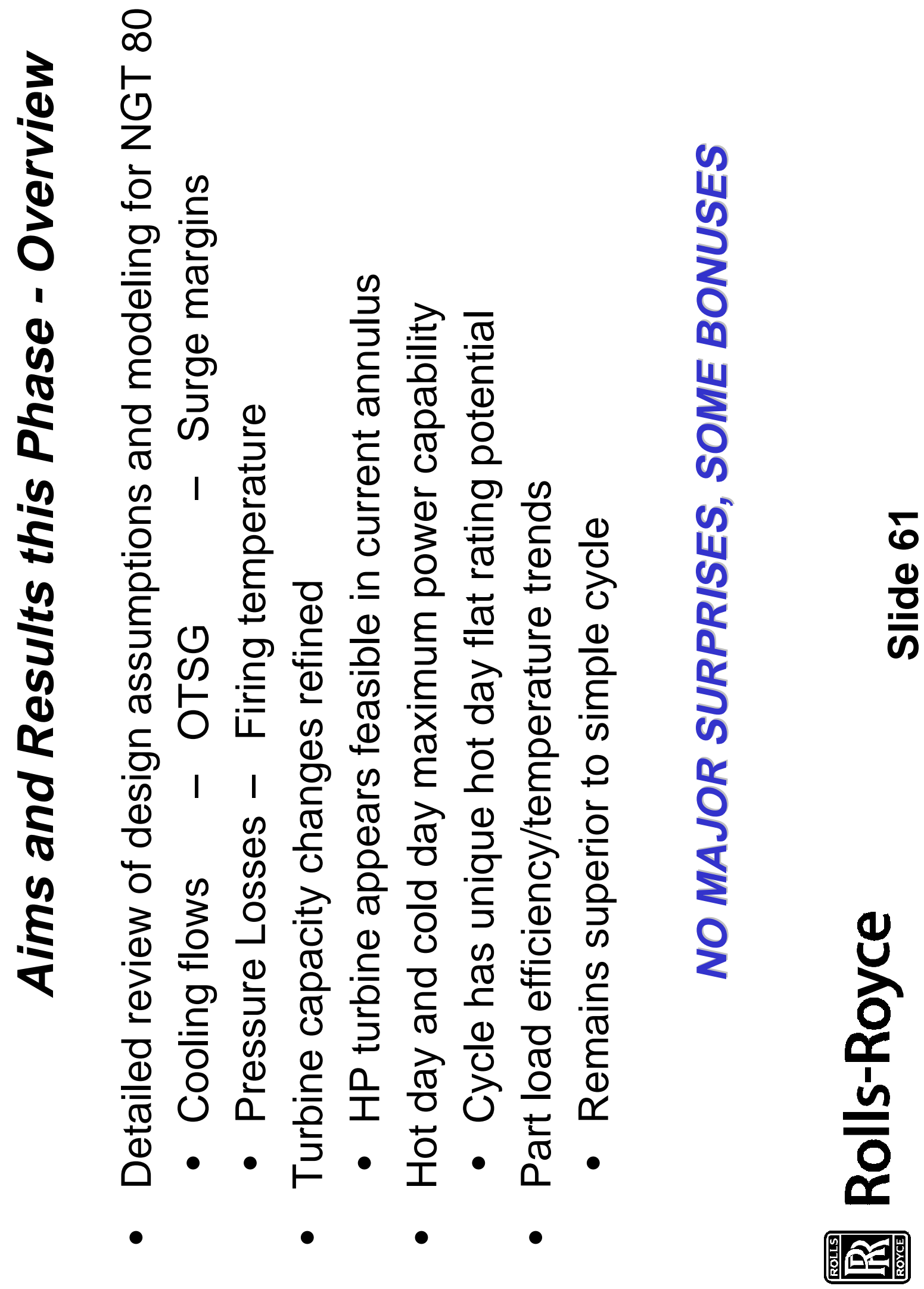




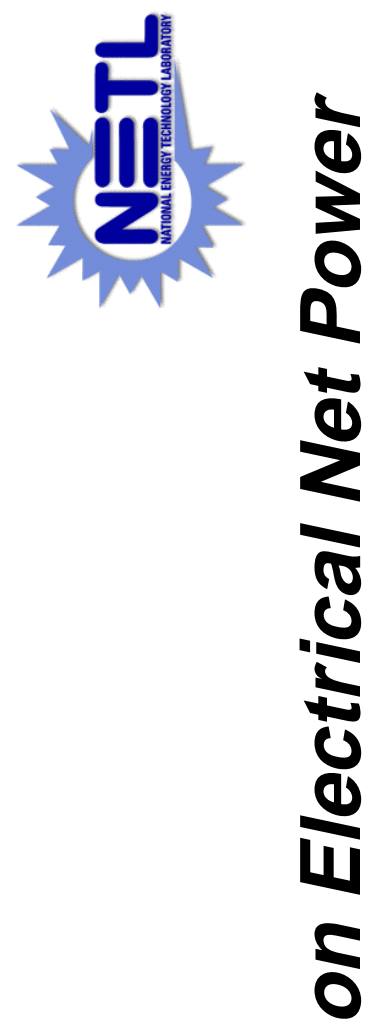

$\stackrel{0}{5}$

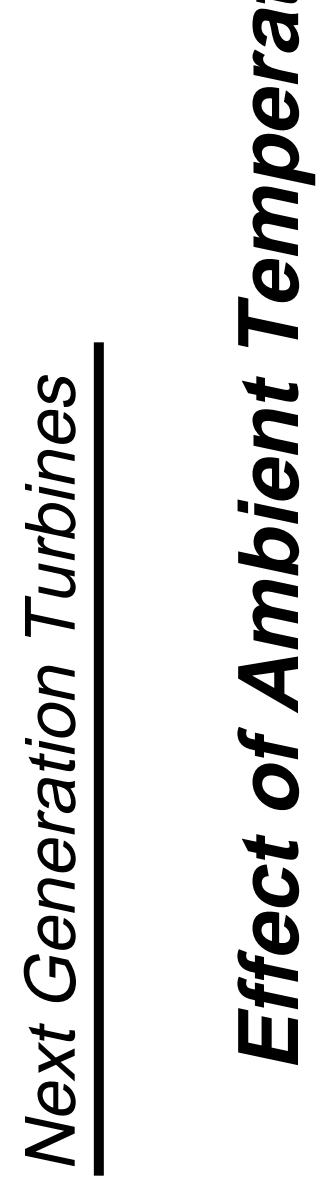

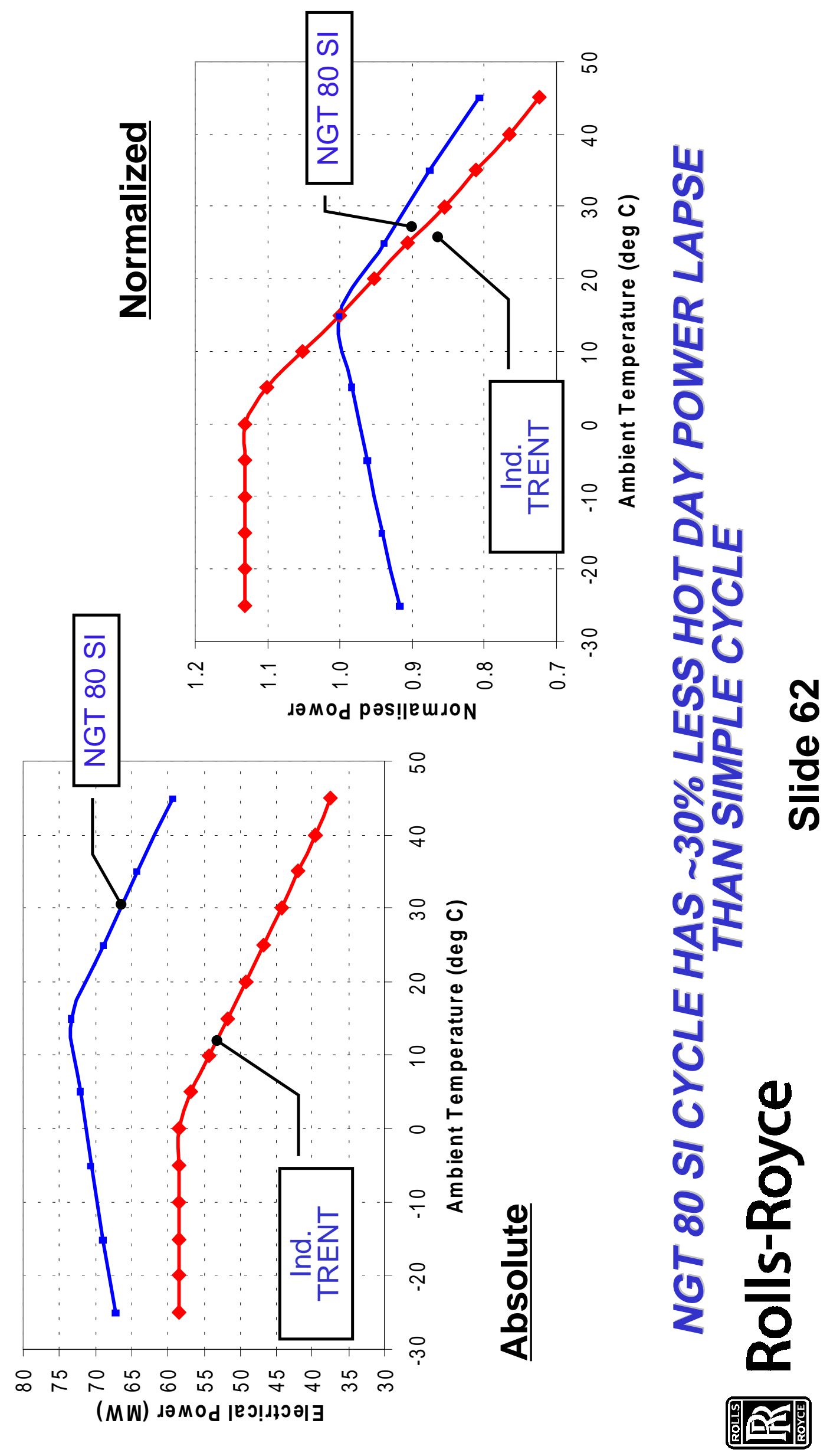




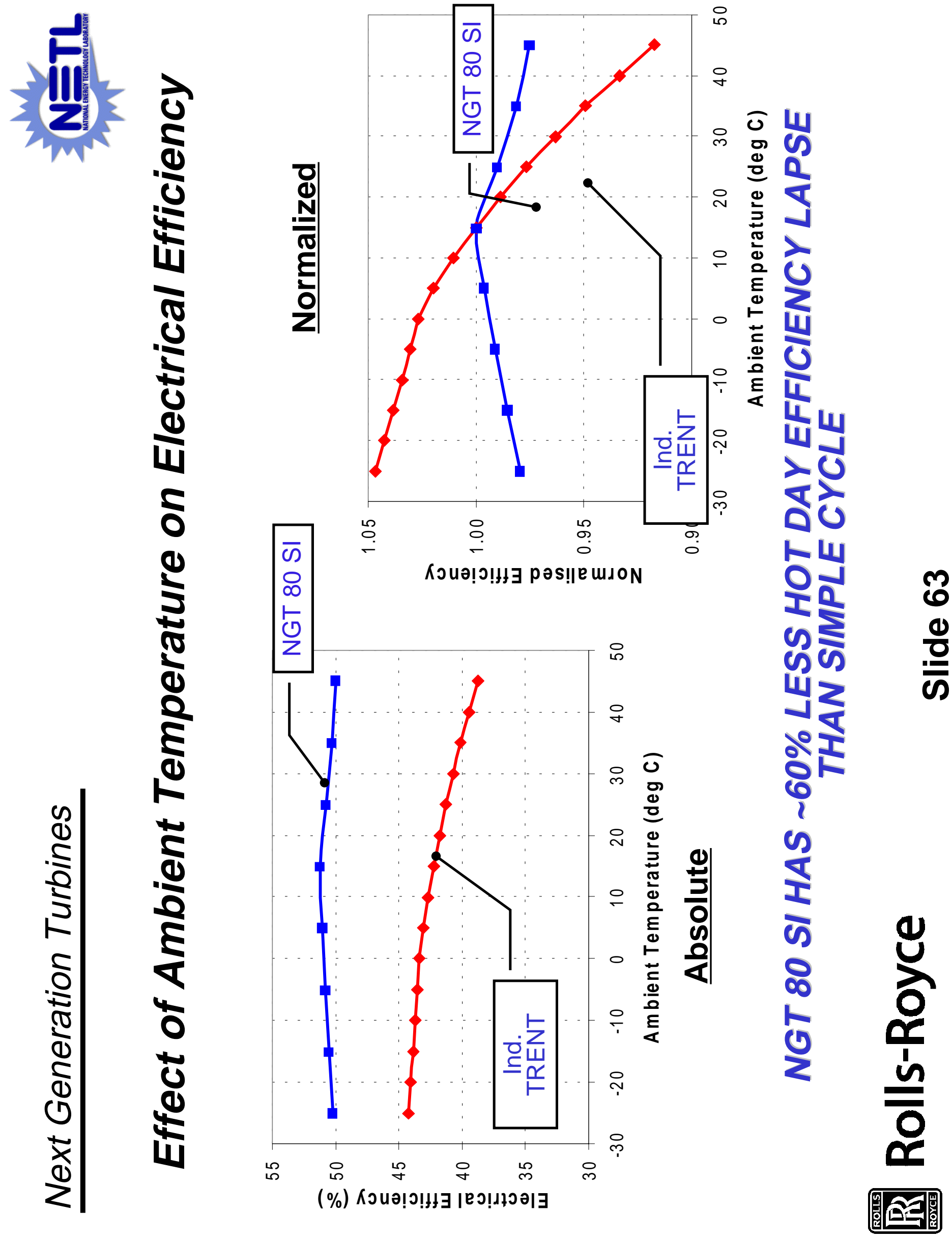



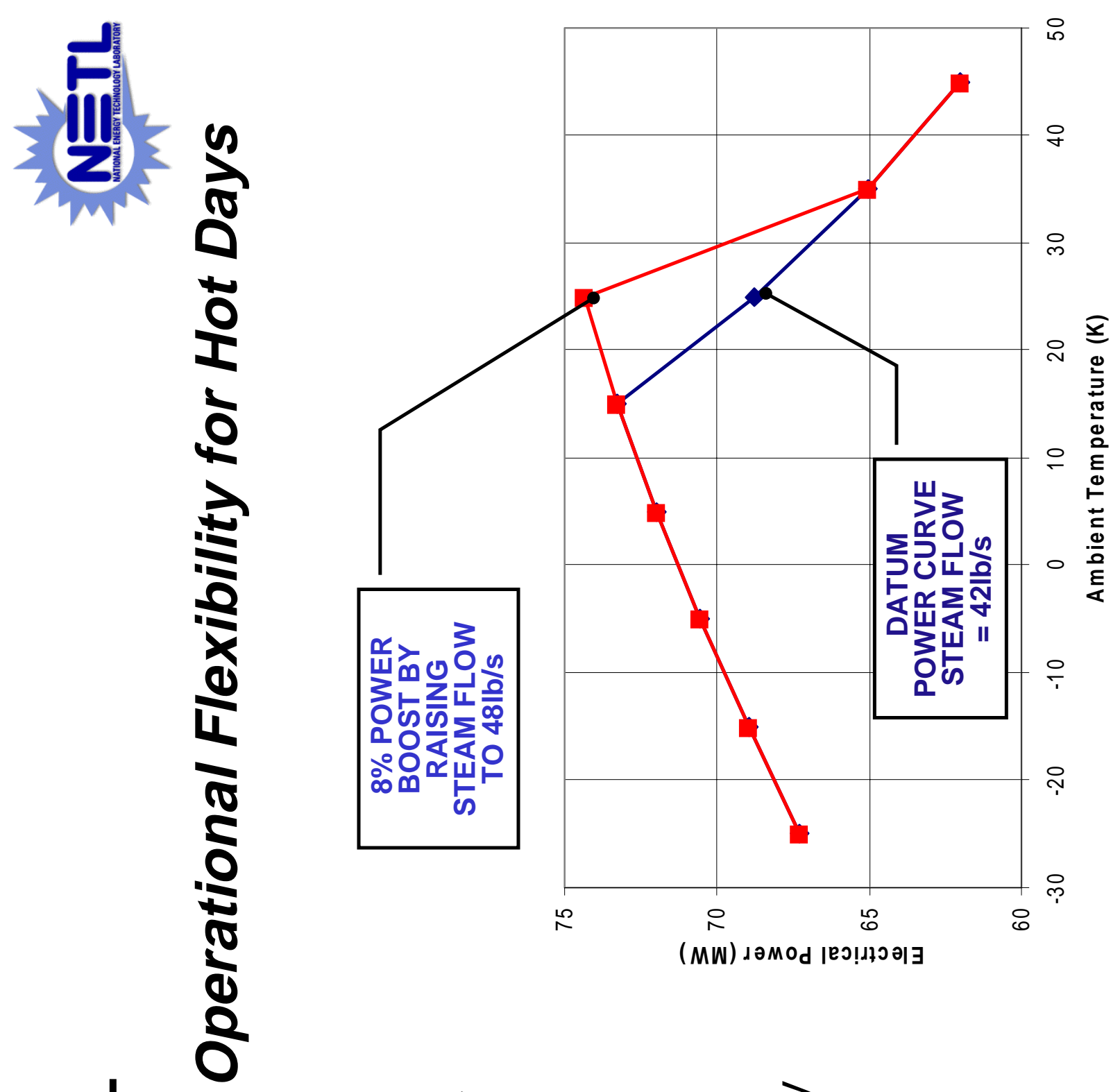

$\stackrel{5}{5}$

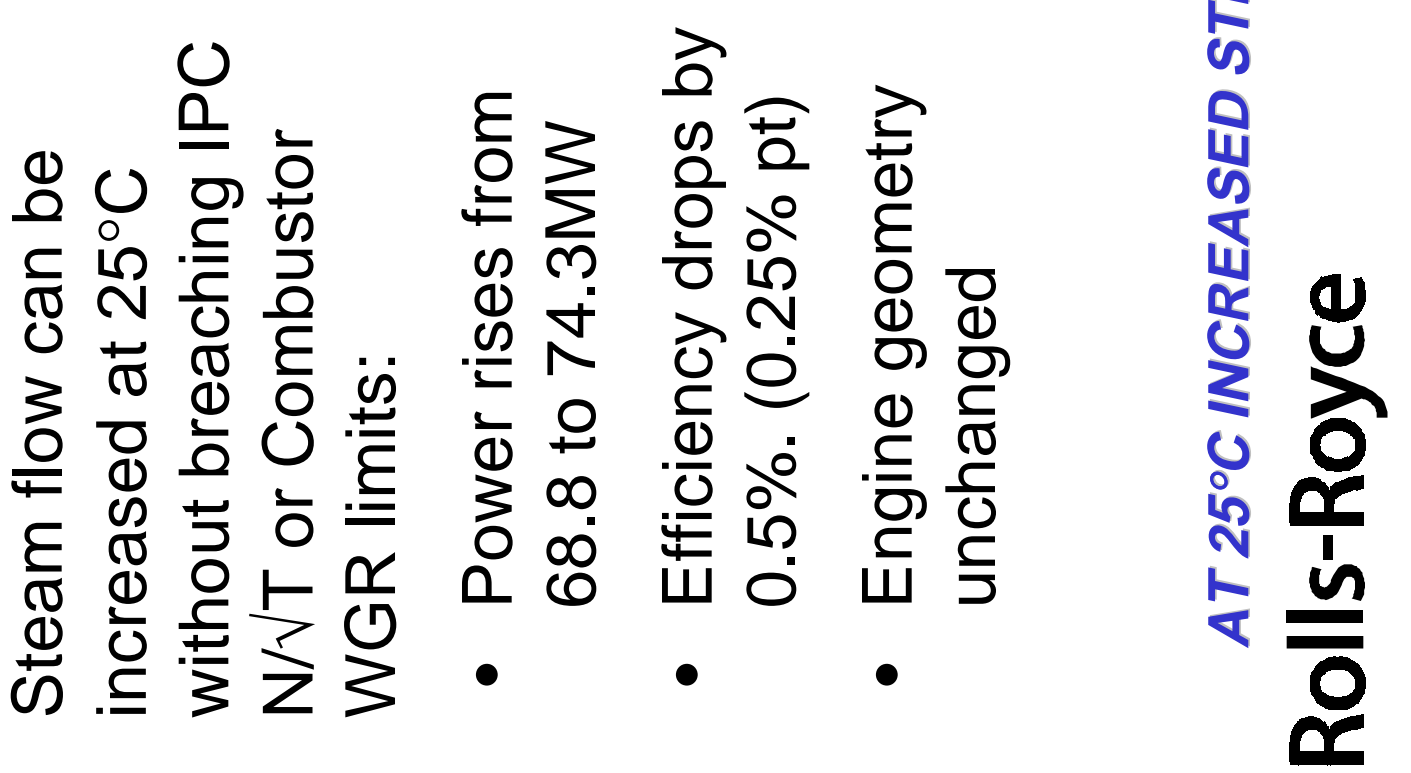
这 


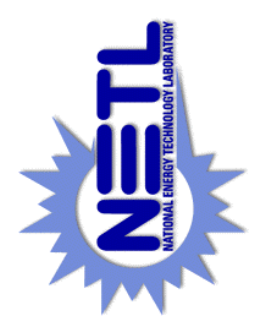

$\frac{\substack{0 \\ \frac{1}{0}}}{0}$
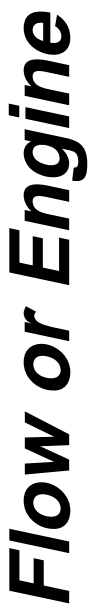

$\frac{\Sigma}{\infty}$

क

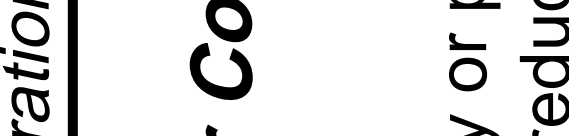

ฮ)

(1)

0

齐

ธั

늘 음

ते

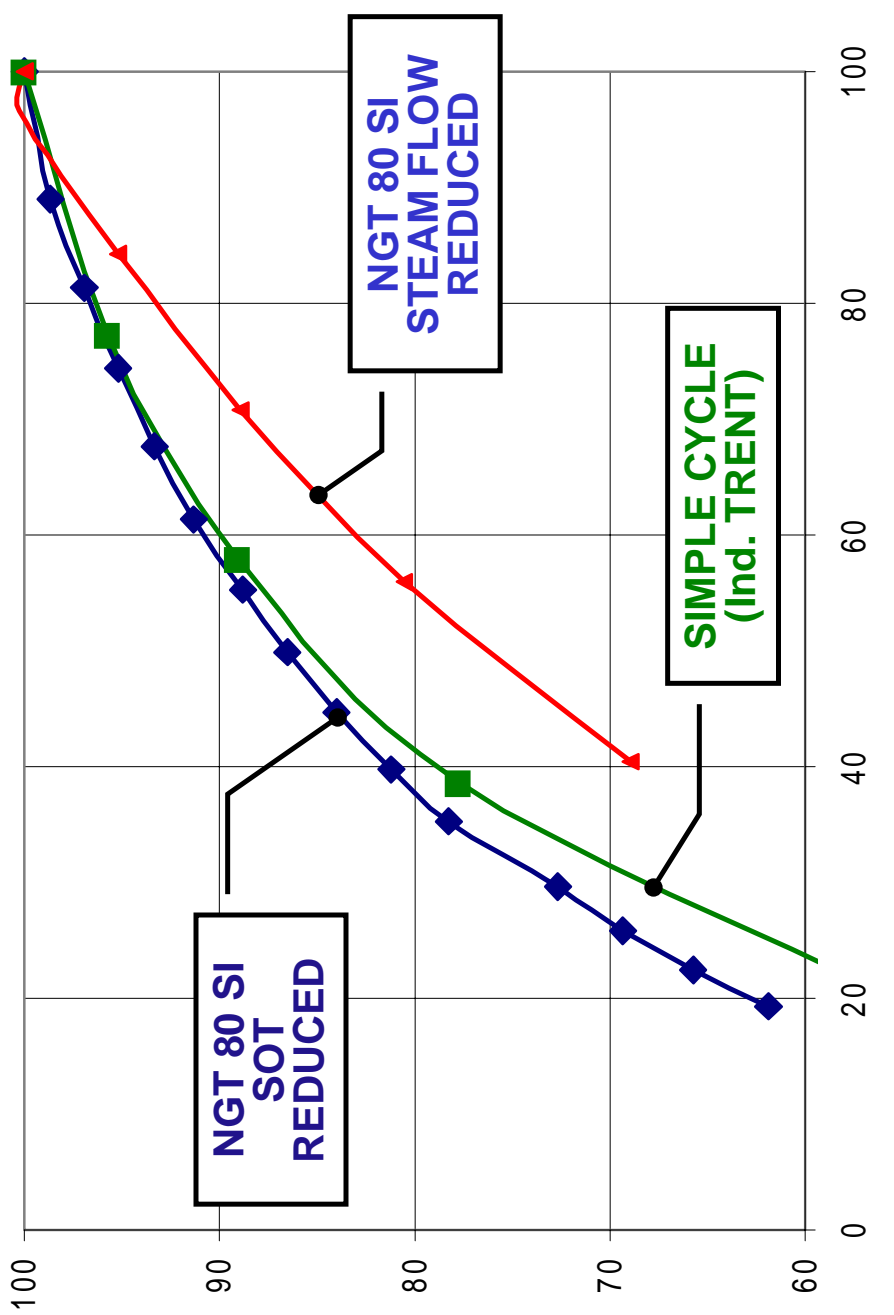

(\%) Кэนә!ग!н

๖ั

3

을

정 일 을

O d

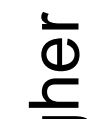

을

a

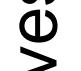

흠

등

Q

$5 \cdot \frac{0}{0}$

踣
응
히웜

ఏ \& $₫$

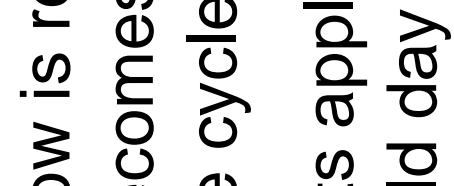

응 응

은 응 엉

ह $\frac{0}{0} \frac{E}{\omega}$ ब

के उ

की

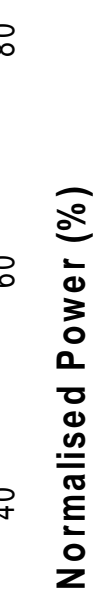

\section{ใ}

핀

님

U

14

올

이

F

U 1

ช

표

을

10

$\infty$

0

ar 

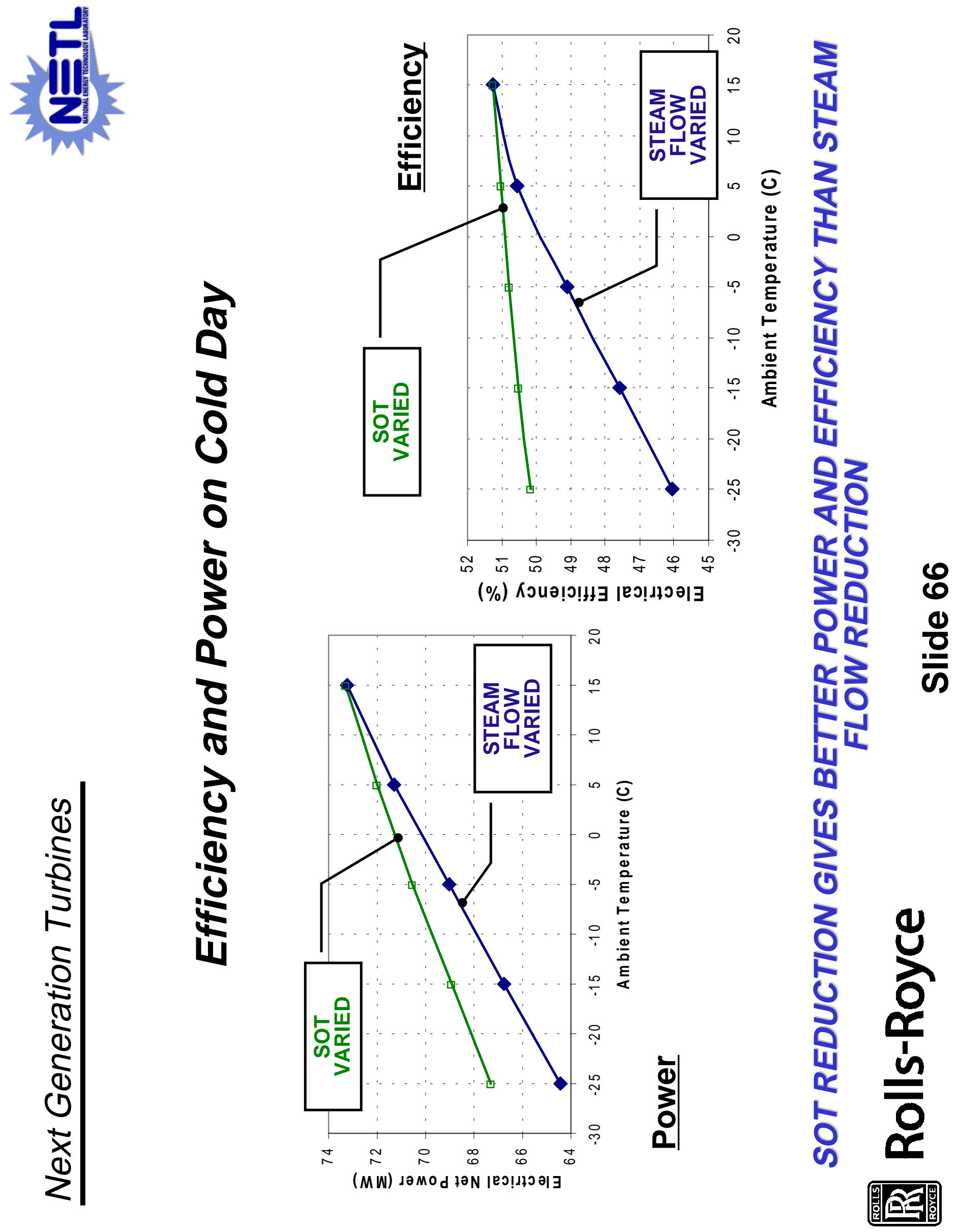

ఏ)
3
0

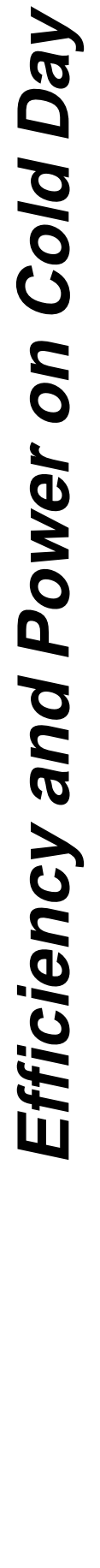



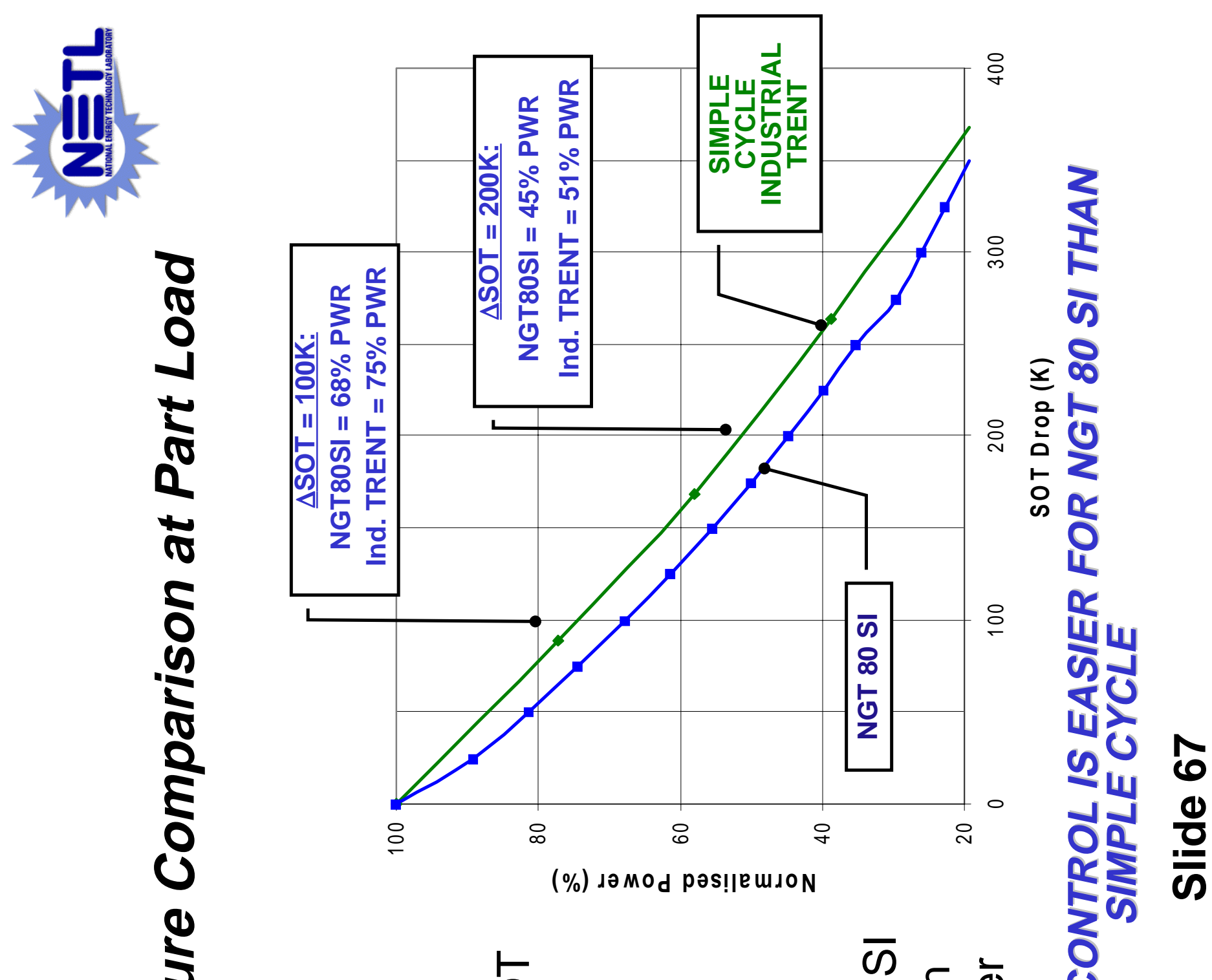

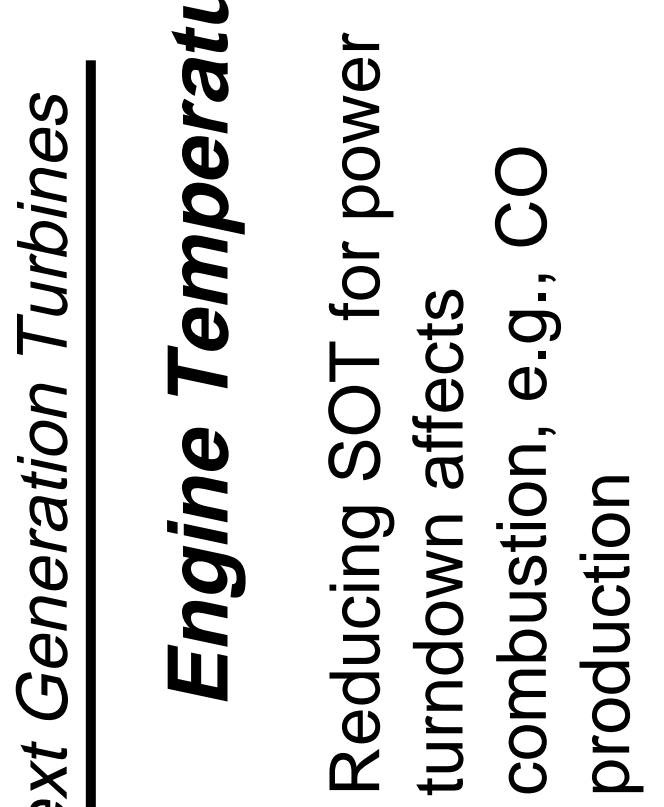

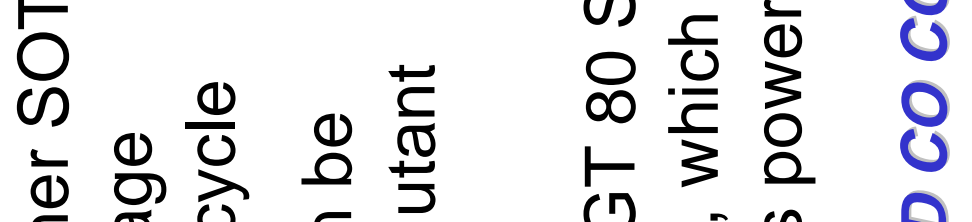

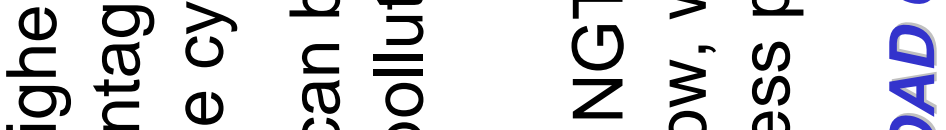

들 잉 응

$\subseteq$ 은

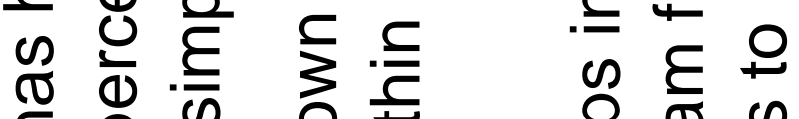

응 ฮิ

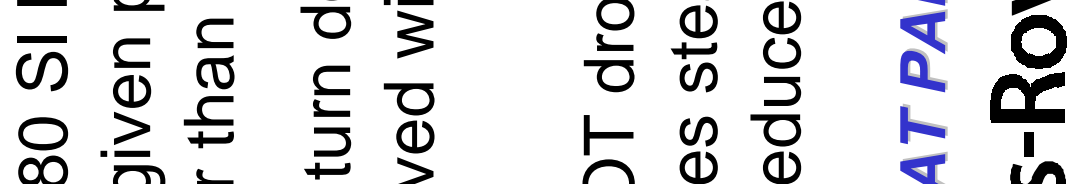

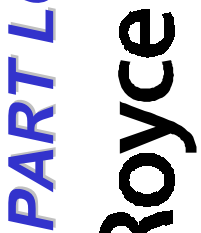

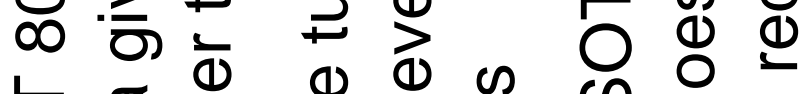

ष

(1)

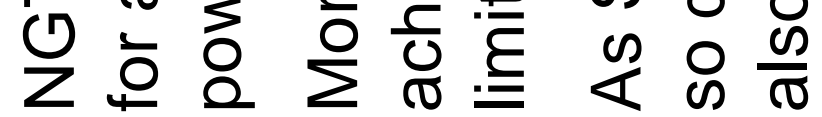

ع

21 


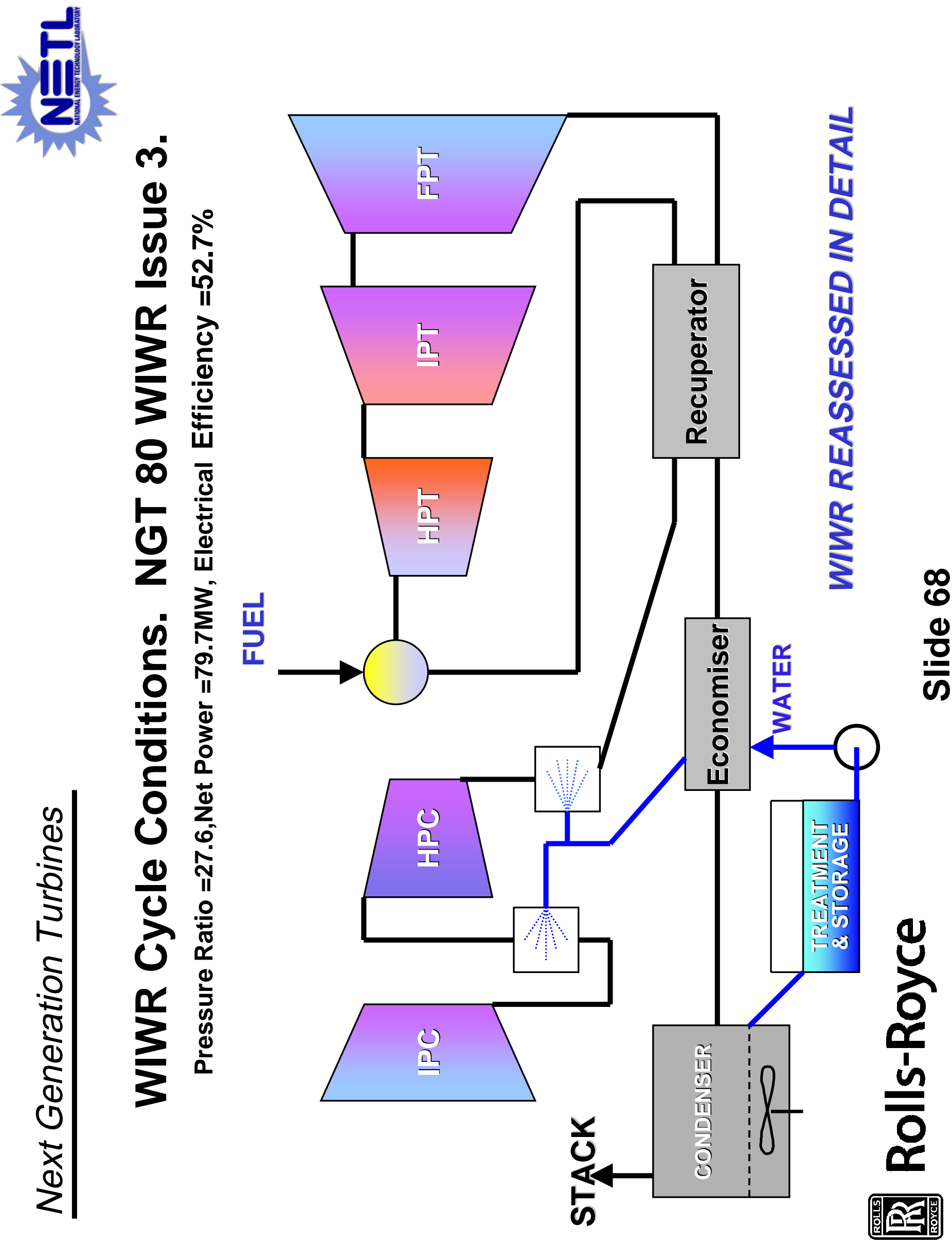



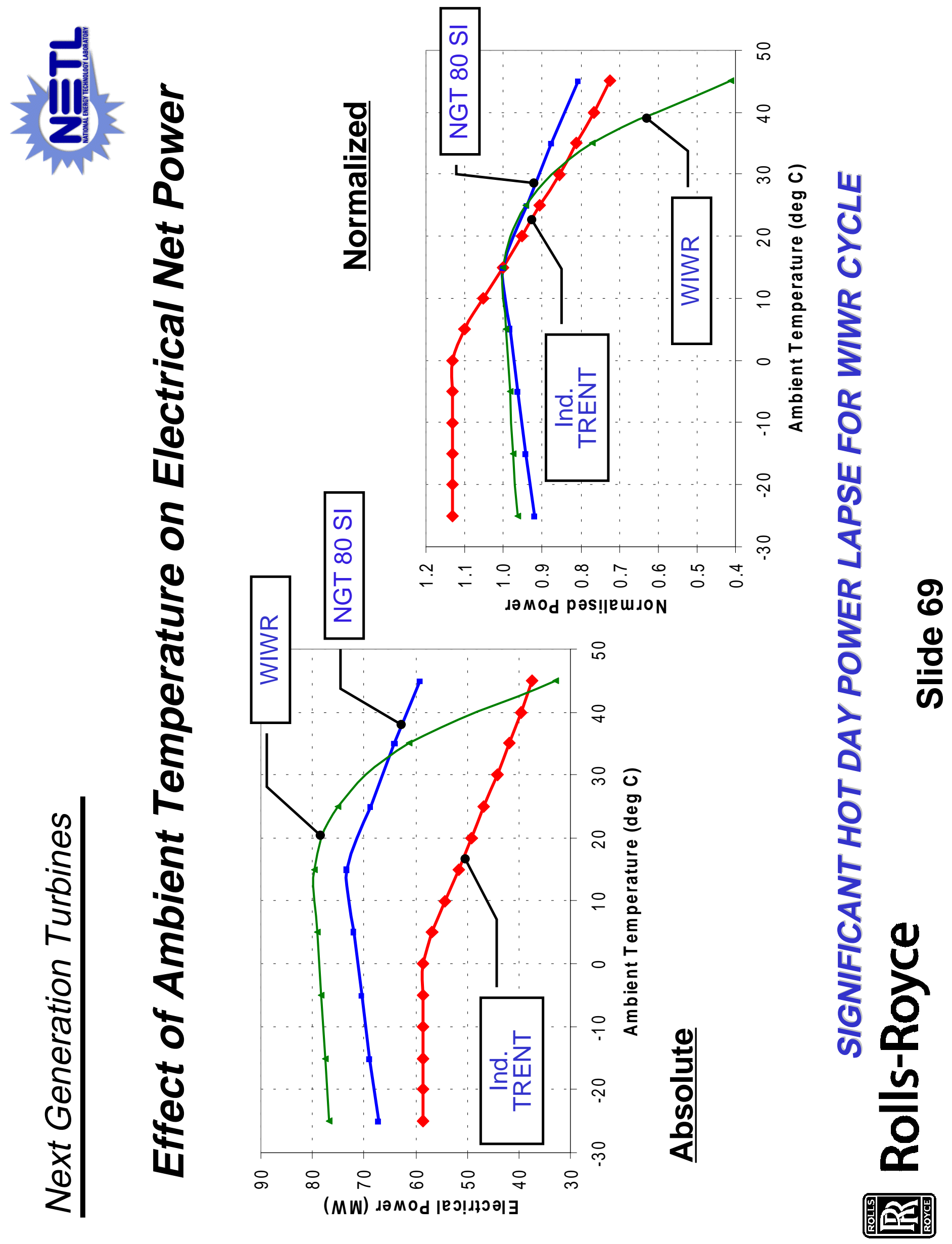

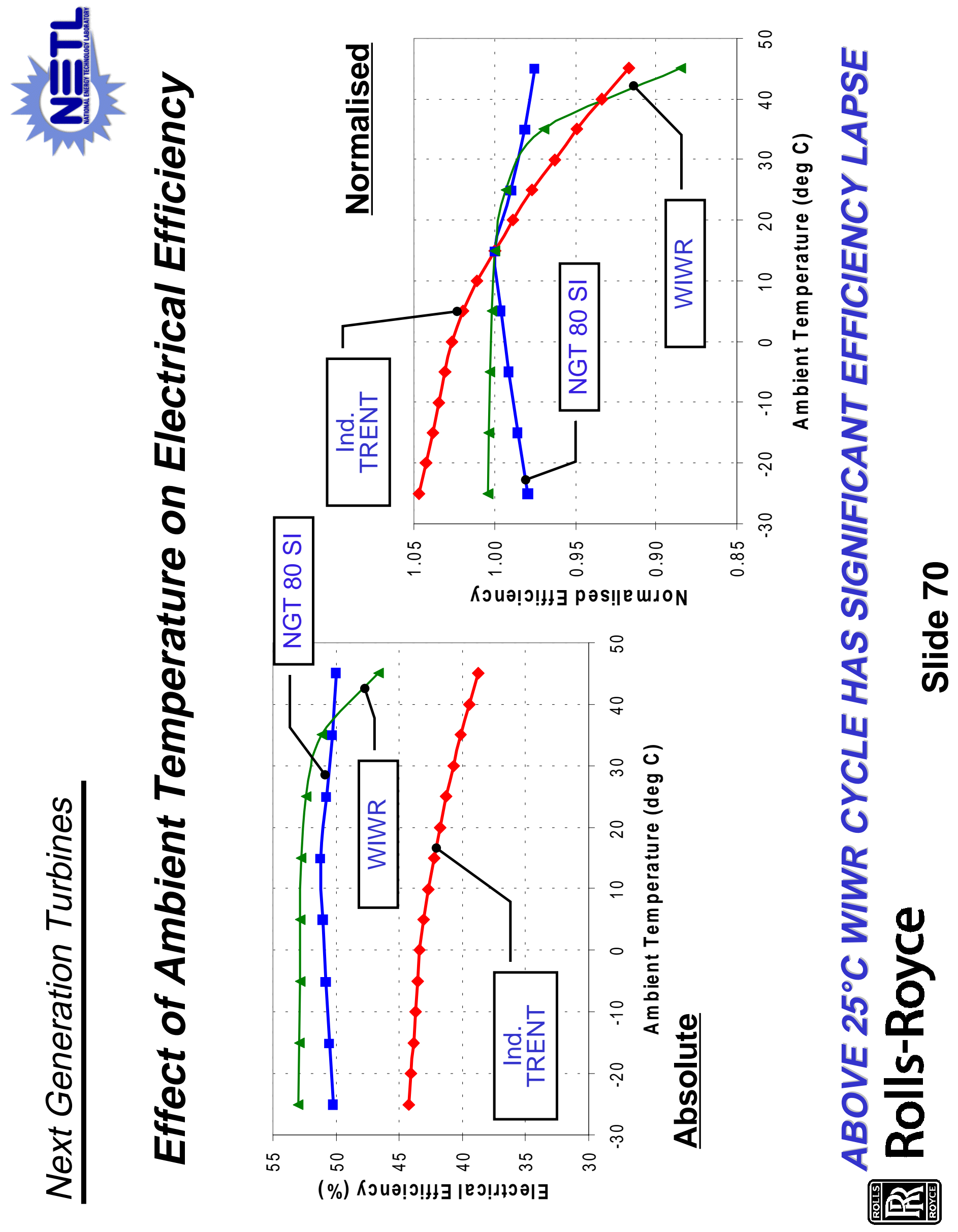


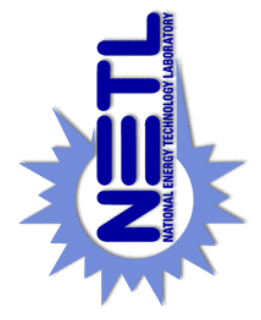

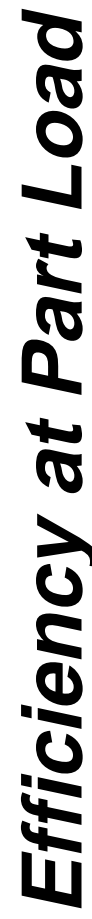

d

क) गे

๖ जे

产 U 带

蛋

$\therefore \gg$ 으의

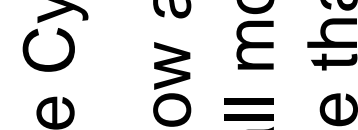

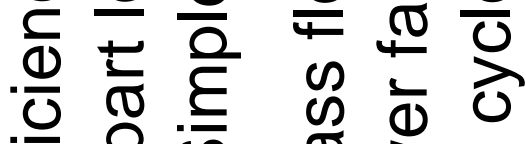

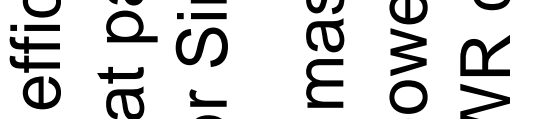

(1) त

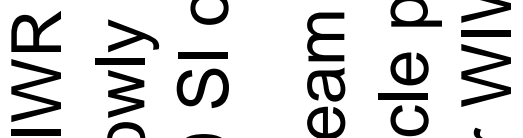

ఠ)

$\bar{x}$

$\stackrel{1}{2}$

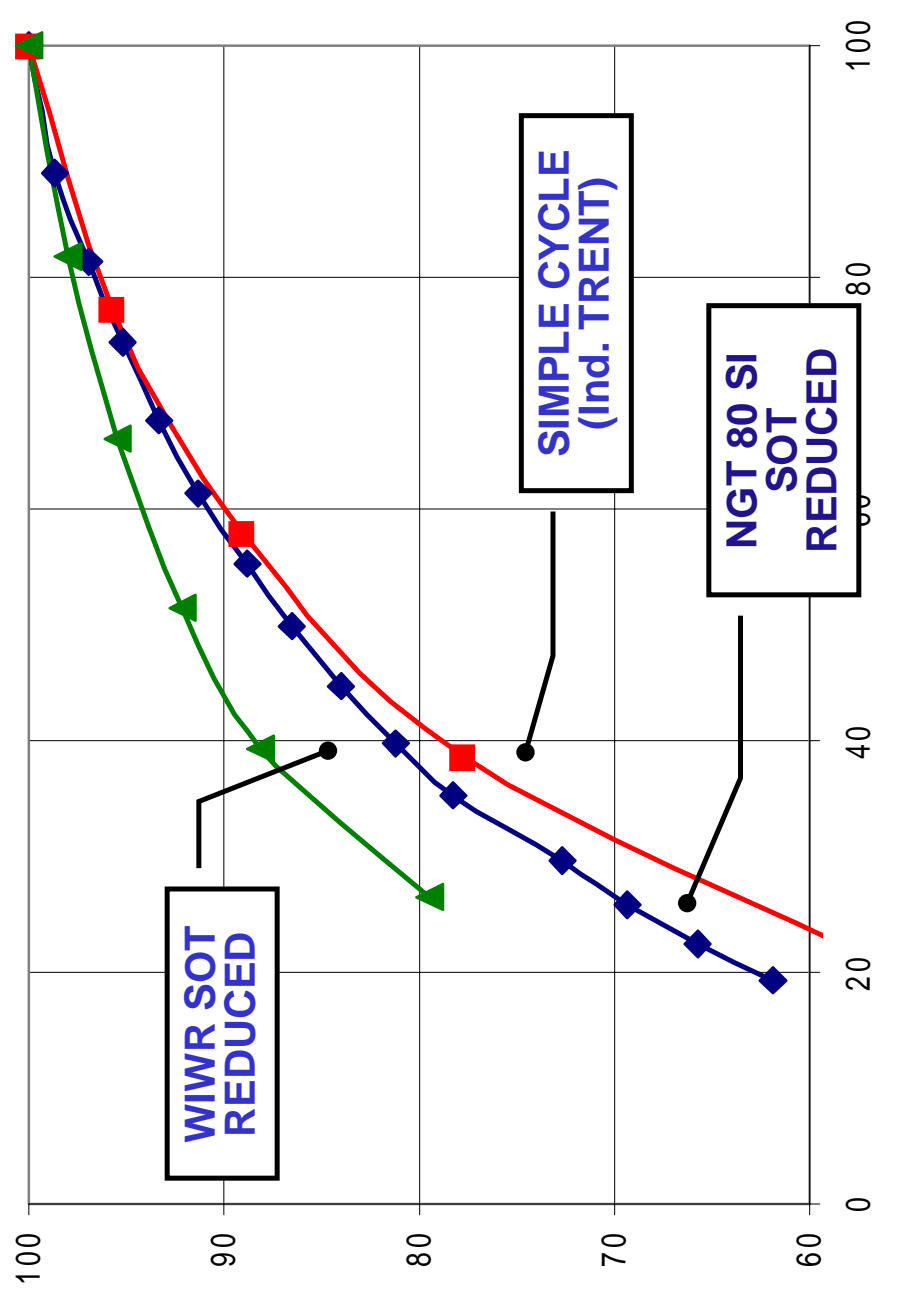

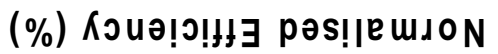

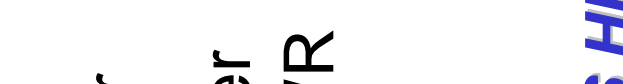

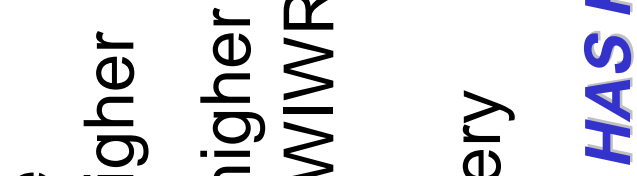

(1) 告 专了。

备.

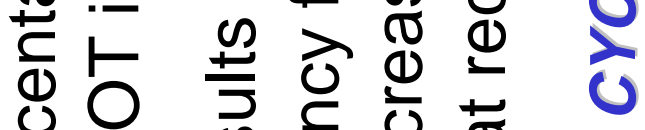

U फ क

ब

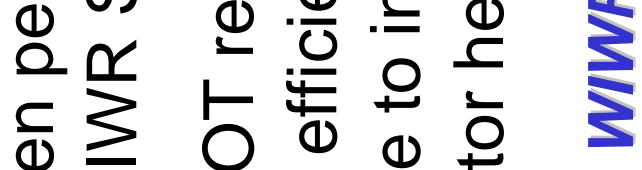

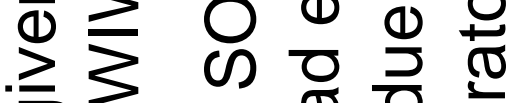

б

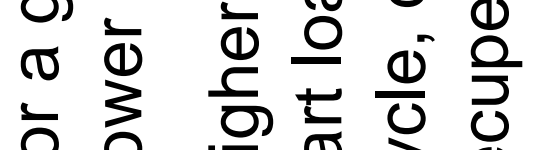

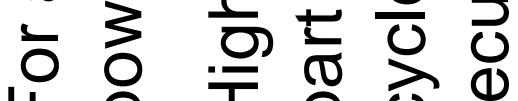

$\underset{1}{\mathbb{2}}$

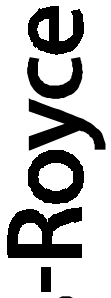

n

음

ar 


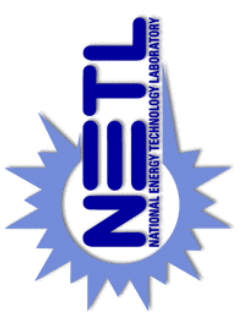

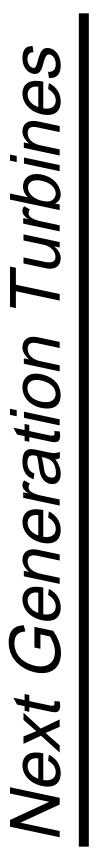
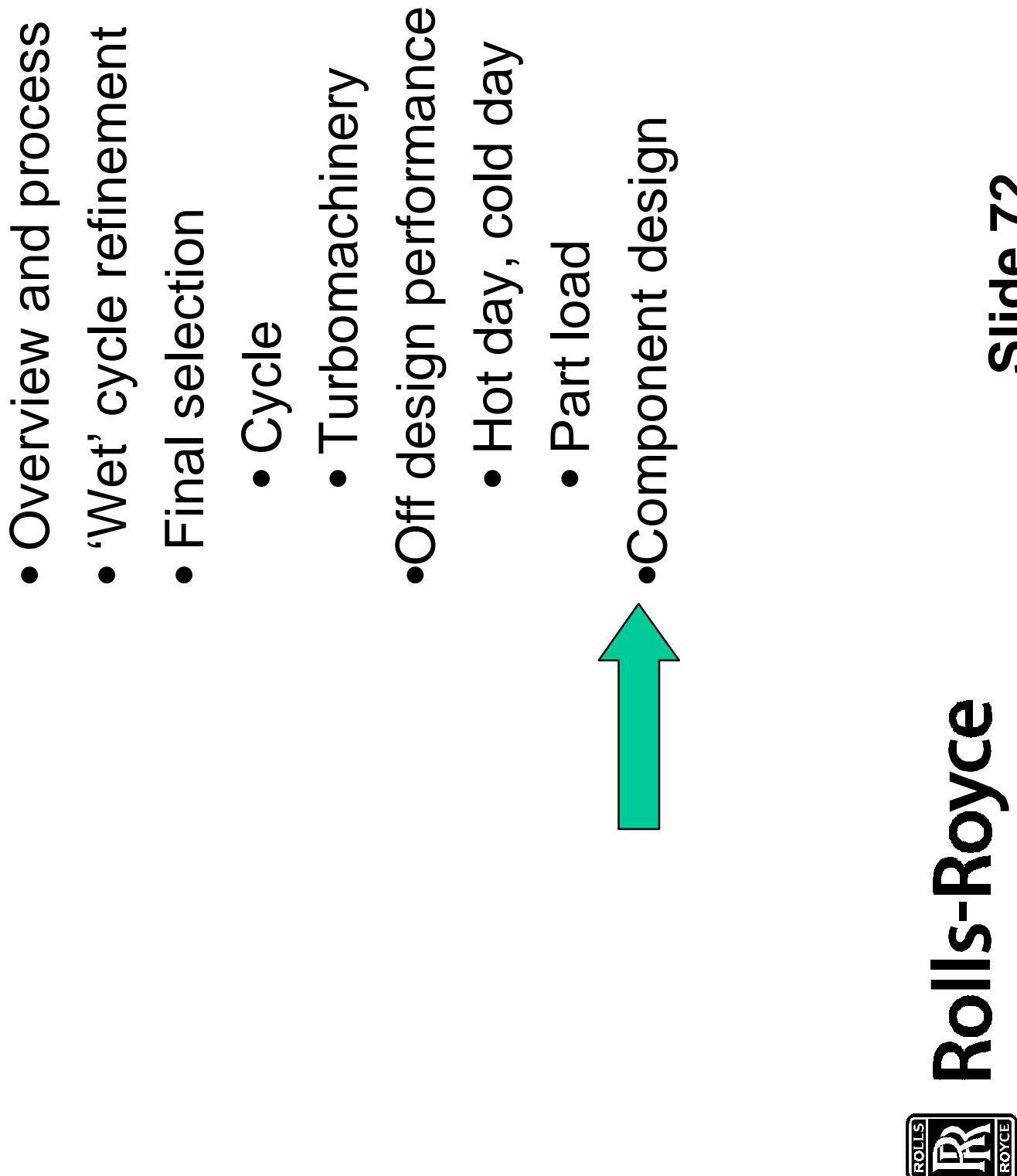

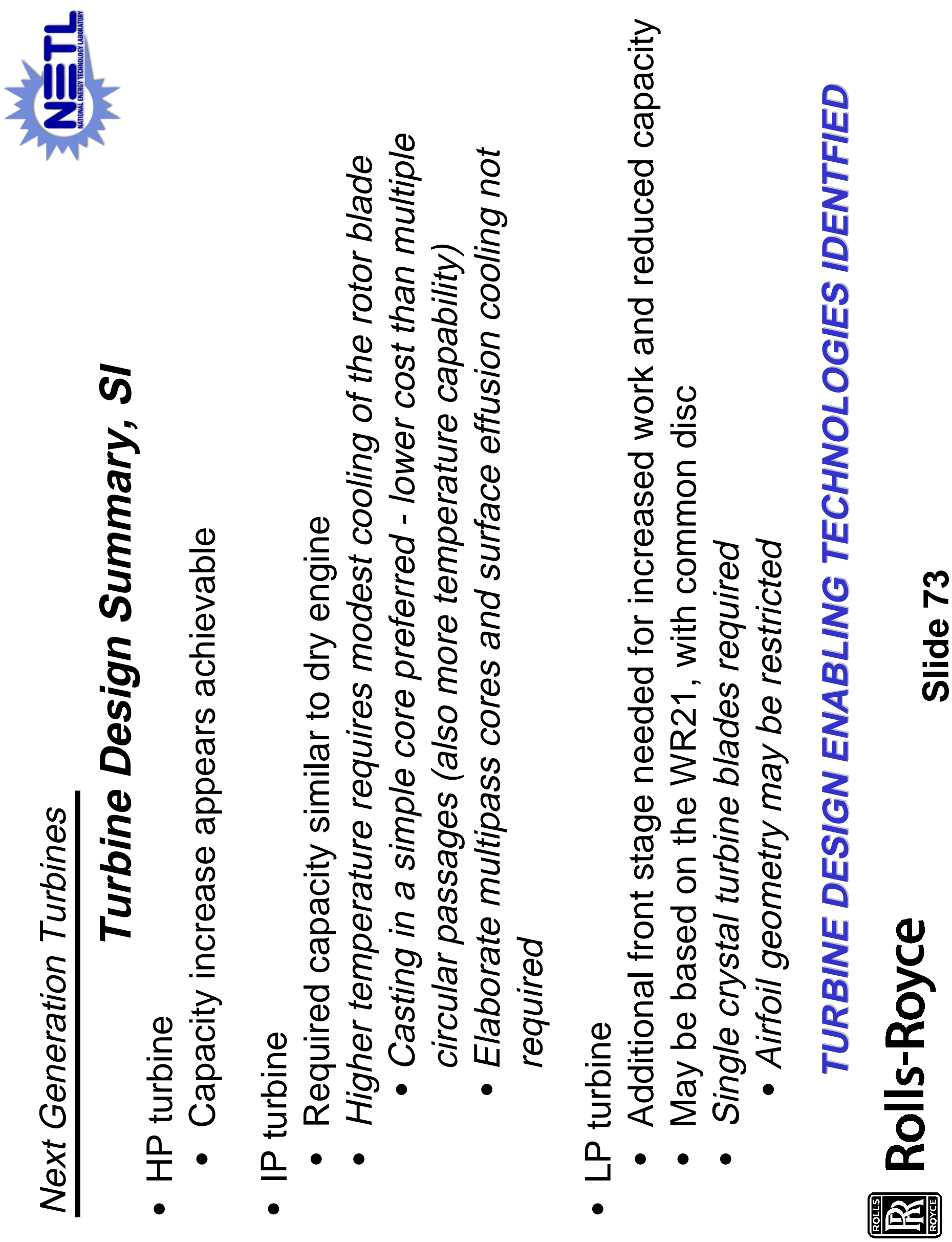


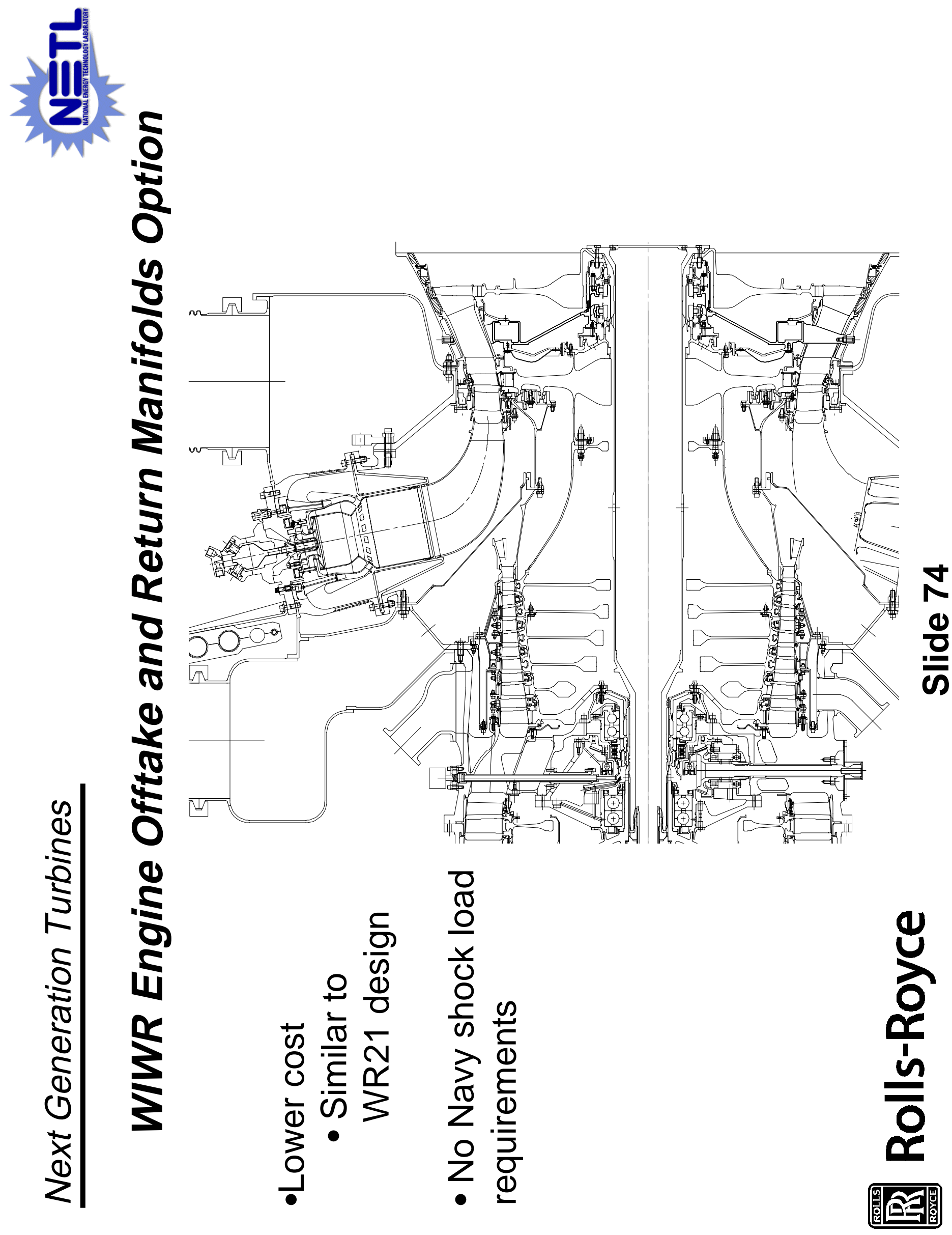



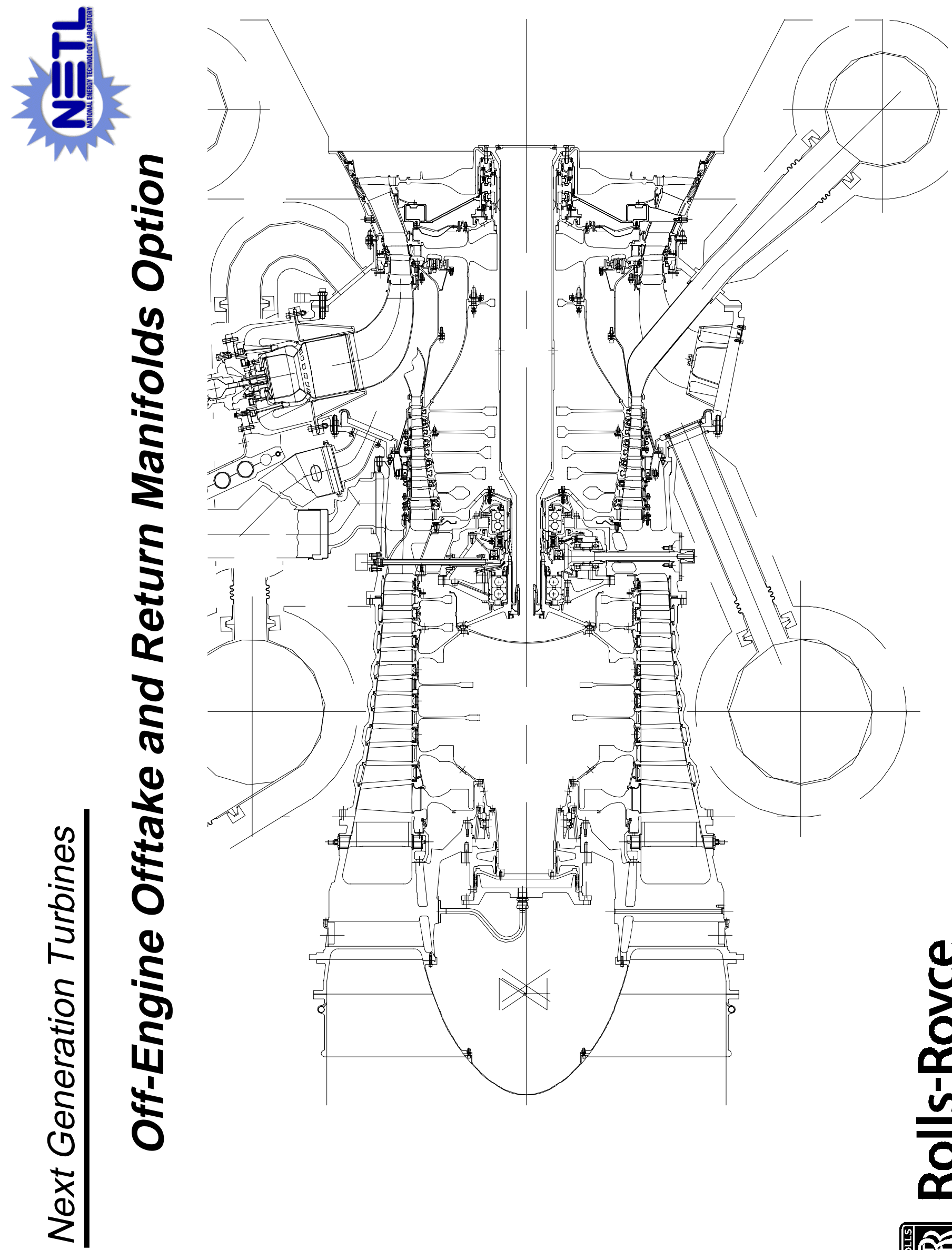

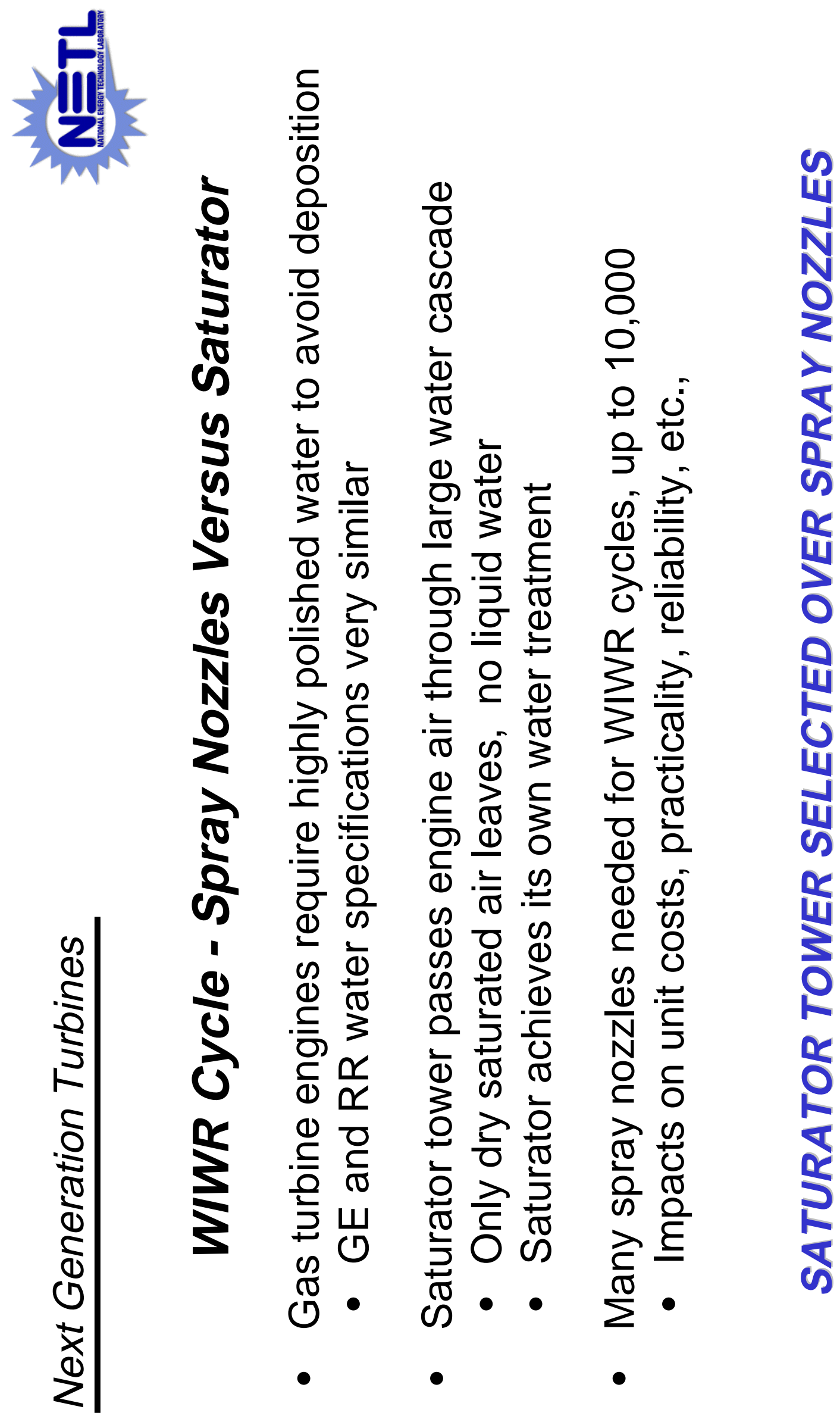

$\frac{0}{0}$
$\frac{0}{0}$
$\frac{0}{0}$

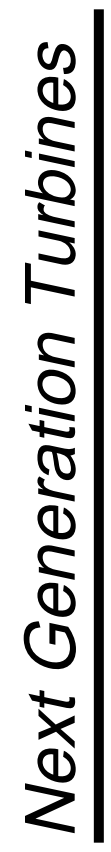

유 क ते 


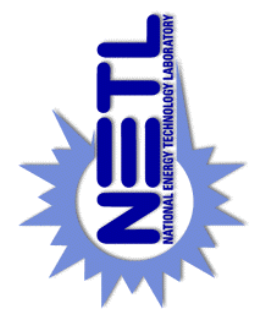

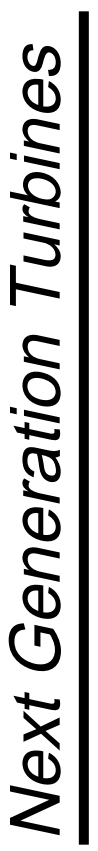
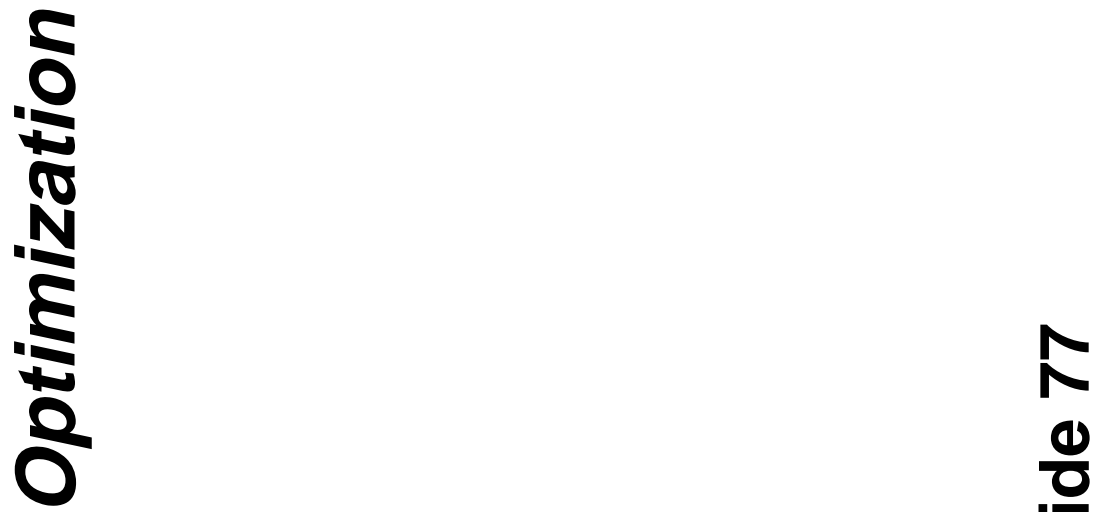

()

(b) क

$\frac{0}{0}$
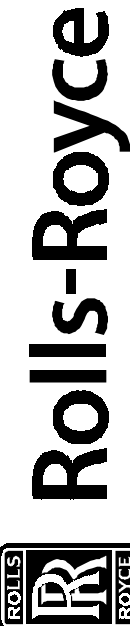

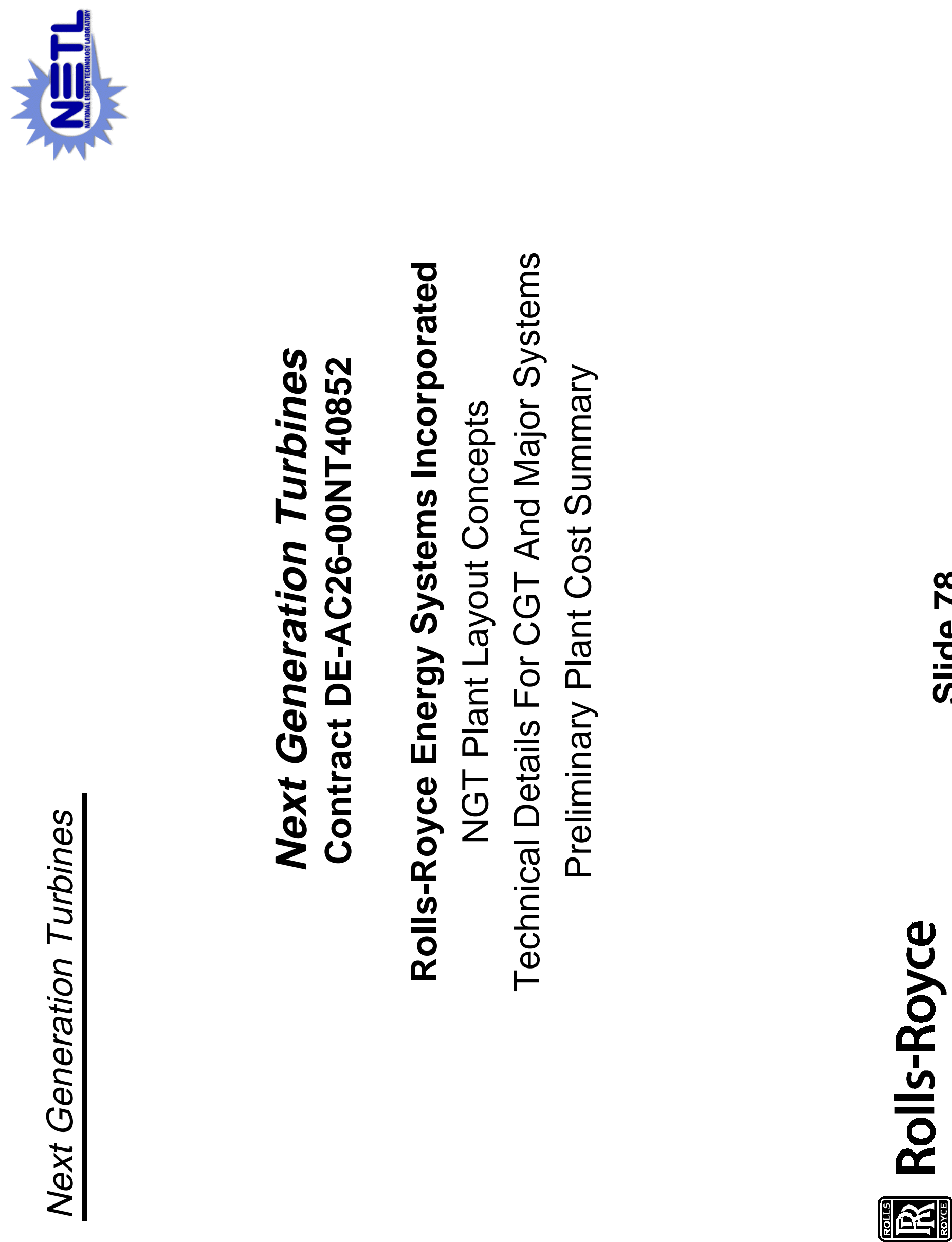

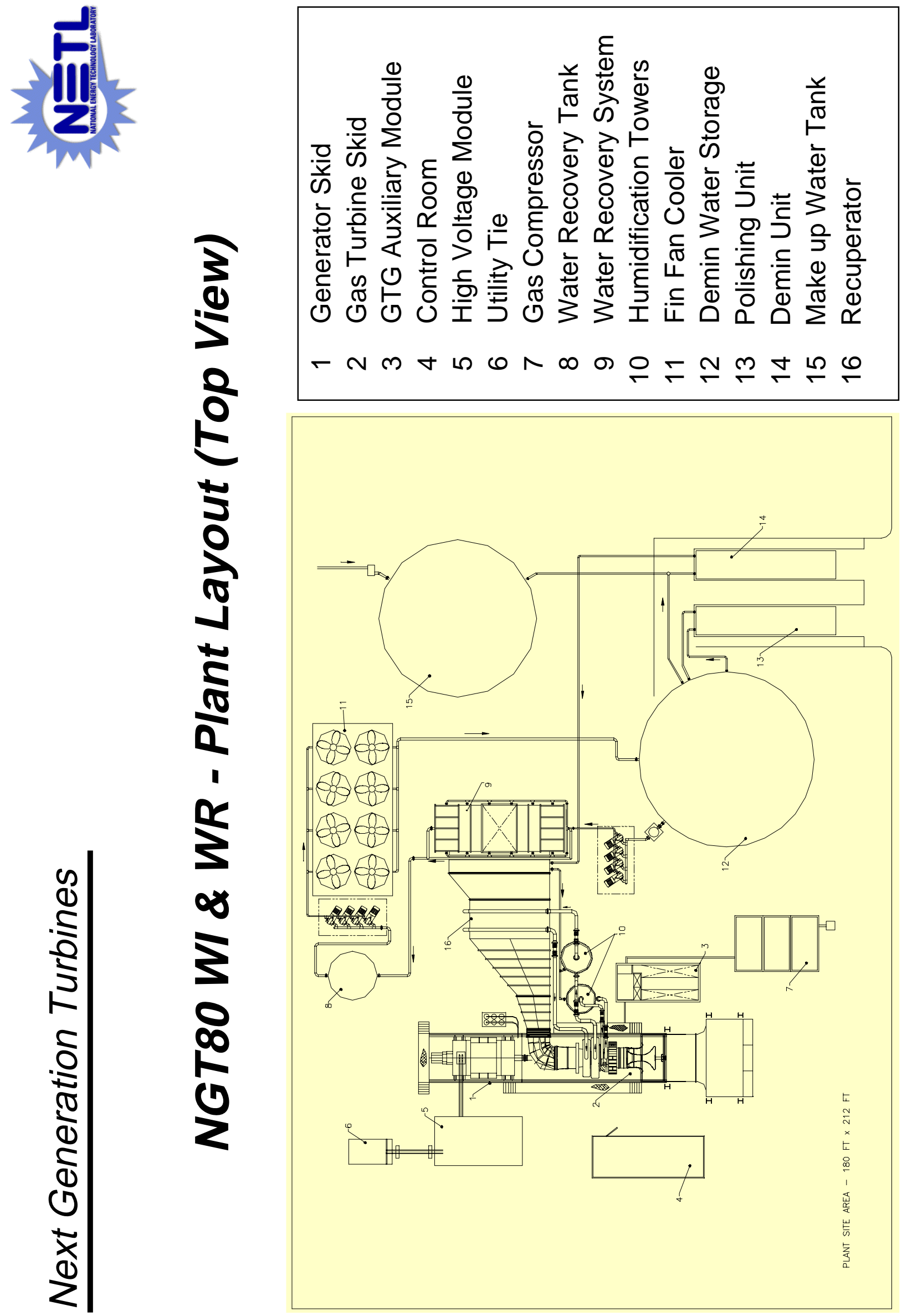

0
0
0

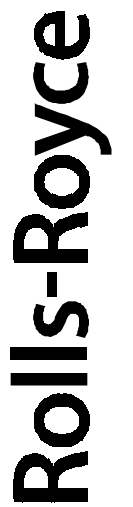




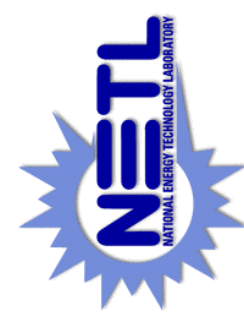

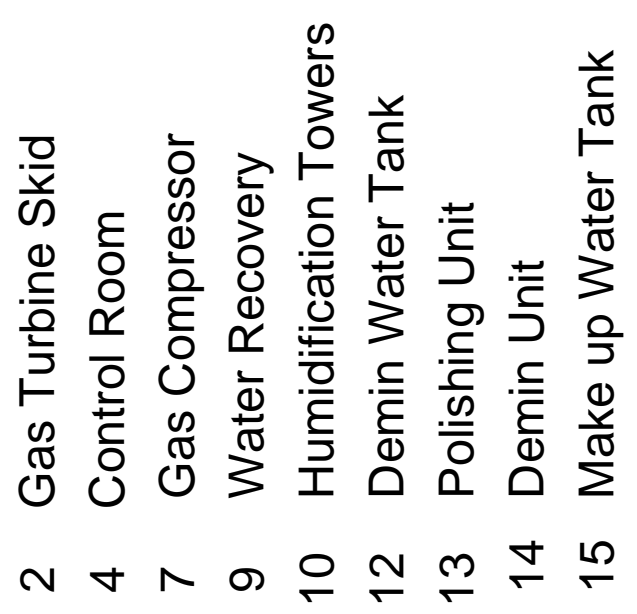
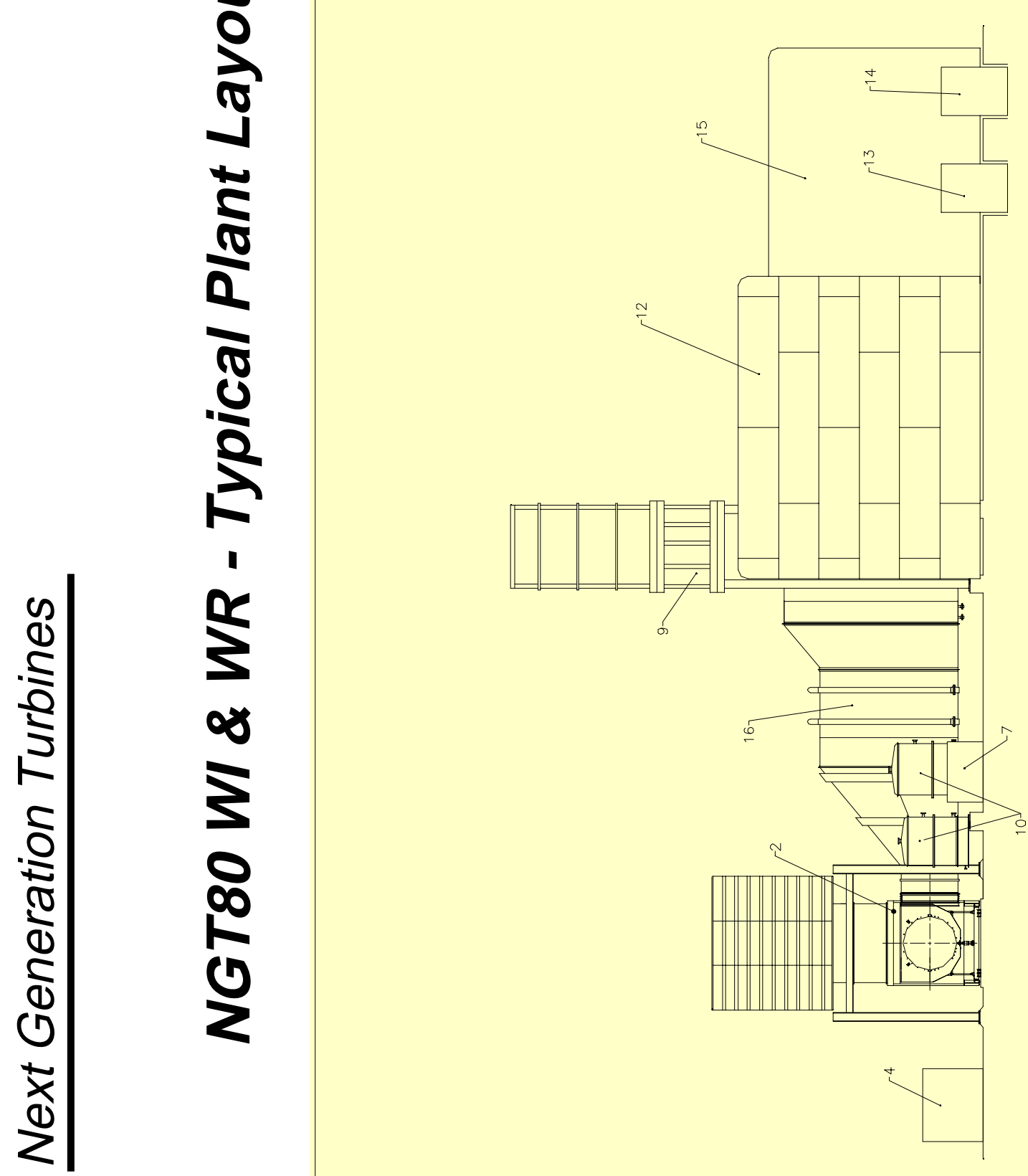

0
0
0
0

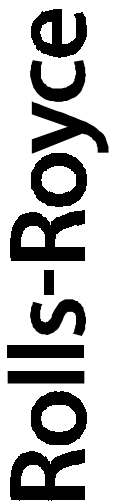



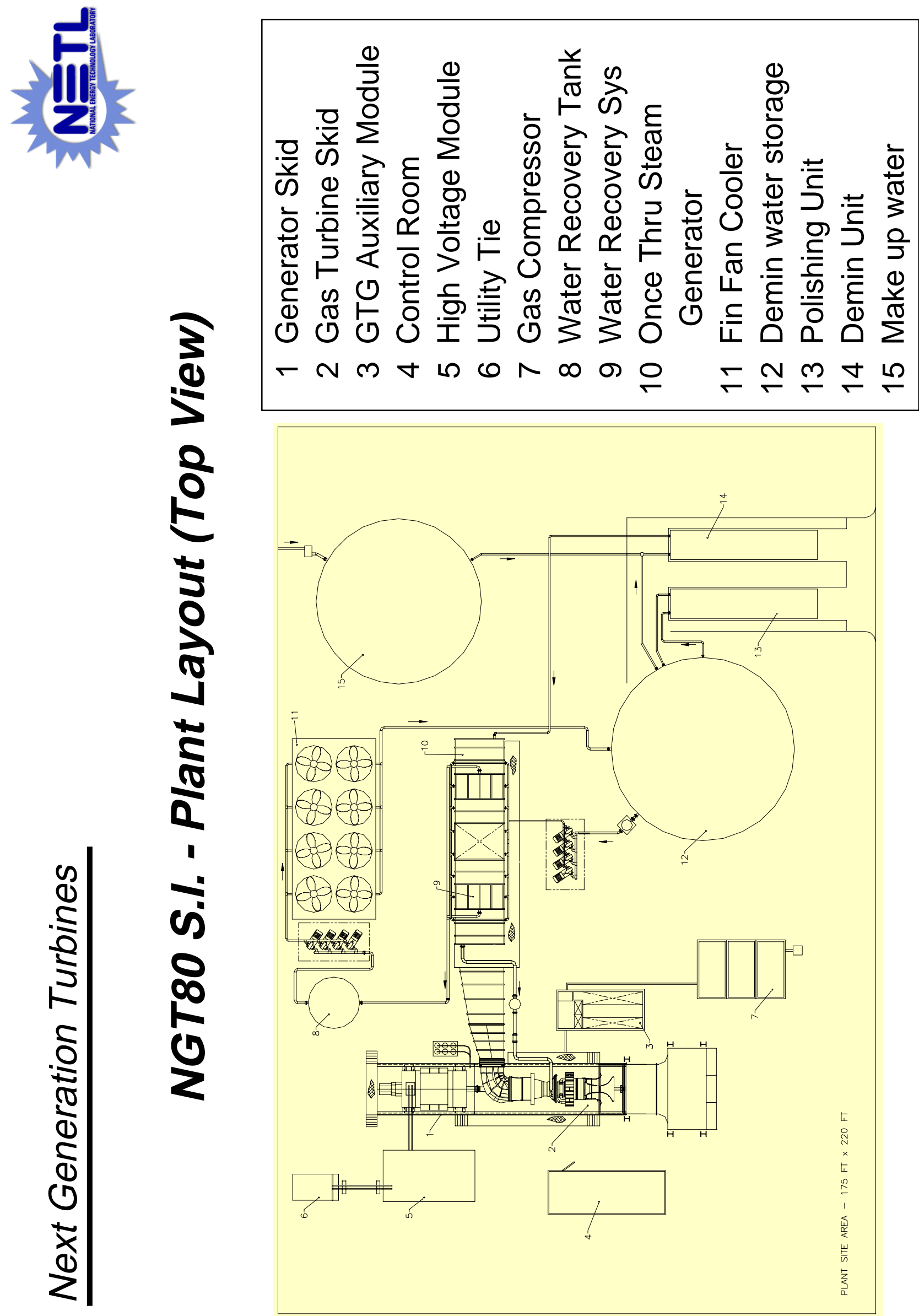

$\bar{\infty}$
$\frac{0}{0}$
$\frac{0}{\infty}$
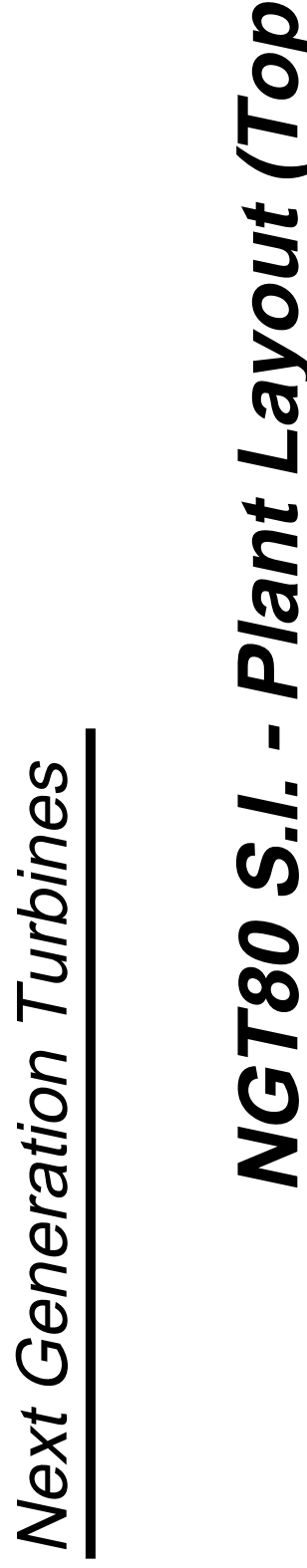

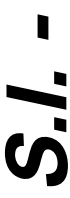

$\frac{0}{2}$ 

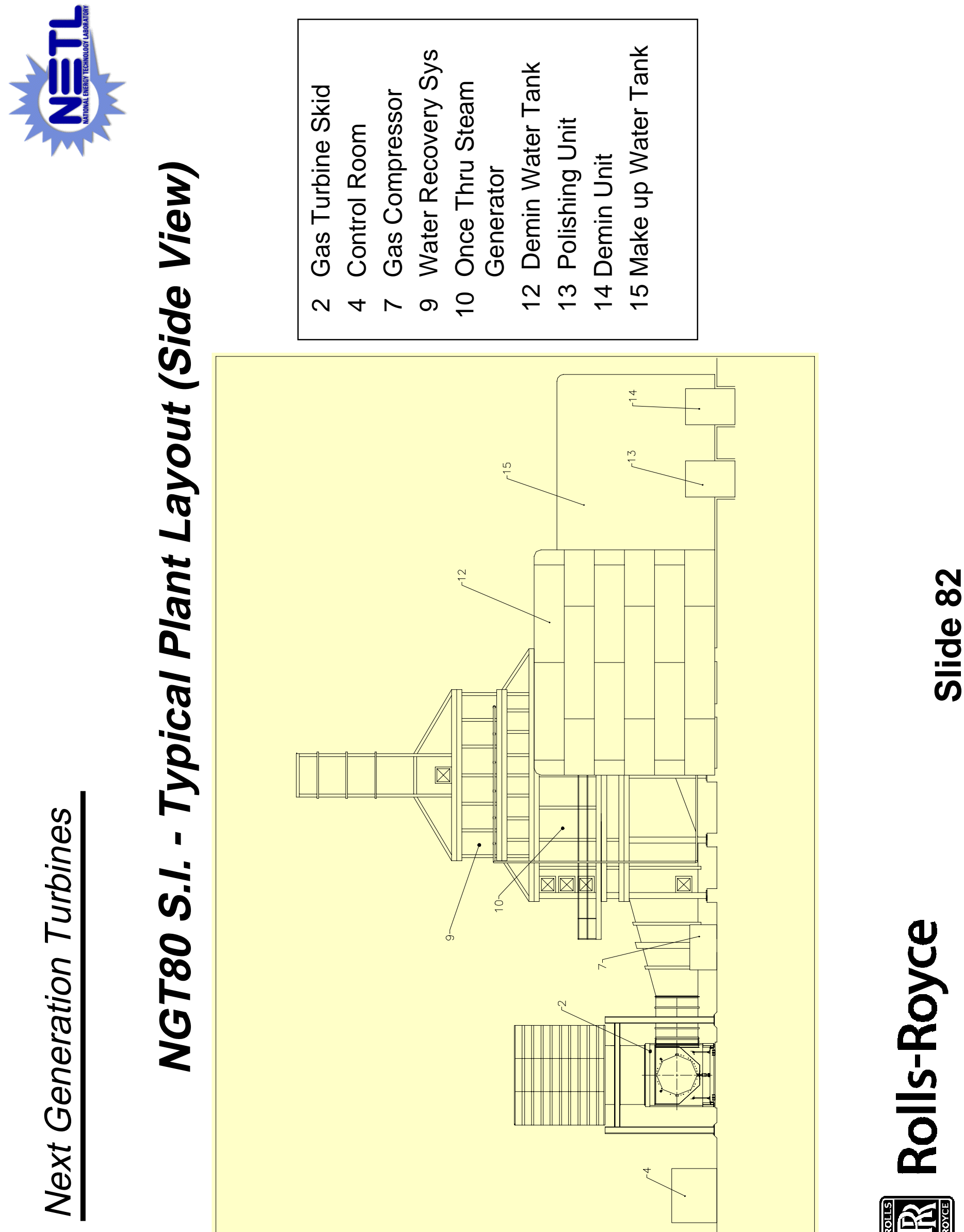


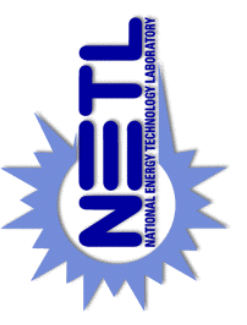

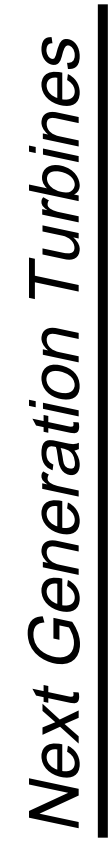
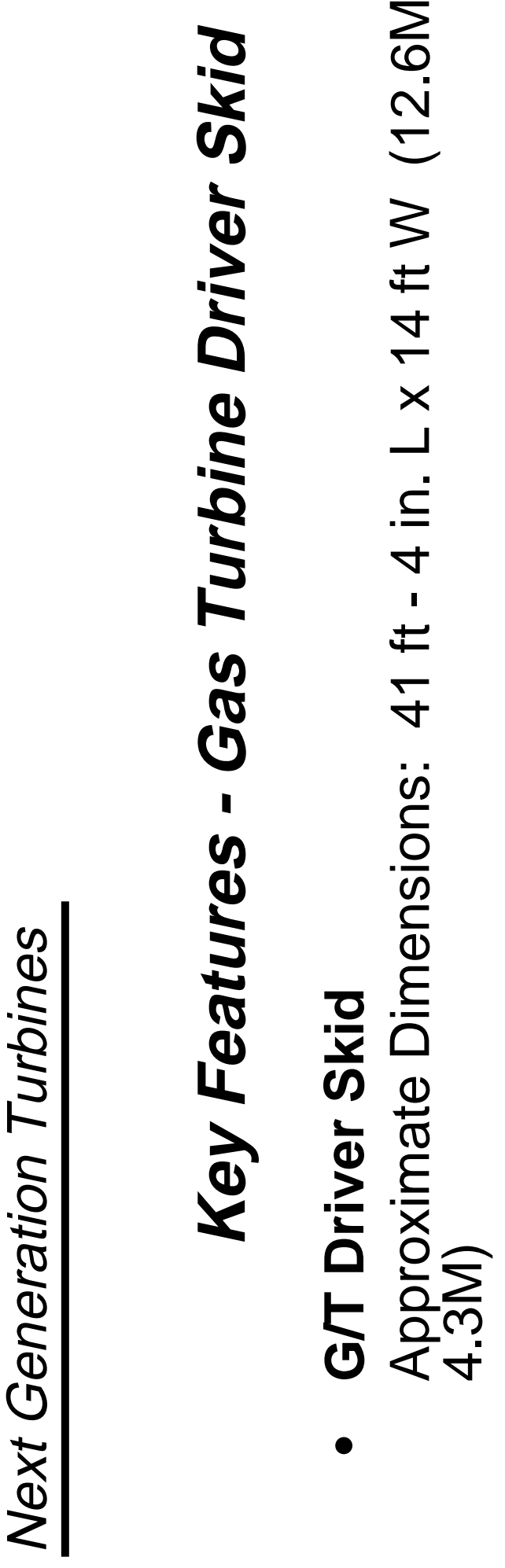

ซ

c

을 엉

ल

윰

잉

므듬

30 ช

○ั

지

Ðㄷㅇ

(1):

잉 응

느을 은

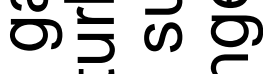

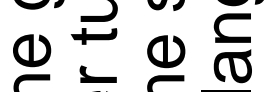

£ ๘

ᄋํำ

$\infty$ 는

돈

(1)

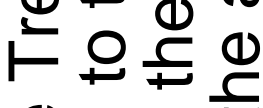

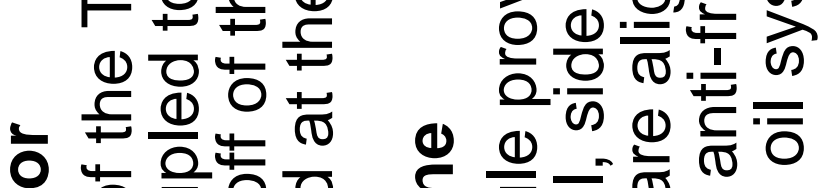

+ $0 \frac{2}{2} 0$

(1) $₫$ ல

ฮ $\geqq ニ$ ত্

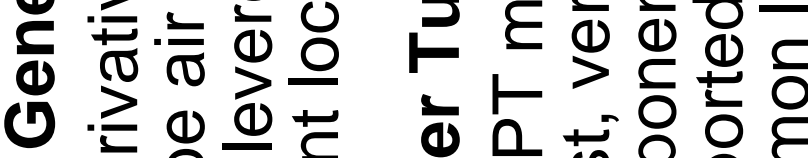

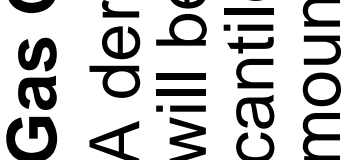

으의.모

엉 응 응

는

ํํㅇำ

듬

읕ㄷ

E三

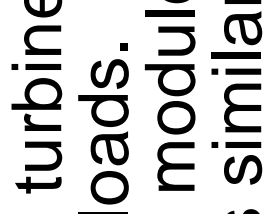

. 듬ํำ

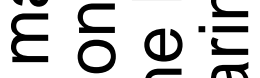

()

드으을 으

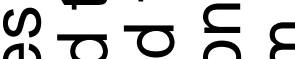

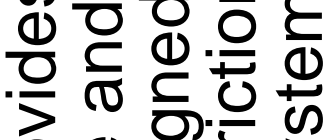

응.

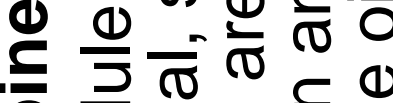

은 긍은 쏭 을

들 드음

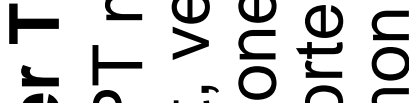

ఏ

으르르으응

ฉЕЕ 잉

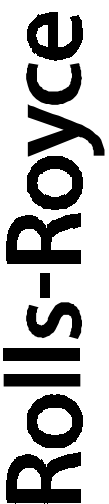


$\varepsilon$

ชั

$\frac{7}{4}$

ఏ

(1)

$\frac{1}{2}$

d

s

(1)

$\frac{1}{2}$

S

$r$

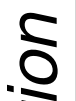

$\approx$

(⿻)

$\frac{1}{9}$

0

文

O

(1)
들 틀

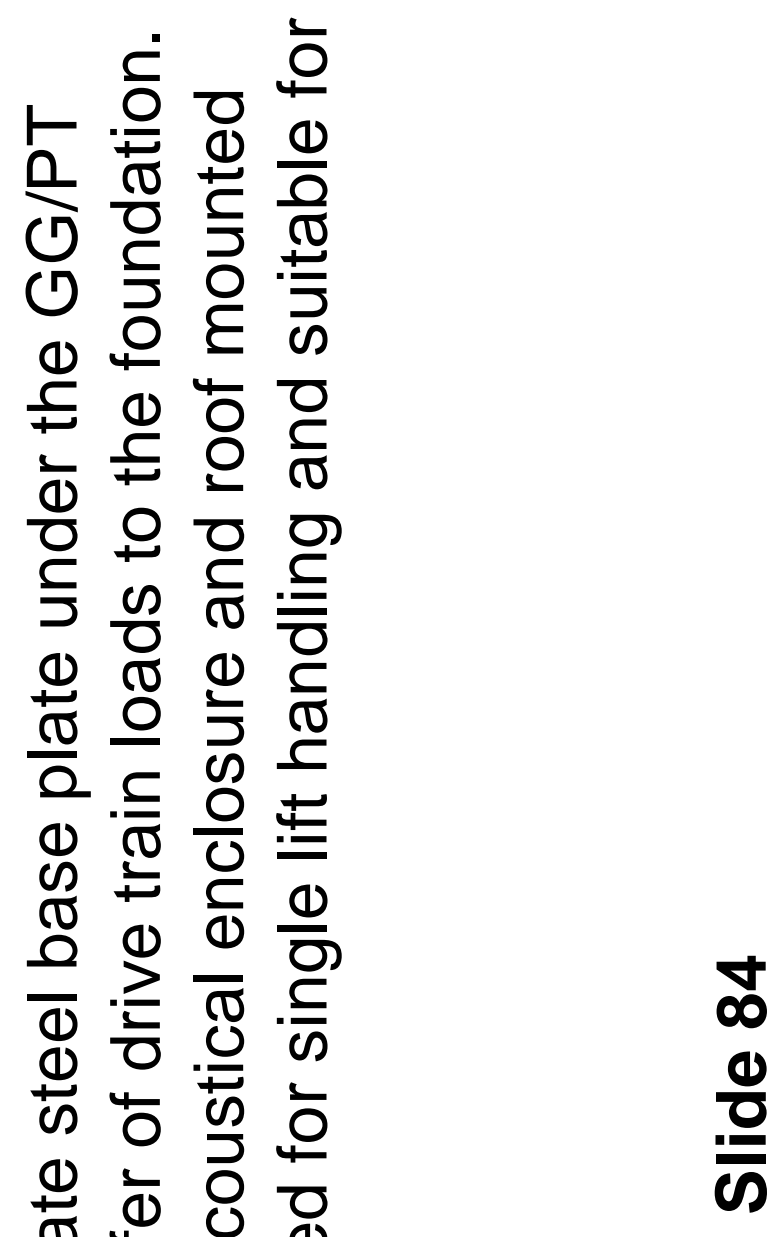

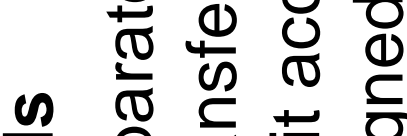

응 웧 志 일 $\frac{\overline{0}}{\mathrm{\sigma}}$

$\overline{1} \pm \frac{1}{(1)}$

$\stackrel{4}{\sim} \stackrel{\frac{\varepsilon}{0}}{0}$

(1) 으 ๘ิ

11 틀 을

$>\quad \frac{2}{0} \frac{1}{1}$

\)응 을

으름

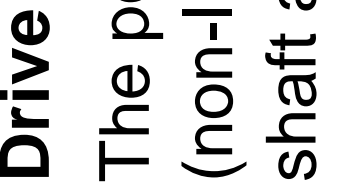

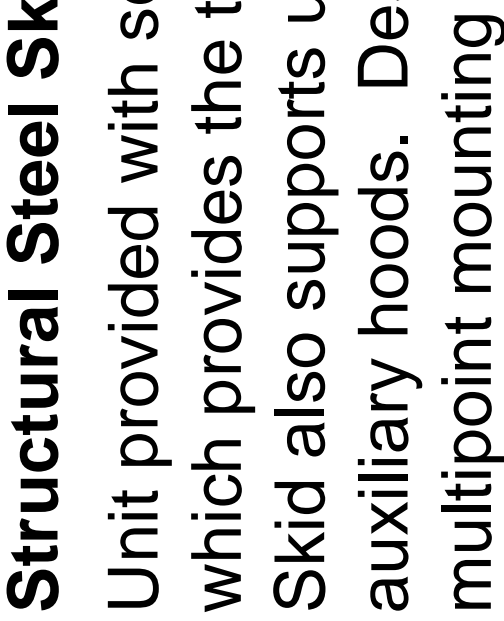

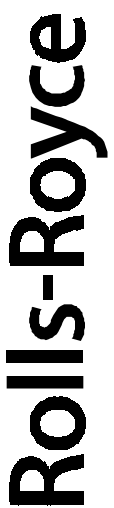



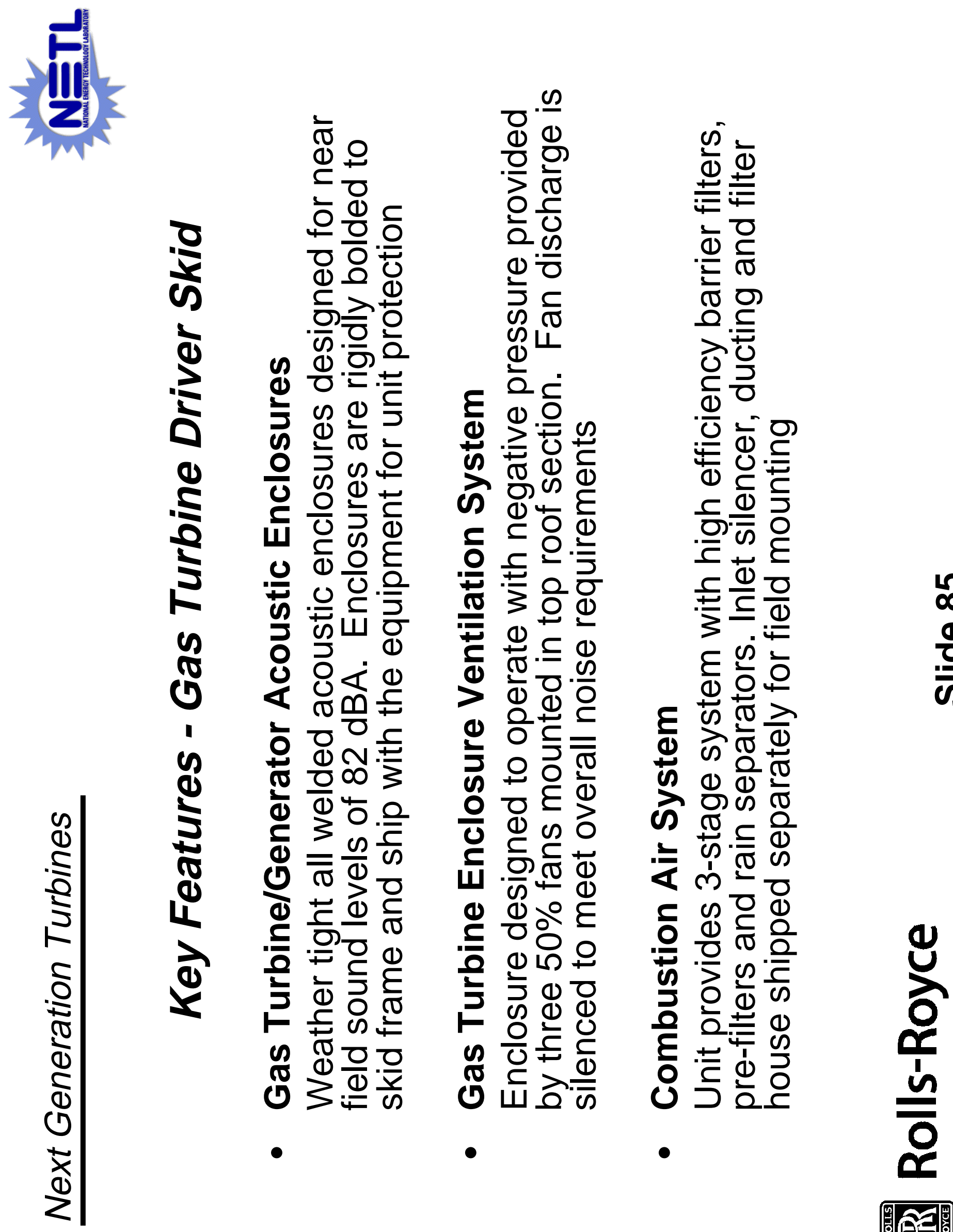

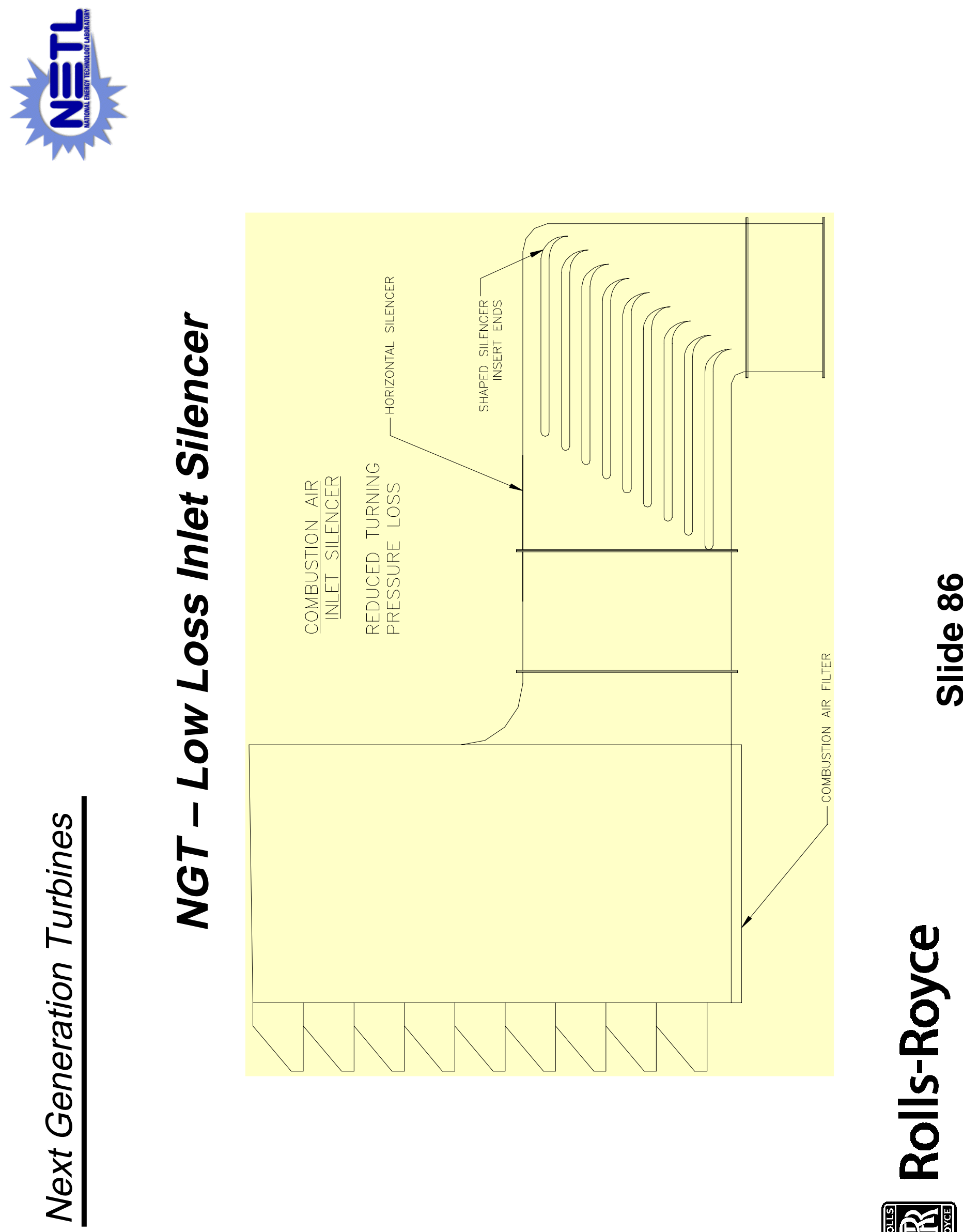

$\frac{0}{\overline{0}}$
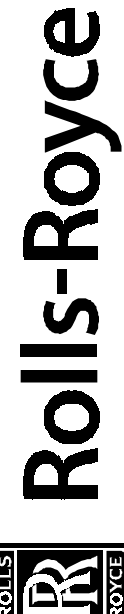


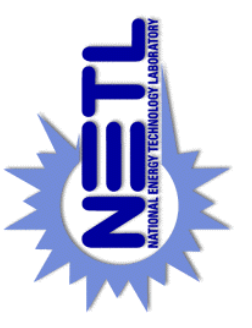

ֻٕ

흘

ह

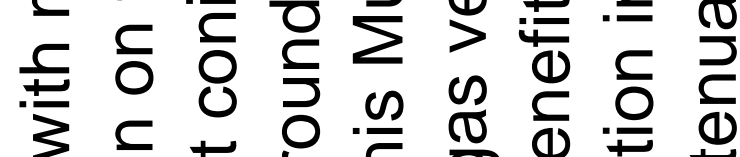

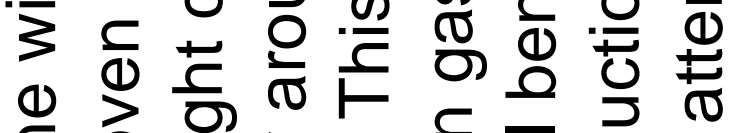

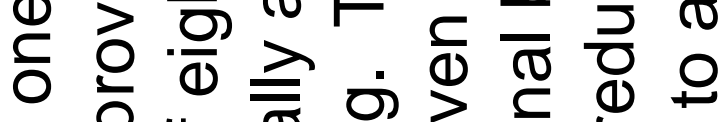

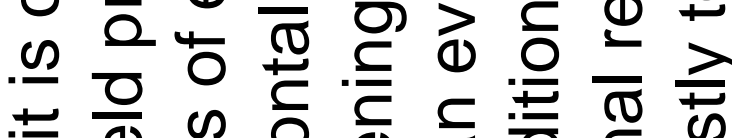

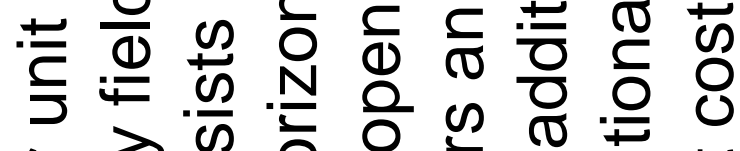

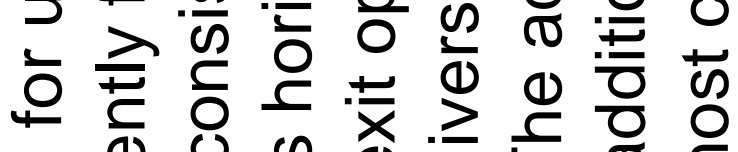

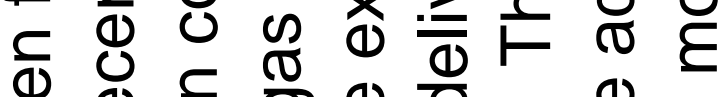

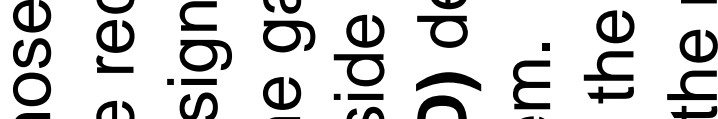

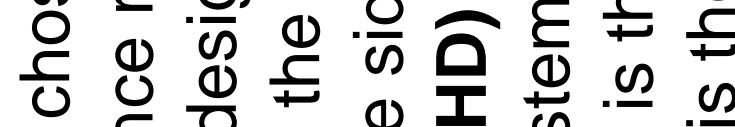

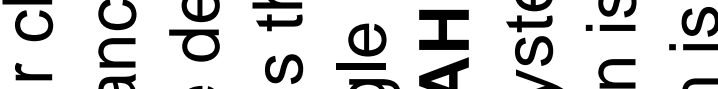

ब ब

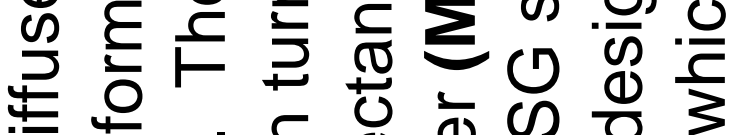

cs

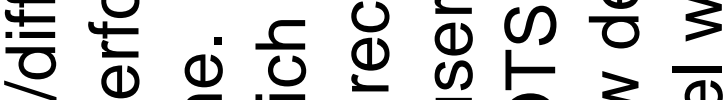

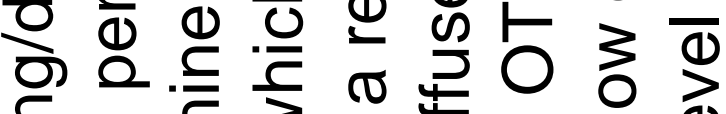

ह 

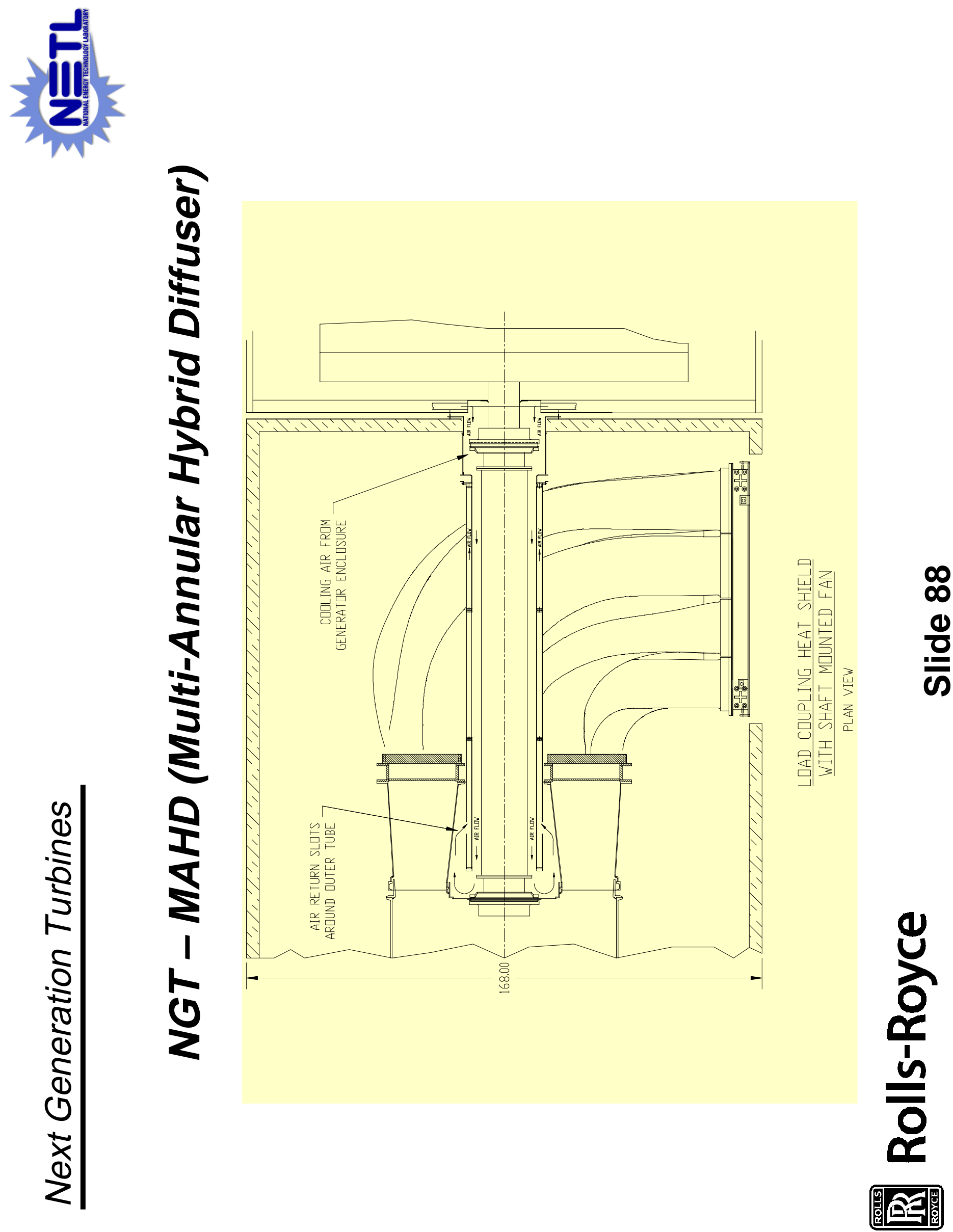

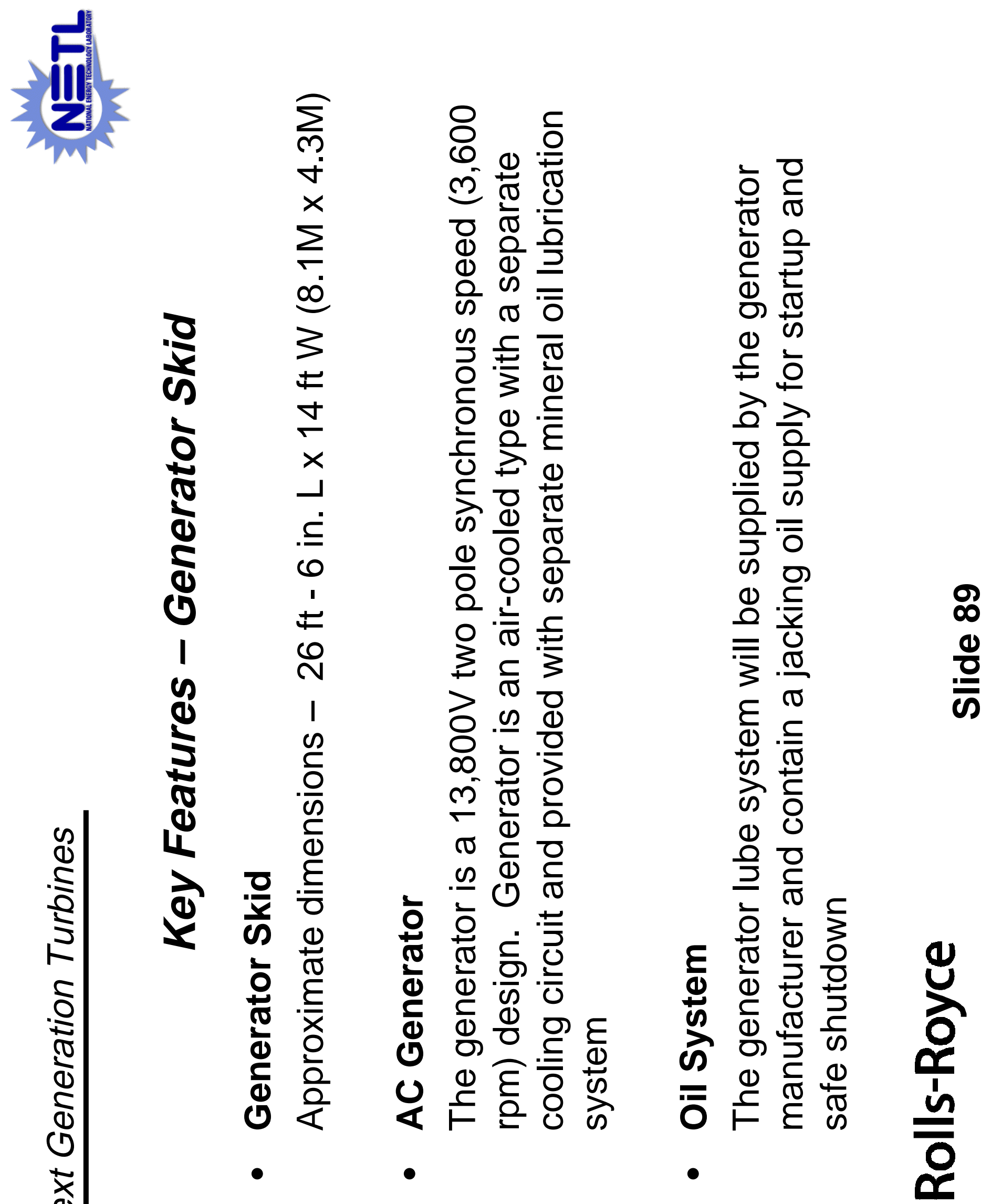


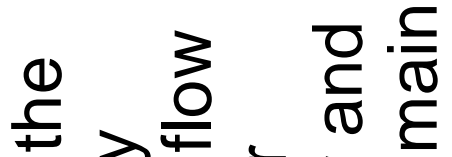

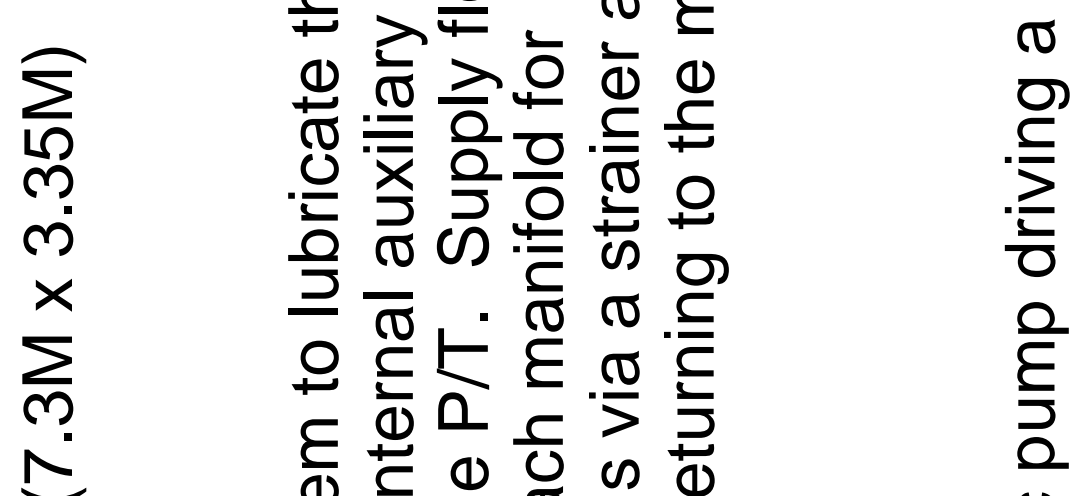

$\frac{1}{2} \sum_{m}^{m}$

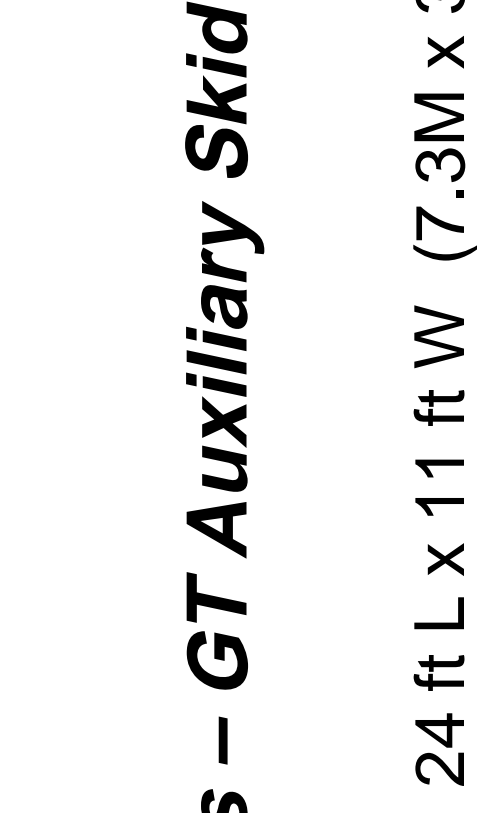

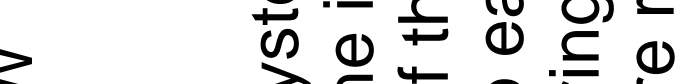

๙

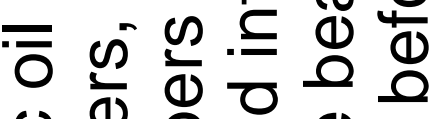

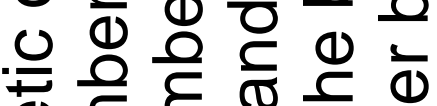

(1) ह ह

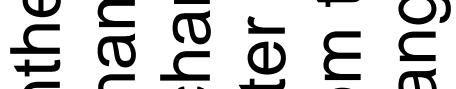

등

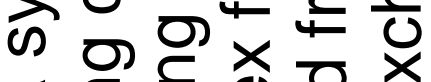

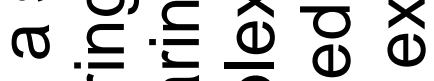

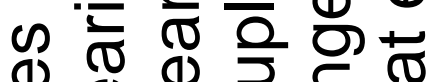

음

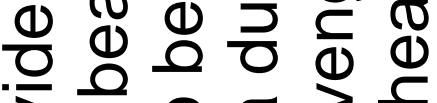

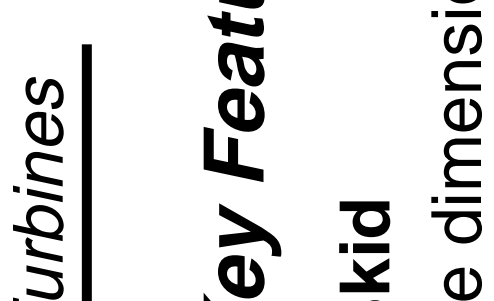

$>\frac{0}{0} 0$ r

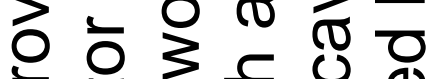

는 눙

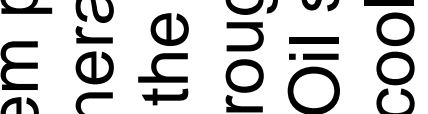

$\bar{\sigma}$

능

ᄃ $\frac{0}{1}$

는

(1) $\frac{x}{\sigma}$

ธิ

(1)

(1)

$\equiv \frac{E}{\mp}$

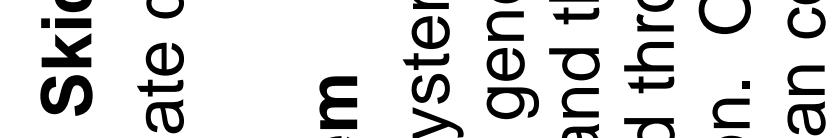

$1 \quad 10$ ब

$\sqrt{-1}$

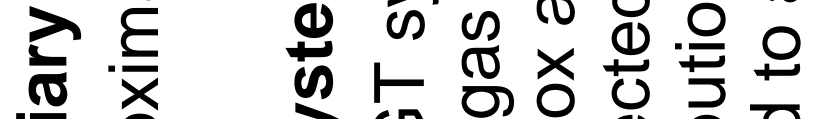

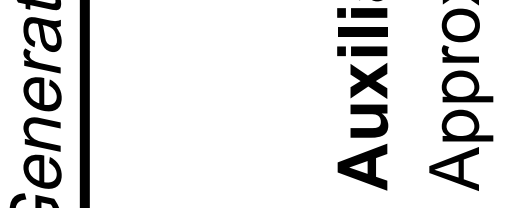

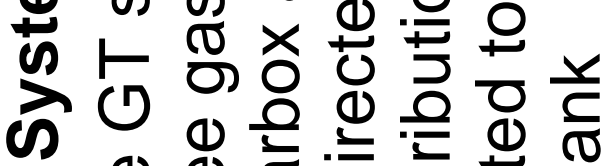

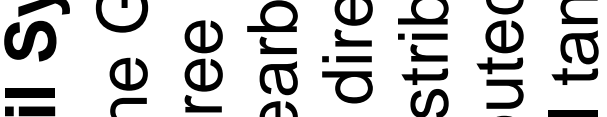

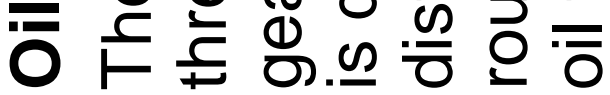

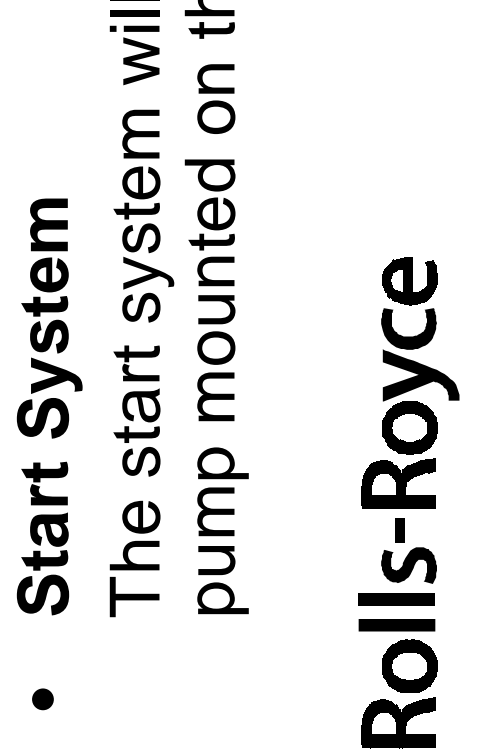



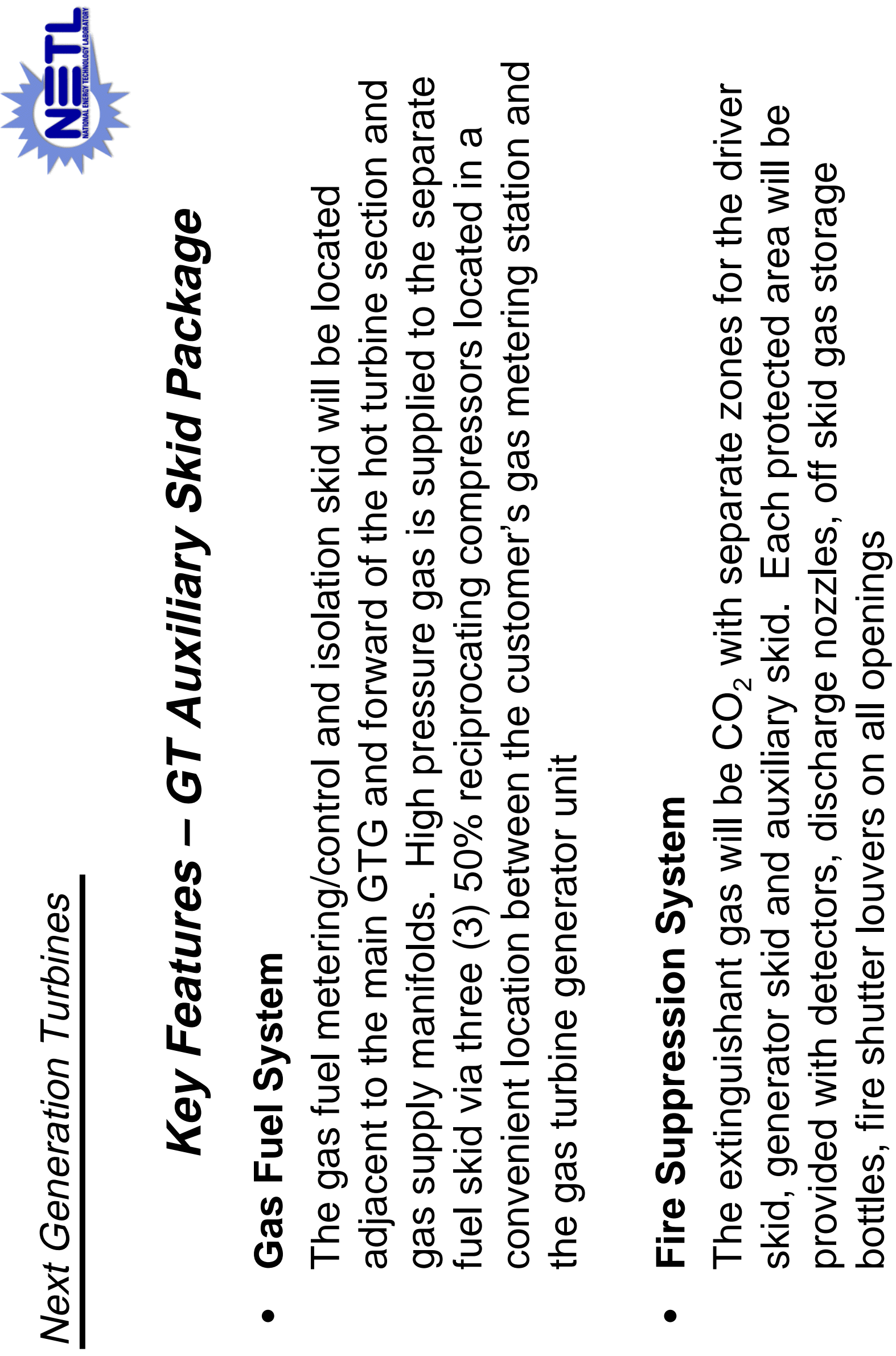

$\bar{\sigma}$

$\frac{0}{0}$

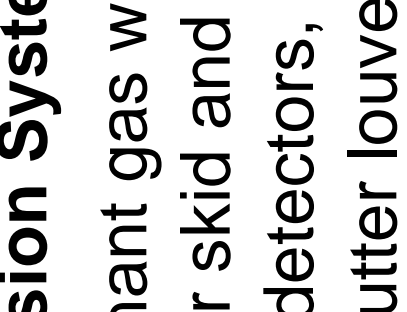

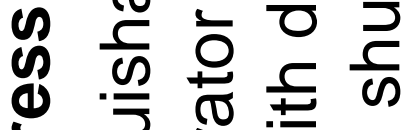

응 긍

윽 言

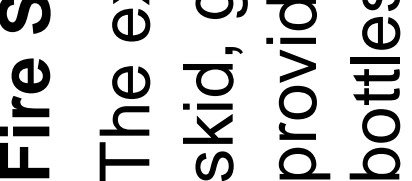

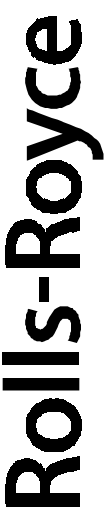




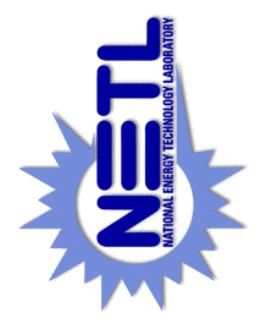

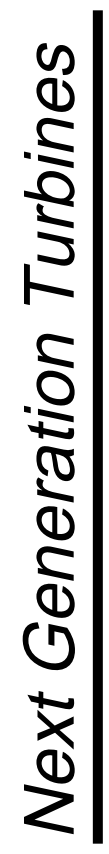

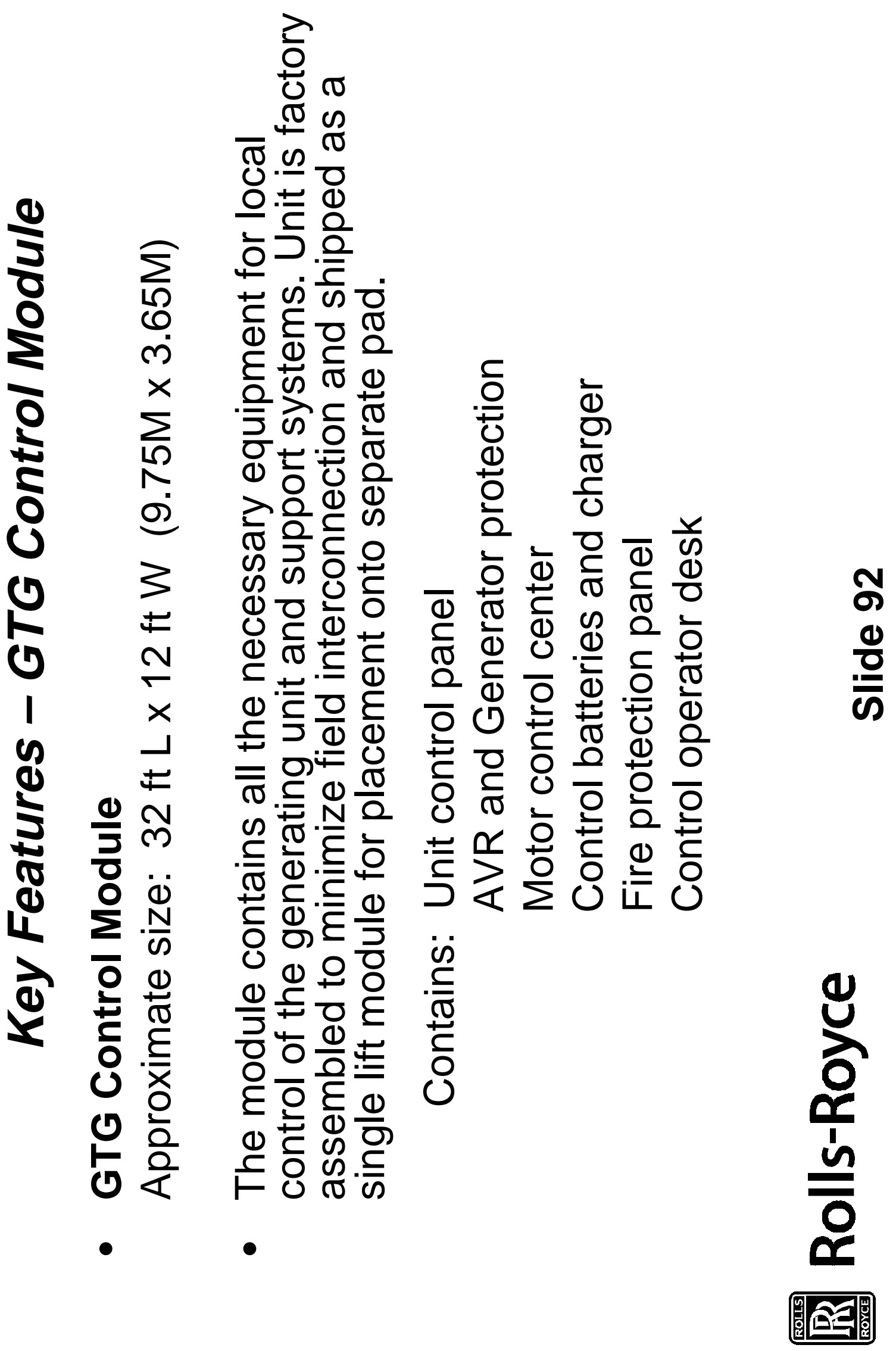



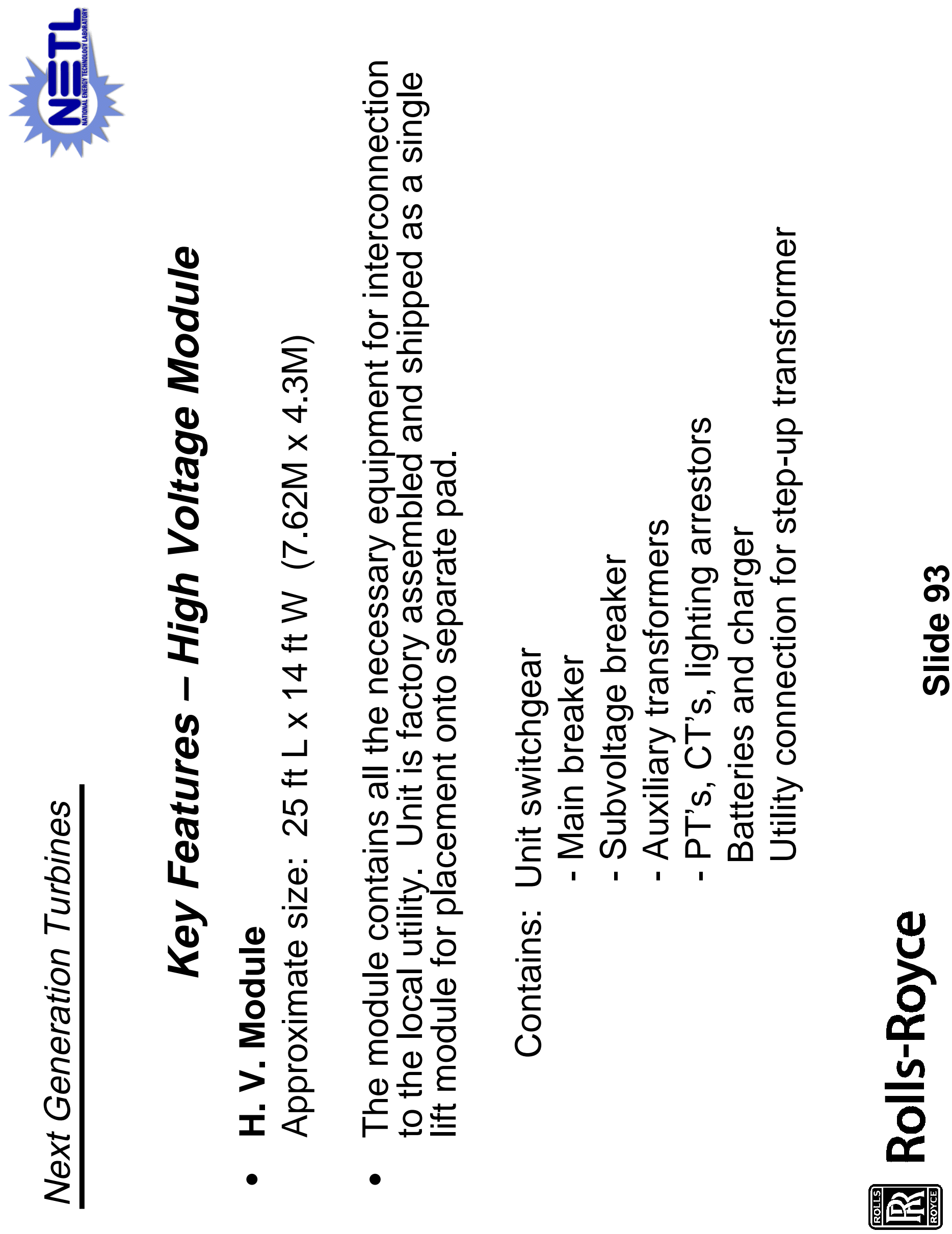

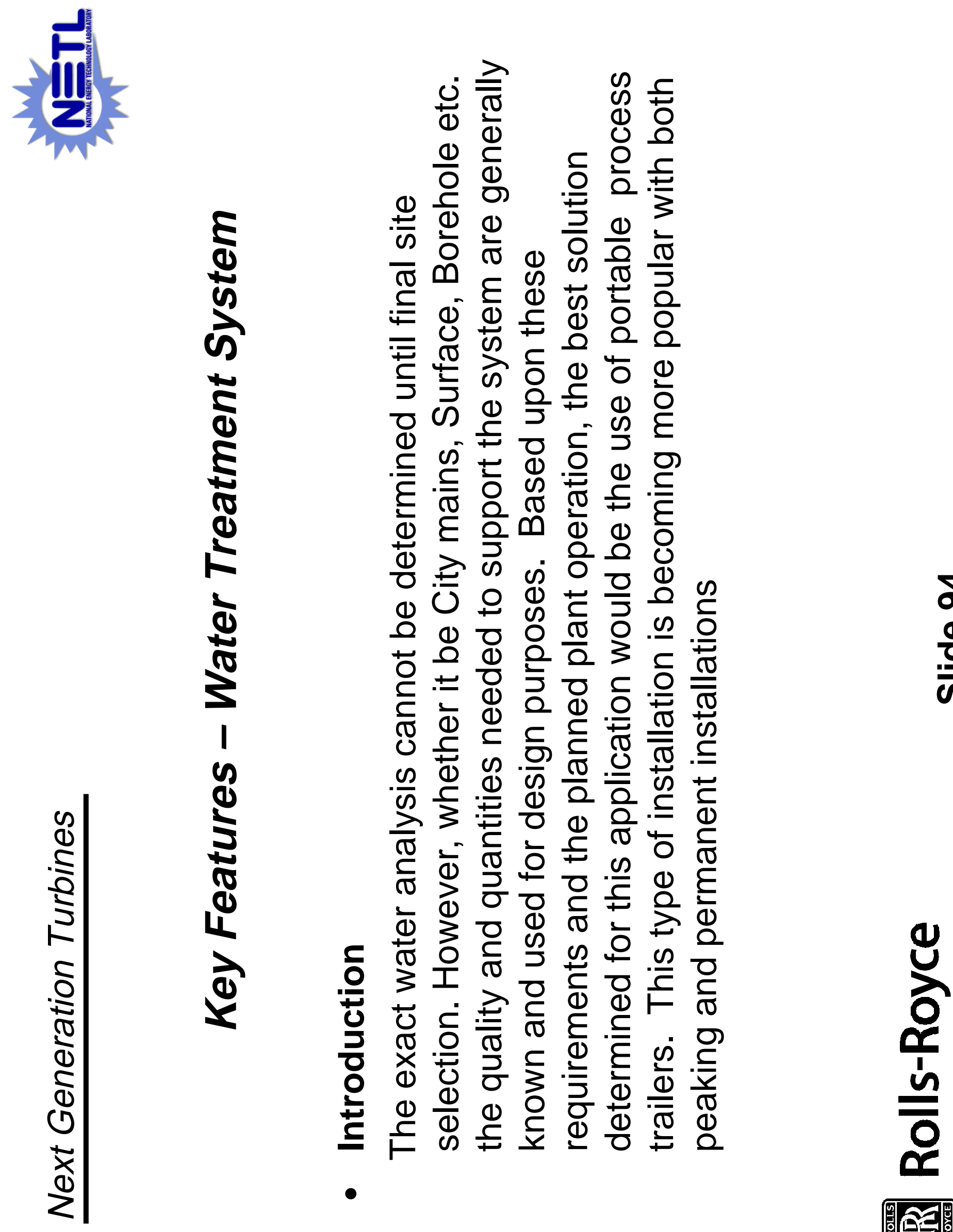


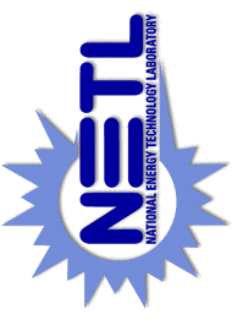

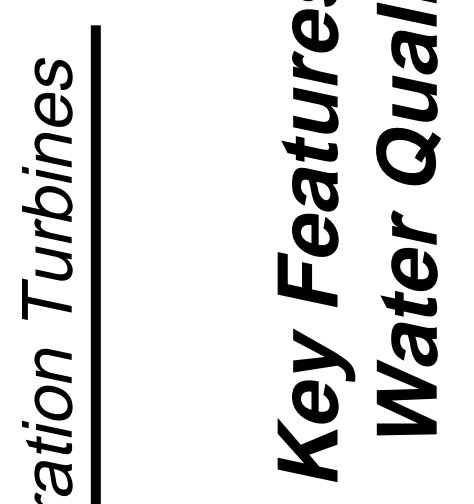
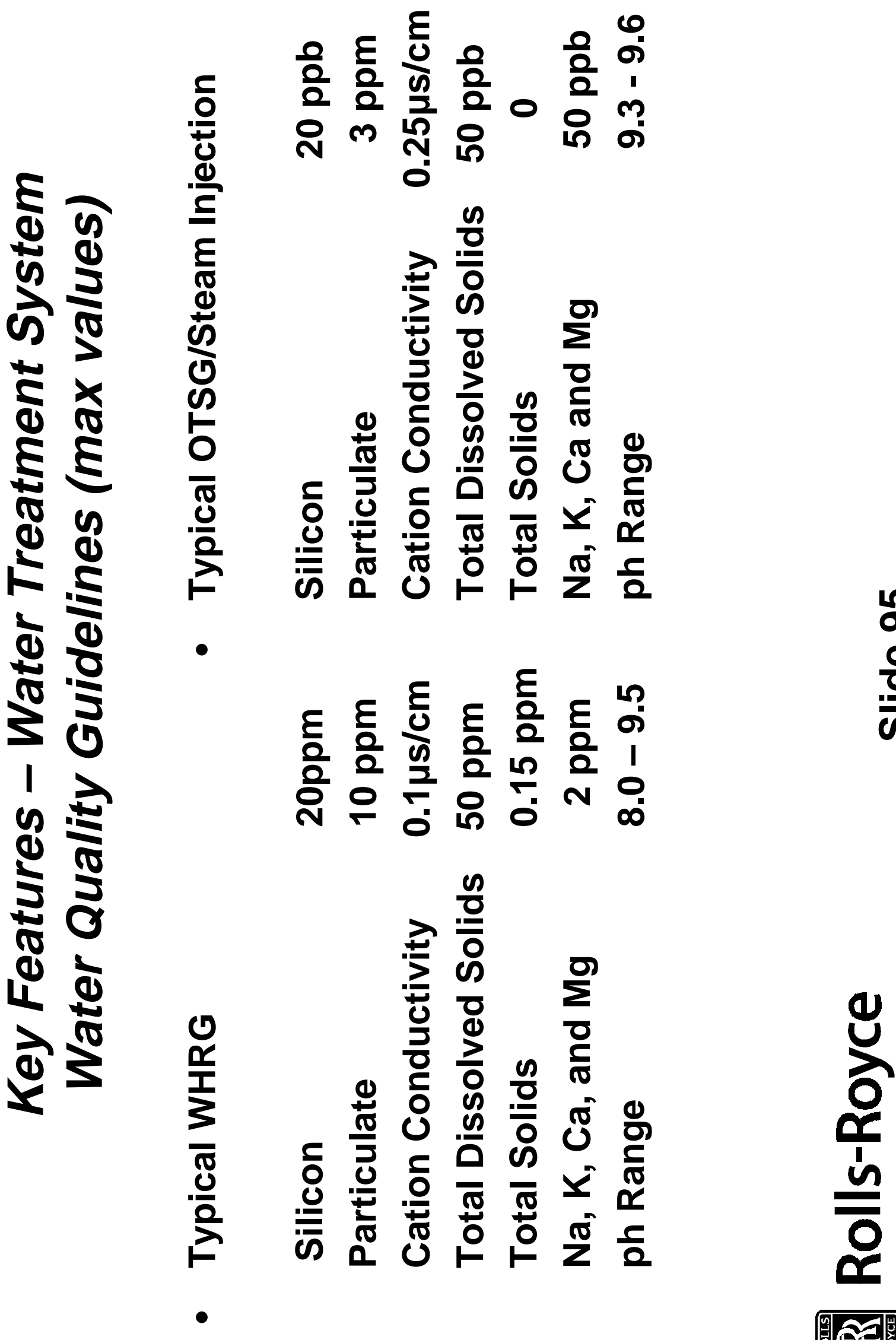

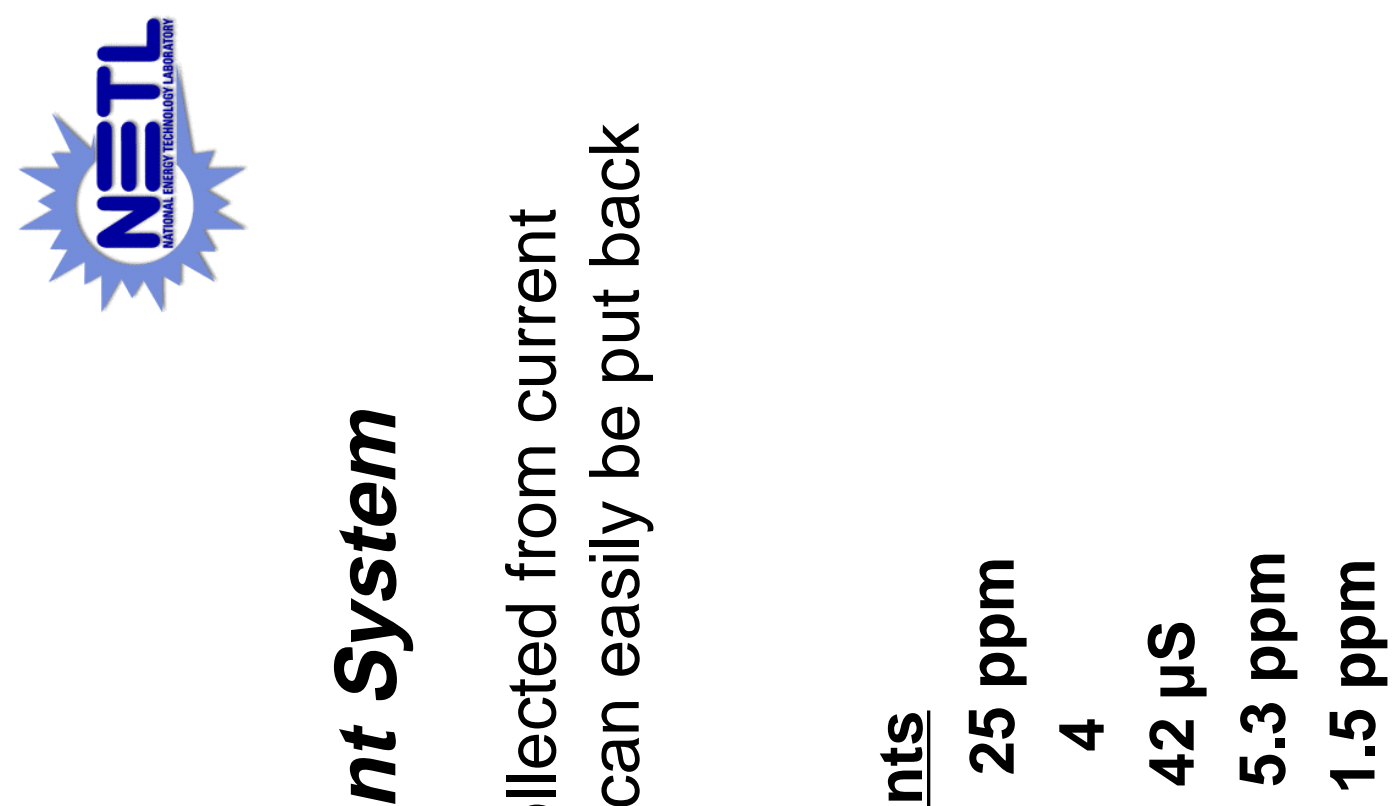

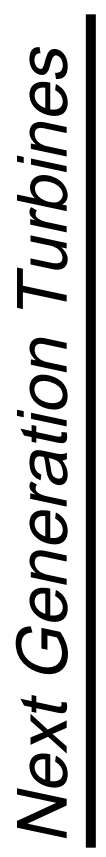

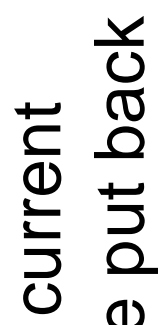

ङ

के ब

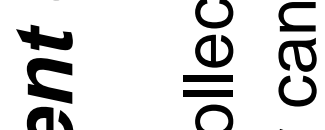

ठิ

نे

$+\quad \stackrel{D}{D}$

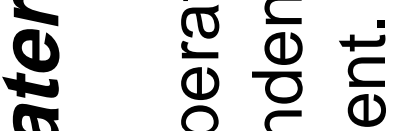

응 응

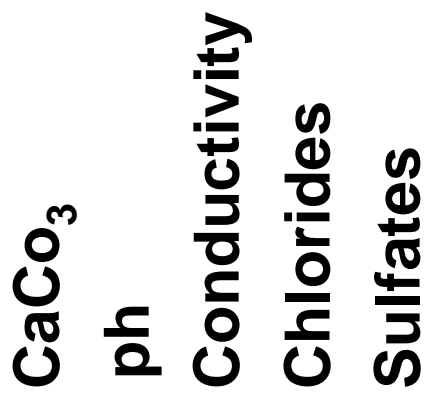

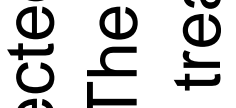

() $D F$

(I) $\cong \dot{\Xi}$

등

里焉了

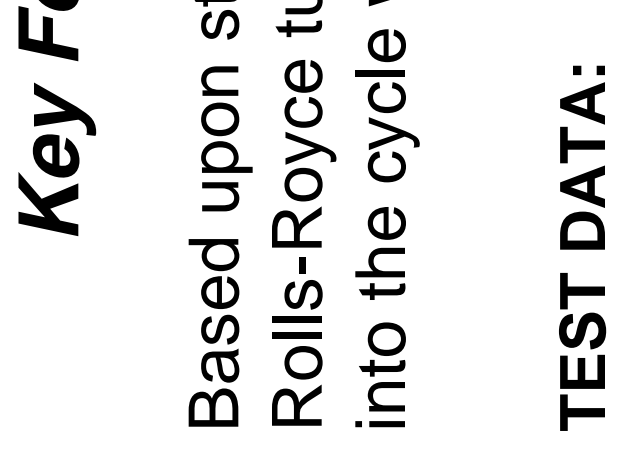

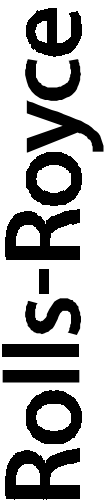

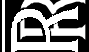




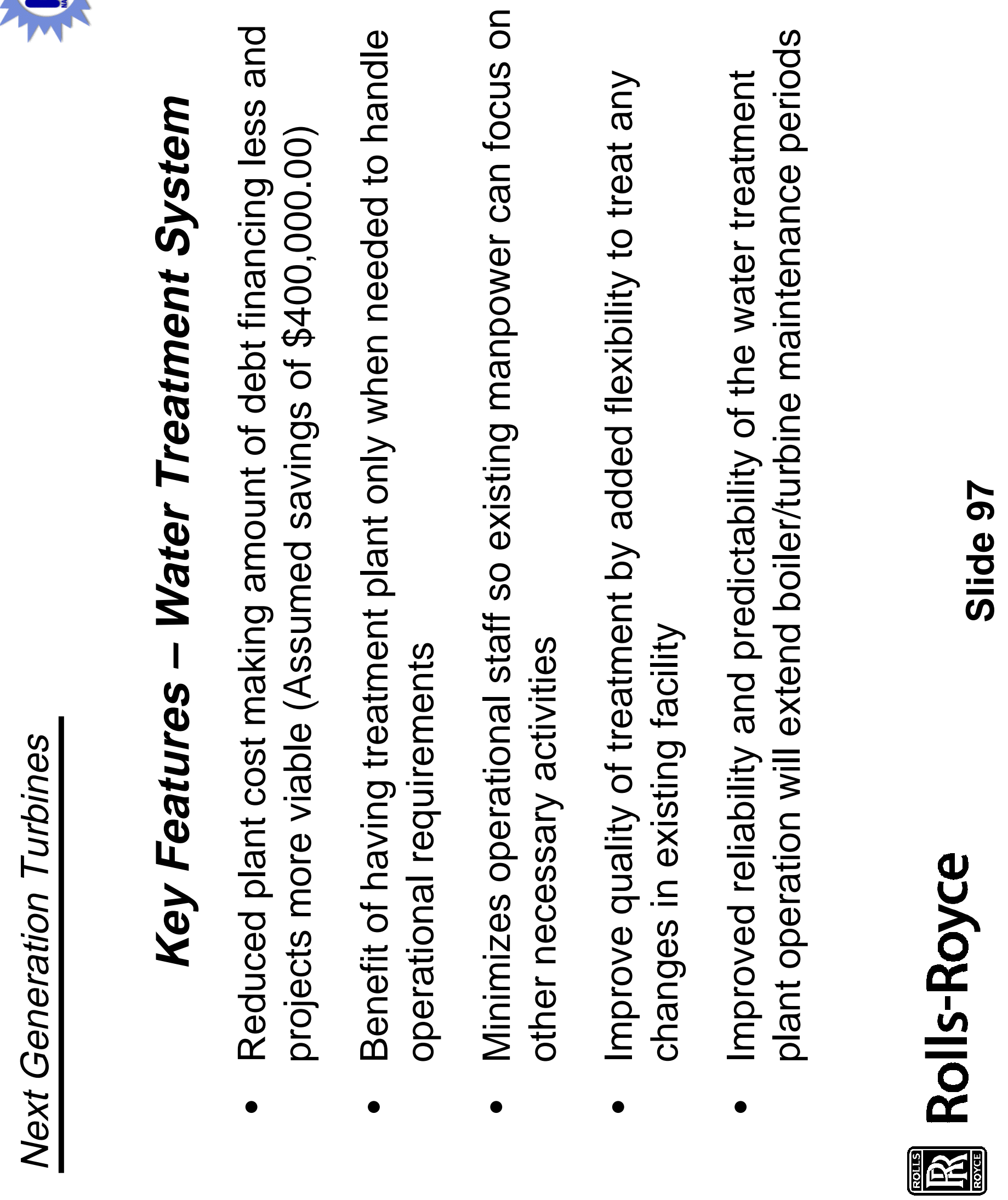



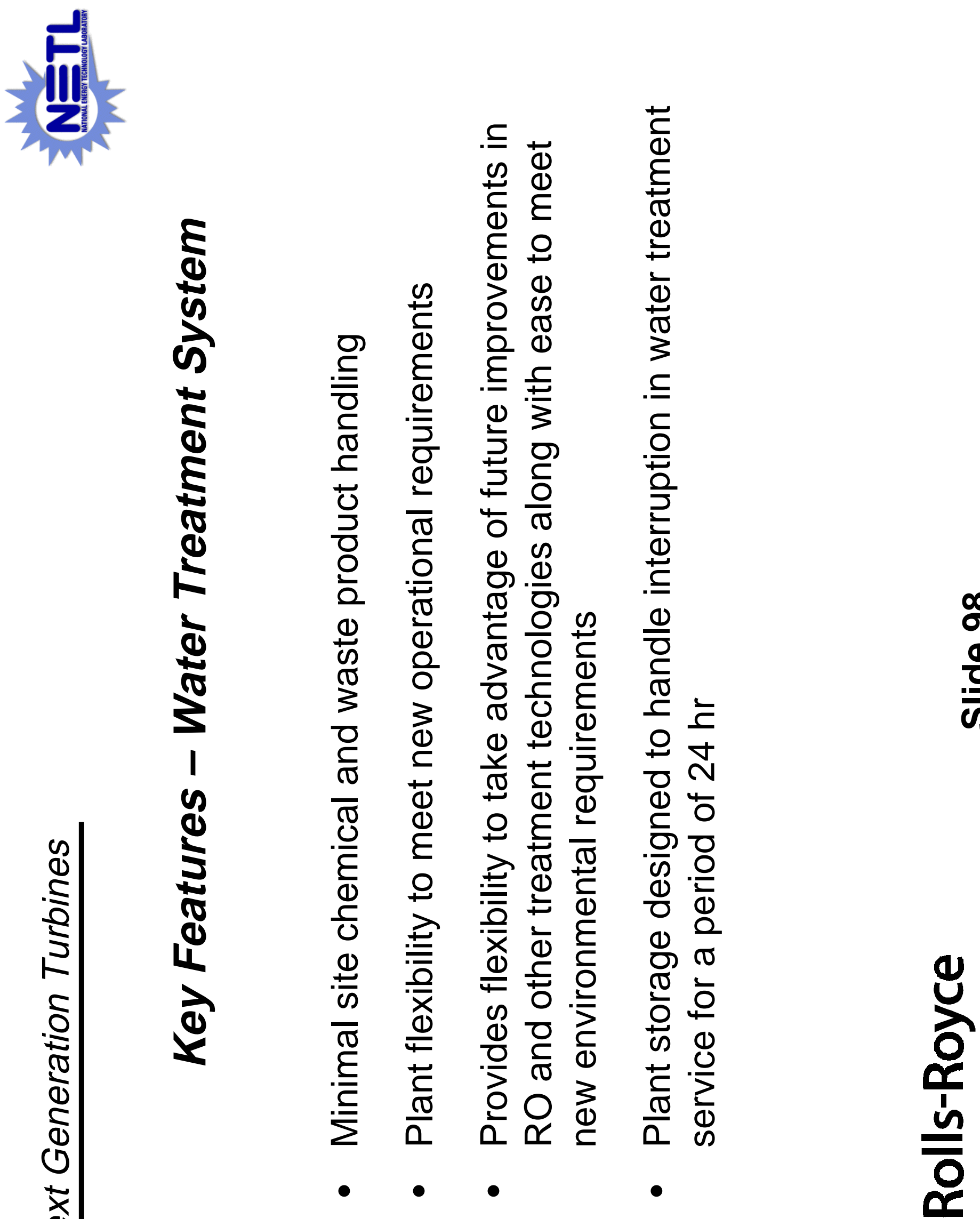
$\overline{\cos } \cos \cos c \mid 1 \cos \cos \cos \cos c$

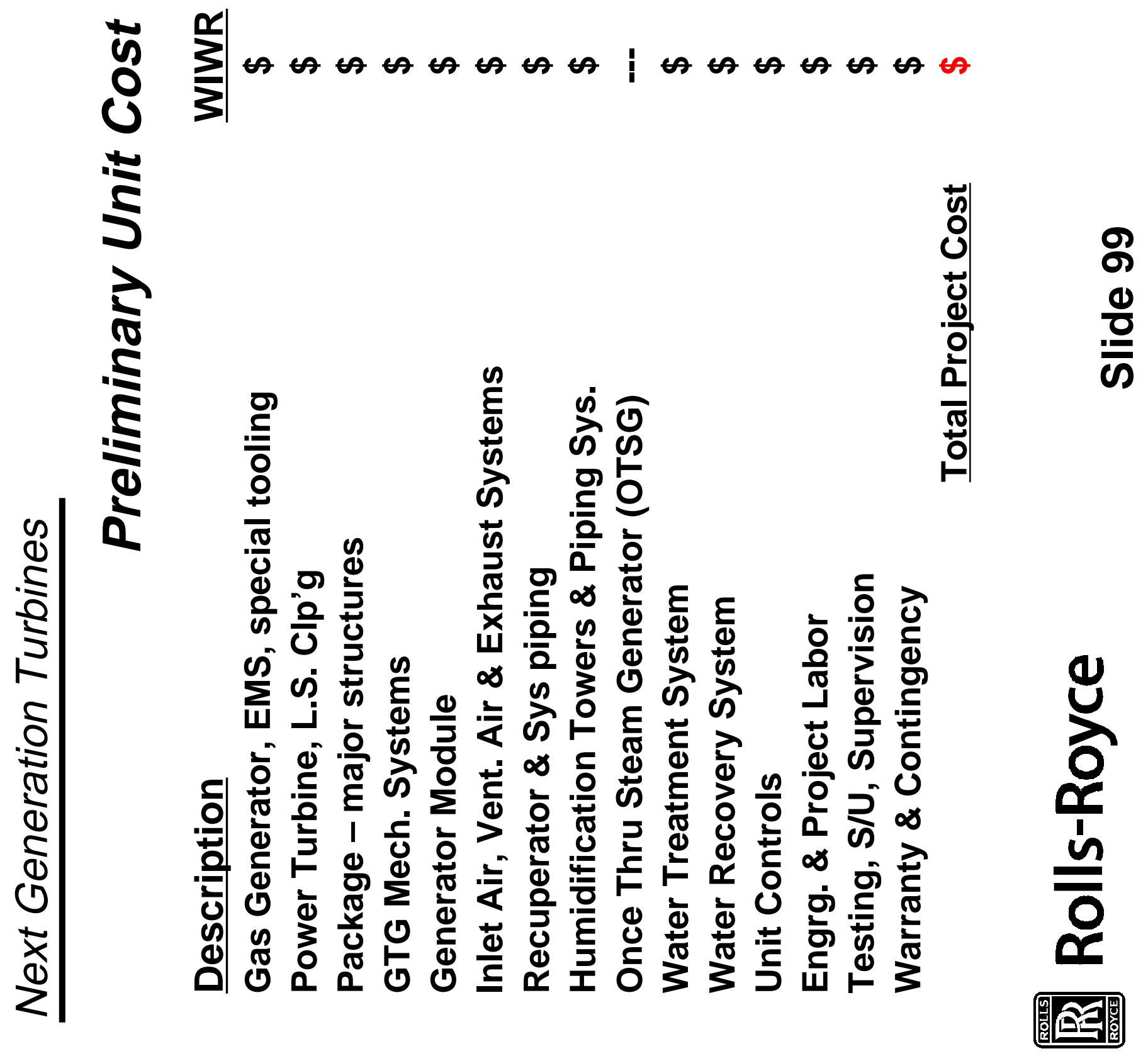




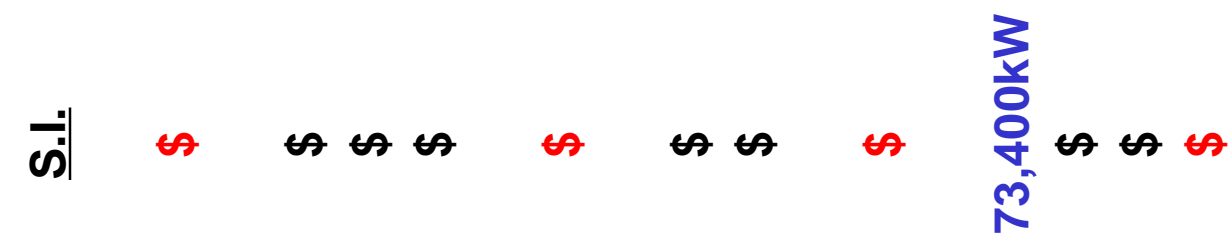

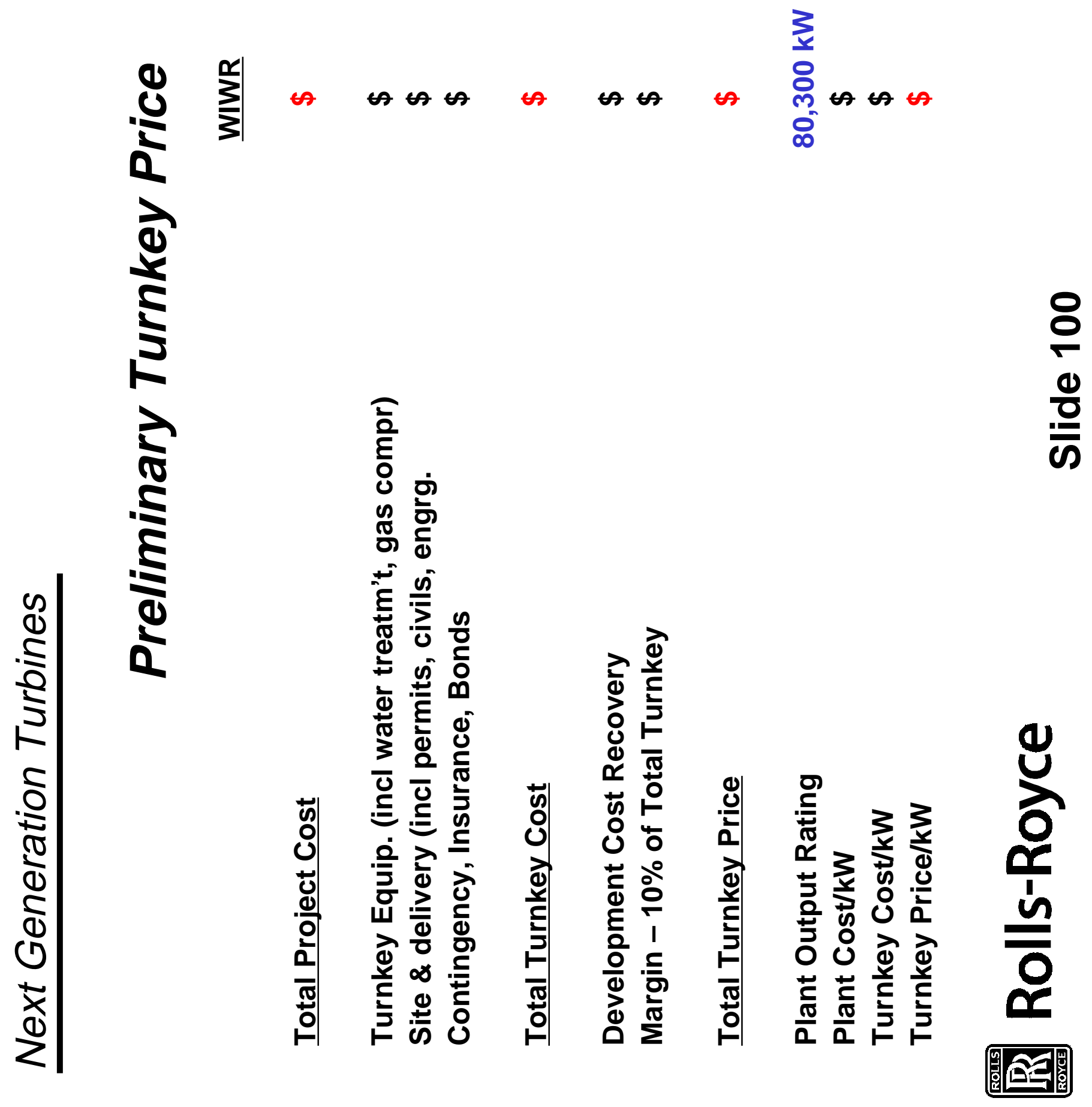



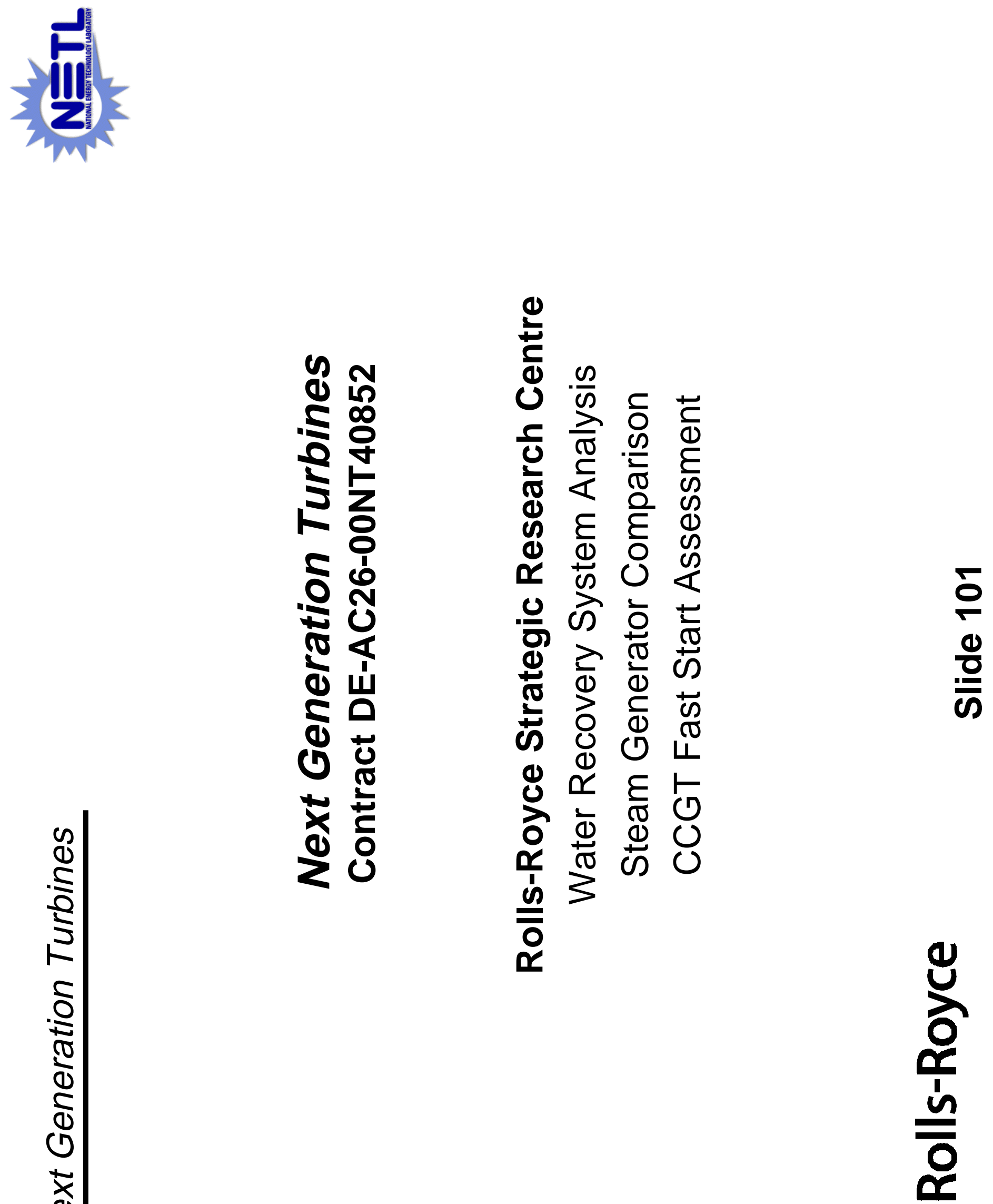

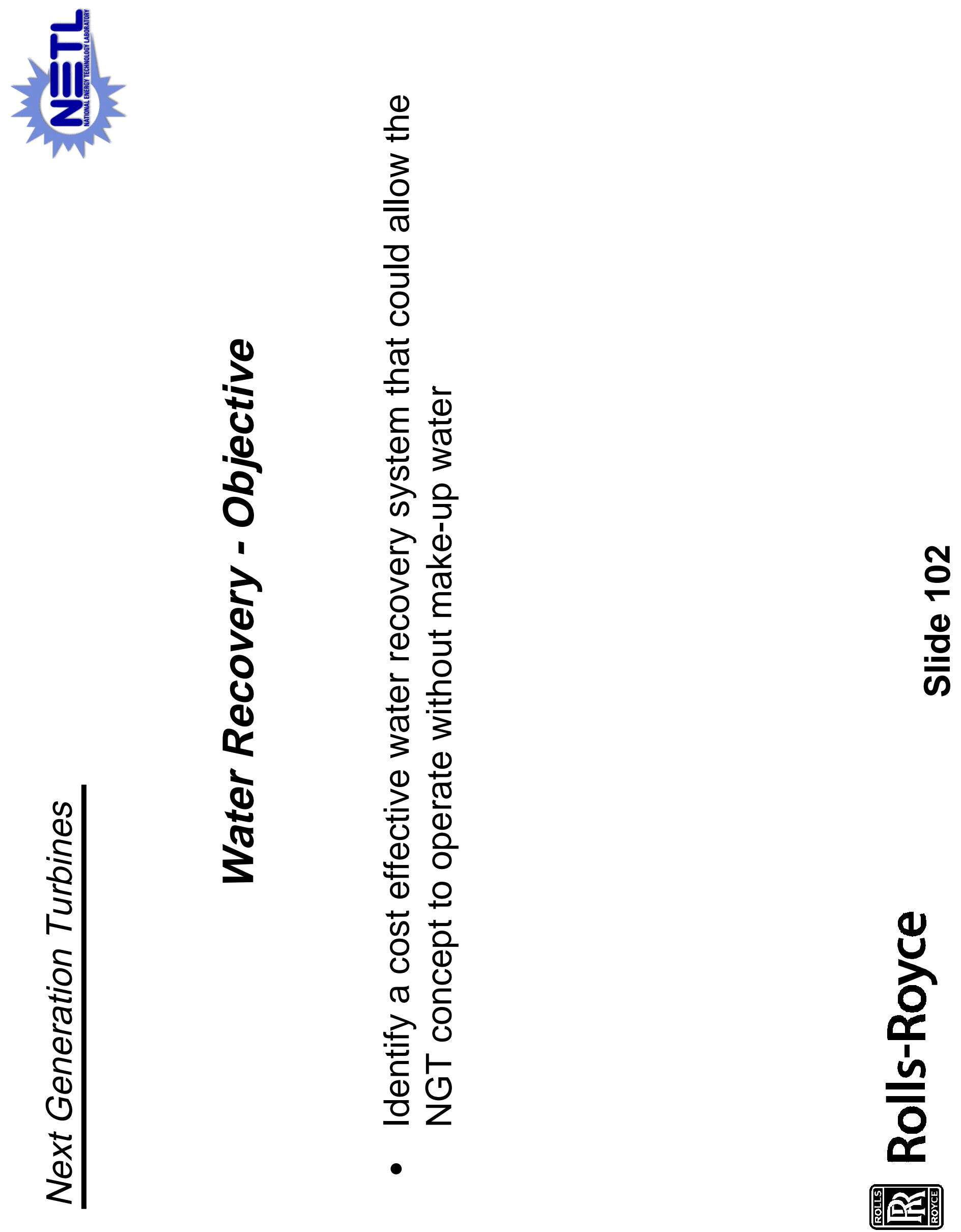


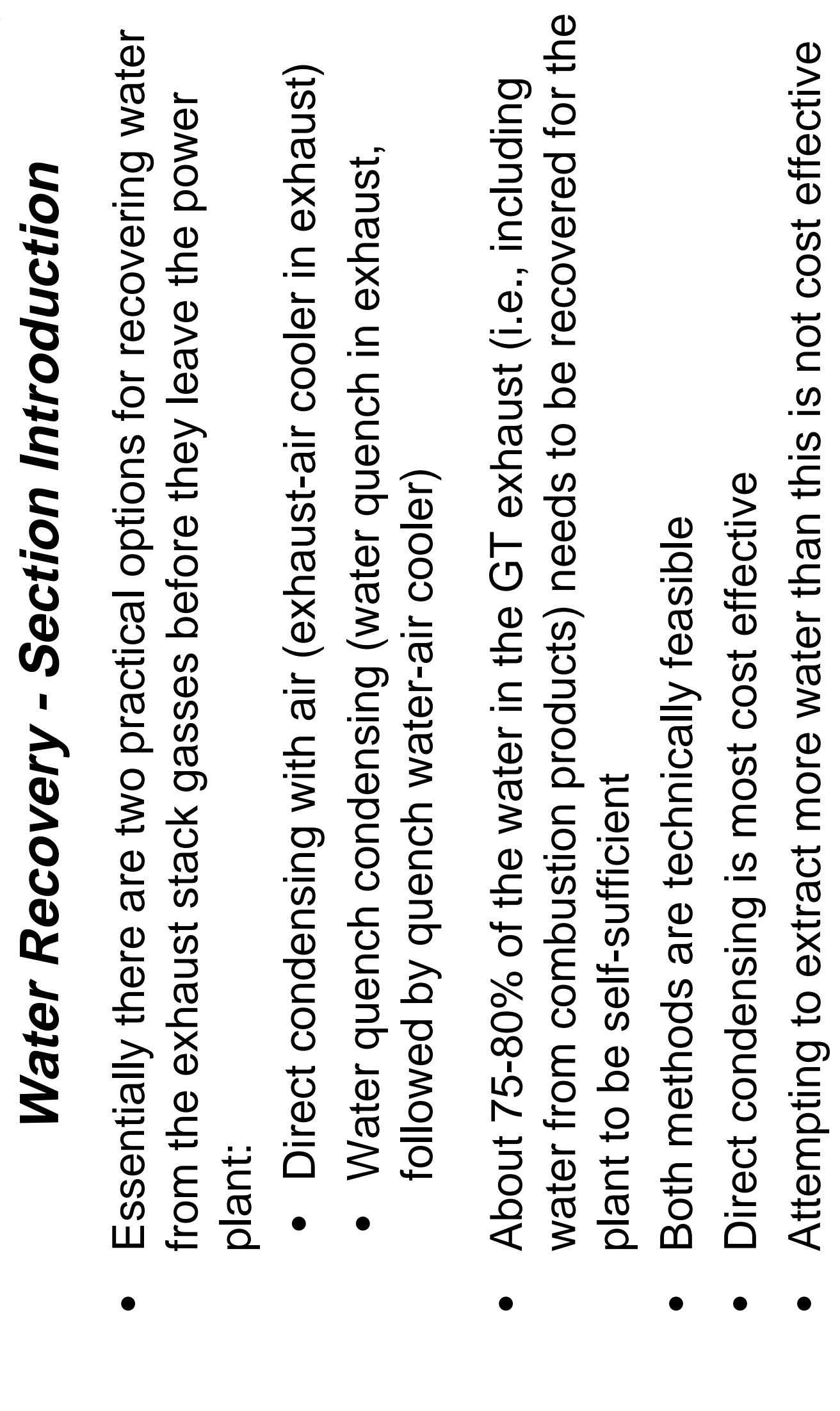



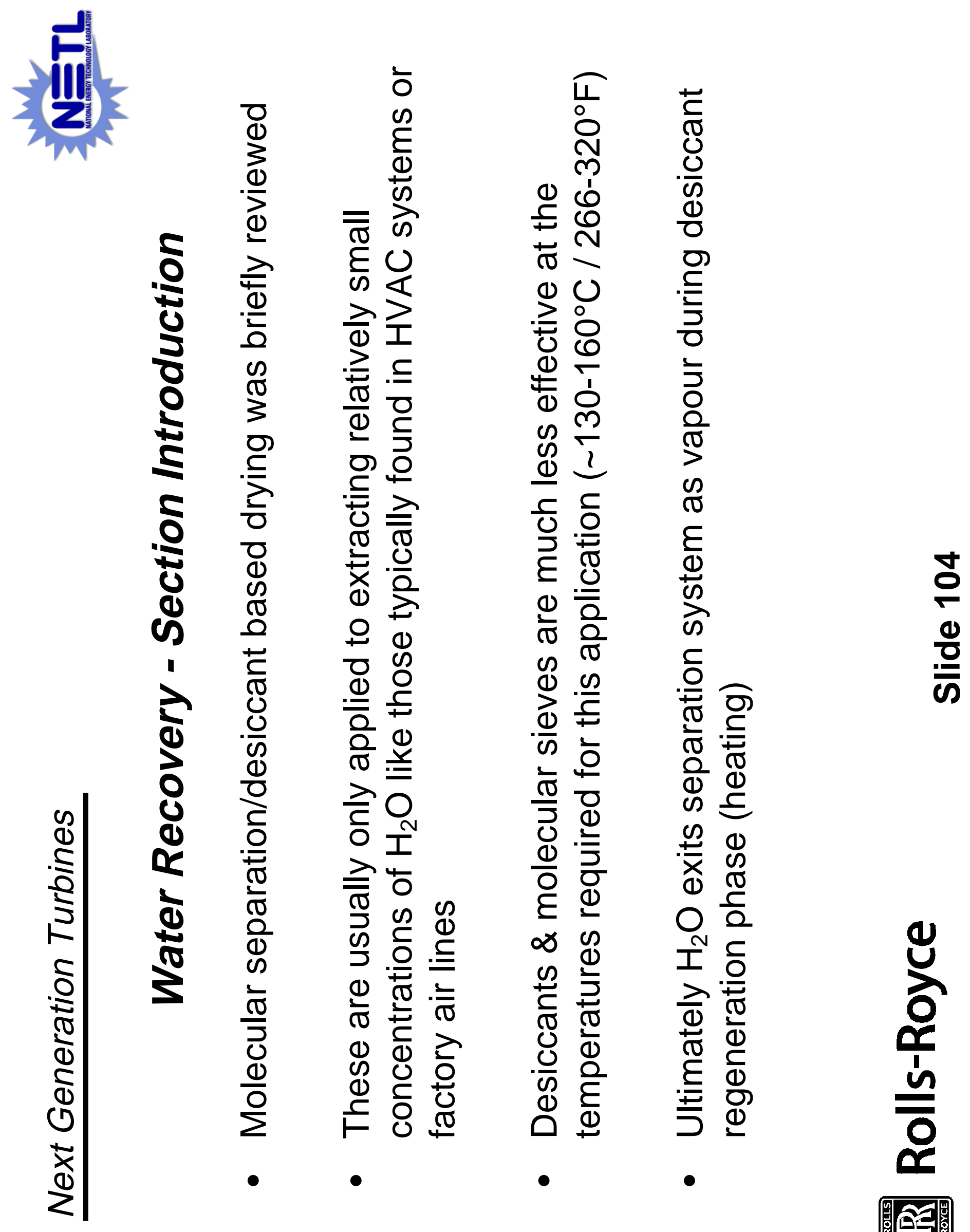

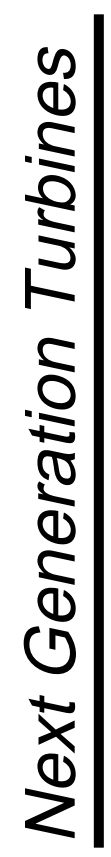




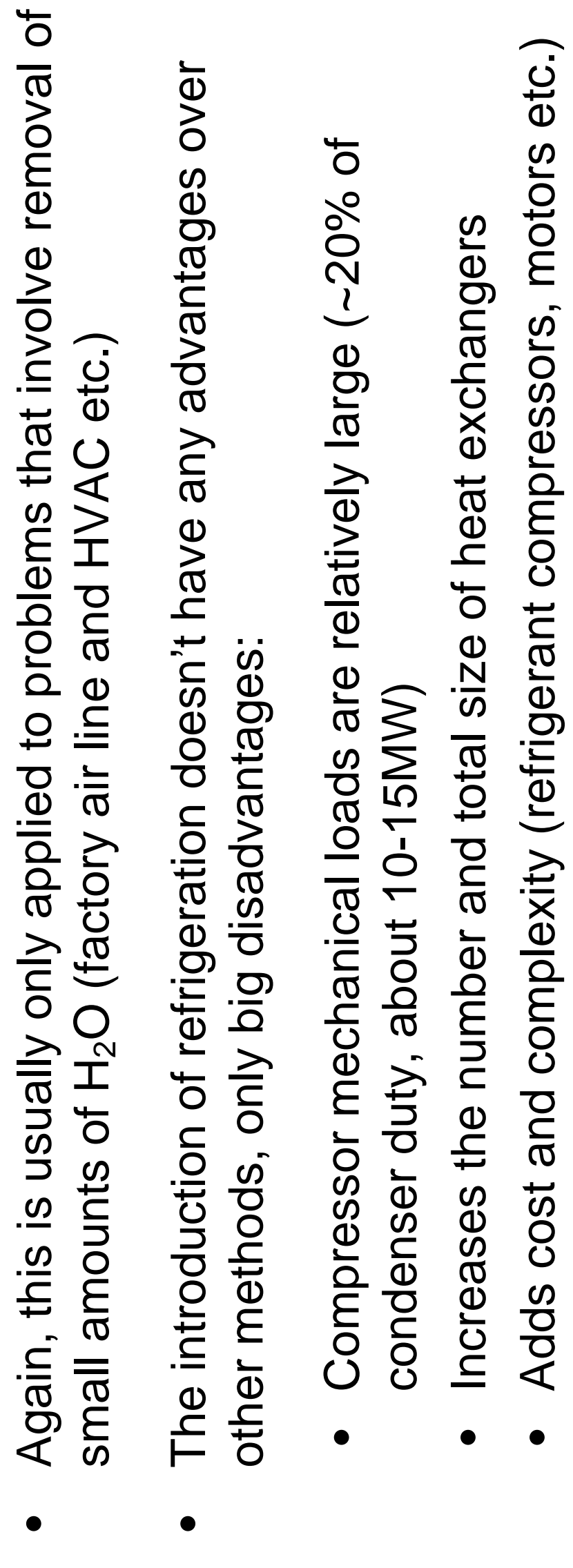

욜

$\leqslant$

$5 \quad 0$ 잉

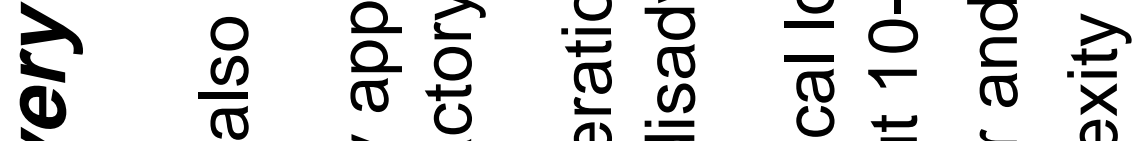
$\$$ o 

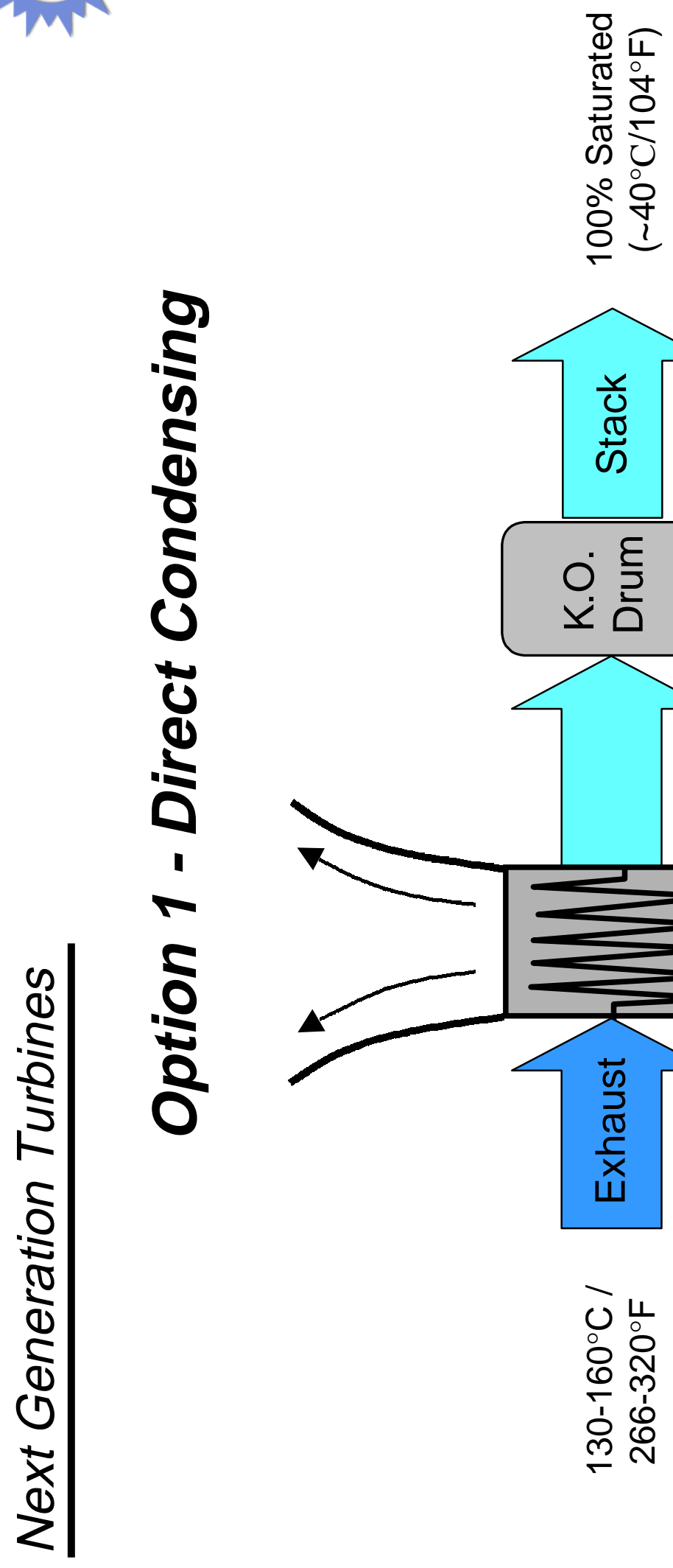

$$
\begin{array}{ll}
0 & 1 \\
0 & 0 \\
0 & N \\
0 & m \\
1 & 0 \\
0 & 0 \\
\text { m }
\end{array}
$$

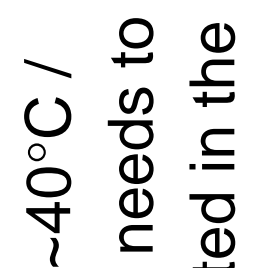

웡

क

\& 0

고

응 त

露

Ð

迹

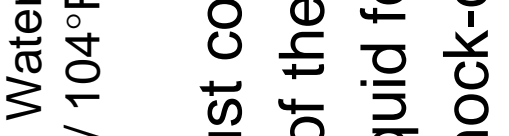

일을

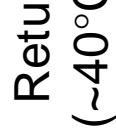

느드

0

$\frac{0}{\circ}$

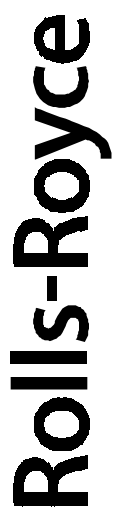




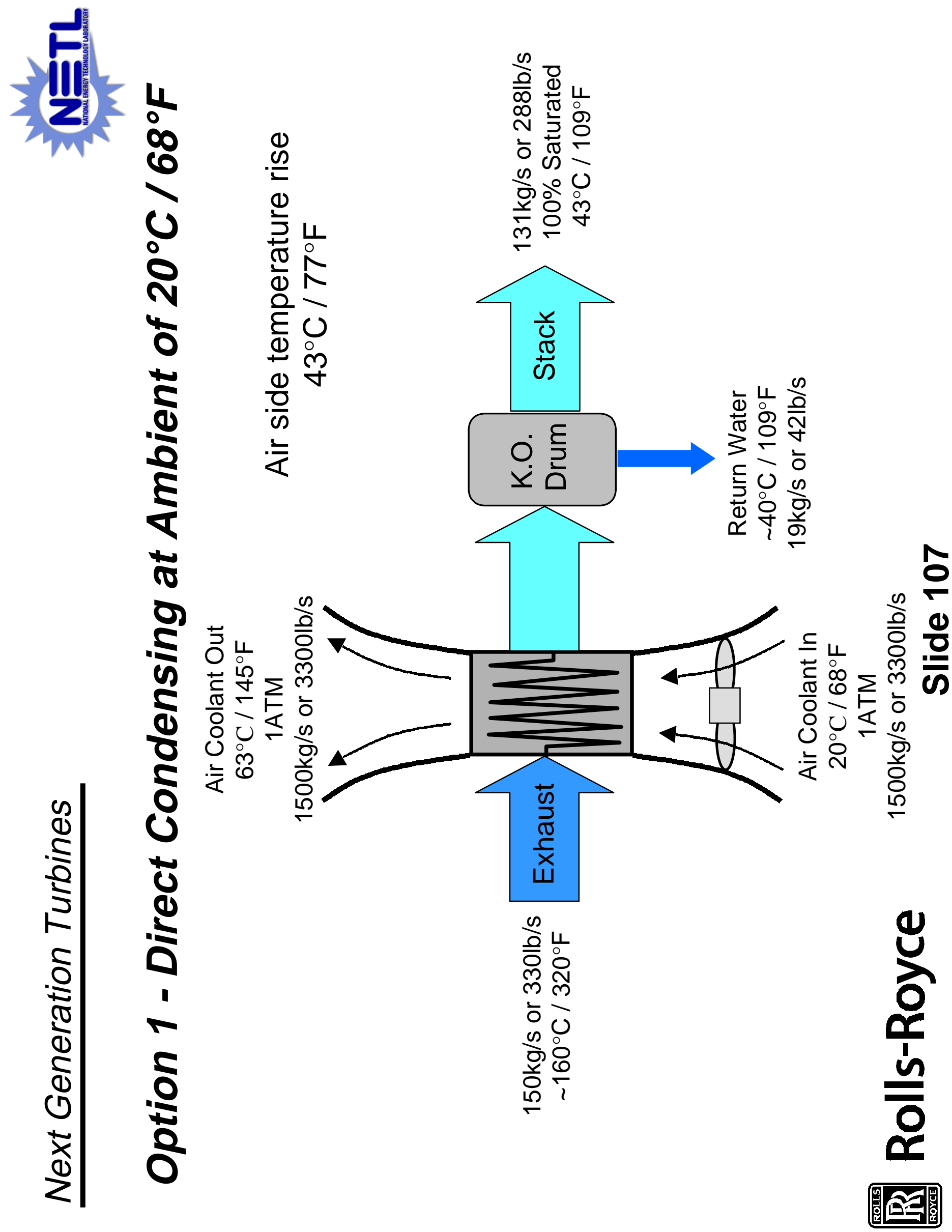




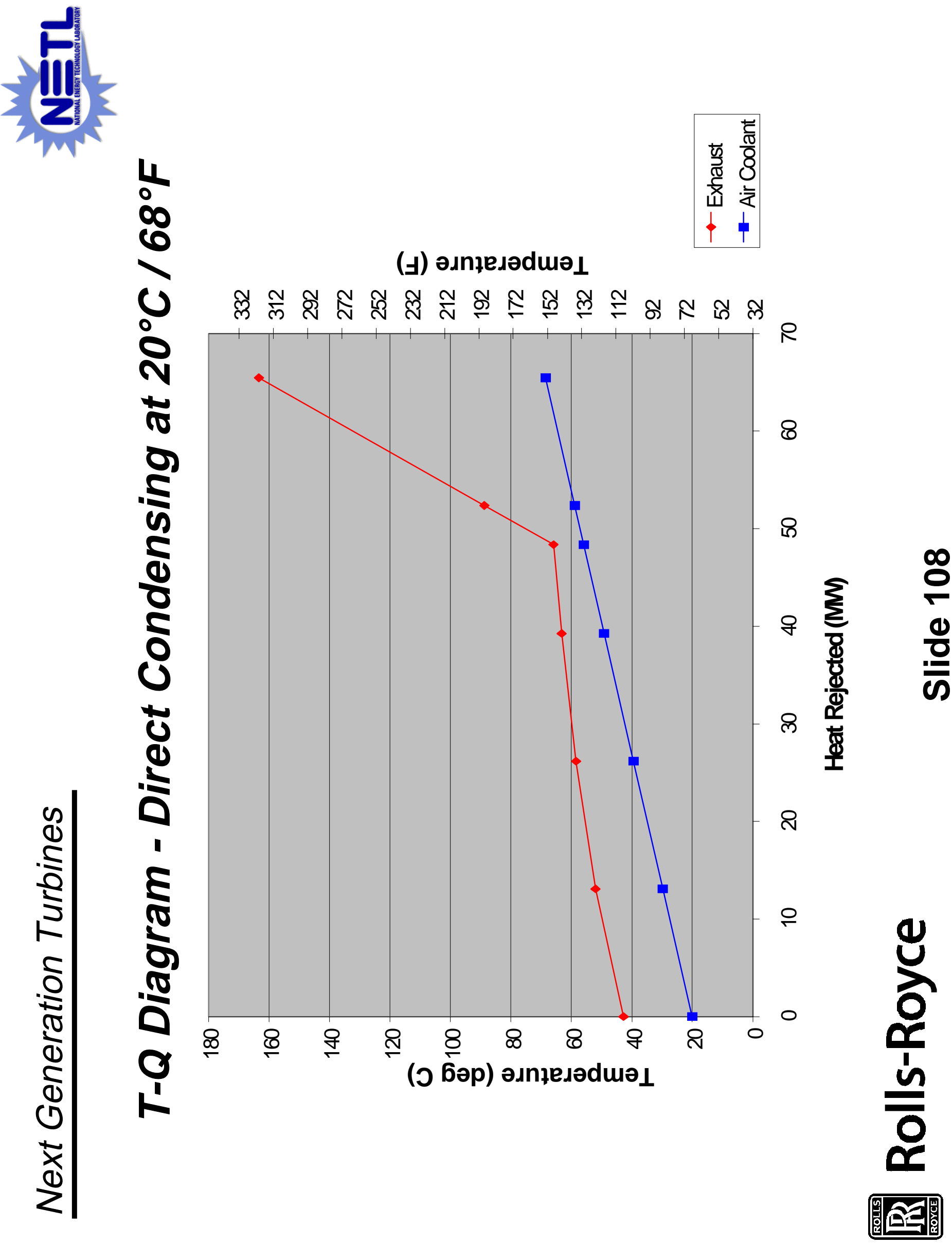




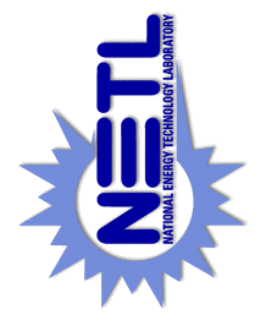

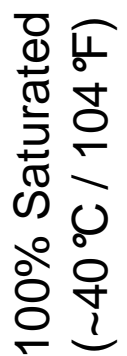
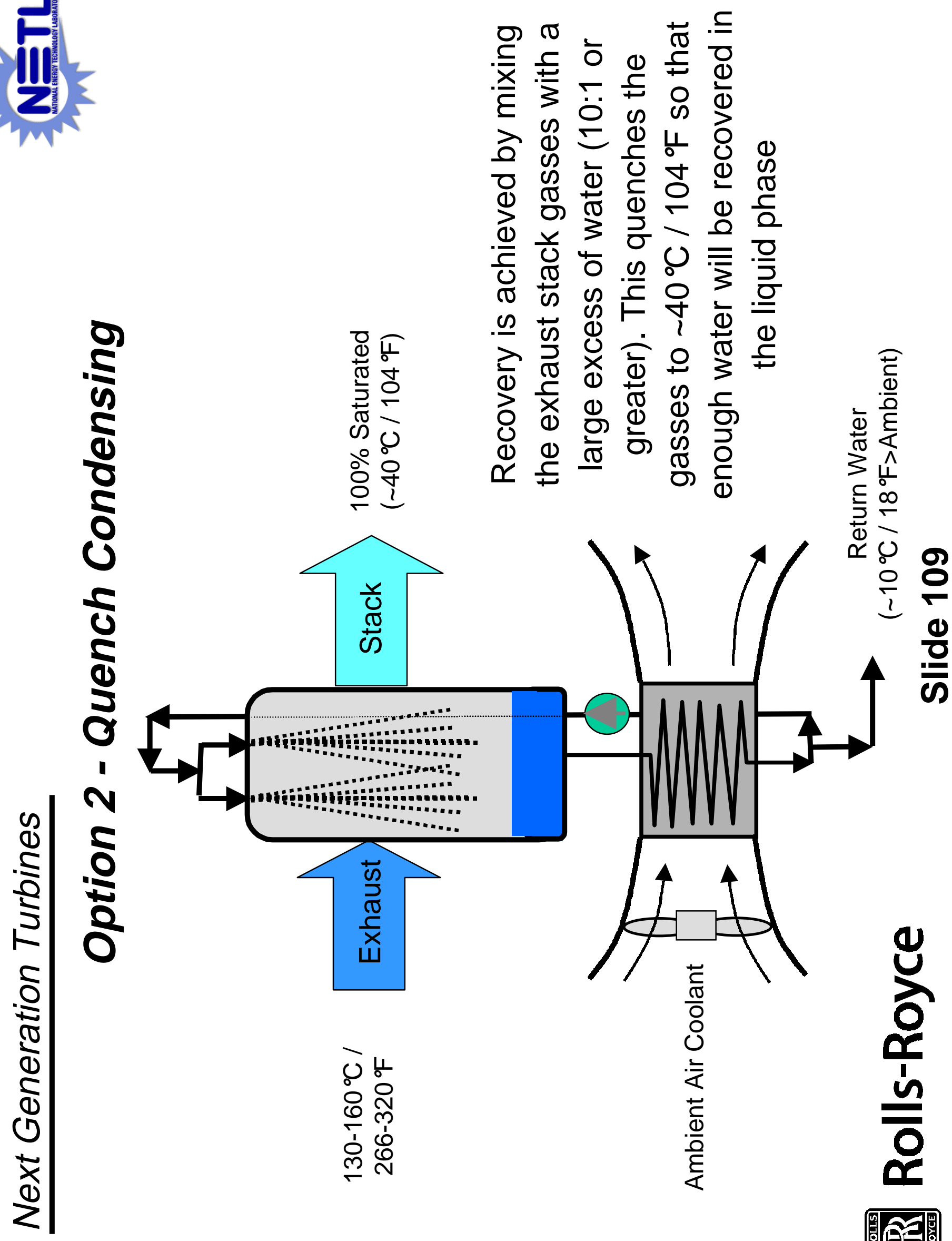


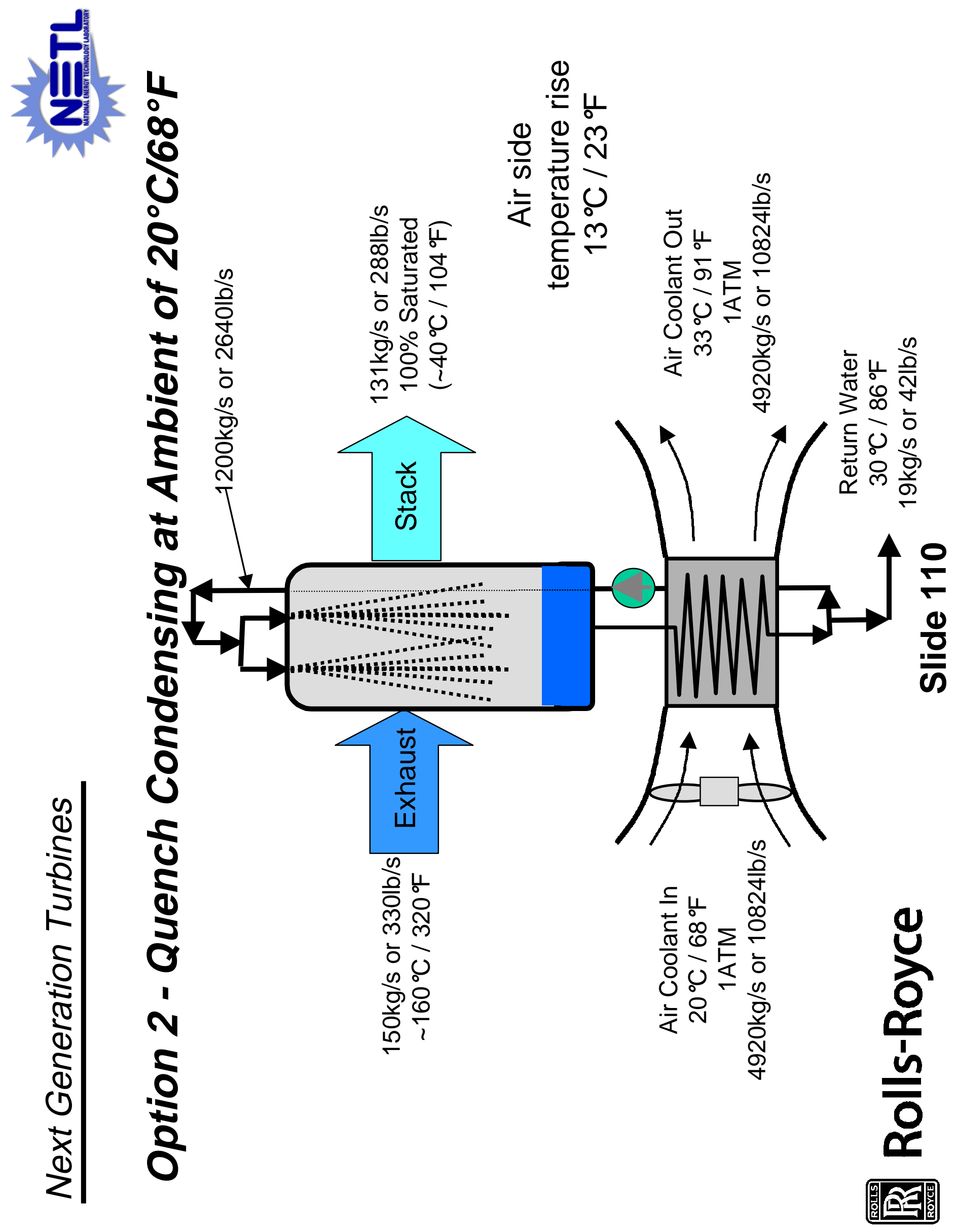




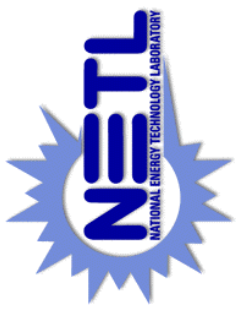

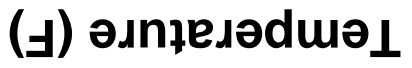
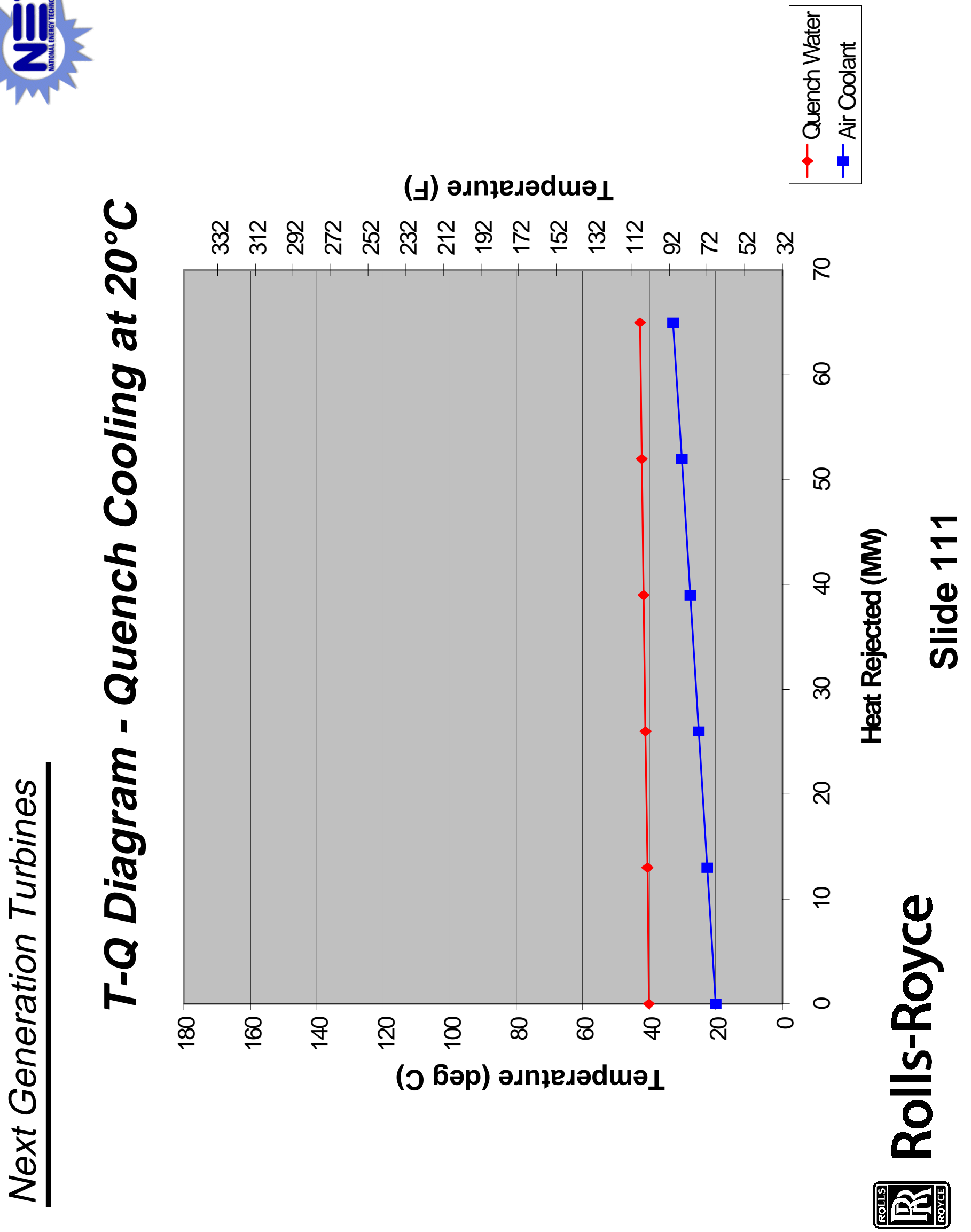

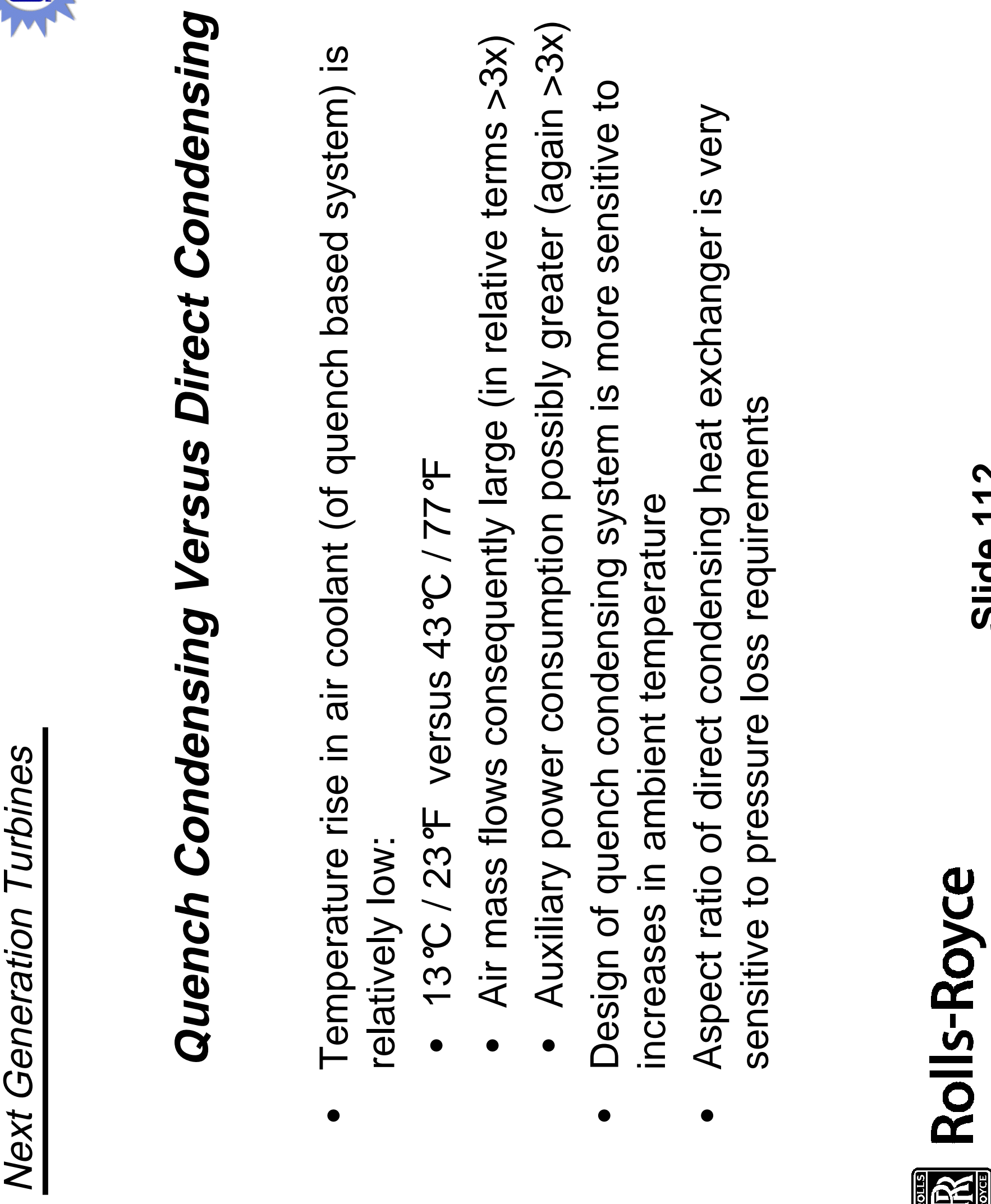

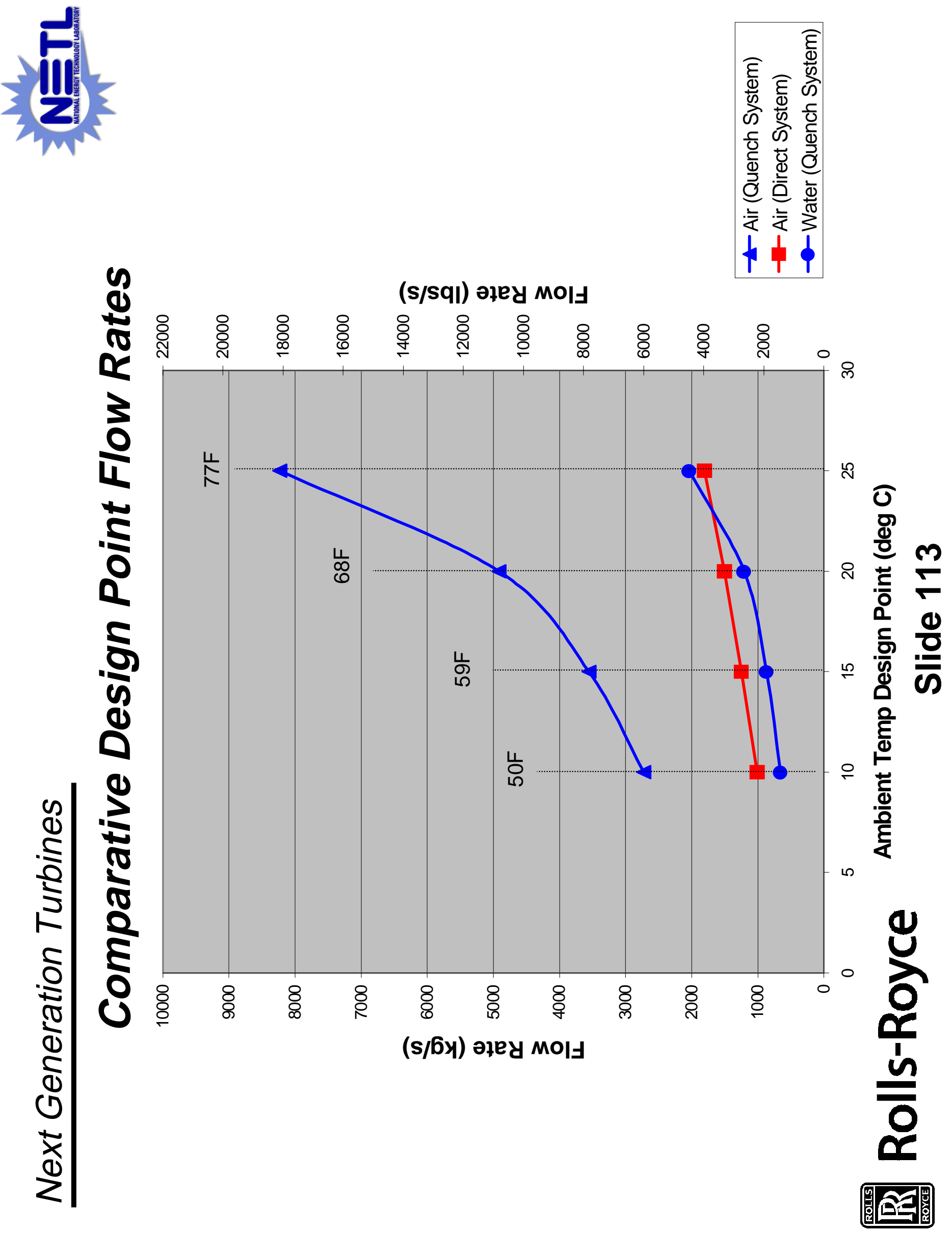


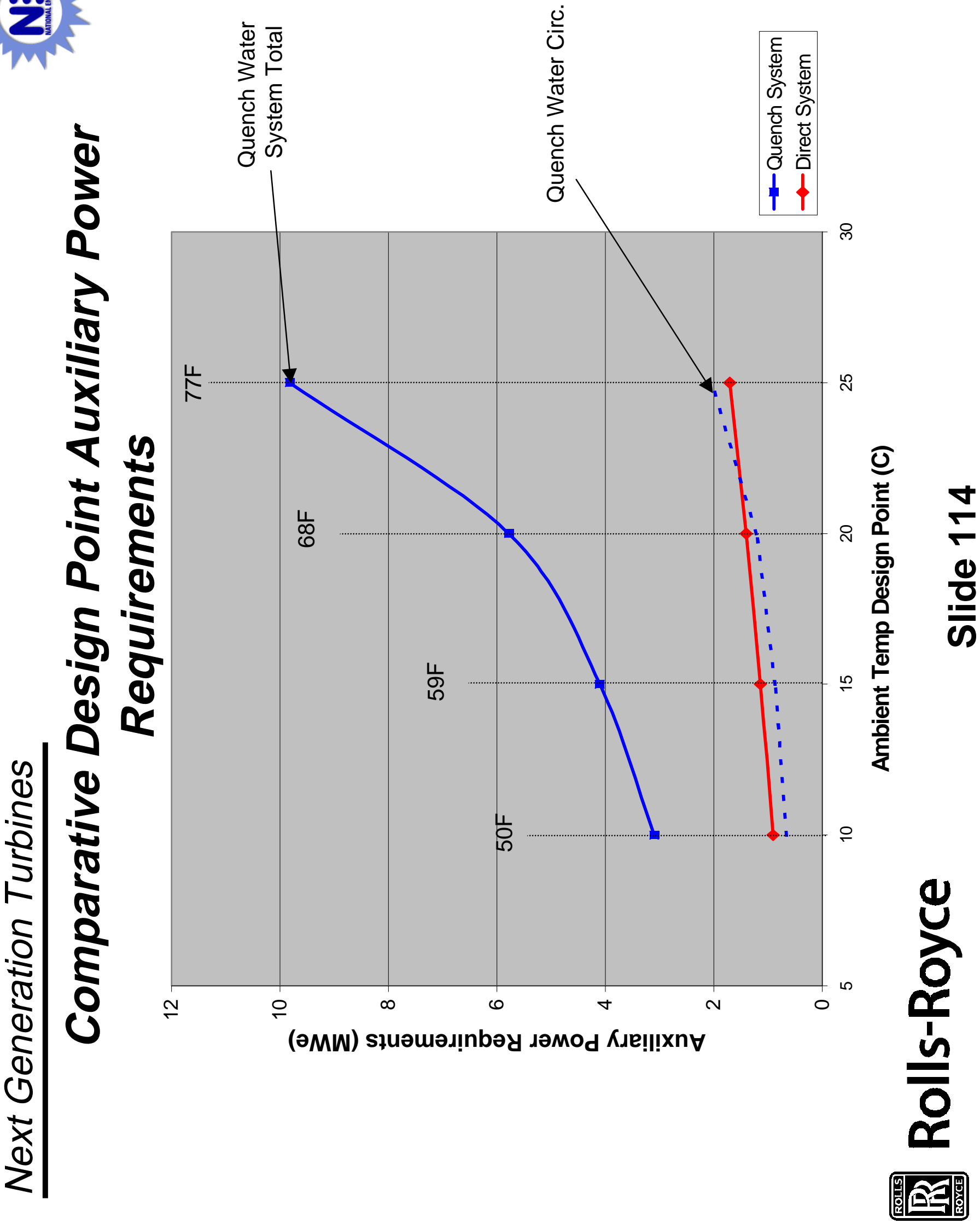



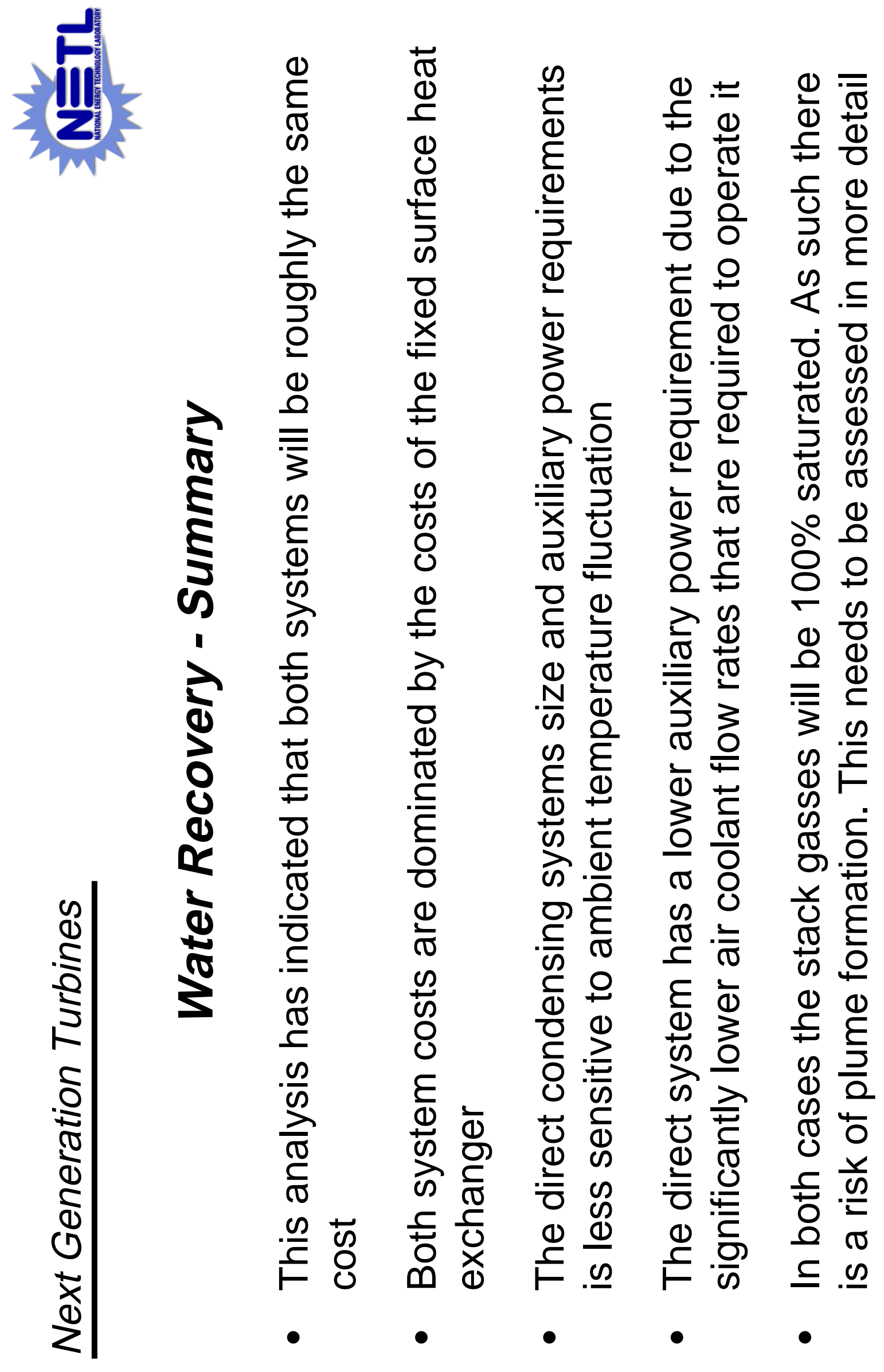

$\frac{10}{\frac{1}{0}}$

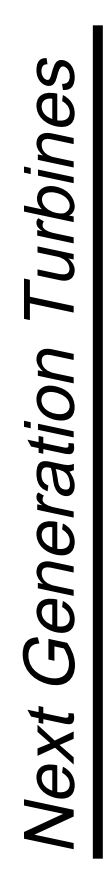




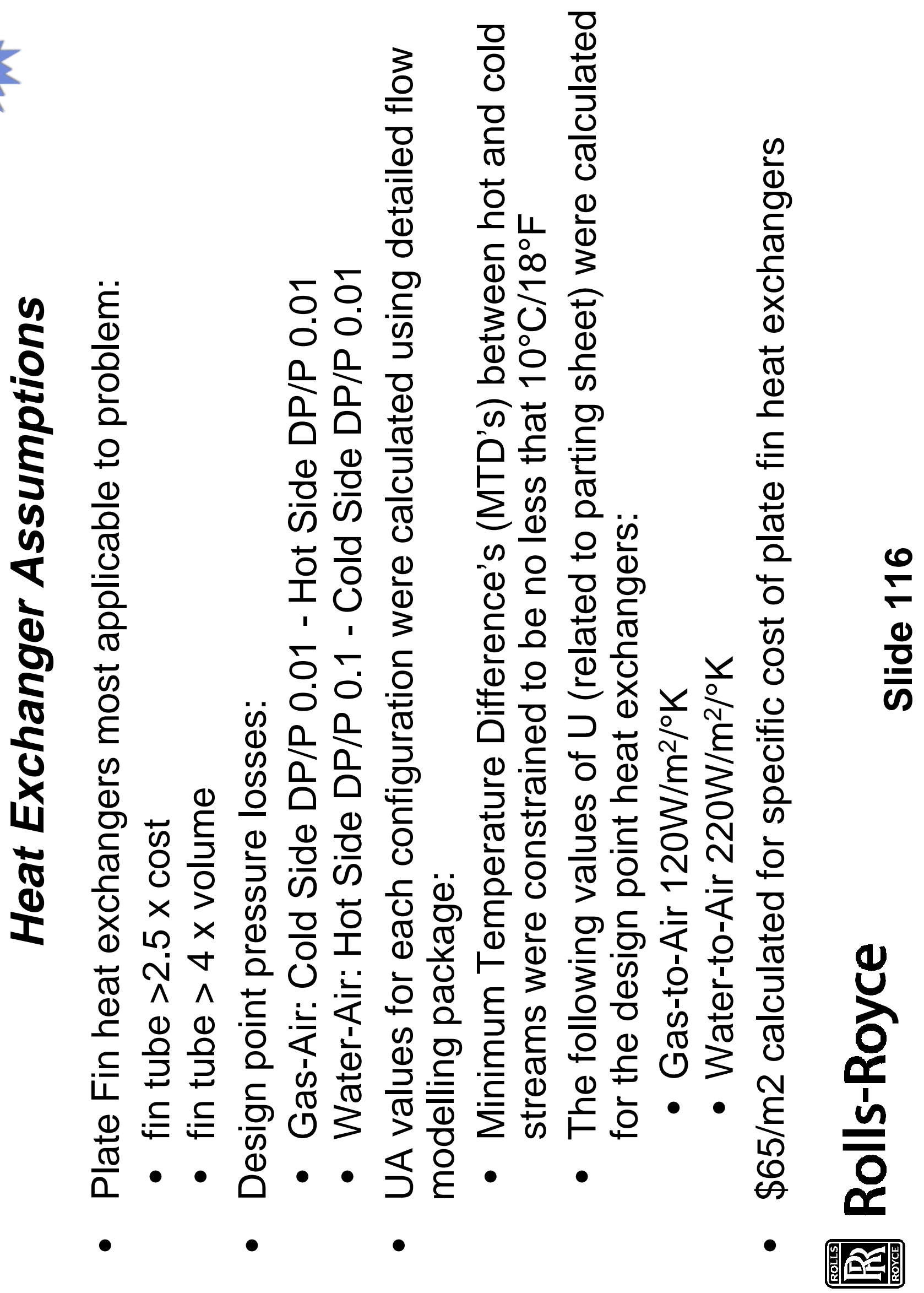




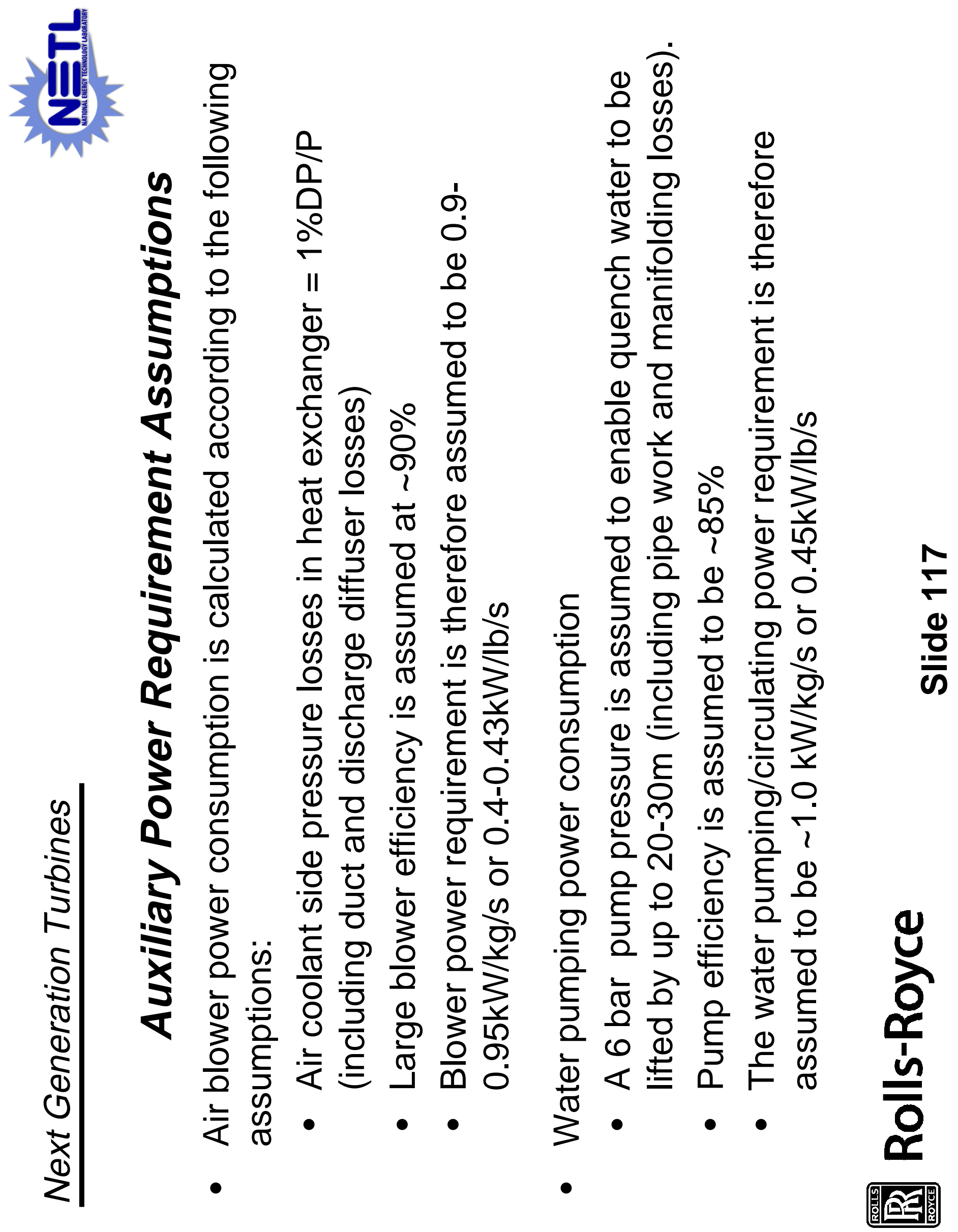




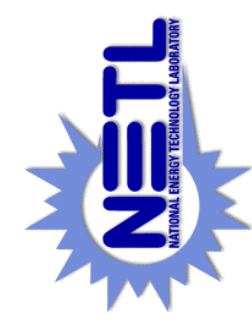

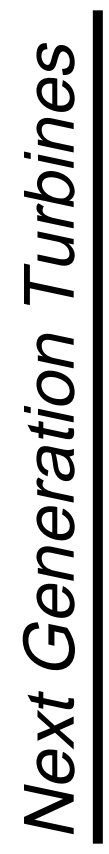
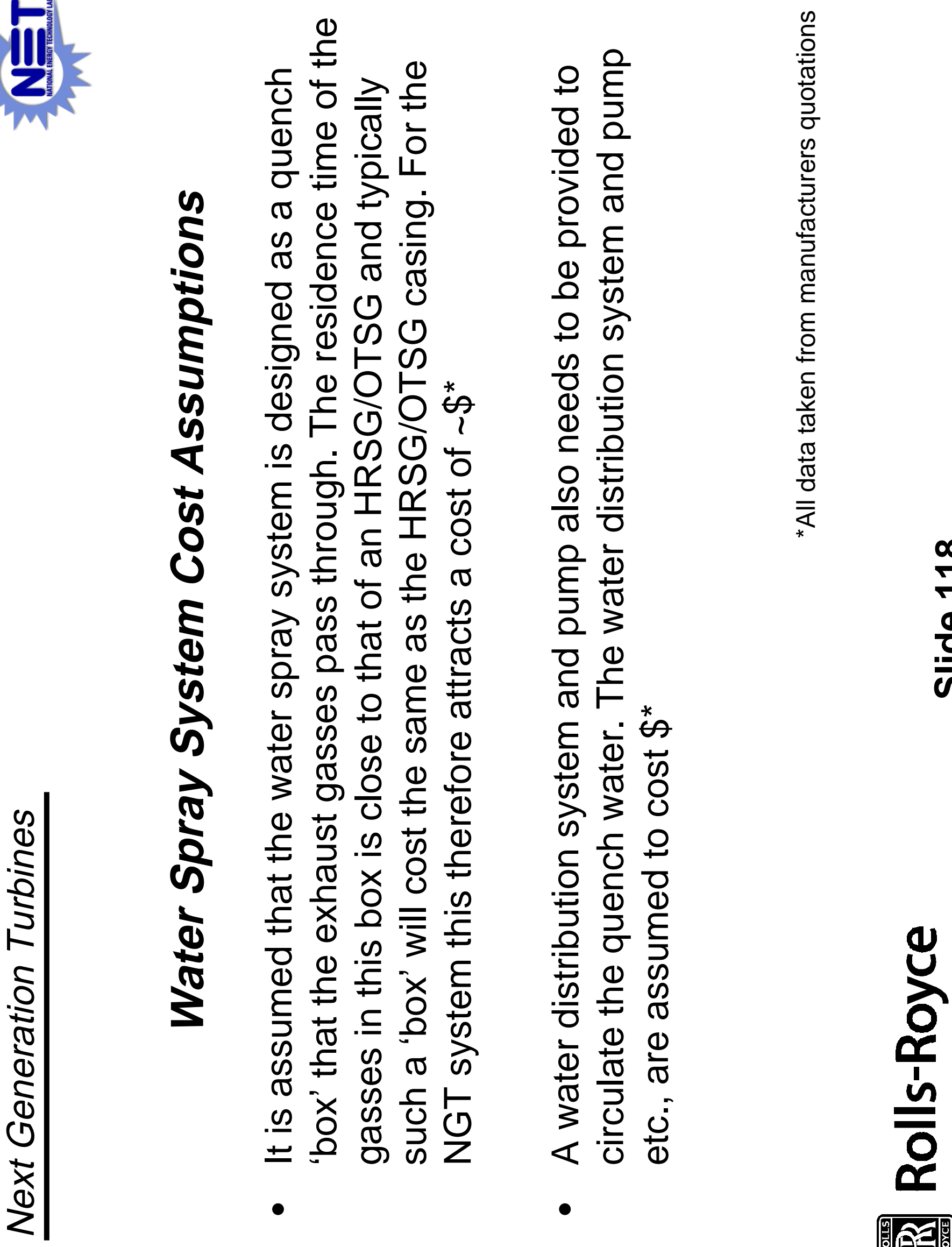


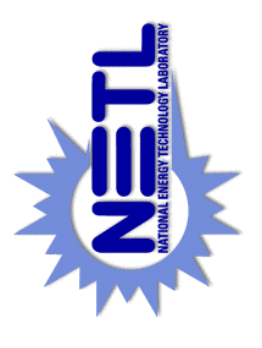

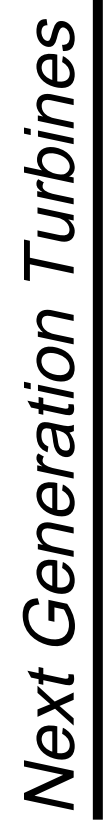
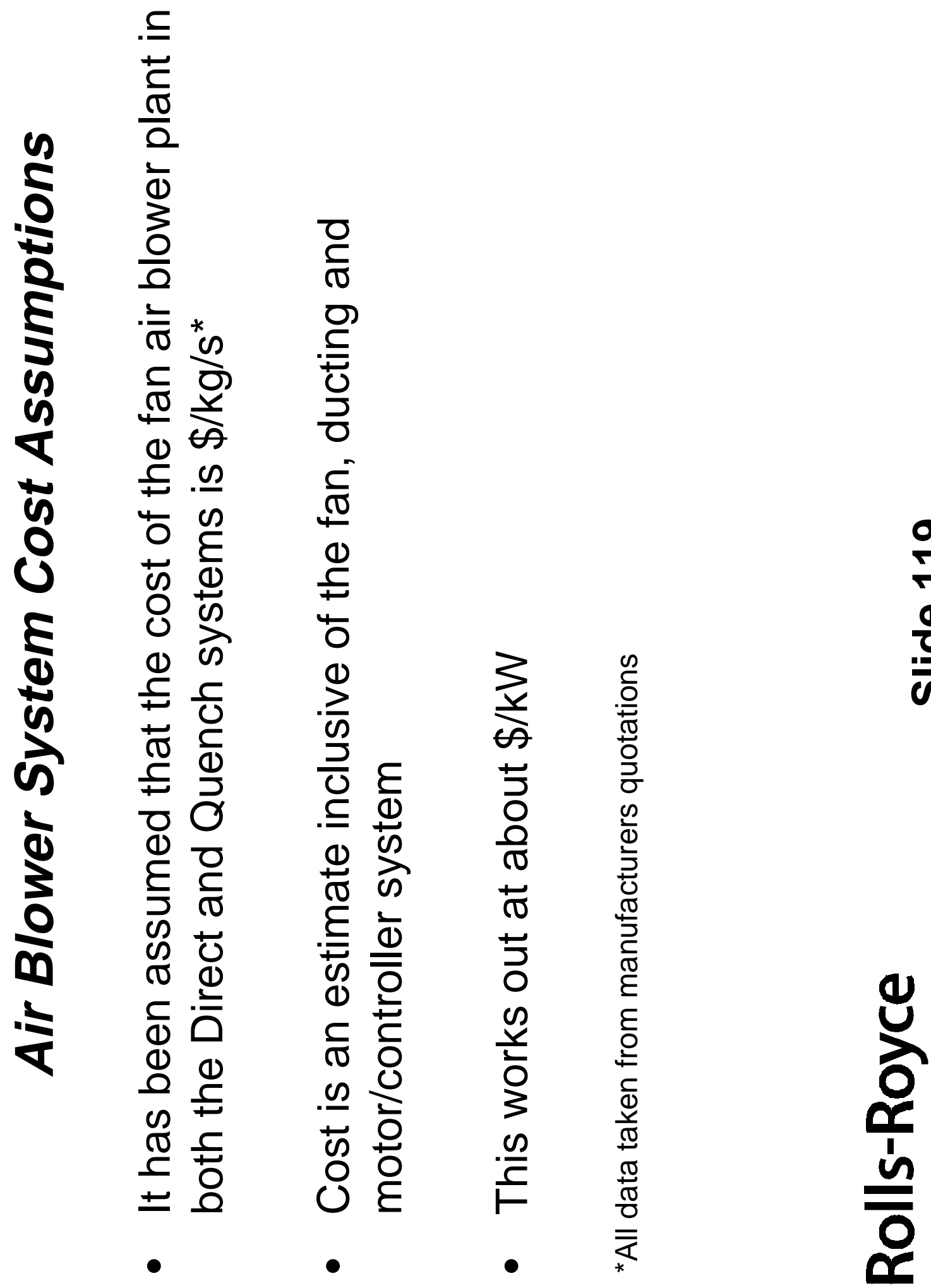

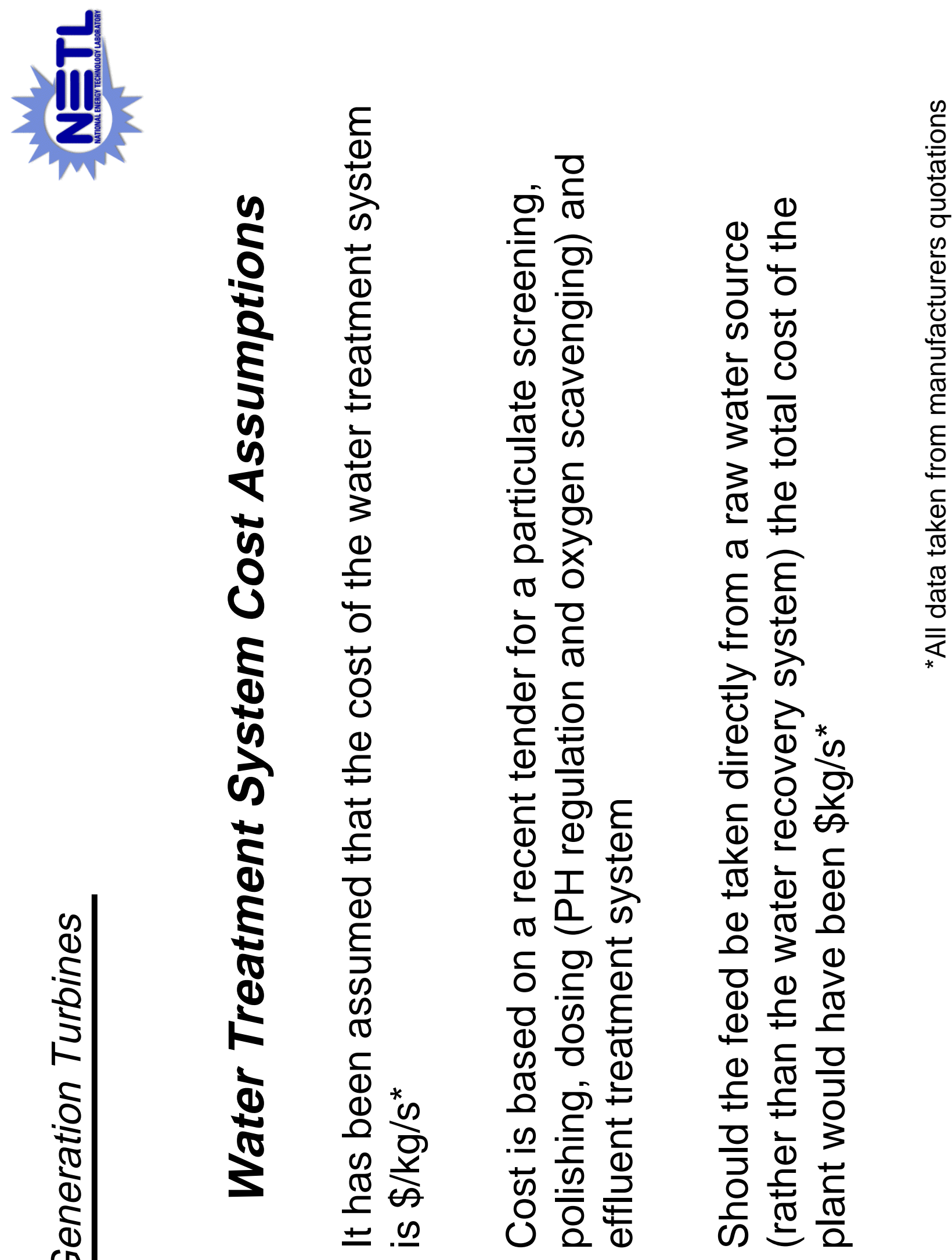

흐 음

ㅎํㅇ 두

들

(1) ত

$\pm \overline{7}$

Ð

它

ब)

त

$3 \div$

띤

ฮั

(1)

U 는

I I

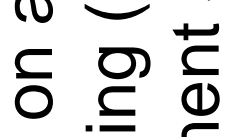

응

$\Phi$ 응 중

路

으.

एक

Oำ

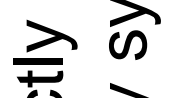



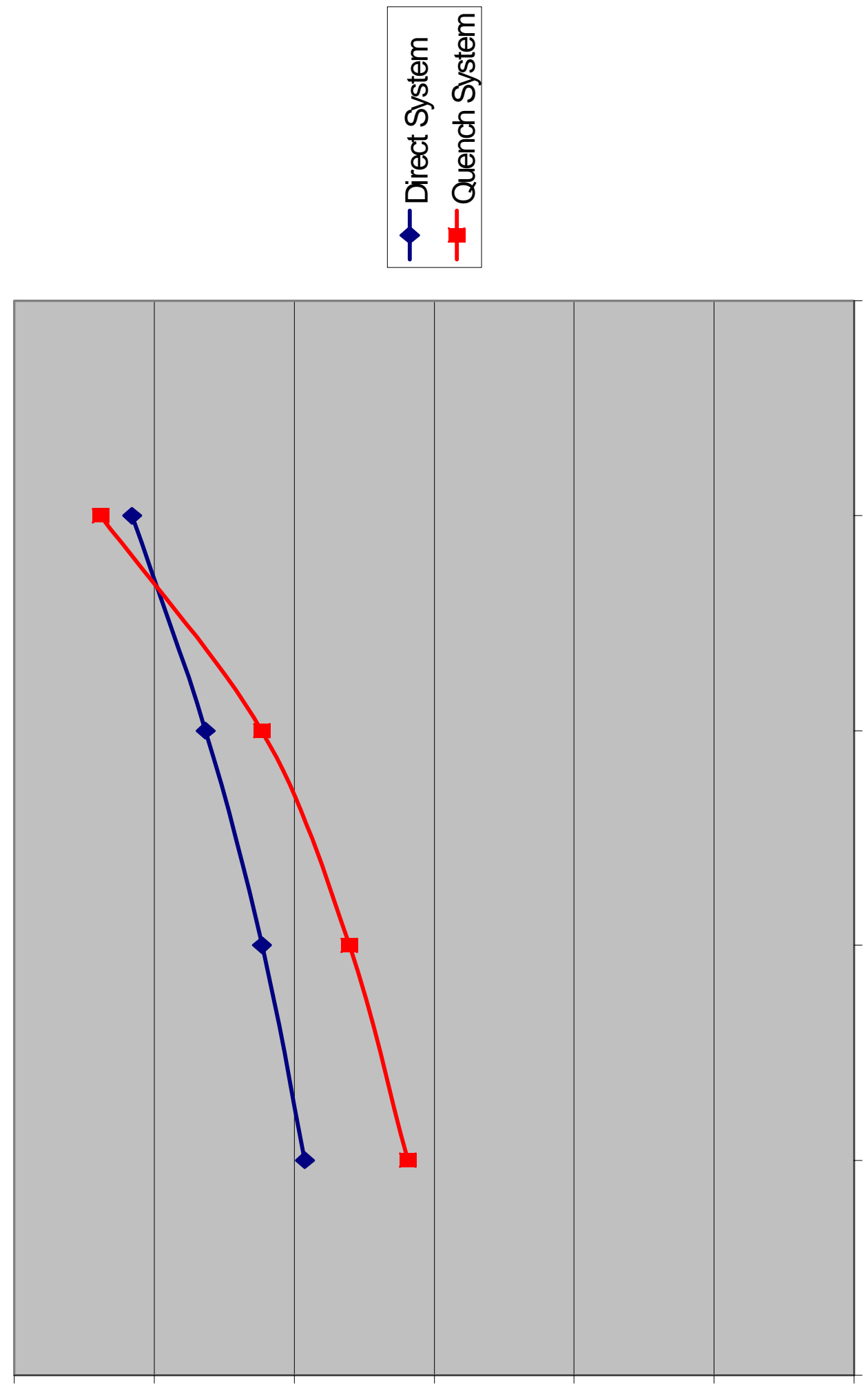

8)

है

$\frac{E}{5}$

है

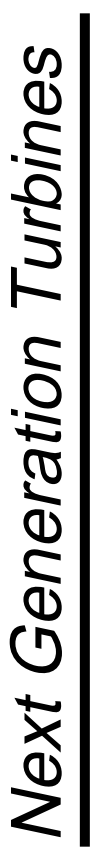

$\stackrel{2}{\sim}$

을

모

등

'क्

융

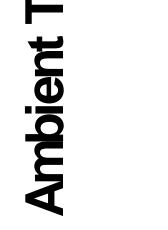

으

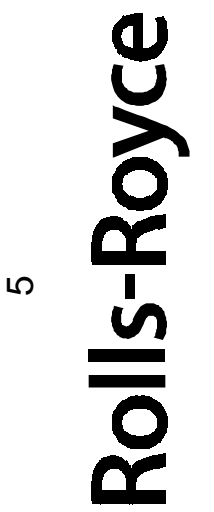

(W\$) słsoo məłsर́s 

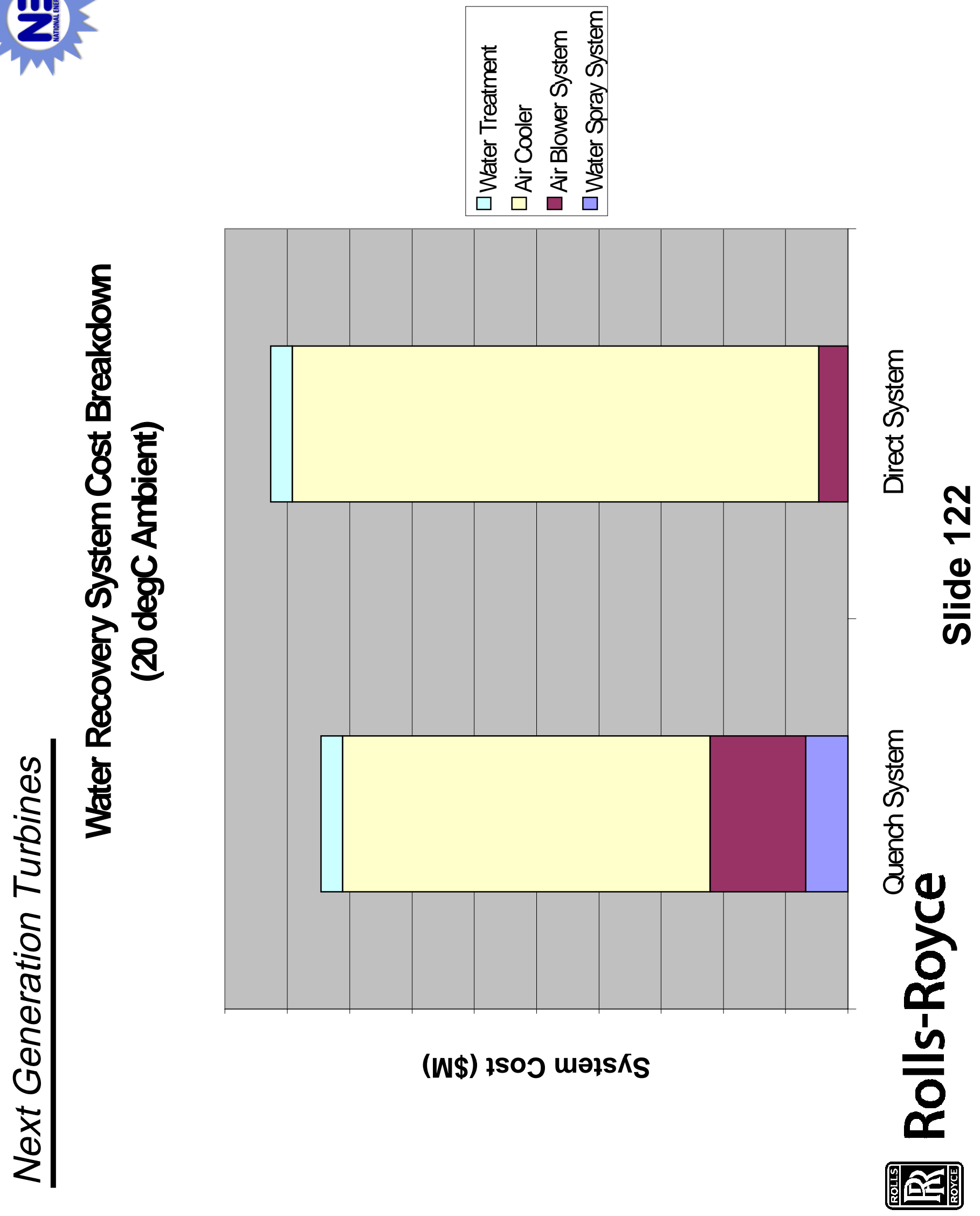

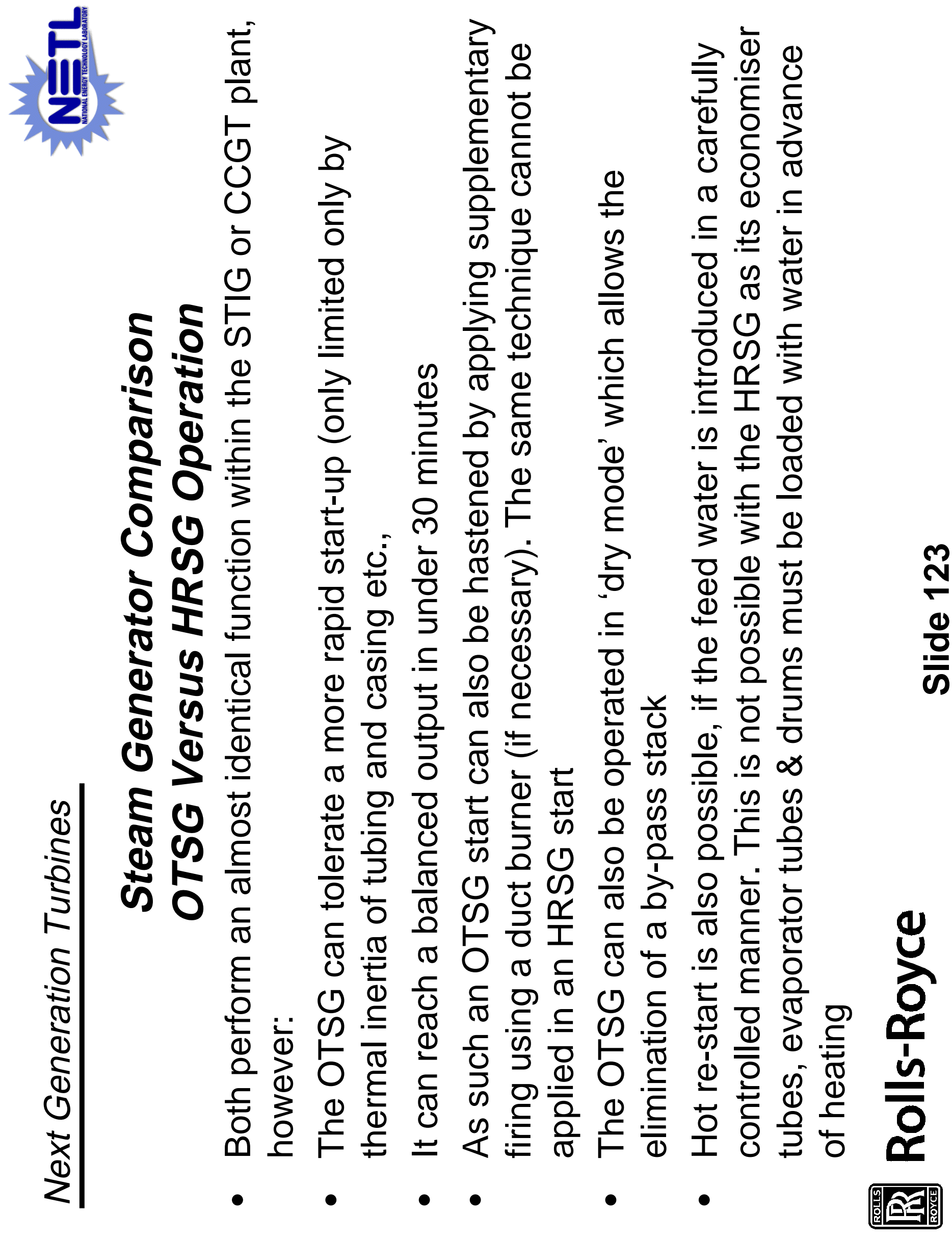


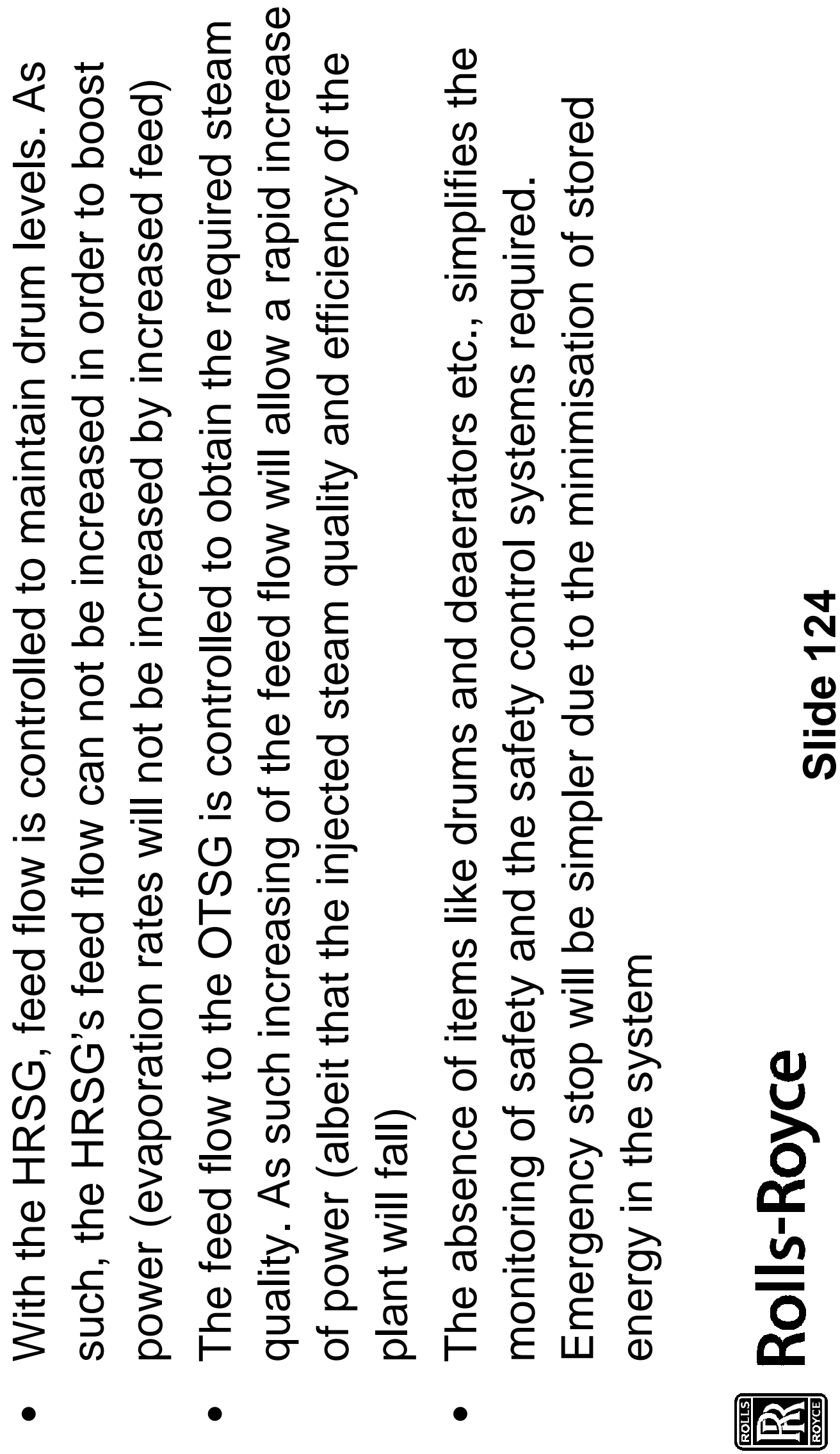



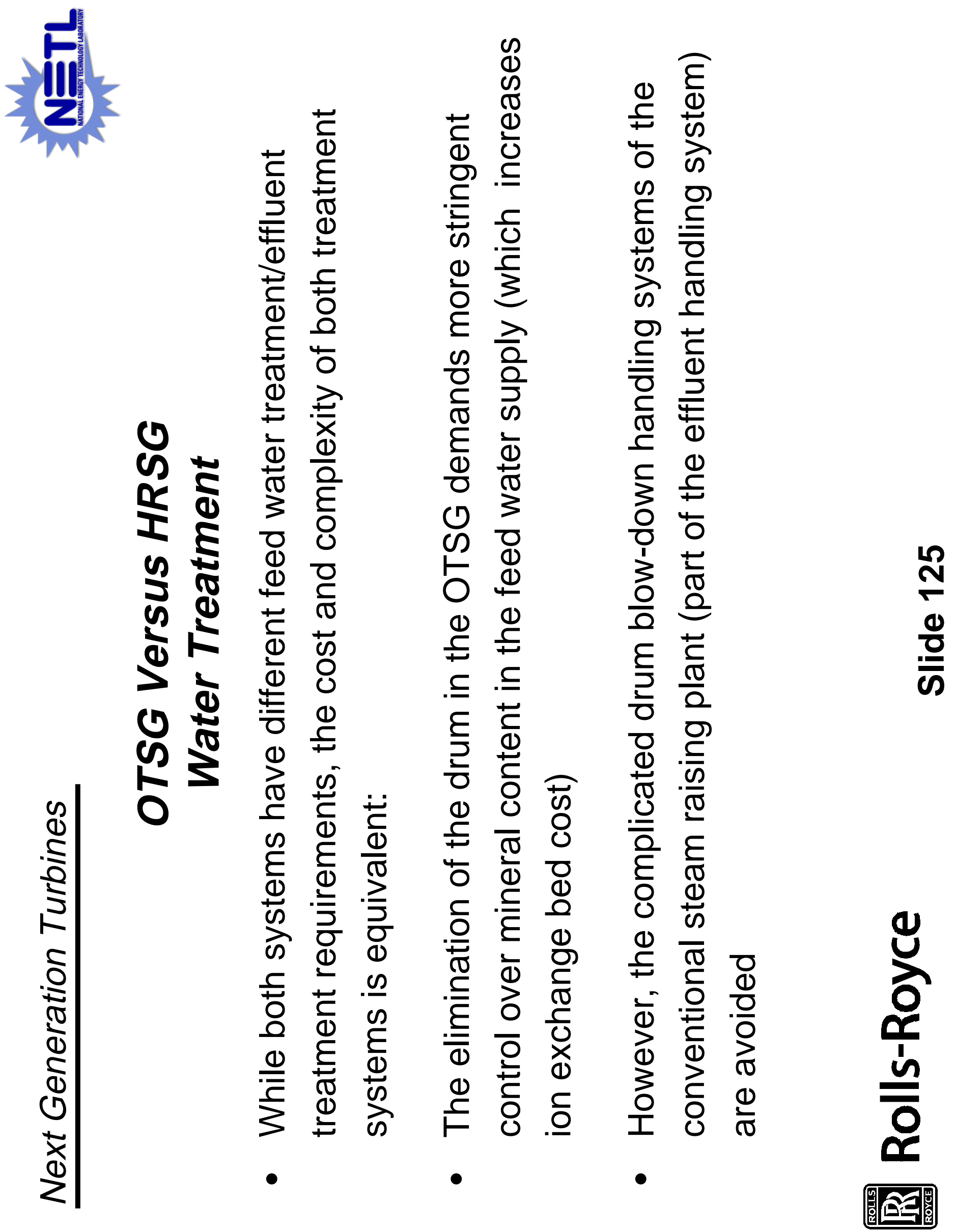


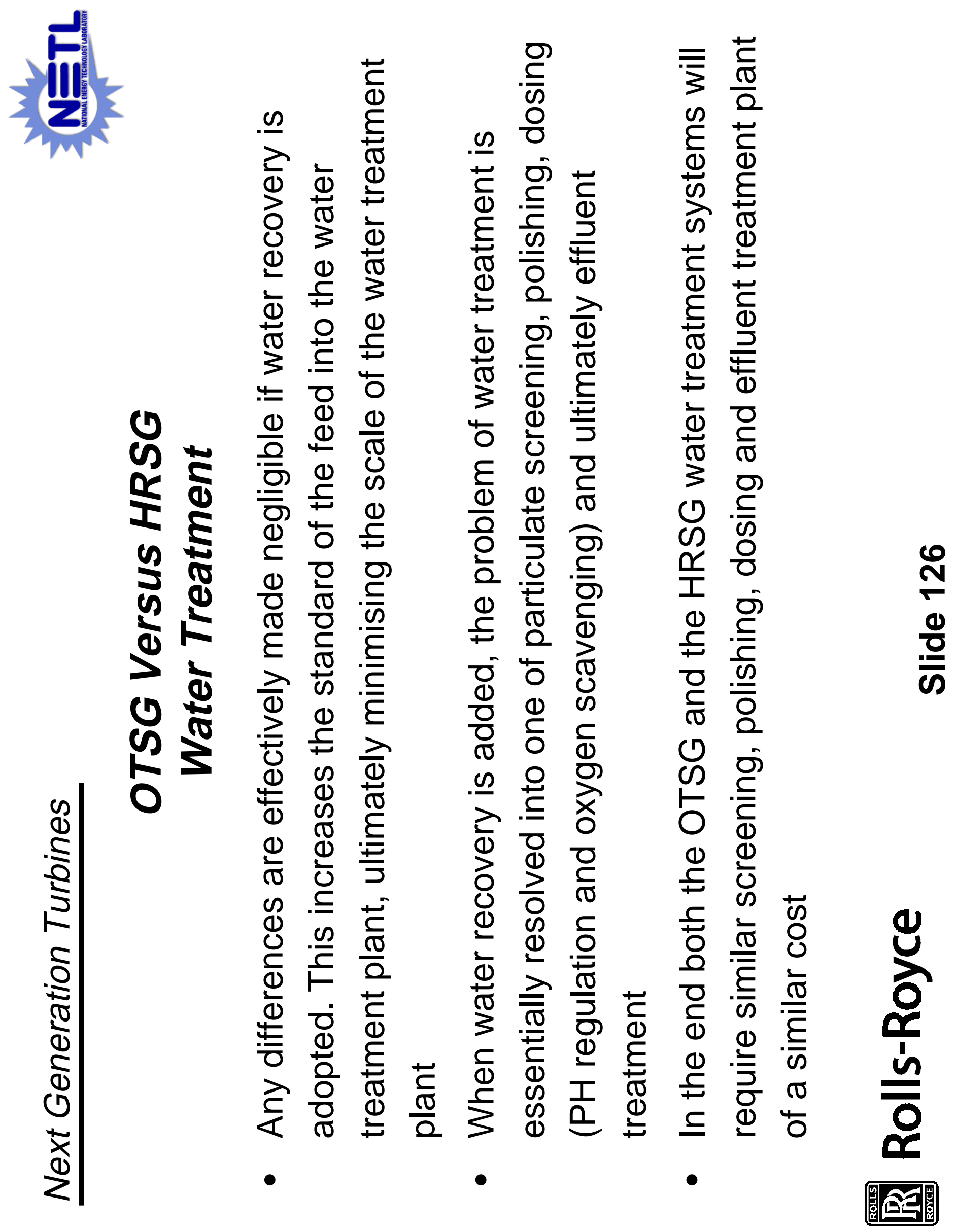




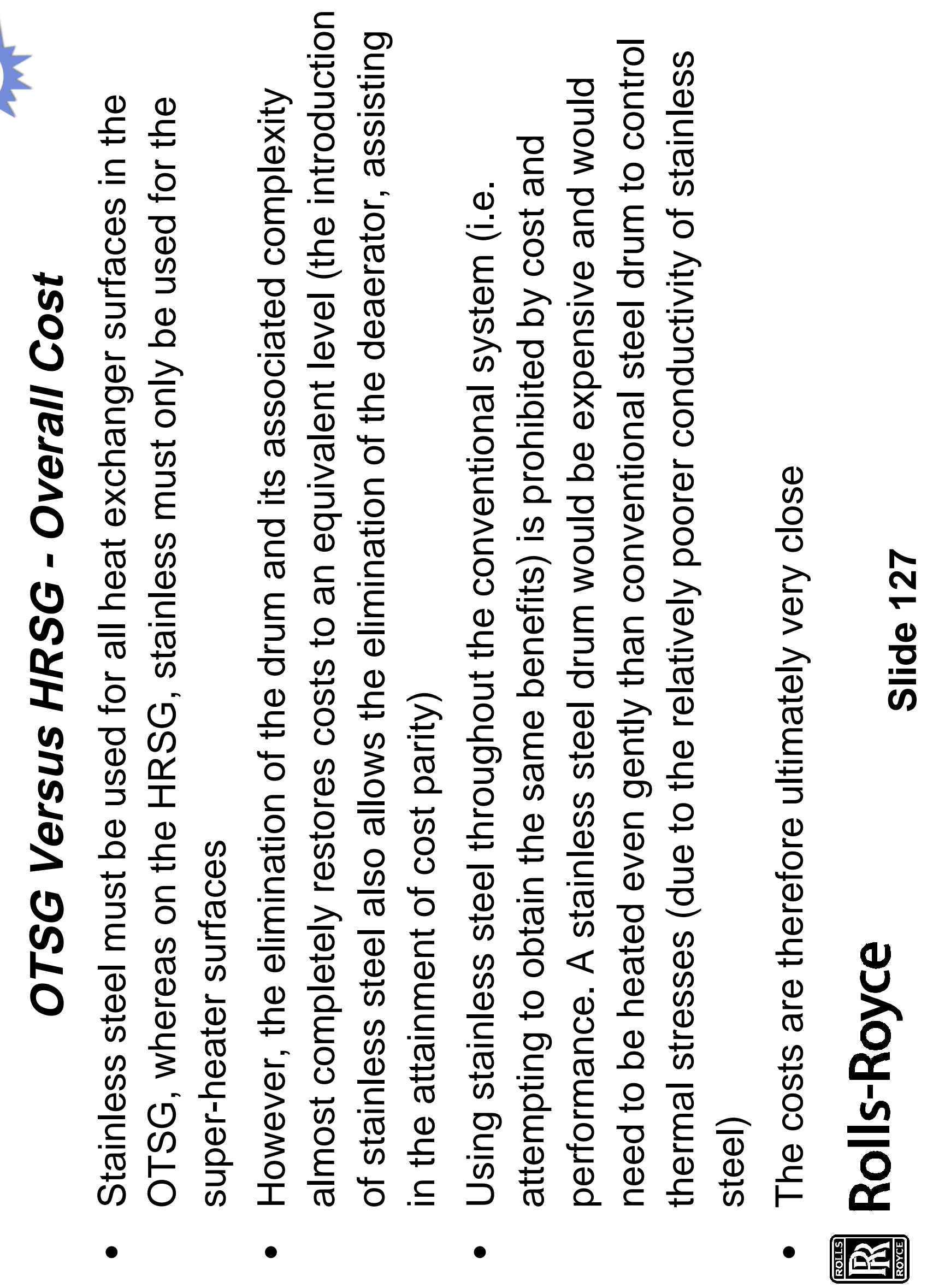




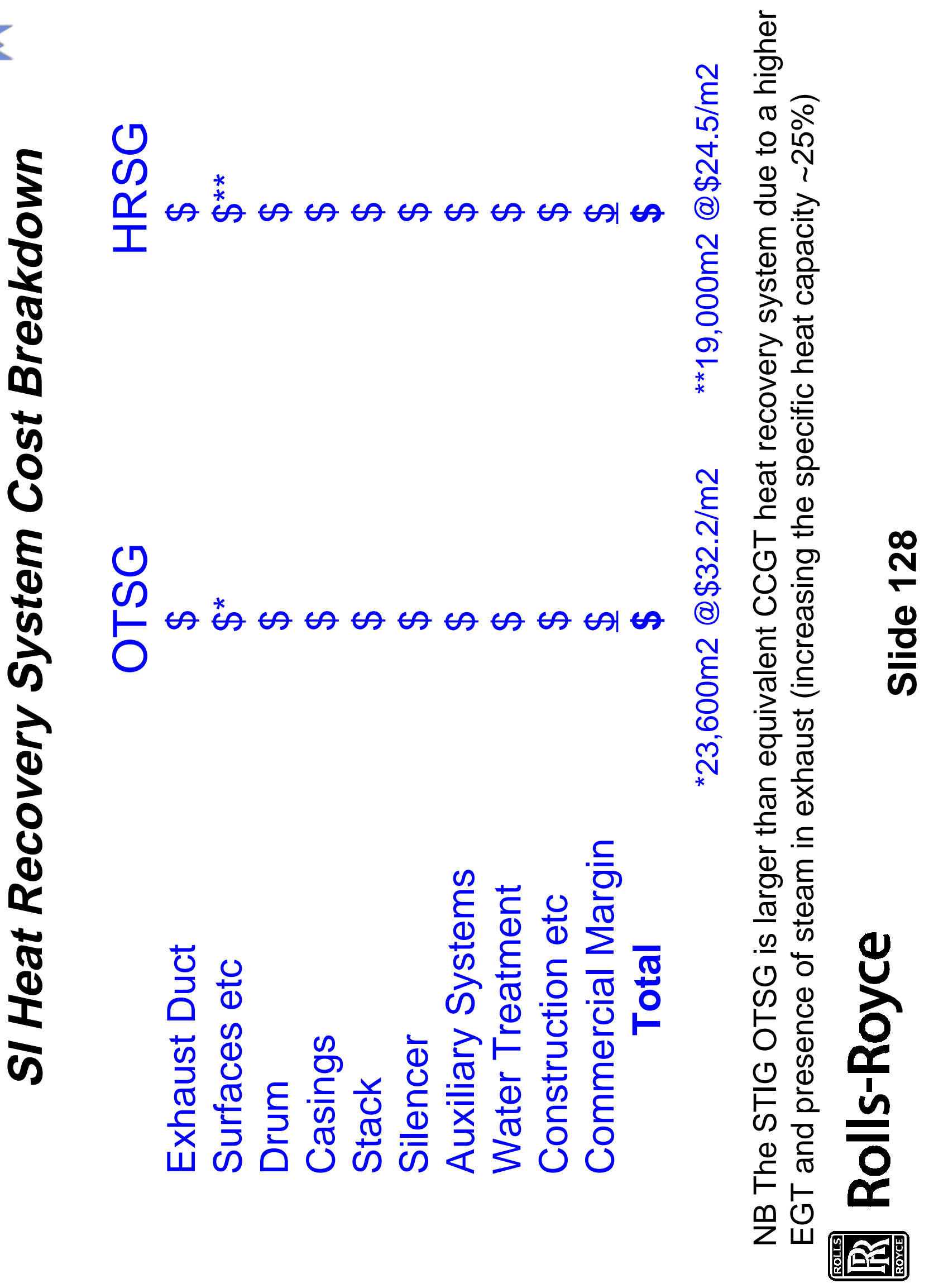



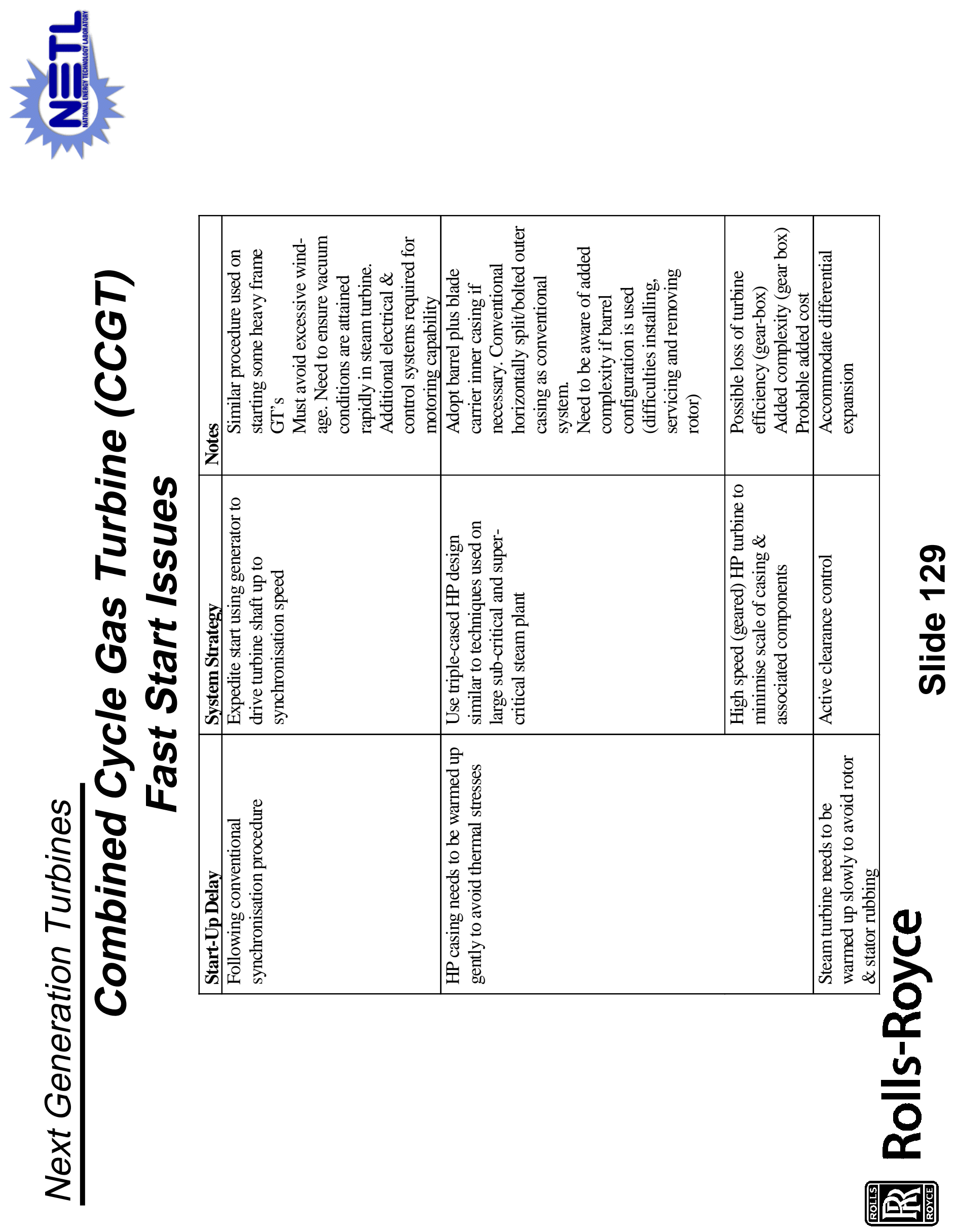


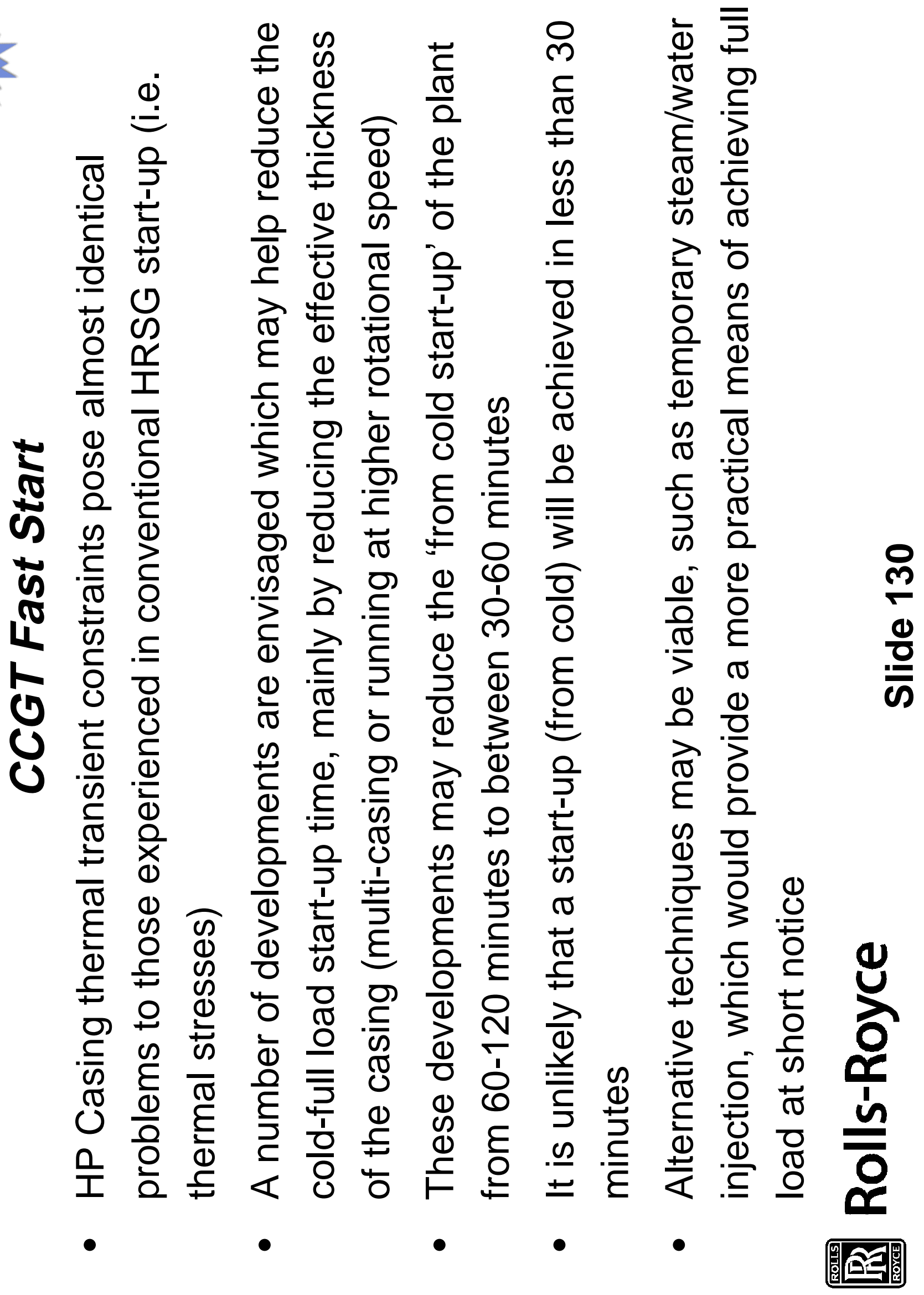



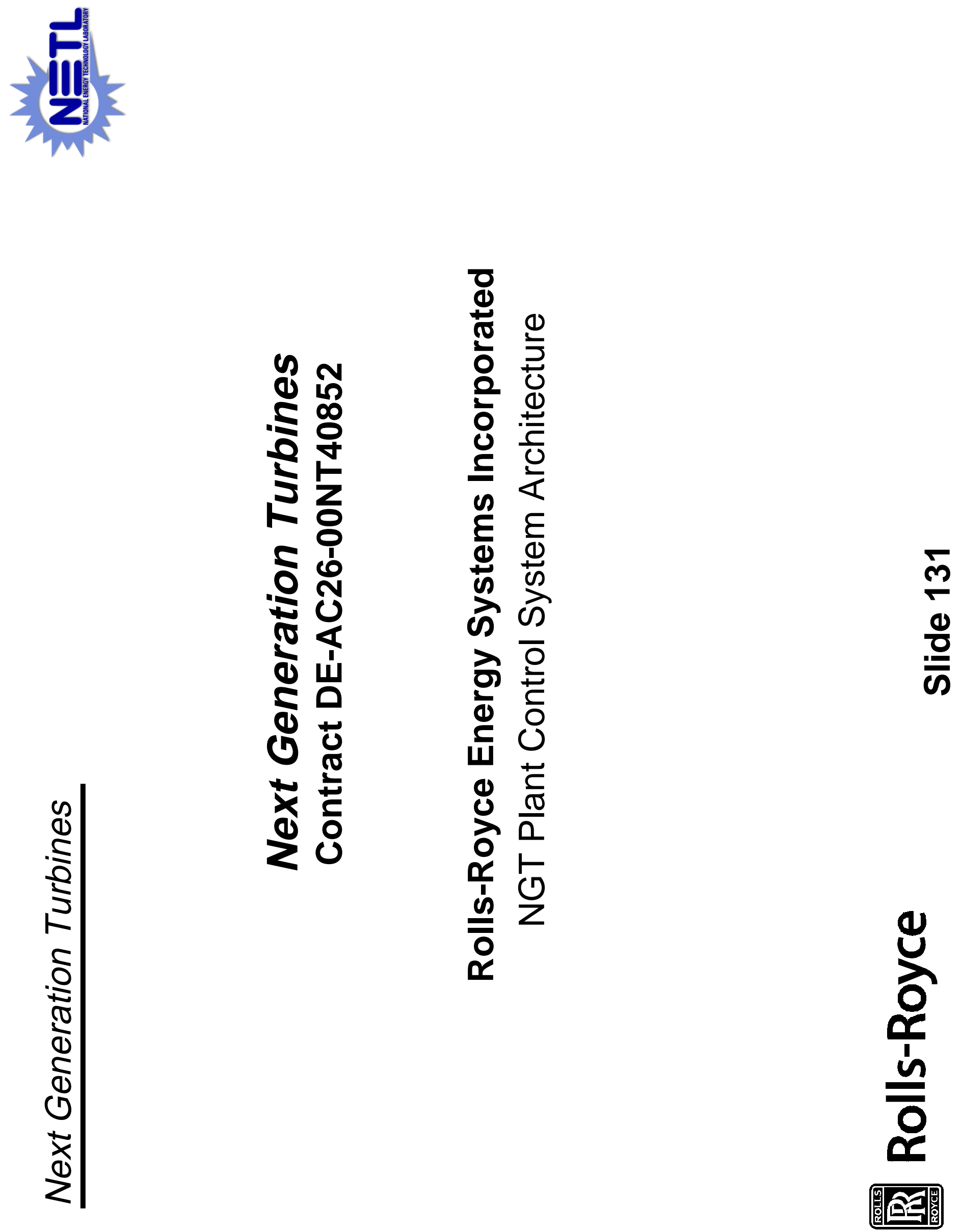


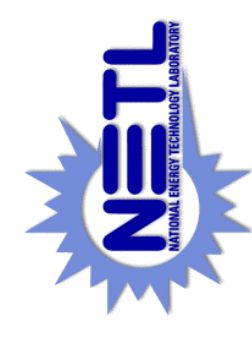

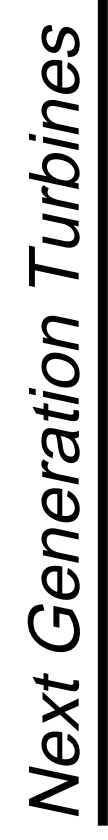

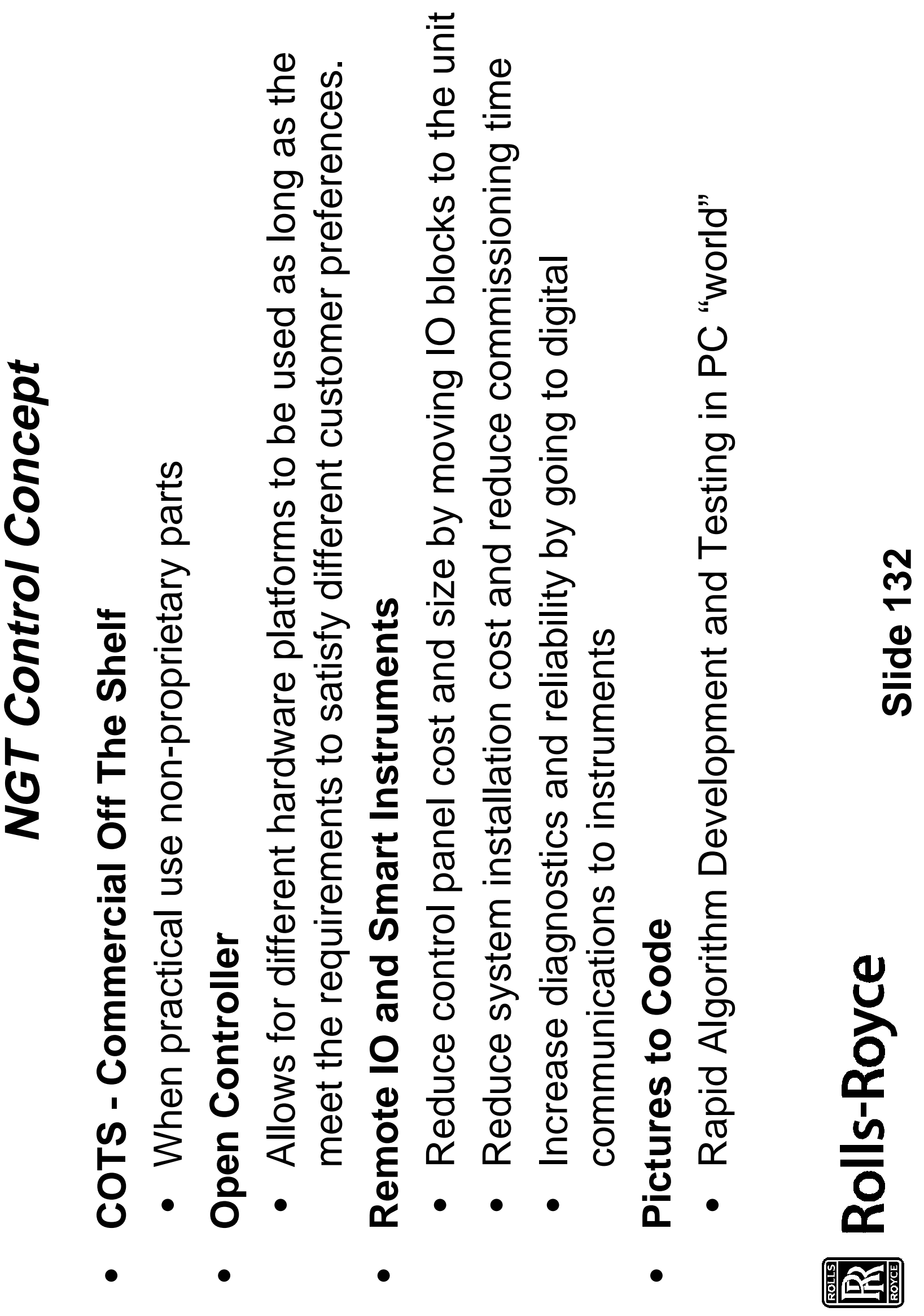



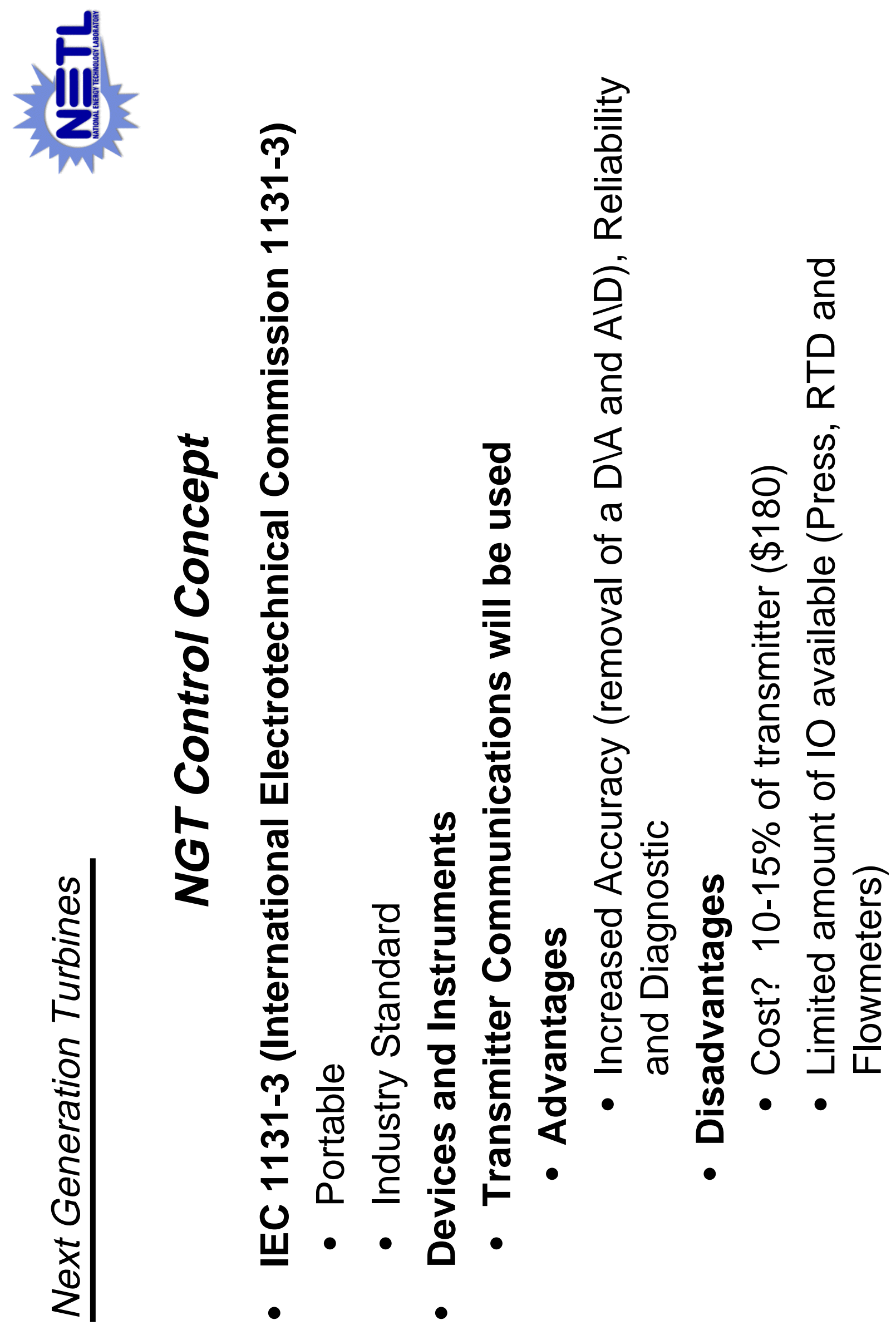

$\frac{m}{\frac{m}{0}}$

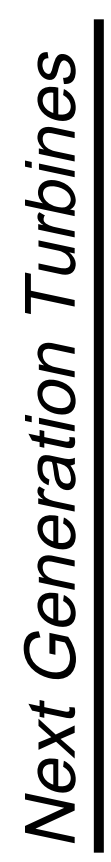




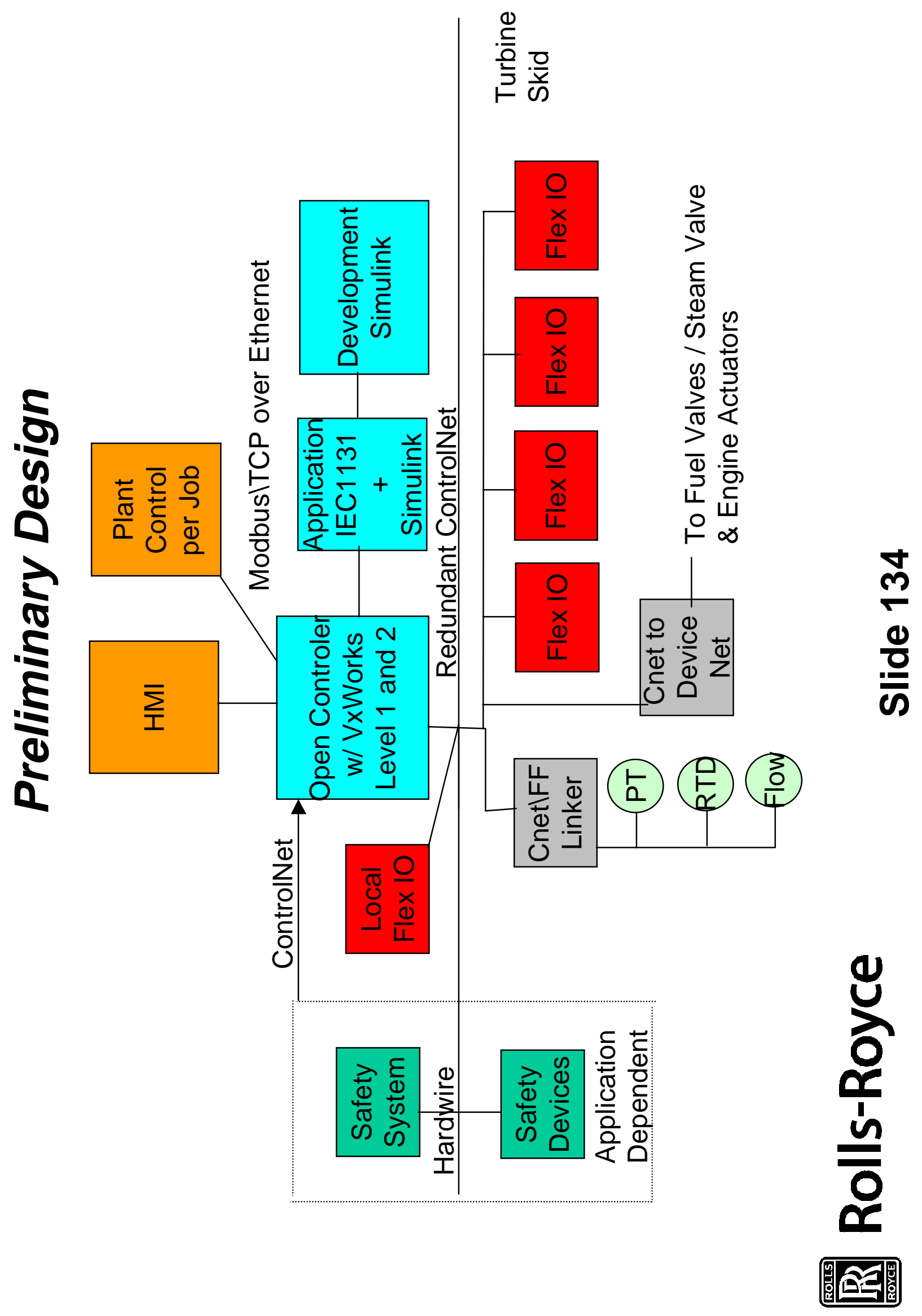



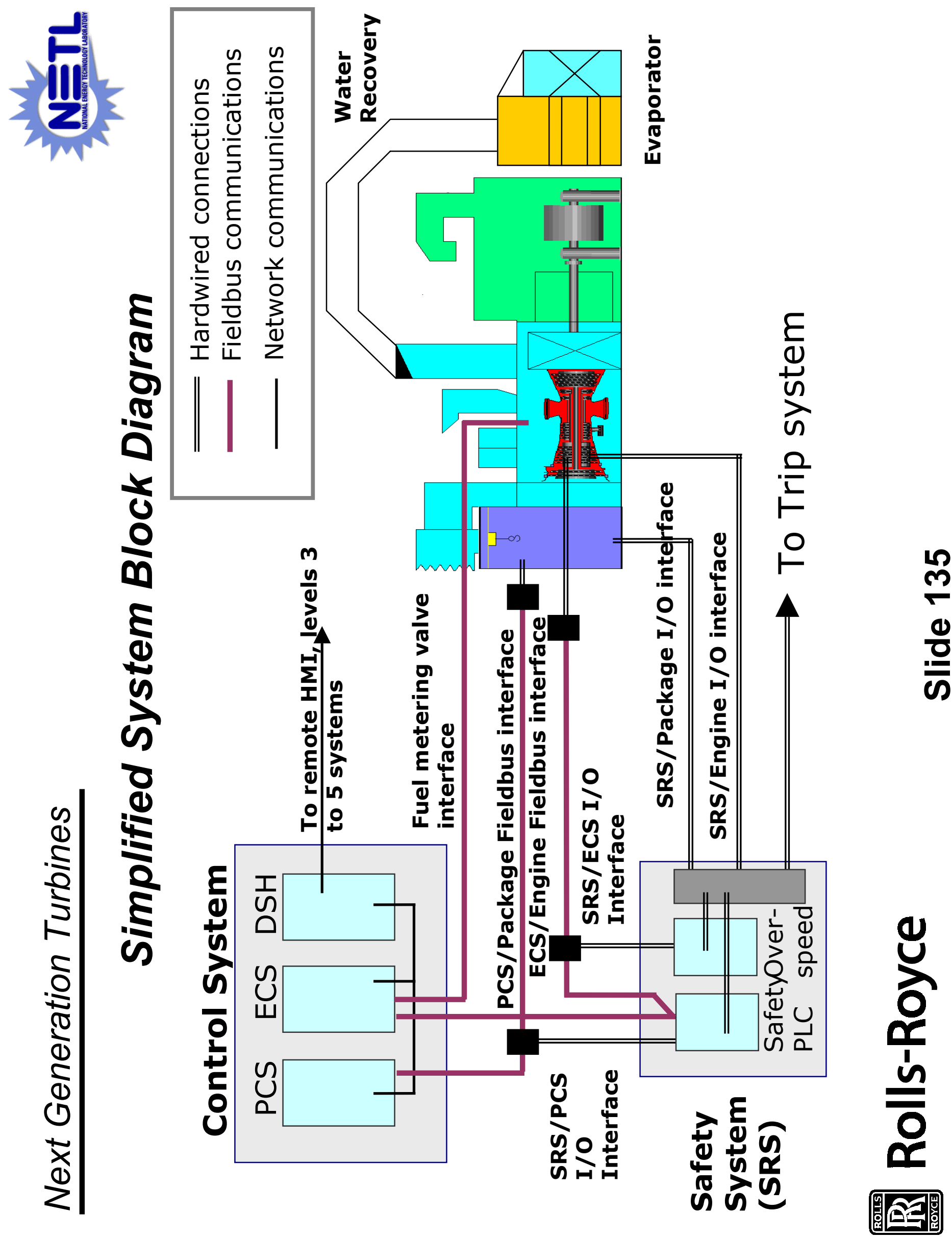

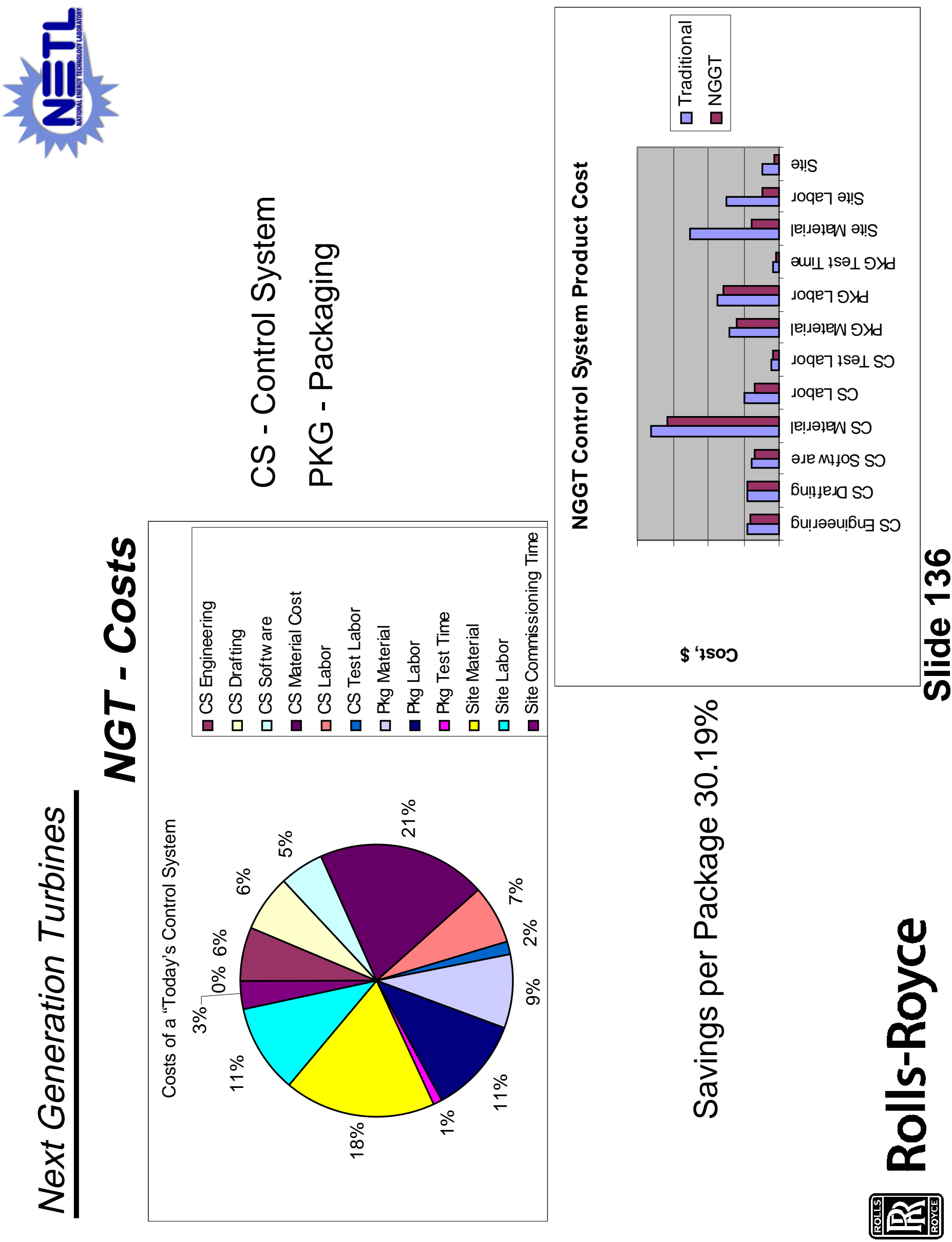


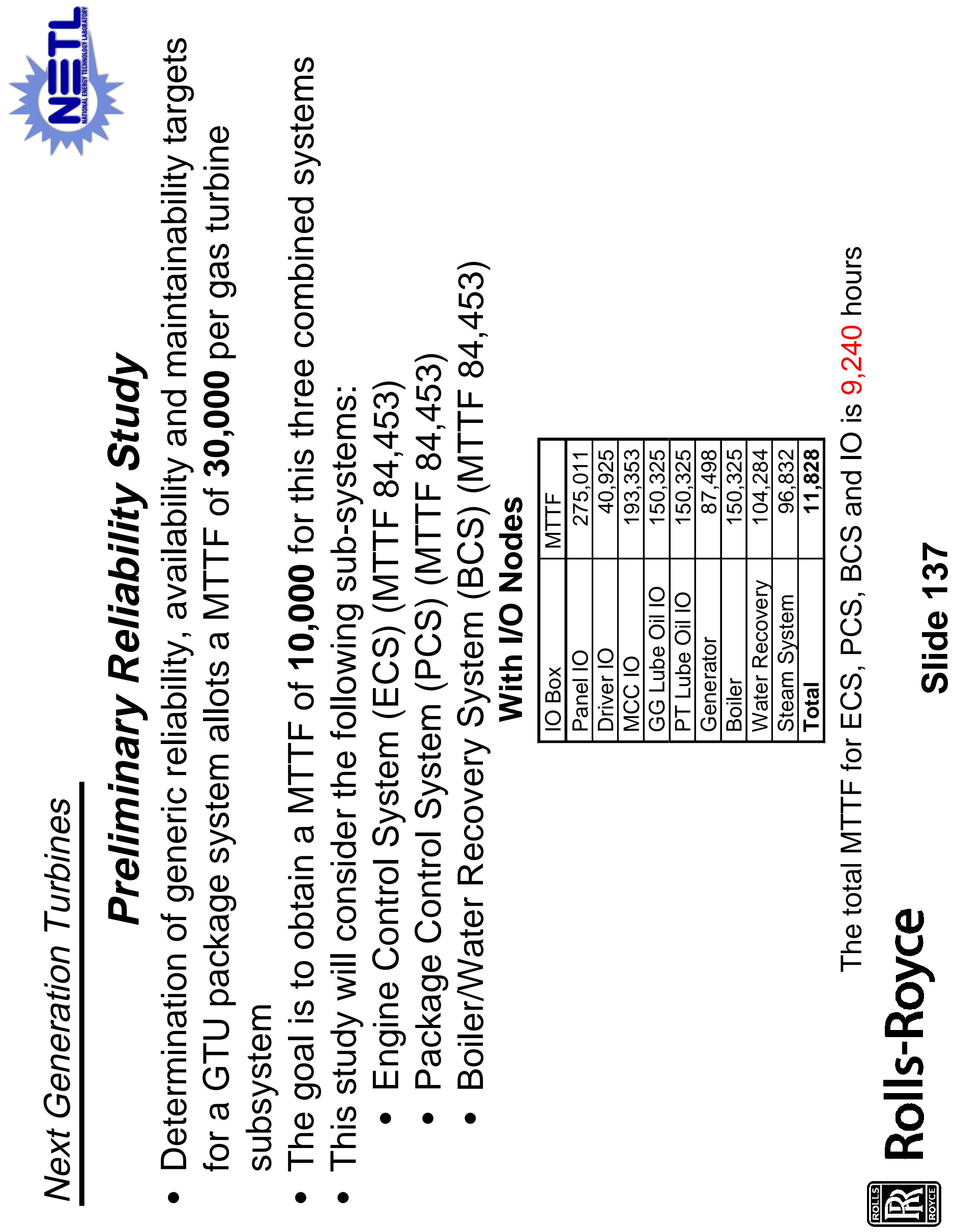




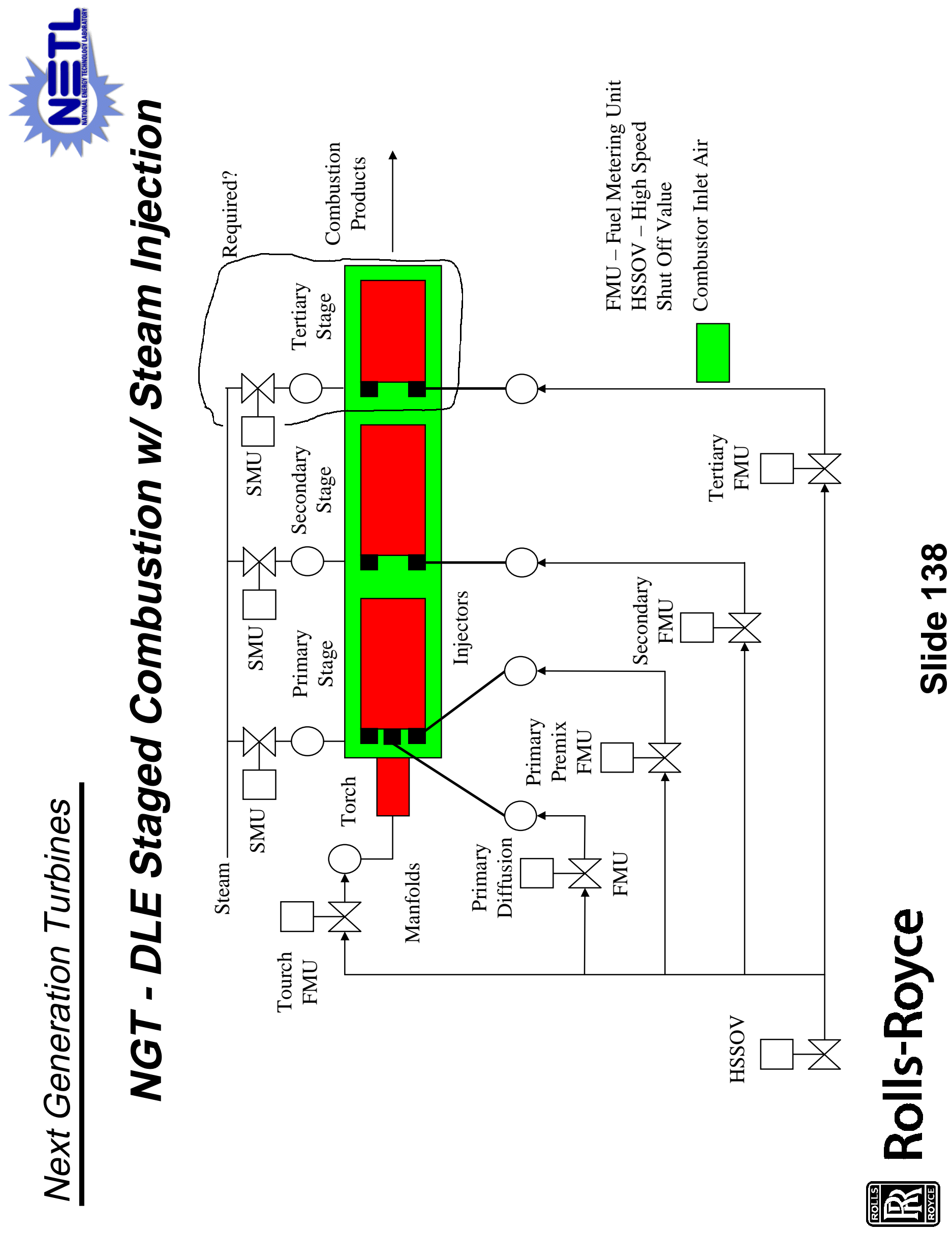




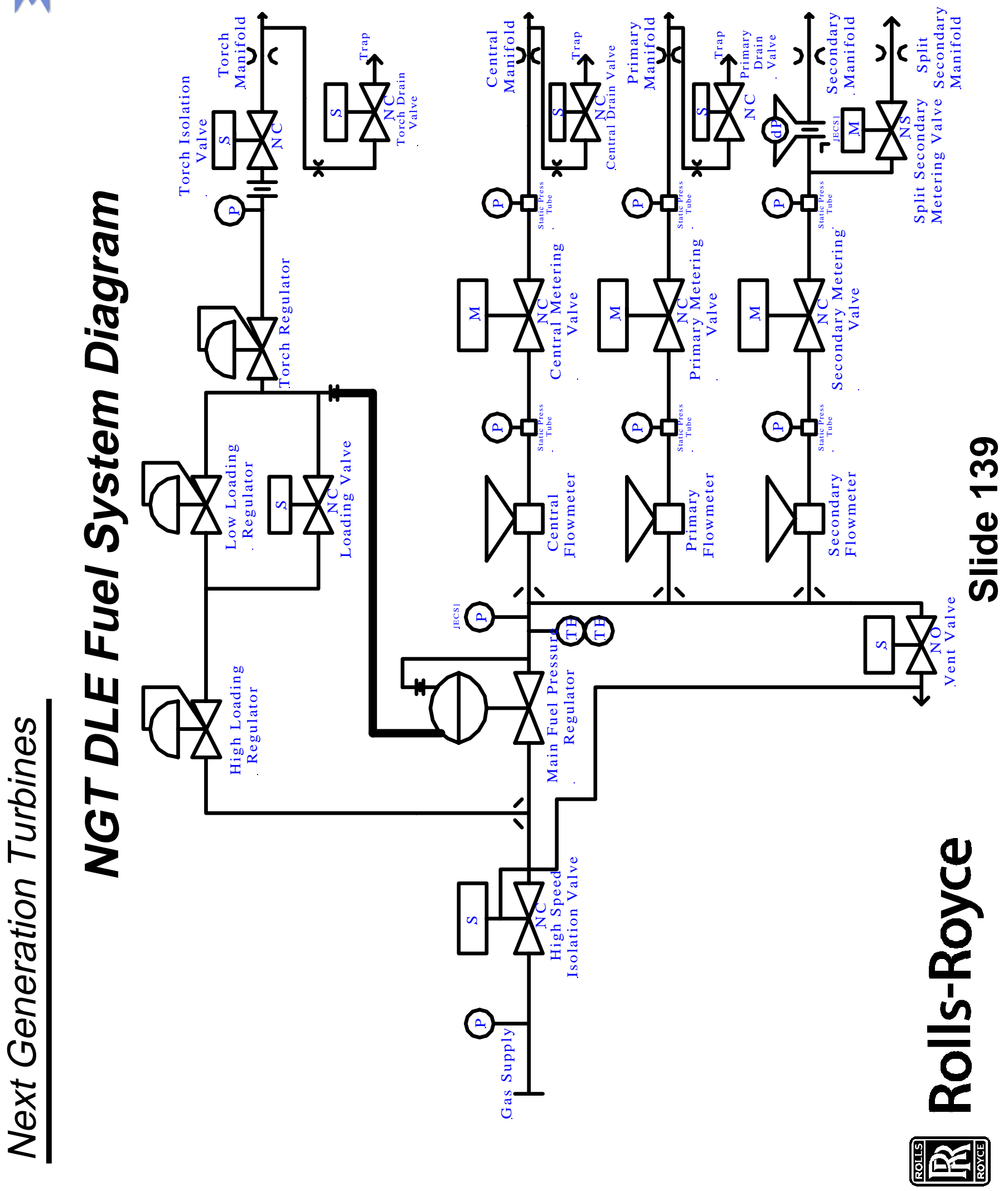



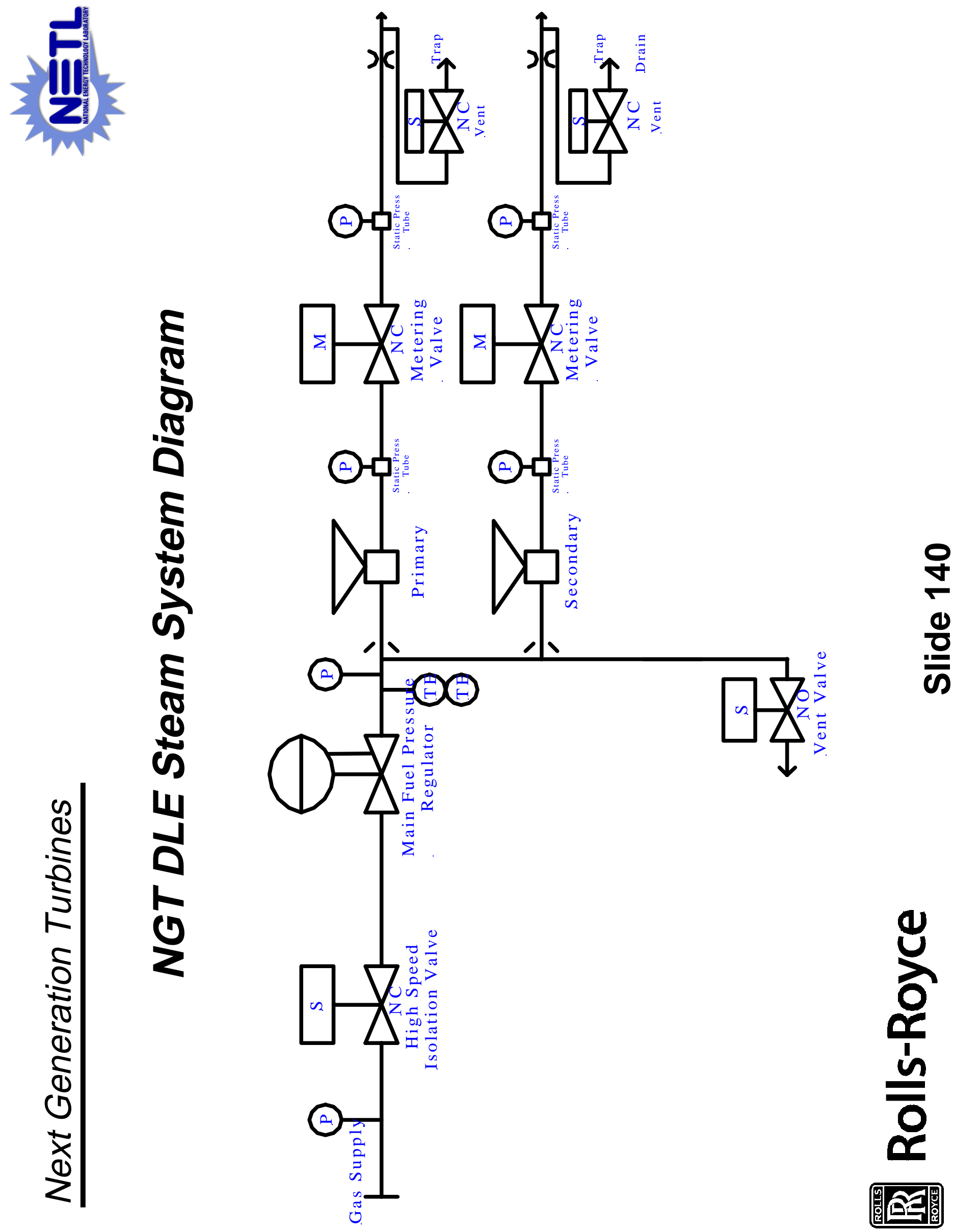

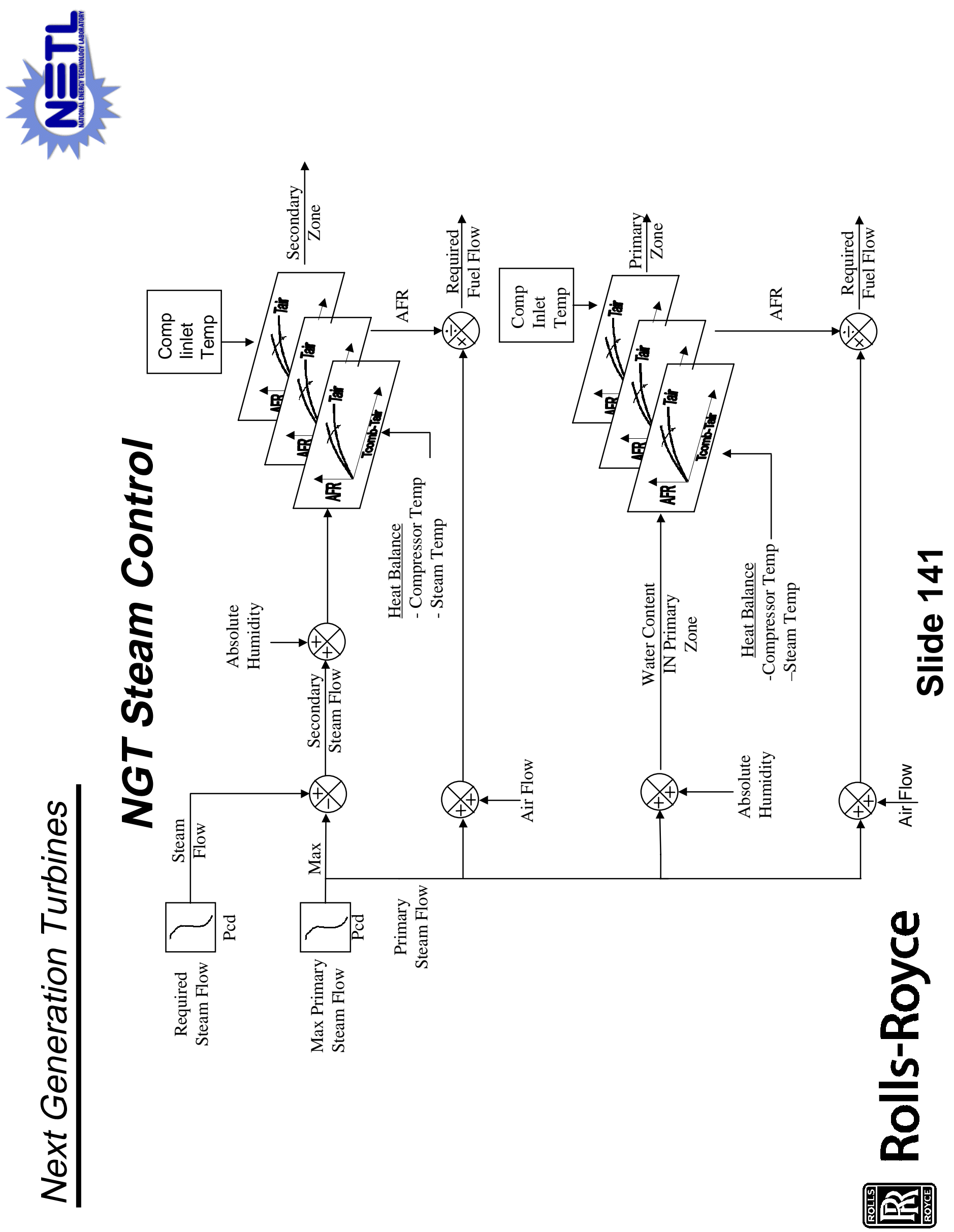


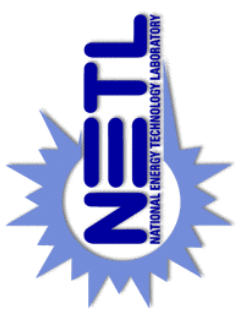

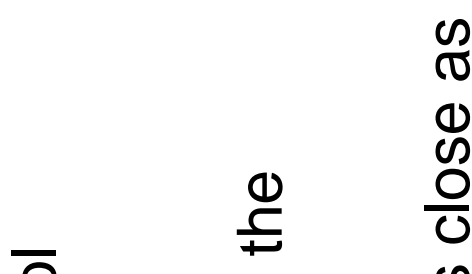

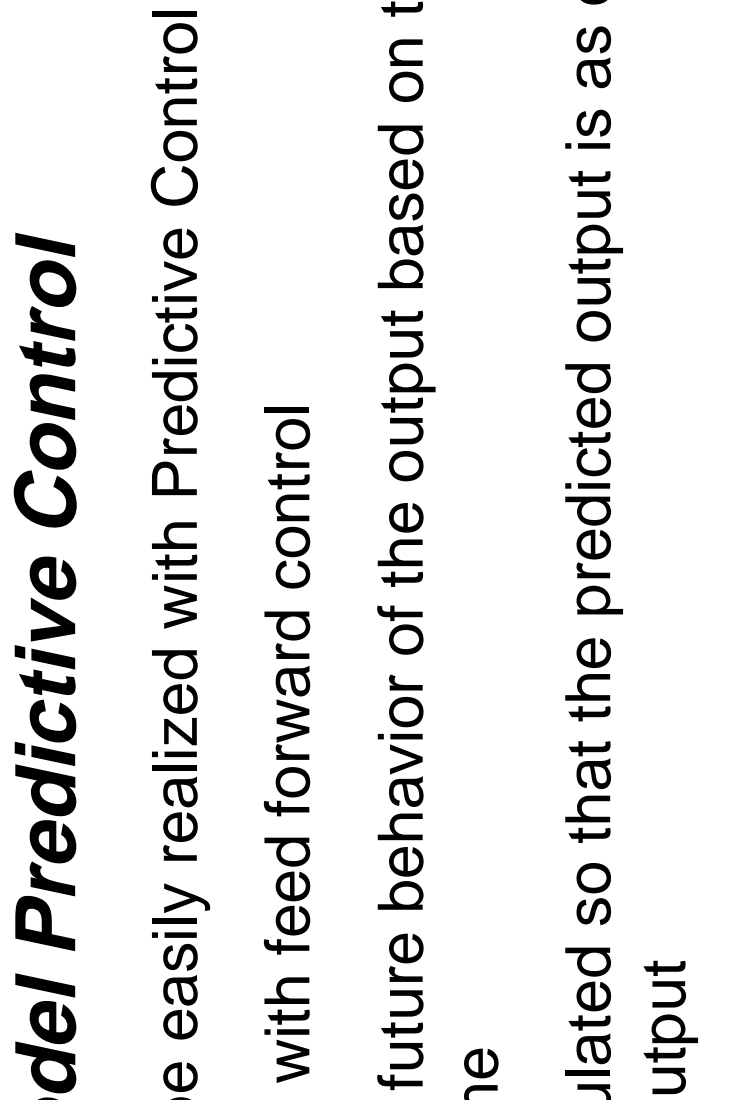

(3)

$\frac{5}{5}$

N

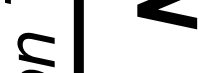

$\frac{1}{10}$

$\pi$

(1)

๘

0

x

$\sum$
๘

()

인

○ 0 ब E गे

ป $\frac{\omega}{0} \cong$

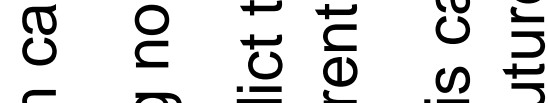

ह 잉 흔

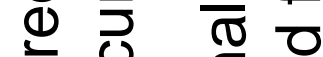

으 0 이

๙ क ज

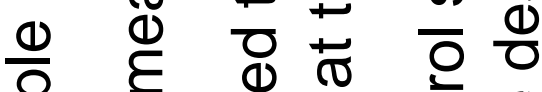

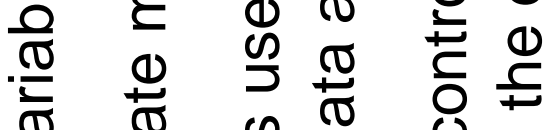

तิ

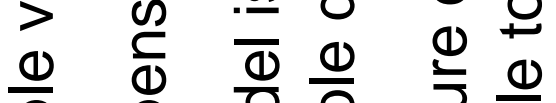

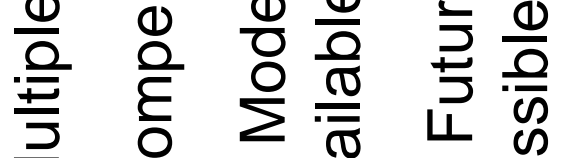

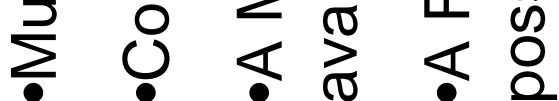

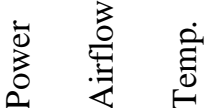

㲔言

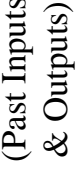

$\overrightarrow{\mathrm{s}} \cdot \stackrel{0}{0}$

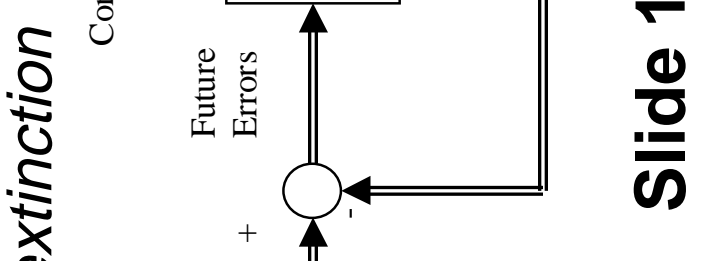

ช

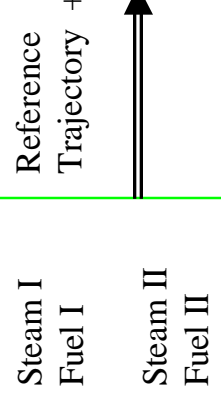

0

$\frac{1}{\infty}$

$\stackrel{1}{3}$

온

ఫั

O

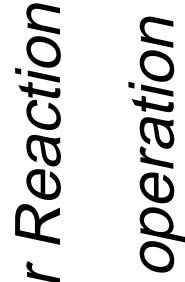

$\mathbf{v}$

ウ

+

(1) $\frac{\pi}{4}$

은

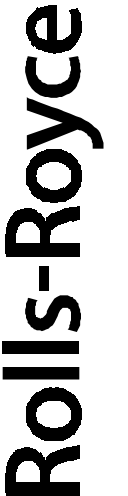



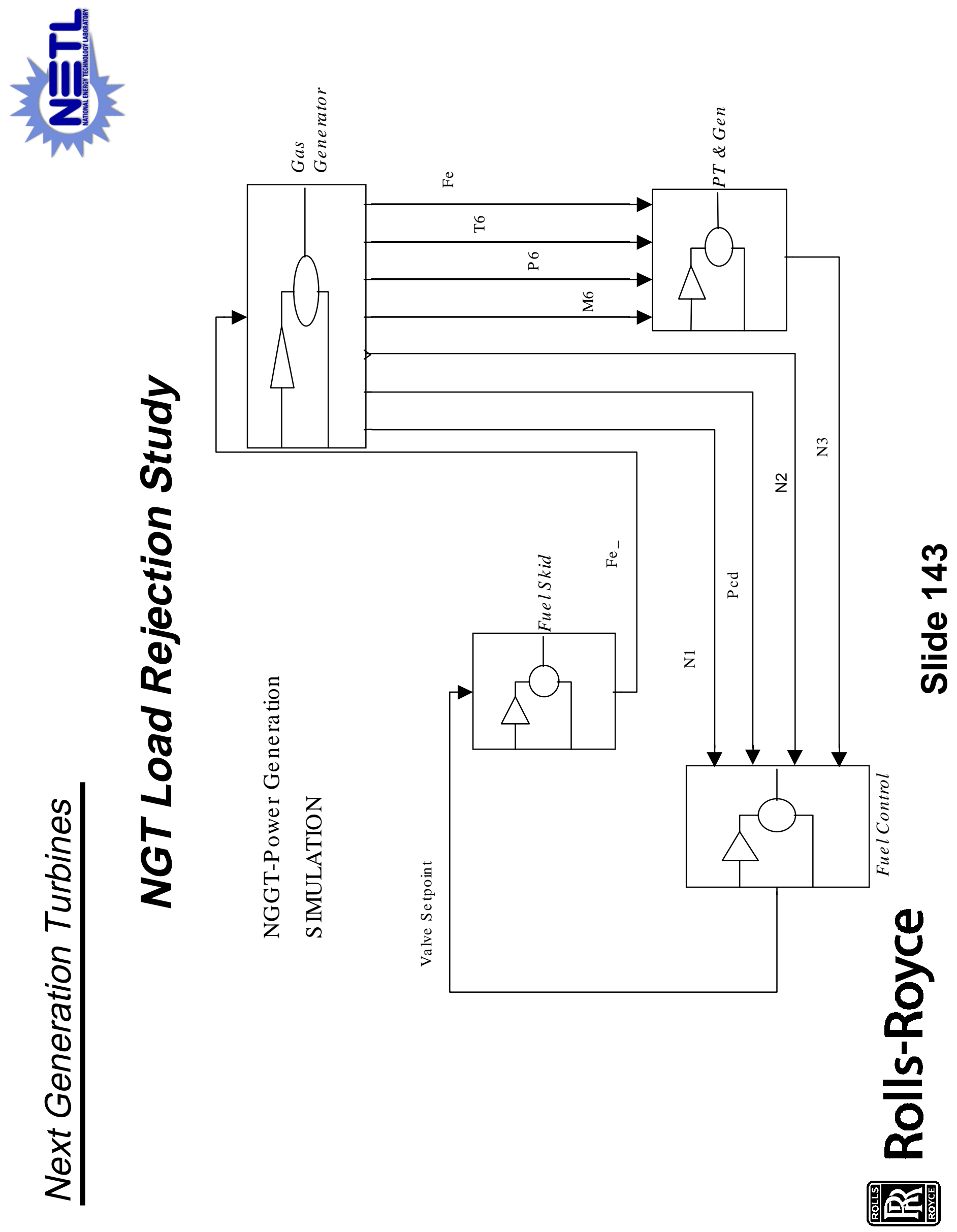


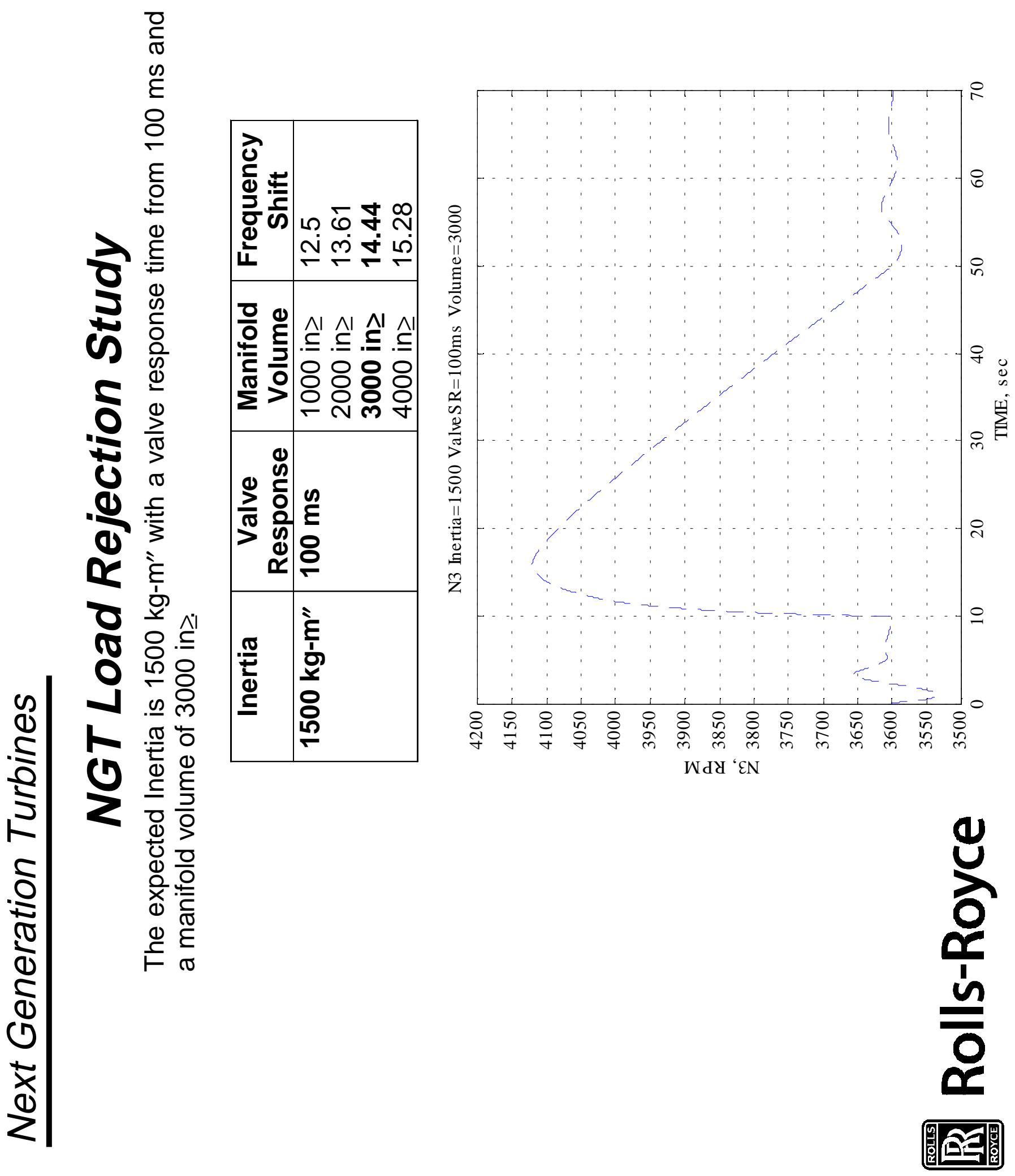




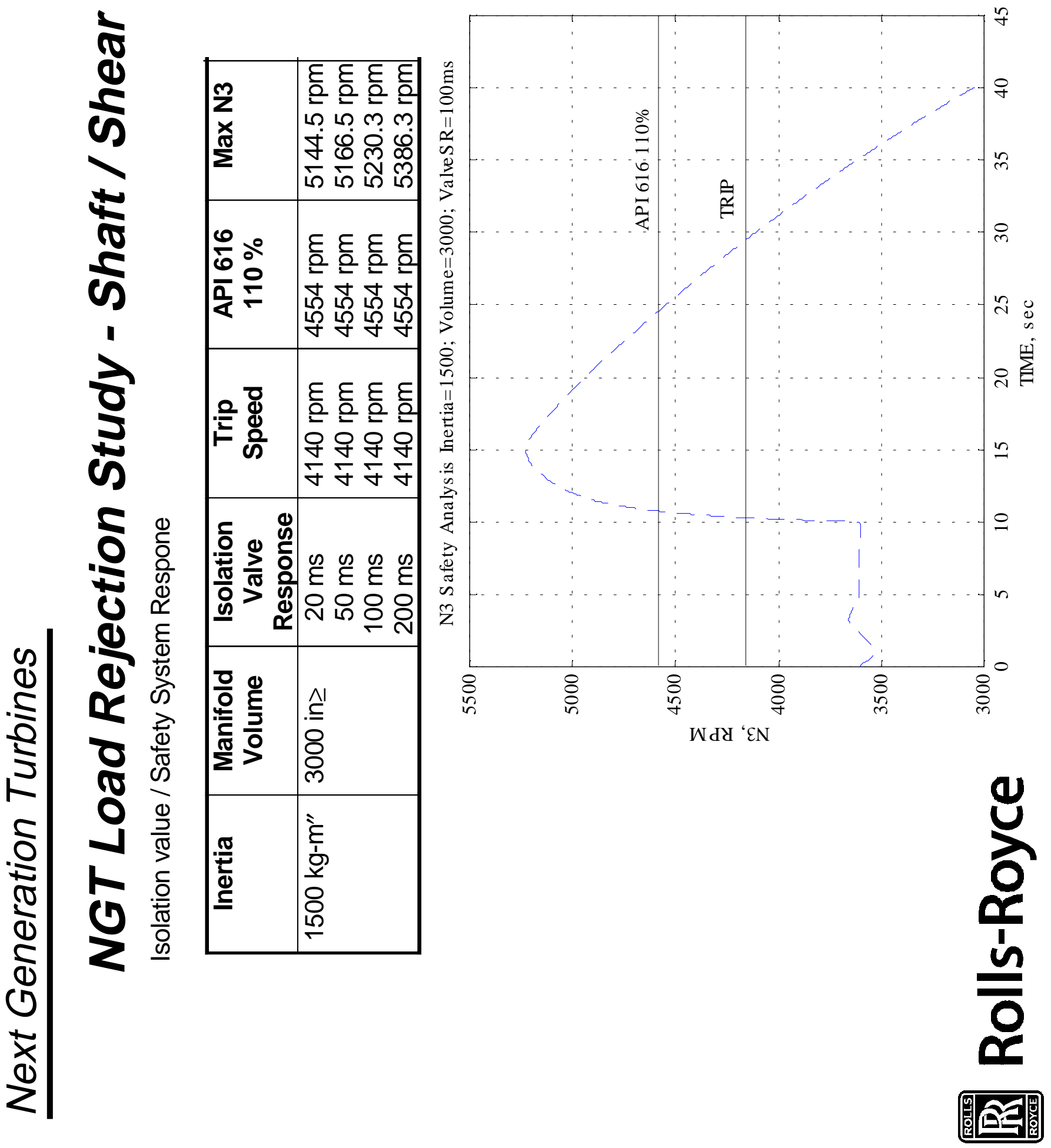



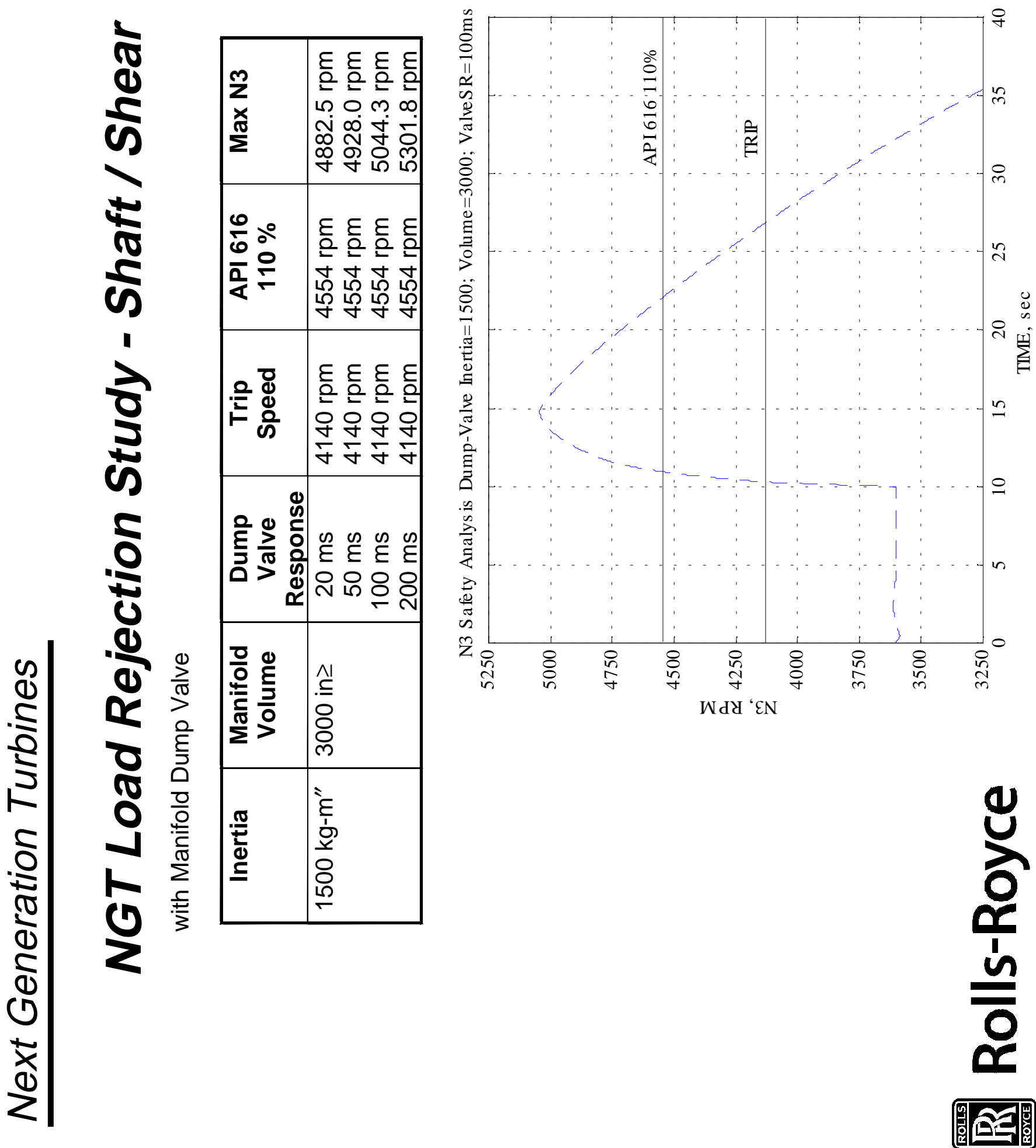


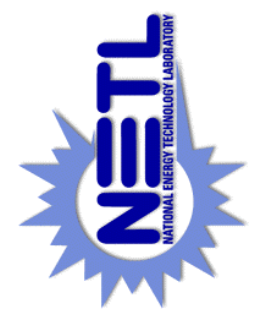

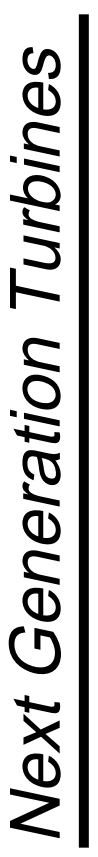
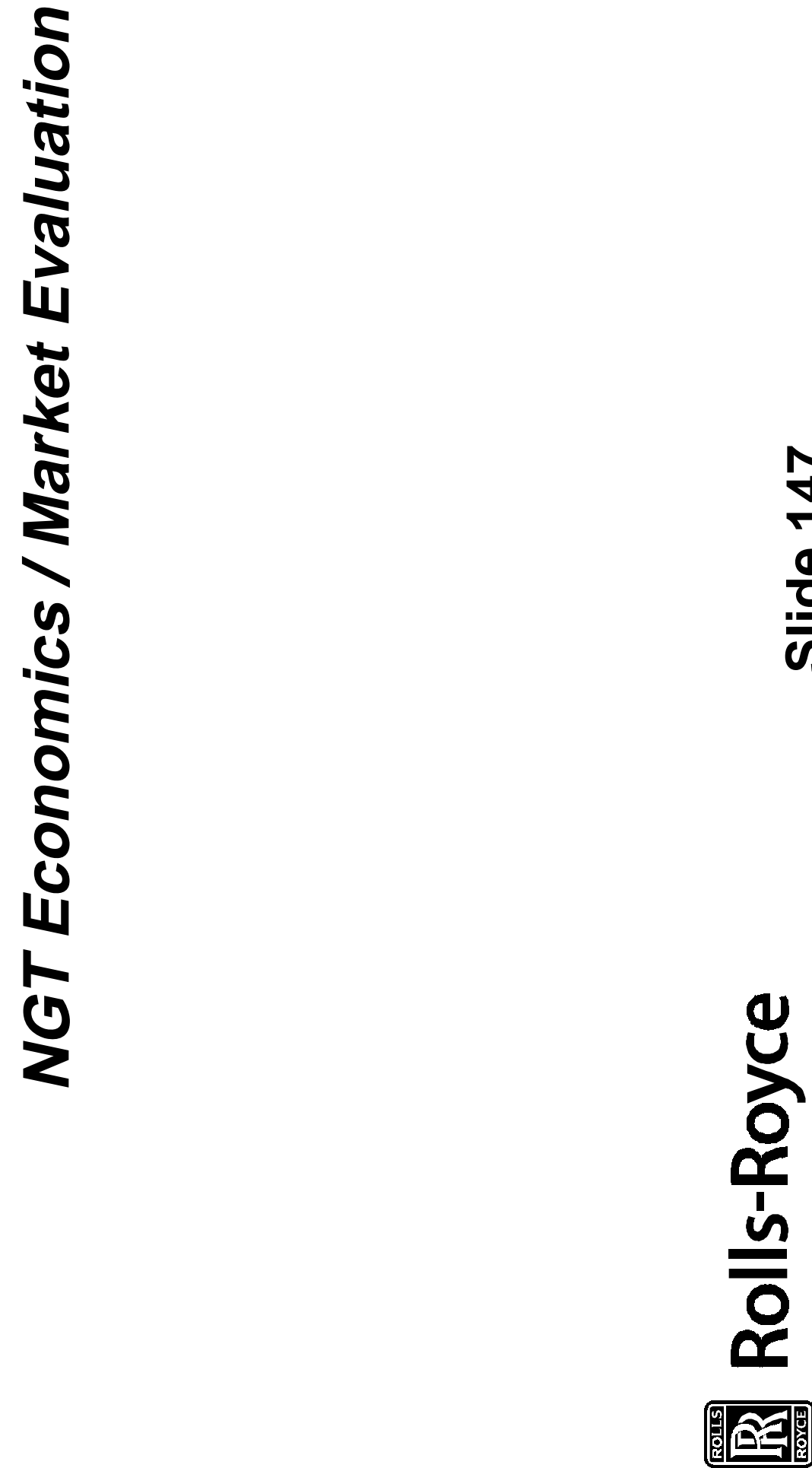

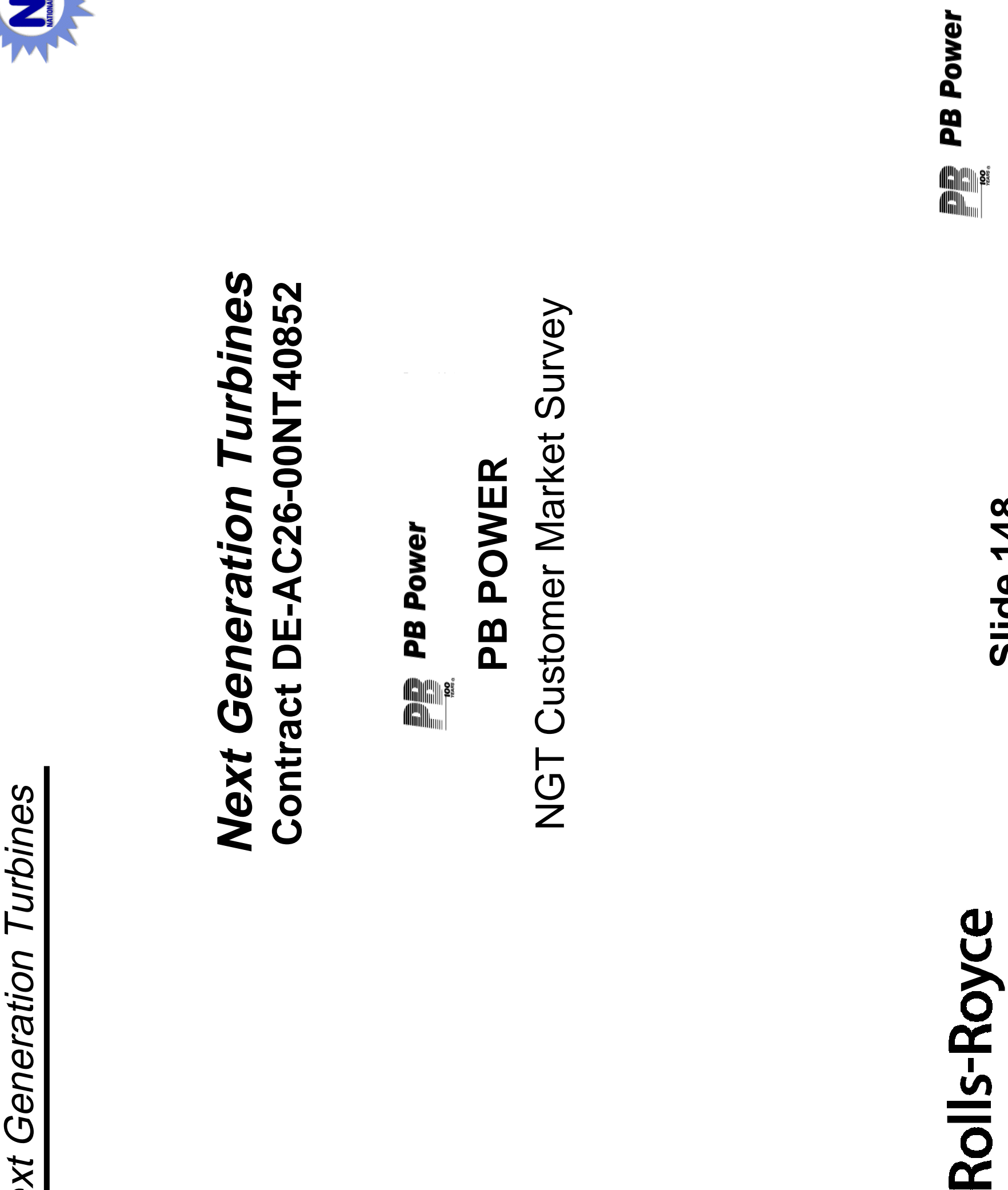


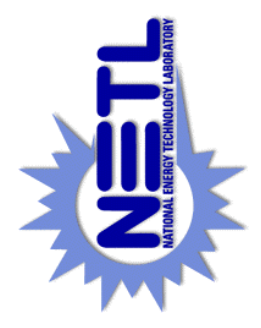

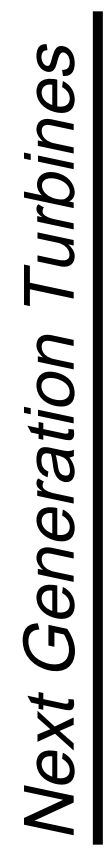
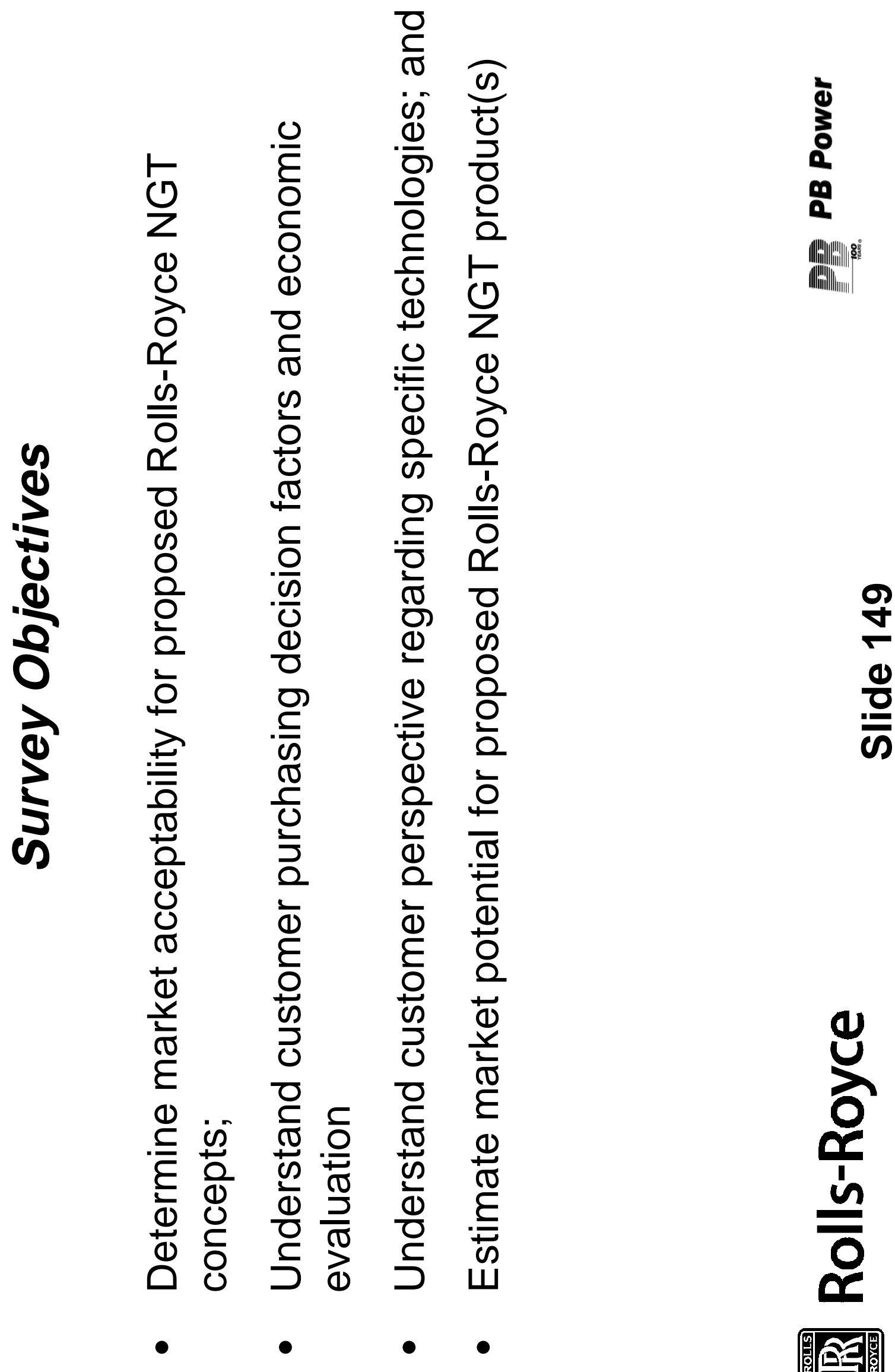


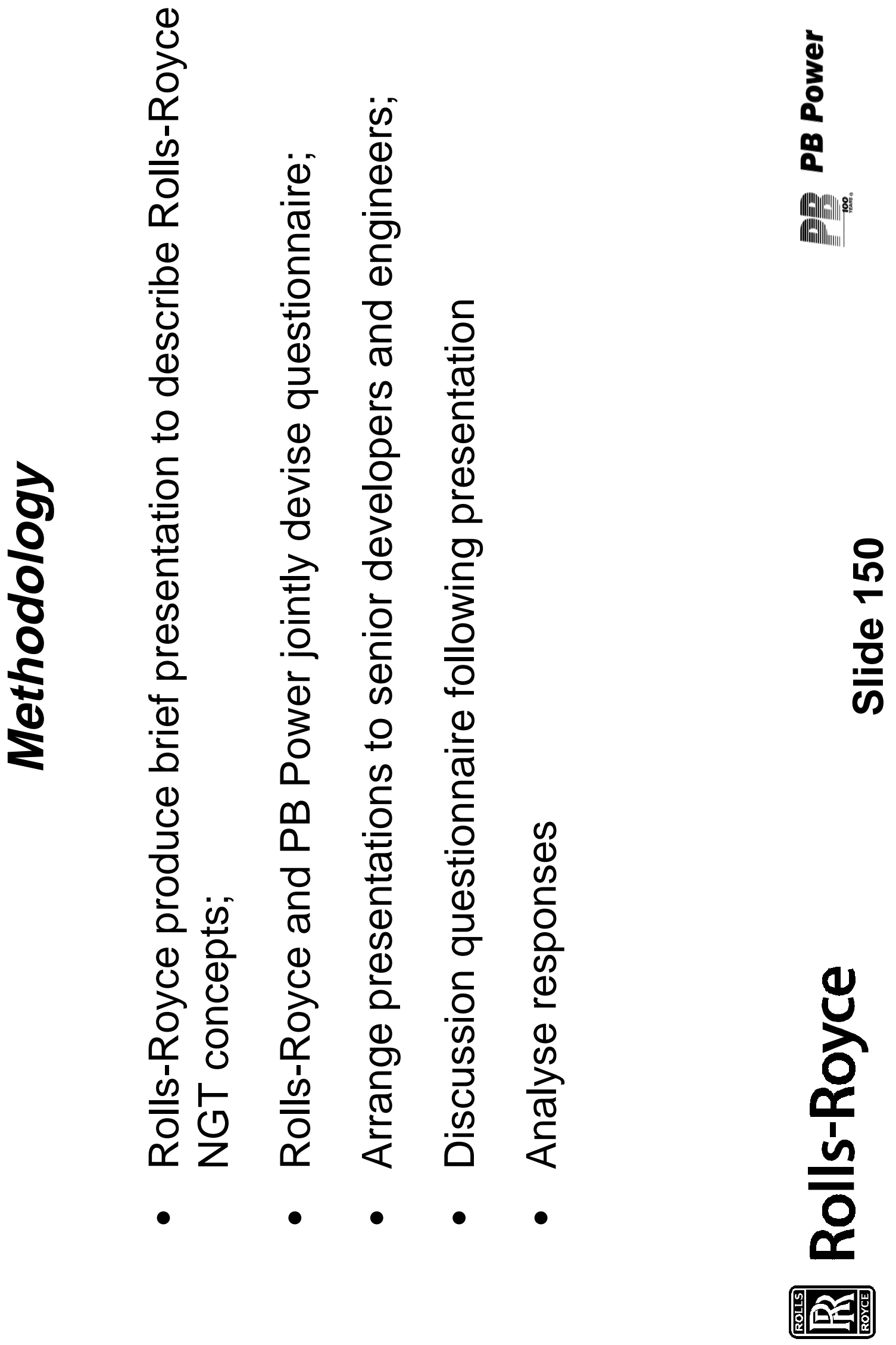




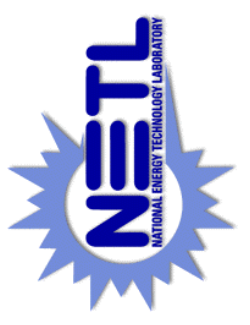

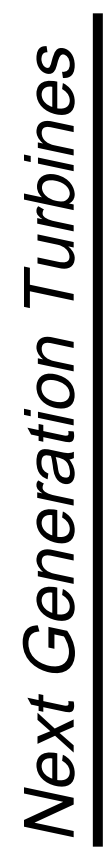

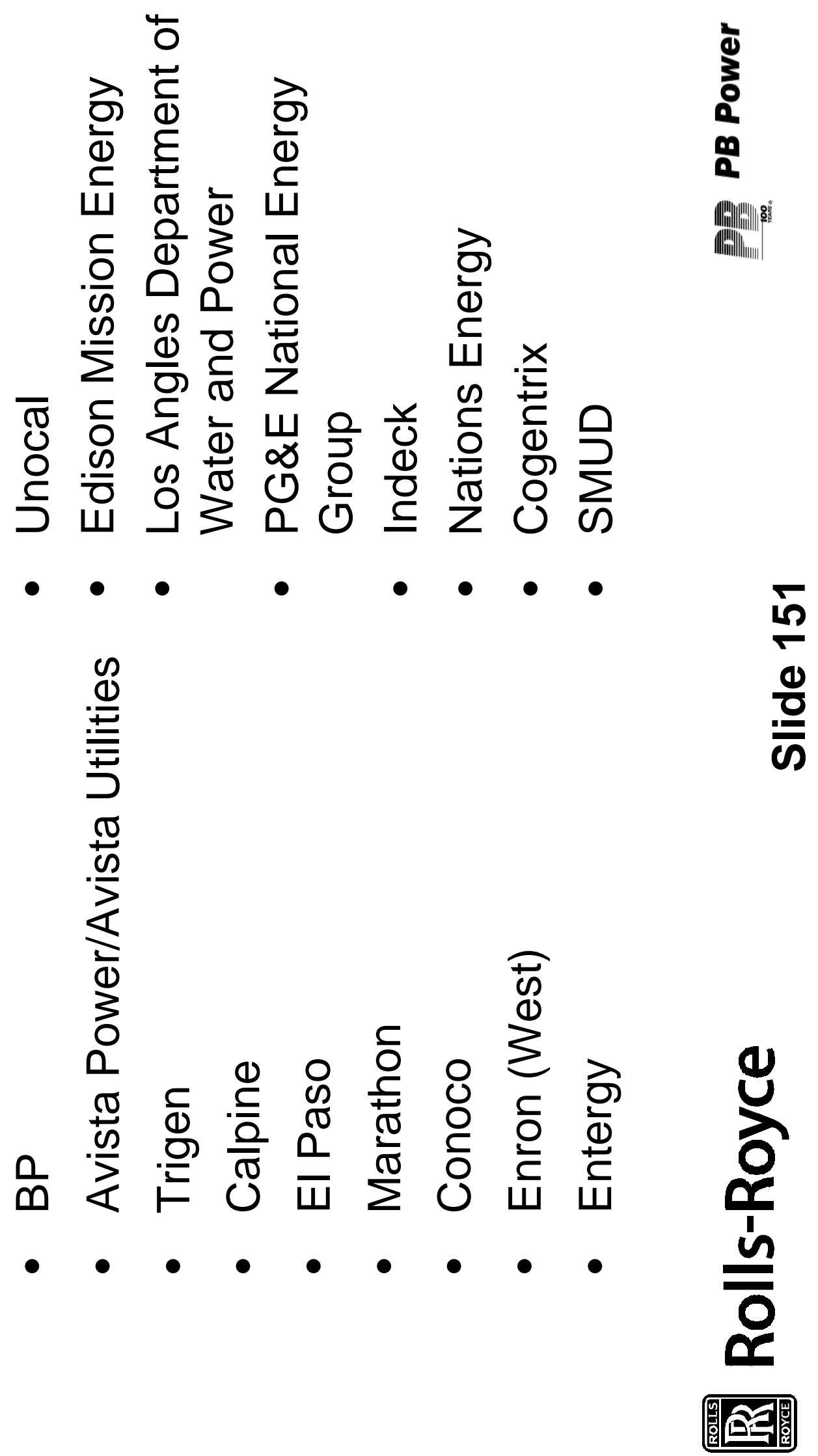




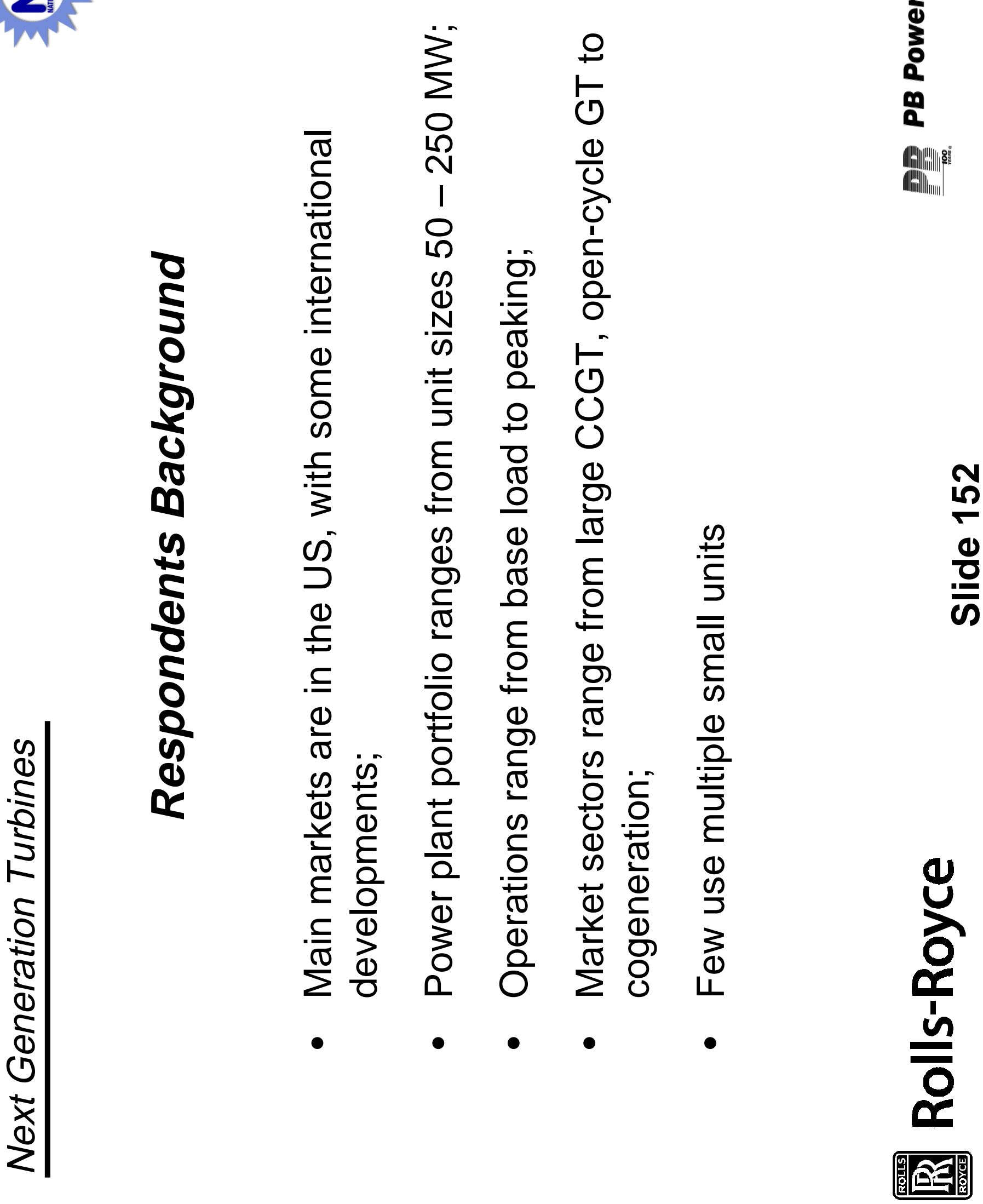




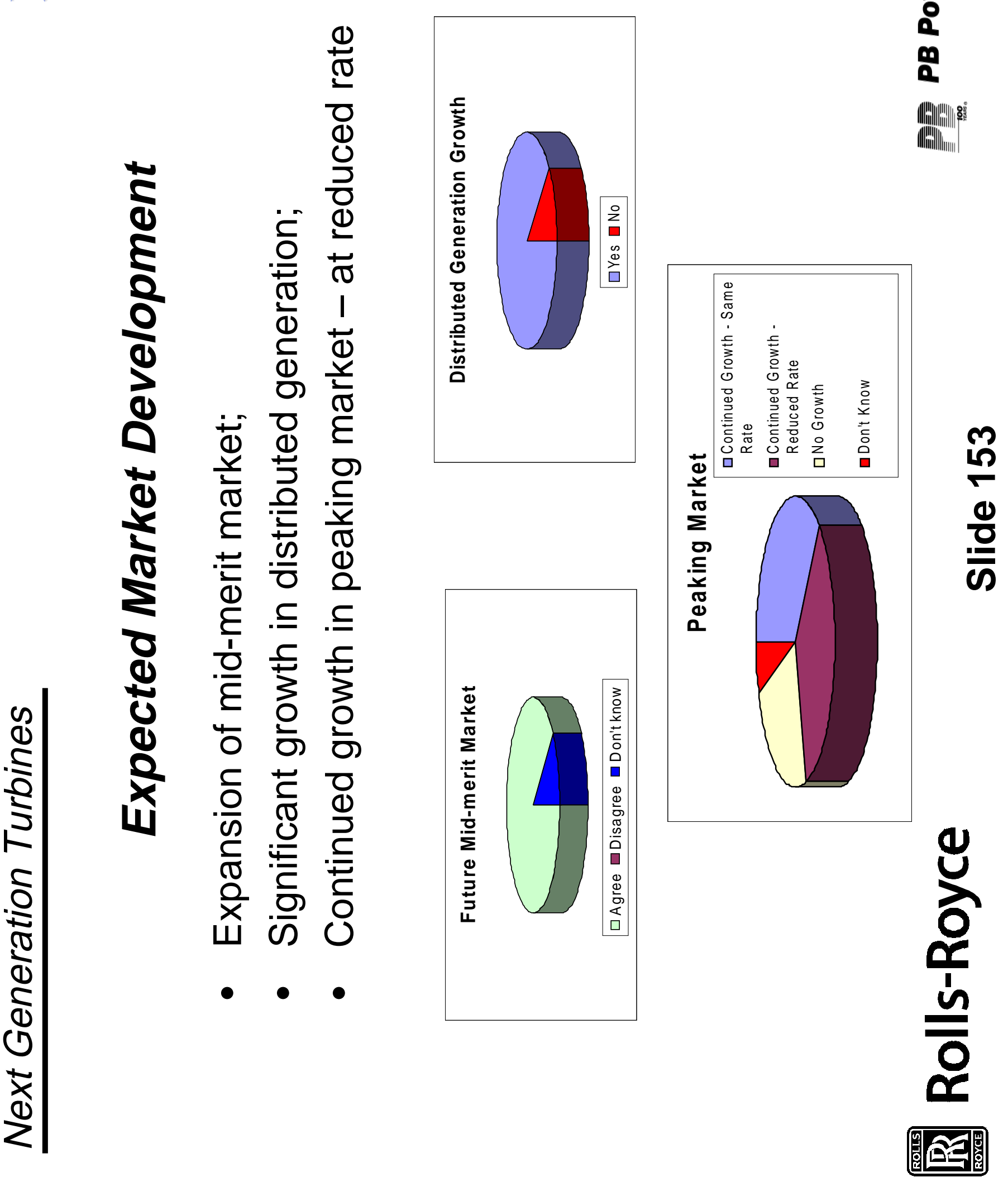




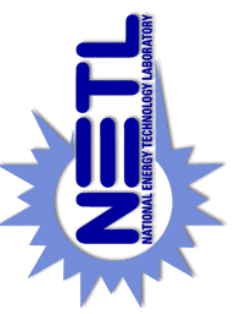

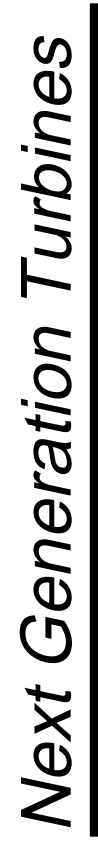
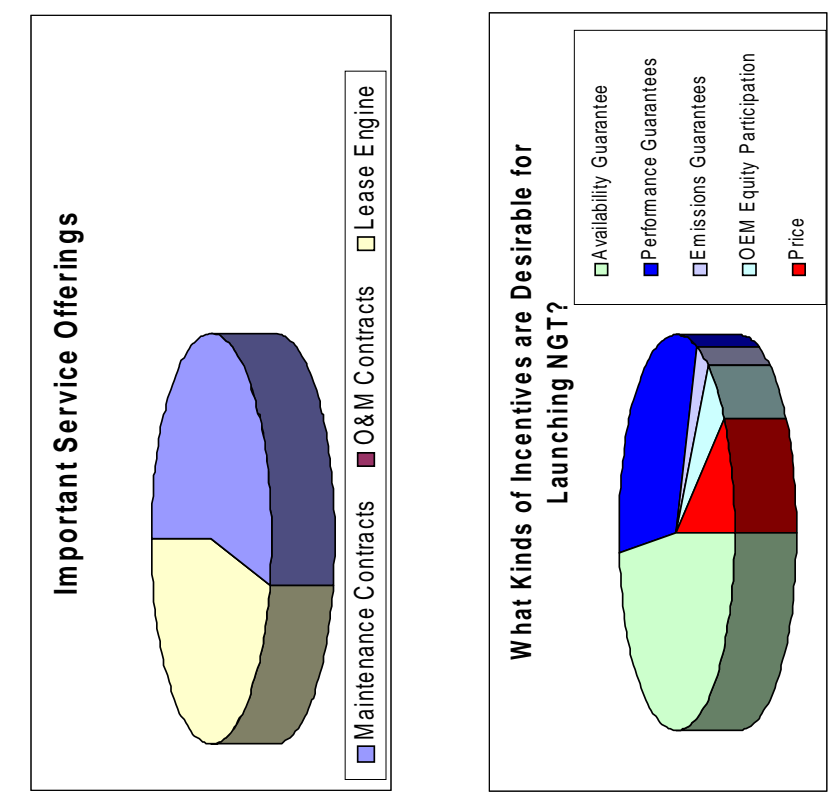

ฐ

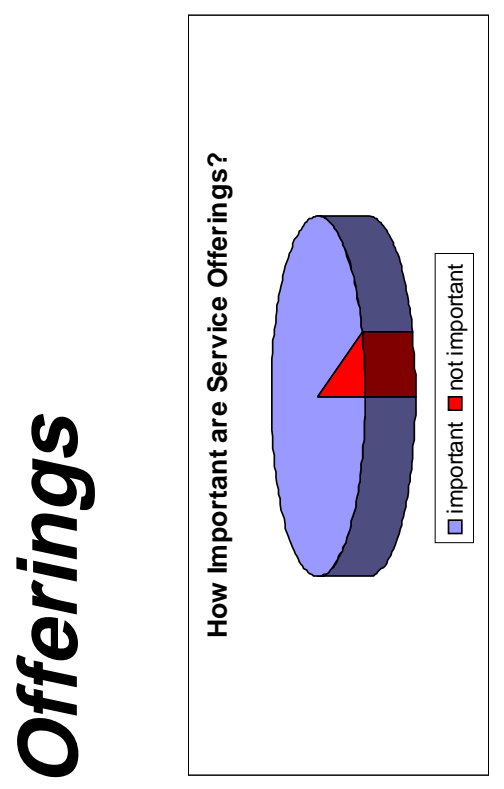

少

ญ

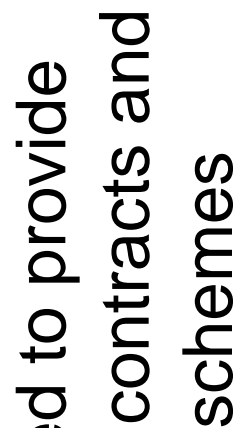

要

$\sum_{\text {ய }}$

$\frac{1}{x}$

(1)

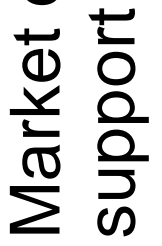

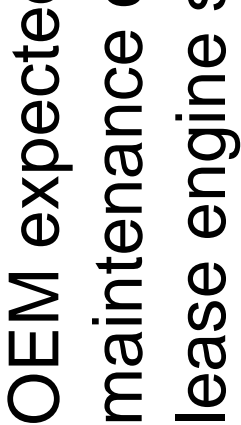

(1)

음

Oे

으 정

우융ㅁㅇ

品

$\Phi$ ㅇ

을 ज

(1) ฮ ฮ

ऐ

$\frac{8}{}$

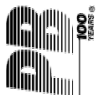




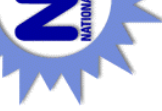

क)
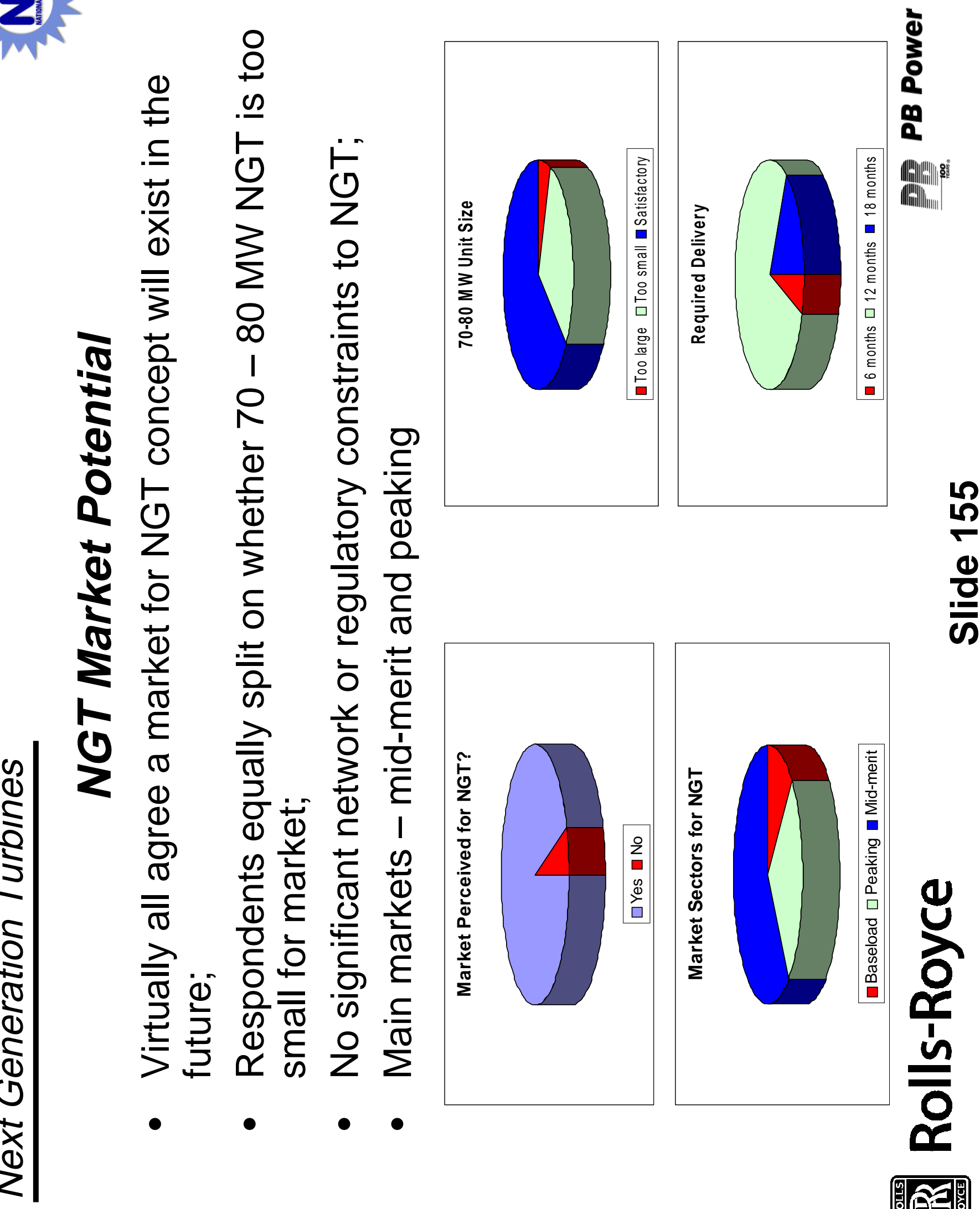

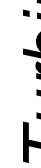

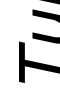

ธิ
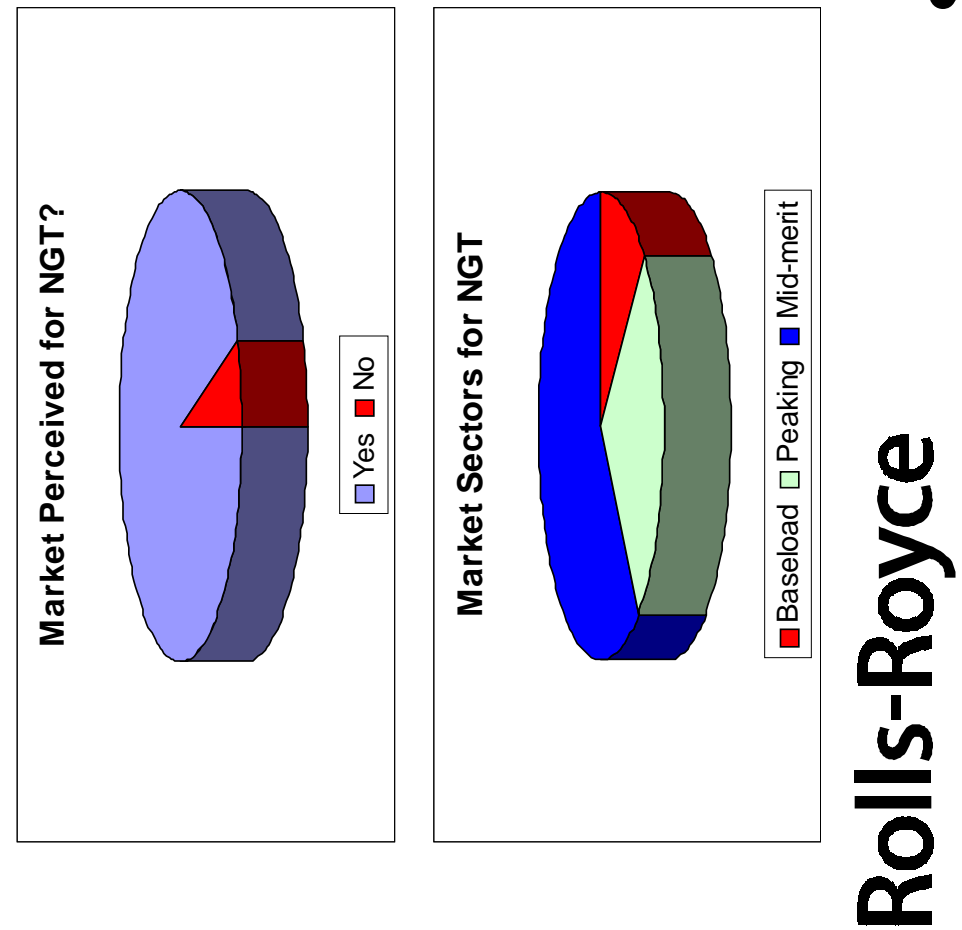

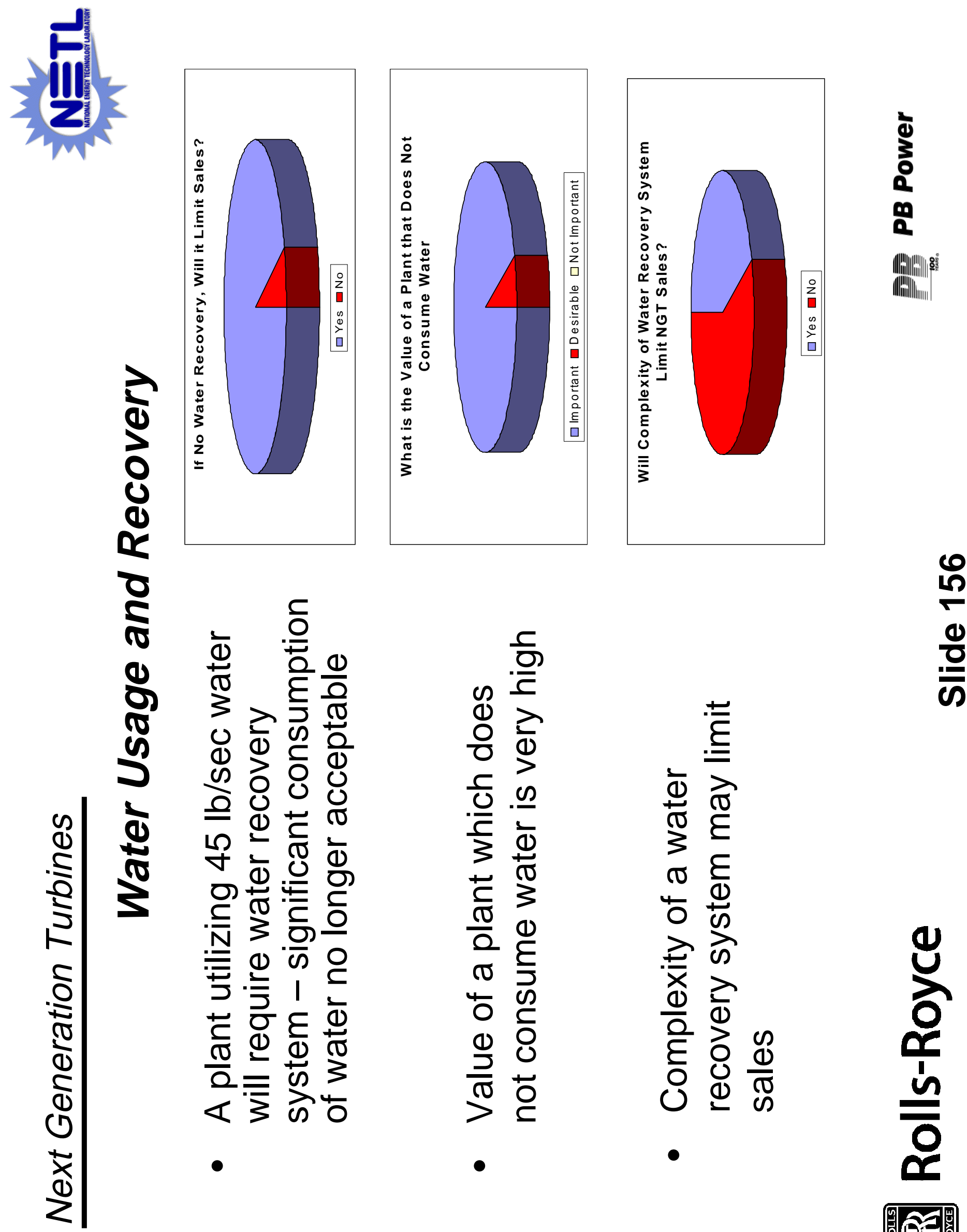

$\frac{0}{0}$

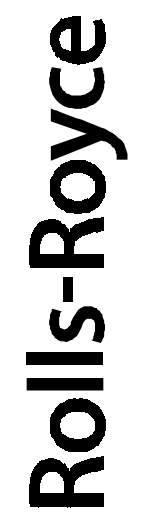




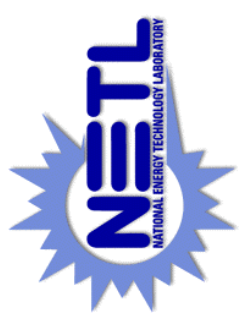

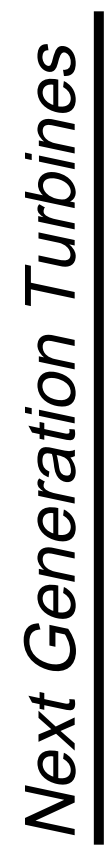
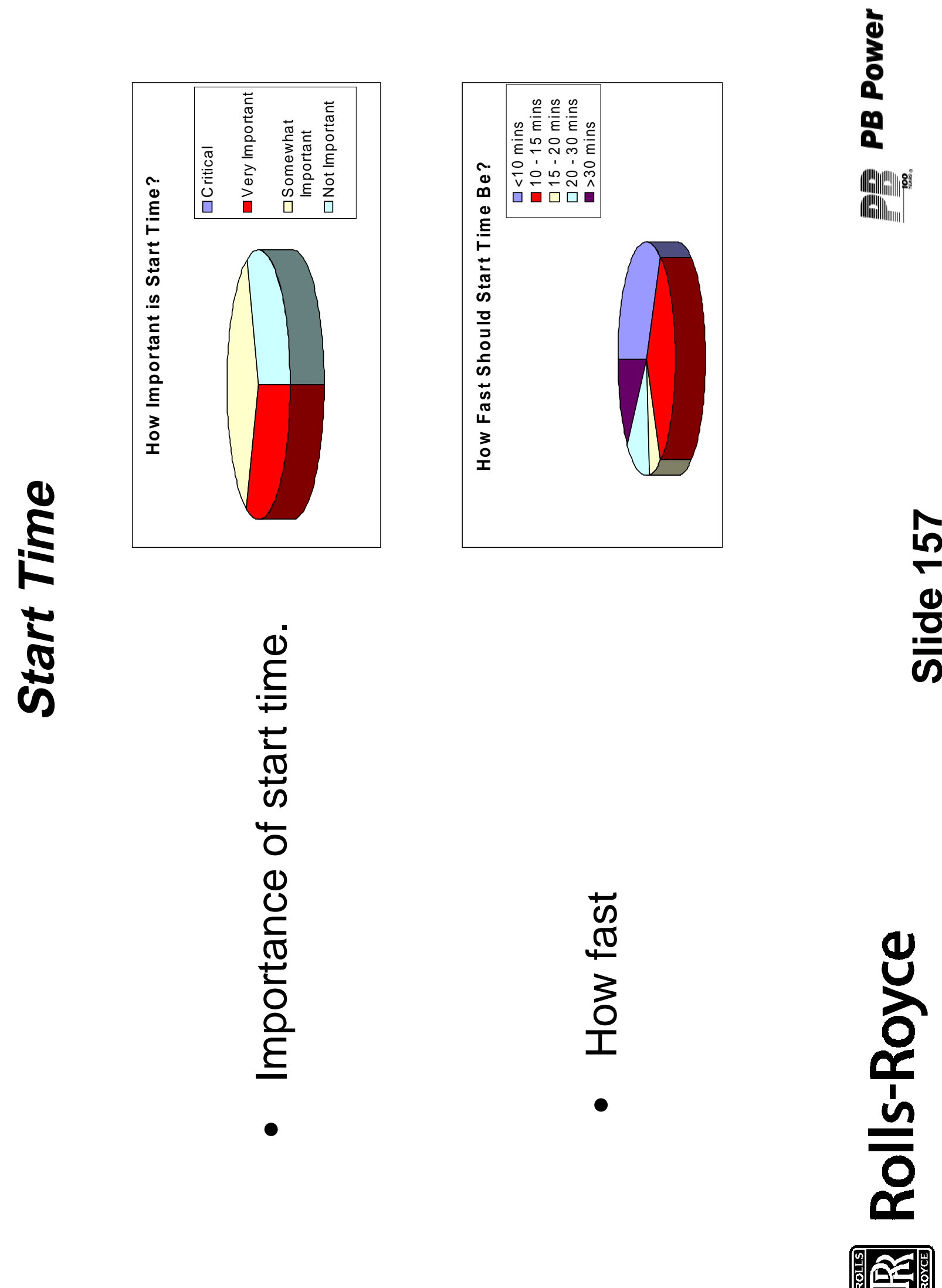

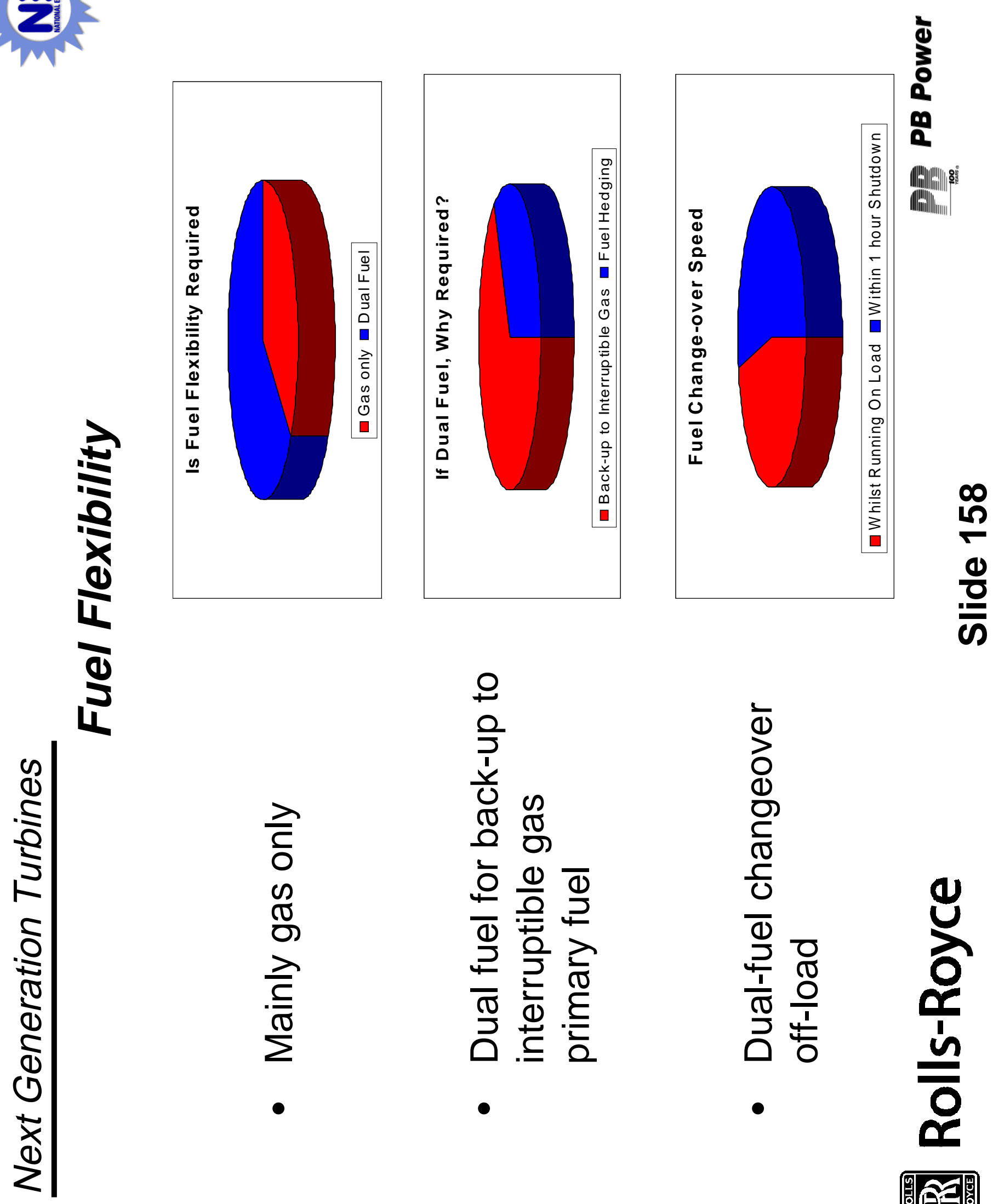


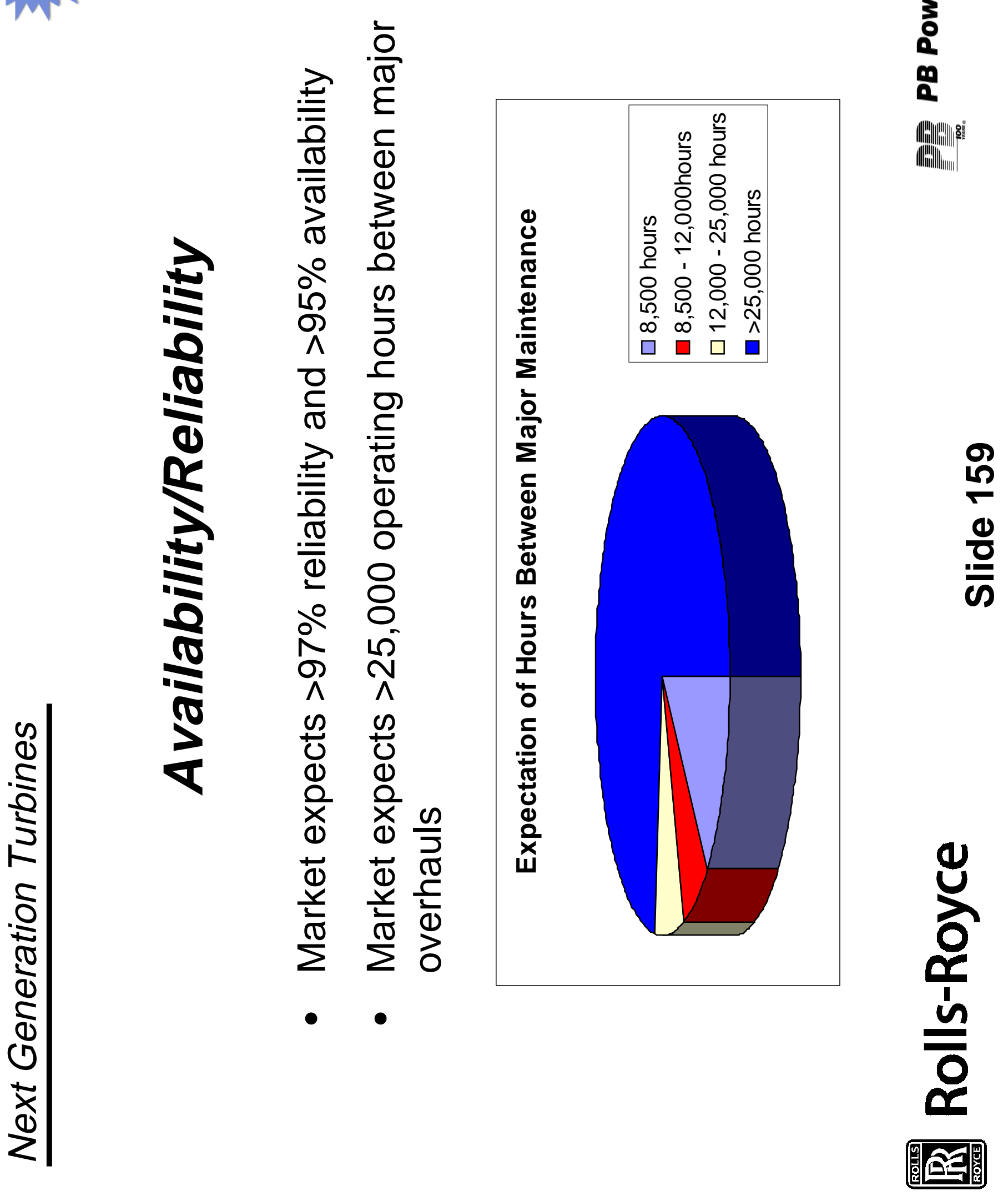



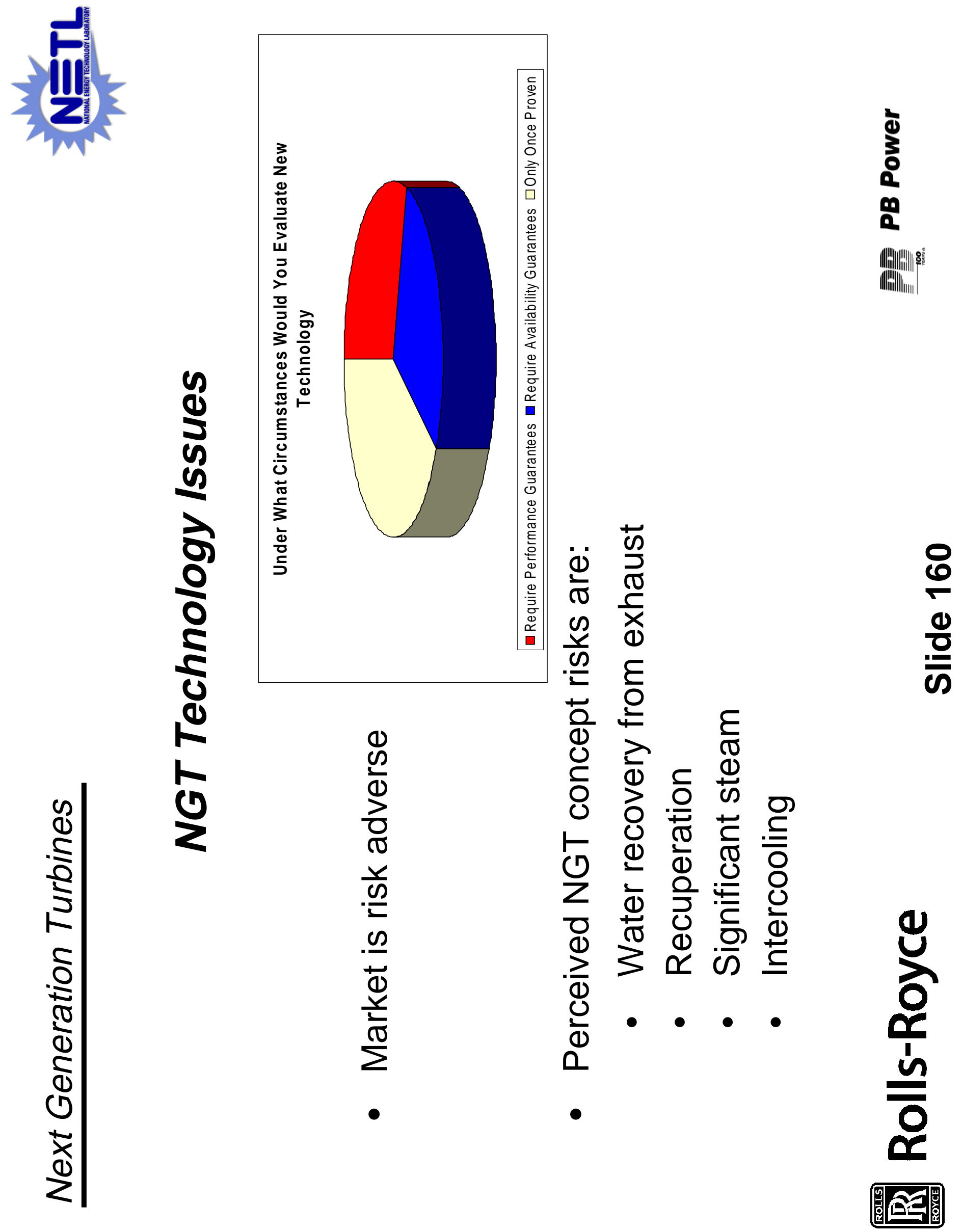

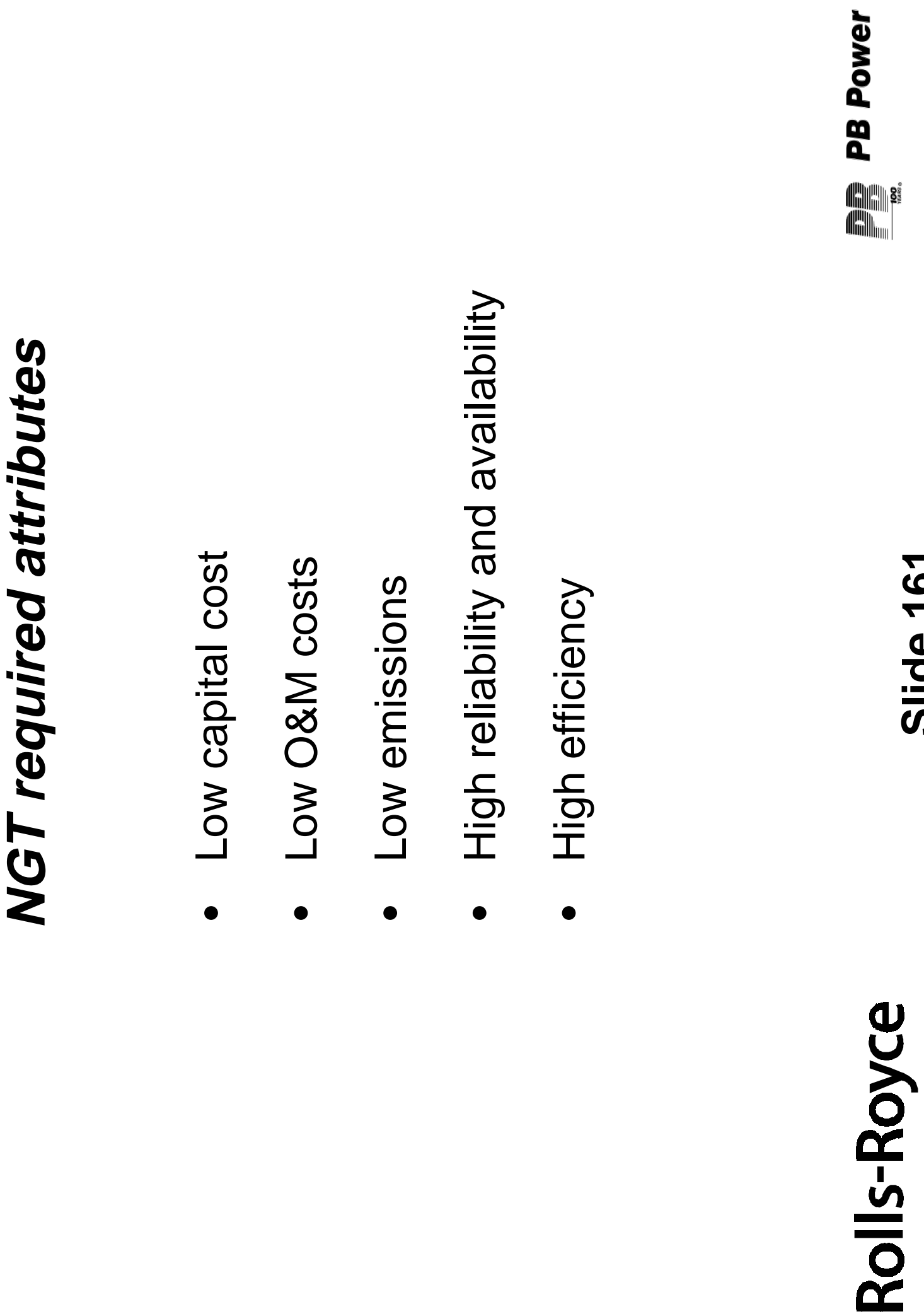


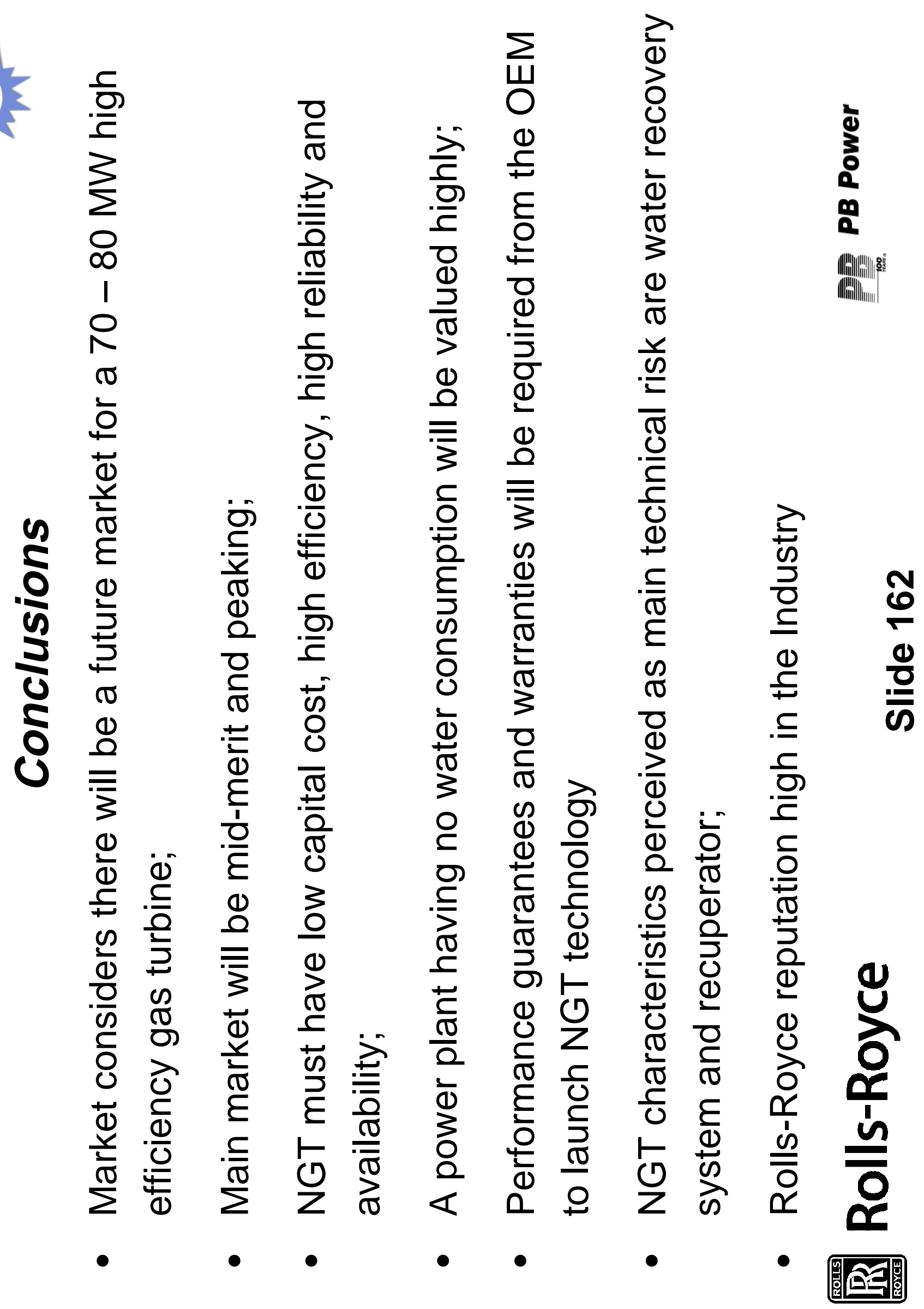




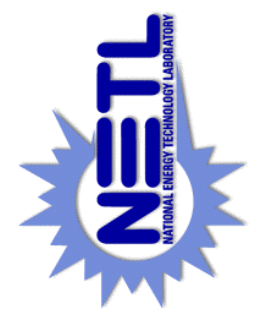

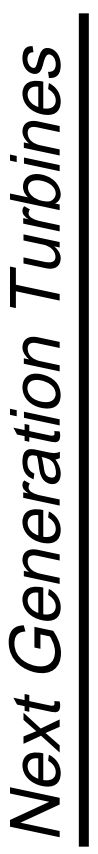
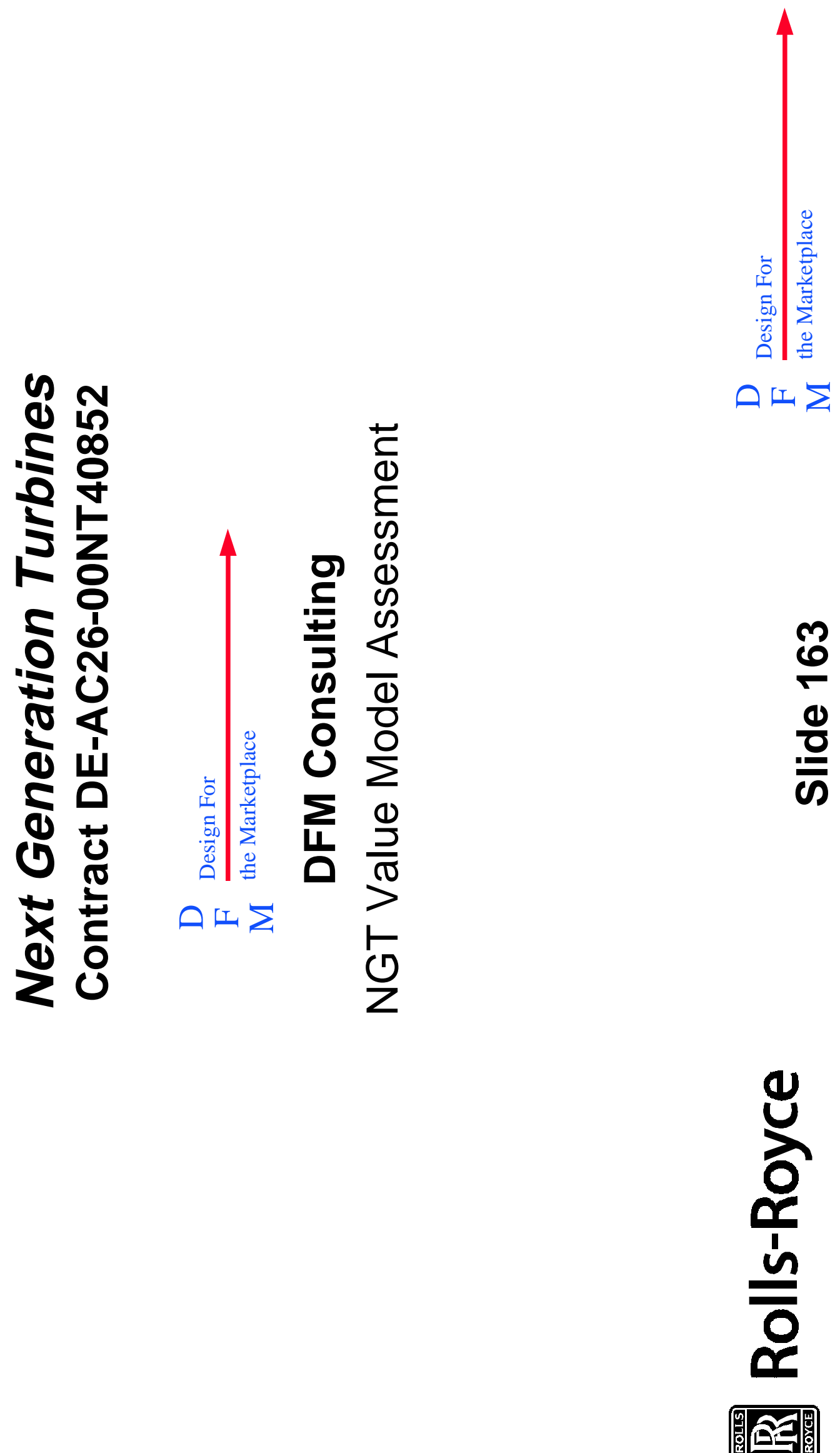


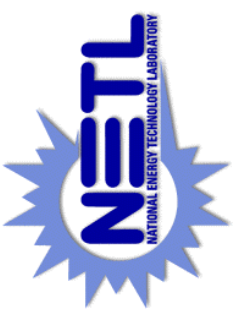

0
0
$\frac{1}{2}$
5
$\frac{1}{2}$
$\frac{0}{0}$
$\frac{0}{0}$
$\frac{1}{0}$
0
$\frac{x}{x}$
$\frac{1}{z}$

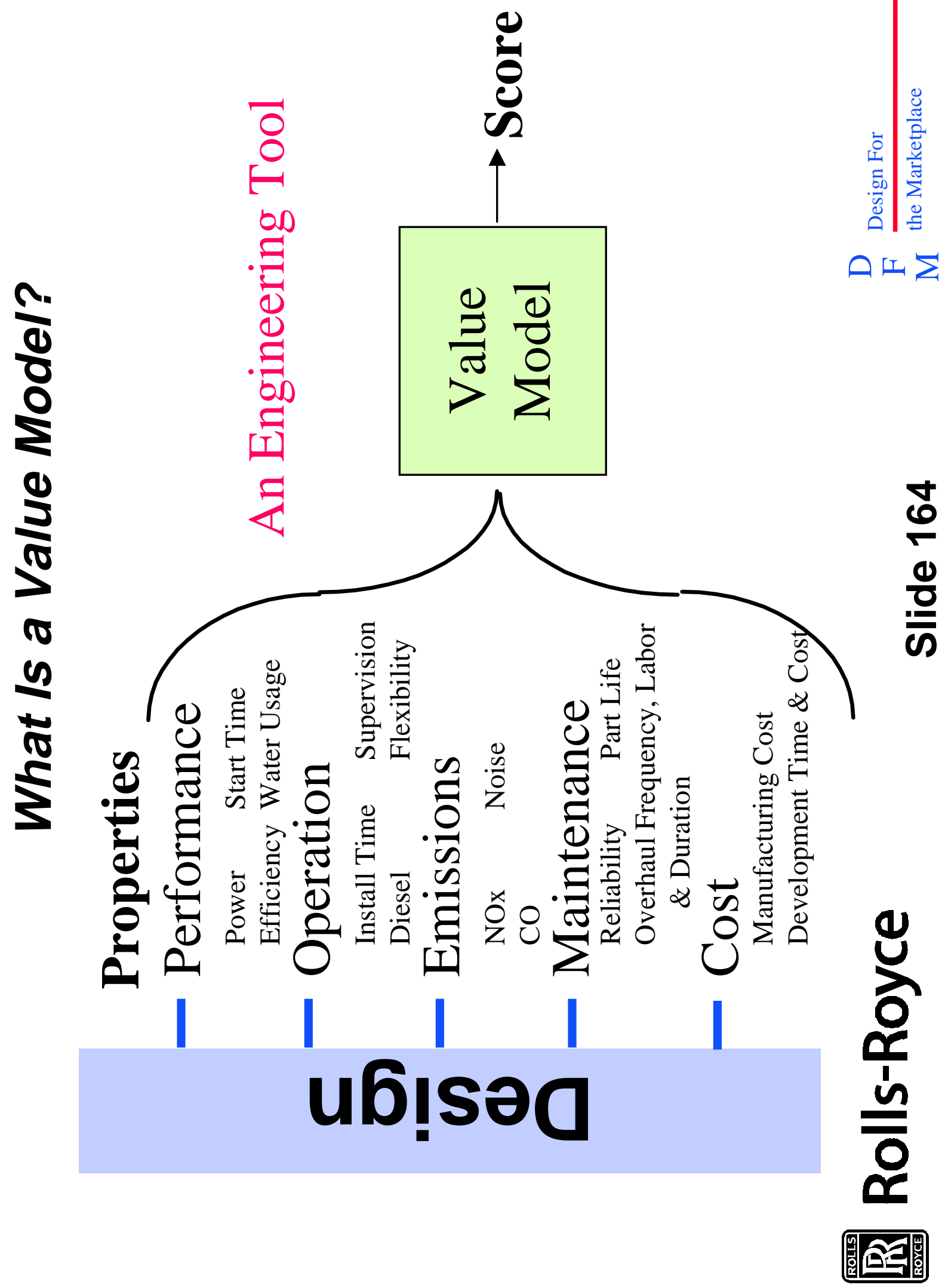



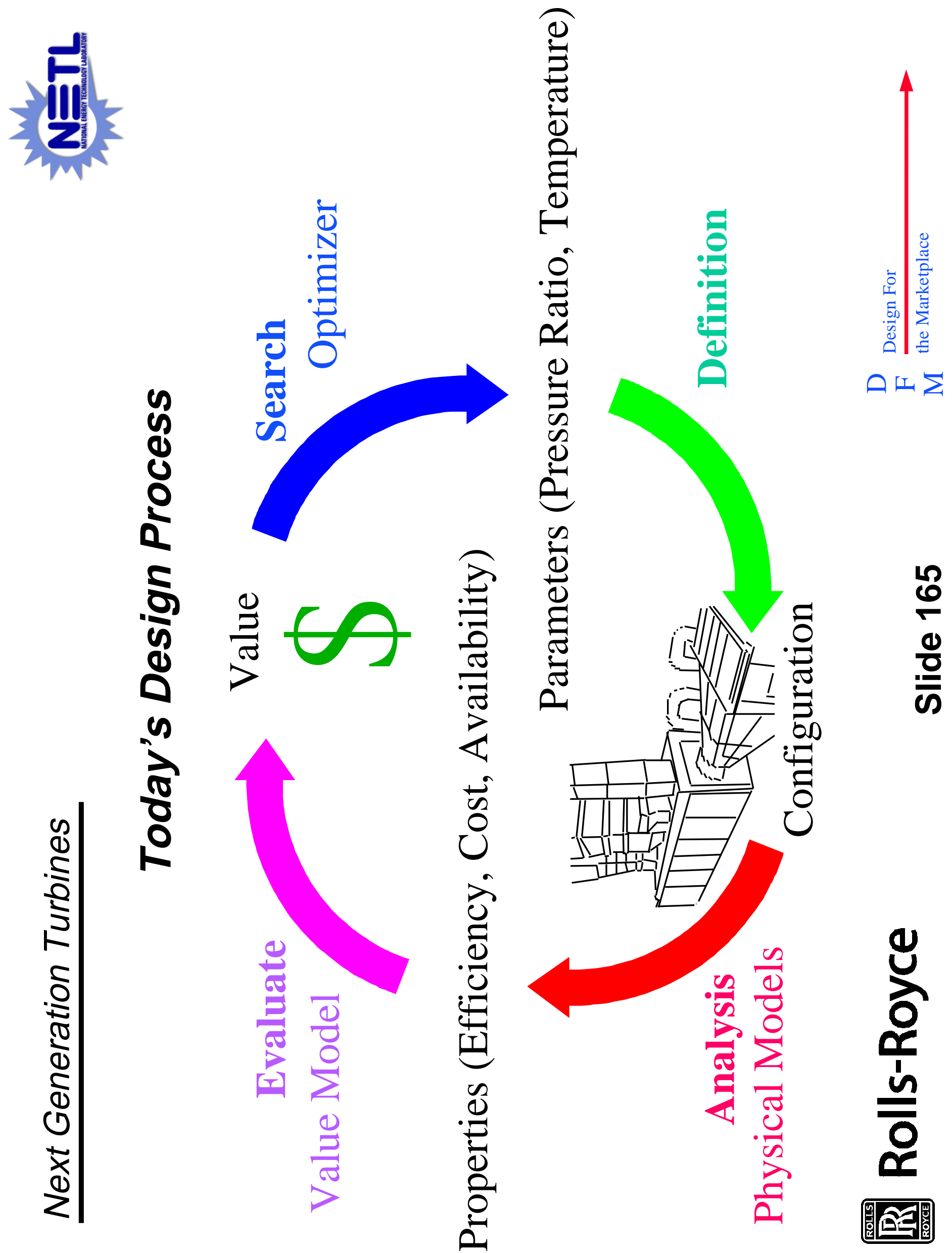

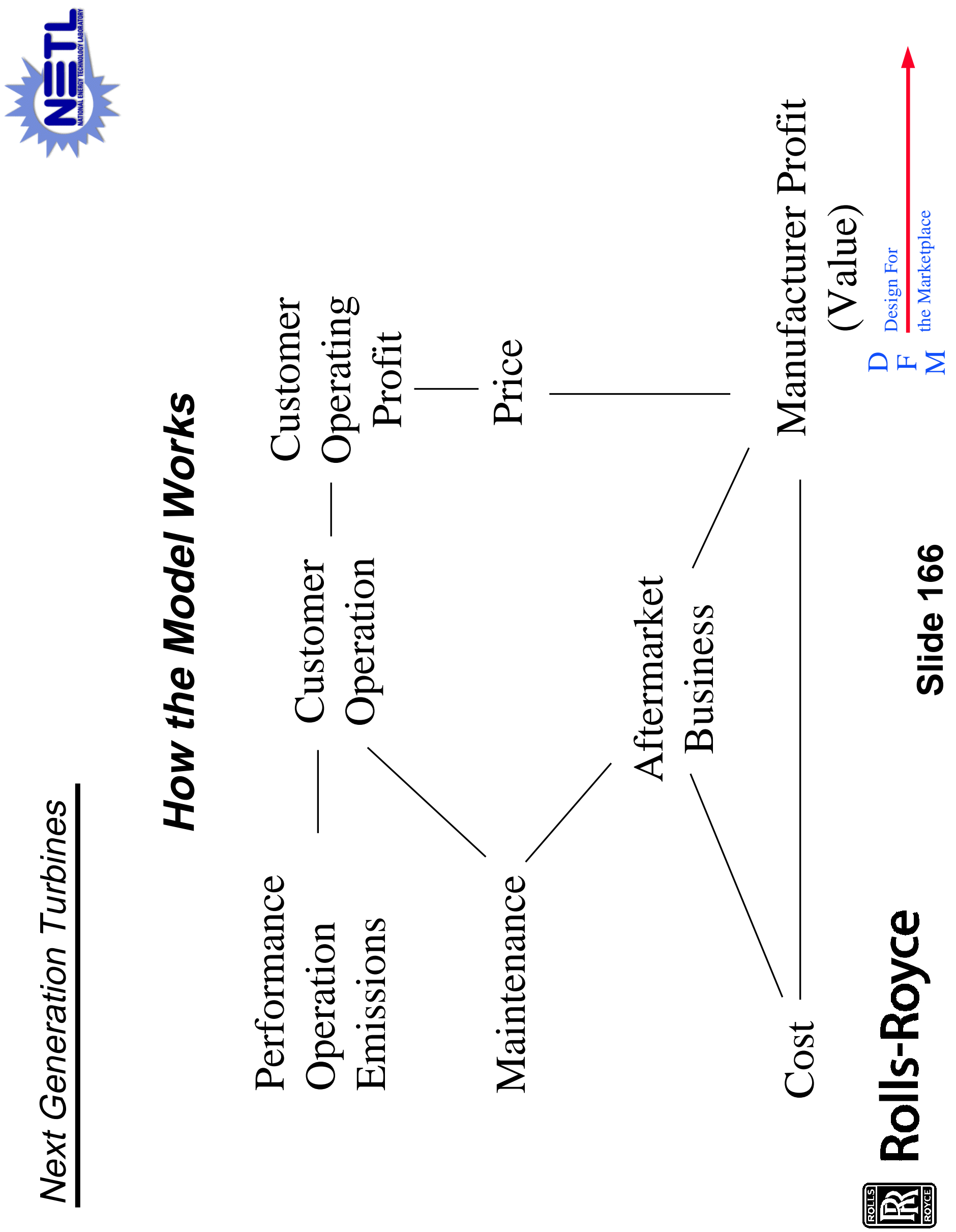


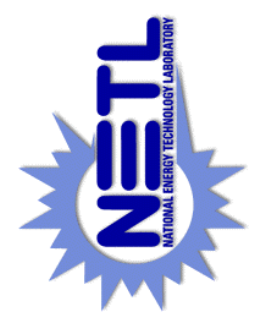

言
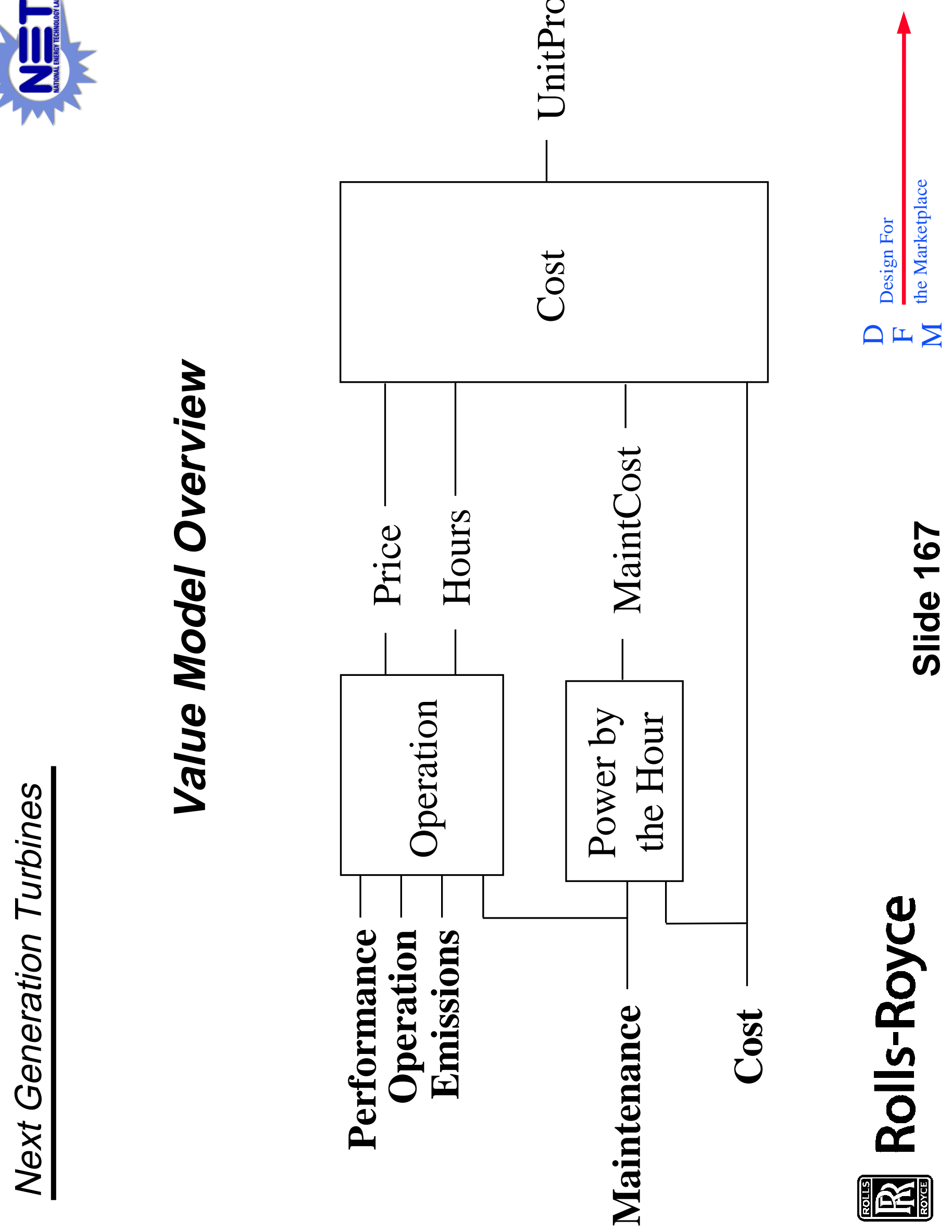

$\frac{\hat{0}}{\frac{0}{0}}$

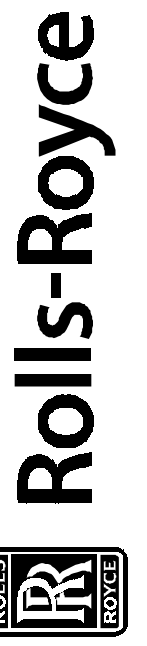



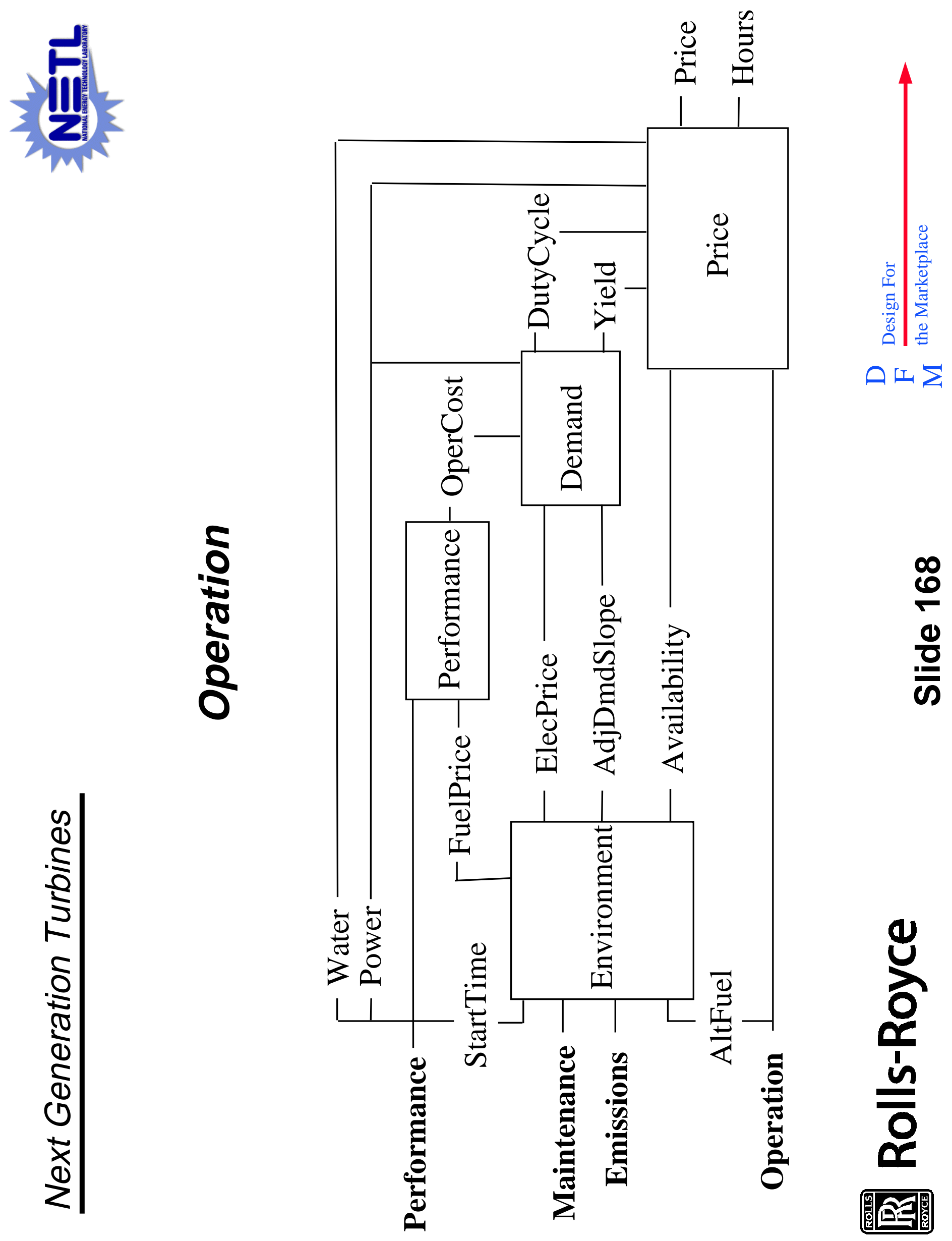

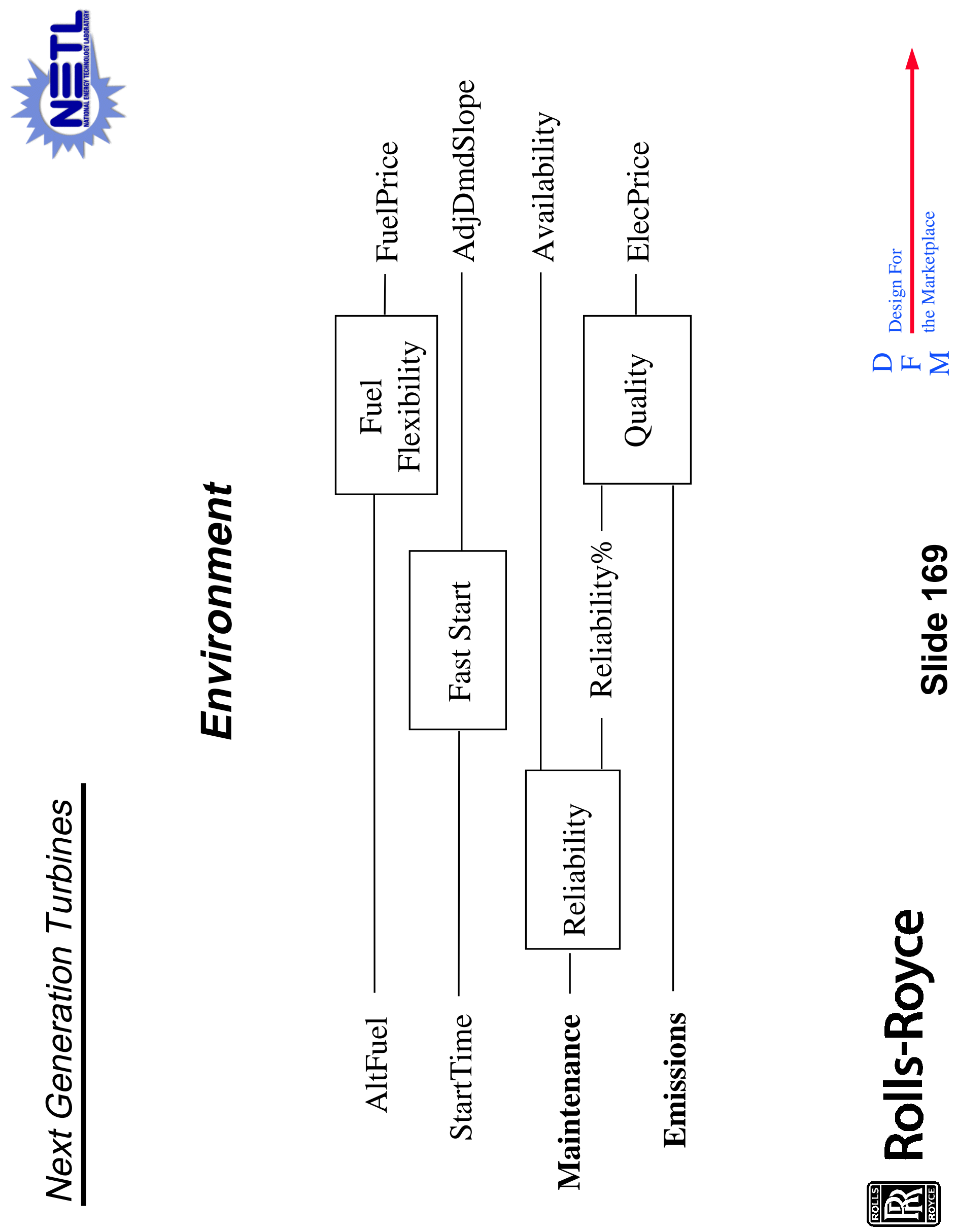

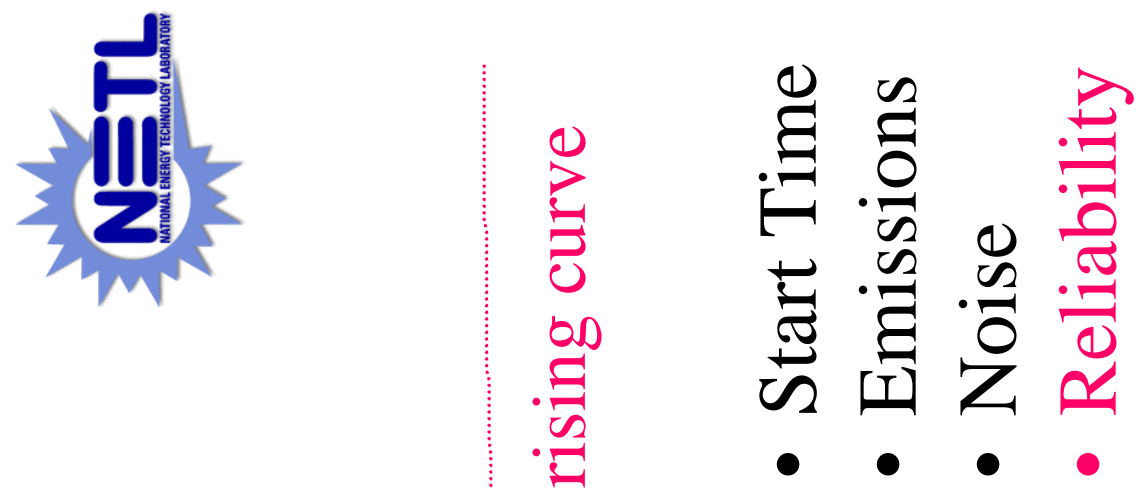

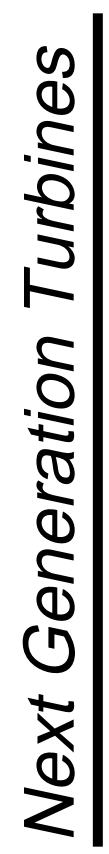
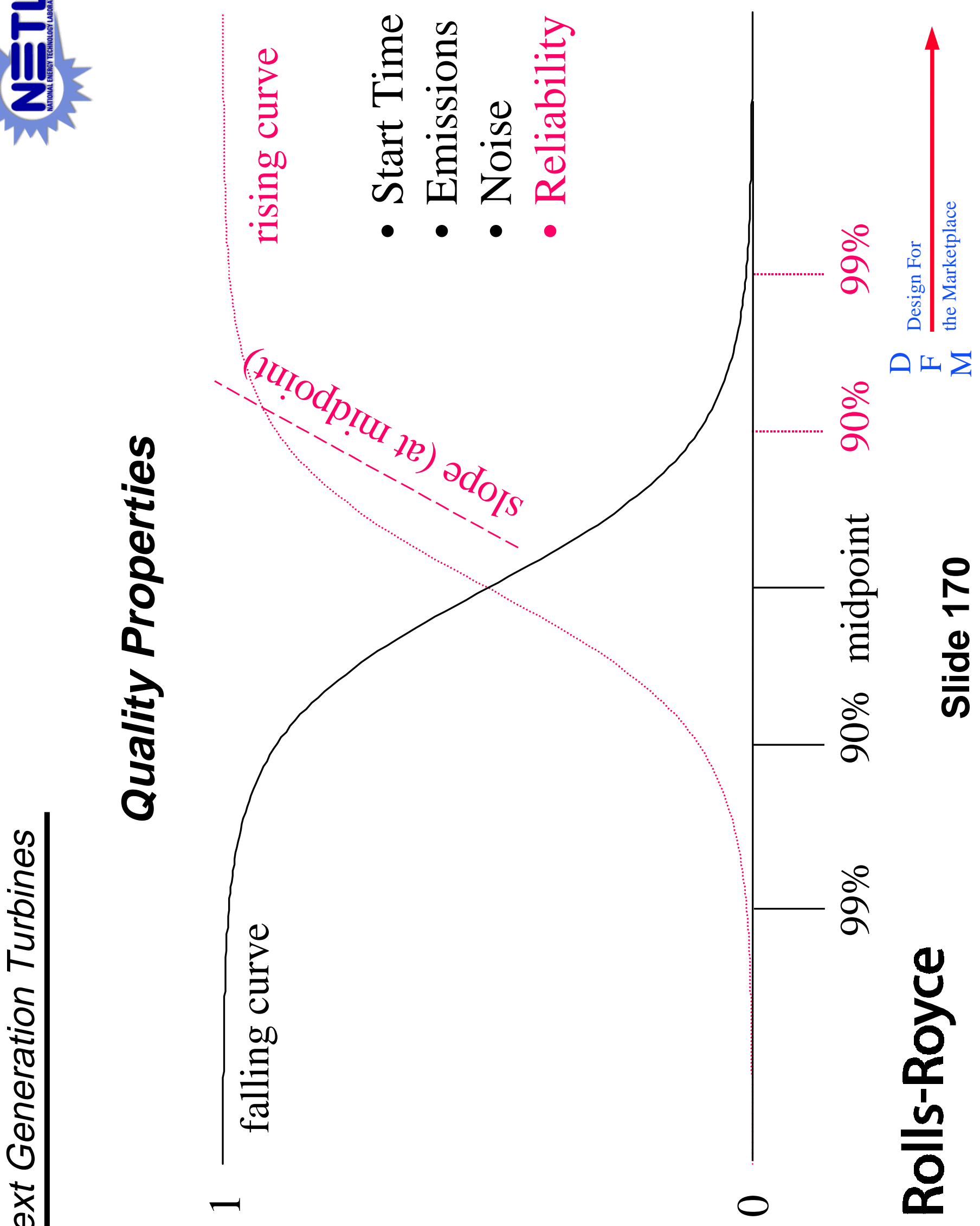

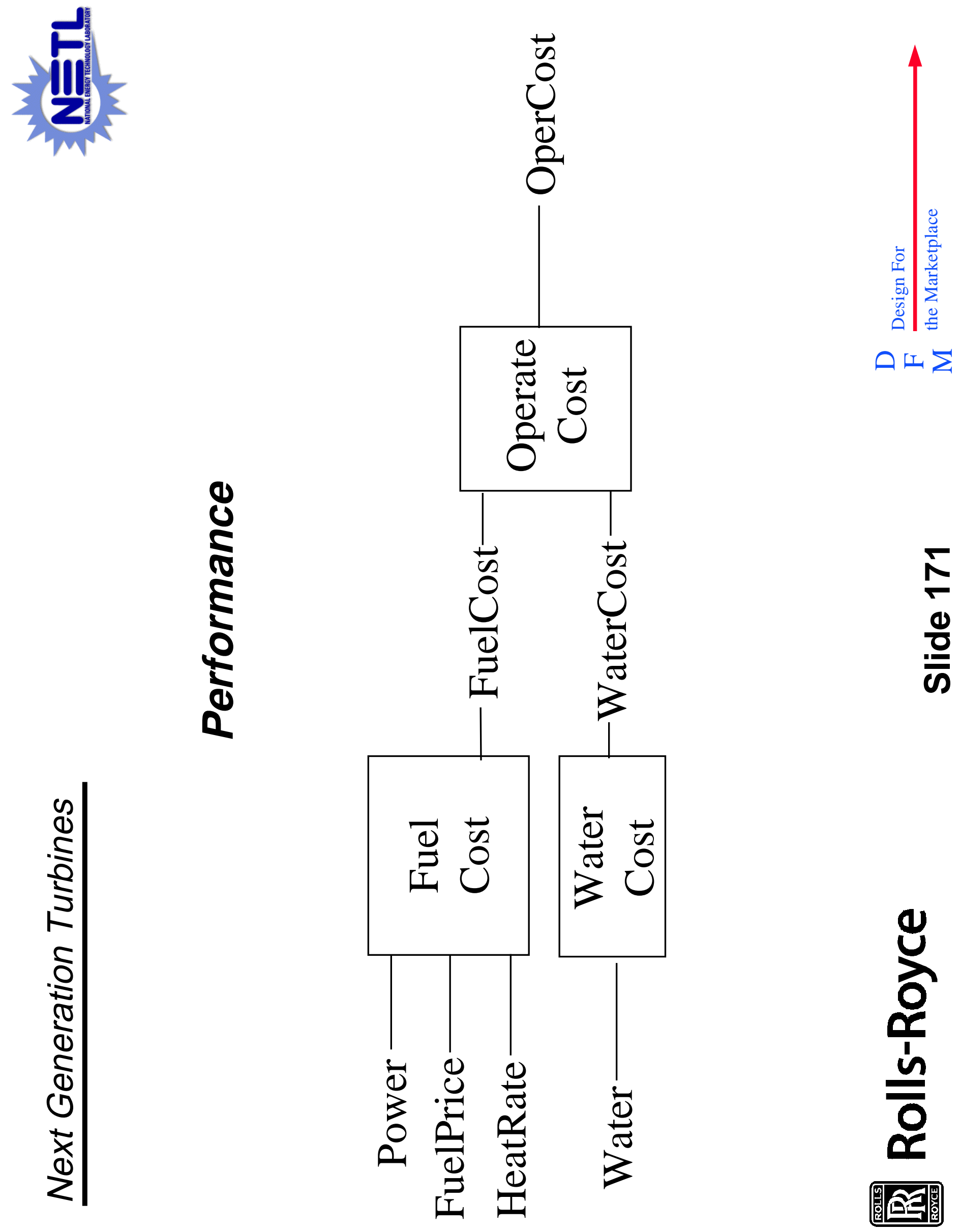

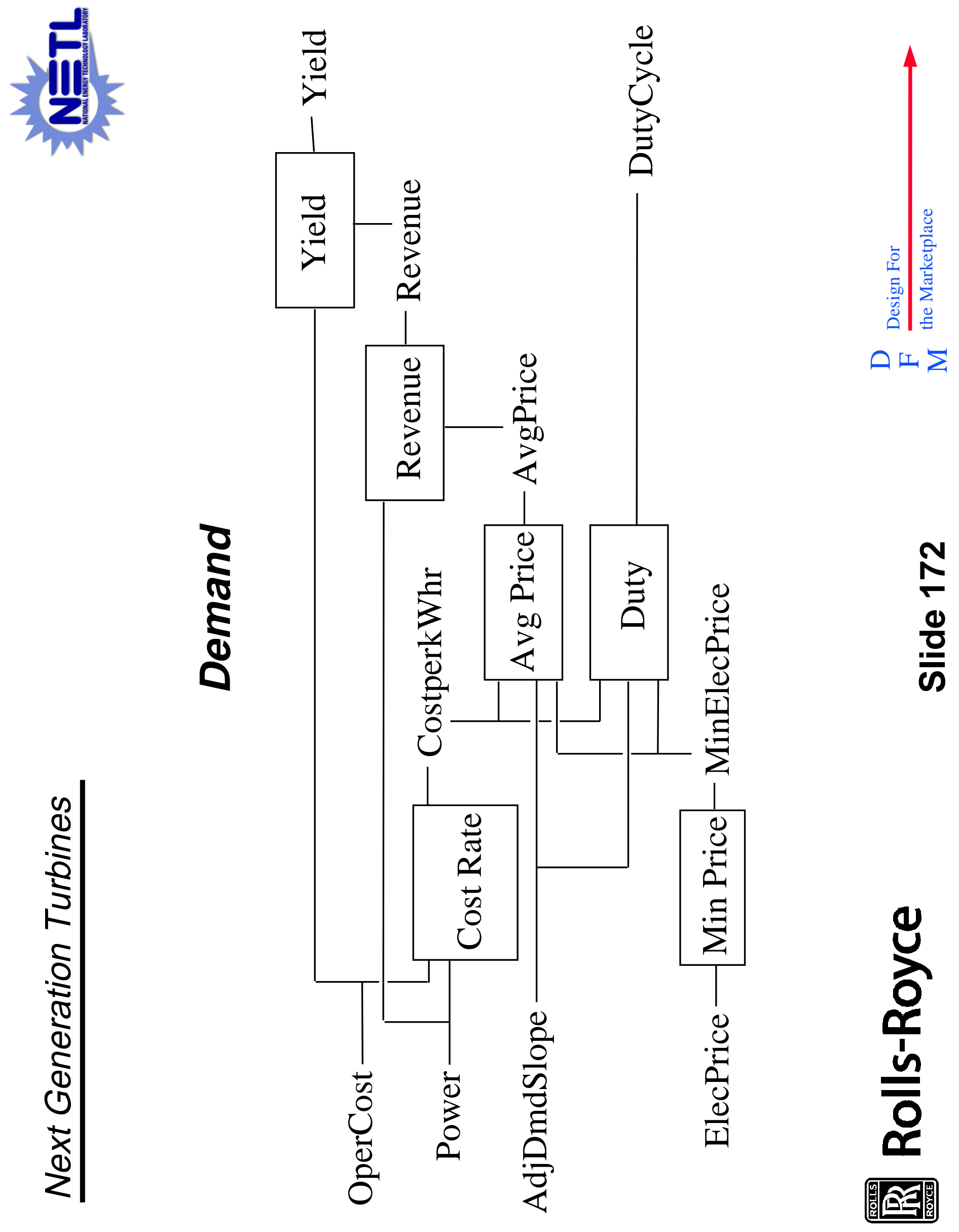


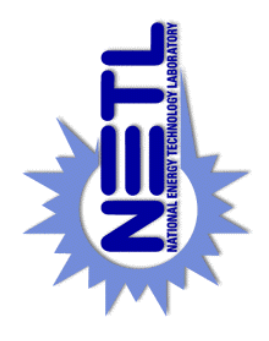

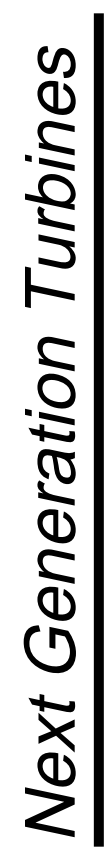

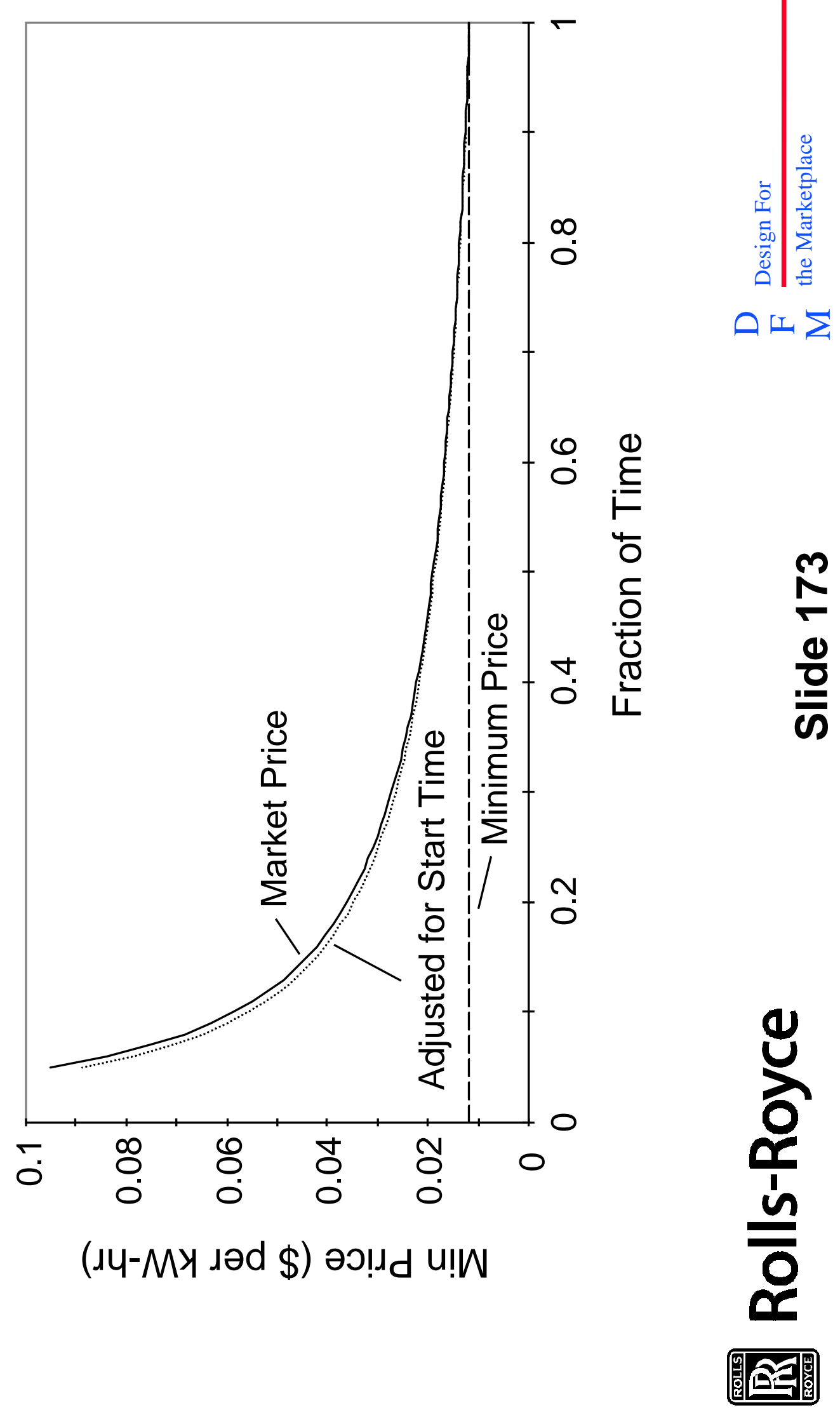



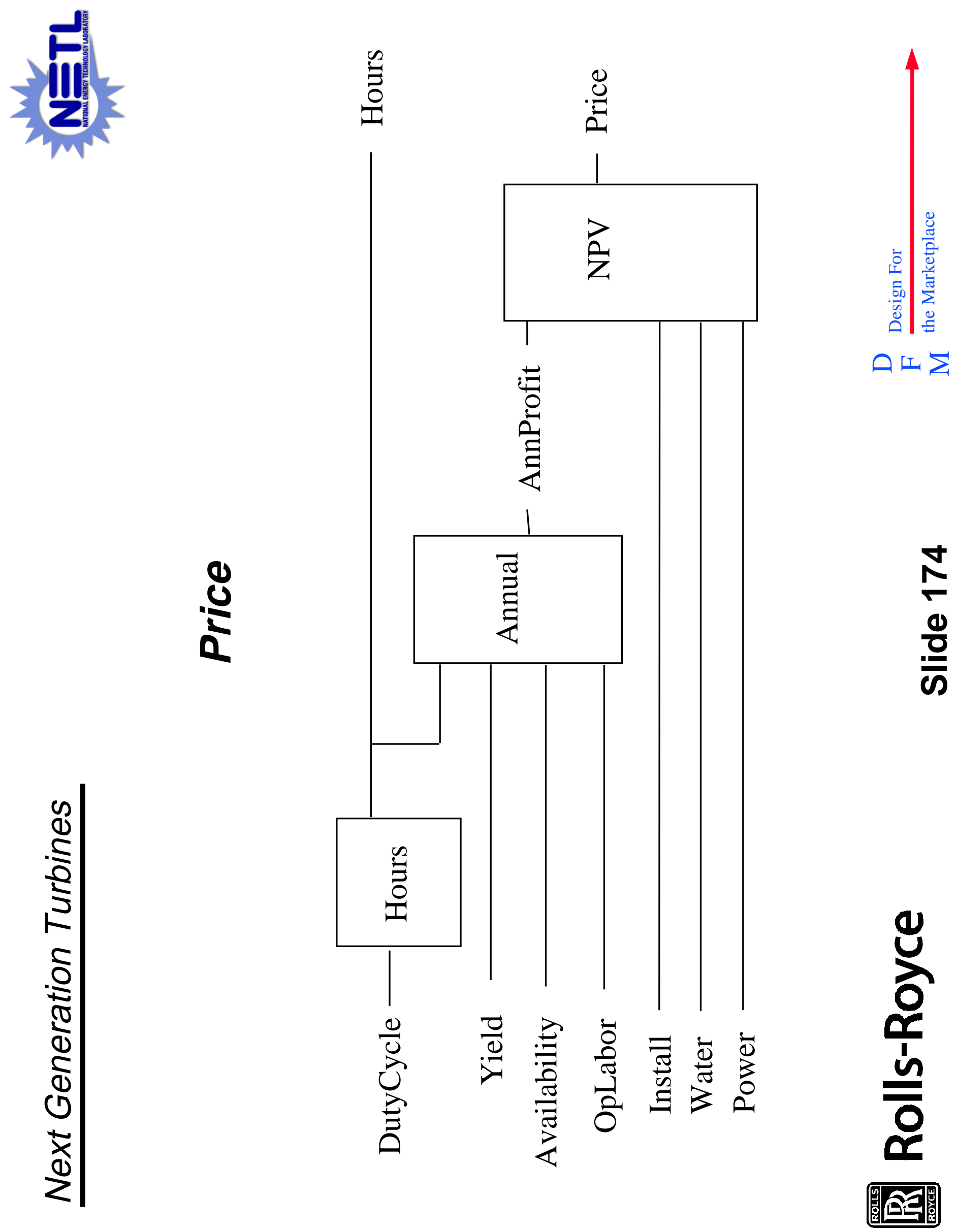

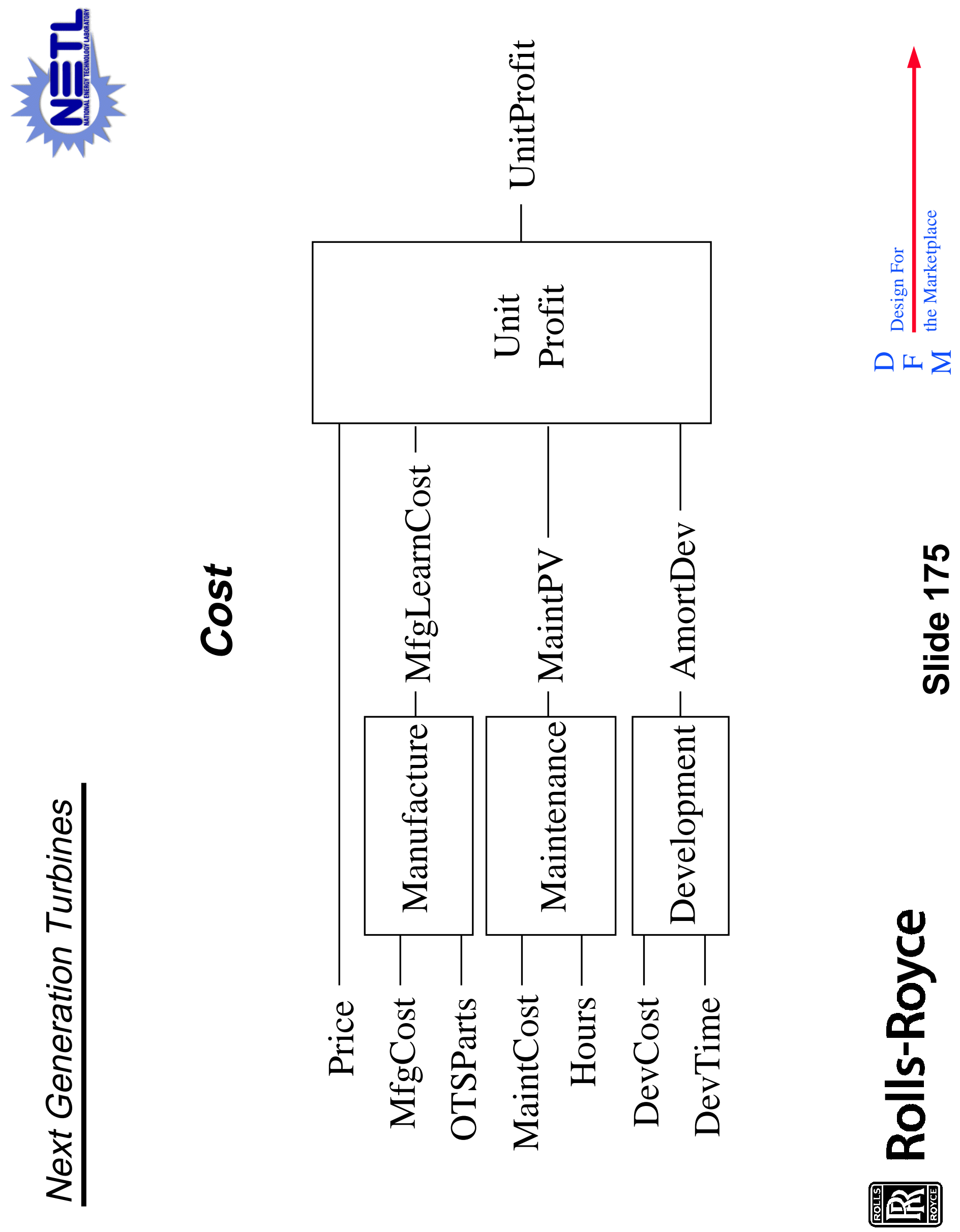


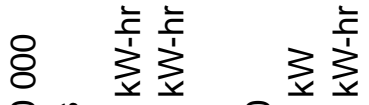
흥 흥

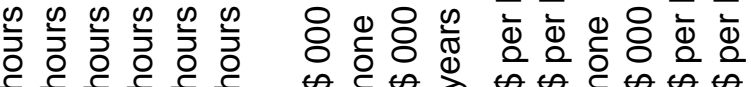

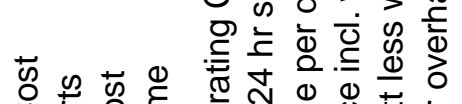
O

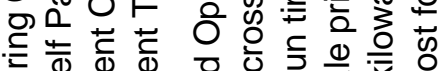

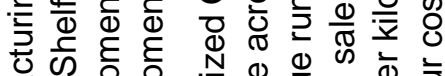
巡 क

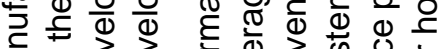

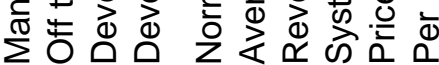

菅嵌

৪

ก

○ัे ०ं

กิ่

$\leftrightarrow \leftrightarrow$

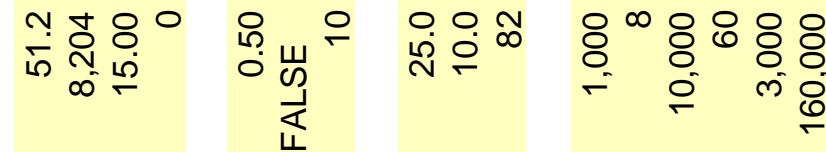

ठें .

$\leftrightarrow$

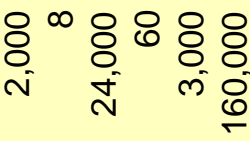

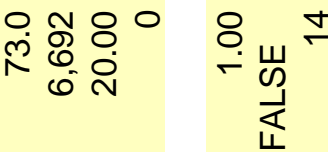

क요

㝋 ๘

$\stackrel{\sim}{\sim}$

$\leftrightarrow$

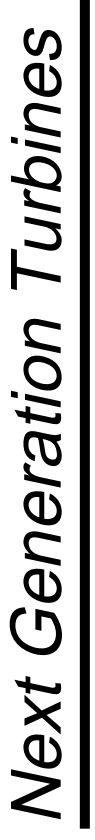

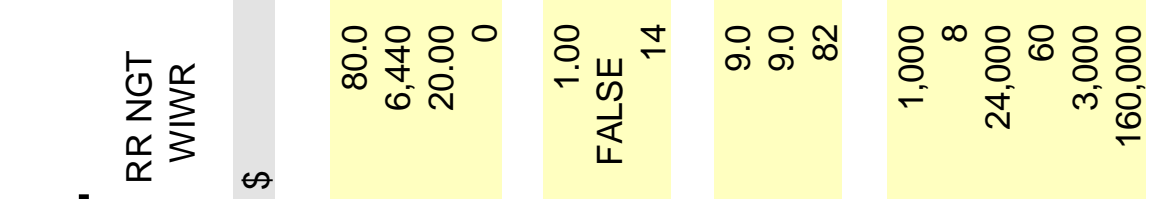

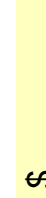
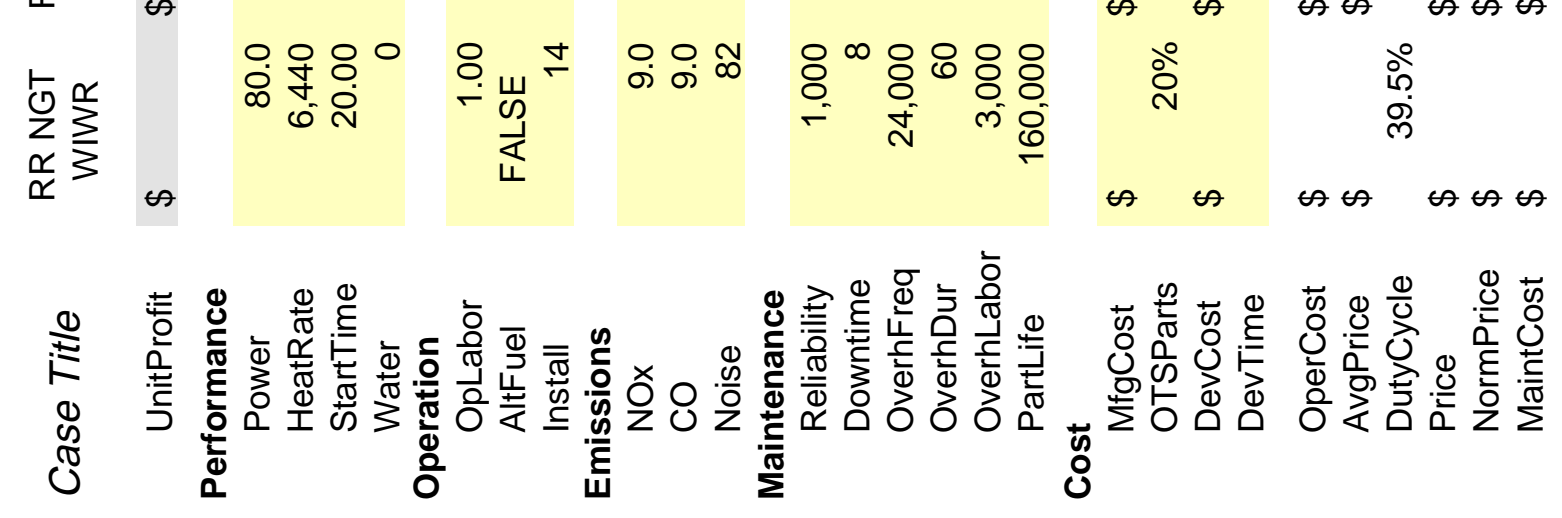

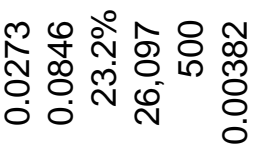

离

단

\%

每

0

$\frac{1}{0}$

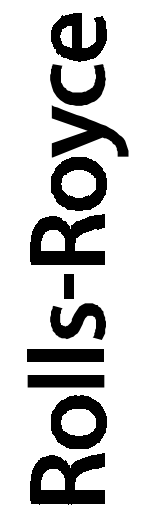



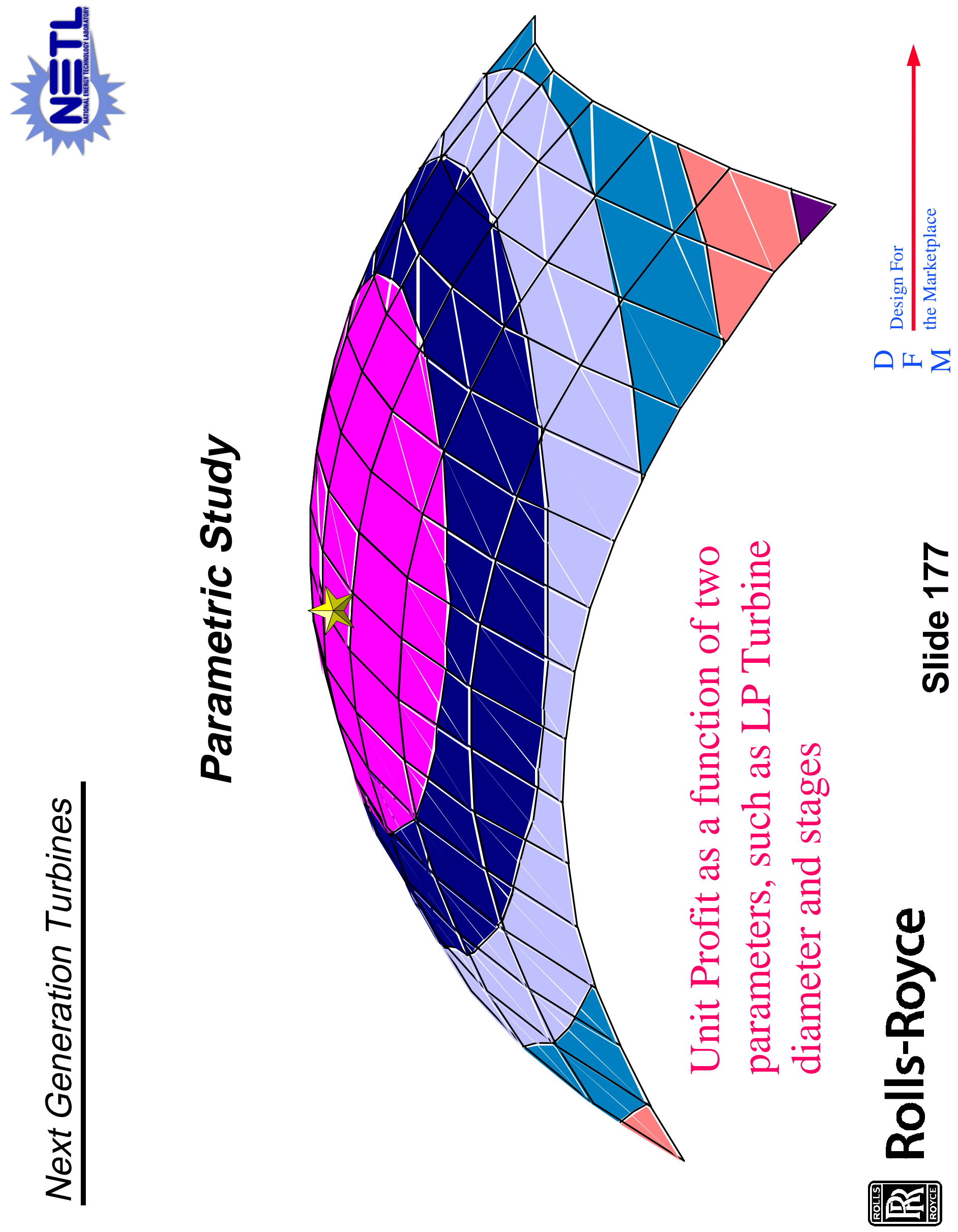


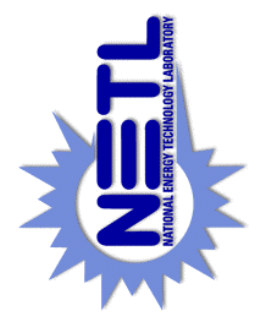

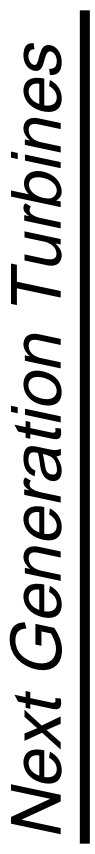

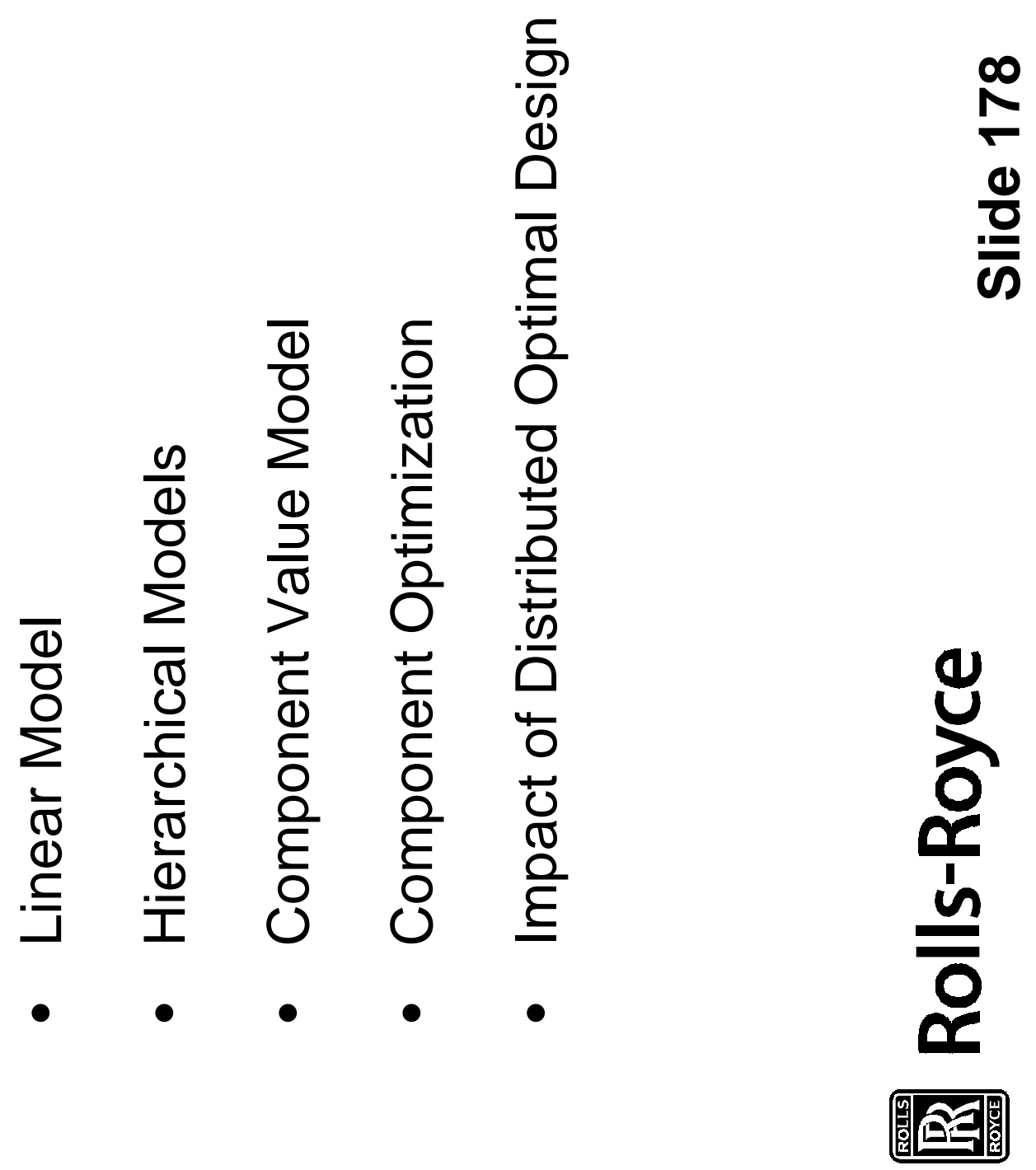




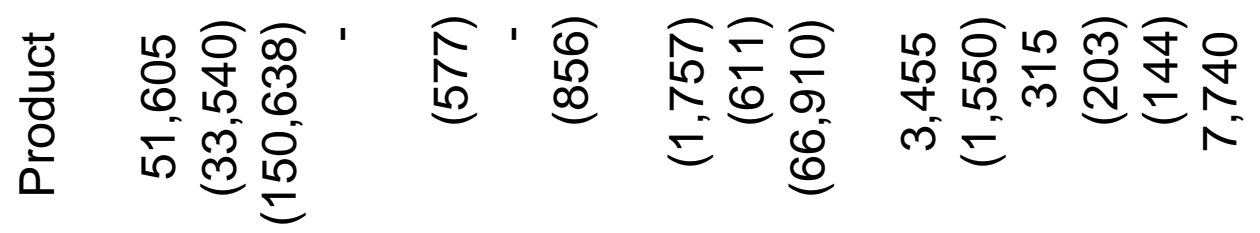

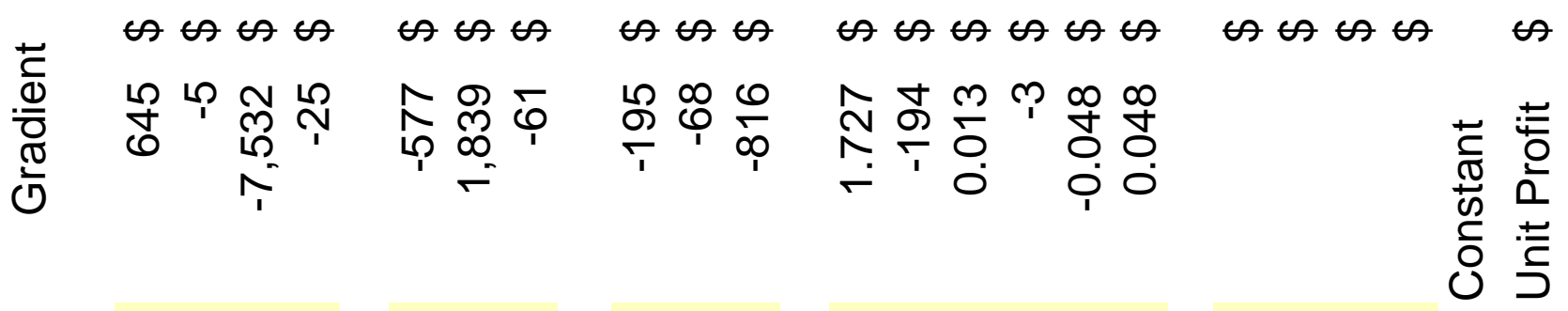

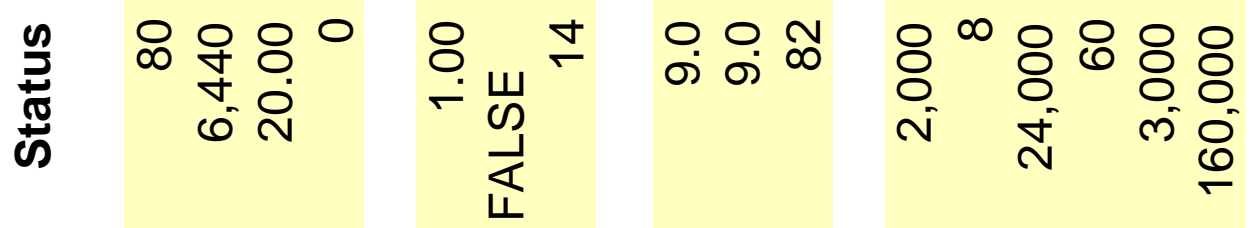

$\leftrightarrow \leftrightarrow N$

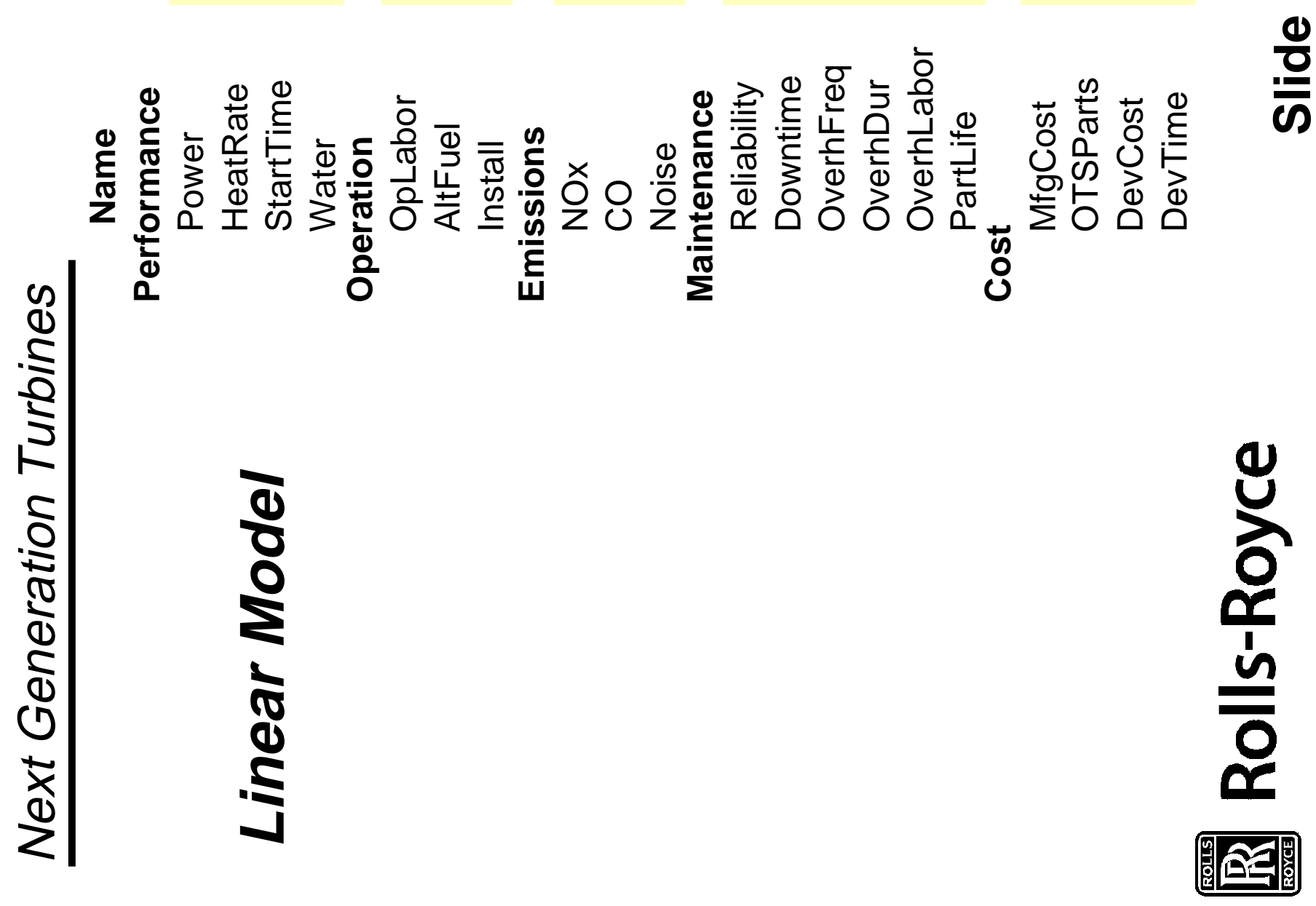



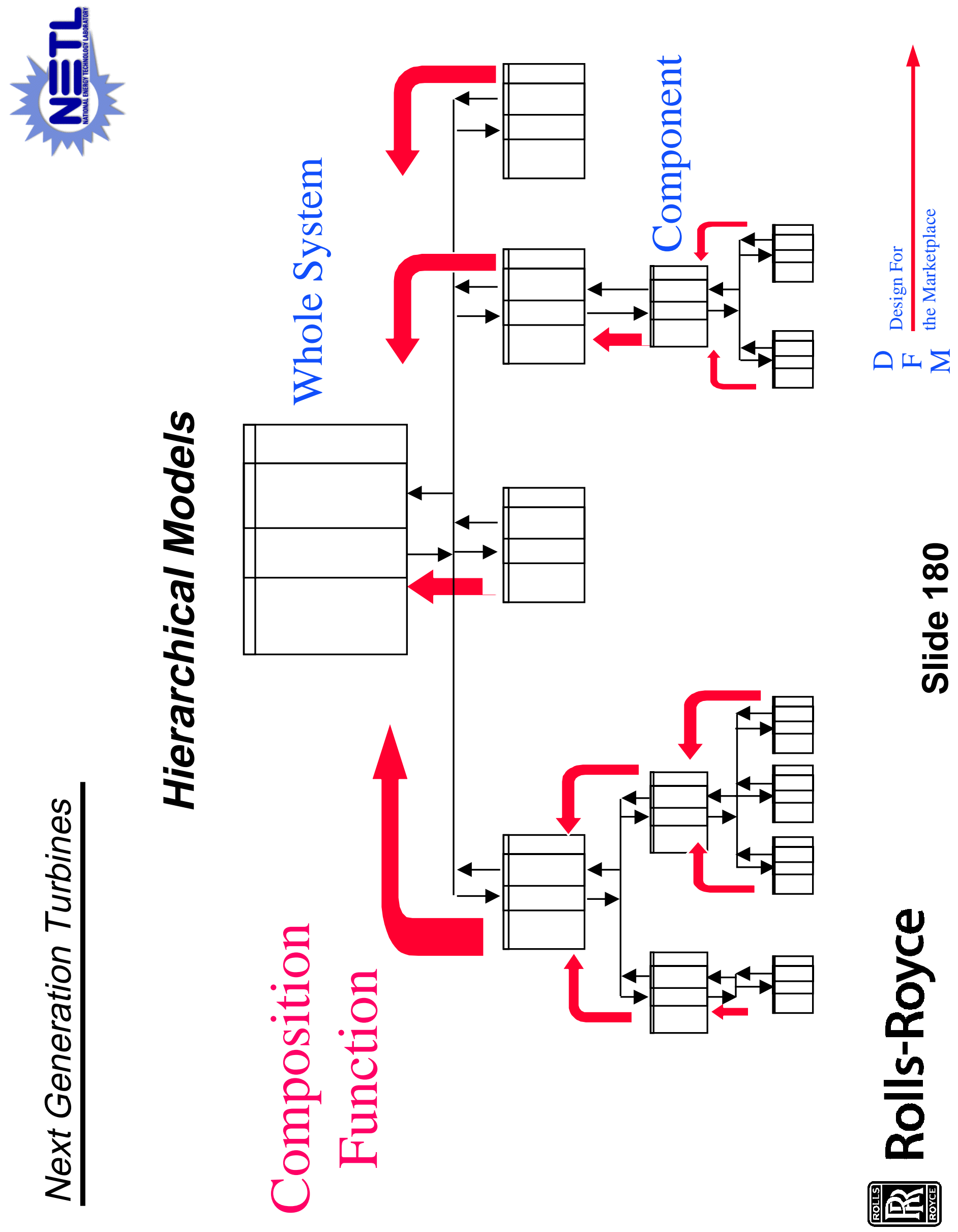


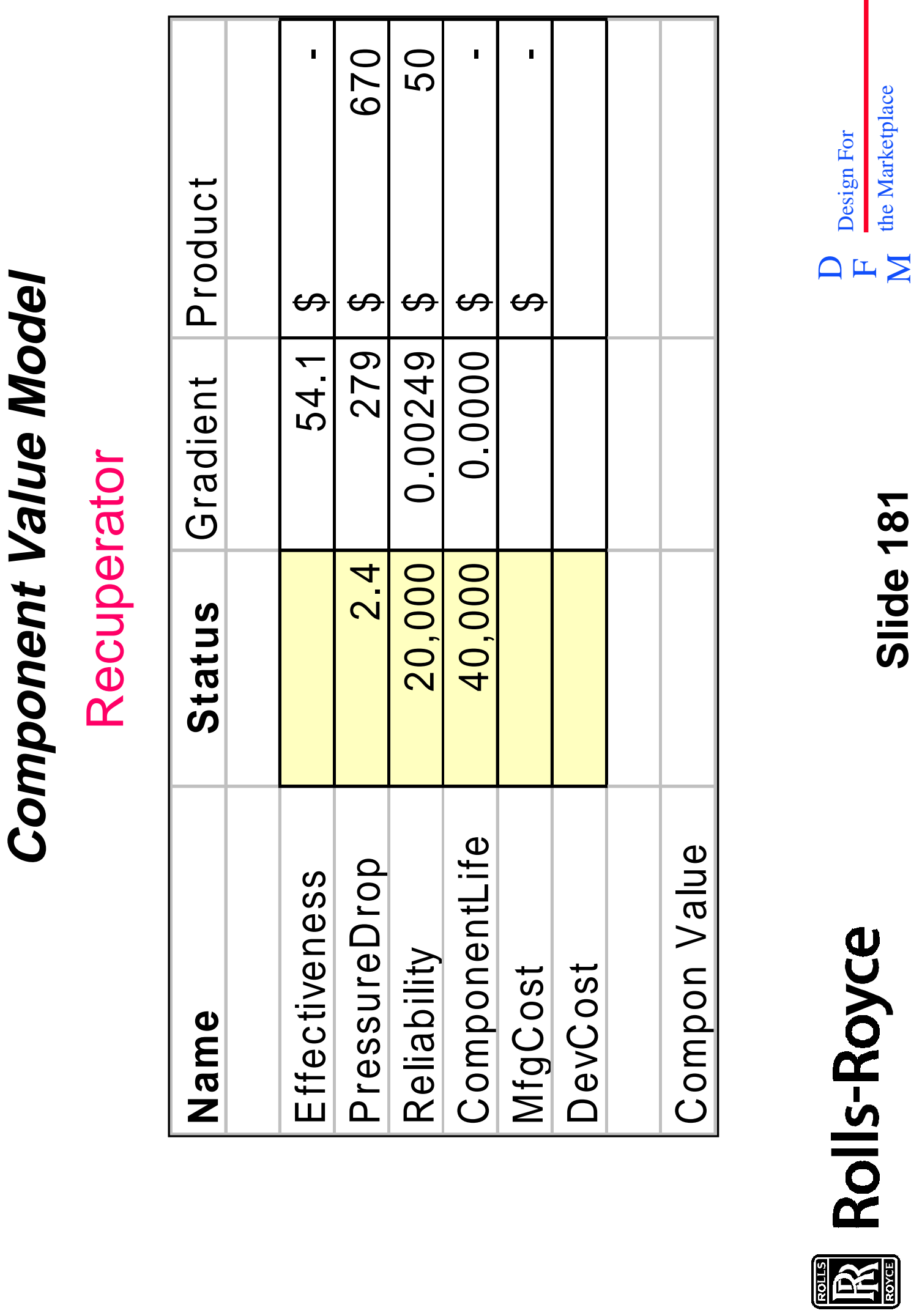



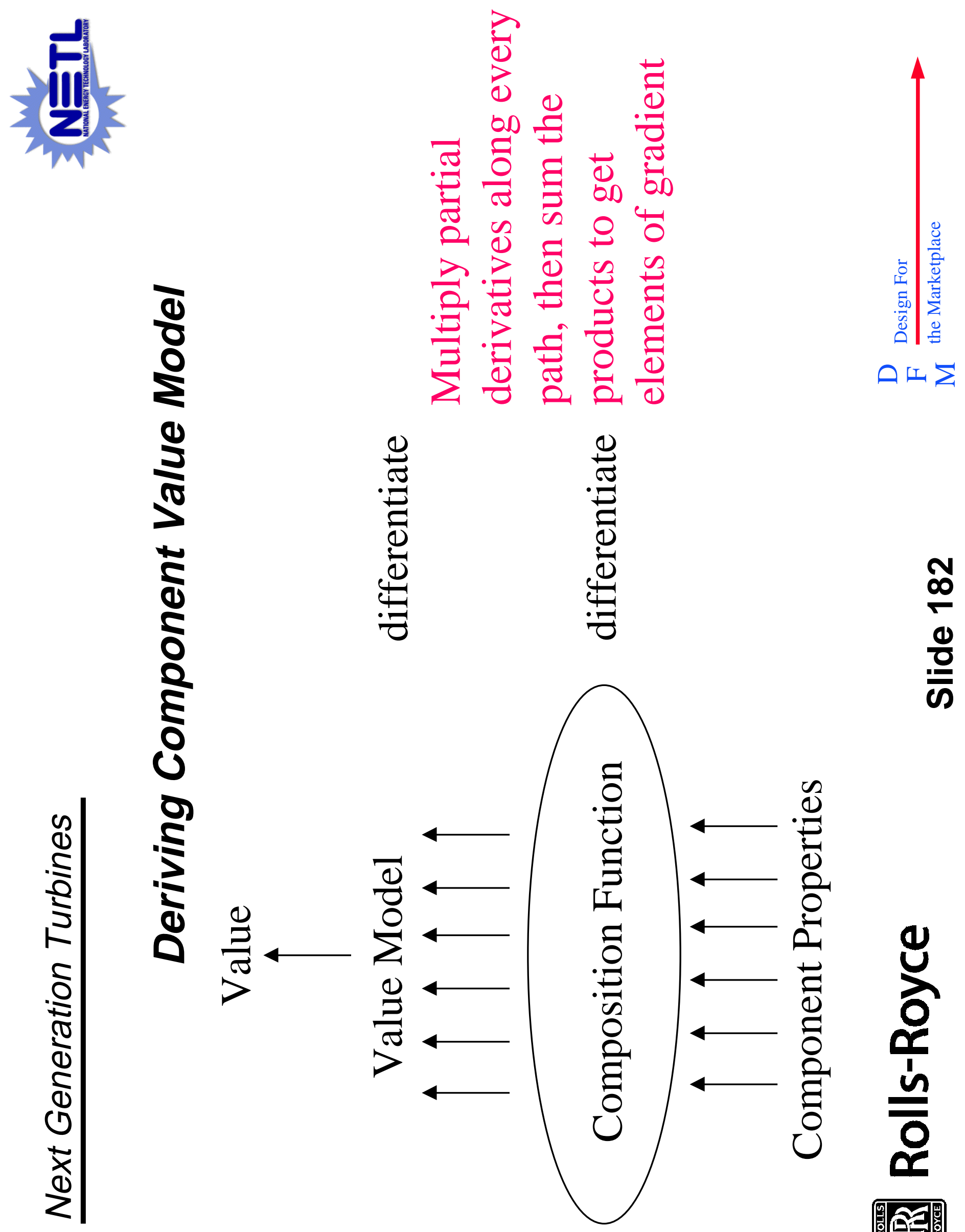


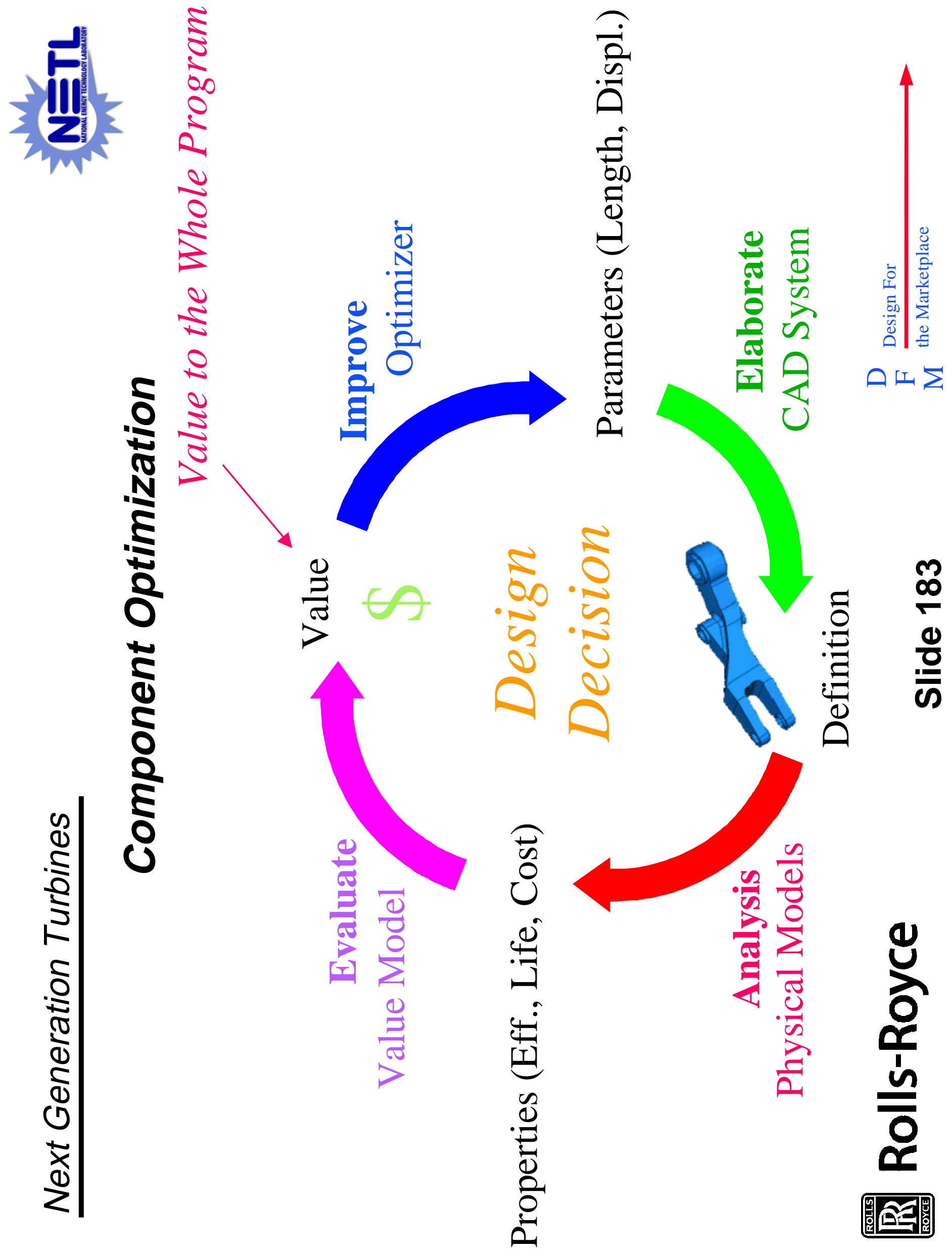



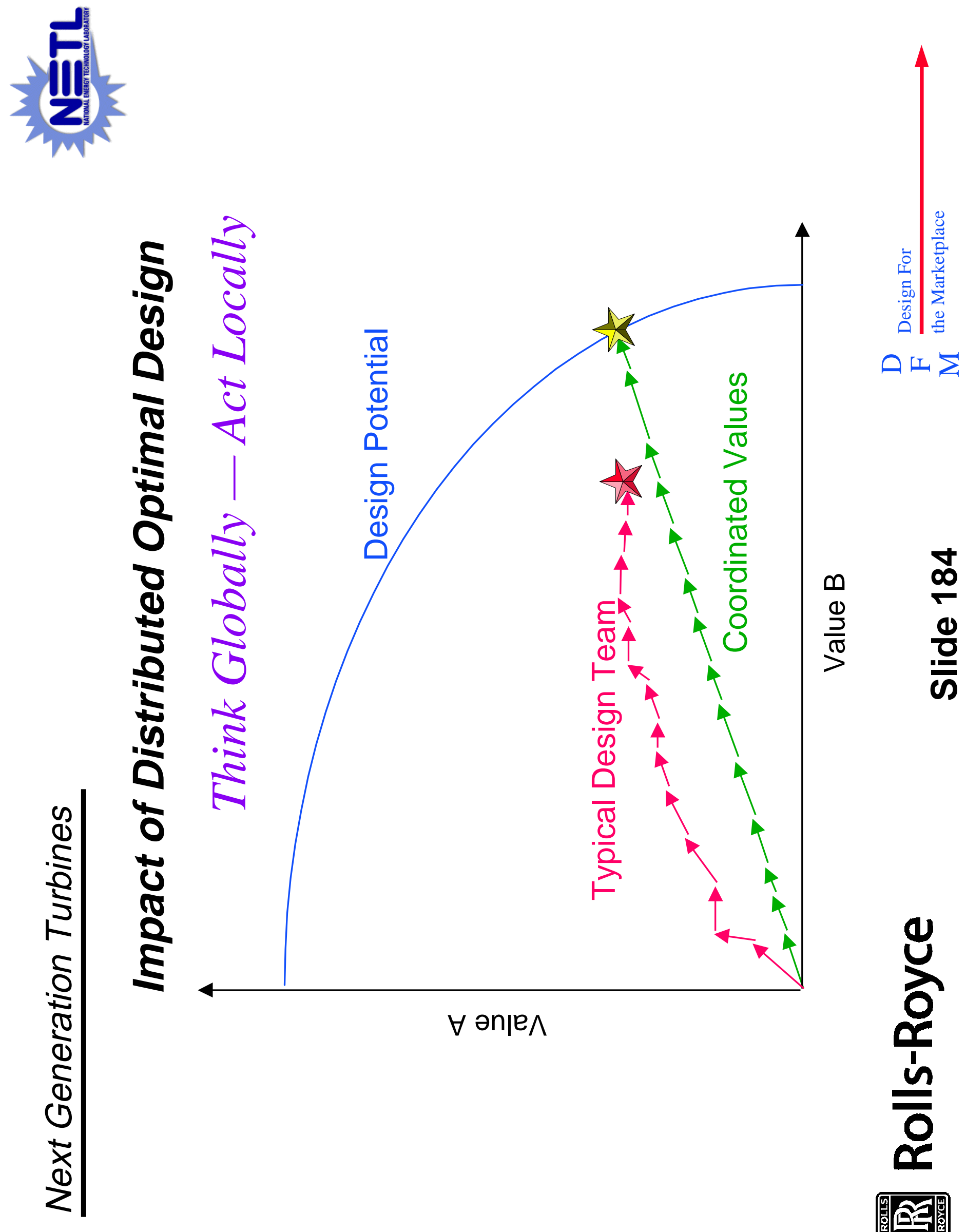

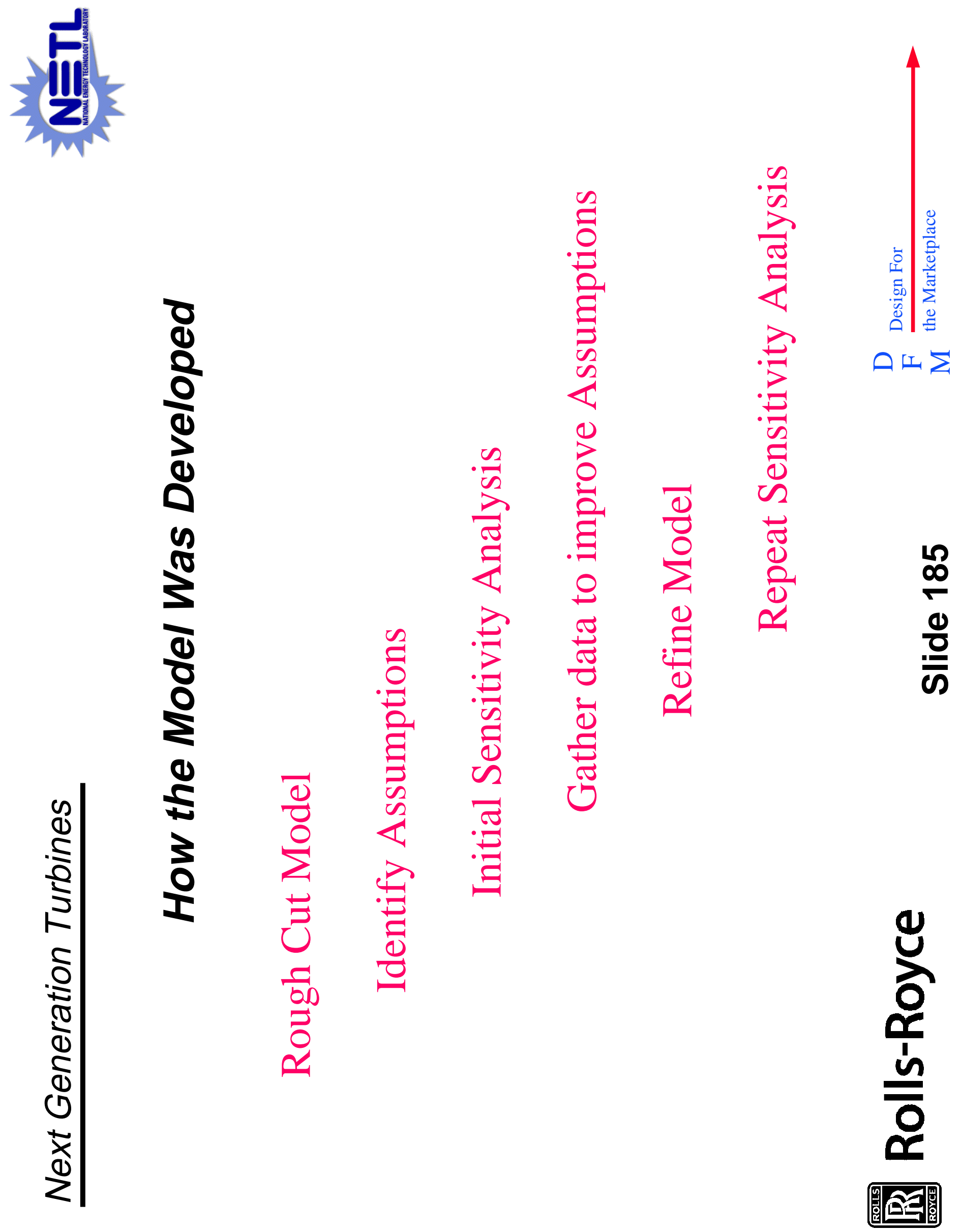


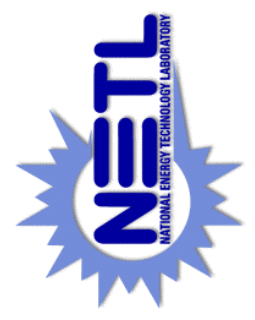

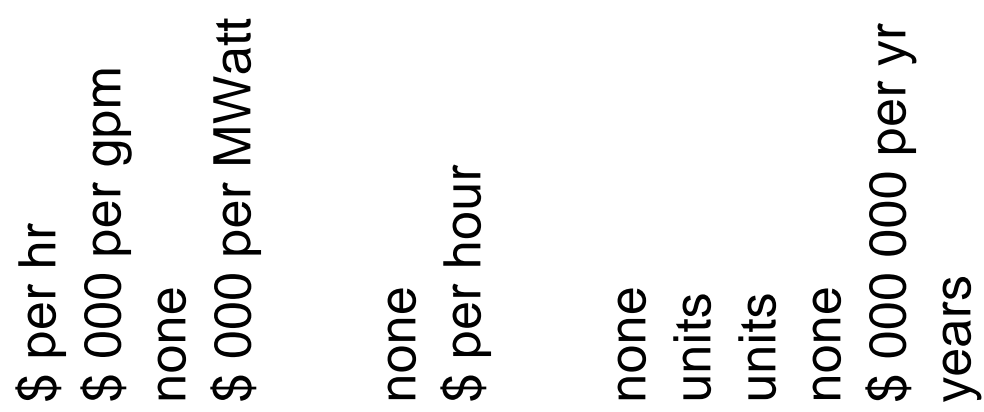
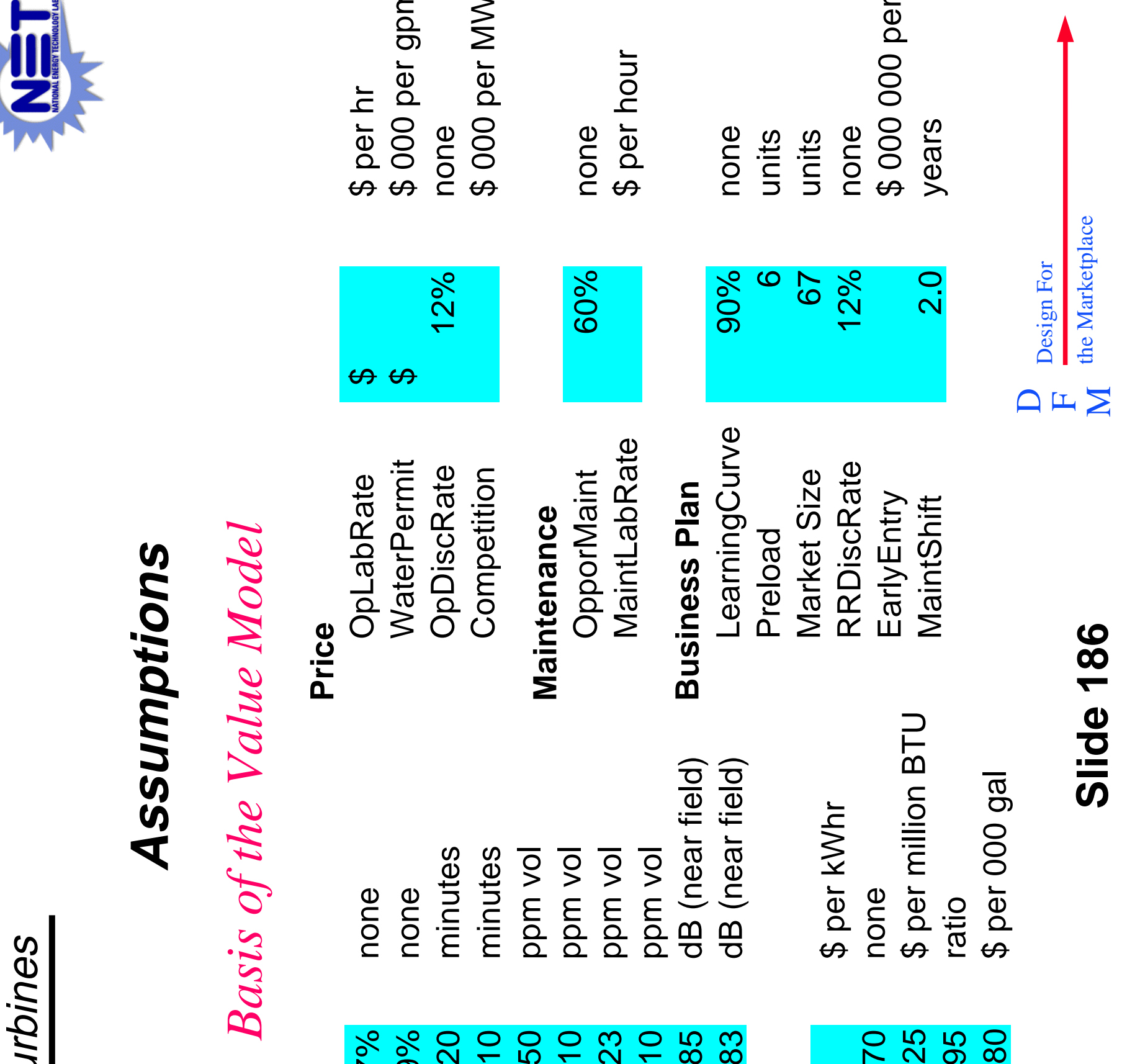

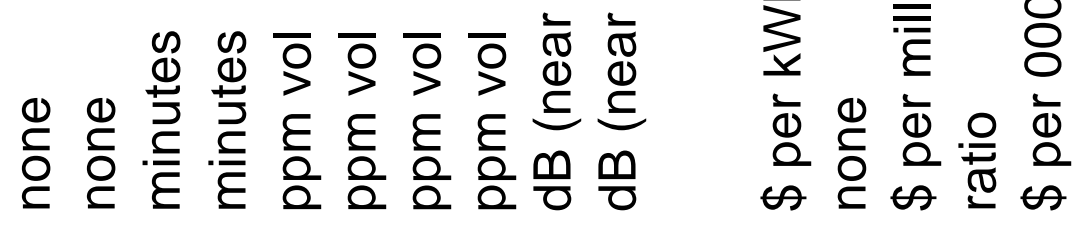

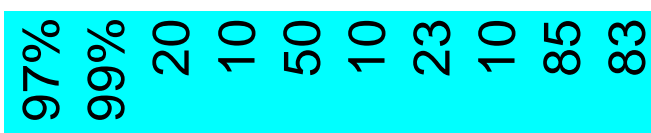

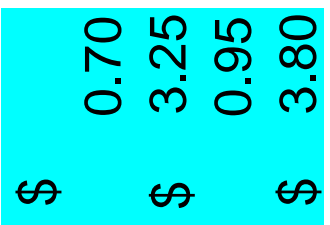

요 \%

至

$\checkmark$

은 흐

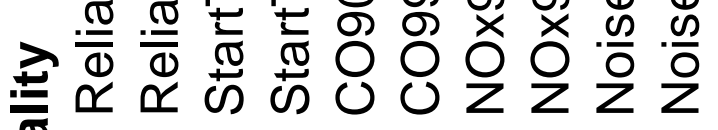

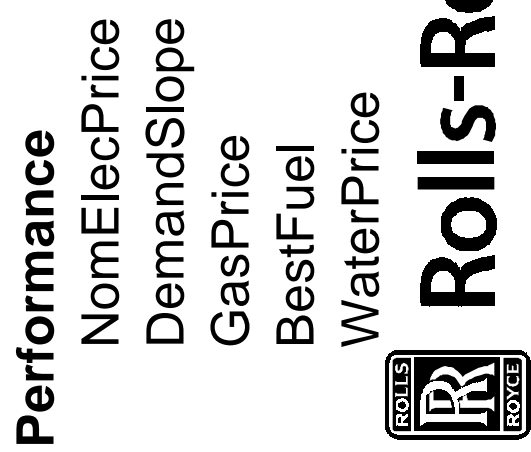



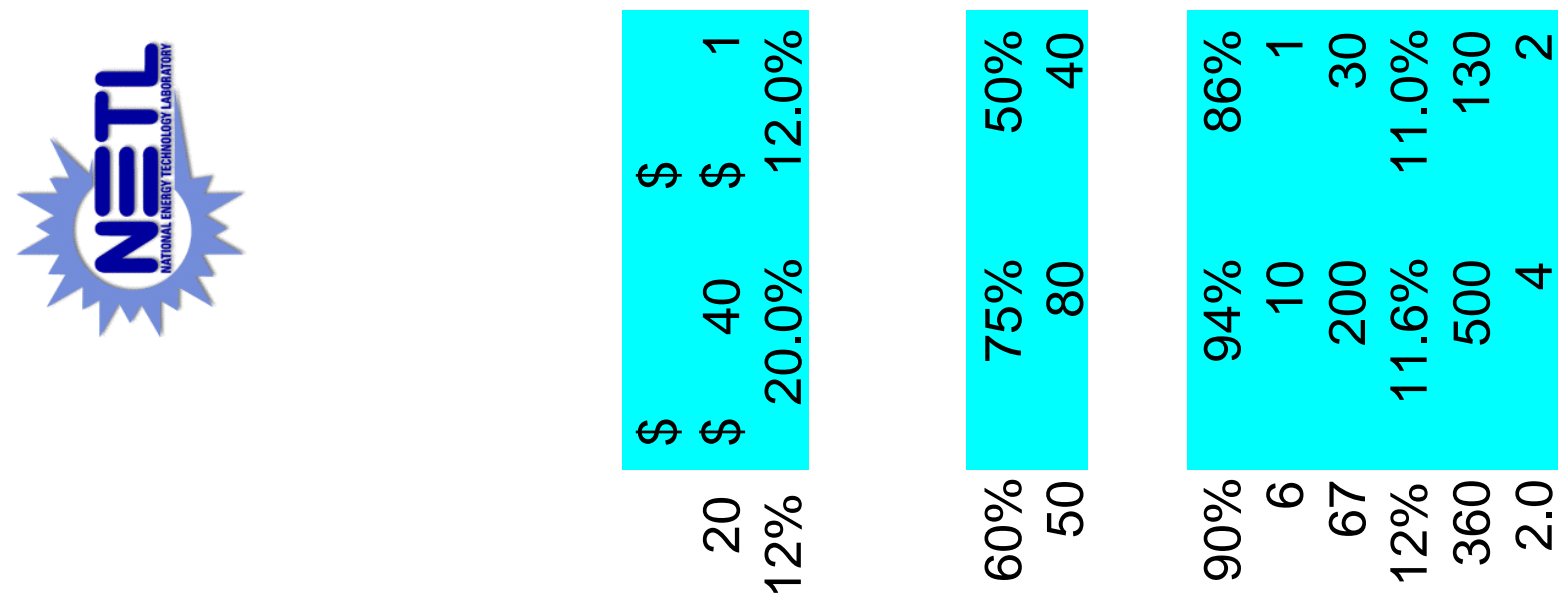

$\leftrightarrow \leftrightarrow$
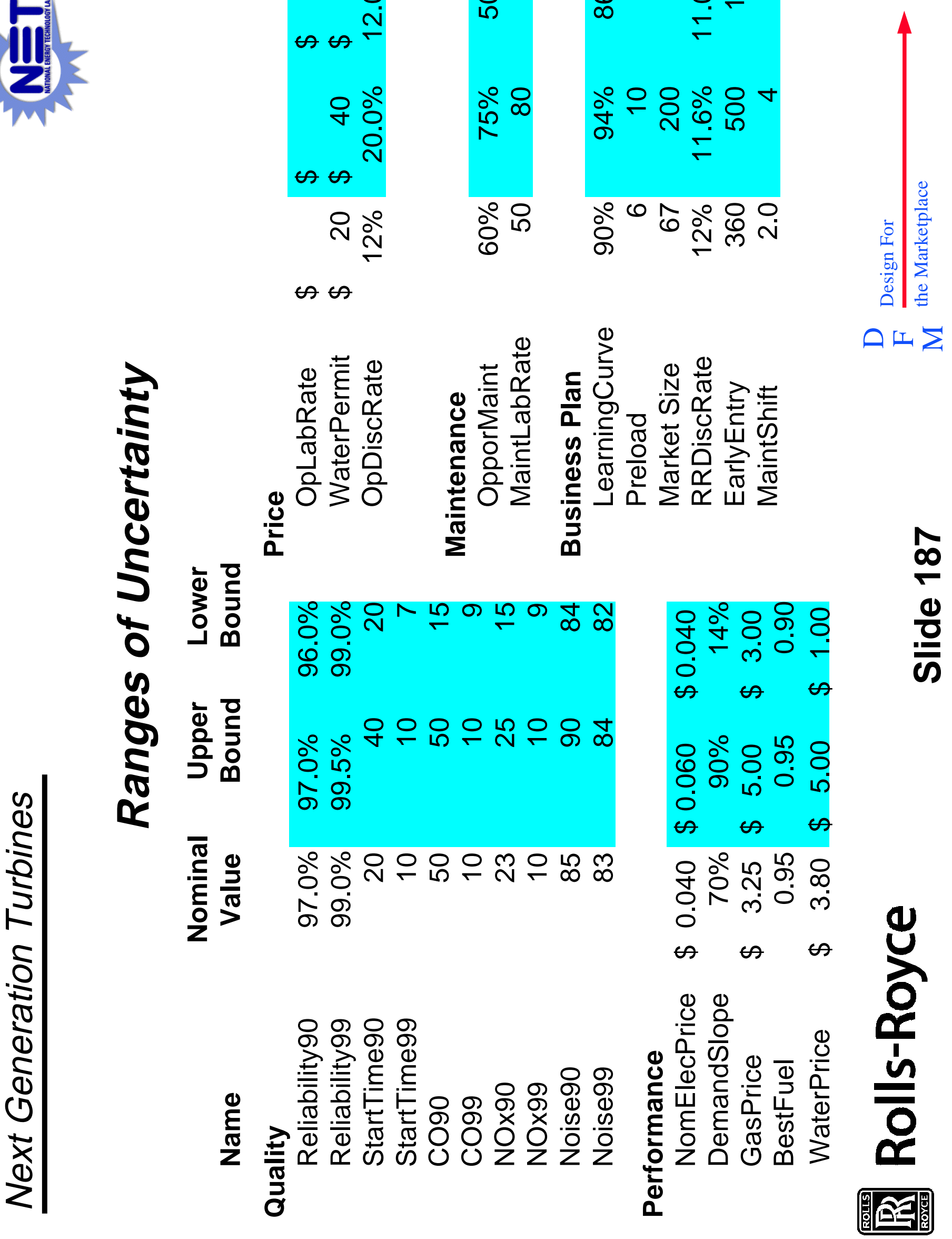


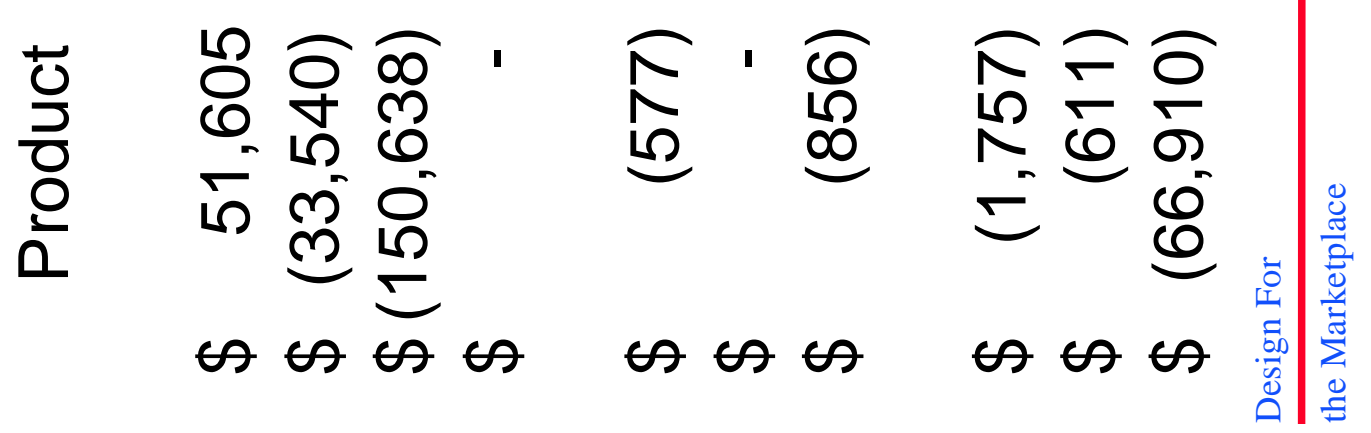

$$
\begin{aligned}
& \text { + } 0 \text { 山 }
\end{aligned}
$$

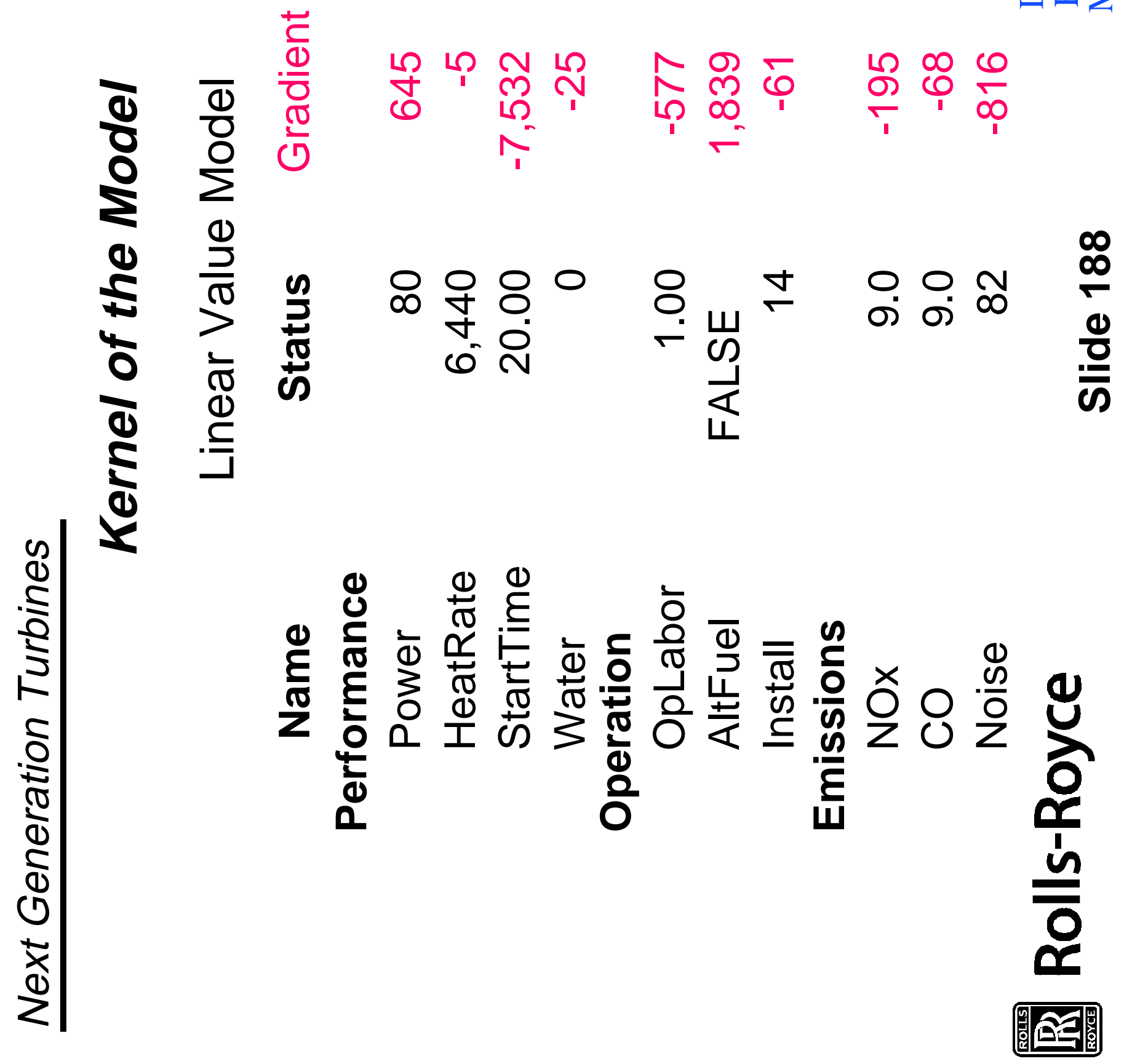



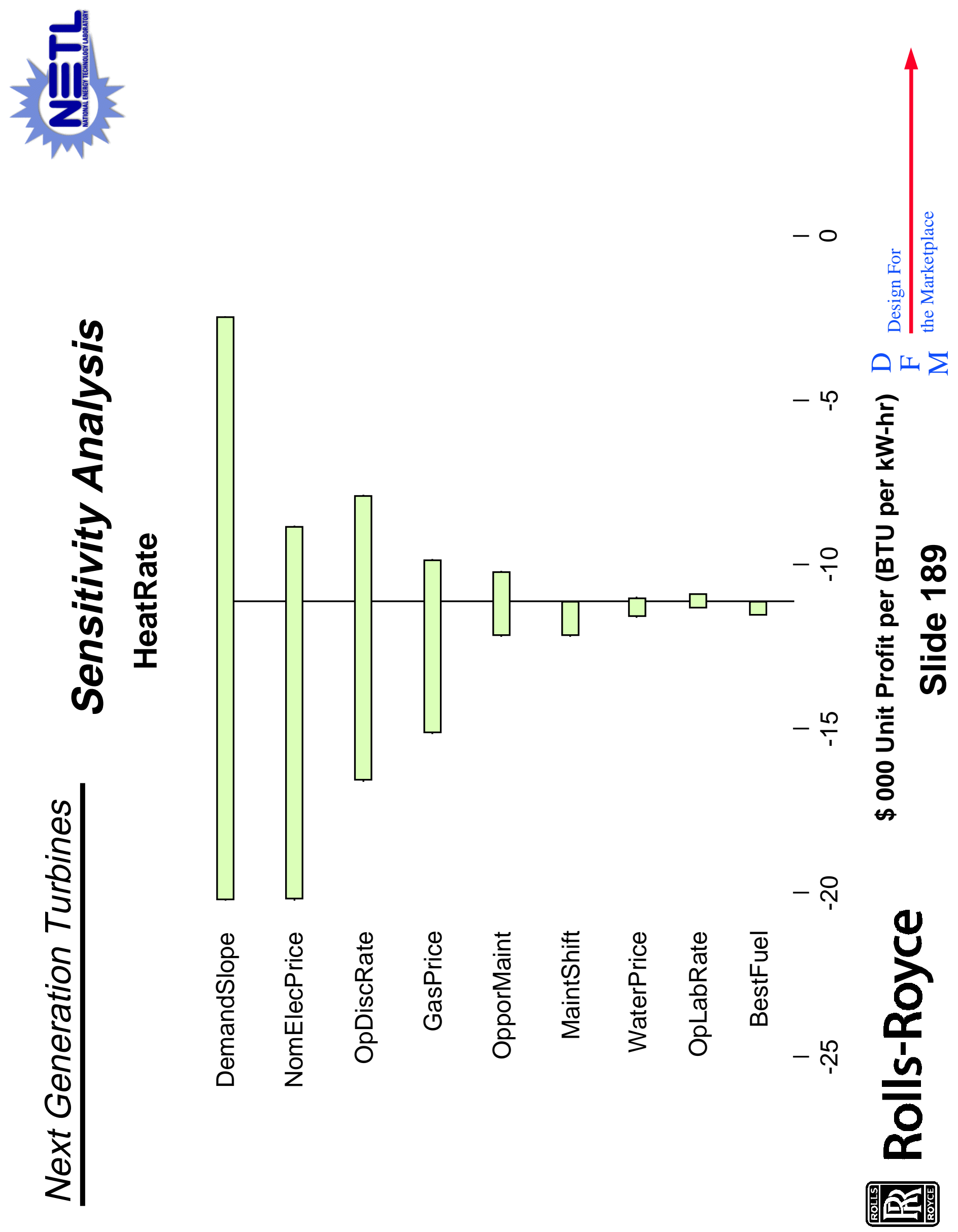


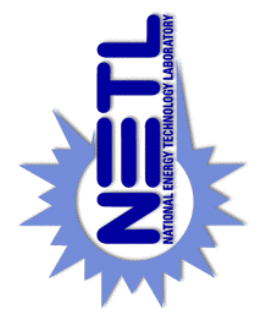

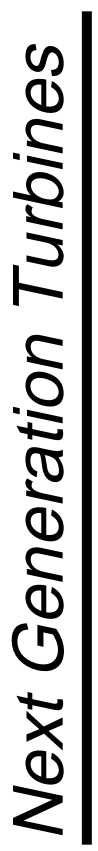
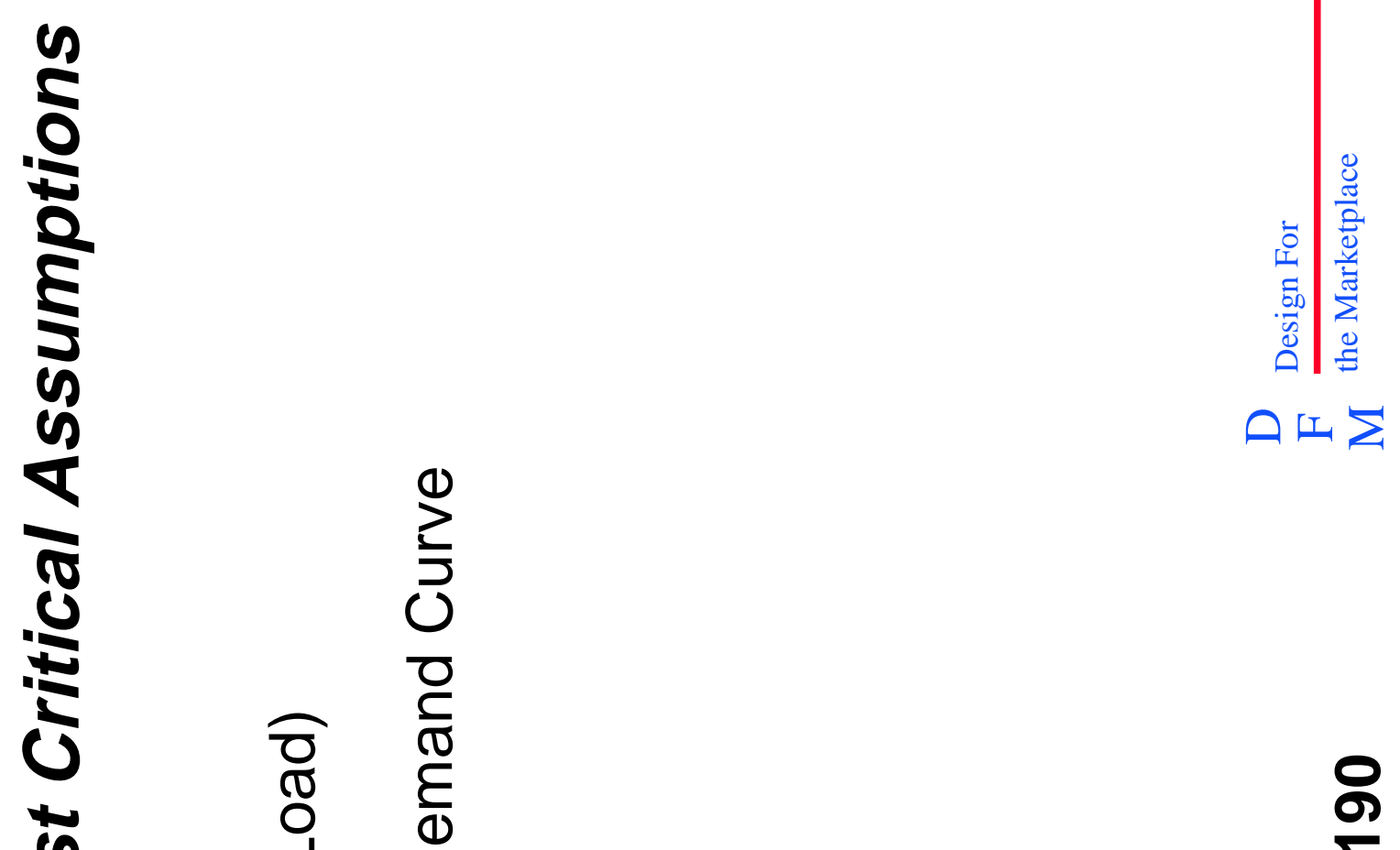

$\frac{0}{\circ}$

$\frac{5}{5}$

9 .

ه D

는

$\overline{0} 0$.

응 응 $\frac{1}{5}$

$\frac{\Phi}{\Psi} \circlearrowleft$

U

บ

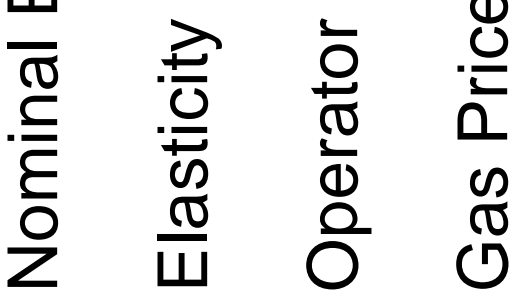

우

0

$n$

잉

r

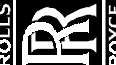




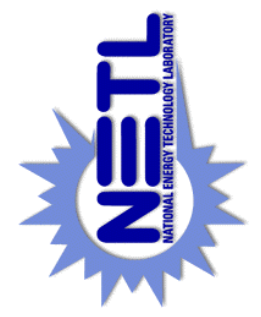

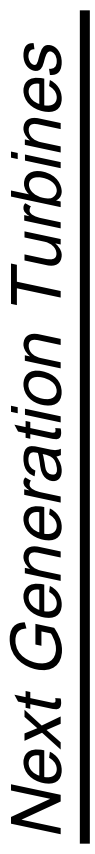

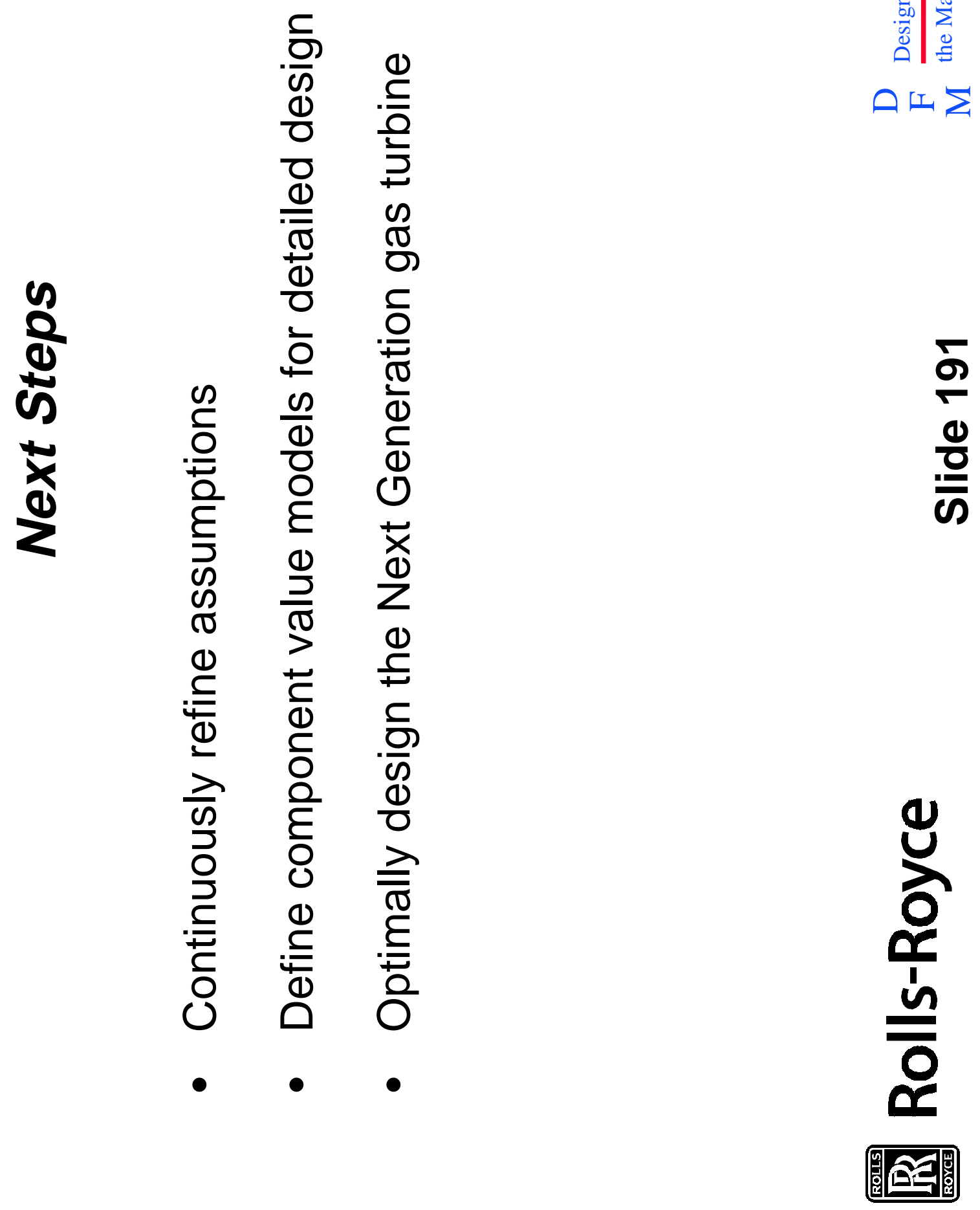



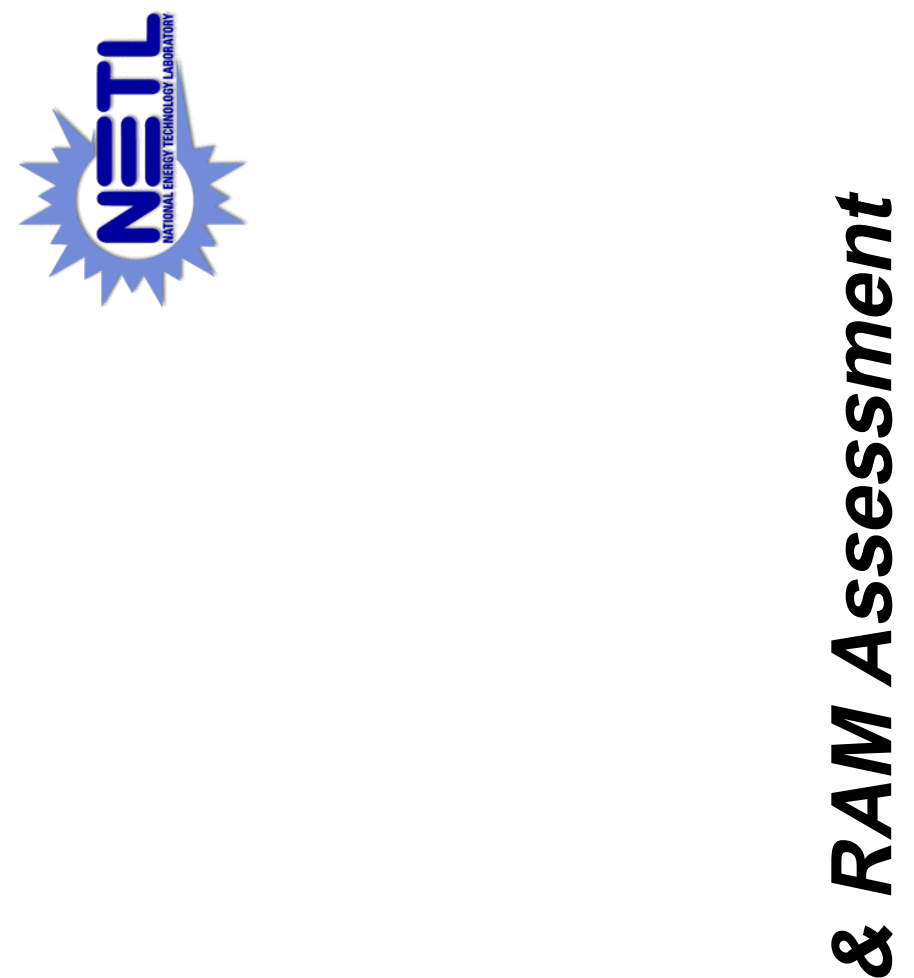

$\frac{9}{8}$

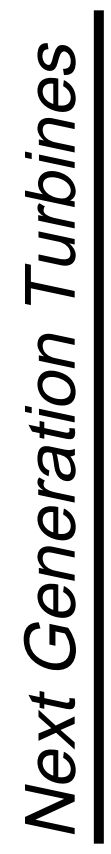

0

$\frac{0}{0}$
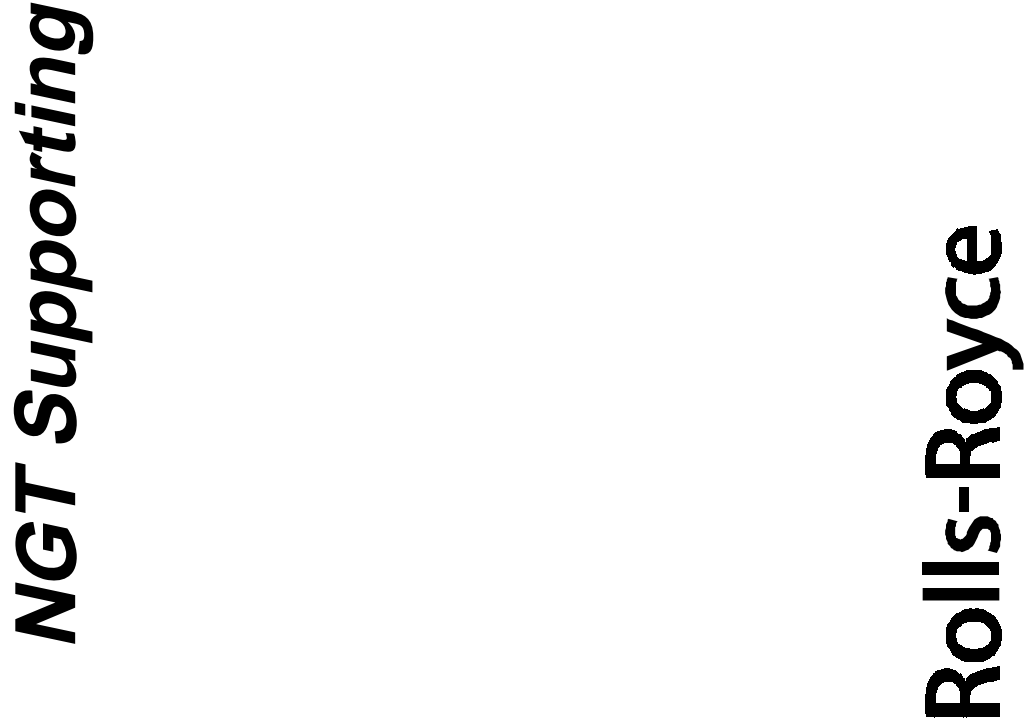

U

a

U 

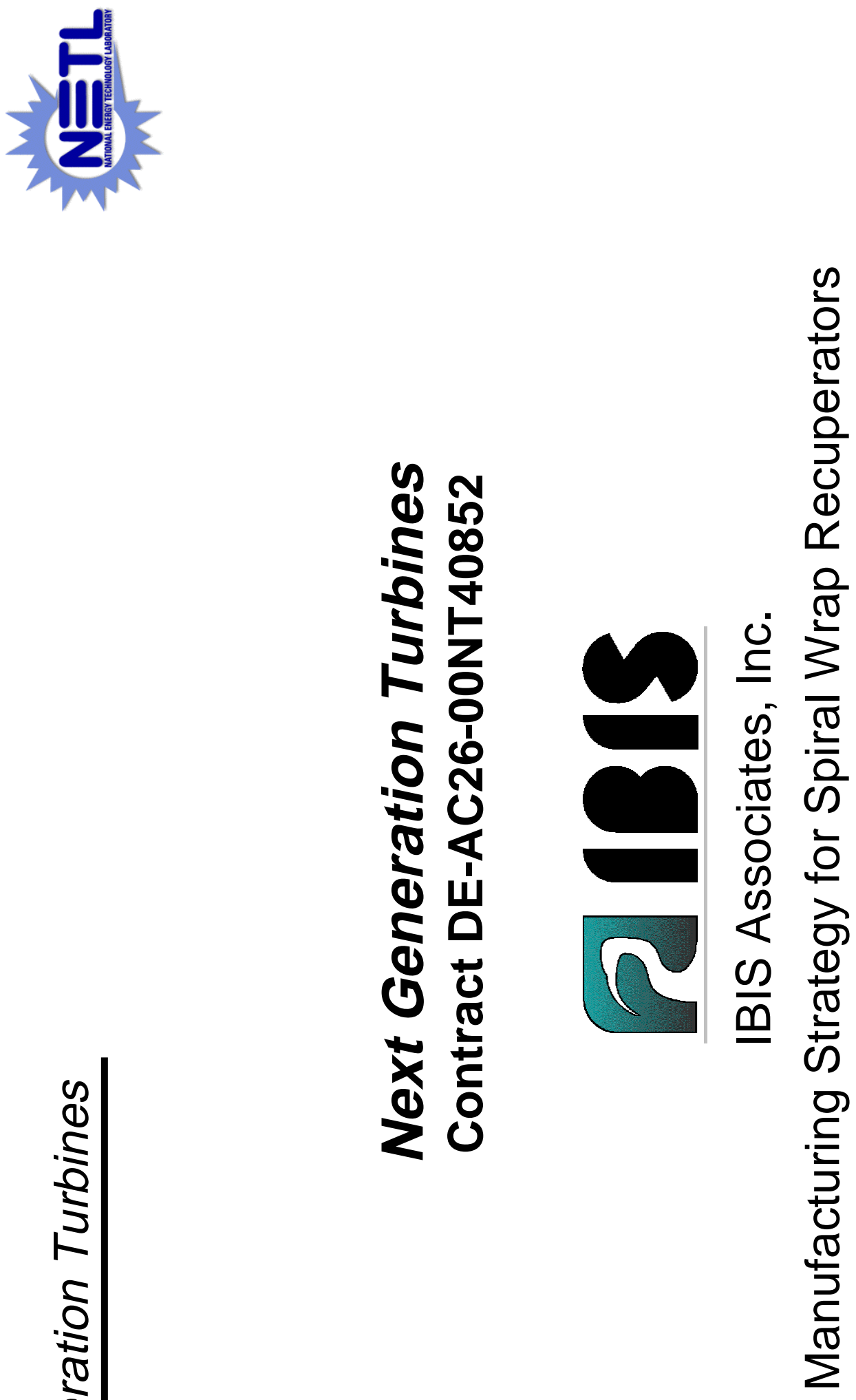

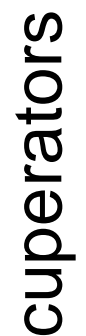

0

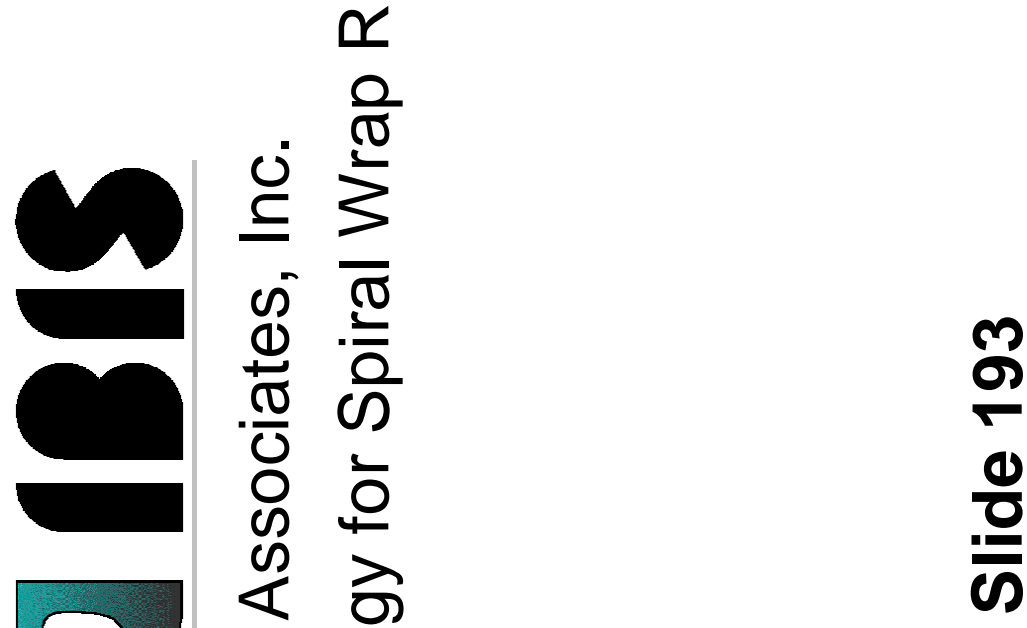

(1)

$\bar{m}$ ত্ত

क

들 () ज

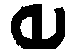

0

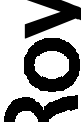

0

$n$

잉

ar

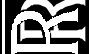




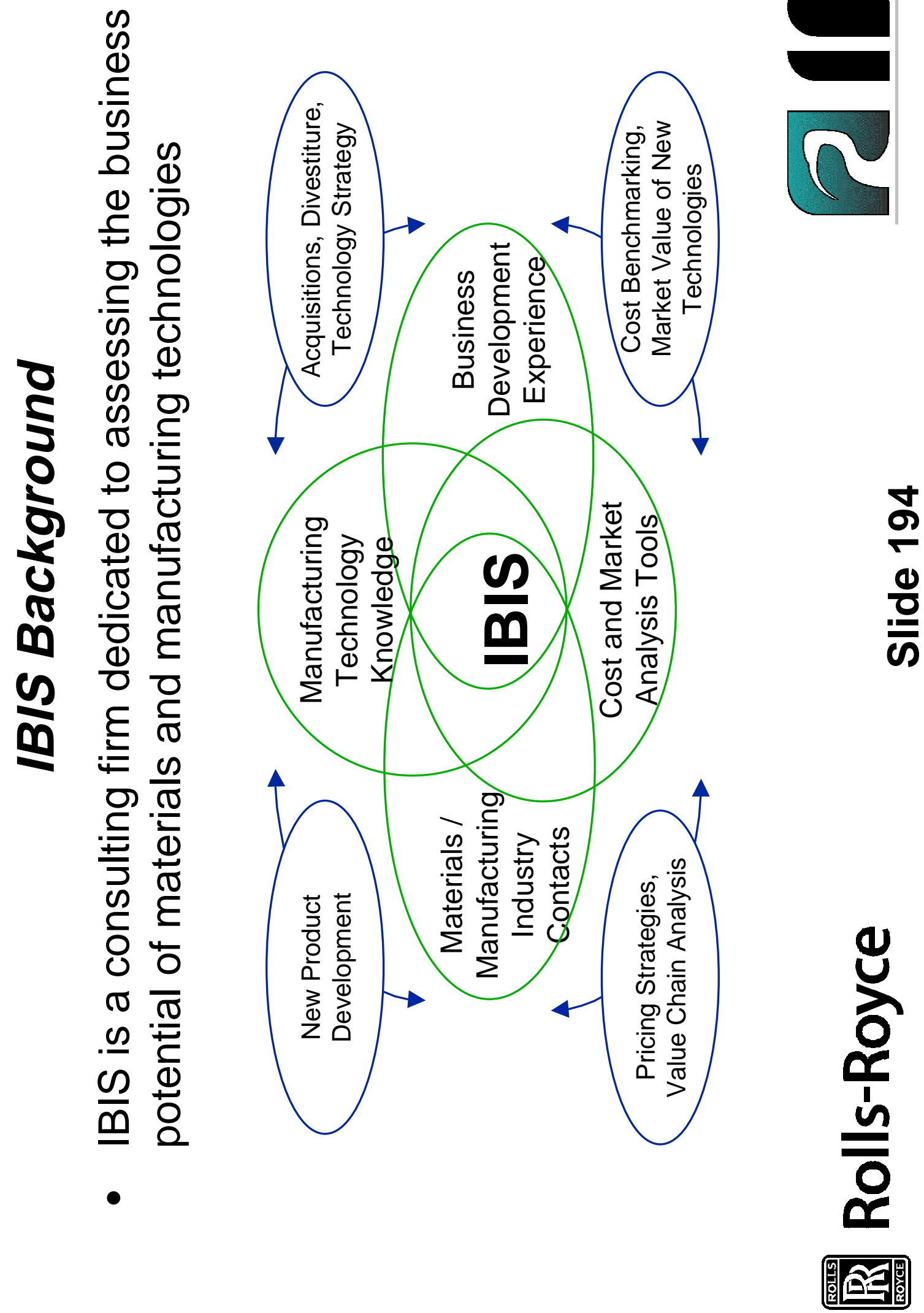



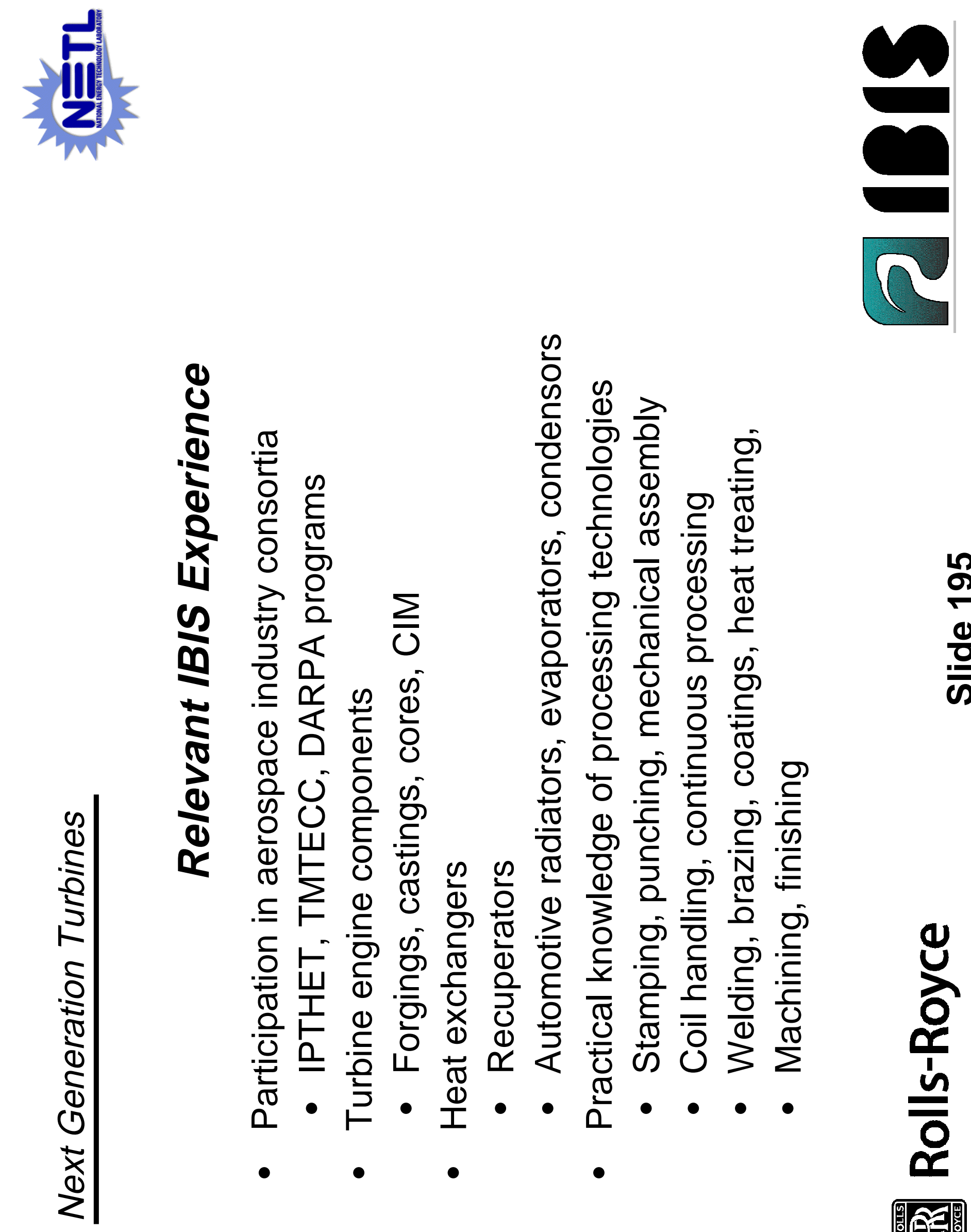


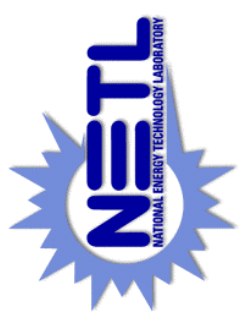

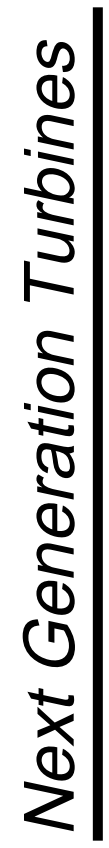
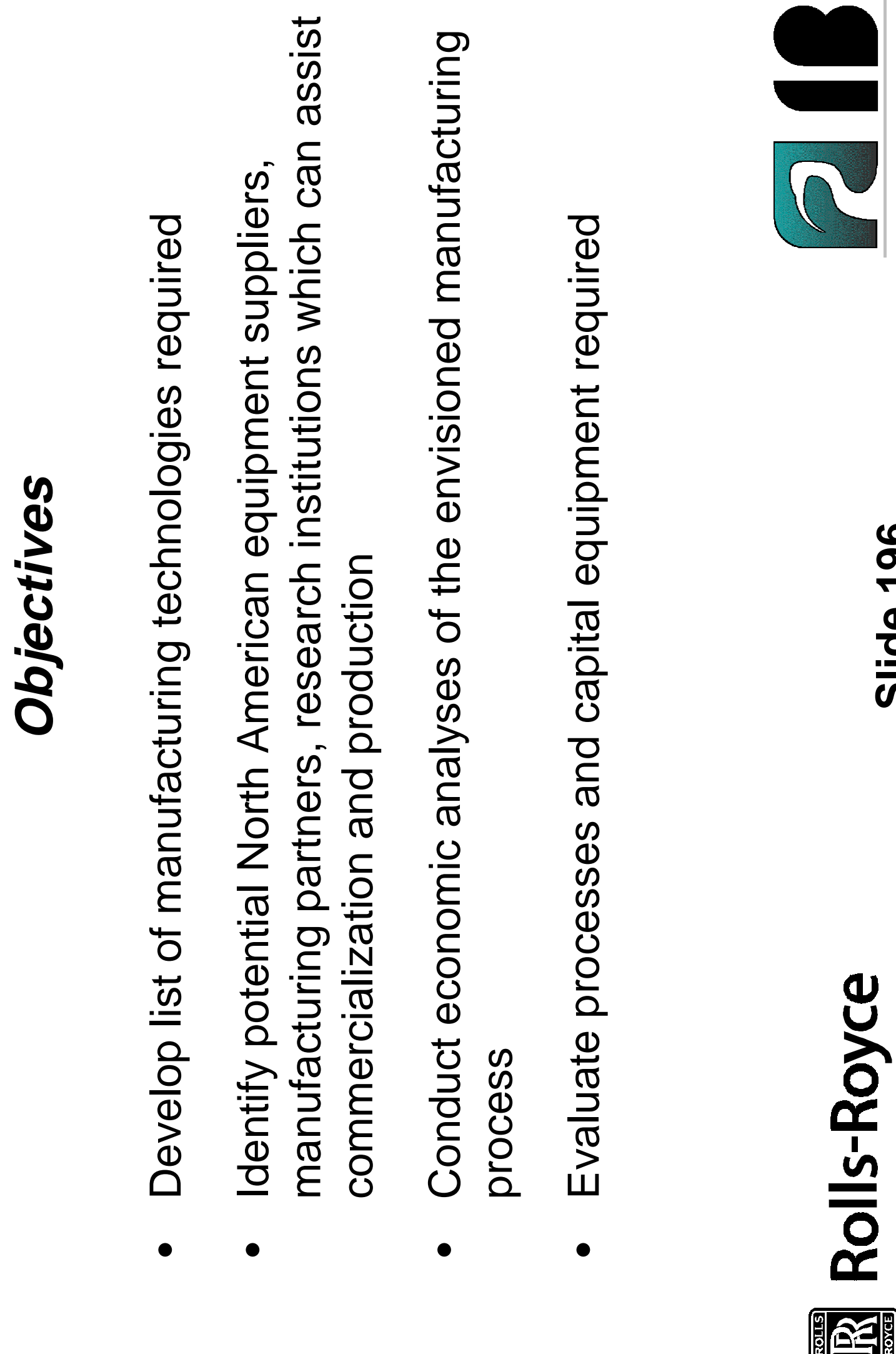


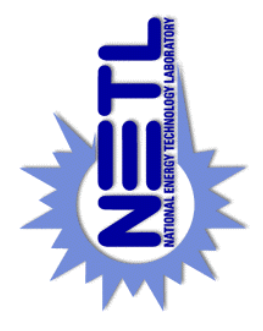

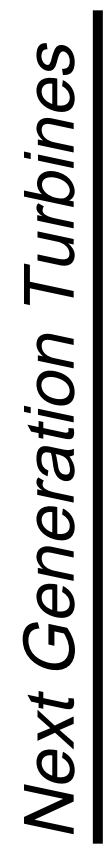

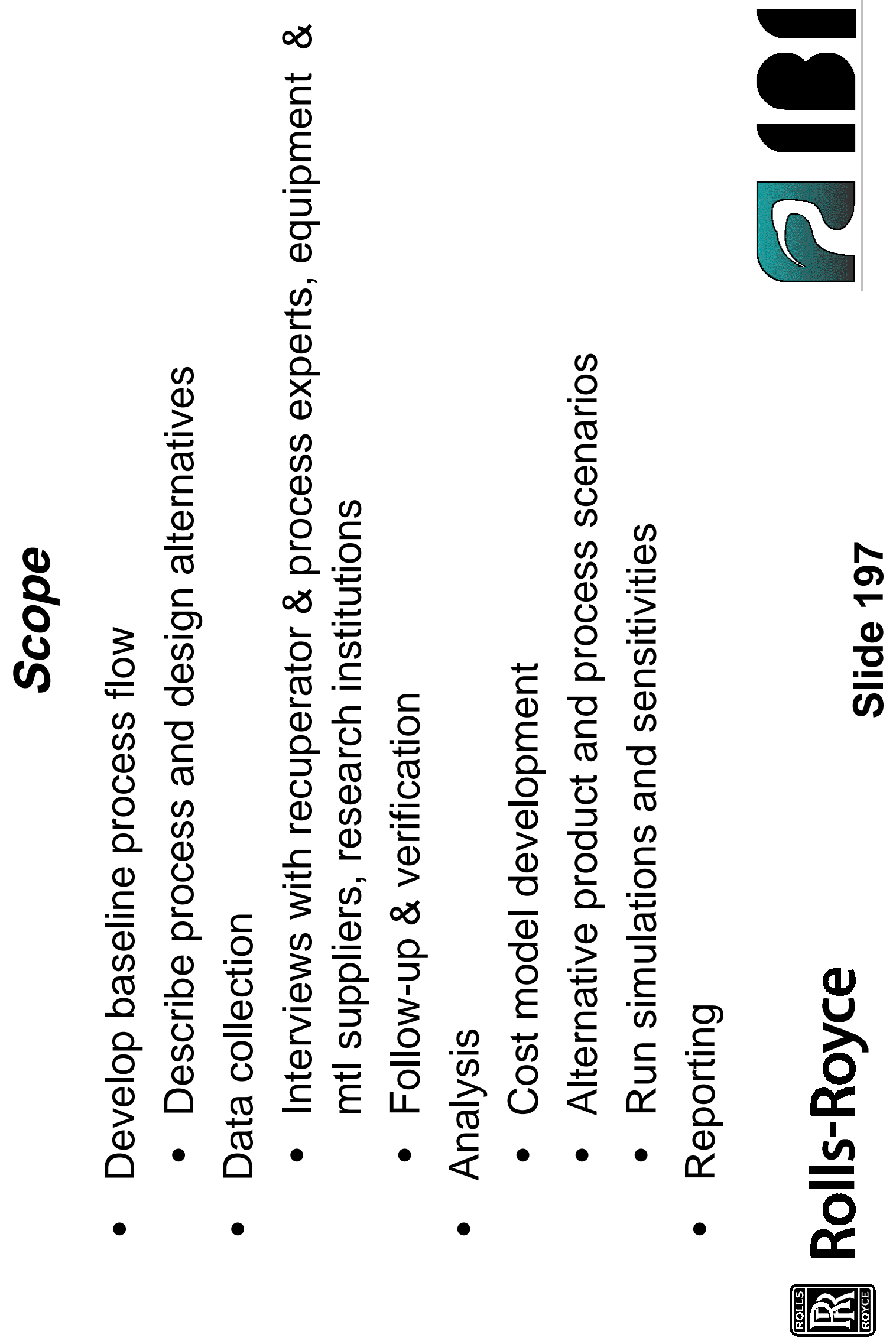



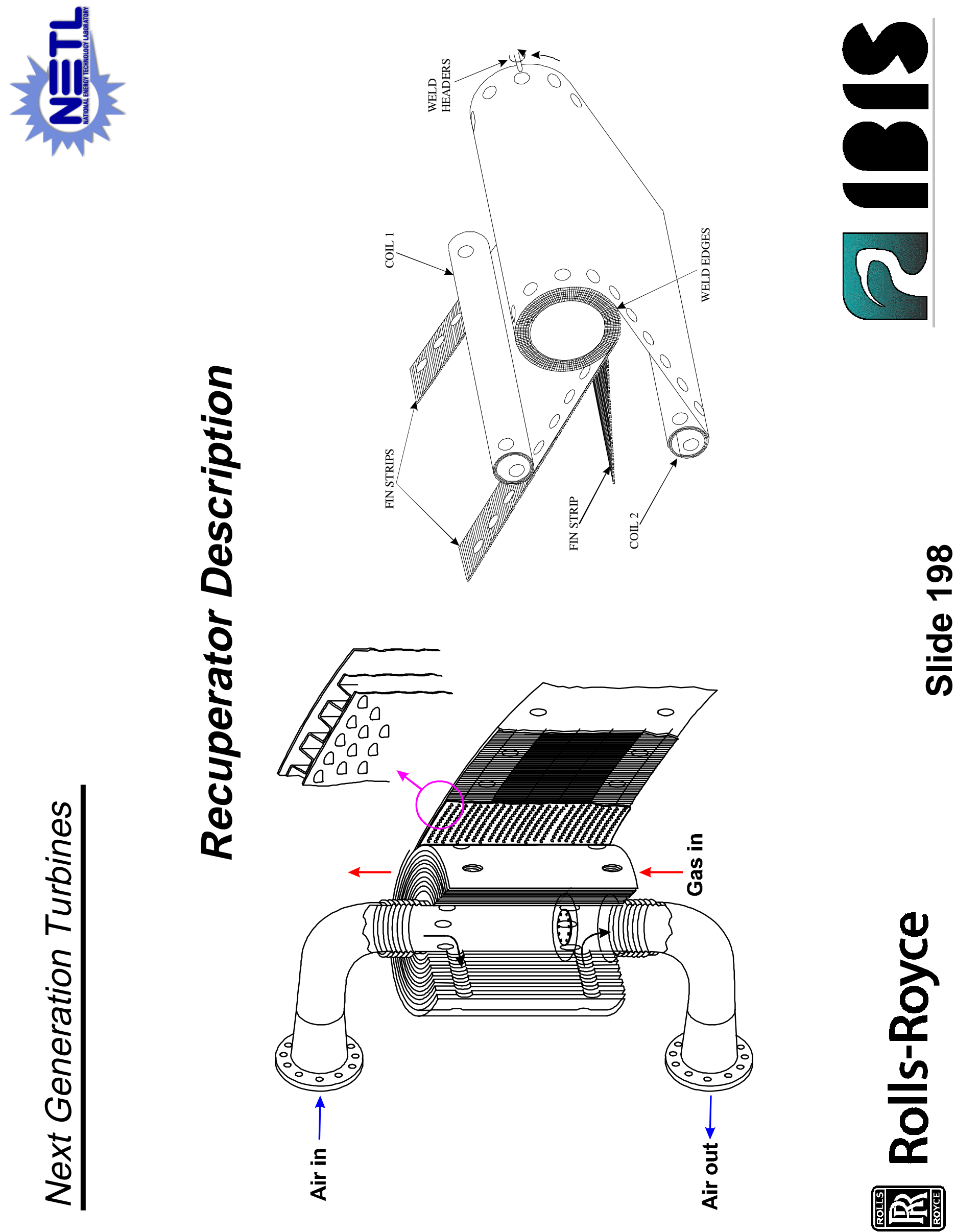
黑

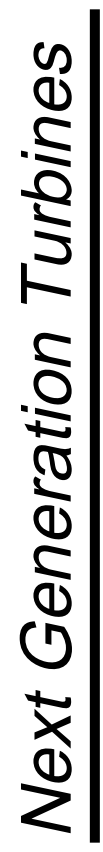

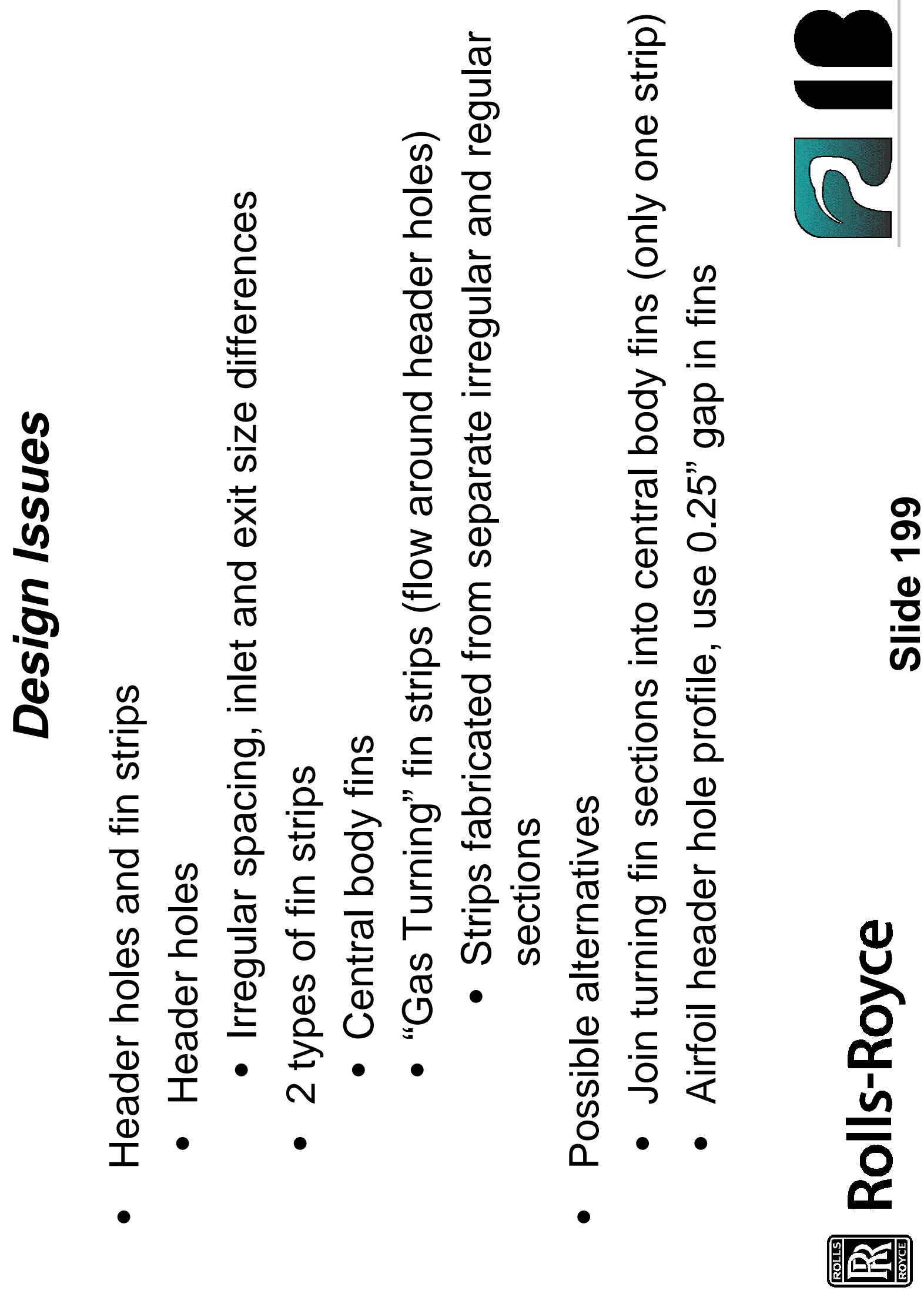



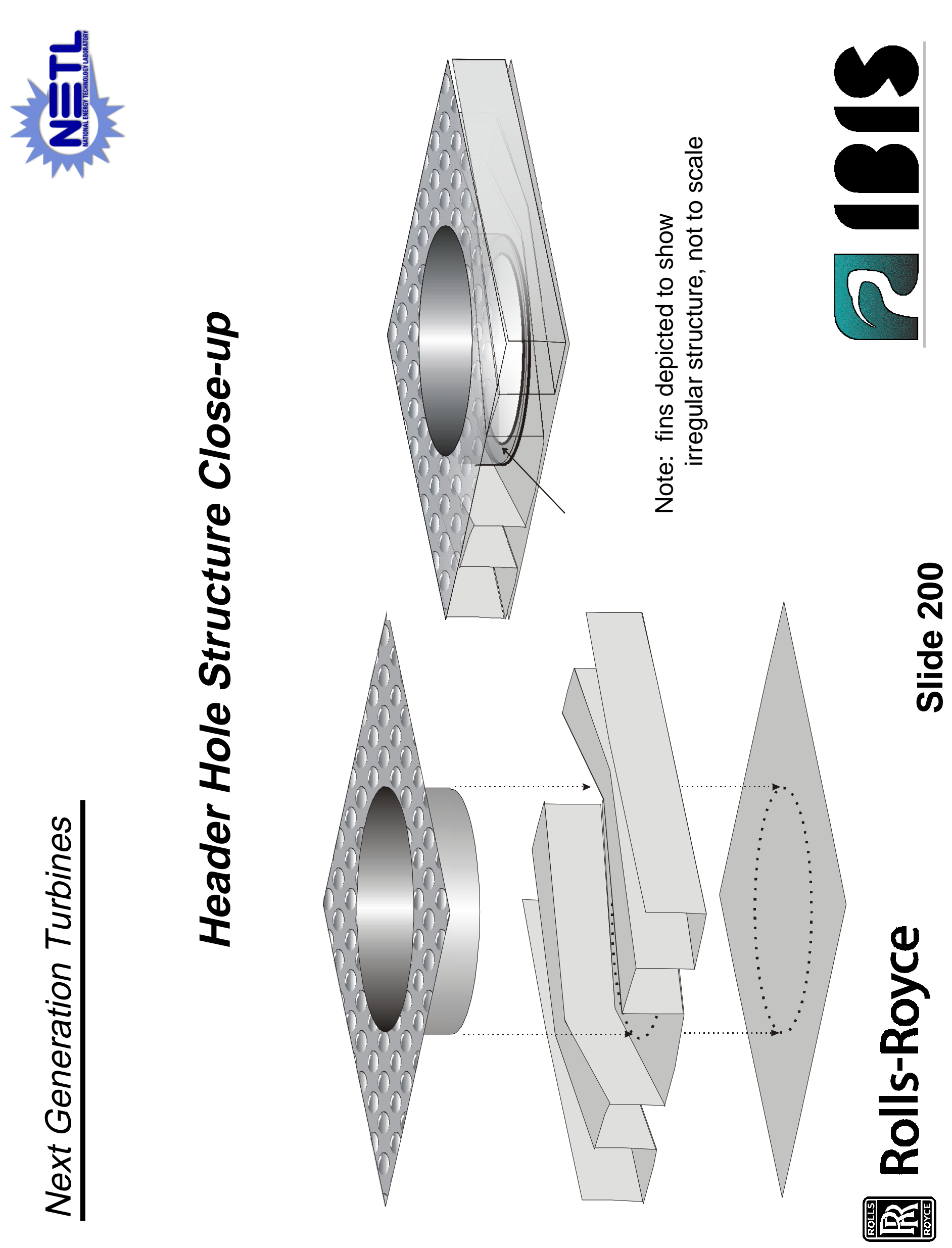


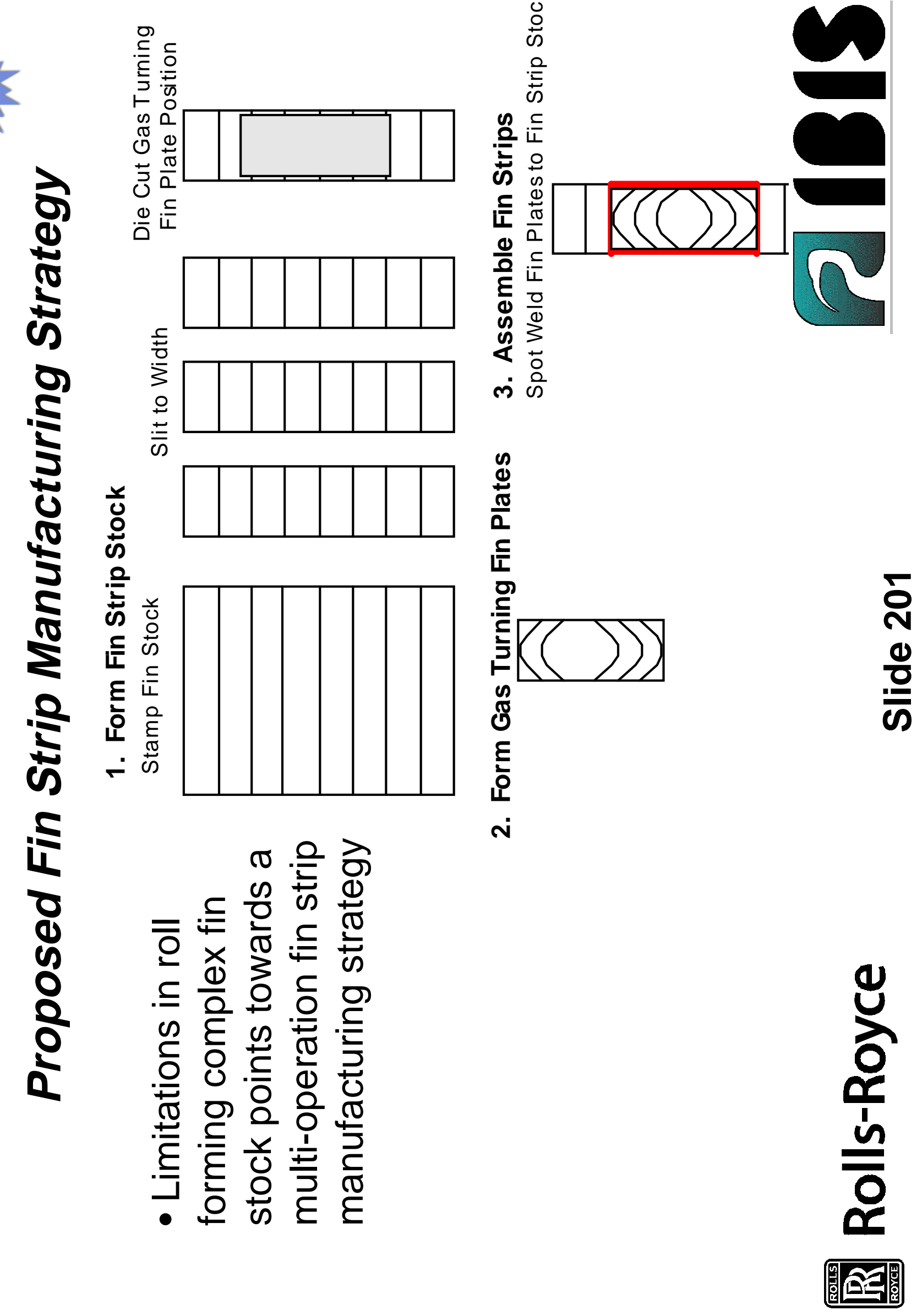



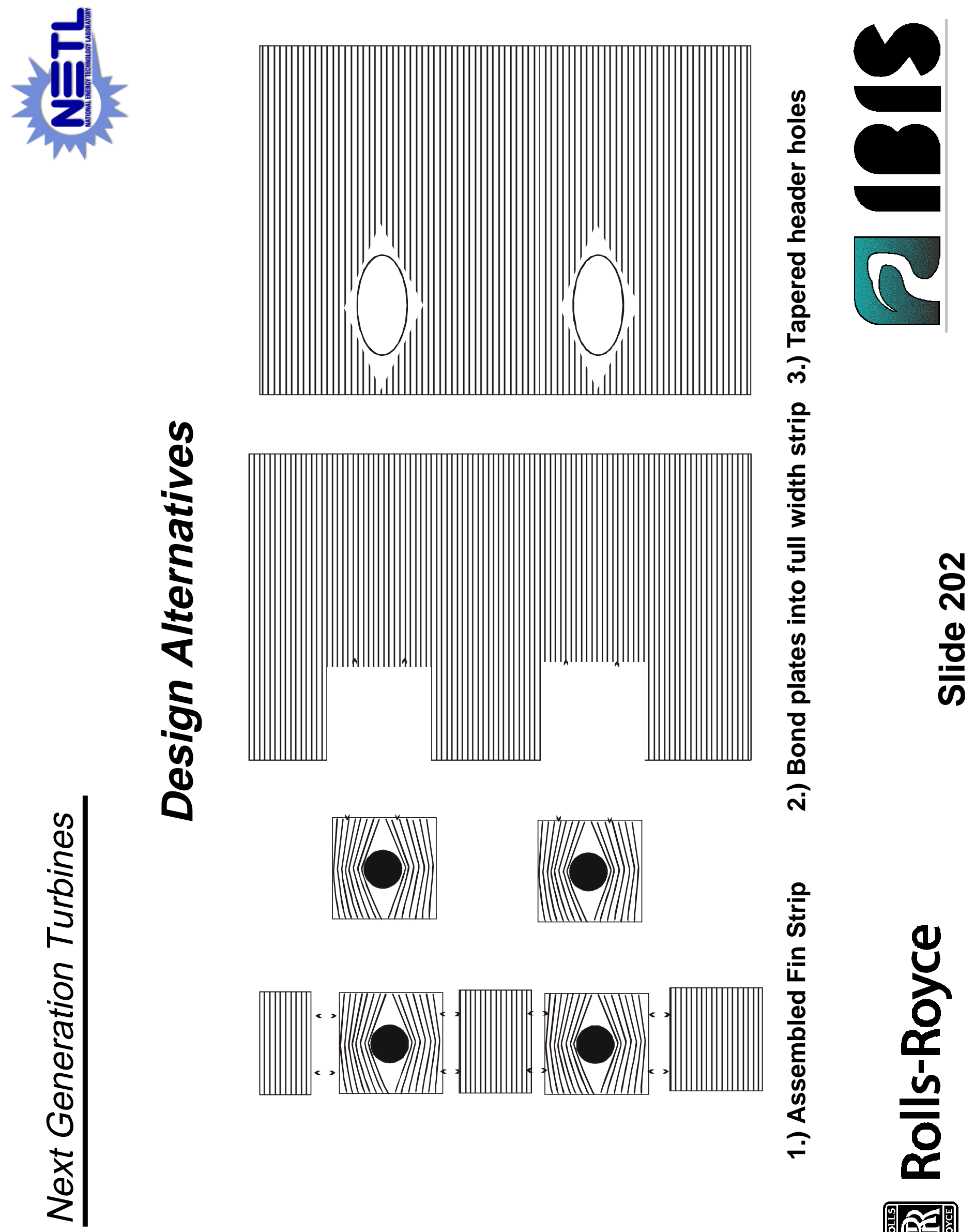

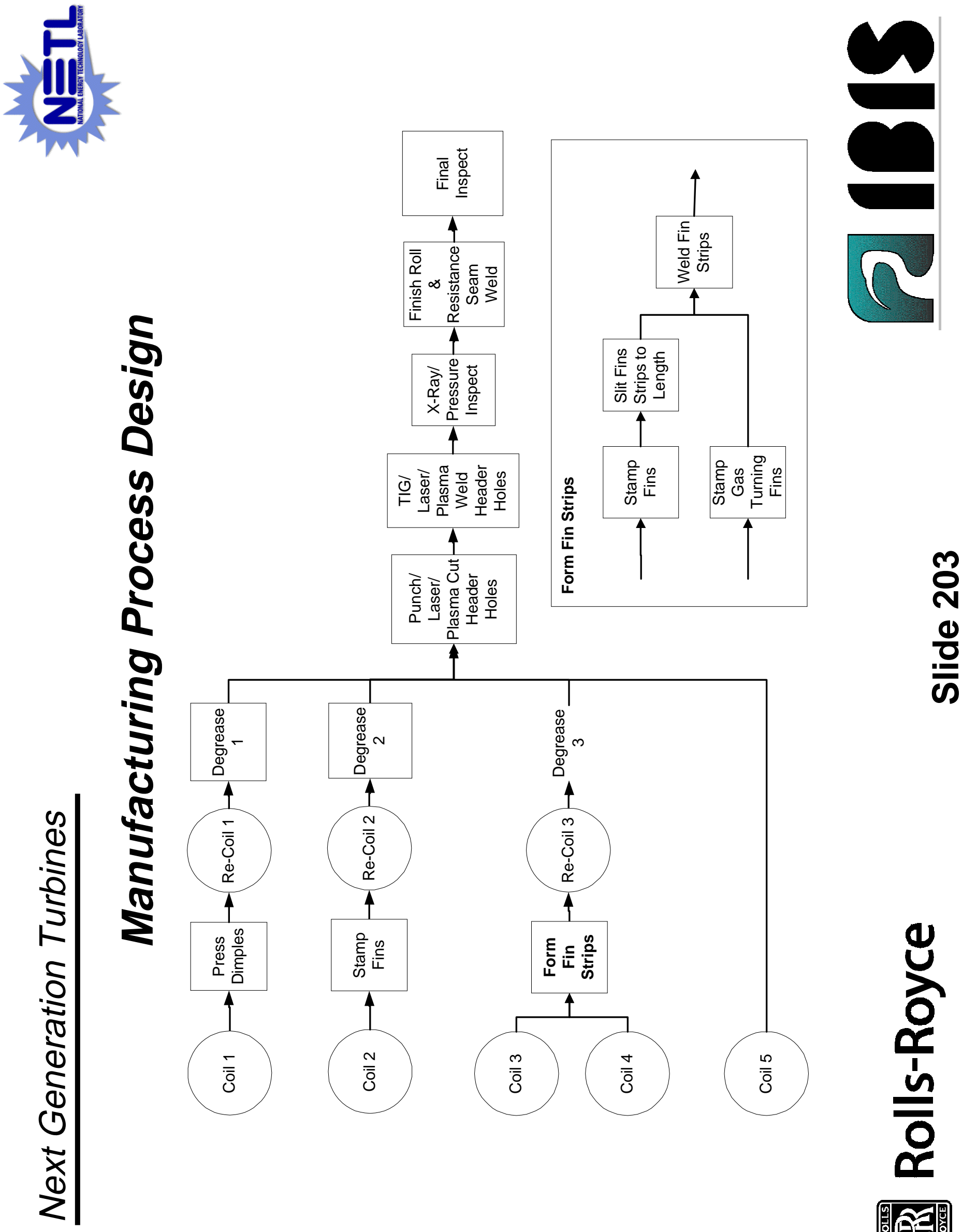

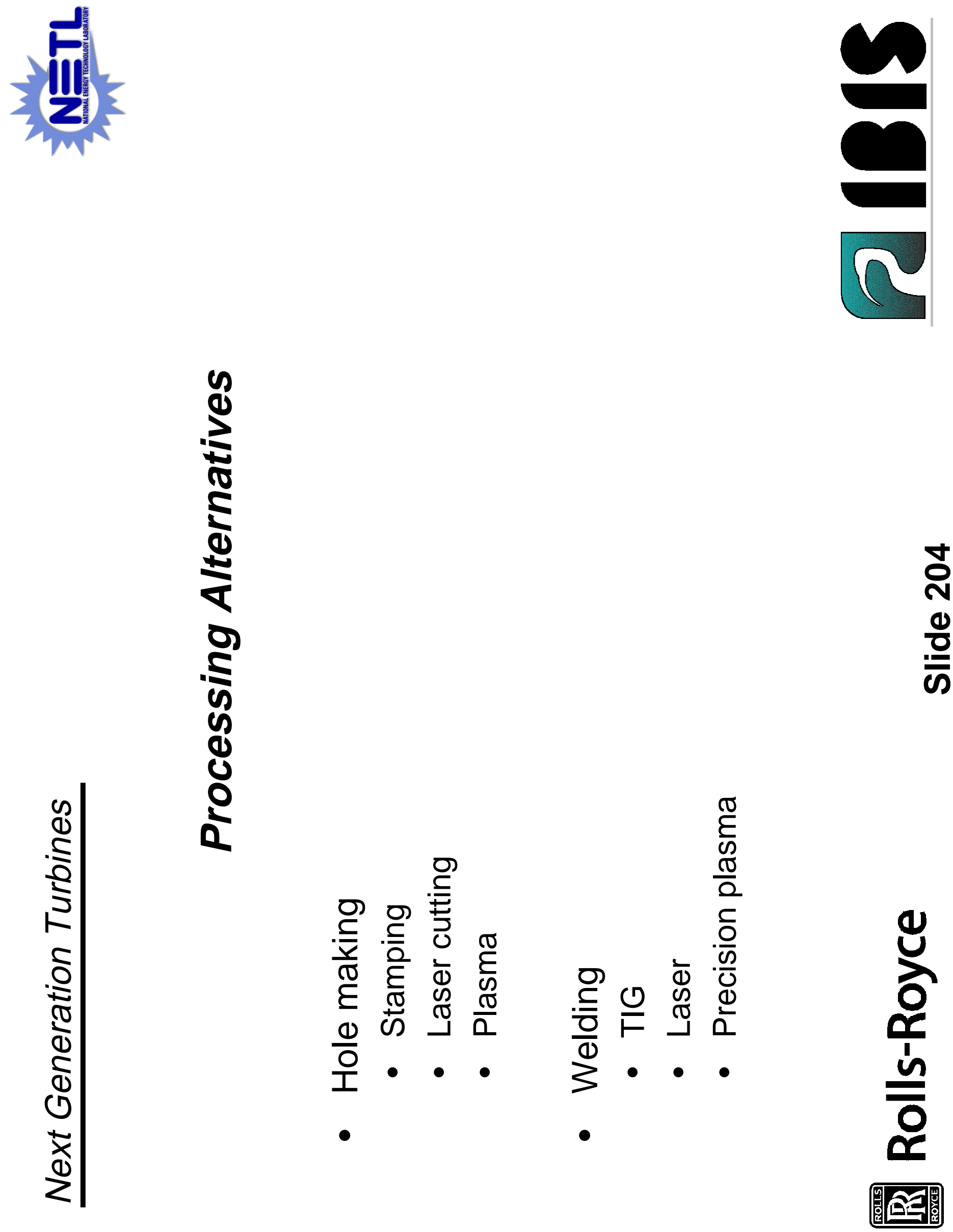

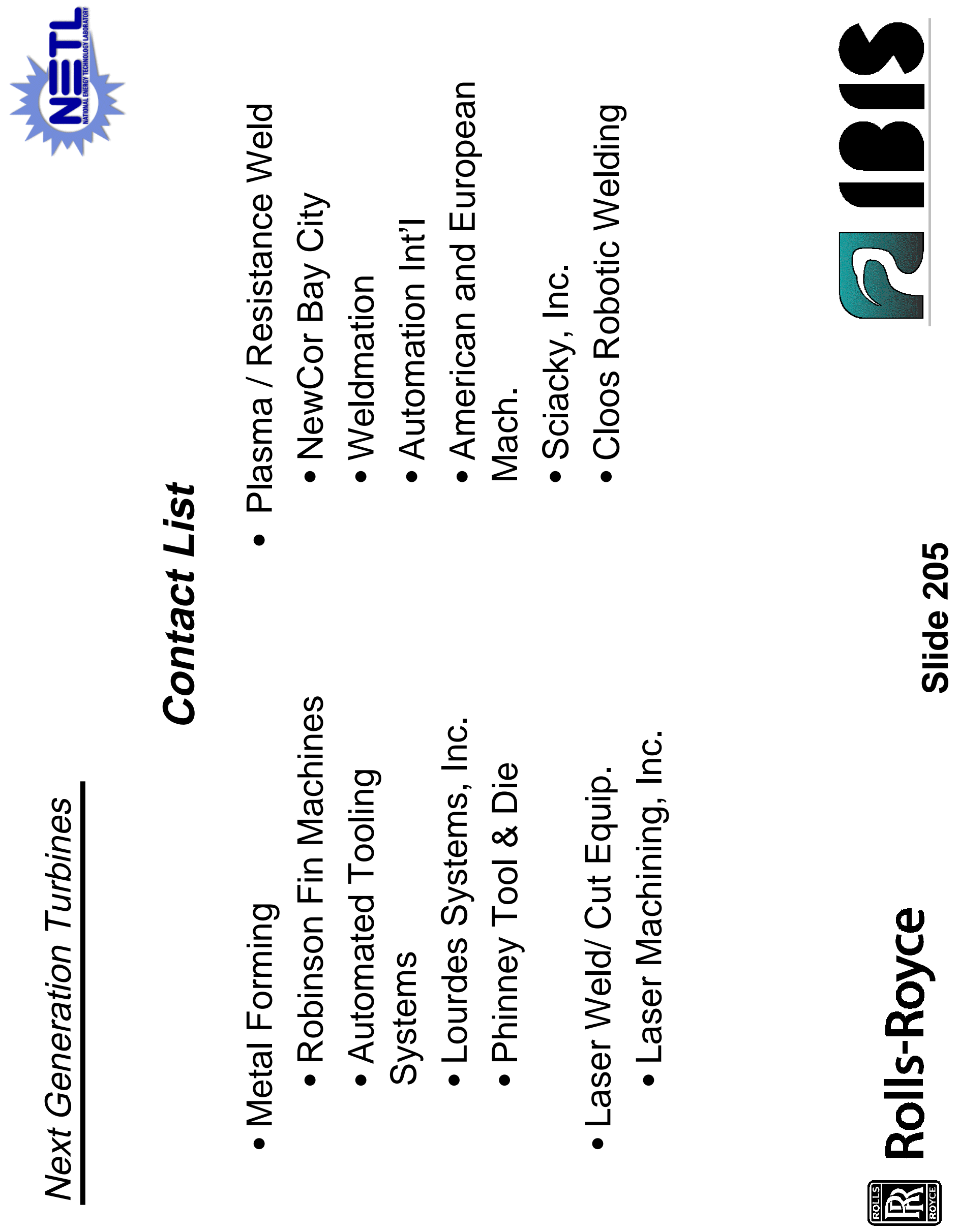

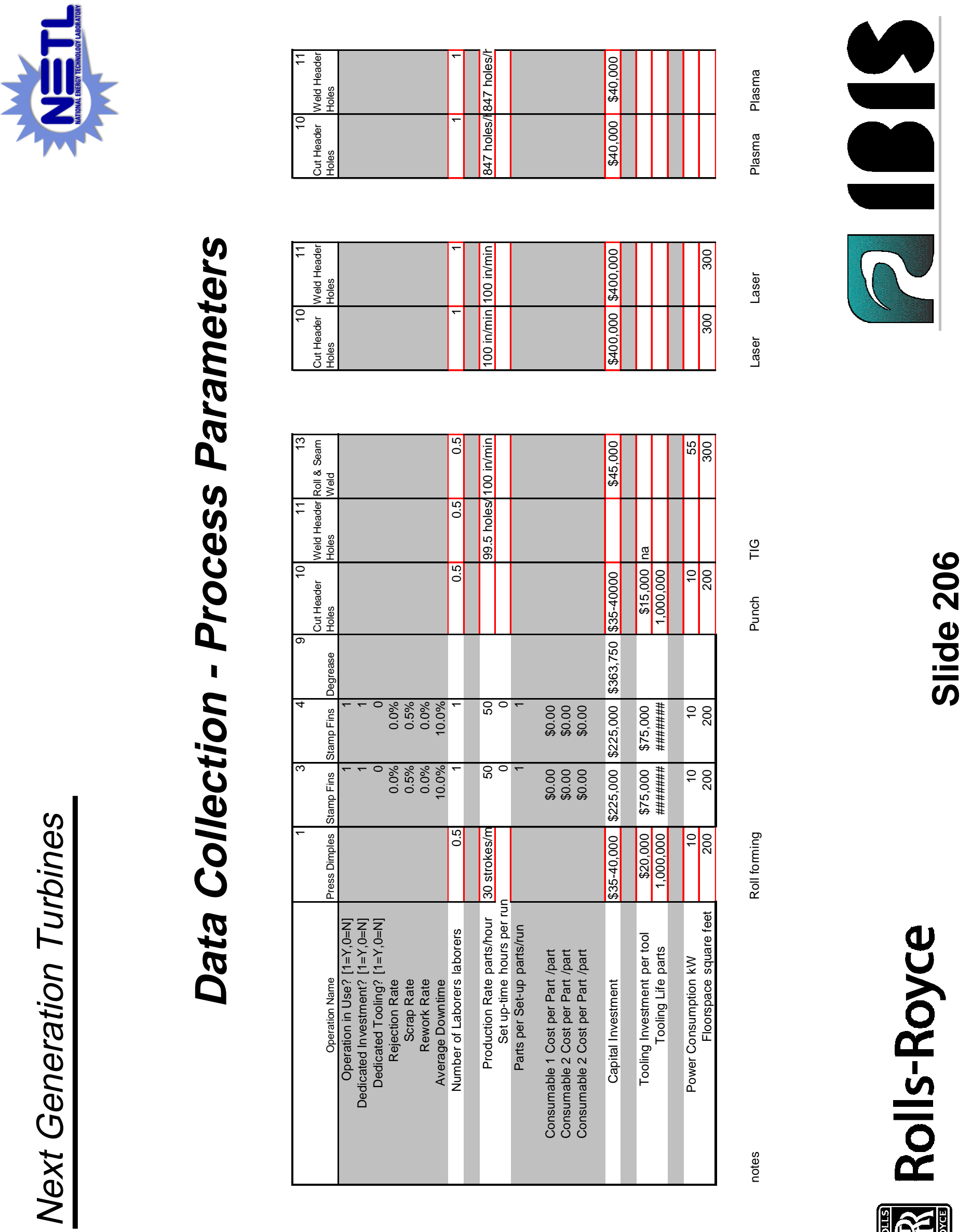

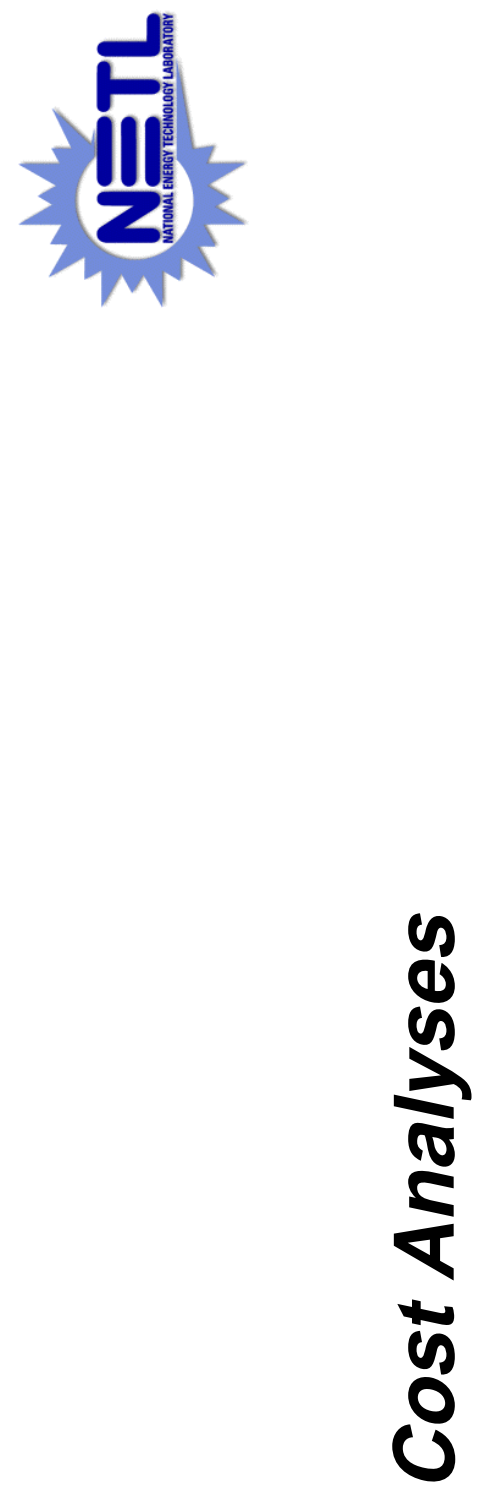

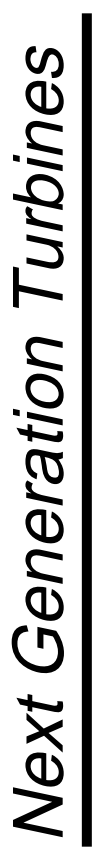
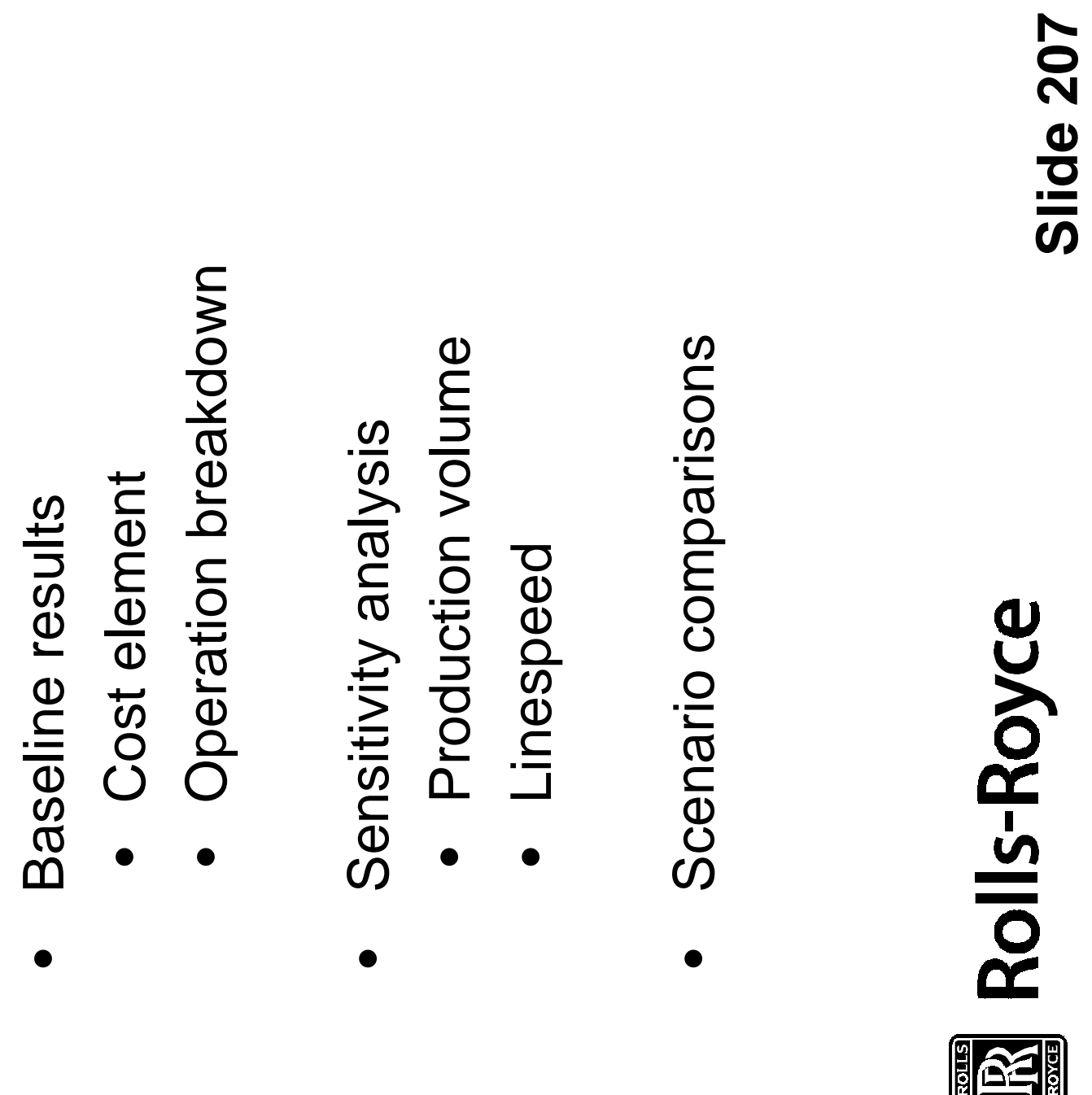

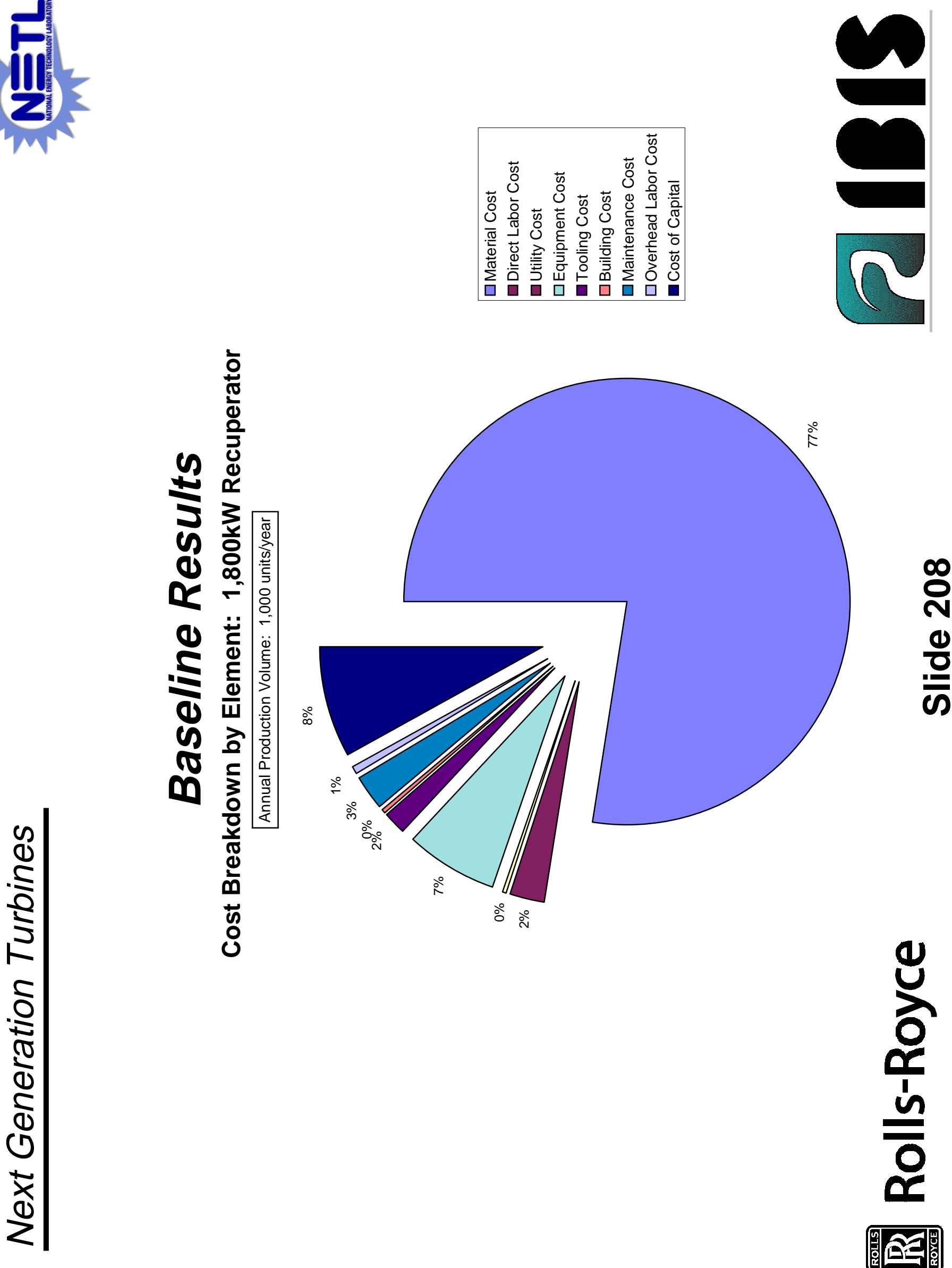

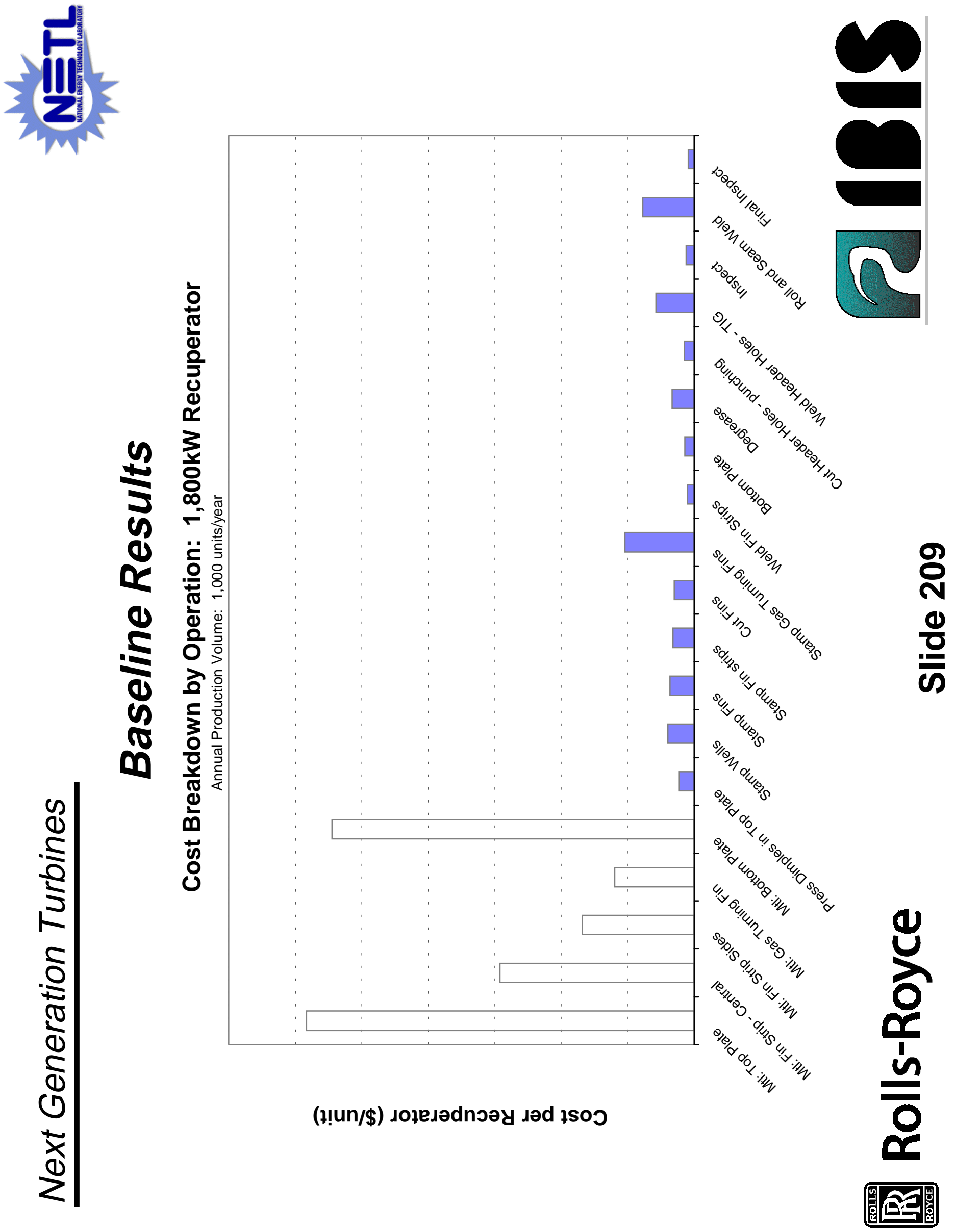

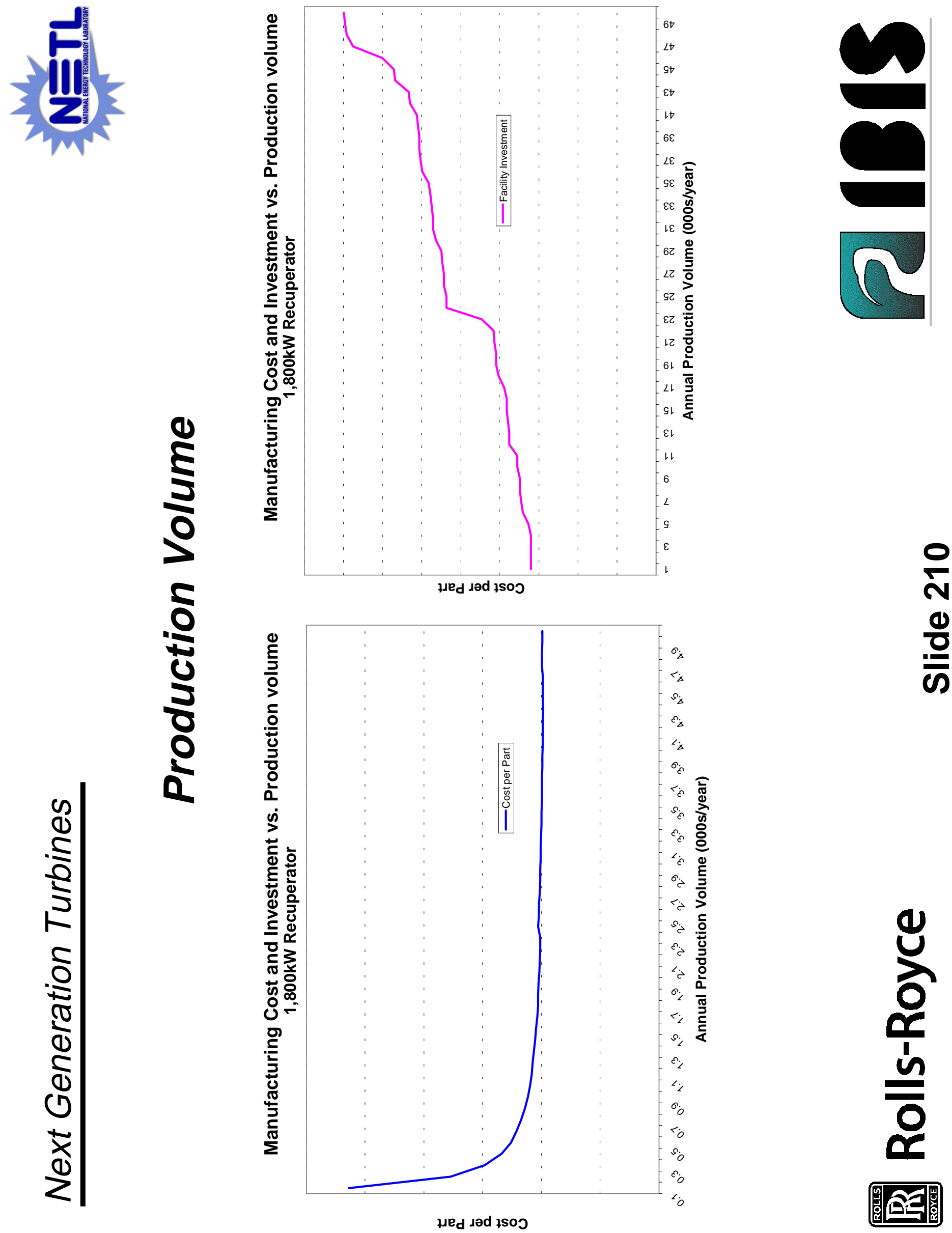

a

$\frac{0}{0}$

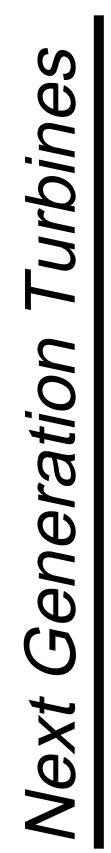




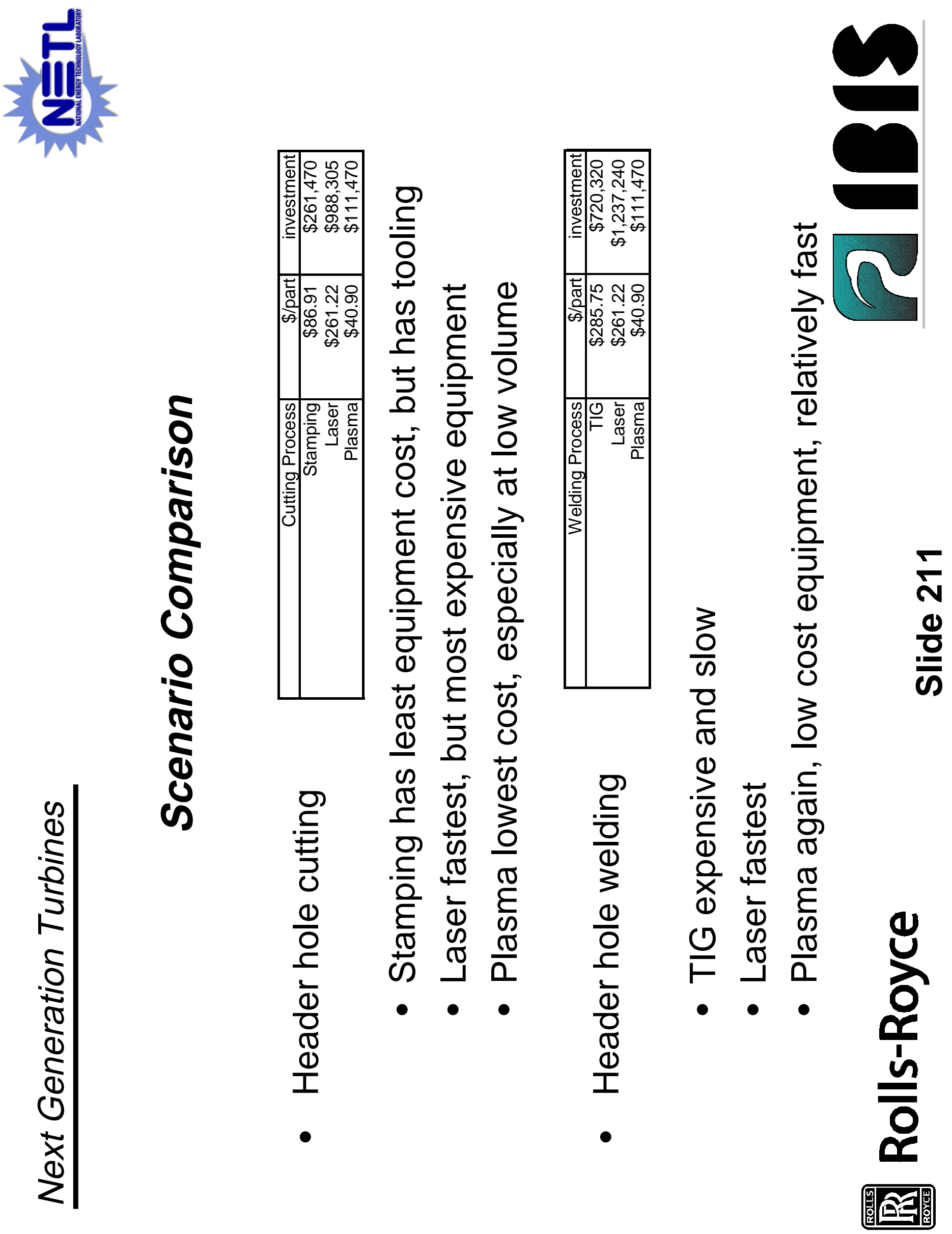




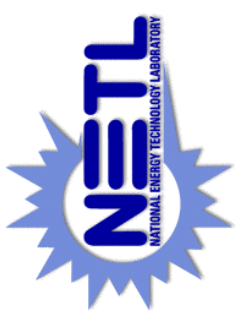

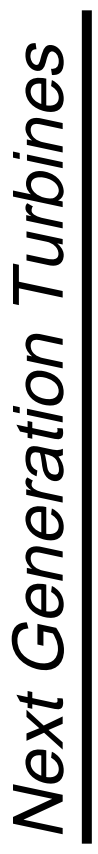
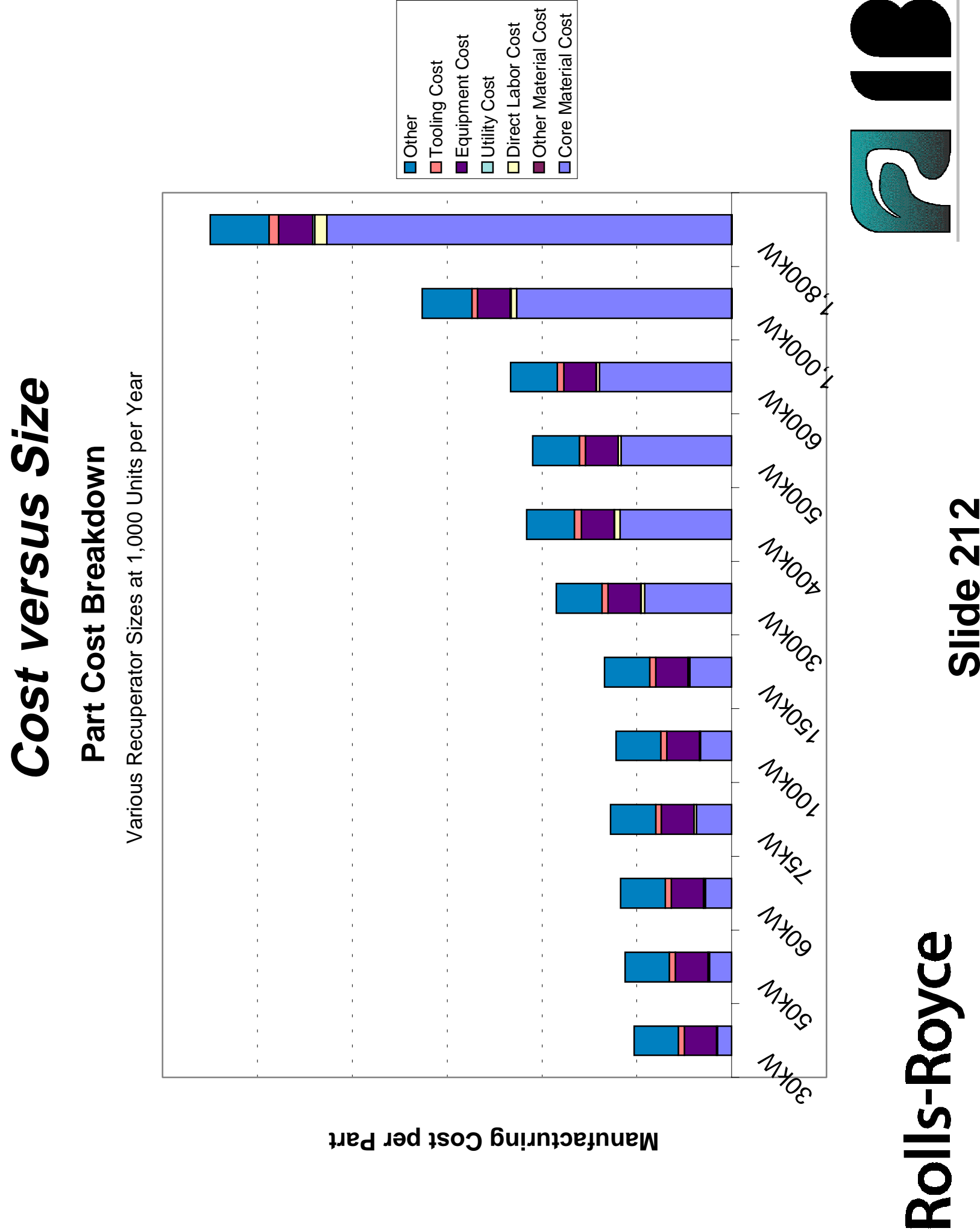


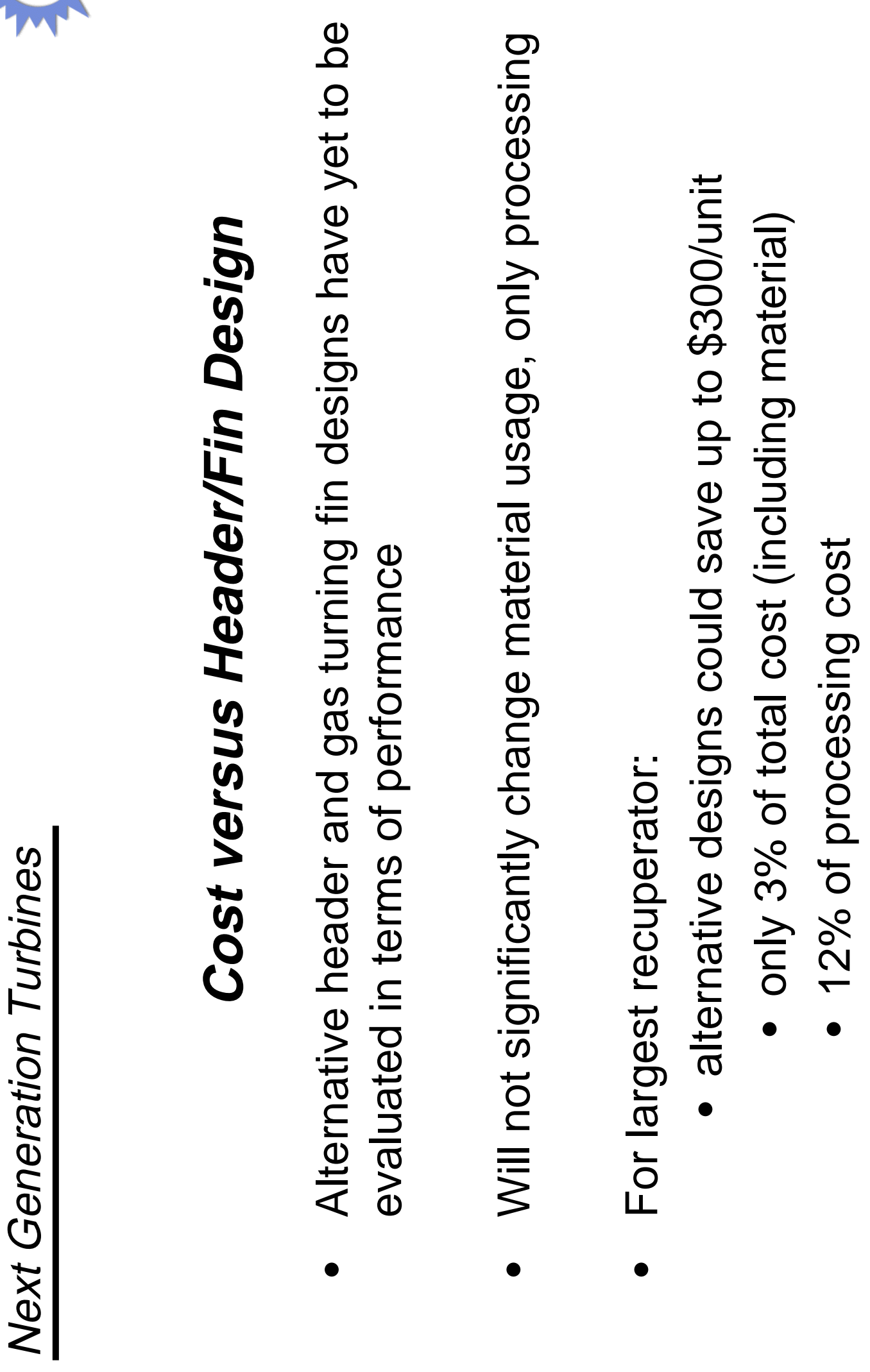

$\frac{m}{N}$
$\frac{0}{0}$
$\frac{0}{\omega}$

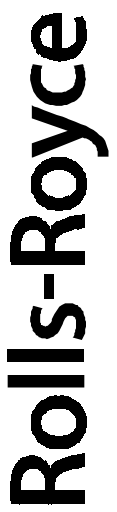




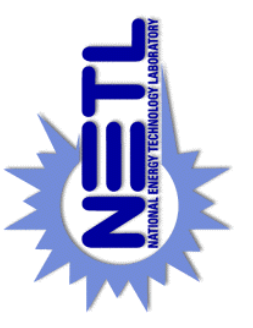

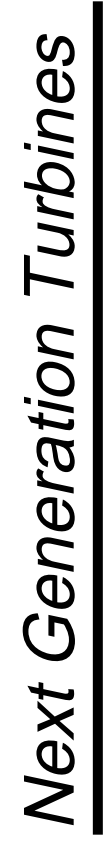

$\frac{9}{2}$

ฮ

$+$

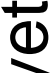

ఏ

흥

$\frac{1}{0}$

g D

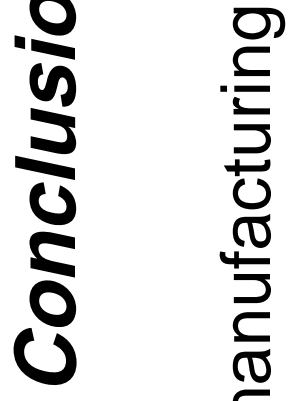

o

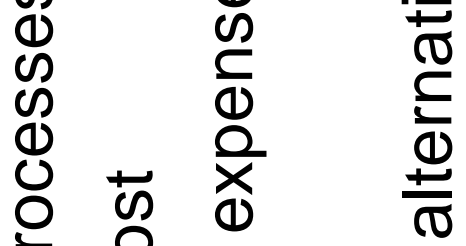

(1)

0

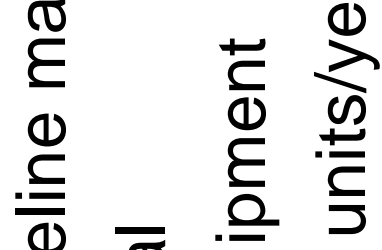

하 $\overline{0}$

응 흉

Ф)

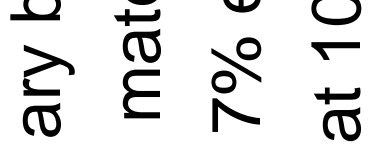

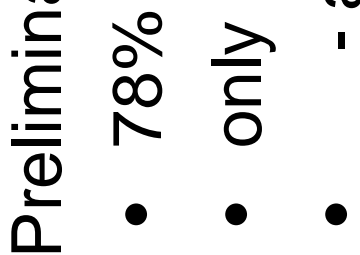

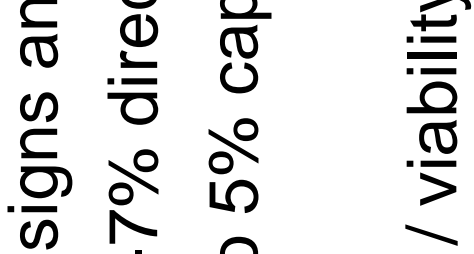

ब 1 क क

क

\) 우 윽

㐫

لِ

¿

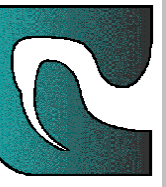

Фथ

U

2

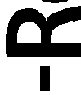

n

0

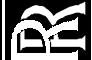




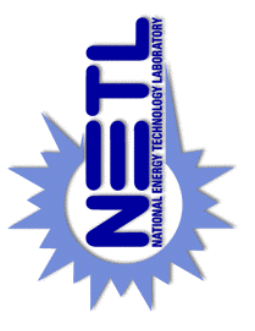

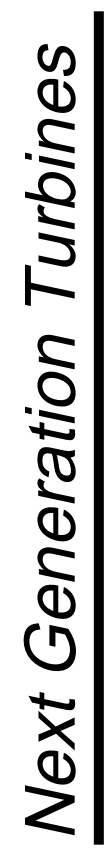
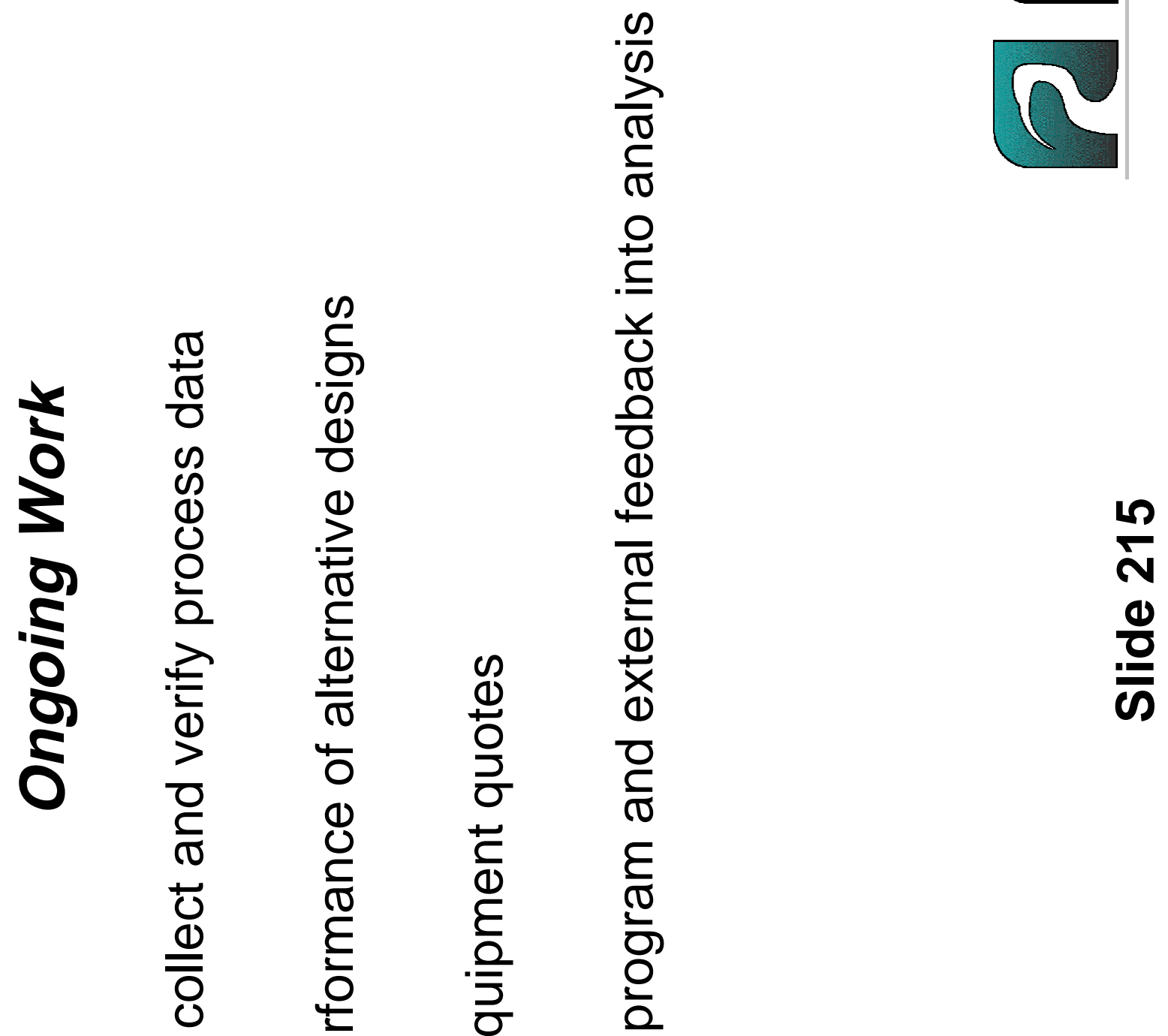

이 (1)
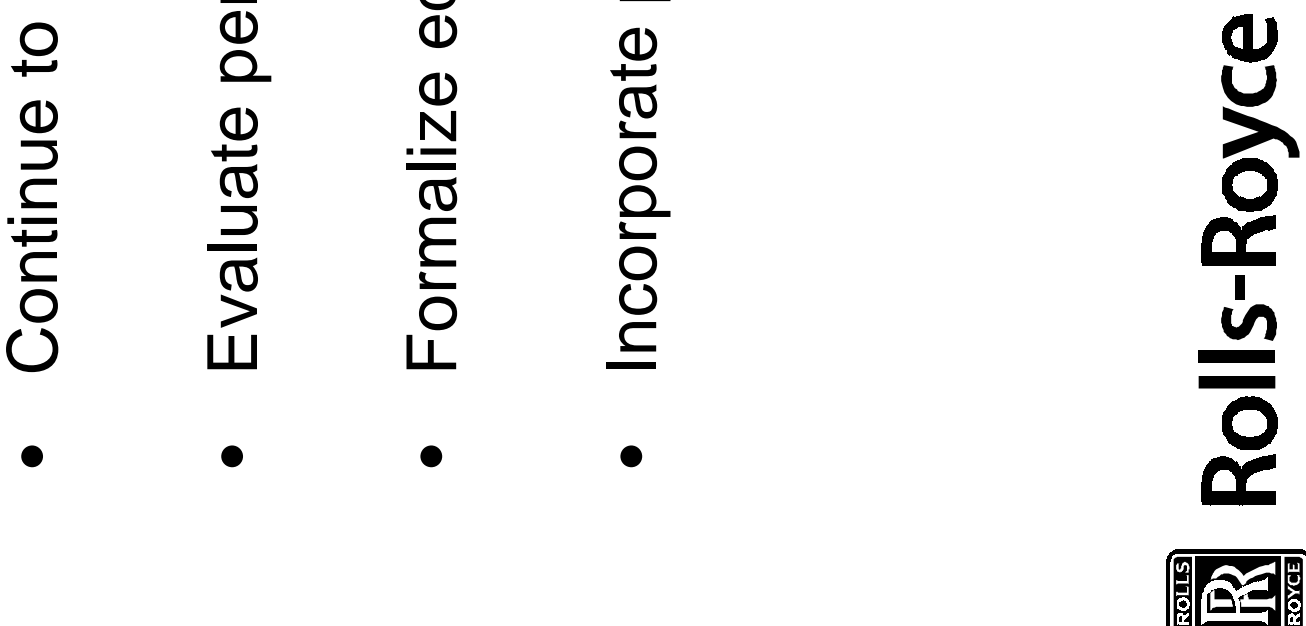


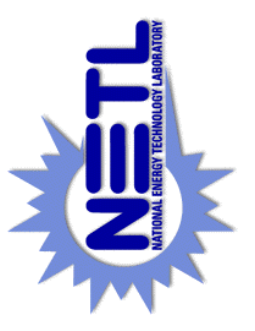

0
0
$\frac{1}{0}$
$\frac{1}{5}$
$\frac{5}{2}$
$\frac{0}{0}$
$\frac{0}{0}$
0
$\frac{x}{0}$
$\frac{1}{z}$
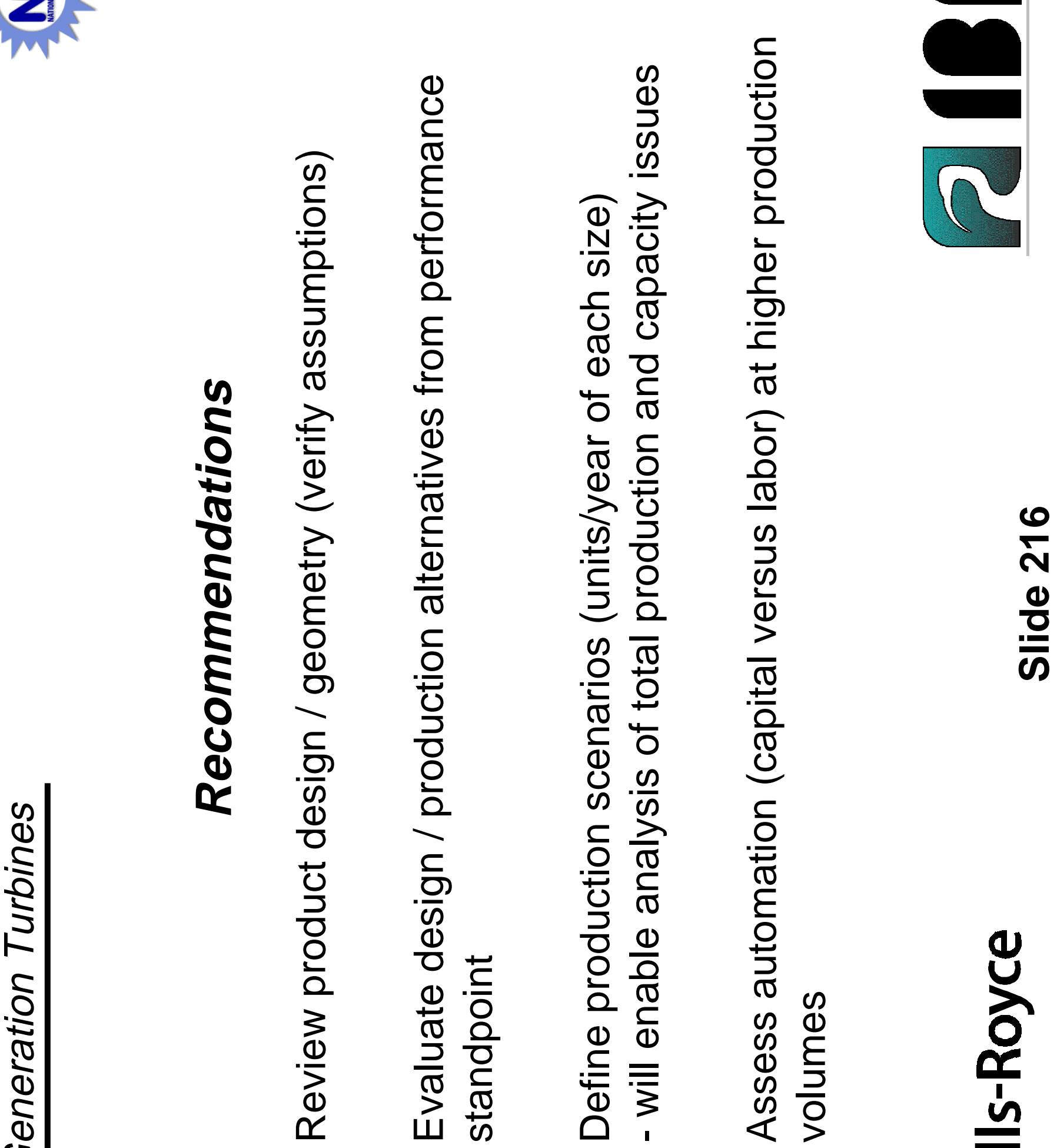

बㅇ

ธั

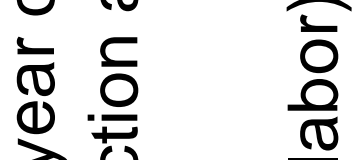

$\stackrel{0}{\supset}$

证 $\frac{n}{n}$

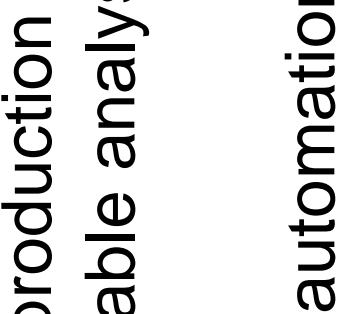

a

की

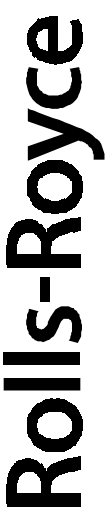



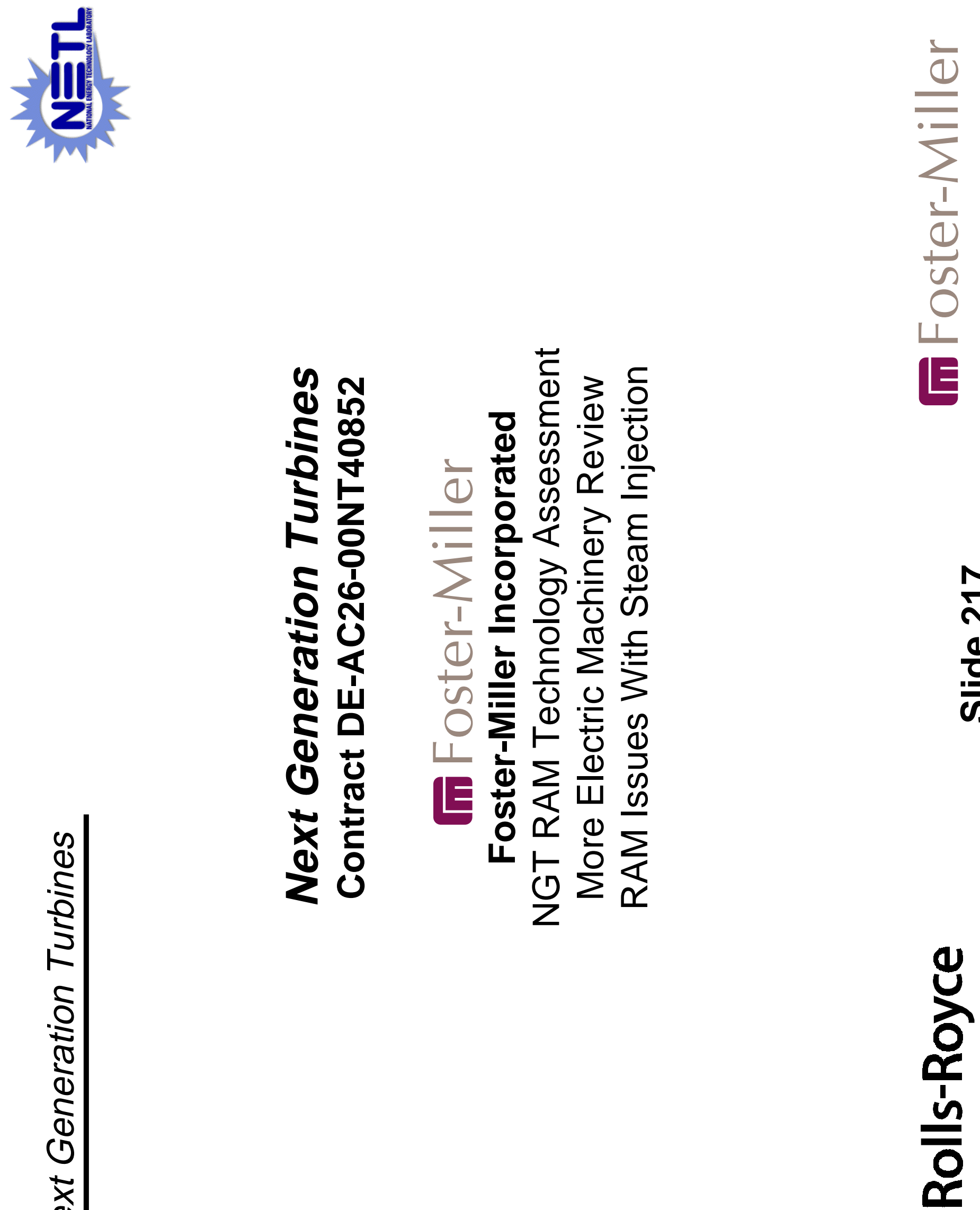


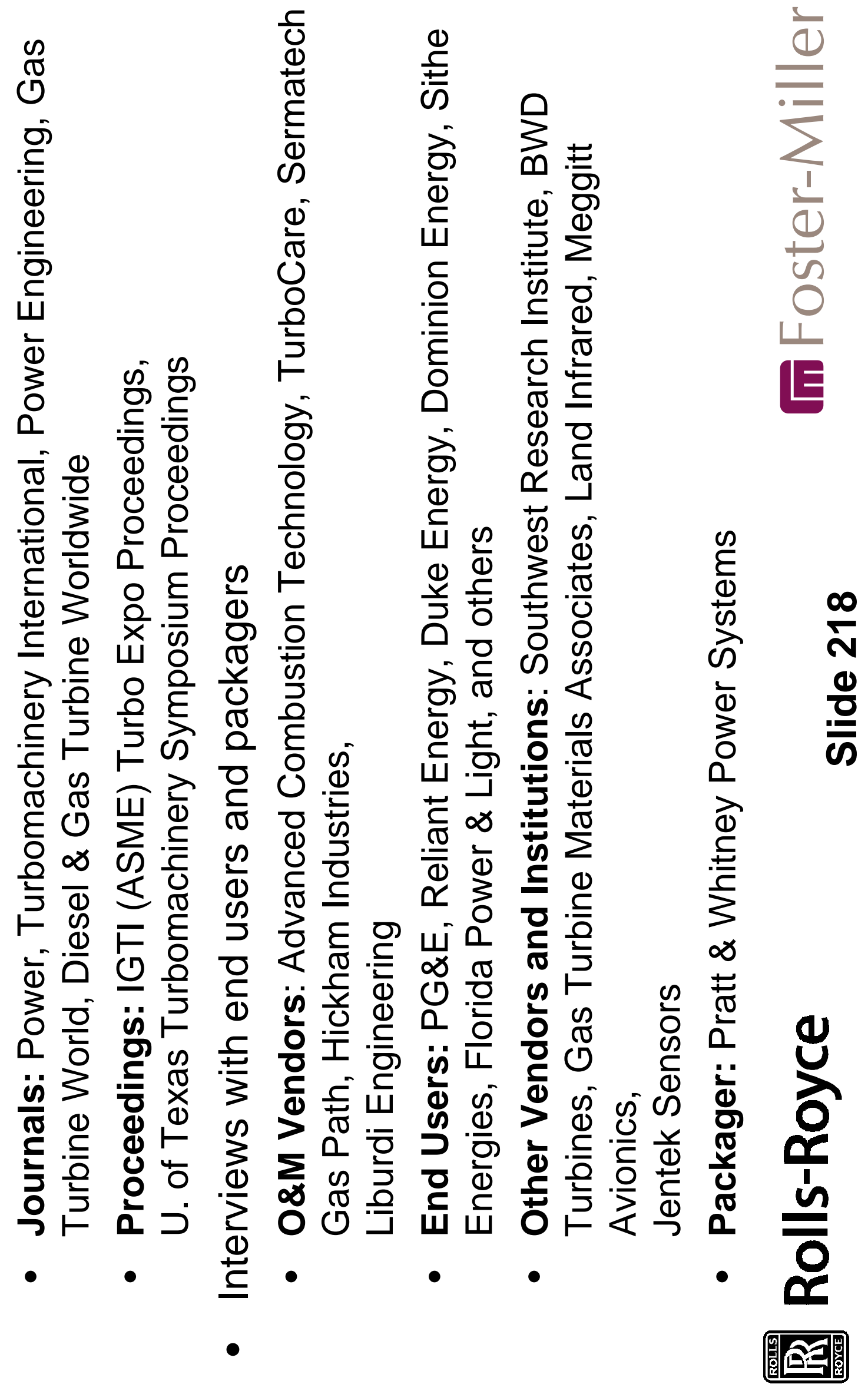



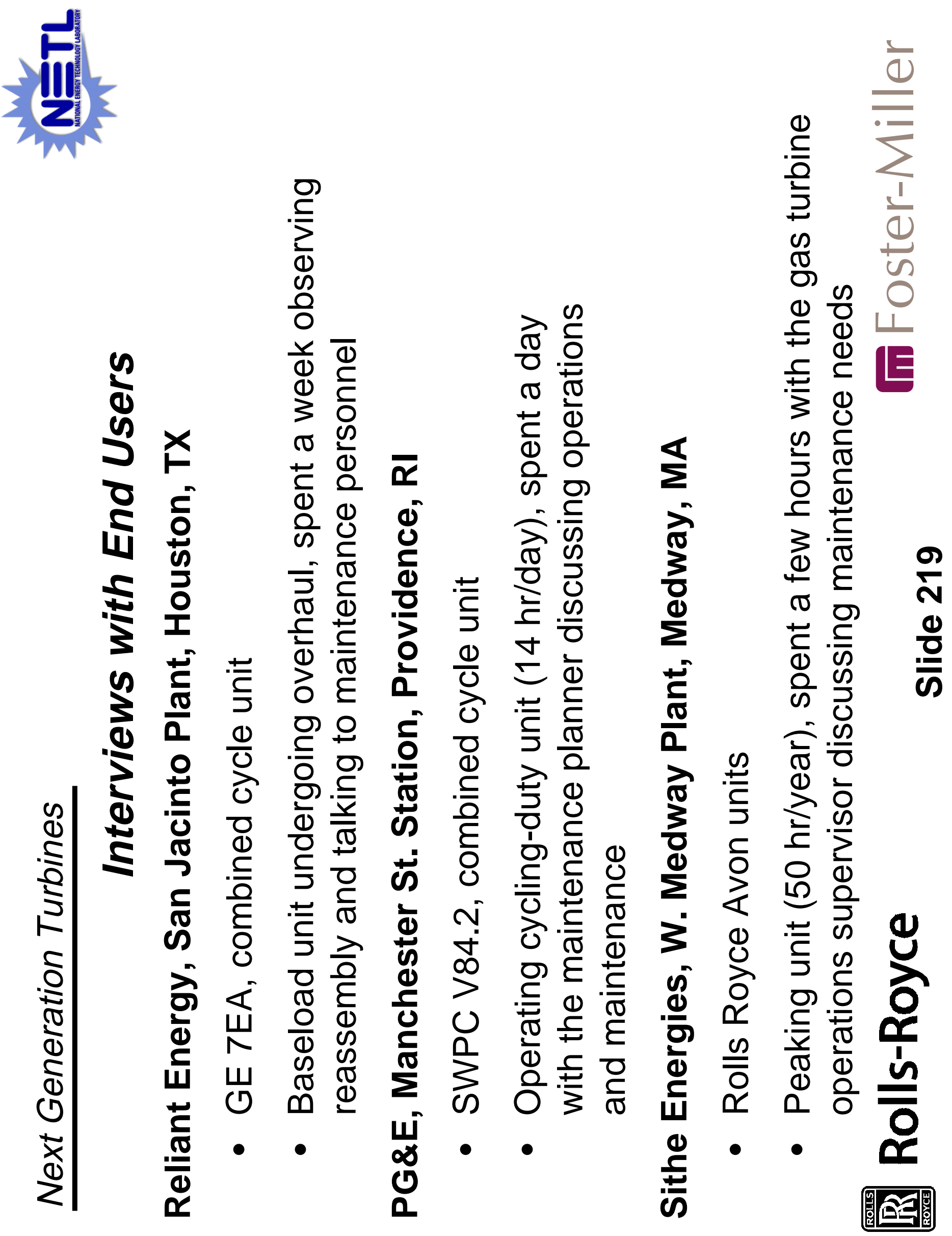

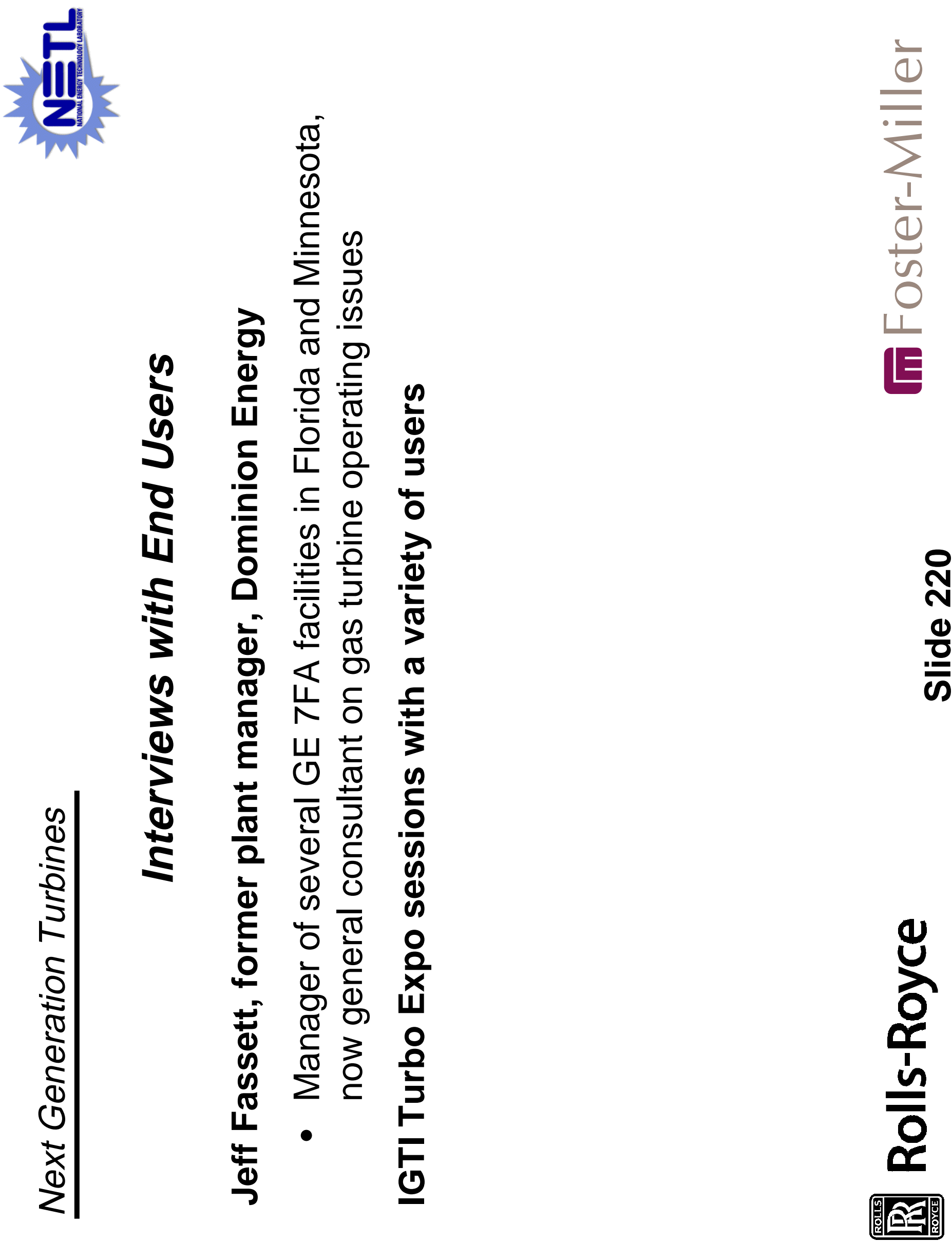

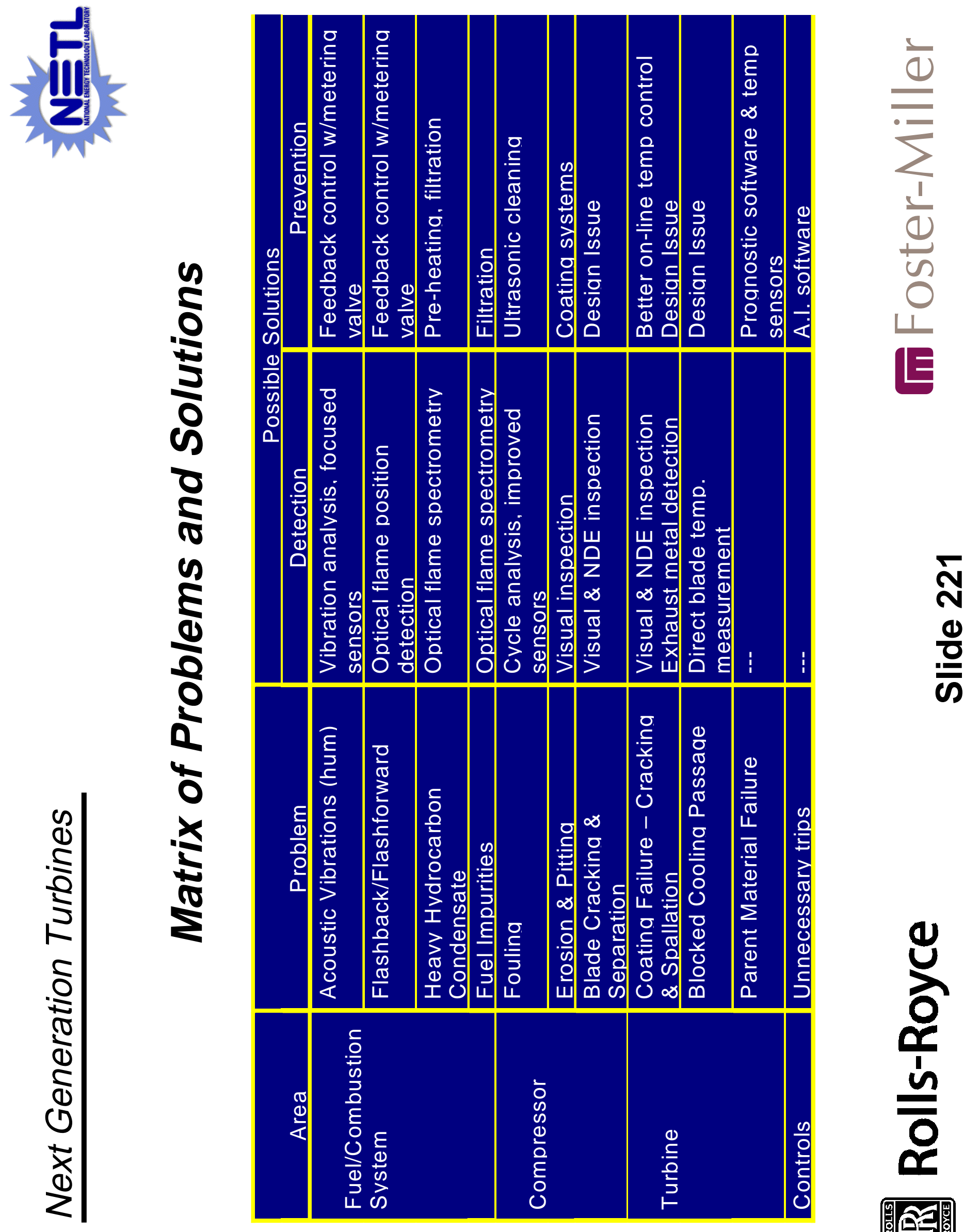


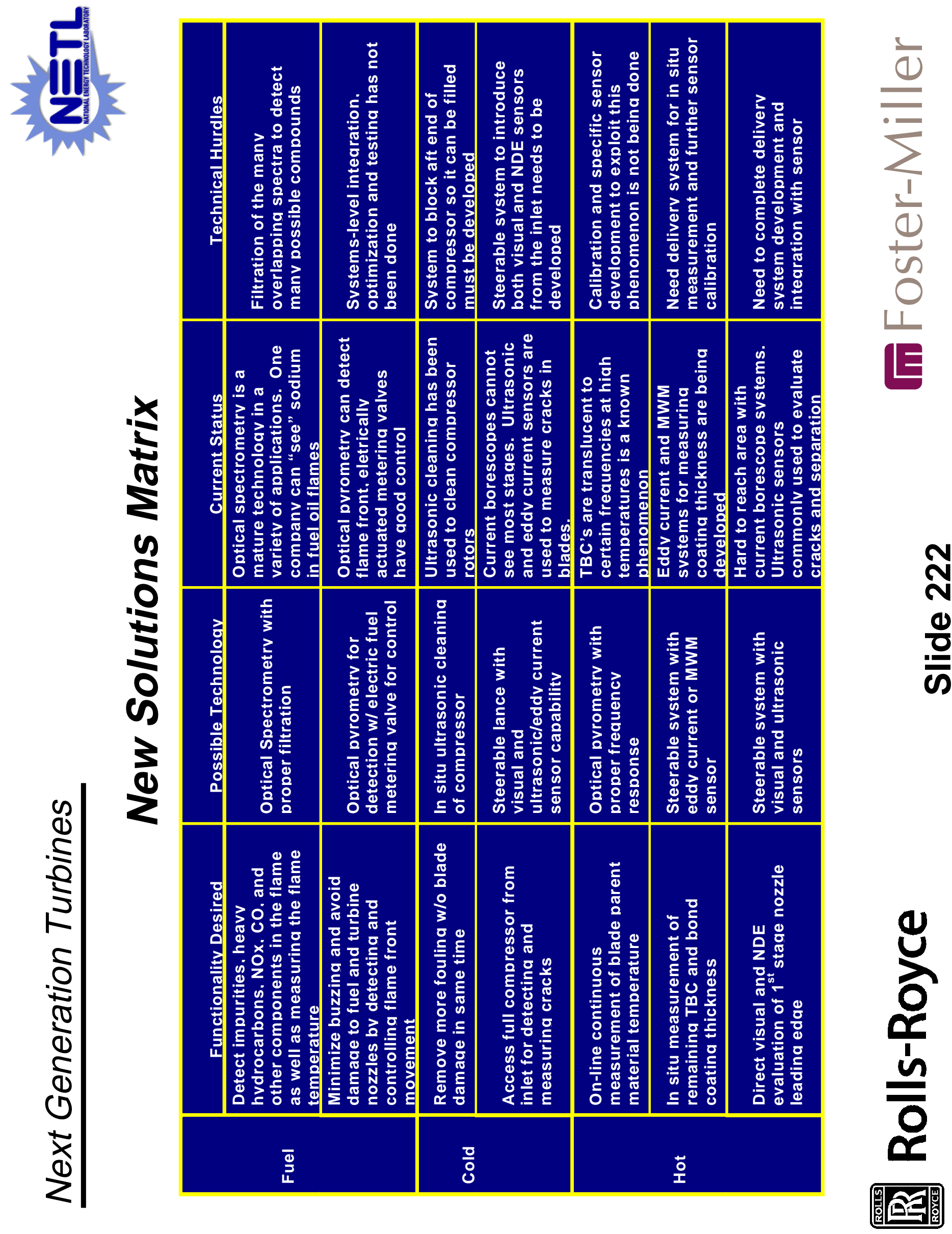



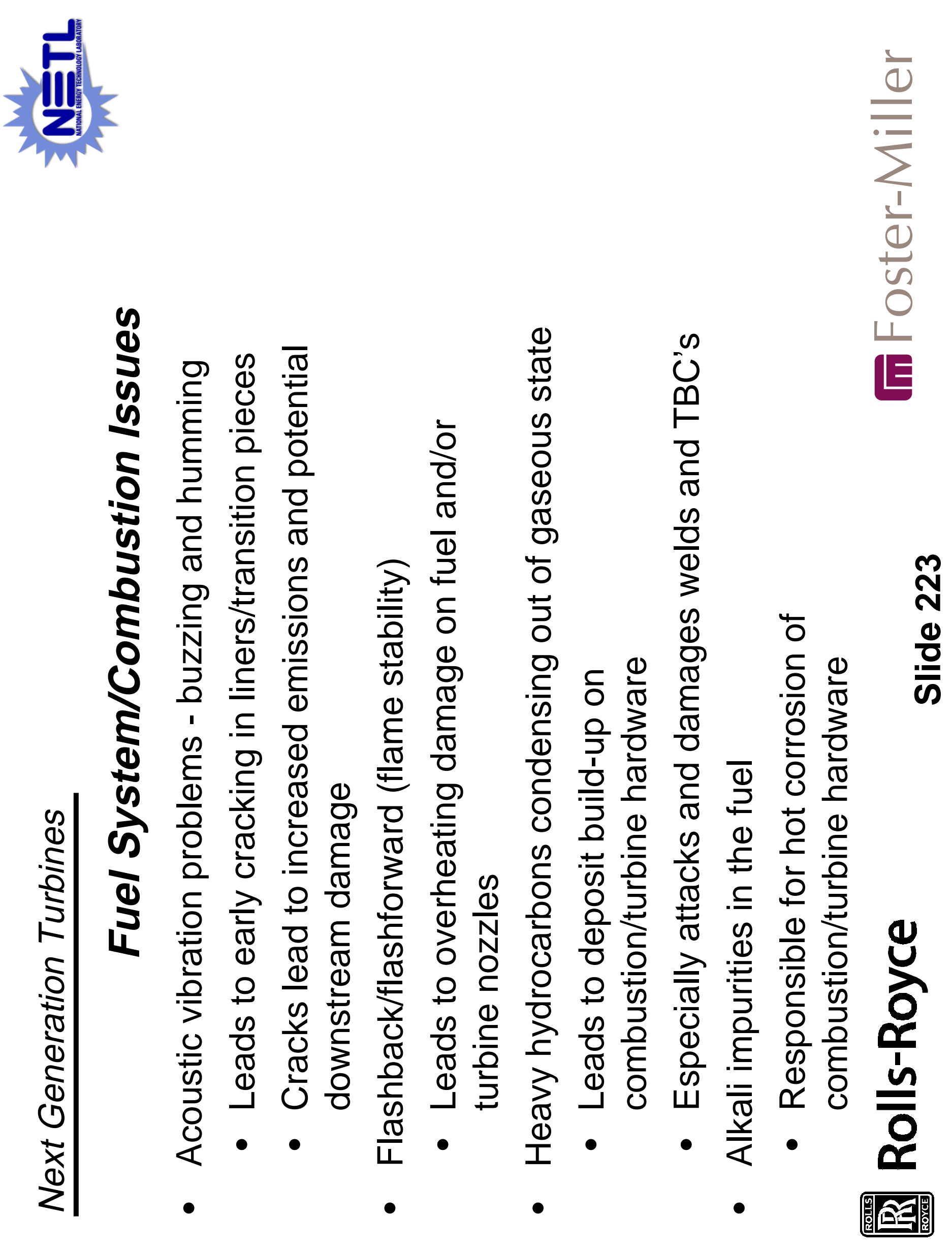


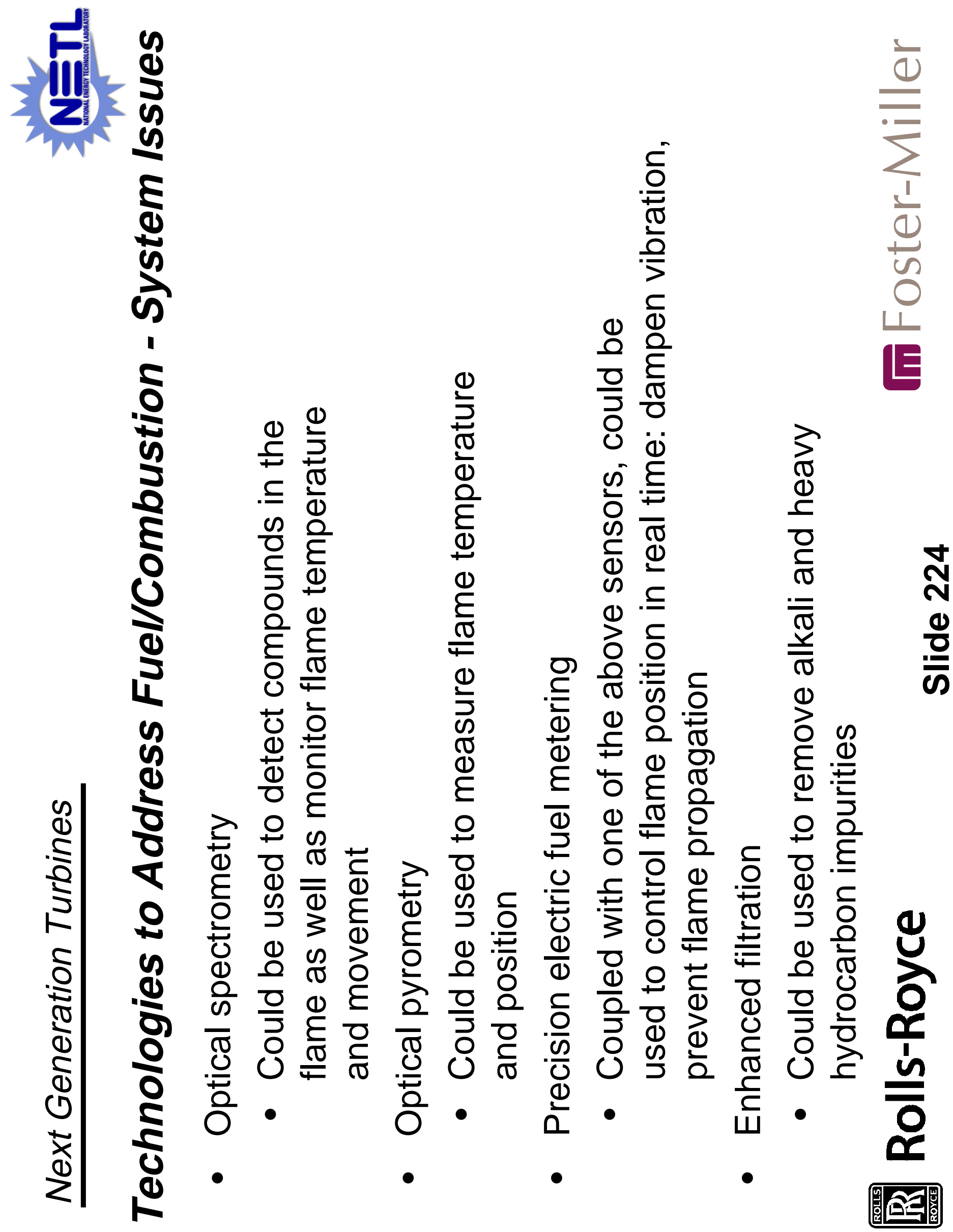




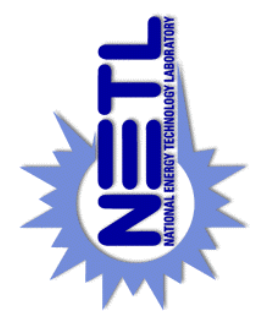

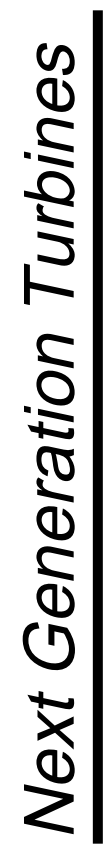
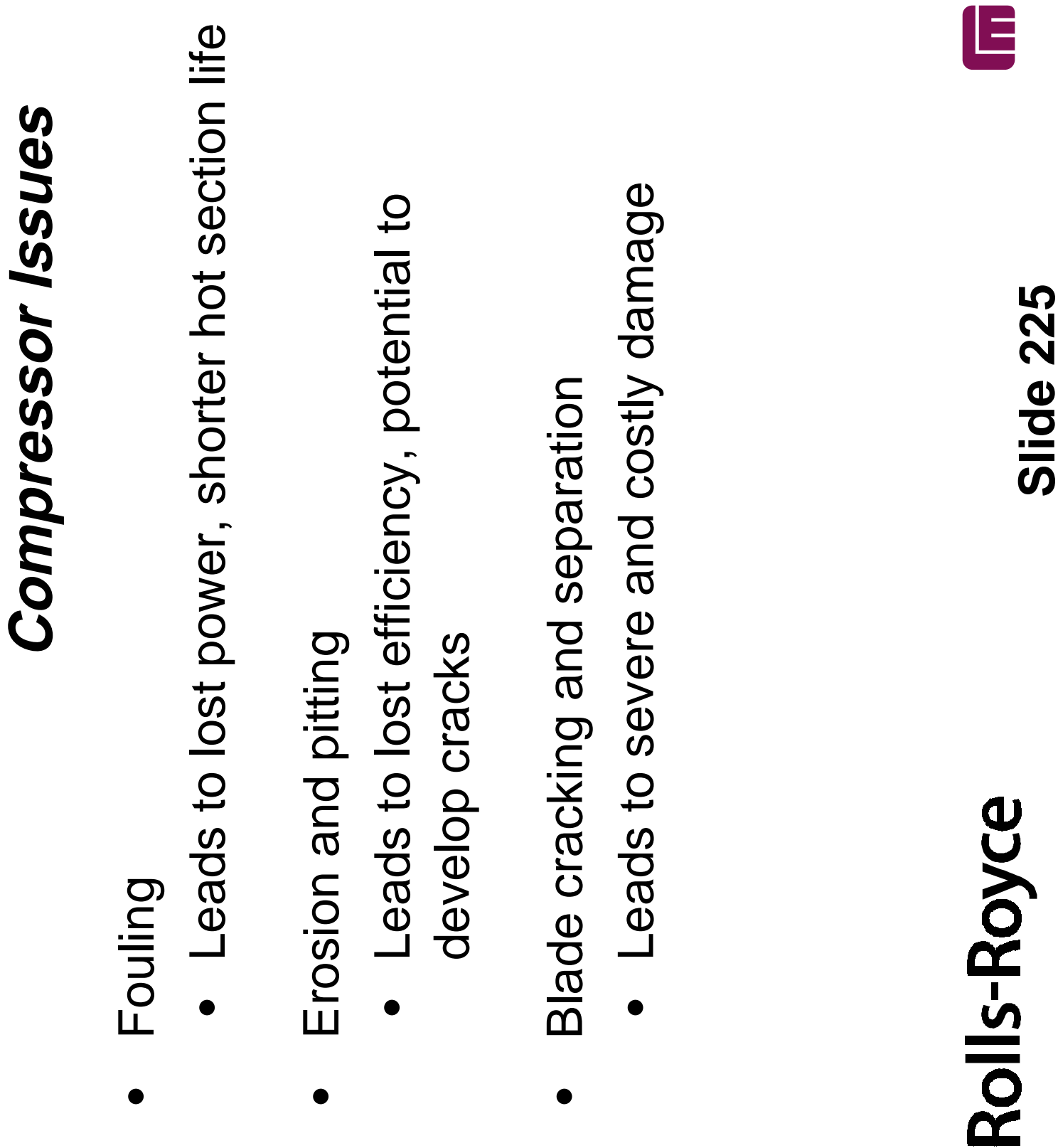

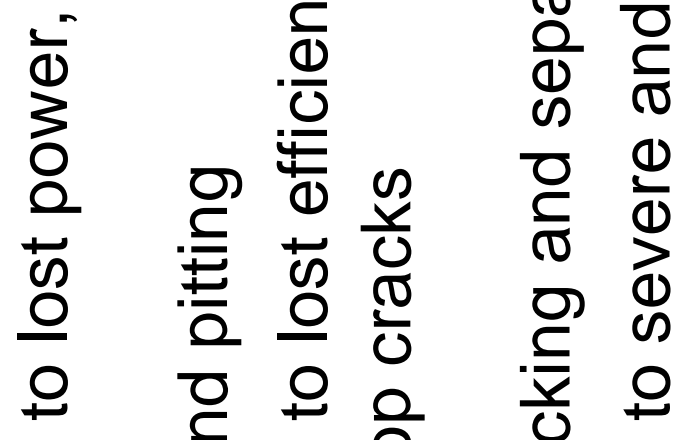

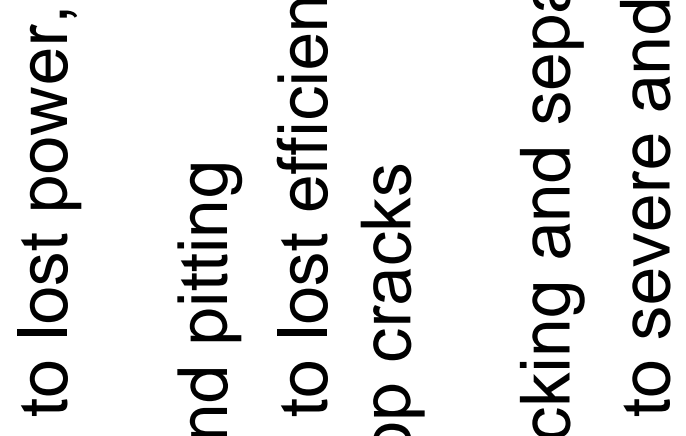

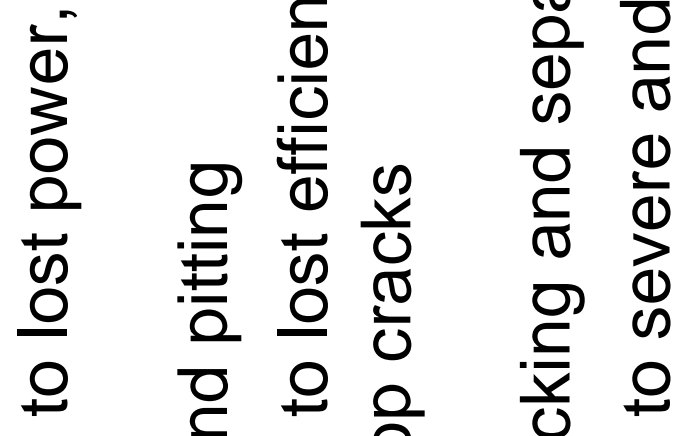

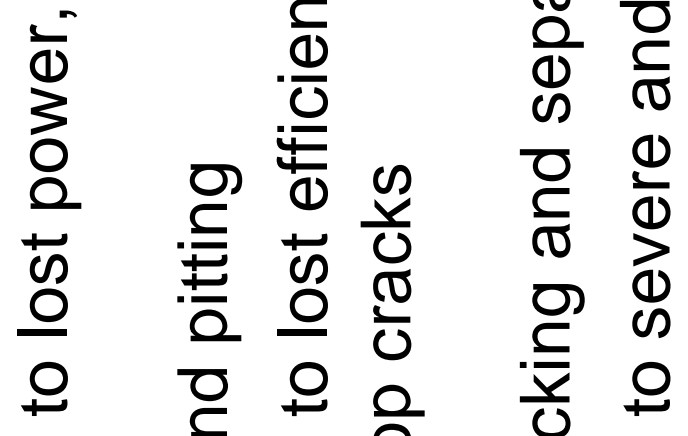

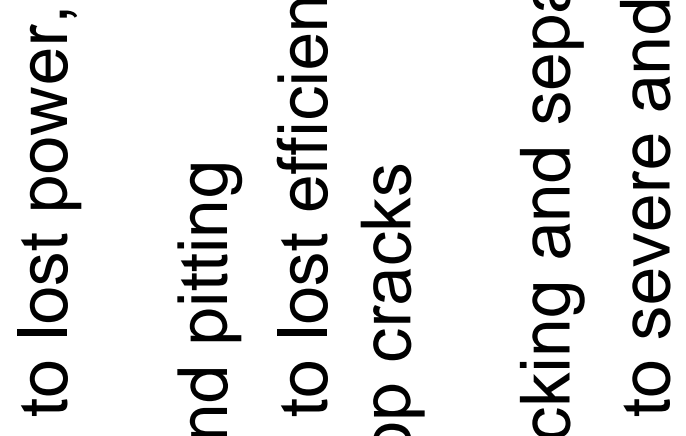

N

$\frac{0}{0}$

U ర্তে

엉

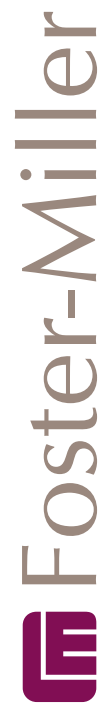




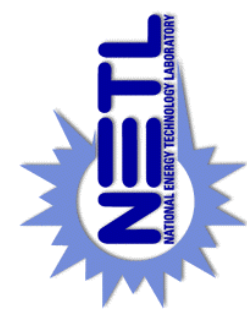

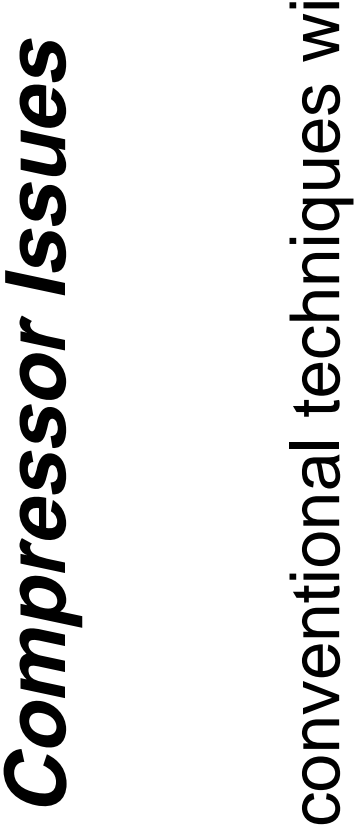

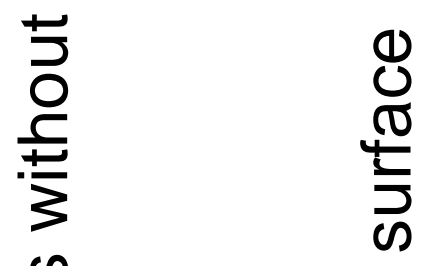

$\stackrel{9}{2}$
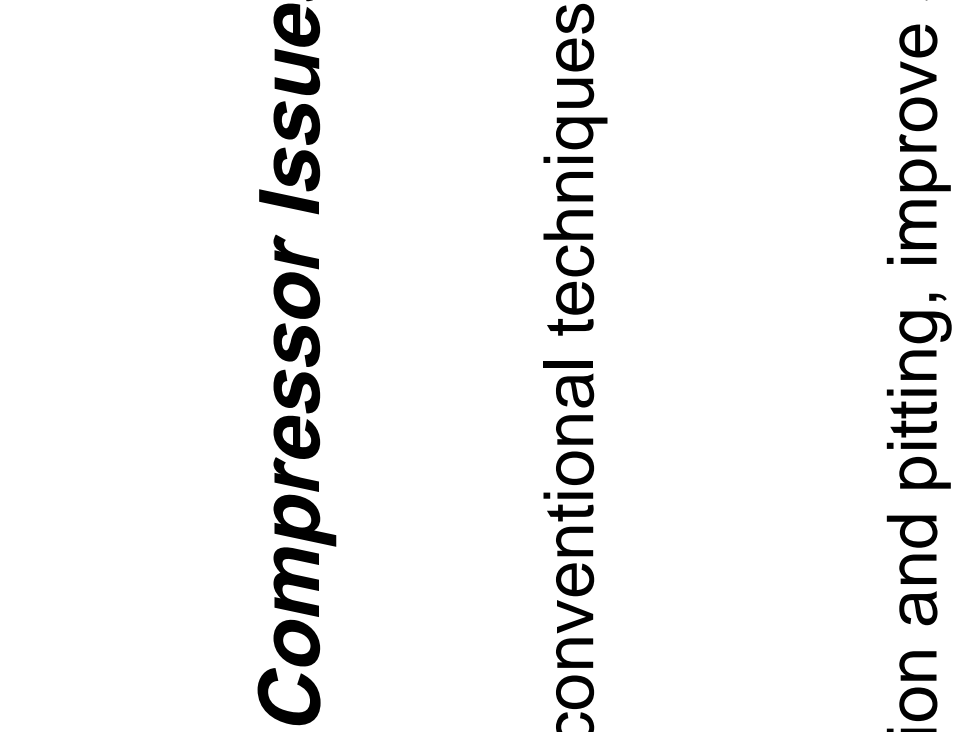

g

\&

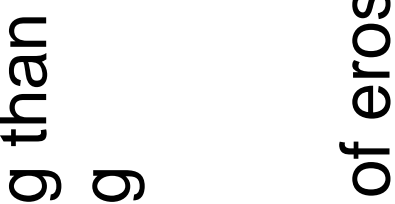

이요

高

은으

ल $\frac{1}{4}$

들

$\frac{1}{0}$
$\frac{0}{0}$
$\frac{0}{1}$
4

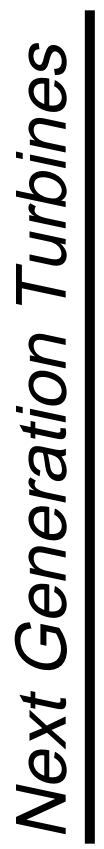

(1) 으 ब ब

(1)

ำ

을 올

E ब

ज ह

ช্

ט.?

(1) ত

ज ह 든

(ธ)

है.

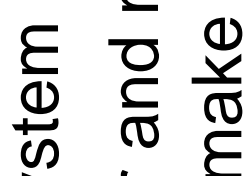

0

N

$\frac{1}{0}$

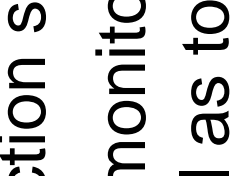

() $\overline{\overline{0}}$

ब

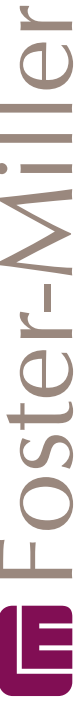

ธัல

உ

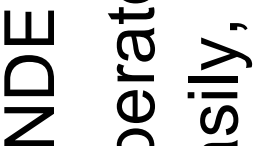

을 ᄃ के

응

ণ

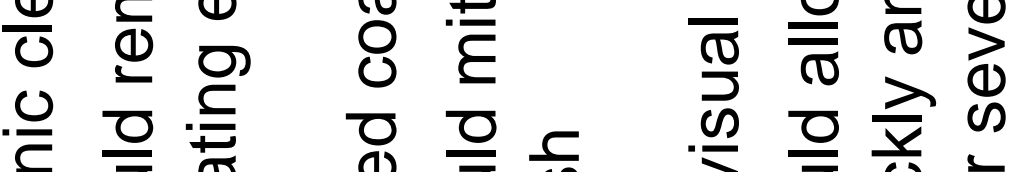

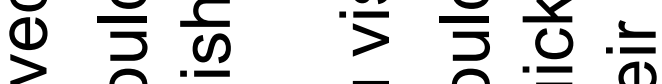

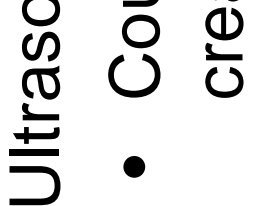

응 을

릉
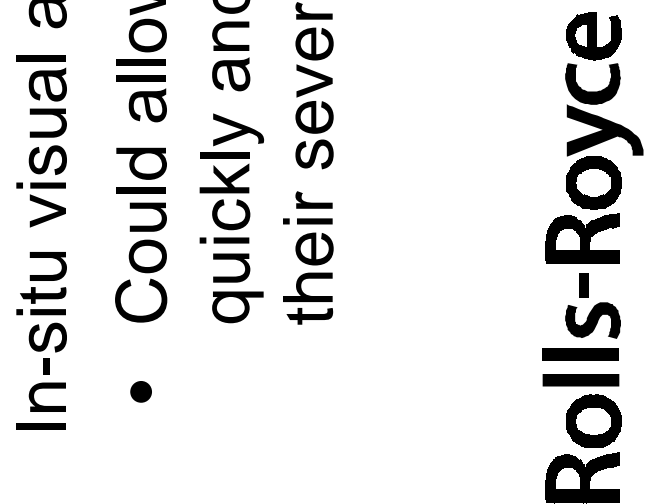


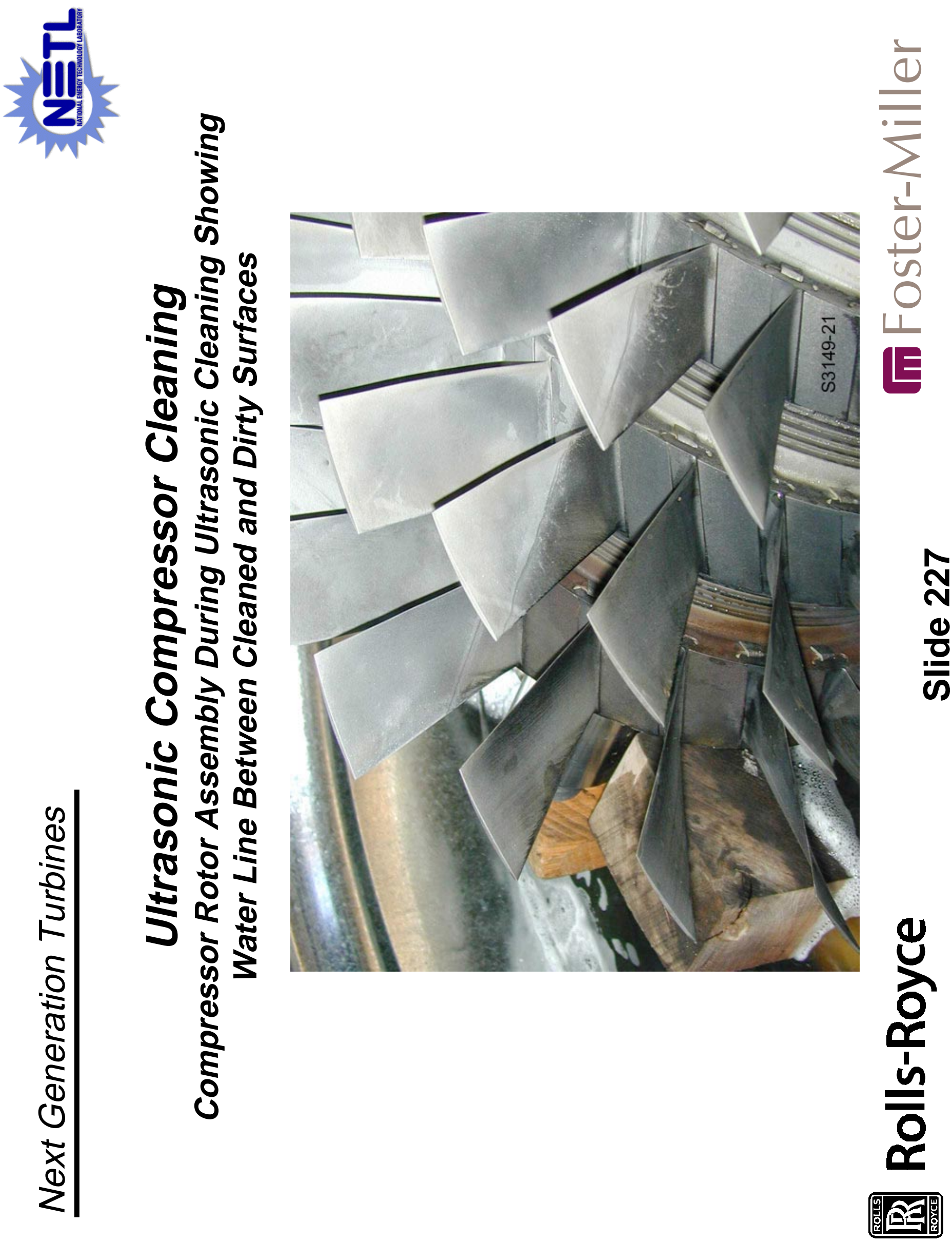




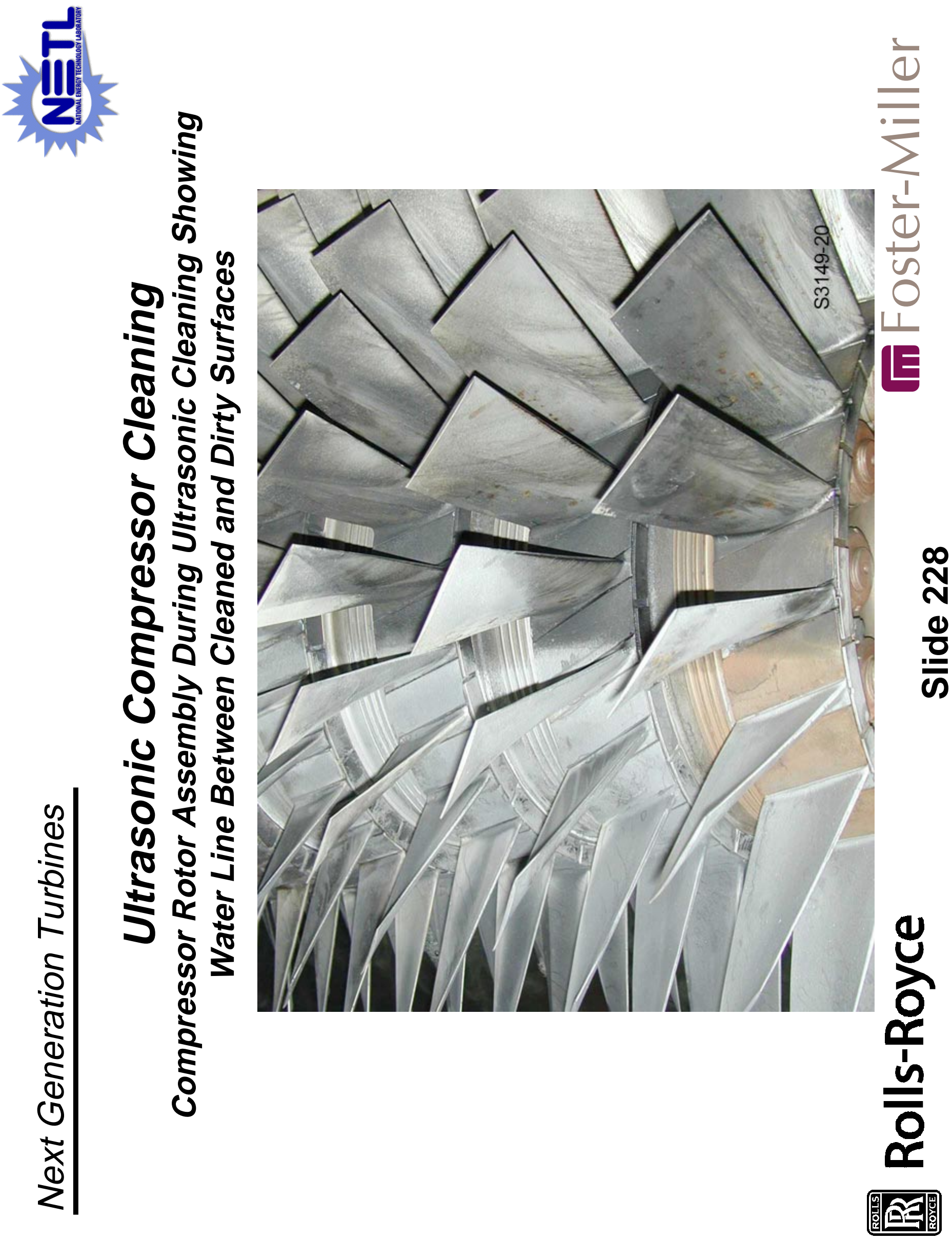



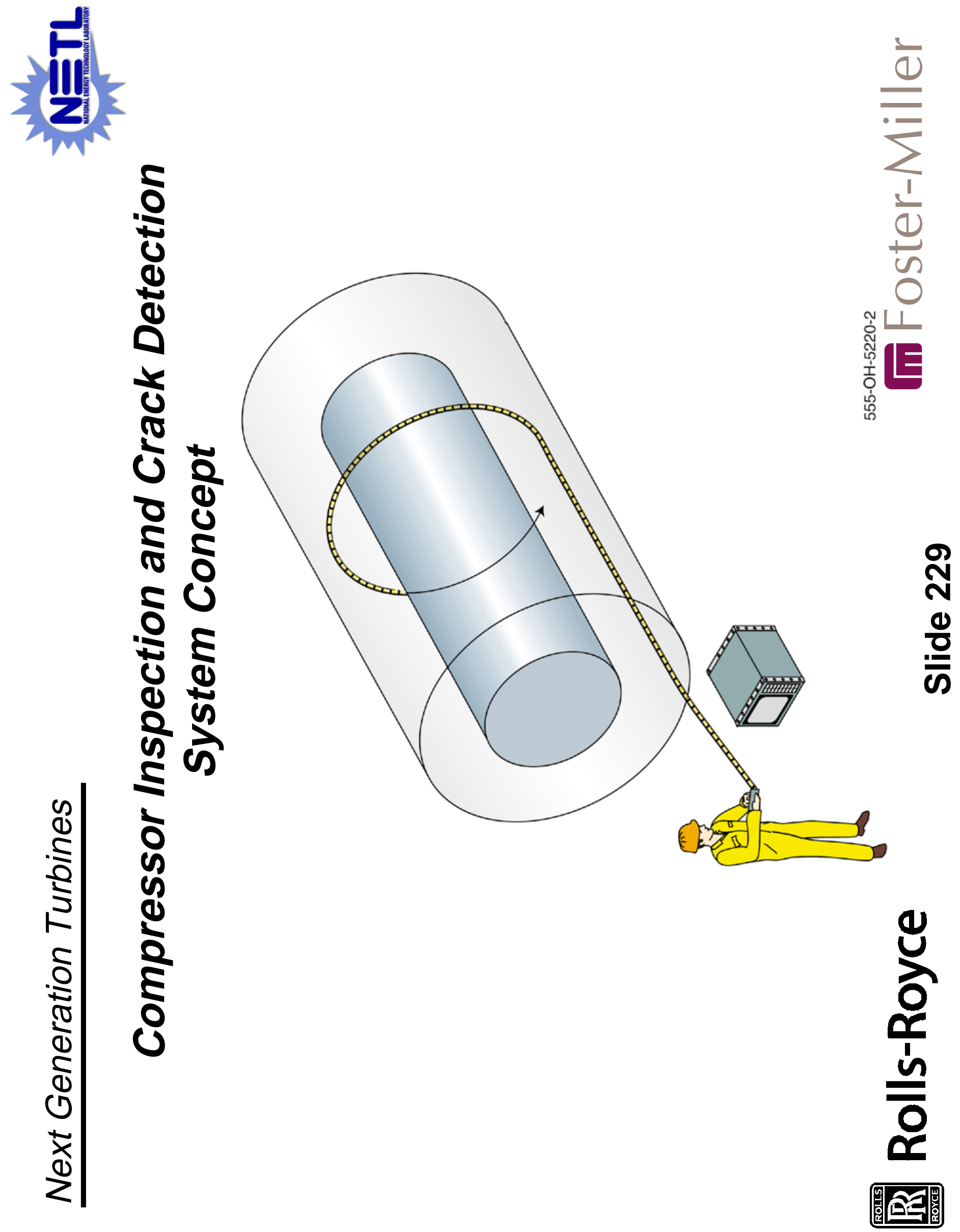


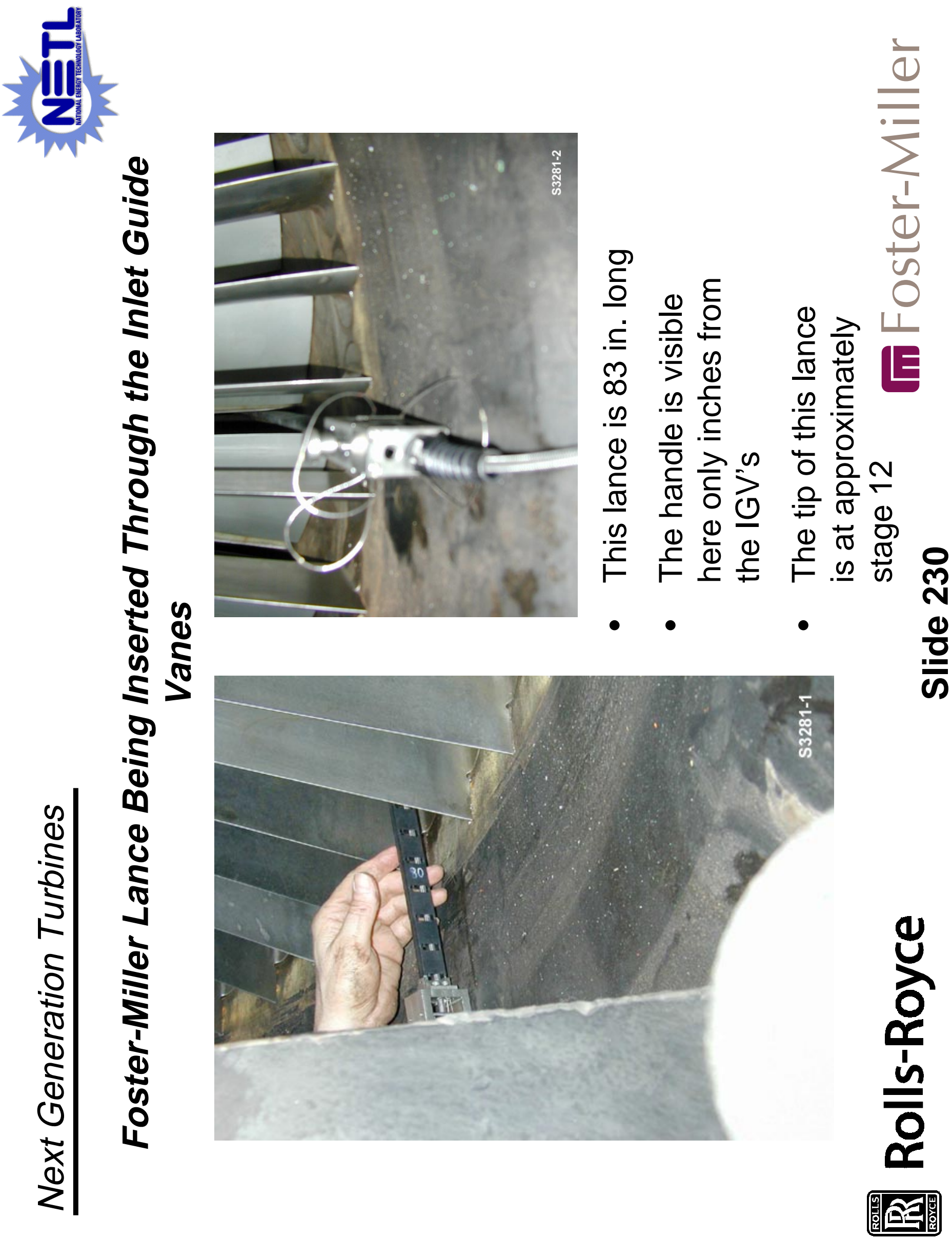




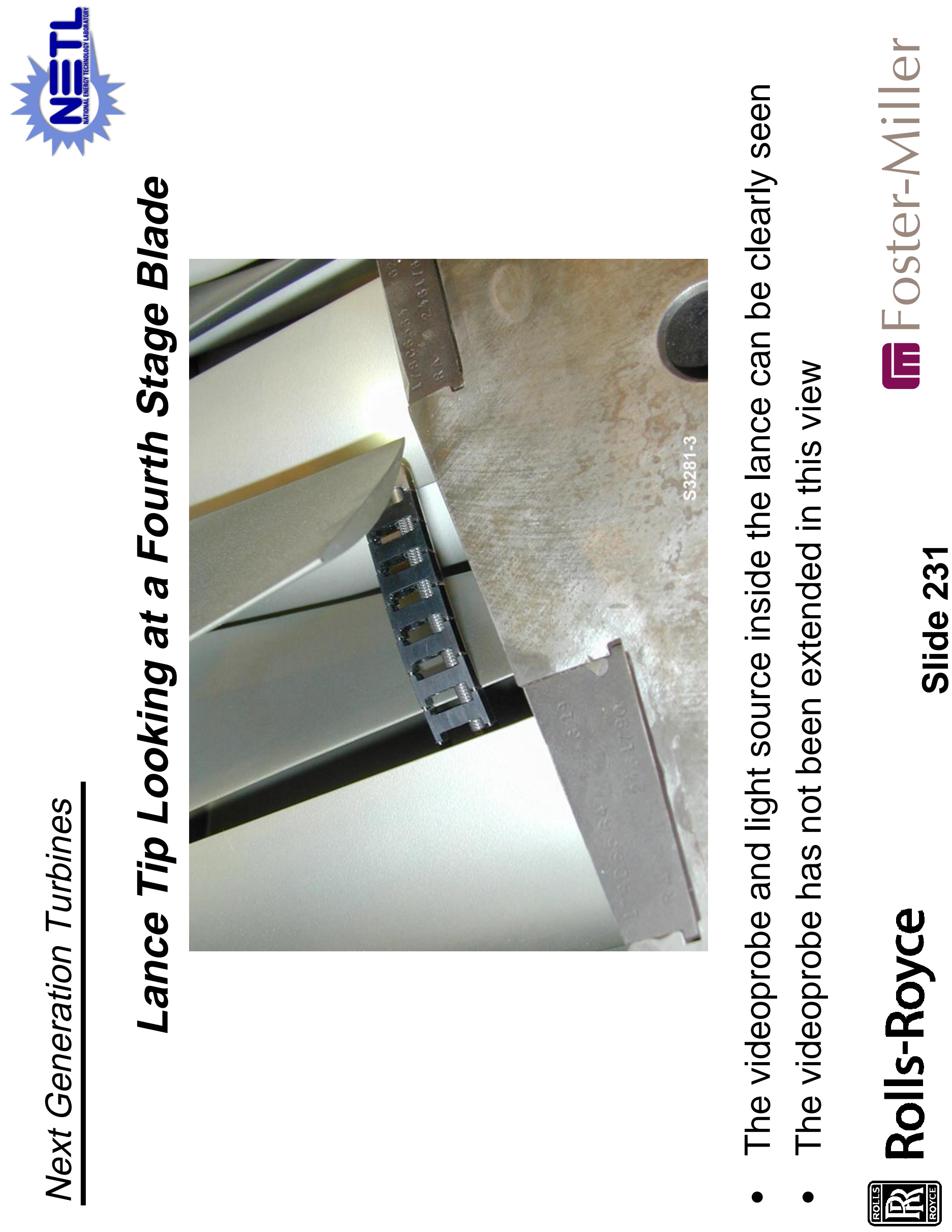




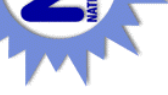

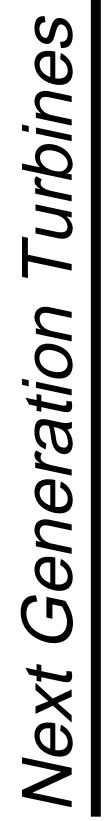

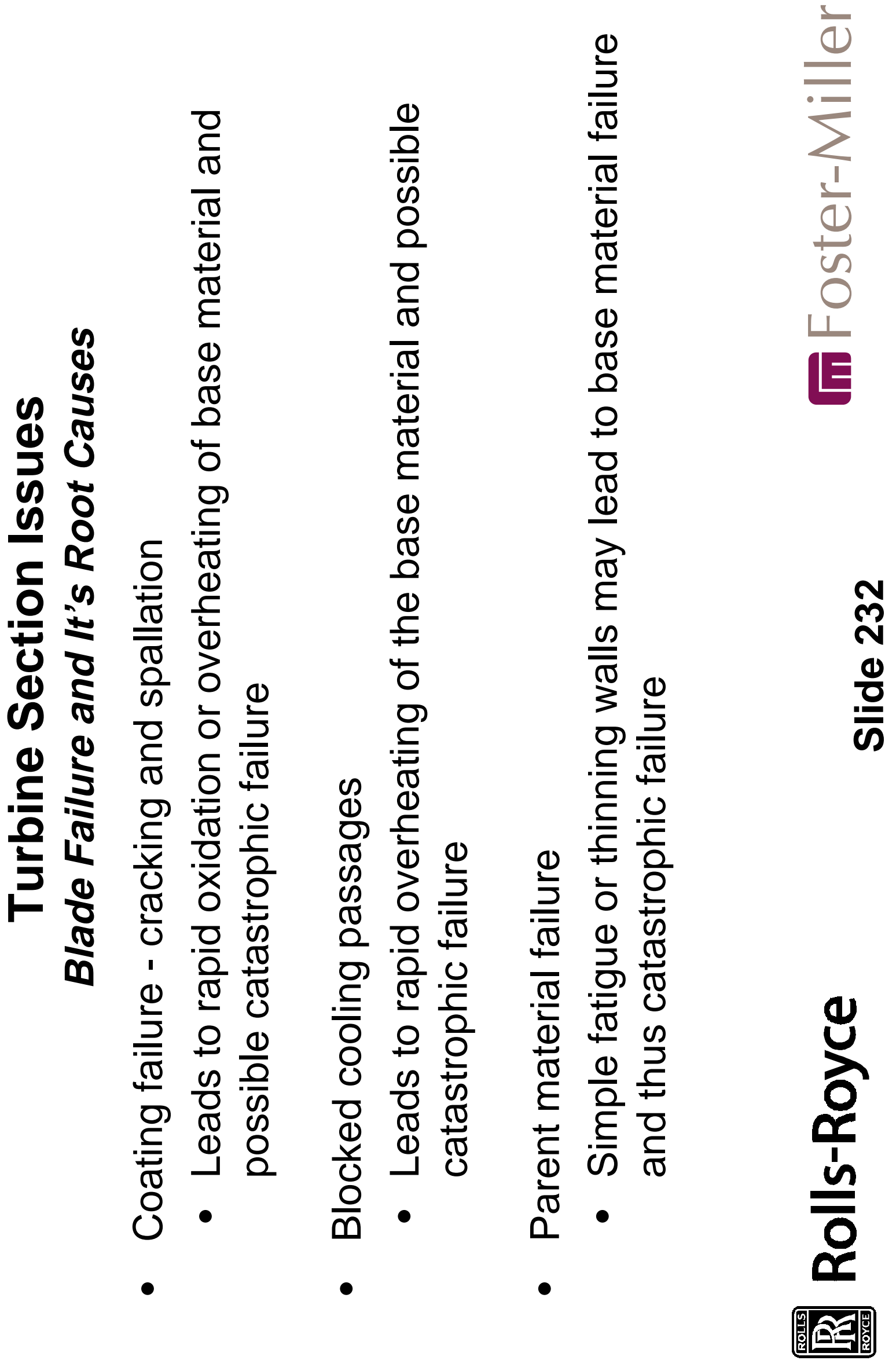




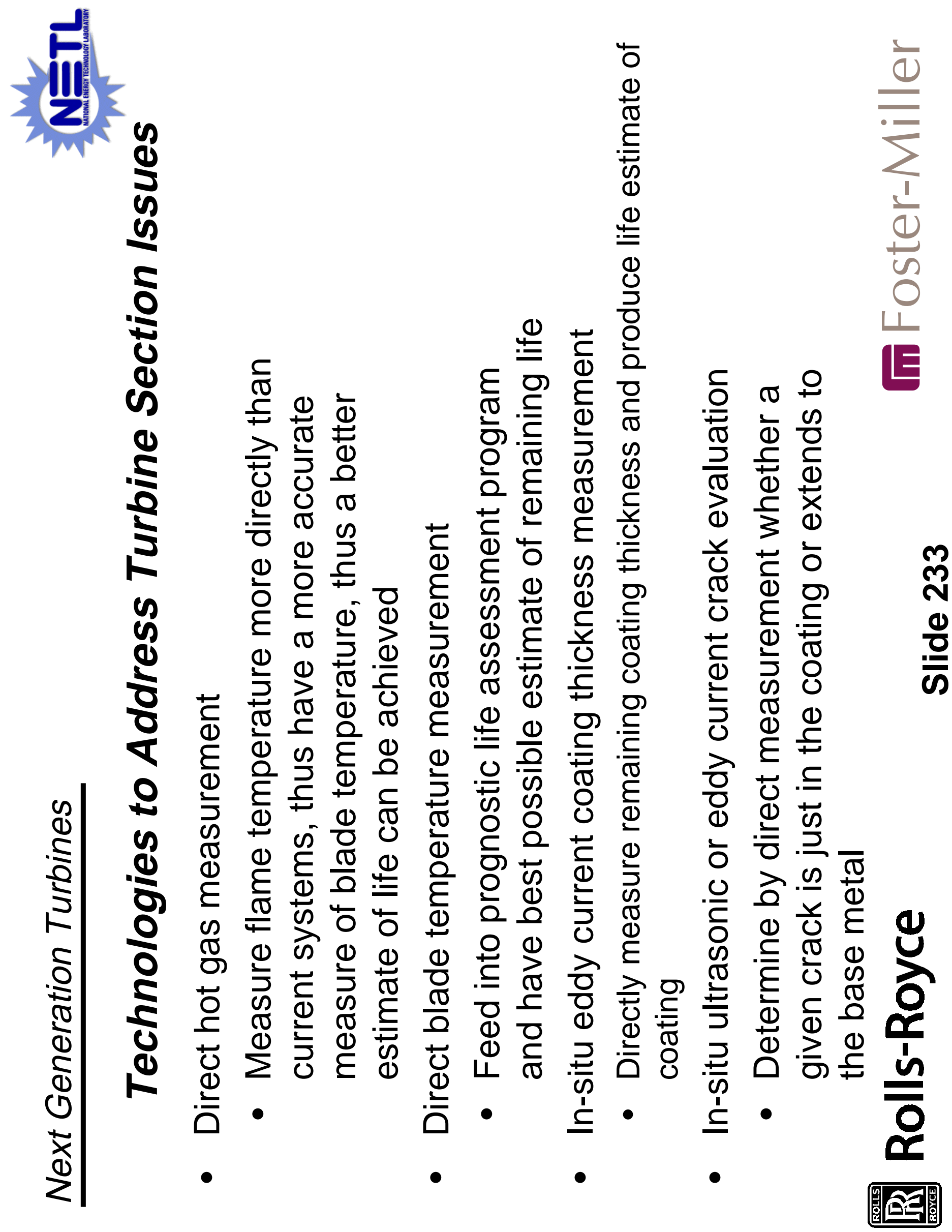




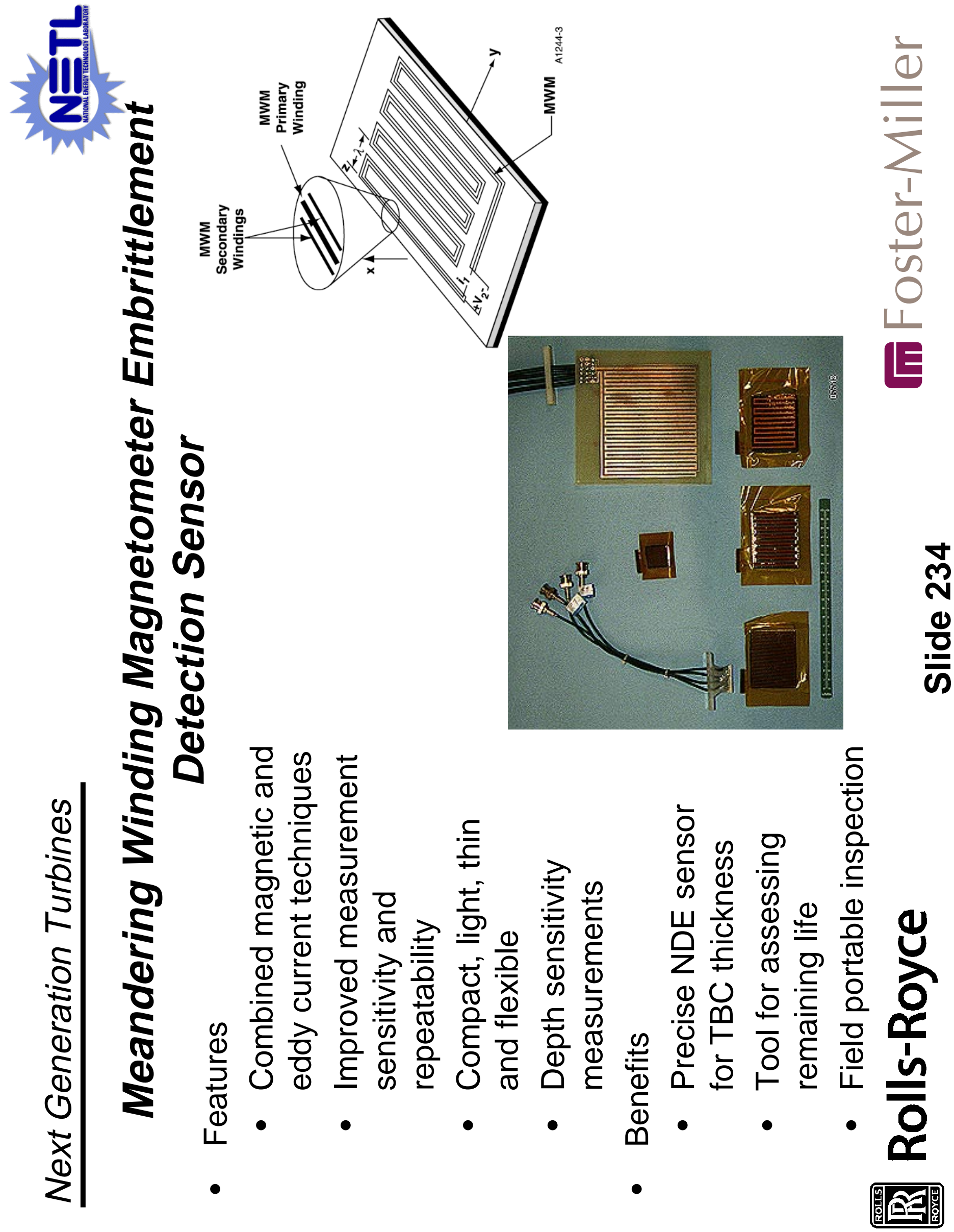




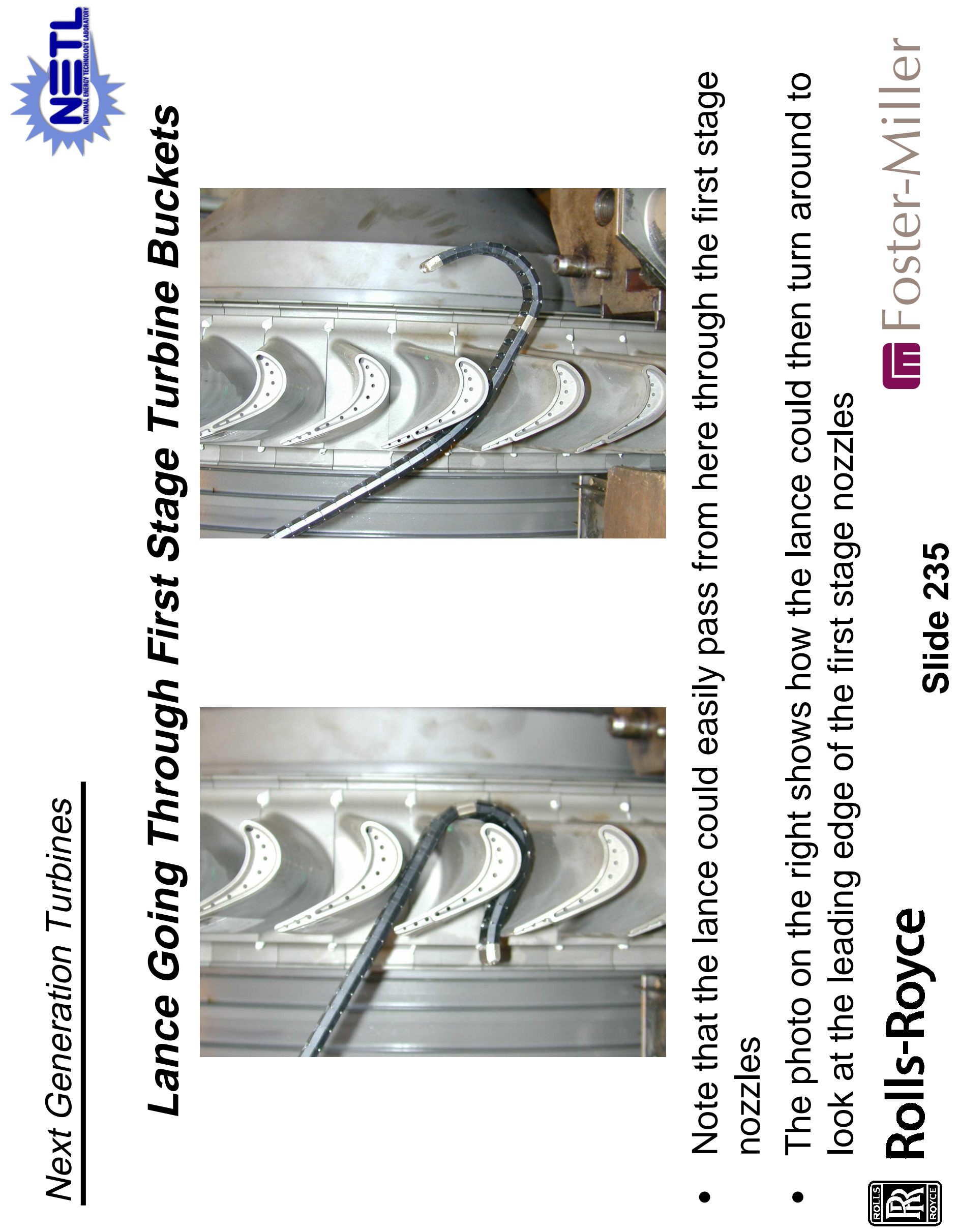



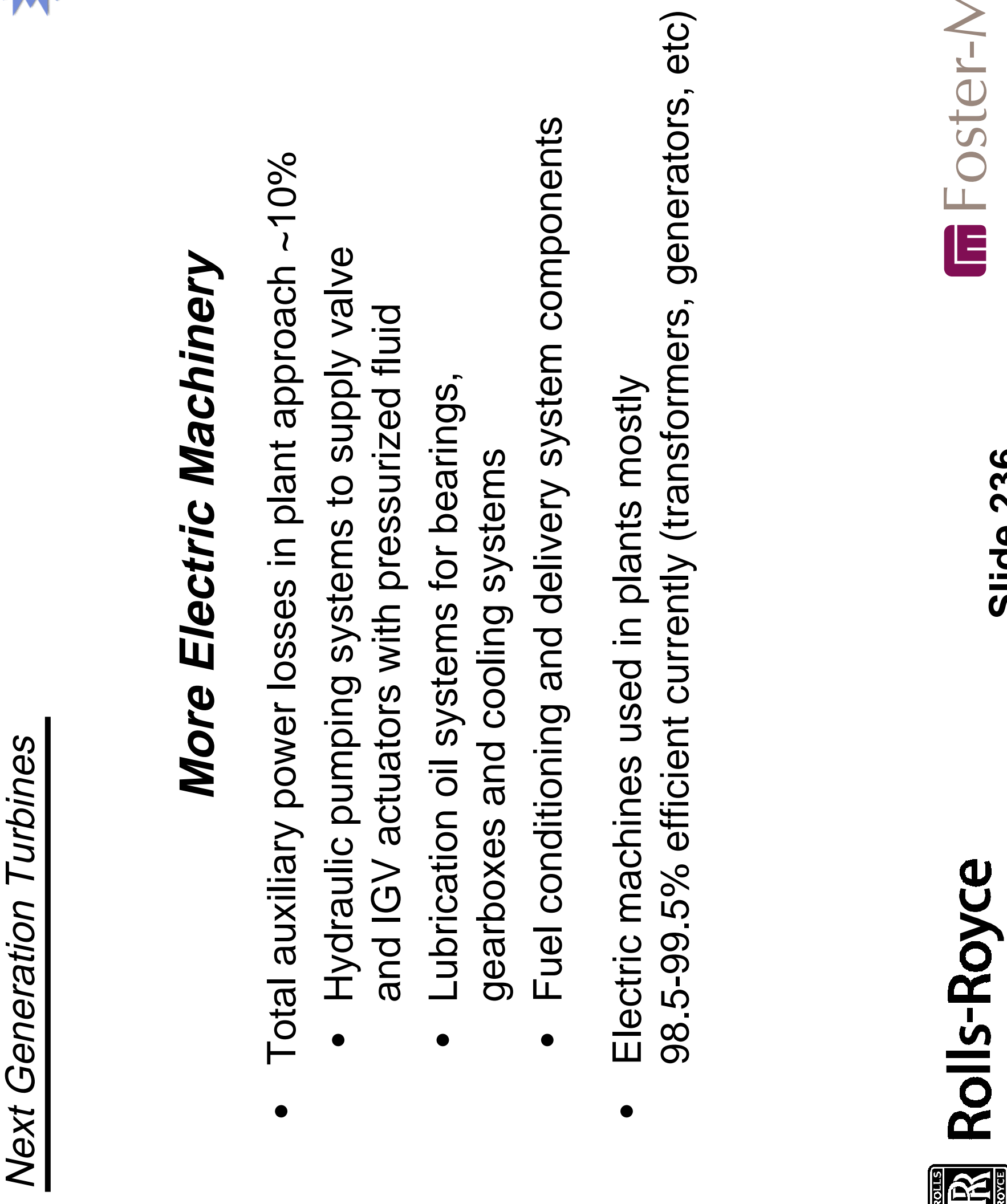


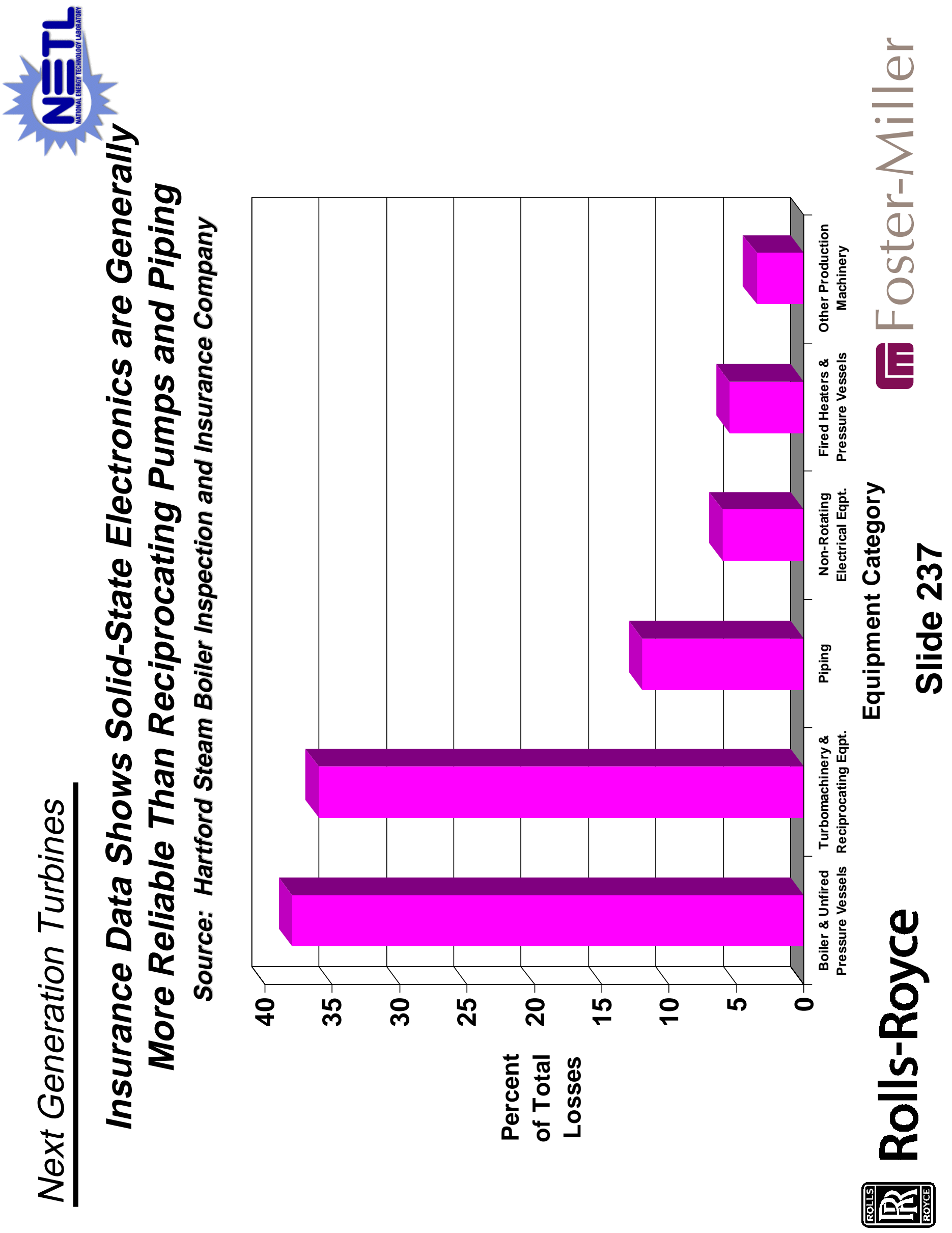




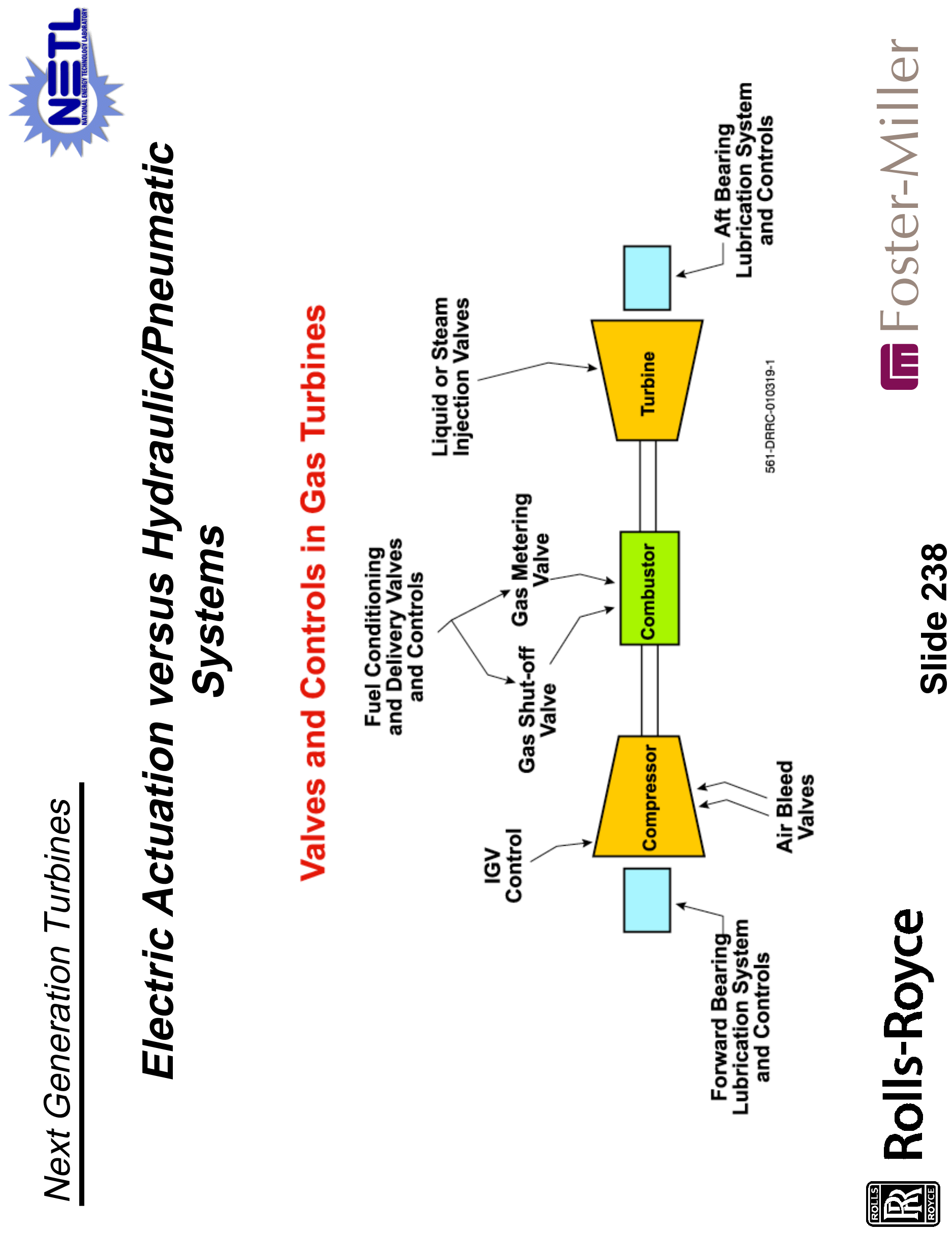




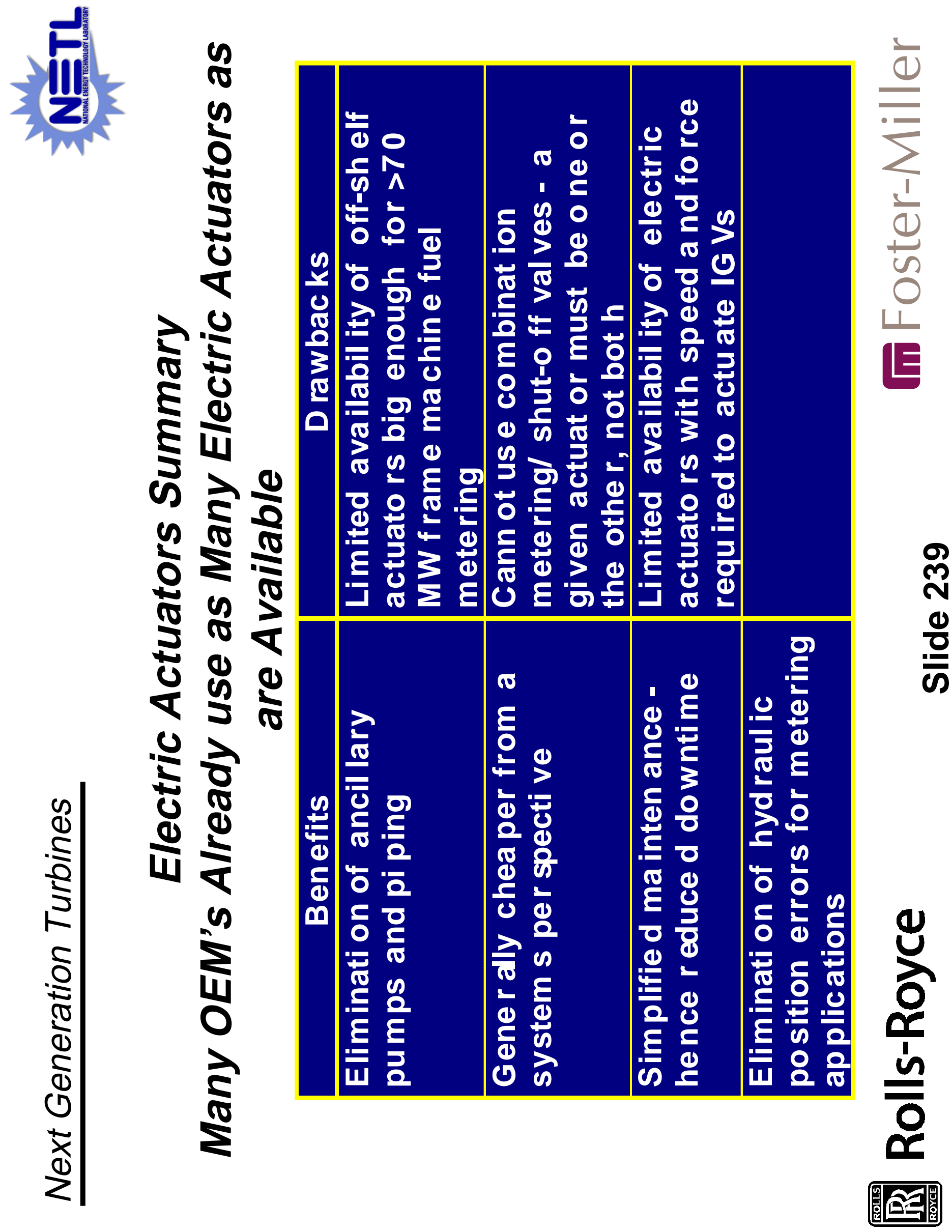




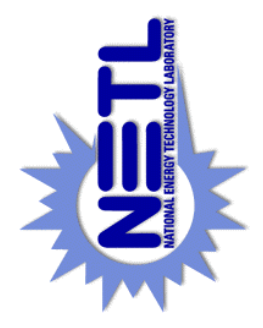

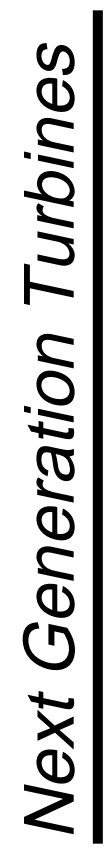

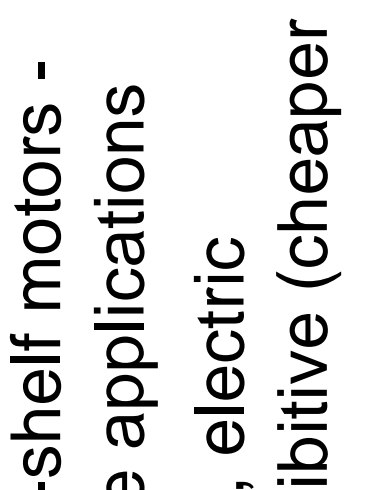

$\stackrel{Ð}{\frac{0}{0}}$

(1)

ఏ हૃ 응 응

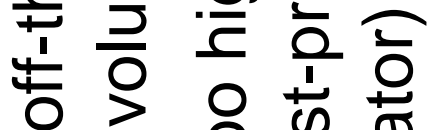

ᄃ $\sum_{0}^{1}$ ○

들 으

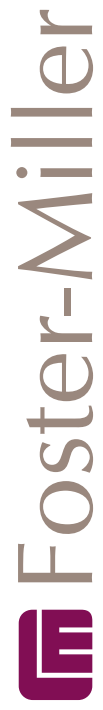

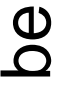

츨

స్

$\begin{array}{ll} \pm & \overline{0} \\ 1 & \frac{1}{0} \\ \frac{1}{0} & \frac{8}{0}\end{array}$

을 음

처 을 을 을

ज

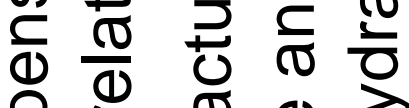

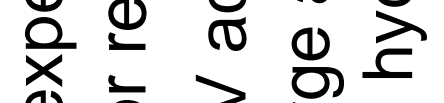

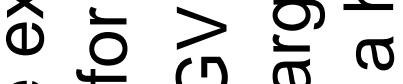

$\frac{0}{\infty}$

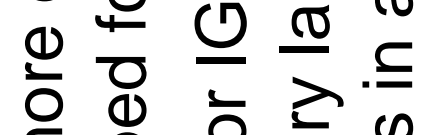

트응 으 d

(1) 은 둔

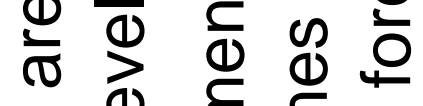

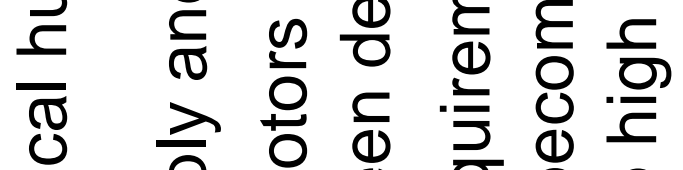

을 응 잉ㅎㅇ용

어 ज ह

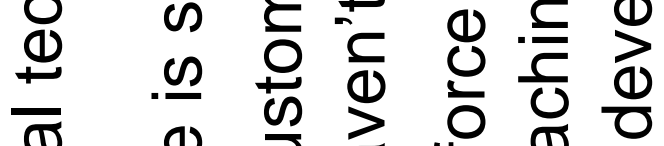

ฮิ

은 고.

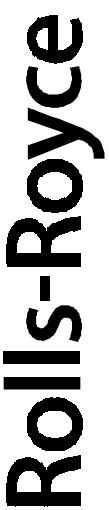




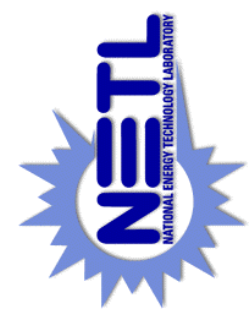

$\frac{8}{3}$
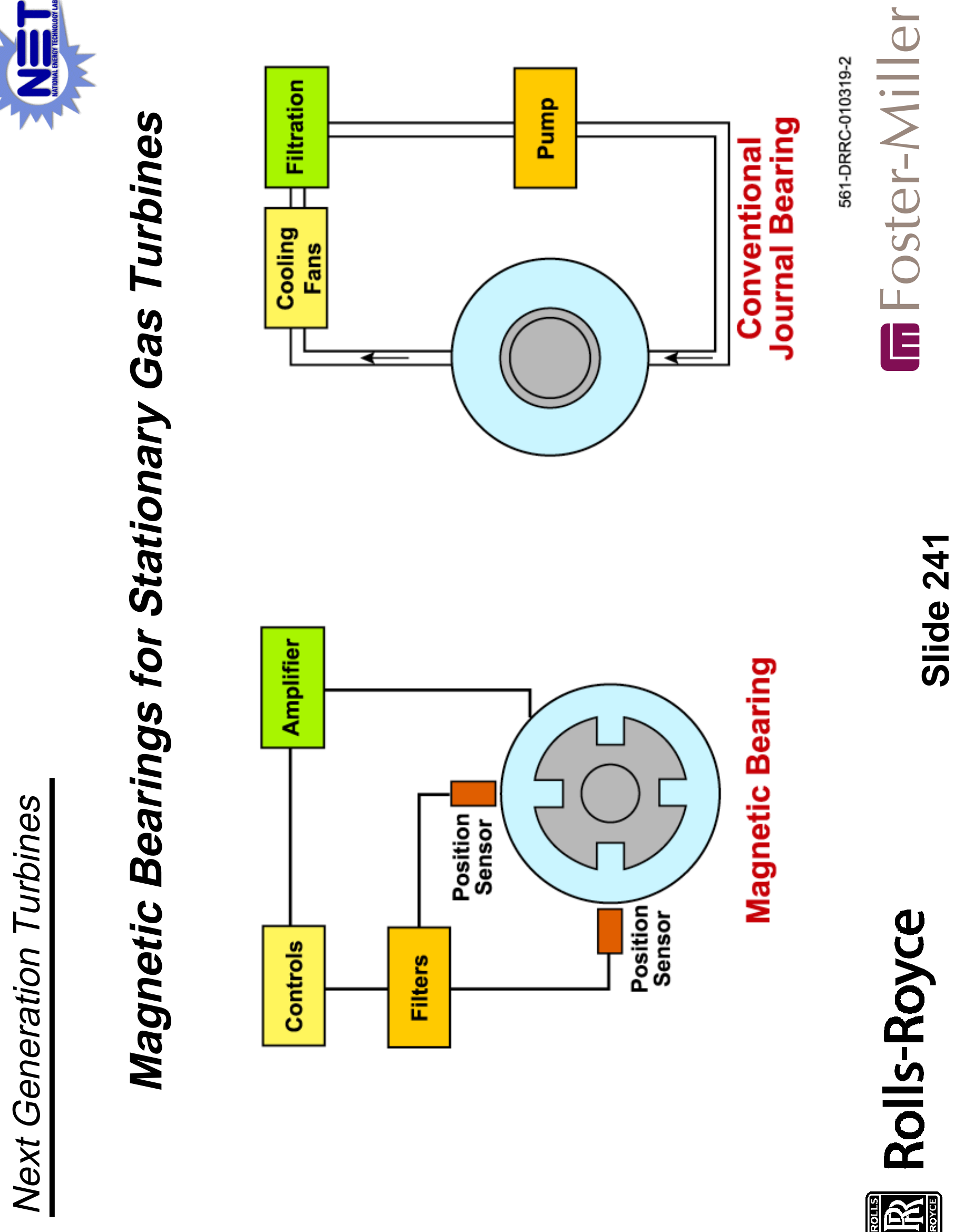


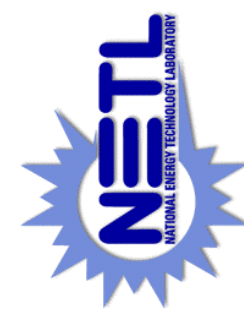

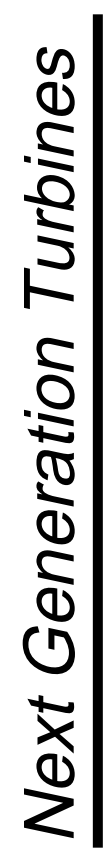
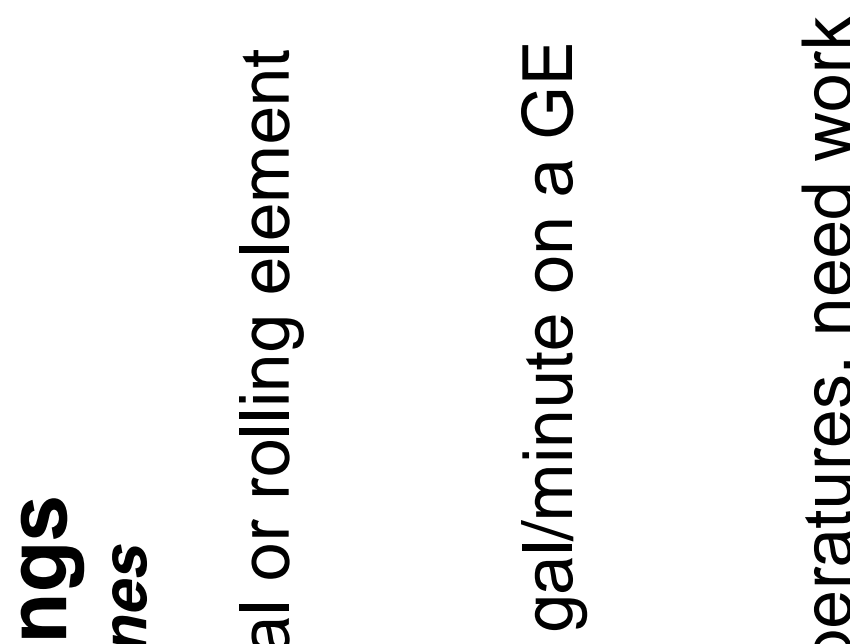

$\frac{+}{\frac{Z}{0}}$

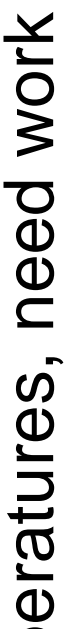

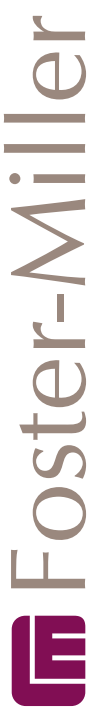

요

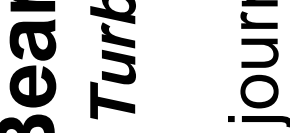

ต

000

$\Rightarrow \geqslant$

ข

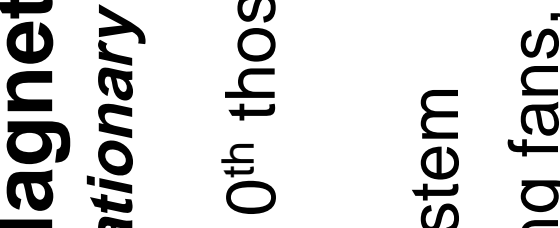

동

का

$\frac{\mathrm{Q}}{\mathrm{E}}$

\begin{tabular}{l}
$\frac{1}{\pi}$ \\
$\frac{0}{10}$ \\
\hline
\end{tabular}

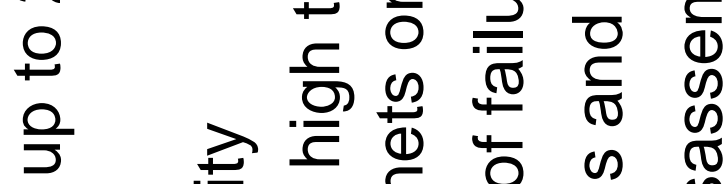

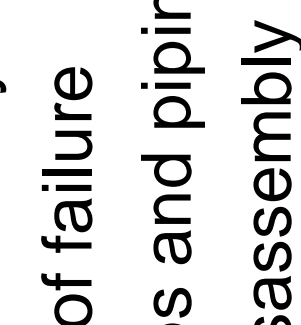

क 융

N
$\frac{0}{O}$
$\frac{0}{\omega}$

tั

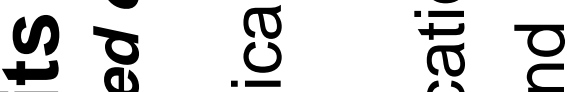

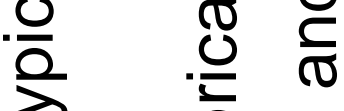

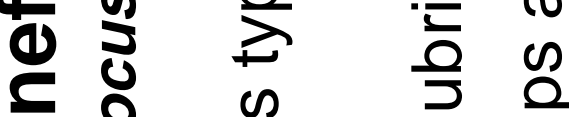

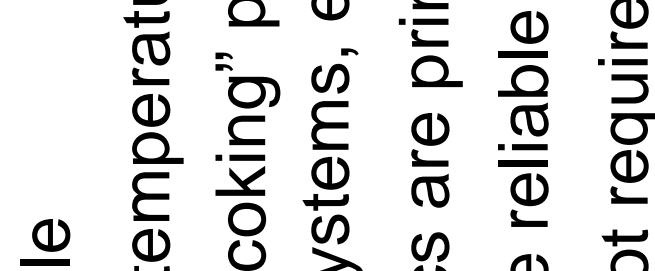

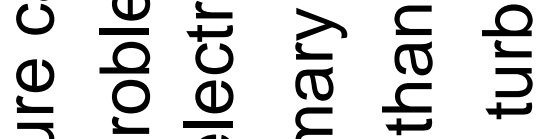

m

그 을 응

ळ

O

를

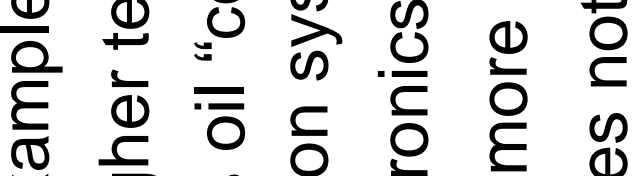

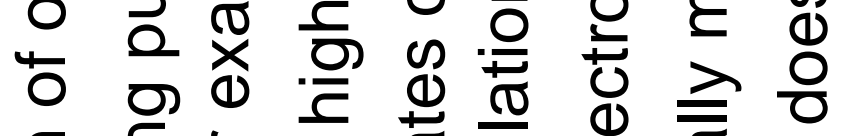

$\overline{\widetilde{J}}$ 든

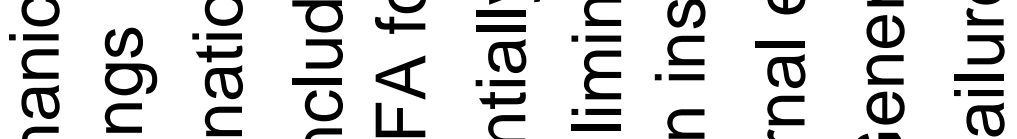

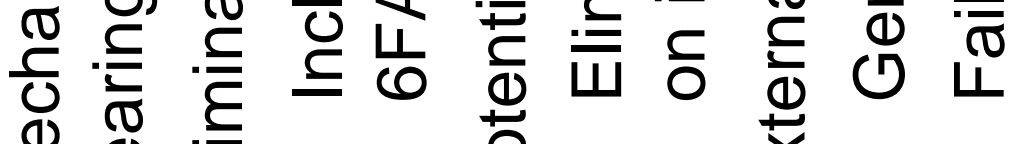

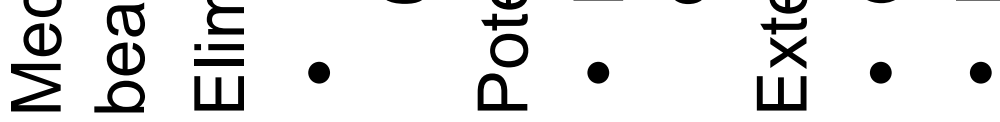

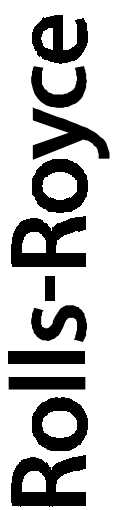




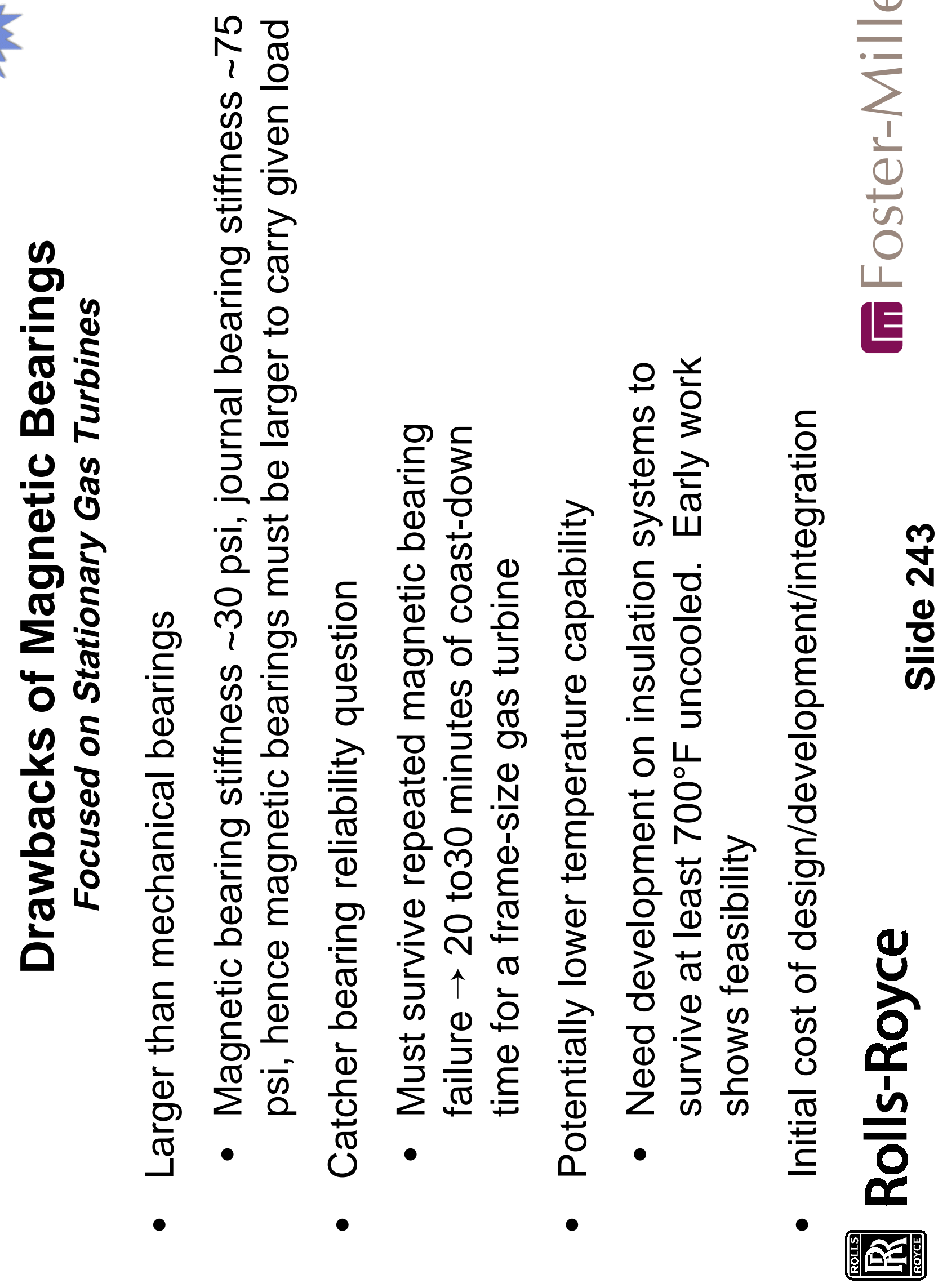




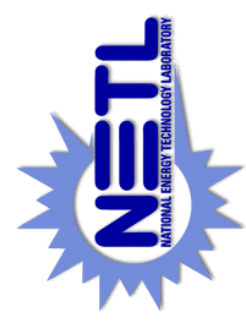

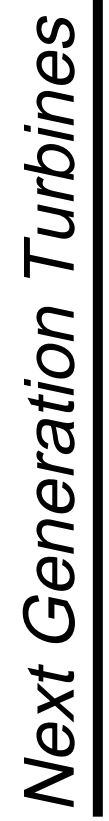
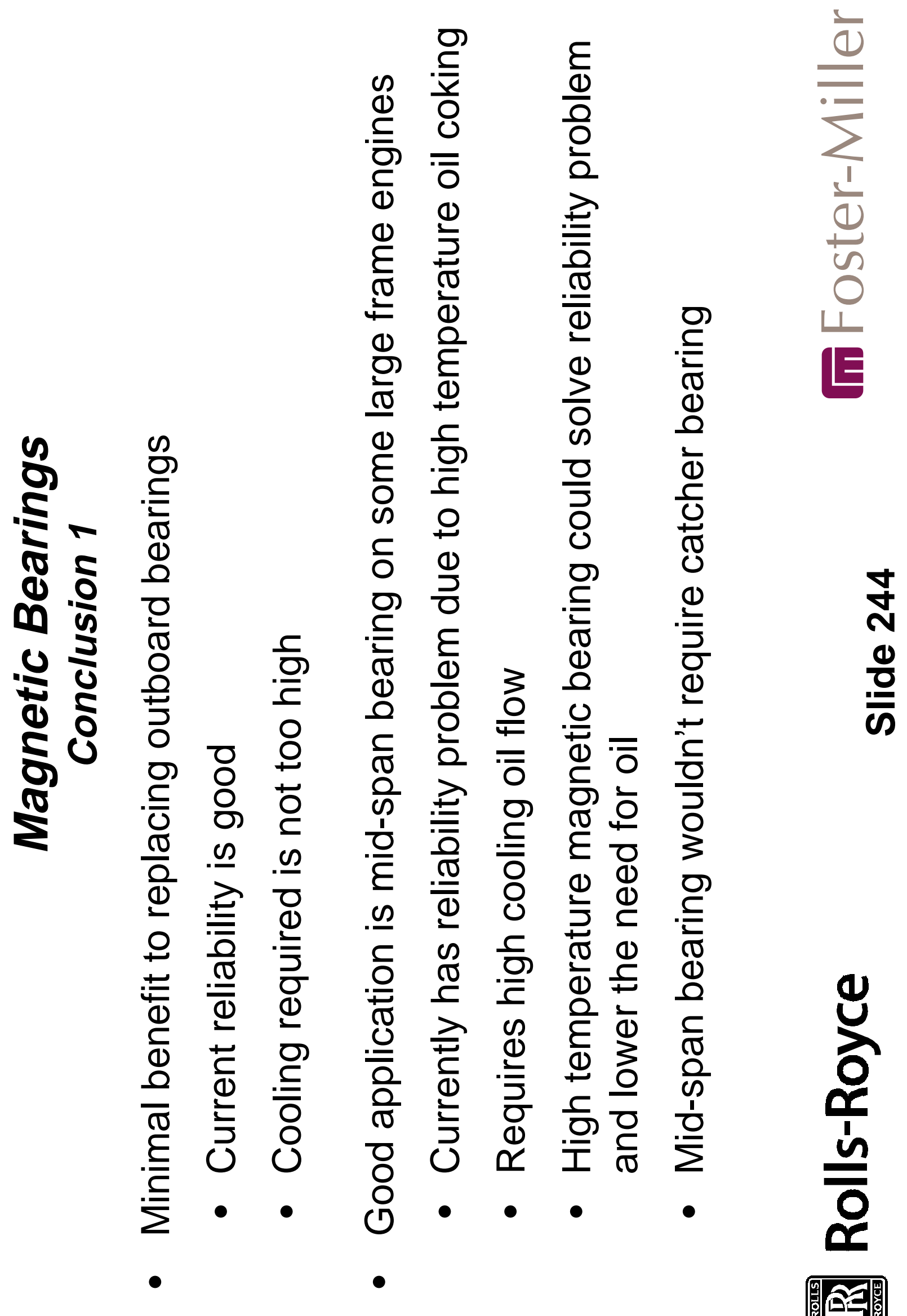


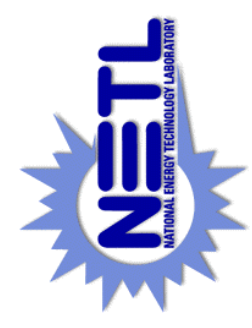

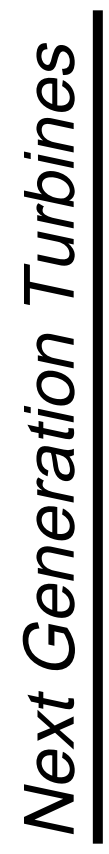
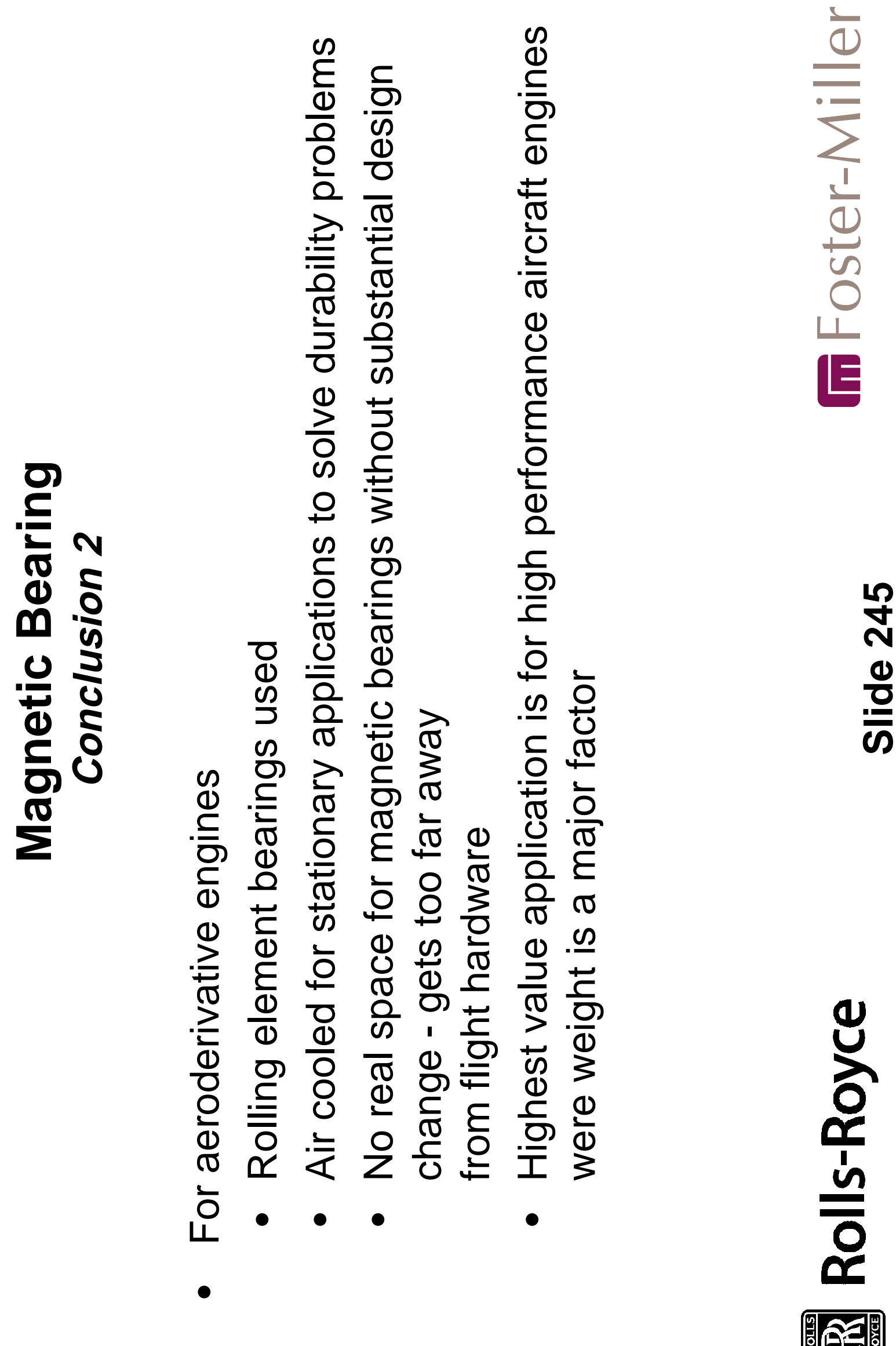

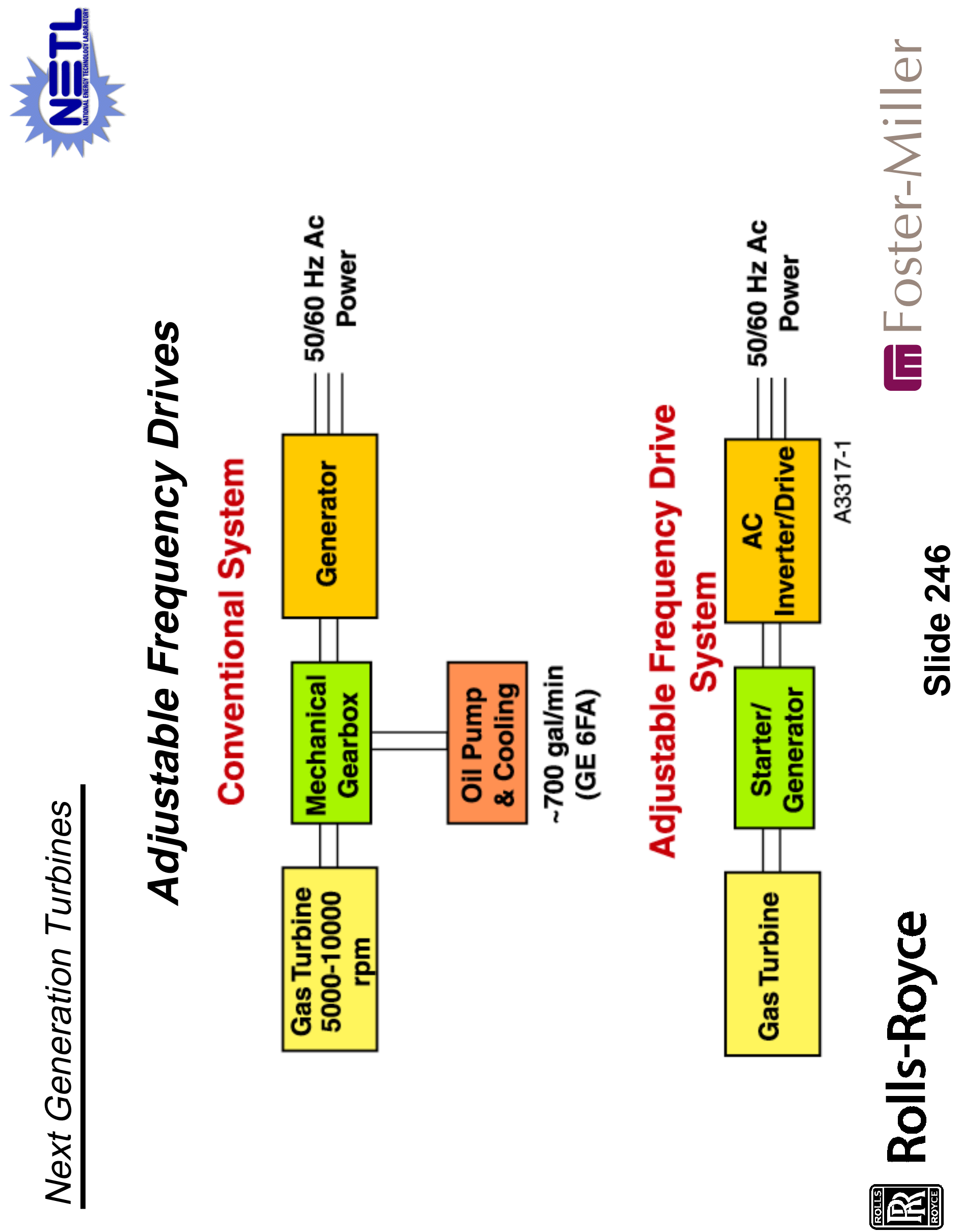


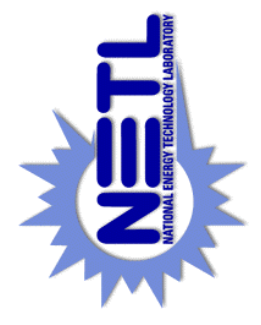

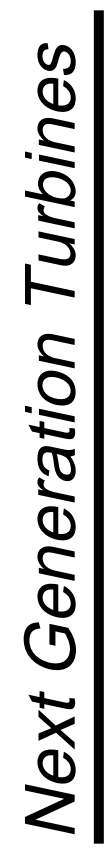

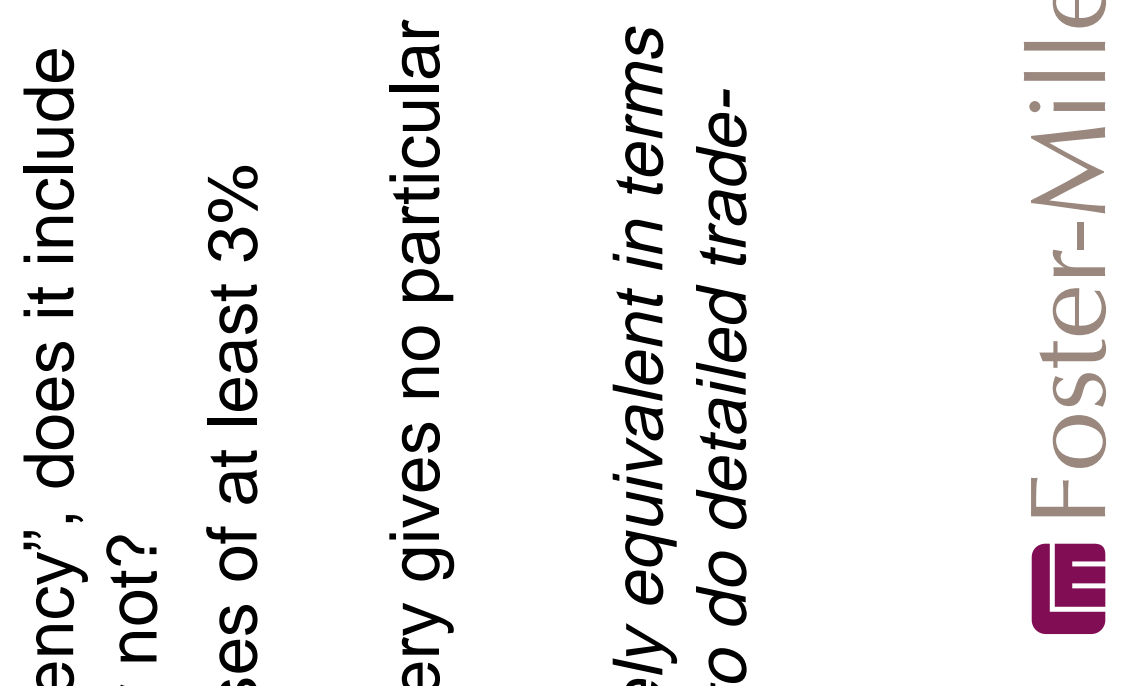

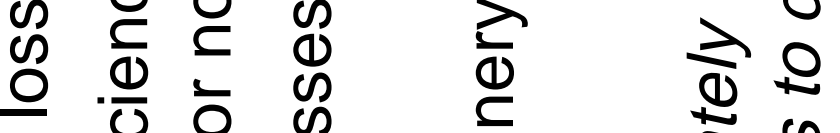

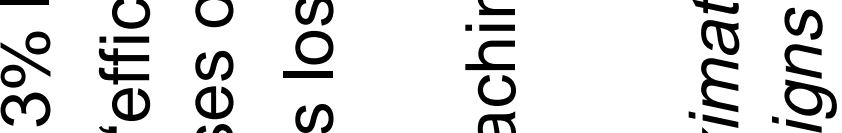

0 क ल ख

잉

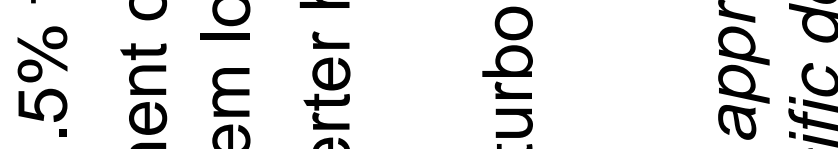

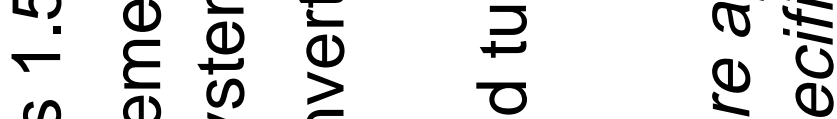

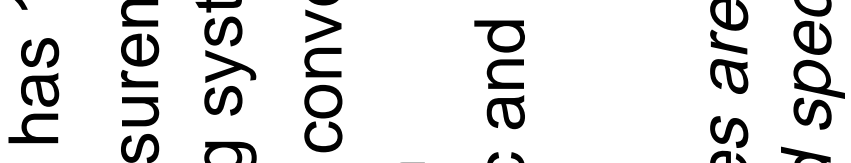

(1) त गे के

(1)

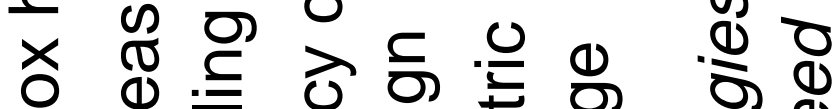

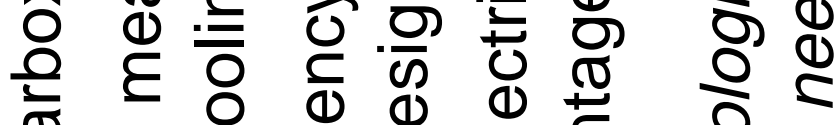

$\underset{\stackrel{N}{*}}{\frac{1}{0}}$

世

d่ $\frac{1}{0}$ 我

ชิ

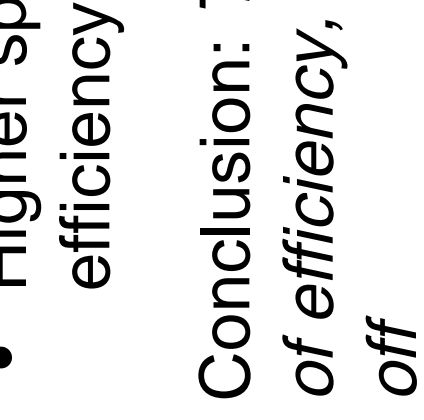

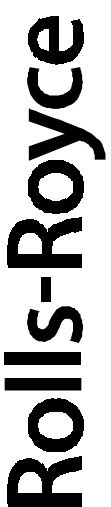

정 등 임 을

$\frac{\bar{d}}{N} \sum^{D} \cdot \frac{\Phi}{\square}$

$\frac{\bar{d}}{\frac{0}{0}} \frac{\mathrm{O}}{0}$ 


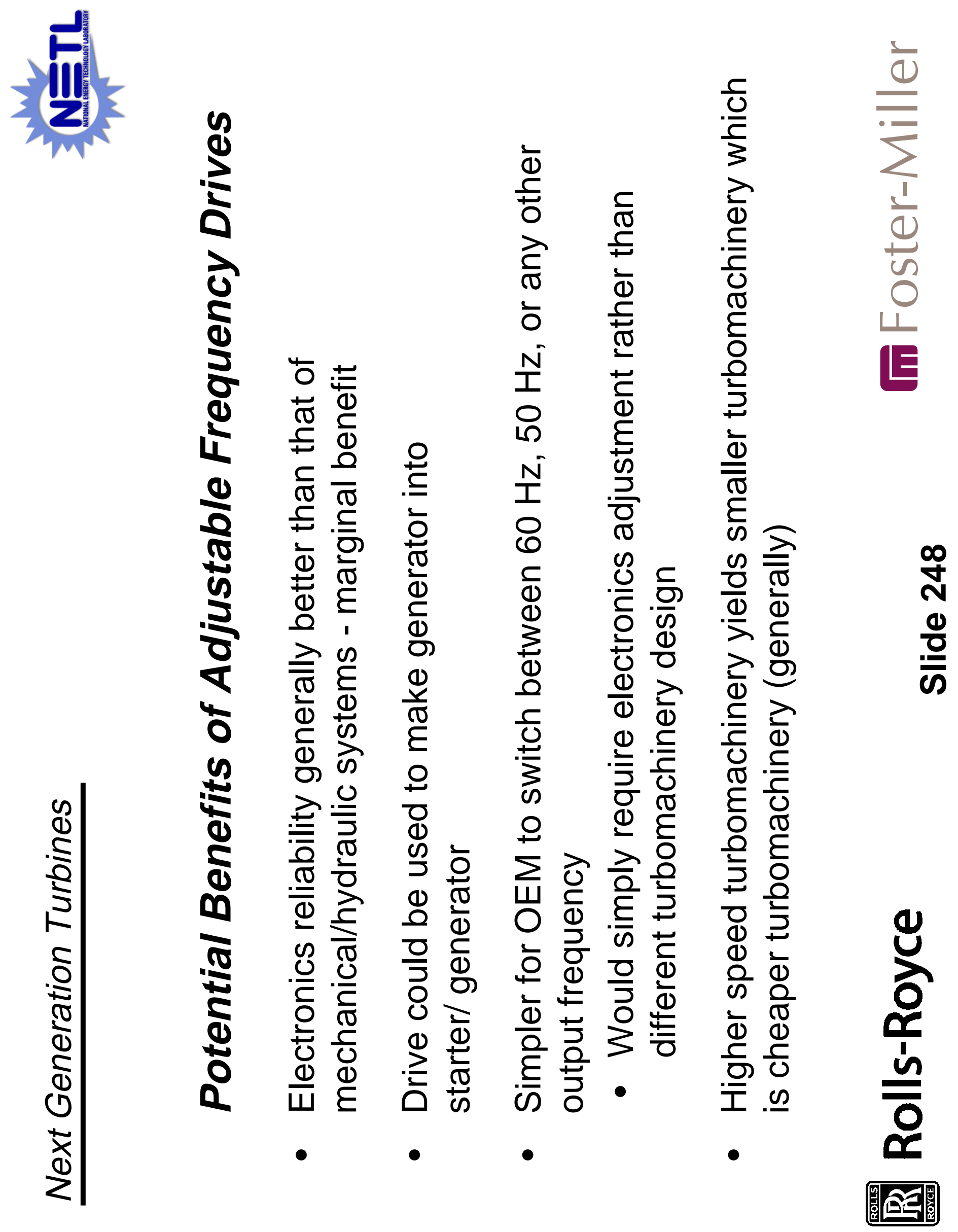




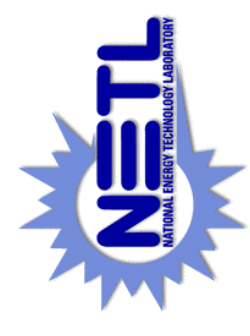

$\pi$

ำ ํํำ

용

궁 우

$\frac{\text { 근 }}{0} \quad \frac{0}{0}$

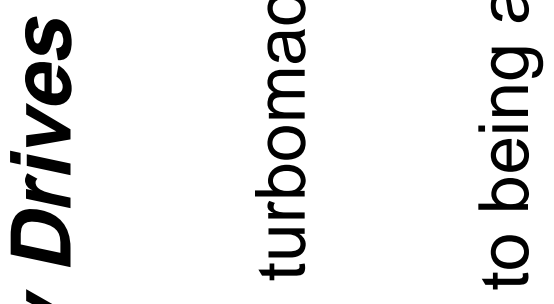

항 으 홍

㐫

(1)

垔

๑ ठ

()

$\stackrel{2}{\mp}$

$\frac{\pi}{2} \quad \frac{1}{2}$

$\frac{\sqrt{2}}{2}$

(2) Ф $\stackrel{0}{ \pm}$

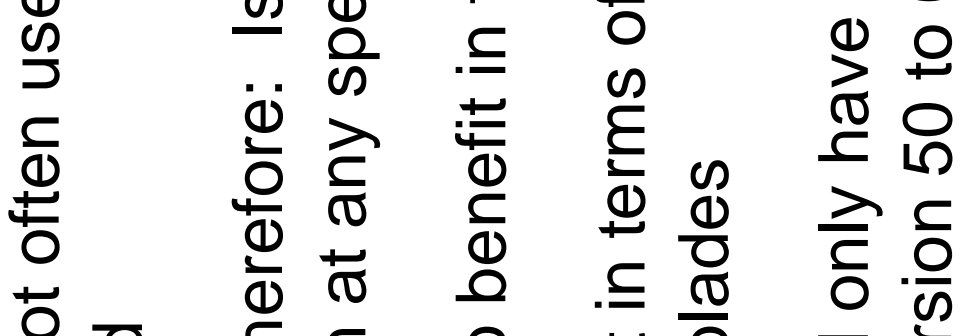

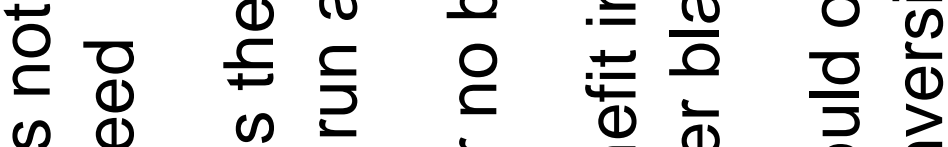

め)

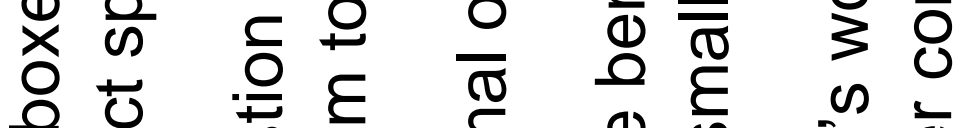

ते

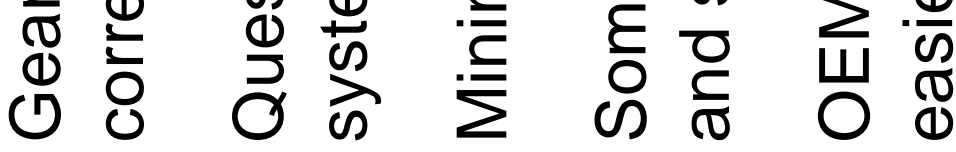

$\sigma$

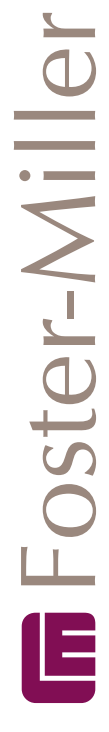

$\stackrel{9}{\mathfrak{N}}$
$\frac{0}{0}$
$\frac{0}{\text { क }}$

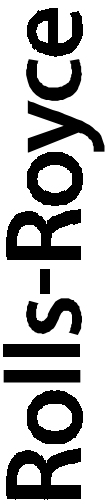



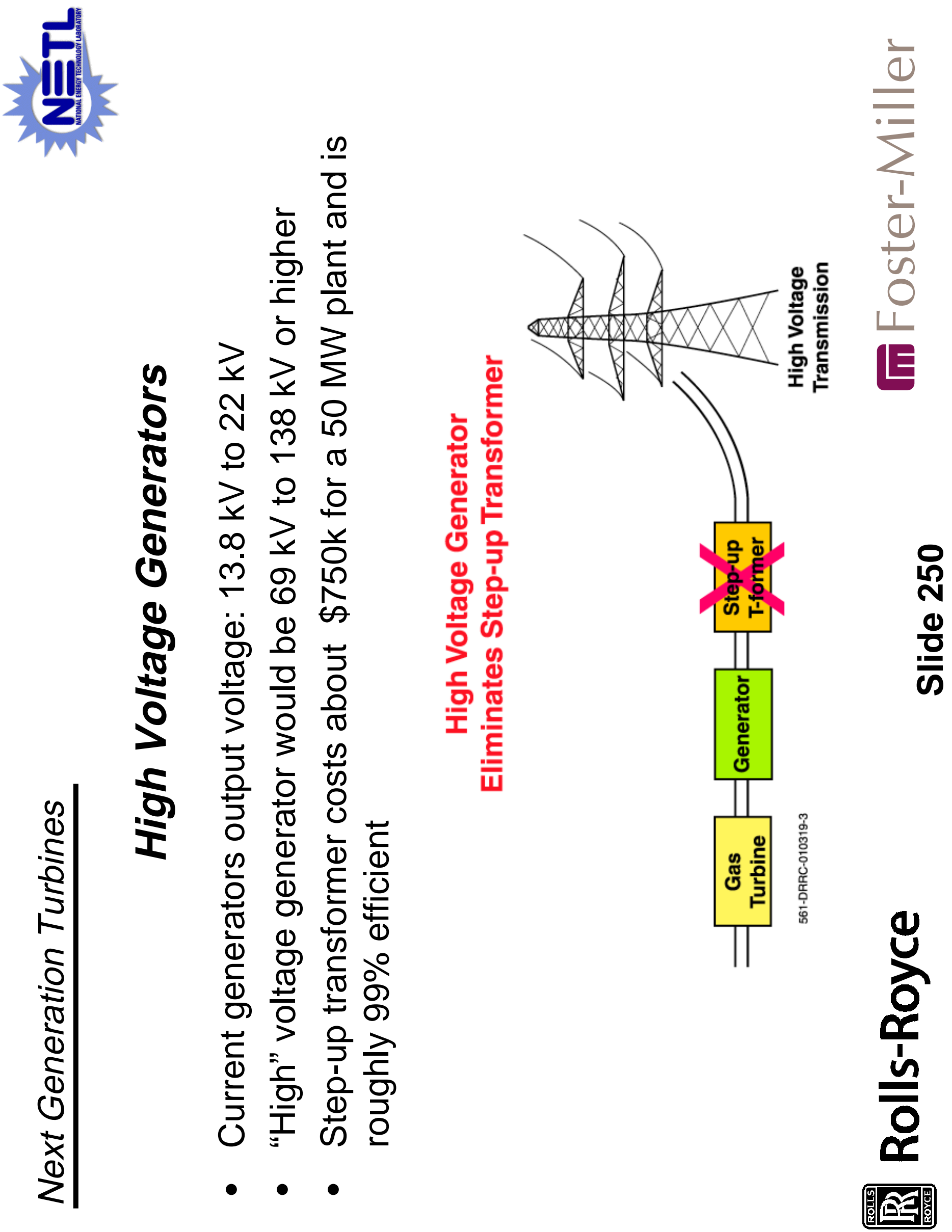

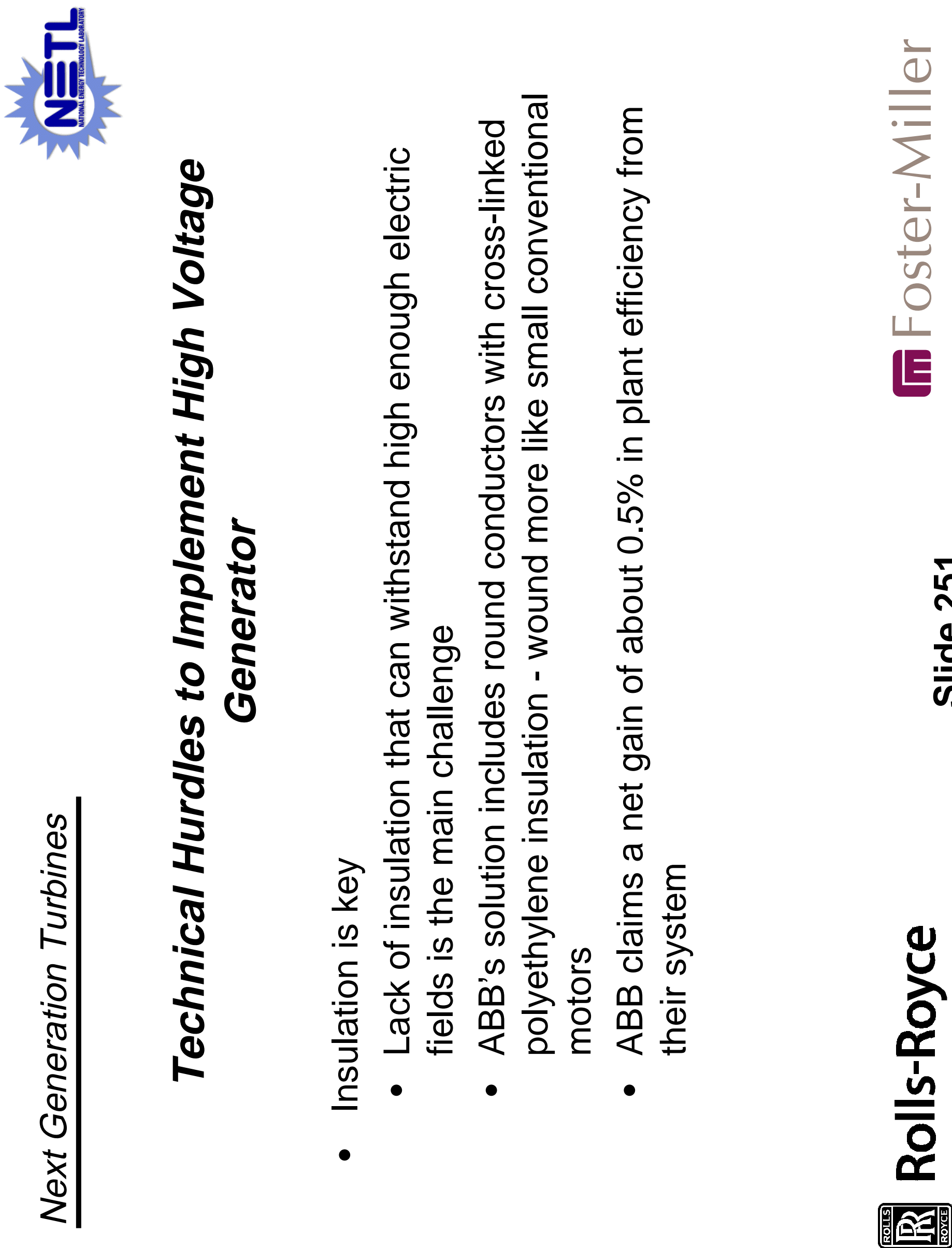

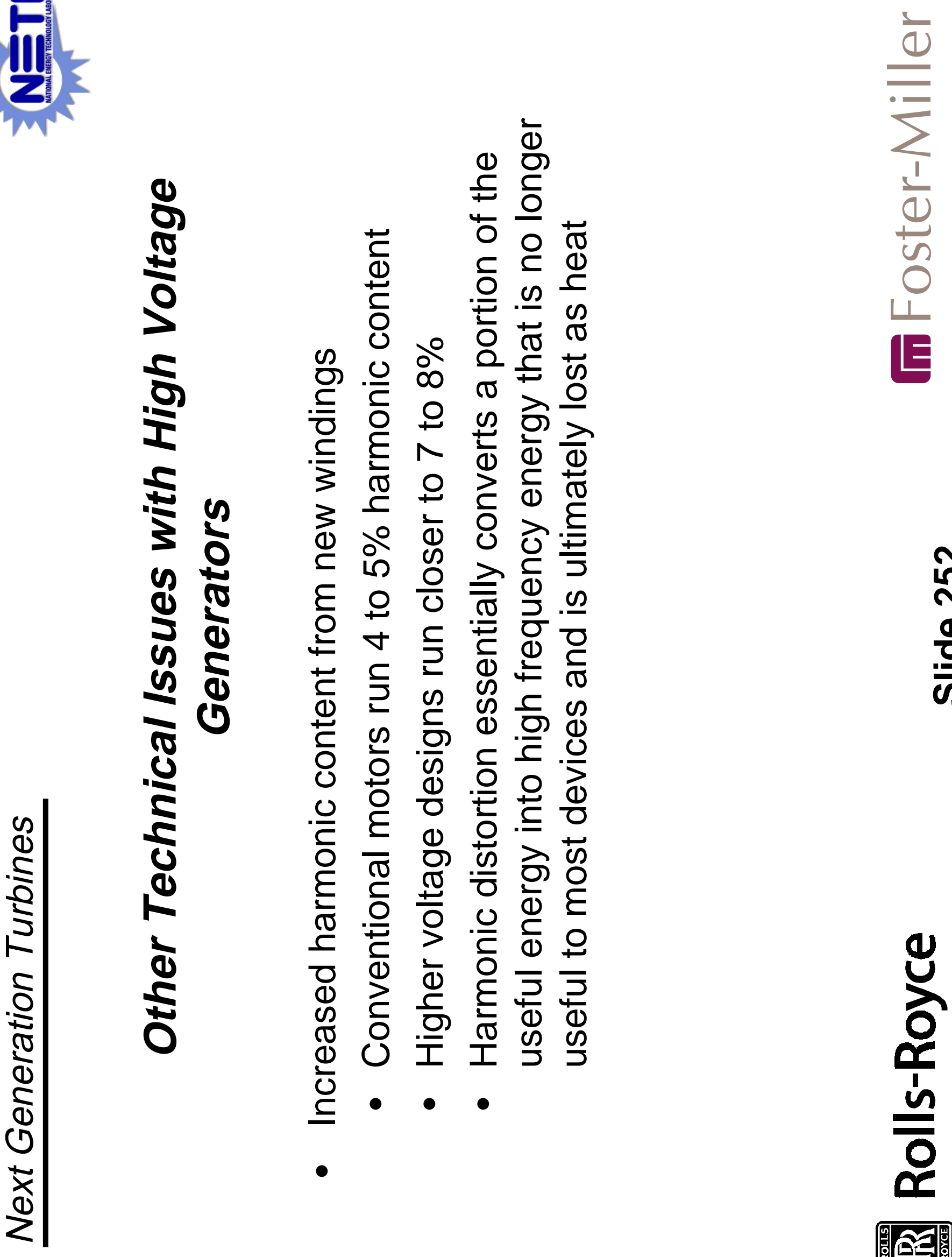


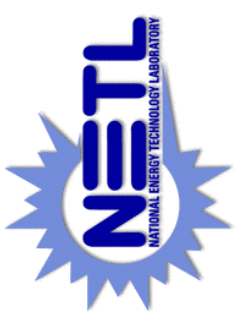

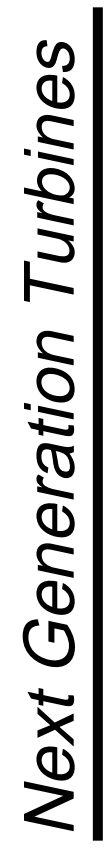
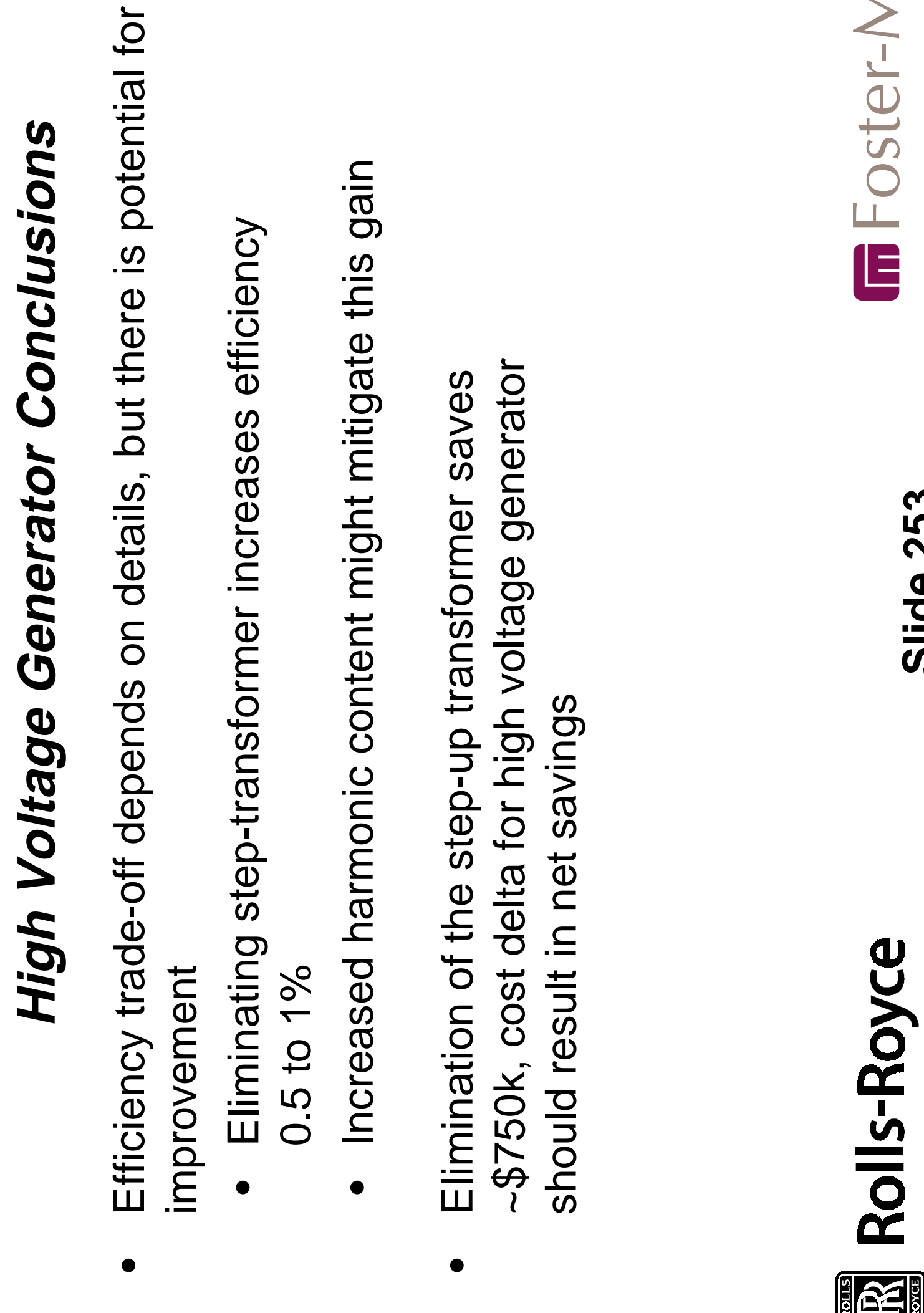


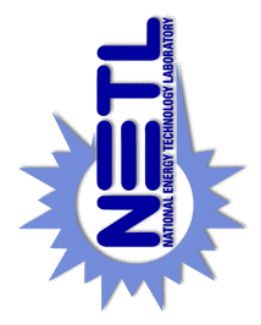

๗)

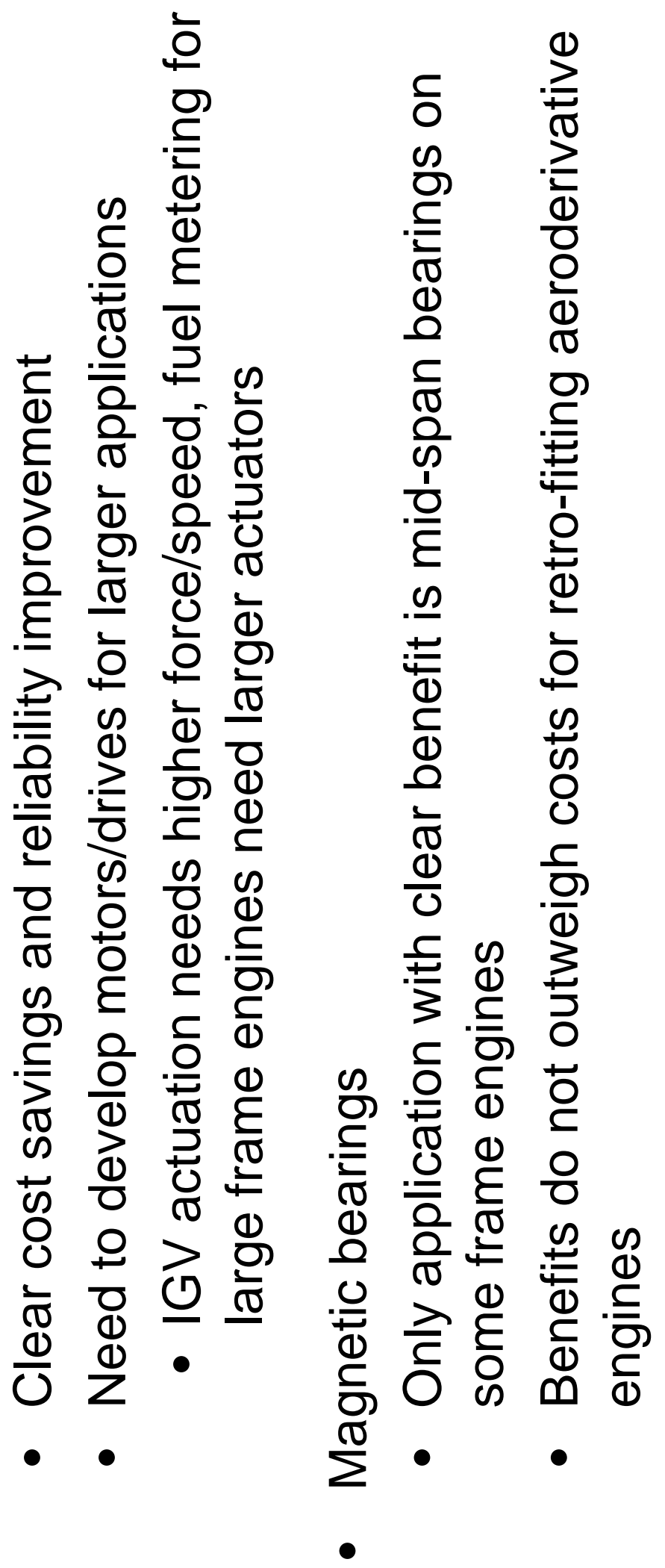

ปั
$\frac{0}{0}$
$\frac{0}{0}$

$\frac{0}{0}$

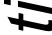

$\frac{\pi}{0}$

$\frac{9}{5}$

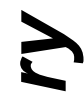

ฮ

క

0

0

$\exists$

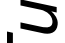

N

옹

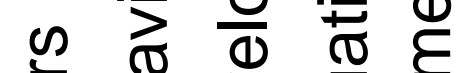

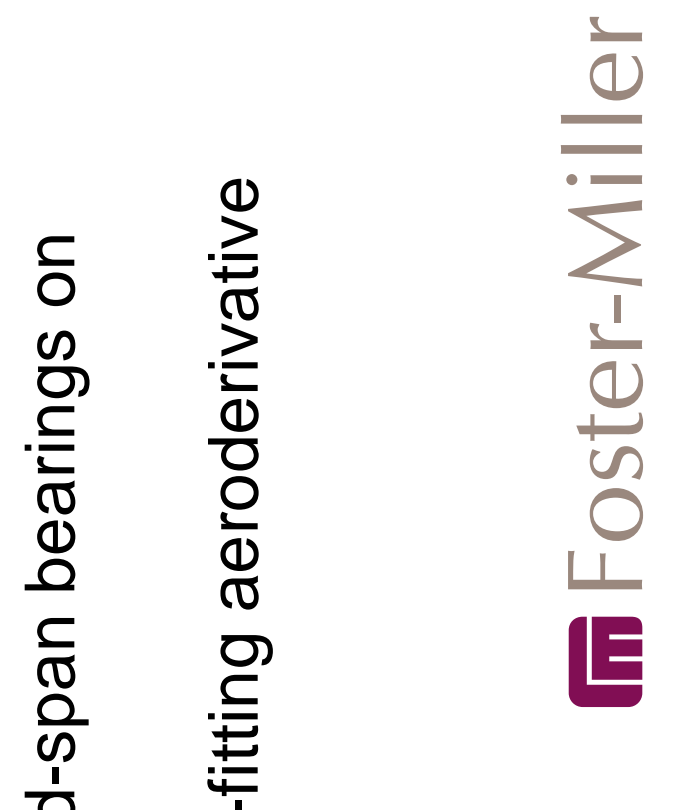

ஸे

ช

$\frac{0}{4}$

$\stackrel{ \pm}{2}$ 


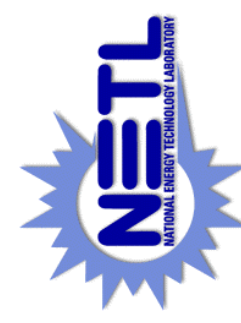

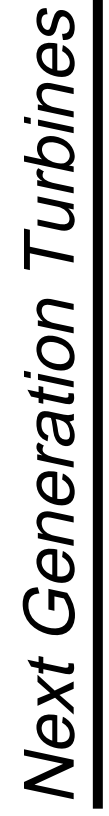

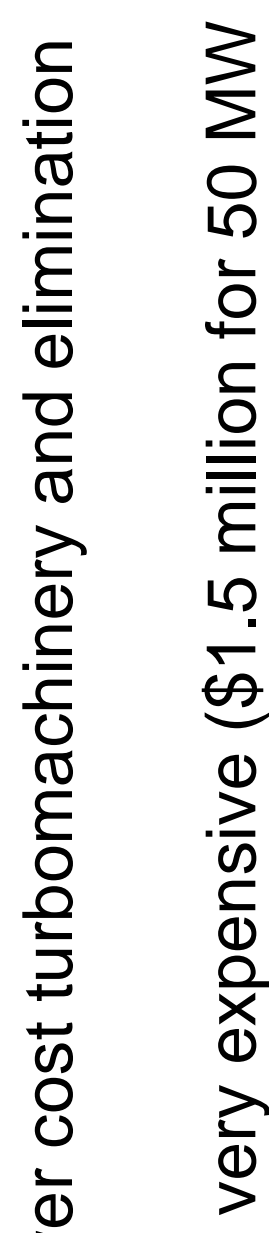

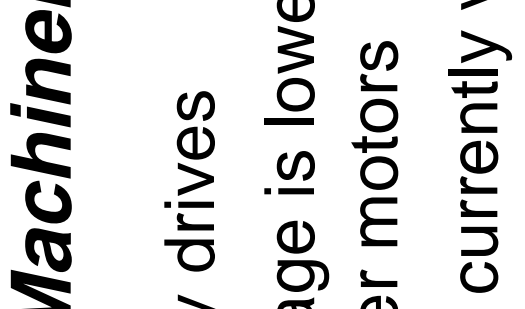

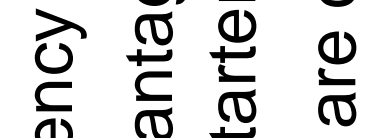

ब $\frac{\pi}{2}$ क

궁 월

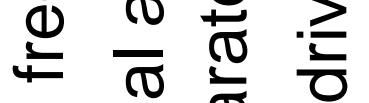

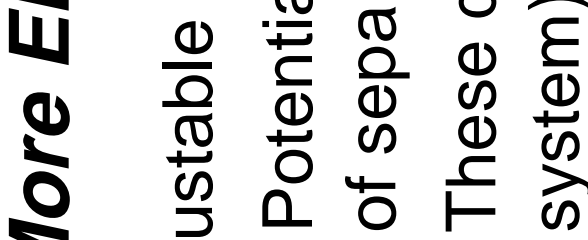

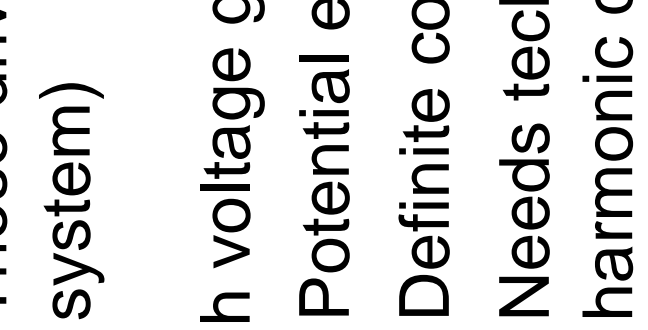

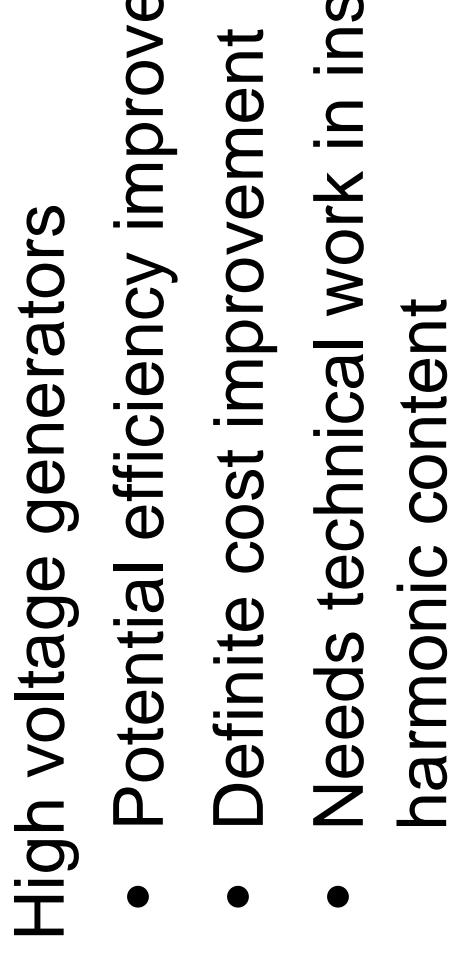

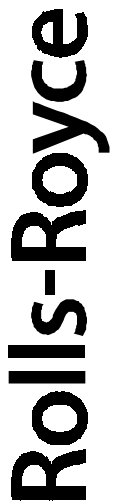



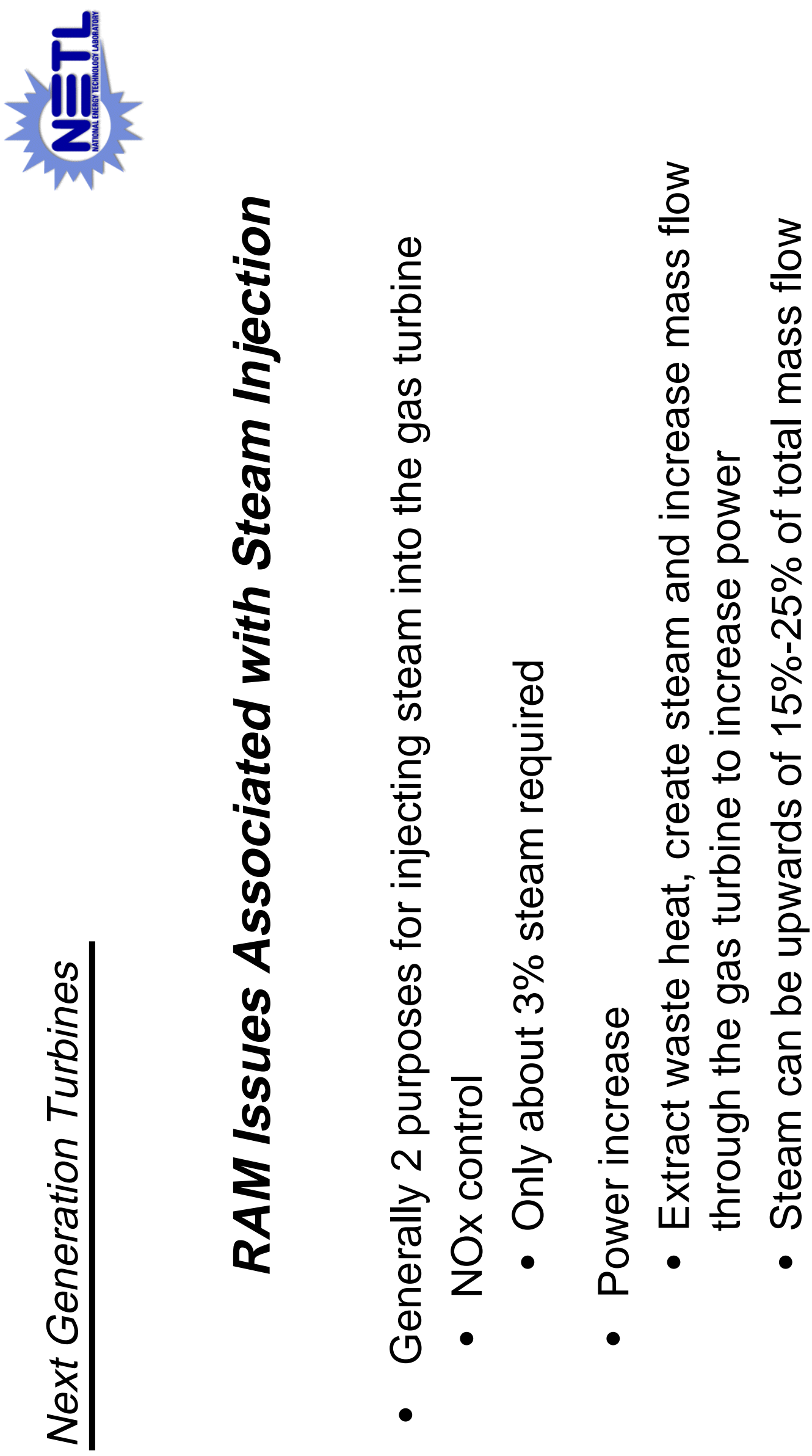

ह क ज

๗ Eี

(1) ד্ত

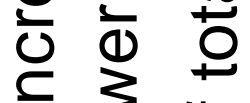

$\stackrel{0}{\mp}$

$\stackrel{ }{\underline{E}}$

$\frac{E}{\sigma}$

$\frac{1}{\infty}$

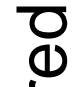

8

(

을 흐

0

0 . 드

5

$\frac{1}{\omega}$

흐

( )

ò

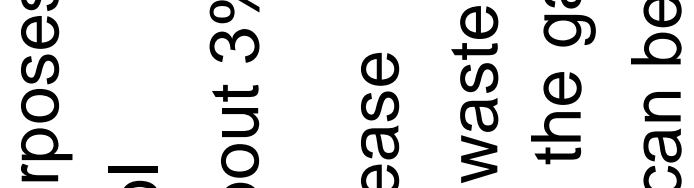

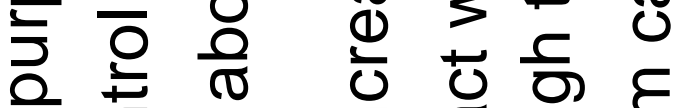

ธช

$\varepsilon \stackrel{\sim}{\sim}$

สู

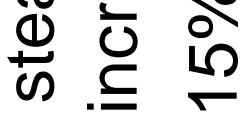

0

ก

$\stackrel{0}{\pi}+\frac{1}{0}$

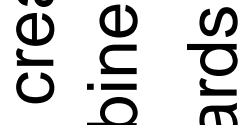

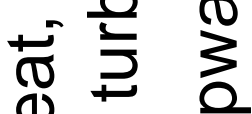

$\stackrel{1}{1} \stackrel{2}{\supset}$

$\frac{0}{0}$

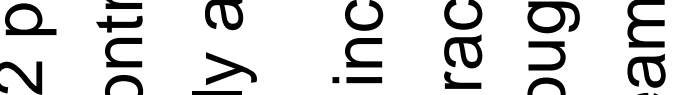

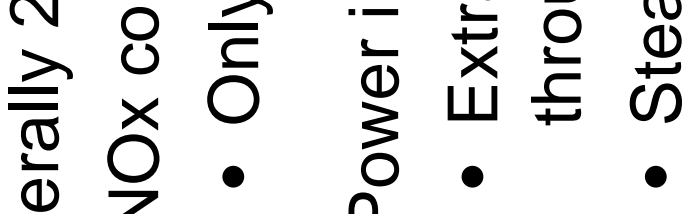

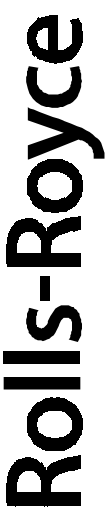

ஸे
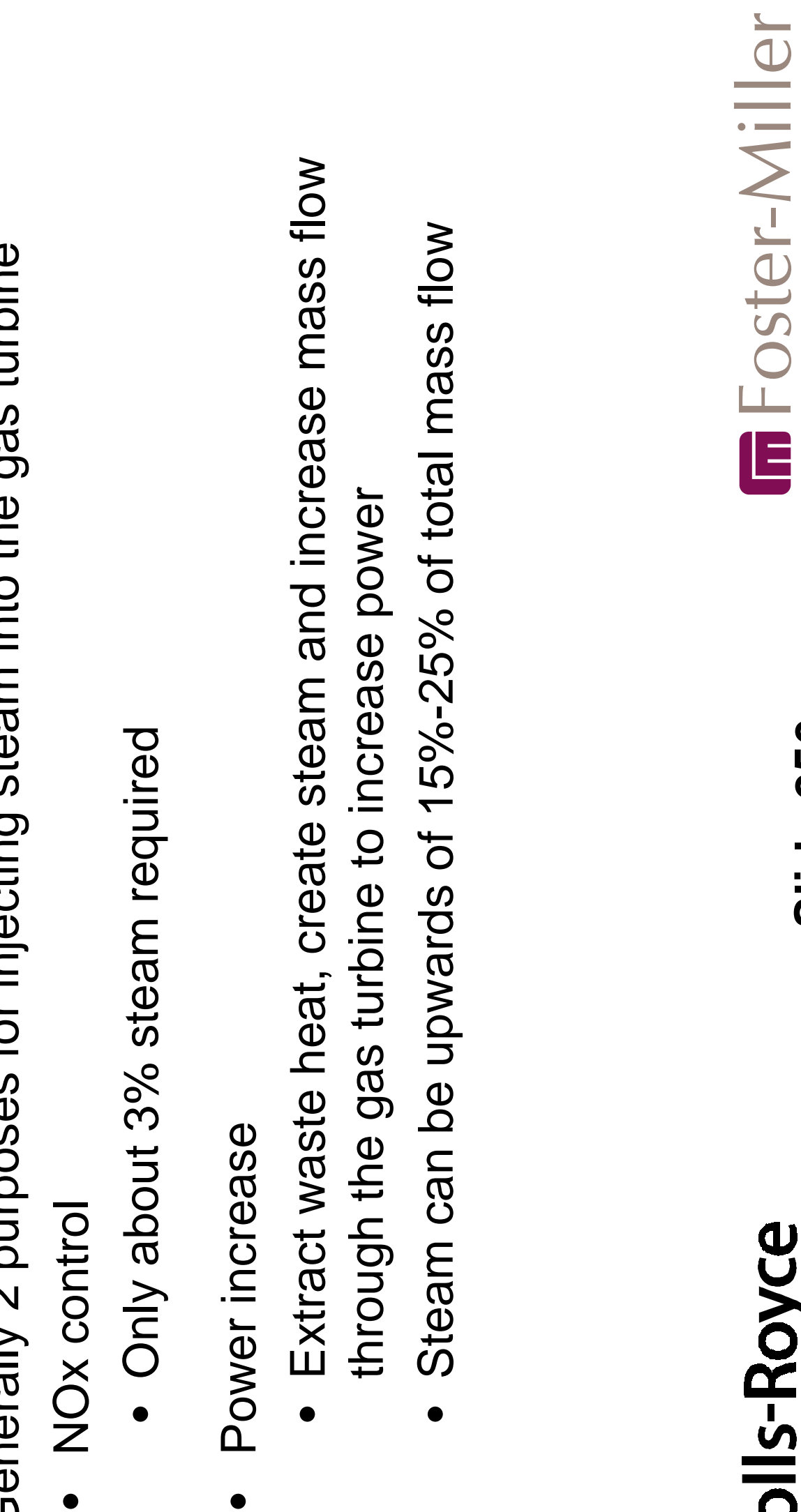


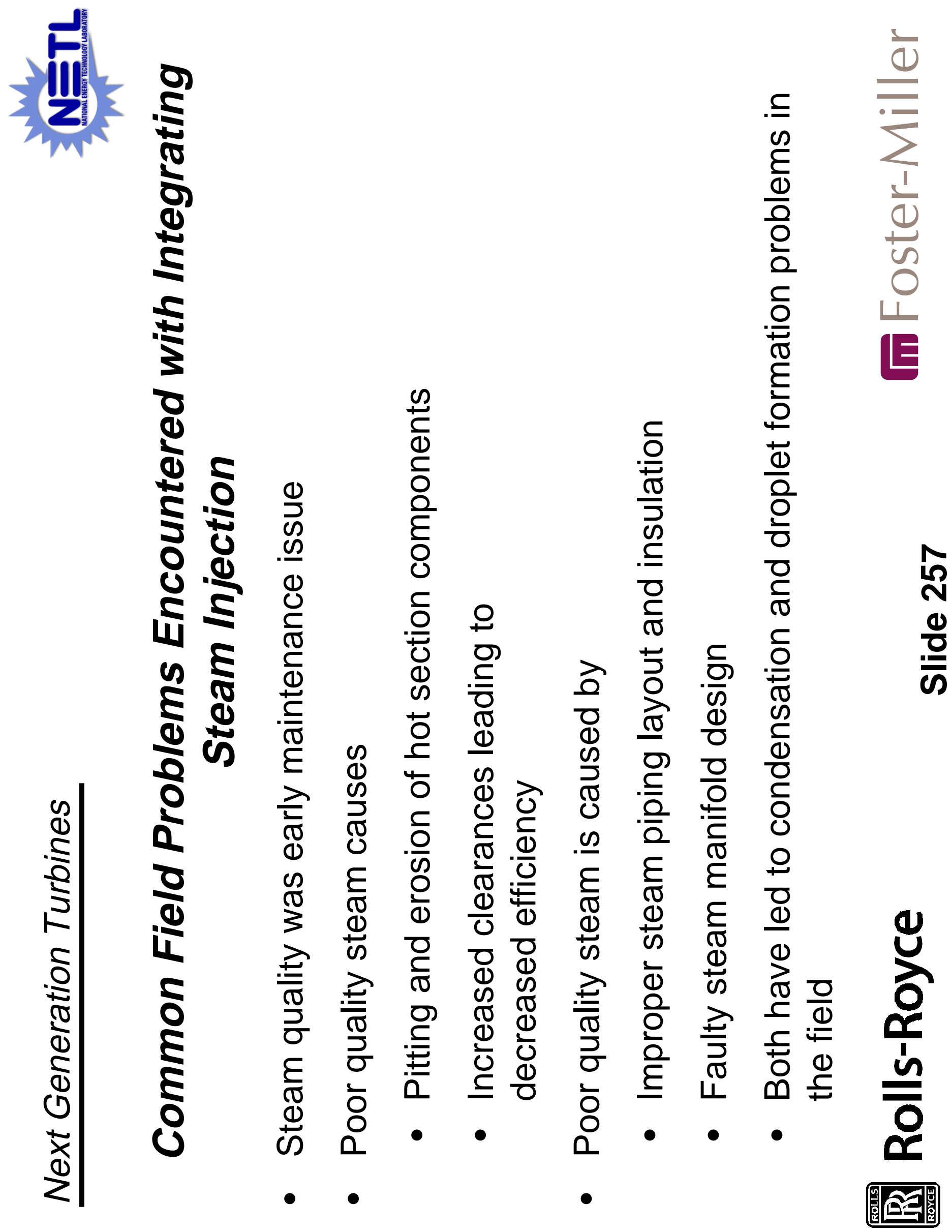



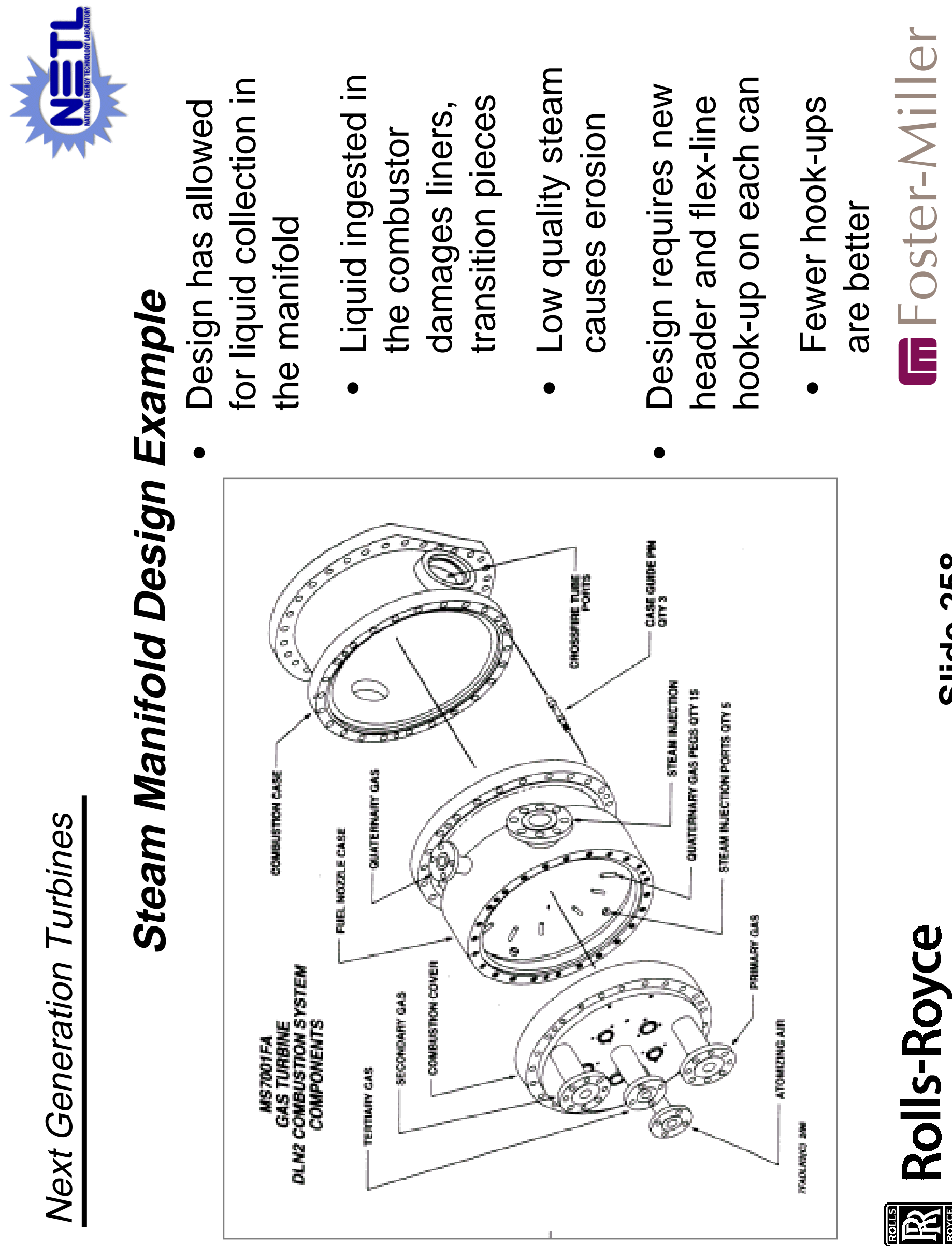

$\infty$
1
$\frac{1}{0}$
$\frac{1}{\infty}$

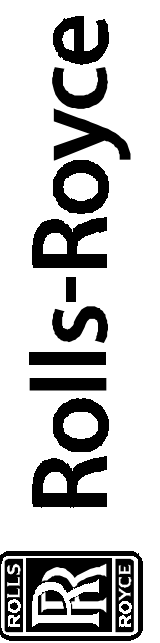



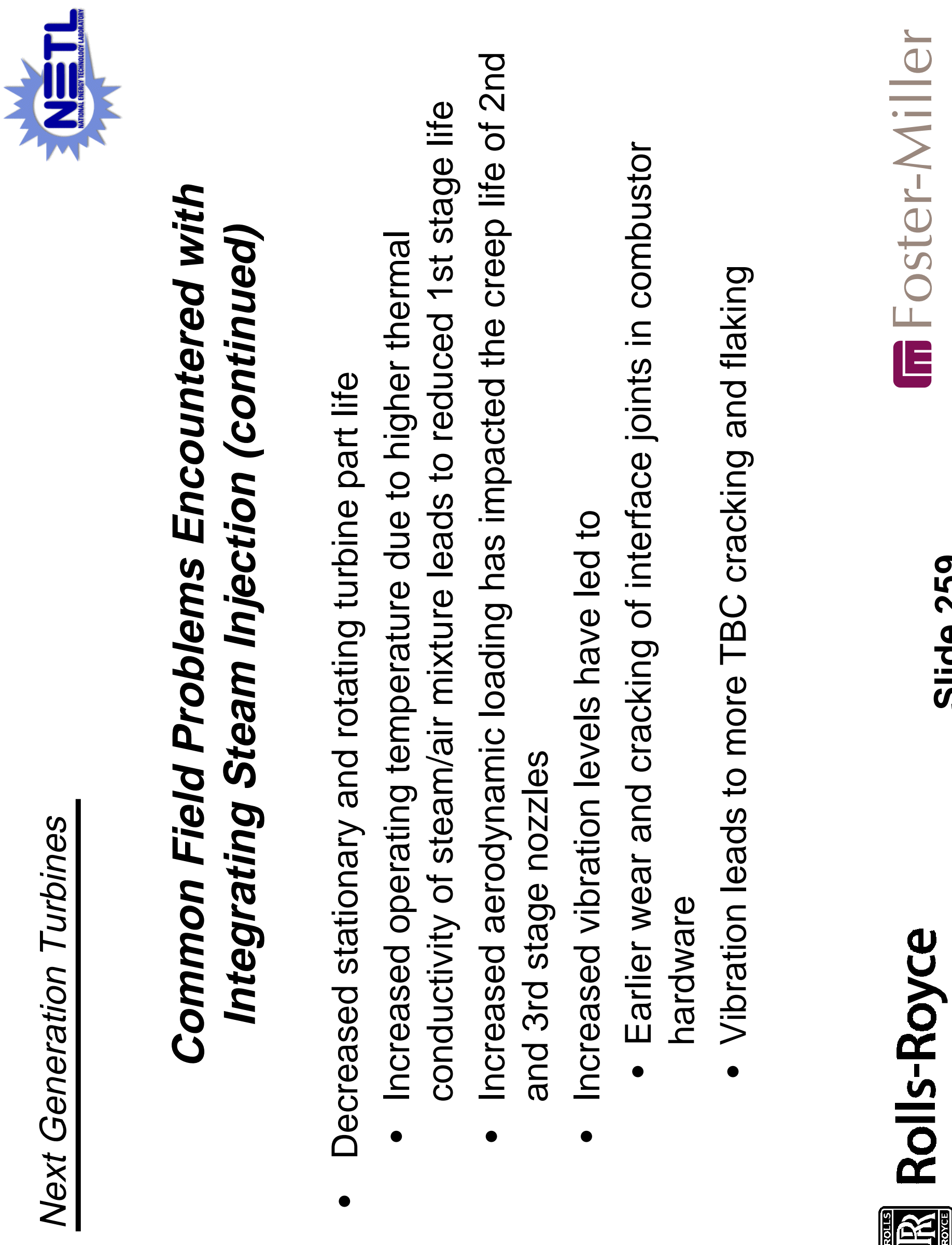


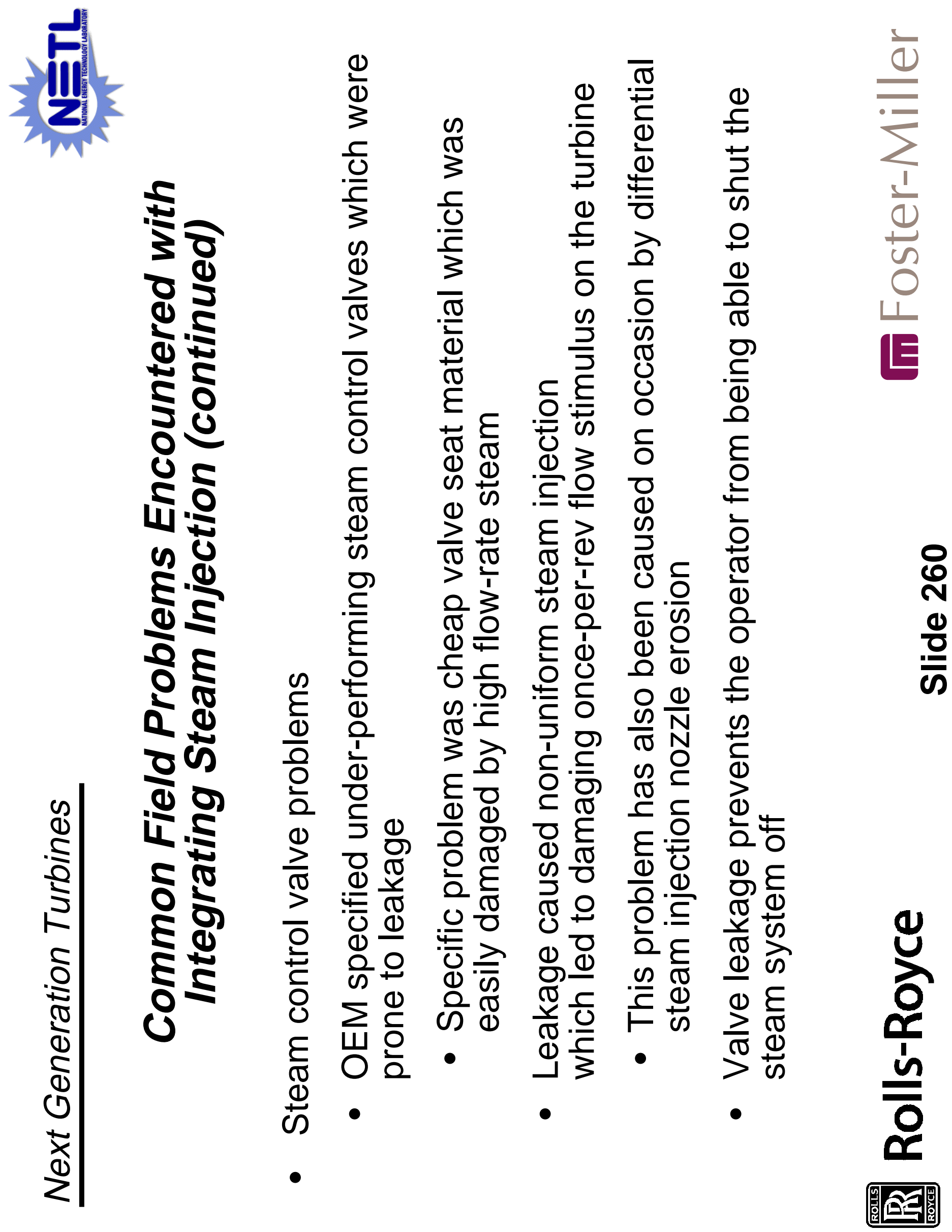



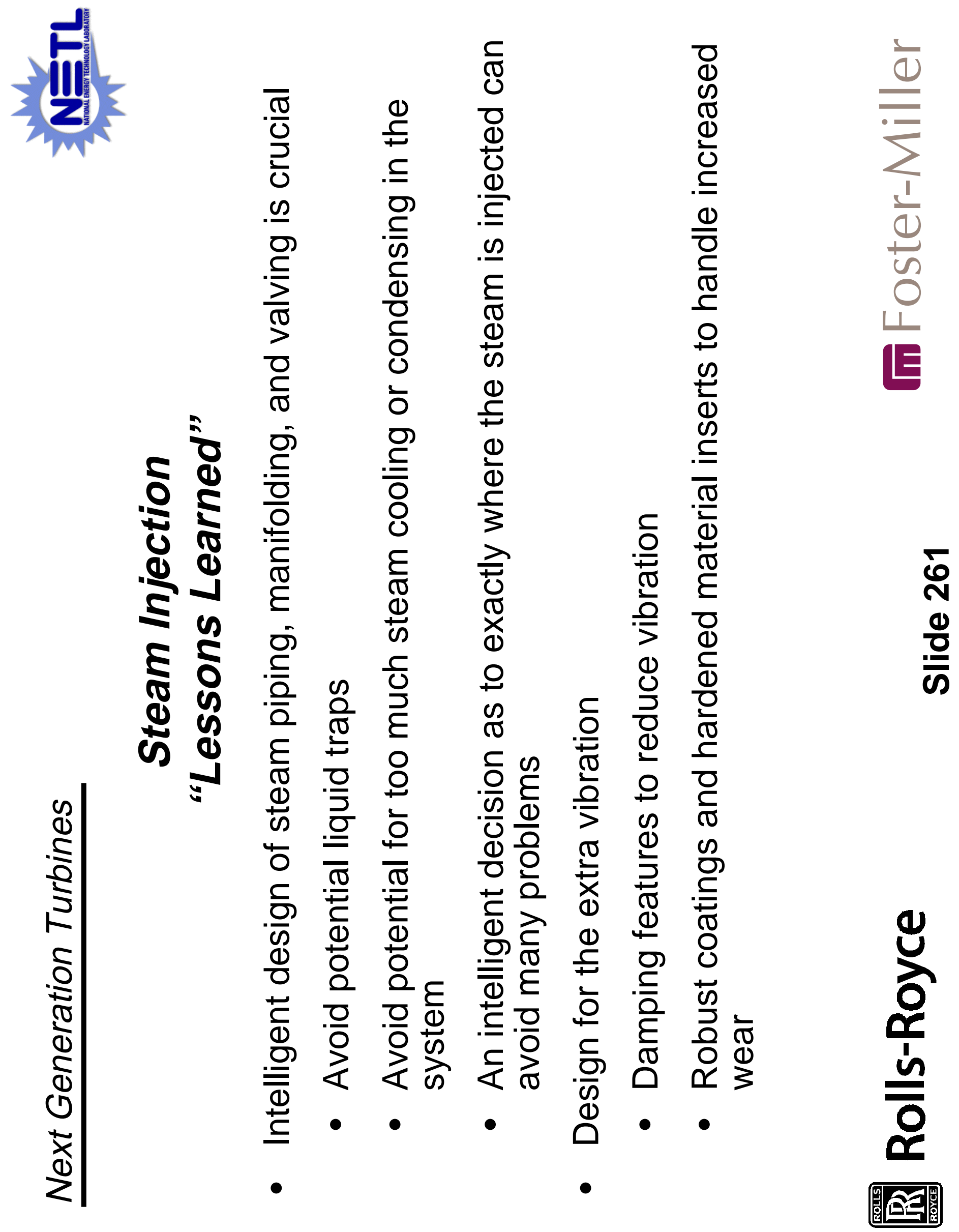

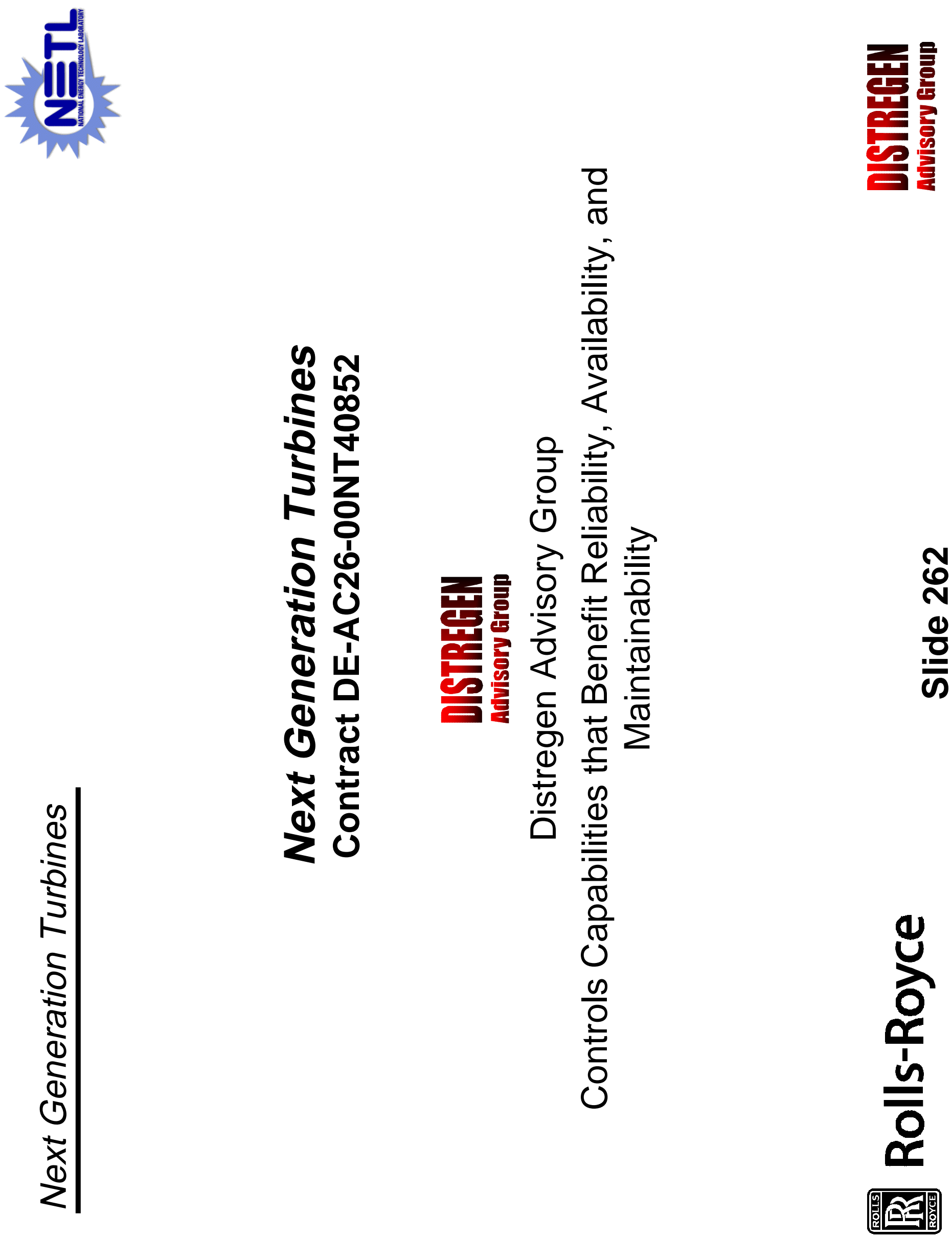

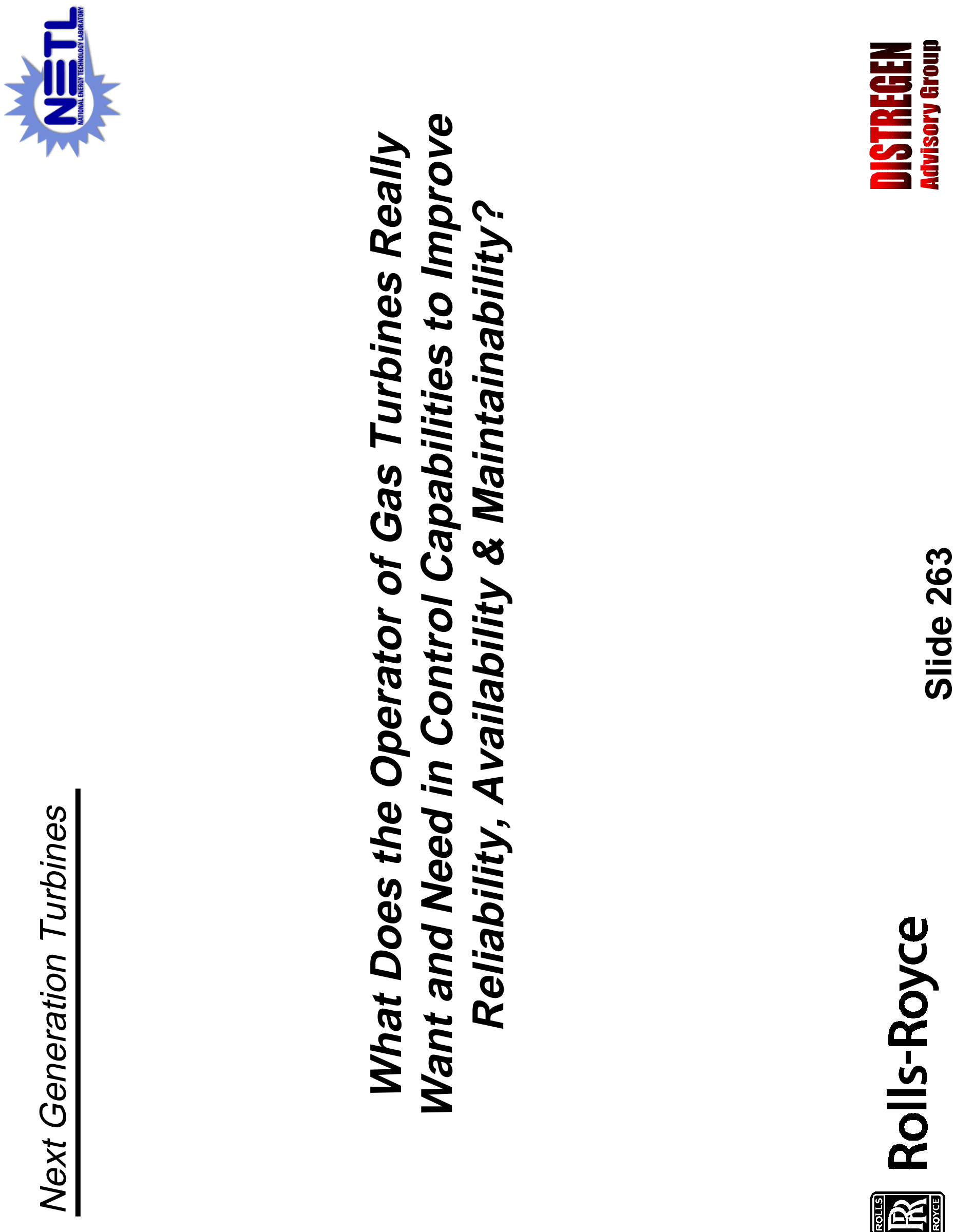

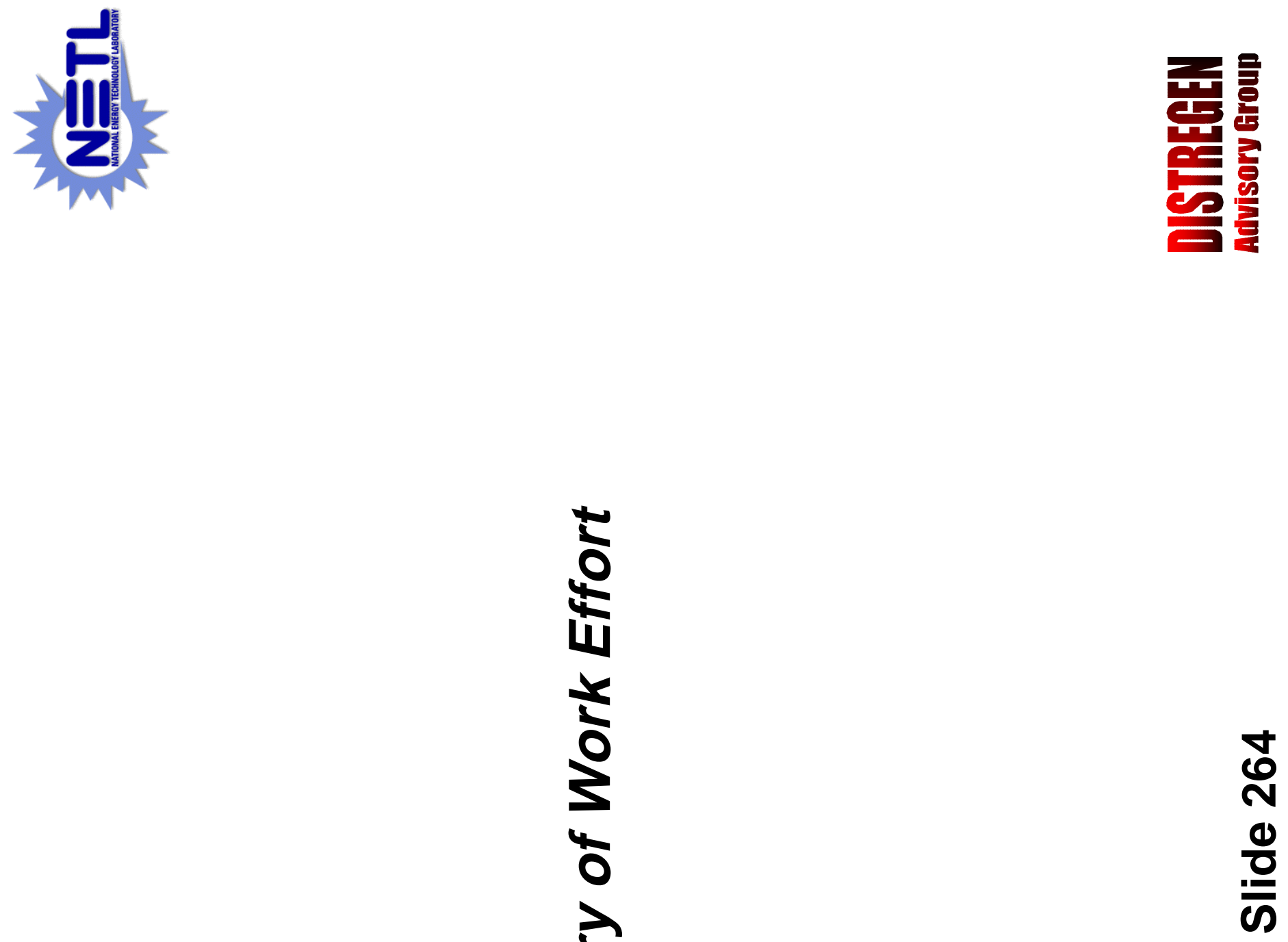

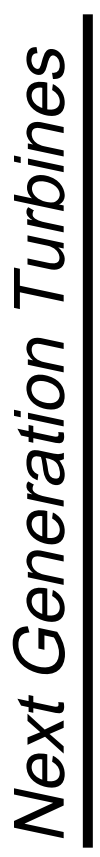
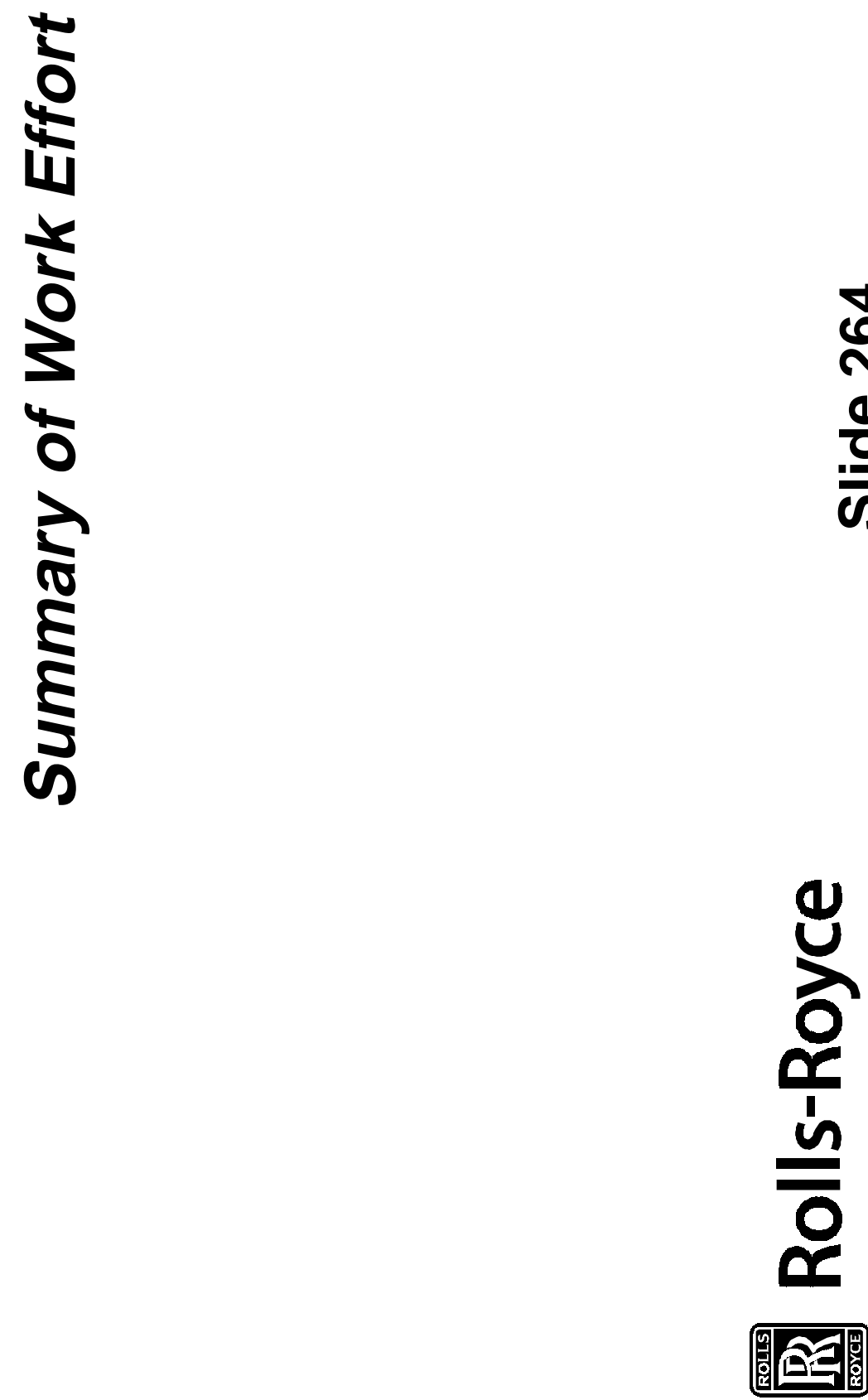

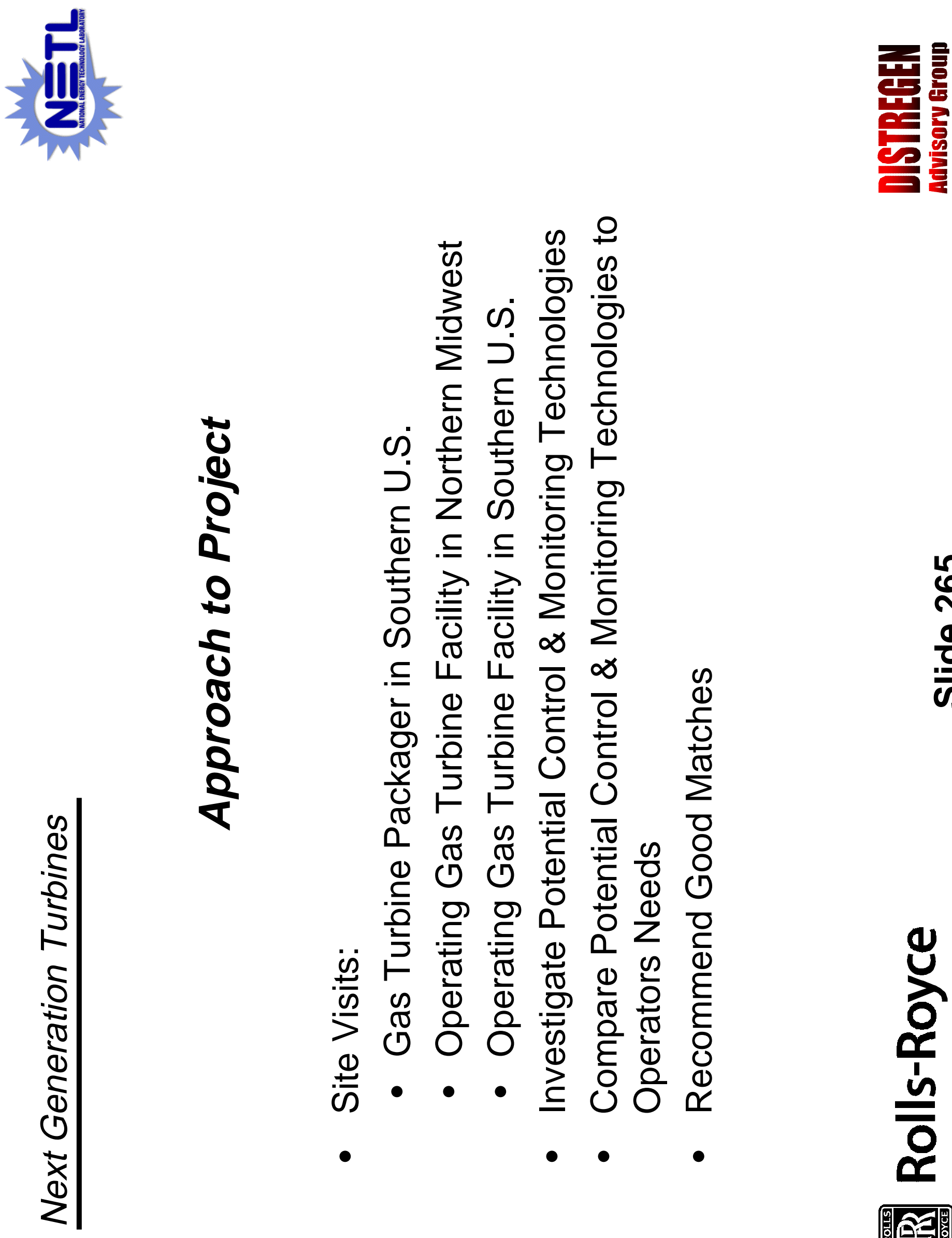

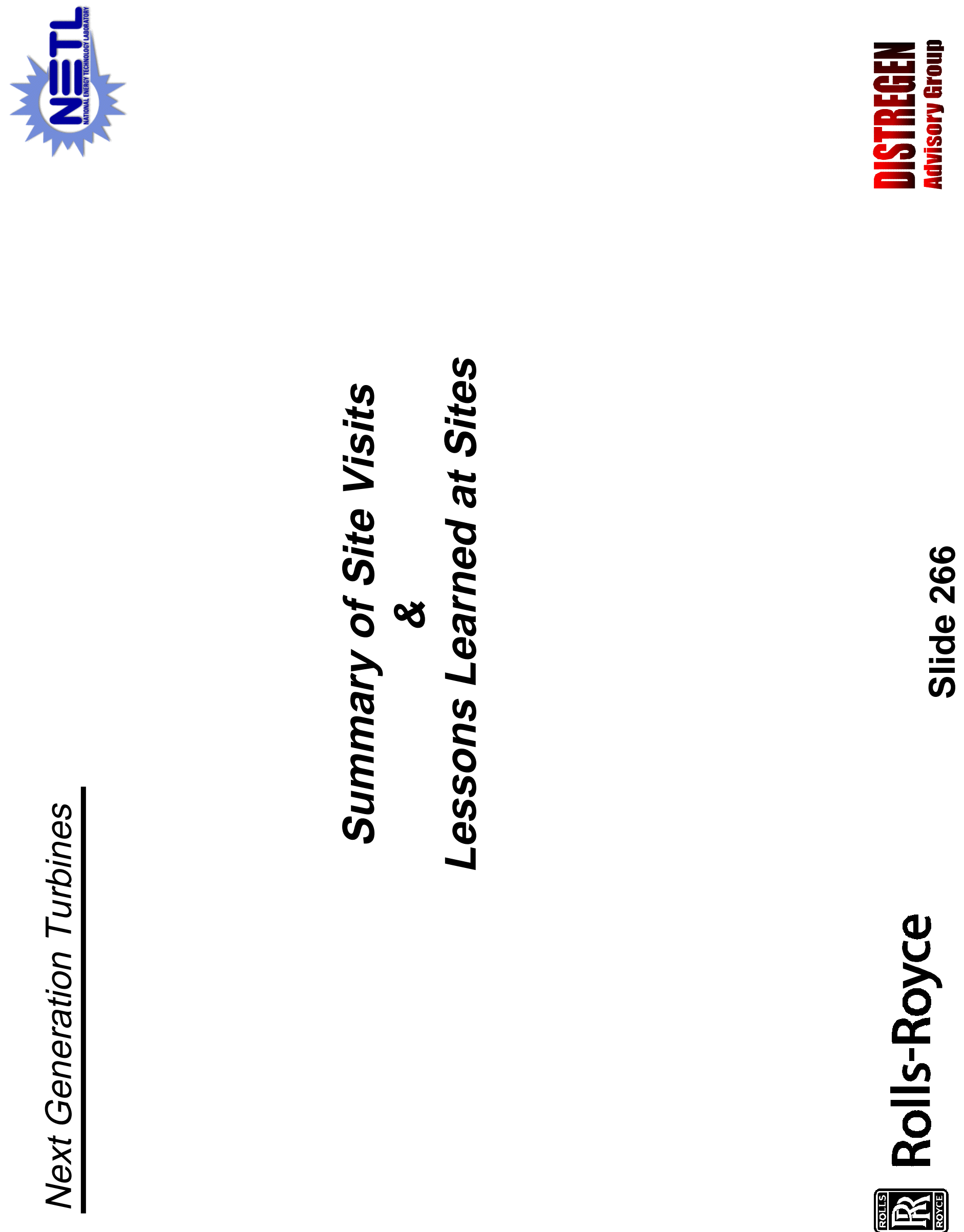

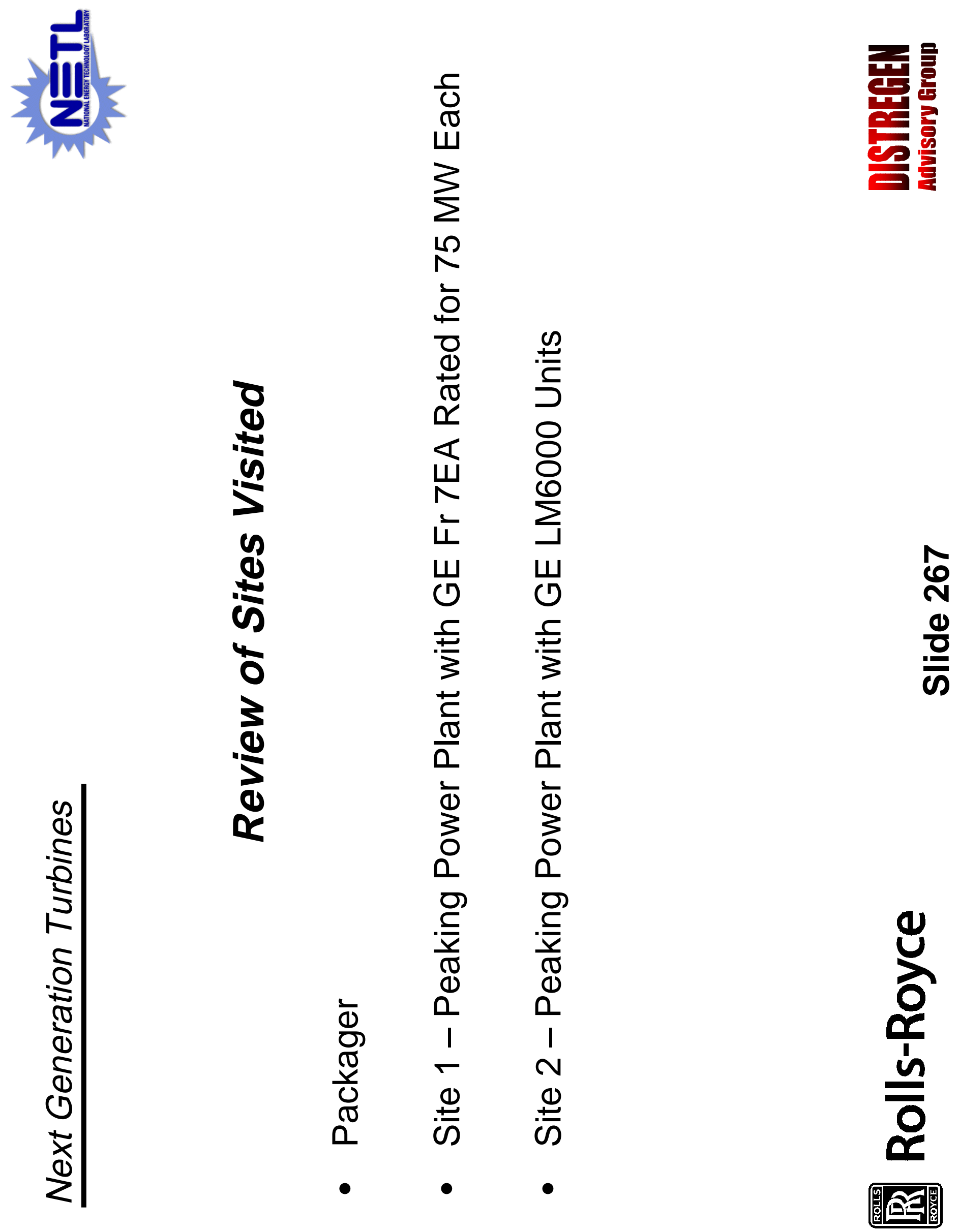

s

4

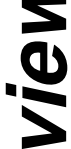

()

a

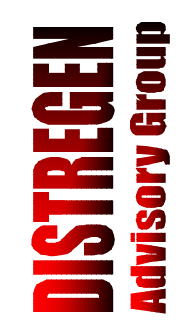



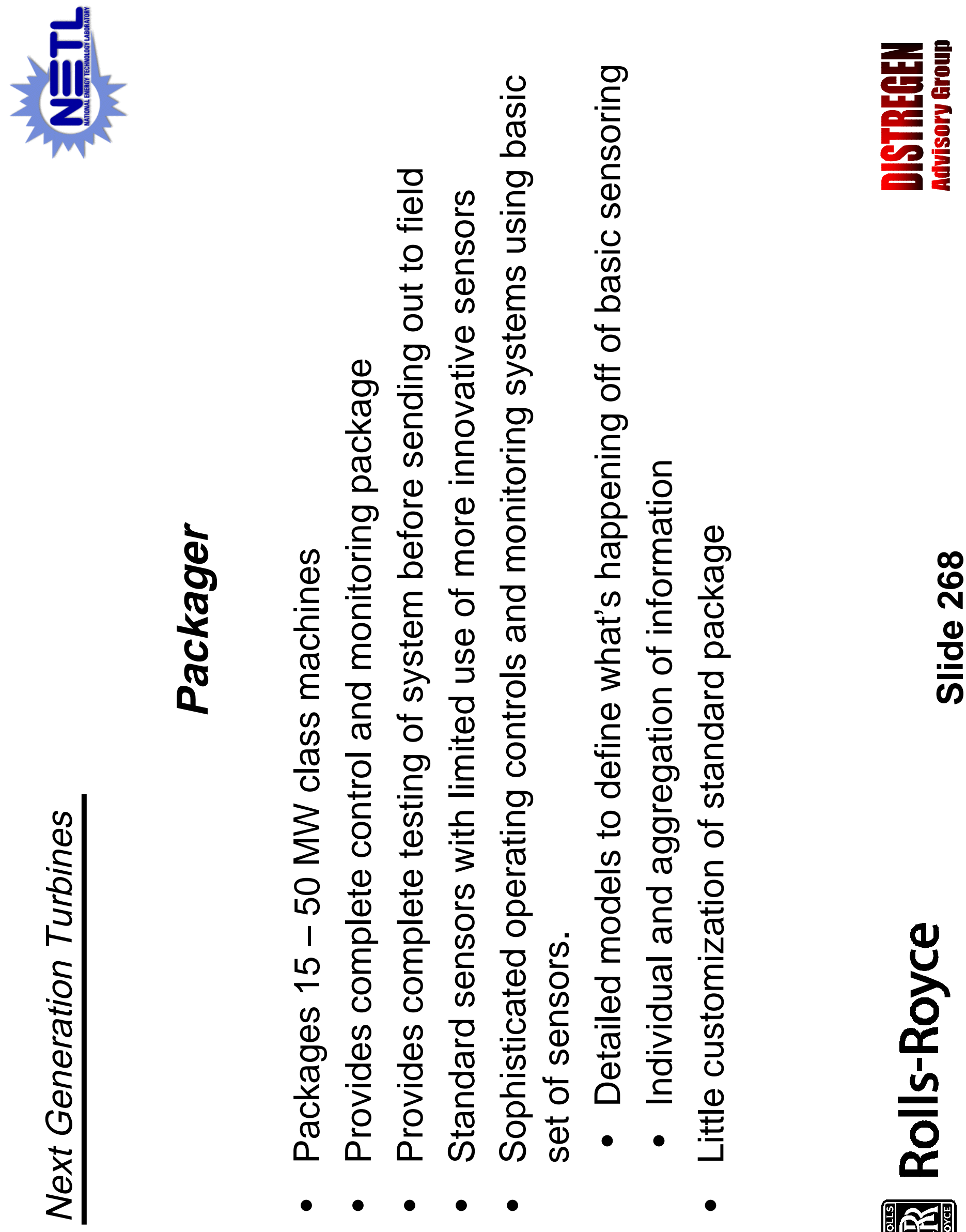

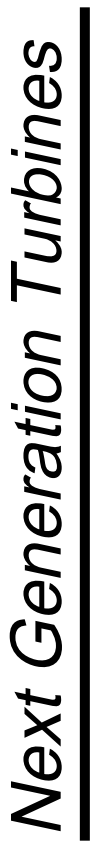



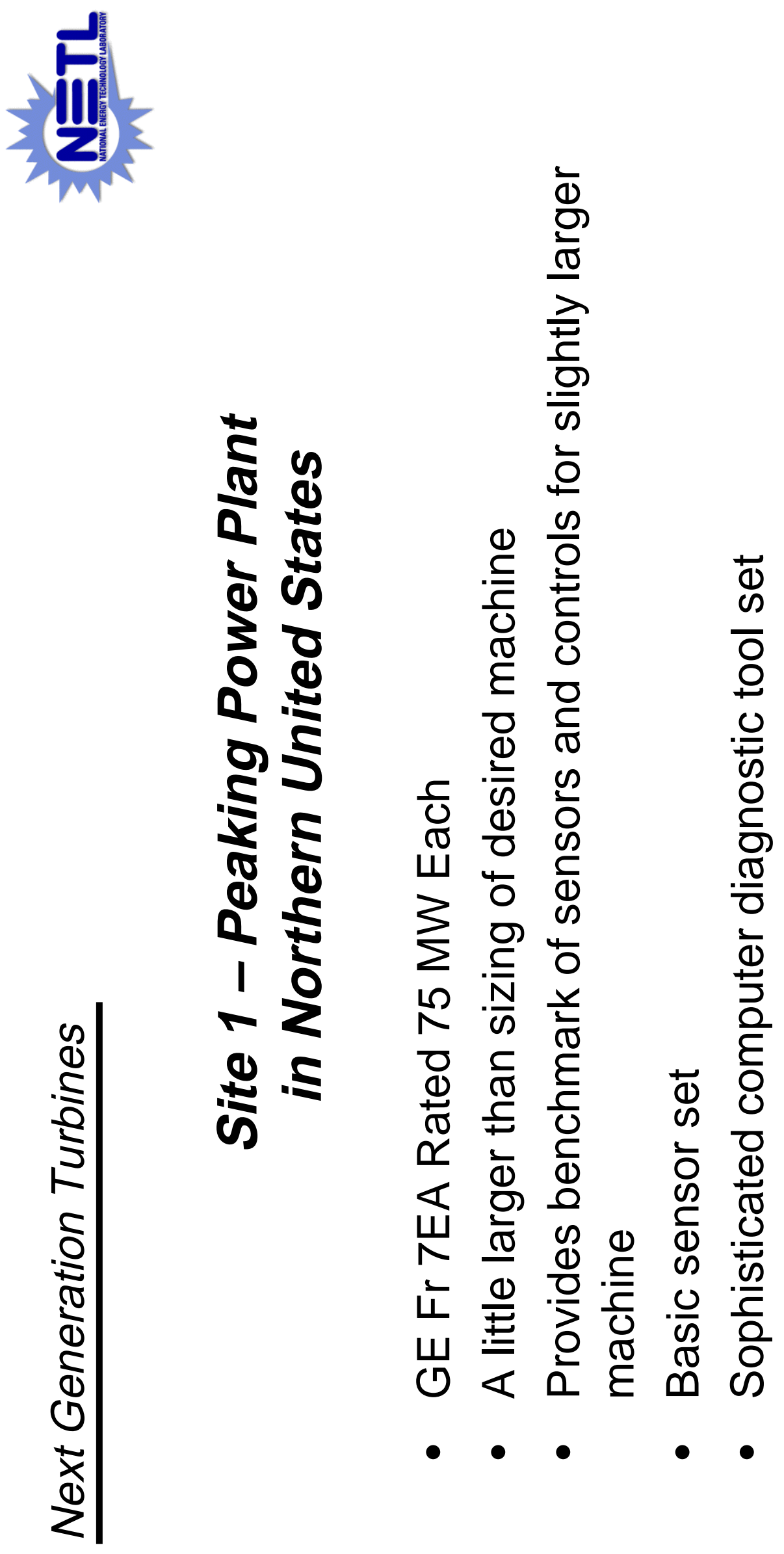

음
ஸ
$\frac{0}{0}$

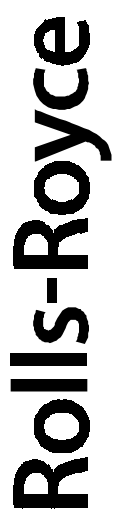

永| 

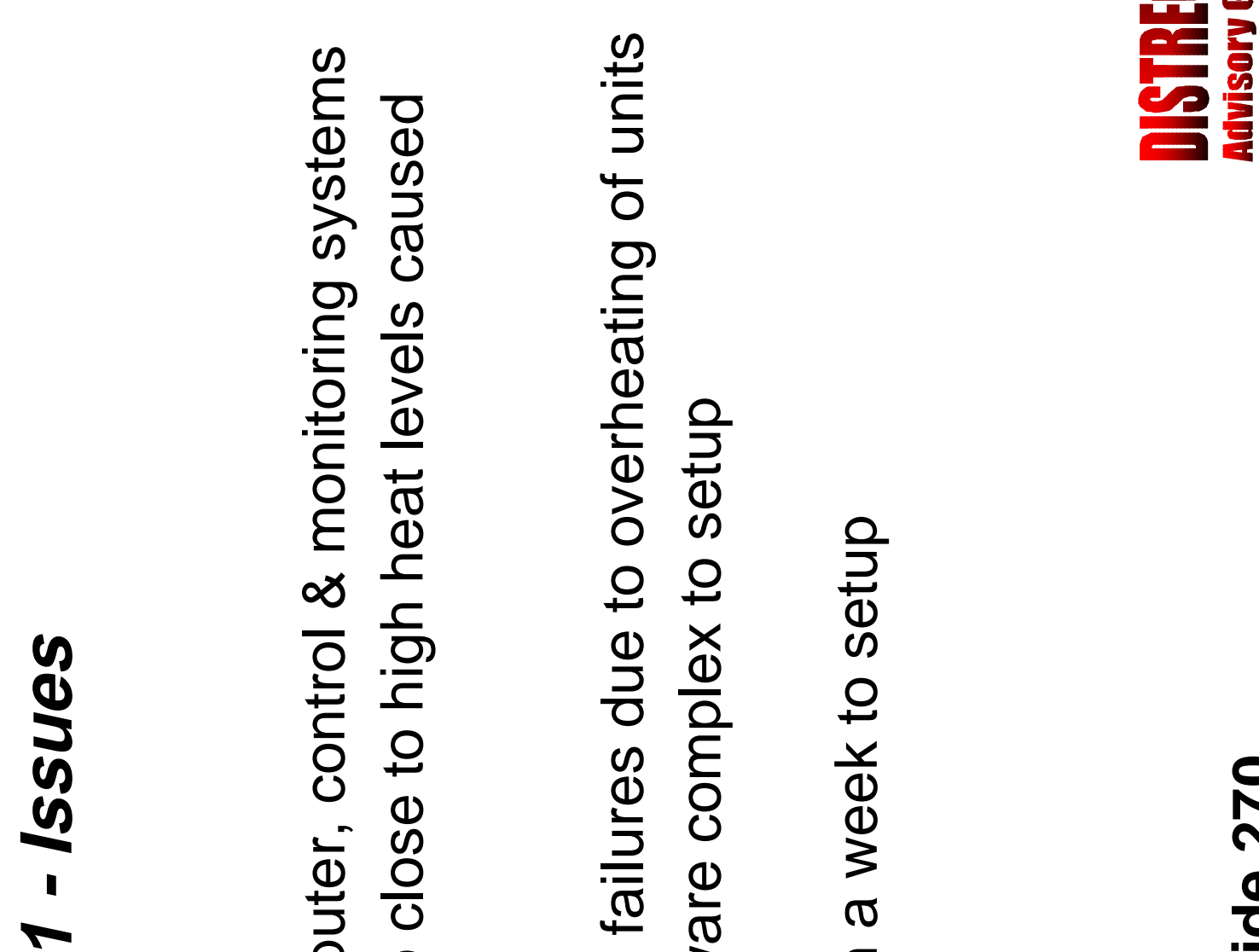

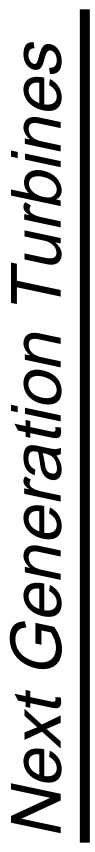

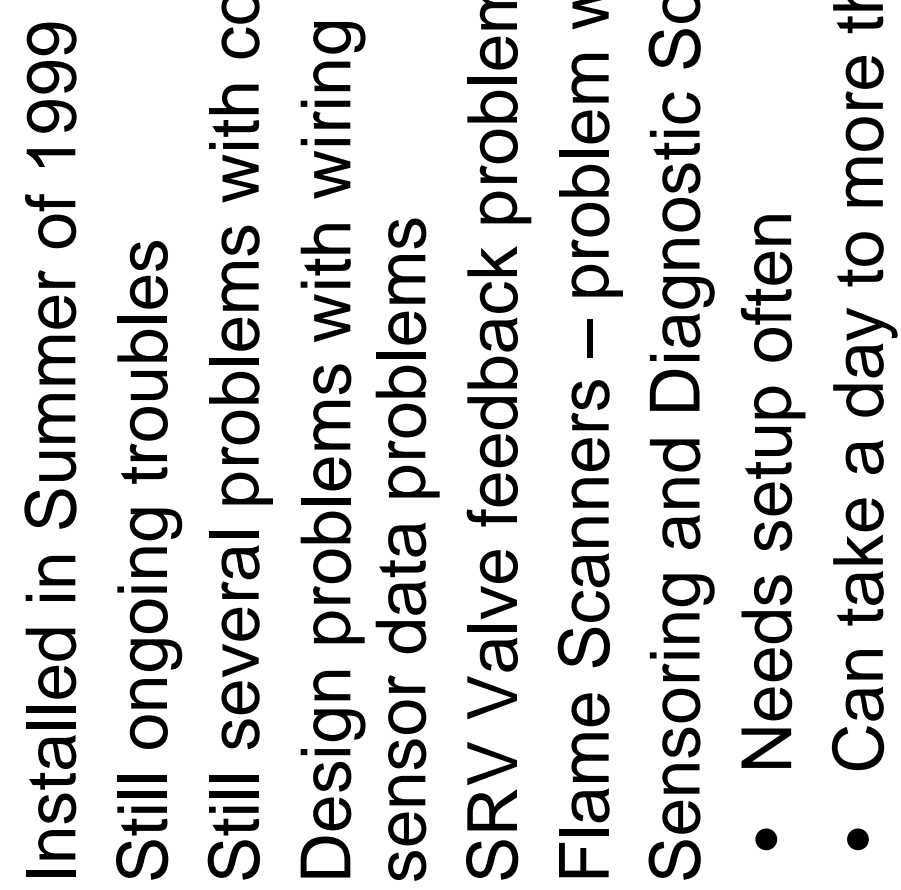

$\frac{2}{2}$

$+$

क ह

$\stackrel{1}{3}$

ธ

$\frac{\bar{\sigma}}{\mathrm{\sigma}}$

드

(1)

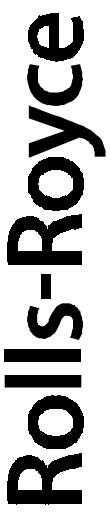



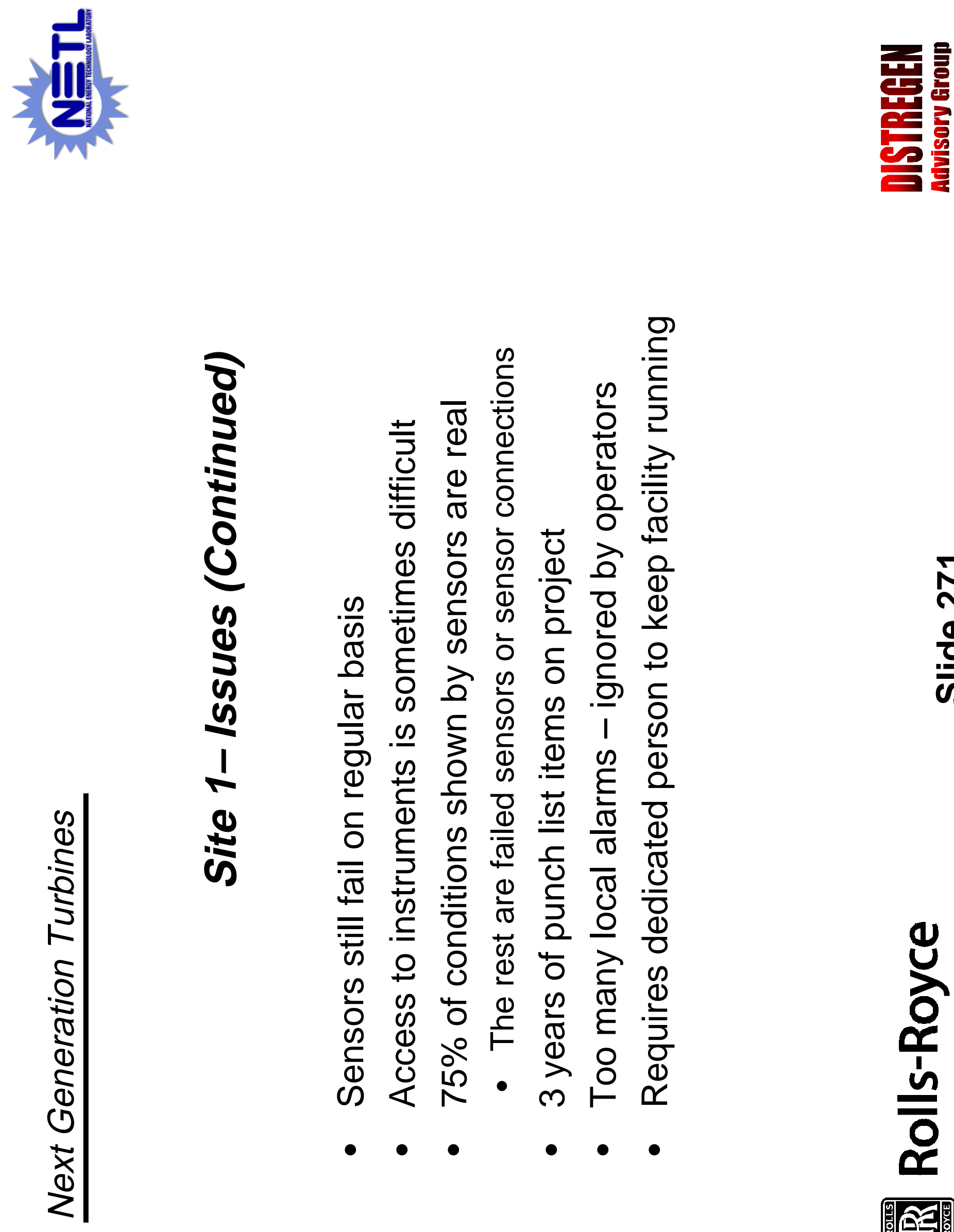


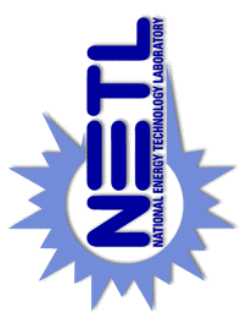

$\frac{5}{3} \frac{a}{2} \frac{1}{\frac{1}{2}}$
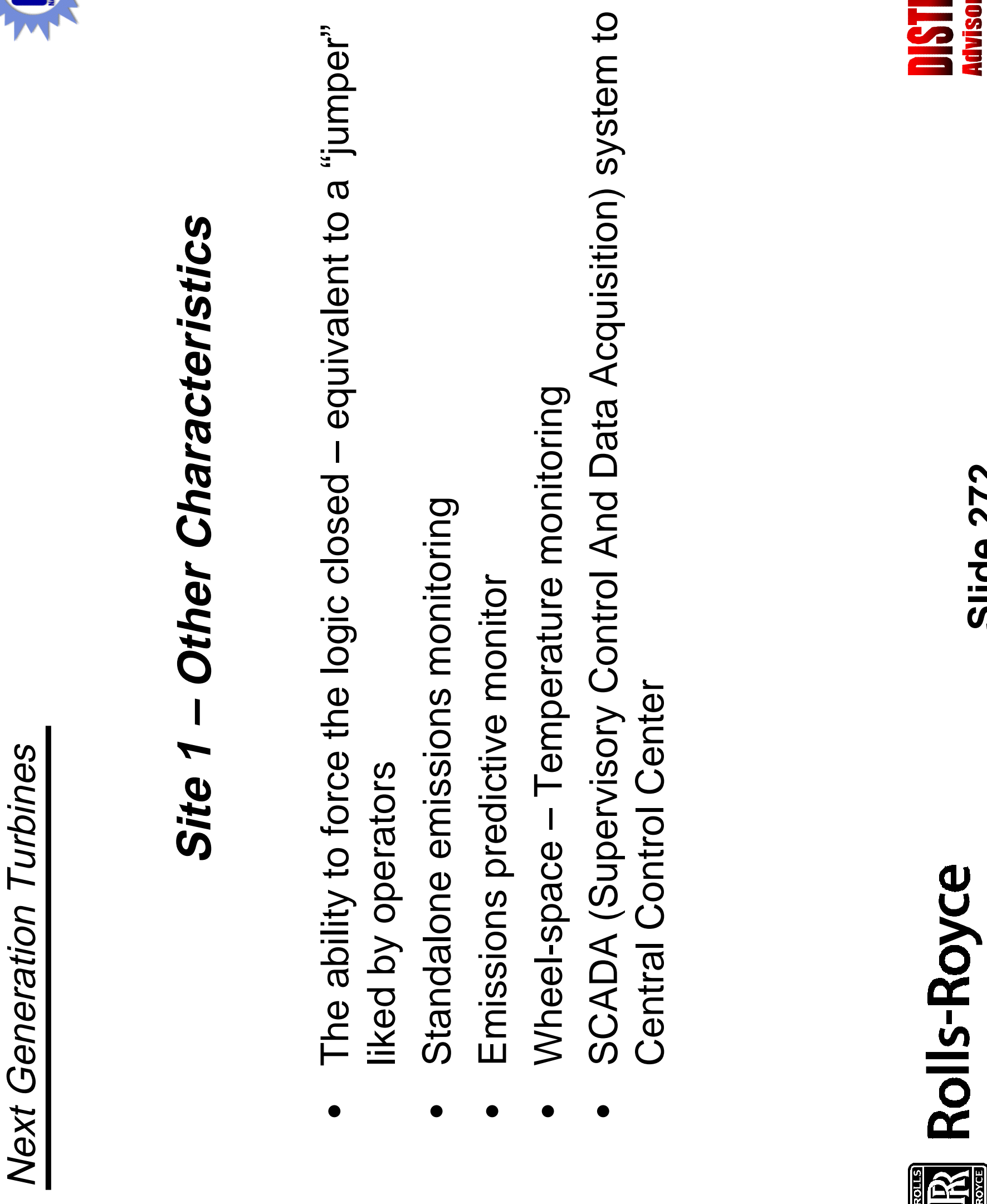

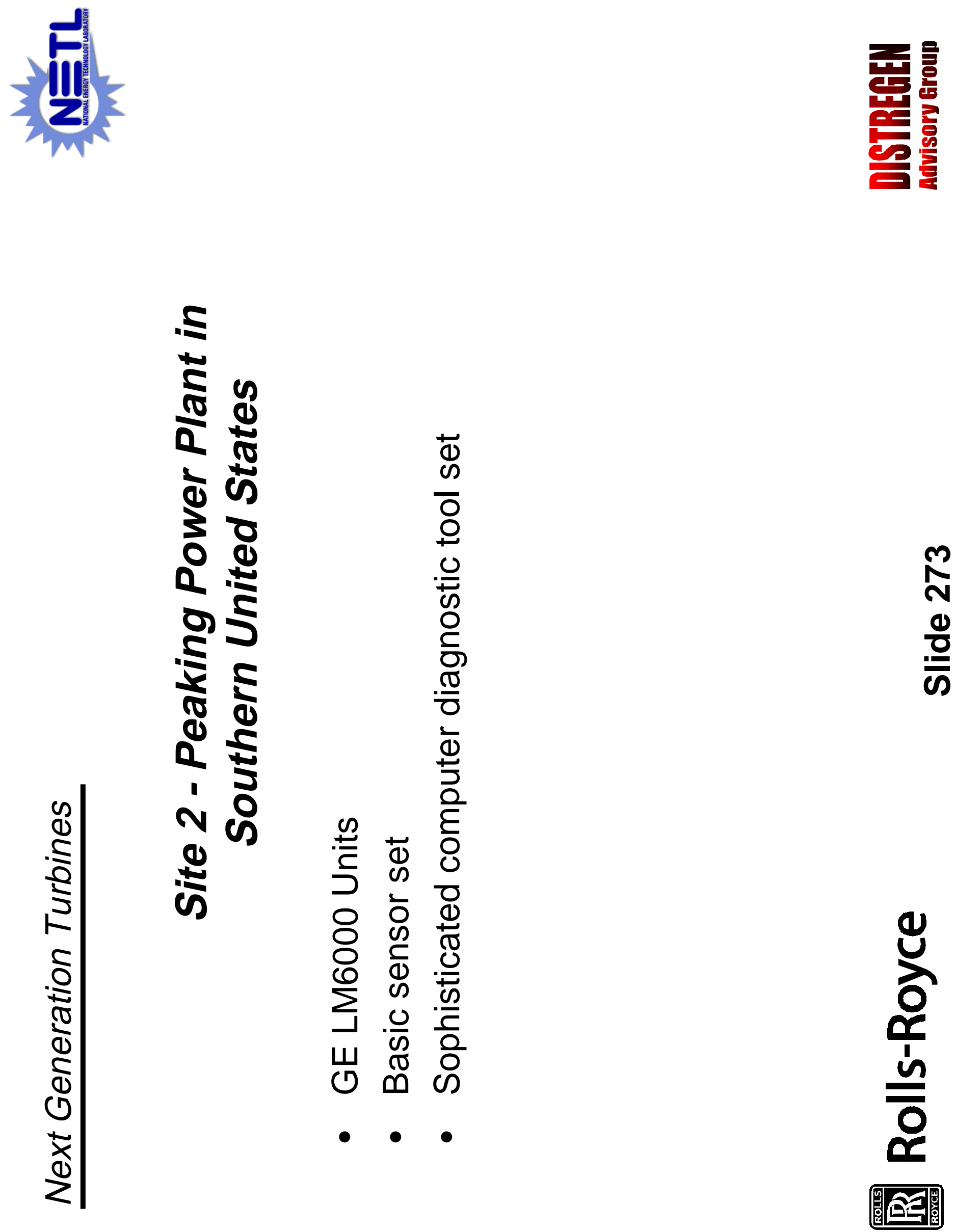

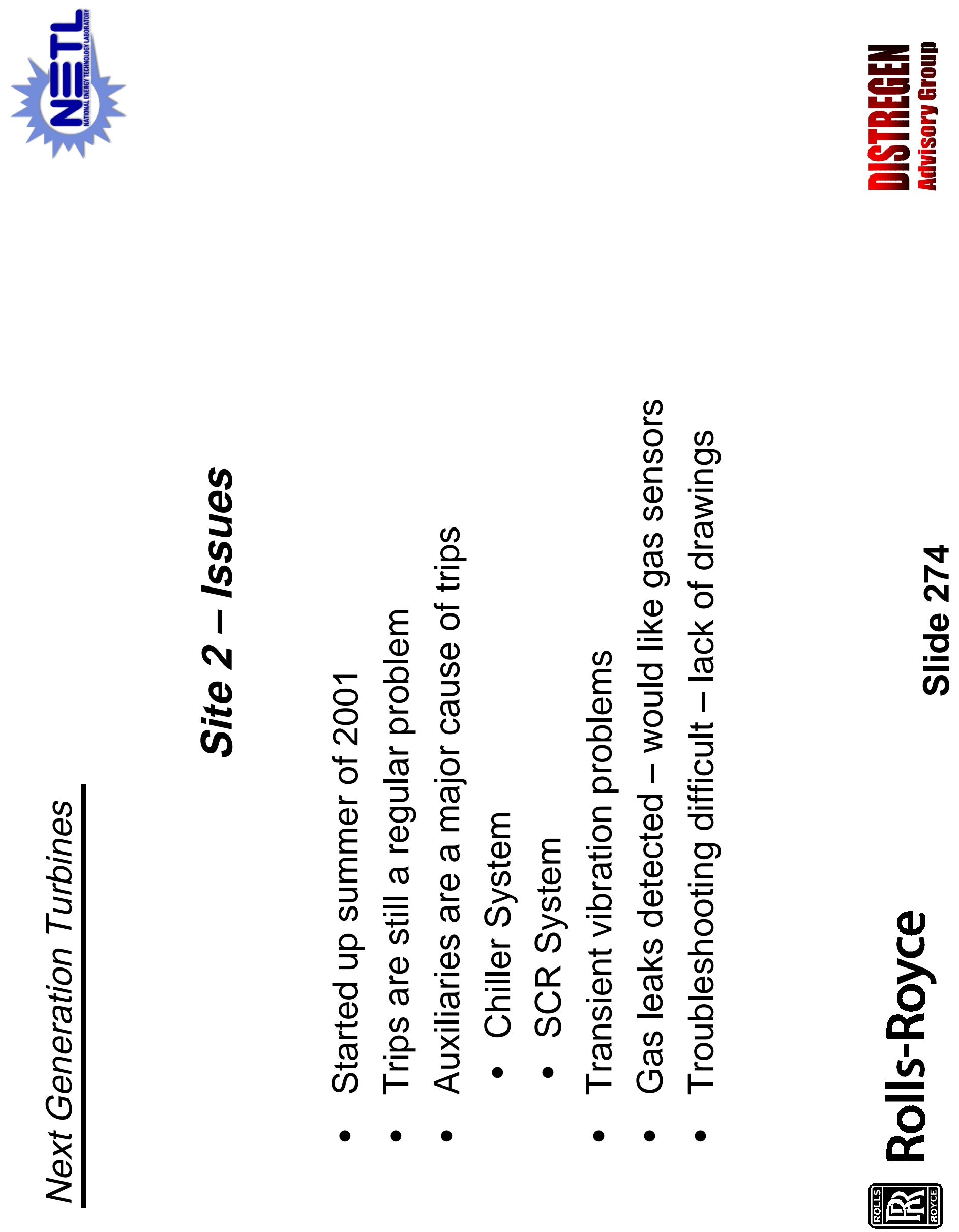

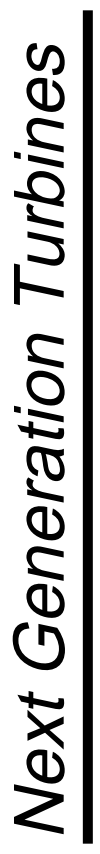



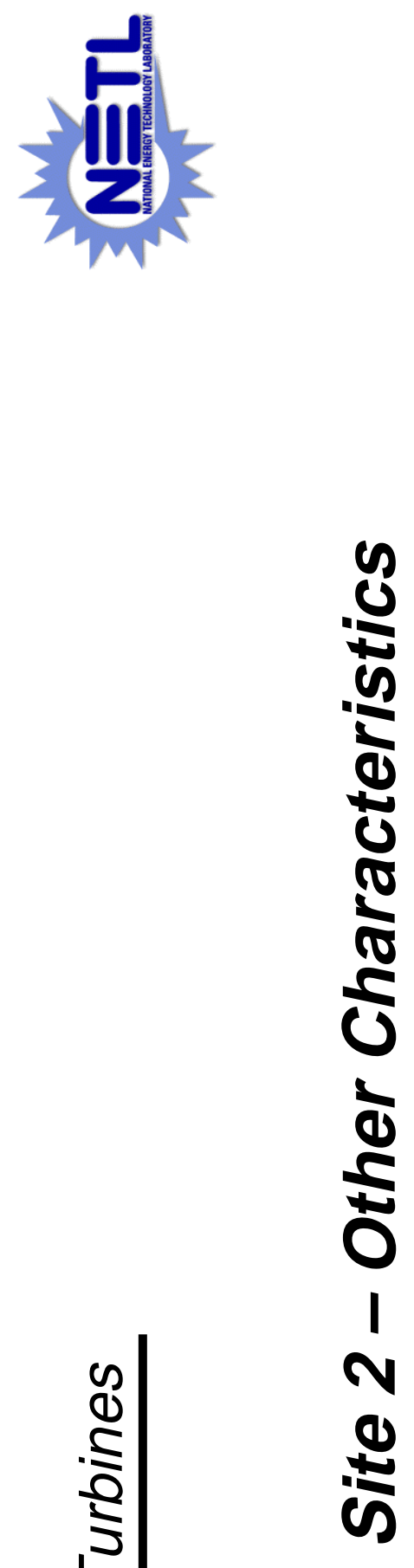

F

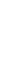

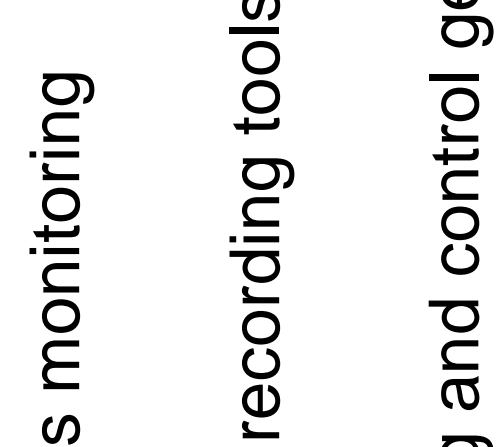

임

$\infty$

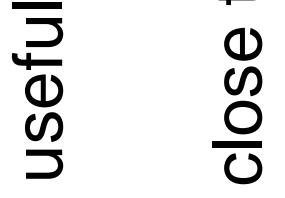

\)

$\frac{1}{0}$ ठ

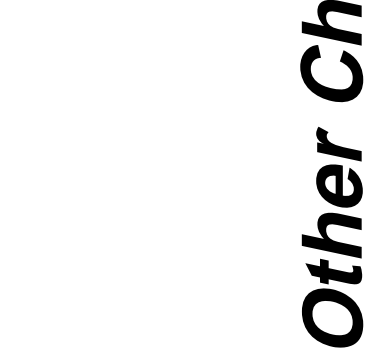

1

J

क

$\frac{.0}{\frac{D}{E}}$

$\frac{\Phi}{\frac{1}{0}}$

$\frac{\bar{c}}{\stackrel{0}{c}}$

$\frac{\frac{0}{2}}{\frac{0}{-0}}$

음

$\overline{0}$ 


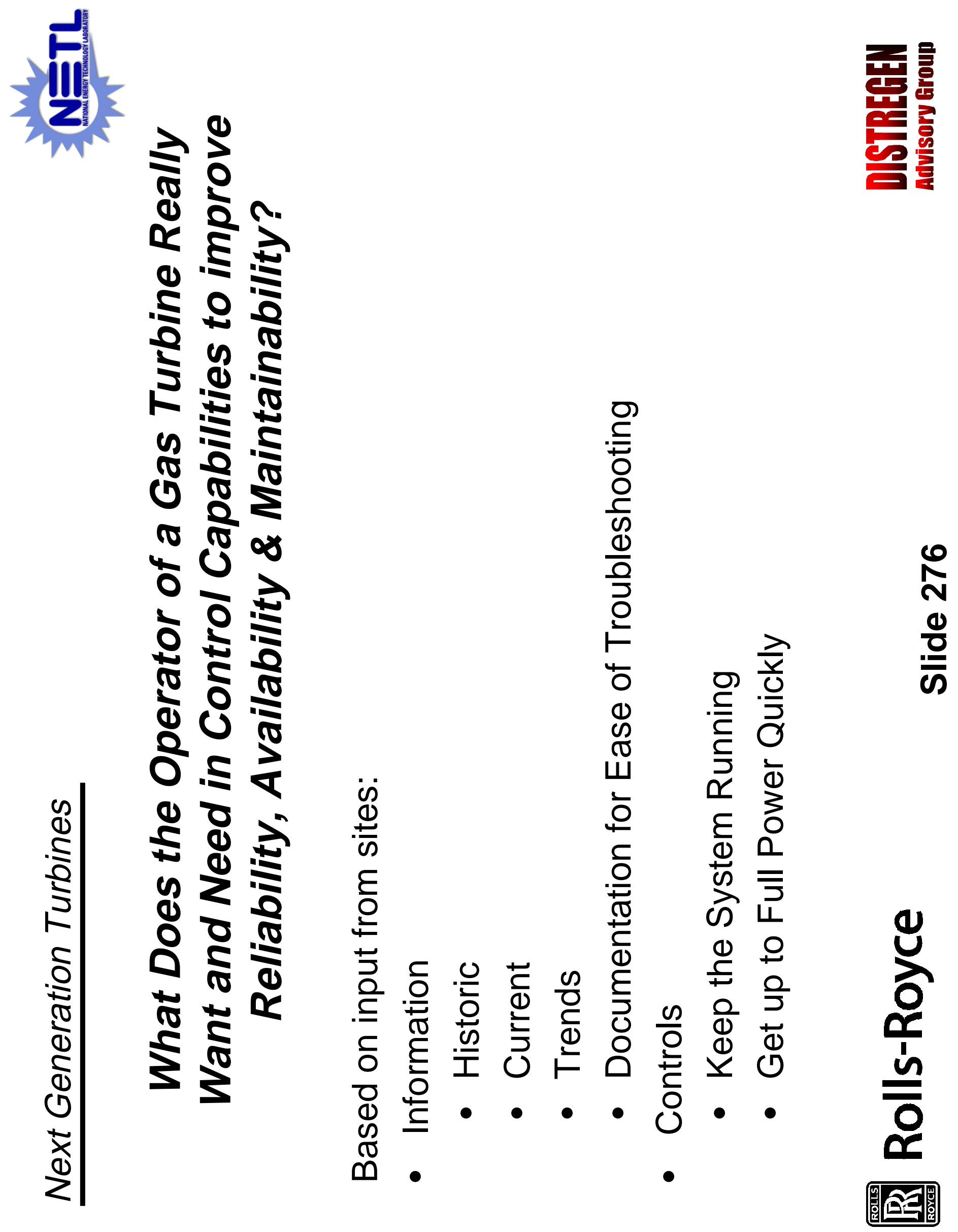




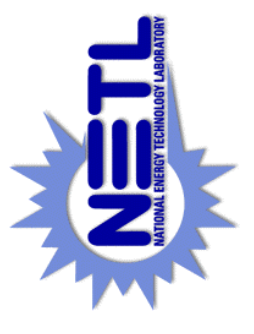

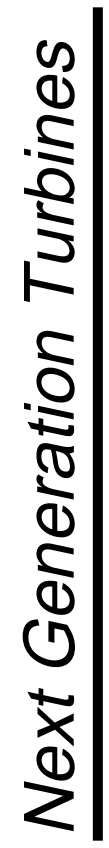
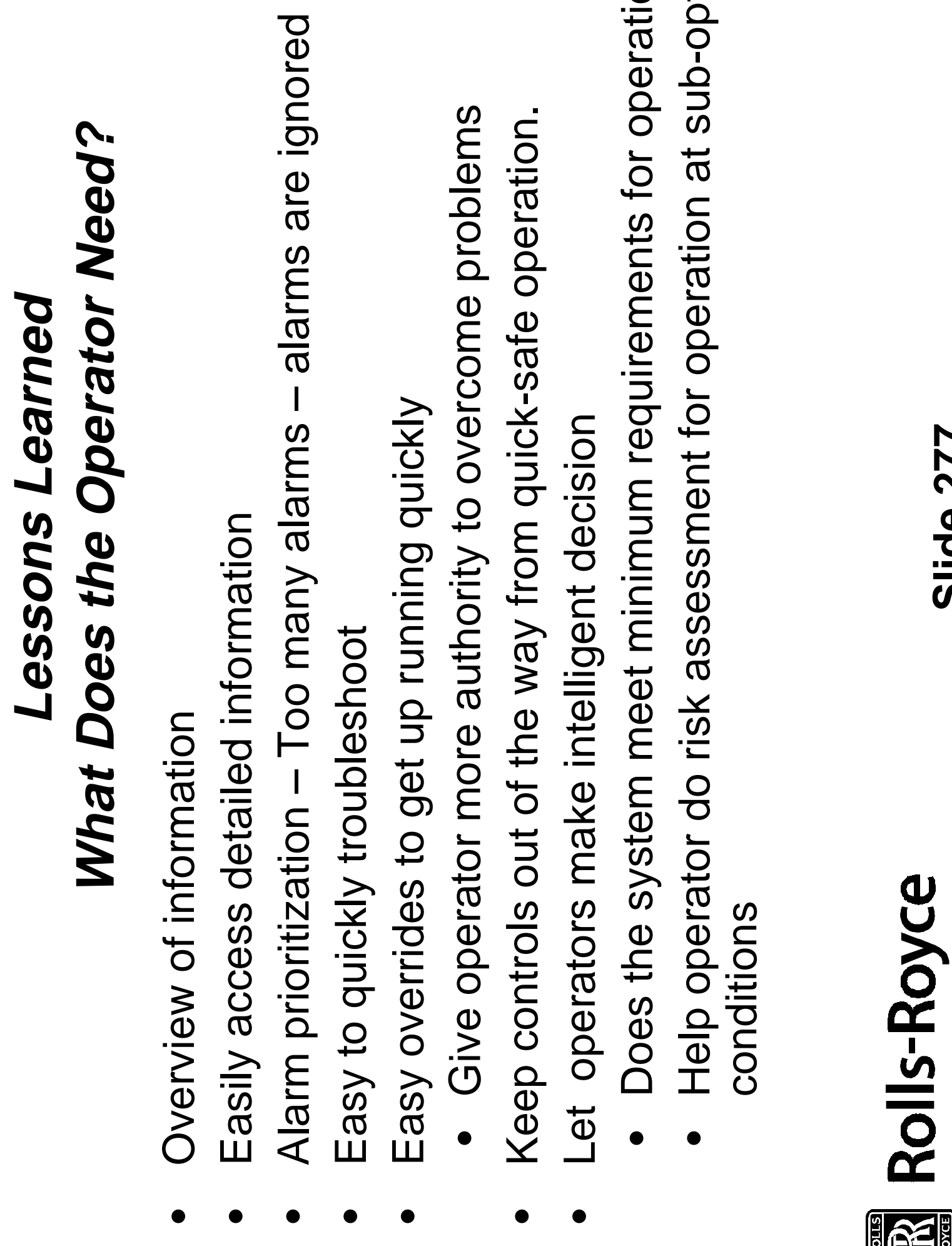


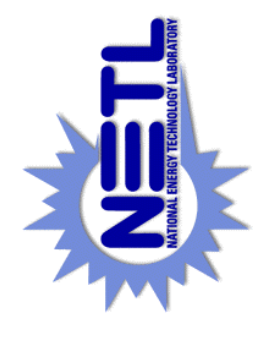

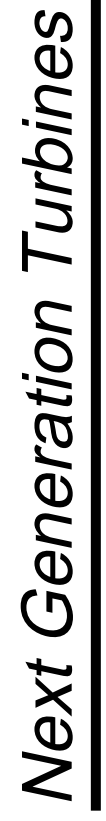

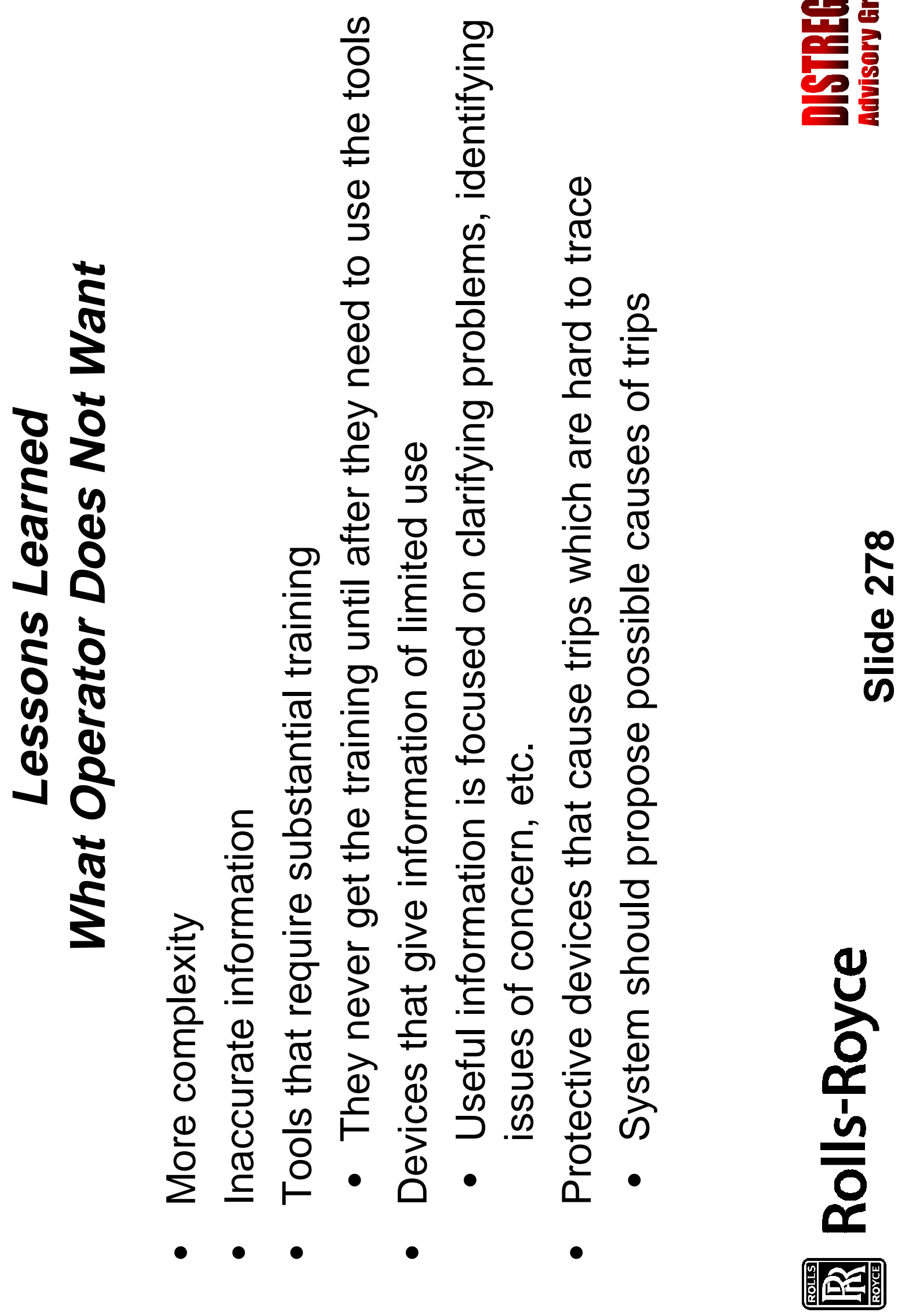




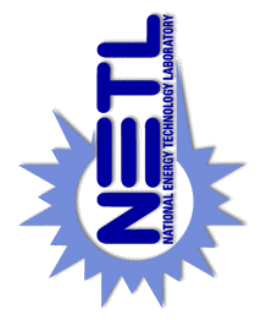

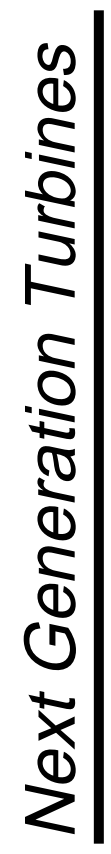

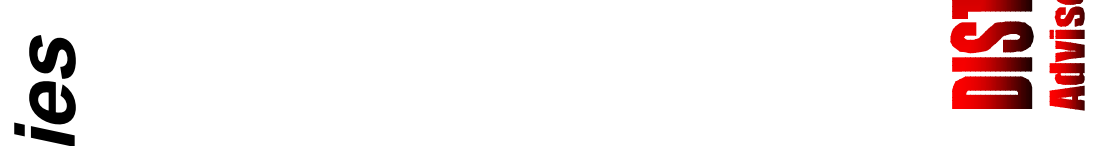

0

$\frac{0}{6}$

(1)

0

$+$

E

0

(

0

oy

N

1

0

(1)

(a)

ज

4

$E$

ज

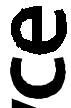

0

S

0

$\frac{0}{\circ}$

1

8

D

U

0

ar

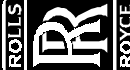




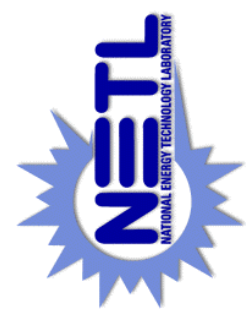

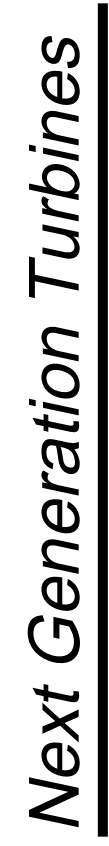

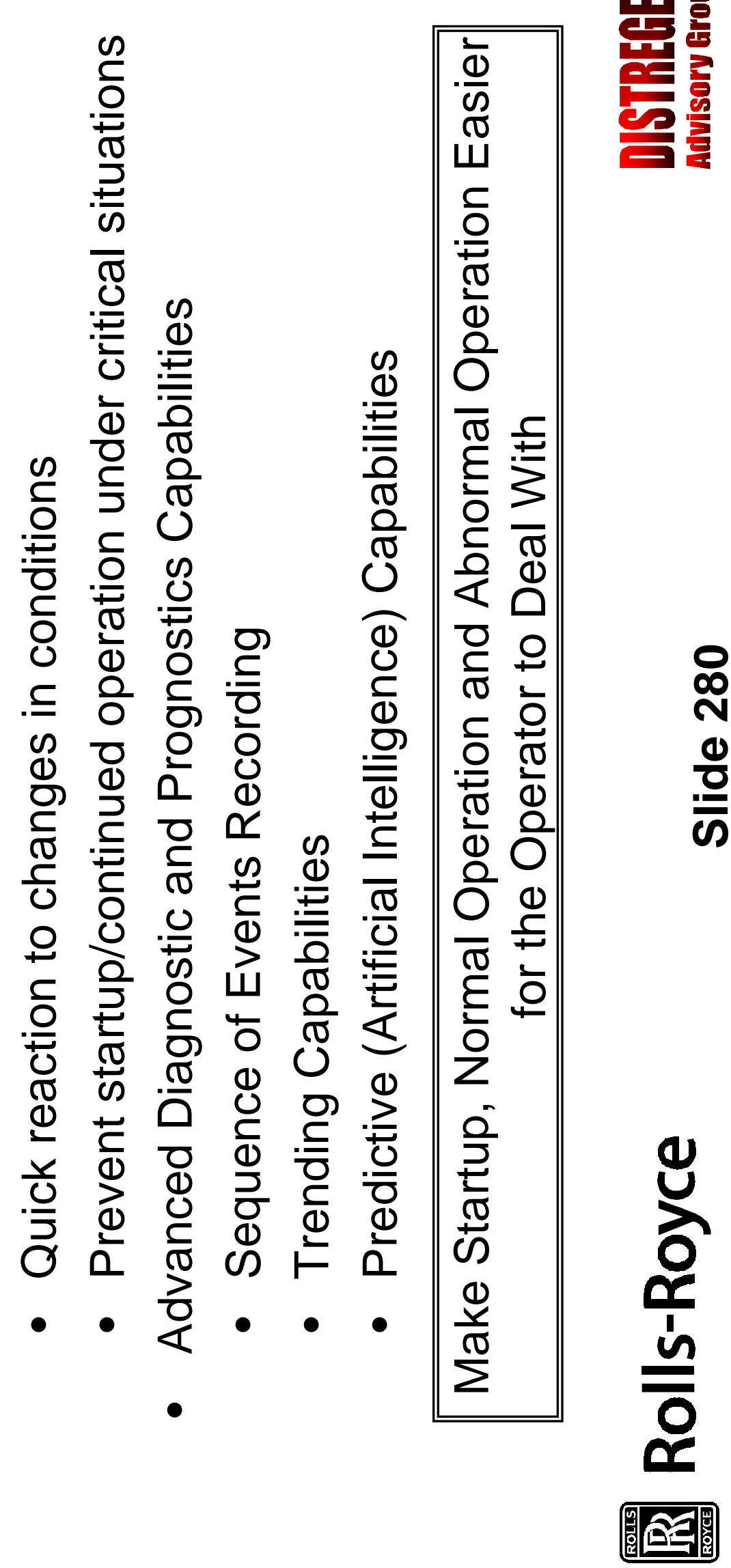




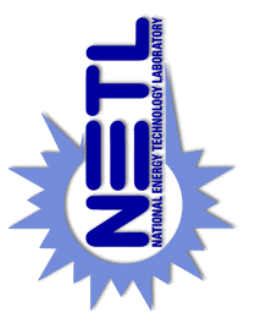

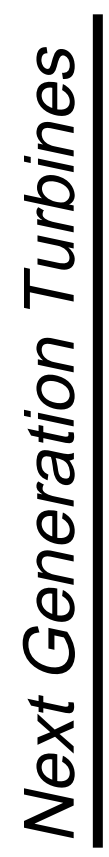

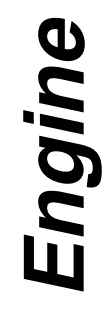

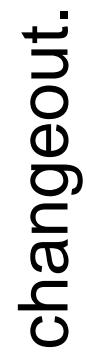

$$
\begin{aligned}
& \text { 흐 } \\
& \frac{\frac{\mathscr{E}}{\Delta}}{\frac{\sigma}{\sigma}} \\
& \pm 0 \\
& \text { ल) } \\
& \text { 응 } \infty \\
& \text { 으으 잉 } \\
& \text { 동 @ }
\end{aligned}
$$

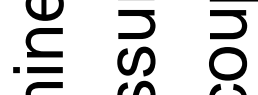

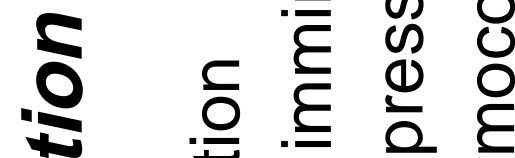

$$
\begin{aligned}
& \text { 을 } \infty \stackrel{\bar{d}}{\varrho} \\
& \text { జ } \\
& \text { Ф) }
\end{aligned}
$$

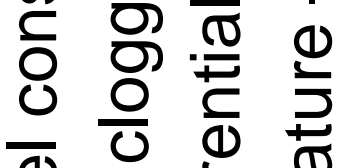

$$
\begin{aligned}
& \text { ब }
\end{aligned}
$$

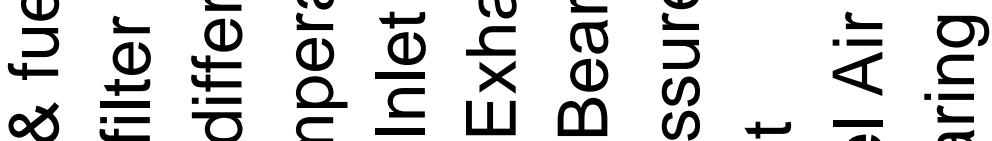

$$
\begin{aligned}
& \text { め }
\end{aligned}
$$

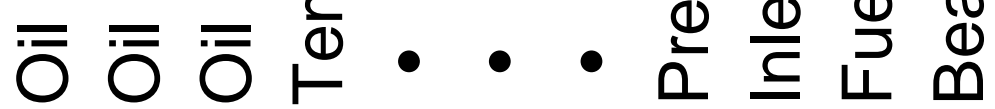

$$
\begin{aligned}
& \text { ป ธั } \\
& \text { ๘ } 3 \\
& \text { ๕ 등 } \\
& \text { 으응 }
\end{aligned}
$$$$
\text { 을 트 }
$$$$
\theta
$$$$
2
$$

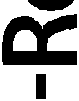$$
\text { U }
$$$$
0
$$$$
\text { a }
$$ 

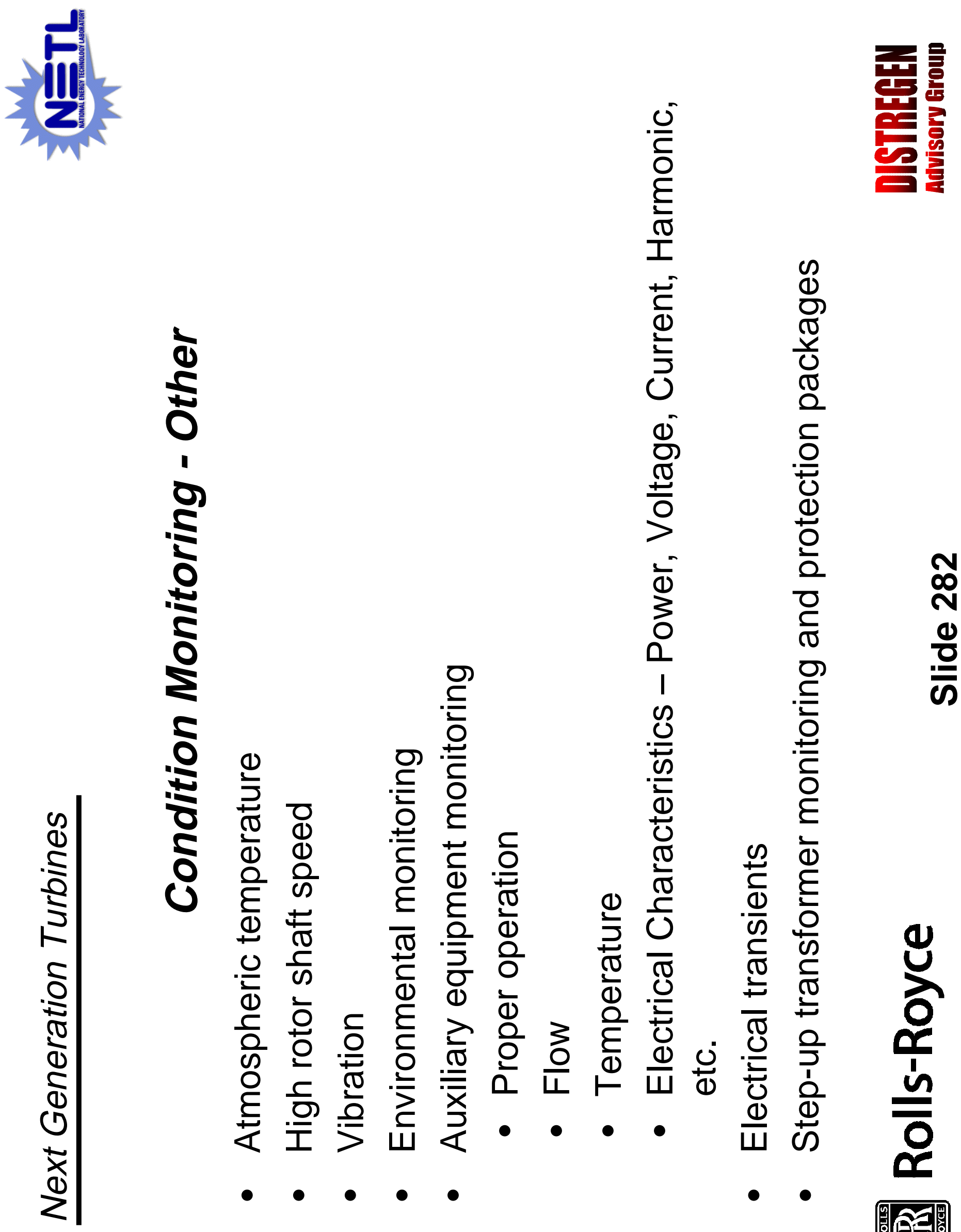


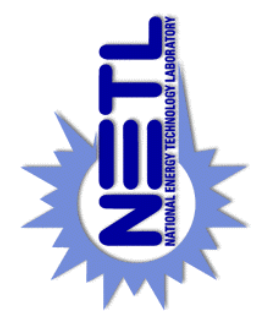

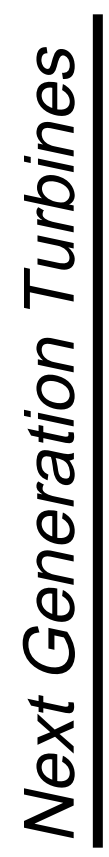
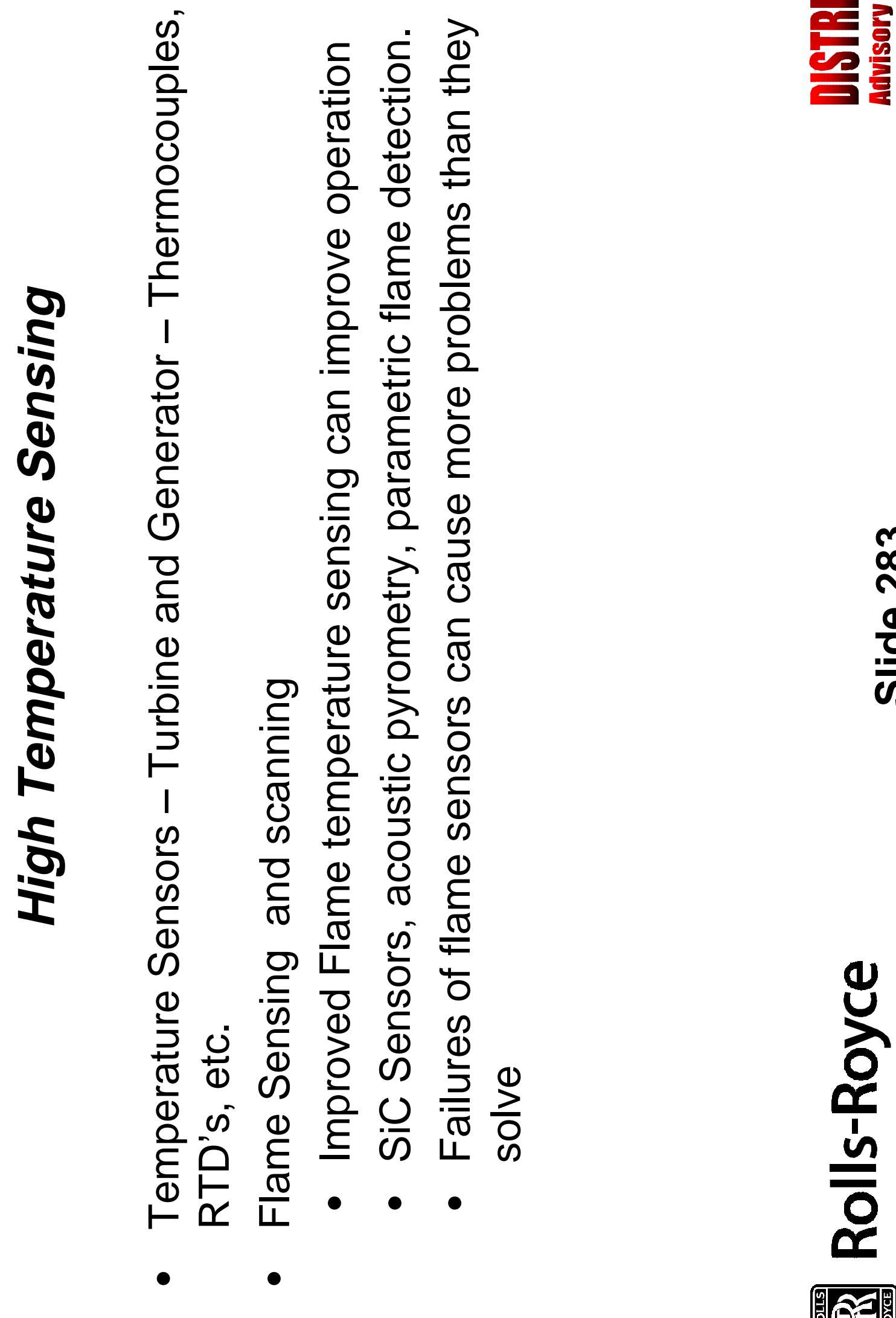


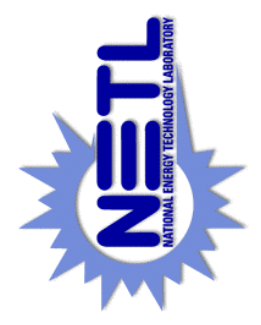

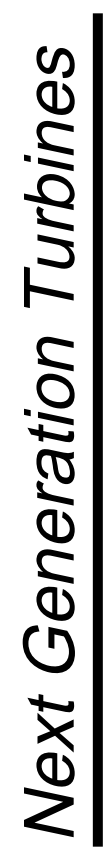

$\frac{\substack{0 \\ \stackrel{N}{N}}}{\frac{0}{\sigma}}$

음

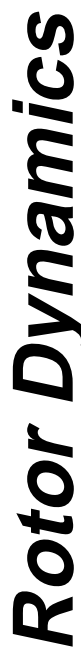

흑

음

它

N

(1)

$\frac{0}{0}$

간

()

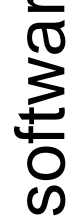

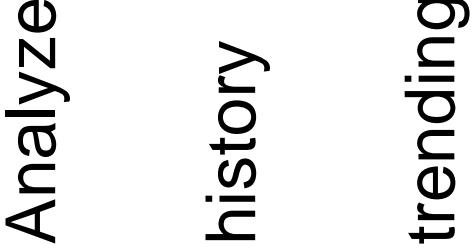

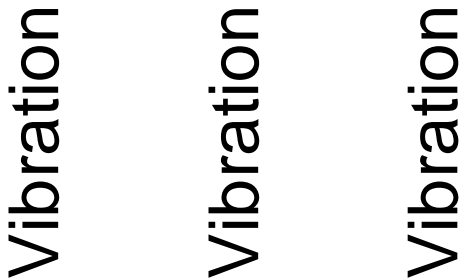

U

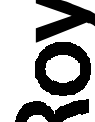

r

$n$

0

人)

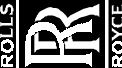




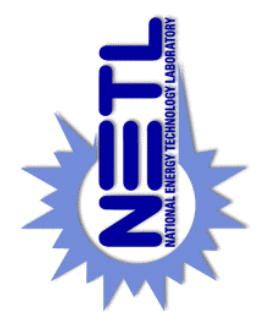

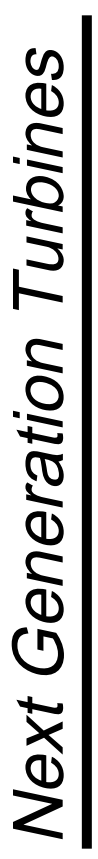
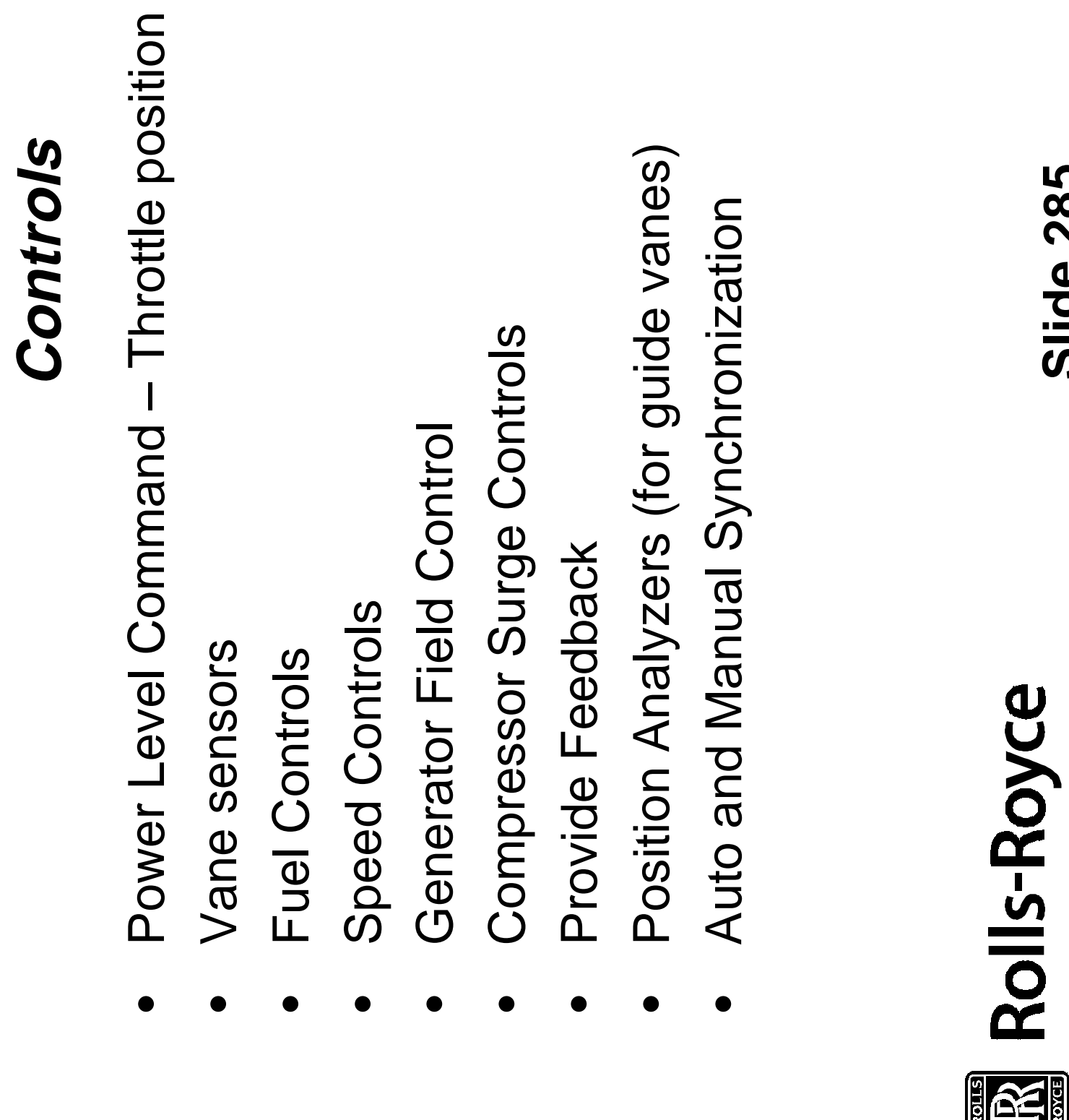

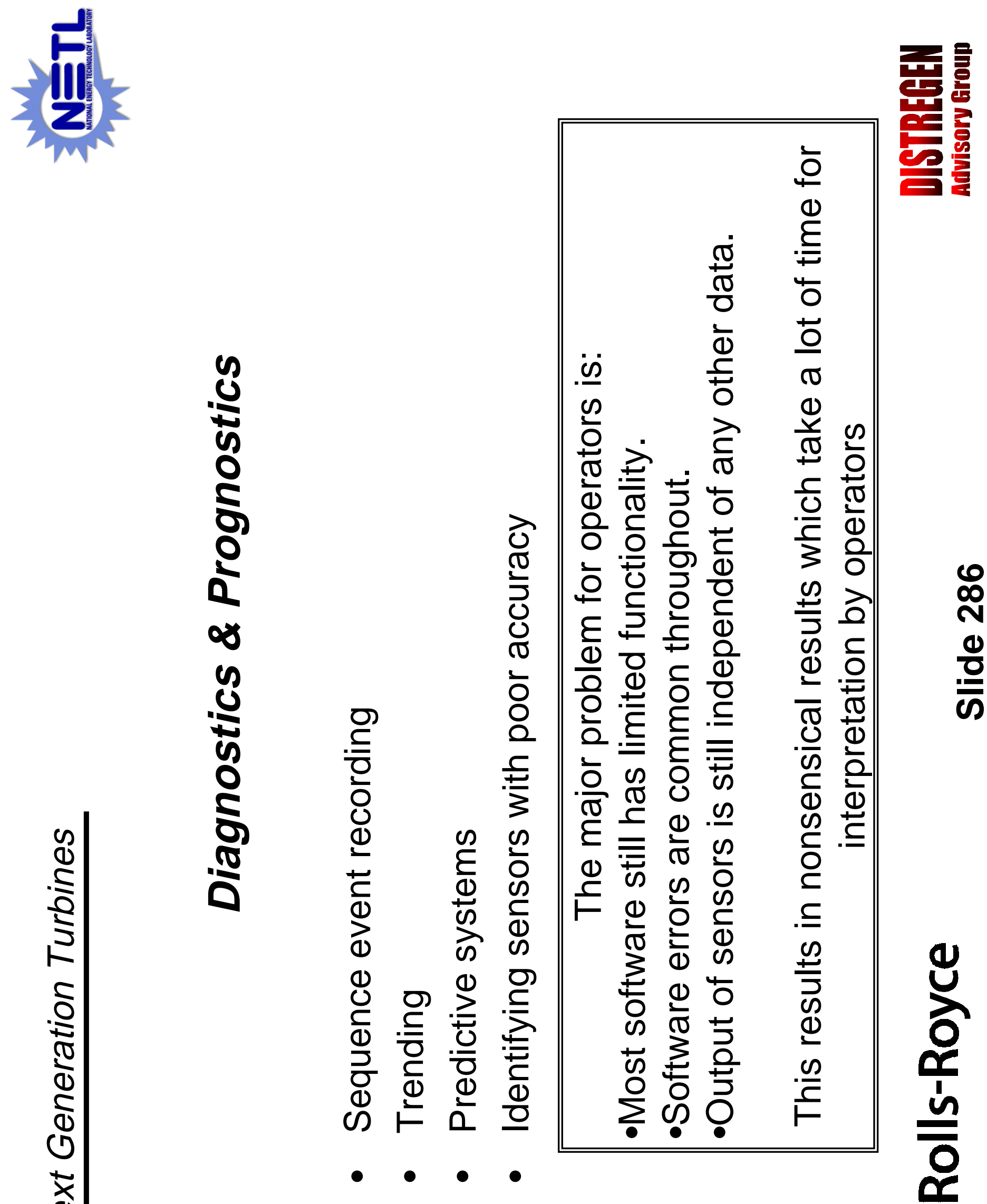

₹ 


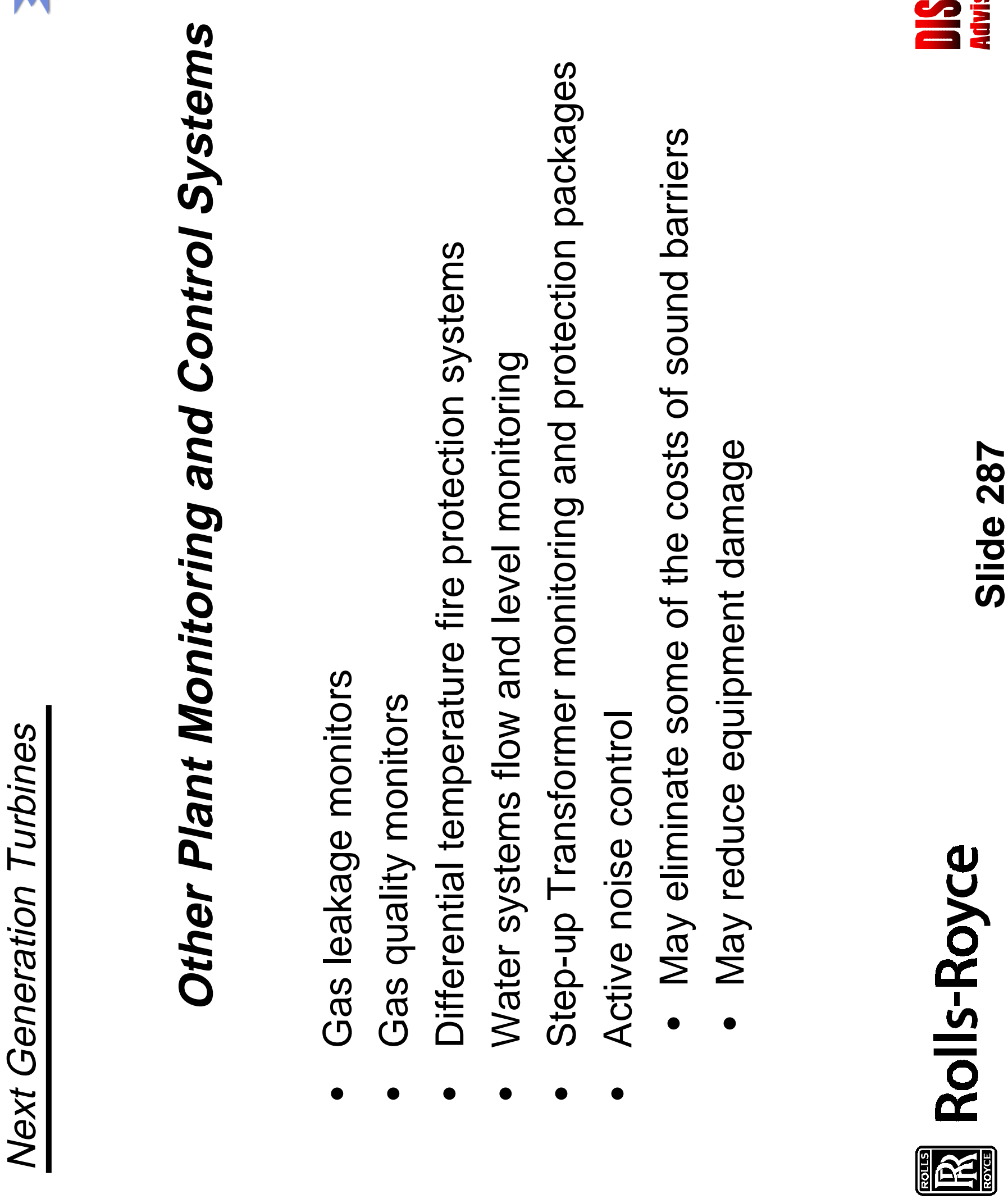




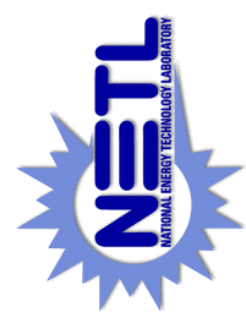

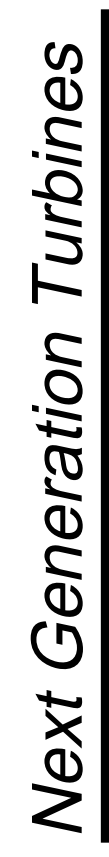

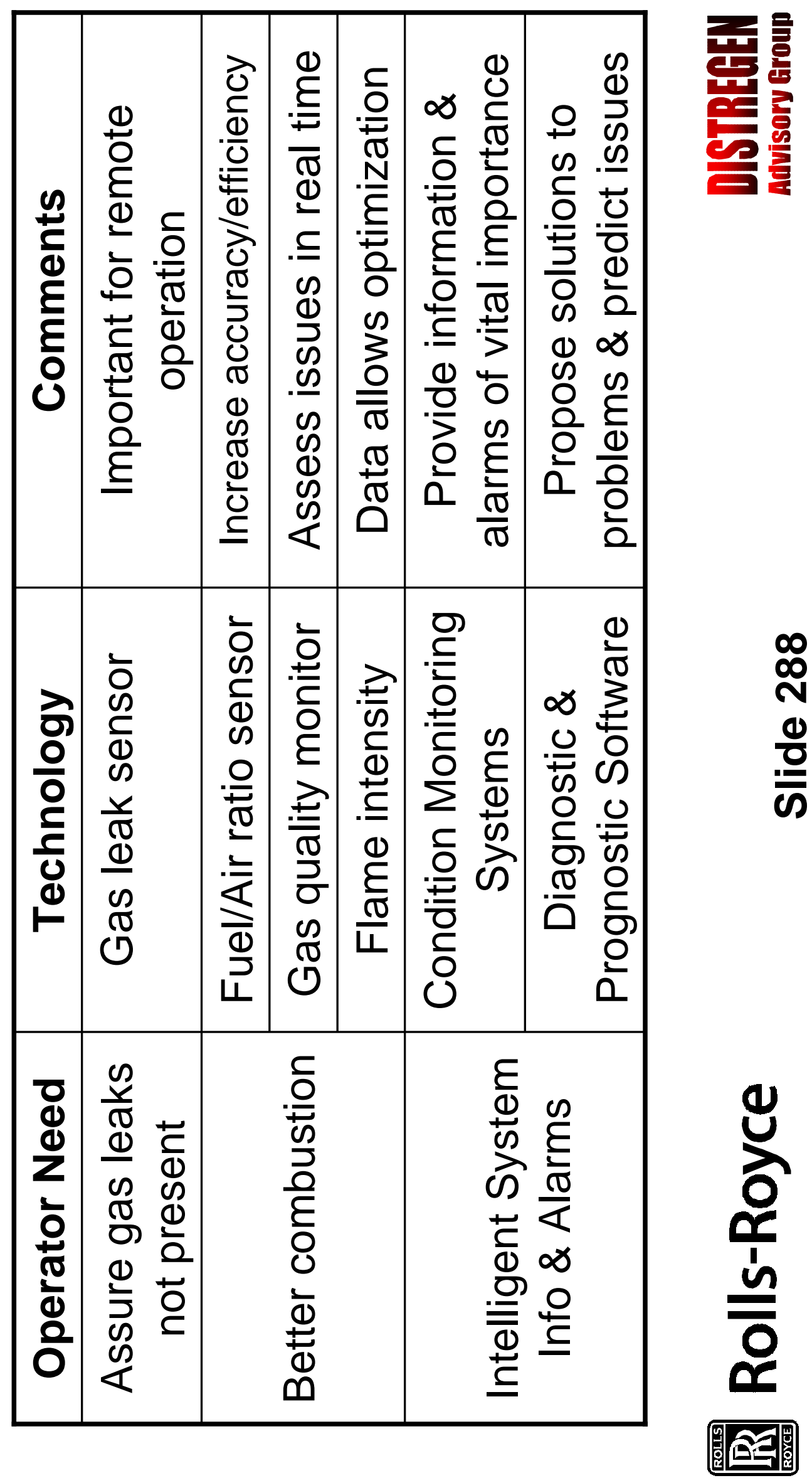




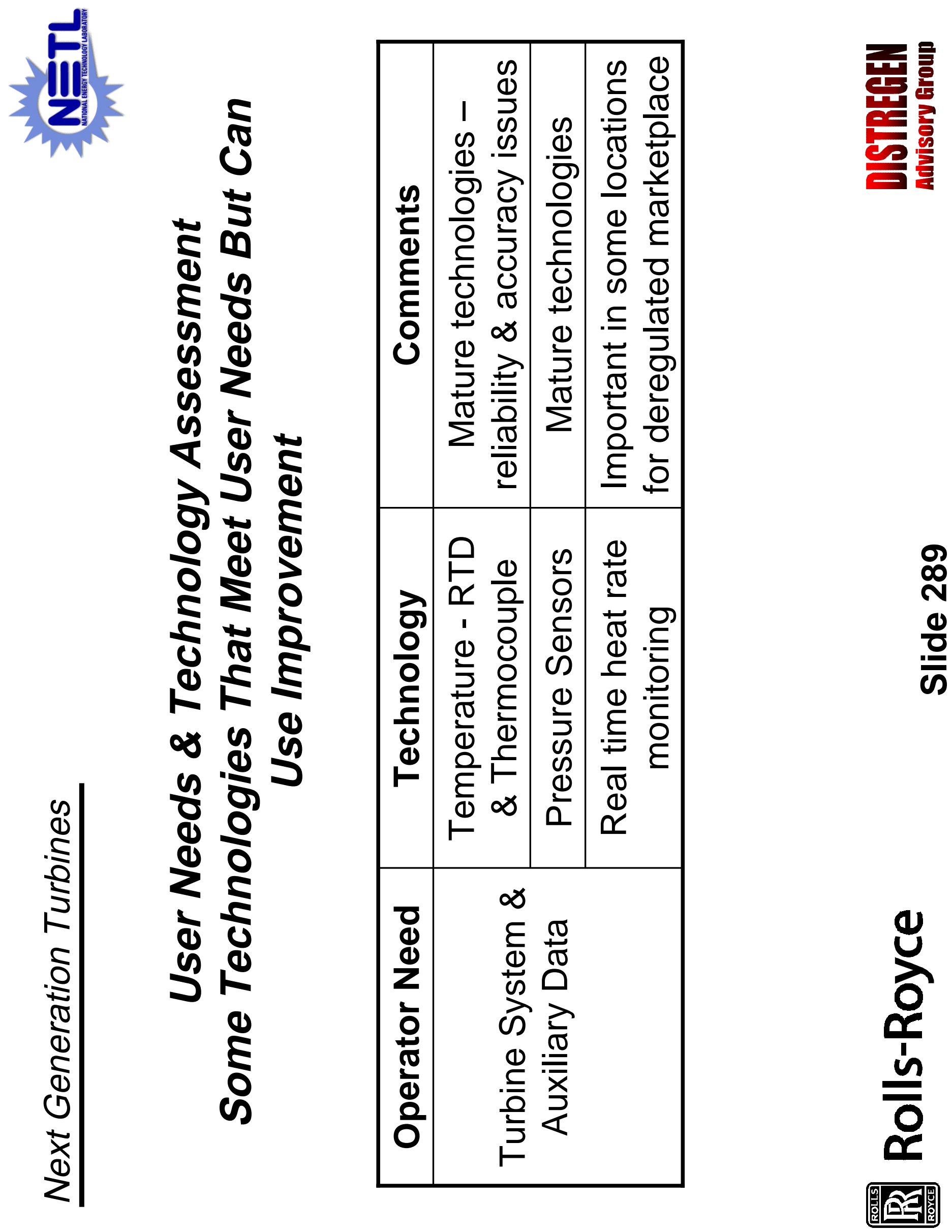



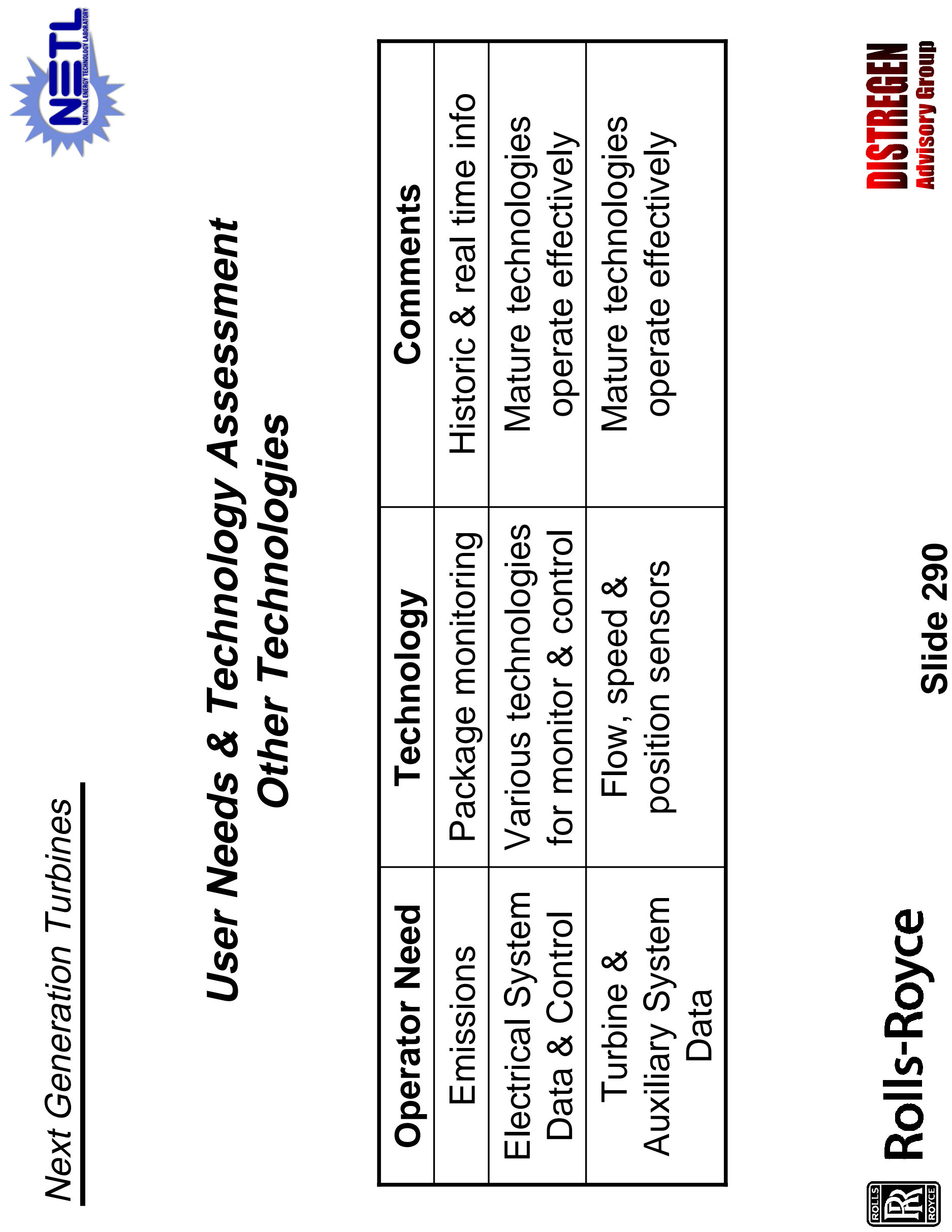


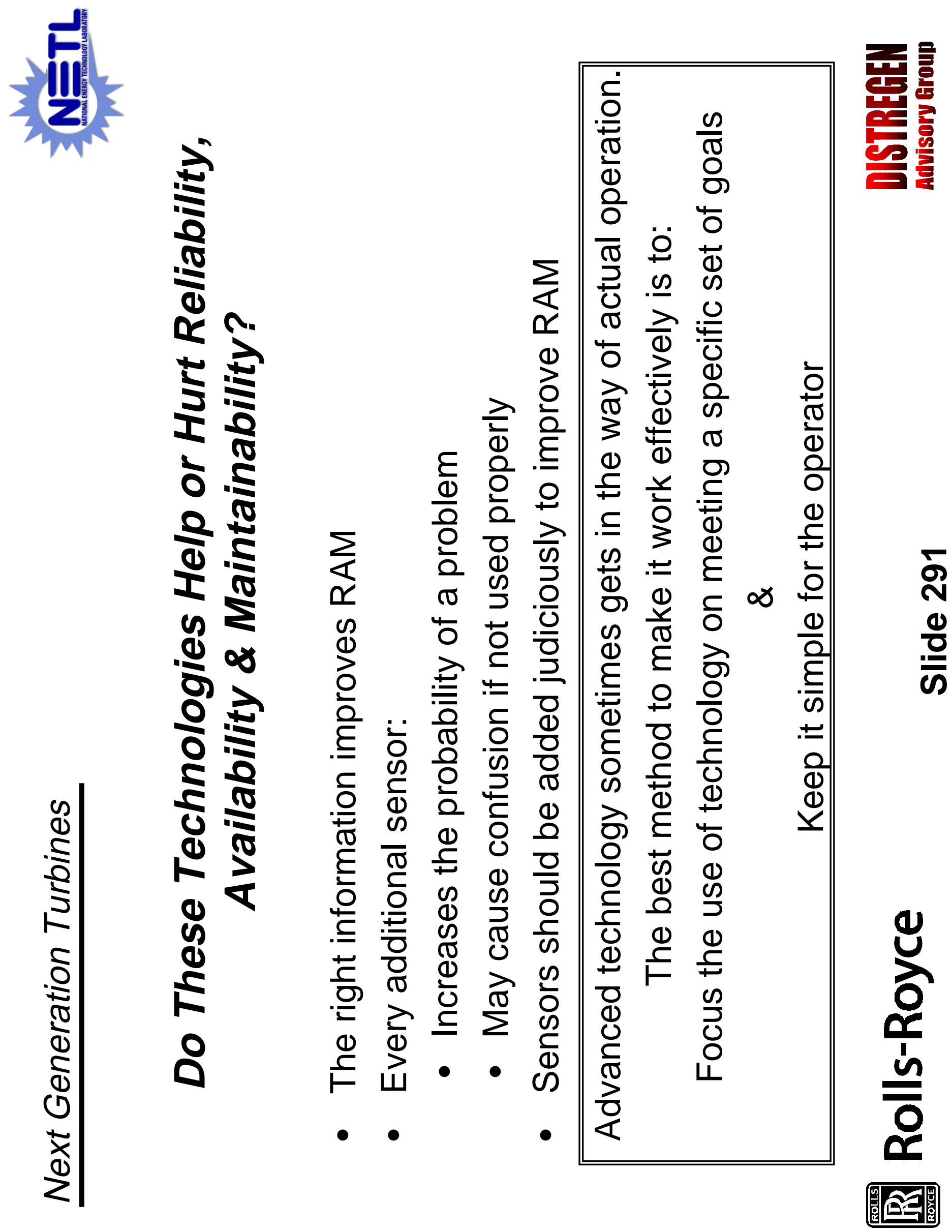




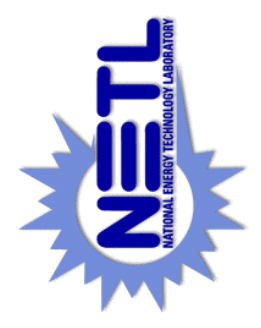

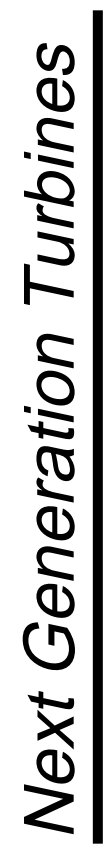

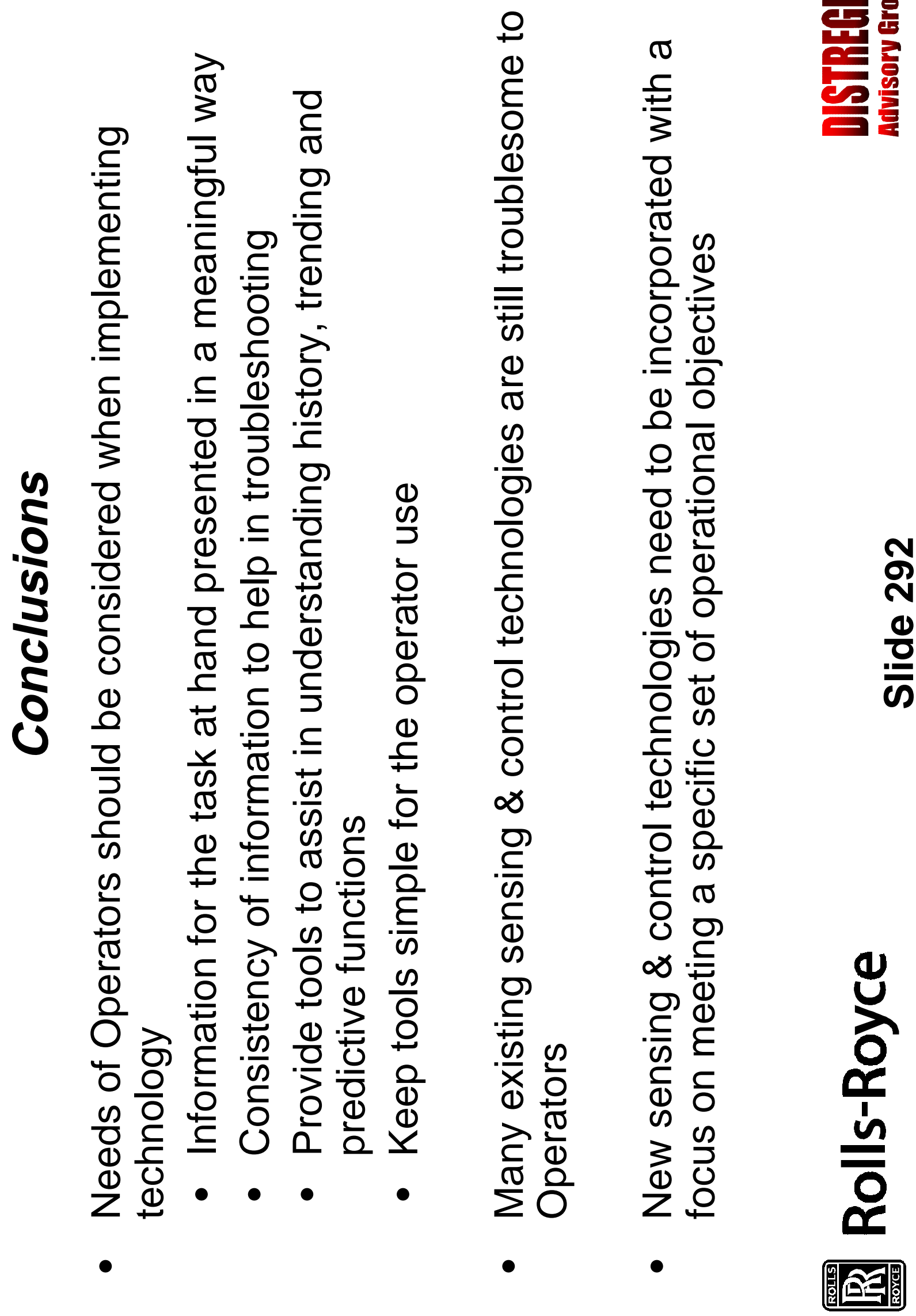




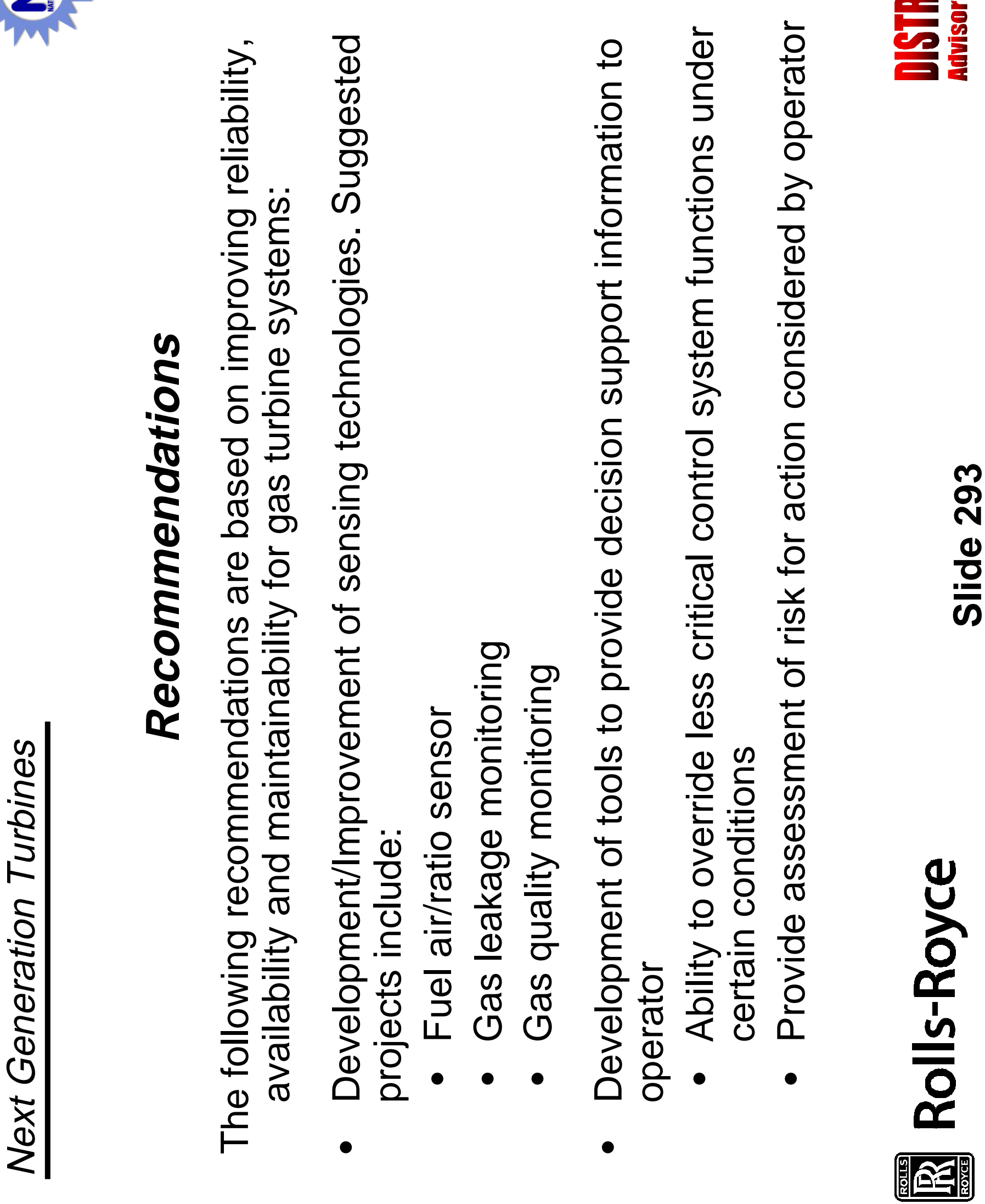




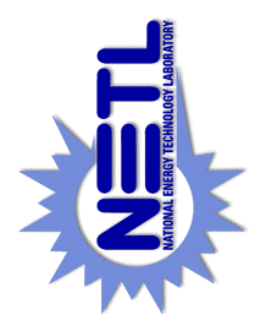

क) है
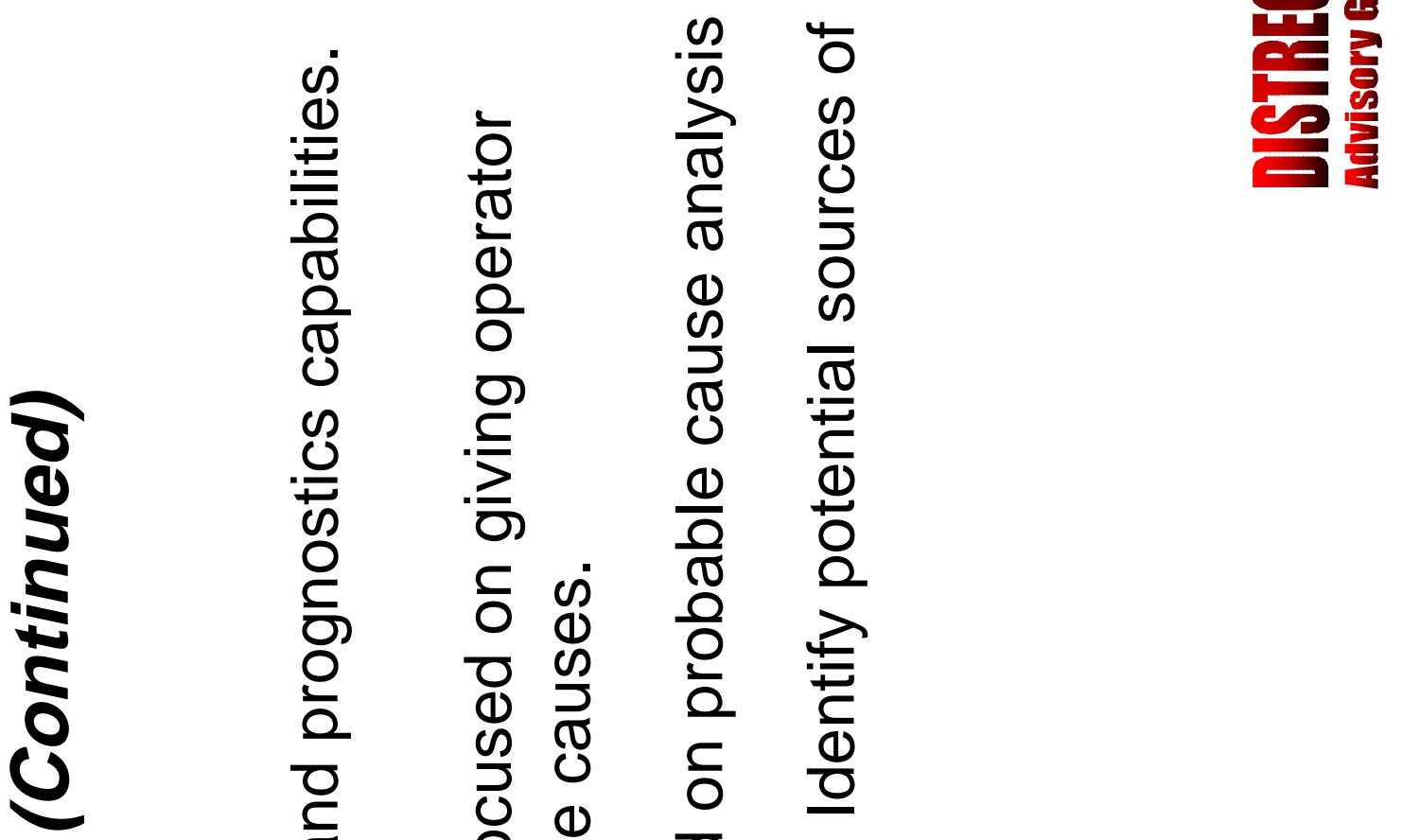

$\frac{1}{8}$

(1)

ธั

융

O O O

产 高 $\frac{0}{\frac{1}{0}}$

ᄃ

잉 월

응 \)

드응 잉 응

ज क त क क है क

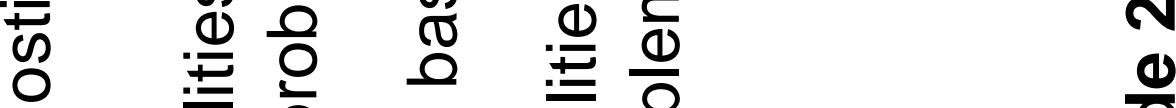

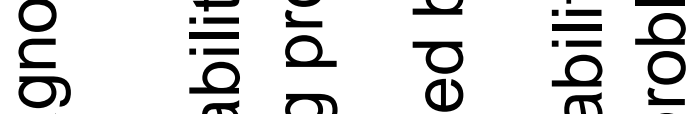

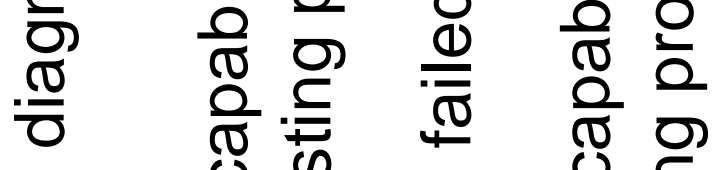

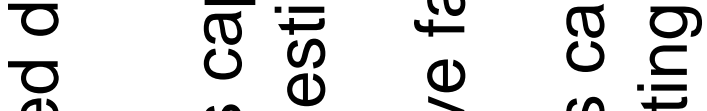

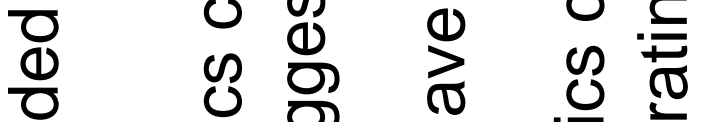

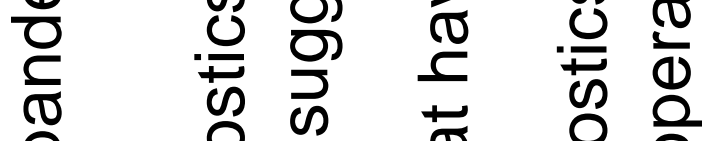

응 ๘

ब ర্ত

प이

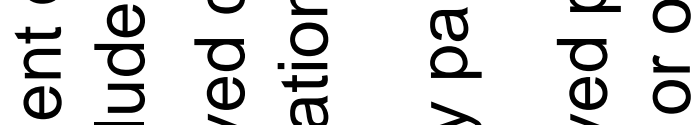

ฮ

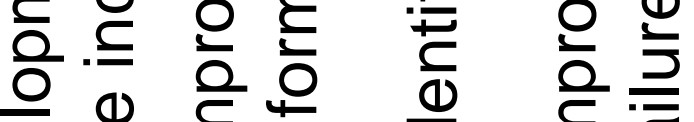

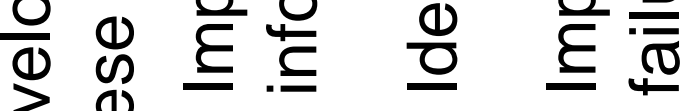

$\sigma$

ญ $\stackrel{\oplus}{上}$ • •

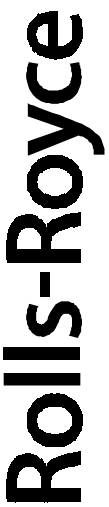




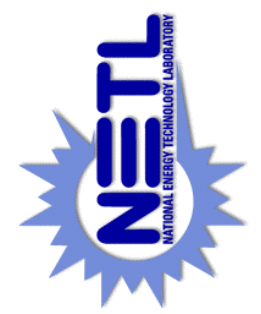

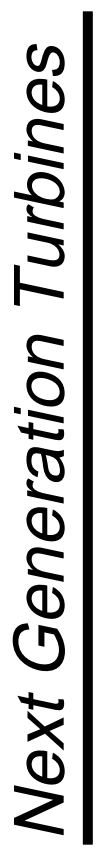
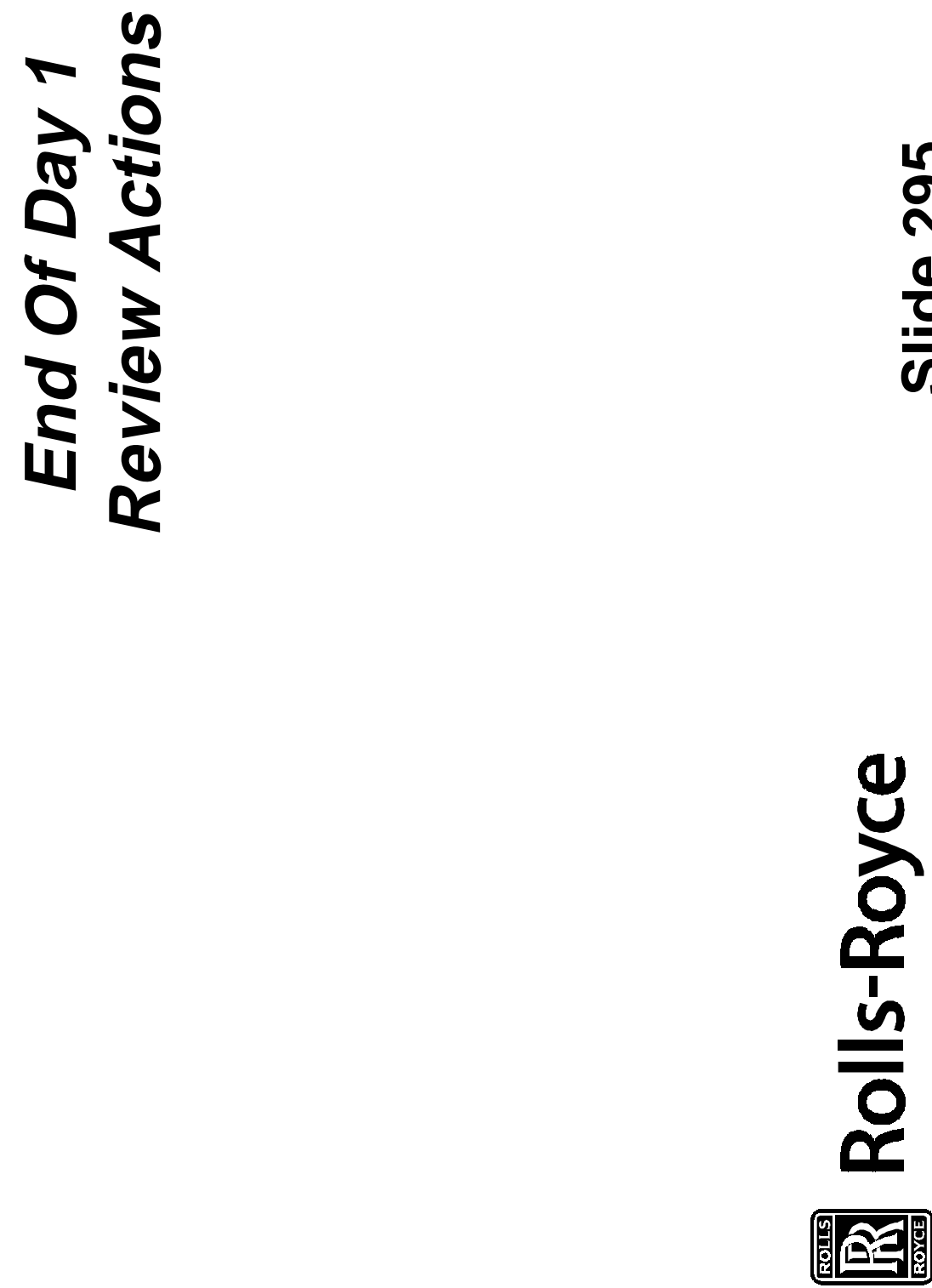

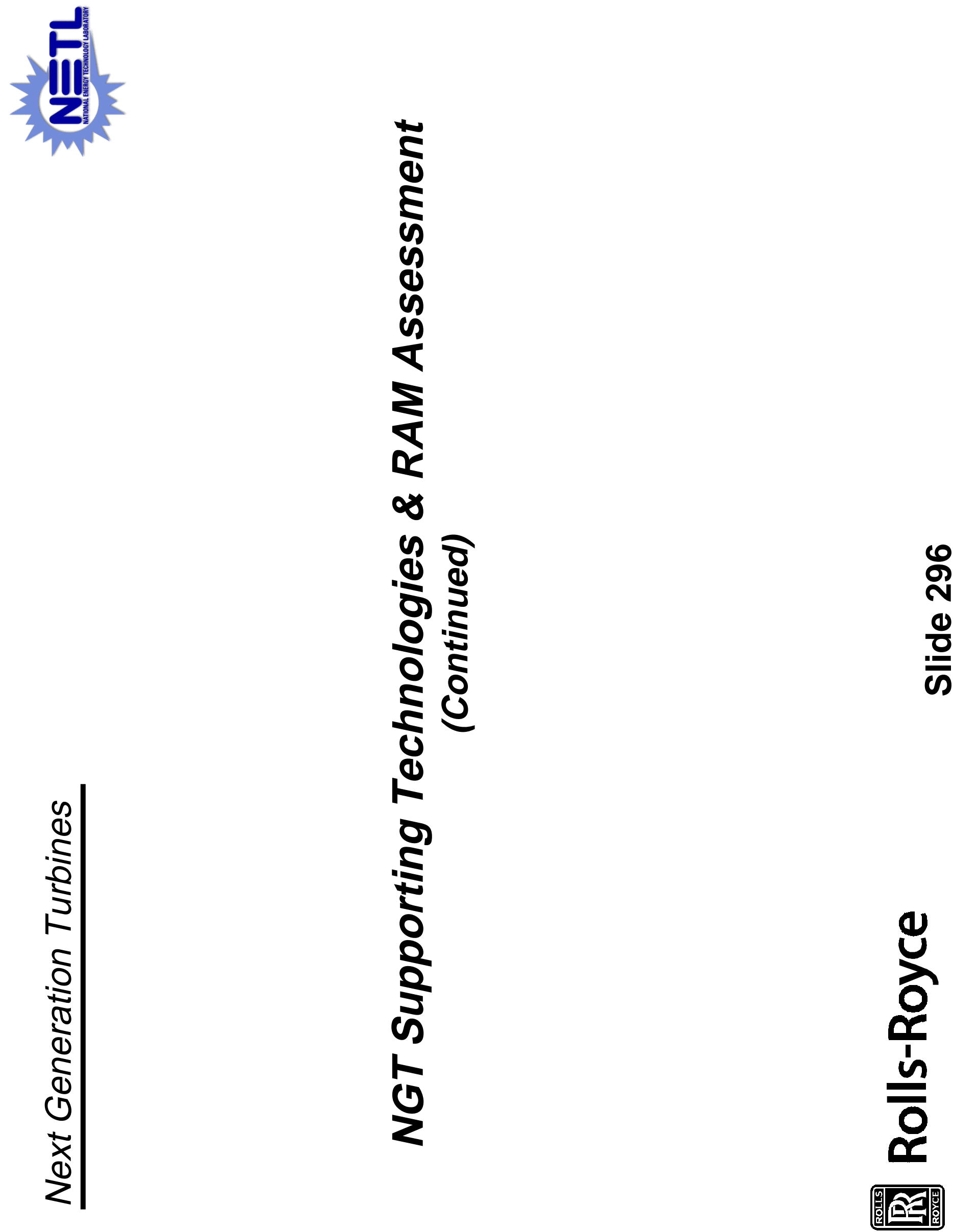

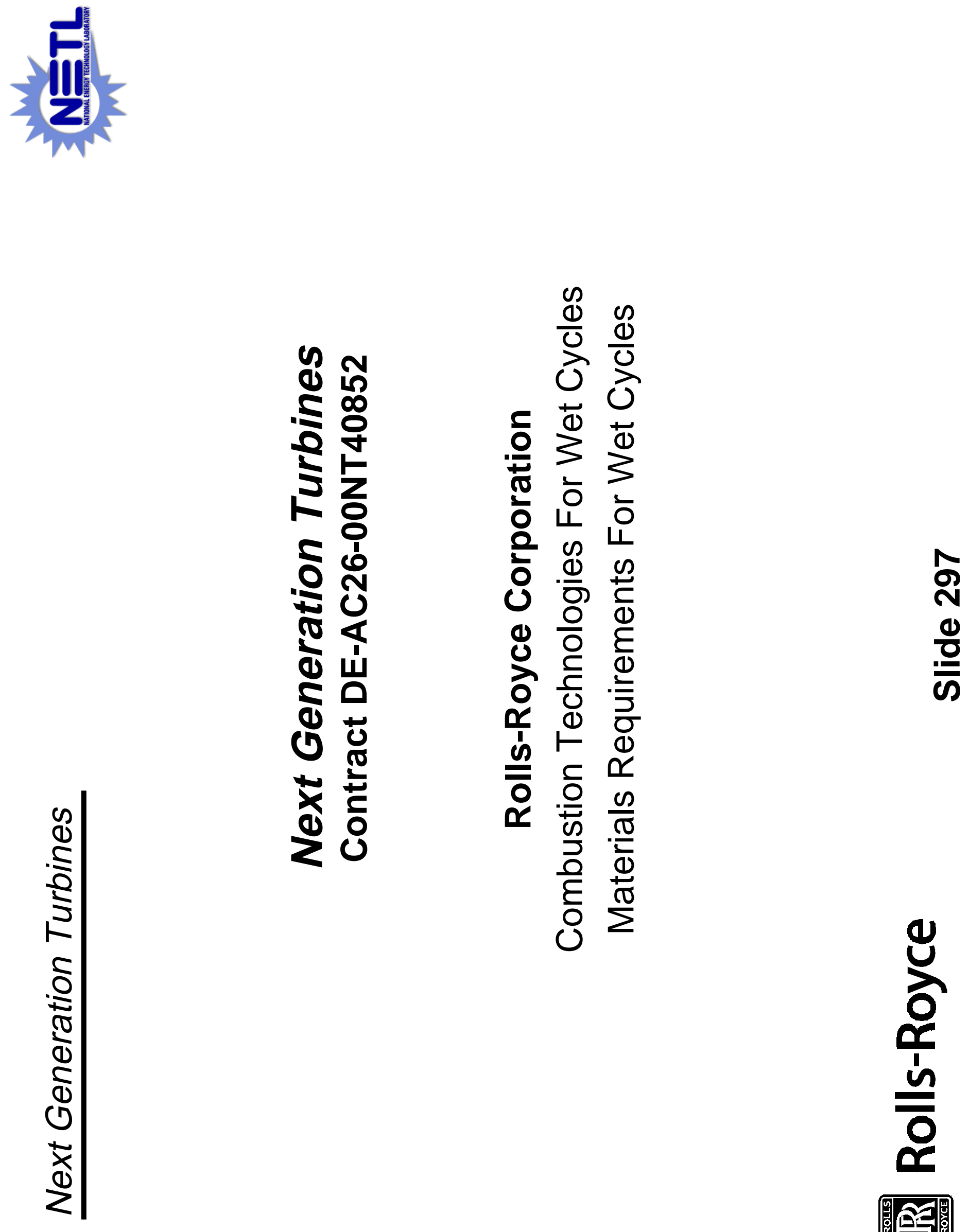


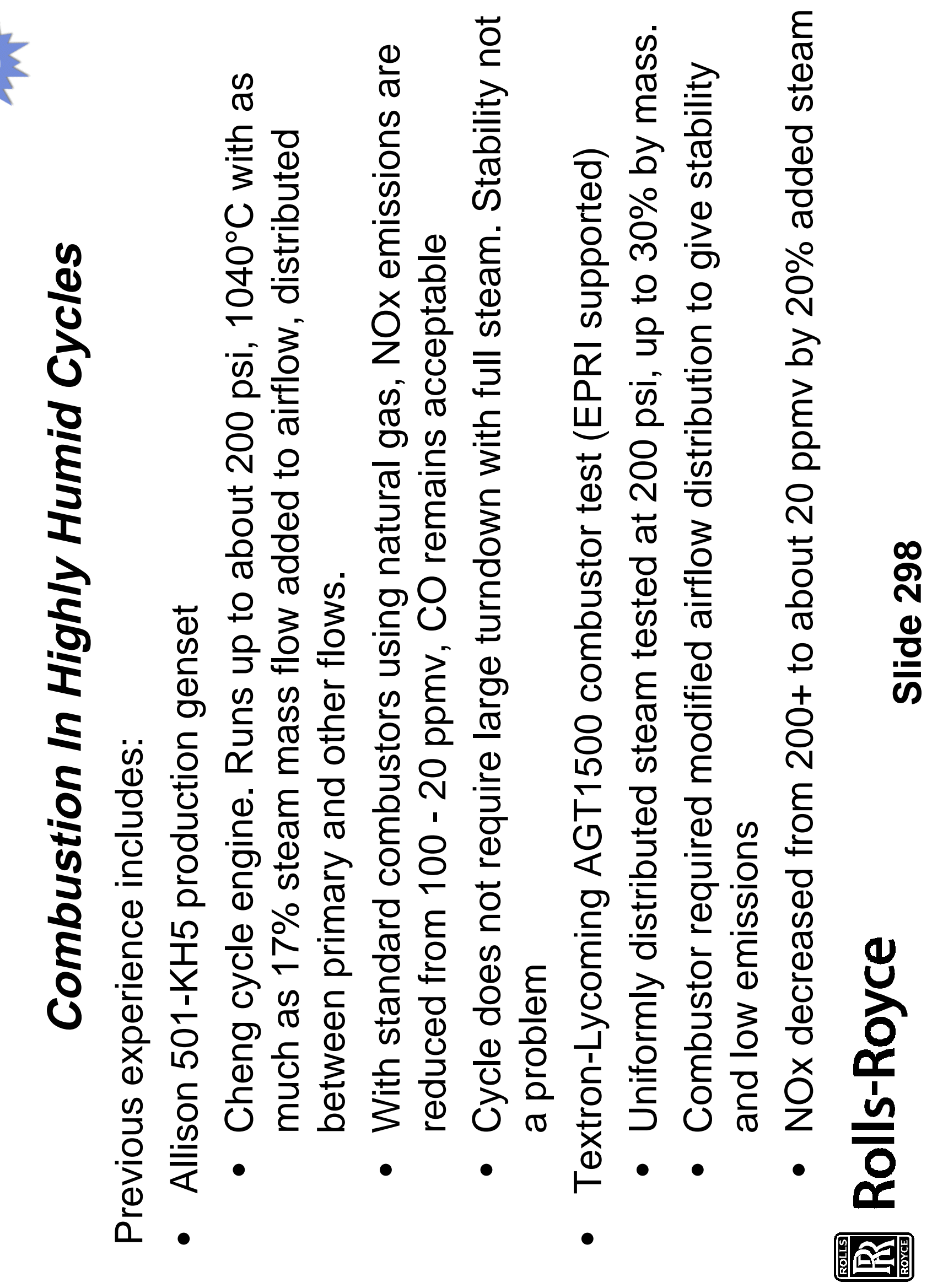




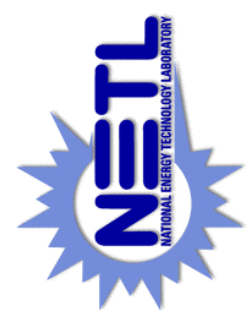

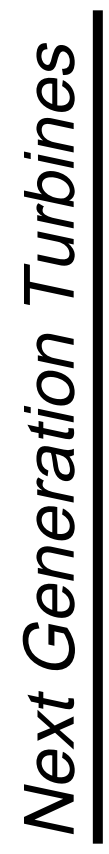

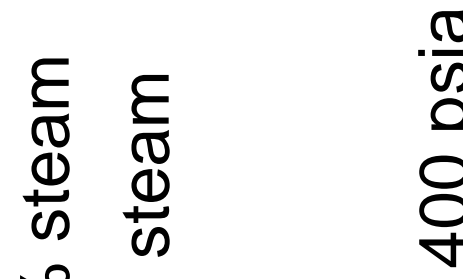

$\frac{9}{0}$

@

$\stackrel{\circ}{\circ}$ 응 응

?.

.

듬

욛

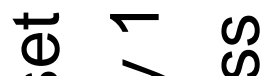

क

ब

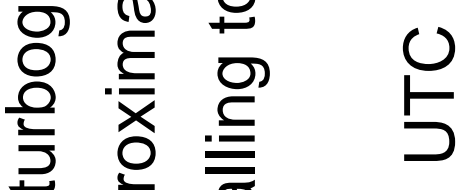

8

으

잉

○े

స

은

윽

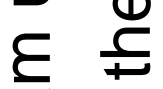

$\frac{\mathbb{\Phi}}{\oplus}$

$\frac{2}{5}$

\%

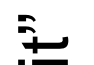

$\omega \geqslant$

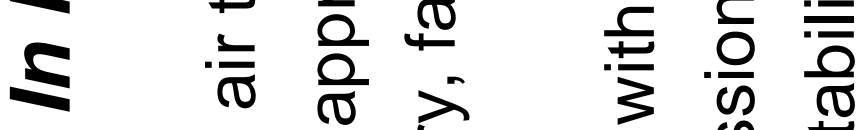
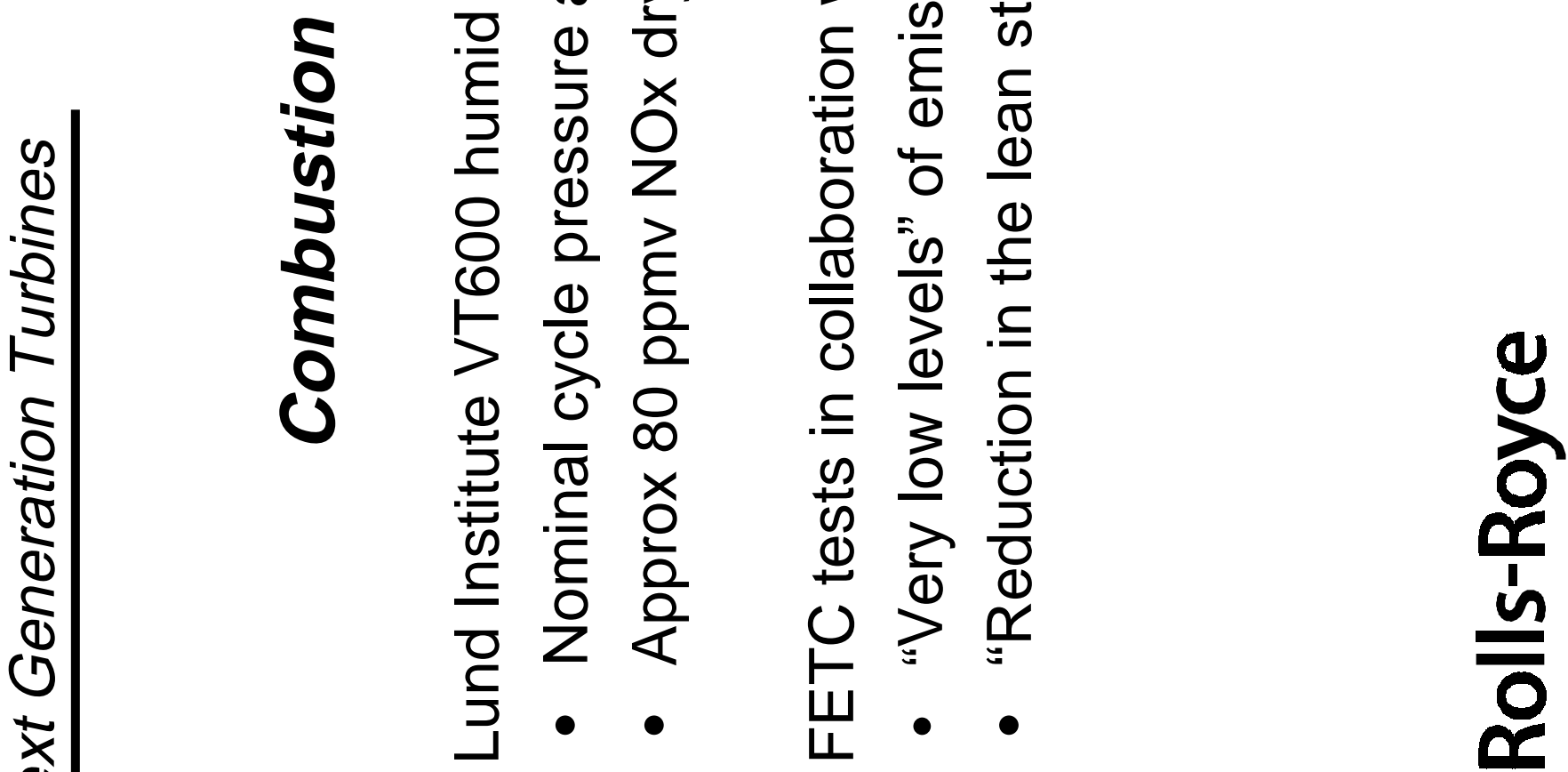


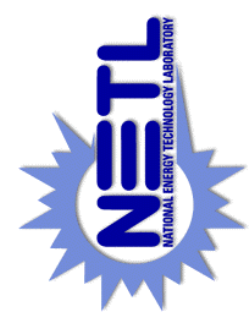

क

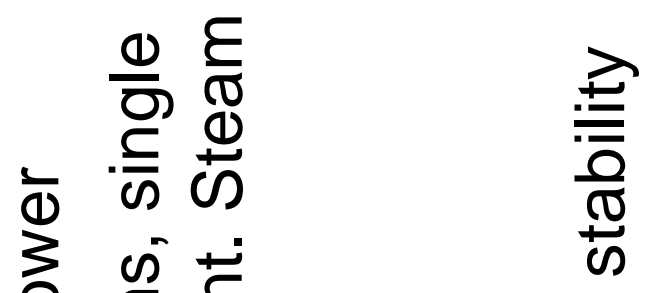

$\frac{1}{6}$

으 है ฮ

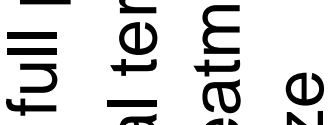

๘

즈도

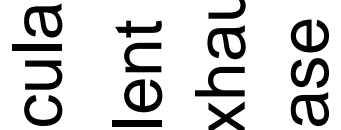

플

สิ

E

(2)

$E$

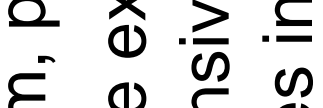

E $₫$ 의

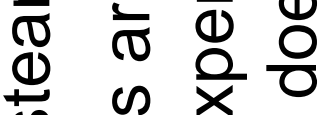

๘

ণ্

이 을 을

웡 ত্য

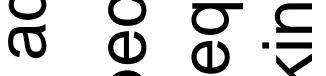

은 ญ

\# $\frac{\omega}{\square}$ ल

떵 을

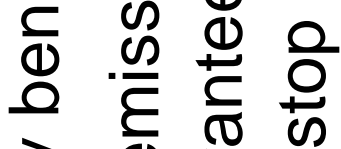

듬

誉

$\frac{\omega}{\frac{0}{0}}$

$\frac{\varepsilon}{\emptyset}$

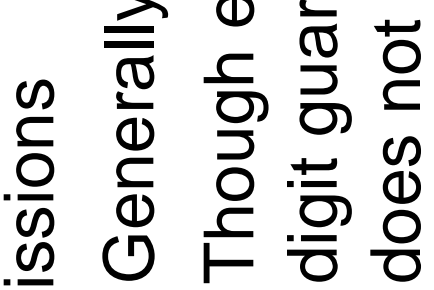

(1)

는

프

(1)

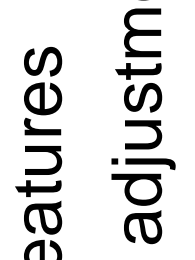

40

(1)

ฮั ฮ

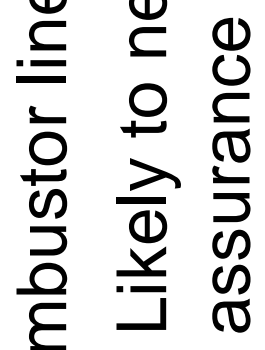

$\frac{0}{0}$

U
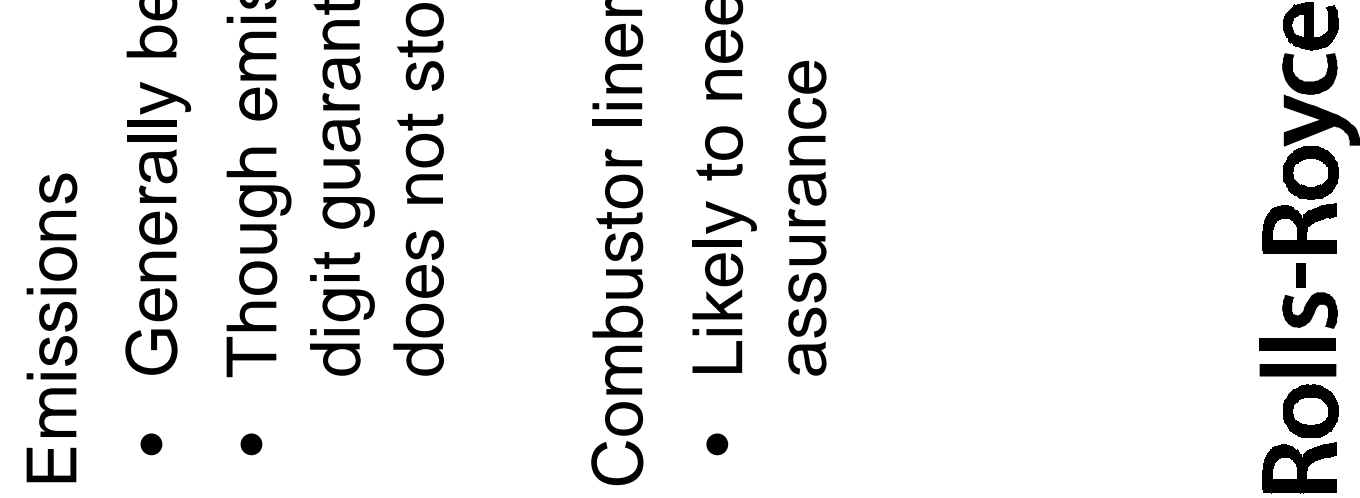


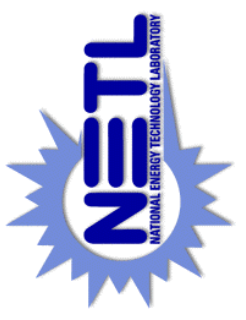

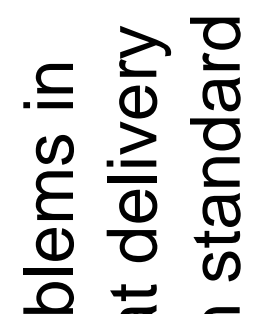

응 흐느

등 힝

음 으 용

을 흥

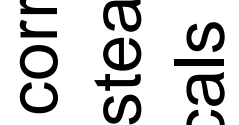

은

仓 ल

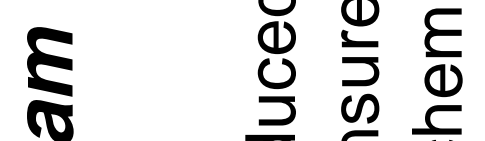

ब

क

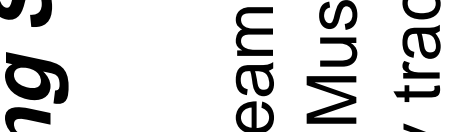

ह $\frac{\Phi}{\omega}$ o

\%

8 ¿

का पे

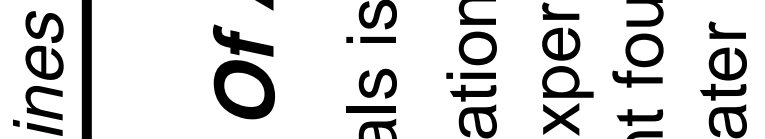

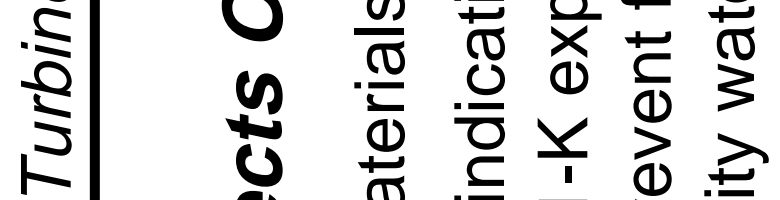

$r$

ธ

(1) $\widetilde{0}$

茂

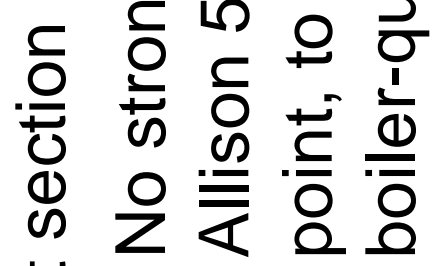

음

ฮั

क्ष

त 흐 ते

$\stackrel{\pi}{=} \sum^{\pi}$

$\pm \Phi$

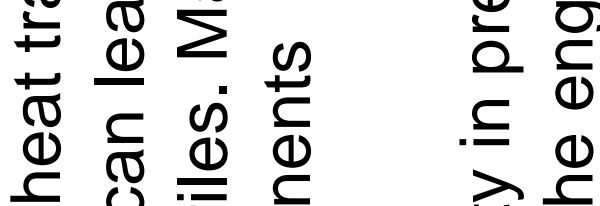

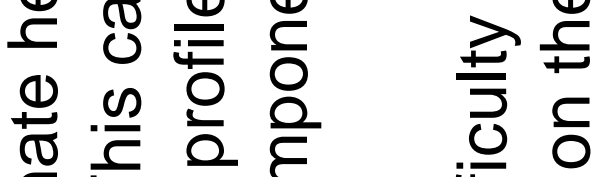

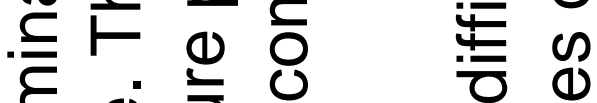

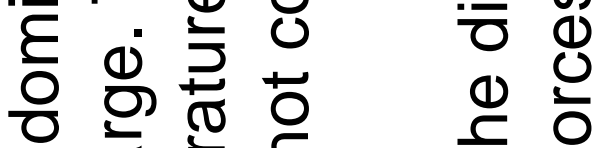

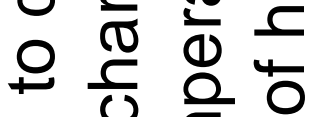

$\stackrel{\oplus}{\mp}$

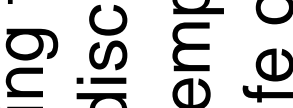

흥 혼

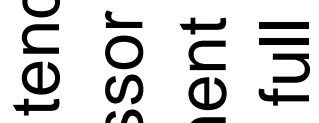

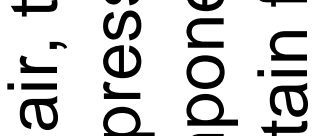

ᄃ है

얻 웡

ن워요

$\overline{0}=\Phi$

등 을 웅 응

Ф

艺.厂

정웡

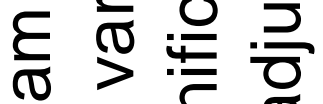

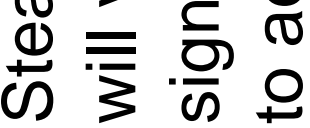

(1) 0

응 웜

드 으

$\overline{0}$
$\frac{0}{0}$

ভ্

(1)

ํㅣㅇ

$\frac{2}{x}$

를 음

응 승

ธே

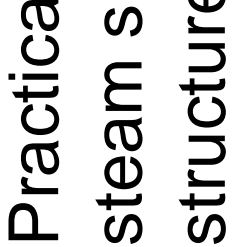

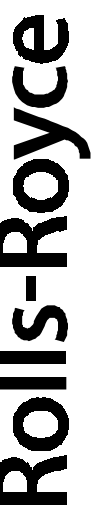

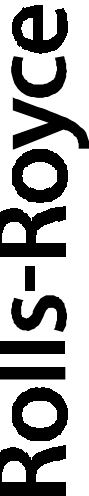




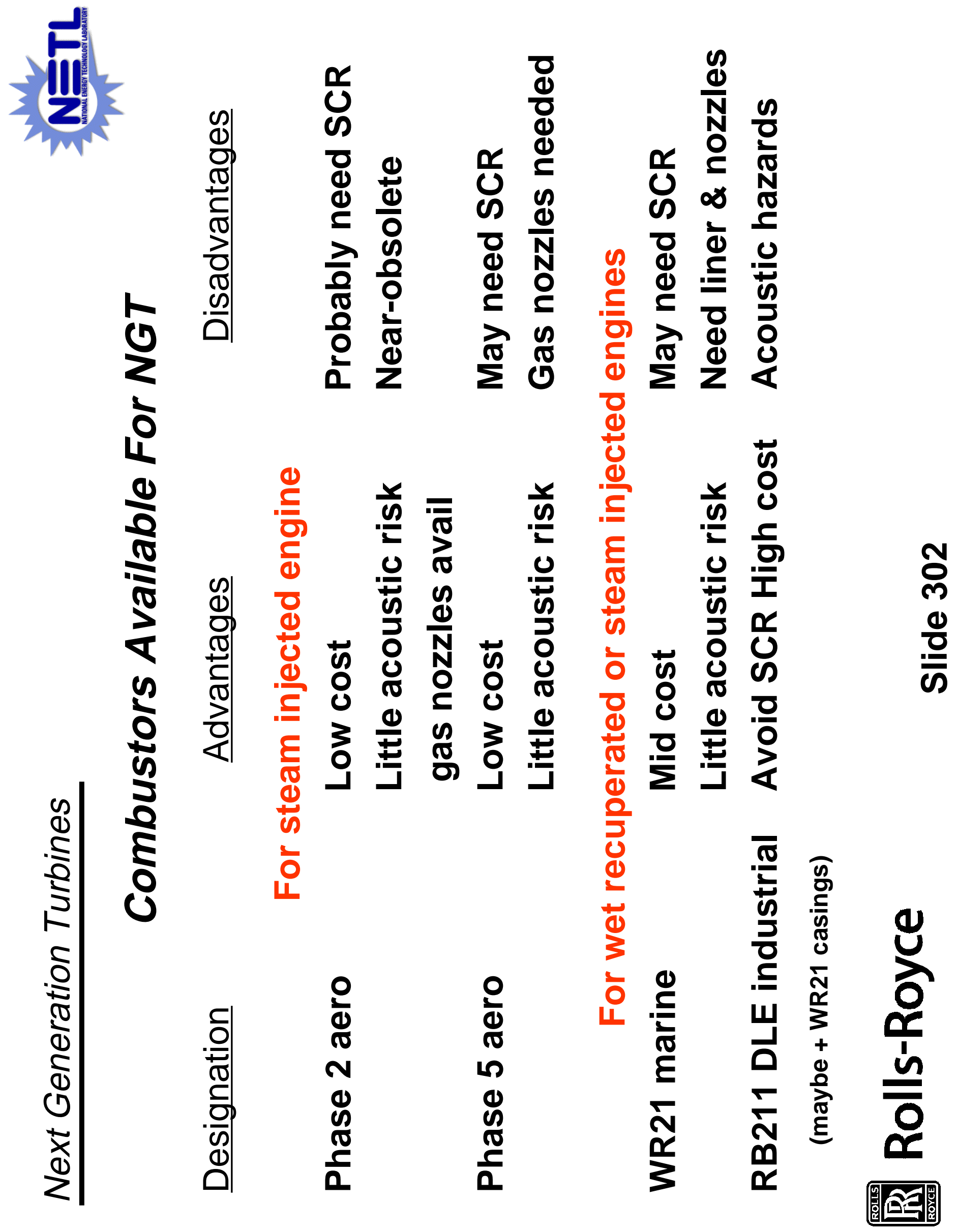



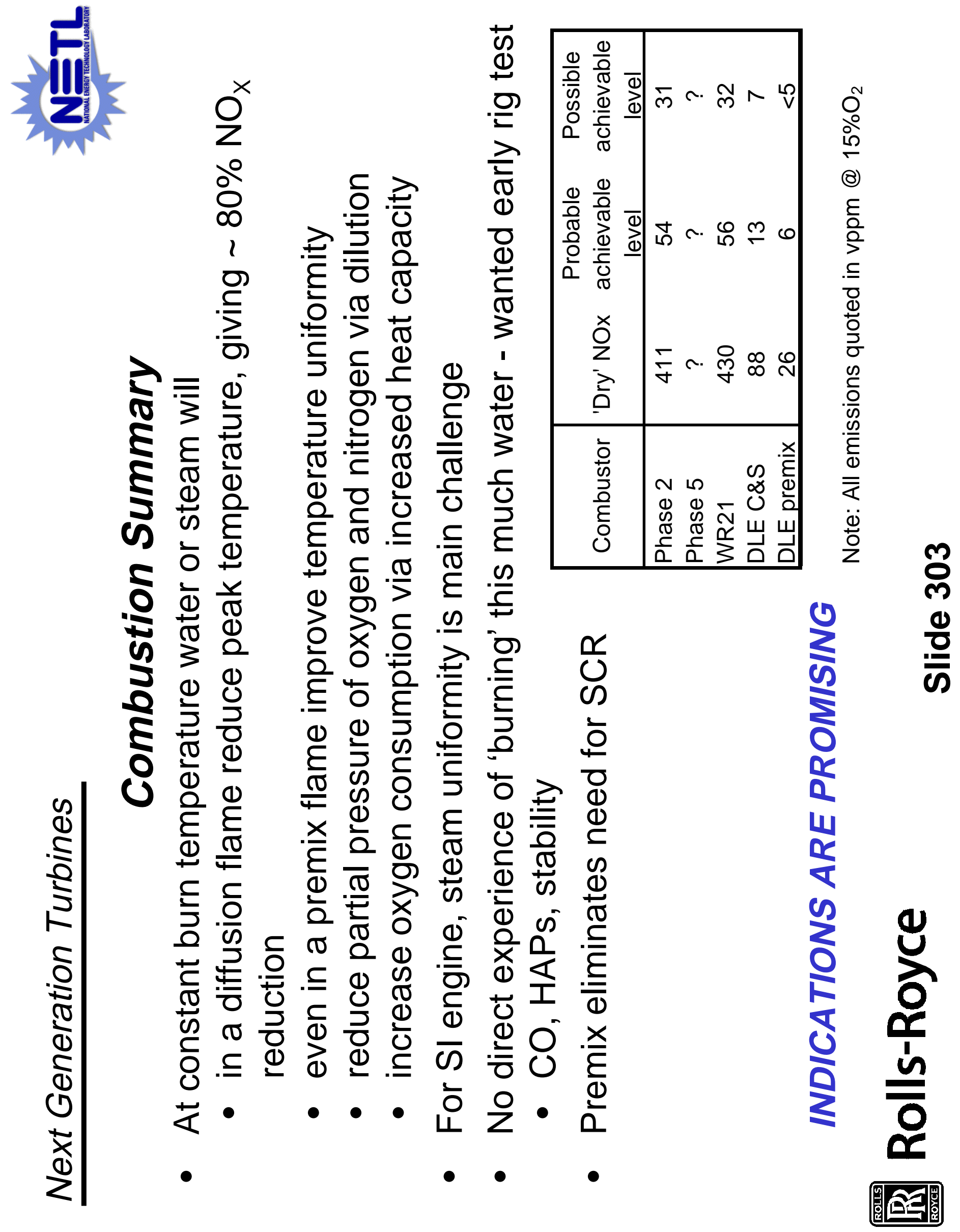


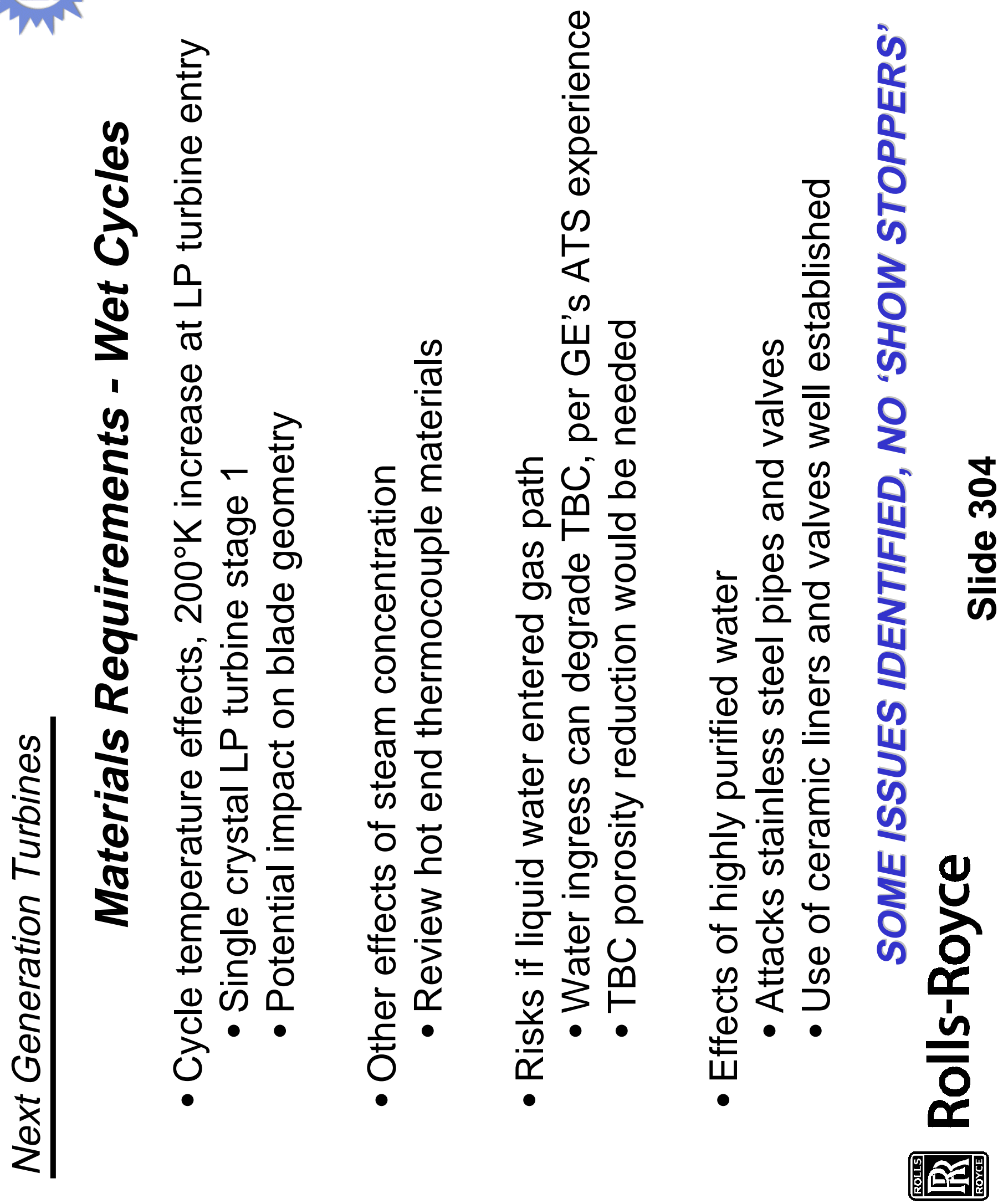




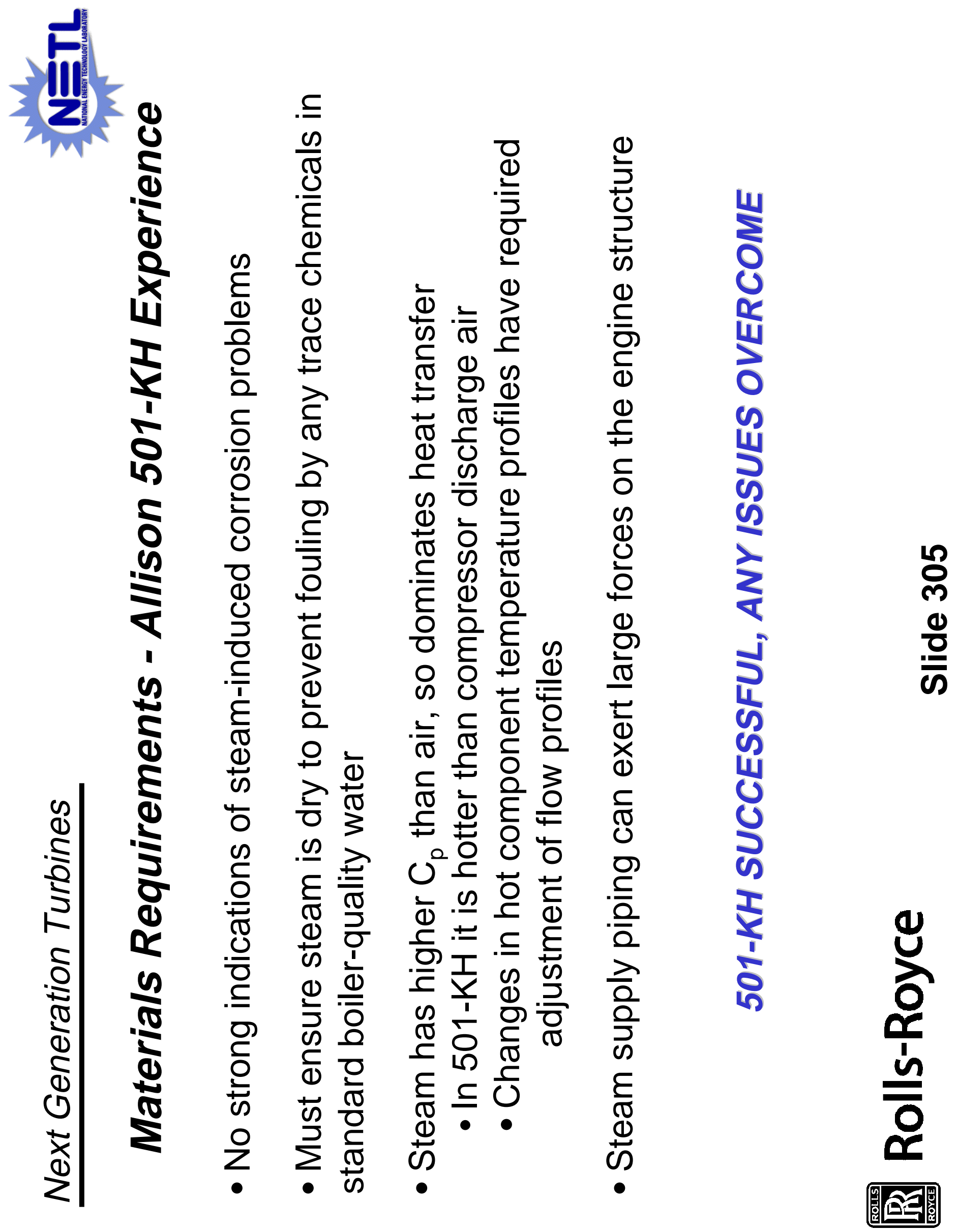




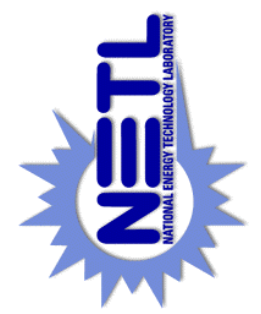

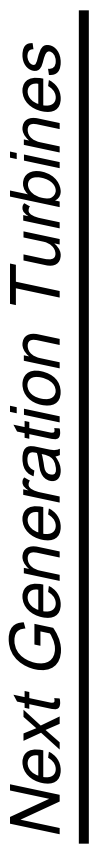

ต

$\frac{0}{\circ}$
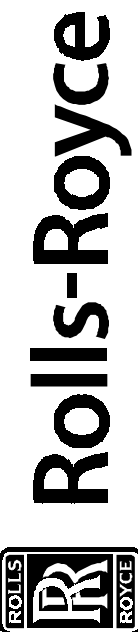

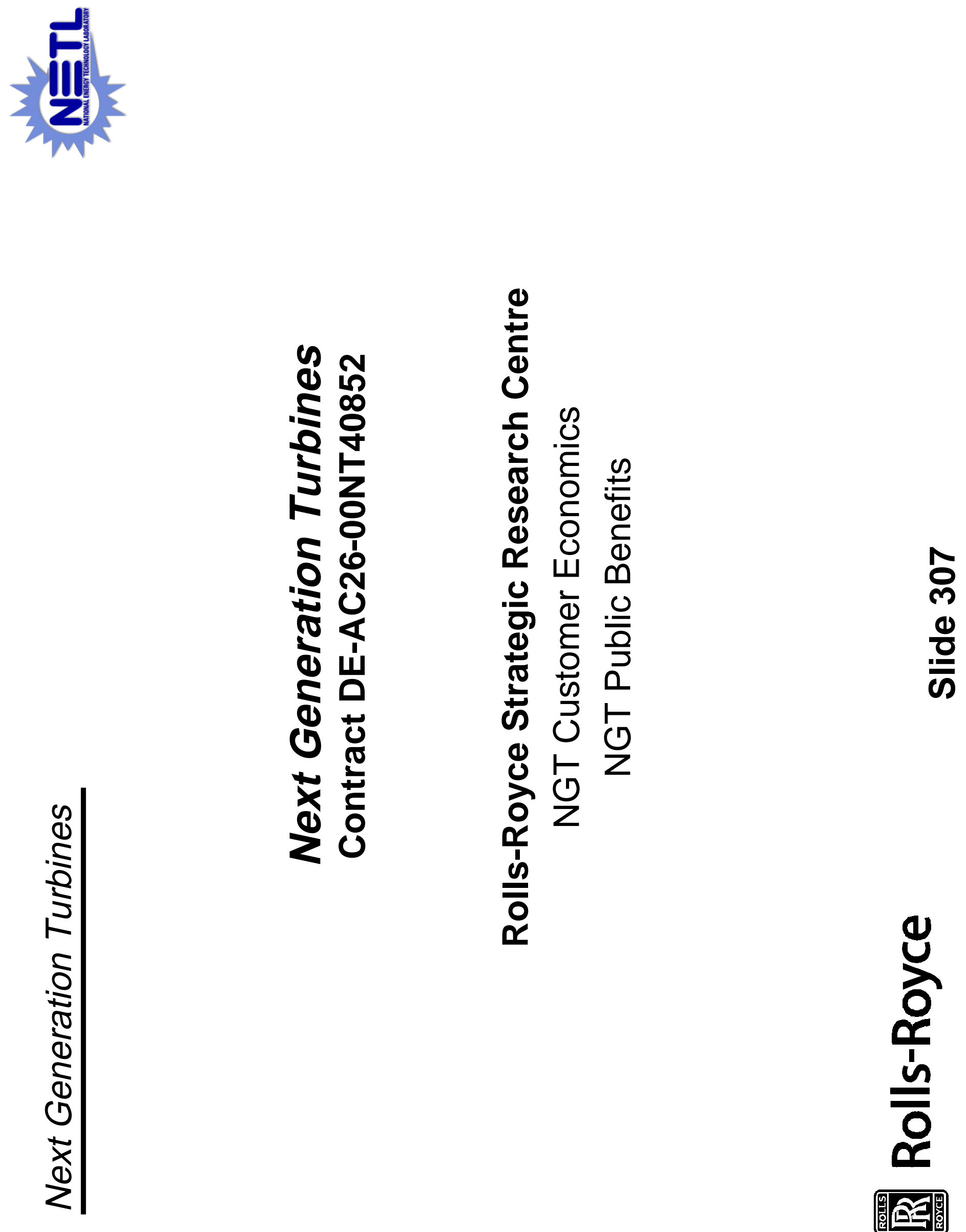


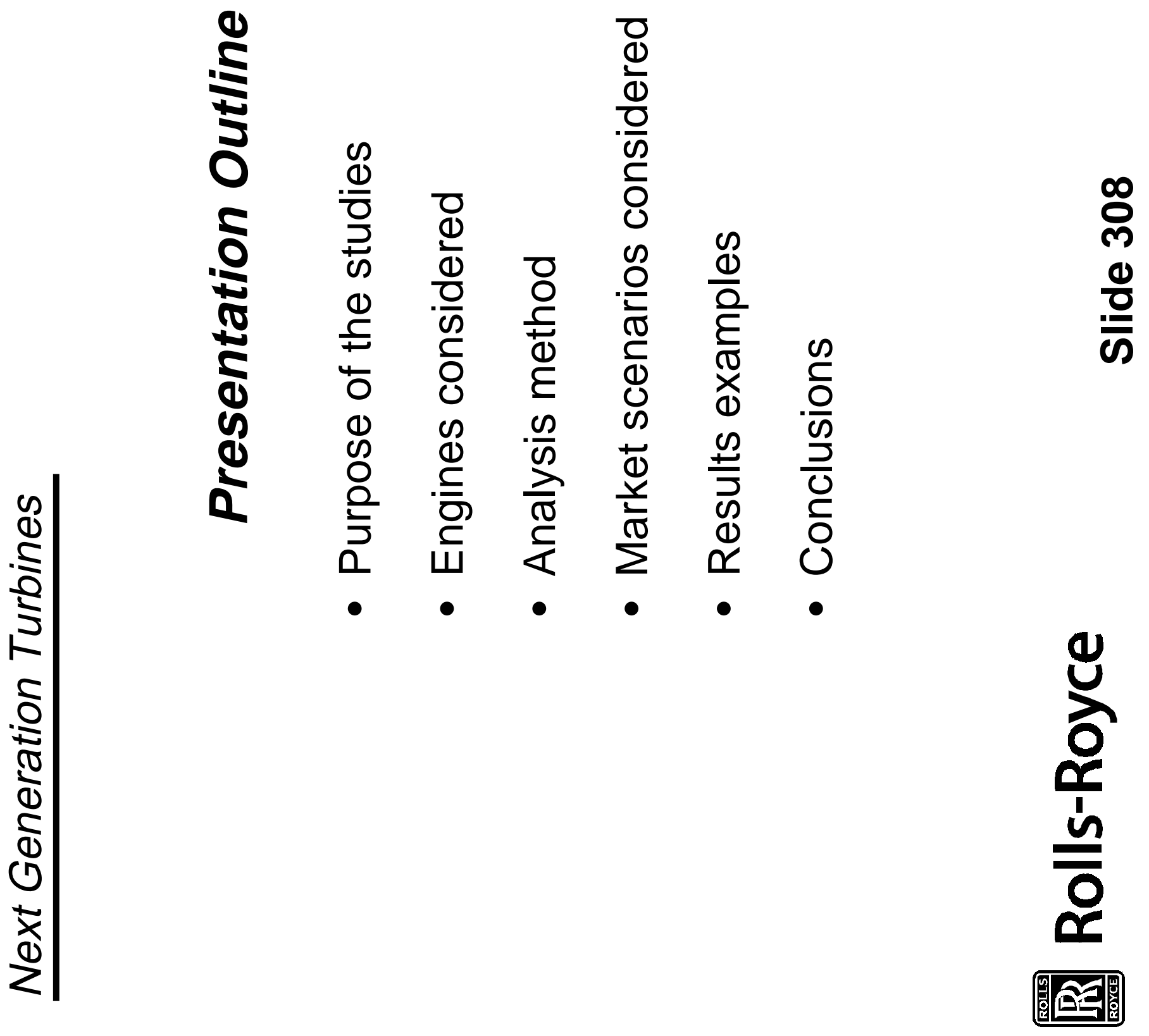




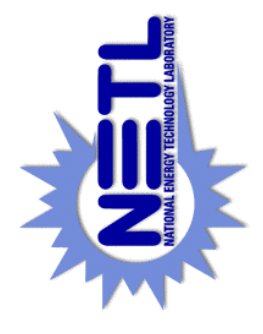

\&
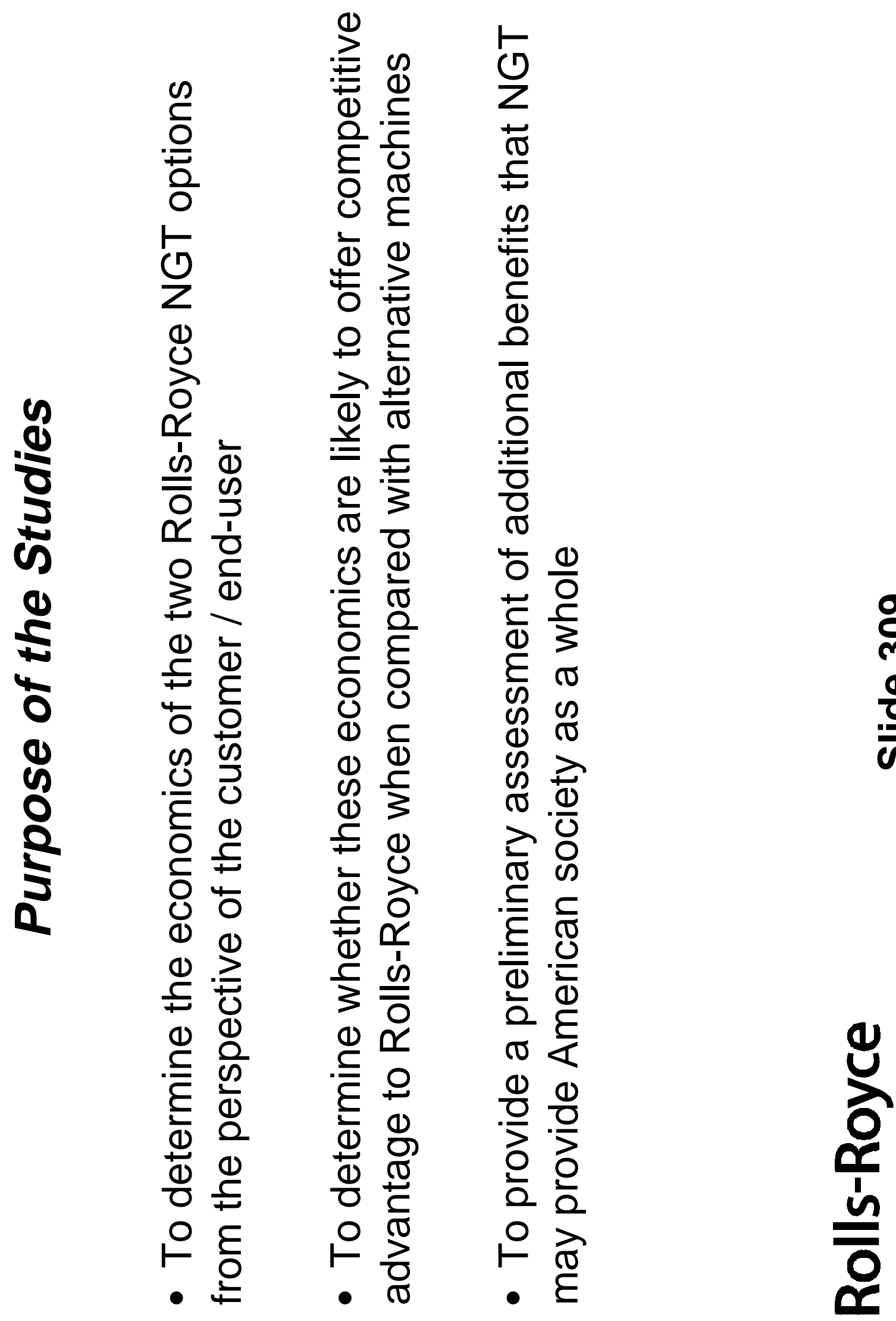


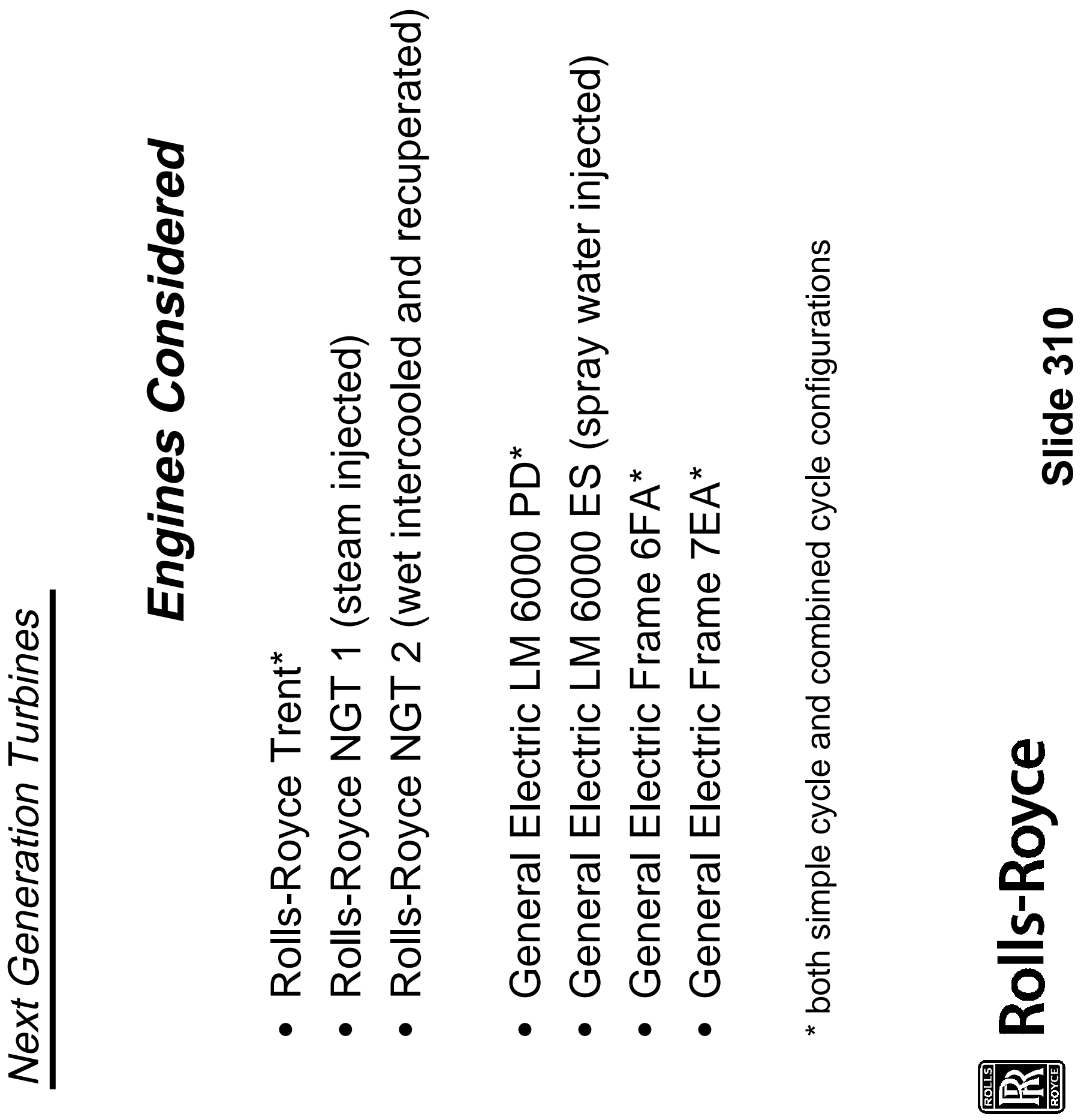



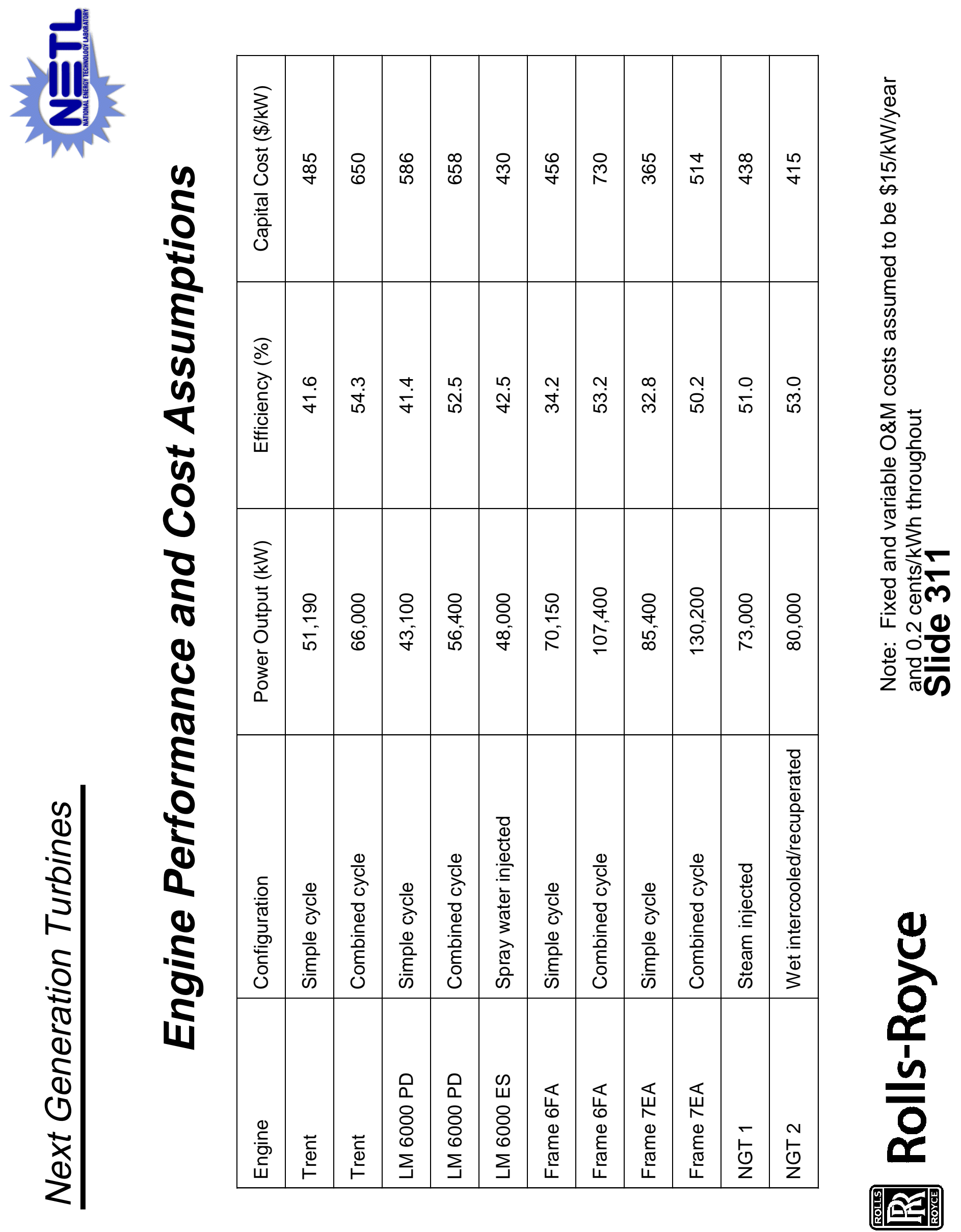


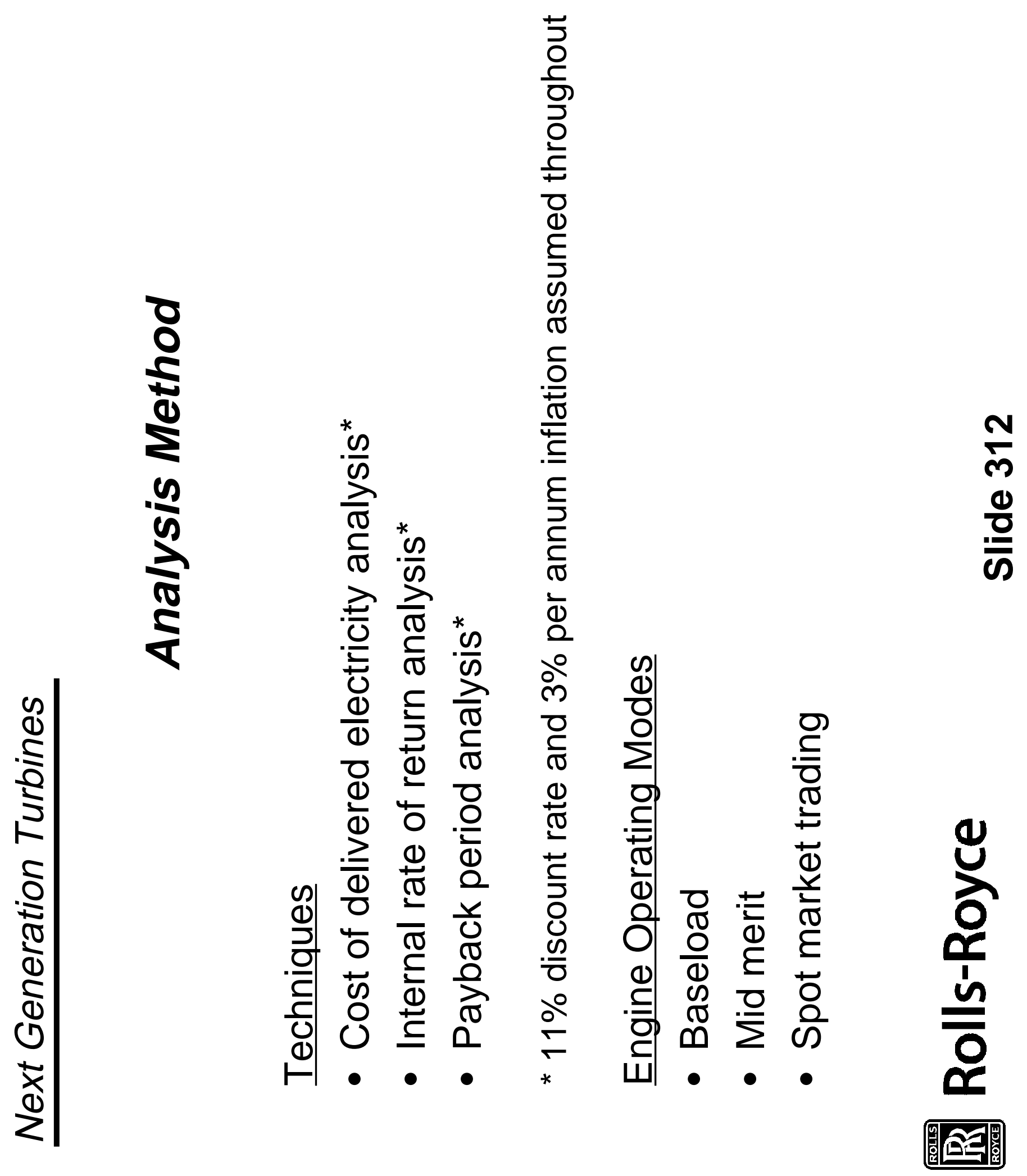




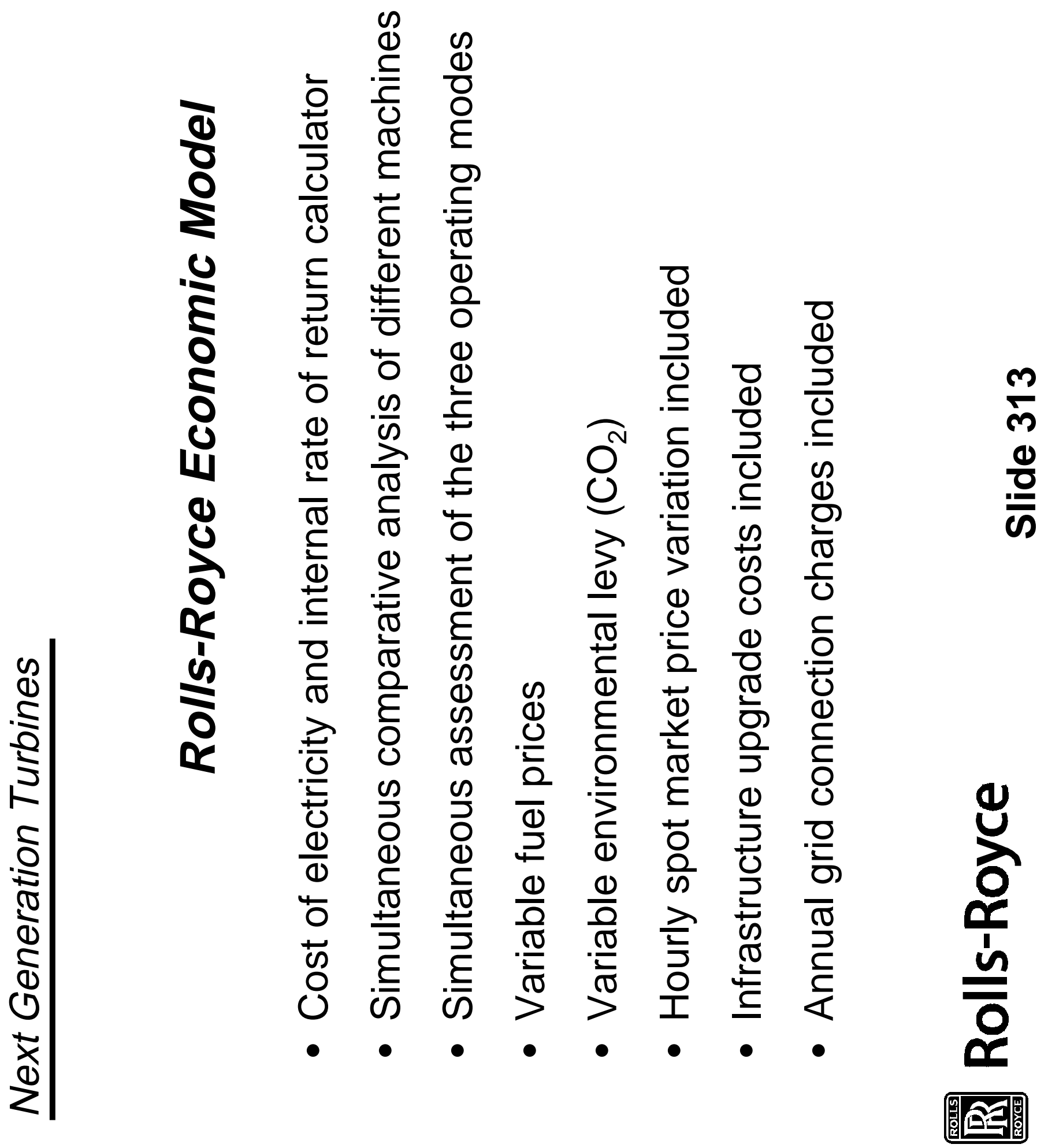




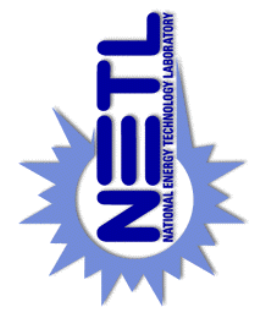

\begin{tabular}{|c|c|c|c|c|c|}
\hline 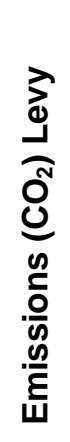 & $\begin{array}{l}0 \\
\stackrel{0}{0} \\
\text { Zे }\end{array}$ & $\begin{array}{l}0 \\
\stackrel{0}{0}\end{array}$ & $\begin{array}{l}0 \\
\stackrel{0}{0} \\
z\end{array}$ & 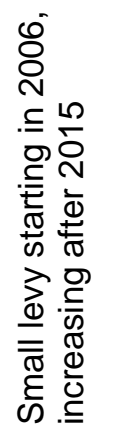 & 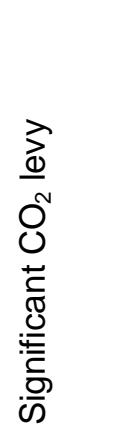 \\
\hline 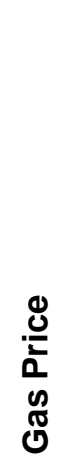 & 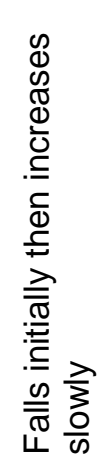 & 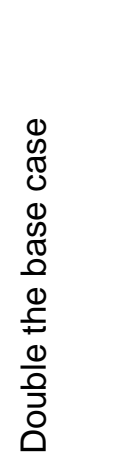 & 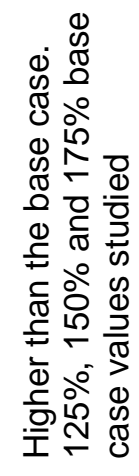 & 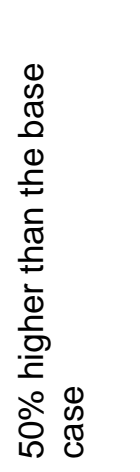 & 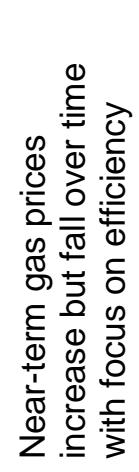 \\
\hline 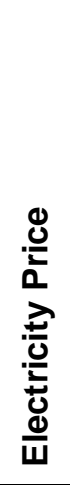 & 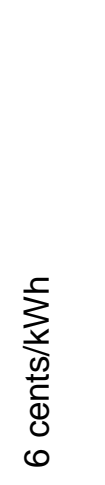 & 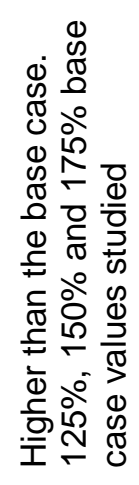 & 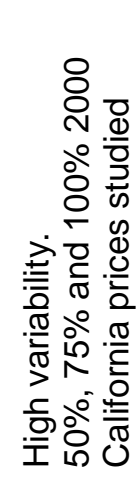 & 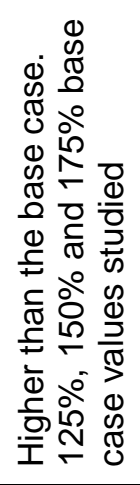 & 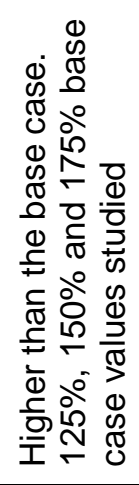 \\
\hline $\begin{array}{l}\frac{3}{2} \\
\frac{2}{0} \\
0 \\
0\end{array}$ & 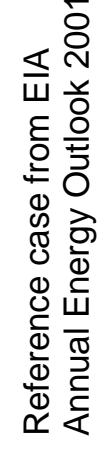 & 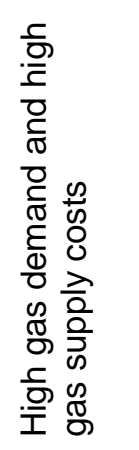 & 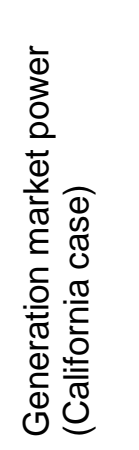 & 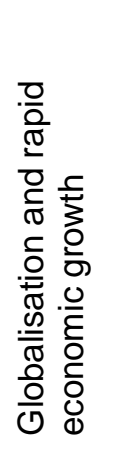 & 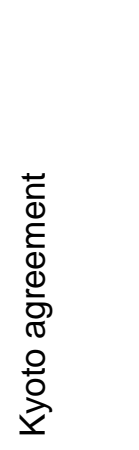 \\
\hline 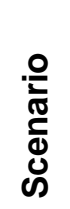 & $\begin{array}{l}\mathbb{D} \\
\mathbb{N} \\
0 \\
\mathbb{D} \\
\mathbb{D} \\
\mathbb{D} \\
0\end{array}$ & 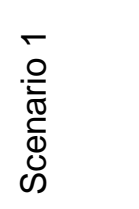 & 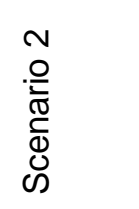 & 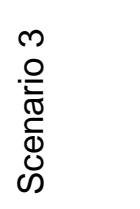 & $\begin{array}{l}\text { 음 } \\
\bar{\varpi} \\
\overline{0} \\
c\end{array}$ \\
\hline
\end{tabular}
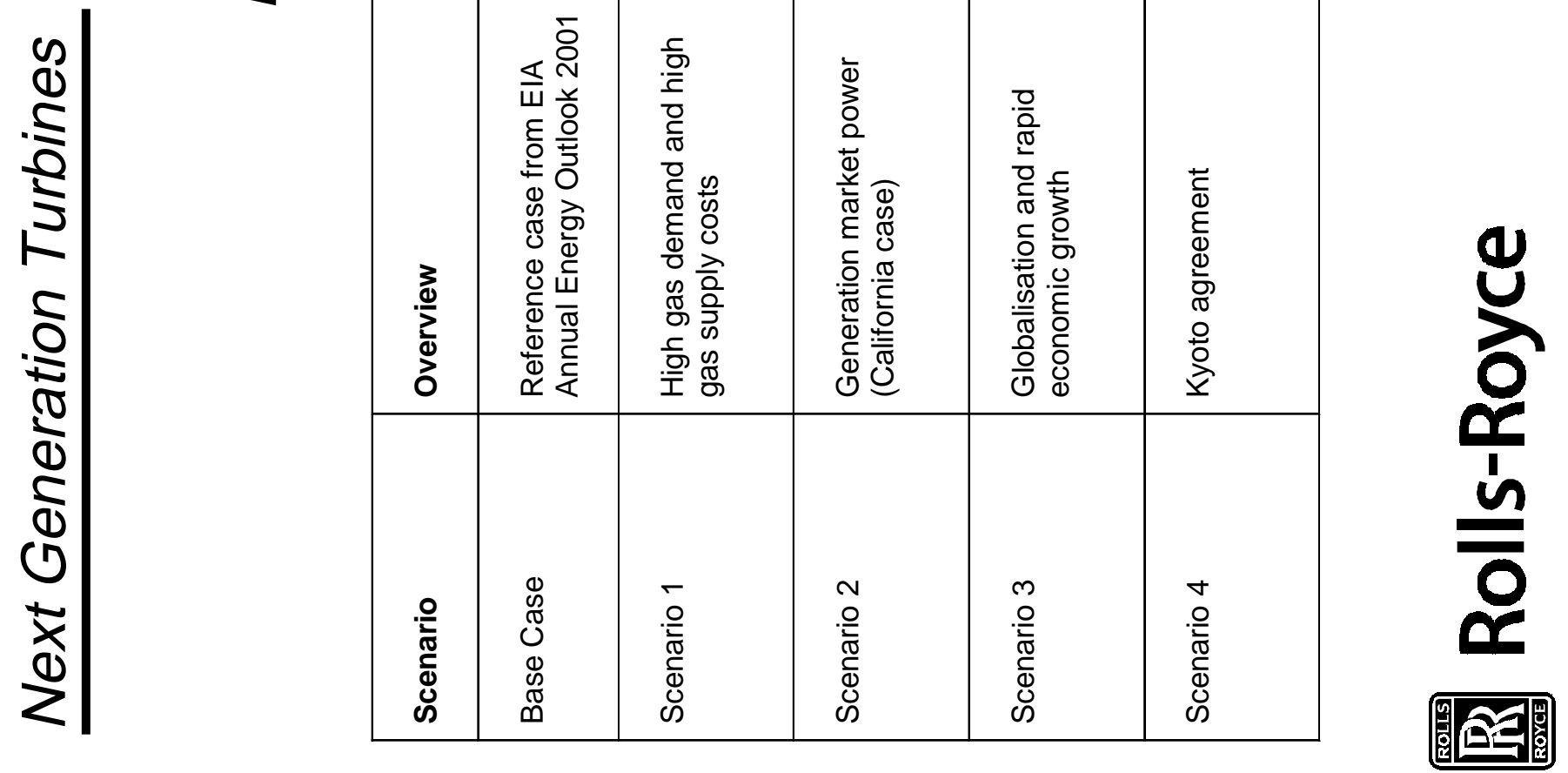

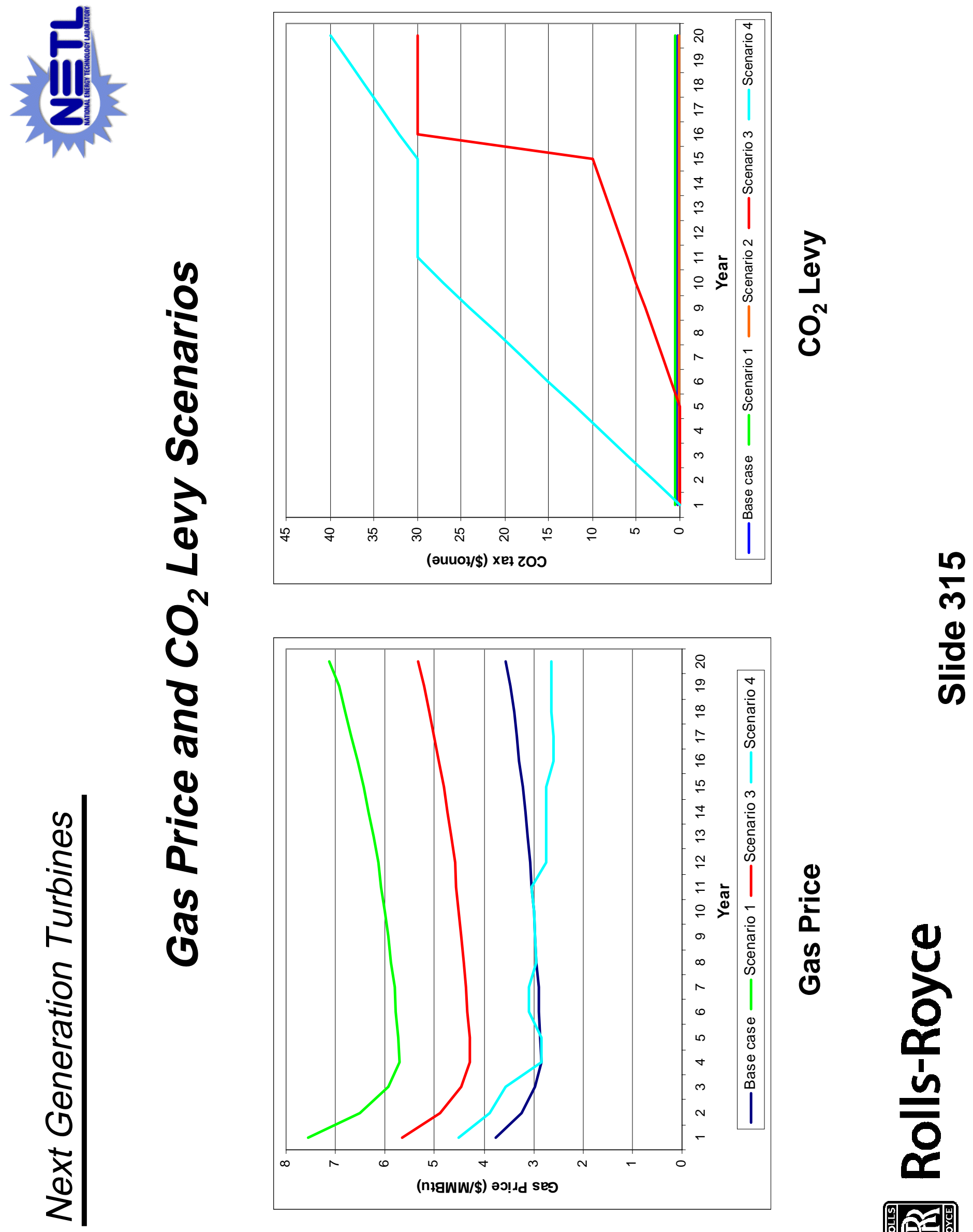

$\frac{1}{6}$

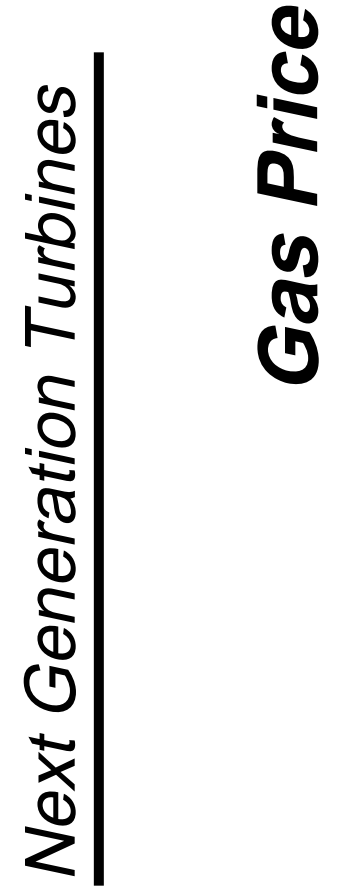




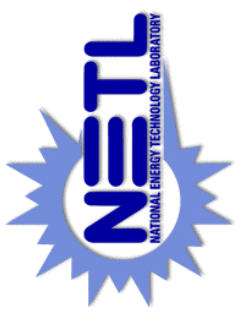

5
$\frac{1}{0}$
$\frac{1}{0}$
0
0
0
0
0
0
0
0
0

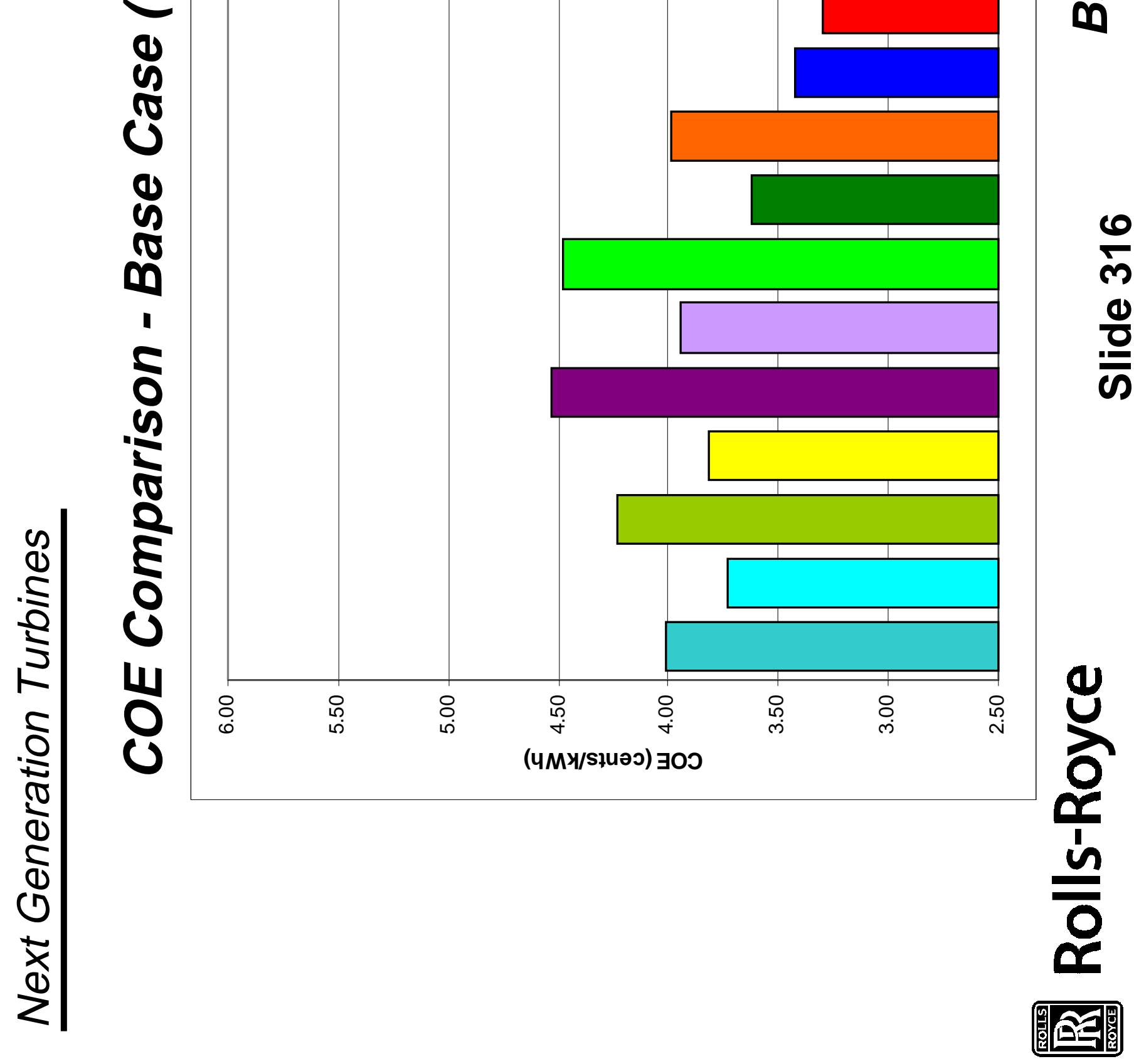



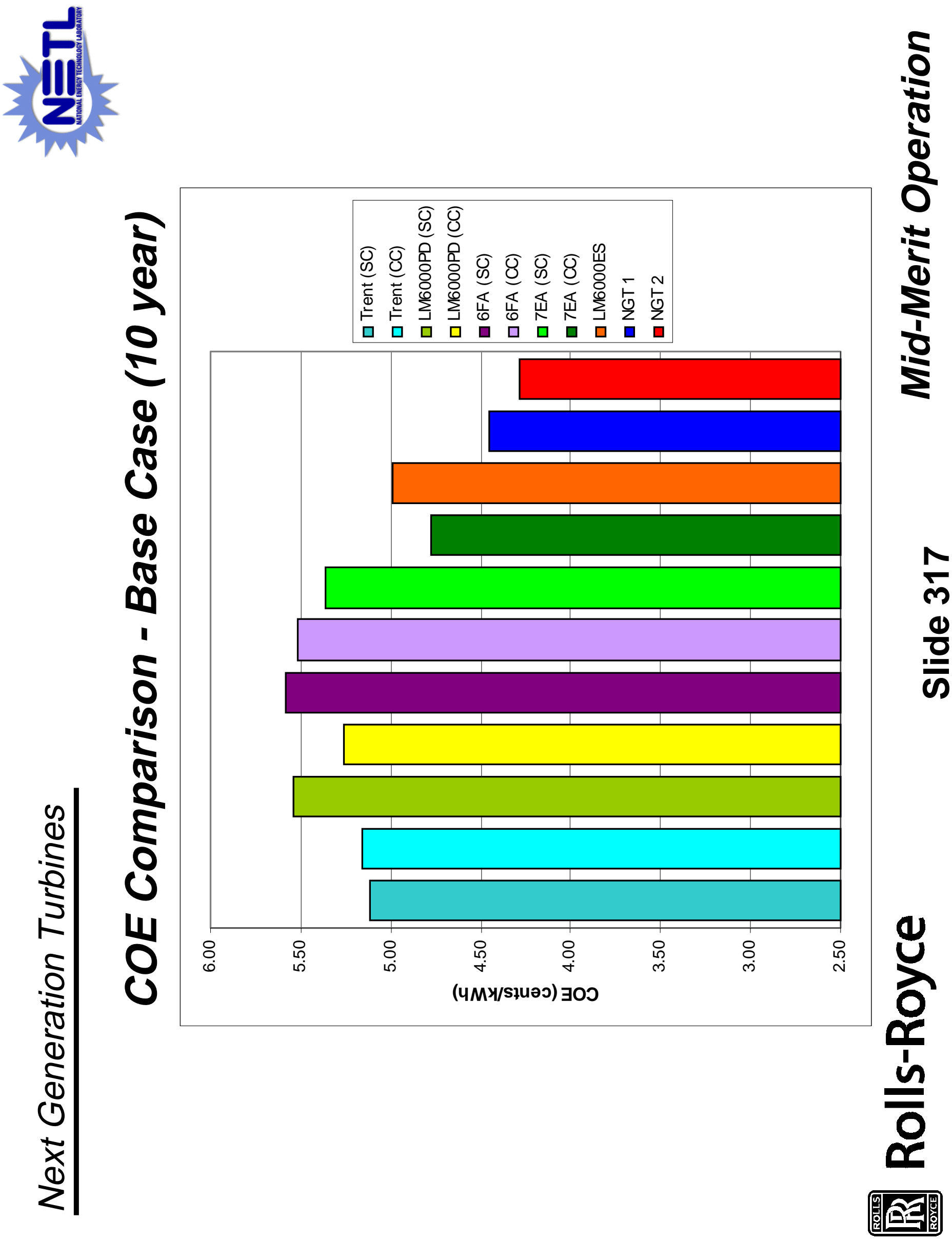


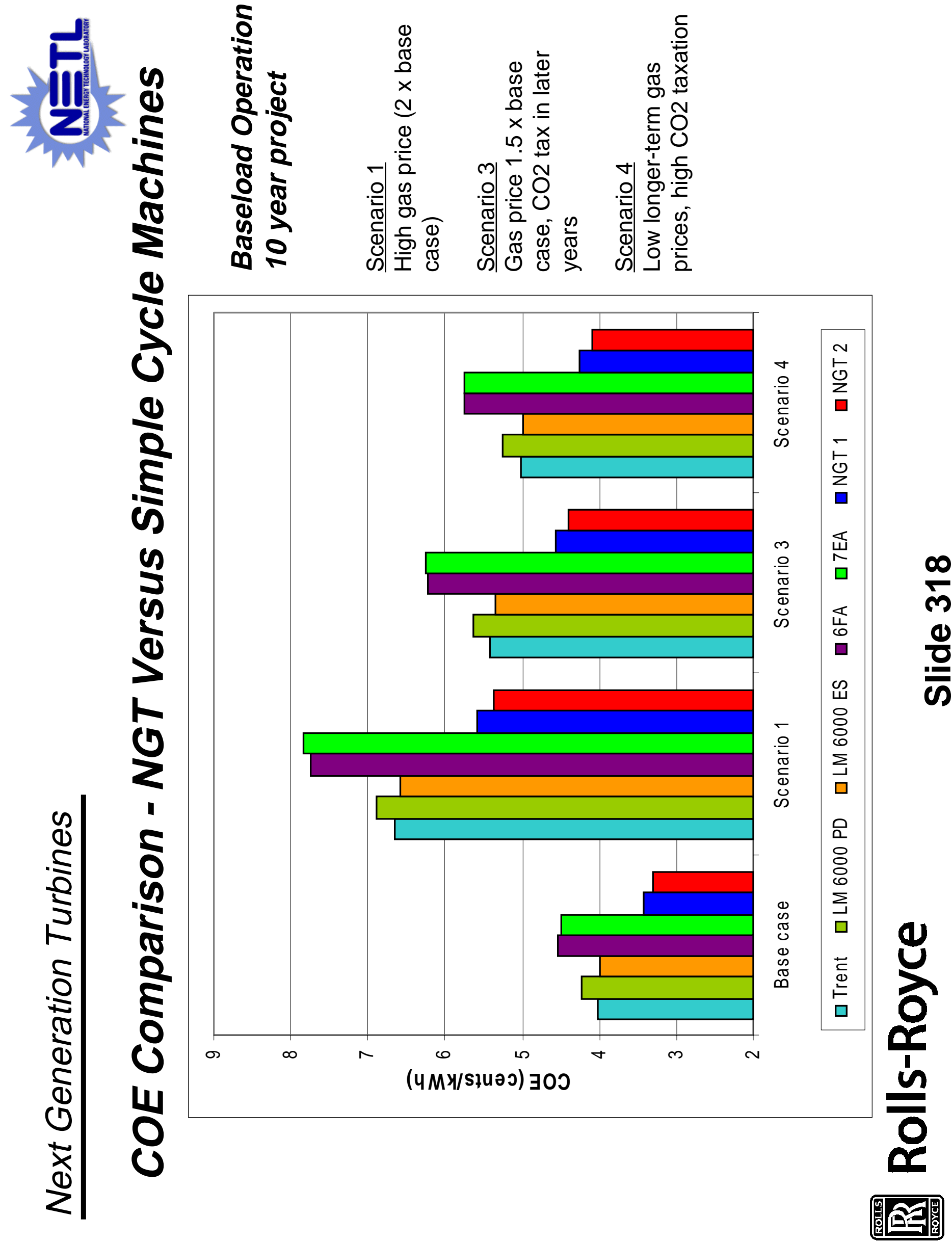




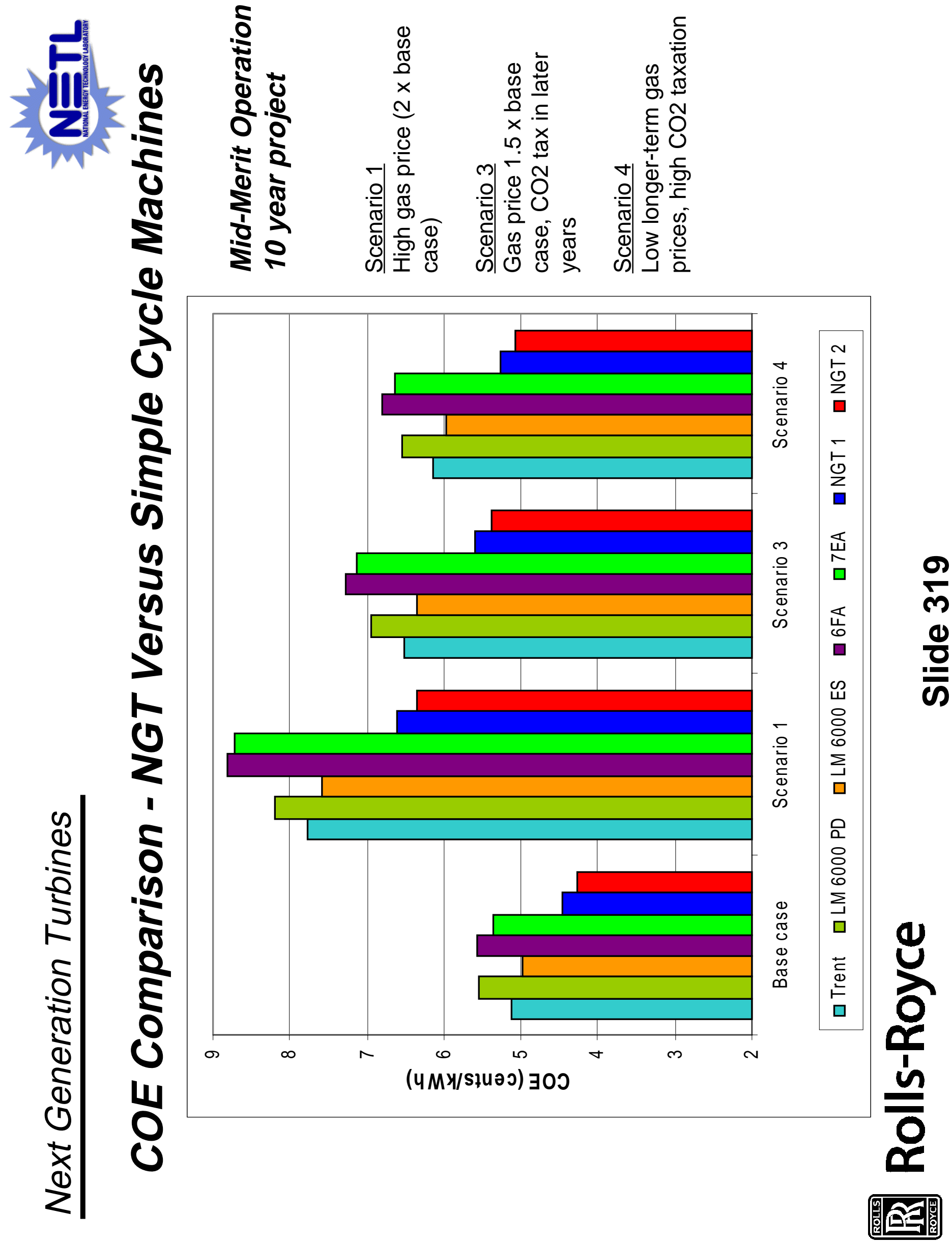




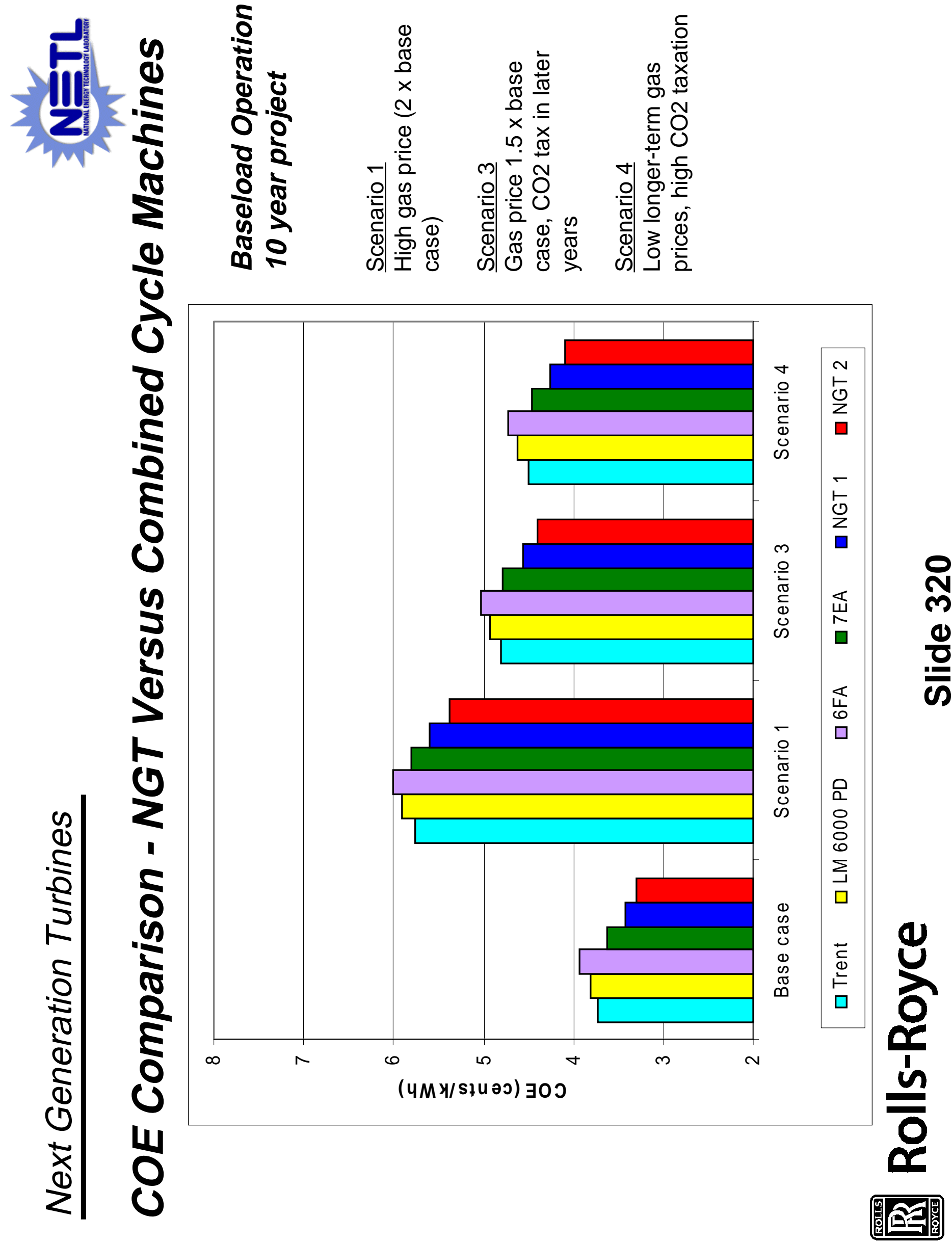




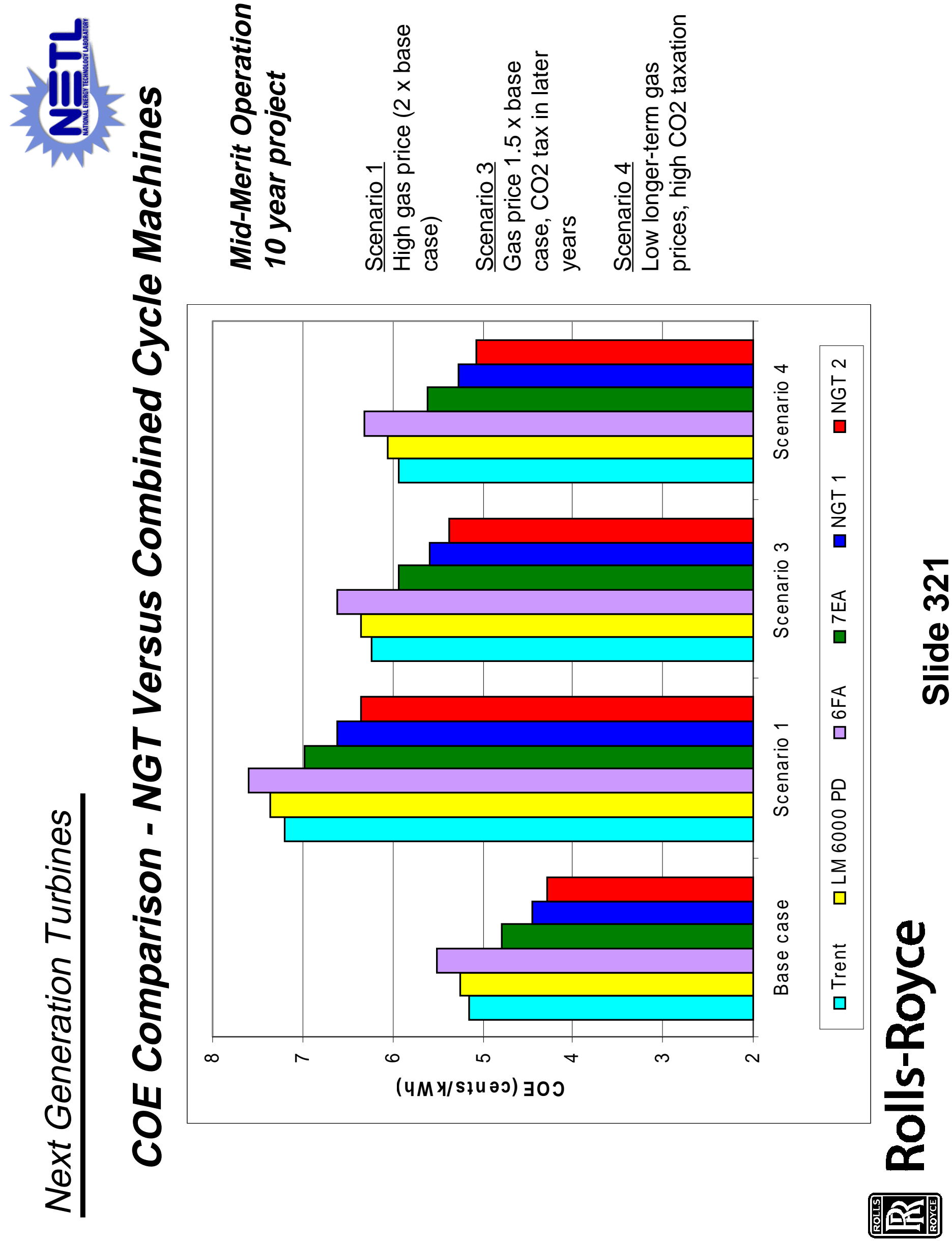




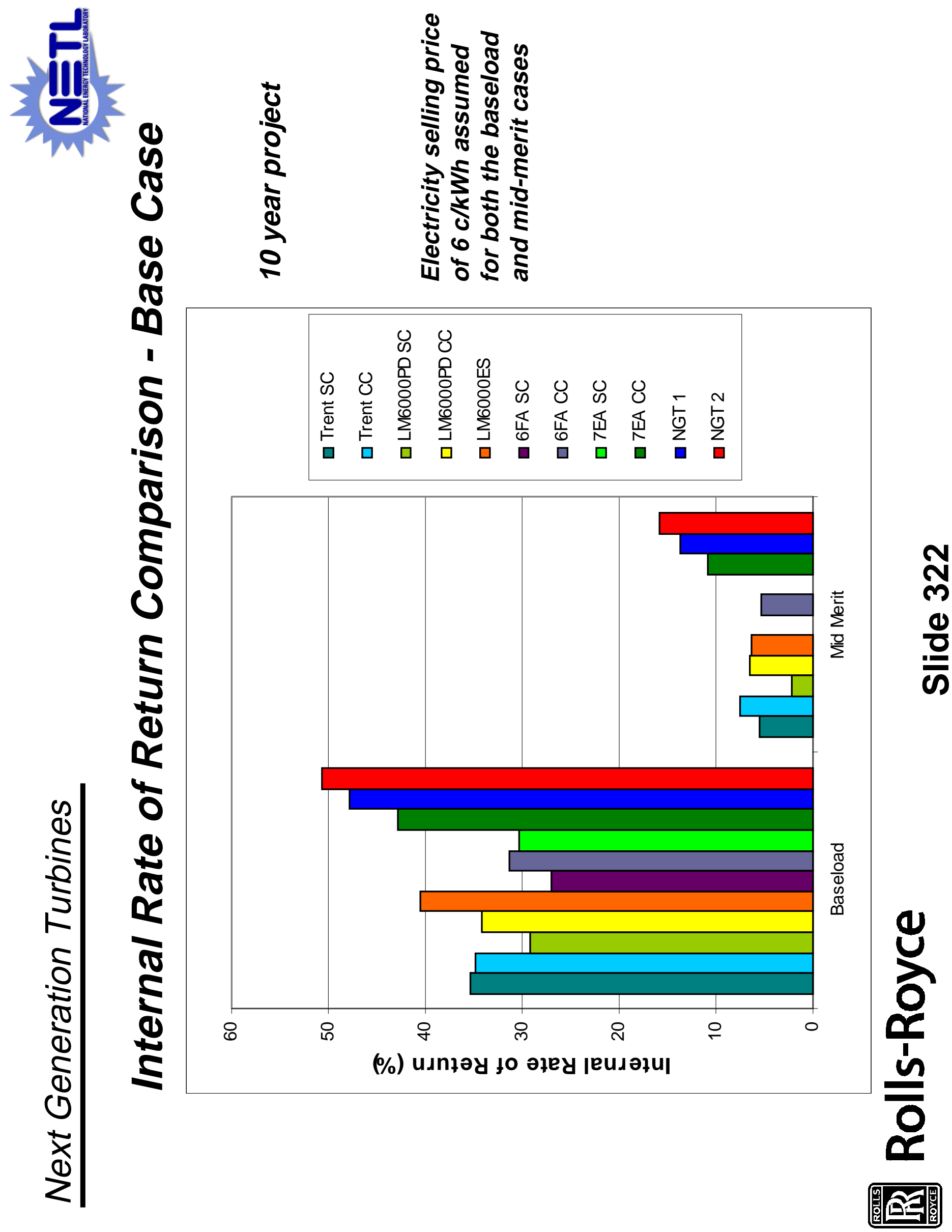




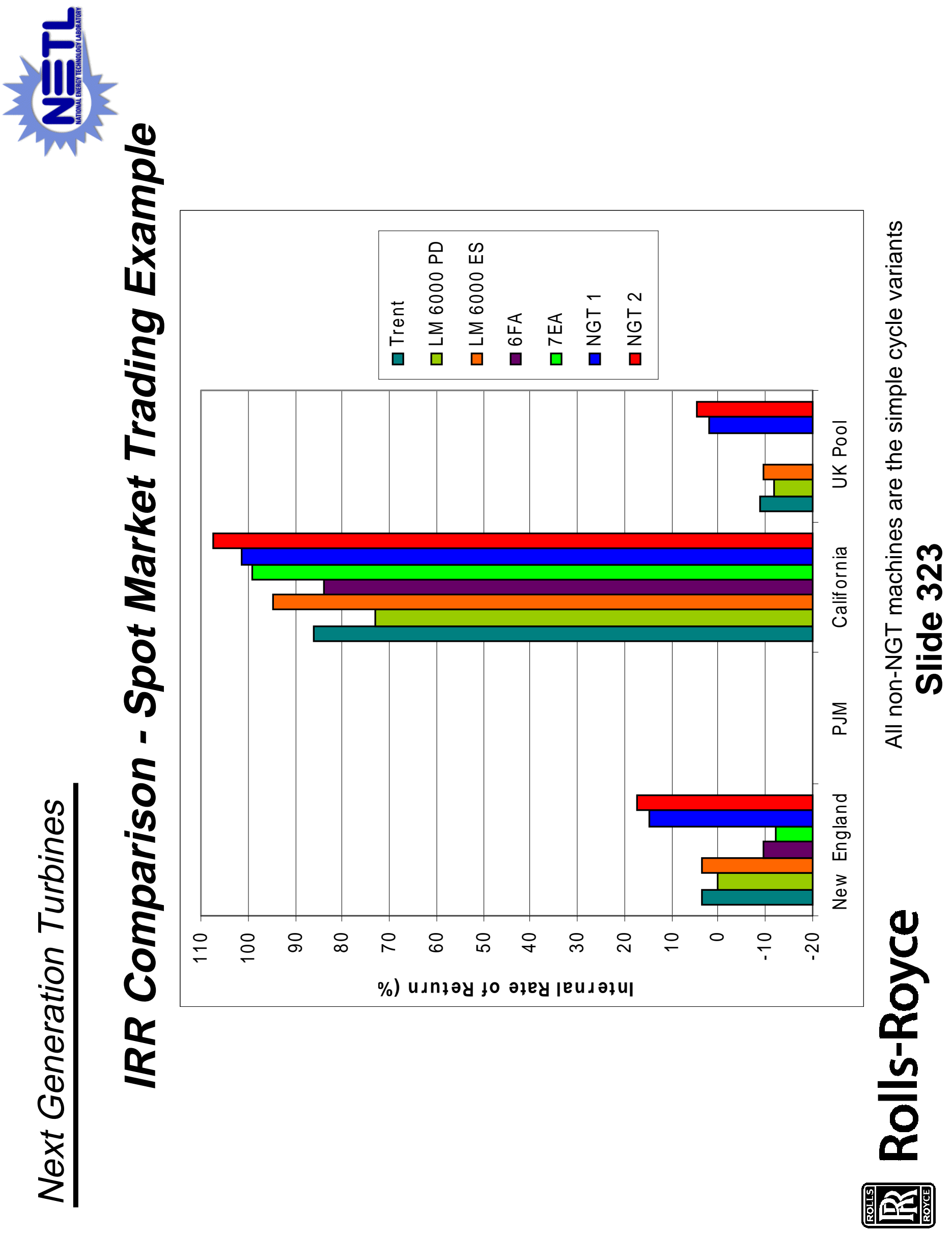



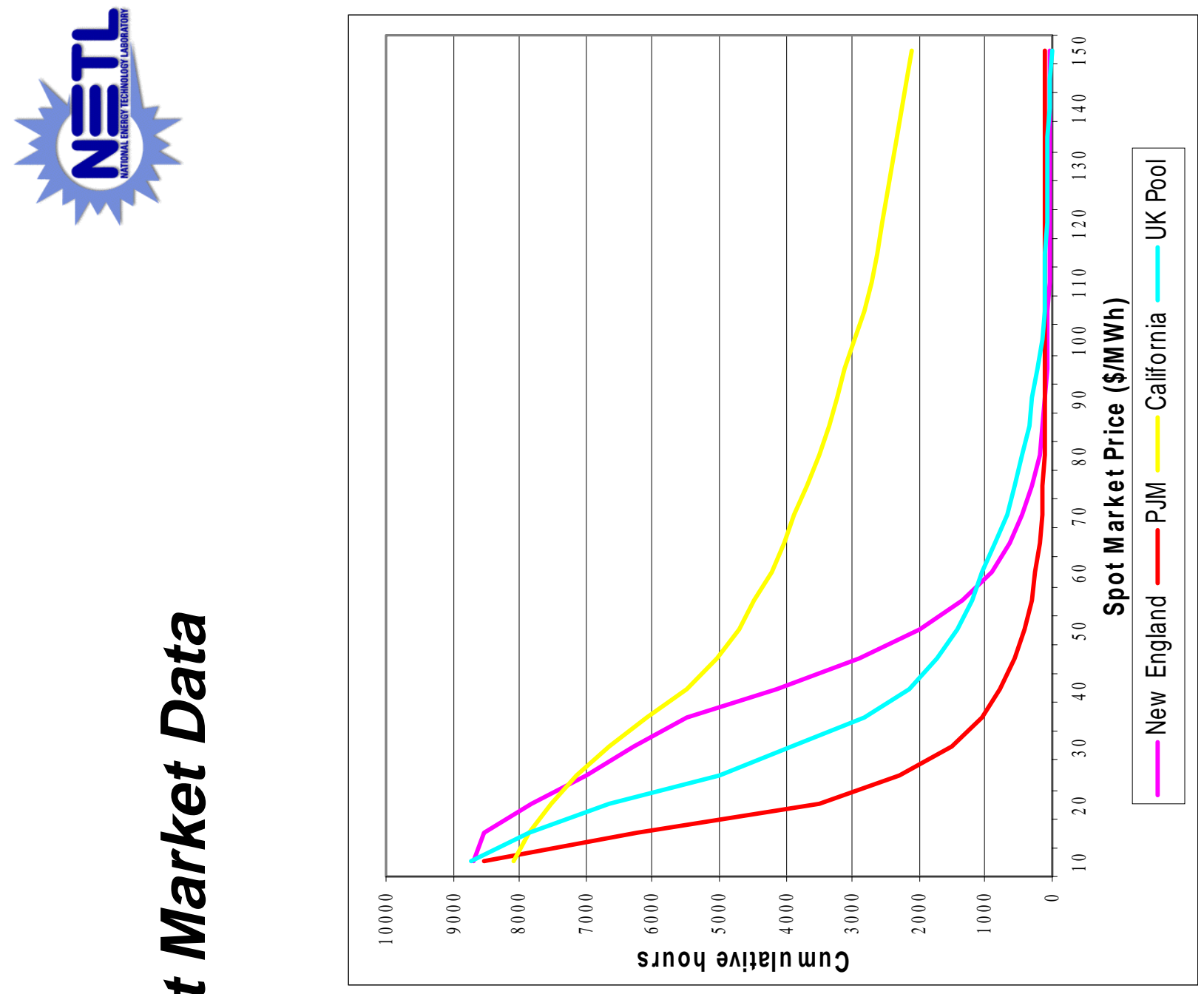

$\frac{\pi}{\pi}$
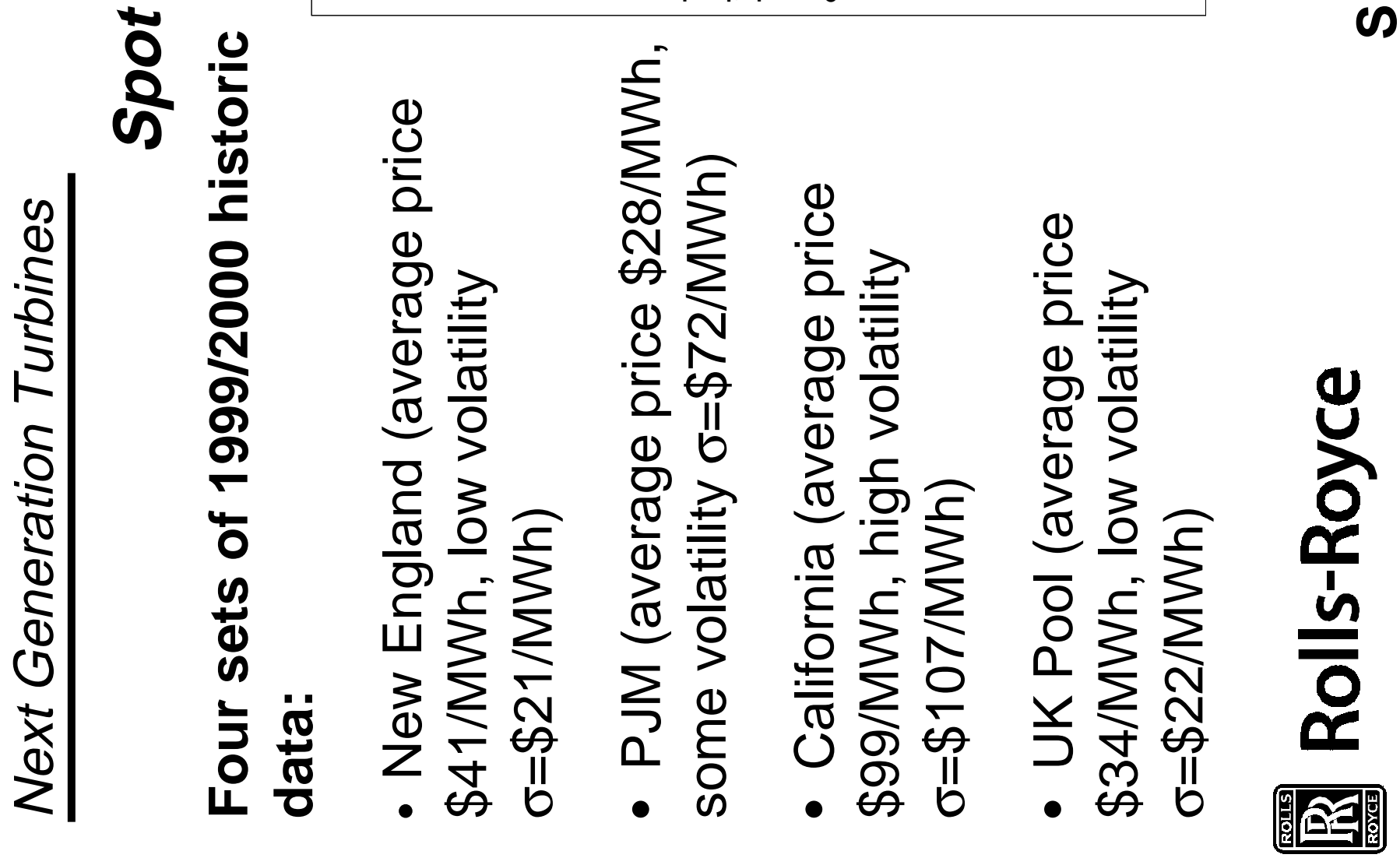


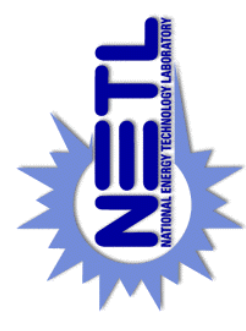

का
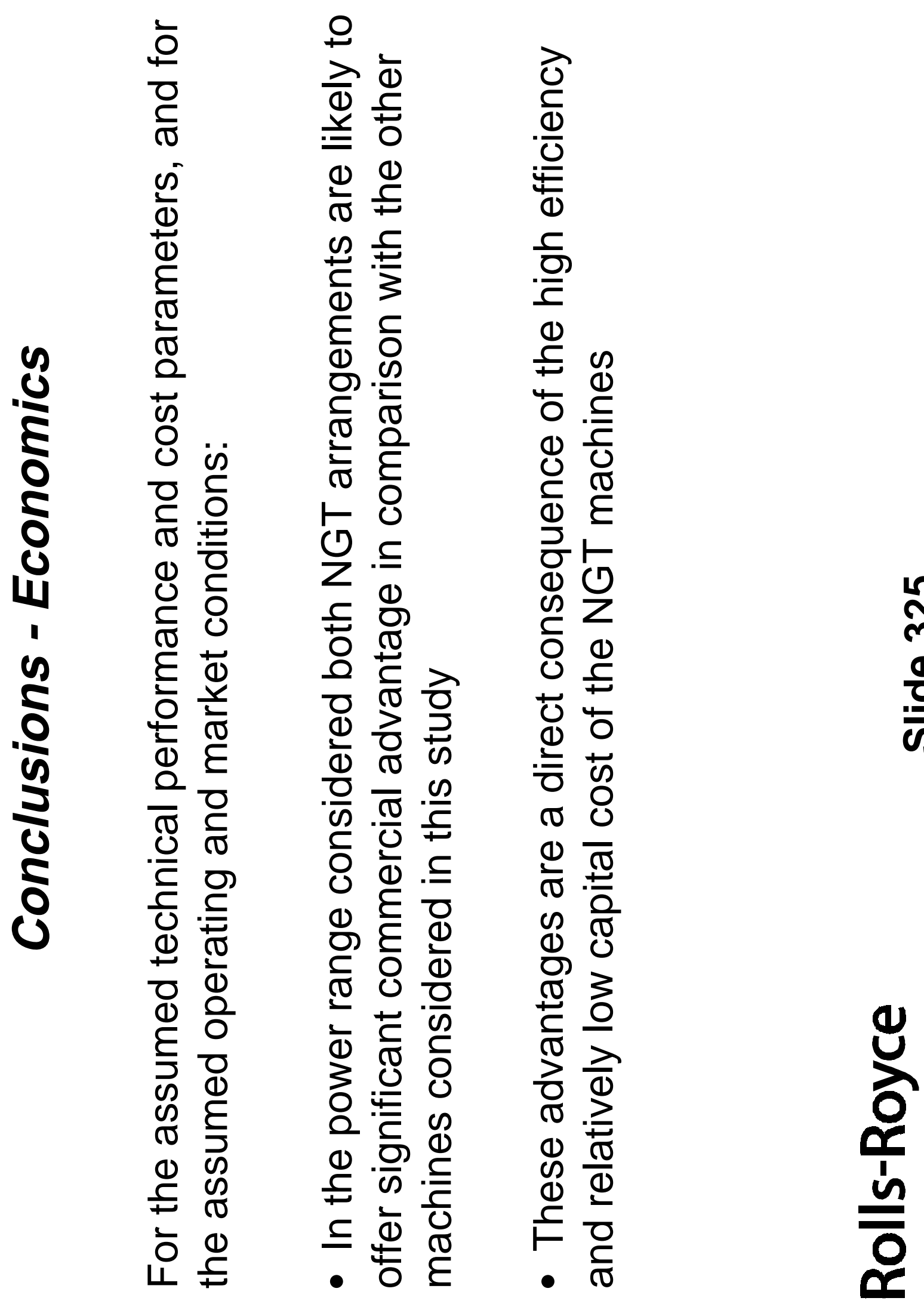

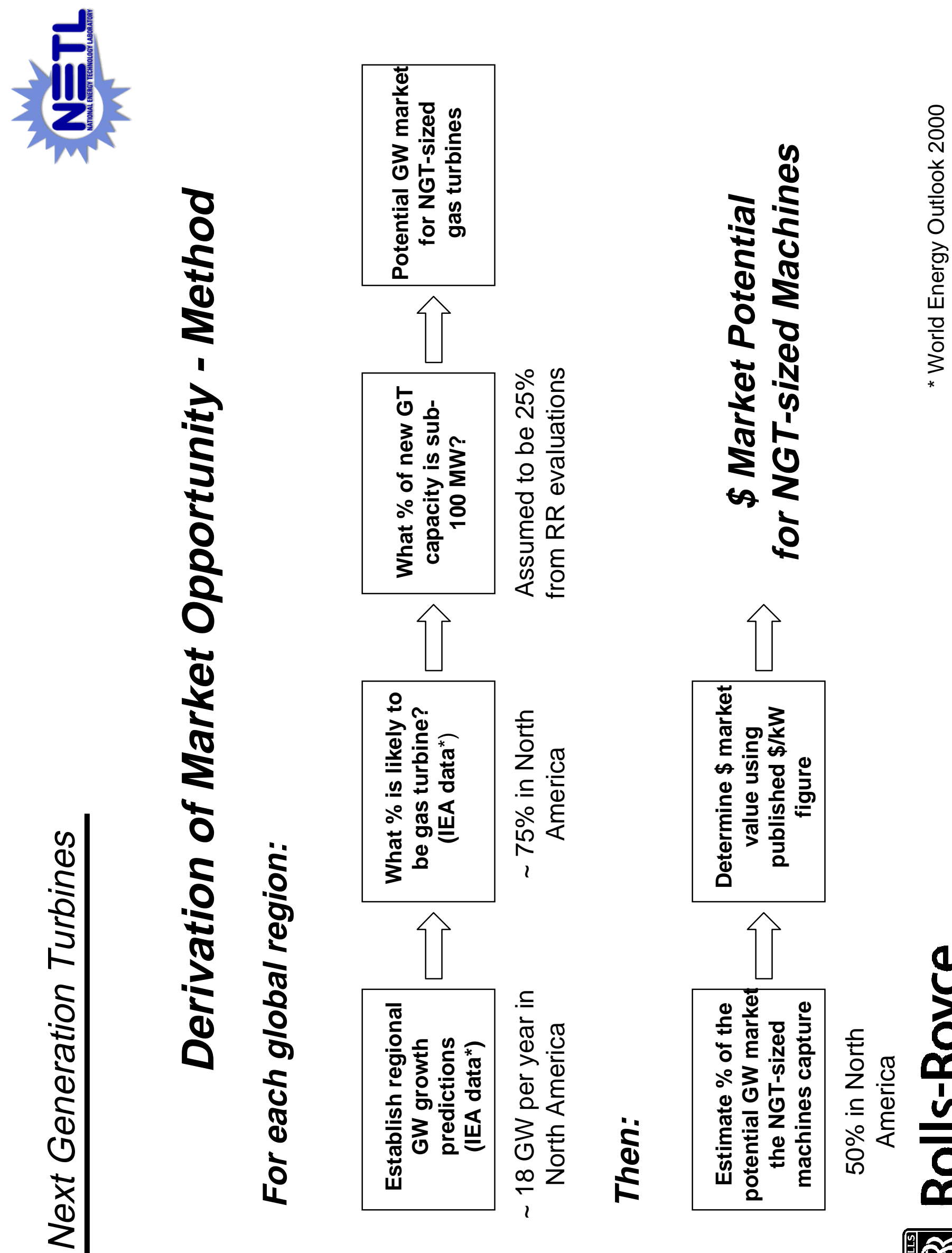

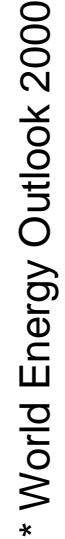
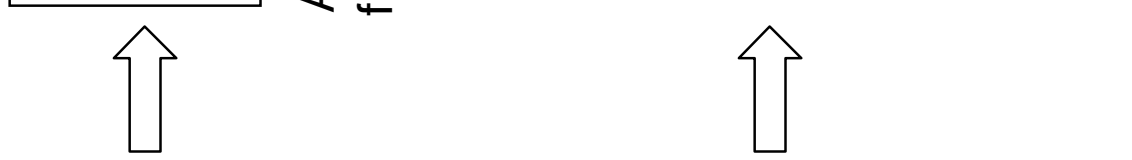

0

N

()
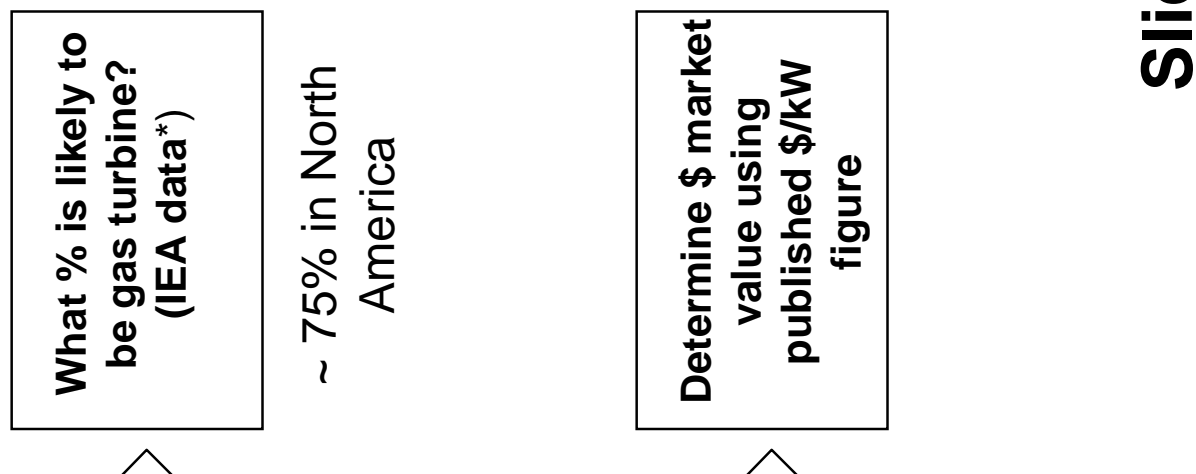

$\frac{10}{6}$

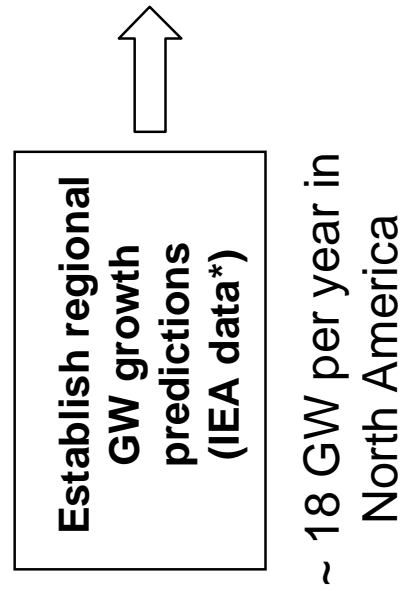

$E$

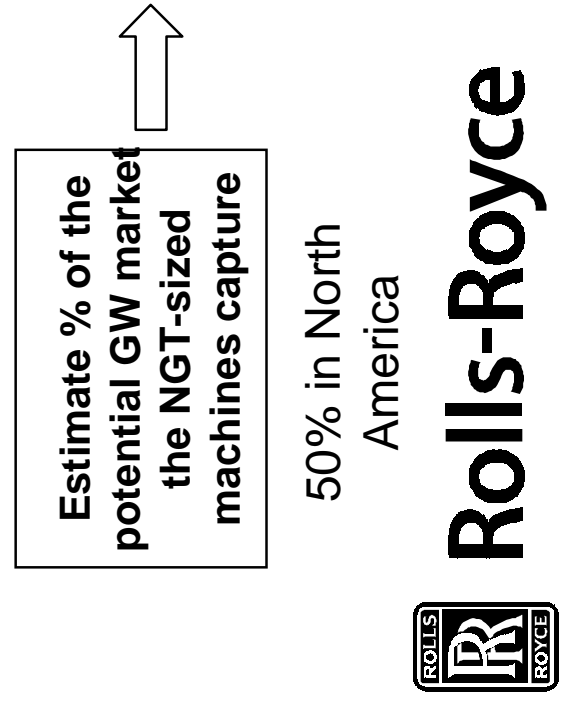



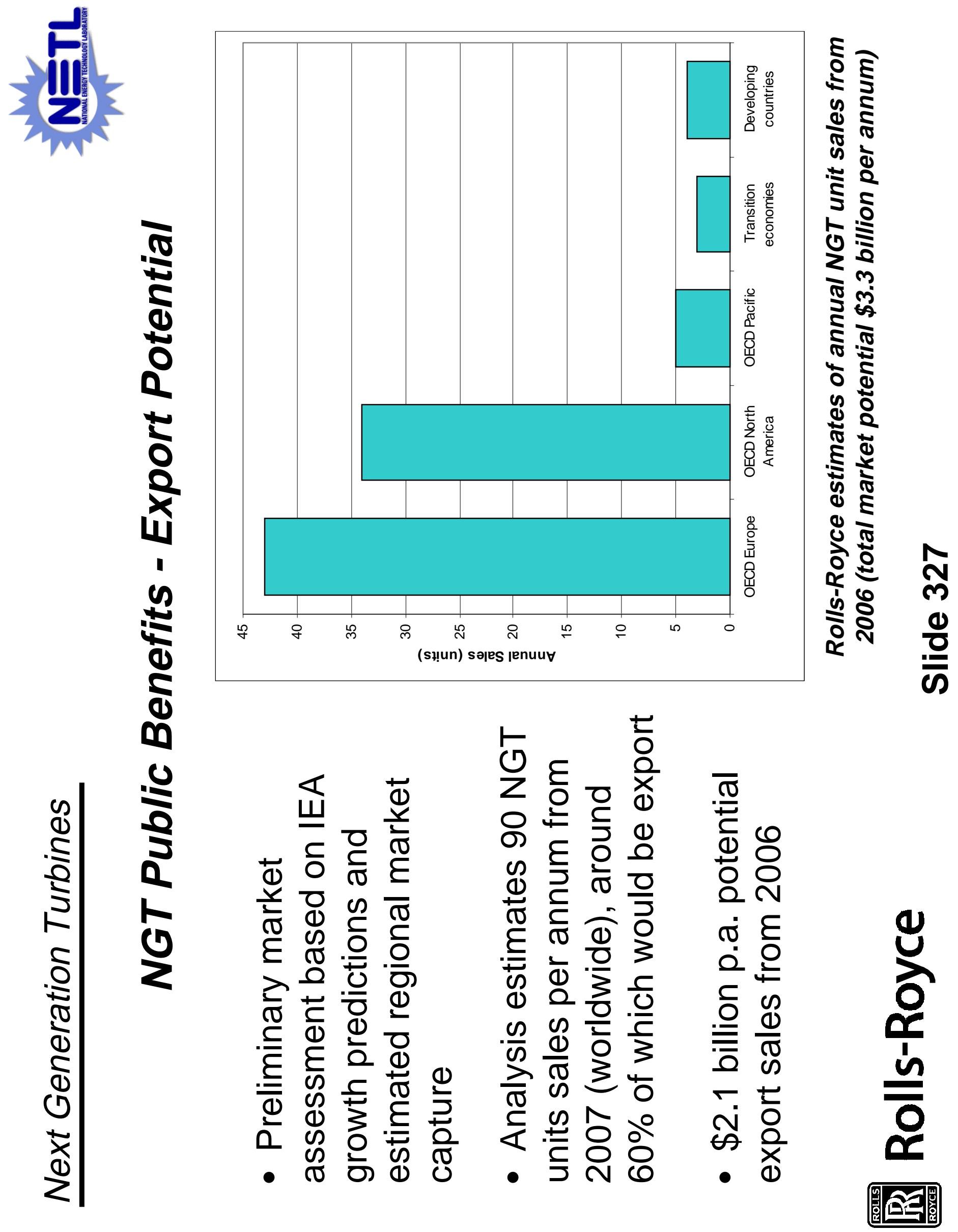


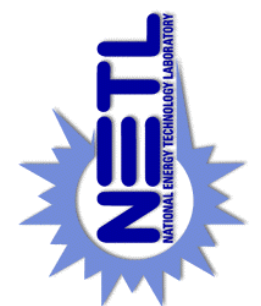

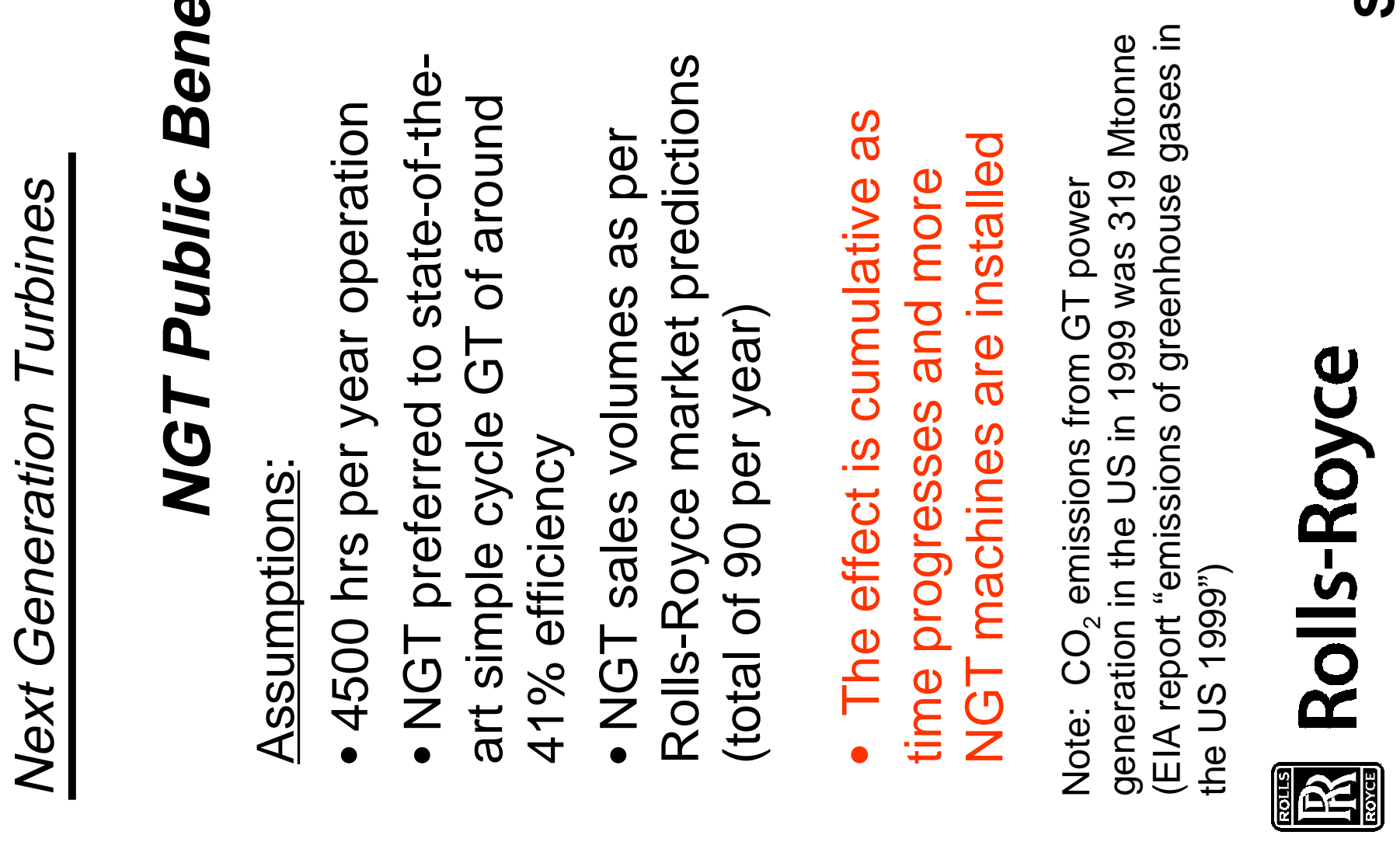

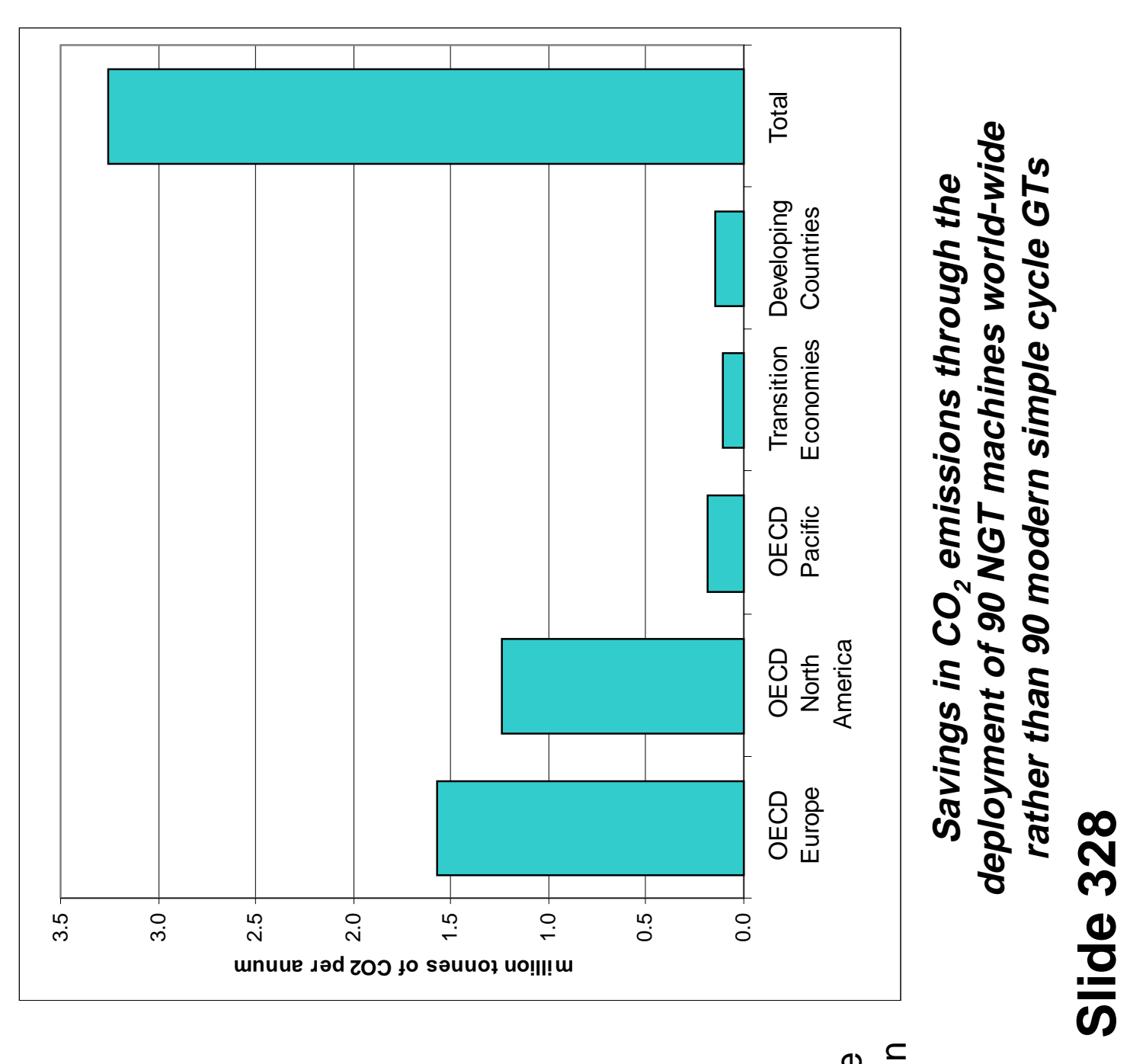




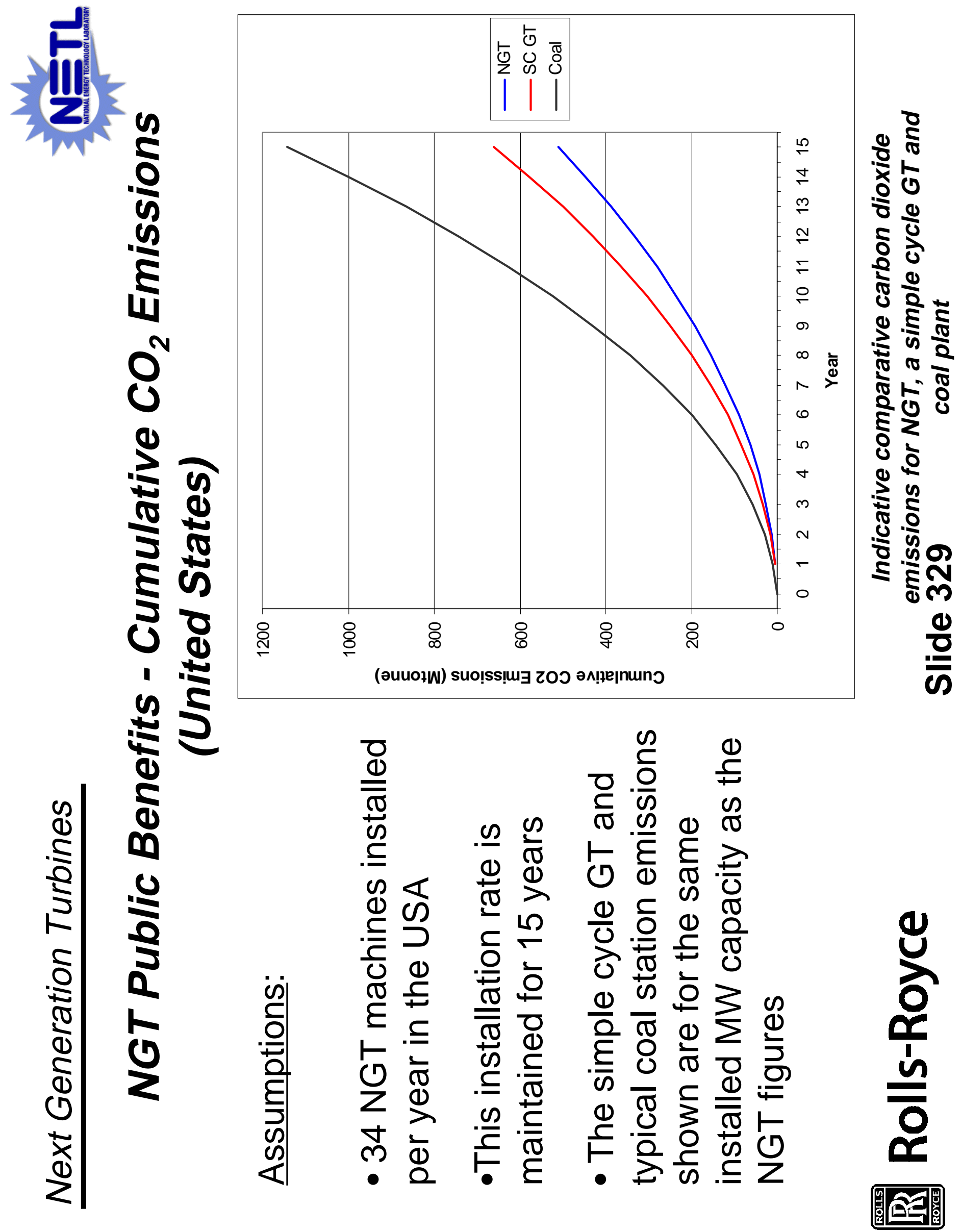



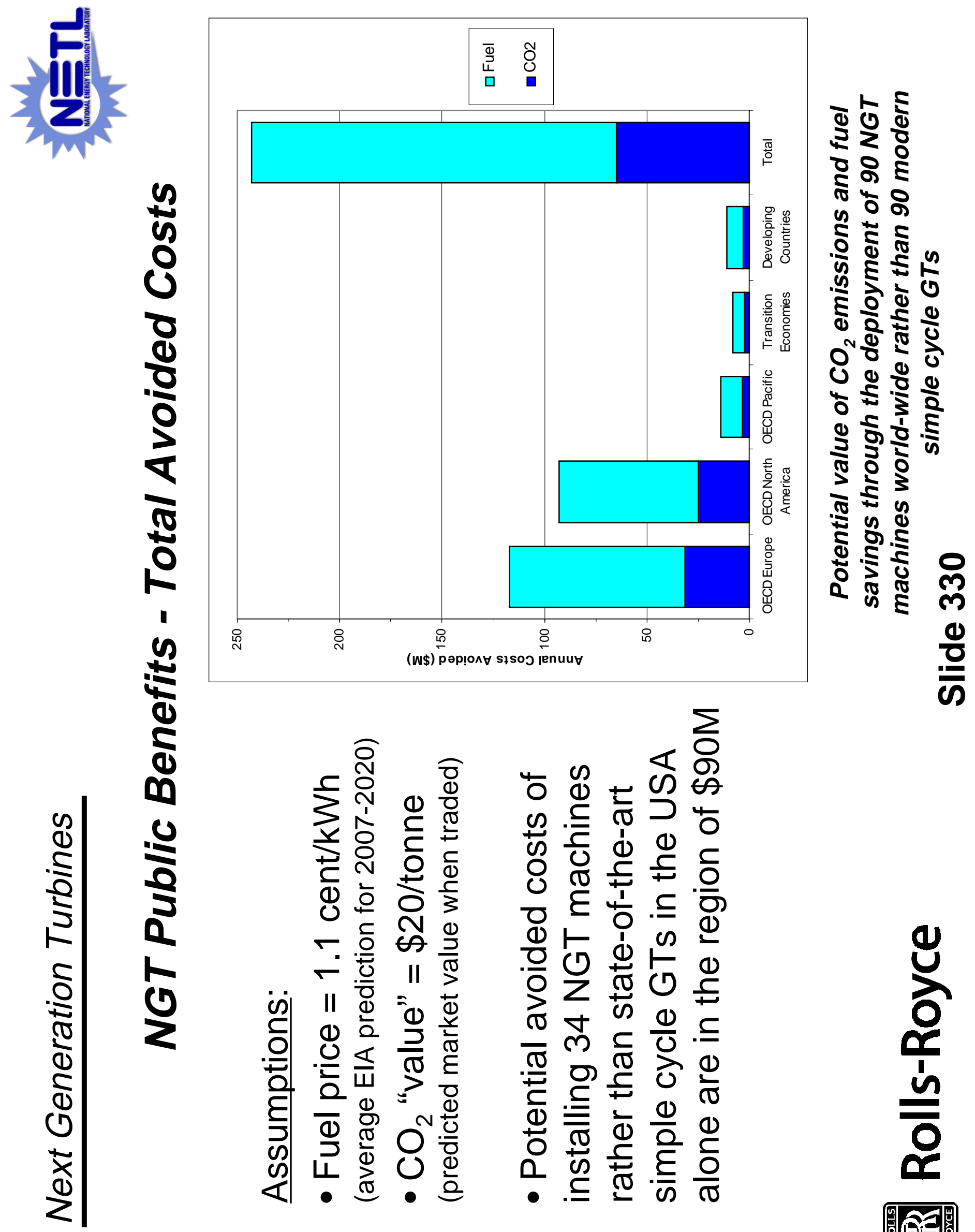


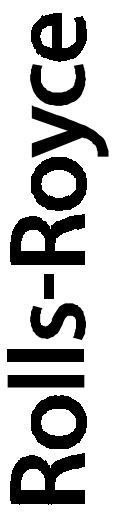




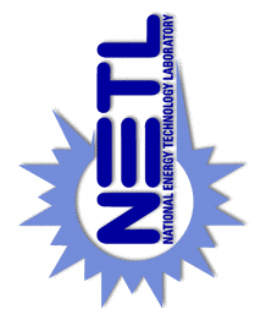

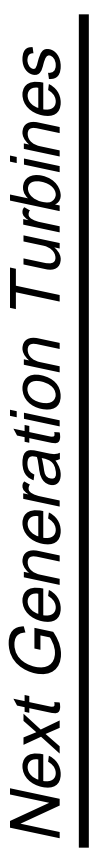
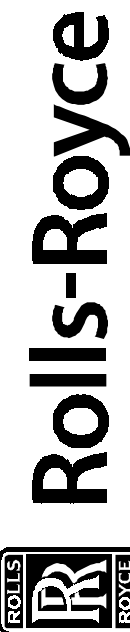

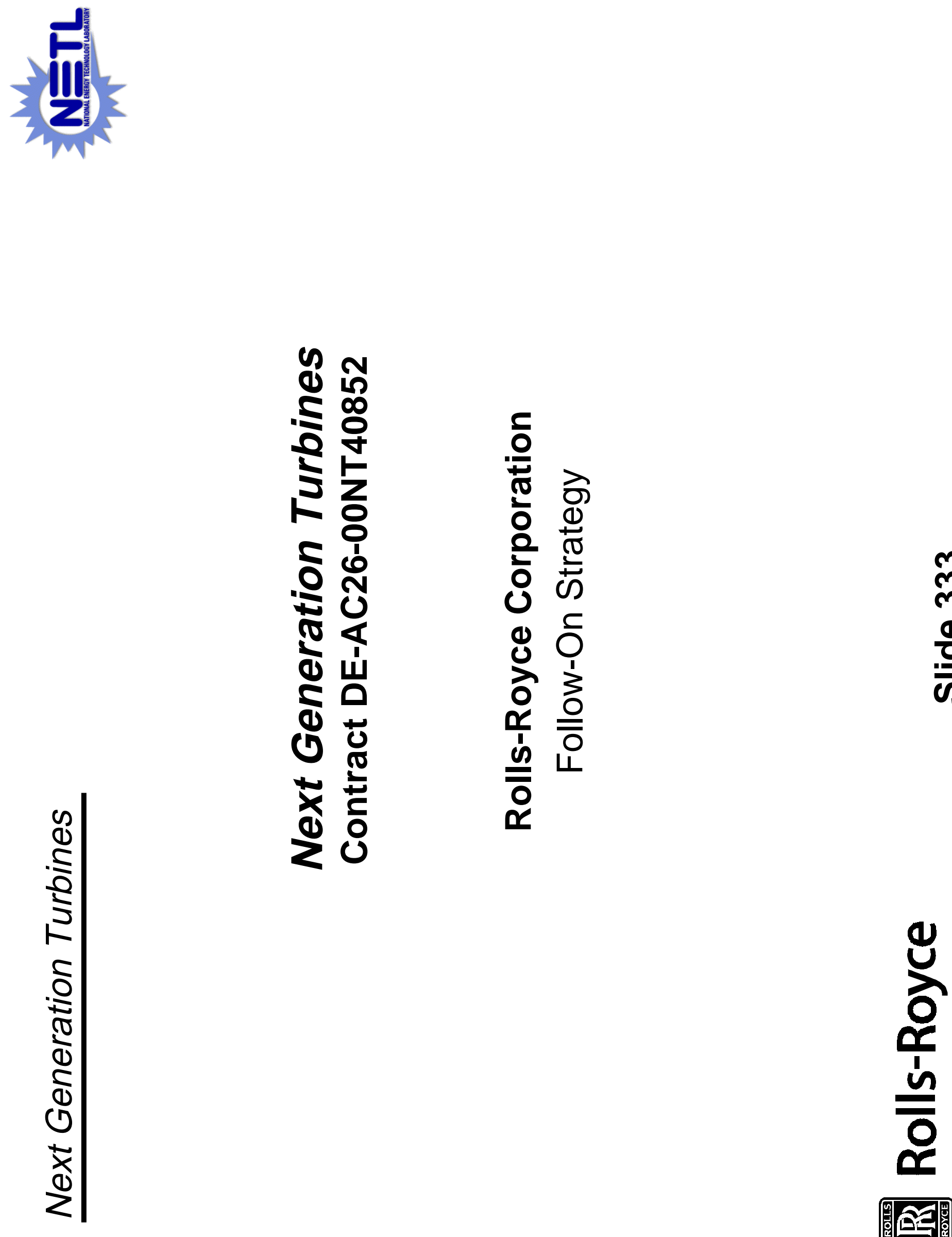


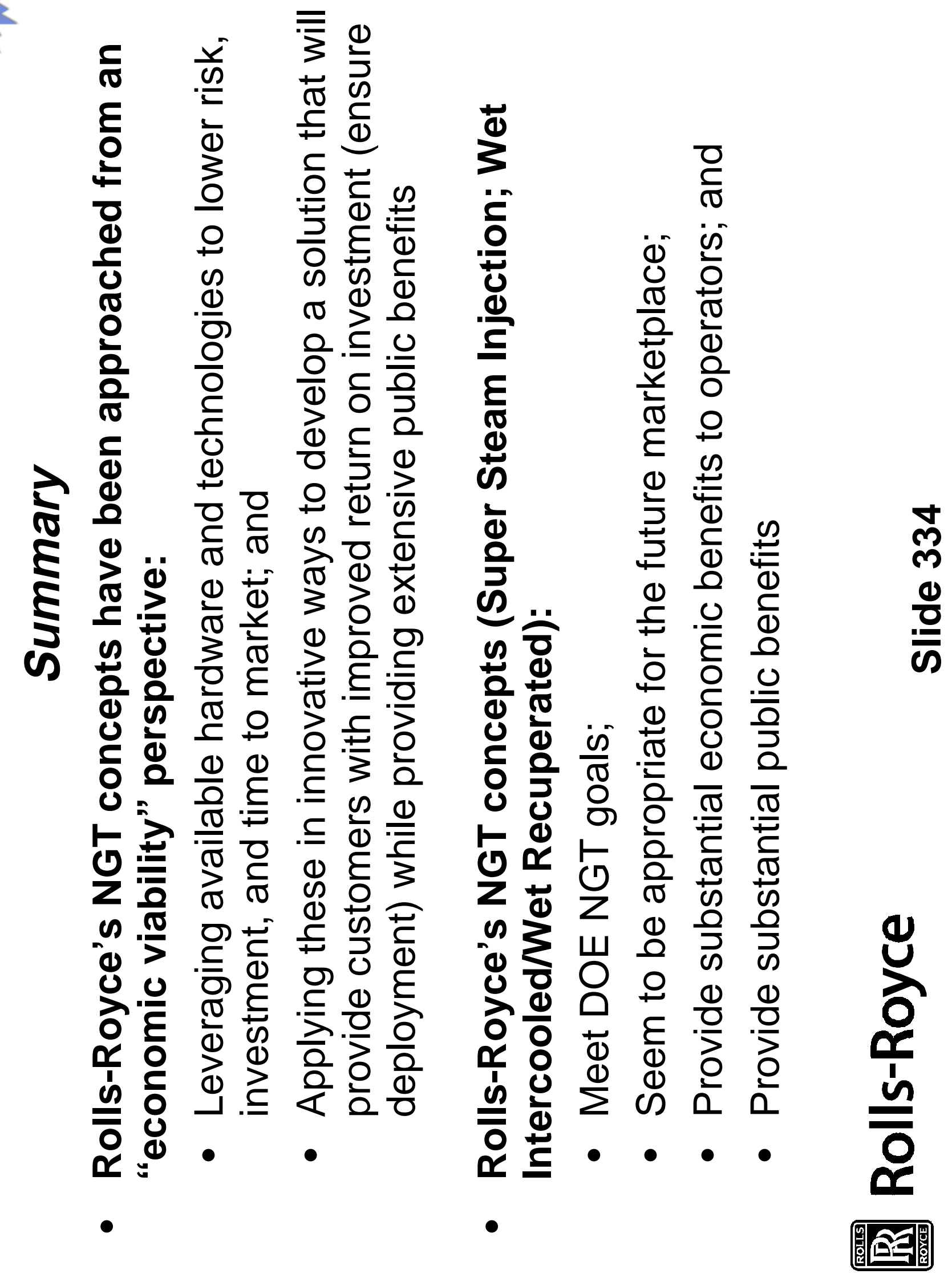




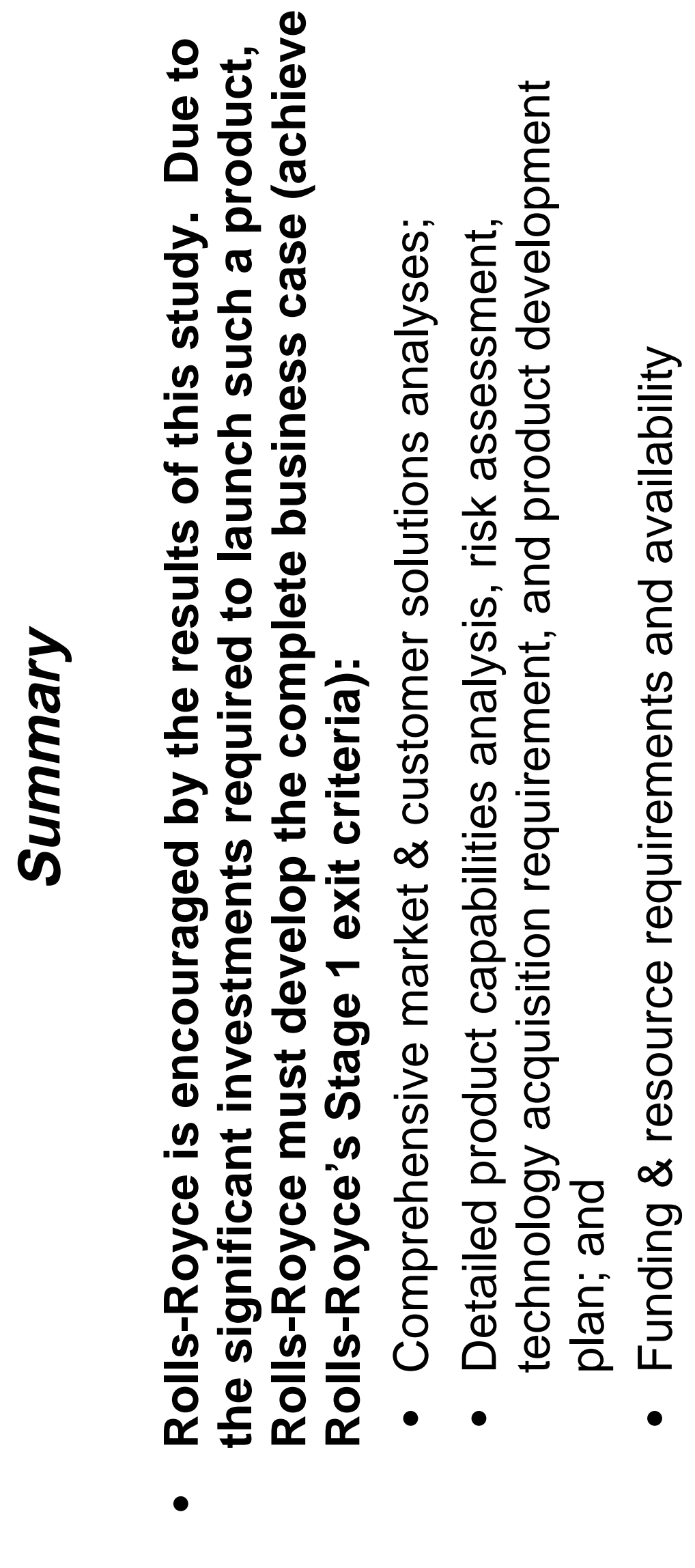

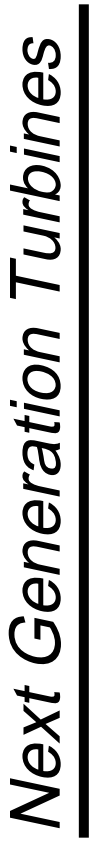

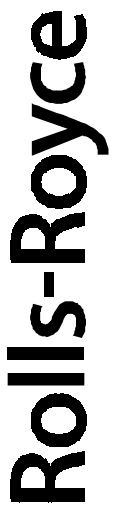



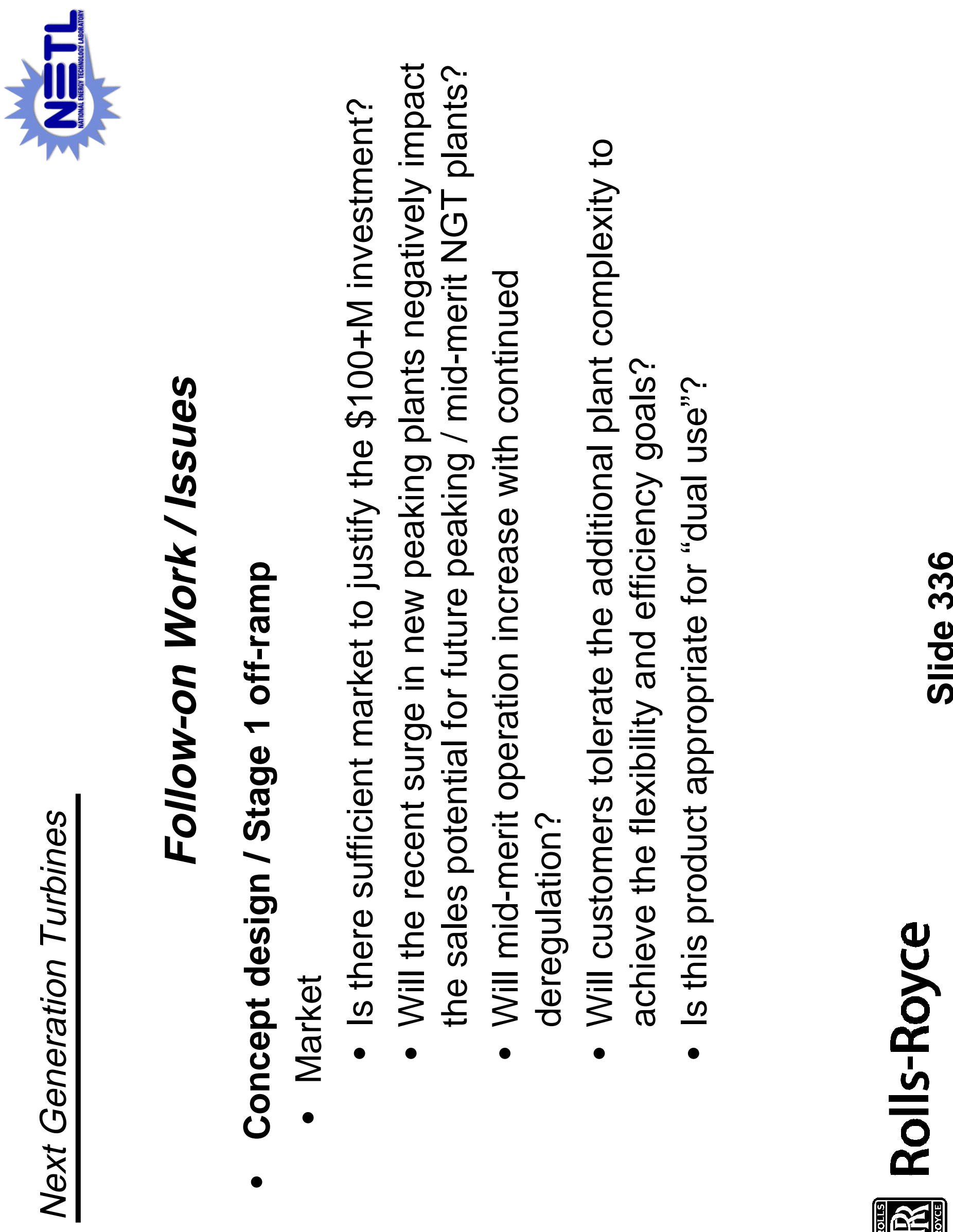


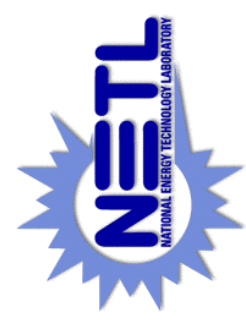

$y$
$\frac{y}{5}$
$y$
$y$
$\frac{1}{5}$
$\frac{1}{3}$
$\frac{5}{0}$
$\frac{1}{0}$
$\frac{0}{0}$
4
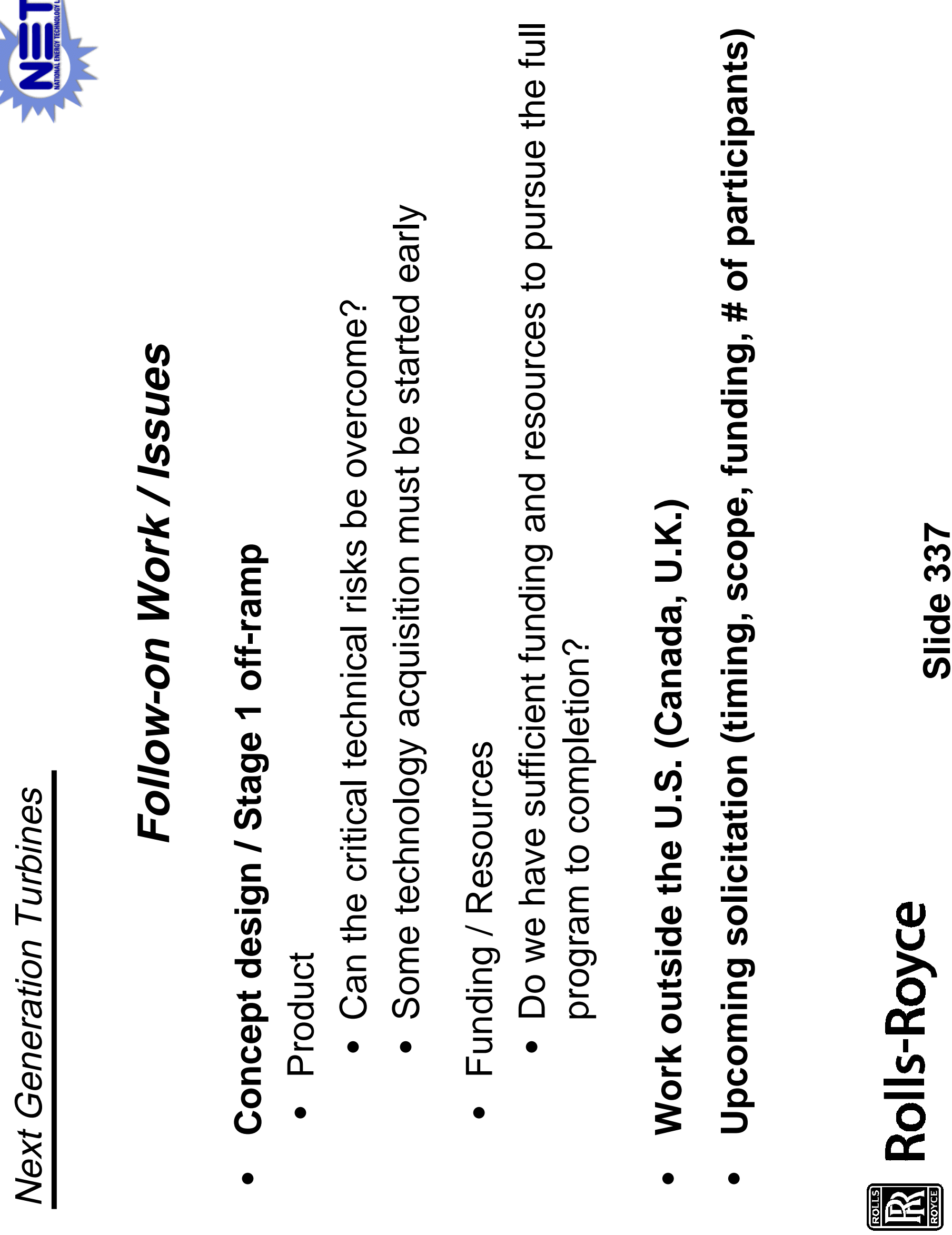

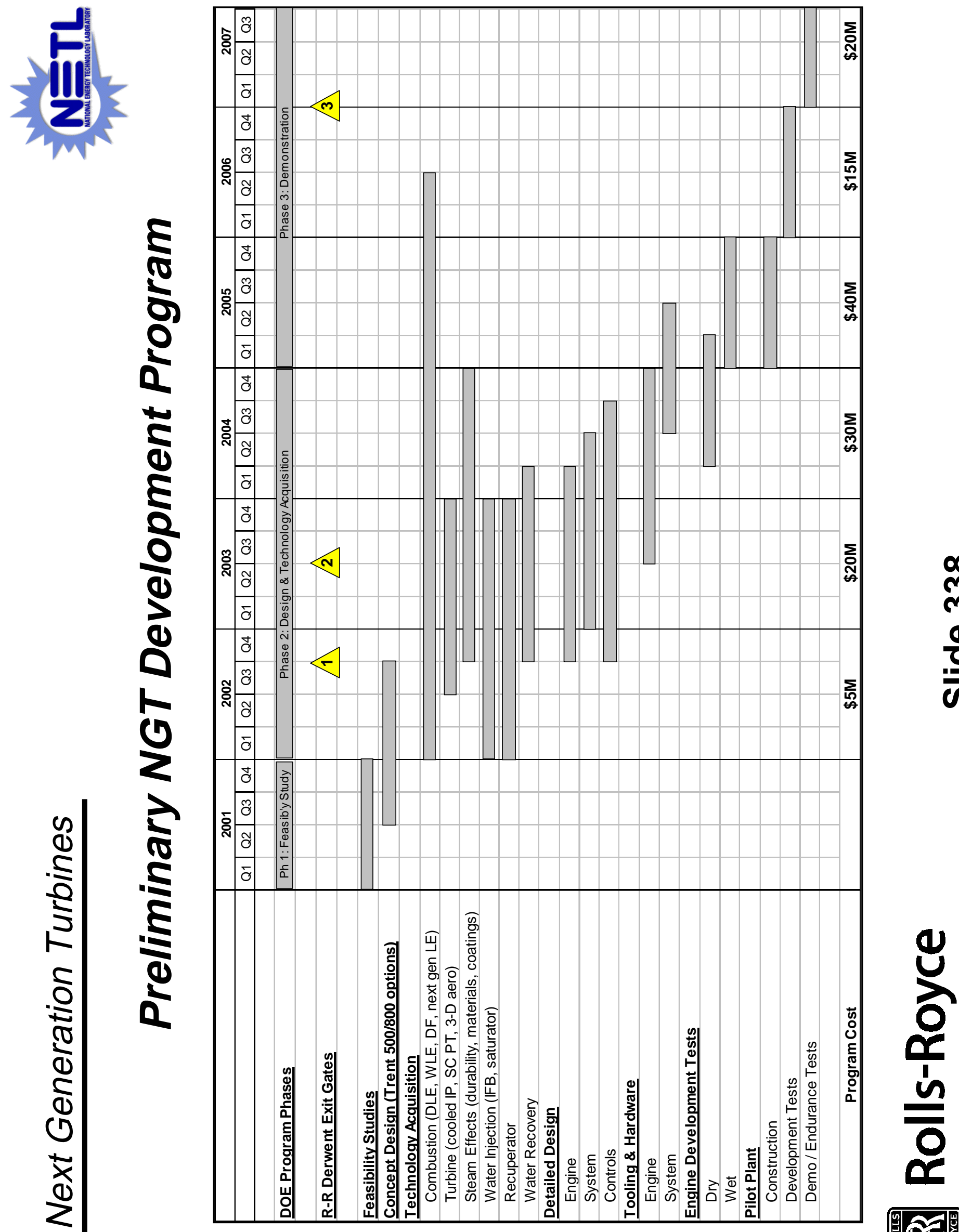

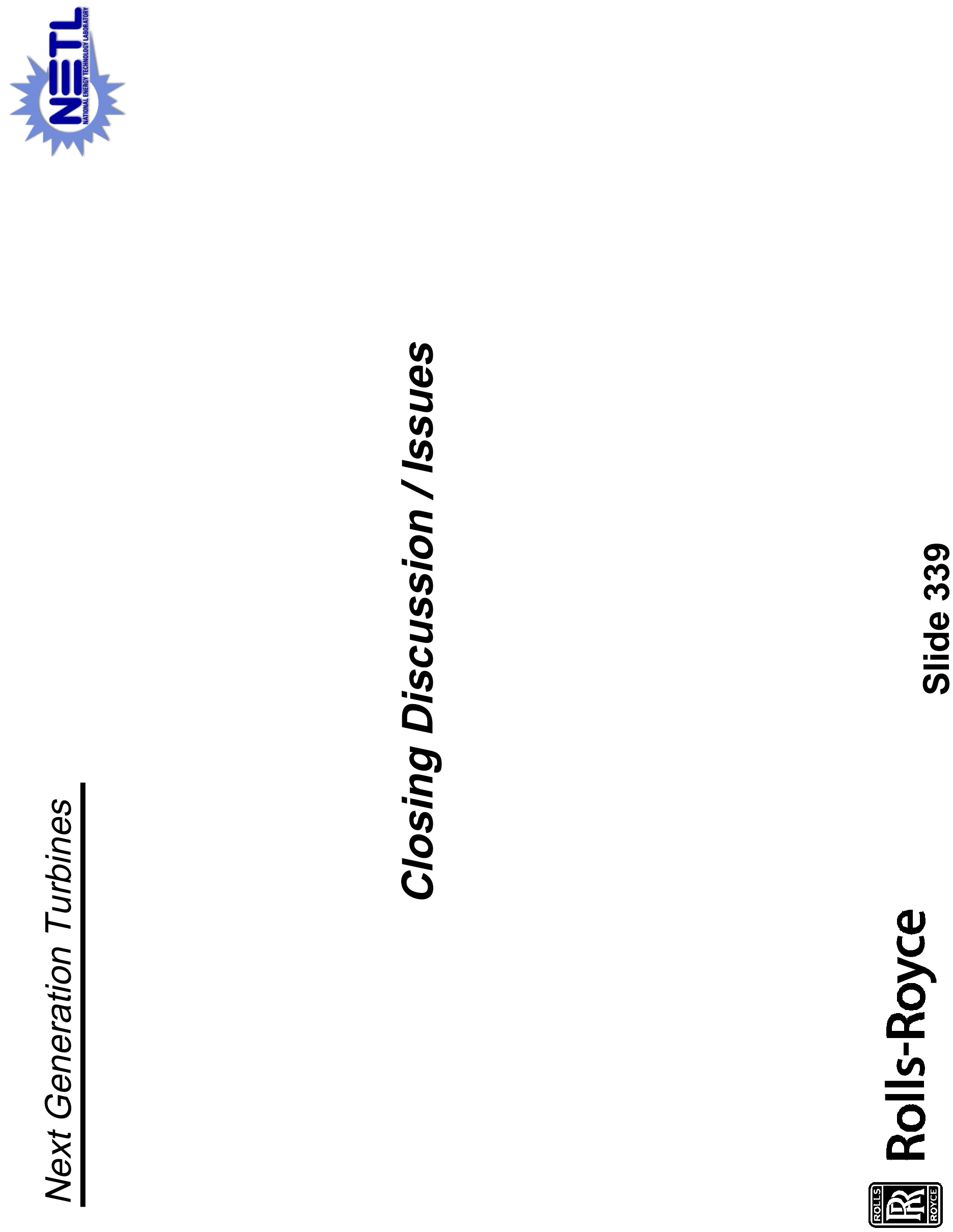